DOE/RL-93-56-3

UC-702

\title{
Quarterly Report of RCRA Groundwater Monitoring Data for Period July 1, 1993 through September 30, 1993
}

Geosciences Function

Westinghouse Hanford Company

Environmental Division

Date Published

January 1994

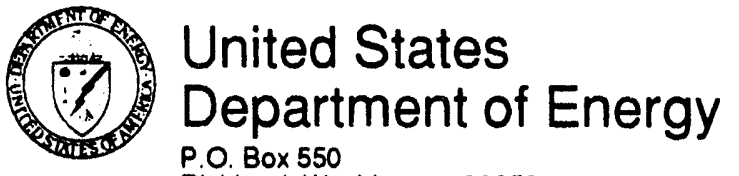

Richland, Washington 99352 


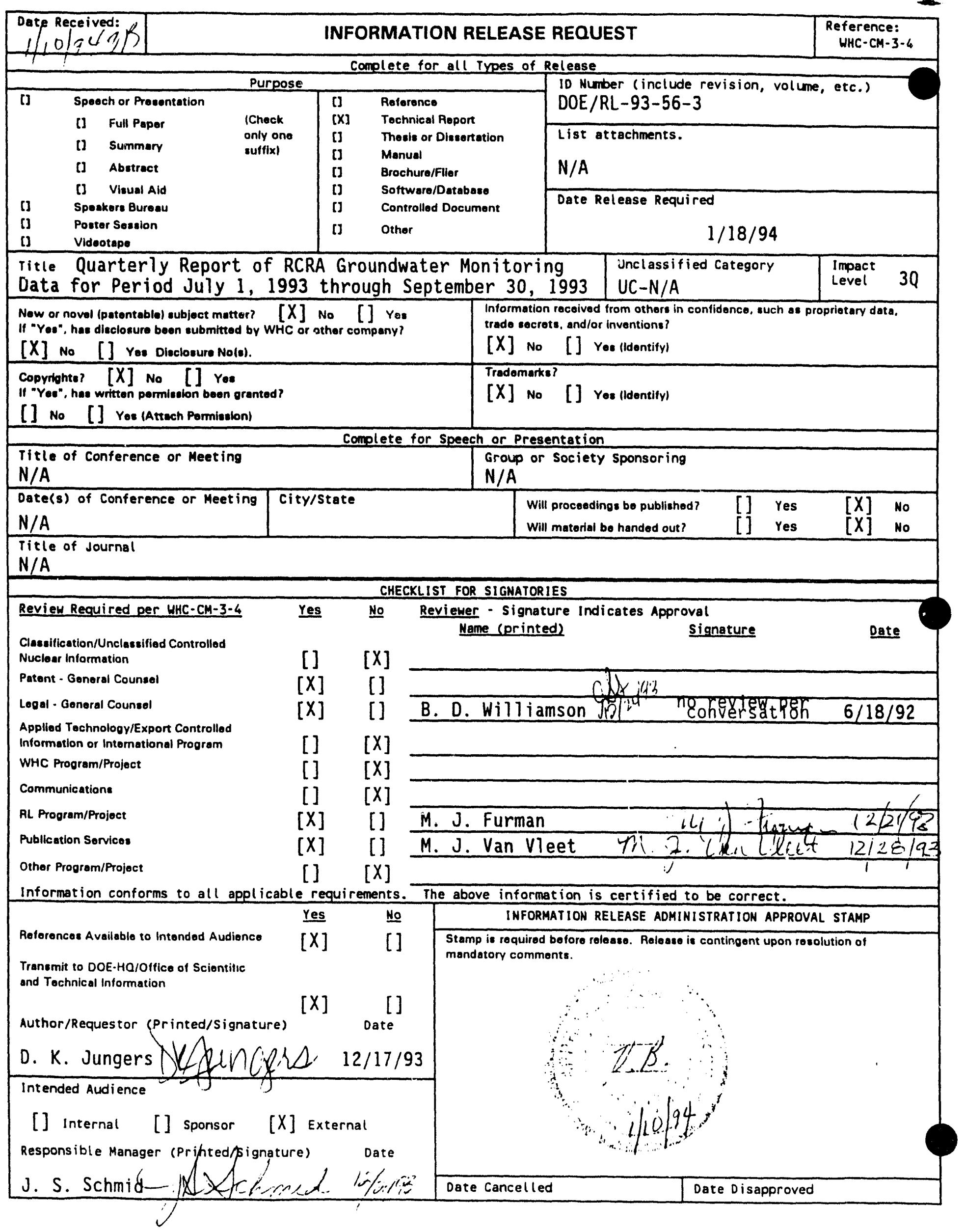


DOE/RL-93-56-3

\section{CONTENTS}

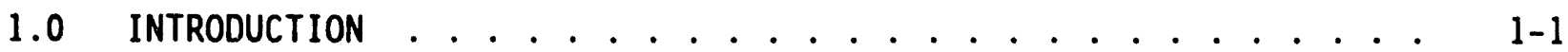

1.1 LABORATORY AND SAMPLING STATUS . . . . . . . . . . . . . . $1-1$

1.2 QUALITY CONTROL PROGRAM . . . . . . . . . . . . . . . 1-1

1.2.1 Introduction . . . . . . . . . . . . . . . . . . 1-1

1.2.2 External Quality Control Program .......... 1-5

1.2.3 Internal Qual ity Control Program . . . . . . . . . . 1-9

1.2.4 Nonconformance/Incident Reports . . . . . . . . . . 1-11

1.2.5 Quality Assurance Occurrences . . . . . . . . . . 1-12

1.3 QUALITY CONTROL DEFINITIONS ...................

1.4 DATA TABLES . . . . . . . . . . . . . . . . . . . . . . . . 1-16

1.4.1 Water Level Measurement Report. . . . . . . . . . 1-16

1.4.2 Constituent List and Summary of Results . . . . . . 1-17

1.4.3 Constituents with at Least One Value Above
the CRQL......... . . . . . . . . . . . . . .

1.4.4 Contamination Indicator Parameters . . . . . . . . 1-18

1.4.5 Codes and Abbreviations ............. 1-20

1.5 DATA EVALUATION ..................... . . . 1-21

2.0 100-D PONDS ......................... . . . . . . . . . .

2.1 INTRODUCTION . . . . . . . . . . . . . . . . 2-1

2.2 WATER LEVEL MEASUREMENTS .................. . . . $2-1$

2.3 WATER CHEMISTRY DATA .................. 2-1

3.0 183-H SOLAR EVAPORATION BASINS ................ . . . . .

3.1 INTRODUCTION . . . . . . . . . . . . . . . . . . 3-1

3.2 WATER LEVEL MEASUREMENTS .................. . . . . .

3.3 WATER CHEMISTRY DATA .................. 3-2

4.0 100-N RCRA SITES ....................... . . . . . 4-1

4.1 INTRODUCTION . . . . . . . . . . . . . . . . . . . . 4-1

4.2 WATER LEVEL MEASUREMENTS .................... . . . . . .

4.3 WATER CHEMISTRY DATA . . . . . . . . . . . . . . . . . . . 4-1

4.3.1 1301-N Liquid Waste Disposal Facility . . . . . . . 4-2

4.3.2 1324-N Surface Impoundment and

1324-NA Percolation Pond .......... 4-2

4.3.3 1325-N Liquid Waste Disposal Facility . . . . . . . 4-2

4.3.4 100-NR-2 Operable Unit............ 4-3

5.0 GROUT TREATMENT FACILITY . . . . . . . . . . . . . . . 5-1

5.1 INTRODUCTION ....................... 5-1

5.2 WATER LEVEL MEASUREMENTS .................... 5 . . . . . .

5.3 WATER CHEMISTRY DATA ................... . . . . 5 . 1

6.0 216-B-3 POND SYSTEM ..................... . . 6-1

6.1 INTRODUCTION ...................... . . . . . $6-1$

6.2 WATER LEVEL DATA .................... 6-1

6.3 WATER CHEMISTRY DATA ................... . . . . 6 . 
7.0 216-A-29 DITCH ..................... . . . . . . . . .

7.1 INTRODUCTION . . . . . . . . . . . . . . . . . 7-1

7.2 WATER LEVEL MEASUREMENTS .................. . . . . $7-1$

7.3 WATER CHEMISTRY DATA . . . . . . . . . . . . . . 7-2

8.0 216-A-10 CRIB ........................ . . 8-1

8.1 INTRODUCTION ...................... 8-1

8.2 WATER LEVEL MEASUREMENTS . . . . . . . . . . . . . . . . 8-1

8.3 WATER CHEMISTRY DATA . . . . . . . . . . . . . . 8 8-2

$9.0216-A-36 B$ CRIB . . . . . . . . . . . . . . . . . . . . . 9-1

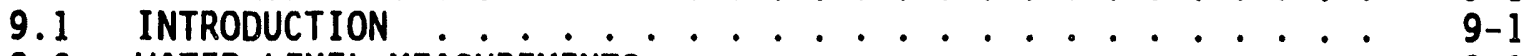

9.2 WATER LEVEL MEASUREMENTS . . . . . . . . . . . . . . . . 9-1

9.3 WATER CHEMISTRY DATA . . . . . . . . . . . . . . . . . . 9-2

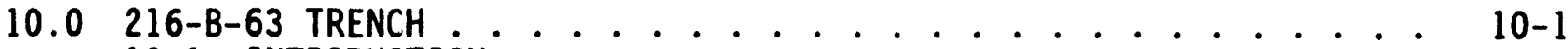

10.1 INTRODUCTION .................... . . 10-1

10.2 WATER LEVEL MEASUREMENTS . . . . . . . . . . . . . . . . . 10-1

10.3 WATER CHEMISTRY DATA . . . . . . . . . . . . . . 10-1

11.0 216-S-10 POND AND DITCH . . . . . . . . . . . . . . . . . . 11-1

11.1 INTRODUCTION ..................... . . . . 11-1

11.2 WATER LEVEL MEASUREMENTS . . . . . . . . . . . . . . . 11-2

11.3 WATER CHEMISTRY DATA . . . . . . . . . . . . . . 11-2

12.0 216-U-12 CRIB . . . . . . . . . . . . . . . . . . . . 12-1

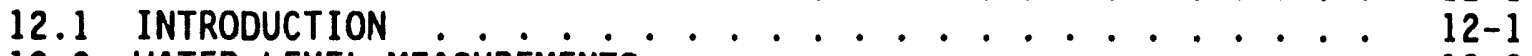

12.2 WATER LEVEL MEASUREMENTS . . . . . . . . . . . . . . 12-2

12.3 WATER CHEMISTRY DATA . . . . . . . . . . . . . . 12-2

13.0 LIQUID EFFLUENT RETENTION FACILITY . . . . . . . . . . . . . 13-1

13.1 INTRODUCTION . . . . . . . . . . . . . . . . . . . 13-1

13.2 WATER LEVEL MEASUREMENTS . . . . . . . . . . . . . . . . . 13-1

13.3 WATER CHEMISTRY DATA . . . . . . . . . . . . . . . 13-1

14.0 2101-M POND . . . . . . . . . . . . . . . . . . . . . 14-1

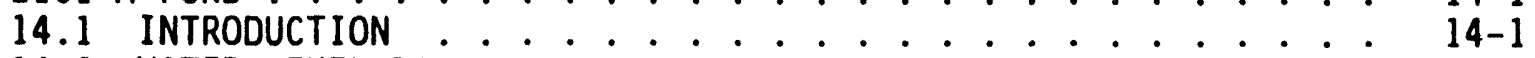

14.2 WATER LEVEL DATA . . . . . . . . . . . . . . . . . 14-1

14.3 WATER CHEMISTRY DATA . . . . . . . . . . . . . . . 14-1

15.0 LOW-LEVEL BURIAL GROUNDS . . . . . . . . . . . . . . . . 15-1

15.1 INTRODUCTION . . . . . . . . . . . . . . . . 15-1

15.2 WATER LEVEL MEASUREMENTS .................. . . 15-1

15.3 WATER CHEMISTRY DATA . . . . . . . . . . . . . 15-1

16.0 SINGLE-SHELL TANKS . . . . . . . . . . . . . . . . 16-1

16.1 INTRODUCTION . . . . . . . . . . . . . . . . . 16-1

16.2 WATER LEVEL MEASUREMENTS . . . . . . . . . . . . . . . 16-2

16.3 WATER CHEMISTRY DATA . . . . . . . . . . . . . . . . . . . 16-3

16.3.1 Waste Management Area A-AX . . . . . . . . . . . 16-4

16.3.2 Waste Management Area B-BX-BY . . . . . . . . . . 16-4 


\section{CONTENTS (cont.)}

16.3.3 Waste Management Area C . . . . . . . . . . 16-4 16.3.4 Waste Management Area S-SX . . . . . . . . . . . . 16-4

16.3.5 Waste Management Area T ... . . . . . . . . . 16-5

16.3.6 Waste Management Area TX-TY . . . . . . . . . . . 16-6

16.3.7 Waste Management Area U. . . . . . . . . . . . . 16-6

17.0300 AREA PROCESS TRENCHES . . . . . . . . . . . . . . . . . 17-1

17.1 INTRODUCTION ..................... . . . 17-1

17.2 WATER LEVEL MEASUREMENTS . . . . . . . . . . . . . . . . 17-1

17.3 WATER CHEMISTRY DATA . . . . . . . . . . . . . . . . . . 17-1

18.0 NONRADIOACTIVE DANGEROUS WASTE LANDFILL . . . . . . . . . . . . 18-1

18.1 INTRODUCTION . . . . . . . . . . . . . . . . . 18-1

18.2 WATER LEVEL DATA .................... . . . 18-1

18.3 WATER CHEMISTRY DATA . . . . . . . . . . . . . . . 18-1

19.0 REFERENCES . . . . . . . . . . . . . . . . . . . 19-1

APPENDIX:

A PREVIOUS DATA FROM 100-NR-2 OPERABLE UNIT . . . . . . . . . . . . A-1 


\section{LIST OF FIGURES}

1-1 Locations of the RCRA Groundwater Monitoring Projects and Landmarks on the Hanford Site .. . . . . . . . . . . . . 1-3

2-1 Monitoring Well Location Map for the 100-D Ponds . . . . . . 2-2

3-1 Monitoring Well Location Map for the 183-H Solar Evaporation Basins Network . . . . . . . . . . . . . . . . 3-3

4-1 Monitoring Well Locations for 1301-N, 1324-N/NA, and 1325-N Facilities ................... . . . 4-4

5-1 Monitoring Well Locations for the Grout Treatment Facility . . . 5-3

6-1 216-B-3 Pond Groundwater Monitoring Network . . . . . . . . . 6-3

7-1 Monitoring Well Locations for the 216-A-29 Ditch . . . . . . . . 7-4

8-1 Monitoring Well Locations for the 216-A-10 Crib . . . . . . 8-3

9-1 Monitoring Well Locations for the 216-A-36B Crib . . . . . . 9-3

10-1 Well Location Map for the 216-B-63 Trench. . . . . . . . . . 10-3

11-1 Monitoring Well Location for the 216-S-10 Facility . . . . . . 11-4

12-1 Well Location Map for 216-U-12 Crib . . . . . . . . . . . 12-3

Monitoring Well Locations for the Liquid Effluent
Retention Facility . . . . . . . . . . . 13-3

14-1 Monitoring Well Locations for the 2101-M Pond . . . . . . . . 14-2

15-1 Monitoring Well Locations for Low-Level Waste Management Area 1............... . . 15-3

Monitoring Well Locations for Low-Level
Waste Management Area 2 . . . . . . . . . . . . . . . . 15-4

15-3 Monitoring Well Locations for Low-Level Waste Management Area 3 . . . . . . . . . . . . . . . 15-5

15-4 Monitoring Well Locations for Low-Level Waste Management Area 4 . . . . . . . . . . . . . . . . 15-6

15-5 Monitoring Well Locations for Low-Leve 1 Waste Management Area 5.................. 15-7 


$$
\vec{\Phi} \vec{i} \quad \vec{T} \quad \vec{i} \quad \vec{i} \quad \vec{i}
$$

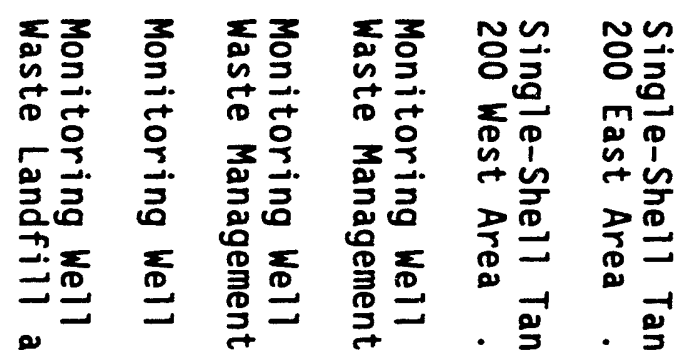

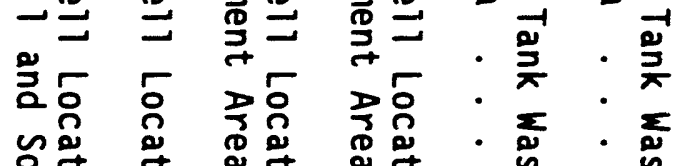

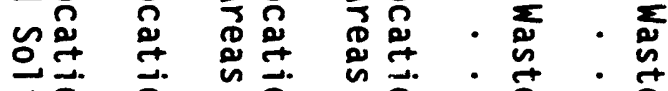

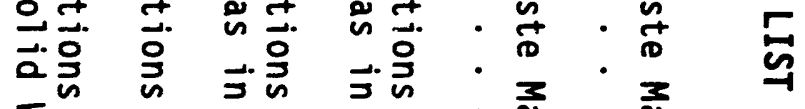

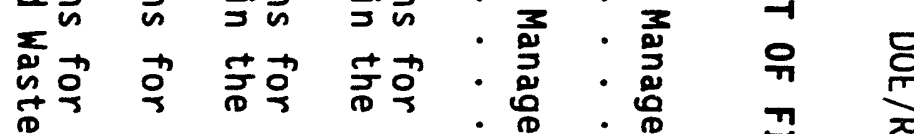

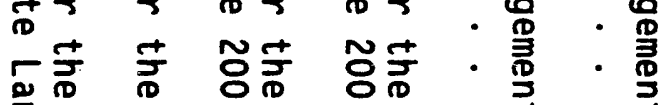$$
\text { 흐를 }
$$

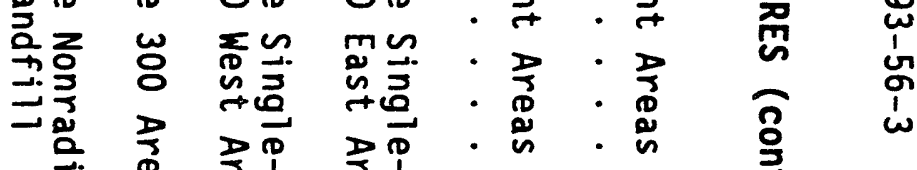

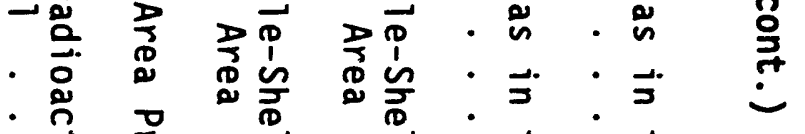

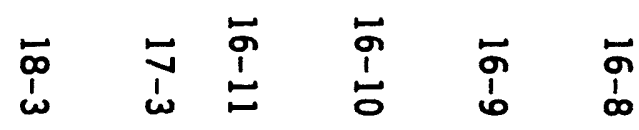


$\mathrm{DOE} / \mathrm{RL}-93-56-3$

\section{LIST OF TABLES}

1-1 Completeness . . . . . . . . . . . . . . . . . . 1-4

1-2 Field Duplicates and Field Blanks Evaluation

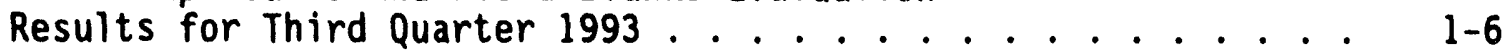

1-3 Breakdown of Field Duplicates Exceeding

Quality Control Limits... . . . . . . . . . . . . . 1-7

1-4 Breakdown of Field Blanks Exceeding Quality Control Limits . . . 1-8

1-5 Frequency of Quality Control Samples for Third Quarter 1993 . . 1-9

1-6 Radiochemistry Quarterly Report Summary . . . . . . . . . . . 1-10

1-7 Summary of EPA Water Pollution, Water Supply, and

Interlaboratory Comparison Study .............. . 1-11

1-8 Analysis Method Code Definitions . . . . . . . . . . . 1-19

1-9 Drinking Water Standards . . . . . . . . . . . . . . 1-22

2-1 Monitoring Well Purpose and Sampling Schedule in the

2-2 RCRA Water Level Measure ient Report 100-D Ponds,

Third Quarter 1993 ................... . 2-4

3-1 Monitoring Well Position and Sampling Schedule for the

183-H Solar Evaporation Basins Network . . . . . . . . . . 3-4

3-2 RCRA Water Level Measurement Report 183-H Solar Evaporation

Basins, Third Quarter 1993 .............. . 3-6

3-3 Constituent List and Summary of Results for 183-H Solar

Evaporation Basins Data for Reporting Period July 1

through September 30, 1993

3-4 Constituents with at Least One Value Above the CRQL for the

183-H Solar Evaporation Basins Data for Reporting Period

July 1 through September 30, 1993

3-5 Contamination Indicator Parameters for the 183-H Solar

Evaporation Basins Data for Reporting Period July 1

through September 30, 1993.

4-1 Monitoring Well Purpose and Sampling Schedule in the 100-N Area . . . . . . . . . . . . . . . . . . . 4-5

4-2 RCRA Water Level Measurement Report 100-N Area, Third Quarter 1993 


\section{LIST OF TABLES (cont.)}

4-3 Constituent List and Summary of Results for 100-N RCRA Sites

Data for Reporting Period July 1 through September 30, 1993

4-4 Constituents with at Least One Value Above the CRQL for the 100-N RCRA Sites Data for Reporting Period July 1 through September 30, 1993 ................... . . 4-18

4-5 Contamination Indicator Parameters for the 100-N RCRA Sites Data for Reporting Period July 1 through September 30, 1993

5-1 Monitoring Well Purpose and Sampling Schedule for the Grout

Treatment Facility Network ................ . 5-4

5-2 RCRA Water Level Measurement Report Grout Treament Facility,

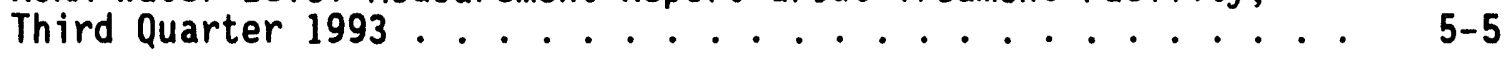

5-3 Constituent List and Summary of Results for the Grout Treatment Facility Data for Reporting Period July 1 through September 30,1993................. 5-6

5-4 Constituents with at Least One Value Above the CRQL for the Grout Treatment Facility Data for Reporting Period July 1 through September 30, 1993 ................

5-5 Contamination Indicator Parameters for the Grout Treatment

Facility Data for Reporting Period July 1 through

September 30, 1993

6-1 Monitoring Well Purpose and Sampling Schedule for the 216-B-3 Pond System Network

6-2 RCRA Water Level Measurement Report 216-B-3 Pond System, Third Quarter 1993

6-3 Constituent List and Summary of Results for the 216-B-3 Pond Data for Reporting Period July 1 through September 30, 1993

6-4 Constituents with at Least One Value Above the CRQL for the 216-B-3 Pond Data for Reporting Period July 1 through September 30, 1993 ...................

6-5 Contamination Indicator Parameters for the 216-B-3 Pond Data for Reporting Period July 1 through September 30, 1993 . . . . 6-28

7-1 Monitoring Well Purpose and Sampling Schedule for the 216-A-29 Ditch..................... . . 7-5

7-2 RCRA Water Level Measurement Report 216-A-29 Ditch, Third Quarter 1993 


\section{LIST OF TABLES (cont.)}

7-3 Constituent List and Summary of Results for the 216-A-29 Ditch Data for Reporting Period July 1 through September 30, 1993 . . 7-9

7-4 Constituents with at Least One Value Above the CRQL for the 216-A-29 Ditch Data for Reporting Period July 1 through

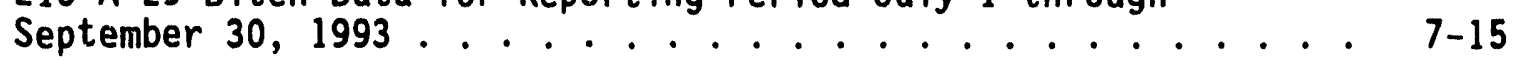

7-5 Contamination Indicator Parameters for the 216-A-29 Ditch Data for Reporting Period July 1 through September 30, 1993 . . . . 7-23

8-1 Well Identification and Sampling Schedule for the 216-A-10 Crib Groundwater Monitoring Network . . . . . . . . . 8-4

8-2 RCRA Water Level Measurement Report 216-A-10 Crib,

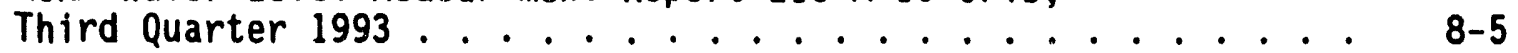

9-1 Well Identification and Sampling Schedule for the 216-A-36B Crib Groundwater Monitoring Network . . . . . . . . 9-4

9-2 RCRA Water Level Measurement Report 216-A-36B Crib,

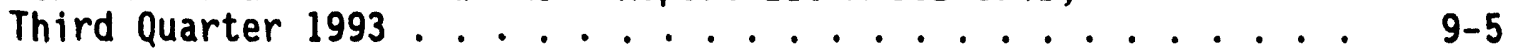

10-1 Monitoring We11 Purpose and Sampling Schedule for the 216-B-63 Trench Network ................. . 10-4

10-2 RCRA Water Level Measurement Report 216-B-63 Trench, Third Quarter $1993 \ldots$

10-3 Constituent List and Summary of Results for the 216-B-63 Trench Data for Reporting Period July 1 through September 30, 1993 . . 10-6

10-4 Constituents with at Least One Value Above the CRQL for the 216-B-63 Trench Data for Reporting Period July 1 through September 30,1993................... 10-12

10-5 Contamination Indicator Parameters for the 216-B-63 Trench Data for Reporting Period July 1 through September 30, 1993 . . . . 10-15

11-1 Monitoring We11 Purpose and Sampling Schedule for the 216-S-10 Facility ................... 11-5

11-2 RCRA Water Level Measurement Report 216-S-10 Facility,

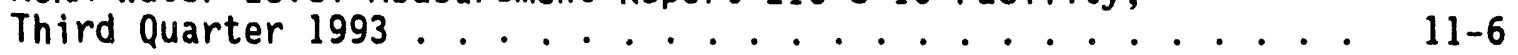

11-3 Constituent List and Summary of Results for the 216-S-10 Facility Data for Reporting Period July 1 through September 30, 1993 


\section{LIST OF TABLES (cont.)}

11-4 Constituents with at Least One Value Above the CRQL for the 216-S-10 Facility Data for Reporting Period July 1 through September 30,1993 ................. 11-13

11-5 Contamination Indicator Parameters for the 216-S-10 Facility Data for Reporting Period July 1 through September 30, 1993 . . 11-16

12-1 Monitoring We11 Purpose and Sampling Schedule for the 216-U-12 Crib . . . . . . . . . . . . . . . . 12-4

12-2 RCRA Water Level Measurement Report 216-U-12 Crib, Third Quarter 1993................ . . 12-5

12-3 Constituent List and Summary of Results for the 216-U-12 Crib Data for Reporting Period July 1 through Sepiember 30, 1993 . . 12-6

12-4 Constituents with at Least One Value Above the CRQL for the 216-U-12 Crib Data for Reporting Period July 1 through

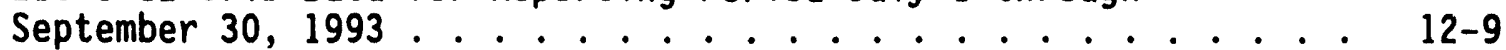

12-5 Contamination Indicator Parameters for the 216-U-12 Crib Data for Reporting Period July 1 through September 30, 1993 . . . . . 12-14

13-1 Monitoring We11 Purpose and Sampling Schedule for the Liquid Effluent Retention Facility Network . . . . . . . . . 13-4

13-2 RCRA Water Leyel Measurement Report LERF, Third Quarter 1993 . . 13-5

13-3 Constituent List and Summary of Results for the Liquid Effluent Retention Facility Data for Reporting Period July 1 through September 30, 1993 . . . . . . . . . . 13-6

13-4 Constituents with at Least One Value Above the CRQL for the Liquid Effluent Retention Facility Data for Reporting Period July 1 through September 30, 1993 . . . . . . . . . 13-8

13-5 Contamination Indicator Parameters for the Liquid Effluent Retention Facility Data for Reporting Period July 1 through September 30,1993 ............. . 13-10

13-6 Critical Means Table for the LERF . . . . . . . . . . . . 13-11

14-1 Monitoring Well Purpose and Sampling Schedule for the 2101-M Pond ...................... 14-3

14-2 RCRA Water Level Measurement Report 2101-M Pond, Third Quarter 1993 ................. 14-4

14-3 Constituent List and Summary of Results for the 2101-M Pond Data for Reporting Period July 1 through September 30, 1993 


\section{LIST OF TABLES (cont.)}

15-1 Low-Level Burial Grounds . . . . . . . . . . . . . . . . . 15-8

15-2 Monitoring Well Purpose and Sampling Schedule for Low-Level Waste Management Area 1.... . . . . . . . . 15-9

15-3 Monitoring We11 Purpose and Sampling Schedule for Low-Level Waste Management Area 2 ............ 15-10

15-4 Monitoring Well Purpose and Sampling Schedule for Low-Level Waste Management Area 3 ............ . 15-11

15-5 Monitoring We11 Purpose and Sampling Schedule for Low-Level Waste Management Area 4 . . . . . . . . . . . . . 15-12

15-6 Monitoring Well Purpose and Sampling Schedule for Low-Leve1 Waste Management Area 5 ............... 15-13

15-7 RCRA Water Level Measurement Report LLWMA 1,

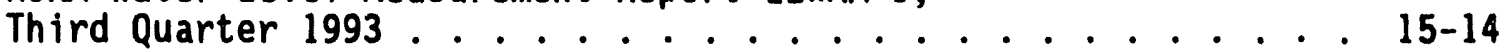

15-8 RCRA Water Level Measurement Report LLWMA 2, Third Quarter 1993 ........... . . . . . . 15-17

15-9 RCRA Water Level Measurement Report LLWMA 3,

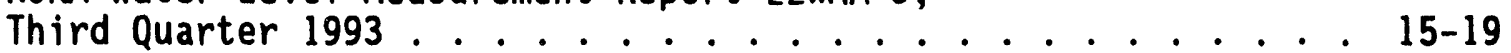

15-10 RCRA Water Level Measurement Report LLWMA 4,

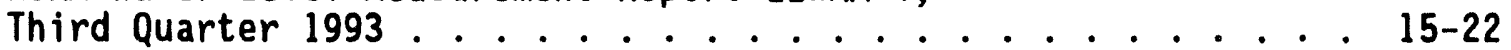

15-11 RCRA Water Level Measurement Report LLWMA 5, Third Quarter 1993 . . . . . . . . . . . . . . . . .

15-12 Constituent List and Summary of Results for the Low-Leve1 Waste Burial Grounds WMA 1 Data for Reporting Period

15-13 Constituents with at Least One Value Above the CRQL for the Low-Level Waste Burial Grounds WMA 1 Data for Reporting Period July 1 through September 30, 1993 . . . . . . . . 15-29

15-14 Contamination Indicator Parameters for the Low-Level Waste Burial Grounds WMA 1 Data for Reporting Period July 1 through September 30, 1993

15-15 Constituent List and Summary of Results for the Low-Level Waste Burial Grounds WMA 2 Data for Reporting Period July 1 through September 30, 1993 
DOE /RL-93-56-3

\section{LIST OF TABLES (cont.)}

15-16 Constituents with at Least One Value Above the CRQL for the Low-Level Waste Burial Grounds WMA 2 Data for Reporting

Period July 1 through September 30, 1993 . . . . . . . . . 15-41

15-17 Contamination Indicator Parameters for the Low-Level Waste Burial Grounds WMA 2 Data for Reporting Period July 1 through September 30,1993............. . . 15-44

15-18 Constituent List and Summary of Results for the Low-Level

Waste Burial Grounds WMA 3 Data for Reporting Period July 1 through September 30, 1993 . . . . . . . . . . 15-45

15-19 Constituents with at Least One Value Above the CRQL for the Low-Level Waste Burial Grounds WMA 3 Data for Reporting Period July 1 through September 30, 1993.......... 15-51

15-20 Contamination Indicator Parameters for the Low-Level Waste Burial Grounds WMA 3 Data for Reporting Period July 1 through September 30,1993............ . . 15-62

15-21 Constituent List and Summary of Results for the Low-Level Waste Burial Grounds WMP 4 Data for Reporting Period July 1 through September 30 , 1993

15-22 Constituents with at Least One Value Above the CRQL for the Low-Level Waste Burial Grounds WMA 4 Data for Reporting Period July 1 through September 30, 1993 . . . . . . . . 15-68

15-23 Contamination Indicator Parameters for the Low-Level Waste Burial Grounds WMA \& Data for Reporting Period July 1

15-24 Constituent List and Summary of Results for the Low-Leve1 Waste Burial Grounds WMA 5 Data for Reporting Period July 1 through September 30, 1993

15-25 Constituents with at Least One Value Above the CRQL for the Low-Level Waste Burial Grounds WMA 5 Data for Reporting Period July 1 through September 30,1993 ...........

15-26 Contamination Indicator Parameters for the Low-Level Waste Burial Grounds WMA 5 Data for Reporting Period July 1 through September 30, 1993 ................

15-27 Water Quality Standards Exceeded at Low-Level Waste Management Area 1. . . . . . . . . . . . . . . 15-90

15-28 Water Quality Standards Exceeded at Low-Level

$$
\text { Waste Management Area } 2 .
$$




\section{LIST OF TABLES (cont.)}

15-29 Water Quality Standards Exceeded at Low-Level

Waste Management Area 3. . . . . . . . . . . . . . . 15-91

15-30 Water Quality Standards Exceeded at Low-Level

Waste Management Area 4. . . . . . . . . . . . . . . 15-92

15-31 Water Quality Standards Exceeded at Low-Level

Waste Management Area 5. . . . . . . . . . . . . . . 15-93

16-1 Groundwater Monitoring Wells for the Single-Shell Tanks . . . 16-12

16-2 RCRA Water Level Measurement Report Single-Shell Tanks, Third Quarter 1993 ...... . . . . . . . . . . . 16-14

16-3 Constituent List and Summary of Results for the Single-Shell

Tank Waste Management Area A-AX Data for Reporting Period July 1 through September 30,1993 . . . . . . . . . . . 16-22

16-4 Constituents with at Least One Value Above the CRQL for the Single-Shell Tank Waste Management Area A-AX Data for Reporting Period July 1 through September 30, 1993 . . . . . . . 16-28

16-5 Contamination Indicator Parameters for the Single-Shel1 Tank Waste Management Area A-AX Data for Reporting Period July 1 through September 30, 1993

16-6 Constituent List and Summary of Results for the Single-Shell Tank Waste Management Area B-BY-BX Data for Reporting Period July 1 through September 30, 1993 . . . . . . . . . . 16-31

16-7 Constituents with at Least One Value Above the CRQL for the Single-Shell rank Waste Management Area B-BY-BX Data for Reporting Period July 1 through September 30, 1993 . . . . . . 16-34

16-8 Contamination Indicator Parameters for the Single-Shell Tank Waste Management Area B-BY-BX Data for Reporting Period July 1 through September 30, 1993 ........... . 16-36

16-9 Constituent List and Summary of Results for the Single-Shell Tank Waste Management Area C Data for Reporting Period July 1 through September 30, 1993 ............. 16-37

16-10 Constituents with at Least One Value Above the CRQL for the Single-Shell Tank Waste Management Area C Data for Reporting Period July 1 through September 30, 1993 . . . . . . . 16-40

16-11 Contamination Indicator Parameters for the Single-She11 Tank Waste Management Area C Data for Reporting Period July 1 through September 30, 1993. 


\section{LIST OF TABLES (cont.)}

16-12 Constituent List and Summary of Results for the Single-Shell Tank Waste Management Area S-SX Data for Reporting Period July 1 through September 30,1993 . . . . . . . . . . . . . 16-42

16-13 Constituents with at Least One Value Above the CRQL for the Single-Shell Tank Waste Management Area S-SX Data for Reporting Period July 1 through September 30, 1993 . . . . . . . 16-48

16-14 Contamination Indicator Parameters for the Single-Shell Tank Waste Management Area S-SX Data for Reporting Period July 1 through September 30,1993 .............. 16-51

16-15 Constituent List and Summary of Results for the Single-Shell Tank Waste Management Area T Data for Reporting Period July 1 through September 30,1993 ... . . . . . . . . 16-52

16-16 Constituents with at Least One Value Above the CRQL for the Single-Shell Tank Waste Management Area T Data for Reporting Period July? through September 30, 1993 . . . . . . 16-55

16-17 Contamination Indicator Parameters for the Singie-She11 Tank Waste Management Area T Data for Reporting Period July 1 through September 30,1993................

16-18 Constituent List and Summary of Results for the Single-Shell Tank Waste Management Area TX-TY Data for Reporting Period July 1 through September 30, 1993 ............ 16-58

16-19 Constituents with at Least One Value Above the CRQL for the Single-Shel1 Tank Waste Management Area TX-TY Data for Reporting Period July 1 through September 30, 1993 . . . . . . 16-61

16-20 Contamination Indicator Parameters for the Single-Shell Tank Waste Management Area TX-TY Data for Reporting Period July 1 through September 30,1993 . . . . . . . . . . 16-64

16-21 Constituent List and Summary of Results for the Single-Shell Tank Waste Management Area U Data for Reporting Period July 1 through September 30, 1993 ............. 16-65

16-22 Constituents with at Least One Value Above the CRQL for the Single-Shell Tank Waste Management Area U Data for Reporting Pcriod July 1 through September 30, 1993 . . . . . . . 16-68

16-23 Contamination Indicator Parameters for the Single-Shell Tank Waste Management Area U Data for Reporting Period July 1 through September 30,1993................ 16-70

17-1 Monitoring Well Purpose and Sampling Schedule for the 300 Area Process Trenches Network 


\section{LIST OF TABLES (cont.)}

17-2 RCRA Water Level Measurement Report 300 Area Process Trenches, Third Quarter 1993................... 17-5

17-3 Constituent List and Summary of Results for the 300 Area

Process Trenches Data for Reporting Period July 1

through September 30,1993 ................ 17-10

17-4 Constituents with at Least One Value Above the CRQL for the

300 Area Process Trenches Data for Reporting Period

July 1 through September 30,1993 ............. 17-13

17-5 Contamination Indicator Parameters for the 300 Area Process

Trenches Data for Reporting Period July 1 through

September 30, 1993 . . . . . . . . . . . . . . . 17-18

18-1 Monitoring Well Purpose and Sampling Schedule for the

Nonradioactive Dangerous Waste Landfill Network . . . . . . . 18-4

18-2 RCRA Water Level Measurement Report NRDWL,

Third Quarter 1993.................. 18-5

18-3 Constituent List and Summary of Results for the NRDW Landfill

Data for Reporting Period July 1 through September 30, 1993 . 18-8

18-4 Constituents with at Least One Value Above the CRQL for the

NRDW Landfill Data for Reporting Period July 1 through

September 30,1993 ............... 18-10

18-5 Contamination Indicator Parameters for the NRDW Landfill Data

for Reporting Period July 1 through September 30, 1993 . . . . 18-13 
DOE/RL-93-56-3

\section{ACRONYMS}

\begin{tabular}{|c|c|}
\hline $\begin{array}{l}\text { A-10 Crib } \\
\text { A-29 Ditch } \\
\text { A-36B Crib } \\
\text { ASD } \\
\text { CFR } \\
\text { CIP } \\
\text { CRQL } \\
\text { CY } \\
\text { DOE } \\
\text { DWS } \\
\text { ECO1 ogy } \\
\text { EPA } \\
\text { FY } \\
\text { GTF } \\
\text { ICP } \\
\text { INC } \\
\text { ITAS } \\
\text { LERF } \\
\text { LLBG } \\
\text { LLWMA } \\
\text { MDC } \\
\text { MDL } \\
\text { NCR } \\
\text { NGVD29 } \\
\text { NRDWL } \\
\text { NTU } \\
\text { PDD } \\
\text { PNL } \\
\text { PUREX } \\
\text { QAPP } \\
\text { QC } \\
\text { RADE } \\
\text { RCRA } \\
\text { REDOX } \\
\text { RPD } \\
\text { S-10 Facility } \\
\text { SMCL } \\
\text { SST } \\
\text { TC } \\
\text { TOC } \\
\text { TOX } \\
\text { TSD } \\
\text { U-12 Crib } \\
\text { WAC } \\
\text { WHC } \\
\text { WMA } \\
\text { WP } \\
\text { WS } \\
\text { W }\end{array}$ & $\begin{array}{l}\text { 216-A-10 Crib } \\
216-A-29 \text { Ditch } \\
216-A-36 \text { B Crib } \\
\text { ammonia scrubber distillate } \\
\text { Code of Federal Regulations } \\
\text { Contamination Indicator Parameter } \\
\text { contractually required quantitation limit } \\
\text { calendar year } \\
\text { U.S. Department of Energy } \\
\text { drinking water standard } \\
\text { Washington State Department of Ecology } \\
\text { U.S. Environmental Protection Agency } \\
\text { fiscal year } \\
\text { Grout Treatment Facility } \\
\text { inductively coupled plasma } \\
\text { Incident (report) } \\
\text { International Technology Analytical Services } \\
\text { Liquid Effluent Retention Facility } \\
\text { Low-Level Burial Grounds } \\
\text { Low-Level Waste Management Area } \\
\text { minimum detectable Concentration } \\
\text { method detection limit } \\
\text { Nonconformance Report } \\
\text { National Geodetic Vertical Datum } 1929 \text { Standard } \\
\text { Nonradioactive Dangerous Waste Landfill } \\
\text { nephelometric turbidity unit } \\
\text { process distillate discharge } \\
\text { Pacific Northwest Laboratory } \\
\text { Plutonium-Uranium Extraction } \\
\text { Quality Assurance Project Plan } \\
\text { quality control } \\
\text { Request for Analytical Data Evaluation } \\
\text { Resource Conservation and Recovery Act of } 1976 \\
\text { Reduction-0xidation (Plant) } \\
\text { relative percent difference } \\
\text { 216-S-10 Pond and Ditch } \\
\text { secondary maximum containment level } \\
\text { single-shell tank } \\
\text { total carbon } \\
\text { total organic carbon } \\
\text { total organic halogen } \\
\text { treatment, storage, and/or disposal } \\
216-U-12 \text { Crib } \\
\text { Washington Administrative Code } \\
\text { Westinghouse Hanford Company } \\
\text { waste management area } \\
\text { Water Pollution } \\
\text { Water Supply }\end{array}$ \\
\hline
\end{tabular}


DOE/RL-93-56-3

This page intentionally left blank. 
DOE/RL-93-56-3

\section{CONTENTS}

1.0 INTRODUCTION . . . . . . . . . . . . . . . . . . . . . . .

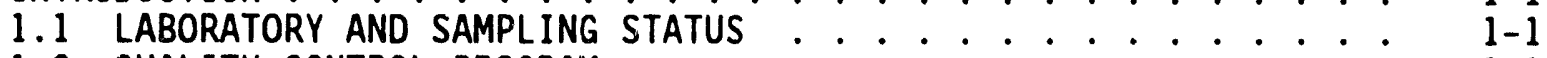

1.2 QUALITY CONTROL PROGRAM . . . . . . . . . . . . . . 1-1

1.2.1 Introduction . . . . . . . . . . . . 1-1

1.2.2 External Quality Control Program ......... 1-5

1.2.3 Internal Quality Control Program ........... 1-9

1.2.4 Nonconformance/Incident Reports . . . . . . . . . . . 1-11

1.2.5 Quality Assurance Occurrences . . . . . . . . . . 1-12

1.3 QUALITY CONTROL DEFINITIONS . . . . . . . . . . . . . . $1-14$

1.4 DATA TABLES . . . . . . . . . . . . . . . . . . 1-16

1.4.1 Water Level Measurement Report . . . . . . . . . . 1-16

1.4.2 Constituent List and Summary of Results . . . . . . 1-17

1.4.3 Constituents with at Least One Value Above

the CRQL ................ 1-18

1.4.4 Contamination Indicator Parameters ........ 1-18

1.4.5 Codes and Abbreviations ............. 1-20

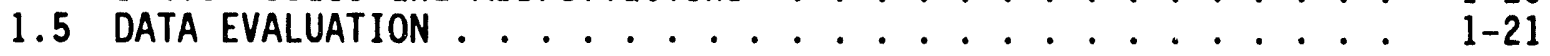




\section{LIST OF FIGURES}

1-1 Locations of the RCRA Groundwater Monitoring Projects and Landmarks on the Hanford Site . . . . . . . . . . . . . 1-3

\section{LIST OF TABLES}

1-1 Completeness . . . . . . . . . . . . . . . . . . . . 1-4

1-2 Field Duplicates and Field Blanks Evaluation Results for Third Quarter 1993 ................. . 1-5

1-3 Breakdown of Field Duplicates Exceeding Quality Control Limits . . 1-7

1-4 Breakdown of Field Blanks Exceeding Quality Control Limits . . . . 1-8

1-5 Frequency of Qualiiy Control Samples for Third Quarter 1993 . . . 1-9

1-6 Radiochemistry Quarterly Report Summary . . . . . . . . . . . 1-10

1-7 Summary of EPA Water Pollution, Water Supply, and Interlaboratory Comparison Study ................. 1-11

1-8 Analysis Method Code Definitions . . . . . . . . . . . . . . 1-19

1-9 Drinking Water Standards . . . . . . . . . . . . . . 1-22 


\section{QUARTERLY REPORT OF RCRA GROUNDWATER MONITORING DATA \\ FOR PERIOD JULY 1, 1993 THROUGH SEPTEMBER 30, 1993}

\subsection{INTRODUCTION}

Hanford Site interim-status groundwater monitoring projects are conducted as either background, indicator parameter evaluation, or groundwater quality assessment monitoring programs as defined in the Resource Conservation and Recovery Act of 1976 (RCRA); and "Interim Status Standards for Owners and Operators of Hazardous Waste Treatment, Storage, and Disposal Facilities," as amended ( 40 Code of Federal Regulations [CFR] 265). Compliance with the 40 CFR 265 regulations is required by the Washington Administrative Code (WAC) 173-303. This report contains data from Hanford Site groundwater monitoring projects. The location of each facility is shown in Figure 1-1.

Westinghouse Hanford Company (WHC) manages the RCRA groundwater monitoring projects for federal facilities on the Hanford Site. Project management, specifying data needs, performing quality control (QC) oversight, managing data, and preparing project sampling schedules are all parts of this responsibility. Pacific Northwest Laboratory (PNL) administers the contract for analytical services and provides groundwater sampling services to WHC for the RCRA groundwater monitoring program.

This quarterly report contains data received between August 21 and November 19, 1993, which are the cutoff dates for this reporting period. This report may contain not only data from samples collected during the July through September quarter but also data from earlier sampling events that were not previousiy reported.

\subsection{LABORATORY AND SAMPLING STATUS}

DataChem Laboratories of Salt Lake City, Utah, performs the hazardous chemicals analyses for the RCRA Program. Analyses for coliform bacteria are performed by Columbia/Biomedical Laboratories and for dioxin by TMS Analytical Services, Inc.; both are under subcontract to DataChem Laboratories. International Technology Analytical Services (ITAS), Richland, Washington, performs the radiochemical analyses.

\subsection{QUALITY CONTROL PROGRAM}

J. C. Johnston/S. M. Kitchka/T. X. Washington Westinghouse Hanford Company

\subsubsection{Introduction}

The QC Program is based on guidance from the U.S. Environmental Protection Agency (EPA), the RCRA Groundwater Monitoring Technical Enforcement 
Guidance Document (EPA 1986a), and Chapter One: Quality Control, from Test Methods for Evaluating Solid Waste (EPA 1986b).

1.2.1.1 Data Quality Objectives. The QC Program uses the five data quality objectives: precision, accuracy, representativeness, completeness, and comparability, along with applicable program-specific quality parameters to evaluate the quality of the data and the analytical laboratories analyzing the samples. Target values for precision and accuracy are specified in Quality Assurance Project Plan (QAPP) for RCRA Groundwater Monitoring Activities (WHC 1992).

1. Precision is evaluated using data results from laboratory duplicates, matrix spike duplicates (see Section 1.2.3), field duplicates, and blind samples (see Section 1.2.2).

2. Accuracy is evaluated using data results from laboratory matrix spikes; laboratory control samples; EPA Water Pollution (WP), Water Supply (WS), and Interlaboratory Performance Evaluation Programs (see Section 1.2.3); and by blind samples (see Section 1.2.2).

3. Representativeness expresses the degree to which RCRA facility groundwater monitoring data represent the real composition of the groundwater in the aquifer. Goals for data representativeness for groundwater monitoring programs are addressed qualitatively by the specification of well construction, sampling locations, sampling intervals, and sampling and analyses techniques in the groundwater monitoring plan for each RCRA facility.

4. Completeness is defined as the percentage of measurements made that are judged to be valid. Completeness is determined by the number of data unflagged during validation divided by the total number of data validated multiplied by 100 . The calculated percentages used in reporting completeness are conservative figures and are based on the data flags $P, F, D, Q$, and/or $H$. Table 1-1 illustrates project completeness for the data presented in this report.

5. Comparability is used to ensure that samples analyzed by different laboratories or by the same laboratory over different time periods are comparable. For the third quarter of 1993, only the primary hazardous chemistry and radiochemistry laboratories were requested to analyze samples and submit data. Samples were analyzed in accordance with Test Methods for Evaluating Solid Waste (EPA 1986b) and other applicable approved methods. Comparability of field measurements is determined by following approved sampling procedures that ensure consistency among sampling events. 
Figure 1-1. Locations of the RCRA Groundwater Monitoring Projects and Landmarks on the Hanford Site.

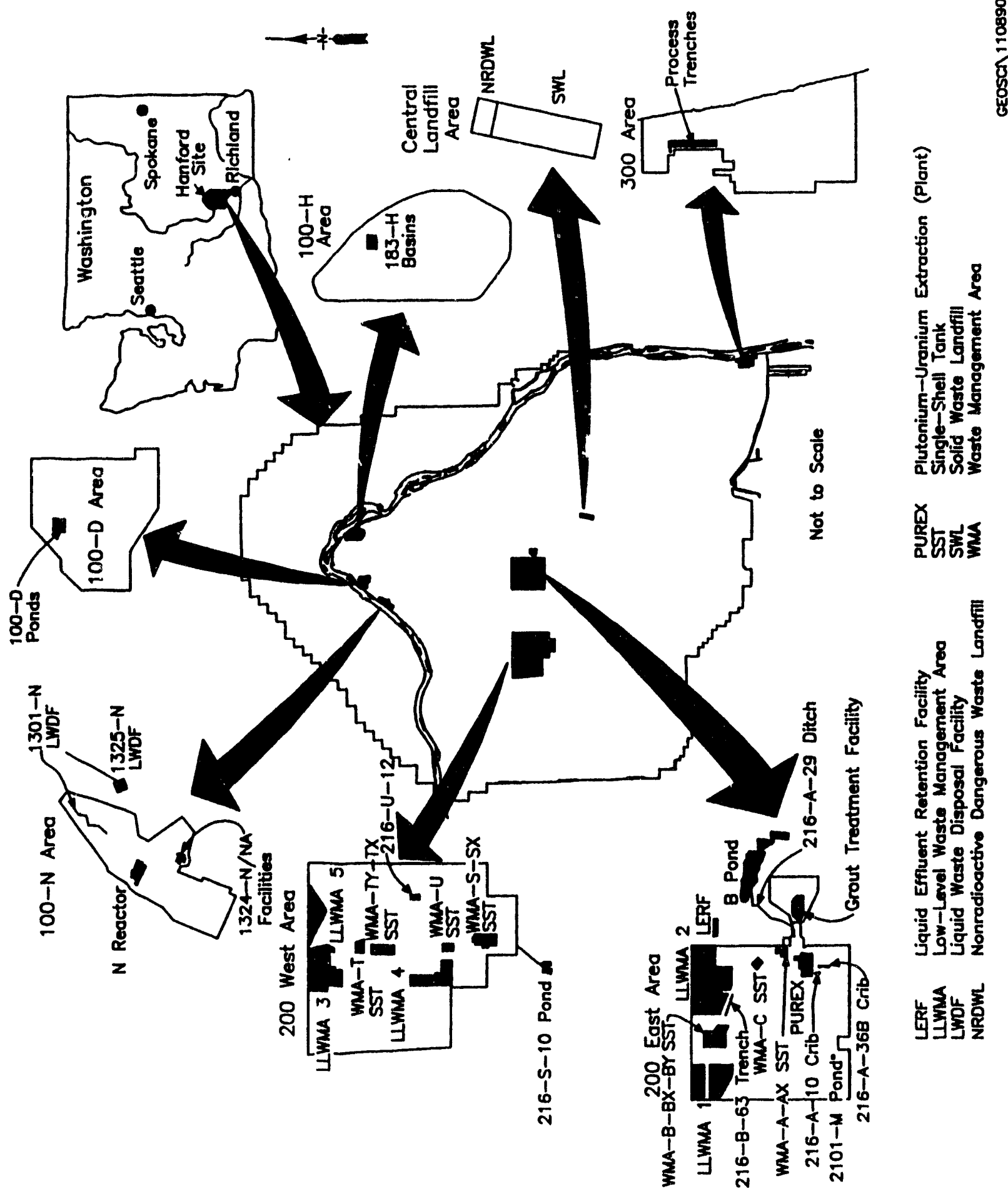


Table 1-1. Completeness.

\begin{tabular}{|c|c|c|c|}
\hline Project & $\begin{array}{c}\text { Total number } \\
\text { of validated } \\
\text { data }\end{array}$ & $\begin{array}{l}\text { Number of } \\
\text { unflagged } \\
\text { results }\end{array}$ & $\begin{array}{l}\text { Percent } \\
\text { complete }\end{array}$ \\
\hline $100-D$ & 0 & 0 & $N / A$ \\
\hline $100-N$ & 2,123 & 2,012 & 95 \\
\hline $183-\mathrm{H}$ & 993 & 981 & 95 \\
\hline $2101-M$ & 19 & 19 & 100 \\
\hline $216-A-10$ & 0 & 0 & $N / A$ \\
\hline $216-A-29$ & 2,405 & 2,385 & 99 \\
\hline $216-A-36 B$ & 0 & 0 & $N / A$ \\
\hline $216-B-3$ & 4,604 & 4,531 & 98 \\
\hline 216-B-63 & 955 & 832 & 87 \\
\hline $216-S-10$ & 695 & 671 & 97 \\
\hline $216-U-12$ & 679 & 670 & 99 \\
\hline 300 APT & 701 & 696 & 99 \\
\hline Grout & 611 & 608 & 100 \\
\hline LERF & 365 & 364 & 100 \\
\hline LLBG WMA-1 & 2,215 & 2,171 & 98 \\
\hline LLBG WMA-2 & 512 & 510 & 100 \\
\hline LLBG WMA-3 & 3,058 & 2,924 & 96 \\
\hline LLBG WMA-4 & 1,606 & 1,485 & 92 \\
\hline LLBG WMA-5 & 1,881 & 1,866 & 99 \\
\hline NRDWL & 257 & 225 & 88 \\
\hline SST $A / A X$ & 405 & 384 & 95 \\
\hline SST $B / B X / B Y$ & 345 & 332 & 96 \\
\hline SST C & 223 & 207 & 93 \\
\hline SST S/SX & 603 & 571 & 95 \\
\hline SST T & 333 & 333 & 100 \\
\hline SST TX/TY & 426 & 410 & 96 \\
\hline SST U & 265 & 259 & 98 \\
\hline
\end{tabular}

APT $=$ area process trench .

LERF = Liquid Effluent Retention Facility.

LLBG = Low-Level Burial Grounds.

$N / A=$ not applicable.

NRDWL $=$ Nonradioactive Dangerous Waste Landfill.

SST = single-shell tank .

WMA = waste management area . 
DOE/RL-93-56-3

1.2.1.2 Program-Specific Validation Flags. Program-specific validation flags are assigned during the process of evaluating RCRA groundwater data. These flags include (1) $H$ flags for exceeding holding times; (2) Q flags for field blank and duplicate data outside of the evaluation criteria; (3) F flags for data under the Request for Analytical Data Evaluation (RADE) process;

(4) D flags for data associated with a Nonconformance Report (NCR)/Incident (INC) report; and (5) P flags for data identified as a "potential problem."

1. Holding time exceedances have been flagged in the quarterly reports with an $\mathrm{H}$ flag. This flag means that the samples were analyzed beyond the method-required holding times as stated in the contract. The $\mathrm{H}$-flagged data may be used qualitatively, but no regulatory decisions should be made based on a single flagged analytical result.

2. Field blanks that indicate contaminations, and field duplicate samples that show greater than $25 \%$ relative percent difference (RPD), are identified in the quarterly project data tables with a $Q$ flag. This flag means that the field QC data associated with the sample data were cutside limits established in the QAPP (WHC 1992). The Q-flagged data can be used qualitatively, but no regulatory decisions should be made based on a single-flagged data point.

3. The RADE system is used to track and evaluate data that appear to be inconsistent with the current pattern or with historical trends (see Section 1.5).

4. NCR and INC reports are received from the laboratory and are used to document occurrences that may affect data quality. Data points affected by these reports are flagged with a 'D.' The D-flagged data may be used qualitatively, but no regulatory decision should be made based on a single-flagged data point (see Section 1.2.4).

5. Data points related to isolated occurrences determined by the specific project scientist are flagged with a ' $P$,' indicating a potential problem with the data. Explanations for use of the flag will be discussed in the specific project chapter.

\subsubsection{External Quality Control Program}

The external $Q C$ program uses three kinds of $Q C$ samples to evaluate quality in the field and laboratory. These are field duplicates, field blanks, and blind samples. (See page 1-14 for definitions of the above QC samples.)

The $Q C$ analytical results are judged to be acceptable if the following evaluation criteria are met.

- Field duplicates--Results of field duplicate pairs must have precision as measured by RPD within $\pm 25 \%$. 
- Blanks--Four kinds of blanks are used to check for contamination resulting from field activities and/or bottle preparation. These are full trip blanks, daily trip blanks, field transfer blanks, and bottle blanks.

Except for common laboratory contaminants, results above the limit of two times the method detection 1 imit (MDL) are identified as suspected contamination. For common laboratory contaminants, such as acetone, methylene chloride, 2-butanone, toluene, and phthalate esters, sample results less than five times the MDL are qualified as nondetects.

- Blind samples--Results must fall within 2.0 standard deviations from the mean recovery of the known concentration of samples submicted to the laboratory.

Table 1-2 provides a summary of the analysis results of QC samples available for the third quarter of 1993.

Samples associated with duplicate or blank analytes that exceeded the QC limits are discussed below and are flagged with a ' $Q$ ' in the data tables.

Precision based on field duplicate analysis was determined to be $94.5 \%$ acceptable. Table 1-3 shows a breakdown of the 128 duplicate results that fell outside the QC limits.

of the 4,585 blanks analyzed during third quarter of 1993, only 82 exceeded the QC limit, for $98.2 \%$ acceptability. Table 1-4 shows a breakdown of the constituents detected in the blanks.

Acetone and methylene chloride are common laboratory contaminants. These two constituents accounted for all but one of the volatile organics that exceeded control limits. For all of the field blanks in which acetone exceeded QC limits and for all but three of the field blanks in which methylene chloride exceeded QC limits, the laboratory's internal QC process identified these as samples contaminated in the laboratory. All of the total organic carbon (TOC) and total carbon (TC) samples were also identified with laboratory contamination. The laboratory assigned and reported these values with B qualifiers.

Table 1-2. Field Duplicates and Field Blanks Evaluation Results for Third Quarter 1993.

\begin{tabular}{|l|c|c|c|}
\hline & $\begin{array}{c}\text { Number of } \\
\text { analyses }\end{array}$ & $\begin{array}{c}\text { Number of results } \\
\text { outside of criteria }\end{array}$ & \% Acceptable \\
\hline Duplicates & 2,314 & 128 & 94.5 \\
\hline Blanks & 4,585 & 82 & 98.2 \\
\hline
\end{tabular}


Table 1-3. Breakdown of Field Duplicates Exceeding Quality Control Limits.

\begin{tabular}{|c|c|c|c|}
\hline $\begin{array}{l}\text { Method } \\
\text { analysis } \\
\text { code }\end{array}$ & Method description & $\begin{array}{l}\text { Number of } \\
\text { occurrences }\end{array}$ & $\begin{array}{l}\text { Percent } \\
\text { of total } \\
\text { out of } \\
\text { limits }\end{array}$ \\
\hline 16 & Volatile organics & 6 & 4.7 \\
\hline 17 & $\begin{array}{l}\text { Pesticides and polychlorinated } \\
\text { biphenyls }\end{array}$ & 2 & 1.6 \\
\hline 19 & Semivolatile organics & 1 & .8 \\
\hline 34 & $\begin{array}{l}\text { Inductively coupled plasma } \\
\text { metals }\end{array}$ & 55 & 43.0 \\
\hline $40,43,48$ & $\begin{array}{l}\text { Graphite furnace atomic } \\
\text { absorption metals }\end{array}$ & 18 & 14.1 \\
\hline 54 & Ammonium & 1 & .8 \\
\hline 63 & Sulfide & 1 & .8 \\
\hline 67 & Total organic halogen & 5 & 3.9 \\
\hline 122 & Total organic carbon & 7 & 5.5 \\
\hline 124 & Anions & 3 & 2.3 \\
\hline 126 & Turbidity & 4 & 3.1 \\
\hline 135 & Gross alpha & 6 & 4.7 \\
\hline 136 & Gross beta & 5 & 3.9 \\
\hline 137 & Radium & 3 & 2.3 \\
\hline 139 & Iodine-129 & 1 & .8 \\
\hline 140 & Radium & 6 & 4.7 \\
\hline 145 & Uranium & 2 & 1.6 \\
\hline 148 & Uranium-235 & 1 & .8 \\
\hline
\end{tabular}


Table 1-4. Breakdown of Field Blanks Exceeding Quality Control Limits.

\begin{tabular}{|c|l|c|c|}
\hline $\begin{array}{c}\text { Method } \\
\text { analysis } \\
\text { code }\end{array}$ & \multicolumn{1}{|c|}{ Method description } & $\begin{array}{c}\text { Number of } \\
\text { occurrences }\end{array}$ & $\begin{array}{c}\text { Percent } \\
\text { of total } \\
\text { out of } \\
\text { ofits }\end{array}$ \\
\hline 16 & Volatile organics & 23 & 28.0 \\
\hline 17 & $\begin{array}{l}\text { Pesticides and polychlorinated } \\
\text { biphenyls }\end{array}$ & 1 & 1.2 \\
\hline 34 & $\begin{array}{l}\text { Inductively coupled plasma } \\
\text { metals }\end{array}$ & 24 & 29.3 \\
\hline 40,43 & $\begin{array}{l}\text { Graphite furnace atomic } \\
\text { absorption metals }\end{array}$ & 4 & 4.9 \\
\hline 54,129 & Ammonium & 5 & 6.1 \\
\hline 122 & Total organic carbon & 4 & 4.9 \\
\hline 124 & Anions & 1 & 1.3 \\
\hline 126 & Turbidity & 1 & 14.6 \\
\hline 127 & Total carbon & 1.2 \\
\hline 136 & Gross beta & 1.2 \\
\hline 145 & Uranium & 1.2 \\
\hline
\end{tabular}

For both field blanks and field duplicates, more results (and a higher percentage) for inductively coupled plasma (ICP) metals fell outside QC limits than did the results for any other analysis. The surveillance conducted August 4-6, 1993 at Datachem Laboratories concluded that the laboratory's system for analysis of ICP metals does not account for the large percentage of unacceptable field results. Based on the conclusion that laboratory analysis is not responsible for the large number of field blank contaminations and field duplicates out of precision for ICP, the QC task team has determined that this situation warrants investigation. Potential sources that should be explored are: sampling methods, sample bottle preparation and preservation, sample shipping, and others. Not excluded from the list of possible causes is the MDL report from the laboratory, upon which the field blanks evaluation procedure is based. The MDL report from the laboratory has not proven to be reliable; therefore, the unreliability is a possible cause for the results falling outside the limits that the QC task team has established, because blank evaluation is based on two times the MDL. 
Table 1-5 shows the required and actual sampling frequencies for QC samples. Sampling frequencies were met for duplicates, field transfer blanks, and bottle blanks. Because of sampler error, the daily trip blank was not taken for two sampling days. The required frequency for full trip blanks was missed by only $0.2 \%$.

Table 1-5. Frequency of Quality Control Samples for Third Quarter 1993.

\begin{tabular}{|l|c|c|c|c|}
\hline \multicolumn{1}{|c|}{$\begin{array}{c}\text { Quality control } \\
\text { samples }\end{array}$} & $\begin{array}{c}\text { Required } \\
\text { frequencies }\end{array}$ & $\begin{array}{c}\text { Number of } \\
\text { wells } \\
\text { sampled }\end{array}$ & $\begin{array}{c}\text { Number of } \\
\text { samples } \\
\text { taken }\end{array}$ & $\begin{array}{c}\text { Actual } \\
\text { frequency }\end{array}$ \\
\hline Duplicates & $5 \%$ & 270 & 15 & $5.6 \%$ \\
\hline Daily trip blank & $\begin{array}{c}\text { One each } \\
\text { sampling day }\end{array}$ & $\begin{array}{c}54 \text { sampling } \\
\text { days }\end{array}$ & 52 & $96.2 \%$ \\
\hline Full trip blank & $5 \%$ & 270 & 13 & $4.8 \%$ \\
\hline Field transfer blank & $5 \%$ & 270 & 21 & $7.8 \%$ \\
\hline Bottle blanks & $\begin{array}{c}\text { Per lot of } \\
\text { bottles }\end{array}$ & N/A & 2 & N/A \\
\hline
\end{tabular}

$N / A=$ not applicable.

\subsubsection{Internal Quality Control Program}

The internal $Q C$ program uses four types of $Q C$ data to establish and monitor performance in the laboratory. These data are laboratory blanks, matrix spikes, matrix duplicates, and EPA studies (WP and WS).

Every quarter the contracted laboratories each supply their own QC report, which includes data quality information on matrix spikes, matrix duplicates, and blanks in the form of precision and accuracy. The contracted laboratories also supply a report of their results on EPA's WP, WS, and radiochemical intercomparison studies on a quarterly basis. The results of EPA studies independently verify the continuation of laboratory performance and are expressed as the percentage of EPA-accepted results. Each half year the contracted laboratories also supply an MDL/minimum detectable concentration (MDC) report. The MDLs and MDCs are required to be below the contractually required quantitation limit (CRQL). The CRQL is not associated with a quantitation limit as the name suggests. It is intended to be the lowest analyte concentration in a given matrix that a laboratory can be expected to detect consistently. The CRQL is agreed on under the contractual statement of work. A laboratory NCR is issued when the MDL is greater than the CRQL. WHC reviews each of these reports and summarizes the results in Table 1-6. 
Table 1-6. Radiochemistry Quarterly Report Summary.

\begin{tabular}{|c|c|c|c|}
\hline $\begin{array}{c}\text { Third } \\
\text { quarter } \\
1993\end{array}$ & $\begin{array}{c}\text { Duplicates } \\
\text { (precision, in } \\
\text { \% acceptable) }\end{array}$ & $\begin{array}{c}\text { Spikes } \\
\text { (accuracy, in } \\
\text { \% acceptable) }\end{array}$ & (in \% acceptable) \\
\hline $\begin{array}{c}\text { July through } \\
\text { September }\end{array}$ & 97.8 & 97.7 & 94.6 \\
\hline
\end{tabular}

Precision and accuracy results from the primary contract laboratories, summarized in Table 1-6, indicate the performance of a11 customers submitting water matrix samples. WHC samples represent only a part of the performance summary. These figures represent samples analyzed during the July through September 1993 timeframe.

Radiochemistry precision and accuracy figures are gleaned from the radiochemistry laboratory's quarterly report, with percent acceptability calculated from duplicates and spikes, respectively. These results, along with radiochemistry blank data, are summarized in Table 1-6.

For the third quarter of 1993, the radiochemistry laboratory analyzed 226 duplicates, of wich 221 were within control limits, for an acceptability of $97.8 \%$.

The radiochemistry laboratory analyzed 301 spikes, of which 294 fell within control limits, for an acceptability of $97.7 \%$. The laboratory reports problems with water spikes in five areas. The data for the spikes exceeding the control limits were reviewed by the laboratory and accepted or reanalyzed based on this review.

Of 408 blanks analyzed for the third quarter of 1993,386 fell within control limits, for an acceptability of $94.6 \%$. The radiochemistry laboratory reported problems with blanks in six areas. The data were reviewed by the laboratory and accepted or reanalyzed based on this review.

A disproportionate and unacceptable number of TOC and TC results were reported by the laboratory with a $B$ qualifier assigned. For this reason, the evaluation of internal (laboratory) QC has focused on these two analyses. The laboratory provided in their quarterly report the results for the matrix spikes and matrix duplicates for TOC and TC, as well as a quarterly summary of blanks, matrix spikes, and matrix duplicates for those analyses. The summary reports that none of the six blanks analyzed for TC during the third quarter exceeded the CRQL. No actual blank results were provided for further evaluation by the QC task team. Control rharts provided show that none of the matrix spike recoveries or matrix duplicate precisions exceeded the laboratory's QC limits for TC. For TOC, of the 74 blanks analyzed during the third quarter, none were reported to have exceeded the CRQL. Of the 96 matrix spikes analyzed for TOC during the third quarter, no spike recoveries fell outside of the laboratory's $(3 \sigma)$ control limit. 
The information provided by the laboratory in its quarterly report suggests that analytical results of TC and TOC generally fall within acceptable control limits. However, analytical results of groundwater samples suggest that a potential problem with TC and TOC analysis exists. Investigation into the $B$ qualifier issue has been initiated by the QC task team through the contract administrator.

EPA WS samples were analyzed by the hazardous chemistry laboratory during August 1993. There were 76 blind samples sent for ICP metals, anions, ammonia ion, $\mathrm{pH}$, conductivity, TOC, cyanide, chemical oxygen demand, total dissolved solids, pesticide, volatile organics, polychlorinated biphenyl, and total phenolic analysis. All results, except one, were in acceptable ranges. The unacceptable result was for alkalinity. The laboratory contractor shall be notified of this deficiency. This results in 75/76 acceptable results for 98.7\% acceptability (Table 1-7).

Table 1-7. Summary of EPA Water Pollution, Water Supply, and Interlaboratory Comparison Study (in percent acceptable).

\begin{tabular}{|c|c|c|c|}
\hline $\begin{array}{c}\text { Third quarter } \\
1993\end{array}$ & $\begin{array}{c}\text { EPA water } \\
\text { pollution }\end{array}$ & $\begin{array}{c}\text { EPA water } \\
\text { supply }\end{array}$ & $\begin{array}{c}\text { EPA interlab } \\
\text { comparison }\end{array}$ \\
\hline $\begin{array}{c}\text { July through } \\
\text { September }\end{array}$ & $\begin{array}{c}\text { Samples not sent } \\
\text { for this quarter }\end{array}$ & $98.7 \%$ & $100 \%$ \\
\hline
\end{tabular}

$E P A=$ U.S. Environmental Protection Agency. "Hazardous chemistry contracted laboratory.

Badiochemistry contracted laboratory.

EPA intercomparison samples were analyzed by the radiochemistry laboratory during August 1993. Six blind samples of gamma-emitting radionuclides were sent to the radiochemistry laboratory; all samples had results within $\pm 25 \%$ bias. This results in six out of six acceptable results for $100 \%$ acceptability (see Table 1-7).

\subsubsection{Nonconformance/Incident Reports}

NCR and INC reports are methods of documentation by which contract laboratories can inform laboratory contractors and their customers of any problems encountered with the analysis, data, and/or data deliverable. This method of documentation identifies occurrences, deficiencies, and/or issues that can potentially have an adverse effect on the data integrity. These may include, but are not limited to, the following:

- Lost sample

- Broken bottle

- Instrument malfunction

- Calibration standard out of acceptable range 
- Laboratory control standard out of acceptable range

- Matrix spike recovery out of acceptable range

- Procedural noncompliance

- Chain of custody discrepancies

- Shipping temperature out of acceptable range

- Misreported data.

During this reporting period, 38 NCR/INC reports were transmitted by the hazardous chemistry laboratory and 5 reports were transmitted by the radiochemistry laboratory. The following is a breakdown of the INC reports received from the hazardous chemistry laboratory: laboratory QC outside of acceptable limits (16 reports); hold time missed (5 reports); insufficient sample for QC (2 reports); incorrect sample preparation ( 8 reports); container received broken or lost in laboratory ( 6 reports); and failure to document comment codes ( 1 report). The reports affect less than $1 \%$ of the data results for the reporting period.

The following is a breakdown of the INC reports received from the radiochemistry laboratory: samples lost in laboratory (1 report); modified method ( 1 report); and matrix effects problems ( 3 reports). The reports affect less than $1 \%$ of the data results for the reporting period.

\subsubsection{Quality Assurance Occurrences}

Quality assurance occurrences are situations that potentially affect the quality of the data. These situations are discussed through summaries of inspections of laboratory services and/or system and performance audits/ surveillances. Section 1.2.5.1 discusses occurrences during the current quarter, while Section 1.2.5.2 updates past occurrences.

Inspection of laboratory services and/or system and performance audits/surveillances for the RCRA groundwater monitoring program are performed throughout the year by the laboratory contractor, WHC, and/or the U.S. Department of Energy (DOE) on various aspects of this program. Quality assurance requirements and data quality objectives are defined in the Quality Assurance Project Plan for RCRA Groundwater Monitoring Activities (WHC 1992). Results of these oversight activities are documented with the audit/ surveillance and/or inspecting organization(s).

1.2.5.1 Occurrences for Current Quarter. There were no occurrences reported during the current quarter.

1.2.5.2 Status of Previous 0ccurrences. An inspection of services of the hazardous chemistry 1aboratory was conducted August 4-6, 1993. The inspection was in response to increasing concern for the quality of ICP metals data and as a followup on the previous inspection of total organic halogen (TOX). A summary of the inspection findings and observations is provided below.

The inspection team found evidence supporting eight (8) findings and three (3) observations; they are as follows:

Finding \#1: Re-profiling of ICP instrumentation was found to have occurred without the required recalibration. 
Finding \#2: For TOX analyses, instances were found when the $B$ column was greater than $10 \%$ of the total (of the $A+B$ columns) without the required reanalysis being performed.

Finding \#3: Documentation of training for a TOX analyst was such that it could not be determined that required training per Rev. 1 of IW-BA-9020 was accomplished before the analys is of samples by the analyst.

Finding \#4: Data were misreported for TOX analyses.

Finding \#5: The February 1993 MDL study included misreported data.

Finding \#6: ICP data generated since implementation of the February 1993 MDL study values have been misreported.

Finding \#7: The hazardous chemistry laboratory and noncompliances with procedure QC-DC-006, Rev. 3, governing NCRs.

Finding \#8: Type II water being used did not comply with the preparation requirements of American Society for Testing and Materials procedure 01193.

Observation \#1: Blank subtraction was not performed when average blank values were less than the MDL per IW-BA-9020, Rev. 1.

Observation \#2: The hazardous chemistry laboratory procedure was not being followed for glassware cleaning.

Observation \#3: Calibration standards were being used past the expiration dates.

The above findings and observations were forwarded to the hazardous laboratory and require a written response to addressing each issue.

Many TOX duplicate results for the second and third quarters of calendar year (CY) 1992 exceeded the RPD. These exceedances corresponded to laboratory blanks containing from 10 to $60 \mathrm{ppb}$ of TOX. These incidents led to an inspection of the TOX procedure, analysis, and records at the hazardous chemistry laboratory during November 1992. An inspection report was issued by the laboratory contractor in December 1992 with three findings and three observations. The findings were (1) lack of appropriate qualifiers and comment codes in reported data, (2) inconsistencies in subtraction of background for TOX analysis, and (3) lack of submission of internal laboratory QC blind samples. The observations were (1) procedural noncompliances for TOX analytical testing, (2) use of a nonstandard equation for calculating RPD, and (3) lack of a system for ensuring that analysts are provided laboratory QC data summaries. It was later discovered that only half of the TOX data were being reported electronically because of miscommunication between the hazardous chemistry laboratory and the laboratory contractor. All affected TOX data from this inspection have been flagged with a ' $P$ ' for potential problem. In future quarterly reports, TOX issues will be updated through the most recent TOX inspection (see Section 1.2.5.1). 


\subsection{QUALITY CONTROL DEFINITIONS}

Accuracy. The closeness of agreement between an observed value and a true value. Accuracy is assessed by means of reference samples and percent recoveries.

Blind sample. A sample that contains a concentration of analyte that is known to the supplier but unknown to the analyzing laboratory. The analyzing laboratory is informed that the sample is a QC sample and not a field sample. The blind, double blind, and matrix matched double blind samples are used to assess accuracy and monitor the performance of the analytical laboratory(ies) with prepared or purchased materials from EPA QC samples/concentrates or primary materials.

Bottle blank. A sample that contains only Type II reagent water. The bottle blank contains one sample for each bottle size, with at least enough bottles to include all constituents analyzed by a specific project, except radionuclides. Bottle blanks shall be submitted to the primary laboratory per lot of bottles. Bottle blanks are filled in the analytical laboratory under the sample preparation procedures. Bottle blanks do not go into the field.

Contractually required quantitation limit. A value intended to be the lowest analyte concentration in a given matrix that the laboratory can be expected to achieve consistently; agreed upon under the contract statement of work.

Daily trip blank. A sample that contains only Type II reagent water. The daily trip blank is used to check for sample contamination by volatile organic compounds arising from conditions encountered during the collection of samples. The daily trip blank is not opened in the field. One daily trip blank is collected for each day that sampling occurs.

Double blind sample. A sample that contains a concentration of analyte that is known to the supplier but is unknown to the analyzing laboratory. The analyzing laboratory is not informed that the sample is a QC sample. All attempts are made to make this sample appear like a field sample. For example, the double blind sample should be submitted to the laboratory within the same time period and with a sample identification number similar to that of the field samples. The double blind sample does not include matrix matching.

External quality control sample. Any QC sample prepared without the knowledge of the analytical laboratory.

Field duplicate sample. A sample used to determine repeatability of an analytical measurement on identical samples collected as close as possible to the same time at the same location. These samples are stored in separate containers and are analyzed independently by the same laboratory.

Field transfer blank. A sample that contains only Type II reagent water. The field transfer blank is used to check for sample contamination by volatile organic compounds arising from conditions encountered during the collection of samples. The field transfer blank is taken during the collection of samples. 
Data that are judged to be suspect by the project scientist are flagged with ' + ' in the table. Before data are designated as suspect, checks are conducted to determine if a transcription error occurred between the field sheet and the electronic database.

\subsubsection{Constituent List and Summary of Results}

The Constituent List and Summary of Results table (Summary table) is the first of three tables that present the results of groundwater sample analyses. The Summary table displays statistics based on the complete data set for the reporting period. The complete data set consists of all analysis results requested by the project that arrived at WHC between the cutoff date for the previous quarterly report and the cutoff date for the current quarterly report, and are associated with samples collected before the end of the current reporting quarter (see Section 1.1 for the current and previous cutoff dates). Therefore, data collected during previous quarters may appear in this document if the laboratory did not report the data until the current reporting period.

The fields in the Summary table are as follows:

- Constituent name--Name of the analyzed constituent being summarized; the Short name is an abbireviation used in the Constituents with at Least One Value Above the CRQL table (Section 1.4.3); the (Method), when present, is a code (Section 1.4.5) used to distinguish between different analytical methods for the same constituent.

- Units--Units in which the CRQL and drinking water standards (DWS) are reported in this table.

- Laboratory CRQL--CRQL imposed on the laboratory. This field is blank for radionuclides where the CRQL is defined as the value of the total error reported by the laboratory for each result.

- Drinking water standard limit/agency--The DWS used for comparison to the reported results; see Section 1.4 .5 for Agency definitions and a complete listing of standards used.

- Number of samples--Total is the number of sample results from all wells in the project contained in the complete data set for that constituent; $\geq C R Q L$ is the number of results that exceed the CRQL; $\geq$ DWS is the number of results that exceed the listed DWS.

Summarized constituents are grouped as Contamination Indicator Parameters (CIP), Drinking Water Parameters, Groundwater Quality Parameters, and SiteSpecific and Other Constituents. Within groups, rows are ordered alphabetically by the full constituent name.

Several constituents have CRQLs that exceed the DWS. In such cases, exceedance of the DWS cannot be determined if the constituent is not detected, so an asterisk $\left(^{*}\right)$ is placed in the $\geq$ DWS field. 


\subsubsection{Constituents with at Least One Value Above the CRQL}

The Constituents with at Least One Value Above the CRQL table (Above CRQL table) is a subset of the complete data set. Only constituents that were detected above the CRQL in at least one sample collected for the project during the current reporting period are reported in the Above CRQL table.

The first three column headings are as follows:

- Well name--Well from which sample was collected.

- Collection date--Date sample was collected.

- Sample number--Unique number assigned to a well sample.

The remaining columns contain analytical results and qualifiers for the constituent. The structure of the column header is as follows.

Constituent short name

Method code/reporting units

CRQL/DWS (suffix).

The Summary table (Section 1.4.2) shows the relationship between the full constituent name and the short name. The analytical method code is defined in Table 1-8. Abbreviations used for reporting units are listed in Section 1.4.5. On the third line, a period appears in place of the CRQL or DWS if a value does not exist. The one-letter suffix that may be added to the DWS identifies the regulation associated with the standard and is described in Section 1.4.5.

Analysis results are displayed with two digits to the right of the decimal point. This is a consequence of the generating software and does not reflect the accuracy of the results. The laboratories normally report the result with three significant digits. Radionuclide values may be negative because the laboratory subtracts a background reading from the measured result. The letter, or group of letters, that may follow the result are data qualifiers and flags. A complete explanation of what each flag means is provided in Section 1.4.5.

\subsubsection{Contamination Indicator Parameters}

The CIP table has a format similar to the Above CRQL table (Section 1.4.3) but includes only data for $\mathrm{pH}$, specific conductance, TOC, and TOX. All results for these parameters, whether above CRQL or not, are listed in the CIP table. The analysis method code is not part of the column header in this report. 
The field transfer blank is filled at the sampling site by pouring Type II reagent water from a cleaned container into a volatile organic analysis vial. At least 1 field transfer blank is collected for each 20 samples, or 1 per sampling batch.

Full trip blank. A sample that contains only Type II reagent water and preservative, as required. A full trip blank is used to check for contamination in sample bottles and sample preparation. The full trip blank is analyzed for all constituents of interest on all types of sample bottles used during that sampling period. The frequency of collection for a full trip blank is 1 per 20 samples, or 1 per sampling batch. A full trip blank is filled in the analytical laboratory under the sample preparation procedures. The full trip blank is not opened in the field.

Internal quality control sample. Any QC sample prepared by the anaiytical laboratory and used to establish and monitor the quality of the analytical laboratory.

Limit of detection. The lowest concentration level that is statistically different from a blank. This is ralculated by the average blank signal plus three standard deviations for the blank analyses.

Matrix matched double blind sample. A matrix matched double blind sample contains a concentration of analyte that is known to the supplier but unknown to the analyzing laboratory. The sample matrix has been altered to closely match that of the field samples.

Method detection limit. The minimum concentration of a substance that can be measured and reported with $99 \%$ confidence that the analyte concentration is greater than zero and is determined from analysis of a sample in a given matrix type containing the analyte.

Minimum detectable concentration. Required level of analytical detection for radiochemical samples.

Precision. The agreement among a set of individual measurements of the same property, usually under prescribed similar conditions. Precision is calculated by using RPD of the duplicate/replicate analyses. These samples should contain concentrations of analyte above the MDL and may involve the use of matrix spikes.

Reliable detection level. A detection limit set at two times the concentration of the MDL, so the risk of both false positives and false negatives falls below $1 \%$.

Iype II reagent water. Distilled or deionized water that is free of contaminants that may interfere with the analytical test in question. 


\subsection{DATA TABLES}

\section{J. A. Serkowski \\ Westinghouse Hanford Company}

Four different formats are used to display water level measurements and groundwater sample analytical results. This section describes the meaning of each table column heading, defines the abbreviations used, explains the data qualifiers and flags, and 1 ists analysis method codes and relevant regulatory standards.

A new hazardous chemical laboratory contract became effective for samples received on or after April 26, 1993. The new contract contains some changes to the CRQLs and a change in how results below the CRQL are reported. In the old contract, analytical results below the CRQL were reported with the CRQL value followed by a $U$ qualifier. In the new contract, results below the CRQL but above the MDL are reported with the measured value followed by an $L$ qualifier. Results below the MDL are reported with the MDL value followed by a $U$ qualifier. This document may contain data processed through both the old and the new contracts and therefore may contain data reported by both methods. The sample collect date is a good indicator of which contract was in place because samples are typically collected no more than 1 day before they are received by the laboratory. Note that all CRQL values shown in the data tables are the new contract values, even if some of the data were processed under the old contract.

\subsubsection{Water Level Measurement Report}

The Water Level Measurement Report has four columns as follows:

- Well--Well in which measurement was made.

- Date--Date of measurement.

- Depth to water--Depth, in feet, from well casing reference point to top of water in well.

- Water level--Elevation, in feet above mean sea level, of water level computed by subtracting depth to water from casing reference elevation.

Wells are grouped according to the zone that they monitor beginning with the top of the unconfined aquifer and continuing downward to the confined aquifer.

Only measurements made during the reporting quarter are included in the tables. In addition to measurements made during sampling, which are marked with an asterisk (*), measurements are routinely performed at other times to permit collection of data for an entire network within a 1- or 2-day time period. 
Table 1-8. Analys is Method Code Definitions.

\begin{tabular}{|c|c|}
\hline Method code & Method name \\
\hline 16 & SW-846 8240 \\
\hline 17 & SH-846 8080 \\
\hline 19 & $S W-8468270^{\circ}$ \\
\hline 25 & SW-846 $8010 / 8020^{\circ}$ \\
\hline 29 & $S W-8468140^{\circ}$ \\
\hline 30 & SW- $8468040^{\circ}$ \\
\hline 34 & SW-846 $6010^{\circ}$ \\
\hline 36 & ASTM D-1385 \\
\hline 40 & SW-846 $7421^{\circ}$ \\
\hline 41 & SW-846 $7470^{\circ}$ \\
\hline 42 & SW-846 7841" \\
\hline 43 & SW-846 $7060^{\circ}$ \\
\hline 48 & SW-846 $7740^{\circ}$ \\
\hline 49 & SH-846 $8150^{\circ}$ \\
\hline 51 & SW-846 8280 \\
\hline 52 & ASTM D-1067-A \\
\hline 54 & ASTM $0-1426-0$ \\
\hline 56 & SH-8469010 \\
\hline 62 & In-house ion chromatography \\
\hline 63 & SH-846 $9030^{\circ}$ \\
\hline 65 & Standerd Methods $\approx 2098^{c}$ \\
\hline 67 & SH-8469020 \\
\hline 69 & SH-846 $9131^{\circ}$ \\
\hline 73 & ASTM $D-1125-A$ \\
\hline 93 & Field probe, pH \\
\hline 94 & Field probe, conductivity \\
\hline 122 & SH-846 $9060^{\circ}$ \\
\hline 124 & ASTM $0-4327-88$ \\
\hline 125 & ASTM $0-1293$ \\
\hline 126 & Standard Methods $\# 214 A^{c}$ \\
\hline 127 & ASTM D-2579-A \\
\hline 130 & EPA Method $300.0^{\circ}$ \\
\hline 135 & SW-846 9310, Alpha" \\
\hline 136 & SW-846 9310, Beta" \\
\hline 137 & SH-846 9315, Radiut \\
\hline 139 & ITAS 1-129 Low level \\
\hline 140 & ITAS Genme scan \\
\hline 141 & ITAS Sr-90 \\
\hline 142 & ITAS H-3 \\
\hline 143 & ITAS TC- 99 \\
\hline 144 & SH-846 9132" \\
\hline 145 & ITAS Gross U \\
\hline 146 & ITAS Isotopic Pu \\
\hline 147 & ITAS Am-241 \\
\hline 148 & ITAS Isotopic U \\
\hline 168 & USEPA HACH COO $^{\circ}$ \\
\hline $35 \%^{\prime}$ & EPA $600,310.2$ \\
\hline
\end{tabular}


$\mathrm{DOE} / \mathrm{RL}-93-56-3$

\subsubsection{Codes and Abbreviations}

Abbreviations--The abbreviations used in the data tables are as follows:

COL coliform colonies per 100 milliliters

CRQL contractually required quantitation limit

DWS drinking water standard

$\mathrm{ft}$ feet

ms 1 mean sea level

NTU nephelometric turbidity unit

$\mathrm{PCi} / \mathrm{L} \quad$ picocuries per liter

ppb parts per billion

ppm parts per million

$\mu$ mho micromhos per centimeter.

Agencies and suffixes--Agency codes and DWS suffixes identify the regulatory origin of the standard of the DWS. Agency codes are used in the Summary table, and DWS suffixes are used in the Above CRQL and CIP tables. The codes are defined as follows:

Agency Suffix Requlatory basis

EPA None Maximum contaminant levels in 40 CFR 141, "National Primary Drinking Water Regulations;" not including subsequent changes made through the Federal Register.

EPAS S Secondary maximum contaminant levels in 40 CFR 143, "National Secondary Drinking Water Regulations."

Data qualifiers and flags--Data qualifiers and flags used in the Above CRQL and CIP tables are assigned by the laboratory and by the WHC QC team, respectively. Qualifiers reflect conditions occurring in the laboratory relating to the analytical procedure. Flags serve a wider function of alerting the data user to the limitations of the reported value. Qualifiers and flags can be appended to each other to form a string of letters when several factors apply to a result. The qualifiers and flags used are as follows:

Laboratory qualifiers:

B - Blank associated with analyte is contaminated

D - Analyzed sample is diluted

E - Concentration is out of instrument calibration range

$\mathrm{J}$ - Concentration is estimated

$L$ - Concentration is below the CRQL but above the MDL

$U$ - Concentration is below the indicated value. 
QC Flags:

D - Result associated with a documented laboratory nonconformance

$H$ - Laboratory holding time exceeded

$P$ - Potential problem; see text associated with table

Q - Result associated with suspect QC data

F - Suspect data currently under review

+ - Suspect water level data currently under review

* - CRQL is greater than DWS, so exceedance of DWS is undetermined.

More complete descriptions of some of these qualifiers and flags are presented in Section 1.2, Quality Control Program.

Analysis method codes--Analys is method codes are used as an abbreviation for the laboratory method used to perform an analysis. A complete listing of the analysis method codes used in the Summary and Above CRQL tables is shown in Table 1-8.

DWSs--DWSs are used in all of the chemistry tables to provide a standard with which to compare sample results. A complete listing of the DWSs used in the Summary, Above CRQL, and CIP tables is shown in Table 1-9.

\subsection{DATA EVALUATION}

Data evaluation is a process through which suspect data are identified and/or investigated. At present, the data evaluation process consists of evaluation of data called out in RADEs, submitted by data users, and the statistical evaluation of CIP data. The statistical evaluations of CIP data are presented within the chapters for individual sites.

Thirty-eight RADEs were submitted during the third quarter of 1993 in time for inclusion in this report. Of the submitted RADEs, 14 are for field parameters ( $\mathrm{pH}$ and specific conductance), 1 is for coliform, 8 are for ICP metals, 3 for various organics, 5 for radionuclides, 1 for water levels, and 1 for selenium. F flags are used to indicate that an RADE has been submitted. After evaluation the $F$ flag may be changed to ' $G$,' indicating acceptable data; ' $Y$,' indicating suspect data; or ' $R$,' indicating unusable data. 
Table 1-9. Drinking Water Standards. (sheet 1 of 2)

\begin{tabular}{|c|c|c|c|c|}
\hline Const ituent name & Short name & DWS & Units & Agency \\
\hline $2,4,5-T P$ & $2,4,5 \mathrm{TP}$ & 10 & ppb & EPA \\
\hline $2,4-0$ & $2,4-D$ & 100 & ppb & EPA \\
\hline Arsenic & ARSENIC & 50 & ppb & EPA \\
\hline Arsenic, filtered & FARSEN I & 50 & ppb & EPA \\
\hline Barium & BARIUM & 1,000 & ppb & EPA \\
\hline Berium, filtered & FBARIUM & 1,000 & ppob & EPA \\
\hline Bronodichlorome thane & BOCM & 100 & ppob & EPA \\
\hline Bromoform & BROMORM & 100 & ppb & EPA \\
\hline Cactnium & CADMIUN & 10 & ppb & EPA \\
\hline Cacmium, filtered & FCADMIU & 10 & ppb & EPA \\
\hline Chloride & CHLORID & 250,000 & ppb & EPAS \\
\hline Chloroform & CHLFORM & 100 & ppb & EPA \\
\hline Chromiun & CHROMUM & 50 & ppb & EPA \\
\hline Chromiun, filtereu & FCHROMI & 50 & ppb & EPA \\
\hline Coliforms, membrane filter & COLI-MFT & 1 & $\mathrm{COL}$ & EPA \\
\hline Copper & COPPER & 1,000 & ppb & EPAS \\
\hline Copper, filtered & FCOPPER & 1,000 & ppb & EPAS \\
\hline Dibromoch loromethane & BRCHLMET & 100 & ppb & EPA \\
\hline Endrin & ENDRIN & 0.2 & ppb & EPA \\
\hline Fluoride & FLUORID & 4,000 & ppb & EPA \\
\hline Gemma-BHC (I indene) & LINDANE & 4 & ppb & EPA \\
\hline Gross olphe & ALPHA & 15 & $\mathrm{PC} i / L$ & EPA \\
\hline Gross beta & BETA & 50 & $\mathrm{PCi} / \mathrm{L}$ & EPA \\
\hline Iron & IRON & 300 & ppb & EPAS \\
\hline Iron, filtered & FIRON & 300 & ppb & EPAS \\
\hline Lead & LEAD & 50 & ppb & EPA \\
\hline Lead, filtered & FLEAD & 50 & ppob & EPA \\
\hline Manganese & MANGESE & 50 & ppb & EPAS \\
\hline Manganese, filtered & FMANGAN & 50 & ppb & EPAS \\
\hline Mercury & MERCURY & 2 & ppb & EPA \\
\hline Mercury, filtered & FMERCUR & 2 & ppb & EPA \\
\hline Methoxychlor & METHLOR & 100 & ppb & EPA \\
\hline Nitrate & NITRATE & 45,000 & ppb & EPA \\
\hline ph, field & PH-FLD & $6.5-8.5$ & $\cdots$ & EPAS \\
\hline pH, lab & PH-LAB & $6.5-8.5$ & $\cdots$ & EPAS \\
\hline Selenium & SELENUM & 10 & ppb & EPA \\
\hline
\end{tabular}




\section{DOE/RL-93-56-3}

Table 1-9. Drinking Water Standards. (sheet 2 of 2)

\begin{tabular}{|l|c|r|r|c|}
\hline \multicolumn{1}{|c|}{ Constituent name } & Short name & DWS & Units & Agency $^{*}$ \\
\hline Selenium, filtered & FSELENI & 10 & Ppb & EPA \\
\hline Silver & SILVER & 50 & ppb & EPA \\
\hline Silver, filtered & FSILVER & 50 & Ppb & EPA \\
\hline Stront ium-90 & SR 90 & 8 & PCi/L & EPA \\
\hline Sulfate & SULFATE & 250,000 & Ppb & EPAS \\
\hline Total radium & RADIUM & 5 & PCi/L & EPA \\
\hline Toxaphene & TOXAENE & 5 & Ppb & EPA \\
\hline Tritium & TRITIUM & 20,000 & PCi/L & EPA \\
\hline Turbidity & TURBID & 1 & NTU & EPA \\
\hline Vinyl chloride & VINYIDE & 2 & Ppb & EPA \\
\hline
\end{tabular}

COL $=$ coliform colonies per 100 milliliters .

NTU = nephelometric turbidity units.

pCi $/ \mathrm{L}=$ picocuries per liter

ppb = parts per billion.

- See section 1.4 .5 for agency listing.

"Turbidity is applicable to surtace water supplies. 


\section{$\mathrm{DOE} / \mathrm{RL}-93-56-3$}

This page intentionally left blank. 
$\mathrm{DOE} / \mathrm{RL}-93-56-3$

\section{CONTENTS}

$2.0100-D$ PONDS . . . . . . . . . . . . . . . . . . . . . 2-1

2.1 INTRODUCTION ...................... . . . . . . . . 2 .

2.2 WATER LEVEL MEASUREMENTS . . . . . . . . . . . . . . . . . . . $2-1$

2.3 WATER CHEMISTRY DATA . . . . . . . . . . . . . . . . . . 2-1 


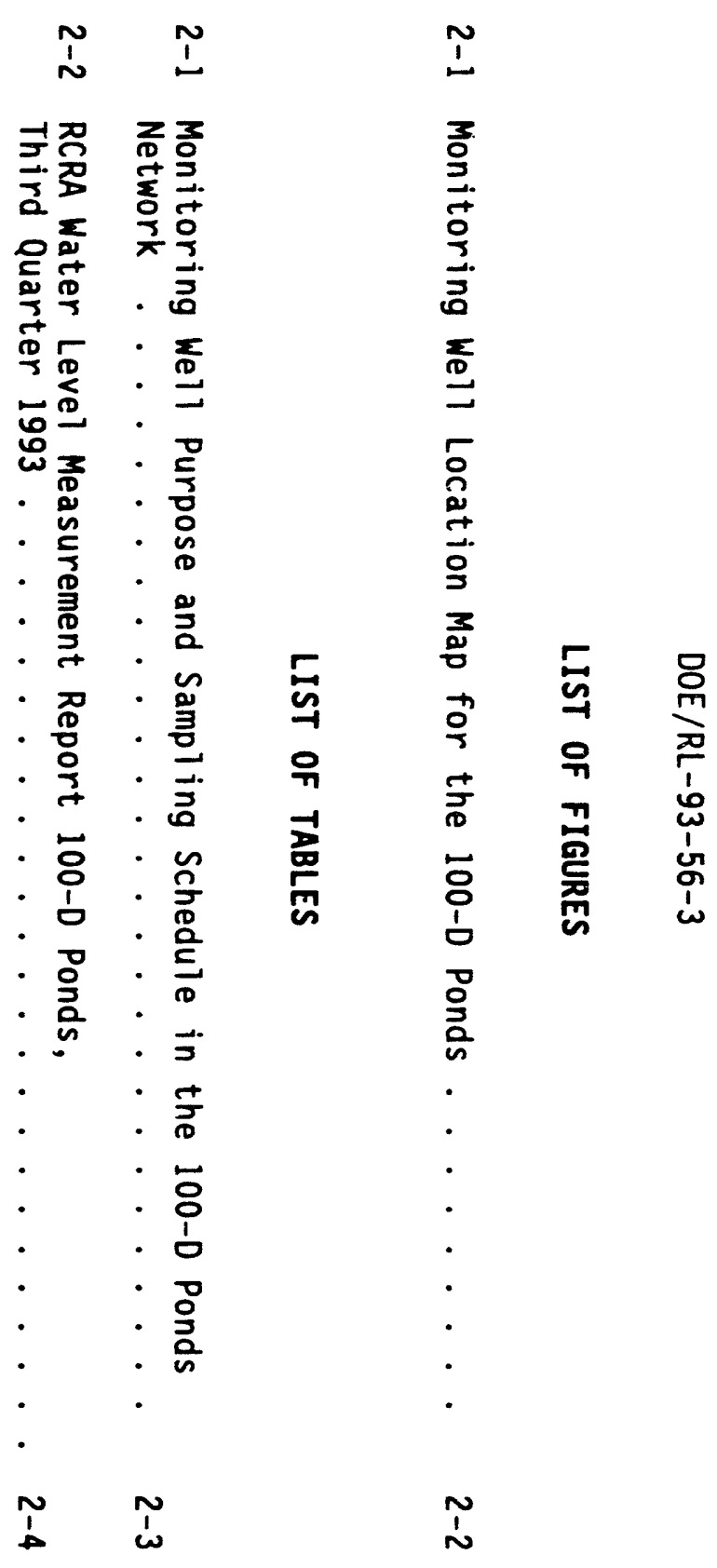


DOE/RL-93-56-3

\subsection{0-D PONDS}

M. J. Hartman

Westinghouse Hanford Company

\subsection{INTRODUCTION}

The 100-D Ponds system is an interim-status RCRA disposal unit located in the 100-D Area of the Hanford Site (Figure 2-1). Four wells make up the 100-D Ponds sampling network (Table 2-1). The 100-D Ponds are monitored under an indicator evaluation program (40 CFR 265).

The 100-D Ponds unit was constructed in 1977 for disposal of nonradioactive effluents derived from 100-D Area operating facilities. The 100-D Ponds are located in the former 188-D ash disposal basin and consist of a settling pond and a percolation pond, separated by a dike.

Effluent to the 100-D Ponds originated from two sources: a filter plant and engineering testing laboratories. Some past discharges may have included dangerous waste (Hartman 1991). The 183-D Filter Plant still discharges low volumes of nondangerous effluent to the 100-D Ponds (WHC 1990a).

\subsection{HATER LEVE: ME,SSUREMENTS}

Water levels are measured monthly in wells in and near the 100-0 Area. Water level data are listed in Table 2-2. The September 1993 measurement for well 699-92-49 is anomalously low compared to a nearby well and historical trends. It has been flagged as a suspected error.

\subsection{WATER CHEMISTRY DATA}

The 100-D Ponds network is sampled semiannually and was not scheduled for sampling this quarter. 


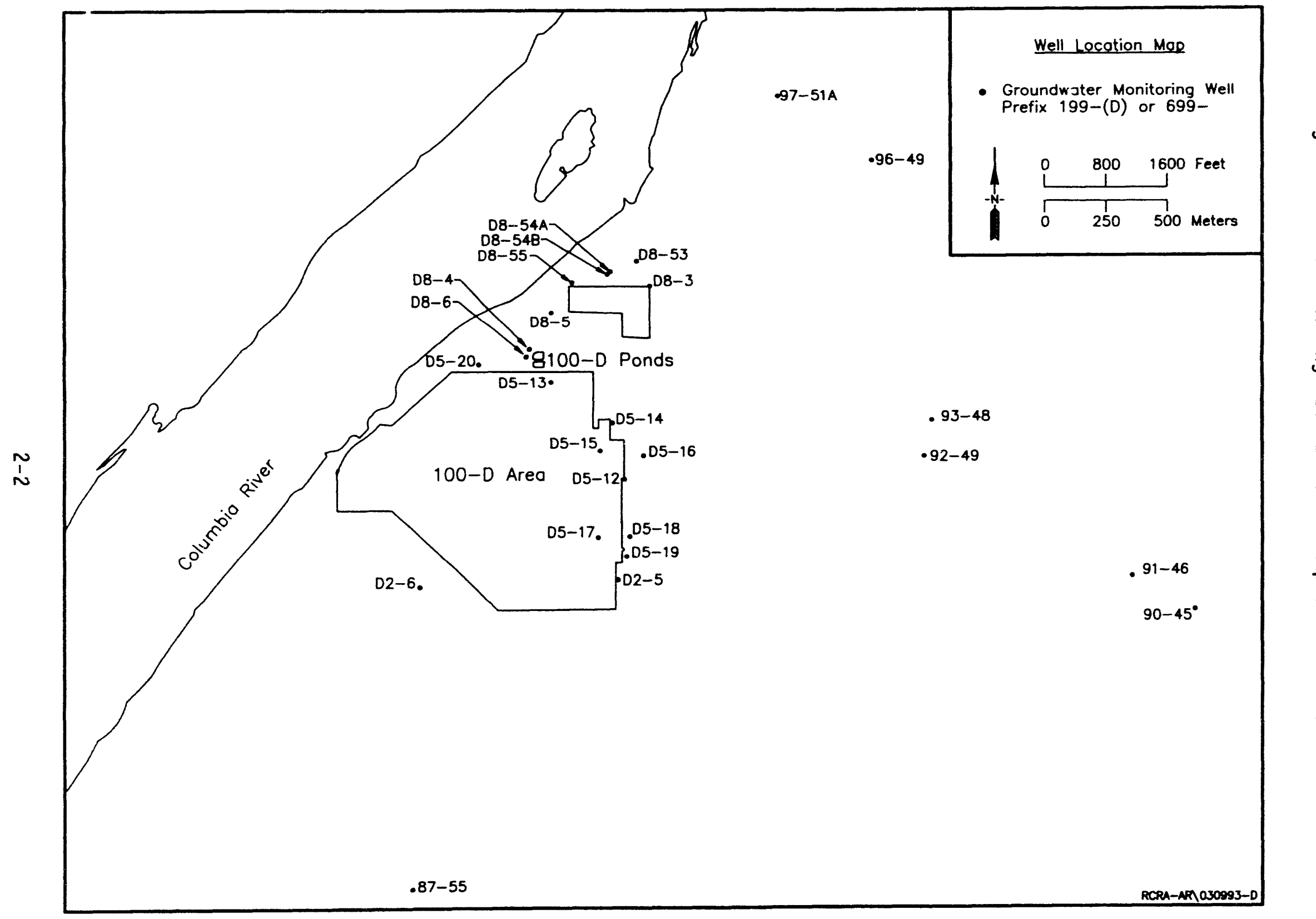

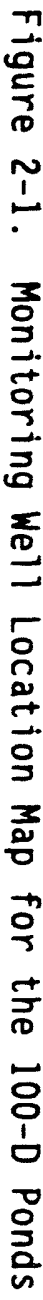


Table 2-1. Monituring Well Purpose and Sampling Schedule in the 100-D Ponds Network.

\begin{tabular}{|c|c|c|c|}
\hline $\begin{array}{c}\text { Well no. } \\
(199-)\end{array}$ & $\begin{array}{c}\text { Relative } \\
\text { position }\end{array}$ & $\begin{array}{c}\text { Sample } \\
\text { frequency }\end{array}$ & $\begin{array}{c}\text { Sample date, } \\
\text { 3rd Qtr 1993 }\end{array}$ \\
\hline D5-13 & Upgradient & Semiannually & Not scheduled \\
\hline D8-4 & Downgradient & Semiannually & Not scheduled \\
\hline D8-5 & Downgradient & Semiannually & Not scheduled \\
\hline D8-6 & Downgradient & Semiannually & Not scheduled \\
\hline
\end{tabular}

Note: The hydrogeologic unit monitored is the uppermost portion of the unconfined aquifer, which comprises unconsolidated sand and gravels of the Ringold Formation.

Well D8-5 will not be used for detection purposes, per agreement with the Washington State Department of Ecology. 
Table 2-2. RCRA Water Level Measurement Report 100-D Ponds, Third Quarter 1993. (sheet 1 of 3)

\begin{tabular}{|c|c|c|c|}
\hline Wel1 & Date & $\begin{array}{l}\text { Depth to } \\
\text { water }(\mathrm{ft})\end{array}$ & $\begin{array}{l}\text { Water level } \\
\text { elevation } \\
\text { above msl (ft) }\end{array}$ \\
\hline \multicolumn{4}{|c|}{ Wells Monitoring the Top of the Unconfined } \\
\hline $199-02-5$ & $\begin{array}{l}7 / 13 / 93 \\
8 / 09 / 93 \\
9 / 13 / 93\end{array}$ & $\begin{array}{l}75.00 \\
74.97 \\
75.19\end{array}$ & $\begin{array}{l}385.30 \\
385.33 \\
385.11\end{array}$ \\
\hline 199-D2-6 & $\begin{array}{l}7 / 13 / 93 \\
8 / 09 / 93 \\
9 / 13 / 93\end{array}$ & $\begin{array}{l}84.29 \\
84.51 \\
85.21\end{array}$ & $\begin{array}{l}384.99 \\
384.77 \\
384.07\end{array}$ \\
\hline $199-05-12$ & $\begin{array}{l}7 / 13 / 93 \\
8 / 09 / 93 \\
9 / 13 / 93\end{array}$ & $\begin{array}{l}85.08 \\
85.11 \\
85.45\end{array}$ & $\begin{array}{l}384.55 \\
384.52 \\
384.18\end{array}$ \\
\hline $199-05-13$ & $\begin{array}{l}7 / 13 / 93 \\
8 / 09 / 93 \\
9 / 13 / 93\end{array}$ & $\begin{array}{l}87.09 \\
87.29 \\
88.04\end{array}$ & $\begin{array}{l}384.40 \\
384.20 \\
383.45\end{array}$ \\
\hline $199-05-14$ & $\begin{array}{l}7 / 13 / 93 \\
8 / 09 / 93 \\
9 / 13 / 93\end{array}$ & $\begin{array}{l}87.32 \\
87.41 \\
87.83\end{array}$ & $\begin{array}{l}384.37 \\
384.28 \\
383.86\end{array}$ \\
\hline 199-D5-15 & $\begin{array}{l}7 / 13 / 93 \\
8 / 09 / 93 \\
9 / 13 / 93\end{array}$ & $\begin{array}{l}86.95 \\
86.99 \\
87.37\end{array}$ & $\begin{array}{l}384.58 \\
384.54 \\
384.16\end{array}$ \\
\hline $199-D 5-16$ & $\begin{array}{l}7 / 13 / 93 \\
8 / 09 / 93 \\
9 / 13 / 93\end{array}$ & $\begin{array}{l}88.64 \\
88.68 \\
89.03\end{array}$ & $\begin{array}{l}384.25 \\
384.21 \\
383.86\end{array}$ \\
\hline 199-D5-17 & $\begin{array}{l}7 / 13 / 93 \\
8 / 09 / 93 \\
9 / 13 / 93\end{array}$ & $\begin{array}{l}84.44 \\
84.42 \\
84.53\end{array}$ & $\begin{array}{l}385.05 \\
385.07 \\
384.96\end{array}$ \\
\hline 199-D5-18 & $\begin{array}{l}7 / 13 / 93 \\
8 / 09 / 93 \\
9 / 13 / 93\end{array}$ & $\begin{array}{l}81.97 \\
81.97 \\
82.24\end{array}$ & $\begin{array}{l}384.71 \\
384.71 \\
384.44\end{array}$ \\
\hline $199-D 5-19$ & $\begin{array}{l}7 / 13 / 93 \\
8 / 09 / 93 \\
9 / 13 / 93\end{array}$ & $\begin{array}{l}79.77 \\
79.72 \\
79.98\end{array}$ & $\begin{array}{l}385.03 \\
385.08 \\
384.82\end{array}$ \\
\hline
\end{tabular}


Table 2-2. RCRA Water Level Measurement Report 100-D Ponds, Third Quarter 1993. (sheet 2 of 3)

\begin{tabular}{|c|c|c|c|}
\hline Well & Date & $\begin{array}{l}\text { Depth to } \\
\text { water }(\mathrm{ft})\end{array}$ & $\begin{array}{l}\text { Water level } \\
\text { elevation } \\
\text { above msl ( } f t)\end{array}$ \\
\hline \multicolumn{4}{|c|}{ Wells Monitoring the Top of the Unconfined } \\
\hline 199-D5-20 & $\begin{array}{l}7 / 13 / 93 \\
8 / 09 / 93 \\
9 / 13 / 93\end{array}$ & $\begin{array}{l}84.27 \\
85.26 \\
86.18\end{array}$ & $\begin{array}{l}383.83 \\
382.84 \\
381.92\end{array}$ \\
\hline $199-08-3$ & $\begin{array}{l}7 / 13 / 93 \\
8 / 09 / 93 \\
9 / 13 / 93\end{array}$ & $\begin{array}{l}66.78 \\
67.86 \\
68.81\end{array}$ & $\begin{array}{l}382.21 \\
381.13 \\
380.18\end{array}$ \\
\hline $199-08-4$ & $\begin{array}{l}7 / 13 / 93 \\
8 / 09 / 93 \\
9 / 13 / 93\end{array}$ & $\begin{array}{l}84.59 \\
85.07 \\
85.98\end{array}$ & $\begin{array}{l}384.14 \\
383.66 \\
382.75\end{array}$ \\
\hline $199-08-5$ & $\begin{array}{l}7 / 13 / 93 \\
8 / 09 / 93 \\
9 / 13 / 93\end{array}$ & $\begin{array}{l}69.36 \\
70.35 \\
71.38\end{array}$ & $\begin{array}{l}383.13 \\
382.14 \\
381.11\end{array}$ \\
\hline $199-08-6$ & $\begin{array}{l}7 / 13 / 93 \\
8 / 09 / 93 \\
9 / 13 / 93\end{array}$ & $\begin{array}{l}92.43 \\
92.95 \\
93.85\end{array}$ & $\begin{array}{l}384.07 \\
383.55 \\
382.65\end{array}$ \\
\hline $199-08-53$ & $\begin{array}{l}7 / 13 / 93 \\
8 / 09 / 93 \\
9 / 13 / 93\end{array}$ & $\begin{array}{l}53.86 \\
55.52 \\
56.40\end{array}$ & $\begin{array}{l}382.17 \\
380.51 \\
379.63\end{array}$ \\
\hline $199-D 8-54 A$ & $\begin{array}{l}7 / 13 / 93 \\
8 / 09 / 93 \\
9 / 13 / 93\end{array}$ & $\begin{array}{l}60.56 \\
62.15 \\
63.02\end{array}$ & $\begin{array}{l}382.22 \\
380.63 \\
379.76\end{array}$ \\
\hline $199-D 8-54 B$ & $\begin{array}{l}7 / 13 / 93 \\
8 / 09 / 93 \\
9 / 13 / 93\end{array}$ & $\begin{array}{l}60.07 \\
61.87 \\
62.49\end{array}$ & $\begin{array}{l}382.44 \\
380.64 \\
380.02\end{array}$ \\
\hline $199-08-55$ & $\begin{array}{l}7 / 13 / 93 \\
8 / 09 / 93 \\
9 / 13 / 93\end{array}$ & $\begin{array}{l}56.93 \\
58.55 \\
59.49\end{array}$ & $\begin{array}{l}382.42 \\
380.80 \\
379.86\end{array}$ \\
\hline $699-87-55$ & $\begin{array}{l}7 / 13 / 93 \\
8 / 09 / 93 \\
9 / 13 / 93\end{array}$ & $\begin{array}{l}72.24 \\
72.35 \\
72.76\end{array}$ & $\begin{array}{l}386.39 \\
386.28 \\
385.87\end{array}$ \\
\hline
\end{tabular}


Table 2-2. RCRA Water Level Measurement Report 100-D Ponds, Third Quarter 1993. (sheet 3 of 3)

\begin{tabular}{|c|c|c|c|}
\hline We11 & Date & $\begin{array}{l}\text { Depth to } \\
\text { water }(f t)\end{array}$ & $\begin{array}{l}\text { Water level } \\
\text { elevation } \\
\text { above msl (ft) }\end{array}$ \\
\hline \multicolumn{4}{|c|}{ Wells Monitoring the Top of the Unconfined Aquifer } \\
\hline $699-90-45$ & $\begin{array}{l}7 / 12 / 93 \\
8 / 09 / 93 \\
9 / 13 / 93\end{array}$ & $\begin{array}{l}37.61 \\
37.65 \\
37.66\end{array}$ & $\begin{array}{l}383.93 \\
383.89 \\
383.88\end{array}$ \\
\hline $699-91-46$ & $\begin{array}{l}7 / 13 / 93 \\
8 / 09 / 93 \\
9 / 13 / 93\end{array}$ & $\begin{array}{l}33.28 \\
33.25 \\
33.31\end{array}$ & $\begin{array}{l}383.78 \\
383.81 \\
383.75\end{array}$ \\
\hline $699-92-49$ & $\begin{array}{l}7 / 13 / 93 \\
8 / 09 / 93 \\
9 / 13 / 93\end{array}$ & $\begin{array}{l}48.69 \\
48.77 \\
49.89\end{array}$ & $\begin{array}{l}383.19 \\
383.11 \\
381.99+\end{array}$ \\
\hline $699-93-48$ & $\begin{array}{l}7 / 13 / 93 \\
8 / 09 / 93 \\
9 / 13 / 93\end{array}$ & $\begin{array}{l}54.92 \\
54.93 \\
55.12\end{array}$ & $\begin{array}{l}382.87 \\
382.86 \\
382.67\end{array}$ \\
\hline $699-96-49$ & $\begin{array}{l}7 / 13 / 93 \\
8 / 09 / 93 \\
9 / 13 / 93\end{array}$ & $\begin{array}{l}37.35 \\
37.45 \\
37.86\end{array}$ & $\begin{array}{l}381.88 \\
381.78 \\
381.37\end{array}$ \\
\hline $699-97-51 A$ & $\begin{array}{l}7 / 13 / 93 \\
8 / 09 / 93 \\
9 / 13 / 93\end{array}$ & $\begin{array}{l}20.64 \\
20.84 \\
21.49\end{array}$ & $\begin{array}{l}381.63 \\
381.43 \\
380.78\end{array}$ \\
\hline
\end{tabular}

Notes: 1. Water level elevations are calculated by subtracting the measured depth to water from the surveyed elevation for the well.

2. Depth-to-water values are transcribed from field records.

3. Elevations marked with a ' + ' are outside of the expected range, and are suspected of error.

4. To convert feet to meters multiply by 0.3048 . 
DOE/RL-93-56-3

\section{CONTENTS}

$3.0 \quad 183-H$ SOLAR EVAPORATION BASINS ............... . . . . $3-1$

3.1 INTRODUCTION . . . . . . . . . . . . . . . . . . . . . . . 3-1

3.2 WATER LEVEL MEASUREMENTS . . . . . . . . . . . . . . . 3-1

3.3 WATER CHEMISTRY DATA .................... $3-2$ 
DOE/RL-93-56-3

LIST OF FIGURES

3-1 Monitoring Well Location Map for the 183-H Solar Evaporation Basins Network . . . . . . . . . . . . . . . . .

\section{LIST OF TABLES}

3-1 Monitoring Well Position and Sampling Schedule for the 183-H Solar Evaporation Basins Network. . . . . . . . . . . . 3-4

3-2 RCRA Water Level Measurement Report 183-H Solar Evaporation Basins, Third Quarter 1993 . . . . . . . . . . . . . . 3-6

3-3 Constituent List and Summary of Results for 183-H Solar Evaporation Basins Data for Reporting Period July 1 through September 30,1993.................. 3-10

3-4 Constituents with at Least One Value Above the CRQL for the 183-H Solar Evaporation Basins Data for Reporting Period July 1 through September 30, 1993

3-5 Contamination Indicator Parameters for the 183-H Solar Evaporation Basins Data for Reporting Period July 1 through September 30,1993................ 3-15 
$\mathrm{DOE} / \mathrm{RL}-93-56-3$

\title{
3.0 183-H SOLAR EVAPORATION BASINS
}

\author{
R. E. Peterson \\ Westinghouse Hanford Company
}

\subsection{INTRODUCTION}

The 183-H Solar Evaporation Basins are a RCRA-regulated treatment, storage, and/or disposal (TSD) facility located along the Columbia River in the 100 Area of the Hanford Site. The 183-H Solar Evaporation Basins were originally part of the water treatment facility that prepared coolant for the 105-H Reactor. Subsequent to that mission, between 1973 and 1985, the 183-H Solar Evaporation Basins were used for storage and volume reduction (by solar evaporation) of liquid waste from nuclear fuel processing activities conducted in the 300 Area. The predominant waste consisted of nitric acid solutions that had been neutralized with sodium hydroxide before discharge into the 183-H Solar Evaporation Basins. The solutions contained various metals and radioactive constituents. Hazardous wastes have been removed from the facility and the basin walls have been partially decontaminated. A soil investigation has been completed. An application for permanent closure is being reviewed by the Washington State Department of Ecology (Ecology). The groundwater monitoring program is being conducted under 40 CFR 265, Subpart F, and is an assessment level program.

When TSD operations began in 1973, only two groundwater wells existed in the 100-H Area, neither of which monitored conditions near the 183-H Solar Evaporation Basins. During the operational life of the 183-H Solar Evaporation Basins, limited groundwater monitoring occurred. In 1974, a single downgradient well was installed (199-H4-3). In 1983, two additional downgradient wells and one upgradient well were installed. Eighteen additional monitoring wells, which were constructed to RCRA standards, were installed in 1986 and 1987 after the facility ceased operation. These wells were constructed in response to a Consent Agreement and Compliance Order entered into by Ecology, EPA, and DOE (Ecology and EPA 1986).

Twenty-three wells are in use during 1993 for obtaining RCRA groundwater samples. One additional well $(199-\mathrm{H} 4-15 \mathrm{C})$ is used exclusively for water level measurements. A location map is shown in Figure 3-1. The purpose of each RCRA well, its sampling frequency, and the dates sampled during the quarter, are summarized in Table 3-1. Nitrate, sodium, gross alpha, and gross beta are used to monitor the extent of the plume attributable to the 183-H Solar Evaporation Basins' wastes. Wells 199-H4-3, 199-H4-4, 199-H4-9, and 199-H4-12A are within the plume.

\subsection{MATER LEVEL MEASUREMENTS}

During this quarter, water levels were measured in the monitoring well network at monthly intervals. Water levels also were recorded at the time of sample collection for water nuality analyses. Water level measurements obtained during the third quarter of 1993 are presented in Table 3-2. These levels are typical of the annual average level. Levels declined slightly in all wells during the third quarter, following the seasonal high that occurred 
duriig the second quarter. The water level in well 199-H4-15CQ is now at or slightly above the top-of-casing reference point. Levels began a steady rise during 1992, reflecting a possible connection between this piezometer and 199-H4-15CP, a deeper piezometer that monitors the uppermost confined aquifer in basalt.

\subsection{HATER CHEMISTRY DATA}

Third quarter sampling was completed in the majority of the 183-H Solar Evaporation Basins' network wells during July 1993. Three wells were also sampled monthly to provide enhanced monitoring of the plume during cleanup and closure activities. A summary of third quarter 1993 results is presented in Table 3-3. Analytical results for constituents with at least one value above the CRQL are presented in Tables 3-4 and 3-5. Blank entries in these tables indicate either the constituent was not included in the analys is list or the results are not yet available. If analytical results for previous sampling episodes were received during the current quarter, they are included in the tables.

For constituents relevant to assessing the 183-H plume, EPA DWSs are exceeded for chromium, gross alpha, gross beta, and nitrate (see Table 3-3). Other constituents that exceed DWSS are iron and manganese. The DWS for turbidity is exceeded in many network wells, although the standard is applicable only to surface water supplies, not to groundwater. One value for $\mathrm{pH}$ exceeds the DWS limits but is suspected to be a measurement error.

The pulse in 183-H indicator constituents that was reported in the previous quarterly report has continued into the third quarter. Increased levels of nitrate, sodium, sulfate, gross alpha, and gross beta are apparent in wells 199-H4-3 and 199-H4-4, which intercept the plume. These wells have al so shown increased turbidity levels during 1993.

Chromium is more widely distributed in the 100-H Area than the plume associated with the 183-H Solar Evaporation Basins (Peterson and Connelly 1992). The highest chronium concentrations are typically found in wells 199-H4-14 and 199-H4-12C, neither of which can be directly associated with the 183-H Solar Evaporation Basins. However, chromium concentrations in plume wells 199- $\mathrm{H4}-3$ and 199- $\mathrm{H4}-4$ are also elevated during 1993, relative to earlier long-term trends.

The quarterly assessment of the groundwater plume has not been influenced significantly by QC problems, although numerous results for chromium, iron, sodium, and zinc have been flagged with a ' $Q$ ' as the result of $Q C$ blank or duplicate samples being out of acceptable limits. A field measurement for $\mathrm{pH}$ appears erroneous and has been flagged accordingly. 
Figure 3-1. Monitoring Well Location Map for the 183-H Solar Evaporation Basins Network.

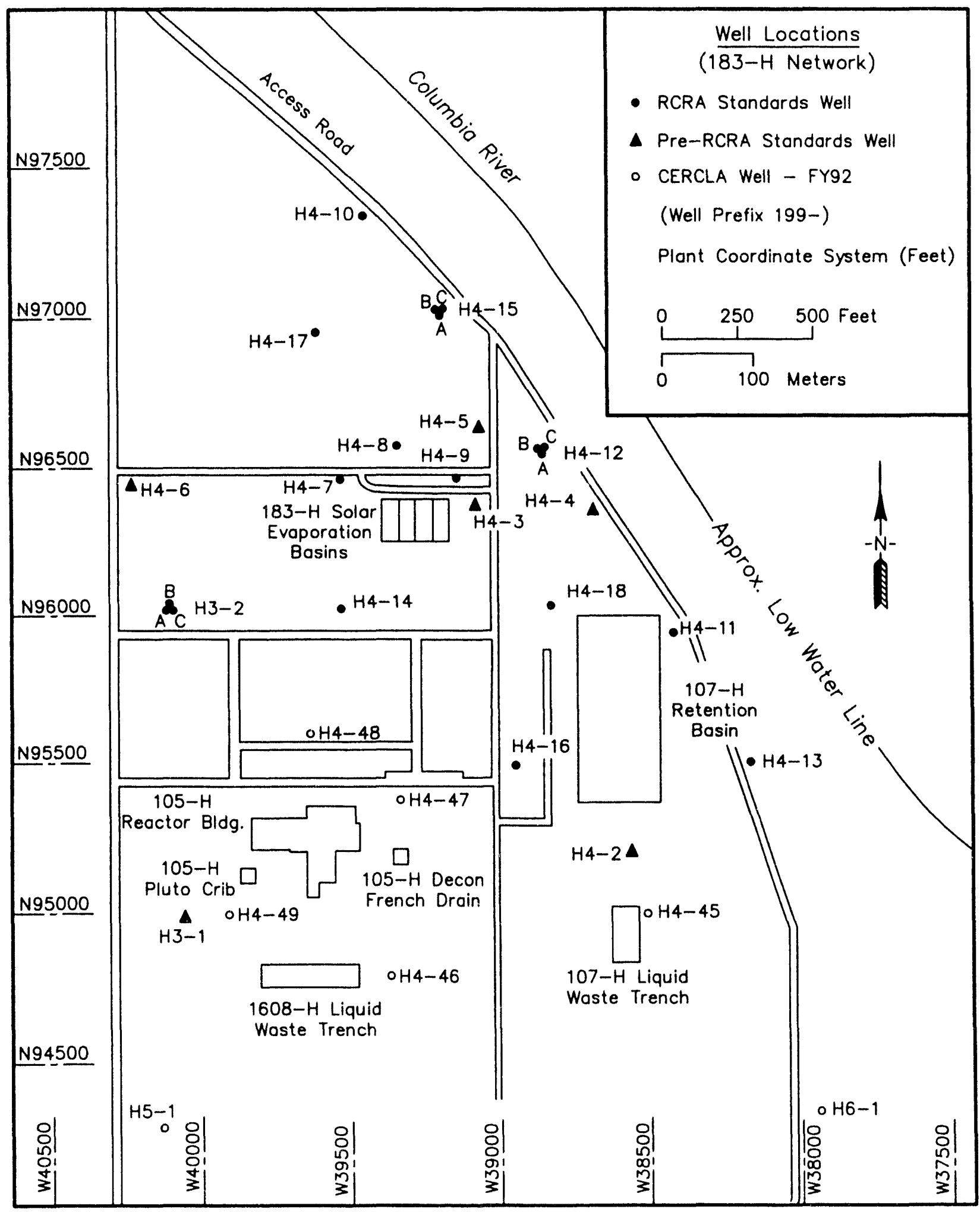

RCRA-AR \122993J 
Table 3-1. Monitoring Well Position and Sampling Schedule for the 183-H Solar Evaporation Basins Network. (sheet 1 of 2)

\begin{tabular}{|c|c|c|c|c|}
\hline $\begin{array}{l}\text { Wel1 no. } \\
(199-)\end{array}$ & $\begin{array}{l}\text { Relative } \\
\text { position }\end{array}$ & Hydrogeologic unit & $\begin{array}{c}\text { Sample } \\
\text { frequency }\end{array}$ & $\begin{array}{l}\text { Sample date, } \\
\text { 3rd Qtr } 1993\end{array}$ \\
\hline $\mathrm{H} 3-1$ & Upgradient & Hanford: Water Table & Annually & $8 / 05 / 93$ \\
\hline$H 3-2 A$ & Upgradient & Hanford: Water Table & Quarterly & $7 / 14 / 93$ \\
\hline$H 3-2 B$ & Upgradient & Hanford: Water Table & Annually & $7 / 14 / 93$ \\
\hline $\mathrm{H} 3-2 \mathrm{C}$ & Upgradient & Ringold: Mid-Depth & Quarterly & $7 / 14 / 93$ \\
\hline $\mathrm{H} 4-10$ & Upgradient & Hanford: Water Table & Annually & $7 / 29 / 93$ \\
\hline $\mathrm{H} 4-11$ & Downgradient & Hanford: Water Table & Quarterly & $7 / 13 / 93$ \\
\hline $\mathrm{H} 4-12 \mathrm{~A}$ & Downgradient & Hanford: Water Table & Quarterly & $7 / 29 / 93$ \\
\hline $\mathrm{H} 4-12 \mathrm{~B}$ & Downgradient & Hanford: Water Table & Annually & $7 / 13 / 93$ \\
\hline $\mathrm{H} 4-12 \mathrm{C}$ & Downgradient & Ringold: Mid-Depth & Quarterly & $7 / 13 / 93$ \\
\hline $\mathrm{H} 4-13$ & Downgradient & Hanford: Water Table & Annually & $7 / 29 / 93$ \\
\hline $\mathrm{H} 4-14$ & Upgradient & Hanford: Water Table & Quarterly & $7 / 13 / 93$ \\
\hline $\mathrm{H} 4-15 \mathrm{~A}$ & Downgradient & Hanford: Water Table & Quarterly & $7 / 13 / 93$ \\
\hline $\mathrm{H} 4-15 \mathrm{~B}$ & Downgradient & Hanford: Water Table & Annually & $7 / 29 / 93$ \\
\hline $\mathrm{H} 4-15 \mathrm{Cq}$ & Downgradient & Ringold: Deep & Monthly & Piezometer \\
\hline $\mathrm{H} 4-15 \mathrm{Cr}$ & Downgradient & Ringold: Mid-Depth & Monthly & Piezometer \\
\hline $\mathrm{H} 4-15 \mathrm{Cs}$ & Downgradient & Ringold: Mid-Depth & Monthlyb & Piezometer \\
\hline H4-16 & Downgradient & Hanford: Water Table & Annually & $7 / 29 / 93$ \\
\hline $\mathrm{H} 4-17$ & Upgradient & Hanford: Water Table & Annually & $8 / 05 / 93$ \\
\hline H4-18 & Downgradient & Hanford: Water Table & Quarterly & $7 / 21 / 93$ \\
\hline $\mathrm{H} 4-3$ & Downgradient & Hanford: Water Table & Monthly & $\begin{array}{l}7 / 13 / 93 \\
8 / 20 / 93 \\
9 / 07 / 93\end{array}$ \\
\hline $\mathrm{H} 4-4$ & Downgradient & Hanford: Water Table & Monthly & $\begin{array}{l}7 / 13 / 93 \\
8 / 18 / 93 \\
9 / 07 / 93\end{array}$ \\
\hline $\mathrm{H} 4-5$ & Downgradient & Hanford: Water Table & Quarterly & $7 / 13 / 93$ \\
\hline $\mathrm{H} 4-6$ & Upgradient & Hanford: Water Table & Quarterly & $7 / 13 / 93$ \\
\hline $\mathrm{H} 4-7$ & Upgradient & Hanford: Water Table & Quarterly & $7 / 13 / 93$ \\
\hline
\end{tabular}


Table 3-1. Monitoring Well Position and Sampling Schedule for the 183-H Solar Evaporation Basins Network. (sheet 2 of 2)

\begin{tabular}{|c|c|c|c|c|}
\hline $\begin{array}{c}\text { Well no. } \\
(199-)\end{array}$ & $\begin{array}{c}\text { Relative } \\
\text { position }\end{array}$ & Hydrogeologic unit & $\begin{array}{c}\text { Sample } \\
\text { freqliency }\end{array}$ & $\begin{array}{c}\text { Sample date, } \\
\text { 3rd Qtr 1993 }\end{array}$ \\
\hline H4-8 & Upgradient & Hanford: Water Table & Quarterly & $7 / 14 / 93$ \\
\hline H4-9 & Downgradient & Hanford: Water Table & Monthly & $\begin{array}{c}7 / 29 / 93 \\
8 / 09 / 93 \\
9 / 03 / 93\end{array}$ \\
\hline
\end{tabular}

aydrogeologic units include the sandy gravels of the Hanford formation and silty sands of the Ringold Formation. Water levels are measured monthly in all wells.

Water level measurement only. 
Table 3-2. RCRA Water Level Measurement Report 183-H Solar Evaporation Basins, Third Quarter 1993. (sheet 1 of 4 )

\begin{tabular}{|c|c|c|c|}
\hline Well & Date & $\begin{array}{l}\text { Depth to } \\
\text { water (ft) }\end{array}$ & $\begin{array}{l}\text { Water level } \\
\text { elevation } \\
\text { above msl (ft) }\end{array}$ \\
\hline \multicolumn{4}{|c|}{$\begin{array}{c}\text { Wells Monitoring the Top of the Unconfined Aquifer } \\
\text { (Hanford formation) }\end{array}$} \\
\hline 199-H3-1 & $\begin{array}{l}7 / 12 / 93 \\
8 / 05 / 93 \\
8 / 09 / 93 \\
9 / 13 / 93\end{array}$ & $\begin{array}{l}45.43 \\
45.55 \\
45.42 \\
45.71\end{array}$ & $\begin{array}{l}376.05 \\
375.93^{\star} \\
376.06 \\
375.77\end{array}$ \\
\hline $199-H 3-2 A$ & $\begin{array}{l}7 / 12 / 93 \\
7 / 14 / 93 \\
8 / 09 / 93 \\
9 / 13 / 93\end{array}$ & $\begin{array}{l}41.98 \\
42.01 \\
42.09 \\
42.54\end{array}$ & $\begin{array}{l}375.85 \\
375.82^{\star} \\
375.74 \\
375.29\end{array}$ \\
\hline $199-H 3-2 B$ & $\begin{array}{l}7 / 12 / 93 \\
7 / 14 / 93 \\
8 / 09 / 93 \\
9 / 13 / 93\end{array}$ & $\begin{array}{l}42.55 \\
42.56 \\
42.69 \\
43.13\end{array}$ & $\begin{array}{l}375.87 \\
375.86 \star \\
375.73 \\
375.29\end{array}$ \\
\hline 199-H4-10 & $\begin{array}{l}7 / 12 / 93 \\
7 / 29 / 93 \\
8 / 09 / 93 \\
9 / 13 / 93\end{array}$ & $\begin{array}{l}30.12 \\
30.05 \\
31.09 \\
31.85\end{array}$ & $\begin{array}{l}374.32 \\
374.39 \star \\
373.35 \\
372.59\end{array}$ \\
\hline $199-H 4-11$ & $\begin{array}{l}7 / 12 / 93 \\
7 / 13 / 93 \\
8 / 09 / 93 \\
9 / 13 / 93\end{array}$ & $\begin{array}{l}43.25 \\
42.98 \\
44.93 \\
45.51\end{array}$ & $\begin{array}{l}373.59 \\
373.86 \star \\
371.91 \\
371.33\end{array}$ \\
\hline $199-\mathrm{H} 4-12 \mathrm{~A}$ & $\begin{array}{l}7 / 12 / 93 \\
7 / 29 / 93 \\
8 / 09 / 93 \\
9 / 13 / 93\end{array}$ & $\begin{array}{l}39.46 \\
39.26 \\
40.64 \\
41.23\end{array}$ & $\begin{array}{l}374.04 \\
374.24^{\star} \\
372.86 \\
372.27\end{array}$ \\
\hline $199-\mathrm{H} 4-12 \mathrm{~B}$ & $\begin{array}{l}7 / 12 / 93 \\
7 / 13 / 93 \\
8 / 09 / 93 \\
9 / 13 / 93\end{array}$ & $\begin{array}{l}39.47 \\
39.16 \\
40.53 \\
41.24\end{array}$ & $\begin{array}{l}374.05 \\
374.36 \star \\
372.99 \\
372.28\end{array}$ \\
\hline $199-\mathrm{H} 4-13$ & $\begin{array}{l}7 / 12 / 93 \\
7 / 29 / 93 \\
8 / 09 / 93 \\
9 / 13 / 93\end{array}$ & $\begin{array}{l}44.83 \\
44.46 \\
46.84 \\
47.37\end{array}$ & $\begin{array}{l}373.37 \\
373.74^{\star} \\
371.36 \\
370.83\end{array}$ \\
\hline
\end{tabular}


Table 3-2. RCRA Water Level Measurement Report 183-H Solar Evaporation Basins, Third Quarter 1993.

(sheet 2 of 4 )

\begin{tabular}{|c|c|c|c|}
\hline Well & Date & $\begin{array}{l}\text { Depth to } \\
\text { water (ft) }\end{array}$ & $\begin{array}{l}\text { Water level } \\
\text { elevation } \\
\text { above ms } 1 \text { (ft) }\end{array}$ \\
\hline \multicolumn{4}{|c|}{$\begin{array}{c}\text { Wells Monitoring the Top of the Unconfined Aquifer } \\
\text { (Hanford formation) }\end{array}$} \\
\hline $199-H 4-14$ & $\begin{array}{l}7 / 12 / 93 \\
7 / 13 / 93 \\
8 / 09 / 93 \\
9 / 13 / 93\end{array}$ & $\begin{array}{l}44.99 \\
44.98 \\
45.17 \\
45.67\end{array}$ & $\begin{array}{l}375.60 \\
375.61 * \\
375.42 \\
374.92\end{array}$ \\
\hline $199-H 4-15 A$ & $\begin{array}{l}7 / 12 / 93 \\
7 / 13 / 93 \\
8 / 09 / 93 \\
9 / 13 / 93\end{array}$ & $\begin{array}{l}32.99 \\
32.64 \\
33.99 \\
34.70\end{array}$ & $\begin{array}{l}374.22 \\
374.57 \star \\
373.22 \\
372.51\end{array}$ \\
\hline $199-\mathrm{H} 4-15 B$ & $\begin{array}{l}7 / 12 / 93 \\
7 / 29 / 93 \\
8 / 09 / 93 \\
9 / 13 / 93\end{array}$ & $\begin{array}{l}32.71 \\
32.54 \\
33.72 \\
34.40\end{array}$ & $\begin{array}{l}374.21 \\
374.38^{\star} \\
373.20 \\
372.52\end{array}$ \\
\hline $199-\mathrm{H} 4-16$ & $\begin{array}{l}7 / 12 / 93 \\
7 / 12 / 93 \\
7 / 29 / 93 \\
8 / 09 / 93 \\
9 / 13 / 93\end{array}$ & $\begin{array}{l}49.07 \\
47.04 \\
49.17 \\
49.32 \\
49.93\end{array}$ & $\begin{array}{l}375.16 \\
377.19 \\
375.06^{\star} \\
374.91 \\
374.30\end{array}$ \\
\hline $199-\mathrm{H} 4-17$ & $\begin{array}{l}7 / 12 / 93 \\
8 / 05 / 93 \\
8 / 09 / 93 \\
9 / 13 / 93\end{array}$ & $\begin{array}{l}44.30 \\
44.78 \\
45.20 \\
45.58\end{array}$ & $\begin{array}{l}374.79 \\
374.31 * \\
373.89 \\
373.51\end{array}$ \\
\hline $199-H 4-18$ & $\begin{array}{l}7 / 12 / 93 \\
7 / 21 / 93 \\
8 / 09 / 93 \\
9 / 13 / 93\end{array}$ & $\begin{array}{l}47.04 \\
46.00 \\
47.57 \\
48.33\end{array}$ & $\begin{array}{l}374.78 \\
375.82^{\star} \\
374.25 \\
373.49\end{array}$ \\
\hline $199-\mathrm{H} 4-3$ & $\begin{array}{l}7 / 12 / 93 \\
7 / 13 / 93 \\
8 / 09 / 93 \\
8 / 20 / 93 \\
9 / 07 / 93 \\
9 / 13 / 93\end{array}$ & $\begin{array}{l}45.61 \\
45.67 \\
46.15 \\
46.52 \\
47.04 \\
47.00\end{array}$ & $\begin{array}{l}374.68 \\
374.62^{\star} \\
374.14 \\
373.77^{\star} \\
373.25^{\star} \\
373.29\end{array}$ \\
\hline
\end{tabular}


Table 3-2. RCRA Water Level Measurement Report 183-H Solar Evaporation Basins, Third Quarter 1993. (sheet 3 of 4 )

\begin{tabular}{|c|c|c|c|}
\hline Well & Date & $\begin{array}{l}\text { Depth to } \\
\text { water (ft) }\end{array}$ & $\begin{array}{l}\text { Water level } \\
\text { elevation } \\
\text { above msl (ft) }\end{array}$ \\
\hline \multicolumn{4}{|c|}{$\begin{array}{l}\text { Wells Monitoring the Top of the Unconfined Aquifer } \\
\text { (Hanford formation) }\end{array}$} \\
\hline $199-\mathrm{H} 4-4$ & $\begin{array}{l}7 / 12 / 93 \\
7 / 13 / 93 \\
8 / 09 / 93 \\
8 / 18 / 93 \\
9 / 07 / 93 \\
9 / 13 / 93\end{array}$ & $\begin{array}{l}39.80 \\
39.51 \\
41.18 \\
40.82 \\
42.10 \\
41.83\end{array}$ & $\begin{array}{l}373.90 \\
374.19 \star \\
372.52 \\
372.88^{\star} \\
371.60^{\star} \\
371.87\end{array}$ \\
\hline $199-\mathrm{H} 4-5$ & $\begin{array}{l}7 / 12 / 93 \\
7 / 13 / 93 \\
8 / 09 / 93 \\
9 / 13 / 93\end{array}$ & $\begin{array}{l}41.88 \\
41.80 \\
42.77 \\
43.67\end{array}$ & $\begin{array}{l}374.33 \\
374.41^{\star} \\
373.44 \\
372.54\end{array}$ \\
\hline $199-\mathrm{H} 4-6$ & $\begin{array}{l}7 / 12 / 93 \\
7 / 13 / 93 \\
8 / 09 / 93 \\
9 / 13 / 93\end{array}$ & $\begin{array}{l}43.73 \\
43.77 \\
43.86 \\
44.30\end{array}$ & $\begin{array}{l}375.85 \\
375.81^{\star} \\
375.72 \\
375.28\end{array}$ \\
\hline $199-\mathrm{H} 4-7$ & $\begin{array}{l}7 / 12 / 93 \\
7 / 13 / 93 \\
8 / 09 / 93 \\
9 / 13 / 93\end{array}$ & $\begin{array}{l}45.33 \\
45.39 \\
45.67 \\
46.33\end{array}$ & $\begin{array}{l}375.26 \\
375.20^{\star} \\
374.92 \\
374.26\end{array}$ \\
\hline $199-\mathrm{H} 4-8$ & $\begin{array}{l}7 / 12 / 93 \\
7 / 14 / 93 \\
8 / 09 / 93 \\
9 / 13 / 93\end{array}$ & $\begin{array}{l}45.02 \\
45.08 \\
45.48 \\
46.32\end{array}$ & $\begin{array}{l}374.98 \\
374.92^{\star} \\
374.52 \\
373.68\end{array}$ \\
\hline $199-\mathrm{H} 4-9$ & $\begin{array}{l}7 / 12 / 93 \\
7 / 29 / 93 \\
8 / 09 / 93 \\
9 / 13 / 93\end{array}$ & $\begin{array}{l}43.35 \\
43.68 \\
43.97 \\
44.93\end{array}$ & $\begin{array}{l}374.73 \\
374.40^{\star} \\
374.11 \\
373.15\end{array}$ \\
\hline
\end{tabular}


Table 3-2. RCRA Water Level Measurement Report 183-H Solar Evaporation Basins, Third Quarter 1993. (sheet 4 of 4 )

\begin{tabular}{cccc}
\hline Well & Date & $\begin{array}{c}\text { Depth to } \\
\text { water (ft) }\end{array}$ & $\begin{array}{c}\text { Water level } \\
\text { elevation } \\
\text { above ms 1 (ft) }\end{array}$ \\
\hline Wel1s Monitoring Upper Levels in the Unconfined Aquifer \\
& (Ringold Formation) & \\
\hline 199-H3-2C & $7 / 12 / 93$ & 42.52 & 375.70 \\
& $7 / 14 / 93$ & 42.50 & $375.72^{\star}$ \\
& $8 / 09 / 93$ & 42.77 & 375.45 \\
$9 / 13 / 93$ & 43.24 & 374.98 \\
$199-\mathrm{H} 4-12 \mathrm{C}$ & $7 / 12 / 93$ & 39.96 & 373.56 \\
& $7 / 13 / 93$ & 39.86 & $373.66 *$ \\
$8 / 09 / 93$ & 41.07 & 372.45 \\
$9 / 13 / 93$ & 41.77 & 371.75 \\
\hline
\end{tabular}

Well Monitoring Mid-Level in the Unconfined Aquifer (Ringold Formation)

\begin{tabular}{llll}
\hline $199-\mathrm{H} 4-15 \mathrm{CS}$ & $7 / 12 / 93$ & 33.60 & 373.84 \\
& $8 / 09 / 93$ & 34.66 & 372.78 \\
& $9 / 13 / 93$ & 35.25 & 372.19 \\
$199-\mathrm{H} 4-15 \mathrm{CR}$ & $7 / 12 / 93$ & 32.70 & 374.67 \\
& $8 / 09 / 93$ & 33.01 & 374.36 \\
& $9 / 13 / 93$ & 33.63 & 373.74 \\
\hline
\end{tabular}

Well Monitoring Bottom of Uppermost Aquifer System (Ringold Formation)

\begin{tabular}{lrrr}
\hline $199-\mathrm{H} 4-15 \mathrm{CQ}$ & $7 / 12 / 93$ & 0.01 & 407.26 \\
& $9 / 13 / 93$ & -0.01 & 407.28 \\
\hline
\end{tabular}

Notes: 1. Water level elevations are calculated by subtracting the measured depth to water from the surveyed elevation for the well.

2. Depth-to-water values are transcribed from field records.

3. Elevations marked with an $1 * 1$ were measured at the time of sampling.

4. To convert feet to meters multiply by 0.3048 . 
Table 3-3. Constituent List and Summary of Results for 183-H Solar Evaporation Basins Data for Reporting Period July 1 through September 30, 1993.

CONTAMINATION INDICATOR PARAMETERS

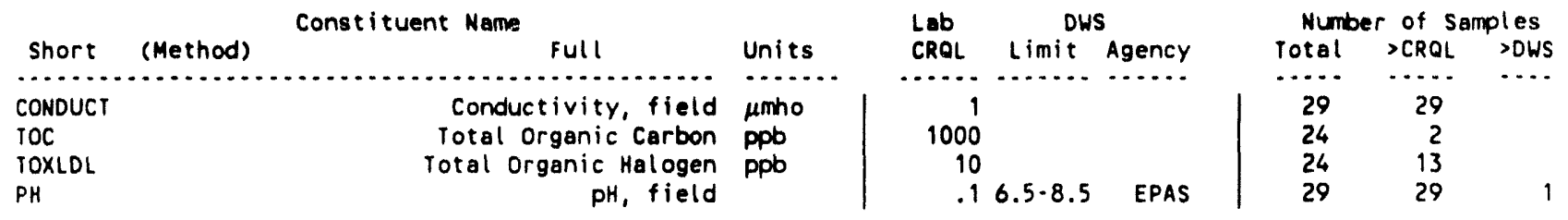

DRINKING WATER PARAMETERS

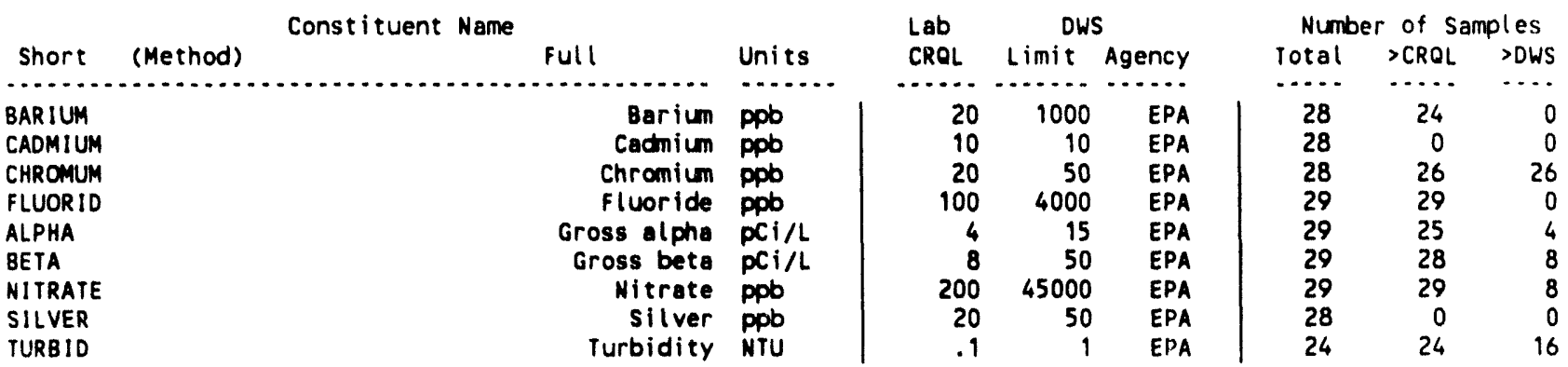

GROUNDWATER QUALITY PARAMETERS

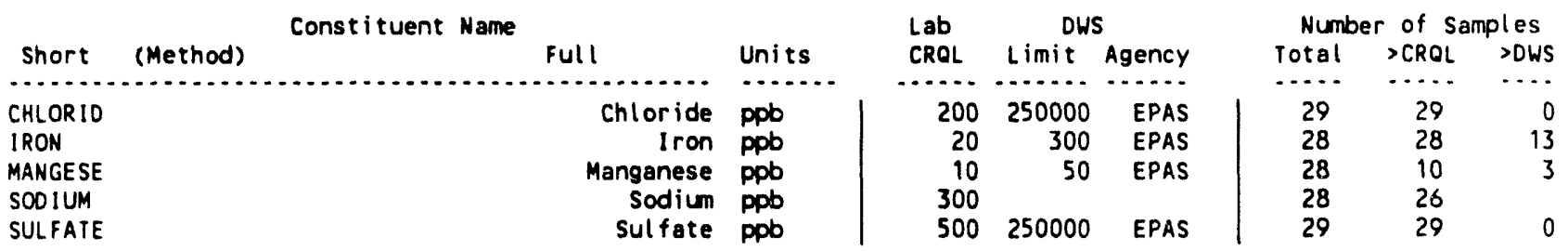

SITE SPECIFIC AND OTHER CONSTITUENTS

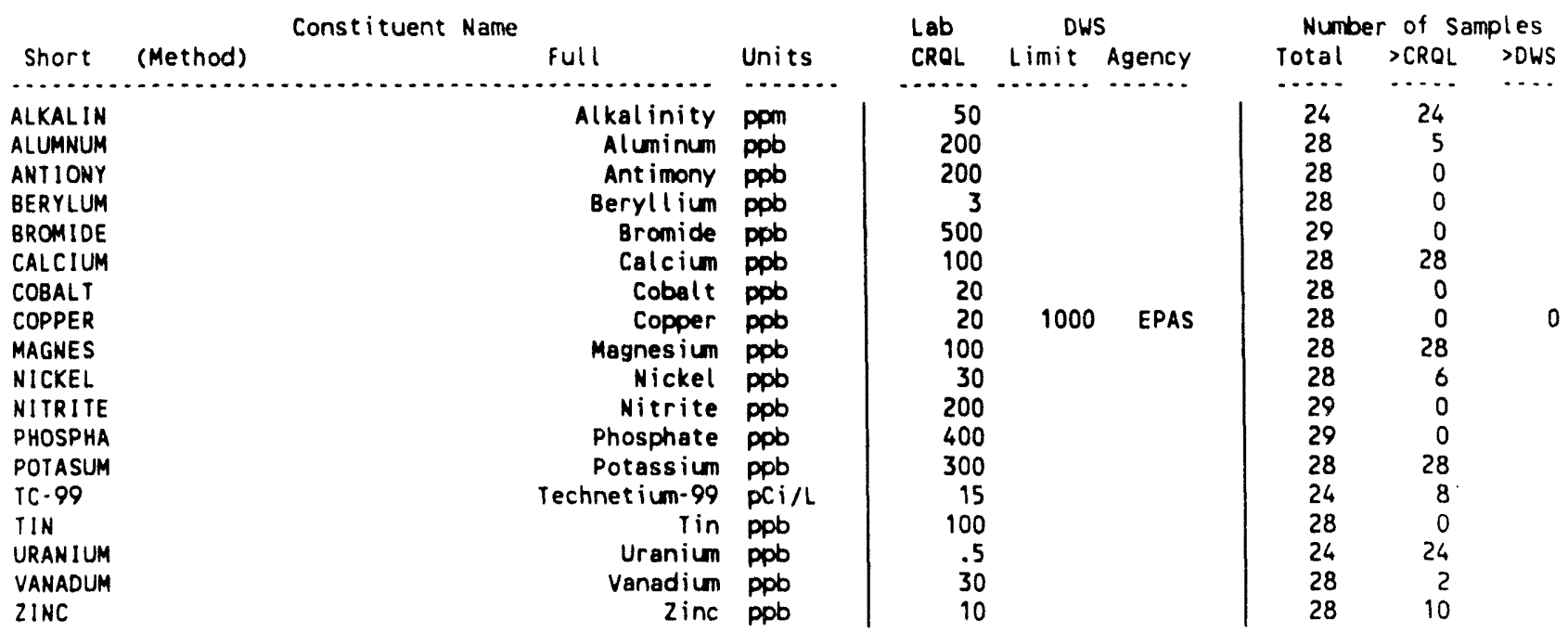

For explanation of this table, see Section 1.4 of report. 
Table 3-4. Constituents with at Least One Value Above the CRQL for the 183-H Solar Evaporation Basins Data for Reporting Period July 1 through September 30, 1993. (sheet 1 of 4)

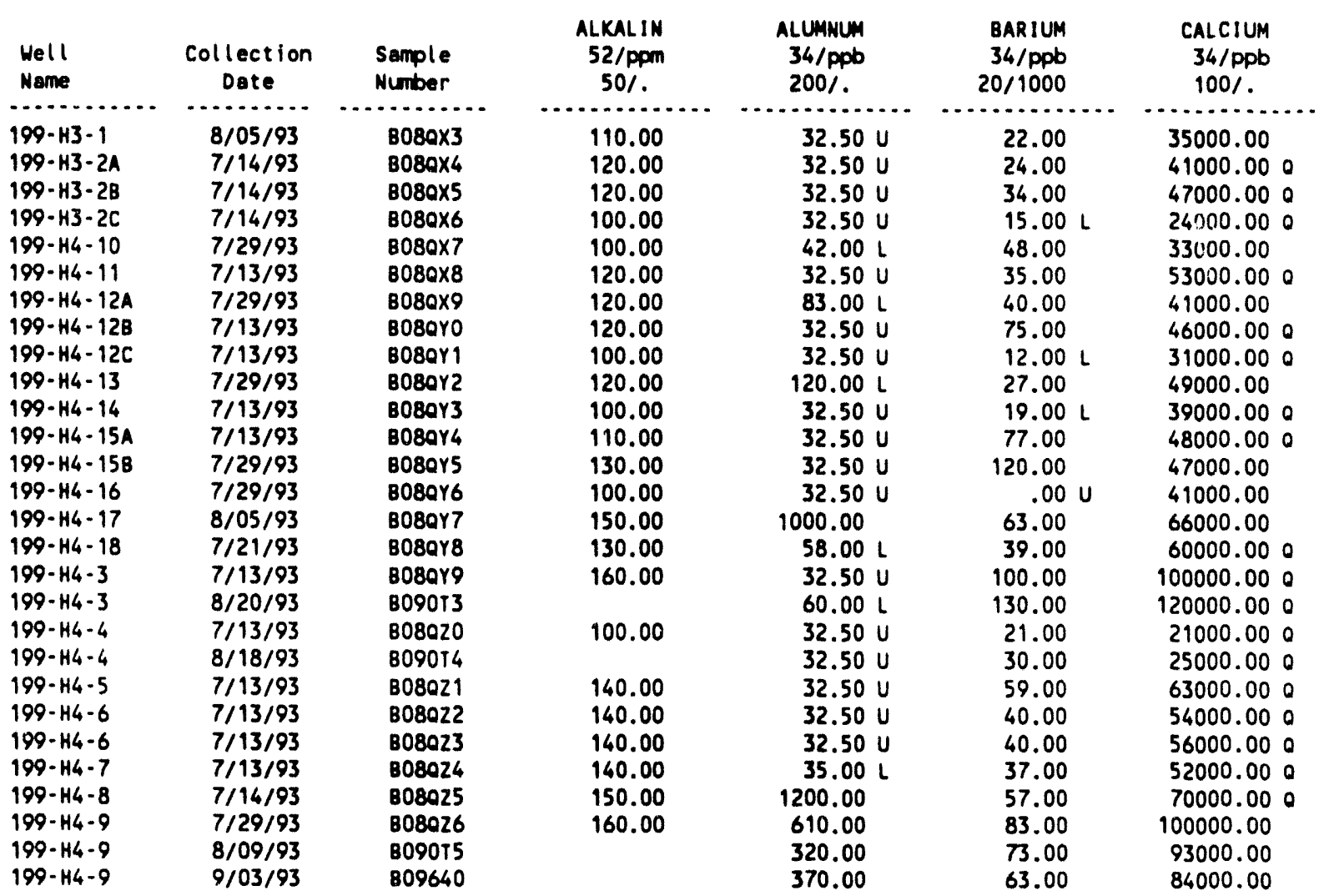

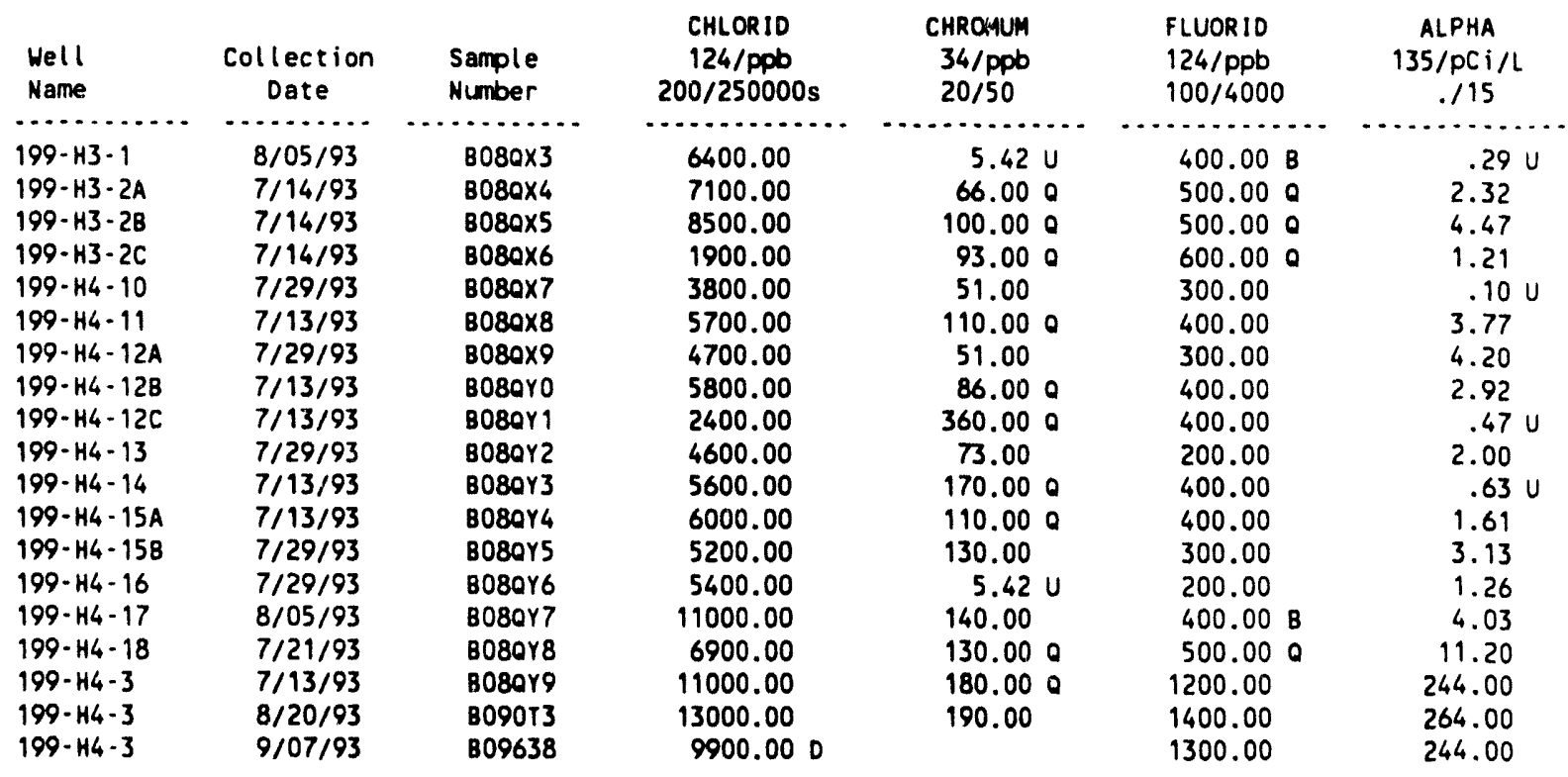


Table 3-4. Constituents with at Least One Value Above the CRQL for the 183-H Solar Evaporation Basins Data for Reporting Period July 1 through September 30, 1993. (sheet 2 of 4 )

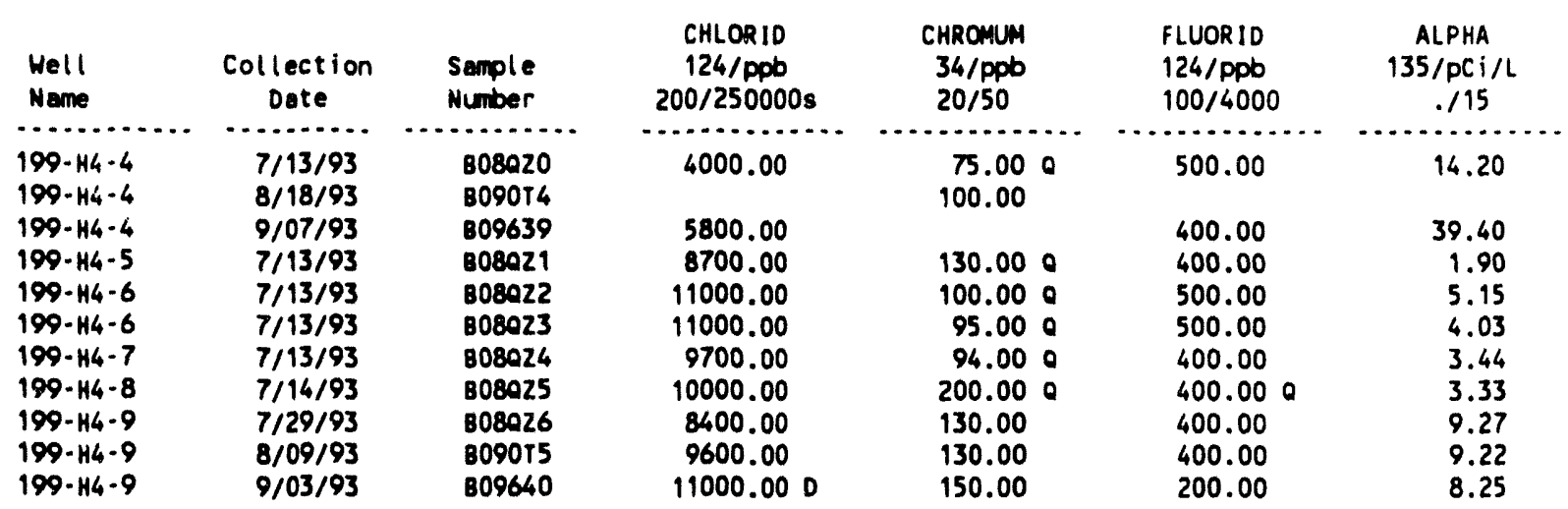

\begin{tabular}{|c|c|c|c|c|c|c|c|}
\hline $\begin{array}{l}\text { Well } \\
\text { Name }\end{array}$ & $\begin{array}{c}\text { Collection } \\
\text { Date }\end{array}$ & $\begin{array}{l}\text { Sample } \\
\text { Number }\end{array}$ & $\begin{array}{c}\text { BETA } \\
136 / \mathrm{pCi} / L \\
. / 50\end{array}$ & $\begin{array}{c}\text { IRON } \\
34 / \mathrm{ppb} \\
20 / 300 \mathrm{~s}\end{array}$ & & $\begin{array}{l}\text { MAGNES } \\
36 / \mathrm{ppb} \\
100 / .\end{array}$ & $\begin{array}{l}\text { MANGESE } \\
34 / \mathrm{ppb} \\
10 / 50 \mathrm{~s}\end{array}$ \\
\hline$\cdots \ldots \ldots \ldots$ & $\cdots \ldots \ldots$ & $\cdots \ldots \ldots \ldots$ & $\ldots \ldots$ & $\cdots$ & $\cdots$ & $\ldots \ldots$ & \\
\hline $\begin{array}{l}199-\mathrm{H} 3-1 \\
199-\mathrm{H}-2 \mathrm{~A}\end{array}$ & $\begin{array}{l}8 / 05 / 93 \\
7 / 14 / 93\end{array}$ & $\begin{array}{l}8080 \times 3 \\
8080 \times 4\end{array}$ & $\begin{array}{l}3.75 \\
5.31\end{array}$ & $\begin{array}{r}1100.00 \\
130.00\end{array}$ & 0 & $\begin{array}{l}8800.00 \\
9500.00\end{array}$ & $\begin{array}{r}17.00 \\
2.50\end{array}$ \\
\hline $199 \cdot \mathrm{H} 3-2 \mathrm{~B}$ & $7 / 14 / 93$ & $8080 \times 5$ & 7.82 & 170.00 & 0 & 11000.00 & 4.80 \\
\hline $199 \cdot \mathrm{H} 3 \cdot 2 \mathrm{C}$ & $7 / 14 / 93$ & $8080 \times 6$ & $2.21 \mathrm{U}$ & 390.00 & 0 & 8300.00 & 8.70 \\
\hline $199 \cdot \mathrm{H} 4 \cdot 10$ & $7 / 29 / 93$ & $8080 \times 7$ & 5.02 & 110.00 & & 7600.00 & 1.35 \\
\hline $199-144-11$ & $7 / 13 / 93$ & $8080 \times 8$ & 39.40 & 78.00 & 0 & 7700.00 & 2.00 \\
\hline $199 \cdot \mathrm{H} 4 \cdot 12 \mathrm{~A}$ & $7 / 29 / 93$ & $8080 \times 9$ & 6.91 & 130.00 & & 6900.00 & 1.35 \\
\hline $199-144-12 B$ & $7 / 13 / 93$ & Bosero & 6.65 & 120.00 & 0 & 7700.00 & 2.10 \\
\hline $199-\mathrm{H} 4-12 \mathrm{C}$ & $7 / 13 / 93$ & Bosar 1 & 3.34 & 350.00 & 0 & 11000.00 & 8.40 \\
\hline $199-H 4-13$ & $7 / 29 / 93$ & Bo80Y2 & 59.10 & 190.00 & & 8300.00 & 1.35 \\
\hline $199-144-14$ & $7 / 13 / 93$ & $8080 Y 3$ & 5.12 & 110.00 & 0 & 7400.00 & 2.30 \\
\hline $199-H 4-15 A$ & $7 / 13 / 93$ & Boser4 & 4.25 & 65.00 & 0 & 9700.00 & 1.60 \\
\hline $199-144-15 B$ & $7 / 29 / 93$ & Bo8er5 & 4.15 & 100.00 & & 11000.00 & 1.35 \\
\hline $199-144-16$ & $7 / 29 / 93$ & Bo8er6 & 11.50 & 77.00 & & 5200.00 & 1.35 \\
\hline $199-\mathrm{H} 4-17$ & $8 / 05 / 93$ & B080Y7 & 7.19 & 1500.00 & & 13000.00 & 33.00 \\
\hline $199-H 4-18$ & $7 / 21 / 93$ & 8080Y8 & 54.10 & 160.00 & 0 & 11000.00 & 4.60 \\
\hline $199-144-3$ & $7 / 13 / 93$ & Bosay9 & 502.00 & 410.00 & 0 & 18000.00 & 10.00 \\
\hline $199-144-3$ & $8 / 20 / 93$ & B090T3 & 764.00 & 450.00 & & 21000.00 & 11.00 \\
\hline $199-114-3$ & $9 / 07 / 93$ & 809638 & 510.00 & & & & \\
\hline $199 \cdot 114-4$ & $7 / 13 / 93$ & 808020 & 36.50 & 250.00 & 0 & 3700.00 & 4.70 \\
\hline $\begin{array}{l}199-114-4 \\
199-144-4\end{array}$ & $\begin{array}{l}8 / 18 / 93 \\
9 / 07 / 93\end{array}$ & $\begin{array}{l}809074 \\
809639\end{array}$ & 130.00 & 290.00 & & 4200.00 & 4.80 \\
\hline $199-114-5$ & $7 / 13 / 93$ & B08021 & 5.25 & 430.00 & 0 & 11000.00 & 5.30 \\
\hline $199-114-6$ & $7 / 13 / 93$ & B08022 & 7.26 & 880.00 & 0 & 13000.00 & 61.00 \\
\hline $199-144-6$ & $7 / 13 / 93$ & 808023 & 7.27 & 850.00 & 0 & 14000.00 & 62.00 \\
\hline $199-1 \mathrm{H} 4-7$ & $7 / 13 / 93$ & B08024 & 7.43 & 120.00 & 0 & $10000 . C 9$ & 2.90 \\
\hline $199-114-8$ & $7 / 14 / 93$ & 808025 & 8.76 & 2700.00 & 0 & 13000.00 & 86.00 \\
\hline $199-114-9$ & $7 / 29 / 93$ & B08026 & 161.00 & 920.00 & & 19000.00 & 22.00 \\
\hline $199-144-9$ & $8 / 09 / 93$ & B09015 & 97.00 & 580.00 & & 17000.00 & 15.00 \\
\hline $199-144-9$ & $9 / 03 / 93$ & 809640 & 40.10 & 840.00 & & 13000.00 & 23.00 \\
\hline
\end{tabular}


Table 3-4. Constituents with at Least One Value Above the CRQL for the 183-H Solar Evaporation Basins Data for Reporting Period July 1 through September 30, 1993. (sheet 3 of 4 )

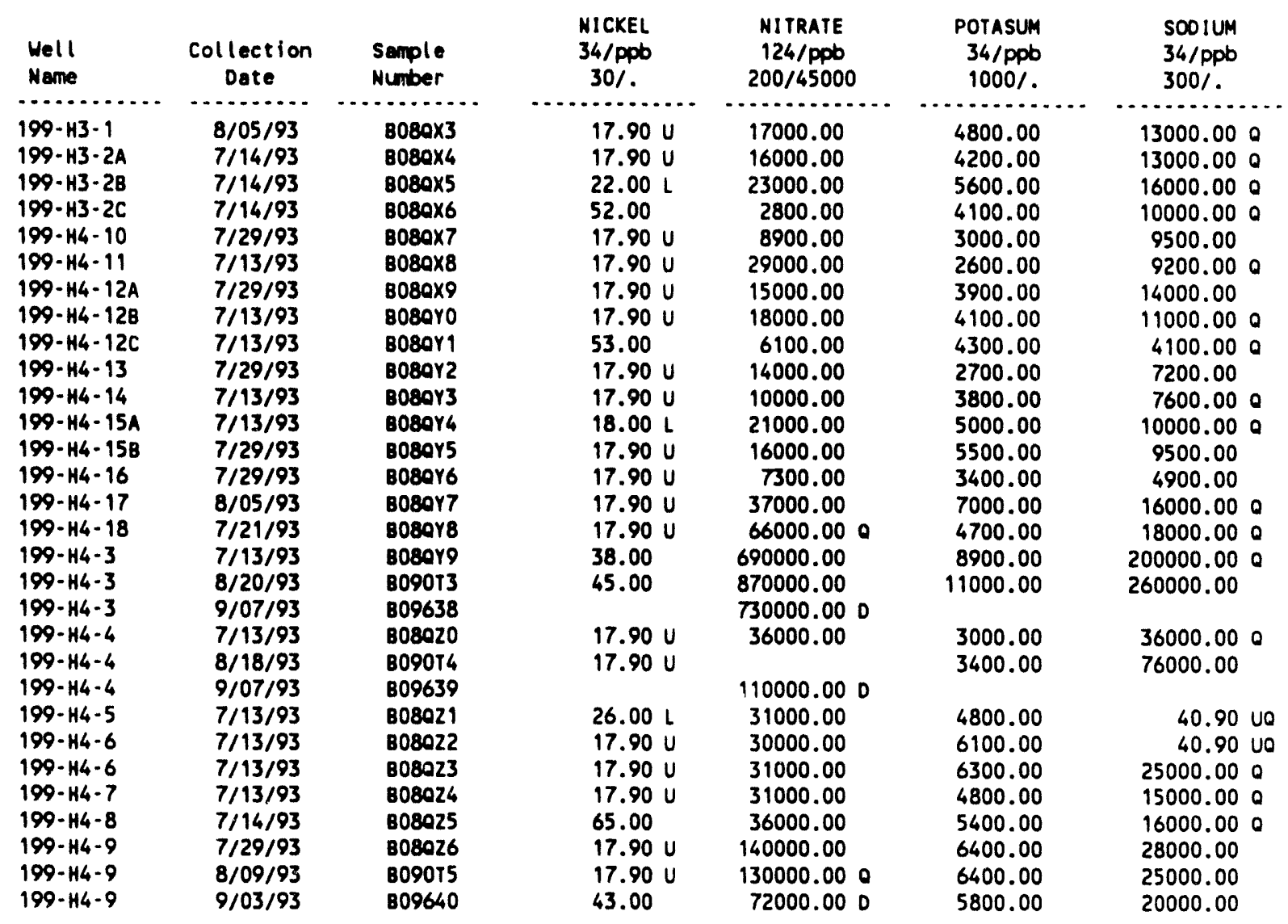

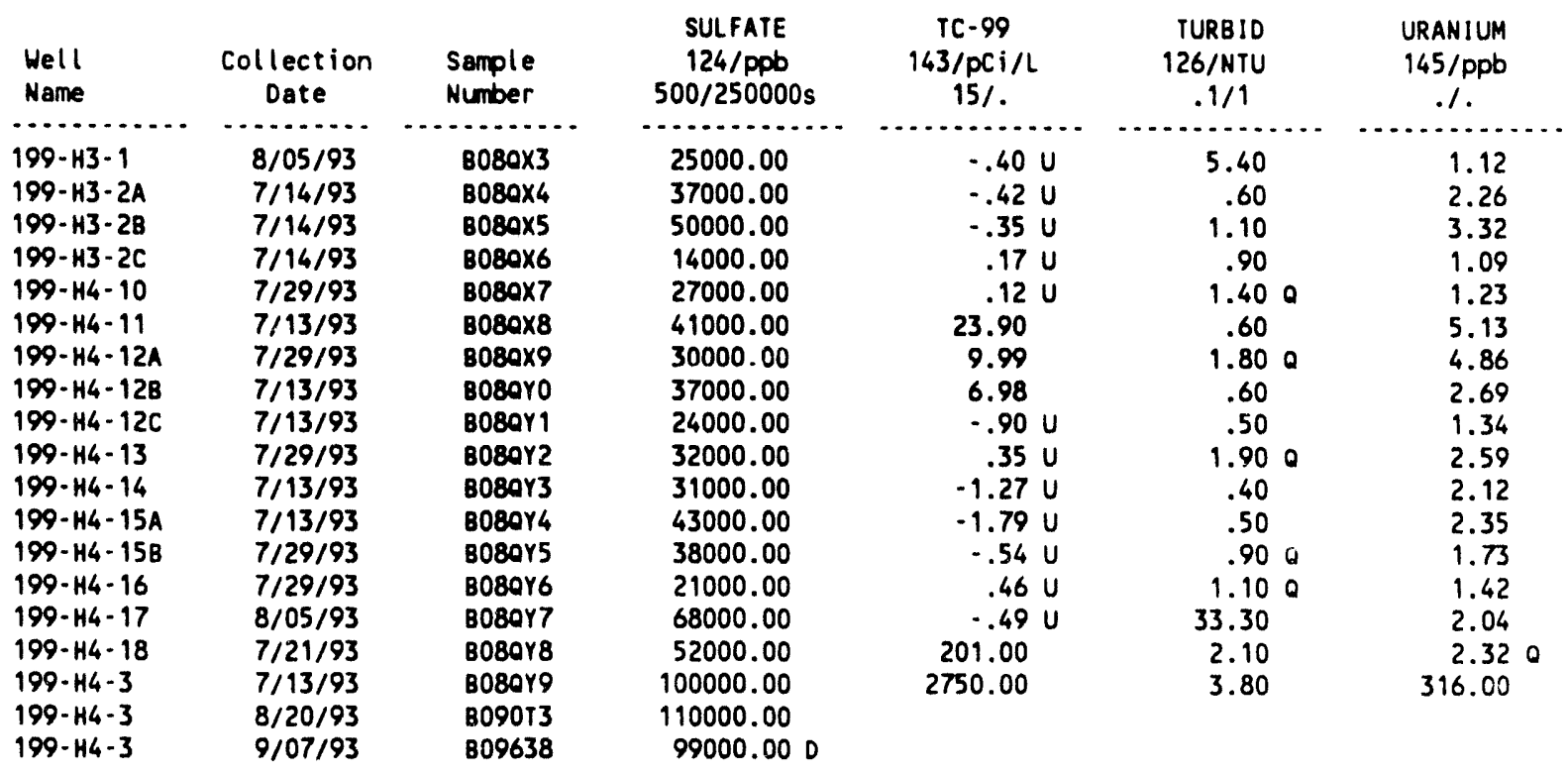


Table 3-4. Constituents with at Least One Value Above the CRQL for the 183-H Solar Evaporation Basins Data for Reporting Period July 1 through September 30,1993 . (sheet 4 of 4 )

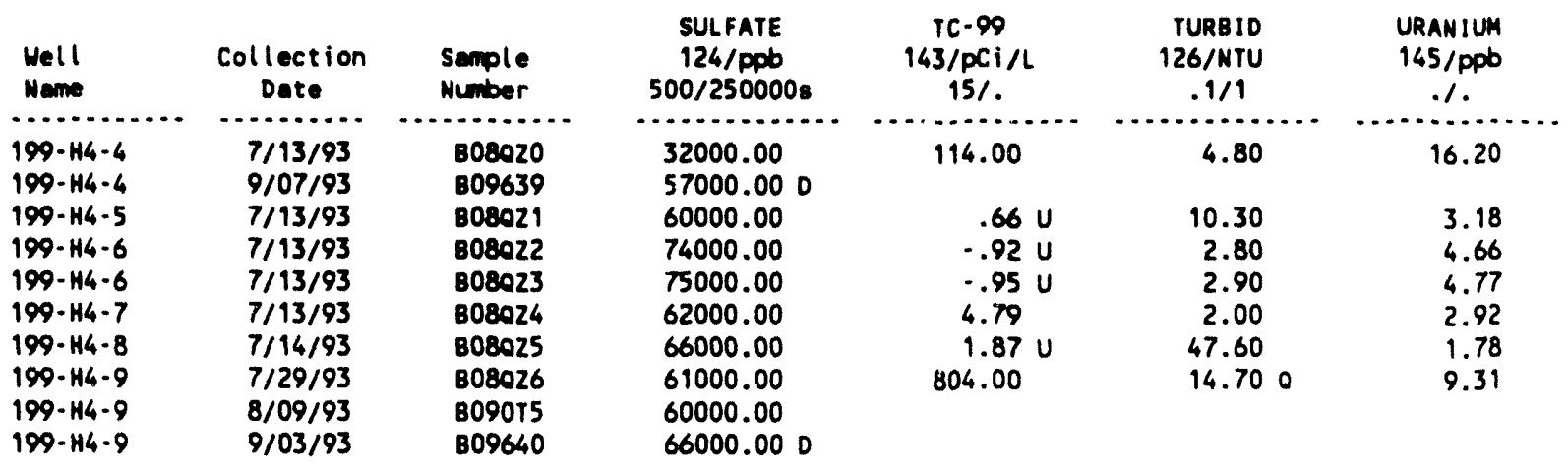

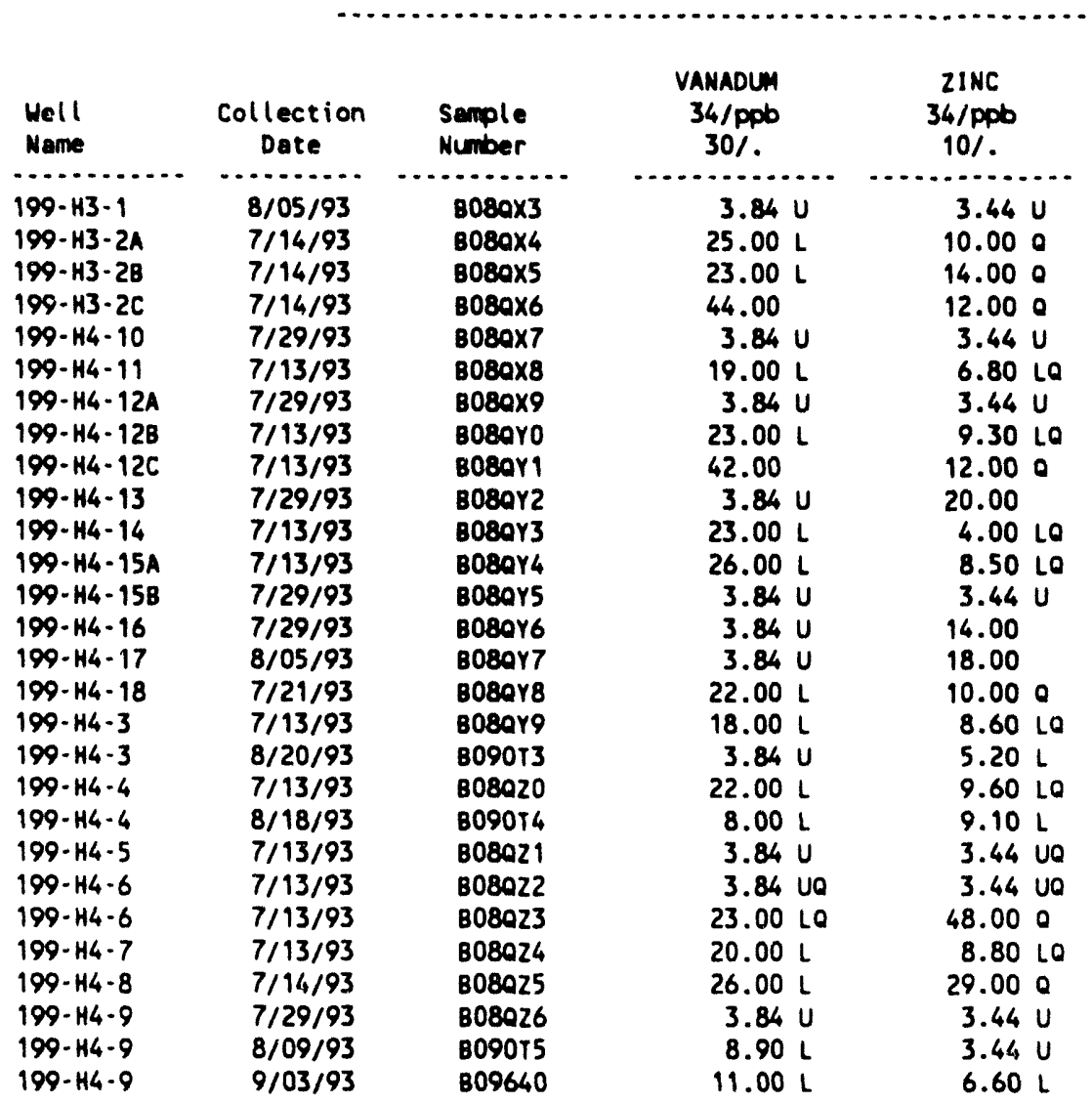


Table 3-5. Contamination Indicator Parameters for the 183-H Solar Evaporation Basins Data for Reporting Period July 1 through September 30, 1993.

\begin{tabular}{|c|c|c|c|c|c|c|c|c|}
\hline $\begin{array}{l}\text { Well } \\
\text { Name }\end{array}$ & $\begin{array}{c}\text { Collection } \\
\text { Date }\end{array}$ & $\begin{array}{l}\text { Sample } \\
\text { Number }\end{array}$ & $\begin{array}{c}\text { COND FIELD } \\
\substack{\mu \text { mho } \\
1 / 700 \mathrm{w}}\end{array}$ & $\begin{array}{c}\text { COND LAB } \\
\mu \text { mho } \\
. / 700_{W}\end{array}$ & $\begin{array}{l}\text { FH FIELD } \\
.01 / 6.5-8.5 \mathrm{~s}\end{array}$ & $\begin{array}{c}\text { PH LAB } \\
.01 / 6.5-8.5 \mathrm{~s}\end{array}$ & $\begin{array}{l}\text { TOC } \\
\text { ppb } \\
1000 \%\end{array}$ & $\begin{array}{c}\text { TOx } \\
\mathrm{ppb} \\
10 \%\end{array}$ \\
\hline $199-43-1$ & (1) & anger & 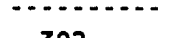 & & 7 & & 500 & \\
\hline $\begin{array}{l}199-H 3-1 \\
199-H 3-2 A\end{array}$ & $\begin{array}{l}8 / 05 / 93 \\
7 / 14 / 93\end{array}$ & $\begin{array}{l}8080 \times 3 \\
8080 \times 4\end{array}$ & $\begin{array}{l}302 \\
356\end{array}$ & & $\begin{array}{l}7.94 \\
7.62\end{array}$ & & $\begin{array}{l}500 \mathrm{~L} \\
500 \mathrm{~L}\end{array}$ & $\begin{array}{l}20.0 \mathrm{P} \\
10.0 \mathrm{P}\end{array}$ \\
\hline $199-H 3-28$ & $7 / 14 / 93$ & $8080 \times 5$ & 403 & & 8.09 & & $600 \mathrm{~L}$ & 8.0 UP \\
\hline $199-H 3-2 C$ & $7 / 14 / 93$ & B080X6 & 232 & & 7.82 & & $200 \mathrm{~L}$ & 8.0 UP \\
\hline $199-H 4-10$ & $7 / 29 / 93$ & $8080 \times 7$ & 272 & & 7.71 & & $500 \mathrm{~L}$ & $20.0 \mathrm{P}$ \\
\hline $199-H 4-11$ & $7 / 13 / 93$ & $8080 \times 8$ & 378 & & 7.87 & & $700 \mathrm{~L}$ & $20.0 \mathrm{P}$ \\
\hline $\begin{array}{l}199-H 4-12 A \\
199-H 4-12 B\end{array}$ & $7 / 29 / 93$ & $\begin{array}{l}8080 \times 9 \\
8080 \times 0\end{array}$ & $\begin{array}{l}322 \\
570\end{array}$ & & 7.96 & & $700 \mathrm{~L}$ & $20.0 P$ \\
\hline $199-144-12 C$ & $7 / 13 / 93$ & B080Y1 & 267 & & $\begin{array}{l}7.46 \\
8.03\end{array}$ & & $300 \mathrm{~L}$ & $\begin{array}{l}10.0 \mathrm{P} \\
8.0 \mathrm{UP}\end{array}$ \\
\hline $199-\mathrm{H} 4-13$ & $7 / 29 / 93$ & B080Y2 & 323 & & 7.80 & & $500 \mathrm{~L}$ & $20.0 \mathrm{P}$ \\
\hline $199-H 4-14$ & $7 / 13 / 93$ & BOBOYJ & 303 & & 7.98 & & $500 \mathrm{~L}$ & $30.0 \mathrm{P}$ \\
\hline $199-H 4-15 A$ & $7 / 13 / 93$ & B080Y4 & 447 & & $6.44 \mathrm{~F}$ & & $500 \mathrm{~L}$ & $20.0 \mathrm{P}$ \\
\hline $199-H 4-15 B$ & $7 / 29 / 93$ & B08OY 5 & 360 & & 7.86 & & $500 \mathrm{~L}$ & 8.0 UP \\
\hline $199-44-16$ & $7 / 29 / 93$ & B080Y 6 & 280 & & 8.25 & & $700 \mathrm{~L}$ & $20.0 \mathrm{P}$ \\
\hline $199-144-17$ & $8 / 05 / 93$ & B080Y7 & 486 & & 7.64 & & $600 \mathrm{~L}$ & 8.0 UP \\
\hline $199-\mathrm{H}_{4}-18$ & $7 / 21 / 93$ & $8080 Y 8$ & 482 & & 7.80 & & 1000 & $20.0 \mathrm{P}$ \\
\hline \multirow{3}{*}{$199-14-3$} & $7 / 13 / 93$ & B080Y9 & 1731 & & 7.58 & & 1000 & $10.0 \mathrm{P}$ \\
\hline & $8 / 20 / 93$ & B090T3 & 2000 & & 7.55 & & & \\
\hline & $9 / 07 / 93$ & 809638 & 1729 & & 7.64 & & & \\
\hline \multirow[t]{2}{*}{$199-\mathrm{H}_{4}-4$} & $7 / 13 / 93$ & $B 08020$ & 355 & & 8.08 & & $700 \mathrm{~L}$ & $8.0 \mathrm{UP}$ \\
\hline & $\begin{array}{l}8 / 18 / 93 \\
9 / 07 / 93\end{array}$ & $\begin{array}{l}809074 \\
\text { B09639 }\end{array}$ & $\begin{array}{l}581 \\
596\end{array}$ & & $\begin{array}{l}8.00 \\
7.91\end{array}$ & & & \\
\hline \multirow{3}{*}{$\begin{array}{l}199-\mathrm{H} 4-5 \\
199-\mathrm{H} 4-6\end{array}$} & $7 / 13 / 93$ & B080Z1 & 478 & & 7.69 & & $800 \mathrm{~L}$ & $10.0 \mathrm{P}$ \\
\hline & $7 / 13 / 93$ & B080z2 & 497 & & 7.68 & & $700 \mathrm{~L}$ & 8.0 UP \\
\hline & & B08023 & & & & & $700 \mathrm{~L}$ & 8.0 UP \\
\hline \multirow{5}{*}{$\begin{array}{l}199-\mathrm{H} 4-7 \\
199-\mathrm{H} 4-8 \\
199-\mathrm{H} 4-9\end{array}$} & $7 / 13 / 93$ & B08024 & 464 & & 7.78 & & $700 \mathrm{~L}$ & $10.0 \mathrm{LP}$ \\
\hline & $7 / 14 / 93$ & B08025 & 497 & & 7.51 & & $800 \mathrm{~L}$ & 8.0 UP \\
\hline & $7 / 29 / 93$ & 808026 & 797 & & 7.69 & & $800 \mathrm{~L}$ & 8.0 UP \\
\hline & $8 / 09 / 93$ & B090T5 & 710 & & 7.85 & & & \\
\hline & $9 / 03 / 93$ & 809640 & 593 & & 7.80 & & & \\
\hline
\end{tabular}

For explanation of this table, see Section 1.4 of report. 
DOE/RL-93-56-3

This page intentionally left blank. 
DOE/RL-93-56-3

\section{CONTENTS}

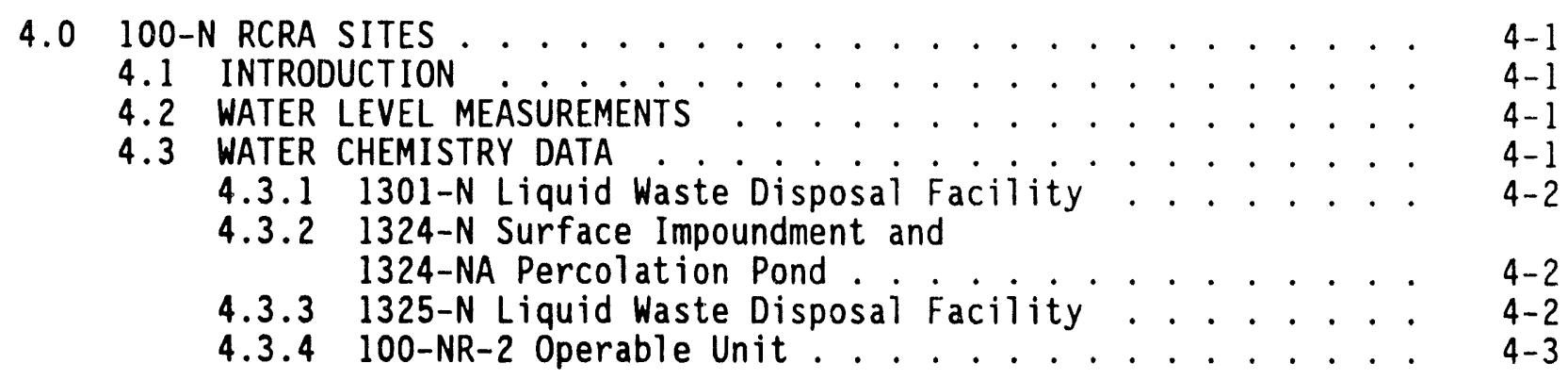


DOE/RL-93-56-3

\section{LIST OF FIGURES}

4-1 Monitoring Well Locations for 1301-N, 1324-N/NA, and 1325-N Facilities ... . . . . . . . . . . . . . . 4-4

\section{LIST OF TABLES}

4-1 Monitoring Well Purpose and Sampling Schedule in the 100-N Area . . . . . . . . . . . . . . . . . . 4-5

4-2 RCRA Water Level Measurement Report 100-N Area, Third Quarter 1993... . . . . . . . . . . . . 4-7

4-3 Constituent List and Summary of Results for 100-N RCRA Sites Data for Reporting Period July 1 through September 30, 1993 . . . 4-12

4-4 Constituents with at Least One Value Above the CRQL for the 100-N RCRA Sites Data for Reporting Period July 1 through September 30,1993............... 4-18

4-5 Contamination Indicator Parameters for the 100-N RCRA Sites Data for Reporting Period July 1 through September 30, 1993 


\title{
4.0 100-N RCRA SITES
}

\author{
M. J. Hartman \\ Westinghouse Hanford Company
}

\subsection{INTRODUCTION}

Four interim-status RCRA disposal units are located in the 100-N Area of the Hanford Site: (1) the 1301-N Liquid Waste Disposal Facility (LWDF), (2) the 1324-N Surface Impoundment, (3) the 1324-NA Percolation Pond, and (4) the 1325-N LWDF (Figure 4-1). The 1324-N and 1324-NA units are monitored as a single site. RCRA monitoring began in late 1987. The 1324-N/NA site has been monitored under a groundwater quality assessment program since 1989 (40 CFR 265.93). The 1325-N site is monitored under an indicator evaluation program (40 CFR 265.92). Background concentrations are being reestablished for the 1301-N LWDF.

The 1301-N and 1325-N units were used for disposal of liquid effluent from the $N$ Reactor and associated facilities. Primary waste constituents were radionuclides, including strontium-90, tritium, and cobalt-60. Minor amounts of hazardous waste also were present in the waste streams (e.g., hydrazine). The 1324-N/NA units received corrosive waste. The 100-N RCRA units are currently not in use.

Data from the 100-NR-2 groundwater operable unit monitoring program are included in the RCRA quarterly report. Sampling is coordinated between RCRA and 100-NR-2 to avoid redundancy. Sampling, analysis, data validation and verification are done according to the same protocols as RCRA.

Well locations in the 100-N Area are illustrated in Figure 4-1. Many of these wells are monitored monthly for water levels. Fewer wells are used for groundwater sampling. Sampling networks are listed in Table 4-1.

\subsection{WATER LEVEL MEASUREMENTS}

Water levels are measured monthly in most of the 100-N Area wells. Data are listed in Table 4-2. The September measurement for well N-59 is anomalously low compared to nearby wells and historical trends. It has been flagged as a suspected error.

\subsection{WATER CHEMISTRY DATA}

This section includes a summary of sampling and analysis activities in the 100-N Area. Groundwater chemistry data are listed in Tables 4-3 through 4-5. The tables include data from samples collected in previous quarters that were not presented in the last qiarterly report. Data flags are discussed in Sections 1.2 and 1.4 . 


\subsubsection{1-N Liquid Waste Disposal Facility}

All of the wells in the 1301-N network were scheduled for sampling during the past quarter. Well N-3 was not sampled because the pump was out of service. Wells N-57 and N-67 were sampled with a bailer because of low water levels. Approximately one borehole volume of water was purged from we $11 \mathrm{~N}-57$ before it was sampled. We11 N-67 was not purged. No filtered samples were collected from either well.

Samples from well N-2 for nitrate and sulfate were diluted for analysis. Potassium was significantly higher in a filtered sample than in an unfiltered sample from well $\mathrm{N}-76$. Tritium was undetected in wells $\mathrm{N}-67$ and N-69, which is not consistent with previous data. Gross alpha and gross beta activity in well N-57 were higher than they have been in the past. The sudden increase in gross beta was not reflected in strontium-90 activity. Data evaluations have been requested and the values are flagged with ' $F$.'

DWSs were exceeded for the following constituents: gross beta and strontium-90 in all the shallow we?ls; gross alpha in well $\mathrm{N}-57$; tritium in wells $\mathrm{N}-2, \mathrm{~N}-14, \mathrm{~N}-34$, and $\mathrm{N}-75$; chromium (unfiltered samples) in wells $\mathrm{N}-57$ and $\mathrm{N}-67$; iron (unfiltered) in wells $\mathrm{N}-34, \mathrm{~N}-57, \mathrm{~N}-67$, and $\mathrm{N}-69$; manganese (unfiltered) in wells $N-57$ and $N-67$; sulfate in well $N-57$; and turbidity in all wells.

\subsubsection{4-N Surface Impoundment and 1324-NA Percolation Pond}

The assessment monitoring program at the 1324-N/NA site has been revised (Hartman 1993). The wells in the revised network were scheduled for sampling during the last quarter. Sampling of well N-59 was delayed until October; data will be presented in the next quarterly report. Data from the other wells are listed in Tables 4-3 through 4-5.

The sample from well $\mathrm{N}-77$ for chloroform was diluted for analysis. An INC report was submitted by the laboratory for chloroform in well N-72. The concentration was estimated and was noted after the analytical holding time. Potassium was significantly higher in a filtered sample than in an unfiltered sample from well $\mathrm{N}-71$. A data evaluation has been requested and the values are flagged with ' $F$.'

DWSs were exceeded for chromium (unfiltered) in well $\mathrm{N}-71$; iron (unfiltered) in wells $\mathrm{N}-71$ and $\mathrm{N}-73$; field conductivity in well $\mathrm{N}-73$; and turbidity in wells $\mathrm{N}-71, \mathrm{~N}-73$, and $\mathrm{N}-77$.

\subsubsection{5-N Liquid Waste Disposal Facility}

The 1325-N monitoring wells were scheduled for sampling during the past quarter. Well $\mathrm{N}-43$ was not accessible and was not sampled. An access gate and road have been installed. Due to an oversight, well $\mathrm{N}-33$ was sampled, although it has been removed from the monitoring network. The well was sampled with a bailer due to low water levels. The well was not purged and no filtered samples were collected. Chemistry data for the other wells are listed in Tables 4-3 through 4-5. 
The sample from well $\mathrm{N}-41$ for sulfate was diluted for analysis. Coliform bacteria was anomalously high in well $\mathrm{N}-41$. There was a large difference between field and laboratory conductivity in we $11 \mathrm{~N}-32$. Turbidity was excessive in well $\mathrm{N}-33$, which was bailed. Other constituents (e.g. metals, radionuclides) were accordingly high, and the data are believed to be unrepresentative of groundwater quality. Data evaluations have been requested and data are flagged with ' $F . '$

DWSs were exceeded for the following constituents: gross beta and strontium-90 in wells $\mathrm{N}-27, \mathrm{~N}-29, \mathrm{~N}-33$, and $\mathrm{N}-81$; tritium in all wells except 81-58; barium, lead, gross alpha, and gross beta in well $\mathrm{N}-33$ (suspect data); chromium (unfiltered) in well $\mathrm{N}-33$; iron in wells $\mathrm{N}-32, \mathrm{~N}-33, \mathrm{~N}-41, \mathrm{~N}-81$, and 81-58; manganese (unfiltered) in wells $\mathrm{N}-32$ and $\mathrm{N}-81$; coliform bacteria in well N-41 (suspect data); and turbidity in most of the wells.

Background values of $\mathrm{pH}$, specific conductance, and TOC have been reestablished using data from the new upgradient well, N-74. Downgradient indicator parameters are compared to background values semiannually. No values exceeded background this quarter. TOX values were not compared due to problems with the data (see Section 1.2.5).

\subsubsection{0-NR-2 Operable Unit}

Most of the 100-NR-2 wells were sampled during the quarter. Well N-18 contained free petroleum product and samplers were instructed not to collect samples. Several of the wells were bailed (see Table 4-1). The wells were not purged and no filtered samples were collected. Data received for the past quarter are listed in Tables 4-3 through 4-5. Some of the wells are sampled only for total petroleum hydrocarbons and $0 i 1$ and grease. These data are not reported electronically, and have not yet been entered into the database. Previously unreported data from past quarters are listed in Appendix $A$.

Turbidity was excessive in well N-49, which was bailed. Other constituents (e.g., metals, radionuclides) were accordingly high, and the data are believed to be unrepresentative of groundwater quality. Tritium in well $\mathrm{N}-64$ was higher than historical values for this well or nearby wells. Specific conductance appears too low in well $\mathrm{N}-16$. There was a 1 arge difference between field and laboratory specific conductance in well $\mathrm{N}-5 \mathrm{l}$; the laboratory value is in line with historical data. Data evaluations were requested and the values are flagged with ' $F . '$

DWSs were exceeded for the following constituents: cadmium, chromium, iron, (unfiltered), gross alpha and gross beta in well $\mathrm{N}-49$ (suspect data); chromium (filtered and unfiltered) in well $\mathrm{N}-80$; iron (unfiltered) in wells $\mathrm{N}-16, \mathrm{~N}-17, \mathrm{~N}-25, \mathrm{~N}-26, \mathrm{~N}-47$, and $\mathrm{N}-64$; iron (filtered and unfiltered) in well $\mathrm{N}-25$; manganese (unfiltered) in wells $\mathrm{N}-25$, and $\mathrm{N}-49$; manganese (filtered) in wells $\mathrm{N}-16$, and $\mathrm{N}-25$; nitrate in wells $\mathrm{N}-26$ and $\mathrm{N}-64$; sulfate in well N-25; tritium in wells $\mathrm{N}-49, \mathrm{~N}-50, \mathrm{~N}-64$ (suspect data), and $\mathrm{N}-80$; and turbidity in most of the wells. 


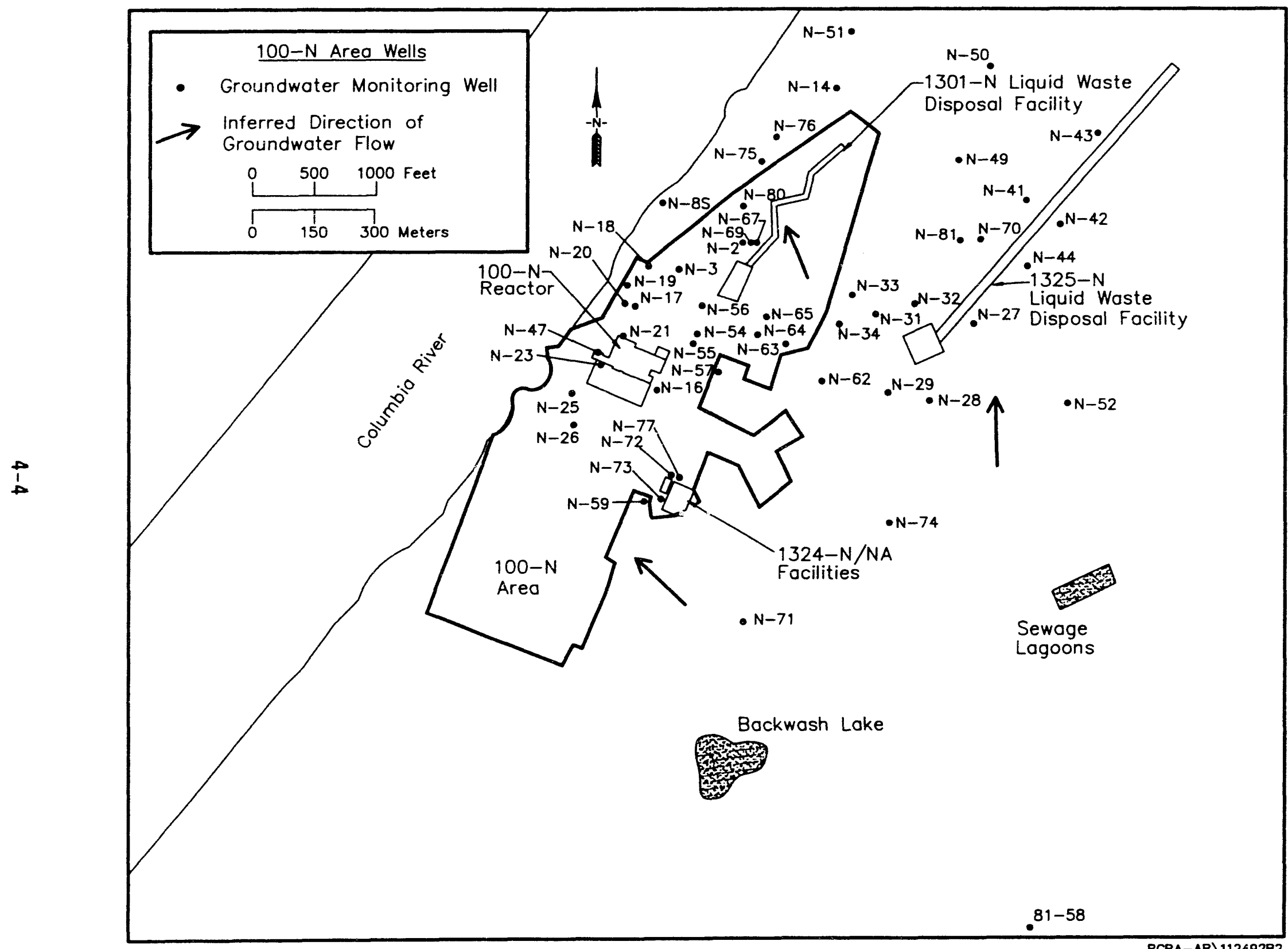

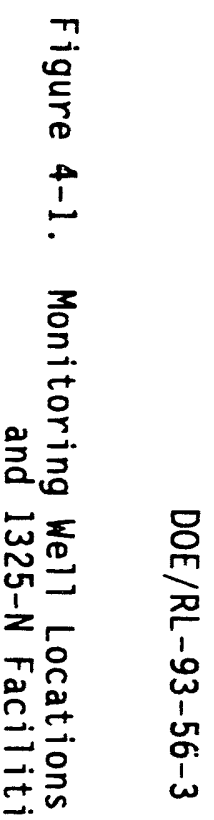


Table 4-1. Monitoring Well Purpose and Sampling Schedule in the 100-N Area. (sheet 1 of 2)

\begin{tabular}{|c|c|c|c|c|}
\hline Well no. & RCRA site & $\begin{array}{l}\text { Relative } \\
\text { position }\end{array}$ & $\begin{array}{c}\text { Sample } \\
\text { frequency }\end{array}$ & $\begin{array}{l}\text { Sample date, } \\
\text { 3rd Qtr } 1993\end{array}$ \\
\hline $199-N-2$ & $1301-N$ & Downgradient & Quarterly & $8 / 24 / 93$ \\
\hline $199-N-3$ & $1301-N, N R-2$ & Downgradient & Quarterly & Not sampled \\
\hline $199-N-14$ & $1301-N$ & Downgradient & Quarterly & $8 / 18 / 93$ \\
\hline $199-N-16$ & NR-2 & Downgradient & Quarterly & $8 / 11 / 93$ \\
\hline $199-N-17$ & NR-2 & Downgradient & Quarterly & Not sampled \\
\hline $199-N-19$ & NR-2 & Downgradient & Quarterly & $8 / 12 / 93$ \\
\hline $199-N-20$ & NR-2 & Downgradient & Quarterly & $8 / 13 / 93^{c}$ \\
\hline $199-N-21$ & NR-2 & Downgradient & Quarterly & $8 / 12 / 93^{c}$ \\
\hline $199-N-25$ & NR-2 & Downgradient & Quarterly & $8 / 13 / 93^{c}$ \\
\hline $199-N-26$ & NR-2 & Downgradient & Quarterly & $8 / 11 / 93$ \\
\hline $199-N-27$ & $1325-N$ & Downgradient & Semiannually & $8 / 10 / 93$ \\
\hline $199-N-29$ & $1325-N$ & Downgradient & Semiannually & $8 / 10 / 93$ \\
\hline $199-N-32$ & $1325-N, N R-2$ & Downgradient & Quarterly & $8 / 09 / 93$ \\
\hline $199-N-33$ & $1325-N^{d}$ & Downgradient & -- & $8 / 10 / 93^{c}$ \\
\hline $199-N-34$ & $1301-N, N R-2$ & Upgradient & Quarterly & $8 / 11 / 93$ \\
\hline $199-N-41$ & $1325-N$ & Downgradient & Semi annual ly & $9 / 01 / 93$ \\
\hline $199-N-43$ & $1325-N$ & Downgradient & Semiannually & Not sampled \\
\hline $199-N-49$ & NR-2 & Downgradient & Quarterly & $8 / 17 / 93^{c}$ \\
\hline $199-N-50$ & NR-2 & Downgradient & Quarterly & $8 / 10 / 93$ \\
\hline $199-N-51$ & NR-2 & Downgradient & Quarterly & $8 / 17 / 93$ \\
\hline $199-N-54$ & NR-2 & Downgradient & Quarterly & $8 / 12 / 93$ \\
\hline $199-N-57$ & $1301-N$ & Upgradient & Quarterly & $8 / 11 / 93^{f}$ \\
\hline $199-N-59$ & $1324-N / N A$ & Downgradient & Quarterly & Not sampled \\
\hline $199-N-64$ & NR-2 & Downgradient & Quarterly & $8 / 16 / 93^{c}$ \\
\hline $199-N-67$ & $1301-N, \quad N R-2$ & Downgradient & Quarterl. & $8 / 19 / 93^{c}$ \\
\hline
\end{tabular}


Table 4-1. Monitoring Well Purpose and Sampling Schedule for 100-N RCRA Sites. (sheet 2 of 2)

\begin{tabular}{|c|c|c|c|c|}
\hline Well no. & RCRA site & $\begin{array}{l}\text { Relative } \\
\text { position }\end{array}$ & $\begin{array}{c}\text { Sample } \\
\text { frequency }\end{array}$ & $\begin{array}{l}\text { Sample date, } \\
\text { 3rd Qtr } 1993\end{array}$ \\
\hline $199-N-69$ & $1301-N$ & $\begin{array}{l}\text { Downgradient; } \\
\text { bottom of } \\
\text { aquifer }\end{array}$ & Quarterly & $8 / 19 / 93$ \\
\hline $199-N-70$ & $1325-N, N R-2$ & $\begin{array}{l}\text { Downgradient; } \\
\text { bottom of } \\
\text { aquifer }\end{array}$ & Quarterly & $8 / 10 / 93$ \\
\hline $199-N-71$ & $1324-N / N A, N R-2$ & Upgradient & Quarterly & $8 / 10 / 93$ \\
\hline $199-N-72$ & $1324-N / N A$ & Downgradient & Quarterly & $8 / 11 / 93$ \\
\hline $199-N-73$ & $1324-N / N A, N R-2$ & Downgradient & Quarterly & $8 / 20 / 93$ \\
\hline $199-N-74$ & $1325-N, N R-2$ & Upgradient & Quarterly & $8 / 10 / 93$ \\
\hline $199-N-75$ & $1301-N, N R-2$ & Downgradient & Quarterly & $8 / 11 / 93$ \\
\hline $199-N-76$ & $1301-N, N R-2$ & Downgradient & Quarterly & $8 / 18 / 93$ \\
\hline $199-N-77$ & 1324-N/NA, NR-2 & $\begin{array}{c}\text { Downgradient; } \\
\text { bottom of } \\
\text { aquifer }\end{array}$ & Quarterly & $8 / 20 / 93$ \\
\hline $199-N-80$ & $N R-2$ & $\begin{array}{c}\text { Downgradient; } \\
\text { first confined } \\
\text { aquifer }\end{array}$ & Quarterly & $8 / 10 / 93$ \\
\hline $199-N-81$ & $1325-N$ & Downgradient & Quarterly & $8 / 11 / 93$ \\
\hline $699-81-58$ & $1325-N$ & Upgradient & Quarterly & $8 / 09 / 93$ \\
\hline
\end{tabular}

Note: The hydrogeologic unit monitored is the unconfined aquifer, which comprises sands and gravels of the Ringold Formation.

"Pump out of service.

${ }^{b}$ Free product in wel1; samples not required.

"Sampled with bailer; well not purged.

Well removed from network; sampled inadvertently.

"No access to well.

'Sampled with bailer; purged approximately 1 borehole volume.

${ }^{9}$ Sampling delayed until October 1993. 
Table 4-2. RCRA Water Level Measurement Report 100-N Area, Third Quarter 1993. (sheet 1 of 5)

\begin{tabular}{|c|c|c|c|}
\hline Well & Date & $\begin{array}{l}\text { Depth to } \\
\text { water (ft) }\end{array}$ & $\begin{array}{l}\text { Water level } \\
\text { elevation } \\
\text { above msl (ft) }\end{array}$ \\
\hline \multicolumn{4}{|c|}{ Wells Monitoring the Top of the Unconfined Aquifer } \\
\hline $199-N-14$ & $\begin{array}{l}7 / 14 / 93 \\
8 / 11 / 93 \\
8 / 18 / 93 \\
9 / 15 / 93\end{array}$ & $\begin{array}{l}68.38 \\
69.29 \\
69.41 \\
70.33\end{array}$ & $\begin{array}{l}385.30 \\
384.39 \\
384.27^{\star} \\
383.35\end{array}$ \\
\hline $199-N-16$ & $\begin{array}{l}7 / 14 / 93 \\
8 / 12 / 93 \\
9 / 16 / 93\end{array}$ & $\begin{array}{l}69.83 \\
70.24 \\
71.28\end{array}$ & $\begin{array}{l}387.41 \\
387.00 \\
385.96\end{array}$ \\
\hline $199-N-17$ & $\begin{array}{l}7 / 14 / 93 \\
8 / 12 / 93 \\
9 / 16 / 93\end{array}$ & $\begin{array}{l}75.71 \\
76.53 \\
77.52\end{array}$ & $\begin{array}{l}386.00 \\
385.18 \\
384.19\end{array}$ \\
\hline $199-N-18$ & $\begin{array}{l}7 / 14 / 93 \\
9 / 16 / 93\end{array}$ & $\begin{array}{l}73.58 \\
76.20\end{array}$ & $\begin{array}{l}385.47 \\
382.85\end{array}$ \\
\hline $199-N-19$ & $\begin{array}{l}7 / 14 / 93 \\
8 / 12 / 93 \\
9 / 16 / 93\end{array}$ & $\begin{array}{l}69.05 \\
70.01 \\
70.54\end{array}$ & $\begin{array}{l}385.39 \\
384.43 \\
383.90\end{array}$ \\
\hline $199-N-2$ & $\begin{array}{l}7 / 15 / 93 \\
8 / 10 / 93 \\
8 / 24 / 93 \\
9 / 15 / 93\end{array}$ & $\begin{array}{l}73.78 \\
74.30 \\
74.75 \\
75.34\end{array}$ & $\begin{array}{l}385.98 \\
385.46 \\
385.01^{\star} \\
384.42\end{array}$ \\
\hline $199-N-20$ & $\begin{array}{l}7 / 14 / 93 \\
8 / 12 / 93 \\
9 / 16 / 93\end{array}$ & $\begin{array}{l}70.47 \\
71.32 \\
72.20\end{array}$ & $\begin{array}{l}385.88 \\
385.03 \\
384.15\end{array}$ \\
\hline $199-N-21$ & $\begin{array}{l}7 / 14 / 93 \\
8 / 12 / 93 \\
9 / 16 / 93\end{array}$ & $\begin{array}{l}71.63 \\
72.48 \\
73.40\end{array}$ & $\begin{array}{l}385.84 \\
384.99 \\
384.07\end{array}$ \\
\hline $199-N-23$ & $\begin{array}{l}7 / 14 / 93 \\
8 / 12 / 93 \\
9 / 16 / 93\end{array}$ & $\begin{array}{l}71.06 \\
72.17 \\
73.13\end{array}$ & $\begin{array}{l}385.74 \\
384.63 \\
383.67\end{array}$ \\
\hline $199-N-25$ & $\begin{array}{l}7 / 14 / 93 \\
8 / 12 / 93 \\
9 / 16 / 93\end{array}$ & $\begin{array}{l}41.81 \\
42.96 \\
43.57\end{array}$ & $\begin{array}{l}384.44 \\
383.29 \\
382.68\end{array}$ \\
\hline $199-N-26$ & $\begin{array}{l}8 / 12 / 93 \\
9 / 16 / 93\end{array}$ & $\begin{array}{l}72.21 \\
73.07\end{array}$ & $\begin{array}{l}384.05 \\
383.19\end{array}$ \\
\hline
\end{tabular}


Table 4-2. RCRA Water Level Measurement Report

100-N Area, Third Quarter 1993. (sheet 2 of 5)

\begin{tabular}{|c|c|c|c|}
\hline Well & Date & $\begin{array}{l}\text { Depth to } \\
\text { water (ft) }\end{array}$ & $\begin{array}{l}\text { Water level } \\
\text { elevation } \\
\text { above msl (ft) }\end{array}$ \\
\hline \multicolumn{4}{|c|}{ Wells Monitoring the Top of the Unconfined Aquifer } \\
\hline $199-N-27$ & $\begin{array}{l}7 / 14 / 93 \\
8 / 10 / 93 \\
8 / 11 / 93 \\
9 / 15 / 93\end{array}$ & $\begin{array}{l}61.62 \\
61.54 \\
61.59 \\
61.92\end{array}$ & $\begin{array}{l}387.98 \\
388.06^{\star} \\
388.01 \\
387.68\end{array}$ \\
\hline $199-N-28$ & $\begin{array}{l}7 / 14 / 93 \\
8 / 11 / 93 \\
9 / 15 / 93\end{array}$ & $\begin{array}{l}76.19 \\
76.13 \\
76.39\end{array}$ & $\begin{array}{l}388.55 \\
388.61 \\
388.35\end{array}$ \\
\hline $199-N-29$ & $\begin{array}{l}7 / 14 / 93 \\
8 / 10 / 93 \\
8 / 11 / 93 \\
9 / 15 / 93\end{array}$ & $\begin{array}{l}77.19 \\
77.42 \\
76.84 \\
77.38\end{array}$ & $\begin{array}{l}388.56 \\
388.33^{\star} \\
388.91 \\
388.37\end{array}$ \\
\hline $199-N-3$ & $\begin{array}{l}7 / 15 / 93 \\
8 / 10 / 93 \\
9 / 15 / 93\end{array}$ & $\begin{array}{l}73.60 \\
74.47 \\
75.70\end{array}$ & $\begin{array}{l}385.79 \\
384.92 \\
383.69\end{array}$ \\
\hline $199-N-32$ & $\begin{array}{l}7 / 14 / 93 \\
8 / 11 / 93 \\
9 / 15 / 93\end{array}$ & $\begin{array}{l}74.91 \\
73.98 \\
75.39\end{array}$ & $\begin{array}{l}387.68 \\
388.61 \\
387.20\end{array}$ \\
\hline $199-N-33$ & $\begin{array}{l}7 / 14 / 93 \\
8 / 10 / 93 \\
8 / 10 / 93 \\
9 / 15 / 93\end{array}$ & $\begin{array}{l}73.06 \\
73.05 \\
73.04 \\
73.57\end{array}$ & $\begin{array}{l}387.31 \\
387.32^{\star} \\
387.33 \\
386.80\end{array}$ \\
\hline $199-N-34$ & $\begin{array}{l}7 / 14 / 93 \\
8 / 10 / 93 \\
8 / 11 / 93 \\
9 / 15 / 93\end{array}$ & $\begin{array}{l}71.99 \\
72.31 \\
72.62 \\
72.78\end{array}$ & $\begin{array}{l}388.16 \\
387.84 \\
387.53^{\star} \\
387.37\end{array}$ \\
\hline $199-N-41$ & $\begin{array}{l}7 / 14 / 93 \\
8 / 11 / 93 \\
9 / 01 / 93 \\
9 / 15 / 93\end{array}$ & $\begin{array}{l}72.24 \\
72.63 \\
73.13 \\
73.51\end{array}$ & $\begin{array}{l}385.87 \\
385.48 \\
384.98 \star \\
384.60\end{array}$ \\
\hline $199-N-42$ & $\begin{array}{l}7 / 14 / 93 \\
8 / 11 / 93 \\
9 / 15 / 93\end{array}$ & $\begin{array}{l}69.39 \\
69.58 \\
70.35\end{array}$ & $\begin{array}{l}386.25 \\
386.06 \\
385.29\end{array}$ \\
\hline
\end{tabular}


Table 4-2. RCRA Water Level Measurement Report 100-N Area, Third Quarter 1993. (sheet 3 of 5)

\begin{tabular}{|c|c|c|c|}
\hline We11 & Date & $\begin{array}{l}\text { Depth to } \\
\text { water (ft) }\end{array}$ & $\begin{array}{l}\text { Water level } \\
\text { elevation } \\
\text { above msl (ft) }\end{array}$ \\
\hline \multicolumn{4}{|c|}{ Wells Monitoring the Top of the Unconfined Aquifer } \\
\hline $199-N-49$ & $\begin{array}{l}7 / 14 / 93 \\
8 / 10 / 93\end{array}$ & $\begin{array}{l}66.83 \\
66.75\end{array}$ & $\begin{array}{l}384.43 \\
384.51\end{array}$ \\
\hline $199-N-50$ & $\begin{array}{l}7 / 14 / 93 \\
8 / 10 / 93 \\
9 / 15 / 93\end{array}$ & $\begin{array}{l}78.78 \\
79.54 \\
80.58\end{array}$ & $\begin{array}{l}385.12 \\
384.36 \\
383.32\end{array}$ \\
\hline $199-N-51$ & $\begin{array}{l}7 / 14 / 93 \\
8 / 10 / 93 \\
9 / 15 / 93\end{array}$ & $\begin{array}{l}78.06 \\
79.09 \\
79.84\end{array}$ & $\begin{array}{l}384.66 \\
383.63 \\
382.88\end{array}$ \\
\hline $199-N-52$ & $\begin{array}{l}7 / 14 / 93 \\
8 / 11 / 93 \\
9 / 15 / 93\end{array}$ & $\begin{array}{l}75.43 \\
75.41 \\
75.62\end{array}$ & $\begin{array}{l}388.77 \\
388.79 \\
388.58\end{array}$ \\
\hline $199-N-54$ & $\begin{array}{l}7 / 14 / 93 \\
8 / 12 / 93 \\
9 / 16 / 93\end{array}$ & $\begin{array}{l}71.07 \\
71.43 \\
72.41\end{array}$ & $\begin{array}{l}386.93 \\
386.57 \\
385.59\end{array}$ \\
\hline $199-N-55$ & $\begin{array}{l}7 / 14 / 93 \\
8 / 12 / 93 \\
9 / 16 / 93\end{array}$ & $\begin{array}{l}71.39 \\
71.73 \\
72.67\end{array}$ & $\begin{array}{l}386.96 \\
386.62 \\
385.68\end{array}$ \\
\hline $199-N-56$ & $\begin{array}{l}7 / 14 / 93 \\
8 / 12 / 93 \\
9 / 16 / 93\end{array}$ & $\begin{array}{l}72.09 \\
72.53 \\
73.55\end{array}$ & $\begin{array}{l}386.50 \\
386.06 \\
385.04\end{array}$ \\
\hline $199-N-57$ & $\begin{array}{l}7 / 14 / 93 \\
8 / 11 / 93 \\
8 / 12 / 93 \\
9 / 16 / 93\end{array}$ & $\begin{array}{l}70.21 \\
70.21 \\
70.30 \\
70.93\end{array}$ & $\begin{array}{l}388.05 \\
388.05^{\star} \\
387.96 \\
387.33\end{array}$ \\
\hline $199-N-59$ & $\begin{array}{l}7 / 14 / 93 \\
8 / 10 / 93 \\
9 / 16 / 93\end{array}$ & $\begin{array}{l}72.11 \\
72.24 \\
75.95\end{array}$ & $\begin{array}{l}387.96 \\
387.83 \\
384.12+\end{array}$ \\
\hline $199-N-62$ & $\begin{array}{l}7 / 14 / 93 \\
8 / 10 / 93 \\
9 / 15 / 93\end{array}$ & $\begin{array}{l}75.47 \\
75.38 \\
75.67\end{array}$ & $\begin{array}{l}388.65 \\
388.74 \\
388.45\end{array}$ \\
\hline
\end{tabular}


Table 4-2. RCRA Water Level Measurement Report 100-N Area, Third Quarter 1993. (sheet 4 of 5)

Well Date $\quad \begin{gathered}\text { Depth to } \\ \text { water (ft) }\end{gathered} \begin{gathered}\text { Water level } \\ \text { elevation } \\ \text { above ms }(\mathrm{ft})\end{gathered}$

Wells Monitoring the Top of the Unconfined Aquifer

\begin{tabular}{|c|c|c|c|}
\hline $199-N-63$ & $\begin{array}{l}7 / 14 / 93 \\
8 / 10 / 93 \\
9 / 15 / 93\end{array}$ & $\begin{array}{l}78.77 \\
78.70 \\
79.04\end{array}$ & $\begin{array}{l}388.46 \\
388.53 \\
388.19\end{array}$ \\
\hline $199-N-64$ & $\begin{array}{l}7 / 15 / 93 \\
8 / 11 / 93 \\
9 / 15 / 93\end{array}$ & $\begin{array}{l}67.01 \\
67.95 \\
67.44\end{array}$ & $\begin{array}{l}388.14 \\
387.20 \\
387.71\end{array}$ \\
\hline $199-N-65$ & $\begin{array}{l}7 / 15 / 93 \\
8 / 11 / 93 \\
9 / 15 / 93\end{array}$ & $\begin{array}{l}69.25 \\
69.26 \\
69.78\end{array}$ & $\begin{array}{l}387.73 \\
387.72 \\
387.20\end{array}$ \\
\hline $199-N-67$ & $\begin{array}{l}7 / 15 / 93 \\
8 / 11 / 93 \\
8 / 19 / 93 \\
9 / 15 / 93\end{array}$ & $\begin{array}{l}72.64 \\
72.93 \\
73.38 \\
73.88\end{array}$ & $\begin{array}{l}386.33 \\
386.04 \\
385.59 * \\
385.09\end{array}$ \\
\hline $199-N-71$ & $\begin{array}{l}7 / 14 / 93 \\
8 / 10 / 93 \\
8 / 10 / 93 \\
9 / 16 / 93\end{array}$ & $\begin{array}{l}73.10 \\
73.01 \\
73.00 \\
73.35\end{array}$ & $\begin{array}{l}389.92 \\
390.01 \\
390.02^{\star} \\
389.67\end{array}$ \\
\hline $199-N-72$ & $\begin{array}{l}7 / 14 / 93 \\
8 / 11 / 93 \\
8 / 12 / 93 \\
9 / 16 / 93\end{array}$ & $\begin{array}{l}69.35 \\
69.39 \\
69.48 \\
70.17\end{array}$ & $\begin{array}{l}389.63 \\
389.59 * \\
389.50 \\
388.81\end{array}$ \\
\hline $199-N-73$ & $\begin{array}{l}7 / 14 / 93 \\
8 / 10 / 93 \\
8 / 20 / 93 \\
9 / 16 / 93\end{array}$ & $\begin{array}{l}74.42 \\
74.47 \\
74.62 \\
75.15\end{array}$ & $\begin{array}{l}388.84 \\
388.79 \\
388.64^{\star} \\
388.11\end{array}$ \\
\hline $199-N-74$ & $\begin{array}{l}7 / 14 / 93 \\
8 / 10 / 93 \\
8 / 10 / 93 \\
9 / 15 / 93\end{array}$ & $\begin{array}{l}68.11 \\
67.98 \\
67.98 \\
68.18\end{array}$ & $\begin{array}{l}389.53 \\
389.66 \\
389.66^{\star} \\
389.46\end{array}$ \\
\hline $199-N-75$ & $\begin{array}{l}7 / 14 / 93 \\
8 / 10 / 93 \\
8 / 11 / 93 \\
9 / 15 / 93\end{array}$ & $\begin{array}{l}71.58 \\
72.67 \\
73.00 \\
73.81\end{array}$ & $\begin{array}{l}385.20 \\
384.11 \\
383.78 * \\
382.97\end{array}$ \\
\hline
\end{tabular}


Table 4-2. RCRA Water Level Measurement Report 100-N Area, Third Quarter 1993. (sheet 5 of 5)

\begin{tabular}{|c|c|c|c|}
\hline Well & Date & $\begin{array}{l}\text { Depth to } \\
\text { water (ft) }\end{array}$ & $\begin{array}{l}\text { Water level } \\
\text { elevation } \\
\text { above msl (ft) }\end{array}$ \\
\hline \multicolumn{4}{|c|}{ Wells Monitoring the Top of the Unconfined Aquifer } \\
\hline $199-N-76$ & $\begin{array}{l}7 / 14 / 93 \\
8 / 10 / 93 \\
8 / 18 / 93 \\
9 / 15 / 93\end{array}$ & $\begin{array}{l}67.03 \\
68.17 \\
68.26 \\
69.23\end{array}$ & $\begin{array}{l}385.09 \\
383.95 \\
383.86^{\star} \\
382.89\end{array}$ \\
\hline $199-N-8 S$ & $\begin{array}{l}7 / 15 / 93 \\
8 / 11 / 93 \\
9 / 15 / 93\end{array}$ & $\begin{array}{l}21.04 \\
22.84 \\
23.26\end{array}$ & $\begin{array}{l}384.08 \\
382.28 \\
381.86\end{array}$ \\
\hline $699-81-58$ & $\begin{array}{l}7 / 15 / 93 \\
8 / 09 / 93 \\
8 / 10 / 93 \\
9 / 16 / 93\end{array}$ & $\begin{array}{l}46.72 \\
46.65 \\
46.74 \\
46.83\end{array}$ & $\begin{array}{l}392.83 \\
392.90^{\star} \\
392.81 \\
392.72\end{array}$ \\
\hline
\end{tabular}

Wells Monitoring the Bottom of the Unconfined Aquife:

\begin{tabular}{llll}
\hline $199-N-69$ & $7 !$ & 73.11 & 386.25 \\
& 8 & 73.48 & 385.88 \\
& $8 /$ & 73.78 & $385.58 *$ \\
$199-N-70$ & 74.46 & 384.90 \\
& $7 / 14 / 93$ & 67.97 & 386.73 \\
$199-N-77$ & $8 / 11 / 93$ & 67.11 & 387.59 \\
& $9 / 15 / 93$ & 68.72 & 385.98 \\
& $8 / 20 / 93$ & 70.35 & $389.09 \star$ \\
& $9 / 16 / 93$ & 70.84 & 388.60 \\
\hline
\end{tabular}

Notes: 1. Water level elevations are calculated by subtracting the measured depth to water from the surveyed elevation for the well.

2. Depth-to-water values are transcribed from field records.

3. Elevations marked with an ' $\star$ ' were measured at the time of sampling.

4. Elevations marked with a ' + ' are outside of the expected range, and are suspected of error.

5. To convert feet to meters multiply by 0.3048 . 
Table 4-3. Constituent List and Summary of Results for 100-N RCRA Sites Data for Reporting Period July 1 through September 30, 1993. (sheet 1 of 6)

CONTAMIMATION INDICATOR PARAMETERS

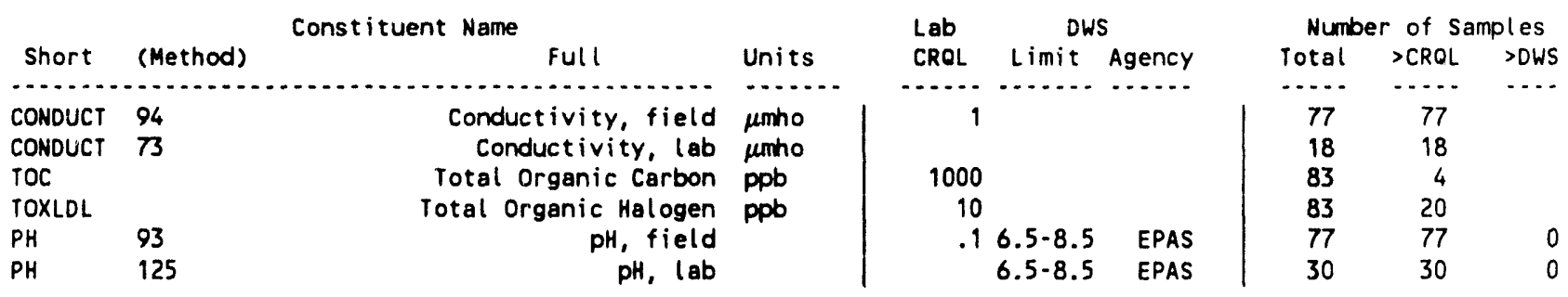

DRINKING WATER PARAMETERS

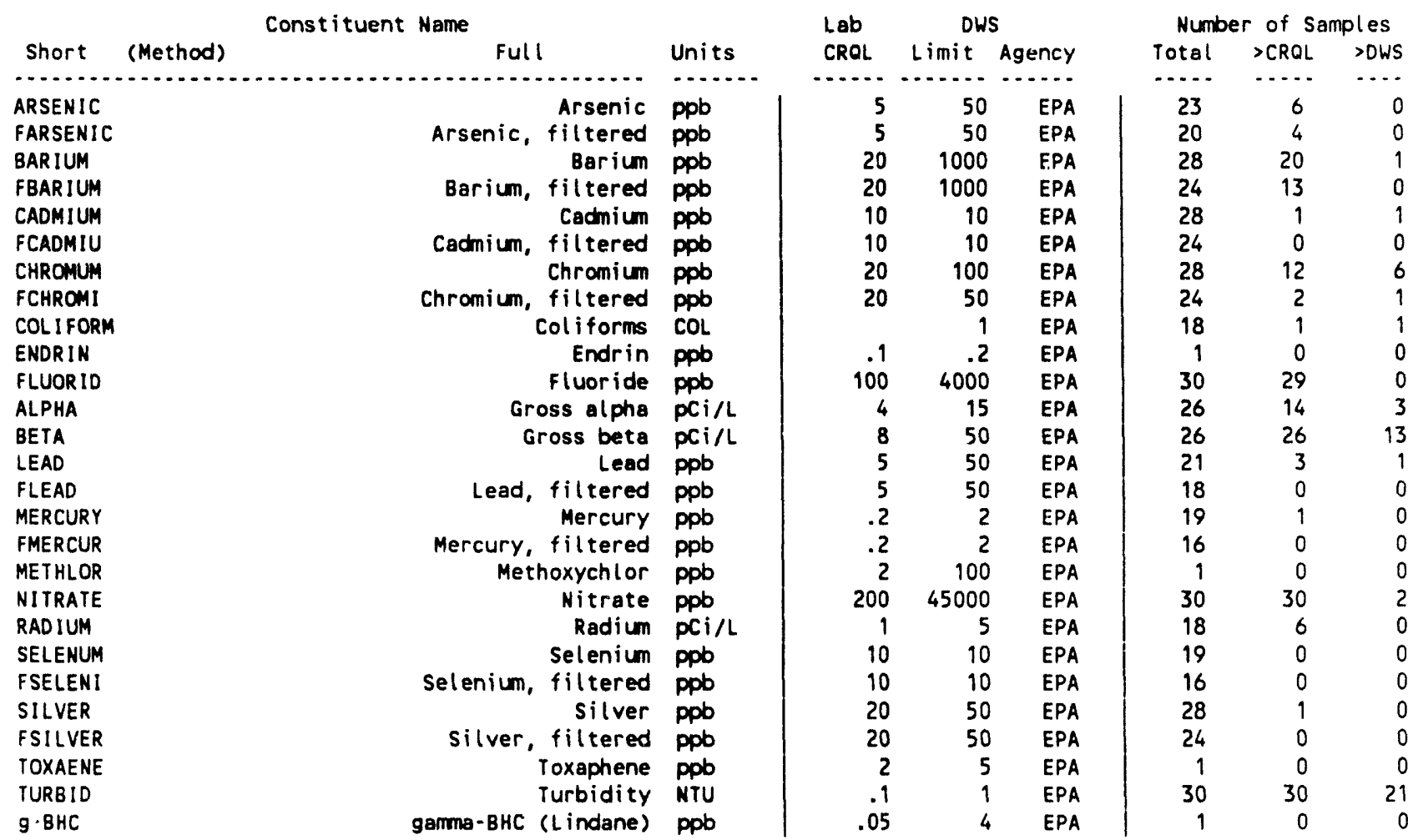

GROUNDWATER QUALITY PARAMETERS

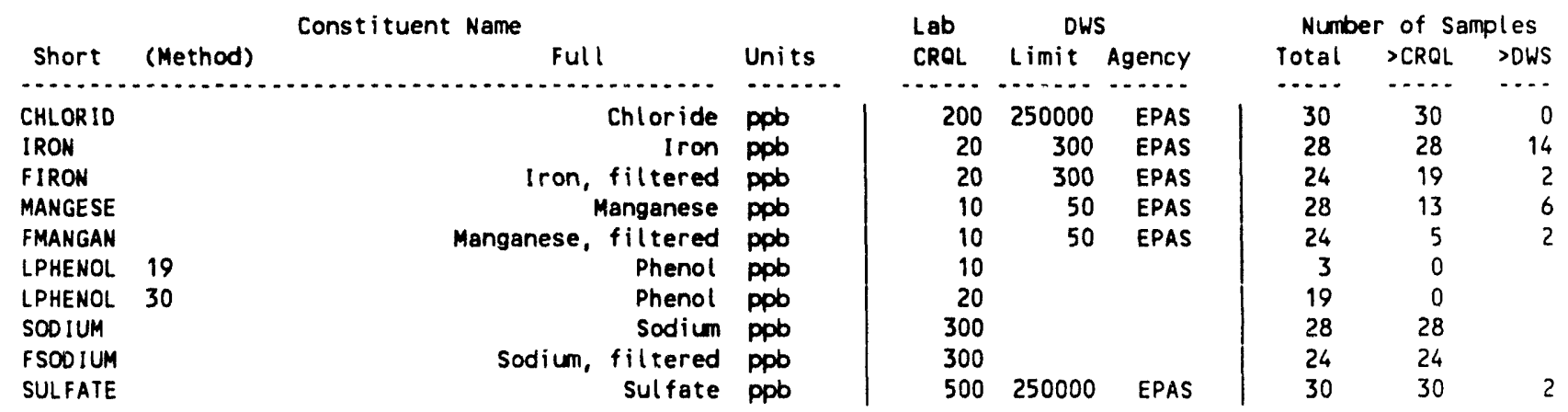


Table 4-3. Constituent List and Summary of Results for 100-N RCRA Sites Data for Reporting Period July 1 through September 30, 1993. (sheet 2 of 6)

SITE SPECIFIC AND OTHER CONSTITUENTS

\begin{tabular}{|c|c|c|c|c|c|c|c|}
\hline & & Constituent Name & & Lab & DWS & Numb & $r$ of $\mathrm{Sa}$ \\
\hline Short & (Method) & Full & Units & CRQL & Limit Agency & Total & $>C R Q L$ \\
\hline$\cdots \ldots \ldots$ & $\ldots \ldots+\cdots$ & 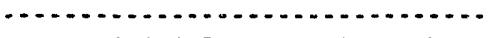 & $\cdots \cdots$ & $\ldots \ldots$ & $\cdots \ldots \ldots$ & $\cdots \cdots$ & $\ldots$. \\
\hline $1112-t c$ & & $1,1,1,2$-Tetrachloroethane & ppb & 5 & & 1 & 0 \\
\hline $1,1,1-T$ & 16 & $1,1,1$-Trichloroethane & ppb & 5 & & 2 & 0 \\
\hline $1,1,1-T$ & 25 & 1,1,1-Trichloroethane & ppb & .5 & & 4 & 0 \\
\hline $1122-t c$ & & $1,1,2,2$-Tetrachloroethane & ppb & 5 & & 1 & 0 \\
\hline $1,1,2-T$ & 16 & 1,1,2-Trichloroethane & ppb & 5 & & 2 & 0 \\
\hline $1,1,2-T$ & 25 & 1,1,2-Trichloroethane & ppb & .5 & & 4 & 0 \\
\hline $1,1-D I C$ & 16 & 1,1-Dichloroethane & ppb & 5 & & 2 & 0 \\
\hline $1,1-D I C$ & 25 & 1,1-Dichloroethane & ppb & 1 & & 4 & 0 \\
\hline DICETHY & & 1,1-Dichloroethene & ppb & 5 & & 1 & 0 \\
\hline 123-trp & & 1,2,3-Trichloropropane & ppb & 5 & & 1 & 0 \\
\hline TETRCHB & & $1,2,4,5-$ Tetrach lorobenzene & ppb & 10 & & 1 & 0 \\
\hline TRICHLB & & 1,2,4-Trichlorobenzene & ppb & 10 & & 1 & 0 \\
\hline DIBRCHL & & 1,2-D ibrono-3-ch loropropane & ppb & 5 & & 1 & 0 \\
\hline DIBRETH & & 1,2-0 ibromoethane & ppb & 5 & & 1 & 0 \\
\hline 12-dben & & 1,2-Dichlorobenzene & ppb & 10 & & 1 & 0 \\
\hline $1,2-D 1 C$ & 16 & 1,2-Dichloroethane & ppb & 5 & & 2 & 0 \\
\hline $1,2-D I C$ & 25 & 1,2-Dichloroethane & ppb & .5 & & 4 & 0 \\
\hline 12-DCE & & 1,2-0 ichloroethene & ppb & 5 & & 2 & 0 \\
\hline DICPANE & & 1,2-Dichloropropane & ppb & 5 & & 1 & 0 \\
\hline 13-dben & & 1,3-0 ichlorobenzene & ppb & 10 & & 1 & 0 \\
\hline 14-dben & 16 & 1,4-Dichlorobenzene & ppb & 5 & & 1 & 0 \\
\hline 14-dben & 19 & 1,4-Dichlorobenzene & ppb & 10 & & 1 & 0 \\
\hline 14 -dben & 25 & 1,4-0 ichlorobenzene & ppb & 2 & & 4 & 0 \\
\hline DIOXANE & & 1,4-Dioxane & $p p b$ & 200 & & 1 & 0 \\
\hline NAPHOUI & & 1,4-Naphtoquinone & ppb & 10 & & 1 & 0 \\
\hline IBUTYN & & 1-Butanol & ppb & 1000 & & 2 & 0 \\
\hline 1-napha & & 1-Naph thylemine & ppb & 10 & & 1 & 0 \\
\hline TETRCHP & & $2,3,4,6$-Tetrachlorophenol & ppb & 10 & & 1 & 0 \\
\hline $245-\operatorname{trp}$ & & 2,4,5-Trichlorophenol & ppb & 10 & & 1 & 0 \\
\hline 246-trp & 19 & 2,4,6-Trichlorophenol & ppb & 10 & & 1 & 0 \\
\hline $246-\operatorname{trp}$ & 30 & $2,4,6$-Trichlorophenol & ppb & 5 & & 19 & 0 \\
\hline $24-d c h p$ & 19 & 2,4-Dichlorophenol & ppb & 10 & & 3 & 0 \\
\hline 24-dchp & 30 & 2,4-Dichlorophenol & ppb & 5 & & 19 & 0 \\
\hline DIMPHEN & 19 & 2,4-D ime thylphenol & ppb & 10 & & 1 & 0 \\
\hline DIMPHEN & 30 & 2,4-D ime thylphenol & ppb & 5 & & 19 & 0 \\
\hline DINPHEN & 19 & 2,4-D initrophenol & ppo & 50 & & 1 & 0 \\
\hline DINPHEN & 30 & 2,4-0 initrophenol & ppb & 150 & & 19 & 0 \\
\hline 24 -dint & & 2,4-D initrotoluene & ppb & 10 & & 1 & 0 \\
\hline 26-dchp & 19 & 2,6-Dichlorophenol & ppb & 10 & & 1 & 0 \\
\hline 26-dchp & 30 & 2,6-D ichl orophenol & ppb & 5 & & 19 & 0 \\
\hline 26-dint & & 2,6-0 initrotoluene & ppb & 10 & & 1 & 0 \\
\hline ACEFENE & & 2-Acetylaminof luorene & ppb & 10 & & 1 & 0 \\
\hline CHLNAPH & & 2-Chloronaphthal ene & ppb & 10 & & 1 & 0 \\
\hline CHLPHEN & 19 & 2-Chlorophenol & ppb & 10 & & 1 & 0 \\
\hline CHLPHEN & 30 & 2-Chlorophenol & ppb & 5 & & 19 & 0 \\
\hline 2HEXANO & & 2-Hexanone & ppb & 50 & & 1 & 0 \\
\hline 2MENAPH & & 2-Methyl naph thal ene & ppb & 10 & & 1 & 0 \\
\hline 2METHPH & & 2-Methylphenol & ppb & 10 & & 3 & 0 \\
\hline 2-napha & & 2-Naphthylamine & ppb & 10 & & 1 & 0 \\
\hline ONITANI & & 2-Nitroaniline & ppb & 50 & & 1 & 0 \\
\hline 2NITPH & 19 & 2-Nitrophenol & ppb & 10 & & 3 & 0 \\
\hline 2NITPH & 30 & 2-Nitrophenol & ppb & 5 & & 19 & 0 \\
\hline PICOLIN & & 2-Picoline & ppb & 10 & & 1 & 0 \\
\hline BUTDINP & & 2-sec-Butyl-4,6-dinitrophenol (DN & ppb & 5 & & 19 & 0 \\
\hline DICHBEN & & 3,3'-Dichlorobenzidine & ppb & 20 & & 1 & 0 \\
\hline DIMEYLB & & 3, $3^{\prime}$-D imethylbenzidine & ppb & 10 & & 1 & 0 \\
\hline METCHAN & & 3-Methylchol anthrene & ppb & 10 & & 1 & 0 \\
\hline
\end{tabular}


Table 4-3. Constituent List and Summary of Results for 100-N RCRA Sites Data for Reporting Period July 1 through September 30, 1993. (sheet 3 of 6)

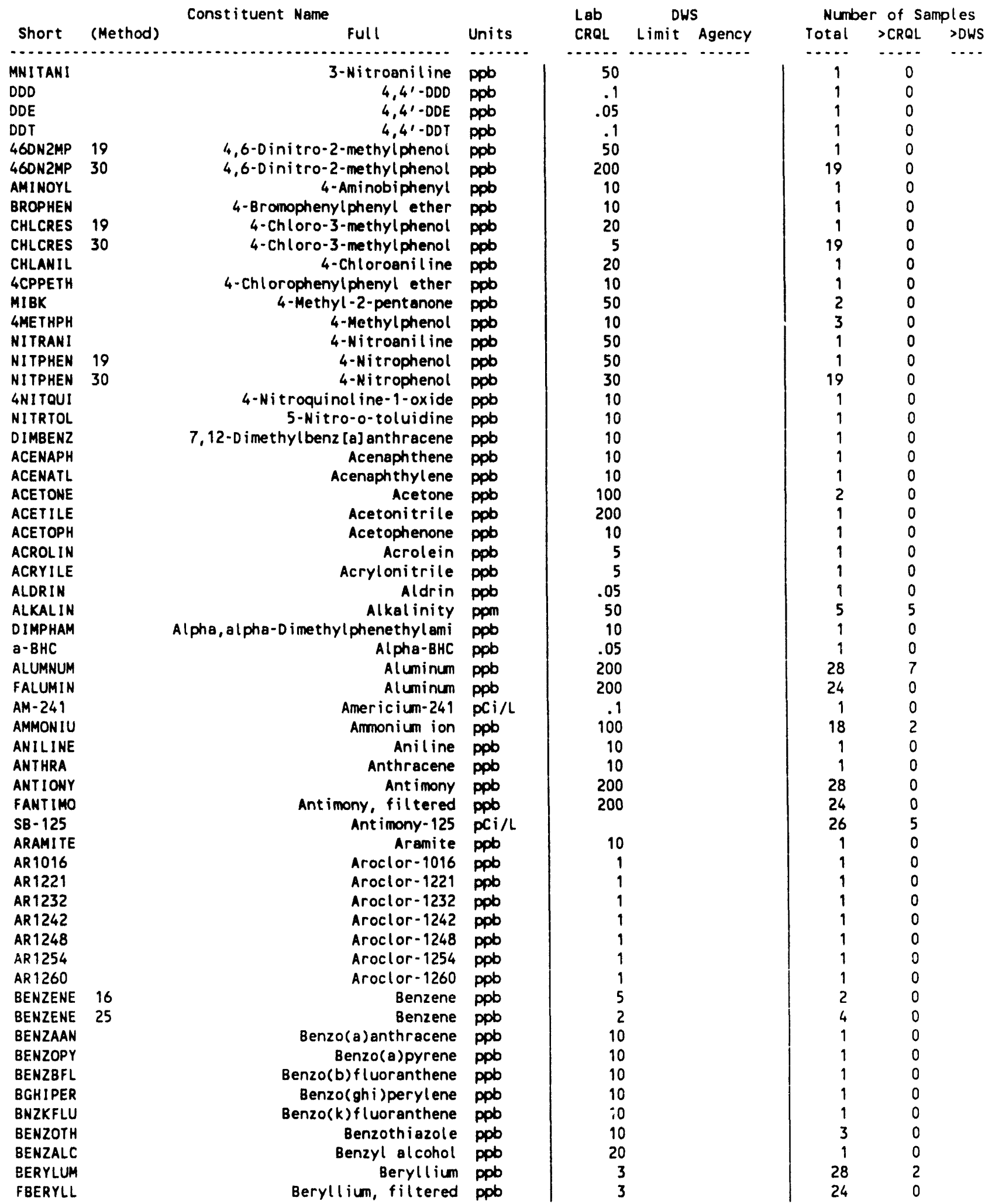


Table 4-3. Constituent List and Summary of Results for 100-N RCRA Sites Data for Reporting Period July 1 through September 30, 1993. (sheet 4 of 6)

\begin{tabular}{|c|c|c|c|c|c|c|c|c|c|}
\hline \multicolumn{4}{|c|}{ Constituent Name } & \multicolumn{3}{|c|}{ DWS } & \multicolumn{3}{|c|}{ Number of Samples } \\
\hline Short & (Method) & Full & Units & CRQL & Limit & Agency & Total & $>C R Q L$ & $>$ DWS \\
\hline$\cdots+\cdots$ & 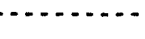 & 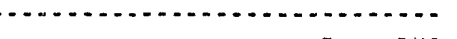 & $\cdots \cdots$ & 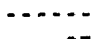 & $\cdots+\cdots-$ & $\cdots \cdots$ & $\cdots \cdots$ & $\cdots+$ & $\cdots$ \\
\hline b-BHC & & Beta-BHC & ppb & .05 & & & 1 & 0 & \\
\hline BIS2CHM & & Bis(2-Choroethoxy)methane & ppb & 10 & & & 1 & 0 & \\
\hline BISZCHE & & Bis (2-chloroethyl) ether & ppb & 10 & & & 1 & 0 & \\
\hline BISZETH & & Bis(2-chloroisopropyl) ether & ppb & 10 & & & 1 & 0 & \\
\hline BIS2EPH & & Bis(2-ethylhexyl) phthalate & ppb & 10 & & & 3 & 0 & \\
\hline BROMIDE & & Bromide & ppb & 500 & & & 30 & 0 & \\
\hline BDCM & & Bromodichlorome thane & ppb & 5 & 100 & EPA & 1 & 0 & \\
\hline BROMORM & & Bromoform & ppb & 5 & 100 & EPA & 1 & 0 & \\
\hline BUTBENP & & Butylbenzylphthalate & ppb & 10 & & & 1 & 0 & \\
\hline CALCIUM & & Calcium & ppb & 100 & & & 28 & 28 & \\
\hline FCALCIU & & Calcium, filtered & ppb & 100 & & & 24 & 24 & \\
\hline CARBIDE & & Carbon disulfide & ppb & 5 & & & 2 & 0 & \\
\hline TETRANE & 16 & Carbon tetrachloride & ppb & 5 & & & 2 & 0 & \\
\hline TETRANE & 25 & Carbon tetrachloride & ppb & 1 & & & 4 & 0 & \\
\hline CS- 137 & & Cesium- 137 & $p C i / L$ & 20 & & & 26 & 4 & \\
\hline $\mathrm{COO}$ & & Chemical Oxygen Demand & ppm & 3 & & & 1 & 0 & \\
\hline CHLOANE & & Chlordane & ppb & .1 & & & 1 & 0 & \\
\hline CHLROB & & Chlorobenzene & ppb & 5 & & & 1 & 0 & \\
\hline CHLLATE & & Chlorobenzilate & ppb & 10 & & & 1 & 0 & \\
\hline CLETHAN & & Chloroethane & ppb & 10 & & & 1 & 0 & \\
\hline CHLFORM & 16 & Chloroform & ppb & 5 & 100 & EPA & 2 & 1 & \\
\hline CHLFORM & 25 & Chloroform & ppb & .5 & 100 & EPA & 4 & 4 & \\
\hline CHLPRENE & & Chloroprene & ppb & 5 & & & 1 & 0 & \\
\hline CHRYSEN & & Chrysene & ppb & 10 & & & 1 & 0 & \\
\hline COBALT & & Cobelt & ppb & 20 & & & 28 & 2 & \\
\hline FCOBALT & & Cobalt, filtered & pob & 20 & & & 24 & 0 & \\
\hline $\mathrm{Co}-60$ & & Cobalt -60 & $\mathrm{pCi} / \mathrm{L}$ & & & & 26 & 9 & \\
\hline COPPER & & Copper & ppb & 20 & 1000 & EPAS & 28 & 4 & c \\
\hline FCOPPER & & Copper, filtered & ppb & 20 & 1000 & EPAS & 24 & 0 & 0 \\
\hline CRESOLS & & Cresols (methylphenols) & ppb & 10 & & & 19 & 0 & \\
\hline CYANIDE & & Cyenide & ppb & 20 & & & 1 & 0 & \\
\hline DECANE & & Decane & ppb & 10 & & & 2 & 0 & \\
\hline d-BHC & & Delta-BHC & ppb & .1 & & & 1 & 0 & \\
\hline DIBPHTH & & Di-n-butylphthalate & ppb & 10 & & & 1 & 0 & \\
\hline DIOPHTH & & Di-n-octylphthalate & ppb & 10 & & & 1 & 0 & \\
\hline DIALLATE & & Diallate & ppb & 10 & & & 1 & 0 & \\
\hline DIBAHAN & & D ibenz $[a, h]$ anthracene & ppb & 10 & & & 1 & 0 & \\
\hline DIBENFR & & Dibenzofuran & ppb & 10 & & & 1 & 0 & \\
\hline BRCHLMET & & Dibromochlorome thane & ppb & 5 & 100 & EPA & 1 & 0 & c \\
\hline DIBRMET & & Dibromomethane & ppb & 5 & & & 1 & 0 & \\
\hline DICDIFM & & Dichlorodifluorome thane & ppb & 5 & & & 1 & 0 & \\
\hline DIELRIN & & Dieldrin & ppb & .05 & & & 1 & 0 & \\
\hline DIEPHTH & & Diethyl phthalate & ppo & 10 & & & 1 & 0 & \\
\hline DIMETHO & & Dimethoote & ppb & 10 & & & 1 & 0 & \\
\hline DIMPHTH & & Dimethyl phthalate & ppb & 10 & & & 1 & 0 & \\
\hline DIPHAMI & & Diphenyl emine & ppb & 10 & & & 1 & 0 & \\
\hline DISULFO & & Disulfoton & ppb & 2 & & & 1 & 0 & \\
\hline DODECAN & & Dodecane & ppb & 10 & & & 2 & 0 & \\
\hline ENDO1 & & Endosulfan I & ppb & .1 & & & 1 & 0 & \\
\hline ENDO2 & & Endosulfan 11 & ppb & .05 & & & 1 & 0 & \\
\hline ENDSFAN & & Endosulfan sulfate & ppb & .5 & & & 1 & 0 & \\
\hline ENDRALD & & Endr in Aldehyde & ppb & .2 & & & 1 & 0 & \\
\hline ETHCYAN & & Ethyl cyanide & ppo & 10 & & & 2 & 0 & \\
\hline ETHMETH & & Ethyl methacrylate & ppb & 5 & & & 1 & 0 & \\
\hline ETHMETS & & Ethyl methanesulfonate & ppb & 10 & & & 1 & 0 & \\
\hline ETHBENZ & 16 & Ethylbenzene & ppb & 5 & & & 1 & 0 & \\
\hline ETHBENZ & 25 & Ethylbenzene & ppb & 2 & & & 4 & 0 & \\
\hline FAMPHUR & & Famphur & ppb & 10 & & & 1 & 0 & \\
\hline
\end{tabular}


Table 4-3. Constituent List and Summary of Results for 100-N RCRA Sites Data for Reporting Period July 1 through September 30, 1993. (sheet 5 of 6)

\begin{tabular}{|c|c|c|c|c|c|c|c|c|}
\hline & & Constituent Name & & Lab & DWS & Numt & $r$ of $\mathrm{Sa}$ & ples \\
\hline Short & (Method) & Full & Units & CRQL & Limit Agency & Total & $>$ CRQL & >OWS \\
\hline$\cdots \ldots$ & 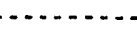 & 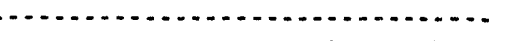 & $\ldots \ldots$ & $\ldots \ldots$ & 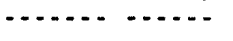 & $\cdots$ & $\cdots$ & $\cdots$ \\
\hline FLUORAN & & Fluoranthene & ppob & 10 & & 1 & 0 & \\
\hline FLRENE & & Fluorene & ppb & 10 & & 1 & 0 & \\
\hline HEPTLOR & & Heptachlor & ppb & .05 & & 1 & 0 & \\
\hline HEPTIDE & & Heptachlor epoxide & ppb & 1 & & 1 & 0 & \\
\hline HEXCBEN & & Hexachlorobenzene & ppb & 10 & & 1 & 0 & \\
\hline HEXCBUT & & Hexachlorobutadiene & ppb & 10 & & 1 & 0 & \\
\hline HEXCCYC & & Hexach lorocyclopentadiene & ppb & 10 & & 1 & 0 & \\
\hline HEXCETH & & Hexachloroethane & ppb & 10 & & 1 & 0 & \\
\hline HEXACHL & & Hexach lorophene & ppb & 10 & & 1 & 0 & \\
\hline HEXAENE & & Hexachloropropene & pplo & 10 & & 1 & 0 & \\
\hline LHYDRAZ & & Hydrazine & ppb & 30 & & 18 & 0 & \\
\hline INDENOP & & Indeno $(1,2,3-\mathrm{cd})$ pyrene & ppob & 10 & & 1 & 0 & \\
\hline ISOBUTY & & Isobutyl alcohol & ppb & 200 & & 1 & 0 & \\
\hline ISOORIN & & Isodrin & ppb & 10 & & 1 & 0 & \\
\hline ISOPHER & & Isophorone & ppob & 10 & & 1 & 0 & \\
\hline ISOSOLE & & Isosafrole & ppb & 10 & & 1 & 0 & \\
\hline KEPONE & & Kepone & ppb & 10 & & 1 & 0 & \\
\hline KEROSEN & & Kerosene & ppb & 10000 & & 1 & 0 & \\
\hline MAGNES & & Magnesium & ppb & 100 & & 28 & 28 & \\
\hline FMAGNES & & Magnesium, filtered & ppb & 100 & & 24 & 24 & \\
\hline METHACR & & Methacrylonitrile & ppb & 5 & & 1 & 0 & \\
\hline METHAPY & & Methapyrilene & ppb & 10 & & 1 & 0 & \\
\hline IODOMET & & Methyl lodide & ppb & 5 & & 1 & 0 & \\
\hline METHBRO & & Methyl bromide & ppob & 10 & & 1 & 0 & \\
\hline METHCHL & & Methyl chloride & ppb & 10 & & 1 & 0 & \\
\hline METHONE & & Methyl ethyl ketone & ppb & 100 & & 2 & 0 & \\
\hline METACRY & & Methyl methacrylate & ppb & 5 & & 1 & 0 & \\
\hline METMSUL & & Methyl methanesulfonate & ppb & 10 & & 1 & 0 & \\
\hline METHPAR & & Methyl parathion & ppob & .5 & & 1 & 0 & \\
\hline METHYCH & 16 & Methylene chloride & ppb & 5 & & 2 & 0 & \\
\hline METHYCH & 25 & Methylene chloride & ppo & 5 & & 4 & 0 & \\
\hline DIPRNIT & & $N$-Nitroso-di-n-dipropylamine & ppb & 10 & & 1 & 0 & \\
\hline NHIBUTY & & $N$-Nitrosodi-n-butylamine & ppb & 10 & & 1 & 0 & \\
\hline NNIDIEY & & $\mathrm{N}$-Nitrosodiethylamine & ppb & 10 & & 1 & 0 & \\
\hline NNIDIME & & $N-N i t r o s o d i m e t h y l$ anine & ppb & 10 & & 1 & 0 & \\
\hline NNDIPHA & & $\mathrm{N}$-Nitrosadiphenylamine & ppb & 10 & & 1 & 0 & \\
\hline NNIMETH & & $\mathrm{N}-\mathrm{N}$ it rosomethylethylamine & ppb & 10 & & 1 & 0 & \\
\hline NNIMORP & & $\mathrm{N}$-Nitrosomorphol ine & ppb & 10 & & 1 & 0 & \\
\hline NNIPIPE & & N-Nitrosopiperidine & ppb & 10 & & 1 & 0 & \\
\hline NAPHTHA & & Naphthalene & ppb & 10 & & 3 & 0 & \\
\hline NICKEL & & Nickel & ppb & 30 & & 28 & 7 & \\
\hline FNICKEL & & Nickel, filtered & ppb & 30 & & 24 & 1 & \\
\hline NITRITE & & Nitrite & pppb & 200 & & 30 & 1 & \\
\hline NITBENZ & & Nitrobenzene & ppb & 10 & & 1 & 0 & \\
\hline NITRPYR & & Nitrosopyrrolidine & ppb & 10 & & 1 & 0 & \\
\hline TRIPHOS & & 0,0,0-Triethyl phosphorothioate & ppb & 10 & & 1 & 0 & \\
\hline DIPKOS & & 0,0 -diethyl0-2-pyrazinyl phosphor & ppb & 10 & & 1 & 0 & \\
\hline PARATHI & & Parathion & ppb & 10 & & 1 & 0 & \\
\hline PENTCHB & & Pentachlorobenzene & ppb & 10 & & 1 & 0 & \\
\hline PENTACH & & Pentachloroethane & ppb & 5 & & 1 & 0 & \\
\hline PENTCHN & & Pentachloronitrobenzene (PCNB) & ppb & 10 & & 1 & 0 & \\
\hline PENTCKP & 19 & Pentachloropienol & ppb & 50 & & 3 & 0 & \\
\hline PENTCHP & 30 & Pentachlorophenol & ppb & 100 & & 19 & 0 & \\
\hline PHENT IN & & Phenacetin & ppob & 10 & & 1 & 0 & \\
\hline PHENANT & & Phenanthrene & ppb & 10 & & 1 & 0 & \\
\hline PHORATE & & Phorate & ppb & 2 & & 1 & 0 & \\
\hline PHOSPHA & & Phosphate & ppo & 400 & & 30 & 0 & \\
\hline PU-238 & & Plutonium-238 & $\mathrm{pCi} / \mathrm{L}$ & .1 & & 1 & 0 & \\
\hline PU39-40 & & Plutonium-239/40 & $\mathrm{pCi} / \mathrm{L}$ & .1 & & 1 & 0 & \\
\hline
\end{tabular}


Table 4-3. Constituent List and Summary of Results for 100-N RCRA Sites Data for Reporting Period July 1 through September 30, 1993. (sheet 6 of 6)

\begin{tabular}{|c|c|c|c|c|c|c|c|c|c|}
\hline \multirow[b]{2}{*}{ Short } & \multirow{3}{*}{ (Method) } & \multirow[t]{2}{*}{ Constituent Name } & \multirow[b]{2}{*}{ Units } & \multirow{2}{*}{$\begin{array}{l}\text { Lab } \\
\text { CRQL }\end{array}$} & \multicolumn{2}{|c|}{ DWS } & \multicolumn{3}{|c|}{ Number of Samples } \\
\hline & & & & & Limit & Agency & Total & $>$ CRQL & $>$ DWS \\
\hline$\ldots \ldots \ldots$ & & $\ldots \ldots \ldots \ldots$ & $\cdots \cdots$ & $\ldots . .$. & -....... & $\ldots \ldots$ & $\cdots$ & $-\ldots$ & $\cdots$ \\
\hline POTASUM & & Potassium & ppb & 300 & & & 28 & 26 & \\
\hline FPOTASS & & Potassium, filtered & ppb & 300 & & & 24 & 22 & \\
\hline PRONIDE & & Pronamide & Ppb & 10 & & & 1 & 0 & \\
\hline PYRENE & & Pyrene & ppb & 10 & & & 1 & 0 & \\
\hline PYRIDIN & & Pyridine & ppb & 10 & & & 1 & 0 & \\
\hline$R U-106$ & & Ruthenium- 106 & $\mathrm{pCi} / \mathrm{L}$ & & & & 26 & 2 & \\
\hline SAFROL & & Safrol & ppb & 10 & & & 1 & 0 & \\
\hline$S R-90$ & & Stront ium-90 & $\mathrm{PCi} / \mathrm{L}$ & 5 & 8 & EPA & 26 & 14 & 12 \\
\hline STYRENE & & Styrene & ppb & 5 & & & 1 & 0 & \\
\hline TC- 99 & & Technetium- 99 & $\mathrm{pCi} / \mathrm{L}$ & 15 & & & 1 & 0 & \\
\hline PERCENE & 16 & Tetrachloroethene & ppb & 5 & & & 2 & 0 & \\
\hline PERCENE & 25 & Tetrachloroethene & ppb & .5 & & & 4 & 0 & \\
\hline TETPHNL & & Tetrachlorophenols & ppb & 10 & & & 19 & 0 & \\
\hline TETRADE & & Tetradecane & ppb & 10 & & & 2 & 0 & \\
\hline PYROPHOS & & Tetraethyldithiopyrophosphate & ppb & 10 & & & 1 & 0 & \\
\hline TAF & & Tetrahydrofuran & ppb & 10 & & & 2 & 0 & \\
\hline THALLIUM & & Thallium & ppb & 5 & & & 14 & 0 & \\
\hline FTHALIUM & & Thallium, filtered & ppb & 5 & & & 11 & 0 & \\
\hline TIN & & Tin & ppb & 100 & & & 28 & 0 & \\
\hline FTIN & & Tin, filtered & ppb & 100 & & & 24 & 0 & \\
\hline TOLUENE & 16 & Tolvene & ppb & 5 & & & 2 & 0 & \\
\hline TOLUENE & 25 & Tolvene & ppb & 2 & & & 4 & 0 & \\
\hline TC & & Total Carbon & ppb & 2000 & & & 4 & 4 & \\
\hline TDS & & Total Dissolved Solids & ppm & 10 & & & 5 & 5 & \\
\hline TRIBUTPH & & Iributyl Phosphate & ppb & 10 & & & 3 & 0 & \\
\hline TRICENE & 16 & Trichloroethene & ppb & 5 & & & 2 & 0 & \\
\hline TRICENE & 25 & Trichloroethene & ppb & 1 & & & 4 & 0 & \\
\hline TRCMHLM & & Tricis! eromonof luorome thane & ppb & 5 & & & 1 & 0 & \\
\hline TRIPHNL & & Trichlorophenols & pph & 5 & & & 19 & 0 & \\
\hline TOICHPH & & Tris-2-chloroethyl phosphate & ppob & 10 & & & 3 & 0 & \\
\hline TRITIUM & & Tritium & $\mathrm{pCi} / \mathrm{L}$ & 500 & 20000 & EPA & 26 & 22 & 17 \\
\hline$U-234$ & & Uranium-234 & $\mathrm{pCi} / \mathrm{L}$ & .1 & & & 1 & 1 & \\
\hline$U-235$ & & Uranium-235 & $\mathrm{pCi} / \mathrm{L}$ & .1 & & & 1 & 1 & \\
\hline$U-238$ & & Uranium-238 & $\mathrm{pCi} / \mathrm{L}$ & .1 & & & 1 & 1 & \\
\hline VANADUM & & Vanadium & ppb & 30 & & & 28 & 3 & \\
\hline FVANADI & & Vanadium, filtered & pob & 30 & & & 24 & 1 & \\
\hline VINYLAC & & Vinyl acetate & ppo & 5 & & & 1 & 0 & \\
\hline VINYIDE & 16 & Vinyl chloride & ppb & 10 & 2 & EPA & 2 & 0 & $0 *$ \\
\hline VINYIDE & 25 & Vinyl chloi ide & ppb & 2 & 2 & EPA & 4 & 0 & 0 \\
\hline XYLENE & 16 & Xylenes (total) & ppb & 5 & & & 2 & 0 & \\
\hline XYLENE & 25 & Xylenes (total) & ppb & 5 & & & 4 & 0 & \\
\hline ZINC & & zinc & ppb & 10 & & & 28 & 15 & \\
\hline FZINC & & zinc, filtered & ppb & 10 & & & 24 & 7 & \\
\hline ALLYLCL & & allylchloride & ppo & 100 & & & 1 & 0 & \\
\hline CIS120E & & cis-1,2-Dichloroethylene & ppob & 1 & & & 4 & 0 & \\
\hline TDICPENE & & cis-1,3-D ichloropropene & ppb & 5 & & & 1 & 0 & \\
\hline MCRESOL & & m-Cresol & ppb & 10 & & & 3 & 0 & \\
\hline DINBENZ & & m-dinitrobenzene & ppb & 10 & & & 1 & 0 & \\
\hline OTOLHYO & & 0-Tolvidine & ppb & 10 & & & 1 & 0 & \\
\hline DIMEAMB & & P-D imethyl aminoazobenzene & ppb & 10 & & & 1 & 0 & \\
\hline PHENDIR & & p-Phenylenediamine & ppb & 10 & & & 1 & 0 & \\
\hline SYMTRIN & & sym-Trinitrobenzene & ppb & 10 & & & 1 & 0 & \\
\hline TRANDCE & & trans-1,2-Dichloroethylene & ppb & 1 & & & 4 & 0 & \\
\hline DICPENE & & trans-1,3-Dichloropropene & ppob & 5 & & & 1 & 0 & \\
\hline TDIBUTEN & & trans-1,4-dichloro-2-butene & ppb & 5 & & & 1 & 0 & \\
\hline
\end{tabular}

For explanation of this table, see Section 1.4 of report. 
Table 4-4. Constituents with at Least One Value Above the CRQL for the 100-N RCRA Sites Data for Reporting Period July 1 through September 30,1993 . (sheet 1 of 16)

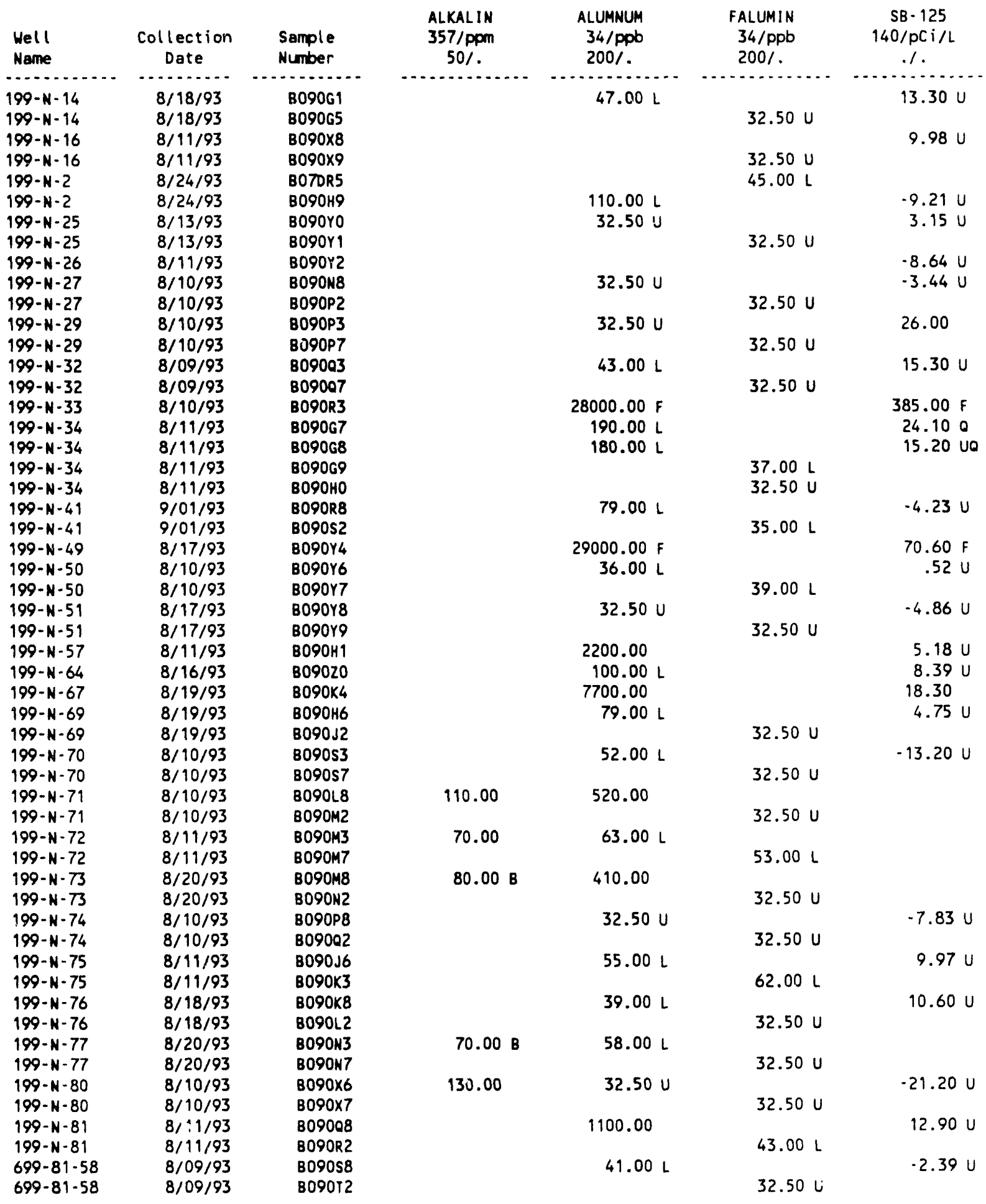


Table 4-4. Constituents with at Least One Value Above the CRQL for the 100-N RCRA Sites Data for Reporting Period July 1 through September 30, 1993. (sheet 2 of 16)

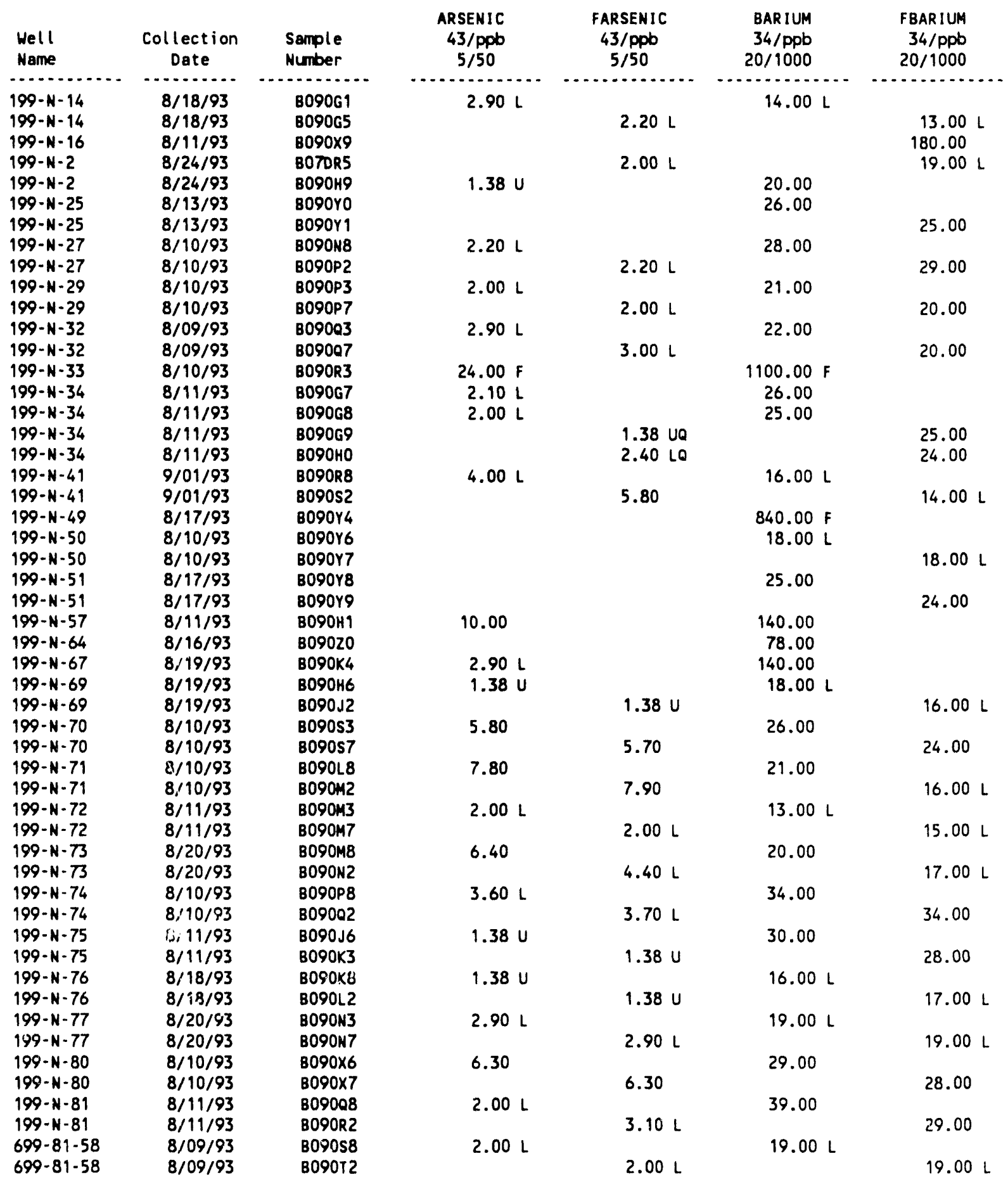


Table 4-4. Constituents with at Least One Value Above the CRQL for the 100-N RCRA Sites Data for Reporting Period July 1 through September 30, 1993. (sheet 3 of 16)

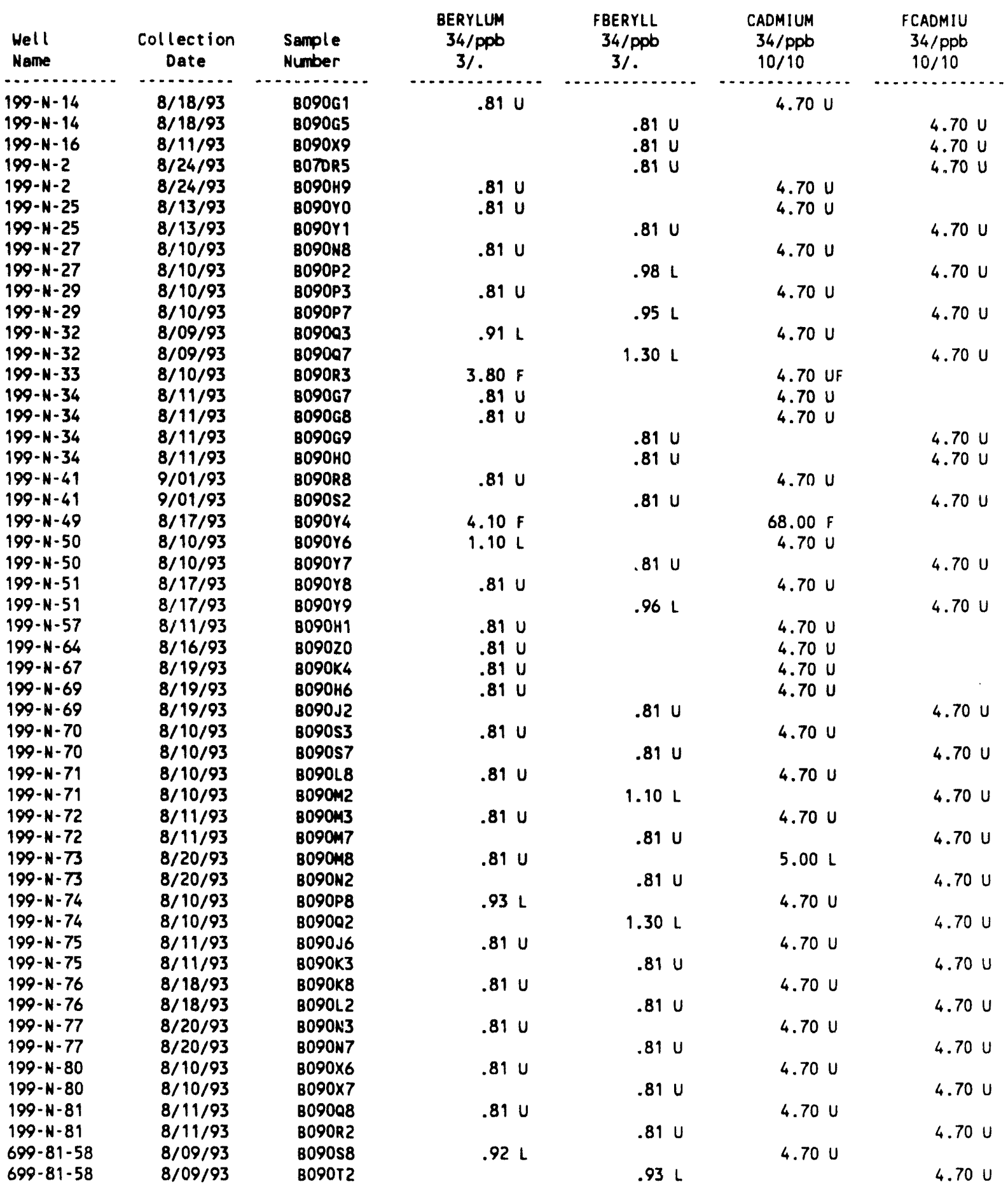


Table 4-4. Constituents with at Least One Value Above the CRQL for the 100-N RCRA Sites Data for Reporting Period July 1 through September 30, 1993. (sheet 4 of 16)

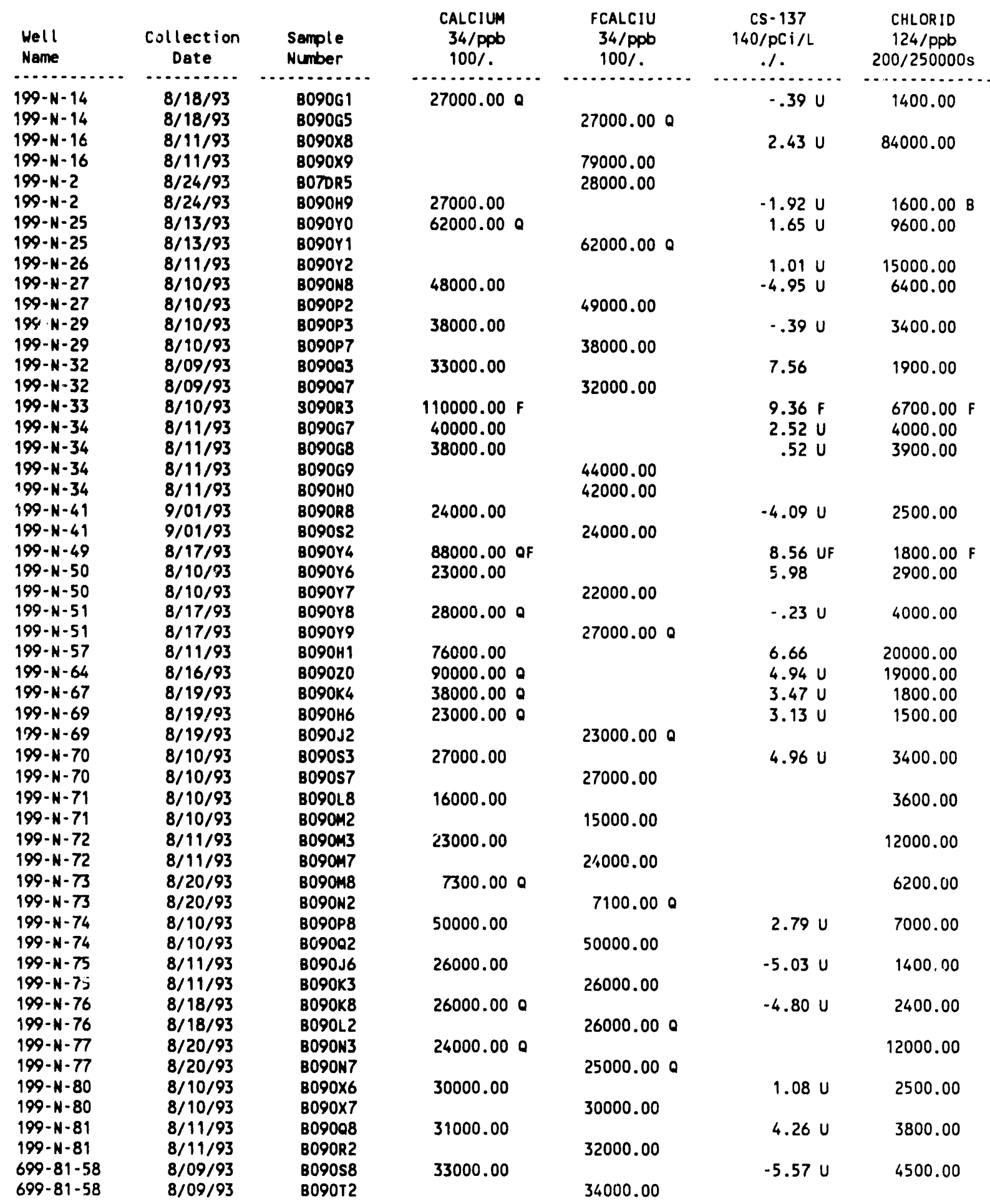


Table 4-4. Constituents with at Least One Value Above the CRQL for the 100-N RCRA Sites Data for Reporting Period July 1 through September 30, 1993. (sheet 5 of 16)

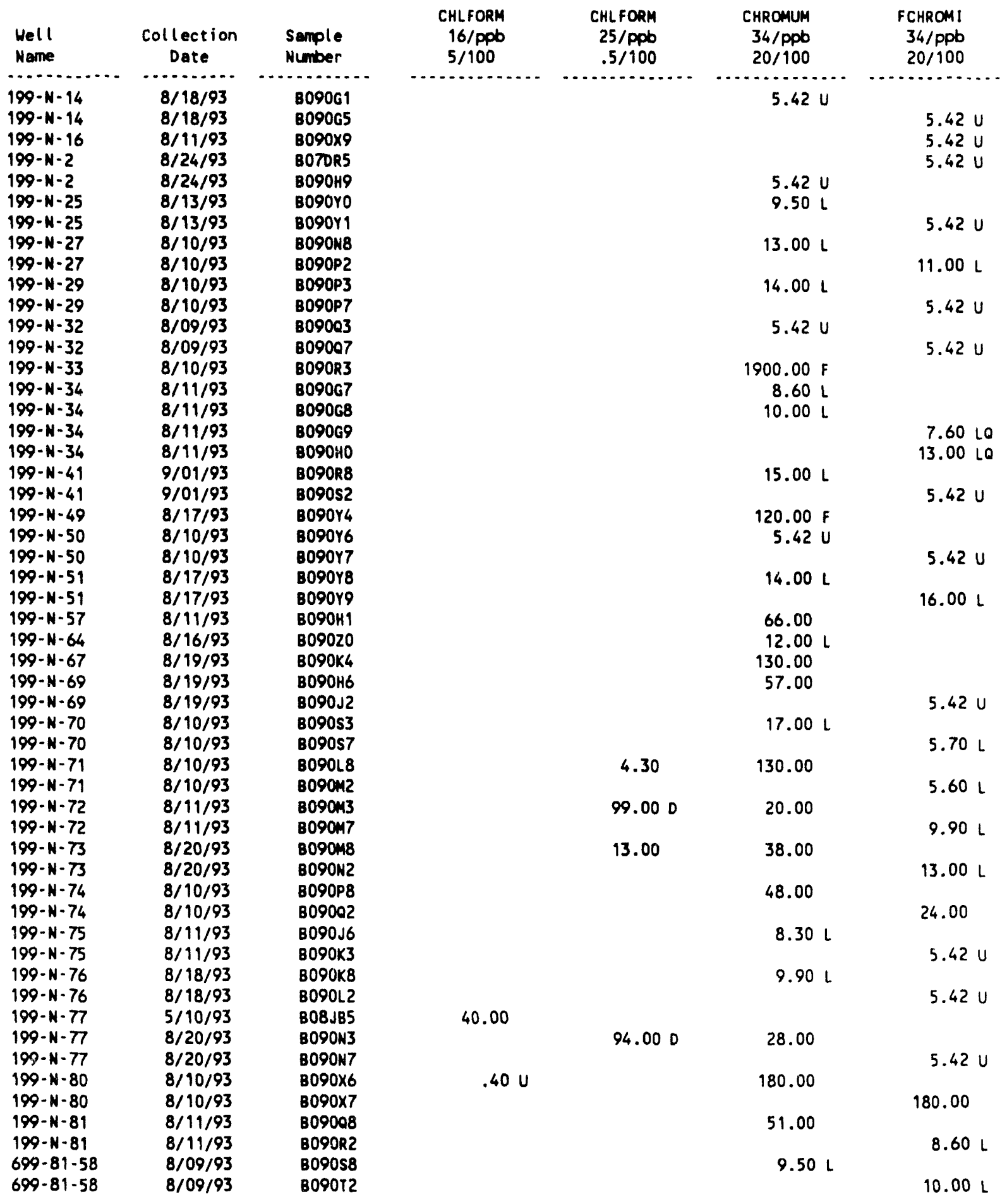


Table 4-4. Constituents with at Least One Value Above the CRQL for the 100-N RCRA Sites Data for Reporting Period July 1 through September 30,1993. (sheet 6 of 16)

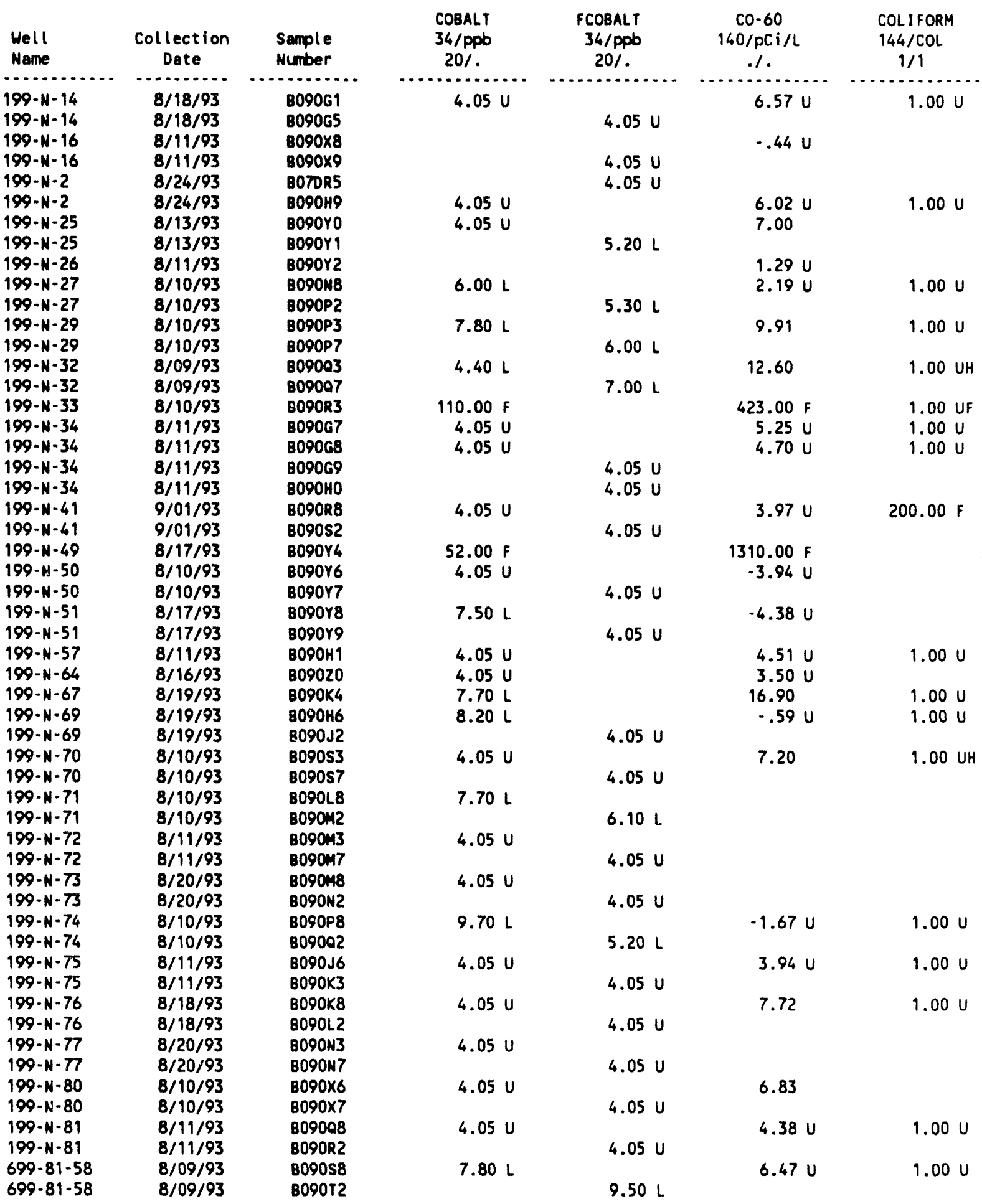


Table 4-4. Constituents with at Least One Value Above the CRQL for the 100-N RCRA Sites Data for Reporting Period July 1 through September 30, 1993. (sheet 7 of 16)

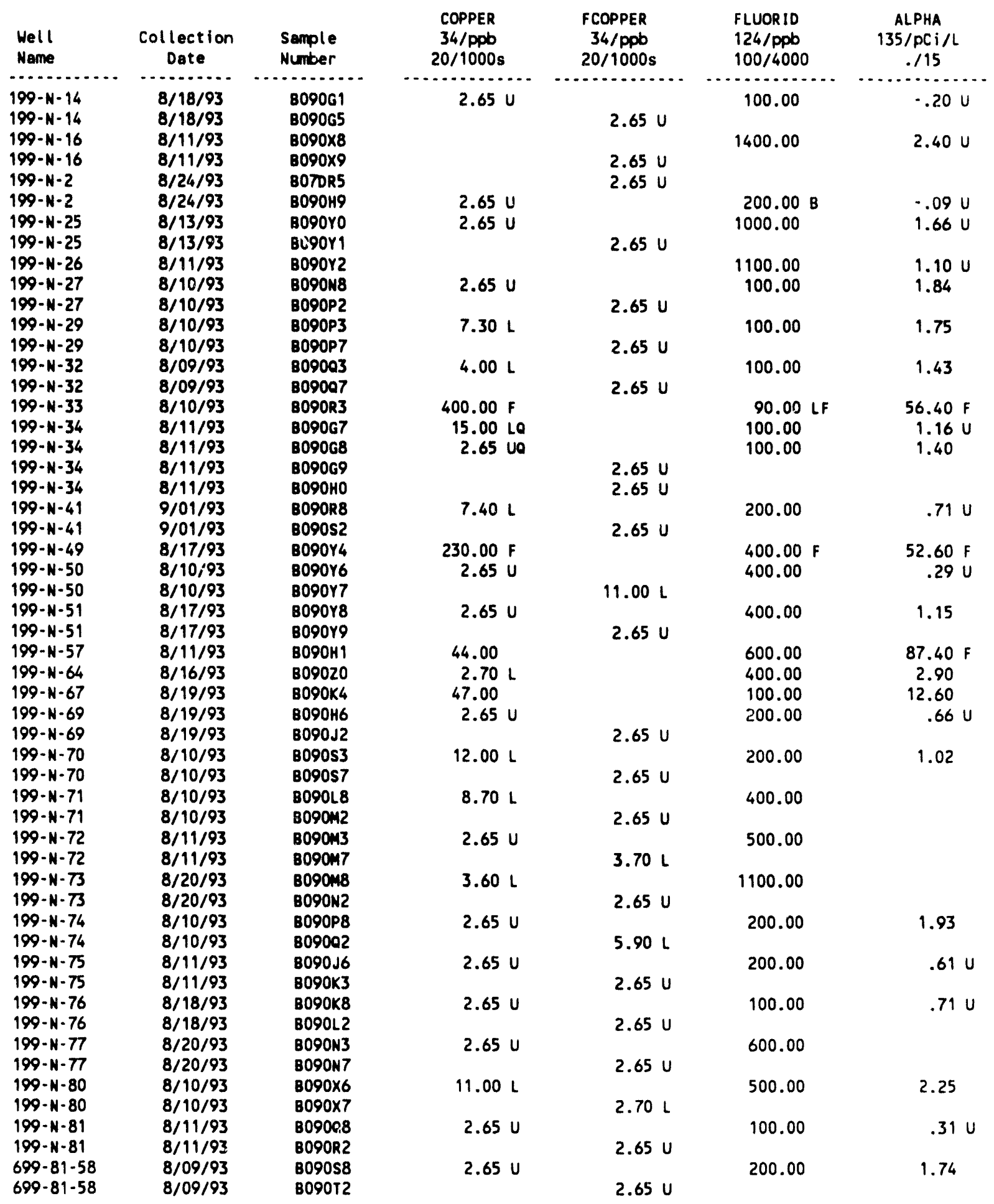


Table 4-4. Constituents with at Least One Value Above the CRQL for the 100-N RCRA Sites Data for Reporting Period July 1 through September 30, 1993. (sheet 8 of 16)

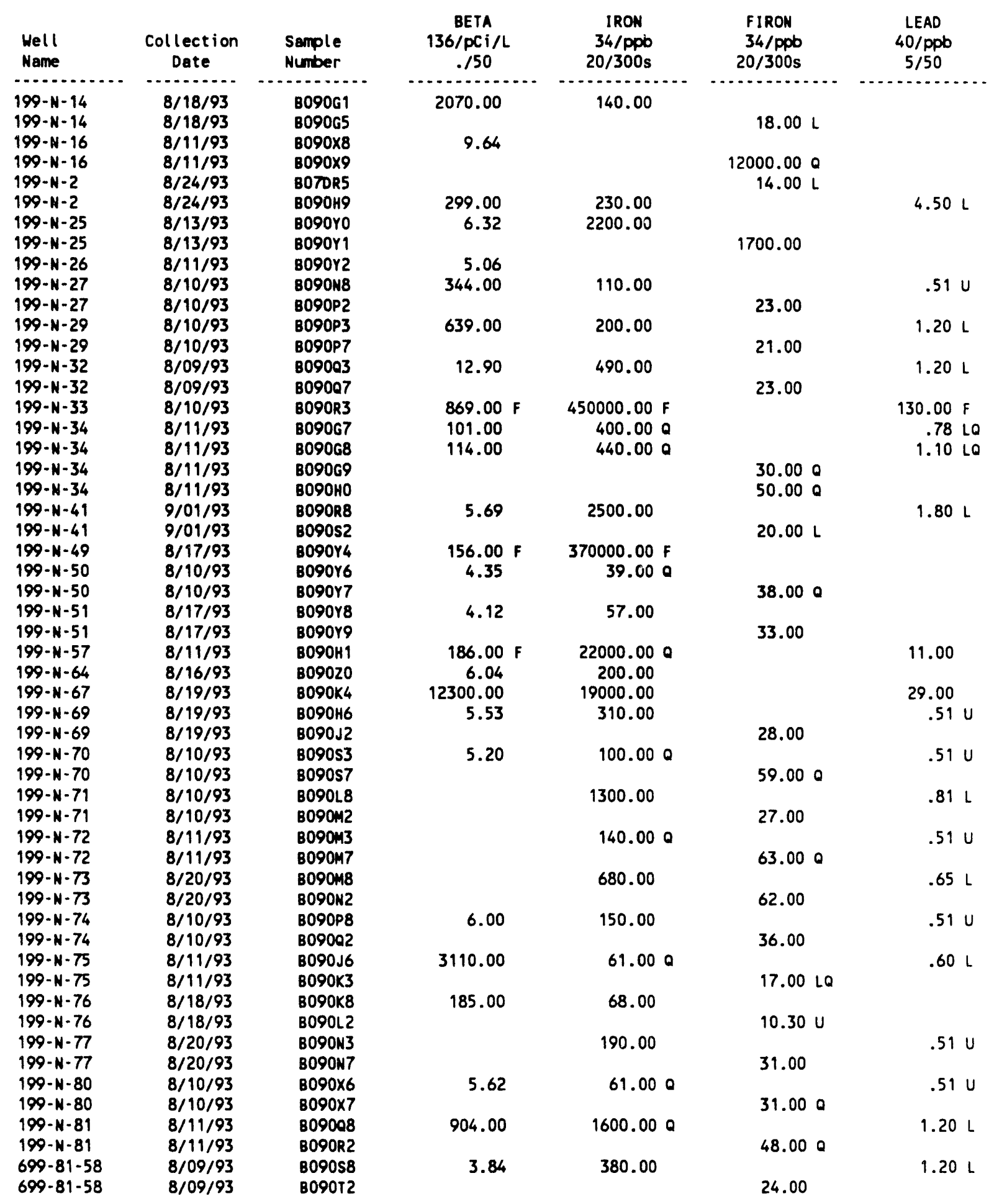


Table 4-4. Constituents with at Least One Value Above the CRQL for the 100-N RCRA Sites Data for Reporting Period July 1 through September 30, 1993. (sheet 9 of 16)

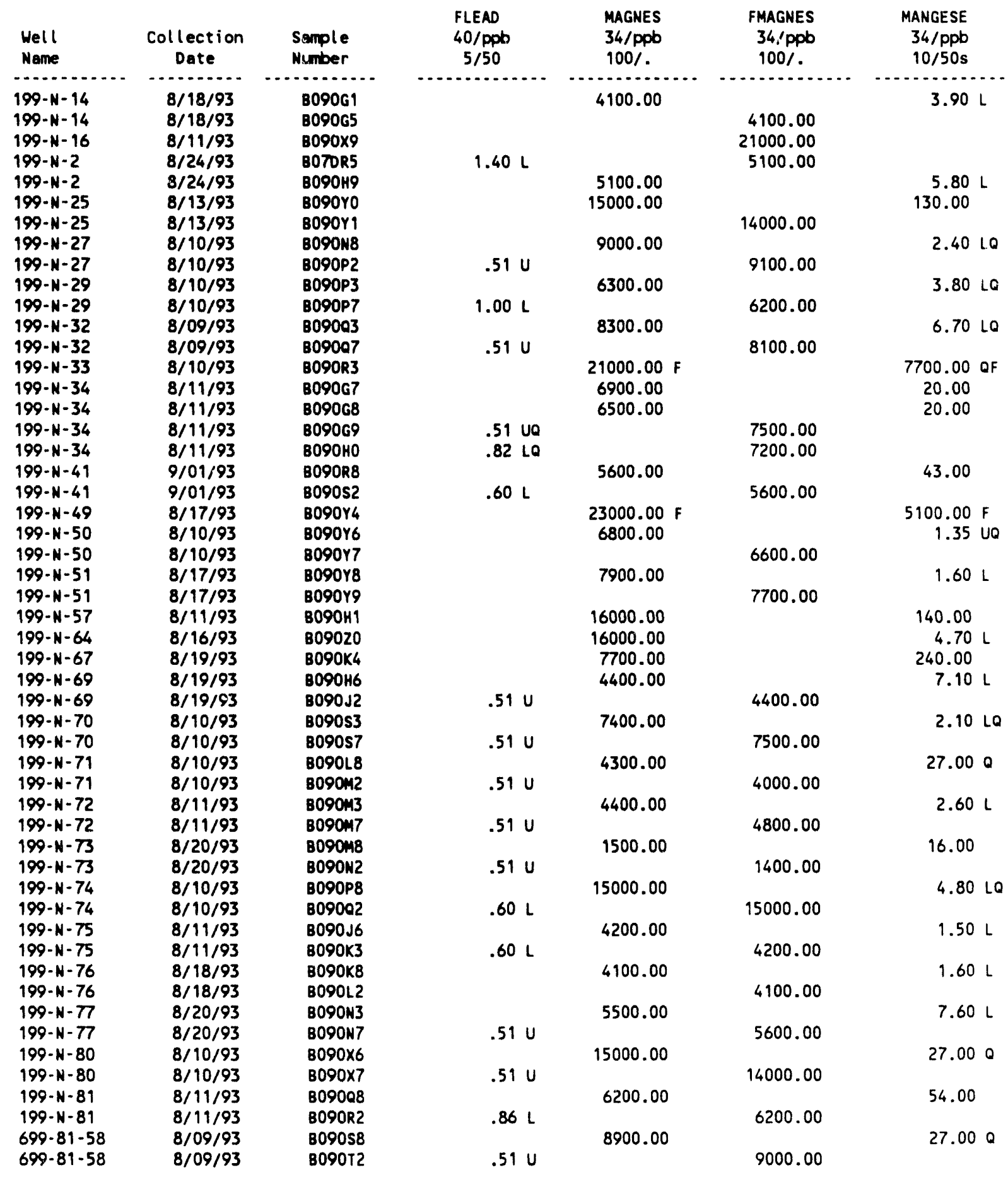


Table 4-4. Constituents with at Least One Value Above the CRQL for the 100-N RCRA Sites Data for Reporting Period July 1 through September 30, 1993. (sheet 10 of 16)

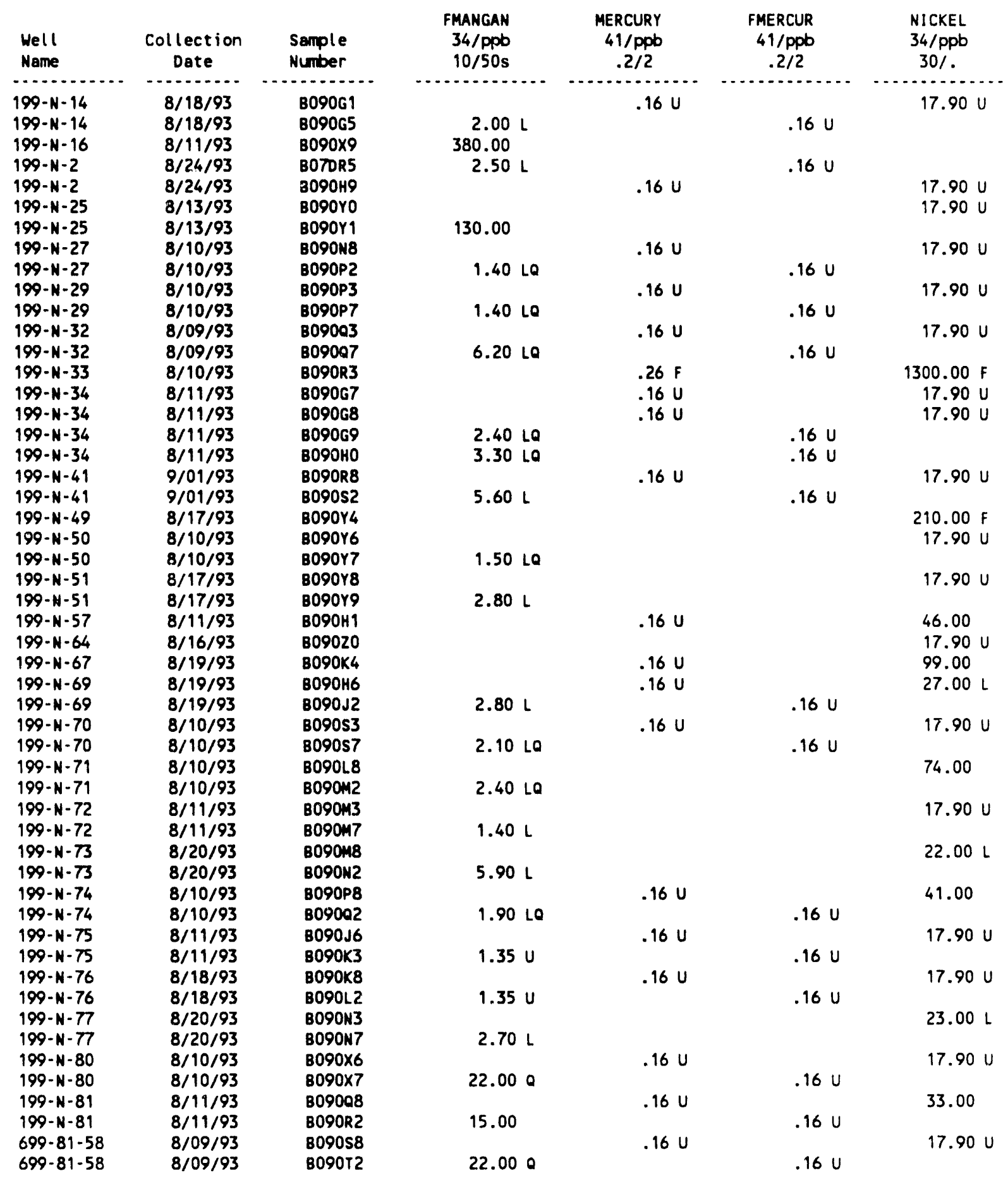


Table 4-4. Constituents with at Least One Value Above the CRQL for the 100-N RCRA Sites Data for Reporting Period July 1 through September 30, 1993. (sheet 11 of 16)

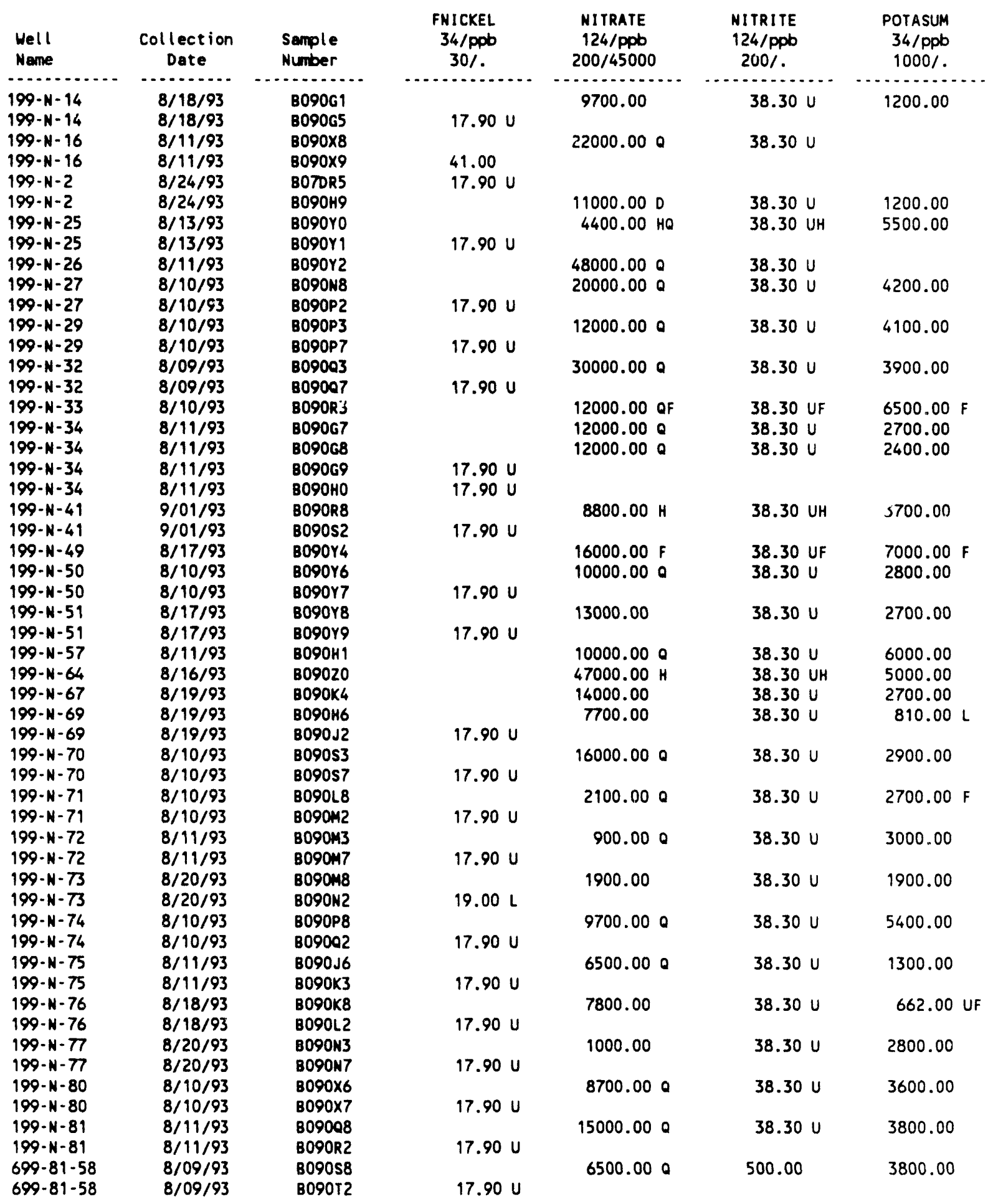


Table 4-4. Constituents with at Least One Value Above the CRQL for the 100-N RCRA Sites Data for Reporting Period July 1 through September 30, 1993. (sheet 12 of 16)

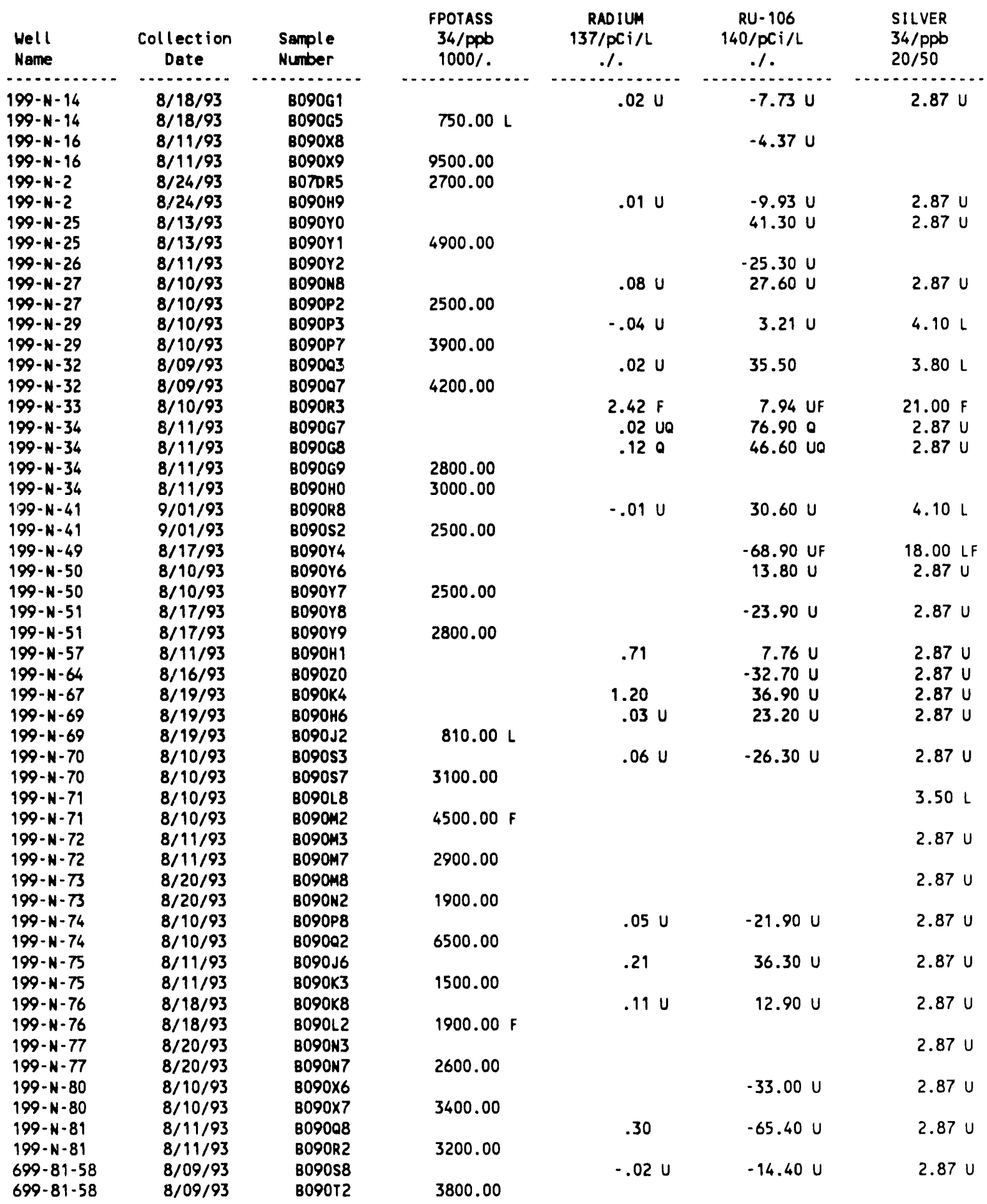


Table 4-4. Constituents with at Least One Value Above the CRQL for the 100-N RCRA Sites Data for Reporting Period July 1 through September 30, 1993. (sheet 13 of 16)

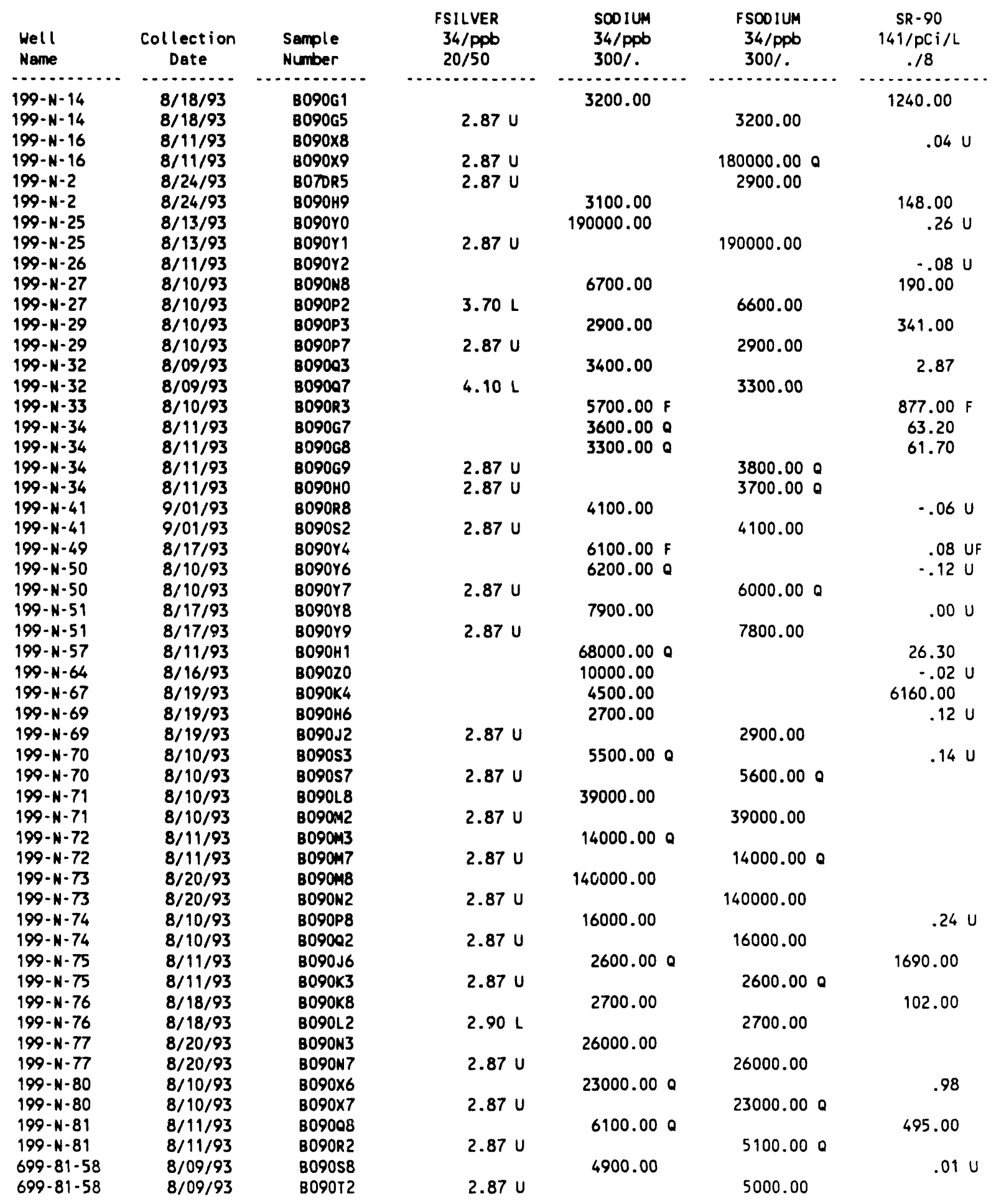


Table 4-4. Constituents with at Least One Value Above the CRQL for the 100-N RCRA Sites Data for Reporting Period July 1 through September 30, 1993. (sheet 14 of 16)

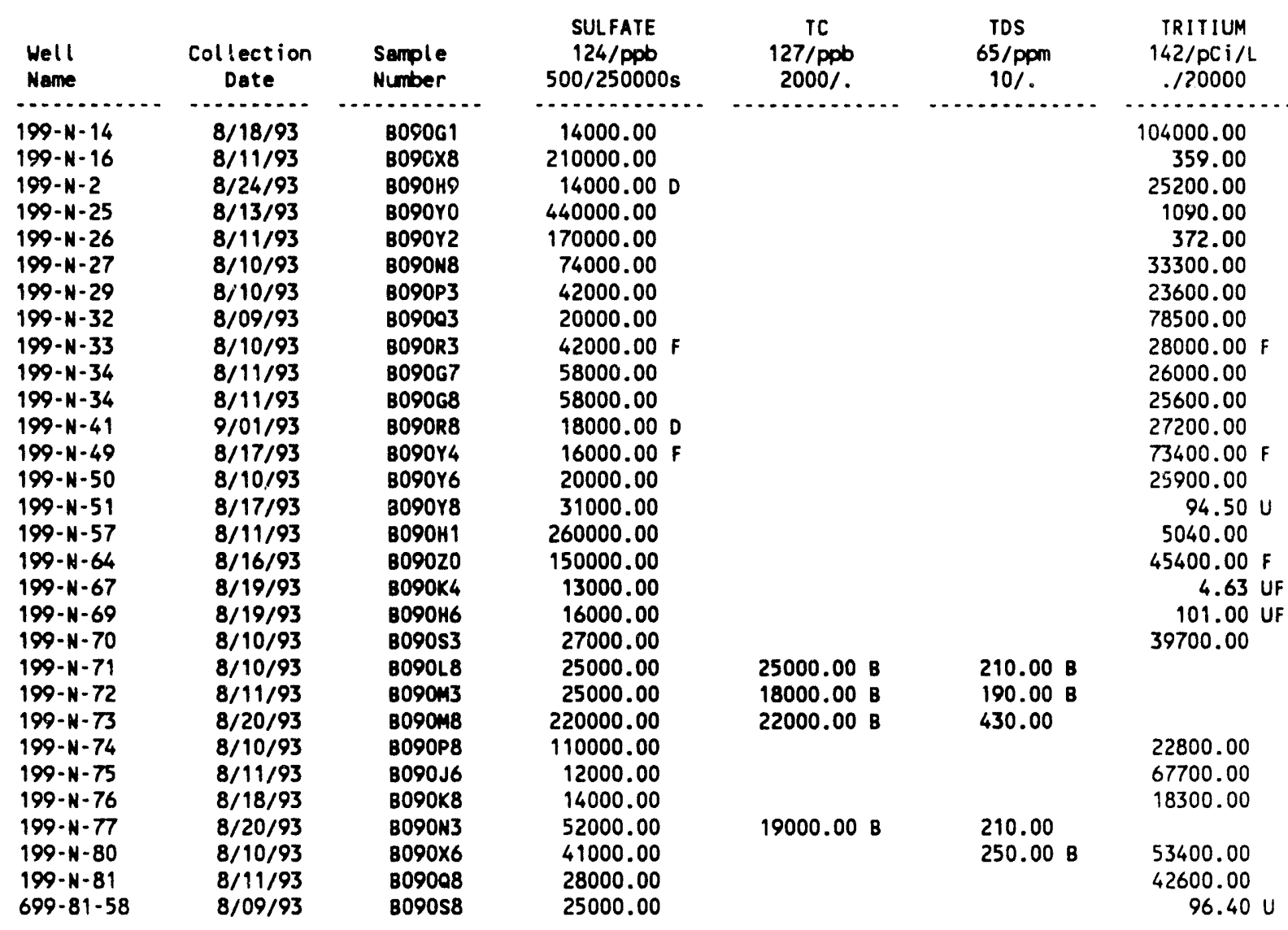

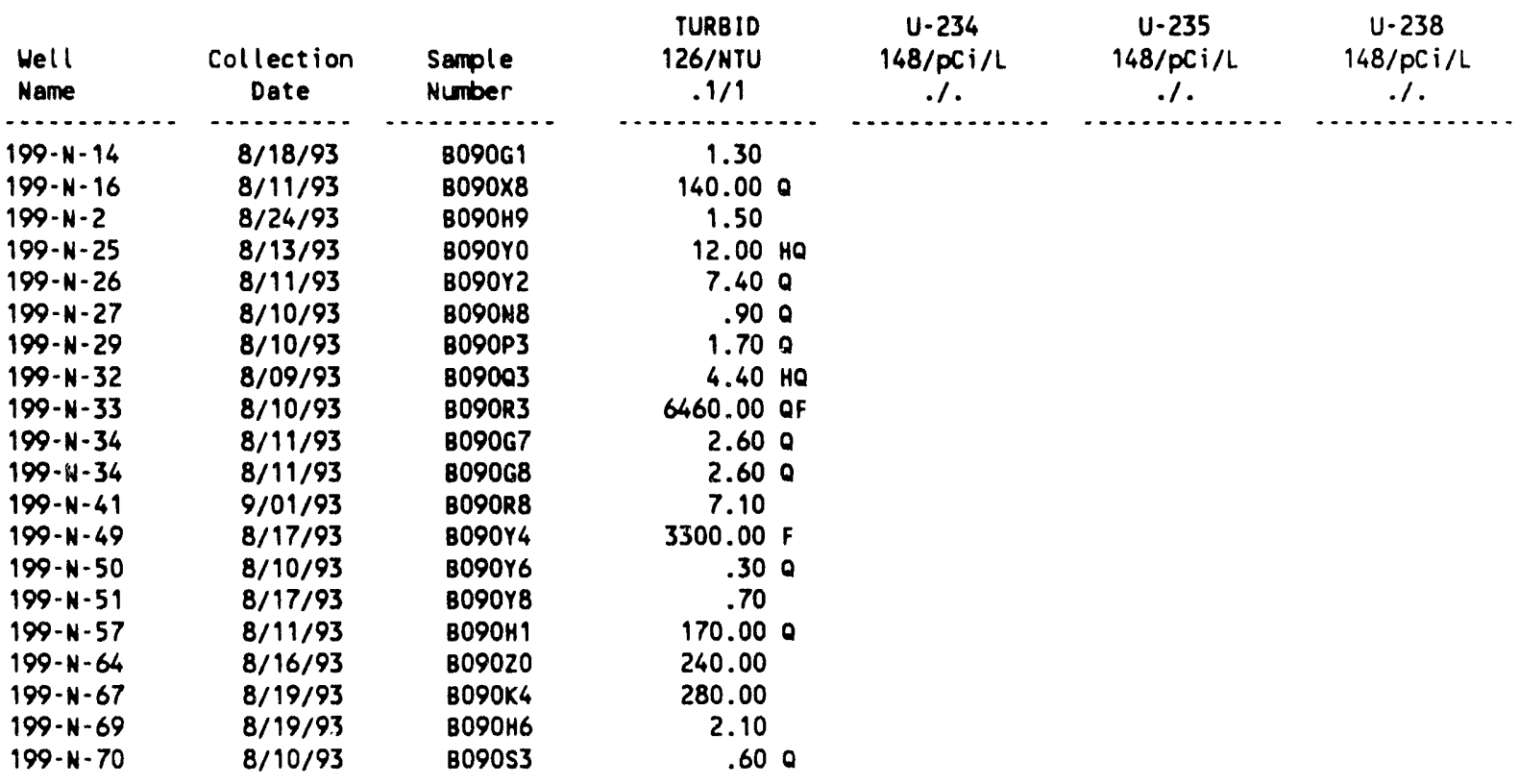


Table 4-4. Constituents with at Least One Value Above the CRQL for the 100-N RCRA Sites Data for Reporting Period July 1 through September 30, 1993. (sheet 15 of 16)

\begin{tabular}{|c|c|c|c|c|c|c|}
\hline $\begin{array}{l}\text { Well } \\
\text { Name }\end{array}$ & $\begin{array}{c}\text { Collection } \\
\text { Date }\end{array}$ & $\begin{array}{l}\text { Sample } \\
\text { Number }\end{array}$ & $\begin{array}{c}\text { TURBID } \\
\text { 126/NTU } \\
.1 / 1\end{array}$ & $\begin{array}{c}U-234 \\
148 / \mathrm{pCi} / \mathrm{L} \\
.1 .\end{array}$ & $\begin{array}{c}U-235 \\
148 / p C i / L \\
. / .\end{array}$ & $\begin{array}{c}U-238 \\
148 / p C i / L \\
. / .\end{array}$ \\
\hline $\begin{array}{l}199-N-71 \\
199-N-72 \\
199-N-73 \\
199-N-74 \\
199-N-75 \\
199-N-76 \\
199-N-77 \\
199-N-80 \\
199-N-81 \\
699-81-58\end{array}$ & $\begin{array}{l}8 / 10 / 93 \\
8 / 11 / 93 \\
8 / 20 / 93 \\
8 / 10 / 93 \\
8 / 11 / 93 \\
8 / 18 / 93 \\
8 / 20 / 93 \\
8 / 10 / 93 \\
8 / 11 / 93 \\
8 / 09 / 93\end{array}$ & $\begin{array}{l}\text { BO9OL8 } \\
\text { B090M3 } \\
\text { B090M8 } \\
\text { B090P8 } \\
\text { B090J6 } \\
\text { B090K8 } \\
\text { B090N3 } \\
\text { B090X6 } \\
\text { B09008 } \\
\text { B090S8 }\end{array}$ & $\begin{array}{c}15.100 \\
.800 \\
11.00 \\
1.000 \\
.600 \\
.80 \\
1.90 \\
.900 \\
22.000 \\
4.70\end{array}$ & 1.93 & .06 & 1.23 \\
\hline
\end{tabular}

\begin{tabular}{|c|c|c|c|c|c|c|}
\hline $\begin{array}{l}\text { Well } \\
\text { Name }\end{array}$ & $\begin{array}{l}\text { Collection } \\
\text { Date }\end{array}$ & $\begin{array}{l}\text { Sample } \\
\text { Number }\end{array}$ & $\begin{array}{c}\text { VANADLM } \\
34 / \text { ppD } \\
30 \% .\end{array}$ & $\begin{array}{l}\text { FVAMADI } \\
34 / \mathrm{ppb} \\
30 \%\end{array}$ & $\begin{array}{c}2 \text { 2INC } \\
34 / \mathrm{ppb} \\
10 \%\end{array}$ & $\begin{array}{c}\text { FZINC } \\
34 / \mathrm{ppb} \\
10 \%\end{array}$ \\
\hline $199-N-14$ & $8 / 18 / 93$ & B090G1 & $10.00 \mathrm{~L}$ & & $5.20 \mathrm{~L}$ & \\
\hline $199-N-14$ & $8 / 18 / 93$ & B090G5 & & $8.20 \mathrm{~L}$ & & $6.50 \mathrm{~L}$ \\
\hline $199-N-16$ & $8 / 11 / 93$ & B090X9 & & $3.84 \mathrm{U}$ & & 3.44 UO \\
\hline $199-N-2$ & $8 / 24 / 93$ & BOTOR5 & & $5.70 \mathrm{~L}$ & & \\
\hline $199-N-2$ & $8 / 24 / 93$ & BO9OH9 & $4.50 \mathrm{~L}$ & & $5.30 \mathrm{~L}$ & \\
\hline $199-N-25$ & $8 / 13 / 93$ & B090YO & $9.20 \mathrm{~L}$ & & 62.00 & \\
\hline $199-N-25$ & $8 / 13 / 93$ & BO90Y1 & & $8.30 \mathrm{~L}$ & & $7.80 \mathrm{~L}$ \\
\hline $199-N-27$ & $8 / 10 / 93$ & BO9ON8 & $8.20 \mathrm{~L}$ & & $3.44 U$ & \\
\hline $199-N-27$ & $8 / 10 / 93$ & B090P2 & & $8.30 \mathrm{~L}$ & & $3.44 \mathrm{U}$ \\
\hline $\begin{array}{l}199-N-29 \\
190-N-29\end{array}$ & $8 / 10 / 93$ & 8090P3 & $8.4 \mathrm{~J} \mathrm{~L}$ & & 11.00 & 5,101 \\
\hline $\begin{array}{l}199-N-29 \\
199-N-32\end{array}$ & $\begin{array}{l}8 / 10 / 93 \\
8 / 09 / 93\end{array}$ & $\begin{array}{l}\text { B090P7 } \\
\text { B09003 }\end{array}$ & $6.90 \mathrm{~L}$ & $5.40 \mathrm{~L}$ & $4.50 \mathrm{~L}$ & $5.10 \mathrm{~L}$ \\
\hline $199-N-32$ & $8 / 09 / 93$ & B09007 & & $11.00 \mathrm{~L}$ & & $3.44 \mathrm{U}$ \\
\hline $199-N-33$ & $8 / 10 / 93$ & B090R3 & $470.00 \mathrm{~F}$ & & $7600.00 \mathrm{~F}$ & \\
\hline $199-N-34$ & $8 / 11 / 93$ & B090G7 & $6.30 \mathrm{~L}$ & & $39.00 \mathrm{Q}$ & \\
\hline $199-N-34$ & $8 / 11 / 93$ & 8090G8 & $7.80 \mathrm{~L}$ & & 28.000 & \\
\hline $199-N-34$ & $8 / 11 / 93$ & B090G9 & & $8.70 \mathrm{LO}$ & & 23.000 \\
\hline $199-N-34$ & $8 / 11 / 93$ & В090HO & & 5.90 LO & & 17.000 \\
\hline $199-N-41$ & 9/01/93 & B090R8 & $22.00 \mathrm{~L}$ & & 13.00 & \\
\hline $199-N-41$ & 9/01/93 & B090S2 & & $18.00 \mathrm{~L}$ & & $3.44 U$ \\
\hline $199-N-49$ & $8 / 17 / 93$ & B090Y4 & $690.00 \mathrm{~F}$ & & $2100.00 \mathrm{~F}$ & \\
\hline $199-N-50$ & $8 / 10 / 93$ & B090Y6 & $14.00 \mathrm{~L}$ & & $7.70 \mathrm{LQ}$ & \\
\hline $199-N-50$ & $8 / 10 / 93$ & B090Y7 & & $13.00 \mathrm{~L}$ & & 18.000 \\
\hline $199-N-51$ & $8 / 17 / 93$ & B090Y8 & $25.00 \mathrm{~L}$ & & 14.00 & \\
\hline $199-N-51$ & $8 / 17 / 93$ & B090Y9 & & $24.00 \mathrm{~L}$ & & $9.20 \mathrm{~L}$ \\
\hline $199-N-57$ & $8 / 11 / 93$ & BO9OHI & 49.00 & & 1000.000 & \\
\hline $199-N-64$ & $8 / 16 / 93$ & B09020 & $7.40 \mathrm{~L}$ & & $8.50 \mathrm{~L}$ & \\
\hline $199-N-67$ & $8 / 19 / 93$ & B090K4 & $28.00 \mathrm{~L}$ & & 320.00 & \\
\hline $199-N-69$ & $8 / 19 / 93$ & B090H6 & $8.00 \mathrm{~L}$ & & $6.50 \mathrm{~L}$ & \\
\hline $199-N-69$ & $8 / 19 / 93$ & B090 J2 & & $6.10 \mathrm{~L}$ & & $9.20 \mathrm{~L}$ \\
\hline $199-N-70$ & $8 / 10 / 93$ & B09053 & $13.00 \mathrm{~L}$ & & 16.000 & \\
\hline $199-N-70$ & $8 / 10 / 93$ & B09057 & & $9.80 \mathrm{~L}$ & & 11.000 \\
\hline $199-N-71$ & $8 / 10 / 93$ & B090L8 & $29.00 \mathrm{~L}$ & & $3.44 \mathrm{U}$ & \\
\hline $199-N-71$ & $8 / 10 / 93$ & 8090M2 & & 32.00 & & $3.44 U$ \\
\hline $199-N-72$ & $8 / 11 / 93$ & BO90M3 & $5.30 \mathrm{~L}$ & & 4.70 LO & \\
\hline $199-N-72$ & 8/11/93 & B090M7 & & $8.40 \mathrm{~L}$ & & 22.000 \\
\hline $199-N-73$ & $8 / 20 / 93$ & B090M8 & $20.00 \mathrm{~L}$ & & $7.10 \mathrm{~L}$ & \\
\hline $199-N-73$ & $8 / 20 / 93$ & B090N2 & & $20.00 \mathrm{~L}$ & & $3.44 \mathrm{U}$ \\
\hline
\end{tabular}


Table 4-4. Constituents with at Least One Value Above the CRQL for the 100-N RCRA Sites Data for Reporting Period July 1 through September 30,1993. (sheet 16 of 16)

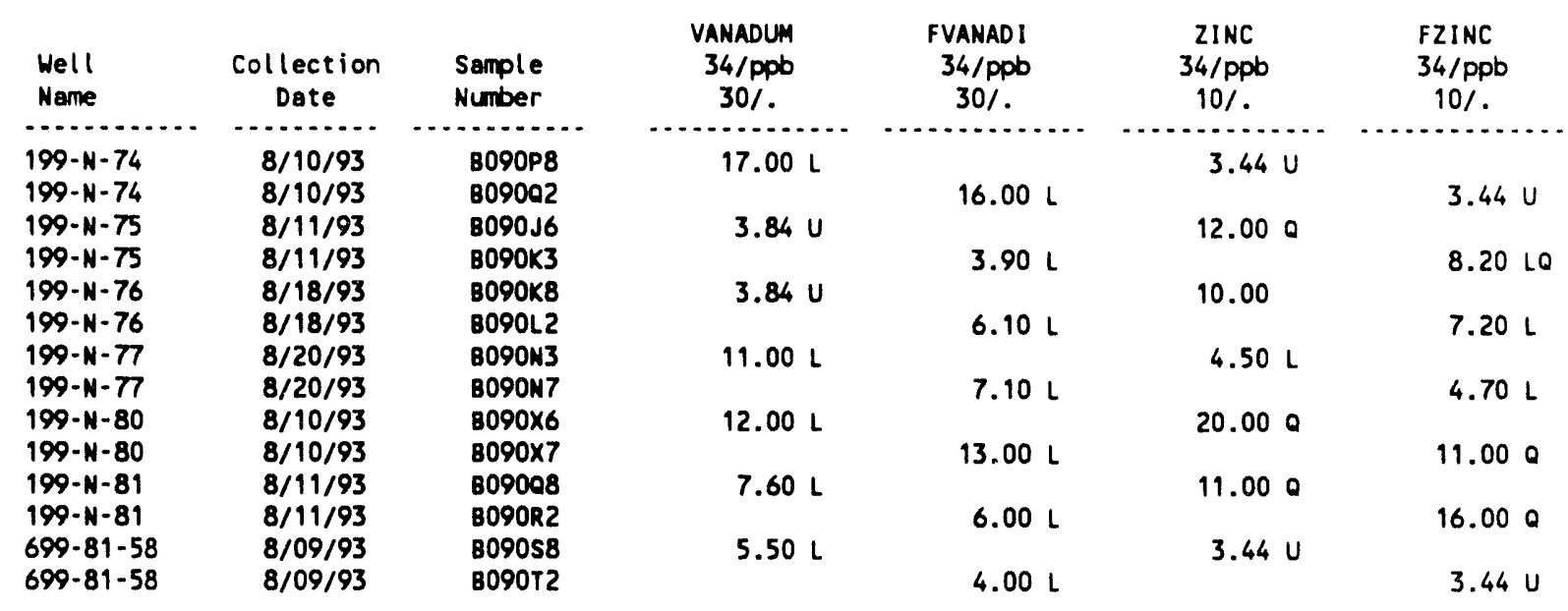

For explanation of this table, see Section 1.4 of report. 
Table 4-5. Contamination Indicator Parameters for the 100-N RCRA Sites Data for Reporting Period July 1 through September 30, 1993. (sheet 1 of 2)

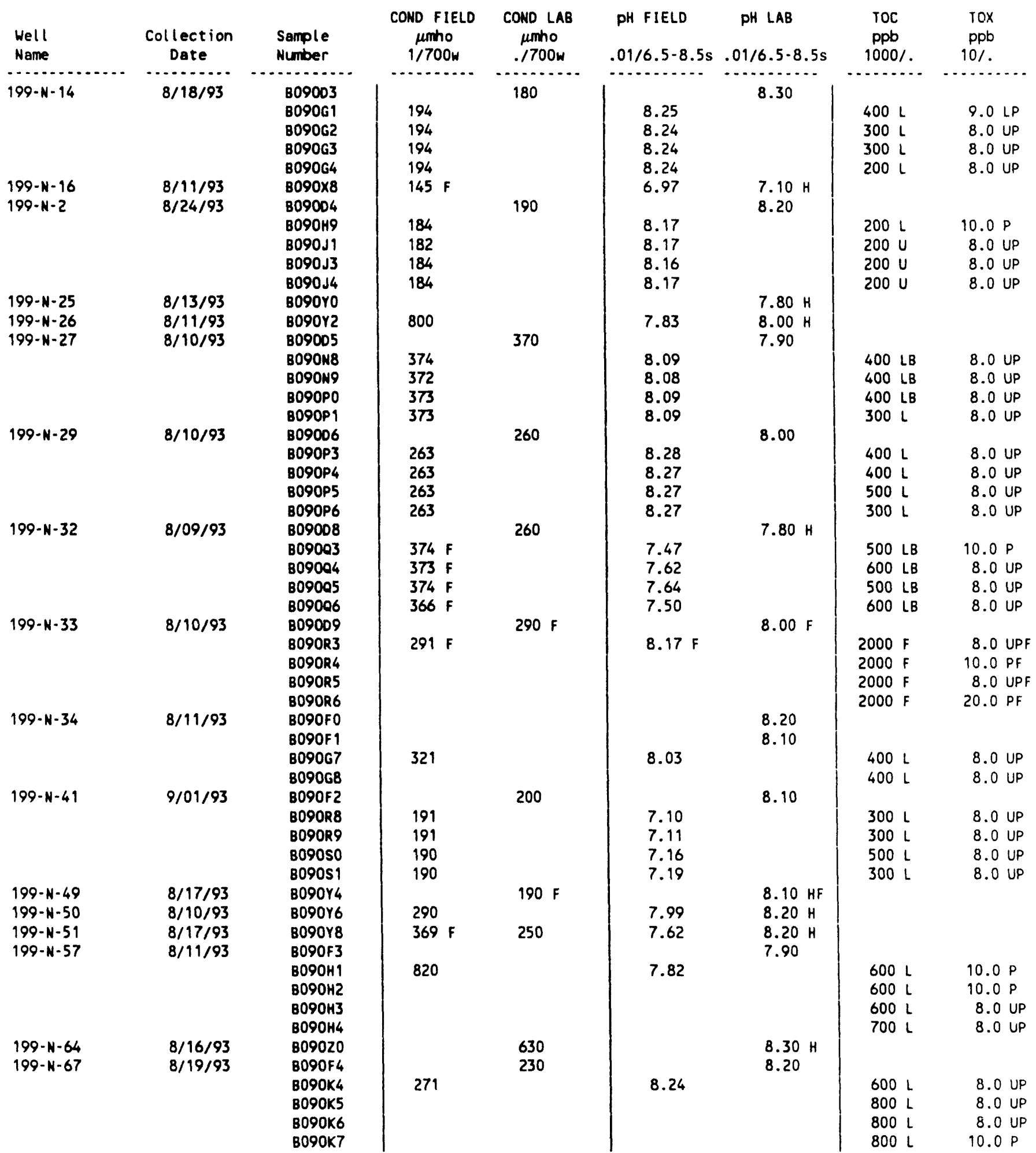


Table 4-5. Contamination Indicator Parameters for the 100-N RCRA Sites Data for Reporting Period July 1 through September 30, 1993. (sheet 2 of 2)

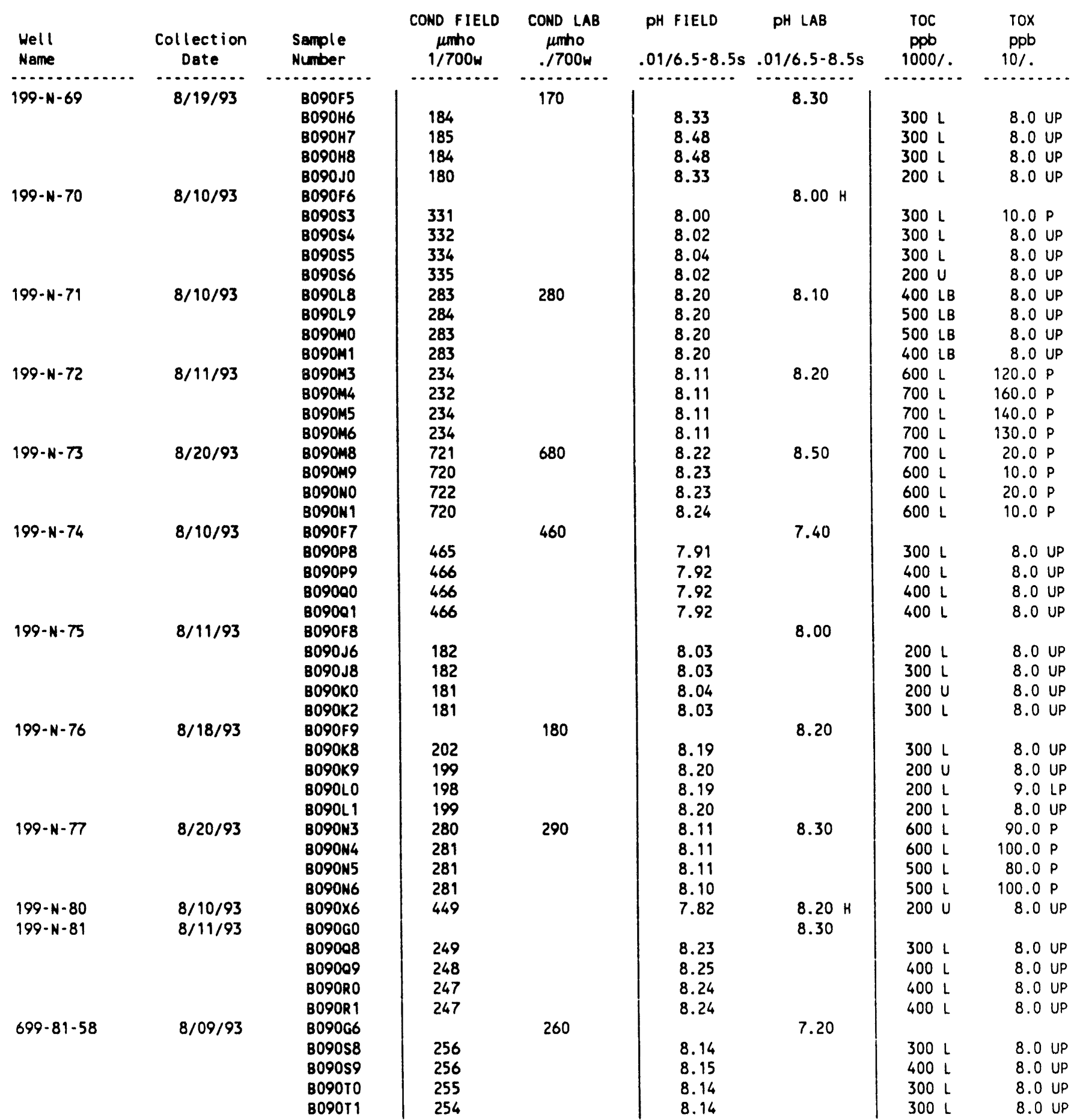

For explanation of this table, see Section 1.4 of report. 


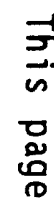

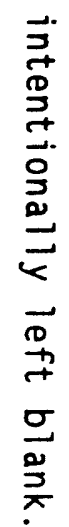


$\mathrm{DOE} / \mathrm{RL}-93-56-3$

CONTENTS

5.0 GROUT TREATMENT FACILITY . . . . . . . . . . . . . . . . . . 5-1

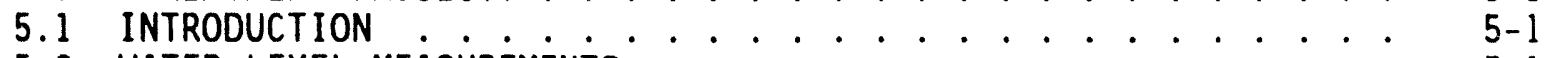

5.2 WATER LEVEL MEASUREMENTS . . . . . . . . . . . . . . . . . . . $5-1$

5.3 WATER CHEMISTRY DATA . . . . . . . . . . . . . . . 5-1 
DOE/RL-93-56-3

\section{LIST OF FIGURES}

5-1 Monitoring Well Locations for the Grout Treatment Facility . . . .

\section{LIST OF TABLES}

5-1 Monitoring Well Purpose and Sampling Schedule for the Grout

Treatment Facility Network..... . . . . . . . . . . 5-4

5-2 RCRA Water Level Measurement Report Grout Treament Facility, Third Quarter 1993 ................... 5-5

5-3 Constituent List and Summary of Results for the Grout Treatment Facility Data for Reporting Period July 1 through September 30, 1993 ................ . . . . . . .

5-4 Constituents with at Least One Value Above the CRQL for the Grout Treatment Facility Data for Reporting Period July 1 through September 30,1993................. 5-11

5-5 Contamination Indicator Parameters for the Grout Treatment Facility Data for Reporting Period July 1 through September 30, 1993 ................ . . 5-14 


\title{
5.0 GROUT TREATMENT FACILITY
}

\author{
L. C. Swanson \\ Westinghouse Hanford Company
}

\subsection{INTRODUCTION}

The Grout Treatment Facility (GTF) is located east of the 200 East Area on the Hanford Site (Figure 5-1). Grout treatment is a process that involves blending a slurry of selected mixed (radioactive and hazardous) liquid waste with grout-forming solids and chemical additives. This slurry is then poured into subterranean vaults for hardening (curing) to produce a massive, stabilized cement-like block suitable for in-place, long-term disposal. Each vault is operated as a surface impoundment while the grout slurry hardens. When the grout hardens, the vault is sealed and closed as a RCRA-permitted landfill. Currently no waste has been placed in this facility.

The RCRA groundwater monitoring network consists of seven downgradient wells and three upgradient wells, as listed in Table 5-1. Three additional groundwater monitoring wells are either scheduled for construction in fiscal year (FY) 1993 or are under construction this quarter. Well locations are depicted in Figure 5-1. A RCRA detection-level monitoring program is now in effect, following 40 CFR 265 and the Interim-Status Ground Water Monitoring Plan for the Grout Treatment Facility (Teel et al. 1989). Baseline concentrations have been established for most wells in the network. Quarterly sampling to establish the groundwater chemistry baseline in the two most recently completed wells (299-E25-44 and 299-E25-45) is now completed. Samples are taken semiannually at the RCRA wells in the network after at least four quarters of baseline data are collected. Table 5-1 lists the sampling network and the monitoring status for each well.

\subsection{WATER LEVEL MEASUREMENIS}

During this quarter, water levels were measured either monthly or quarterly in the 10 network wells and several other monitoring wells located in the area of the GTF. Water level measurements were also recorded when the network wells were sampled.

Water level measurements for this quarter are consistent with past water level measurements. However, one water level measurement at well 299-E25-32P is flagged with a ' + ,' indicating that this value is suspect. The reported value is about $0.3 \mathrm{~m}$ ( $1 \mathrm{ft}$ ) higher than expected. Water levels for all of the network wells are listed in Table 5-2.

\subsection{WATER CHEMISTRY DATA}

Groundwater sample results are reported for four wells this quarter. All other wells are either sampled on a semiannual schedule (after at least one full year of quarterly sampling), or are sampled as part of other RCRA facility groundwater monitoring networks. Table 5-1 lists each GTF well and the sampling frequency and dates for this quarter. Table 5-3 is a summary 
listing of the analysis results for all of the wells. Table 5-4 contains the analysis results for any constituents that had at least one detected value. Table 5-5 lists the analysis results for the four indicator parameters: specific conductance, field $\mathrm{pH}$, TOC, and TOX.

Almost all of the constituent concentrations for this quarter are below the DWSs. Those that did exceed the DWSs followed historical trends, such as unfiltered chromium, unfiltered iron, and turbidity; however, all of the filtered species for these constituents did fall below the DWS. A turbidity reading of 6.1 NTUs was higher than normal at well 299-E25-31, and probably reflects wellhead remediation work performed at this well the previous quarter.

Constituents that exceeded the CRQL al so followed historical trends, including arsenic, chloride, fluoride, and gross alpha. Unfiltered aluminum was higher than generally observed at well 299-E25-45 (450 ppb). The concentration of filtered aluminum was below the detection limit. The indicator parameters fell below the critical mean for all reported wells. TOX was not evaluated because of quality problems identified in the laboratory. Therefore, the data are flagged with a ' $P$,' showing that suspicions remain concerning the quality of the TOX data. Some calcium, fluoride, potassium, sodium, and zinc values are flagged with a ' $Q$ ' to reflect a problem with the quality of the $Q C$ data. The $B$ flag for the TOC results indicates a problem with blank sample contamination. Even so, the reported TOC data still fall in the expected range. 
Figure 5-1. Monitoring Well Locations for the Grout Treatment Facility.

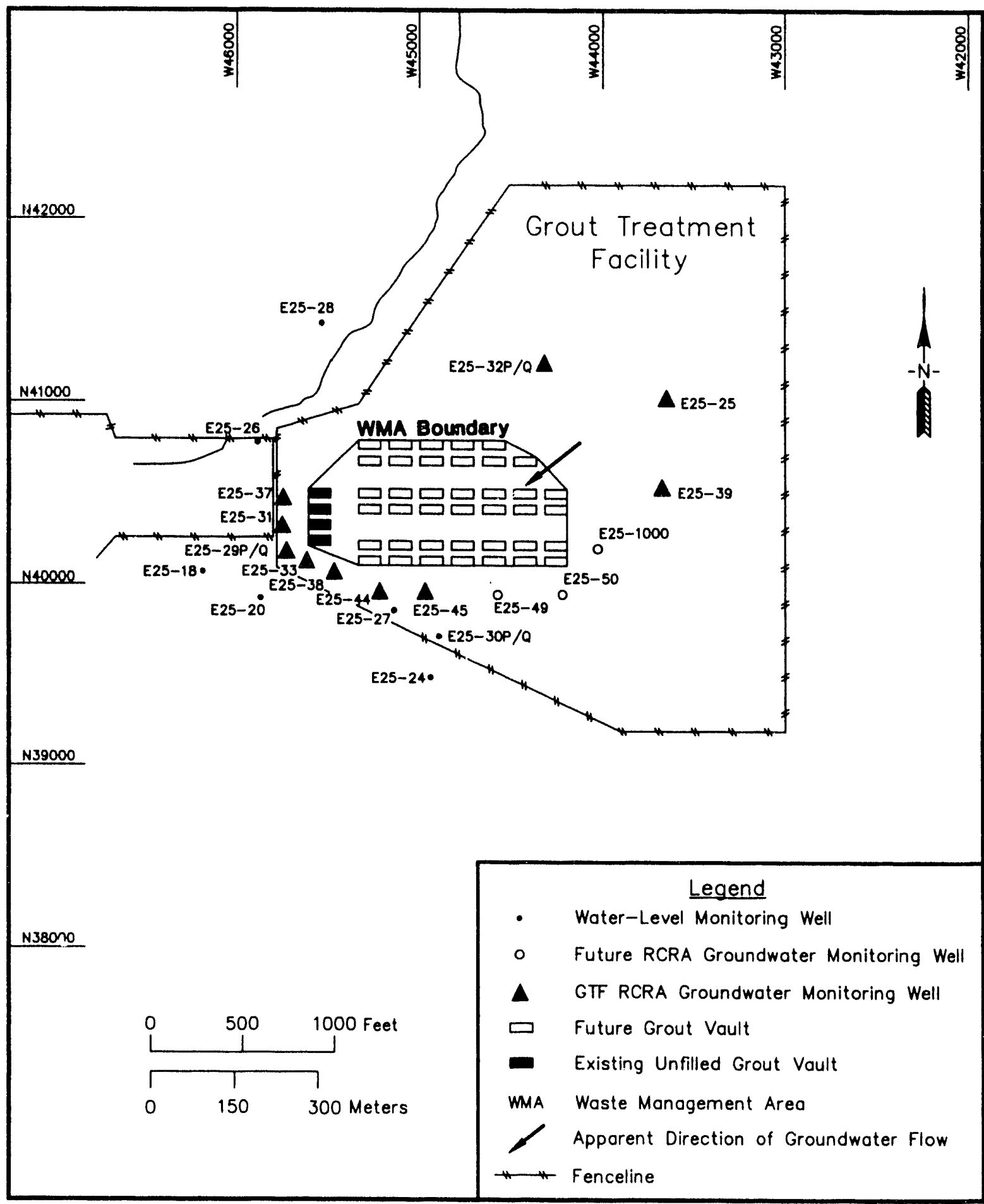


Table 5-1. Monitoring Well Purpose and Sampling Schedule for the Grout Treatment Facility Network.

\begin{tabular}{|c|c|l|c|c|}
\hline $\begin{array}{c}\text { Well no. } \\
(299-)\end{array}$ & $\begin{array}{c}\text { Relative } \\
\text { position }\end{array}$ & Hydrogeologic unit & $\begin{array}{c}\text { Sample } \\
\text { frequency }\end{array}$ & $\begin{array}{c}\text { Sample date, } \\
\text { 3rd Qtr 1993 }\end{array}$ \\
\hline E25-25 & Upgradient & Hanford: Water Table & Semiannually & N/A \\
\hline E25-32P & Upgradient & Hanford: Water Table & Quarterly & 7/15/93 \\
\hline E25-39 & Upgradient & Hanford: Water Table & Semiannually & N/A \\
\hline E25-29P & Downgradient & Hanford: Water Table & Semiannually & N/A \\
\hline E25-31 & Downgradient & Hanford: Water Table & Quarterly & 7/16/93 \\
\hline E25-33 & Downgradient & Hanford: Water Table & Semiannually & N/A \\
\hline E25-37 & Downgradient & Hanford: Water Table & Semiannually & N/A \\
\hline E25-38 & Downgradient & Hanford: Water Table & Semiannually & N/A \\
\hline E25-44 & Downgradient & Hanford: Water Table & Quarterly & 7/22/93 \\
\hline E25-45 & Downgradient & Hanford: Water Table & Quarterly & 7/22/93 \\
\hline
\end{tabular}

$N / A=$ not available.

aydrogeologic units include the sandy gravels of the Hanford formation. 
Table 5-2. RCRA Water Level Measurement Report Grout Treament Facility, Third Quarter 1993.

\begin{tabular}{|c|c|c|c|}
\hline Well & Date & $\begin{array}{l}\text { Depth to } \\
\text { water }(f t)\end{array}$ & $\begin{array}{l}\text { Water level } \\
\text { elevation } \\
\text { above msl (ft) }\end{array}$ \\
\hline 299-E25-18 & $\begin{array}{l}8 / 03 / 93 \\
9 / 07 / 93\end{array}$ & $\begin{array}{l}277.22 \\
277.19\end{array}$ & $\begin{array}{l}401.83 * \\
401.86\end{array}$ \\
\hline $299-E 25-20$ & $\begin{array}{l}7 / 15 / 93 \\
9 / 07 / 93\end{array}$ & $\begin{array}{l}274.70 \\
274.62\end{array}$ & $\begin{array}{l}401.60 \star \\
401.68\end{array}$ \\
\hline 299-E25-24 & $9 / 10 / 93$ & 277.77 & $401.78^{\star}$ \\
\hline $299-E 25-26$ & $\begin{array}{l}7 / 08 / 93 \\
8 / 02 / 93 \\
8 / 06 / 93 \\
9 / 03 / 93\end{array}$ & $\begin{array}{l}266.38 \\
266.05 \\
266.35 \\
266.61\end{array}$ & $\begin{array}{l}402.14 \\
402.47^{\star} \\
402.17 \\
401.91\end{array}$ \\
\hline $299-E 25-28$ & $\begin{array}{l}7 / 08 / 93 \\
8 / 02 / 93 \\
8 / 05 / 93 \\
9 / 03 / 93\end{array}$ & $\begin{array}{l}260.01 \\
260.55 \\
260.06 \\
260.17\end{array}$ & $\begin{array}{l}402.43 \\
401.89 \star \\
402.38 \\
402.27\end{array}$ \\
\hline $299-E 25-31$ & $9 / 07 / 93$ & 272.47 & 400.06 \\
\hline 299-E25-32P & $\begin{array}{l}7 / 08 / 93 \\
7 / 15 / 93 \\
8 / 05 / 93 \\
9 / 03 / 93\end{array}$ & $\begin{array}{l}267.89 \\
267.75 \\
267.81 \\
267.04\end{array}$ & $\begin{array}{l}402.15 \\
402.29 \star \\
402.23 \\
403.00 t\end{array}$ \\
\hline 299-E25-39 & $9 / 02 / 93$ & 268.63 & 402.60 \\
\hline $299-E 25-44$ & $7 / 22 / 93$ & 272.88 & $402.41^{*}$ \\
\hline $299-E 25-45$ & $7 / 22 / 93$ & 275.96 & $402.49 *$ \\
\hline
\end{tabular}

Notes: 1. Water level elevations are calculated by subtracting the measured depth to water from the surveyed elevation for the well.

2. Depth-to-water values are transcribed from field records.

3. Elevations marked with an ' $*$ ' were measured at the time of sampling.

4. Elevations marked with a ' + ' are outside of the expected range, and are suspected of error.

5. To convert feet to meters multiply by 0.3048 . 
Table 5-3. Constituent List and Summary of Results for the Grout Treatment Facility Data for Reporting Period July 1 through September 30, 1993. (sheet 1 of 5)

CONTAMINATION INDICATOR PARAMETERS

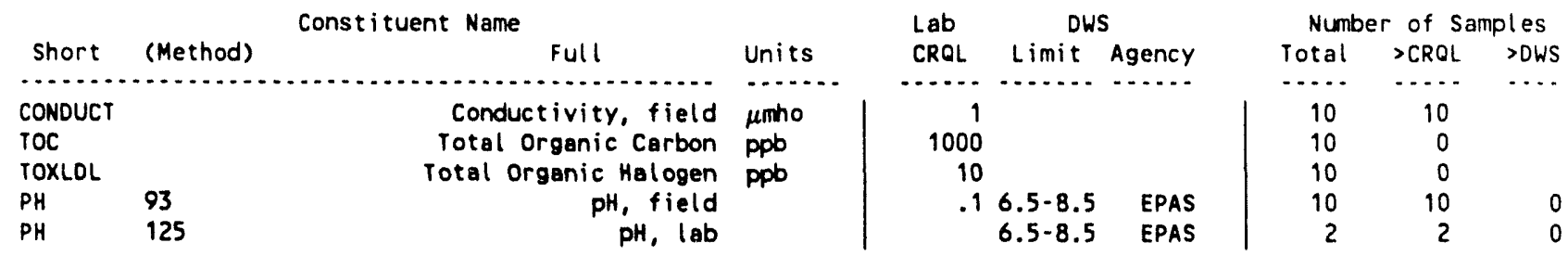

DRINKING HATER PARAMETERS

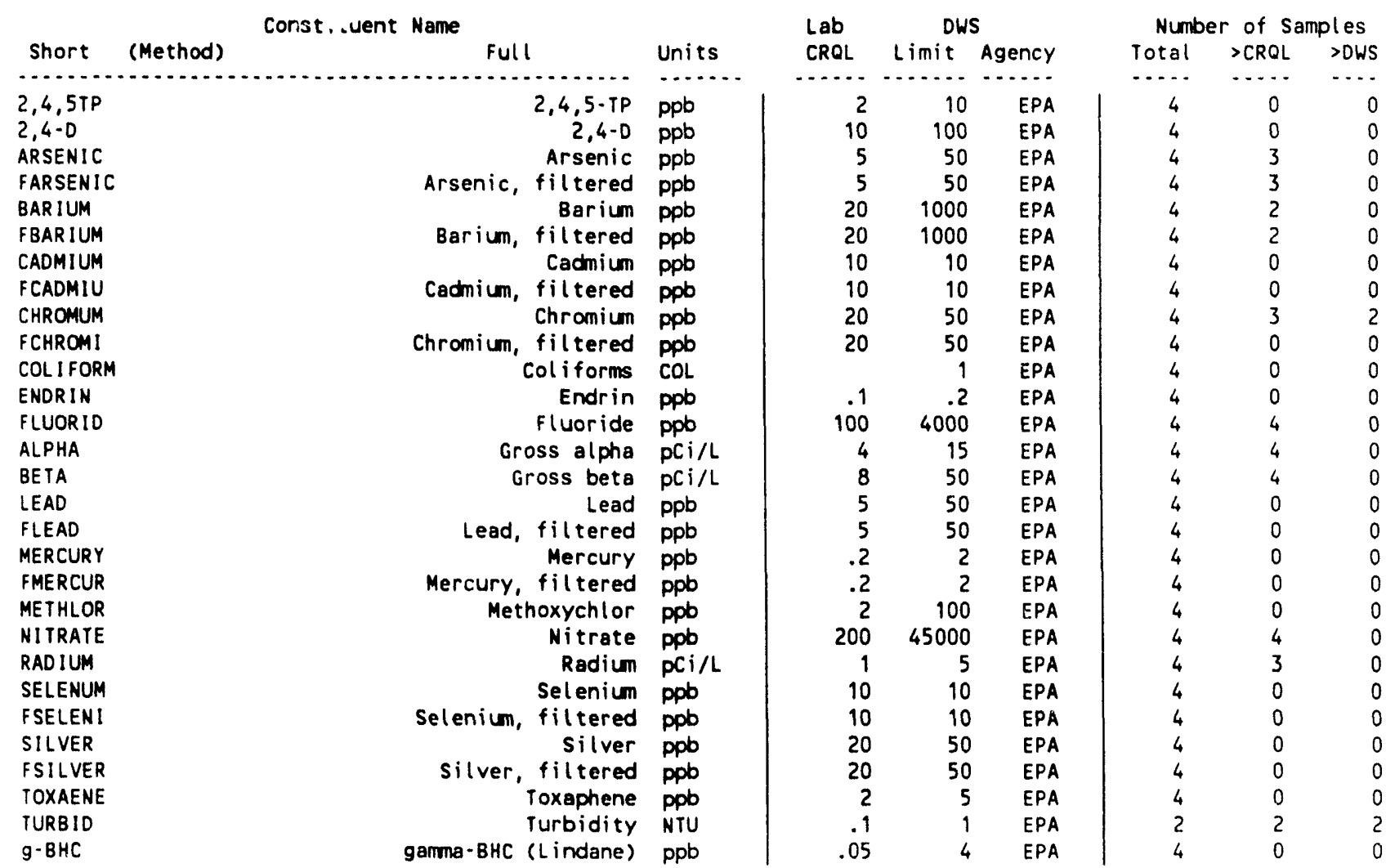

GROUNDWATER QUAL ITY PARAMETERS

\begin{tabular}{|c|c|c|c|c|c|c|c|c|c|}
\hline \multirow[b]{2}{*}{ Short } & \multirow{3}{*}{ (Method) } & \multirow[t]{2}{*}{ Constituent Name } & \multirow[b]{2}{*}{ Units } & \multirow{2}{*}{$\begin{array}{l}\text { Lab } \\
\text { CROL }\end{array}$} & \multicolumn{2}{|c|}{ DWS } & \multicolumn{3}{|c|}{ Number of Samples } \\
\hline & & & & & Limit & Agency & Total & $>C R Q L$ & $>0 W S$ \\
\hline$\ldots \ldots \ldots$ & & $\ldots \ldots \ldots$ & $\ldots \ldots$ & $\ldots \ldots$ & $\ldots \ldots$ & $\ldots \ldots$ & $\ldots \ldots$ & $\ldots \ldots$ & $\ldots$ \\
\hline CHLORID & & Chloride & ppb & 200 & 250000 & EPAS & 4 & 4 & 0 \\
\hline IRON & & Iron & ppb & 20 & 300 & EPAS & 4 & 4 & 3 \\
\hline FIRON & & Iron, filtered & ppb & 20 & 300 & EPAS & 4 & 3 & 0 \\
\hline MANGESE & & Manganese & ppb & 10 & 50 & EPAS & 4 & 3 & 0 \\
\hline FMANGAN & & Manganese, filtered & ppb & 10 & 50 & EPAS & 4 & 0 & 0 \\
\hline LPHENOL & 19 & Phenol & ppb & 10 & & & 1 & 0 & \\
\hline LPHENOL & 30 & Phenol & ppb & 20 & & & 4 & 0 & \\
\hline SOOIUM & & Sodium & ppb & 300 & & & 4 & 4 & \\
\hline FSODIUM & & Sodium, filtered & ppb & 300 & & & 4 & 4 & \\
\hline SULFATE & & Sulfate & $\mathrm{ppb}$ & 500 & 250000 & EPAS & 4 & 4 & 0 \\
\hline
\end{tabular}


Table 5-3. Constituent List and Summary of Results for the Grout Treatment Facility Data for Reporting Period July 1 through September 30, 1993. (sheet 2 of 5)

SITE SPECIFIC AND OTHER CONSTITUENTS

\begin{tabular}{|c|c|c|c|c|c|c|c|c|}
\hline \multirow[b]{2}{*}{ Short } & \multirow[b]{2}{*}{ (Method) } & \multirow{2}{*}{ Constituent Name Ful! } & \multirow[b]{2}{*}{ Units } & \multirow{2}{*}{$\begin{array}{l}\text { Lab } \\
\text { CRQL }\end{array}$} & DWS & \multicolumn{3}{|c|}{ Num'ser of Samples } \\
\hline & & & & & Limit Agency & Total & $>$ CRQL & $>$ DWS \\
\hline $1,1,1-T$ & & 1,1,1-Trichloroethane & ppo & 5 & & 2 & 0 & \\
\hline $1,1,2-1$ & & $1,1,2-T r i c h l o r o e t h a n e$ & ppb & 5 & & 2 & 0 & \\
\hline $1,1-01 C$ & & i,1-Dichloroethane & ppb & 5 & & 2 & 0 & \\
\hline TETRCHB & & $1,2,4,5$-Tetrachlorobenzene & ppb & 10 & & 1 & 0 & \\
\hline TRICHLB & & 1,2,4-Trichlorobenzene & $\mathrm{ppb}$ & 10 & & 1 & 0 & \\
\hline 12-aben & & 1,2-Dichlorobenzene & ppb & 10 & & 1 & 0 & \\
\hline $1,2-01 C$ & & 1,2-Dicnloroe thane & $\mathrm{ppb}$ & 5 & & 2 & 0 & \\
\hline 12-DCE & & 1,2-Dichloroethene & ppb & 5 & & 2 & 0 & \\
\hline 13-dben & & 1,3-Dichlorobenzene & ppb & 10 & & 1 & 0 & \\
\hline 14-dben & 16 & 1,4-Dichlorobenzene & ppob & 5 & & 2 & 0 & \\
\hline 14 -dben & 19 & 1,4-Dichlorobenzene & ppb & 10 & & 1 & 0 & \\
\hline NAPHQUI & & 1,4-Naph toquinone & ppb & 10 & & 1 & 0 & \\
\hline 1BUTYN & & 1-Butanol & ppob & 1000 & & 2 & 0 & \\
\hline 1-napha & & 1-Naphthylamine & ppb & 10 & & 1 & 0 & \\
\hline TETRCHP & & 2,3,4,6-Tetrachlorophenol & ppob & 10 & & 1 & 0 & \\
\hline $2,4,5 \cdot T$ & & $2,4,5-T$ & ppb & 2 & & 4 & 0 & \\
\hline $245-\operatorname{trp}$ & & 2,4,5-Trichlorophenol & ppb & 10 & & 1 & 0 & \\
\hline $246-\mathrm{trp}$ & 19 & $2,4,6$-Trichlorophenol & ppb & 10 & & 1 & 0 & \\
\hline $246-t r p$ & 30 & 2,4,6-Trichlorophenol & ppb & 5 & & 4 & 0 & \\
\hline $24-d c h p$ & 19 & 2,4-Dichlorophenol & ppb & 10 & & 1 & 0 & \\
\hline $24-d c h p$ & 30 & 2,4-Dichlorophenol & ppb & 5 & & 4 & 0 & \\
\hline DIMPHEN & 19 & 2,4-Dime thylphenol & ppb & 10 & & 1 & 0 & \\
\hline DIMPHEN & 30 & 2,4-D ime thylphenol & ppb & 5 & & 4 & 0 & \\
\hline DINPHEN & 19 & 2,4-Dinitrophenol & ppb & 50 & & 1 & 0 & \\
\hline DINPHEN & 30 & 2,4-D initrophenol & ppb & 150 & & 4 & 0 & \\
\hline 24 -dint & & 2,4-Dinitrotoluene & ppb & 10 & & 1 & 0 & \\
\hline 26-dchp & 19 & 2,6-D ichlorophenol & ppb & 10 & & 1 & 0 & \\
\hline 26-dchp & 30 & 2,6-Dichlorophenol & ppb & 5 & & 4 & 0 & \\
\hline 26-dint & & 2,6-Dinitrotoluene & ppob & 10 & & 1 & 0 & \\
\hline ACEFENE & & 2-Acetylaminof luorene & ppob & 10 & & 1 & 0 & \\
\hline CHLNAPH & & 2-Chloronaph thalene & ppb & 10 & & 1 & 0 & \\
\hline CHLPHEN & 19 & 2-Chlorophenol & ppb & 10 & & 1 & 0 & \\
\hline CHLPHEN & 30 & 2-Chlorophenol & ppb & 5 & & 4 & 0 & \\
\hline 2MENAPH & & 2-Methylnaph thalene & $\mathrm{ppb}$ & 10 & & 1 & 0 & \\
\hline 2METHPH & & 2-Methylphenol & $\mathrm{ppb}$ & 10 & & 1 & 0 & \\
\hline 2-napha & & 2-Naph thylamine & $\mathrm{ppb}$ & 10 & & 1 & 0 & \\
\hline ONITAN! & & 2-Nitroaniline & $\mathrm{ppb}$ & 50 & & 1 & 0 & \\
\hline 2NITPH & 19 & 2-Nitrophenol & ppb & 10 & & 1 & 0 & \\
\hline 2NITPH & 30 & 2-Nitrophenol & ppb & 5 & & 4 & 0 & \\
\hline PICOLIN & & 2-Picoline & ppb & 10 & & 1 & 0 & \\
\hline BUTDINP & 30 & 2-sec-Butyl-4,6-dinitrophenol(DN & $\mathrm{ppb}$ & 5 & & 4 & 0 & \\
\hline BUTDINP & 49 & 2-sec-Butyl-4,6-dinitrophenol (DN & ppb & 1 & & 4 & 0 & \\
\hline DICHBEN & & 3,3'-Dichlorobenz idine & ppb & 20 & & 1 & 0 & \\
\hline DIMEYLB & & 3,3'-D imethylbenzidine & ppb & 10 & & 1 & 0 & \\
\hline METCHAN & & 3-Methylcholanthrene & ppb & 10 & & 1 & 0 & \\
\hline MNITANI & & 3-Nitroaniline & ppb & 50 & & 1 & 0 & \\
\hline DDD & & $4,4^{\prime}-D D D$ & ppb & .1 & & 4 & 0 & \\
\hline DDE & & $4,4^{\prime} \cdot \mathrm{DDE}$ & ppb & .05 & & 4 & 0 & \\
\hline DDT & & $4,4^{\prime}-D D T$ & ppb & .1 & & 4 & 0 & \\
\hline 460N2MP & 19 & 4,6-D initro-2-methyl phenol & ppb & 50 & & 1 & 0 & \\
\hline 46DN2MP & 30 & 4,6-0 initro-2-methylphenol & ppb & 200 & & 4 & 0 & \\
\hline AMINOYL & & 4-Aminobiphenyl & ppb & 10 & & 1 & 0 & \\
\hline BROPHEN & & 4-Bromophenylphenyl ether & ppb & 10 & & 1 & 0 & \\
\hline CHLCRES & 19 & 4-Chloro-3-methylphenol & ppb & 20 & & 1 & 0 & \\
\hline CHLCRES & 30 & 4-Chloro-3-methylphenol & ppo & 5 & & 4 & 0 & \\
\hline
\end{tabular}


Table 5-3. Constituent List and Summary of Results for the Grout Treatment Facility Data for Reporting Period July 1 through September 30, 1993. (sheet 3 of 5)

\begin{tabular}{|c|c|c|c|c|c|c|c|c|c|}
\hline \multicolumn{4}{|c|}{ Const ituent Name } & \multicolumn{3}{|l|}{ Lab } & \multicolumn{3}{|c|}{ Number of Samples } \\
\hline Short & (Method) & Full & Units & CRQL & Limit $A$ & Agency & Total & $>C R Q L$ & $>$ DWS \\
\hline$\cdots+\cdots$ & $\cdots$ & $\ldots \ldots \ldots \ldots$ & $\ldots \ldots$ & $\cdots \cdots$ & $\cdots \ldots$. & $\ldots .$. & $\cdots \cdots$ & $\cdots$ & $\cdots$ \\
\hline CHLANIL & & 4-Chloroeniline & ppb & 20 & & & 1 & 0 & \\
\hline 4CPPETH & & 4-Chlorophenylphenyl ether & ppb & 10 & & & 1 & 0 & \\
\hline MIBK & & 4-Methyl-2-pent anone & ppo & 50 & & & 2 & 0 & \\
\hline 4METHPH & & 4-Methylphenol & ppb & 10 & & & 1 & 0 & \\
\hline NITRANI & & 4-Nitroaniline & ppo & 50 & & & 1 & 0 & \\
\hline NITPHEN & 19 & 4-Nitrophenol & ppob & 50 & & & 1 & 0 & \\
\hline NITPHEN & 30 & 4-Nitrophenol & ppb & 30 & & & 4 & 0 & \\
\hline 4NITQUI & & 4-Nitroquinol ine-1-oxide & ppob & 10 & & & 1 & 0 & \\
\hline NITRTOL & & 5-Nitro-O-toluidine & ppb & 10 & & & 1 & 0 & \\
\hline DIMBENZ & & 7, 12-D ime thylbenz [a] anthracene & ppb & 10 & & & 1 & 0 & \\
\hline ACENAPH & & Acenaph thene & ppb & 10 & & & 1 & 0 & \\
\hline ACENATL & & Acenaphthylene & ppb & 10 & & & 1 & 0 & \\
\hline ACETONE & & Acetone & ppb & 100 & & & 2 & 0 & \\
\hline ACETOPH & & Acetophenone & ppb & 10 & & & 1 & 0 & \\
\hline ALDR IN & & Aldrin & ppo & .05 & & & 4 & 0 & \\
\hline DIMPHAM & & Alpha, alpha-D imethyl phenethylami & ppo & 10 & & & 1 & 0 & \\
\hline a-BHC & & Alpha-8HC & ppb & .05 & & & 4 & 0 & \\
\hline ALUMNUM & & Aluminum & ppb & 200 & & & 4 & 1 & \\
\hline FALUMIN & & Aluminum & ppb & 200 & & & 4 & 0 & \\
\hline AMMONIU & & Ammonium ion & ppo & 100 & & & 2 & 0 & \\
\hline ANILINE & & Aniline & ppb & 10 & & & 1 & 0 & \\
\hline ANTHRA & & Anthracene & ppb & 10 & & & 1 & 0 & \\
\hline ANTIONY & & Ant imony & ppb & 200 & & & 4 & 0 & \\
\hline FANTIMO & & Ant imony, filtered & ppb & 200 & & & 4 & 0 & \\
\hline ARAMITE & & Aramite & ppb & 10 & & & 1 & 0 & \\
\hline AR 1016 & & Aroclor -1016 & ppb & 1 & & & 1 & 0 & \\
\hline AR1221 & & Aroclor-1221 & ppb & 1 & & & 1 & 0 & \\
\hline AR1232 & & Aroclor- 1232 & ppb & 1 & & & 1 & 0 & \\
\hline AR 1242 & & Aroclor-1242 & ppo & 1 & & & 1 & 0 & \\
\hline AR 1248 & & Aroclor -1248 & ppb & 1 & & & 1 & 0 & \\
\hline AR 1254 & & Aroclor-1254 & ppb & 1 & & & 1 & 0 & \\
\hline AR 1260 & & Aroclor -1260 & ppb & 1 & & & 1 & 0 & \\
\hline BENZENE & & Benzene & ppo & 5 & & & 2 & 0 & \\
\hline BENZAAN & & Benzo(a)anthracene & ppb & 10 & & & 1 & 0 & \\
\hline BENZOPY & & Benzo(a)pyrene & ppob & 10 & & & 1 & 0 & \\
\hline BENZBFL & & Benzo(b)f luoranthene & ppo & 10 & & & 1 & 0 & \\
\hline BGHIPER & & Benzo(ghi)perylene & ppo & 10 & & & 1 & 0 & \\
\hline BNZKFLU & & Benzo $(k) f$ luoranthene & ppb & 10 & & & 1 & 0 & \\
\hline BENZOTH & & Benzothiazole & ppb & 10 & & & 1 & 0 & \\
\hline BENZALC & & Benzyl alcohol & ppb & 20 & & & 1 & 0 & \\
\hline BERYLUM & & Beryllium & ppb & 3 & & & 4 & 0 & \\
\hline FBERYLL & & Beryllium, filtered & ppb & 3 & & & 4 & 0 & \\
\hline $\mathrm{b}-\mathrm{BHC}$ & & Beta-BHC & ppb & .05 & & & 4 & 0 & \\
\hline BIS2CHM & & Bis (2-Choroethoxy)methane & ppob & 10 & & & 1 & 0 & \\
\hline BIS2CHE & & Bis(2-chloroethyl) ether & ppb & 10 & & & 1 & 0 & \\
\hline BIS2ETH & & Bis(2-chloroisopropyl) ether & ppb & 10 & & & 1 & 0 & \\
\hline BISZEPH & & Bis(2-ethylhexyl) phthalate & ppb & 10 & & & 1 & 0 & \\
\hline BROMIDE & & Bromide & ppo & 500 & & & 4 & 0 & \\
\hline BUTBENP & & Butylbenzylphthalate & ppb & 10 & & & 1 & 0 & \\
\hline CALCIUM & & Calcium & ppb & 100 & & & 4 & 4 & \\
\hline FCALCIU & & Calcium, filtered & ppb & 100 & & & 4 & 4 & \\
\hline CARBIDE & & Carbon disulfide & ppb & 5 & & & 2 & 0 & \\
\hline TETRANE & & Carbon tetrachloride & ppb & 5 & & & 2 & 0 & \\
\hline CHLOANE & & Chlordane & ppb & .1 & & & 4 & 0 & \\
\hline CHLLATE & & Chlorobenzilate & ppb & 10 & & & 1 & 0 & \\
\hline CHLFORM & & Chloroform & ppb & 5 & 100 & EPA & 2 & 0 & 0 \\
\hline CHRYSEN & & Chrysene & ppo & 10 & & & 1 & 0 & \\
\hline COBALT & & Cobalt & ppb & 20 & & & 4 & 0 & \\
\hline
\end{tabular}


Table 5-3. Constituent List and Summary of Results for the Grout Treatment Facility Data for Reporting Period July 1 through September 30, 1993. (sheet 4 of 5)

\begin{tabular}{|c|c|c|c|c|c|c|c|c|}
\hline \multicolumn{3}{|c|}{ Constituent Name } & \multicolumn{3}{|c|}{ DWS } & \multicolumn{3}{|c|}{ Number of Samples } \\
\hline Short & (Method) & Units & CROL & Limit & Agency & Total & $>C R Q L$ & $>$ DWS \\
\hline$\ldots \ldots$ & 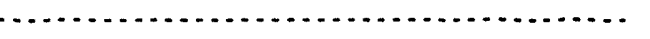 & $\ldots \ldots$ & $\ldots .$. & $\ldots \ldots$ & $\ldots .$. & $\cdots$ & $\cdots \cdots$ & $\cdots$ \\
\hline FCOBALT & Cobalt, filtered & ppob & 20 & & & 4 & 0 & \\
\hline COPPER & Copper & ppo & 20 & 1000 & EPAS & 4 & 0 & 0 \\
\hline FCOPPER & Copper, filtered & pob & 20 & 1000 & EPAS & 4 & 0 & 0 \\
\hline CRESOLS & Cresols (methylphenols) & ppb & 10 & & & 4 & 0 & \\
\hline d-BHC & Delta-BHC & ppb & .1 & & & 4 & 0 & \\
\hline DIBPHTH & Di-n-butylphthalate & ppb & 10 & & & 1 & 0 & \\
\hline DIOPHTH & Di-n-octylphthalate & ppb & 10 & & & 1 & 0 & \\
\hline DIALLATE & Diallate & ppb & 10 & & & 1 & 0 & \\
\hline DIBAHAN & D ibenz $[a, h]$ anthracene & ppob & 10 & & & 1 & 0 & \\
\hline DIBENFR & Dibenzofuran & ppb & 10 & & & 1 & 0 & \\
\hline DIELRIN & Dieldrin & ppb & .05 & & & 4 & 0 & \\
\hline DIEPHTH & Diethyl phthalate & ppb & 10 & & & 1 & 0 & \\
\hline DIMETHO & Dimethoate & ppb & 10 & & & 1 & 0 & \\
\hline DIMPHTH & Dimethyl phthalate & ppo & 10 & & & 1 & 0 & \\
\hline DIPHAMI & Diphenylamine & ppb & 10 & & & 1 & 0 & \\
\hline ENDO1 & Endosulfan I & ppo & .1 & & & 4 & 0 & \\
\hline ENDO2 & Endosulfan II & ppo & .05 & & & 4 & 0 & \\
\hline ENDSFAN & Endosulfan sulfate & ppb & .5 & & & 4 & 0 & \\
\hline ENDRALD & Endrin Aldehyde & ppob & .2 & & & 4 & 0 & \\
\hline ETHCYAN & Ethyl cyanide & ppb & 10 & & & 2 & 0 & \\
\hline ETHMETS & Ethyl methanesulfonate & ppo & 10 & & & 1 & 0 & \\
\hline FAMPHUR & Famphur & ppb & 10 & & & 1 & 0 & \\
\hline FLUORAN & Fluoranthene & ppb & 10 & & & 1 & 0 & \\
\hline FLRENE & Fluorene & ppb & 10 & & & 1 & 0 & \\
\hline HEPTLOR & Heptachl or & ppb & .05 & & & 4 & 0 & \\
\hline HEPT IDE & Heptachlor epoxide & ppb & 1 & & & 4 & 0 & \\
\hline HEXCBEN & Hexachlorobenzene & ppb & 10 & & & 1 & 0 & \\
\hline HEXCBUT & Hexachlorobutadiene & ppb & 10 & & & 1 & 0 & \\
\hline HEXCCYC & Hexachlorocyclopentadiene & ppb & 10 & & & 1 & 0 & \\
\hline HEXCETH & Hexachloroe thane & ppb & 10 & & & 1 & 0 & \\
\hline HEXACHL & Hexachlorophene & ppb & 10 & & & 1 & 0 & \\
\hline HEXAENE & Hexach loropropene & ppb & 10 & & & 1 & 0 & \\
\hline LHYDRAZ & Hydrazine & ppb & 30 & & & 2 & 0 & \\
\hline INOENOP & Indeno $(1,2,3-c d)$ pyrene & ppb & 10 & & & 1 & 0 & \\
\hline ISOOR IN & Isodr in & ppb & 10 & & & 1 & 0 & \\
\hline ISOPHER & Isophorone & ppb & 10 & & & 1 & 0 & \\
\hline ISOSOLE & Isosafrole & ppb & 10 & & & 1 & 0 & \\
\hline KEPONE & Kepone & ppb & 10 & & & 1 & 0 & \\
\hline KEROSEN & Kerosene & ppb & 10000 & & & 1 & 0 & \\
\hline MAGNES & Magnesium & ppb & 100 & & & 4 & 4 & \\
\hline FMAGNES & Magnesium, filtered & ppo & 100 & & & 4 & 4 & \\
\hline METHAPY & Methapyrilene & ppob & 10 & & & 1 & 0 & \\
\hline METHONE & Methyl ethyl ketone & ppb & 100 & & & 2 & 0 & \\
\hline METMSUL & Methyl methanesulfonate & ppb & 10 & & & 1 & 0 & \\
\hline METHYCH & Methylene chloride & ppb & 5 & & & 2 & 0 & \\
\hline DIPRNIT & $N-N i$ troso-di-n-dipropylamine & ppo & 10 & & & 1 & 0 & \\
\hline NNIBUTY & $N-N i t r o s o d i-n$-butylamine & ppb & 10 & & & 1 & 0 & \\
\hline NNIDIEY & N-Nitrosodiethyl amine & ppb & 10 & & & 1 & 0 & \\
\hline NNIDIME & $N$-Nitrosodimethyl amine & ppb & 10 & & & 1 & 0 & \\
\hline NNDIPHA & $\mathrm{N}-\mathrm{Nitrosodiphenylamine}$ & ppb & 10 & & & 1 & 0 & \\
\hline NHIMETH & $N-N i$ trosome thylethylamine & ppb & 10 & & & 1 & 0 & \\
\hline NNIMORP & $\mathrm{N}$-Nitrosomorphol ine & ppo & 10 & & & 1 & 0 & \\
\hline NNIPIPE & N-Nitrosopiperidine & ppb & 10 & & & 1 & 0 & \\
\hline NAPHTHA & Naphthalene & ppb & 10 & & & 1 & 0 & \\
\hline NICKEL & Nickel & ppb & 30 & & & 4 & 2 & \\
\hline FNICKEL & Nickel, filtered & ppb & 30 & & & 4 & 0 & \\
\hline
\end{tabular}


Table 5-3. Constituent List and Summary of Results for the Grout Treatment Facility Data for Reporting Period July 1 through September 30,1993 . (sheet 5 of 5 )

\begin{tabular}{|c|c|c|c|c|c|c|c|c|c|}
\hline \multicolumn{4}{|c|}{ Const ituent Name } & \multicolumn{3}{|c|}{ DWS } & \multicolumn{3}{|c|}{ Number of Samples } \\
\hline Short & (Method) & Full & Units & CRQL & Limit $A$ & Agency & Total & $>$ CRQL & $>$ DWS \\
\hline$\ldots \ldots \ldots$ & $\ldots \ldots$ & $\ldots \ldots \ldots \ldots$ & $\cdots \ldots$ & $\cdots \cdots$ & $\ldots \ldots$. & $\ldots .$. & $\cdots \cdot$ & $\cdots \cdots$ & $\cdots$ \\
\hline NITRITE & & Nitrite & ppob & 200 & & & 4 & 0 & \\
\hline NITBENZ & & Nitrobenzene & ppob & 10 & & & 1 & 0 & \\
\hline NITRPYR & & Nitrosopyrrolidine & pob & 10 & & & 1 & 0 & \\
\hline TRIPHOS & & $0,0,0$-Triethyl phosphorothioate & ppb & 10 & & & 1 & 0 & \\
\hline DIPHOS & & 0,0 -diethyl0-2-pyraz inylphosphor & ppb & 10 & & & 1 & 0 & \\
\hline PARATHI & & Parathion & pob & 10 & & & 1 & 0 & \\
\hline PENTCHB & & Pentachlorobenzene & ppo & 10 & & & 1 & 0 & \\
\hline PENTCHN & & Pentachloronitrobenzene (PCNB) & ppo & 10 & & & 1 & 0 & \\
\hline PENTCHP & 19 & Pentachlorophenol & ppb & 50 & & & 1 & 0 & \\
\hline PENTCHP & 30 & Pent ach l orophenol & ppo & 100 & & & 4 & 0 & \\
\hline PHENTIN & & Phenacet in & ppb & 10 & & & 1 & 0 & \\
\hline PHENANT & & Phenanthrene & ppo & 10 & & & 1 & 0 & \\
\hline PHOSPHA & & Phosphate & ppb & 400 & & & 4 & 0 & \\
\hline POTASUM & & Potassium & ppob & 300 & & & 4 & 4 & \\
\hline FPOTASS & & Potassium, filtered & ppb & 300 & & & 4 & 4 & \\
\hline PRONIDE & & Pronamide & $p p b$ & 10 & & & 1 & 0 & \\
\hline PYRENE & & Pyrene & ppo & 10 & & & 1 & 0 & \\
\hline PYRIDIN & & Pyridine & ppo & 10 & & & 1 & 0 & \\
\hline SAFROL & & Safrol & ppb & 10 & & & 1 & 0 & \\
\hline TC- 99 & & Technetium-99 & $\mathrm{pCi} / \mathrm{L}$ & 15 & & & 2 & 0 & \\
\hline PERCENE & & Tetrachloroethene & ppb & 5 & & & 2 & 0 & \\
\hline TETPHNL & & Tetrachlorophenols & ppb & 10 & & & 4 & 0 & \\
\hline PYROPHOS & & Tetraethyldi thiopyrophosphate & ppb & 10 & & & 1 & 0 & \\
\hline TAF & & Tetrahydrofuran & pob & 10 & & & 2 & 0 & \\
\hline TIN & & Tin & ppob & 100 & & & 4 & 0 & \\
\hline FTIN & & Tin, filtered & ppo & 100 & & & 4 & 0 & \\
\hline TOLUENE & & Toluene & pob & 5 & & & 2 & 0 & \\
\hline TR!BUTPH & & Tributyl Phosphate & ppb & 10 & & & 1 & 0 & \\
\hline TRICENE & & Trichloroethene & ppb & 5 & & & 2 & 0 & \\
\hline TRIPHNL & & Trichlorophenols & ppb & 5 & & & 4 & 0 & \\
\hline TOICHPH & & Iris-2-chloroethyl phosphate & ppb & 10 & & & 1 & 0 & \\
\hline TRITIUM & & Tritium & $\mathrm{pCi} / \mathrm{L}$ & 500 & 20000 & EPA & 4 & 4 & 0 \\
\hline VANADUM & & Vanadium & ppo & 30 & & & 4 & 3 & \\
\hline FVANAD 1 & & Vanadium, filtered & ppo & 30 & & & 4 & 2 & \\
\hline VINYIDE & & Vinyl chloride & ppo & 10 & 2 & EPA & 2 & 0 & $0^{*}$ \\
\hline XYLENE & & Xylenes (total) & ppob & 5 & & & 2 & 0 & \\
\hline ZINC & & 2 inc & ppb & 10 & & & 4 & 2 & \\
\hline FZINC & & zinc, filtered & $p p b$ & 10 & & & 4 & 0 & \\
\hline MCRESOL & & m-Cresol & pob & 10 & & & 1 & 0 & \\
\hline DINBENZ & & m-dinitrobenzene & pob & 10 & & & 1 & 0 & \\
\hline OTOLHYD & & o-Toluidine & ppb & 10 & & & 1 & 0 & \\
\hline DIMEAMB & & P-D ime thylaminoazobenzene & ppb & 10 & & & 1 & 0 & \\
\hline PHENDIA & & p-Phenylenediamine & ppb & 10 & & & 1 & 0 & \\
\hline SYMTR IN & & sym-Irinitrobenzene & ppb & 10 & & & 1 & 0 & \\
\hline
\end{tabular}

For explanation of this table, see Section 1.4 of report. 
Table 5-4. Constituents with at Least One Value Above the CRQL for the Grout Treatment Facility Data for Reporting Period July 1 through September 30, 1993. (sheet 1 of 3)

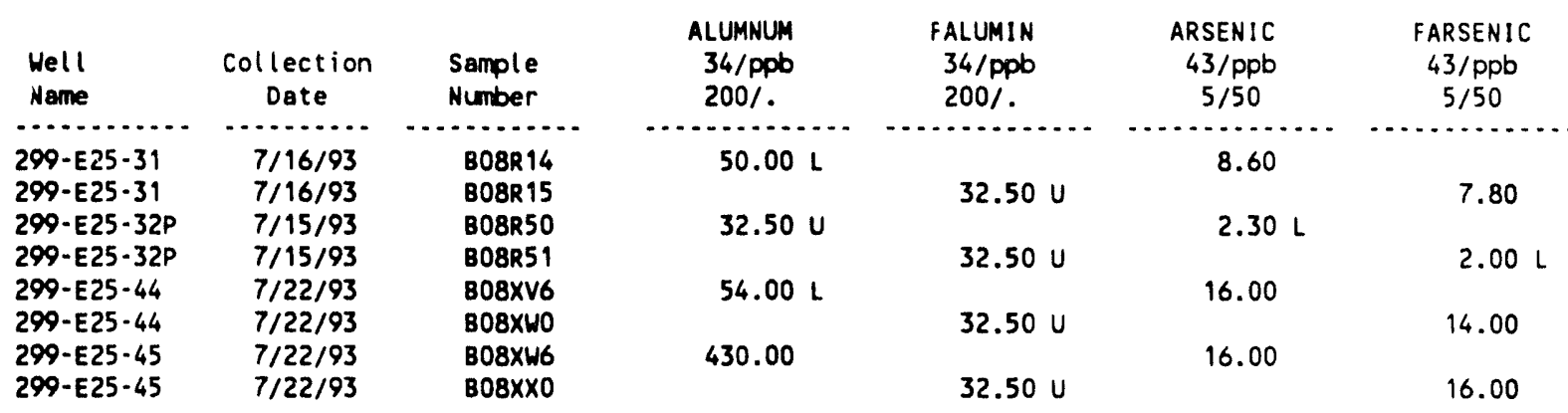

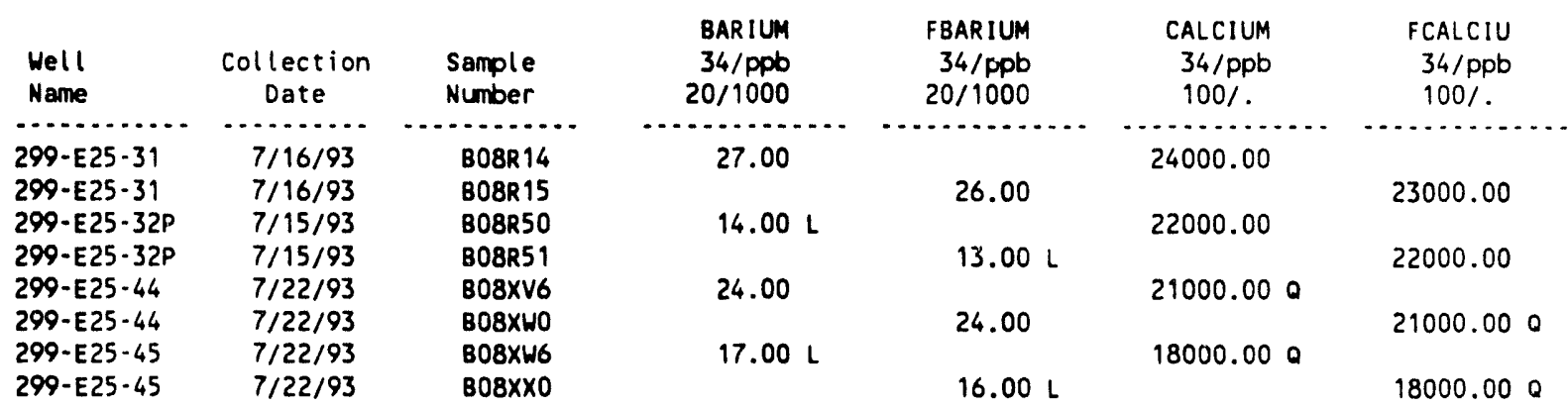

\begin{tabular}{|c|c|c|c|c|c|c|}
\hline $\begin{array}{l}\text { Well } \\
\text { Name }\end{array}$ & $\begin{array}{c}\text { Collection } \\
\text { Date }\end{array}$ & $\begin{array}{l}\text { Sample } \\
\text { Number }\end{array}$ & $\begin{array}{c}\text { CHLORID } \\
124 / \text { Ppb } \\
200 / 250000 \text { s }\end{array}$ & $\begin{array}{l}\text { CHROMUM } \\
34 / \text { ppb } \\
20 / 50\end{array}$ & $\begin{array}{l}\text { FCHROMI } \\
34 / \text { ppb } \\
20 / 50\end{array}$ & $\begin{array}{l}\text { FLUORID } \\
124 / \mathrm{ppb} \\
100 / 4000\end{array}$ \\
\hline 299-E25-31 & $7 / 16 / 93$ & B08R 14 & 2100.00 & 150.00 & & 500.00 \\
\hline $299-E 25-31$ & $7 / 16 / 93$ & B08R15 & & & $7.30 \mathrm{~L}$ & \\
\hline $\begin{array}{l}299-E 25-32 P \\
299-E 25-32 P\end{array}$ & $\begin{array}{l}7 / 15 / 93 \\
7 / 15 / 93\end{array}$ & $\begin{array}{l}\text { B08R50 } \\
\text { B08R51 }\end{array}$ & 1600.00 & 230.00 & $5.42 \mathrm{U}$ & 200.00 \\
\hline $\begin{array}{l}299-E 25-44 \\
299-E 25-44\end{array}$ & $\begin{array}{l}7 / 22 / 93 \\
7 / 22 / 93\end{array}$ & $\begin{array}{l}808 \times 16 \\
808 \times 40\end{array}$ & 3500.00 & $18.00 \mathrm{~L}$ & $5.42 \mathrm{U}$ & 600.000 \\
\hline $\begin{array}{l}299-E 25-45 \\
299-E 25-45\end{array}$ & $\begin{array}{l}7 / 22 / 93 \\
7 / 22 / 93\end{array}$ & $\begin{array}{l}\text { B08XW6 } \\
\text { B08XX0 }\end{array}$ & 3000.00 & 33.00 & $5.42 \mathrm{U}$ & 600.000 \\
\hline
\end{tabular}

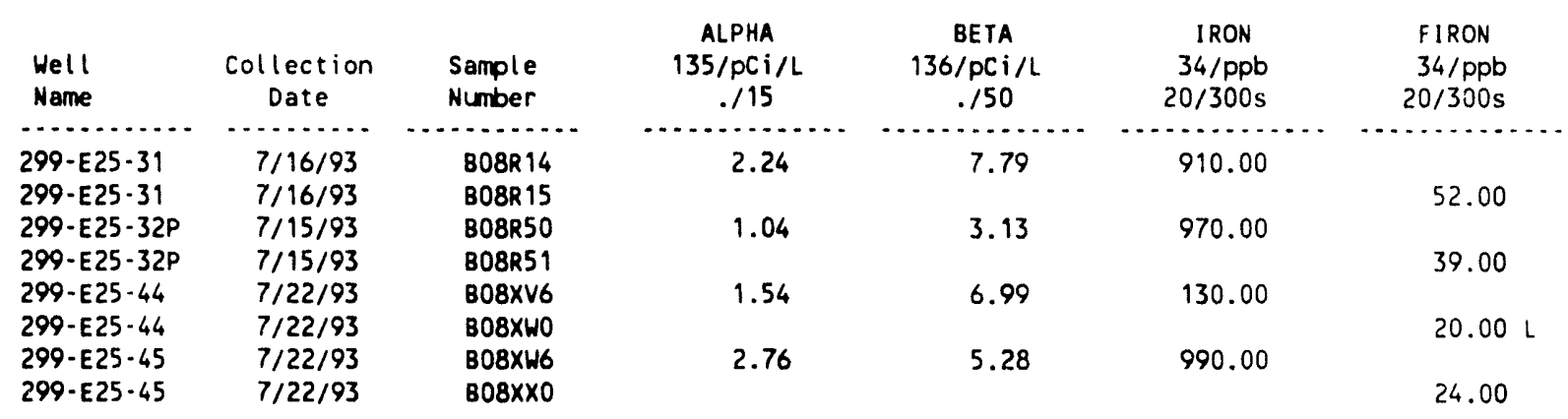


Table 5-4. Constituents with at Least One Value Above the CRQL for the Grout Treatment Facility Data for Reporting Period July 1 through September 30, 1993. (sheet 2 of 3)

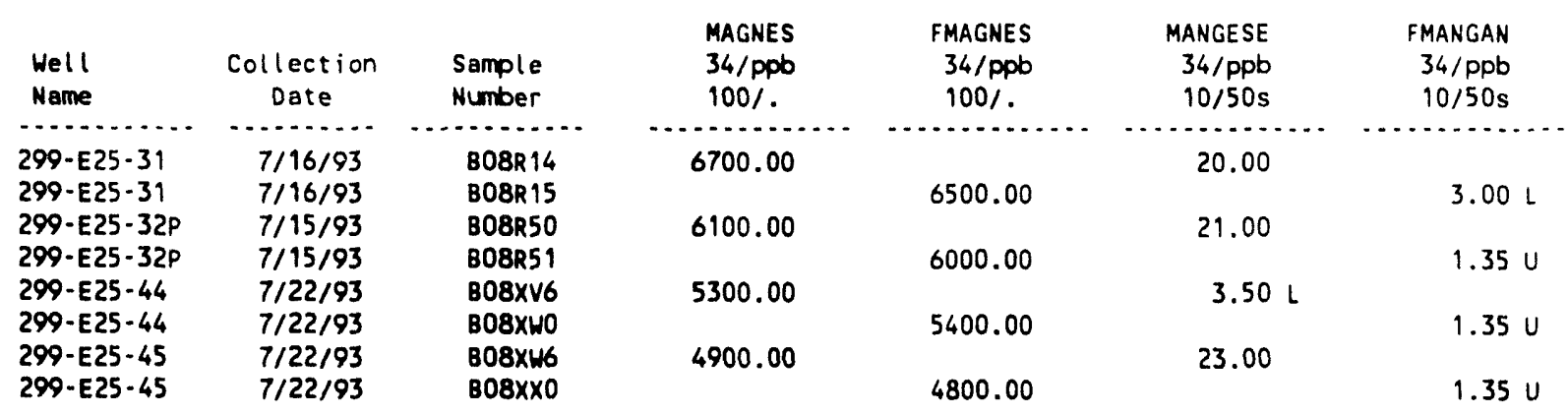

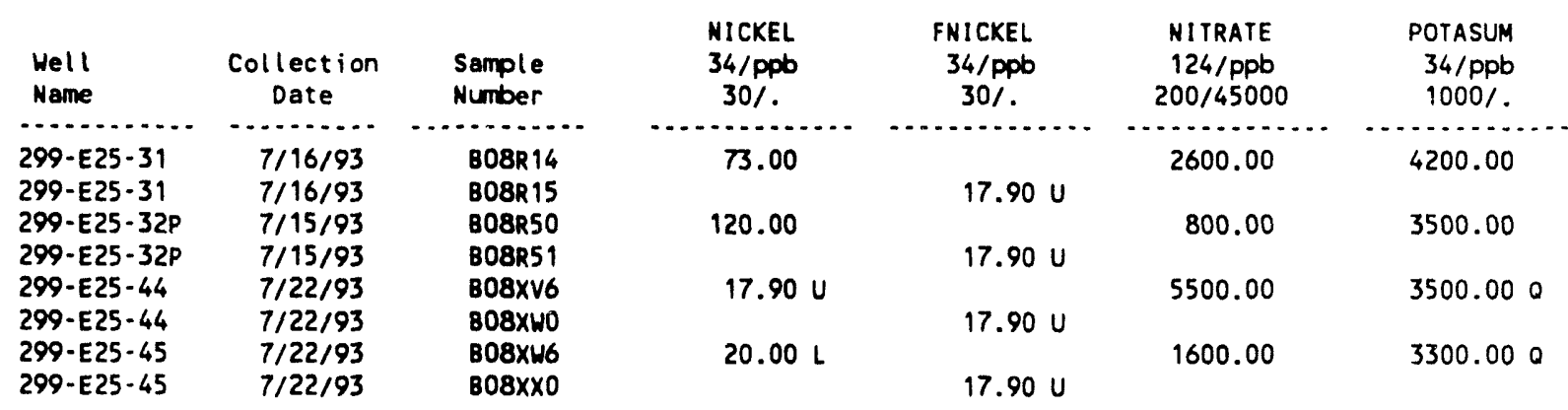

\begin{tabular}{|c|c|c|c|c|c|c|}
\hline $\begin{array}{l}\text { Well } \\
\text { Nane }\end{array}$ & $\begin{array}{c}\text { Collection } \\
\text { Date }\end{array}$ & $\begin{array}{l}\text { Sample } \\
\text { Number }\end{array}$ & $\begin{array}{l}\text { FPOTASS } \\
34 / \text { ppb } \\
1000 / .\end{array}$ & $\begin{array}{c}\text { RADIUM } \\
137 / \mathrm{pCi} / \mathrm{L} \\
. / .\end{array}$ & $\begin{array}{l}\text { SODIUM } \\
34 / \mathrm{ppb} \\
300 \%\end{array}$ & $\begin{array}{l}\text { FSODIUM } \\
34 / \mathrm{ppb} \\
300 \%\end{array}$ \\
\hline $\begin{array}{l}299-E 25-31 \\
299-E 25-31 \\
299-E 25-32 P \\
299-E 25-32 P \\
299-E 25-44 \\
299-E 25-44 \\
299-E 25-45 \\
299-E 25-45\end{array}$ & $\begin{array}{l}7 / 16 / 93 \\
7 / 16 / 93 \\
7 / 15 / 93 \\
7 / 15 / 93 \\
7 / 22 / 93 \\
7 / 22 / 93 \\
7 / 22 / 93 \\
7 / 22 / 93\end{array}$ & $\begin{array}{l}\text { B08R } 14 \\
\text { B08R 15 } \\
\text { B08R50 } \\
\text { B08R51 } \\
\text { B08XV6 } \\
\text { B08X10 } \\
\text { B08XW6 } \\
\text { B08XX0 }\end{array}$ & $\begin{array}{l}3600.00 \\
4100.00 \\
3400.000 \\
3200.000\end{array}$ & $\begin{array}{l}.14 \\
.03 \mathrm{U} \\
.30 \\
.23\end{array}$ & $\begin{array}{l}15000.00 \\
5700.00 \\
16000.000 \\
15000.000\end{array}$ & $\begin{array}{l}15000.00 \\
5700.00 \\
16000.000 \\
15000.000\end{array}$ \\
\hline
\end{tabular}

\begin{tabular}{|c|c|c|c|c|c|c|}
\hline $\begin{array}{l}\text { Well } \\
\text { Name }\end{array}$ & $\begin{array}{c}\text { Collection } \\
\text { Date }\end{array}$ & $\begin{array}{l}\text { Sample } \\
\text { Number }\end{array}$ & $\begin{array}{c}\text { SULFATE } \\
124 / \mathrm{ppb} \\
500 / 250000 \mathrm{~s}\end{array}$ & $\begin{array}{l}\text { TRITIUM } \\
142 / \mathrm{pCi} / \mathrm{L} \\
. / 20000\end{array}$ & $\begin{array}{c}\text { TURBID } \\
126 / \text { NTU } \\
.1 / 1\end{array}$ & $\begin{array}{c}\text { VANADUM } \\
34 / \mathrm{ppb} \\
30 \%\end{array}$ \\
\hline $\begin{array}{l}299-E 25-31 \\
299-E 25-32 P \\
299-E 25-44 \\
299-E 25-45\end{array}$ & $\begin{array}{l}7 / 16 / 93 \\
7 / 15 / 93 \\
7 / 22 / 93 \\
7 / 22 / 93\end{array}$ & $\begin{array}{l}\text { BOBR } 14 \\
\text { BO8R50 } \\
\text { B08XV6 } \\
\text { B08XW6 }\end{array}$ & $\begin{array}{l}20000.00 \\
10000.00 \\
26000.00 \\
13000.00\end{array}$ & $\begin{array}{r}8990.00 \\
969.00 \\
7470.00 \\
3430.00\end{array}$ & $\begin{array}{l}6.10 \\
1.10\end{array}$ & $\begin{array}{l}31.00 \\
15.00 \\
37.00 \\
39.00\end{array}$ \\
\hline
\end{tabular}


Table 5-4. Constituents with at Least One Value Above the CRQL for the Grout Treatment Facility Data for Reporting Period July 1 through September 30, 1993. (sheet 3 of 3)

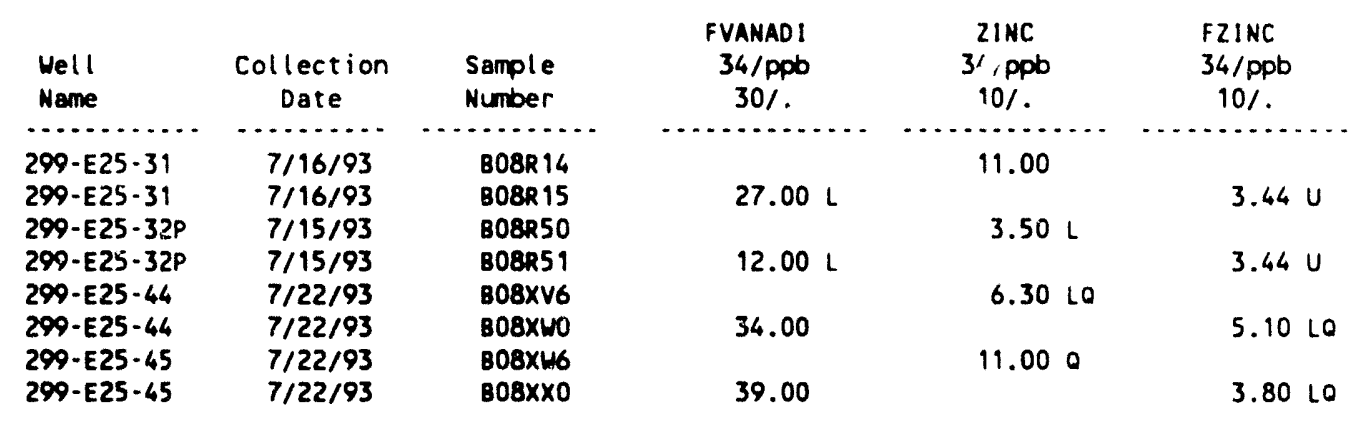

For explanation of this table, see Section 1.4 of report. 
Table 5-5. Contamination Indicator Parameters for the Grout Treatment Facility Data for Reporting Period July l through September 30, 1993.

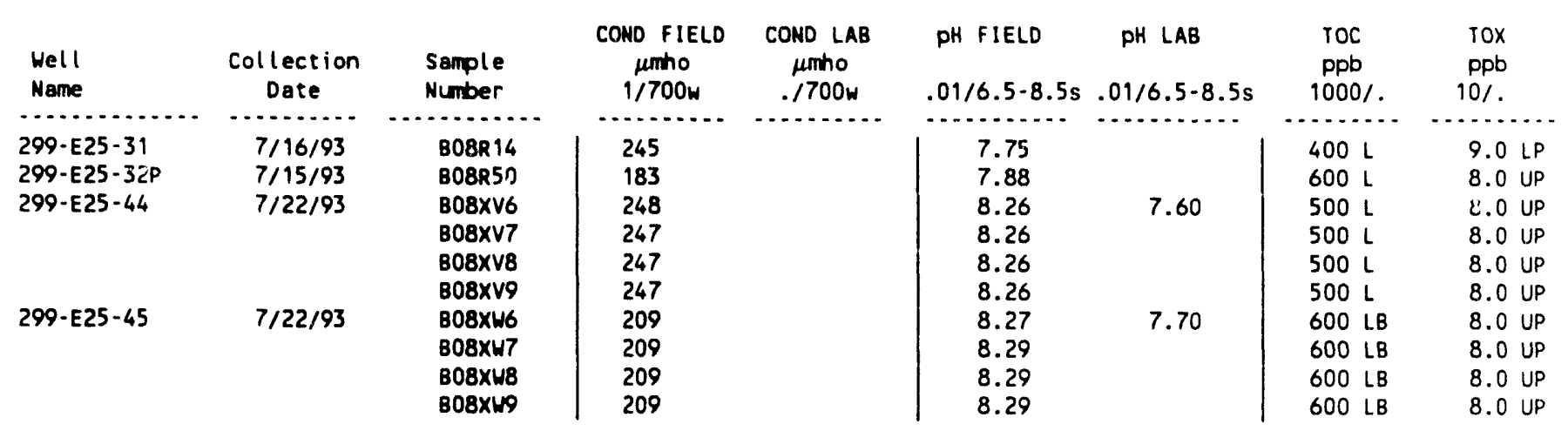

For explanation of this table, see section 1.4 of report. 
DOE/RL-93-56-3

\section{CONTENTS}

6.0 216-B-3 POND SYSTEM . . . . . . . . . . . . . . . . . . 6-1

6.1 INTRODUCTION .................... . . . . . .

6.2 WATER LEVEL DATA . . . . . . . . . . . . . . . . . . 6-1

6.3 WATER CHEMISTRY DATA . . . . . . . . . . . . 6-1 
$\mathrm{DOE} / \mathrm{RL}-93-56-3$

\section{LIST OF FIGURES}

6-1 216-B-3 Pond Groundwater Monitoring Network . . . . . . . . . . . 6-3

\section{LIST OF TABLES}

6-1 Monitoring Well Purpose and Sampling Schedule for the 216-B-3 Pond System Network . . . . . . . . . . . . . . . . 6-4

6-2 RCRA Water Level Measurement Report 216-B-3 Pond System,

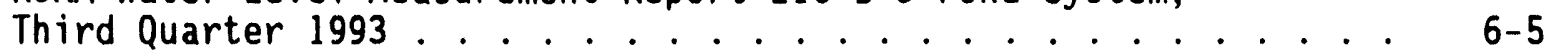

6-3 Constituent List and Summary of Results for the 216-B-3 Pond Data for Reporting Period July 1 through September 30, 1993 . . . 6-9

6-4 Constituents with at Least One Value Above the CRQL for the 216-B-3 Pond Data for Reporting Period July 1 through September 30, 1993 ................ 6- . . . . 5

6-5 Contamination Indicator Parameters for the 216-B-3 Pond Data for Reporting Period July 1 through September 30, 1993 . . . . . . 6-28 


\title{
6.0 216-B-3 POND SYSTEM
}

\author{
D. B. Barnett
}

Westinghouse Hanford Company

\subsection{INTRODUCTION}

The 216-B-3 Pond System is located east of the 200 East Area and consists of a main pond, three interconnected lobes for waste water disposal, and several ditches leading to the ponds (Figures $1-1$ and $6-1$ ). These surface impoundments cover approximately 41.3 hectares (102 acres). Table 6-1 lists the groundwater wells and their monitoring status.

The 216-B-3 Pond System groundwater monitoring well locations are shown in Figure 6-1. Detection monitoring began at the B Pond System in November 1988 and continued through June 1990, when assessment monitoring was scheduled to begin because of elevated levels of TOX in two downgradient monitoring wells (699-43-41E and 699-43-41F). Assessment monitoring actually began in June 1991 when groundwater sampling on the Hanford Site resumed, following a hiatus, as described in the Groundwater Quality Assessment Plan for the 216-B-3 Pond System (Harris 1990). Thus far, no specific constituents have been linked to the elevated TOX results. It is anticipated that the groundwater monitoring program will return to detection level monitoring in CY 1994.

\subsection{WATER LEVEL DATA}

Water levels were measured in all wells during the July through September 1993 period, as shown in Table 6-2. The water level in well 669-41-40 (measured August 5, 1993) is outside of the expected range and may be in error.

\subsection{WATER CHEMISTRY DATA}

All wells scheduled for the July through September 1993 sampling were sampled during all three months of the period. Analytical results for this period that are unavailable as of the writing of this report will be reported in future quarterly reports. Unavailable data for wells 699-40-36, 699-41-35, and 699-42-37 from the April through June 1993 sampling period are also included here.

The list of constituents for which data were received and summary of results available are given in Table 6-3. The results of the available analyses, for constituents with at least one value above the CRQL, are reported in Table 6-4. Results of analyses for CIPs are reported in Table 6-5. Explanations of data flags and qualifiers are given in Section 1.4. 
Constituents exceeding DWS during the July through September 1993 period include chromium in unfiltered samples from wells 699-40-39, 699-40-40A, 699-41-40, 699-42-39A, 699-42-39B, 699-42-42B, and 699-43-42 J; col iform in well 699-44-39B (RADE submitted for this result); iron in unfiltered samples from all wells in the network except 699-41-35, 699-43-41E, 699-43-43, and 699-44-43B; manganese from both unfiltered and filtered samples in wells 699-40-36, 699-40-40A, 699-40-40B, 699-41-35, 699-42-37, 699-42-39B, and 699-43-41G; manganese from unfiltered samples only in well 699-40-39; tritium from wells 699-41-40, 699-41-42, 699-42-39A, 699-42-39B, 699-42-42B, 699-43-40, 699-43-41E, 699-43-41F, and 699-43-41G; and turbidity in all wells in the network sampled for this constituent except 699-42-41, 699-43-43, and 699-44-42. Results in Table 6-4 show an unusually large number of $Q$ flags, especially for results of ammonium, turbidity, and ICP metals (e.g., sodium). The reasons for this flag are discussed in Section 1.2.

During the April through June 1993 period a result for tris-2-chloroethyl phosphate of $44 \mathrm{ppb}$ was reported for well 699-40-40A. This constituent was once again reported in the July through September 1993 period as detectable in this same well (2l ppb) and four other wells: 699-40-40B (14 ppb), 699-40-36 (18 ppb), 699-41-35 (25 ppb), and 699-42-37 (18 ppb). A RADE was submitted for these results to assist in determining likely sources for this compound.

Results for several constituents from well 699-42-40A exceed or fall below historical ranges for these constituents. The constituents include several metals (unfiltered and some filtered samples), gross alpha, gross beta, tritium, TOC, and turbidity. It is likely that the high turbidity (555 NTU) is responsible for these departures. The affected constituents are flagged in Tables 6-4 and 6-5, and a RADE has been submitted to confirm the reason for the anomalies.

Additional RADEs were submitted for tin in well 699-40-36 and nitrate in we 11 699-42-37. These results exceeded historical values for tin and nitrate. A RADE was also submitted for results of barium in wells 699-43-42J and 699-44-39B, because of uncertainty associated with the reported values ( $0.00 \mathrm{ppb}$, in both cases). Apparently, the MDL is less than $0.00 \mathrm{ppb}$, and the reporting format of Table 6-4 will not accommodate the additional characters to the right of the decimal.

${ }^{1}$ The DWS for chromium is $100 \mathrm{ppb}$. The previous chromium DWS of $50 \mathrm{ppb}$ is still shown in Tables 1-10,6-3, and 6-4. 


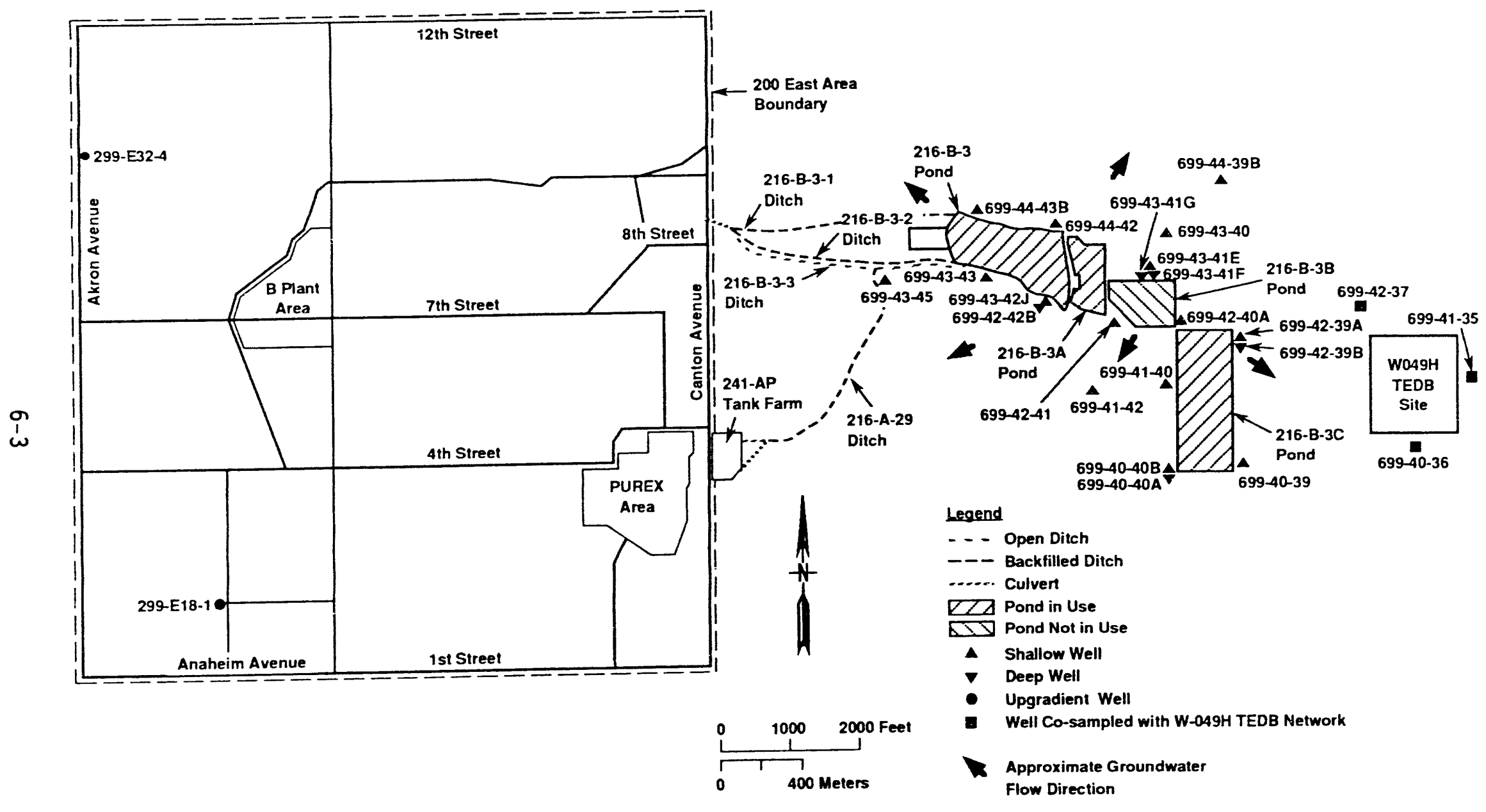

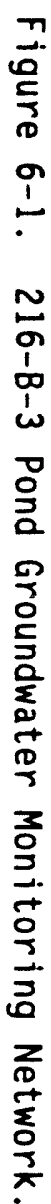

H9312019.1 
Table 6-1. Monitoring Well Purpose and Sampling Schedule for the 216-8-3 Pond System Network.

\begin{tabular}{|c|c|c|c|c|}
\hline Well no. & $\begin{array}{l}\text { Relative } \\
\text { position }\end{array}$ & Hydrogeologic unit & $\begin{array}{c}\text { Sample } \\
\text { frequency }\end{array}$ & $\begin{array}{l}\text { Sample date, } \\
\text { 3rd Qtr } 1993\end{array}$ \\
\hline $299-E 18-1^{8}$ & Upgradient & Hanford: Water Table & Semiannually & Not scheduled \\
\hline $299-E 32-4^{b}$ & Upgradient & Hanford: Water Table & Semiannualiy & $9 / 02 / 93$ \\
\hline $699-40-36^{c}$ & Downgradient & Ringold: Top of Confined & Quarterly & $8 / 09 / 93$ \\
\hline $699-40-39$ & Downgradient & Ringold: Upper Semiconfined & Quarterly & $8 / 05 / 93$ \\
\hline $699-40-40 A$ & Downgradient & Ringold: Lower Semiconfined & Quarterly & $7 / 27 / 93$ \\
\hline $699-40-40 B$ & Downgradient & Ringold: Upper Semiconfined & Quarterly & $7 / 27 / 93$ \\
\hline $699-41-35^{c}$ & Downgradient & Ringold: Top of Confined & Quarterly & $8 / 09 / 93$ \\
\hline $699-41-40$ & Downgradient & Ringold: Upper Semiconfined & Quarterly & $8 / 05 / 93$ \\
\hline $699-41-42$ & Downgradient & Ringold: Top of Confined & Quarterly & $7 / 28 / 93$ \\
\hline $699-42-37^{c}$ & Downgradient & Ringold: Top of Confined & Quarterly & $8 / 30 / 93$ \\
\hline $699-42-39 A$ & Downgradient & Ringold: Upper Semiconfined & Quarterly & $7 / 29 / 93$ \\
\hline $699-42-39 B$ & Downgradient & Ringold: Lower Semiconfined & Quarterly & $7 / 29 / 93$ \\
\hline $699-42-40 A$ & Downgradient & Ringold: Upper Semiconfined & Semiannually & $7 / 27 / 93$ \\
\hline $699-42-41$ & Downgradient & Ringold: Water Table & Quarterly & $7 / 28 / 93$ \\
\hline $699-42-428$ & Downgradient & Ringold: Lower Unconfined & Semiannually & $7 / 28 / 93$ \\
\hline $699-43-40$ & Downgradient & Ringold: Water Table & Quarterly & $7 / 30 / 93$ \\
\hline $699-43-41 E$ & Downgradient & Ringold: Upper Semiconfined & Quarterly & $7 / 30 / 93$ \\
\hline $699-43-41 F$ & Downgradient & Ringold: Lower Semiconfined & Quarterly & $7 / 28 / 93$ \\
\hline $699-43-41 G$ & Downgradient & \begin{tabular}{|ll} 
Ringold: & $\begin{array}{l}\text { Bottom of } \\
\text { Semiconfined }\end{array}$ \\
\end{tabular} & Quarterly & $7 / 30 / 93$ \\
\hline $699-43-42 J$ & Downgradient & Ringold: Water Table & Semiannually & $8 / 05 / 93$ \\
\hline $699-43-43^{d}$ & Downgradient & Hanford/Ringold: Water Table & Semiannual1y & $7 / 28 / 93$ \\
\hline $699-43-45^{d}$ & Downgradient & Hanford/Ringold: Water Table & Quarterly & $7 / 27 / 93$ \\
\hline $699-44-39 B$ & Downgradient & Ringold: Water Table & Quarterly & $7 / 29 / 93$ \\
\hline $699-44-42$ & Downgradient & Ringold: Water Table & Semiannually & $7 / 28 / 93$ \\
\hline $699-44-43 B$ & Downgradient & Ringold: Water Table & Quarterly & $7 / 30 / 93$ \\
\hline
\end{tabular}

Well shared with the 2101-M Pond network.

Well shared with the Low-Level Waste Burial Grounds network.

Wells shared with the W-O49H Treated Effluent Disposal Basin network.

Wells shared with the 216-A-29 Ditch network. 
Table 6-2. RCRA Water Level Measurement Report 216-B-3 Pond System, Third Quarter 1993.

(sheet 1 of 4 )

\begin{tabular}{|c|c|c|c|}
\hline Well & Date & $\begin{array}{l}\text { Depth to } \\
\text { water (ft) }\end{array}$ & $\begin{array}{l}\text { Water level } \\
\text { elevation } \\
\text { above msl (ft) }\end{array}$ \\
\hline \multicolumn{4}{|c|}{ Wells Monitoring } \\
\hline $299-E 18-1$ & $\begin{array}{l}7 / 19 / 93 \\
8 / 16 / 93 \\
9 / 20 / 93\end{array}$ & $\begin{array}{l}318.27 \\
318.34 \\
318.32\end{array}$ & $\begin{array}{l}401.97 \\
401.90 \\
401.92\end{array}$ \\
\hline 299-E32-4 & $\begin{array}{l}7 / 27 / 93 \\
8 / 20 / 93 \\
9 / 02 / 93 \\
9 / 22 / 93\end{array}$ & $\begin{array}{l}284.35 \\
284.37 \\
284.48 \\
284.45\end{array}$ & $\begin{array}{l}401.53 \\
401.51 \\
401.40^{\star} \\
401.43\end{array}$ \\
\hline $699-41-42$ & $\begin{array}{l}7 / 08 / 93 \\
7 / 28 / 93 \\
8 / 06 / 93 \\
9 / 03 / 93\end{array}$ & $\begin{array}{l}232.19 \\
232.78 \\
233.18 \\
233.44\end{array}$ & $\begin{array}{l}411.72 \\
411.13^{\star} \\
410.73 \\
410.47\end{array}$ \\
\hline $699-42-40 A$ & $\begin{array}{l}7 / 27 / 93 \\
7 / 29 / 93 \\
8 / 06 / 93 \\
9 / 03 / 93\end{array}$ & $\begin{array}{l}125.34 \\
125.45 \\
124.25 \\
125.56\end{array}$ & $\begin{array}{l}420.19 \star \\
420.08 \\
421.28 \\
419.97\end{array}$ \\
\hline $699-42-41$ & $\begin{array}{l}7 / 08 / 93 \\
7 / 28 / 93 \\
8 / 06 / 93 \\
9 / 03 / 93\end{array}$ & $\begin{array}{l}147.59 \\
147.40 \\
146.75 \\
147.47\end{array}$ & $\begin{array}{l}419.71 \\
419.90^{\star} \\
420.55 \\
419.83\end{array}$ \\
\hline $699-43-40$ & $\begin{array}{l}7 / 08 / 93 \\
7 / 30 / 93 \\
8 / 06 / 93 \\
9 / 03 / 93\end{array}$ & $\begin{array}{l}122.90 \\
123.69 \\
123.53 \\
123.68\end{array}$ & $\begin{array}{l}419.30 \\
418.51^{\star} \\
418.67 \\
418.52\end{array}$ \\
\hline $699-43-42 \mathrm{~J}$ & $\begin{array}{l}7 / 08 / 93 \\
8 / 05 / 93 \\
8 / 06 / 93 \\
9 / 03 / 93\end{array}$ & $\begin{array}{l}163.71 \\
163.28 \\
163.30 \\
163.52\end{array}$ & $\begin{array}{l}417.97 \\
418.40^{\star} \\
418.38 \\
418.16\end{array}$ \\
\hline $699-43-43$ & $\begin{array}{l}7 / 08 / 93 \\
7 / 28 / 93 \\
8 / 06 / 93 \\
9 / 03 / 93\end{array}$ & $\begin{array}{l}164.83 \\
164.75 \\
164.54 \\
164.59\end{array}$ & $\begin{array}{l}414.54 \\
414.62^{\star} \\
414.83 \\
414.78\end{array}$ \\
\hline
\end{tabular}


Table 6-2. RCRA Water Level Measurement Report 216-B-3 Pond System, Third Quarter 1993.

(sheet 2 of 4 )

\begin{tabular}{|c|c|c|c|}
\hline Well & Date & $\begin{array}{l}\text { Depth to } \\
\text { water (ft) }\end{array}$ & $\begin{array}{l}\text { Water level } \\
\text { elevation } \\
\text { above msl (ft) }\end{array}$ \\
\hline \multicolumn{4}{|c|}{ Wells Monitoring } \\
\hline $699-43-45$ & $\begin{array}{l}7 / 08 / 93 \\
7 / 27 / 93 \\
8 / 06 / 93 \\
9 / 03 / 93\end{array}$ & $\begin{array}{l}194.62 \\
194.64 \\
194.64 \\
194.76\end{array}$ & $\begin{array}{l}403.06 \\
403.04^{\star} \\
403.04 \\
402.92\end{array}$ \\
\hline $699-44-39 B$ & $\begin{array}{l}7 / 08 / 93 \\
7 / 29 / 93 \\
8 / 06 / 93 \\
9 / 03 / 93\end{array}$ & $\begin{array}{l}94.45 \\
95.04 \\
95.14 \\
95.18\end{array}$ & $\begin{array}{l}418.95 \\
418.36^{\star} \\
418.26 \\
418.22\end{array}$ \\
\hline $699-44-42$ & $\begin{array}{l}7 / 08 / 93 \\
7 / 28 / 93 \\
8 / 06 / 93 \\
9 / 03 / 93\end{array}$ & $\begin{array}{l}159.03 \\
158.10 \\
158.68 \\
158.88\end{array}$ & $\begin{array}{l}420.19 \\
421.12^{\star} \\
420.54 \\
420.34\end{array}$ \\
\hline $699-44-43 B$ & $\begin{array}{l}7 / 08 / 93 \\
7 / 30 / 93 \\
8 / 06 / 93 \\
9 / 03 / 93\end{array}$ & $\begin{array}{l}164.76 \\
164.88 \\
164.68 \\
164.73\end{array}$ & $\begin{array}{l}415.36 \\
415.24^{\star} \\
415.44 \\
415.39\end{array}$ \\
\hline
\end{tabular}

Wells Monitoring Confined or Semi-Confined Aquifer

\begin{tabular}{llll}
\hline $699-40-36$ & $7 / 08 / 93$ & 118.03 & 410.89 \\
& $8 / 06 / 93$ & 118.07 & 410.85 \\
& $8 / 09 / 93$ & 118.15 & $410.77^{\star}$ \\
$9 / 03 / 93$ & 118.28 & 410.64 \\
$699-40-39$ & $7 / 08 / 93$ & 129.71 & 412.13 \\
& $8 / 05 / 93$ & 129.82 & $412.02 \star$ \\
& $8 / 06 / 93$ & 129.78 & 412.06 \\
$699-40-40 A$ & $9 / 03 / 93$ & 129.98 & 411.86 \\
& $7 / 08 / 93$ & 130.42 & 410.79 \\
& $7 / 27 / 93$ & 130.51 & $410.70 \star$ \\
& $8 / 06 / 93$ & 130.39 & 410.82 \\
& $9 / 03 / 93$ & 130.66 & 410.55
\end{tabular}


Table 6-2. RCRA Water Level Measurement Report 216-B-3 Pond System, Third Quarter 1993.

(sheet 3 of 4 )

\begin{tabular}{|c|c|c|c|}
\hline Well & Date & $\begin{array}{l}\text { Depth to } \\
\text { water (ft) }\end{array}$ & $\begin{array}{l}\text { Water level } \\
\text { elevation } \\
\text { above msl (ft) }\end{array}$ \\
\hline \multicolumn{4}{|c|}{ Wells Monitoring Confined o } \\
\hline $699-40-40 B$ & $\begin{array}{l}7 / 08 / 93 \\
7 / 27 / 93 \\
8 / 06 / 93 \\
9 / 03 / 93\end{array}$ & $\begin{array}{l}131.10 \\
130.89 \\
131.09 \\
131.32\end{array}$ & $\begin{array}{l}411.08 \\
411.29 \star \\
411.09 \\
410.86\end{array}$ \\
\hline $699-41-35$ & $\begin{array}{l}7 / 08 / 93 \\
8 / 06 / 93 \\
8 / 09 / 93 \\
9 / 03 / 93\end{array}$ & $\begin{array}{l}108.47 \\
108.47 \\
108.59 \\
108.74\end{array}$ & $\begin{array}{l}411.91 \\
411.91 \\
411.79 \star \\
411.64\end{array}$ \\
\hline $699-41-40$ & $\begin{array}{l}7 / 08 / 93 \\
8 / 05 / 93 \\
8 / 06 / 93 \\
9 / 03 / 93\end{array}$ & $\begin{array}{l}131.39 \\
131.73 \\
131.34 \\
131.68\end{array}$ & $\begin{array}{l}414.55 \\
414.21^{\star}+ \\
414.60 \\
414.26\end{array}$ \\
\hline $699-42-37$ & $\begin{array}{l}7 / 08 / 93 \\
8 / 06 / 93 \\
8 / 30 / 93 \\
9 / 03 / 93\end{array}$ & $\begin{array}{l}104.74 \\
104.85 \\
105.20 \\
105.18\end{array}$ & $\begin{array}{l}414.68 \\
414.57 \\
414.22 \star \\
414.24\end{array}$ \\
\hline $699-42-39 A$ & $\begin{array}{l}7 / 08 / 93 \\
7 / 29 / 93 \\
8 / 06 / 93 \\
9 / 03 / 93\end{array}$ & $\begin{array}{l}140.14 \\
140.62 \\
140.28 \\
140.61\end{array}$ & $\begin{array}{l}418.00 \\
417.52^{\star} \\
417.86 \\
417.53\end{array}$ \\
\hline $699-42-39 B$ & $\begin{array}{l}7 / 08 / 93 \\
7 / 29 / 93 \\
8 / 06 / 93 \\
9 / 03 / 93\end{array}$ & $\begin{array}{l}140.55 \\
140.92 \\
140.67 \\
141.01\end{array}$ & $\begin{array}{l}417.77 \\
417.40^{\star} \\
417.65 \\
417.31\end{array}$ \\
\hline $699-43-41 E$ & $\begin{array}{l}7 / 08 / 93 \\
7 / 30 / 93 \\
8 / 06 / 93 \\
9 / 03 / 93\end{array}$ & $\begin{array}{l}130.83 \\
131.27 \\
130.68 \\
131.11\end{array}$ & $\begin{array}{l}420.03 \\
419.59 \star \\
420.18 \\
419.75\end{array}$ \\
\hline $699-43-41 F$ & $\begin{array}{l}7 / 08 / 93 \\
7 / 28 / 93 \\
8 / 06 / 93 \\
9 / 03 / 93\end{array}$ & $\begin{array}{l}130.93 \\
130.79 \\
130.74 \\
131.19\end{array}$ & $\begin{array}{l}420.08 \\
420.22^{\star} \\
420.27 \\
419.82\end{array}$ \\
\hline
\end{tabular}


Table 6-2. RCRA Water Level Measurement Report 216-B-3 Pond System, Third Quarter 1993. (sheet 4 of 4 )

\begin{tabular}{|c|c|c|c|}
\hline We11 & Date & $\begin{array}{l}\text { Depth to } \\
\text { water }(\mathrm{ft})\end{array}$ & $\begin{array}{l}\text { Water level } \\
\text { elevation } \\
\text { above msl (ft) }\end{array}$ \\
\hline \multicolumn{4}{|c|}{ Wells Monitoring Confined } \\
\hline $699-43-41 G$ & $\begin{array}{l}7 / 08 / 93 \\
7 / 30 / 93 \\
8 / 06 / 93 \\
9 / 03 / 93\end{array}$ & $\begin{array}{l}135.52 \\
135.91 \\
135.90 \\
136.10\end{array}$ & $\begin{array}{l}415.82 \\
415.43^{\star} \\
415.44 \\
415.24\end{array}$ \\
\hline
\end{tabular}

Well Monitoring the Unconfined Aquifer Below the Water Table

\begin{tabular}{llll}
\hline $699-42-42 B$ & $7 / 08 / 93$ & 166.89 & 416.34 \\
& $7 / 28 / 93$ & 166.70 & $416.53^{\star}$ \\
& $8 / 06 / 93$ & 166.54 & 416.69 \\
& $9 / 03 / 93$ & 166.75 & 416.48 \\
\hline
\end{tabular}

Notes: 1. Water level elevations are calculated by subtracting the measured depth to water from the surveyed elevation for the well.

2. Depth-to-water values are transcribed from field records.

3. Elevations marked with an $1 * 1$ were measured at the time of sampling.

4. Elevations marked with a ' + ' are outside of the expected range, and are suspected of error.

5. To convert feet to meters multiply by 0.3048 . 
Table 6-3. Constituent List and Summary of Results for the 216-B-3 Pond Data for Reporting Period July 1 through September 30, 1993. (sheet 1 of 6)

CONTAMINATION INDICATOR PARAMETERS

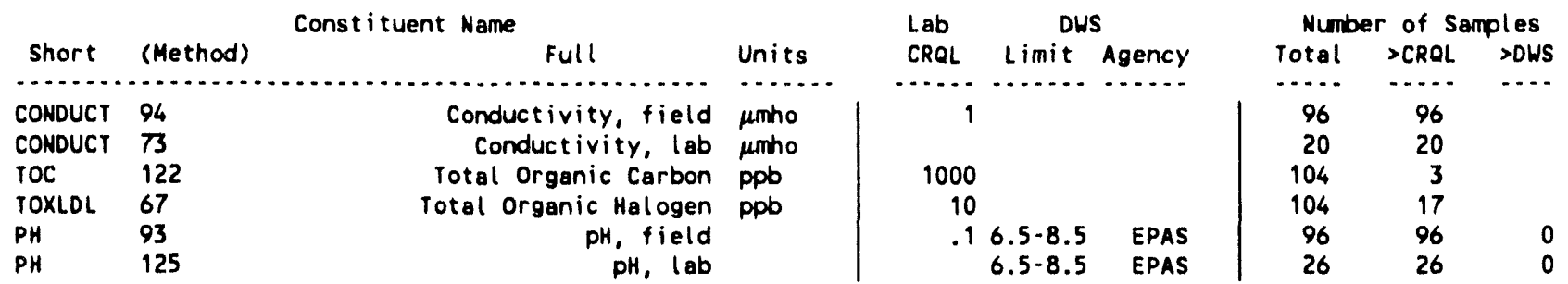

DRINKING WATER PARAMETERS

\begin{tabular}{|c|c|c|c|c|c|c|c|c|c|}
\hline \multirow[b]{2}{*}{ Short } & \multirow{2}{*}{\multicolumn{2}{|c|}{ Constituent Name }} & \multirow[b]{2}{*}{ Units } & \multirow{2}{*}{$\begin{array}{l}\text { Lab } \\
\text { CRQL }\end{array}$} & \multicolumn{2}{|c|}{ DWS } & \multicolumn{3}{|c|}{ Number of Samples } \\
\hline & & & & & Limit & Agency & Total & $>\mathrm{CRQL}$ & $>$ DWS \\
\hline$\ldots \ldots$ & $\ldots$ & $\ldots \ldots \ldots$ & $\ldots \ldots$ & $\ldots .$. & $\ldots \ldots$ & $\ldots \ldots$ & $\ldots$ & $\ldots .$. & $\ldots$ \\
\hline $2,4,5 \mathrm{TP}$ & 49 & $2,4,5 \cdot T P$ & ppb & 2 & 10 & EPA & 22 & 0 & 0 \\
\hline $2,4-0$ & 49 & $2,4-D$ & ppb & 10 & 100 & EPA & 22 & 0 & 0 \\
\hline ARSENIC & 43 & Arsenic & ppb & 5 & so & EPA & 26 & 11 & 0 \\
\hline FARSENIC & 43 & Arsenic, filtered & ppb & 5 & 50 & EPA & 26 & 8 & 0 \\
\hline BARIUM & 34 & Barium & ppob & 20 & 1000 & EPA & 26 & 22 & 0 \\
\hline FBARIUM & 34 & Barium, filtered & ppb & 20 & 1000 & EPA & 26 & 21 & 0 \\
\hline CADMIUM & 34 & Cadmium & ppb & 10 & 10 & EPA & 26 & 0 & 0 \\
\hline FCADMIU & 34 & Cadmium, filtered & ppb & 10 & 10 & EPA & 26 & 0 & 0 \\
\hline CHROMUM & 34 & Chromium & ppb & 20 & 50 & EPA & 26 & 26 & 23 \\
\hline FCHROMI & 34 & Chromium, filtered & ppb & 20 & 50 & EPA & 26 & 2 & 0 \\
\hline COLIFORM & 144 & Coliforms & $\mathrm{COL}$ & & 1 & EPA & 26 & 2 & 1 \\
\hline ENDRIN & 17 & Endrin & ppb & .1 & .2 & EPA & 27 & 0 & 0 \\
\hline FLUOR ID & & Fluoride & ppb & 100 & 4000 & EPA & 26 & 26 & 0 \\
\hline ALPHA & 135 & Gross alpha & $p C i / L$ & 4 & 15 & EPA & 26 & 22 & 0 \\
\hline BETA & 136 & Gross beta & $\mathrm{pCi} / \mathrm{L}$ & 8 & 50 & EPA & 26 & 26 & 0 \\
\hline LEAD & 40 & Lead & ppb & 5 & 50 & EPA & 26 & 2 & 0 \\
\hline FLEAD & 40 & Lead, filtered & ppb & 5 & 50 & EPA & 26 & 0 & 0 \\
\hline MERCURY & 41 & Mercury & ppb & .2 & 2 & EPA & 26 & 0 & 0 \\
\hline FMERCUR & 41 & Mercury, filtered & ppb & .2 & 2 & EPA & 25 & 0 & 0 \\
\hline METHLOR & 17 & Methoxychlor & ppb & 2 & 100 & EPA & 27 & 0 & 0 \\
\hline NITRATE & & Nitrate & ppb & $20 \mathrm{C}$ & 45000 & EPA & 26 & 25 & 0 \\
\hline RADIUM & 137 & Radium & $p C i / L$ & 1 & 5 & EPA & 22 & 10 & 0 \\
\hline SELENUM & 48 & Selenium & ppb & 10 & 10 & EPA & 26 & 0 & 0 \\
\hline FSELENI & 48 & Selenium, filtered & ppb & 10 & 10 & EPA & 26 & 0 & 0 \\
\hline SILVER & 34 & silver & ppb & 20 & 50 & EPA & 26 & 0 & 0 \\
\hline FSILVER & 34 & silver, filtered & ppb & 20 & 50 & EPA & 26 & 0 & 0 \\
\hline TOXAENE & 17 & Toxaphene & ppb & 2 & 5 & EPA & 27 & 0 & 0 \\
\hline TURBID & 126 & Turbidity & NTU & .1 & 1 & EPA & 22 & 22 & 19 \\
\hline g-BHC & & garma-BHC (L indane) & ppb & .05 & 4 & EPA & 27 & 0 & 0 \\
\hline
\end{tabular}

GROUNDWATER OUALITY PARAMETERS

\begin{tabular}{|c|c|c|c|c|c|c|c|c|c|}
\hline \multirow[b]{2}{*}{ Short } & \multirow[b]{2}{*}{ (Method) } & \multirow[t]{2}{*}{ Const ituent Name } & \multirow[b]{2}{*}{ Units } & \multirow{2}{*}{$\begin{array}{l}\text { Lab } \\
\text { CROL }\end{array}$} & \multicolumn{2}{|c|}{ DWS } & \multicolumn{3}{|c|}{ Number of Samples } \\
\hline & & & & & Limit & Agency & Total & $>$ CRQL & $>$ DWS \\
\hline$\ldots \ldots$ & $\ldots$ & $\ldots \ldots \ldots$ & $\ldots .$. & $\ldots \ldots$ & $\ldots \ldots$ & $\ldots \ldots$ & $\ldots$. & $\ldots \ldots$ & $\ldots$ \\
\hline CHLOR ID & & Chloride & ppb & 200 & 250000 & EPAS & 26 & 26 & 0 \\
\hline IRON & 34 & Iron & $\mathrm{ppb}$ & 20 & 300 & EPAS & 26 & 26 & 21 \\
\hline FIRON & 34 & Iron, filtered & $\mathrm{ppb}$ & 20 & 300 & EPAS & 26 & 23 & 1 \\
\hline MANGESE & 34 & Manganese & ppb & 10 & 50 & EPAS & 26 & 18 & 10 \\
\hline FMANGAN & 34 & Manganese, filtered & $p p b$ & 10 & 50 & EPAS & 26 & 12 & 8 \\
\hline LPHENOL & 19 & Phenol & ppb & 10 & & & 13 & 0 & \\
\hline LPHENOL & 30 & Phenol & ppb & 20 & & & 20 & 0 & \\
\hline SODIUM & 34 & Sodium & ppb & 300 & & & 26 & 26 & \\
\hline FSOOIUM & 34 & Sodium, filtered & ppb & 300 & & & 26 & 26 & \\
\hline SULFATE & & Sulfate & ppb & 500 & 250000 & EPAS & 26 & 26 & 0 \\
\hline
\end{tabular}


Table 6-3. Constituent List and Summary of Results for the 216-B-3 Pond Data for Reporting Period July 1 through September 30, 1993. (sheet 2 of 6)

SITE SPECIFIC AND OTHER CONSTITUENTS

\begin{tabular}{|c|c|}
\hline Short & \\
\hline & \\
\hline $1112-t c$ & 16 \\
\hline $1,1,1-1$ & 16 \\
\hline $1,1,1-T$ & 25 \\
\hline $1122-t c$ & 16 \\
\hline $1,1,2-T$ & 16 \\
\hline $1,1,2-1$ & 25 \\
\hline $1,1-D I C$ & 16 \\
\hline $1,1-D \perp C$ & 25 \\
\hline DICETHY & 16 \\
\hline $123-t r p$ & 16 \\
\hline TETRCHB & 19 \\
\hline TRICHLB & 19 \\
\hline DIBRCHL & 16 \\
\hline DIBRETH & 16 \\
\hline 12-dben & 19 \\
\hline $\begin{array}{l}1,2-D / C \\
1,2-D 1 C\end{array}$ & \\
\hline 12-DCE & \\
\hline DICPANE & 16 \\
\hline 13-dben & \\
\hline 14-dben & 16 \\
\hline 14 -dben & 19 \\
\hline 14-dben & 25 \\
\hline DIOXANE & 16 \\
\hline NAPHQUI & \\
\hline $\begin{array}{l}\text { IBUTYM } \\
1 \text {-napha }\end{array}$ & 16 \\
\hline TETRCHP & 19 \\
\hline $2,4,5 \cdot 1$ & 49 \\
\hline $245-\operatorname{trp}$ & 19 \\
\hline 246-trp & 19 \\
\hline $246-t r p$ & 30 \\
\hline 24 -dchp & 19 \\
\hline $24 \cdot d c h p$ & 30 \\
\hline DIMPHEN & 19 \\
\hline DIMPHEN & 30 \\
\hline DINPHEN & \\
\hline DINPHEN & \\
\hline 24-dint & 19 \\
\hline 26-dchp & 19 \\
\hline $\begin{array}{l}\text { 26-dchp } \\
26-\text { dint }\end{array}$ & 30 \\
\hline ACEFENE & \\
\hline CHLNAPH & 19 \\
\hline CHLPHEN & 19 \\
\hline CHLPHEN & 3 \\
\hline 2HEXANO & \\
\hline 2MENAPH & 15 \\
\hline 2METHPH & 19 \\
\hline 2-napha & 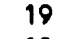 \\
\hline ONITANI & 19 \\
\hline 2NITPH & 1 \\
\hline 2NITPH & 30 \\
\hline PICOLIN & \\
\hline
\end{tabular}

Constituent Name

Full

1,1,1,2-Tetrachloroethane 1,1,1-Trichloroethane 1,1,1-Trichloroethane

$1,1,2,2-$ Tetrachloroethane 1,1,2-Trichloroethane ppb

1,1,2-Trichloroethane ppb

1,1-Dichloroethane ppb

1,1-Dichloroethane ppb

1,1-Dichloroethene ppb

1,2,3-Trichloropropane ppb

1,2,4,5-Tetrachlorobenzene ppb

1,2,4-Trichlorobenzene ppb

1,2-Dibromo-3-chloropropane ppb

1,2-Dibromoethane ppb

1,2-Dichlorobenzene ppb

1,2-Dichloroethane ppb

1,2-Dichloroethane ppb

1,2-Dichloroethene ppb

1,2-Dichloropropane ppb

1,3-Dichlorobenzene ppb

1,4-Dichlorobenzene ppb

1,4-Dichlorobenzene ppb

1,4-Dichlorobenzene ppb 1,4-Dioxane ppb

1,4-Naphtoquinone ppb 1-Butanol ppb

1-Naphthylamine ppb

2,3,4,6-Tetrachlorophenol ppb $2,3,7,8-$ TCDD ppb $2,4,5-T$

2,4,5-Trichlorophenol

2,4,6-Trichlorophenol

2,4,6-Trichlorophenol

2,4-Dichlorophenol

2,4-Dichlorophenol

2,4-0 ime thylphenol

2,4-0 ime thylphenol

2,4-Dinitrophenol

2,4-Dinitrophenol

2,4-Dinitrotoluene

2,6-Dichtorophenol

2,6-Dichlorophenol

2,6-0 initrotolvene

2-Acetyl aminofluorene

2-Chloronaph thalene

2-Chlorophenol

2-Chlorophenol 2-Hexanone

2-Methylnaph thal ene 2-Methylphenol 2-Naphthylamine 2-Nitroaniline

2-Nitrophenol

2-Nitrophenol 2-Picoline
Number of Samples Total >CRQL >DWS TOEA

110

120

40

110

120

$\begin{array}{rr}4 & 0 \\ 12 & 0\end{array}$

$\begin{array}{cc}12 & 0 \\ 4 & 0\end{array}$

$11 \quad 0$

110

$\begin{array}{ll}11 & 0 \\ 11 & 0\end{array}$

110

110

$\begin{array}{rr}11 & 0 \\ 4 & 0\end{array}$

$\begin{array}{rl}4 & 0 \\ 12 & 0\end{array}$

$\begin{array}{ll}12 & 0 \\ 11 & 0\end{array}$

$\begin{array}{rl}11 & 0 \\ 1 & 0\end{array}$

110

40

$\begin{array}{ll}11 & 0 \\ 11 & 0\end{array}$

12

110

110

$\begin{array}{rr}2 & 0 \\ 22 & 0\end{array}$

$\begin{array}{ll}11 & 0 \\ 11 & 0\end{array}$

$20-0$

130

$\begin{array}{ll}20 & 0 \\ 11 & 0\end{array}$

20

110

$20 \quad 0$

110

$\begin{array}{ll}11 & 0 \\ 20 & 0\end{array}$

110

110

110

$\begin{array}{ll}11 & 0 \\ 20 & 0\end{array}$

110

110

130

110

$\begin{array}{ll}11 & 0 \\ 13 & 0\end{array}$

$20 \quad 0$ 
Table $6-3$. Constituent List and Summary of Results for the 216-B-3 Pond Data for Reporting Period July 1 through September 30, 1993. (sheet 3 of 6)

\begin{tabular}{|c|c|c|c|c|c|c|c|c|}
\hline \multirow[b]{2}{*}{ Short } & \multicolumn{3}{|c|}{ Constituent Name } & \multirow{2}{*}{$\begin{array}{l}\text { Lab } \\
\text { CROL }\end{array}$} & DuS & \multicolumn{3}{|c|}{ Nimber of Samples } \\
\hline & (Method) & Full & Units & & Limit Agency & Total & $>$ CROL & $>$ DWS \\
\hline$\ldots \ldots \ldots$ & $\cdots$ & $\ldots \ldots \ldots \ldots$ & $\ldots \ldots$ & $\ldots \ldots$ & $\ldots \ldots \ldots$ & $\ldots$. & $\ldots \ldots$ & $\cdots$ \\
\hline BUTOINP & 30 & 2-sec-Butyl-4,6-dinitrophenol CON & ppb & 5 & & 20 & 0 & \\
\hline BUTDINP & 49 & 2-sec-Butyl-4,6-dinitrophenol CDN & ppb & 1 & & 22 & 0 & \\
\hline DICHBEN & 19 & 3,3'-Dichlorobenzidine & ppb & 20 & & 11 & 0 & \\
\hline DIMEYLB & 19 & 3,3'-0 ime thylbenzidine & ppb & 10 & & 11 & 0 & \\
\hline METCHAN & & 3-Methyl chol anthrene & ppb & 10 & & 11 & 0 & \\
\hline MNITAHI & 19 & 3-Nitrosniline & ppb & 50 & & 11 & 0 & \\
\hline DOD & 17 & $4,4^{\prime}-D D D$ & ppb & .1 & & 27 & 0 & \\
\hline DDE & 17 & $4,4^{\prime}-$ DDE & ppb & .05 & & 27 & 0 & \\
\hline DDI & & $4,4^{\prime} \cdot 00 T$ & ppb & .1 & & 27 & 0 & \\
\hline 460N2MP & & 4,6-0 initro-2-methylphenol & ppb & 50 & & 11 & 0 & \\
\hline 460N2MP & & 4,0-0 initro-2-methylphenol & ppb & 200 & & 20 & 0 & \\
\hline AMINOYL & 19 & 4-Aminobiphenyl & ppb & 10 & & 11 & 0 & \\
\hline BROPHEN & & 4-Bromophenylphenyl ether & ppb & 10 & & 11 & 0 & \\
\hline CHLCRES & & 4-Chloro-3-methylphenol & ppb & 5 & & 20 & 0 & \\
\hline CHLCRES & & 6-Chloro-3-methylphenol & ppo & 20 & & 11 & 0 & \\
\hline CHLANIL & 19 & 4-Chloroenil ine & ppb & 20 & & 11 & 0 & \\
\hline 4CPPETH & 9 & 4-Chlorophenylphenyl ether & ppb & 10 & & 11 & 0 & \\
\hline MIBK & 16 & 4-Methyl-2-pentanone & ppb & 50 & & 12 & 0 & \\
\hline ४METHPH & & 4-Methylphenol & ppb & 10 & & 13 & 0 & \\
\hline NITRANI & 19 & 4-Nitroaniline & ppb & 50 & & 11 & 0 & \\
\hline NITPHEN & 19 & 4-Nitrophenol & ppb & 50 & & 11 & 0 & \\
\hline MITPHEN & 30 & 4-Nitrophenol & ppb & 30 & & 20 & 0 & \\
\hline 4N1TOUI & & 4-Nitroquinol ine-1-oxide & ppb & 10 & & 11 & 0 & \\
\hline NITRTOL & 19 & 5-Nitro-o-toluidine & ppb & 10 & & 11 & 0 & \\
\hline DIMBENZ & & $7,12-0$ ime thyl benz $[a]$ anthracene & ppb & 10 & & 11 & 0 & \\
\hline ACENAPH & 19 & Acenaph thene & ppb & 10 & & 11 & 0 & \\
\hline ACENATL & & Acenaphthylene & mb & 10 & & 11 & 0 & \\
\hline ACETONE & 16 & Acetone & ppb & 100 & & 12 & 0 & \\
\hline ACETILE & 16 & Acetonitrile & ppb & 200 & & 11 & 0 & \\
\hline ACETOPH & 19 & Acetophenone & ppb & 10 & & 11 & 0 & \\
\hline ACROL IN & 16 & Acrolein & ppb & 5 & & 11 & 0 & \\
\hline ACRYILE & 16 & Acrylonitrile & ppb & 5 & & 11 & 0 & \\
\hline ALDR IN & & Aldrin & ppb & .05 & & 27 & 0 & \\
\hline DIMPHAM & 19 & Alpha, alpha-Dime thyl phene thyl ami & ppb & 10 & & 11 & 0 & \\
\hline$\triangle-B H C$ & & Alpha-BHC & ppb & .05 & & 27 & 0 & \\
\hline ALUANUM & 34 & Aluminum & ppb & 200 & & 26 & 7 & \\
\hline FALUMIN & 34 & Aluminum & ppb & 200 & & 26 & 0 & \\
\hline AMMONIU & & Ammoniun ion & ppb & 100 & & 8 & 0 & \\
\hline NMMONIU & & Ammonium ion & ppb & 100 & & 18 & 1 & \\
\hline ANILINE & & Aniline & ppb & 10 & & 11 & 0 & \\
\hline ANITKRA & 19 & Anthrocene & ppb & 10 & & 11 & 0 & \\
\hline ANTIONY & 34 & Ant imony & ppb & 200 & & 26 & 0 & \\
\hline FANTIMO & 34 & Ant imony, filtered & ppb & 200 & & 26 & 0 & \\
\hline ARAMITE & & Aramite & ppb & 10 & & 11 & 0 & \\
\hline AR 1016 & & Aroclor-1016 & ppb & 1 & & 7 & 0 & \\
\hline AR 1221 & 17 & Aroclor -1221 & ppb & 1 & & 7 & 0 & \\
\hline AR 1232 & 17 & Aroclor- 1232 & ppb & 1 & & 7 & 0 & \\
\hline AR 1242 & & Aroclor -1242 & ppo & 1 & & 7 & 0 & \\
\hline AR 1248 & & Aroclor- 1248 & ppb & 1 & & 7 & 0 & \\
\hline AR 1254 & 17 & Aroclor-1254 & ppb & 1 & & 7 & 0 & \\
\hline AR 1260 & 17 & Aroclor -1260 & ppb & 1 & & 7 & 0 & \\
\hline BENZENE & 16 & Benzene & ppb & 5 & & 12 & 0 & \\
\hline BENZENE & 25 & Benzene & ppb & 2 & & 4 & 0 & \\
\hline BENZAAN & & Benzo( $\theta$ )anthracene & ppo & 10 & & 11 & 0 & \\
\hline BENZOPY & & Benzo(a)pyrene & ppo & 10 & & 11 & 0 & \\
\hline BENZBFL & & Benzo(b) f luoranthene & ppb & 10 & & 11 & 0 & \\
\hline BGHIPER & & Benzo(ghi)perylene & ppb & 10 & & 11 & 0 & \\
\hline
\end{tabular}


Table 6-3. Constituent List and Summary of Results for the 216-8-3 Pond Data for Reporting Period July 1 through September 30, 1993. (sheet 4 of 6)

\begin{tabular}{|c|c|c|c|c|c|c|c|c|c|}
\hline \multirow[b]{2}{*}{ Short } & \multirow[b]{2}{*}{ (Method) } & \multirow[t]{2}{*}{ Constituent Name } & \multirow[b]{2}{*}{ Units } & \multirow{2}{*}{$\begin{array}{l}\text { Lab } \\
\text { CRQL }\end{array}$} & \multicolumn{2}{|c|}{ DUS } & \multicolumn{3}{|c|}{ Number of Semples } \\
\hline & & & & & Limit & Agency & Total & $>\mathrm{CROL}$ & $>$ DUS \\
\hline$\ldots \ldots \ldots$ & $\cdots$ & $\ldots \ldots \ldots \ldots$ & $\ldots \ldots$ & $\ldots \ldots$ & $\ldots \ldots$ & $\ldots .$. & $\ldots$. & $\ldots$. & $\ldots$ \\
\hline BNZKFLU & & Benzo(k)f luoranthene & ppb & 10 & & & 11 & 0 & \\
\hline BENZOTH & 19 & Benzothiazole & ppo & 10 & & & 13 & 0 & \\
\hline BENZALC & 19 & Benzyl alcohol & ppb & 20 & & & 11 & 0 & \\
\hline BERYLUM & 34 & Beryllium & ppb & 3 & & & 26 & 0 & \\
\hline FBERYLL & 34 & Beryllium, filtered & ppb & 3 & & & 26 & 0 & \\
\hline b- $8 \mathrm{HC}$ & & Beta-BHC & ppb & .05 & & & 27 & 0 & \\
\hline BIS2CHM & 19 & Bis(2-Chor oethoxy)me thane & ppob & 10 & & & 11 & 0 & \\
\hline BIS2CHE & 19 & Bis(2-chloroethyl) ether & ppb & 10 & & & 11 & 0 & \\
\hline BISZETH & 19 & Bis(2-chloroisopropyl) ether & ppo & 10 & & & 11 & 0 & \\
\hline BISZEPH & 19 & Bis(2-ethylhexyl) phthalate & ppb & 10 & & & 13 & 0 & \\
\hline BROMIDE & & Bromide & ppb & 500 & & & 26 & 0 & \\
\hline BDCM & 16 & Bromodichlorome thane & ppb & 5 & 100 & EPA & 11 & 0 & 0 \\
\hline BROMORM & 16 & Bromoform & ppb & 5 & 100 & EPA & 11 & 0 & 0 \\
\hline BUTBENP & 19 & Butylbenzylphthalate & ppob & 10 & & & 11 & 0 & \\
\hline CALCIUM & 34 & Calcium & ppo & 100 & & & 26 & 26 & \\
\hline FCALCIU & 34 & Calcium, filtered & ppb & 100 & & & 26 & 26 & \\
\hline CARBIDE & 16 & Carbon disulfide & ppo & 5 & & & 12 & 0 & \\
\hline TETRANE & & Carbon tetrachloride & ppo & 1 & & & 4 & 0 & \\
\hline TETRANE & & Carbon tetrachloride & ppb & 5 & & & 12 & 0 & \\
\hline CHLOANE & & Chlordane & ppb & .1 & & & 27 & 0 & \\
\hline CHLROB & 16 & Chlorobenzene & ppb & 5 & & & 11 & 0 & \\
\hline ChLLATE & & Chlorobenzilate & ppb & 10 & & & 11 & 0 & \\
\hline CLETHAN & 16 & Chloroethane & ppo & 10 & & & 11 & 0 & \\
\hline CHLFORM & 16 & Chloroform & ppb & 5 & 100 & EPA & 12 & 0 & 0 \\
\hline CHLFORM & 25 & Chloroform & ppb & .5 & 100 & EPA & 4 & 0 & 0 \\
\hline CHLPRENE & & Chloroprene & ppb & 5 & & & 11 & 0 & \\
\hline CHRYSEN & & Chrysene & ppb & 10 & & & 11 & 0 & \\
\hline COBALT & 34 & Cobalt & ppb & 20 & & & 26 & 0 & \\
\hline FCOBALT & 34 & Cobslt, filtered & ppb & 20 & & & 26 & 0 & \\
\hline COPPER & 34 & Copper & ppo & 20 & 1000 & EPAS & 26 & 1 & 0 \\
\hline FCOPPER & 34 & Copper, filtered & ppb & 20 & 1000 & EPAS & 26 & 0 & 0 \\
\hline CRESOLS & & Cresols (methylphenols) & ppb & 10 & & & 20 & 0 & \\
\hline CYANIDE & & Cyanide & ppb & 20 & & & 7 & 0 & \\
\hline DECANE & 19 & Decane & ppb & 10 & & & 2 & 0 & \\
\hline d-BHC & & Delta-BHC & ppb & .1 & & & 27 & 0 & \\
\hline DIBPHTH & 19 & Di-n-butylphthal ate & ppb & 10 & & & 11 & 0 & \\
\hline DIOPHTH & 19 & Di-n-octylphthalate & ppb & 10 & & & 11 & 0 & \\
\hline DIALLATE & & Diallate & ppo & 10 & & & 11 & 0 & \\
\hline DIBAHAN & & Dibenz $[a, h]$ anthracene & ppo & 10 & & & 11 & 0 & \\
\hline DIBENFR & & Dibenzofuran & ppb & 10 & & & 11 & 0 & \\
\hline BRCHLMET & & D ibromochlorome thane & ppb & 5 & 100 & EPA & 11 & 0 & 0 \\
\hline DIBRMET & 16 & D ibromome thane & ppb & 5 & & & 11 & 0 & \\
\hline DICDIFM & 16 & D ichlorod ifluorome thane & ppb & 5 & & & 11 & 0 & \\
\hline DIELRIN & & Dieldrin & ppb & .05 & & & 27 & 0 & \\
\hline DIEPHTH & 19 & Diethyl phthalate & ppb & 10 & & & 11 & 0 & \\
\hline DIMETHO & & Dimethoate & ppb & 10 & & & 11 & 0 & \\
\hline DIMPHTH & & Dimethyl phthalate & ppb & 10 & & & 11 & 0 & \\
\hline DIPHAMI & & Diphenylamine & ppb & 10 & & & 11 & 0 & \\
\hline DISULFO & & Disulfoton & ppb & 2 & & & 6 & 0 & \\
\hline DOOECAN & 19 & Dodecane & $\mathrm{ppb}$ & 10 & & & 2 & 0 & \\
\hline ENDO1 & 17 & Endosul fan 1 & ppb & .1 & & & 27 & 0 & \\
\hline ENDO2 & & Endosulfan 11 & ppb & .05 & & & 27 & 0 & \\
\hline ENDSFAN & & Endosulfan sulfate & ppb & .5 & & & 27 & 0 & \\
\hline ENORALD & 17 & Endr in Aldehyde & ppo & .2 & & & 27 & 0 & \\
\hline ETHCYAN & 16 & Ethyl cyanide & ppb & 10 & & & 12 & 0 & \\
\hline ETHME TH & 16 & Ethyl methacrylate & ppob & 5 & & & 11 & 0 & \\
\hline ETHMETS & 19 & Ethyl methanesulfonate & ppo & 10 & & & 11 & 0 & \\
\hline ETHBENZ & 16 & Ethylbenzene & ppo & 5 & & & 11 & 0 & \\
\hline ETHBENZ & 25 & Ethylbenzene & ppo & 2 & & & 4 & 0 & \\
\hline
\end{tabular}


Table 6-3. Constituent List and Summary of Results for the 216-B-3 Pond Data for Reporting Period July 1 through September 30, 1993. (sheet 5 of 6)

\begin{tabular}{|c|c|c|c|c|c|c|c|c|}
\hline \multirow[b]{2}{*}{ Short } & \multicolumn{2}{|r|}{ Const ituent Name } & \multirow[b]{2}{*}{ Units } & \multirow{2}{*}{$\begin{array}{l}\text { Lab } \\
\text { CROL }\end{array}$} & DWS & \multicolumn{3}{|c|}{ Number of Samples } \\
\hline & (Method) & Full & & & Limit Agency & Total & $>C R Q L$ & $>$ DWS \\
\hline & & n. & & $\cdots \cdots$ & $\cdots \ldots$ & $\cdots \cdot$ & $\ldots$. & $\cdots$ \\
\hline FAMPHUR & & Famphur & ppb & 10 & & 11 & 0 & \\
\hline FLUORAN & & Fluoranthene & $\mathrm{ppb}$ & 10 & & 11 & 0 & \\
\hline FLRENE & 19 & Fluorene & ppb & 10 & & 11 & 0 & \\
\hline HEPTLOR & 17 & Heptachlor & ppb & .05 & & 27 & 0 & \\
\hline HEPTIDE & & Heptachlor epoxide & $\mathrm{ppb}$ & 1 & & 27 & 0 & \\
\hline HEXCBEN & 19 & Hexachlorobenzene & $\mathrm{ppb}$ & 10 & & 11 & 0 & \\
\hline HEXCBUT & 19 & Hexachlorobutadiene & $\mathrm{ppb}$ & 10 & & 11 & 0 & \\
\hline HEXCCYC & 19 & Hexachlorocyclopentadiene & $\mathrm{ppb}$ & 10 & & 11 & 0 & \\
\hline HEXCETH & 19 & Hexachloroethane & ppb & 10 & & 11 & 0 & \\
\hline HEXACHL & 19 & Hexach lorophene & ppb & 10 & & 11 & 0 & \\
\hline HEXAENE & & Hexachloropropene & ppb & 10 & & 11 & 0 & \\
\hline LKYORAZ & & Hydraz ine & ppb & 30 & & 26 & 0 & \\
\hline INDENOP & & Indeno( $1,2,3-\mathrm{cd})$ pyrene & ppb & 10 & & 11 & 0 & \\
\hline $1-129$ & & lodine-129 & $\mathrm{pCi} / \mathrm{L}$ & 1 & & 2 & 0 & \\
\hline I SOBUTY & 16 & Isobutyl alcohol & $\mathrm{ppb}$ & 200 & & 11 & 0 & \\
\hline ISOORIN & & Isadrin & ppb & 10 & & 11 & 0 & \\
\hline ISOPHER & 19 & Isophorone & ppb & 10 & & 11 & 0 & \\
\hline ISOSOLE & 19 & Isosafrole & ppb & 10 & & 11 & 0 & \\
\hline KEPONE & & Kepone & $\mathrm{ppb}$ & 10 & & 11 & 0 & \\
\hline KEROSEN & 19 & Kerosene & $\mathrm{ppb}$ & 10000 & & 11 & 0 & \\
\hline MAGNES & 34 & Magnesium & $\mathrm{ppb}$ & 100 & & 26 & 26 & \\
\hline FMAGNES & 34 & Magnesium, filtered & ppb & 100 & & 26 & 26 & \\
\hline METHACR & & Methacrylonitrile & ppb & 5 & & 11 & 0 & \\
\hline METHAPY & 19 & Methapyrilene & $\mathrm{ppb}$ & 10 & & 11 & 0 & \\
\hline IOOOMET & 16 & Methyl lodide & ppb & 5 & & 11 & 0 & \\
\hline METHBRO & 16 & Methyl bromide & ppb & 10 & & 11 & 0 & \\
\hline METHCHL & 16 & Methyl chloride & $\mathrm{ppb}$ & 10 & & 11 & 0 & \\
\hline METHONE & 16 & Methyl ethyl ketone & $\mathrm{ppb}$ & 100 & & 12 & 0 & \\
\hline METACRY & 16 & Methyl methacrylate & $\mathrm{ppb}$ & 5 & & 11 & 0 & \\
\hline METMSUL & 19 & Methyl methanesul fonate & ppb & 10 & & 11 & 0 & \\
\hline METHPAR & & Methyl parathion & $\mathrm{ppb}$ & .5 & & 6 & 0 & \\
\hline METHYCH & 16 & Methylene chloride & ppb & 5 & & 12 & 0 & \\
\hline METHYCH & 25 & Methylene chloride & $\mathrm{ppb}$ & 5 & & 4 & 0 & \\
\hline DIPRNIT & & N-Nitroso-di-n-dipropylamine & ppb & 10 & & 11 & 0 & \\
\hline NNIBUTY & 19 & N-Nitrosodi-n-butylamine & $\mathrm{ppb}$ & 10 & & 11 & 0 & \\
\hline NNIDIEY & & N-Nitrosodiethylamine & $\mathrm{ppb}$ & 10 & & 11 & 0 & \\
\hline NNIDIME & & N-Nitrosodimethylamine & $\mathrm{ppb}$ & 10 & & 11 & 0 & \\
\hline NNDIPHA & 19 & N-Nitrosodiphenylamine & $\mathrm{ppb}$ & 10 & & 11 & 0 & \\
\hline NNIMETH & 19 & N-Nitrosome thylethyl amine & $\mathrm{ppb}$ & 10 & & 11 & 0 & \\
\hline NNIMORP & 19 & N-Nitrosomorphol ine & $\mathrm{ppb}$ & 10 & & 11 & 0 & \\
\hline NNIPIPE & 19 & N-Nitrosopiperidine & $\mathrm{ppb}$ & 10 & & 11 & 0 & \\
\hline NAPHTHA & 19 & Naph thal ene & $\mathrm{ppb}$ & 10 & & 13 & 0 & \\
\hline NICKEL & 34 & Nickel & $\mathrm{ppb}$ & 30 & & 26 & 20 & \\
\hline FNICKEL & 34 & Nickel, filtered & $\mathrm{ppb}$ & 30 & & 26 & 1 & \\
\hline NITRITE & & Nitrite & ppb & 200 & & 26 & 0 & \\
\hline NITBENZ & 19 & Nitrobenzene & ppb & 10 & & 11 & 0 & \\
\hline NITRPYR & 19 & Nitrosopyrrolidine & $\mathrm{ppb}$ & 10 & & 11 & 0 & \\
\hline TRIPHOS & & $0,0,0-$ Iriethyl phosphorothioate & $\mathrm{ppb}$ & 10 & & 11 & 0 & \\
\hline DIPHOS & & 0,0-diethyl0-2-pyrazinylphosphor & $\mathrm{ppb}$ & 10 & & 11 & 0 & \\
\hline PCDOS & 51 & PCODS & $\mathrm{ppb}$ & .01 & & 2 & 0 & \\
\hline PCDFS & 51 & PCDFs & $\mathrm{ppb}$ & .01 & & 2 & 0 & \\
\hline PARATHI & & Parathion & $\mathrm{ppb}$ & 10 & & $1:$ & 0 & \\
\hline PENTCHB & 19 & Pentachlorobenzene & $\mathrm{ppb}$ & 10 & & 11 & 0 & \\
\hline PENTACH & 16 & Pentachloroethane & $\mathrm{ppb}$ & 5 & & 11 & 0 & \\
\hline PENTCHN & 19 & Pentachloronitrobenzene (PCNB) & $\mathrm{ppb}$ & 10 & & 11 & 0 & \\
\hline PENTCHP & 19 & Pentachlorophenol & $\mathrm{ppb}$ & 50 & & 13 & 0 & \\
\hline PENTCHP & 30 & Pentachlorophenol & $\mathrm{ppb}$ & 100 & & 20 & 0 & \\
\hline
\end{tabular}


Table 6-3. Constituent List and Summary of Results for the 216-B-3 Pond Data for Reporting Period July 1 through September 30, 1993. (sheet 6 of 6)

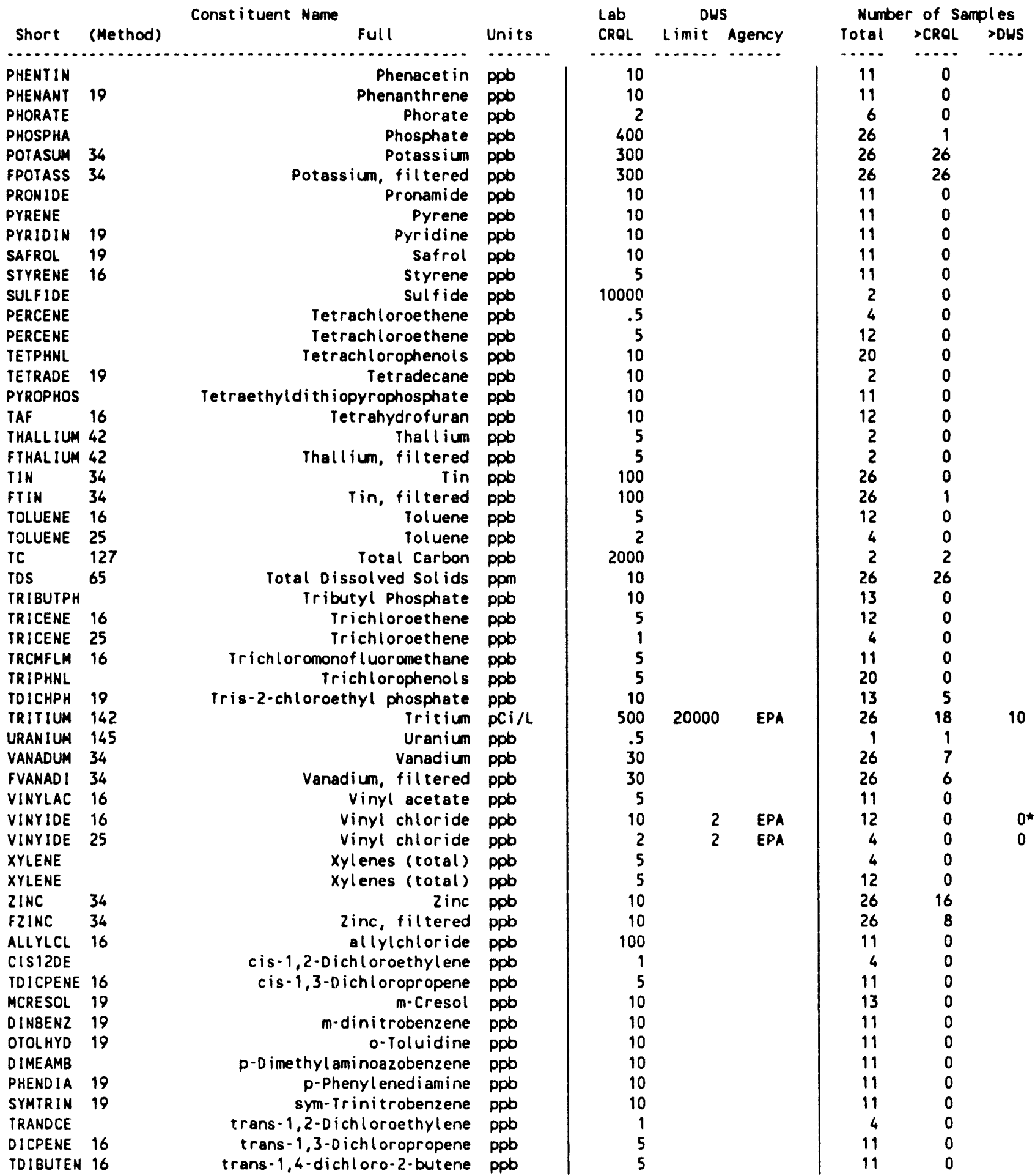

For explanation of this table, see Section 1.4 of report. 
Table 6-4. Constituents with at Least One Value Above the CRQL for the 216-B-3 Pond Data for Reporting Period July 1

through September 30, 1993. (sheet 1 of 13)

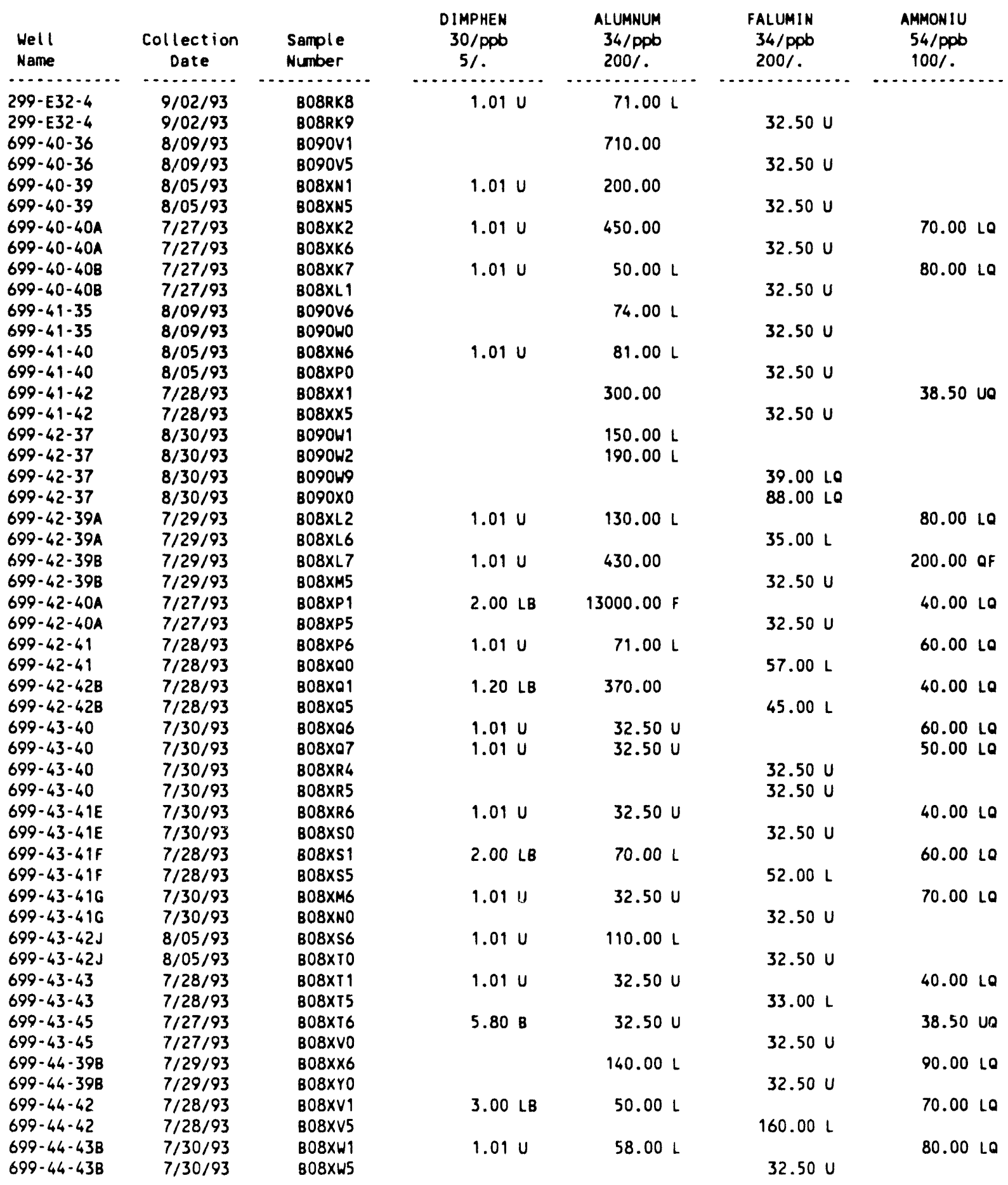


Table 6-4. Constituents with at Least One Value Above the CRQL for the 216-B-3 Pond Data for Reporting Period July 1

through September 30,1993 . (sheet 2 of 13)

\begin{tabular}{|c|c|c|c|c|c|c|}
\hline $\begin{array}{l}\text { Well } \\
\text { Name }\end{array}$ & $\begin{array}{c}\text { Collection } \\
\text { Date }\end{array}$ & $\begin{array}{l}\text { Sample } \\
\text { Number }\end{array}$ & $\begin{array}{c}\text { ARSENIC } \\
43 / \mathrm{ppb} \\
5 / 50\end{array}$ & $\begin{array}{l}\text { FARSENIC } \\
43 / \mathrm{ppb} \\
5 / 50\end{array}$ & $\begin{array}{l}\text { BARIUM } \\
34 / \mathrm{ppb} \\
20 / 1000\end{array}$ & $\begin{array}{l}\text { FBARIUM } \\
34 / \mathrm{ppD} \\
20 / 1000\end{array}$ \\
\hline $299-$ E32-4 & $9 / 02 / 93$ & B08RK8 & $2.00 \mathrm{~L}$ & $\cdots$ & 66.00 & \\
\hline $299-E 32-4$ & $9 / 02 / 93$ & B08RK9 & & $2.90 \mathrm{~L}$ & & 65.00 \\
\hline $699-40-36$ & $8 / 09 / 93$ & B090V1 & $1.38 \mathrm{U}$ & & 72.00 & \\
\hline $699-40-36$ & $8 / 09 / 93$ & 8090V5 & & $2.30 \mathrm{~L}$ & & 68.00 \\
\hline $699-40-39$ & $8 / 05 / 93$ & B08XN1 & $2.20 \mathrm{LO}$ & & 110.00 & \\
\hline $699-40-39$ & $8 / 05 / 93$ & B08XN5 & & $2.00 \mathrm{LQ}$ & & 110.00 \\
\hline $699-40-40 \mathrm{~A}$ & $7 / 27 / 93$ & B08XK2 & $2.90 \mathrm{~L}$ & & 110.00 & \\
\hline $699-40-40 \mathrm{~A}$ & $7 / 27 / 93$ & B08XK6 & & $2.80 \mathrm{~L}$ & & 110.00 \\
\hline $699-40-40 B$ & $7 / 27 / 93$ & B08XK7 & $1.38 \mathrm{U}$ & & 87.00 & \\
\hline $699-40-40 B$ & $7 / 27 / 93$ & B08XL1 1 & & $1.38 \mathrm{U}$ & & 86.00 \\
\hline $699-49-35$ & $8 / 09 / 93$ & 8090V6 & $3.20 \mathrm{~L}$ & & 130.00 & \\
\hline $699-41-35$ & $8 / 09 / 93$ & BO90WO & & $3.10 \mathrm{~L}$ & & 130.00 \\
\hline $\begin{array}{l}699-41-40 \\
699-41-40\end{array}$ & $\begin{array}{l}8 / 05 / 93 \\
8 / 05 / 93\end{array}$ & $\begin{array}{l}\text { B08XN6 } \\
\text { B08XP0 }\end{array}$ & 4.5010 & $4.00 \mathrm{LQ}$ & 67.00 & 66.00 \\
\hline $699-41-42$ & $7 / 28 / 93$ & $B 08 \times \times 1$ & 8.60 & 9.00 & 42.00 & \\
\hline $699-41-42$ & $7 / 28 / 93$ & $B 08 \times \times 5$ & & 8.70 & & 29.00 \\
\hline $\begin{array}{l}699-42-37 \\
699-42-37\end{array}$ & $\begin{array}{l}8 / 30 / 93 \\
8 / 30 / 93\end{array}$ & $\begin{array}{l}\text { B090W1 } \\
\text { B090W2 }\end{array}$ & $\begin{array}{l}3.70 \mathrm{~L} \\
2.90 \mathrm{~L}\end{array}$ & & $\begin{array}{l}56.00 \\
55.00\end{array}$ & \\
\hline $\begin{array}{l}699-42-37 \\
699-42-37\end{array}$ & $\begin{array}{l}8 / 30 / 93 \\
8 / 30 / 93\end{array}$ & $\begin{array}{l}\text { 809049 } \\
8090 \times 0\end{array}$ & & $\begin{array}{l}2.00 \text { LQ } \\
1.38 \text { UQ }\end{array}$ & & $\begin{array}{l}54.00 \\
57.00\end{array}$ \\
\hline $699-42-39 A$ & $7 / 29 / 93$ & B08XL2 & $3.80 \mathrm{~L}$ & & 58.00 & \\
\hline $699-42-39 A$ & $7 / 29 / 93$ & B08XL6 & & $4.70 \mathrm{~L}$ & & 58.00 \\
\hline $699-42-398$ & $7 / 29 / 93$ & $808 \times 17$ & $2.30 \mathrm{~L}$ & & 81.00 & \\
\hline $699-42-398$ & $7 / 29 / 93$ & B08XM5 & & $2.40 \mathrm{~L}$ & & 78.00 \\
\hline $699-42-40 A$ & $7 / 27 / 93$ & B08XP1 & 11.00 & & 160.00 & \\
\hline $699-42-40 A$ & $7 / 27 / 93$ & B08XP5 & & $1.38 \mathrm{U}$ & & $6.30 \mathrm{~L}$ \\
\hline $699-42-41$ & $7 / 28 / 93$ & B08XP6 & $2.80 \mathrm{~L}$ & & $10.00 \mathrm{~L}$ & \\
\hline $699-42-41$ & $7 / 28 / 93$ & $B 08 \times 00$ & & $2.20 \mathrm{~L}$ & & $6.90 \mathrm{~L}$ \\
\hline $699-42-42 B$ & $7 / 28 / 93$ & $808 \times 01$ & 8.20 & & 27.00 & \\
\hline $699-42-42 B$ & $7 / 28 / 93$ & $808 \times 05$ & & 7.50 & & 24.00 \\
\hline $\begin{array}{l}699-43-40 \\
699-43-40\end{array}$ & $\begin{array}{l}7 / 30 / 93 \\
7 / 30 / 93\end{array}$ & $\begin{array}{l}808 \times 06 \\
808 \times 07\end{array}$ & $\begin{array}{l}2.70 \mathrm{LO} \\
2.60 \mathrm{LO}\end{array}$ & & $\begin{array}{l}58.00 \\
58.00\end{array}$ & \\
\hline $699-43-40$ & $7 / 30 / 93$ & B08XR4 & $2.60 \mathrm{LQ}$ & $3.00 \mathrm{LO}$ & 38.00 & 60.00 \\
\hline $699-43-40$ & $7 / 30 / 93$ & $808 \times R 5$ & & $3.00 \mathrm{LO}$ & & 60.00 \\
\hline $699-43-41 E$ & $7 / 30 / 93$ & B08XR6 & 5.400 & & 37.00 & \\
\hline $699-43-41 E$ & $7 / 30 / 93$ & B08XSO & & $4.90 \mathrm{LQ}$ & & 37.00 \\
\hline $699-43-41 F$ & $7 / 28 / 93$ & B08X51 & 5.00 & & 33.00 & \\
\hline $699-43-41 F$ & $7 / 28 / 93$ & $808 \times 55$ & & $4.80 \mathrm{~L}$ & & 35.00 \\
\hline $699-43-496$ & $7 / 30 / 93$ & $808 \times M 6$ & 7.300 & & 54.00 & \\
\hline $699 \cdot 43-416$ & $7 / 30 / 93$ & BO8XNO & & 6.100 & & 53.00 \\
\hline $699-43-42 J$ & $8 / 05 / 93$ & $808 \times 56$ & 19.000 & & $.00 \mathrm{UF}$ & \\
\hline $699-43-42 \mathrm{~J}$ & $8 / 05 / 93$ & B08XTO & & 21.000 & & .00 UF \\
\hline $699 \cdot 43 \cdot 43$ & $7 / 28 / 93$ & $808 \times 11$ & 8.70 & & $15.00 \mathrm{~L}$ & \\
\hline $699-43-43$ & $7 / 28 / 93$ & B08XT5 & & 9.60 & & $12.00 \mathrm{~L}$ \\
\hline $699-43-45$ & $7 / 27 / 93$ & B08X16 & 10.00 & & 34.00 & \\
\hline $699-43-45$ & $7 / 27 / 93$ & B08XV0 & & 9.20 & & 33.00 \\
\hline $699-44-398$ & $7 / 29 / 93$ & $808 \times \times 6$ & $4.40 \mathrm{~L}$ & & $.00 \mathrm{UF}$ & \\
\hline $699 \cdot 44-398$ & $7 / 29 / 93$ & B08XYO & & $4.20 \mathrm{~L}$ & & .00 UF \\
\hline $699-44-42$ & $7 / 28 / 93$ & $808 \times V 1$ & 13.00 & & 32.00 & \\
\hline $699-44-42$ & $7 / 28 / 93$ & B08XV5 & & 14.00 & & 37.00 \\
\hline $699-44-43 B$ & $7 / 30 / 93$ & B08XW1 & 7.700 & & 46.00 & \\
\hline $699-44-438$ & $7 / 30 / 93$ & $808 \times 65$ & & 9.500 & & 44.00 \\
\hline
\end{tabular}


Table 6-4. Constituents with at Least One Value Above the CRQL for the 216-B-3 Pond Data for Reporting Period July 1 through September 30, 1993. (sheet 3 of 13)

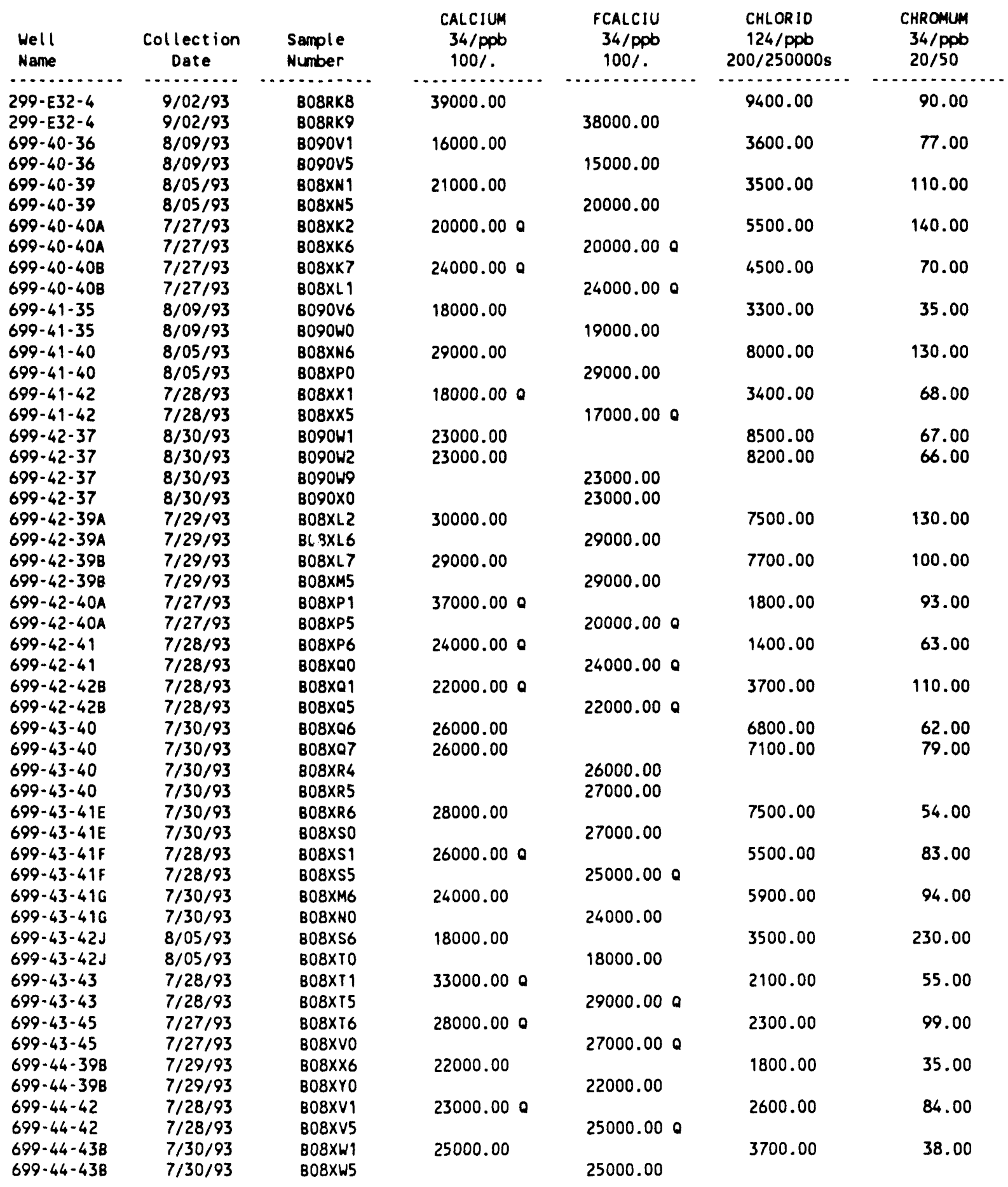


Table 6-4. Constituents with at Least One Value Above the CRQL for the 216-B-3 Pond Data for Reporting Period July 1 through September 30,1993 . (sheet 4 of 13)

\begin{tabular}{|c|c|c|c|c|c|c|}
\hline $\begin{array}{l}\text { Well } \\
\text { Name }\end{array}$ & $\begin{array}{c}\text { Collection } \\
\text { Date }\end{array}$ & $\begin{array}{l}\text { Sample } \\
\text { Number }\end{array}$ & $\begin{array}{l}\text { FCHROMI } \\
34 / \mathrm{ppb} \\
20 / 50\end{array}$ & $\begin{array}{c}\text { COLIFORM } \\
144 / \mathrm{COL} \\
1 / 1\end{array}$ & $\begin{array}{c}\text { COPPER } \\
34 / \text { PPb } \\
20 / 1000 \mathrm{~s}\end{array}$ & $\begin{array}{c}\text { FCOPPER } \\
34 / \text { ppb } \\
20 / 1000 \mathrm{~s}\end{array}$ \\
\hline $299-E 32-4$ & $9 / 02 / 93$ & BO8RK8 & $\cdots$ & $1.00 \mathrm{u}$ & $2.65 \mathrm{u}$ & \\
\hline 299-E32-4 & $9 / 02 / 93$ & BO8RK9 & $5.42 \mathrm{U}$ & 1.000 & 2.650 & $2.65 \mathrm{U}$ \\
\hline $699-40-36$ & $8 / 09 / 93$ & B090V1 & & $1.00 \mathrm{U}$ & $6.00 \mathrm{~L}$ & \\
\hline $699-40-36$ & $8 / 09 / 93$ & $8090 \mathrm{VS}$ & $8.40 \mathrm{~L}$ & & & $2.65 \mathrm{U}$ \\
\hline $699 \cdot 40-39$ & $8 / 05 / 93$ & B08XN1 & & $1.00 \mathrm{U}$ & $2.65 \mathrm{U}$ & \\
\hline $699-40-39$ & $8 / 05 / 93$ & B08XN5 & $5.42 \mathrm{U}$ & & & $2.65 \mathrm{U}$ \\
\hline $699-40-40 A$ & $7 / 27 / 93$ & B08XK2 & & $1.00 \mathrm{U}$ & $6.90 \mathrm{~L}$ & \\
\hline $699-40-40 \mathrm{~A}$ & $7 / 27 / 93$ & B08XK6 & $5.42 \mathrm{U}$ & & & $2.65 \mathrm{U}$ \\
\hline $699-40-40 B$ & $7 / 27 / 93$ & B08XK7 & & 1.00 & $6.20 \mathrm{~L}$ & \\
\hline $699-40-40 B$ & $7 / 27 / 93$ & B08XL1 1 & $5.42 \mathrm{U}$ & & & $4.00 \mathrm{~L}$ \\
\hline $699-41 \cdot 35$ & $8 / 09 / 93$ & B090V6 & & $1.00 \mathrm{U}$ & $4.60 \mathrm{~L}$ & \\
\hline $699 \cdot 41 \cdot 35$ & $8 / 09 / 93$ & Bo9040 & $8.60 \mathrm{~L}$ & & & $6.00 \mathrm{~L}$ \\
\hline $\begin{array}{l}699-41-40 \\
699-41-40\end{array}$ & $\begin{array}{l}8 / 05 / 93 \\
8 / 05 / 93\end{array}$ & $\begin{array}{l}\text { B08XN6 } \\
\text { B08XP0 }\end{array}$ & $5.42 \mathrm{U}$ & $1.00 \mathrm{U}$ & $2.65 \mathrm{U}$ & $2.65 \mathrm{U}$ \\
\hline $699-41-42$ & $7 / 28 / 93$ & $B 08 \times \times 1$ & & $1.00 \mathrm{U}$ & $6.80 \mathrm{~L}$ & \\
\hline $699-41-42$ & $7 / 28 / 93$ & $808 \times \times 5$ & $5.42 \mathrm{U}$ & & & $2.65 \mathrm{U}$ \\
\hline $\begin{array}{l}699-42-37 \\
699-42 \cdot 37\end{array}$ & $\begin{array}{l}8 / 30 / 93 \\
8 / 30 / 93\end{array}$ & $\begin{array}{l}\text { B090W1 } \\
\text { B090W2 }\end{array}$ & & $\begin{array}{l}1.00 \mathrm{U} \\
1.00 \mathrm{U}\end{array}$ & $\begin{array}{l}2.65 U \\
2.65 U\end{array}$ & \\
\hline $\begin{array}{l}699-42-37 \\
699-42-37\end{array}$ & $\begin{array}{l}8 / 30 / 93 \\
8 / 30 / 93\end{array}$ & $\begin{array}{l}\text { B090W9 } \\
\text { B090X0 }\end{array}$ & $\begin{array}{l}5.42 U \\
5.42 U\end{array}$ & & & $\begin{array}{l}2.65 U \\
2.65 U\end{array}$ \\
\hline $699-42-39 A$ & $7 / 29 / 93$ & B08XL2 & & $1.00 \mathrm{U}$ & $2.65 \mathrm{U}$ & \\
\hline $699-42-39 A$ & $7 / 29 / 93$ & $808 \times 16$ & $5.42 \mathrm{U}$ & & & $2.65 U$ \\
\hline $699-42 \cdot 398$ & $7 / 29 / 93$ & B08XL7 & & $1.00 \mathrm{U}$ & $2.65 \mathrm{U}$ & \\
\hline $699-42-398$ & $7 / 29 / 93$ & B08XM5 & $5.42 \mathrm{U}$ & & & $2.65 \mathrm{U}$ \\
\hline $699-42-40 A$ & $7 / 27 / 93$ & B08XP1 & & $1.00 \mathrm{U}$ & $22.00 \mathrm{~F}$ & \\
\hline $699-42-40 \mathrm{~A}$ & $7 / 27 / 93$ & B08XP5 & $5.42 \mathrm{U}$ & & & $2.65 \mathrm{U}$ \\
\hline $699-42-41$ & $7 / 28 / 93$ & B08XP6 & & $1.00 \mathrm{U}$ & $2.65 \mathrm{U}$ & \\
\hline $699-42-41$ & $7 / 28 / 93$ & $808 \times 00$ & $5.42 \mathrm{U}$ & & & $2.65 \mathrm{U}$ \\
\hline $699-42-42 B$ & $7 / 28 / 93$ & B08X01 & & $1.00 \mathrm{U}$ & $5.20 \mathrm{~L}$ & \\
\hline $699-42-428$ & $7 / 28 / 93$ & B08X05 & $7.50 \mathrm{~L}$ & & & $2.65 U$ \\
\hline $699-43 \cdot 40$ & $7 / 30 / 93$ & B08X06 & & $1.00 \mathrm{U}$ & $4.50 \mathrm{~L}$ & \\
\hline $699-43-40$ & $7 / 30 / 93$ & $808 \times 07$ & & $1.00 \mathrm{U}$ & $5.70 \mathrm{~L}$ & \\
\hline $699-43-40$ & $7 / 30 / 93$ & B08XR4 & 22.00 & & & $2.65 \mathrm{U}$ \\
\hline $699-43-40$ & $7 / 30 / 93$ & B08XR5 & $5.60 \mathrm{~L}$ & & & $2.65 \mathrm{U}$ \\
\hline $699-43-41 E$ & $7 / 30 / 93$ & B08XR6 & & $1.00 \mathrm{U}$ & $2.65 \mathrm{U}$ & \\
\hline $699-43-41 E$ & $7 / 30 / 93$ & B08x50 & $11.00 \mathrm{~L}$ & & & 9.501 \\
\hline $699-43-41 F$ & $7 / 28 / 93$ & B08Xs1 & & $1.00 \mathrm{U}$ & $2.80 \mathrm{~L}$ & \\
\hline $699-43-41 F$ & $7 / 28 / 93$ & B08X55 & $15.00 \mathrm{~L}$ & & & $2.65 \mathrm{U}$ \\
\hline $699-43-410$ & $7 / 30 / 93$ & B08XM6 & & $1.00 \mathrm{U}$ & $6.10 \mathrm{~L}$ & \\
\hline $699-43-416$ & $7 / 30 / 93$ & B08XNO & $5.70 \mathrm{~L}$ & & & $2.65 U$ \\
\hline $699-43-42 \mathrm{~J}$ & $8 / 05 / 93$ & B08XS6 & & $1.00 \mathrm{U}$ & $2.65 \mathrm{U}$ & \\
\hline $699-43-42 J$ & $8 / 05 / 93$ & B08XTO & $5.42 \mathrm{U}$ & & & $2.65 U$ \\
\hline $699-43-43$ & $7 / 28 / 93$ & $\begin{array}{l}B 08 \times 11 \\
808 \times T 5\end{array}$ & & $1.00 \mathrm{U}$ & $2.65 \mathrm{U}$ & 265.11 \\
\hline $\begin{array}{l}699-43-43 \\
699-43-45\end{array}$ & $\begin{array}{l}7 / 28 / 93 \\
7 / 27 / 93\end{array}$ & $\begin{array}{l}808 \times 15 \\
808 \times 16\end{array}$ & $7.30 \mathrm{~L}$ & $1.00 \mathrm{U}$ & $6.20 \mathrm{~L}$ & 2.050 \\
\hline $699-43-45$ & $7 / 27 / 93$ & B08XV0 & $5.42 \mathrm{U}$ & & & $2.65 \mathrm{U}$ \\
\hline $699-44-39 B$ & $7 / 29 / 93$ & $808 \times \times 6$ & & $21.00 \mathrm{~F}$ & $2.65 \mathrm{U}$ & \\
\hline $699-44-39 B$ & $7 / 29 / 93$ & B08XYO & $5.42 \mathrm{U}$ & & & $2.65 \mathrm{U}$ \\
\hline $699-44-42$ & $7 / 28 / 93$ & B08XV1 & & $1.00 \mathrm{U}$ & $4.50 \mathrm{~L}$ & \\
\hline $699-44-42$ & $7 / 28 / 93$ & B08XV5 & 27.00 & & & $6.30 \mathrm{~L}$ \\
\hline $699-44-43 B$ & $7 / 30 / 93$ & B08XW1 & & $1.00 \mathrm{U}$ & $4.10 \mathrm{~L}$ & \\
\hline $699-44-43 B$ & $7 / 30 / 93$ & B08XW5 & $5.80 \mathrm{~L}$ & & & $2.65 \mathrm{U}$ \\
\hline
\end{tabular}


Table 6-4. Constituents with at Least One Value Above the CRQL for the 216-B-3 Pond Data for Reporting Period July 1 through September 30, 1993. (sheet 5 of 13)

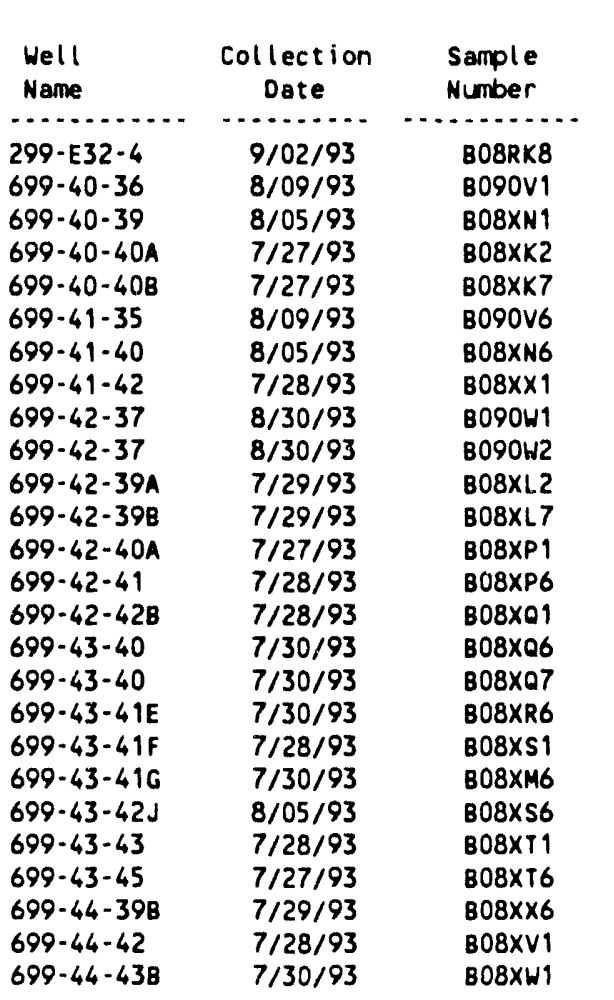

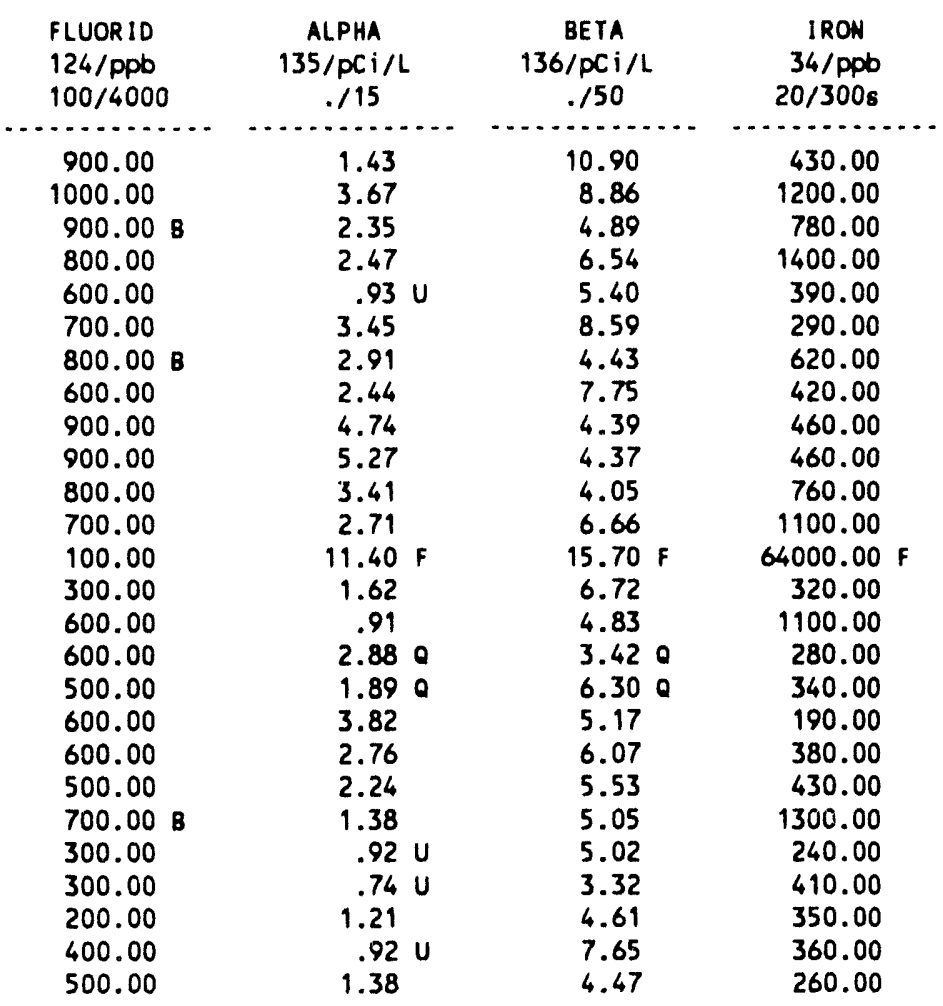

\begin{tabular}{|c|c|c|c|c|c|c|}
\hline $\begin{array}{l}\text { Hell } \\
\text { Name }\end{array}$ & $\begin{array}{c}\text { Collection } \\
\text { Date }\end{array}$ & $\begin{array}{l}\text { Sample } \\
\text { Number }\end{array}$ & $\begin{array}{c}\text { FIRON } \\
34 / \mathrm{ppb} \\
20 / 300 \mathrm{~s}\end{array}$ & $\begin{array}{l}\text { LEAD } \\
40 / p p b \\
5 / 50\end{array}$ & $\begin{array}{l}\text { FLEAD } \\
40 / \mathrm{ppb} \\
5 / 50\end{array}$ & $\begin{array}{l}\text { MAGNES } \\
34 / \mathrm{ppb} \\
100 /\end{array}$ \\
\hline$\cdots+\ldots$ & $\ldots \ldots$ & $\ldots \ldots$ & 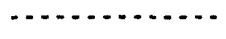 & $-\ldots$ & $\ldots$ & 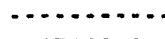 \\
\hline $299-E 32-4$ & $9 / 02 / 93$ & B08RK8 & & $1.30 \mathrm{~L}$ & & 13000.00 \\
\hline $299-E 32-4$ & $9 / 02 / 93$ & B08RK9 & 26.00 & & $.82 \mathrm{~L}$ & \\
\hline $699-40-36$ & $8 / 09 / 93$ & B090V1 & & $.89 \mathrm{~L}$ & & 5200.00 \\
\hline $699 \cdot 40-36$ & $8 / 09 / 93$ & B090V5 & 51.00 & & $.51 \mathrm{U}$ & \\
\hline $699-40-39$ & $8 / 05 / 93$ & B08XN1 & & $1.70 \mathrm{~L}$ & & 8200.00 \\
\hline $699-40-39$ & $8 / 05 / 93$ & B08XN5 & 73.00 & & $.97 \mathrm{~L}$ & \\
\hline $699-40-40 A$ & $7 / 27 / 93$ & B08XK2 & & $.73 \mathrm{~L}$ & & 7300.00 \\
\hline $699-40-40 A$ & $7 / 27 / 93$ & B08XK6 & 140.00 & & $.51 \mathrm{U}$ & \\
\hline $699-40-408$ & $7 / 27 / 93$ & $808 \times K 7$ & & $.51 \mathrm{U}$ & & 6100.00 \\
\hline $699-40-40 B$ & $7 / 27 / 93$ & B08XL1 & 49.00 & & $.51 \mathrm{U}$ & \\
\hline $699-41-35$ & $8 / 09 / 93$ & B090V6 & & $1.20 \mathrm{~L}$ & & 6600.00 \\
\hline $699-41-35$ & $8 / 09 / 93$ & B090W0 & 69.00 & & $.51 \mathrm{U}$ & \\
\hline $699-41-40$ & $8 / 05 / 93$ & B08XN6 & & $.66 \mathrm{~L}$ & & 11000.00 \\
\hline $699 \cdot 41 \cdot 40$ & $8 / 05 / 93$ & $B 08 \times P 0$ & 44.00 & & $.60 \mathrm{~L}$ & \\
\hline $699-41-42$ & $7 / 28 / 93$ & $808 \times \times 1$ & & 9.40 & & 6400.00 \\
\hline $699-41-42$ & $7 / 28 / 93$ & $808 \times \times 5$ & $16.00 \mathrm{~L}$ & & $1.60 \mathrm{~L}$ & \\
\hline $699-42 \cdot 37$ & $8 / 30 / 93$ & B090WT & & $.77 \mathrm{LO}$ & & 9900.00 \\
\hline $699-42-37$ & $8 / 30 / 93$ & B090W2 & & .51 vo & & 10000.00 \\
\hline $699 \cdot 42 \cdot 37$ & $8 / 30 / 93$ & Bo90w9 & $20.00 \mathrm{~L}$ & & $.51 \mathrm{U}$ & \\
\hline $699 \cdot 42 \cdot 37$ & $8 / 30 / 93$ & $8090 \times 0$ & 23.00 & & $.51 \mathrm{U}$ & \\
\hline $699 \cdot 42-39 A$ & $7 / 29 / 93$ & B08XL2 & & $1.50 \mathrm{~L}$ & & 12000.00 \\
\hline $699-42-39 A$ & $7 / 29 / 93$ & $808 \times 16$ & 44.00 & & $.51 \mathrm{U}$ & \\
\hline $699-42-39 B$ & $7 / 29 / 93$ & $808 \times 17$ & & $.75 \mathrm{~L}$ & & 11000.00 \\
\hline $699 \cdot 42 \cdot 398$ & $7 / 29 / 93$ & $808 \times 115$ & 32.00 & & $1.10 \mathrm{~L}$ & \\
\hline
\end{tabular}


Table 6-4. Constituents with at Least One Value Above the CRQL for the 216-B-3 Pond Data for Reporting Period July 1

through September 30, 1993. (sheet 6 of 13)

\begin{tabular}{|c|c|c|c|c|c|c|}
\hline $\begin{array}{l}\text { Well } \\
\text { Name }\end{array}$ & $\begin{array}{c}\text { Collection } \\
\text { Date }\end{array}$ & $\begin{array}{l}\text { Sample } \\
\text { Number }\end{array}$ & $\begin{array}{c}\text { FIRON } \\
34 / \mathrm{ppb} \\
20 / 300 \mathrm{~s}\end{array}$ & $\begin{array}{l}\text { LEAD } \\
40 / \mathrm{ppb} \\
5 / 50\end{array}$ & $\begin{array}{l}\text { FLEAD } \\
40 / p p b \\
5 / 50\end{array}$ & $\begin{array}{l}\text { MAGNES } \\
34 / \text { ppb } \\
100 \%\end{array}$ \\
\hline $699-42-401$ & $7 / 27 / 93$ & в08xp1 & …..... & 21.00 & ........ & 13000.00 \\
\hline $699-42-40 A$ & $7 / 27 / 93$ & B08XP5 & 52.00 & 21.00 & $.73 \mathrm{~L}$ & 15000.00 \\
\hline $699-42-41$ & $7 / 28 / 93$ & B08XP6 & & $1.30 \mathrm{~L}$ & & 5500.00 \\
\hline $699-42-41$ & $7 / 28 / 93$ & $B 08 \times 00$ & 36.00 & & $1.70 \mathrm{~L}$ & \\
\hline $699-42-42 B$ & $7 / 28 / 93$ & $808 \times 01$ & & $2.10 \mathrm{~L}$ & & 7300.00 \\
\hline $699-42-42 B$ & $7 / 28 / 93$ & $808 \times 05$ & 49.00 & & $1.30 \mathrm{~L}$ & \\
\hline $\begin{array}{l}699-43 \cdot 40 \\
699-43-40\end{array}$ & $\begin{array}{l}7 / 30 / 93 \\
7 / 30 / 93\end{array}$ & $\begin{array}{l}808 \times 06 \\
808 \times 07\end{array}$ & & $\begin{array}{l}.60 \mathrm{LQ} \\
.78 \mathrm{LO}\end{array}$ & & $\begin{array}{l}11000.00 \\
11000.00\end{array}$ \\
\hline $\begin{array}{l}699-43-40 \\
699-43-40\end{array}$ & $\begin{array}{l}7 / 30 / 93 \\
7 / 30 / 93\end{array}$ & $\begin{array}{l}808 \times R 4 \\
808 \times R 5\end{array}$ & $\begin{array}{r}100.00 \\
46.00\end{array}$ & & $\begin{array}{l}.60 \mathrm{~L} \\
.51 \mathrm{U}\end{array}$ & \\
\hline $699-43-41 E$ & $7 / 30 / 93$ & $808 \times R 6$ & & $.99 \mathrm{~L}$ & & 10000.00 \\
\hline $699-43-41 E$ & $7 / 30 / 93$ & $808 \times 50$ & 27.00 & & $1.50 \mathrm{~L}$ & \\
\hline $699-43-41 F$ & $7 / 28 / 93$ & B08XS1 & & $3.30 \mathrm{~L}$ & & 9800.00 \\
\hline $699-43-41 F$ & $7 / 28 / 93$ & B08XS5 & 81.00 & & $.92 \mathrm{~L}$ & \\
\hline $699-43-416$ & $7 / 30 / 93$ & B08XM6 & & $.62 \mathrm{~L}$ & & 9900.00 \\
\hline $699-43-416$ & $7 / 30 / 93$ & BO8XNO & 22.00 & & $.71 \mathrm{~L}$ & \\
\hline $699-43-42 J$ & $8 / 05 / 93$ & B08X56 & & $2.30 \mathrm{~L}$ & & 4900.00 \\
\hline $699-43-42 J$ & $8 / 05 / 93$ & B08X10 & $10.30 \mathrm{U}$ & & $.72 \mathrm{~L}$ & \\
\hline $699-43-43$ & $7 / 28 / 93$ & B08XT1 & & $1.30 \mathrm{~L}$ & & 7300.00 \\
\hline $699-43-43$ & $7 / 28 / 93$ & B08XT5 & 32.00 & & $.90 \mathrm{~L}$ & \\
\hline $699-43-45$ & $7 / 27 / 93$ & B08X16 & & $.85 \mathrm{~L}$ & & 6500.00 \\
\hline $699-43-45$ & $7 / 27 / 93$ & B08xvo & 31.00 & & $1.20 \mathrm{~L}$ & \\
\hline $699-44-39 B$ & $7 / 29 / 93$ & $808 \times \times 6$ & & $.51 \mathrm{U}$ & & 8200.00 \\
\hline $699-44-398$ & $7 / 29 / 93$ & B08XYO & 26.00 & & $.66 \mathrm{l}$ & \\
\hline $699-44-42$ & $7 / 28 / 93$ & B08XV1 & & $1.30 \mathrm{~L}$ & & 6500.00 \\
\hline $699-44 \cdot 42$ & $7 / 28 / 93$ & B08XV5 & 330.00 & & $.88 \mathrm{~L}$ & \\
\hline $699-44-43 B$ & $7 / 30 / 93$ & B08XW1 & & $2.30 \mathrm{~L}$ & & 9400.00 \\
\hline $699-44-43 B$ & $7 / 30 / 93$ & B08xw5 & 24.00 & & .60 & \\
\hline
\end{tabular}

\begin{tabular}{|c|c|c|c|c|c|c|}
\hline $\begin{array}{l}\text { Well } \\
\text { Name }\end{array}$ & $\begin{array}{c}\text { Collection } \\
\text { Date }\end{array}$ & $\begin{array}{l}\text { Sample } \\
\text { Number }\end{array}$ & $\begin{array}{l}\text { FMAGNES } \\
34 / \mathrm{ppb} \\
100 \%\end{array}$ & $\begin{array}{r}\text { MANGESE } \\
34 / \text { ppb } \\
10 / 50 \mathrm{~s}\end{array}$ & $\begin{array}{l}\text { FMANGAN } \\
34 / \text { ppb } \\
10 / 50 \mathrm{~s}\end{array}$ & $\begin{array}{c}\text { NICKEL } \\
34 / \mathrm{ppb} \\
30 \%\end{array}$ \\
\hline $299-E 32-4$ & $9 / 02 / 93$ & B08RK8 & $\cdots$ & $9.00 \mathrm{~L}$ & ............ & 43.00 \\
\hline $299-E 32-4$ & $9 / 02 / 93$ & BO8RK9 & 13000.00 & & $2.10 \mathrm{~L}$ & 45.00 \\
\hline $699-40 \cdot 36$ & $8 / 09 / 93$ & Bo90V1 & & 150.000 & & 43.00 \\
\hline $699-40-36$ & $8 / 09 / 93$ & B090V5 & 5000.00 & & 130.000 & \\
\hline $699-40-39$ & $8 / 05 / 93$ & B08XN1 & & 56.00 & & 63.00 \\
\hline $699-40-39$ & $8 / 05 / 93$ & B08XN5 & 8000.00 & & 34.00 & \\
\hline $699-40-40 A$ & $7 / 27 / 93$ & B08XK2 & & 190.00 & & 65.00 \\
\hline $699 \cdot 40.40 \mathrm{~A}$ & $7 / 27 / 93$ & B08XK6 & 7200.00 & & 140.00 & \\
\hline $699-40-40 B$ & $7 / 27 / 93$ & B08XK7 & & 200.00 & & 45.00 \\
\hline $699-40-40 B$ & $7 / 27 / 93$ & B08XL1 & 6000.00 & & 190.00 & \\
\hline $699-41 \cdot 35$ & $8 / 09 / 93$ & $8090 \mathrm{~V} 6$ & & 130.000 & & $23.00 \mathrm{~L}$ \\
\hline $699-41 \cdot 35$ & $8 / 09 / 93$ & Bo90wO & 6400.00 & & 160.000 & \\
\hline $699-41 \cdot 40$ & $8 / 05 / 93$ & $808 \times N 6$ & & 17.00 & & 66.00 \\
\hline $699-41 \cdot 40$ & $8 / 05 / 93$ & BOBXPO & 11000.00 & & $1.35 \mathrm{U}$ & \\
\hline $699-41-42$ & $7 / 28 / 93$ & $808 \times \times 1$ & & $7.90 \mathrm{~L}$ & & 110.00 \\
\hline $699-41 \cdot 42$ & $7 / 28 / 93$ & $808 \times \times 5$ & 6200.00 & & $1.35 \mathrm{U}$ & \\
\hline $699 \cdot 42 \cdot 37$ & $8 / 30 / 93$ & BO9OH1 & & 71.00 & & 35.00 \\
\hline $699-42-37$ & $8 / 30 / 93$ & B090W2 & & 65.00 & & 35.00 \\
\hline $699-42 \cdot 37$ & $8 / 30 / 93$ & B090W9 & 10000.00 & & 62.00 & \\
\hline $699 \cdot 42 \cdot 37$ & $8 / 30 / 93$ & B090X0 & 10000.00 & & 63.00 & \\
\hline
\end{tabular}


Table 6-4. Constituents with at Least One Value Above the CRQL for the 216-B-3 Pond Data for Reporting Period July 1 through September 30, 1993. (sheet 7 of 13)

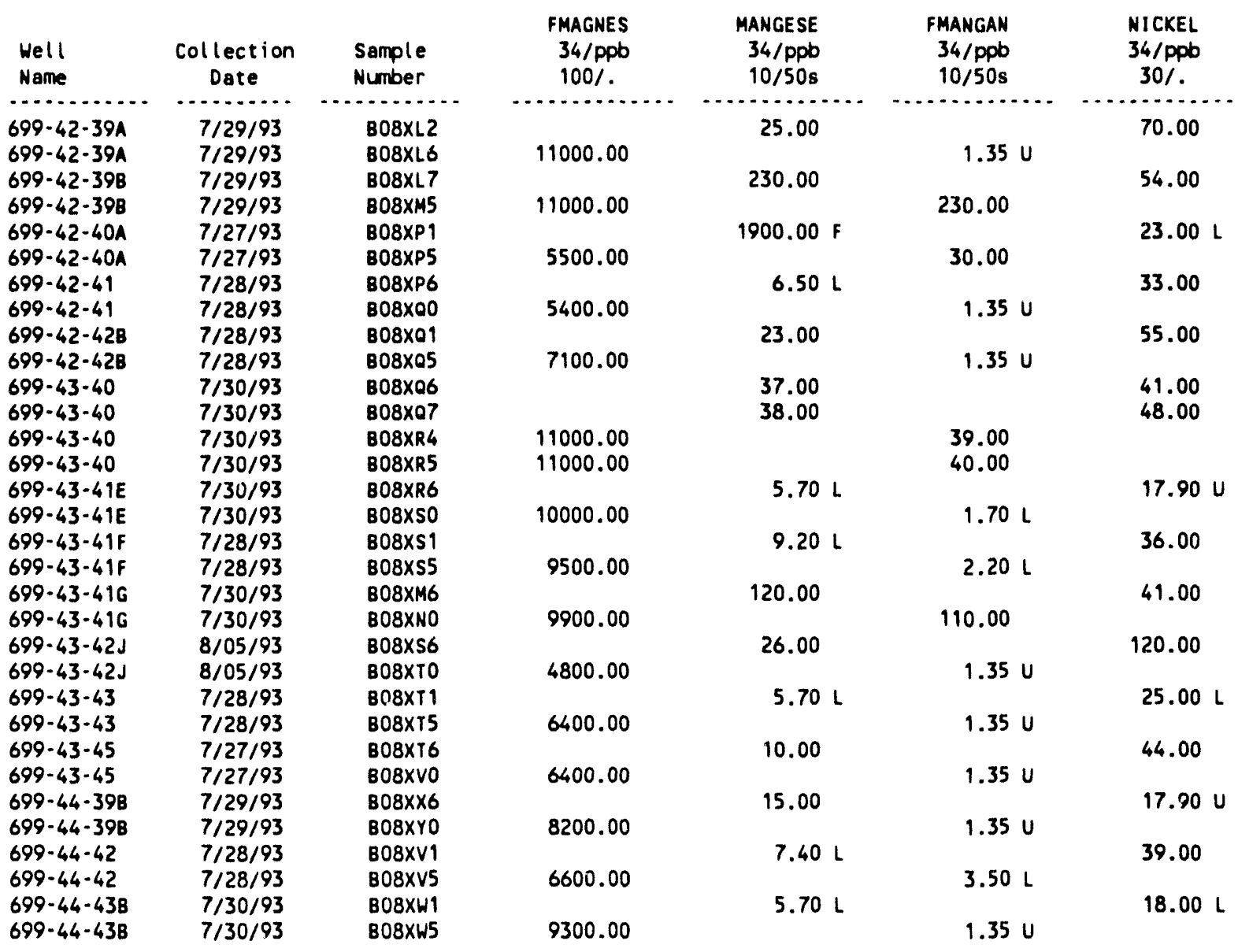

\begin{tabular}{|c|c|c|c|c|c|c|}
\hline $\begin{array}{l}\text { Well } \\
\text { Name }\end{array}$ & $\begin{array}{c}\text { Collection } \\
\text { Date }\end{array}$ & $\begin{array}{l}\text { Sample } \\
\text { Number }\end{array}$ & $\begin{array}{c}\text { FNICKEL } \\
34 / \mathrm{ppb} \\
30 \%\end{array}$ & $\begin{array}{l}\text { NITRATE } \\
124 / \mathrm{ppb} \\
200 / 45000\end{array}$ & $\begin{array}{l}\text { PHOSPHA } \\
124 / \text { ppb } \\
500 \%\end{array}$ & $\begin{array}{c}\text { POTASUM } \\
34 / \text { ppb } \\
1000 \%\end{array}$ \\
\hline$\cdots \cdots$ & $-\ldots$ & $\cdots \cdots$ & & 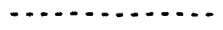 & & \\
\hline $\begin{array}{l}299-E 32-4 \\
299-E 32-4\end{array}$ & $9 / 02 / 93$ & $\begin{array}{l}\text { BO8RKB } \\
\text { B08RK9 }\end{array}$ & $17.90 \mathrm{U}$ & $25000.00 \mathrm{DH}$ & $147.00 \mathrm{UH}$ & 6000.00 \\
\hline $699-40-36$ & $8 / 09 / 93$ & Bogovi & & 200.00 Lo & $147.00 \mathrm{U}$ & 5800.00 \\
\hline $699-40-36$ & $8 / 09 / 93$ & $8090 \mathrm{~V} 5$ & $17.90 \mathrm{U}$ & & & \\
\hline $699-40 \cdot 39$ & $8 / 05 / 93$ & B08XN1 & & 700.00 & $147.00 \mathrm{U}$ & 4200.00 \\
\hline $699-40-39$ & $8 / 05 / 93$ & B08XN5 & $17.90 \mathrm{U}$ & & & \\
\hline $699 \cdot 40-40 \mathrm{~A}$ & $7 / 27 / 93$ & 808XK2 & & 3400.00 & $147.00 \mathrm{U}$ & 6700.000 \\
\hline $699-40-40 \mathrm{~A}$ & $7 / 27 / 93$ & B08XK6 & $17.90 \mathrm{U}$ & & & \\
\hline $699-40-40 B$ & $7 / 27 / 93$ & B08XK7 & & 800.00 & $147.00 \mathrm{U}$ & 5000.000 \\
\hline $699-40-408$ & $7 / 27 / 93$ & B08XL1 & $17.90 \mathrm{U}$ & & & \\
\hline $699-41-35$ & $8 / 09 / 93$ & B090V6 & & 200.000 & $147.00 \mathrm{U}$ & 7300.00 \\
\hline $699-41-35$ & $8 / 09 / 93$ & BO9OWO & $17.90 \mathrm{U}$ & & & \\
\hline $699-41-40$ & $8 / 05 / 93$ & BOBXNG & & 18000.00 & $147.00 \mathrm{U}$ & 4800.00 \\
\hline $699-41-40$ & $8 / 05 / 93$ & BO8XPO & $17.90 \mathrm{U}$ & & & \\
\hline $699-41-42$ & $7 / 28 / 93$ & B08XX1 & & 4000.00 & $147.00 \mathrm{U}$ & 6300.00 \\
\hline $699-41-42$ & $7 / 28 / 93$ & B08XX5 & $17.90 \mathrm{U}$ & & & \\
\hline
\end{tabular}


Table 6-4. Constituents with at Least One Value Above the CRQL for the 216-B-3 Pond Data for Reporting Period July 1 through September 30, 1993. (sheet 8 of 13)

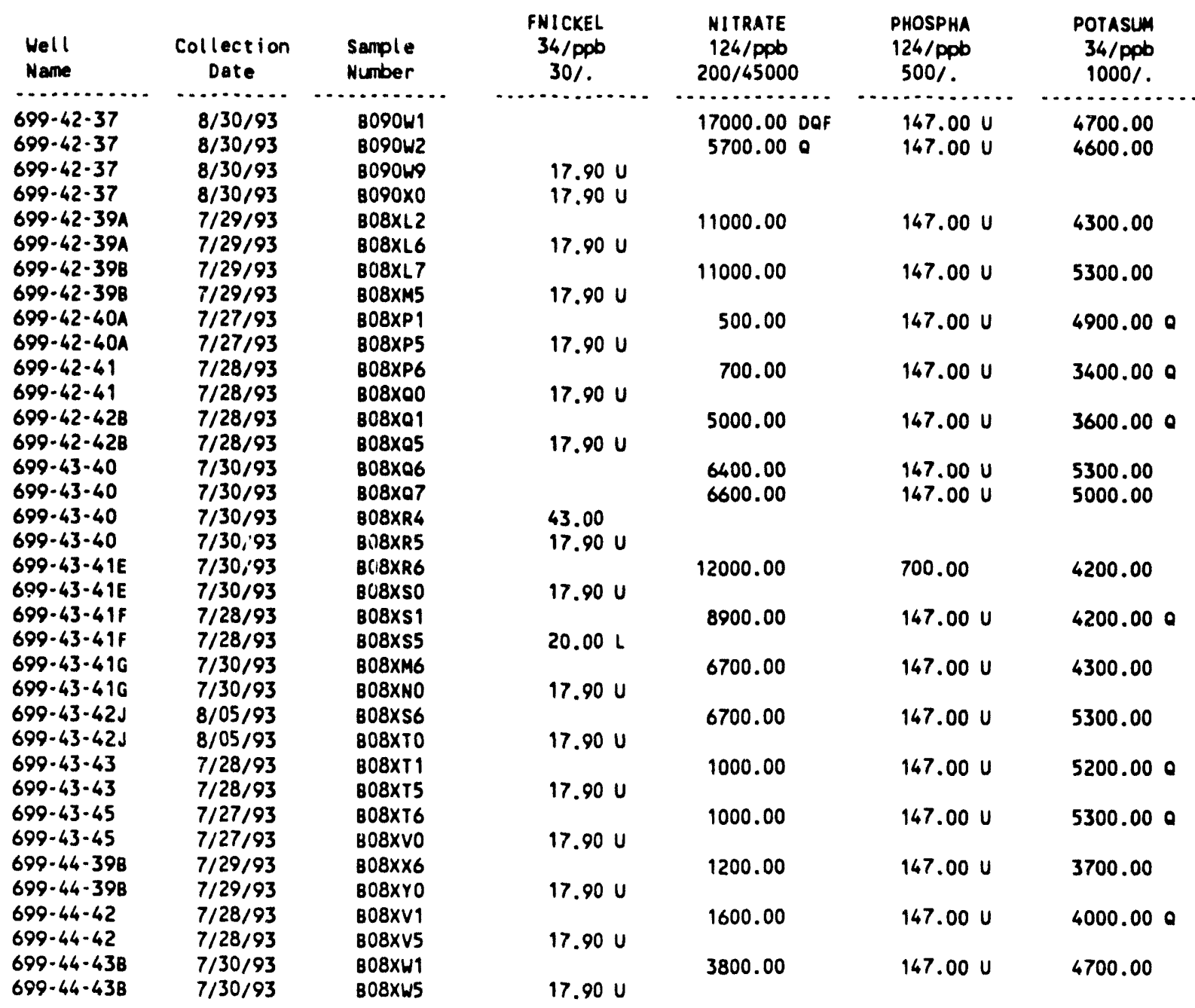

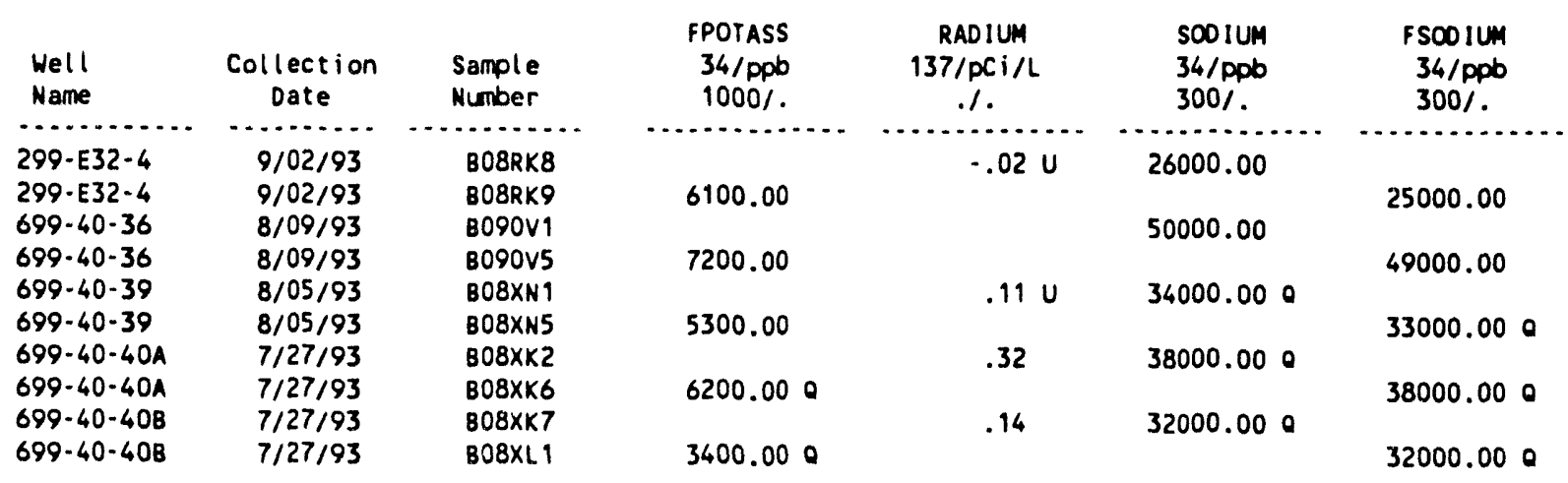


Table 6-4. Constituents with at Least One Value Above the CRQL for the 216-B-3 Pond Data for Reporting Period July 1

through September 30, 1993. (sheet 9 of 13)

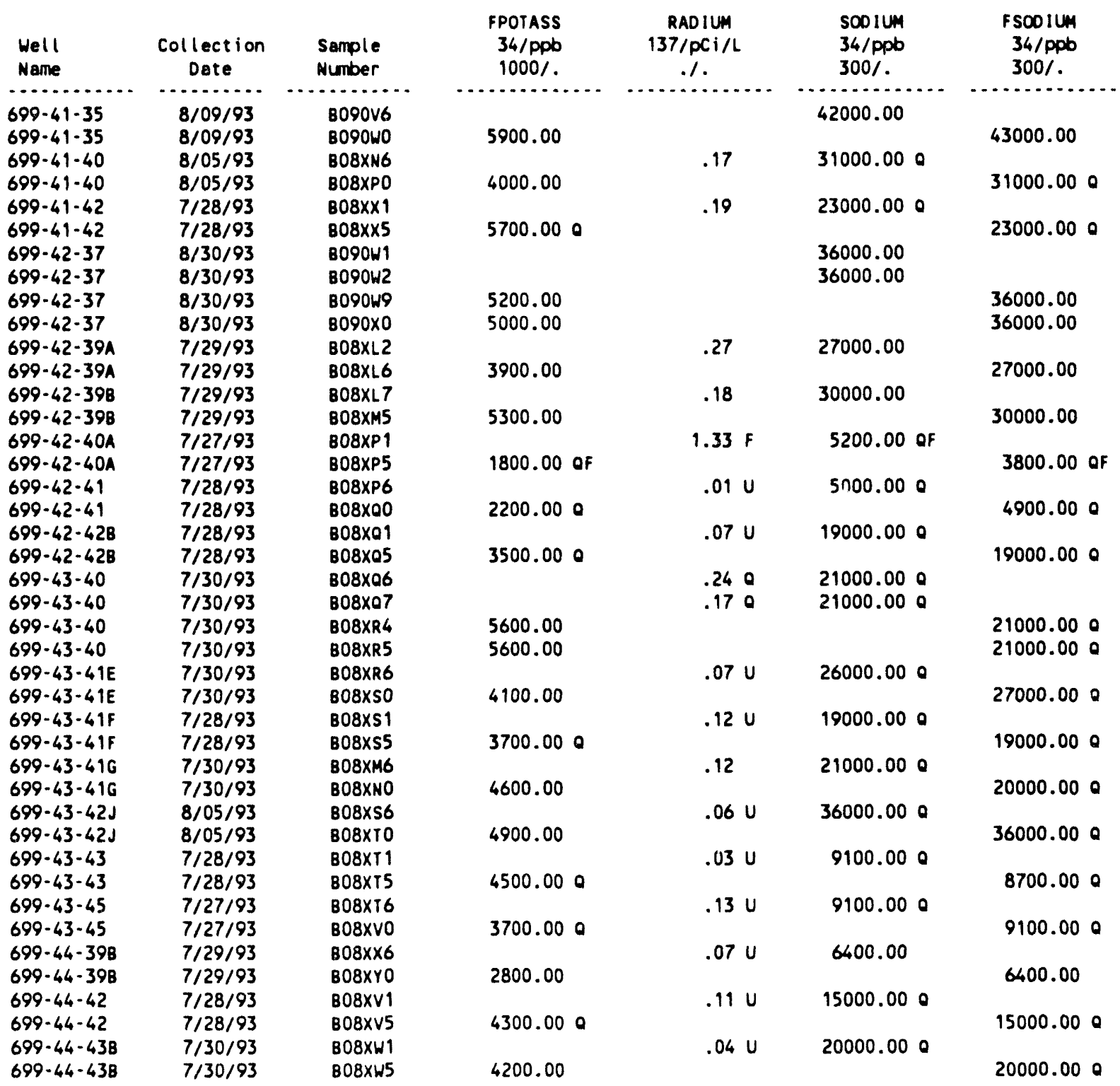

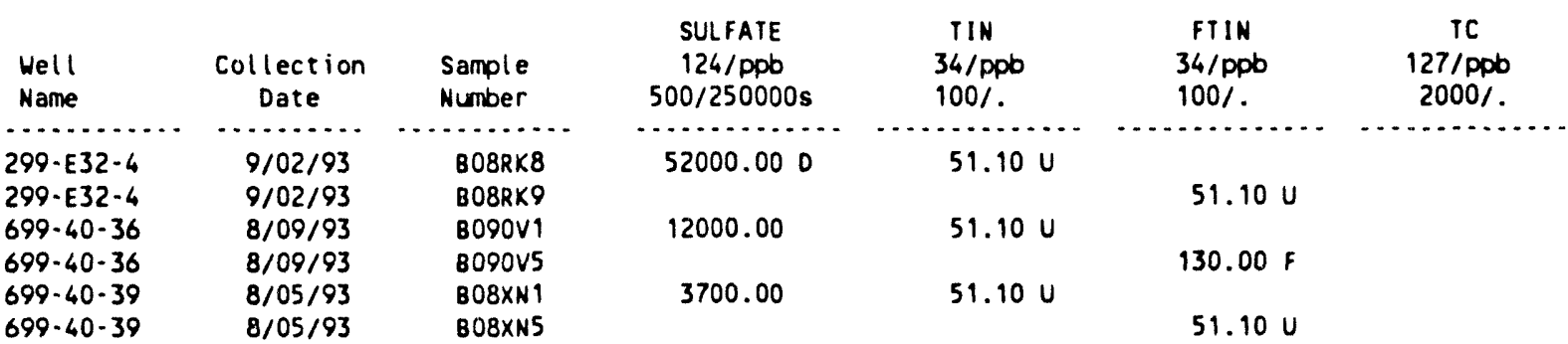


Table 6-4. Constituents with at Least One Value Above the CRQL for the 216-B-3 Pond Data for Reporting Period July 1 through September 30, 1993. (sheet 10 of 13)

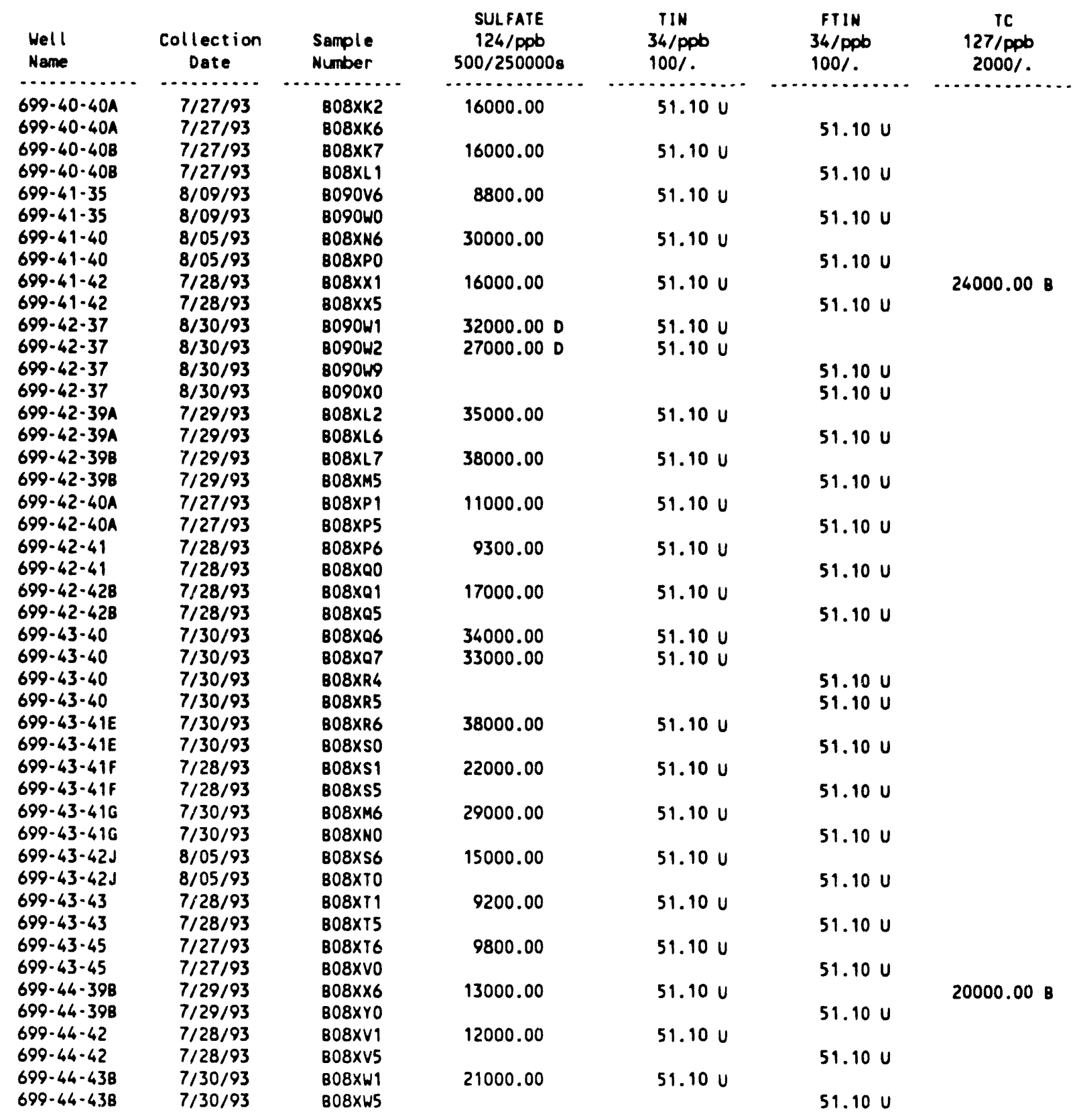


Table 6-4. Constituents with at Least One Value Above the CRQL for the 216-B-3 Pond Data for Reporting Period July 1 through September 30,1993 . (sheet 11 of 13)

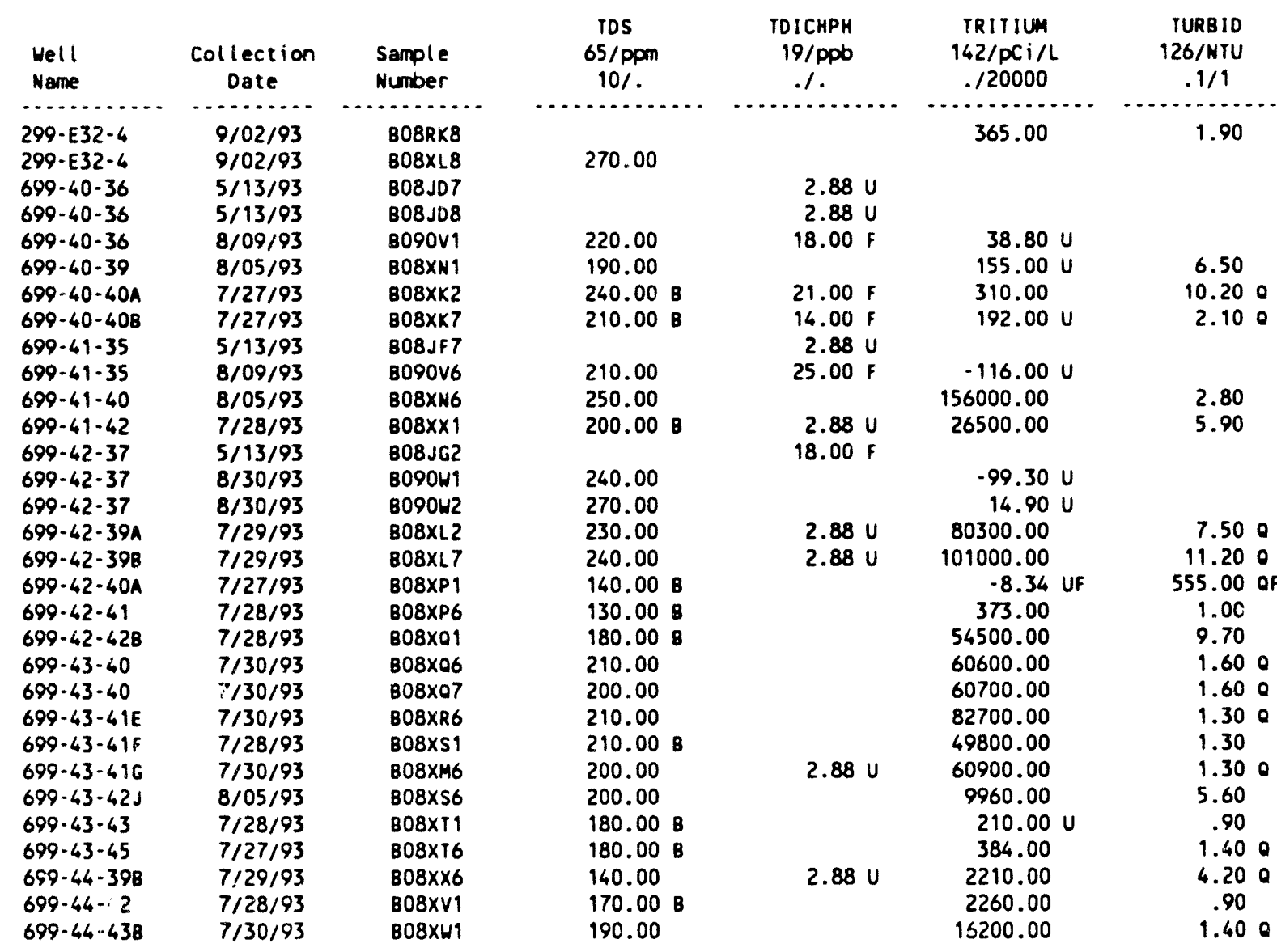

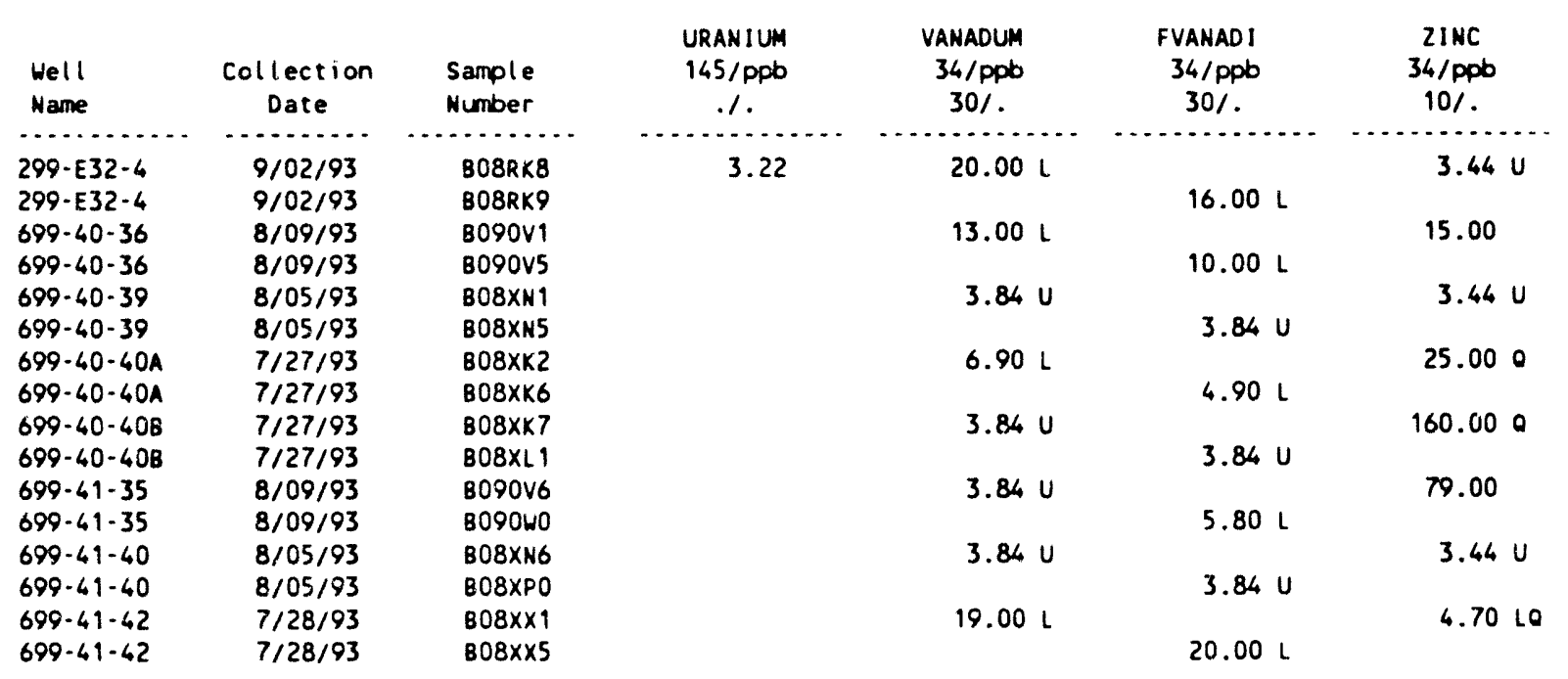


Table 6-4. Constituents with at Least One Value Above the CRQL for the 216-B-3 Pond Data for Reporting Period July 1 through September 30, 1993. (sheet 12 of 13)

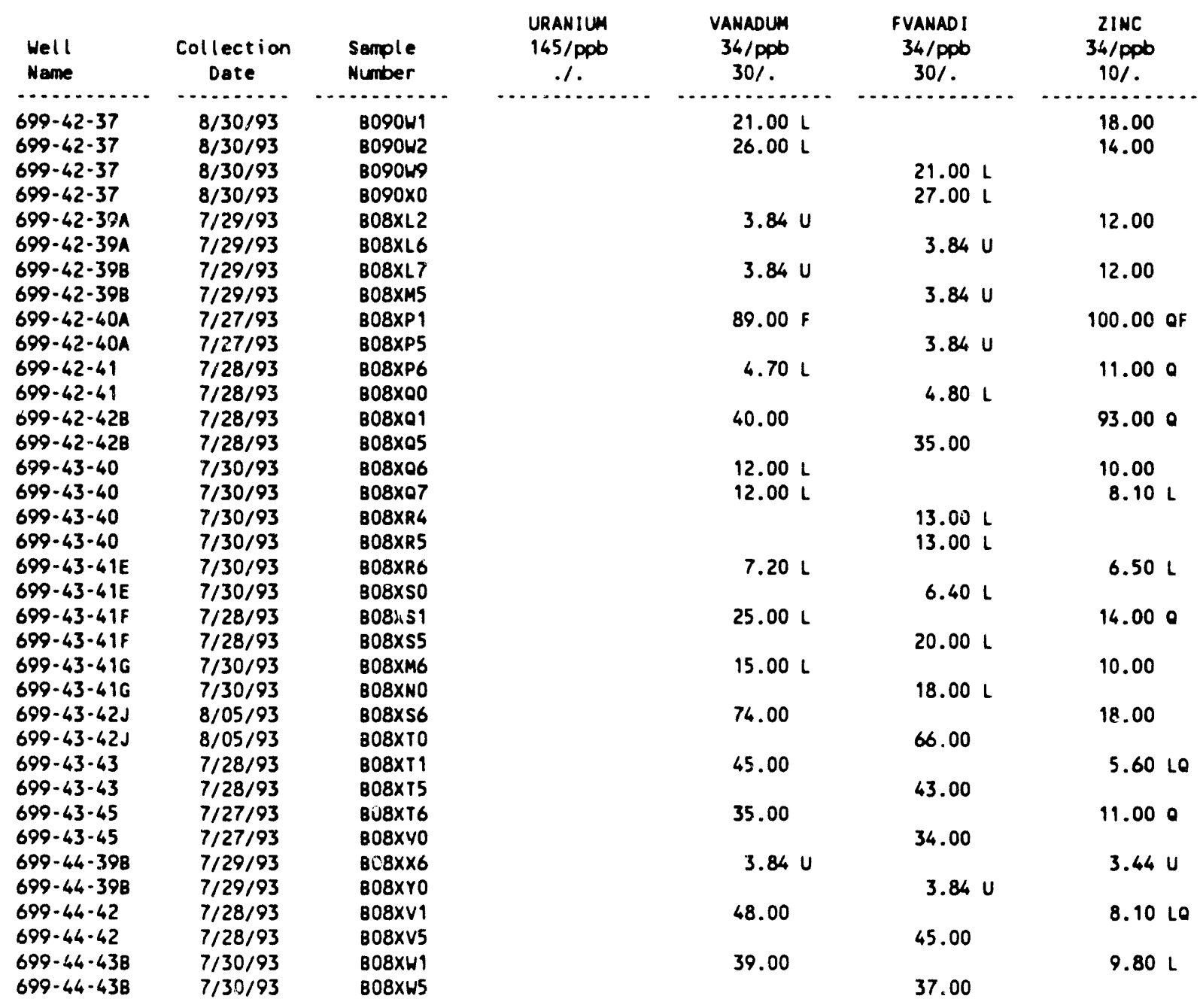

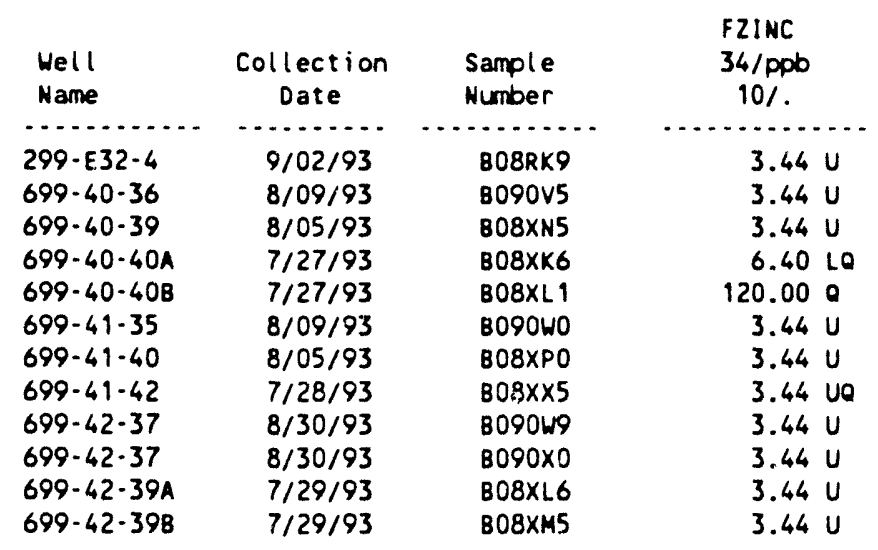


Table 6-4. Constituents with at Least One Value Above the CRQL for the 216-B-3 Pond Data for Reporting Period July 1 through September 30, 1993. (sheet 13 of 13)

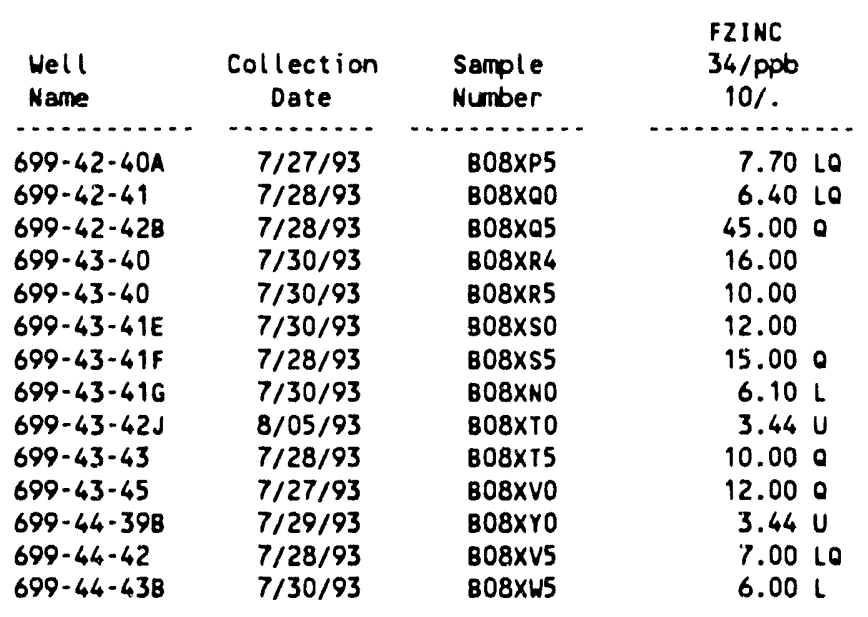

For explanation of this table, see Section 1.4 of report. 
Table 6-5. Contamination Indicator Parameters for the 216-B-3 Pond Data for Reporting Period July 1 through September 30, 1993. (sheet 1 of 2)

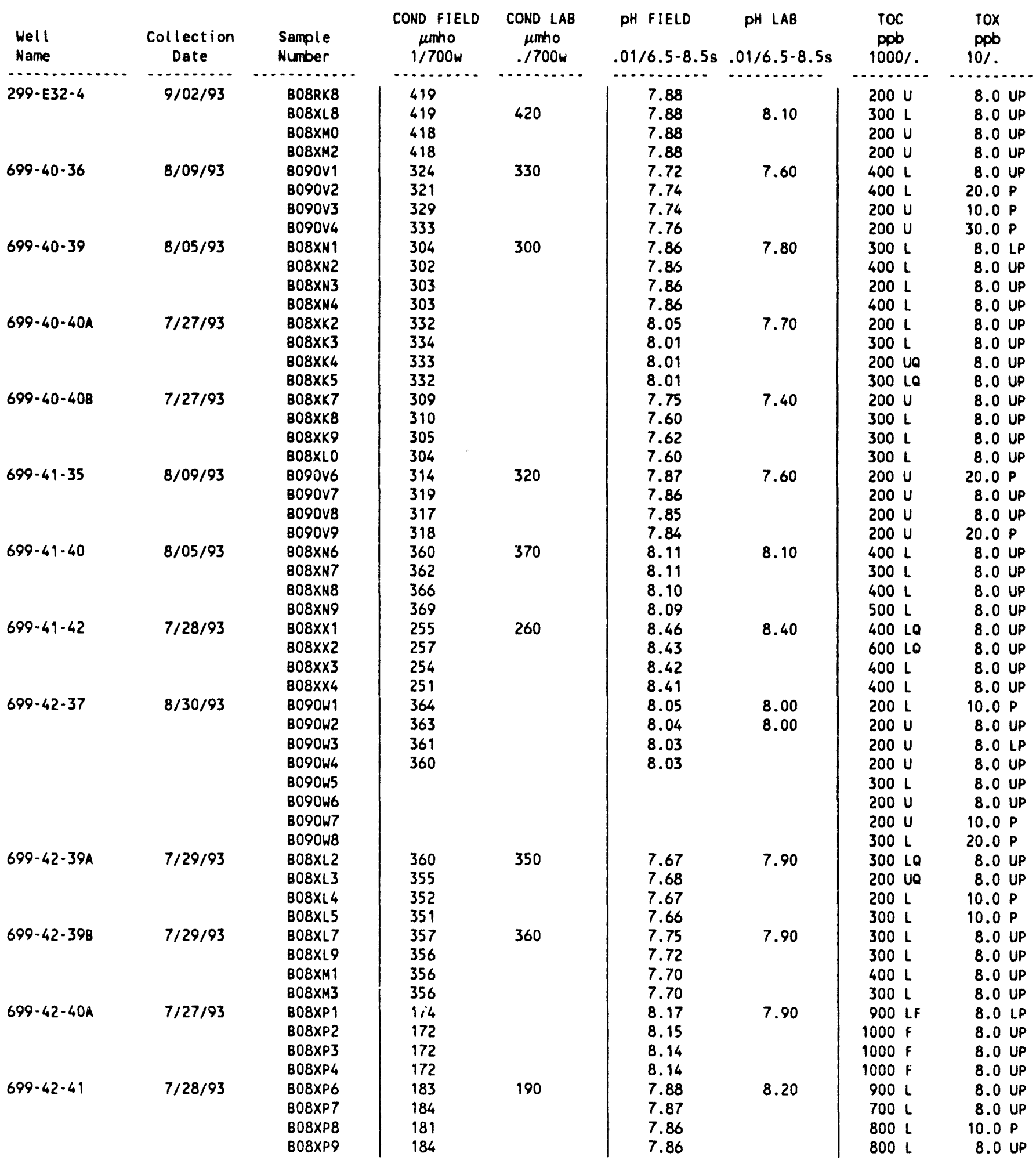


Table 6-5. Contamination Indicator Parameters for the 216-B-3 Pond Data for Reporting Period July 1 through September 30, 1993. (sheet 2 of 2)

\begin{tabular}{|c|c|c|c|c|c|c|c|c|c|}
\hline $\begin{array}{l}\text { Well } \\
\text { Name }\end{array}$ & $\begin{array}{c}\text { Collection } \\
\text { Date }\end{array}$ & $\begin{array}{l}\text { Sample } \\
\text { Number }\end{array}$ & $\begin{array}{c}\text { COND FIELD } \\
\mu \mathrm{mho} \\
1 / 700 \mathrm{~W}\end{array}$ & $\begin{array}{c}\text { COND LAB } \\
\mu \text { mho } \\
. / 700 \mathrm{w}\end{array}$ & $\begin{array}{l}\text { PH FIELD } \\
.01 / 6.5-8.5 \mathrm{~s}\end{array}$ & $\begin{array}{c}\text { PH LAB } \\
.01 / 6.5-8.5 \mathrm{~s}\end{array}$ & $\begin{array}{l}\text { TOC } \\
\text { ppb } \\
1000 \%\end{array}$ & $\begin{array}{c}10 x \\
p p b \\
10 \%\end{array}$ & \\
\hline $699-42-42 B$ & $7 / 28 / 93$ & $\begin{array}{l}808 \times 01 \\
808 \times 02 \\
808 \times 03 \\
808 \times 04\end{array}$ & $\begin{array}{l}260 \\
260 \\
262 \\
263\end{array}$ & 260 & $\begin{array}{l}7.91 \\
7.92 \\
7.91 \\
7.91\end{array}$ & 7.70 & $\begin{array}{l}300 \mathrm{~L} \\
300 \mathrm{~L} \\
400 \mathrm{~L} \\
300 \mathrm{~L}\end{array}$ & $\begin{array}{l}8.0 \\
8.0 \\
8.0 \\
8.0\end{array}$ & $\begin{array}{l}\text { UP } \\
\text { UP } \\
\text { UP } \\
\text { UP }\end{array}$ \\
\hline $699-43-40$ & $7 / 30 / 93$ & $\begin{array}{l}\text { B08X06 } \\
\text { B08X07 } \\
\text { B08X08 } \\
\text { B08X09 } \\
\text { B08XR0 } \\
\text { B08XR1 } \\
\text { B08XR2 } \\
\text { B08XR3 }\end{array}$ & $\begin{array}{l}290 \\
290 \\
291 \\
291\end{array}$ & $\begin{array}{l}310 \\
310\end{array}$ & $\begin{array}{l}8.07 \\
8.05 \\
8.03 \\
8.03\end{array}$ & $\begin{array}{l}8.10 \\
8.10\end{array}$ & $\begin{array}{l}500 \mathrm{~L} \\
500 \mathrm{~L} \\
400 \mathrm{~L} \\
400 \mathrm{~L} \\
400 \mathrm{~L} \\
500 \mathrm{~L} \\
400 \mathrm{~L} \\
400 \mathrm{~L}\end{array}$ & $\begin{array}{l}8.0 \\
8.0 \\
8.0 \\
8.0 \\
8.0 \\
8.0 \\
8.0 \\
8.0\end{array}$ & $\begin{array}{l}\text { UP } \\
\text { UP } \\
\text { UP } \\
\text { UP } \\
\text { UP } \\
\text { UP } \\
\text { UP } \\
\text { UP }\end{array}$ \\
\hline $699-43-41 E$ & $7 / 30 / 93$ & $\begin{array}{l}\text { B08XR6 } \\
\text { B08XR7 } \\
\text { B08XR8 } \\
\text { B08XR9 }\end{array}$ & $\begin{array}{l}318 \\
313 \\
316 \\
317\end{array}$ & 330 & $\begin{array}{l}8.47 \\
8.45 \\
8.43 \\
8.43\end{array}$ & 8.40 & $\begin{array}{l}500 \mathrm{~L} \\
400 \mathrm{~L} \\
400 \mathrm{~L} \\
500 \mathrm{~L}\end{array}$ & $\begin{array}{r}8.0 \\
10.0 \\
10.0 \\
10.0\end{array}$ & $\begin{array}{l}U P \\
P \\
P \\
P\end{array}$ \\
\hline $699-43-41 F$ & $7 / 28 / 93$ & $\begin{array}{l}\text { B08XS1 } \\
\text { B08X52 } \\
\text { B08XS3 } \\
\text { B08XS4 }\end{array}$ & $\begin{array}{l}300 \\
301 \\
303 \\
302\end{array}$ & 290 & $\begin{array}{l}7.96 \\
7.95 \\
7.95 \\
7.95\end{array}$ & 7.70 & $\begin{array}{l}300 L \\
300 L \\
300 L \\
300 L\end{array}$ & $\begin{array}{l}8.0 \\
8.0 \\
8.0 \\
8.0\end{array}$ & $\begin{array}{l}\text { UP } \\
\text { UP } \\
\text { UP } \\
\text { UP }\end{array}$ \\
\hline $699-43-416$ & $7 / 30 / 93$ & $\begin{array}{l}\text { B08XM6 } \\
\text { B08XM7 } \\
\text { B08XM8 } \\
\text { B08XM9 }\end{array}$ & $\begin{array}{l}286 \\
287 \\
286 \\
285\end{array}$ & 290 & $\begin{array}{l}8.37 \\
8.36 \\
8.36 \\
8.35\end{array}$ & 8.10 & $\begin{array}{l}600 L \\
300 L \\
300 L \\
300 L\end{array}$ & $\begin{array}{r}8.0 \\
8.0 \\
8.0 \\
20.0\end{array}$ & $\begin{array}{l}\text { UP } \\
\text { UP } \\
\text { UP }\end{array}$ \\
\hline $699-43-42 \mathrm{~J}$ & $8 / 05 / 93$ & $\begin{array}{l}B 08 \times 56 \\
B 08 \times 57 \\
B 08 \times 58 \\
B 08 \times 59\end{array}$ & $\begin{array}{l}284 \\
283 \\
286 \\
286\end{array}$ & 290 & $\begin{array}{l}8.23 \\
8.23 \\
8.24 \\
8.24\end{array}$ & 8.20 & $\begin{array}{l}400 \mathrm{~L} \\
300 \mathrm{~L} \\
400 \mathrm{~L} \\
300 \mathrm{~L}\end{array}$ & $\begin{array}{l}8.0 \\
8.0 \\
8.0 \\
8.0\end{array}$ & $\begin{array}{l}\text { UP } \\
\text { UP } \\
\text { LP } \\
\text { UP }\end{array}$ \\
\hline $699-43-43$ & $7 / 28 / 93$ & $\begin{array}{l}\text { B08XT1 } \\
\text { B08XT2 } \\
\text { B08XT3 } \\
808 \times T 4\end{array}$ & $\begin{array}{l}240 \\
242 \\
243 \\
242\end{array}$ & 240 & $\begin{array}{l}8.07 \\
8.07 \\
8.07 \\
8.07\end{array}$ & 8.20 & $\begin{array}{l}500 \mathrm{~L} \\
500 \mathrm{~L} \\
500 \mathrm{~L} \\
600 \mathrm{~L}\end{array}$ & $\begin{array}{l}8.0 \\
8.0 \\
8.0 \\
8.0\end{array}$ & $\begin{array}{l}\text { UP } \\
\text { UP } \\
\text { UP } \\
\text { UP }\end{array}$ \\
\hline $699-43-45$ & $7 / 27 / 93$ & $\begin{array}{l}\text { B08X16 } \\
\text { B08X17 } \\
\text { B08 } 518 \\
808 \times 19\end{array}$ & $\begin{array}{l}233 \\
234 \\
234 \\
235\end{array}$ & & $\begin{array}{l}8.01 \\
8.00 \\
8.00 \\
8.01\end{array}$ & 7.90 & $\begin{array}{l}400 \mathrm{~L} \\
400 \mathrm{~L} \\
400 \mathrm{~L} \\
500 \mathrm{~L}\end{array}$ & $\begin{array}{l}8.0 \\
8.0 \\
8.0 \\
8.0\end{array}$ & $\begin{array}{l}\text { UP } \\
\text { UP } \\
\text { UP } \\
\text { UP }\end{array}$ \\
\hline $699-44-398$ & $7 / 29 / 93$ & $\begin{array}{l}808 \times \times 6 \\
808 \times \times 7 \\
808 \times \times 8 \\
808 \times \times 9\end{array}$ & $\begin{array}{l}200 \\
200 \\
198 \\
197\end{array}$ & 200 & $\begin{array}{l}7.88 \\
7.89 \\
7.88 \\
7.90\end{array}$ & 8.00 & $\begin{array}{l}600 L \\
500 L \\
500 L \\
500 L\end{array}$ & $\begin{array}{r}8.0 \\
8.0 \\
30.0 \\
8.0\end{array}$ & $\begin{array}{l}\text { UP } \\
\text { UP } \\
\text { UP }\end{array}$ \\
\hline $699-44-42$ & $7 / 28 / 93$ & $\begin{array}{l}\text { B08XV1 } \\
\text { B08XV2 } \\
\text { B08XV3 } \\
\text { B08XV4 }\end{array}$ & $\begin{array}{l}253 \\
250 \\
249 \\
251\end{array}$ & 240 & $\begin{array}{l}8.18 \\
8.18 \\
8.19 \\
8.17\end{array}$ & 8.20 & $\begin{array}{l}500 \mathrm{~L} \\
600 \mathrm{~L} \\
400 \mathrm{~L} \\
500 \mathrm{~L}\end{array}$ & $\begin{array}{r}8.0 \\
8.0 \\
8.0 \\
10.0\end{array}$ & $\begin{array}{l}\text { UP } \\
\text { UP } \\
\text { UP }\end{array}$ \\
\hline $699-44-438$ & $7 / 30 / 93$ & $\begin{array}{l}\text { B08XW1 } \\
\text { B08XW2 } \\
\text { B08XW3 } \\
\text { B08XW4 }\end{array}$ & $\begin{array}{l}276 \\
277 \\
277 \\
277\end{array}$ & 280 & $\begin{array}{l}7.80 \\
7.80 \\
7.80 \\
7.80\end{array}$ & 8.30 & $\begin{array}{l}300 \mathrm{~L} \\
400 \mathrm{~L} \\
400 \mathrm{~L} \\
400 \mathrm{~L}\end{array}$ & $\begin{array}{l}8.0 \\
8.0 \\
8.0 \\
8.0\end{array}$ & $\begin{array}{l}0 \text { UP } \\
0 \text { UP } \\
0 \text { UP } \\
0 \text { UP }\end{array}$ \\
\hline
\end{tabular}

For explanation of this table, see section 1.4 of report. 
DOE/RL-93-56-3

This page intentionally left blank. 
$\mathrm{DOE} / \mathrm{RL}-93-56-3$

CONTENTS

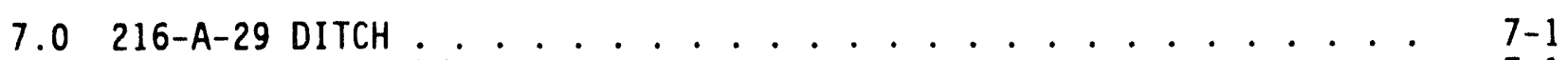

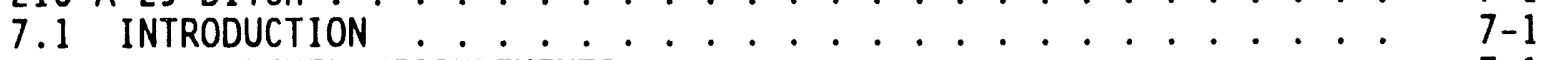

7.2 WATER LEVEL MEASUREMENTS . . . . . . . . . . . . . . . . . . 7-1

7.3 WATER CHEMISTRY DATA . . . . . . . . . . . . . . . . 7-2 
$\mathrm{DOE} / \mathrm{RL}-93-56-3$

LIST OF FIGURES

7-1 Monitoring Well Locations for the 216-A-29 Ditch . . . . . . . . . 7-4

\section{LIST OF TABLES}

7-1 Monitoring Well Purpose and Sampling Schedule for the 216-A-29 Ditch.................... . . 7-5

7-2 RCRA Water Level Measurement Report 216-A-29 Ditch,

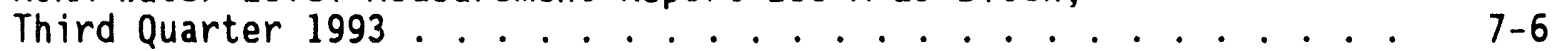

7-3 Constituent List and Summary of Results for the 216-A-29 Ditch Data for Reporting Period July 1 through September 30,1993 . . . 7-9

7-4 Constituents with at Least One Value Above the CRQL for the 216-A-29 Ditch Data for Reporting Period July 1 through

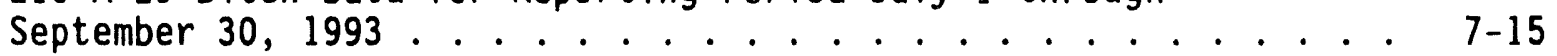

7-5 Contamination Indicator Parameters for the 216-A-29 Ditch Data for Reporting Period July 1 through September 30, $1993 .$. . . . 7-23 


\title{
$7.0 \quad 216-A-29$ DITCH
}

\author{
G. L. Kasza \\ Westinghouse Hanford Company
}

\subsection{INTRODUCTION}

The 216-A-29 Ditch (A-29 Ditch) is located east of the 200 East Area in the central portion of the Hanford Site. From 1955 to July 1991, this excavated, unlined ditch received the effluent from the Plutonium-Uranium Extraction (PUREX) Plant Chemical Sewer Line and conducted it to the 216-B-3-3 Ditch and the B Pond System. After July 15, 1991, the flow to the A-29 Ditch was rerouted to the PUREX Cooling Water Line, and the site of the ditch was backfilled, graded, and stabilized as part the Hanford Federal Facility Agreement and Consent Order (Ecology et al. 1992), Milestone M-17-10. Because the A-29 Ditch will not receive additional hazardous substances, the DOE proposed that the ditch be permanently closed under RCRA interim status (WAC 173-303-400; 40 CFR 265 Subpart G; and DOE 1987).

Groundwater beneath the A-29 Ditch has been monitored by a RCRA groundwater monitoring network since 1988 because of earlier discharges of caustic and potentially hazardous chemicals to the ditch. The A-29 Ditch is currently in a groundwater quality assessment monitoring program because of elevated specific conductance levels in one monitoring well (299-E25-35) (Chou et a1. 1990). The groundwater monitoring activities for the A-29 Ditch also are directed by the Groundwater Monitoring Plan for the 216-A-29 Ditch (Kasza and Goodwin 1991).

The RCRA groundwater monitoring network consists of 3 upgradient and 10 downgradient wells. Nine additional wells in the surrounding area are used to provide supplementary data for the groundwater quality assessment investigation. Figure 7-1 shows the locations of the wells used to monitor groundwater quality for the A-29 Ditch. Table 7-1 presents pertinent data about these wells.

\subsection{WATER LEVEL MEASUREMENTS}

Water levels are measured monthly in the 13 wells comprising the A-29 Ditch monitoring network, and are measured quarterly in the 9 additional wells that provide supplemental analytical data for the groundwater quality assessment investigation. Water level measurements are also recorded whenever the wells are sampled.

Water level measurements obtained during this quarter are presented in Table 7-2. Several inconsistencies were noted when recent data were compared to the water levels previously measured. Water levels reported for well 299-E25-32P on September 3, 1993, well 299-E25-35 for the October and November 1993 measurements, well 299-E25-48 on July 27, 1993, and well 299-E26-12 on July 22, 1993, fell outside of the historic ranges. 
Inspection of the field report sheets does not reveal any data transcription or retrieval errors. An attempt will be made to reconcile these suspected errors after additional data are received from future measurements. These data are flagged in Table 7-2.

\subsection{WATER CHEMISTRY DATA}

Groundwater beneath the A-29 Ditch is sampled quarterly in accordance with the groundwater quality assessment plan (Chou et a1. 1990). The constituent list and summary of results are provided in Table 7-3. Reported analytical data are included in Tables 7-4 and 7-5. Table 7-4 provides a list of the wells and analytical results for chemical constituents with at least one value above CRQL. In many cases, the detected values are from the wells that are monitored to provide comparative regional data for the groundwater quality assessment investigation. Results of analyses for CIPs are reported in Table 7-5.

Constituents reported in concentrations that exceed the DWS for the A-29 Ditch detection network wells include unfiltered chromium in we11s 299-E25-32P, 299-E25-34, 299-E25-35, 299-E25-42, and 299-E25-43; unfiltered iron in wells 299-E25-32P, 299-E25-34, 299-E25-35, 299-E25-42, 299-E25-43, 299-E25-47, 299-E25-48, 299-E26-12, and 699-43-45; manganese in well 299-E25-48; tritium in wells 299-E25-35 and 299-E25-42; and turbidity in wells 299-E25-32P, 299-E25-34, 299-E25-35, 299-E25-42, 299-E25-43, 299-E25-47, 299-E25-48, 299-E26-12, and 699-43-45.

Constituents reported in concentrations that exceed the DWS at the wells that provide regional data for the A-29 Ditch groundwater quality assessment investigation include unfiltered chromium in well 299-E25-31; unfiltered iron in wells 299-E25-19, 299-E25-20, 299-E25-21, and 299-E25-31; nitrate in wells 299-E25-19 and 299-E25-20; tritium in wells 299-E25-11, 299-E25-18, 299-E25-19, and 299-E25-20; and turbidity in wells 299-E25-11, 299-E25-18, 299-E25-19, 299-E25-20, 299-E25-21, 299-E25-31, and 299-E25-36.

The increase in the number of wells where the turbidity in the groundwater exceeds the DWS has been recognized in several areas onsite. An effort to determine the causes of this increase is planned for a future report period.

Field-measured $\mathrm{pH}$ at wells 299-E25-26 and 299-E25-21 fell outside the acceptable range of the DWS on August 2, 1993. Review of the field records for the sampling activity indicates no data transcription errors. The recorded value in both cases is outside the historic trend for the wells. Resampling of well 299-E25-26 has been requested to determine the validity of the measurement and a RADE has been prepared. Because well 299-E25-21 is an assessment investigation well, no resampling has been requested.

Field-measured specific conductance at well 299-E25-35 remains below the critical mean value $(455.3 \mu$ mhos $/ \mathrm{cm})$ established when groundwater quality assessment monitoring was initiated in 1990 due to high specific conductance at this well. Laboratory measurement of specific conductivity confirms the 
field measurement. Efforts are currently in progress to revise the Groundwater Quality Assessment Plan to accommodate this improvement in groundwater quality.

Suspect data are flagged in Tables 7-4 and 7-5 according to the explanation found in Section 1.4.

Analytical data that are unavailable at time of preparation of this report will be included in a future quarterly report. 
DOE/RL-93-56-3

Figure 7-1. Monitoring Well Locations for the 216-A-29 Ditch.

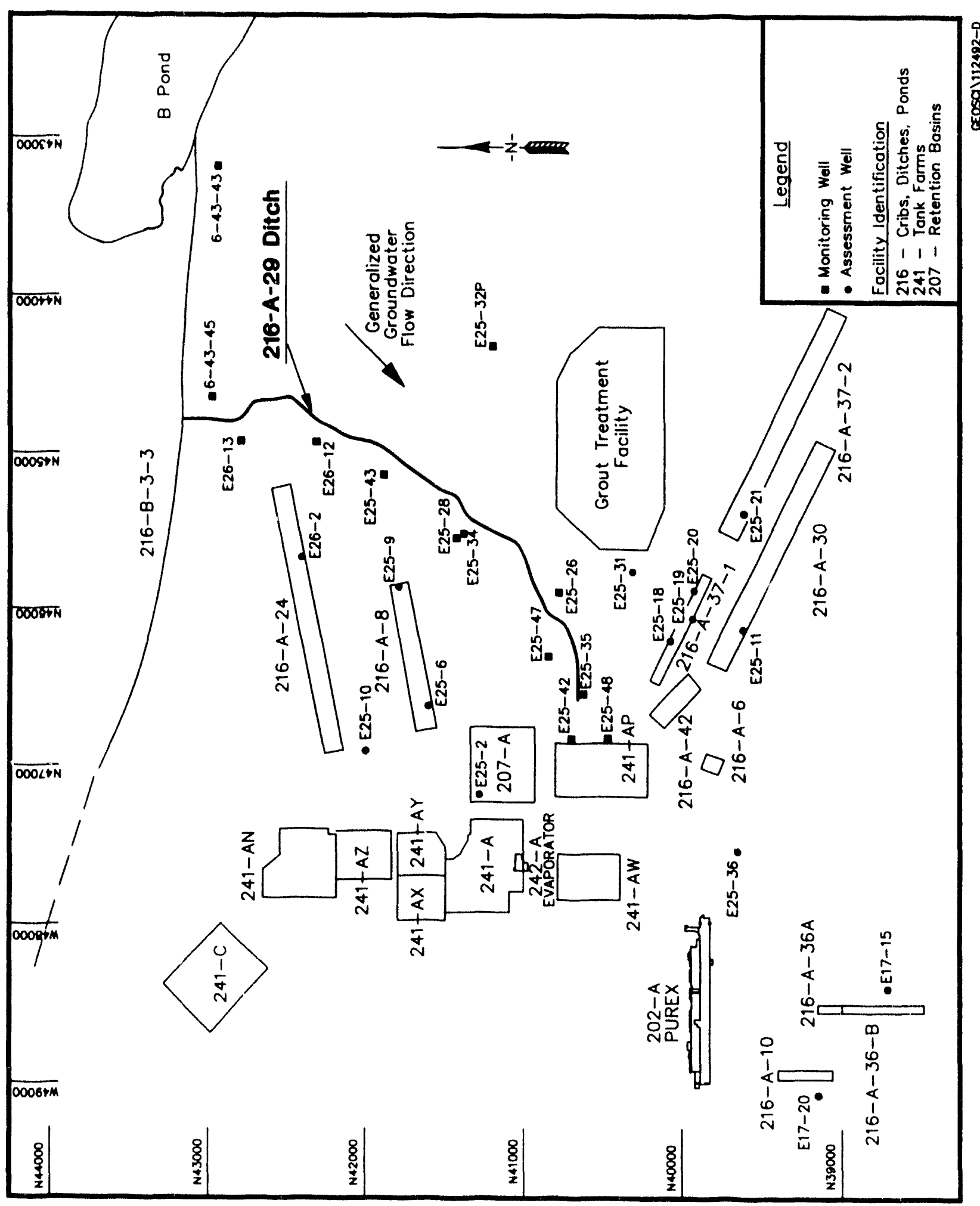


Table 7-1. Monitoring Well Purpose and Sampling Schedule for the 216-A-29 Ditch.

\begin{tabular}{|c|c|c|c|c|}
\hline Well no. & $\begin{array}{l}\text { Relative } \\
\text { position }\end{array}$ & Hydrogeologic unit & $\begin{array}{c}\text { Sample } \\
\text { frequency }\end{array}$ & $\begin{array}{l}\text { Sample date, } \\
\text { 3rd Qtr } 1993\end{array}$ \\
\hline $699-43-43^{b}$ & Upgradient & Hanford: Water Table & Quarterly & $7 / 28 / 93$ \\
\hline $699-43-45^{b}$ & Upgradient & Hanford: Water Table & Quarterly & $7 / 27 / 93$ \\
\hline $299-E 25-32 P^{C}$ & Upgradient & Hanford: Water Table & Quarterly & $7 / 15 / 93$ \\
\hline $299-E 25-26$ & Downgradient & Hanford: Mid-Depth & Quarterly & $8 / 02 / 93$ \\
\hline $299-E 25-28$ & Downgradient & Ringold: Deep & Quarterly & $8 / 02 / 93$ \\
\hline 299-E25-34 & Downgradient & Hanford: Water Table & Quarterly & $8 / 04 / 93$ \\
\hline 299-E25-35 & Dow & Hanford: Water Table & Quarterly & $8 / 04 / 93$ \\
\hline $299-E 25-42$ & Downgradient & Hanford: Water Table & Quarterly & $7 / 16 / 93$ \\
\hline $299-E 25-43$ & Downgr & Hanford: Water Table & Quarterly & $7 / 15 / 93$ \\
\hline 299-E25-47 & Downgradient & Hanford: Water Table & Quarterly & $7 / 16 / 93$ \\
\hline 299-E25-48 & Downgra & Hanford: Water Table & terly & $7 / 27 / 93$ \\
\hline 299-E26-12 & Downgradient & Hanford: Water Table & Quarterly & $7 / 22 / 93$ \\
\hline 299-E26-13 & Downgradient & Hanford: Water Table & rly & $7 / 23 / 93$ \\
\hline $299-E 17-15^{d}$ & Asses sment & Ringold: Water Table & Quarterly & 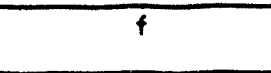 \\
\hline $299-E 17-20^{\circ}$ & As ses sment & Ringold: Water Table & Quarterly & 7 \\
\hline $299-E 25-11$ & As se s sment & $\begin{array}{l}\text { Undifferentiated } \\
\text { sediments: Mid-Depth }\end{array}$ & Quarterly & $8 / 03 / 93$ \\
\hline $299-E 25-18$ & Asse & $\begin{array}{l}\text { Undifferentiated } \\
\text { sediments: Mid-Depth }\end{array}$ & Quarterly & $8 / 03 / 93$ \\
\hline 299-E25-19 & Asses sment & $\begin{array}{l}\text { Undifferentiated } \\
\text { sediments: Mid-Depth }\end{array}$ & Quarterly & $8 / 03 / 93$ \\
\hline $299-E 25-20$ & Assessment & $\begin{array}{l}\text { Undifferentiated } \\
\text { sediments: Mid-Depth }\end{array}$ & Quarterly & $7 / 15 / 93$ \\
\hline 299-E25-21 & Assessment & Hanford: Water Table & Quarterly & $8 / 02 / 93$ \\
\hline 299-E25-31 & Assessment & Hanford: Water Table & Quarterly & $7 / 16 / 93$ \\
\hline $299-E 25-36^{\circ}$ & Assessment & Ringold: Water Table & Quarterly & $f$ \\
\hline
\end{tabular}

"Hydrogeologic units include the sandy gravels of the Hanford formation and the silty sands of the Ringold Formation.

Wells shared with the 216-B-3 Pond monitoring network.

"Wells shared with the Grout Treatment Facility monitoring network.

Wells shared with the 216-A-36B monitoring network.

"Wells shared with the 216-A-10 monitoring network.

these wells were sampled twice in the previous report period. 
Table 7-2. RCRA Water Level Measurement Report 216-A-29 Ditch, Third Quarter 1993. (sheet 1 of 3)

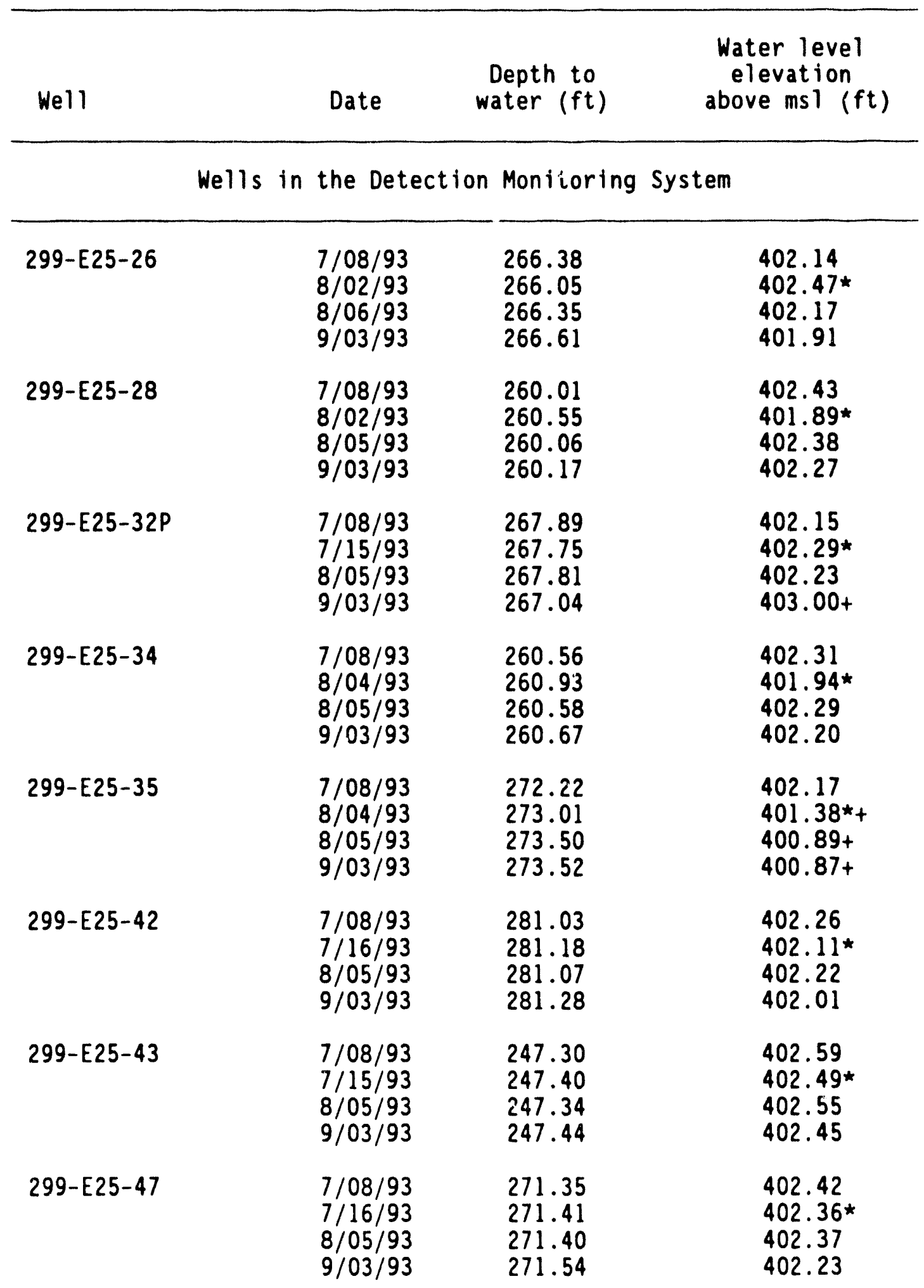


Table 7-2. RCRA Water Level Measurement Report 216-A-29 Ditch, Third Quarter 1993. (sheet 2 of 3)

Well Date $\quad \begin{gathered}\text { Depth to } \\ \text { water ( } \mathrm{ft})\end{gathered} \quad \begin{gathered}\text { Water level } \\ \text { elevation } \\ \text { above msl (ft) }\end{gathered}$

Wells in the Detection Monitoring System

\begin{tabular}{llll}
\hline $299-E 25-48$ & $7 / 08 / 93$ & 279.92 & 402.39 \\
& $7 / 27 / 93$ & 280.79 & $401.52^{\star}+$ \\
& $8 / 05 / 93$ & 279.94 & 402.37 \\
& $9 / 03 / 93$ & 280.14 & 402.17 \\
$299-E 26-12$ & $7 / 08 / 93$ & 227.98 & 402.76 \\
& $7 / 22 / 93$ & 227.31 & $403.43^{\star}+$ \\
& $8 / 05 / 93$ & 227.99 & 402.75 \\
& $9 / 03 / 93$ & 228.09 & 402.65 \\
& $7 / 08 / 93$ & 202.33 & 402.69 \\
& $7 / 23 / 93$ & 202.37 & $402.65 \star$ \\
$699-E 26-13$ & $8 / 05 / 93$ & 202.35 & 402.67 \\
& $9 / 03 / 93$ & 202.44 & 402.58 \\
& $7 / 08 / 93$ & 164.83 & 414.54 \\
& $7 / 28 / 93$ & 164.75 & $414.62^{\star}$ \\
& $8 / 06 / 93$ & 164.54 & 414.83 \\
& $9 / 03 / 93$ & 164.59 & 414.78 \\
& $7 / 08 / 93$ & 194.62 & 403.06 \\
& $7 / 27 / 93$ & 194.64 & $403.04 \star$ \\
& $8 / 06 / 93$ & 194.64 & 403.04 \\
& $9 / 03 / 93$ & 194.76 & 402.92 \\
\hline
\end{tabular}

Supplemental Wells for Assessment Monitoring

\begin{tabular}{llll}
\hline $299-E 17-15$ & $9 / 02 / 93$ & 321.04 & 400.74 \\
$299-E 17-20$ & $9 / 02 / 93$ & 318.05 & 401.18 \\
$299-E 25-11$ & $8 / 03 / 93$ & 278.99 & $402.29 \star$ \\
& $9 / 07 / 93$ & 279.54 & 401.74 \\
& $9 / 30 / 93$ & 279.49 & $401.79 \star$ \\
$299-E 25-18$ & $8 / 03 / 93$ & 277.22 & $401.83 \star$ \\
& $9 / 07 / 93$ & 277.19 & 401.86
\end{tabular}


Table 7-2. RCRA Water Level Measurement Report 216-A-29 Ditch, Third Quarter 1993. (sheet 3 of 3)

\begin{tabular}{|c|c|c|c|}
\hline Well & Date & $\begin{array}{l}\text { Depth to } \\
\text { water (ft) }\end{array}$ & $\begin{array}{l}\text { Water level } \\
\text { elevation } \\
\text { above msl (ft) }\end{array}$ \\
\hline \multicolumn{4}{|c|}{ Supplemental Wells for Assessment Monitoring } \\
\hline 299-E25-19 & $\begin{array}{l}8 / 03 / 93 \\
9 / 07 / 93\end{array}$ & $\begin{array}{l}275.58 \\
275.50\end{array}$ & $\begin{array}{l}401.62^{\star} \\
401.70\end{array}$ \\
\hline $299-E 25-20$ & $\begin{array}{l}7 / 15 / 93 \\
9 / 07 / 93\end{array}$ & $\begin{array}{l}274.70 \\
274.62\end{array}$ & $\begin{array}{l}401.60^{\star} \\
401.68\end{array}$ \\
\hline $299-E 25-21$ & $8 / 02 / 93$ & 275.55 & $401.72^{\star}$ \\
\hline $299-E 25-31$ & $9 / 07 / 93$ & 272.47 & 400.06 \\
\hline 299-E25-36 & $9 / 02 / 93$ & 305.76 & 401.63 \\
\hline 299-E25-6 & $\begin{array}{l}9 / 07 / 93 \\
9 / 10 / 93\end{array}$ & $\begin{array}{l}259.06 \\
258.94\end{array}$ & $\begin{array}{l}399.25 \\
399.37 \star\end{array}$ \\
\hline 299-E25-9 & $\begin{array}{l}9 / 07 / 93 \\
9 / 10 / 93\end{array}$ & $\begin{array}{l}255.14 \\
254.92\end{array}$ & $\begin{array}{l}399.72 \\
399.94^{*}\end{array}$ \\
\hline $299-E 26-2$ & 9/07/93 & 233.15 & 402.15 \\
\hline
\end{tabular}

Notes: 1. Water level elevations are calculated by subtracting the measured depth to water from the surveyed elevation for the well.

2. Depth-to-water values are transcribed from field records.

3. Elevations marked with an ' $*$ ' were measured at the time of sampling.

4. Elevations marked with a ' + ' are outside of the expected range, and are suspected of error.

5. To convert feet to meters multiply by 0.3048 . 
Table 7-3. Constituent List and Summary of Results for the 216-A-29 Ditch Data for Reporting Period July 1 through September 30, 1993. (sheet 1 of 6)

CONTAMINATION INDICAYOR PARAMETERS

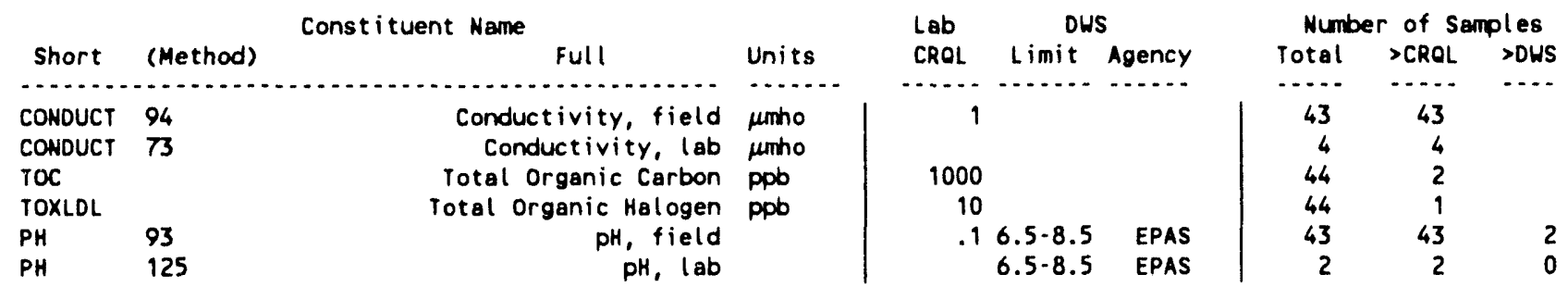

DRINKING WATER PARAMETERS

\begin{tabular}{|c|c|c|c|c|c|c|c|c|c|}
\hline \multirow[b]{2}{*}{ Short } & \multirow{2}{*}{\multicolumn{2}{|c|}{ Const ituent Name }} & \multirow[b]{2}{*}{ Units } & \multirow{2}{*}{$\begin{array}{l}\text { Lab } \\
\text { CRQL }\end{array}$} & \multicolumn{2}{|c|}{ DWS } & \multicolumn{3}{|c|}{ Number of Samples } \\
\hline & & & & & Limit & Agency & Total & 2 CRQL & $>$ DWS \\
\hline 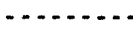 & 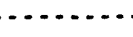 & $\ldots \ldots \ldots$ & $\ldots \ldots$ & $\cdots \ldots$ & $\cdots \ldots$ & $\ldots \ldots$ & $\ldots$. & $\ldots \ldots$ & $\cdots$ \\
\hline $2,4,5 \mathrm{TP}$ & & $2,4,5-T P$ & ppb & 2 & 10 & EPA & 20 & 0 & 0 \\
\hline $2,4-0$ & & $2,4-0$ & ppb & 10 & 100 & EPA & 20 & 0 & 0 \\
\hline ARSENIC & & Arsenic & ppb & 5 & 50 & EPA & 20 & 19 & 0 \\
\hline FARSENIC & & Arsenic, filtered & ppb & 5 & 50 & EPA & 20 & 19 & 0 \\
\hline BARIUM & & Barium & ppb & 20 & 1000 & EPA & 20 & 11 & 0 \\
\hline FBARIUM & & Barium, filtered & ppb & 20 & 1000 & EPA & 20 & 11 & 0 \\
\hline CADMIUM & & Cadmium & ppb & 10 & 10 & EPA & 20 & 0 & 0 \\
\hline FCADMIU & & Cadmium, filtered & ppob & 10 & 10 & EPA & 20 & 0 & 0 \\
\hline CHROAUM & & Chromium & ppob & 20 & 50 & EPA & 20 & 12 & 6 \\
\hline FCHROMI & & Chromium, filtered & ppb & 20 & 50 & EPA & 20 & 0 & 0 \\
\hline COL IFORM & & Coliforms & $\mathrm{COL}$ & & 1 & EPA & 20 & 0 & 0 \\
\hline ENDRIN & & Endrin & ppb & .1 & .2 & EPA & 20 & 0 & 0 \\
\hline FLUORID & & Fluoride & ppb & 100 & 4000 & EPA & 19 & 19 & 0 \\
\hline ALPHA & & Gross alpha & $\mathrm{pCi} / \mathrm{L}$ & 4 & 15 & EPA & 20 & 13 & 0 \\
\hline BETA & & Gross beta & $\mathrm{pCi} / \mathrm{L}$ & 8 & 50 & EPA & 20 & 19 & 0 \\
\hline LEAD & & Lead & ppb & 5 & 50 & EPA & 20 & 1 & 0 \\
\hline FLEAD & & Lead, filtered & ppb & 5 & 50 & EPA & 20 & 1 & 0 \\
\hline MERCURY & & Mercury & ppb & .2 & 2 & EPA & 20 & 0 & 0 \\
\hline FMERCUR & & Mercury, filtered & ppb & .2 & 2 & EPA & 19 & 0 & 0 \\
\hline METHLOR & & Methoxychlor & ppb & 2 & 100 & EPA & 20 & 0 & 0 \\
\hline NITRATE & & Nitrate & ppb & 200 & 45000 & EPA & 20 & 20 & 2 \\
\hline RADIUM & & Radium & $\mathrm{pCi} / \mathrm{L}$ & 1 & 5 & EPA & 20 & 2 & 0 \\
\hline SELENUM & & Selenium & ppb & 10 & 10 & EPA & 20 & 0 & 0 \\
\hline FSELENI & & Selenium, filtered & ppb & 10 & 10 & EPA & 20 & 0 & 0 \\
\hline SILVER & & Silver & cpb & 20 & 50 & EPA & 20 & 0 & 0 \\
\hline FSILVER & & silver, filtered & ppb & 20 & 50 & EPA & 20 & 0 & 0 \\
\hline TOXAENE & & Toxaphene & ppb & 2 & 5 & EPA & 20 & 0 & 0 \\
\hline TURBID & & Turbidity & NTU & .1 & 1 & EPA & 20 & 20 & 16 \\
\hline g-BHC & & gamma-BHC (Lindane) & ppb & .05 & 4 & EPA & 20 & 0 & 0 \\
\hline
\end{tabular}

GROUNDWATER QUALITY PARAMETERS

\begin{tabular}{|c|c|c|c|c|c|c|c|c|c|}
\hline \multirow[b]{2}{*}{ Short } & \multirow[b]{2}{*}{ (Method) } & \multirow[t]{2}{*}{ Constituent Name } & \multirow[b]{2}{*}{ Units } & \multirow{2}{*}{$\begin{array}{l}\text { Lab } \\
\text { CRQL }\end{array}$} & \multicolumn{2}{|c|}{ DWS } & \multicolumn{3}{|c|}{ Number of Samples } \\
\hline & & & & & Limit & Agency & Total & $>C R Q L$ & $>$ DWS \\
\hline$\ldots \ldots$. & $\ldots \ldots$ & $\ldots \ldots \ldots$ & $\ldots \ldots$ & $\ldots \ldots$ & $\ldots \ldots$ & $\ldots \ldots$ & $\cdots$ & $\cdots \cdots$ & $\cdots$ \\
\hline CHLORID & & Chloride & ppb & 200 & 250000 & EPAS & 20 & 20 & 0 \\
\hline IRON & & Iron & ppb & 20 & 300 & EPAS & 20 & 18 & 13 \\
\hline FIRON & & Iron, filtered & ppb & 20 & 300 & EPAS & 20 & 13 & 0 \\
\hline MANGESE & & Manganese & ppb & 10 & 50 & EPAS & 20 & 11 & 1 \\
\hline FMANGAN & & Manganese, filtered & ppb & 10 & 50 & EPAS & 20 & 0 & 0 \\
\hline LPHENOL & 19 & Phenol & $\mathrm{ppb}$ & 10 & & & 1 & 0 & \\
\hline LPHENOL & 30 & Phenol & ppb & 20 & & & 20 & 0 & \\
\hline SODIUM & & Sodium & ppb & 300 & & & 20 & 20 & \\
\hline FSOOIUM & & Sodium, filtered & ppb & 300 & & & 20 & 20 & \\
\hline SULFATE & & Sulfate & ppb & 500 & 250000 & EPAS & 20 & 20 & 0 \\
\hline
\end{tabular}


Table 7-3. Constituent List and Summary of Results for the 216-A-29 Ditch Data for Reporting Period July 1 through September 30, 1993. (sheet 2 of 6)

SITE SPECIFIC AND OTHER CONSTITUENTS

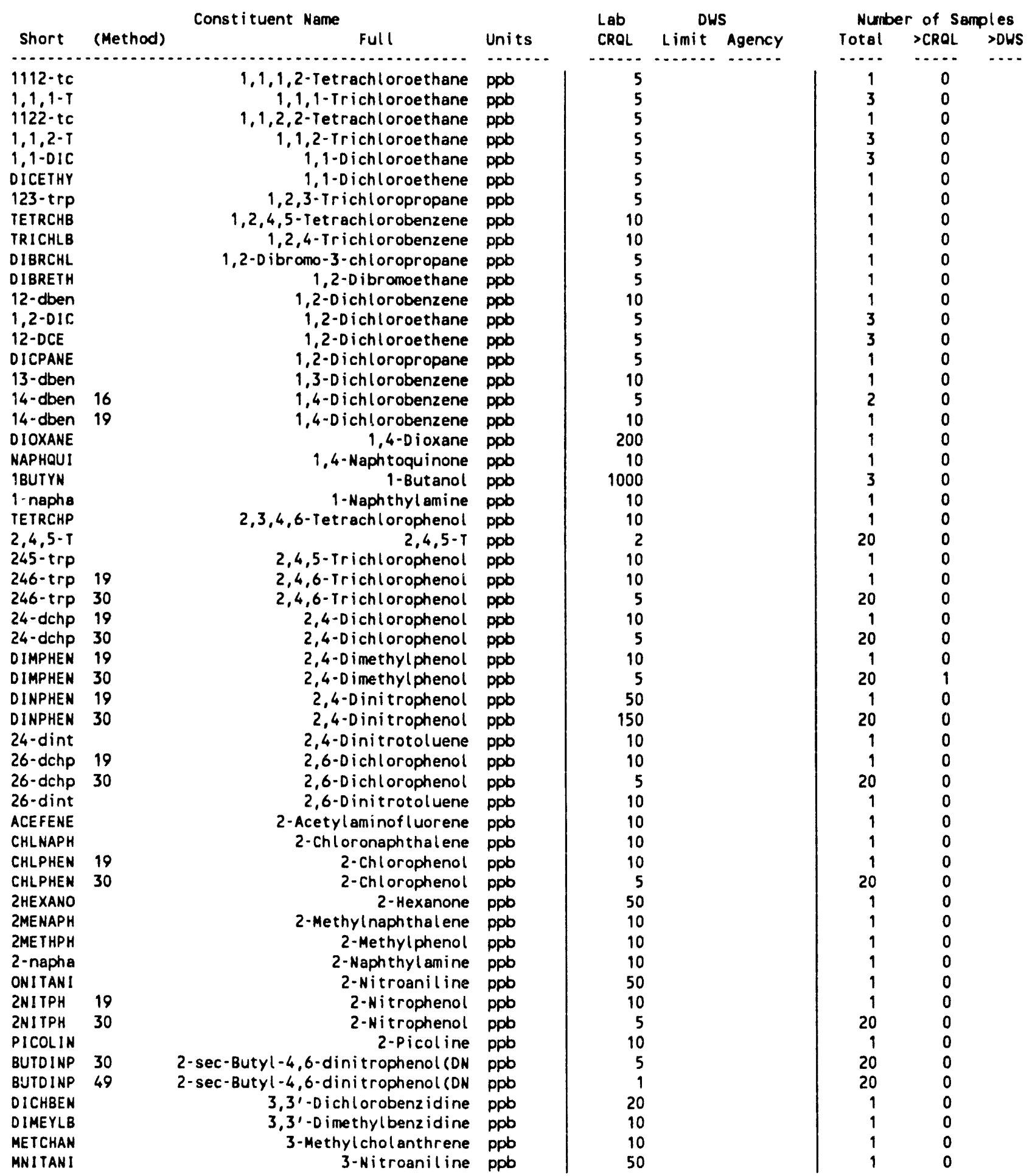


Table 7-3. Constituent List and Summary of Results for the 216-A-29 Ditch Data for Reporting Period July 1 through September 30, 1993. (sheet 3 of 6)

\begin{tabular}{|c|c|c|c|c|c|c|c|c|}
\hline & & Const ituent Name & & \multicolumn{2}{|l|}{ Lab } & \multicolumn{3}{|c|}{ Number of Samples } \\
\hline Short & (Method) & Full & Units & CROL & Limit Agency & Total & $>\mathrm{CROL}$ & $>$ DWS \\
\hline ........ & $\cdots$ & (n) & (........ & -..... & (n....... & …. & & $\cdots$ \\
\hline DDO & & $4,4^{\prime}-D 0 D$ & ppb & .1 & & 20 & 0 & \\
\hline DDE & & $4,44^{\prime}-D D E$ & ppo & .05 & & 20 & 0 & \\
\hline DDT & & $4,4^{\prime}-D D T$ & ppb & .1 & & 20 & 0 & \\
\hline 460N2MP & 19 & 4,6-D initro-2-methylphenol & ppb & 50 & & 1 & 0 & \\
\hline 460N2MP & 30 & 4,6-D initro-2-methylphenol & Ppb & 200 & & 20 & 0 & \\
\hline AMINOYL & & 4-Aminobiphenyl & ppb & 10 & & 1 & 0 & \\
\hline BROPHEN & & 4-Bromophenylphenyl ether & $\mathrm{ppb}$ & 10 & & 1 & 0 & \\
\hline CHLCRES & 19 & 4-Chloro-3-methylphenol & ppob & 20 & & 1 & 0 & \\
\hline CHLCRES & 30 & 4-Chloro-3-methylphenol & ppb & 5 & & 20 & 0 & \\
\hline CHLANIL & & 4-Chloroaniline & ppb & 20 & & 1 & 0 & \\
\hline 4СРPETH & & 4-Chlorophenylphenyl ether & $\mathrm{ppb}$ & 10 & & 1 & 0 & \\
\hline MIBK & & 4-Methyl-2-pentanone & ppo & 50 & & 3 & 0 & \\
\hline 4METHPH & & 4-Methylphenol & ppb & 10 & & 1 & 0 & \\
\hline NITRANI & & 4-Nitroaniline & ppb & 50 & & 1 & 0 & \\
\hline NITPHEN & 19 & 4-Nitrophenol & ppb & 50 & & 1 & 0 & \\
\hline NITPHEN & 30 & 4-Nitrophenol & ppb & 30 & & 20 & 0 & \\
\hline 4NITOUI & & 4-Nitroquinol ine-1-oxide & ppb & 10 & & 1 & 0 & \\
\hline NITRTOL & & 5-Nitro-o-toluidine & ppb & 10 & & 1 & 0 & \\
\hline DIMBENZ & & $7,12-0$ ime thy lbenz $[\theta]$ anthracene & ppb & 10 & & 1 & 0 & \\
\hline ACENAPH & & Acenaph thene & ppb & 10 & & 1 & 0 & \\
\hline ACENATL & & Acenaphthylene & ppb & 10 & & 1 & 0 & \\
\hline ACETONE & & Acetone & ppb & 100 & & 3 & 0 & \\
\hline ACETILE & & Acetonitrile & ppb & 200 & & 1 & 0 & \\
\hline ACETOPH & & Acetophenone & ppb & 10 & & 1 & 0 & \\
\hline ACROLIN & & Acrolein & $\mathrm{ppb}$ & 5 & & 1 & 0 & \\
\hline ACRYILE & & Acrylonitrile & ppb & 5 & & 1 & 0 & \\
\hline ALDRIN & & Aldrin & $\mathrm{ppb}$ & .05 & & 20 & 0 & \\
\hline DIMPHAM & & Alpha, alpha-D imethylphenethyl ami & $p p b$ & 10 & & 1 & 0 & \\
\hline$a-B H C$ & & Alpha-BHC & ppb & .05 & & 20 & 0 & \\
\hline ALUMNUM & & Aluminum & ppb & 200 & & 20 & 2 & \\
\hline FALUMIN & & Aluminum & ppb & 200 & & 20 & 0 & \\
\hline AMMONIU & & Ammonium ion & ppob & 100 & & 20 & 0 & \\
\hline ANILINE & & Aniline & ppb & 10 & & 1 & 0 & \\
\hline ANTHRA & & Anthracene & ppob & 10 & & 1 & 0 & \\
\hline ANTIONY & & Antimony & ppb & 200 & & 20 & 0 & \\
\hline FANTIMO & & Ant imony, filtered & ppo & 200 & & 20 & 0 & \\
\hline ARAMITE & & Aramite & ppb & 10 & & 1 & 0 & \\
\hline AR1016 & & Aroclor -1016 & ppb & 1 & & 1 & 0 & \\
\hline AR1221 & & Aroclor -1221 & ppob & 1 & & 1 & 0 & \\
\hline AR 1232 & & Aroclor -1232 & ppb & 1 & & 1 & 0 & \\
\hline AR 1242 & & Aroclor -1242 & $\mathrm{ppb}$ & 1 & & 1 & 0 & \\
\hline AR 1248 & & Aroclor -1248 & $\mathrm{ppb}$ & 1 & & 1 & 0 & \\
\hline AR 1254 & & Aroclor -1254 & ppb & 1 & & 1 & 0 & \\
\hline AR1260 & & Aroclor -1260 & $\mathrm{ppb}$ & 1 & & 1 & 0 & \\
\hline BENZENE & & Benzene & $\mathrm{ppb}$ & 5 & & 3 & 0 & \\
\hline BENZAAN & & Benzo(a)anthracene & $\mathrm{ppb}$ & 10 & & 1 & 0 & \\
\hline BENZOPY & & Benzo(a)pyrene & $\mathrm{ppb}$ & 10 & & 1 & 0 & \\
\hline BENZBFL & & Benzo(b) fluoranthene & $\mathrm{ppb}$ & 10 & & 1 & 0 & \\
\hline BCHIPER & & Benzo(ghi)perylene & ppb & 10 & & 1 & 0 & \\
\hline BNZKFLU & & Benzo(k)fluoranthene & $p p b$ & 10 & & 1 & 0 & \\
\hline BENZOTH & & Benzothiazole & ppb & 10 & & 1 & 0 & \\
\hline BENZALC & & Benzyl alcohol & $\mathrm{ppb}$ & 20 & & 1 & 0 & \\
\hline BERYLUM & & Beryllium & $\mathrm{ppb}$ & 3 & & 20 & 0 & \\
\hline FBERYLL & & Beryllium, illtered & $\mathrm{ppb}$ & 3 & & 20 & 0 & \\
\hline b-BHC & & Beta-BHC & ppb & .05 & & 20 & 0 & \\
\hline BISZCHM & & Bis (2-Choroethoxy)methane & $\mathrm{ppb}$ & 10 & & 1 & 0 & \\
\hline BISZCHE & & Bis(2-chloroethyl) ether & $\mathrm{ppb}$ & 10 & & 1 & 0 & \\
\hline
\end{tabular}


Table 7-3. Constituent List and Summary of Results for the 216-A-29 Ditch Data for Reporting Period July 1 through September 30, 1993. (sheet 4 of 6)

\begin{tabular}{|c|c|c|c|c|c|c|c|c|c|}
\hline \multicolumn{4}{|c|}{ Constituent Name } & \multicolumn{3}{|c|}{ DWS } & \multicolumn{3}{|c|}{ Number of Samples } \\
\hline Short & (Method) & Full & Units & CROL & Limit & Agency & Total & $>\mathrm{CRQL}$ & $>$ DWS \\
\hline$\ldots \ldots$ & 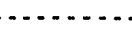 & - & $\ldots . .$. & $\ldots .$. & $\ldots \ldots$ & $\ldots .$. & $\ldots$. & $\ldots$. & $\ldots$ \\
\hline BIS2ETH & & Bis (2-chloroisopropyl) ether & ppb & 10 & & & 1 & 0 & \\
\hline BIS2EPH & & Bis(2-ethylhexyl) phthalate & ppo & 10 & & & 1 & 0 & \\
\hline BROMIDE & & Bromide & ppb & 500 & & & 20 & 0 & \\
\hline BDCM & & Bromodich lorome thane & ppb & 5 & 100 & EPA & 1 & 0 & 0 \\
\hline BROMORM & & Bromoform & ppb & 5 & 100 & EPA & 1 & 0 & 0 \\
\hline BUTBENP & & Butylbenzylphthalate & ppb & 10 & & & 1 & 0 & \\
\hline CALCIUM & & Calcium & ppob & 100 & & & 20 & 20 & \\
\hline FCALCIU & & Calcium, filtered & ppob & 100 & & & 20 & 20 & \\
\hline CAR8IDE & & Carbon disulfide & ppo & 5 & & & 3 & 0 & \\
\hline TETRANE & & Carbon tetrachloride & ppb & 5 & & & 3 & 0 & \\
\hline CHLOANE & & Chlordane & ppb & .1 & & & 20 & 0 & \\
\hline CHLROB & & Chlorobenzene & ppb & 5 & & & 1 & 0 & \\
\hline CHLLATE & & Chlorobenzilate & ppb & 10 & & & 1 & 0 & \\
\hline CLETHAN & & Chloroethane & ppb & 10 & & & 1 & 0 & \\
\hline CHLFORM & & Chloroform & ppb & 5 & 100 & EPA & 3 & 0 & 0 \\
\hline CHLPRENE & & Chloroprene & proth & 5 & & & 1 & 0 & \\
\hline CHRYSEN & & Chrysene & ppb & 10 & & & 1 & 0 & \\
\hline COBALT & & Cobalt & ppb & 20 & & & 20 & 0 & \\
\hline FCOBALT & & Cobalt, filtered & ppb & 20 & & & 20 & 0 & \\
\hline COPPER & & Copper & ppb & 20 & 1000 & EPAS & 20 & 0 & 0 \\
\hline FCOPPER & & Copper, filtered & ppb & 20 & 1000 & EPAS & 20 & 0 & 0 \\
\hline CRESOLS & & Cresols (methylphenols) & ppb & 10 & & & 20 & 0 & \\
\hline d-BHC & & Delta-BHC & ppb & .1 & & & 20 & 0 & \\
\hline DIBPHTH & & Di-n-butylphthalate & ppb & 10 & & & 1 & 0 & \\
\hline DIOPHTH & & Di-n-octylphthalate & ppb & 10 & & & 1 & 0 & \\
\hline DIALLATE & & Diallate & ppb & 10 & & & 1 & 0 & \\
\hline DIBAHAN & & Dibenz $[a, h]$ anthracene & ppb & 10 & & & 1 & 0 & \\
\hline DIBENFR & & Dibenzofuran & ppb & 10 & & & 1 & 0 & \\
\hline BRCHLMET & & Dibromochlorome thane & ppb & 5 & 100 & EPA & 1 & 0 & 0 \\
\hline DIBRMET & & D ibromome thane & ppb & 5 & & & 1 & 0 & \\
\hline DICDIFM & & Dichlorodifluoromethane & ppb & 5 & & & 1 & 0 & \\
\hline DIELRIN & & Dieldrin & ppb & .05 & & & 20 & 0 & \\
\hline DIEPHTH & & Diethyl phthalate & ppb & 10 & & & 1 & 0 & \\
\hline DIMETHO & & Dimethoate & ppb & 10 & & & 9 & 0 & \\
\hline DIMPHTH & & Dimethyl phthalate & ppb & 10 & & & 1 & 0 & \\
\hline DIPHAMI & & Diphenylamine & ppb & 10 & & & 1 & 0 & \\
\hline DISULFO & & Disulfoton & ppb & 2 & & & 1 & 0 & \\
\hline ENDO1 & & Endosulfan I & ppo & .1 & & & 20 & 0 & \\
\hline ENDO2 & & Endosul fan II & ppb & .05 & & & 20 & 0 & \\
\hline ENDSFAN & & Endosulfan sulfate & ppb & .5 & & & 20 & 0 & \\
\hline ENDRALD & & Endr in Aldehyde & ppb & .2 & & & 20 & 0 & \\
\hline ETHCYAN & & Ethyl cyanide & ppb & 10 & & & 3 & 0 & \\
\hline ETHMETH & & Ethyl methacrylate & ppo & 5 & & & 1 & 0 & \\
\hline ETHMETS & & Ethyl methanesul fonate & ppb & 10 & & & 1 & 0 & \\
\hline ETHBENZ & & Ethylbenzene & ppb & 5 & & & 1 & 0 & \\
\hline FAMPHUR & & Famphur & ppb & 10 & & & 1 & 0 & \\
\hline FLUORAN & & Fluoranthene & ppb & 10 & & & 1 & 0 & \\
\hline FLRENE & & Fluorene & ppb & 10 & & & 1 & 0 & \\
\hline HEPTLOR & & Heptachlor & ppb & .05 & & & 20 & 0 & \\
\hline HEPT IDE & & Heptachlor epoxide & ppb & 1 & & & 20 & 0 & \\
\hline HEXCBEN & & Hexach lorobenzene & ppb & 10 & & & 1 & 0 & \\
\hline HEXCBUT & & Hexachlorobutadiene & ppb & 10 & & & 1 & 0 & \\
\hline HEXCCYC & & Hexachlorocyclopentadiene & ppb & 10 & & & 1 & 0 & \\
\hline HEXCETH & & Hexachlor oethane & ppb & 10 & & & 1 & 0 & \\
\hline HEXACHL & & Hexach lorophene & ppb & 10 & & & 1 & 0 & \\
\hline HEXAENE & & Hexach loropropene & ppb & 10 & & & 1 & 0 & \\
\hline LHYORAZ & & Hydrazine & $\mathrm{ppb}$ & 30 & & & 20 & 0 & \\
\hline
\end{tabular}


Table 7-3. Constituent List and Summary of Results for the 216-A-29 Ditch Data for Reporting Period July 1 through September 30, 1993. (sheet 5 of 6)

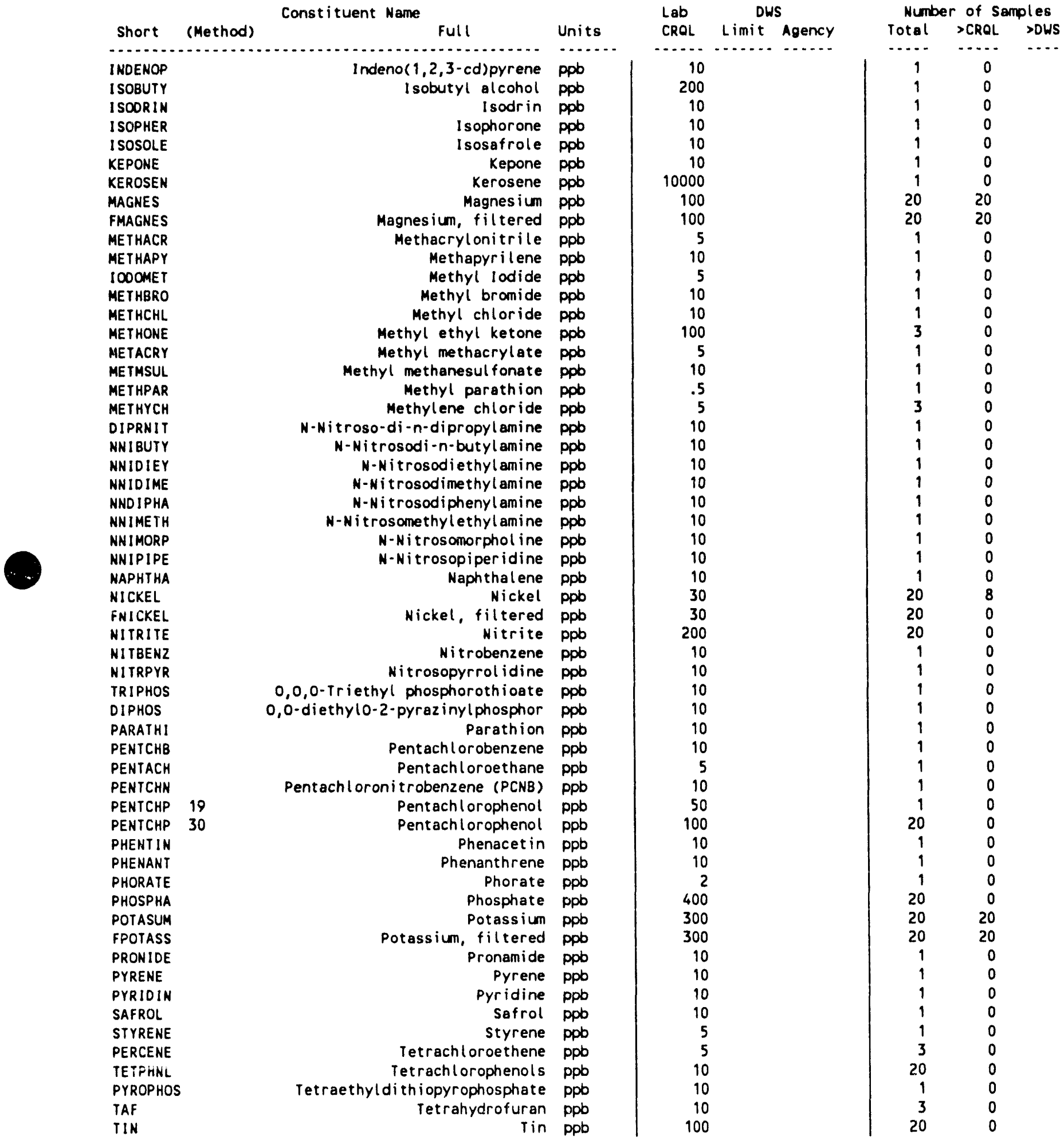


Table 7-3. Constituent List and Summary of Results for the 216-A-29 Ditch Data for Reporting Period July 1 through September 30, 1993. (sheet 6 of 6)

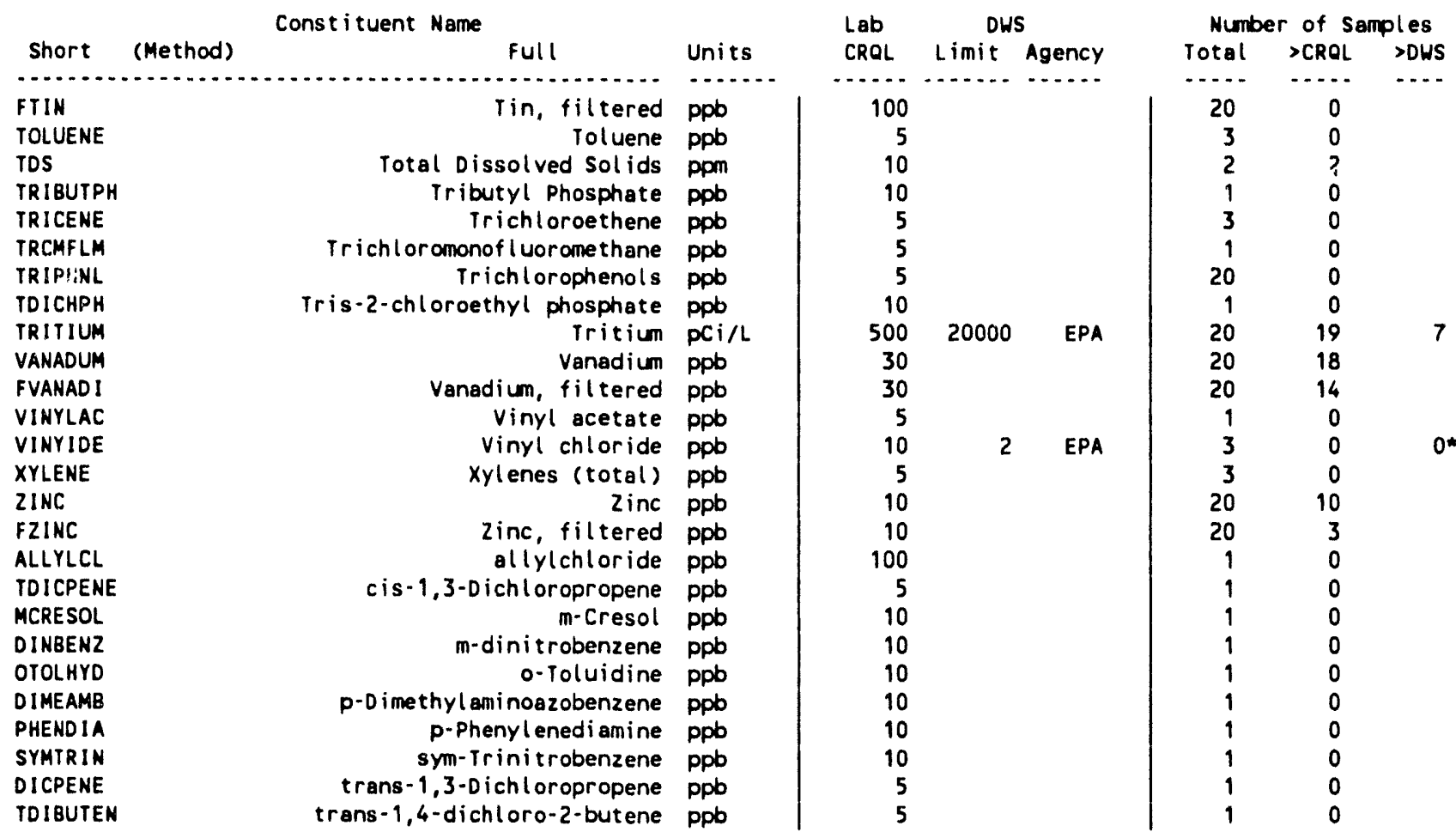

For explanation of this table, see section 1.4 of report. 
Table 7-4. Constituents with at Least One Value Above the CRQL for the 216-A-29 Ditch Data for Reporting Period July 1 through September 30, 1993. (sheet 1 of 8)

\begin{tabular}{|c|c|c|c|c|c|c|}
\hline $\begin{array}{l}\text { Well } \\
\text { Name }\end{array}$ & $\begin{array}{c}\text { Collection } \\
\text { Date }\end{array}$ & $\begin{array}{l}\text { Sample } \\
\text { Number }\end{array}$ & $\begin{array}{c}\text { DIMPHEN } \\
30 / \mathrm{ppb} \\
5 /\end{array}$ & $\begin{array}{l}\text { ALUMNUM } \\
34 / \mathrm{ppb} \\
200 /\end{array}$ & $\begin{array}{l}\text { FALUMIN } \\
34 / \mathrm{ppb} \\
200 /\end{array}$ & $\begin{array}{c}\text { ARSENIC } \\
43 / \mathrm{ppb} \\
5 / 50\end{array}$ \\
\hline $\begin{array}{l}299-E 25-11 \\
299-E 25-11\end{array}$ & $8 / 03 / 93$ & $\begin{array}{l}808028 \\
808029\end{array}$ & $1.01 \mathrm{U}$ & $32.50 \mathrm{U}$ & & 13.00 \\
\hline $\begin{array}{l}299-E 25-11 \\
299-E 25-18 \\
299-E 25-18\end{array}$ & $\begin{array}{l}8 / 03 / 93 \\
8 / 03 / 93 \\
8 / 03 / 93\end{array}$ & $\begin{array}{l}\text { B08Q29 } \\
\text { B08R00 } \\
\text { B08R01 }\end{array}$ & $\begin{array}{l}1.01 \mathrm{U} \\
1.01 \mathrm{U}\end{array}$ & $\begin{array}{l}32.50 u \\
32.50 u\end{array}$ & $32.50 \mathrm{U}$ & $\begin{array}{l}11.00 \\
12.00\end{array}$ \\
\hline $\begin{array}{l}299-E 25-18 \\
299-E 25-18\end{array}$ & $\begin{array}{l}8 / 03 / 93 \\
8 / 03 / 93\end{array}$ & $\begin{array}{l}\text { B08R02 } \\
\text { B08R03 }\end{array}$ & & & $\begin{array}{l}32.50 \mathrm{U} \\
32.50 \mathrm{U}\end{array}$ & \\
\hline $\begin{array}{l}299-E 25-19 \\
299-E 25-19\end{array}$ & $\begin{array}{l}8 / 03 / 93 \\
8 / 03 / 93\end{array}$ & $\begin{array}{l}\text { B08R04 } \\
\text { B08R05 }\end{array}$ & $1.01 \mathrm{U}$ & $33.00 \mathrm{~L}$ & $32.50 \mathrm{U}$ & 7.40 \\
\hline $\begin{array}{l}299-E 25-20 \\
299-E 25-20\end{array}$ & $\begin{array}{l}7 / 15 / 93 \\
7 / 15 / 93\end{array}$ & $\begin{array}{l}\text { B08R06 } \\
\text { B08R07 }\end{array}$ & $1.01 \mathrm{U}$ & $32.50 \mathrm{U}$ & $32.50 \mathrm{U}$ & 13.00 \\
\hline $\begin{array}{l}299-E 25-21 \\
299-E 25-21\end{array}$ & $\begin{array}{l}8 / 02 / 93 \\
8 / 02 / 93\end{array}$ & $\begin{array}{l}\text { B08R08 } \\
\text { BO8R09 }\end{array}$ & $1.01 \mathrm{U}$ & $32.50 \mathrm{U}$ & $38.00 \mathrm{~L}$ & 14.00 \\
\hline $\begin{array}{l}299-E 25-26 \\
299-E 25-26\end{array}$ & $\begin{array}{l}8 / 02 / 93 \\
8 / 02 / 93\end{array}$ & $\begin{array}{l}\text { BO8R 10 } \\
\text { B08R1! }\end{array}$ & $1.01 \mathrm{U}$ & $32.50 \mathrm{U}$ & $32.50 \mathrm{U}$ & 7.40 \\
\hline $\begin{array}{l}299-E 25-28 \\
299-E 25-28\end{array}$ & $\begin{array}{l}8 / 02 / 93 \\
8 / 02 / 93\end{array}$ & $\begin{array}{l}\text { BO8R 12 } \\
\text { BO8R 13 }\end{array}$ & $1.01 \mathrm{U}$ & $32.50 \mathrm{U}$ & $32.50 \mathrm{U}$ & 11.00 \\
\hline $\begin{array}{l}299-E 25-31 \\
299-E 25-31\end{array}$ & $\begin{array}{l}7 / 16 / 93 \\
7 / 16 / 93\end{array}$ & $\begin{array}{l}\text { B08R 14 } \\
\text { B08R15 }\end{array}$ & $1.01 \mathrm{U}$ & $50.00 \mathrm{~L}$ & $32.50 \mathrm{U}$ & 8.60 \\
\hline $\begin{array}{l}299-E 25-32 P \\
299-E 25-32 P\end{array}$ & $\begin{array}{l}7 / 15 / 93 \\
7 / 15 / 93\end{array}$ & $\begin{array}{l}\text { B08R50 } \\
\text { B08R51 }\end{array}$ & $1.01 \mathrm{U}$ & $32.50 \mathrm{U}$ & $32.50 \mathrm{U}$ & $2.30 \mathrm{~L}$ \\
\hline $\begin{array}{l}299-E 25-34 \\
299-E 25-34\end{array}$ & $\begin{array}{l}8 / 04 / 93 \\
8 / 04 / 93\end{array}$ & $\begin{array}{l}\text { B08R 16 } \\
\text { B08R 17 }\end{array}$ & $1.01 \mathrm{U}$ & $32.50 \mathrm{U}$ & $32.50 \mathrm{U}$ & 8.600 \\
\hline $\begin{array}{l}299-E 25-35 \\
299-E 25-35\end{array}$ & $\begin{array}{l}8 / 04 / 93 \\
8 / 04 / 93\end{array}$ & $\begin{array}{l}\text { BOSR 18 } \\
\text { BO8R } 19\end{array}$ & $1.01 \mathrm{U}$ & $35.00 \mathrm{~L}$ & & 15.000 \\
\hline $\begin{array}{l}299-E 25-42 \\
299-E 25-42\end{array}$ & $\begin{array}{l}7 / 16 / 93 \\
7 / 16 / 93\end{array}$ & $\begin{array}{l}\text { B08R20 } \\
\text { B08R24 }\end{array}$ & $1.01 \mathrm{U}$ & $150.00 \mathrm{~L}$ & $32.50 \mathrm{U}$ & 10.00 \\
\hline $\begin{array}{l}299-E 25-43 \\
299-E 25-43\end{array}$ & $\begin{array}{l}7 / 15 / 93 \\
7 / 15 / 93\end{array}$ & $\begin{array}{l}\text { B08R25 } \\
\text { B08R29 }\end{array}$ & $1.01 \mathrm{U}$ & 200.00 & $32.50 \mathrm{U}$ & 7.10 \\
\hline $\begin{array}{l}299-E 25-47 \\
299-E 25-47\end{array}$ & $\begin{array}{l}7 / 16 / 93 \\
7 / 16 / 93\end{array}$ & $\begin{array}{l}\text { B08R30 } \\
\text { B08R34 }\end{array}$ & $1.01 \mathrm{U}$ & $59.00 \mathrm{~L}$ & $32.50 \mathrm{U}$ & 13.00 \\
\hline $\begin{array}{l}299-E 25-48 \\
299-E 25-48\end{array}$ & $\begin{array}{l}7 / 27 / 93 \\
7 / 27 / 93\end{array}$ & $\begin{array}{l}\text { BO8R35 } \\
\text { B08R39 }\end{array}$ & $1.01 \mathrm{U}$ & 490.00 & $32.50 \mathrm{U}$ & 7.80 \\
\hline $\begin{array}{l}299-E 26-12 \\
299-E 26-12\end{array}$ & $\begin{array}{l}7 / 22 / 93 \\
7 / 22 / 93\end{array}$ & $\begin{array}{l}\text { B08R40 } \\
\text { B08R44 }\end{array}$ & $1.01 \mathrm{U}$ & $40.00 \mathrm{~L}$ & $32.50 \mathrm{U}$ & 9.40 \\
\hline $\begin{array}{l}299-E 26-13 \\
299-E 26-13\end{array}$ & $\begin{array}{l}7 / 23 / 93 \\
7 / 23 / 93\end{array}$ & $\begin{array}{l}808 R 45 \\
808 R 49\end{array}$ & $1.01 \mathrm{U}$ & $32.50 \mathrm{U}$ & $32.50 \mathrm{U}$ & 11.00 \\
\hline $\begin{array}{l}699-43-43 \\
699-43-43\end{array}$ & $\begin{array}{l}7 / 28 / 93 \\
7 / 28 / 93\end{array}$ & $\begin{array}{l}808 \times T 1 \\
808 \times T 5\end{array}$ & $1.01 \mathrm{U}$ & $32.50 \mathrm{U}$ & $33.00 \mathrm{~L}$ & 8.70 \\
\hline $\begin{array}{l}699-43-45 \\
699-43-45\end{array}$ & $\begin{array}{l}7 / 27 / 93 \\
7 / 27 / 93\end{array}$ & $\begin{array}{l}\text { B08XT6 } \\
\text { B08XV0 }\end{array}$ & $5.80 \mathrm{~B}$ & $32.50 \mathrm{U}$ & $32.50 \mathrm{U}$ & 10.00 \\
\hline
\end{tabular}

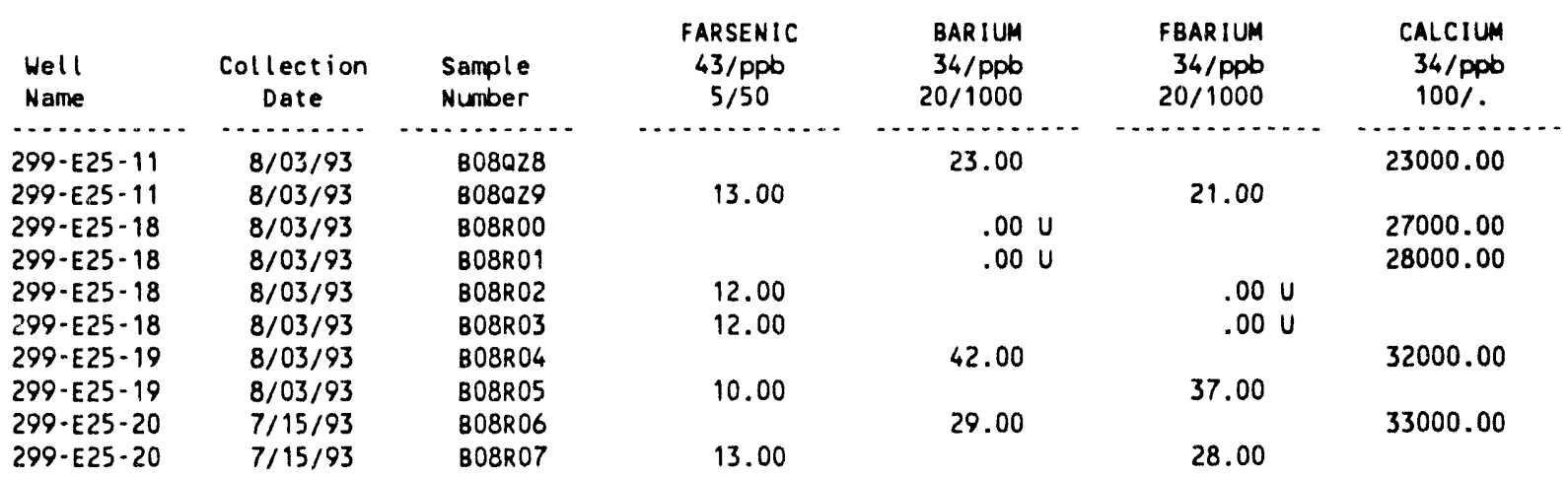


Table 7-4. Constituents with at Least One Value Above the CRQL for the 216-A-29 Ditch Data for Reporting Period July 1 through September 30, 1993. (sheet 2 of 8)

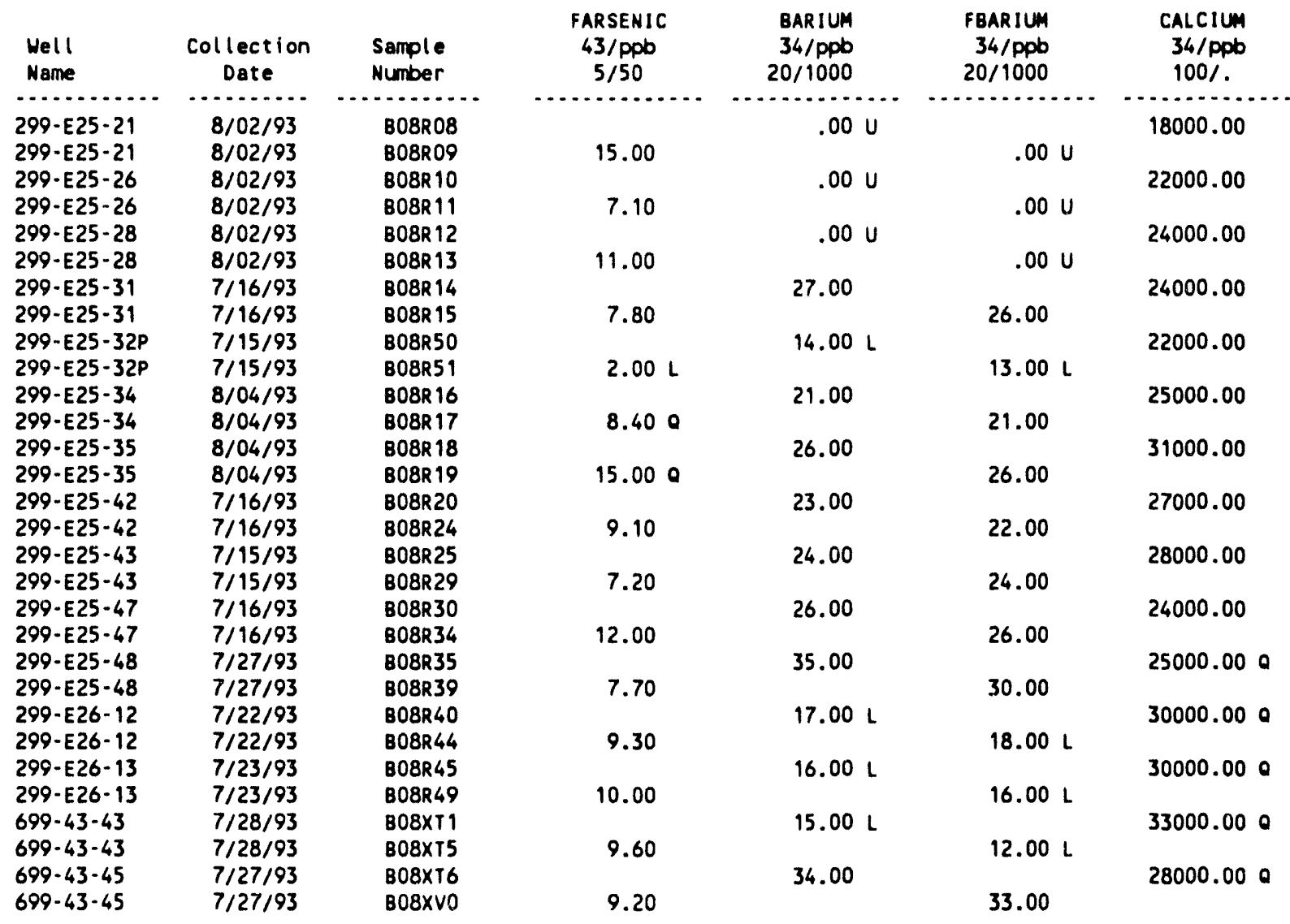

\begin{tabular}{|c|c|c|c|c|c|c|}
\hline $\begin{array}{l}\text { Hell } \\
\text { Name }\end{array}$ & $\begin{array}{c}\text { Collection } \\
\text { Dote }\end{array}$ & $\begin{array}{l}\text { Sample } \\
\text { Number }\end{array}$ & $\begin{array}{l}\text { FCALCIU } \\
34 / \mathrm{ppb} \\
100 \%\end{array}$ & $\begin{array}{c}\text { CHLORID } \\
124 / \mathrm{ppb} \\
200 / 250000 \mathrm{~s}\end{array}$ & $\begin{array}{r}\text { CHROMUM } \\
34 / \mathrm{ppb} \\
20 / 100\end{array}$ & $\begin{array}{r}\text { FCHROMI } \\
34 / \text { ppb } \\
20 / 100\end{array}$ \\
\hline$\cdots \cdot \cdot$ & $\cdots$ & , & n......... & ............. & & \\
\hline 299-E25-11 & $8 / 03 / 93$ & 808028 & & 2700.00 & $5.42 \mathrm{U}$ & \\
\hline $299-E 25-11$ & $8 / 03 / 93$ & 908029 & 22000.00 & & & $5.42 U$ \\
\hline $299-E 25-18$ & $8 / \cup 3 / 93$ & BO8R0O & & 2900.00 & $5.42 \mathrm{U}$ & \\
\hline $299-E 25-18$ & $8 / 03 / 9$ & 78R01 & & 2900.00 & $5.42 \mathrm{U}$ & \\
\hline $299-E 25-18$ & $8 / 03 / 9$. & $8 R 02$ & 27000.00 & & & $5.42 \mathrm{U}$ \\
\hline 299-E25-18 & 8/03/9, & $B 08 R 03$ & 27000.00 & & & $5.42 \mathrm{U}$ \\
\hline $299-E 25-19$ & $8 / 03 / 93$ & B08R04 & & 2900.00 & $5.42 \mathrm{U}$ & \\
\hline $299-E 25-19$ & $8 / 03 / 93$ & B08R05 & 32000.00 & & & $5.42 \mathrm{U}$ \\
\hline 299-E25 20 & $7 / 15 / 93$ & B08R06 & & 3600.00 & $5.42 \mathrm{U}$ & \\
\hline $299-E 25-20$ & $7 / 15 / 93$ & B08R07 & 33000.00 & & & $5.42 \mathrm{U}$ \\
\hline $299-E 25-21$ & $8 / 02 / 93$ & BO8R08 & & 280000 & $5.42 \mathrm{U}$ & \\
\hline 299-E25-21 & $8 / 02 / 93$ & B08R09 & 18000.00 & & & $5.42 \mathrm{U}$ \\
\hline $299-E 25-26$ & $8 / 02 / 93$ & B08R 10 & & 2000.00 & $5.42 \mathrm{U}$ & \\
\hline 299-E25-26 & $8 / 02 / 93$ & B08R11 & 22000.00 & & & $5.42 U$ \\
\hline $299-E 25-28$ & $8 / 02 / 93$ & B08R 12 & & 2800.00 & $5.42 \mathrm{U}$ & \\
\hline 299-E25-28 & $8 / 02 / 93$ & B08R 13 & 23000.00 & & & $5.42 \mathrm{U}$ \\
\hline 299-E25-31 & $7 / 16 / 93$ & B08R 14 & & 2100.00 & 150.00 & \\
\hline $299-E 25-31$ & $7 / 16 / 93$ & B08R 15 & 23000.00 & & & $7.30 \mathrm{~L}$ \\
\hline 299-E25-32P & $7 / 15 / 93$ & 808R50 & & 1600.00 & 230.00 & \\
\hline 299-E25-32P & $7 / 15 / 93$ & B08R51 & 22000.00 & & & $5.42 U$ \\
\hline
\end{tabular}


Table 7-4. Constituents with at Least One Value Above the CRQL for the 216-A-29 Ditch Data for Reporting Period July 1 through September 30, 1993. (sheet 3 of 8)

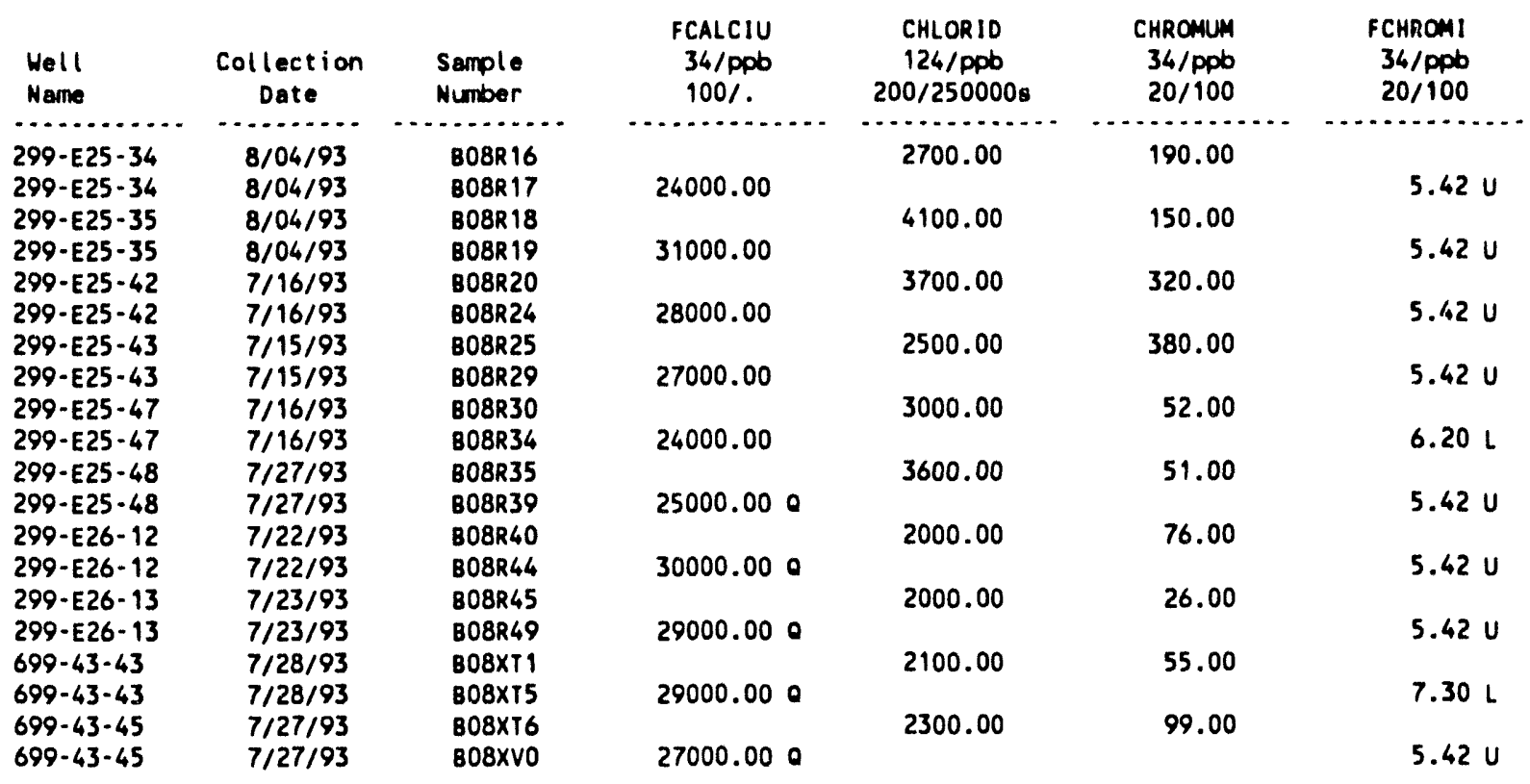

\begin{tabular}{|c|c|c|c|c|c|c|}
\hline $\begin{array}{l}\text { Well } \\
\text { Name }\end{array}$ & $\begin{array}{c}\text { Collection } \\
\text { Date }\end{array}$ & $\begin{array}{l}\text { Sample } \\
\text { Numbur }\end{array}$ & $\begin{array}{l}\text { FLUORID } \\
124 / \text { ppb } \\
100 / 4000\end{array}$ & $\begin{array}{c}\text { ALPHA } \\
135 / P C i / L \\
. / 15\end{array}$ & $\begin{array}{c}\text { BETA } \\
136 / \mathrm{pCi} / \mathrm{L} \\
. / 50\end{array}$ & $\begin{array}{c}\text { IROW } \\
34 / \mathrm{Ppb} \\
20 / 300 \mathrm{~s}\end{array}$ \\
\hline$\cdots \ldots$ & (............. & $\ldots$ & $\cdots$ & & $\cdots \cdot \cdot$ & \\
\hline $\begin{array}{l}299-E 25-11 \\
299-E 25-18\end{array}$ & $\begin{array}{l}8 / 03 / 93 \\
8 / 03 / 93\end{array}$ & $\begin{array}{l}\text { B08028 } \\
\text { B08R00 }\end{array}$ & $\begin{array}{l}500.00 \\
600.00\end{array}$ & $\begin{array}{l}1.11 \\
1.690\end{array}$ & $\begin{array}{l}4.73 \\
5.63\end{array}$ & $\begin{array}{l}240.00 \\
180.00\end{array}$ \\
\hline $299-E 25-18$ & $8 / 03 / 93$ & BO8R01 & 700.00 & .85 va & 6.50 & 190.00 \\
\hline 299-E25-19 & $8 / 03 / 93$ & B08R04 & 800.00 & 1.97 & 10.40 & 3400.00 \\
\hline $299-$ E25-20 & $7 / 15 / 93$ & B08R06 & 700.00 & $1.20 \mathrm{U}$ & 11.20 & 360.00 \\
\hline $299-E 25-21$ & $8 / 02 / 93$ & BO8R08 & 600.00 & $.05 U$ & 7.12 & 470.00 \\
\hline 299-E25-26 & $8 / 02 / 93$ & BOBR 10 & 400.00 & $.75 \mathrm{U}$ & 3.23 & $10.30 \mathrm{U}$ \\
\hline 299-E25-28 & $8 / 02 / 93$ & BO8R 12 & 400.00 & 1.15 & 2.66 & $10.30 \mathrm{U}$ \\
\hline $299-E 25-31$ & $7 / 16 / 93$ & B08R 14 & 500.00 & 2.24 & 7.79 & 910.00 \\
\hline $299-E 25-32 P$ & $7 / 15 / 93$ & 808R50 & 200.00 & 1.04 & 3.13 & 970.00 \\
\hline $299-E 25-34$ & $8 / 04 / 93$ & BO8R 16 & $300.00 \mathrm{~B}$ & .89 & 5.84 & 960.00 \\
\hline $299-E 25 \cdot 35$ & $8 / 04 / 93$ & BOBR 18 & 800.008 & 3.46 & $1.95 \mathrm{U}$ & 950.00 \\
\hline $299-E 25-42$ & $7 / 16 / 93$ & BO8R2O & 900.00 & 3.00 & 4.96 & 1600.00 \\
\hline $299-E 25-43$ & $7 / 15 / 93$ & B08R25 & 200.00 & 2.73 & 2.68 & 1900.00 \\
\hline $299-E 25-47$ & $7 / 16 / 93$ & BO8R30 & 500.00 & 1.67 & 6.99 & 330.00 \\
\hline $299-E 25-48$ & $7 / 27 / 93$ & BO8R35 & 300.00 & $.99 \mathrm{U}$ & 5.50 & 1500.00 \\
\hline $299-E 26-12$ & $7 / 22 / 93$ & B08R40 & 400.000 & 1.89 & 6.39 & 370.00 \\
\hline $299-\varepsilon 26-13$ & $7 / 23 / 93$ & $808 R 45$ & & 1.85 & 7.64 & 120.00 \\
\hline $699-43-43$ & $7 / 28 / 93$ & $B 08 \times 11$ & 300.00 & $.92 \mathrm{U}$ & 5.02 & 240.00 \\
\hline $699-43-45$ & $7 / 27 / 93$ & $808 \times 16$ & 300.00 & $.74 \mathrm{U}$ & 3.32 & 410.00 \\
\hline
\end{tabular}


Table 7-4. Constituents with at Least One Value Above the CRQL for the 216-A-29 Ditch Data for Reporting Period July 1 through September 30, 1993. (sheet 4 of 8 )

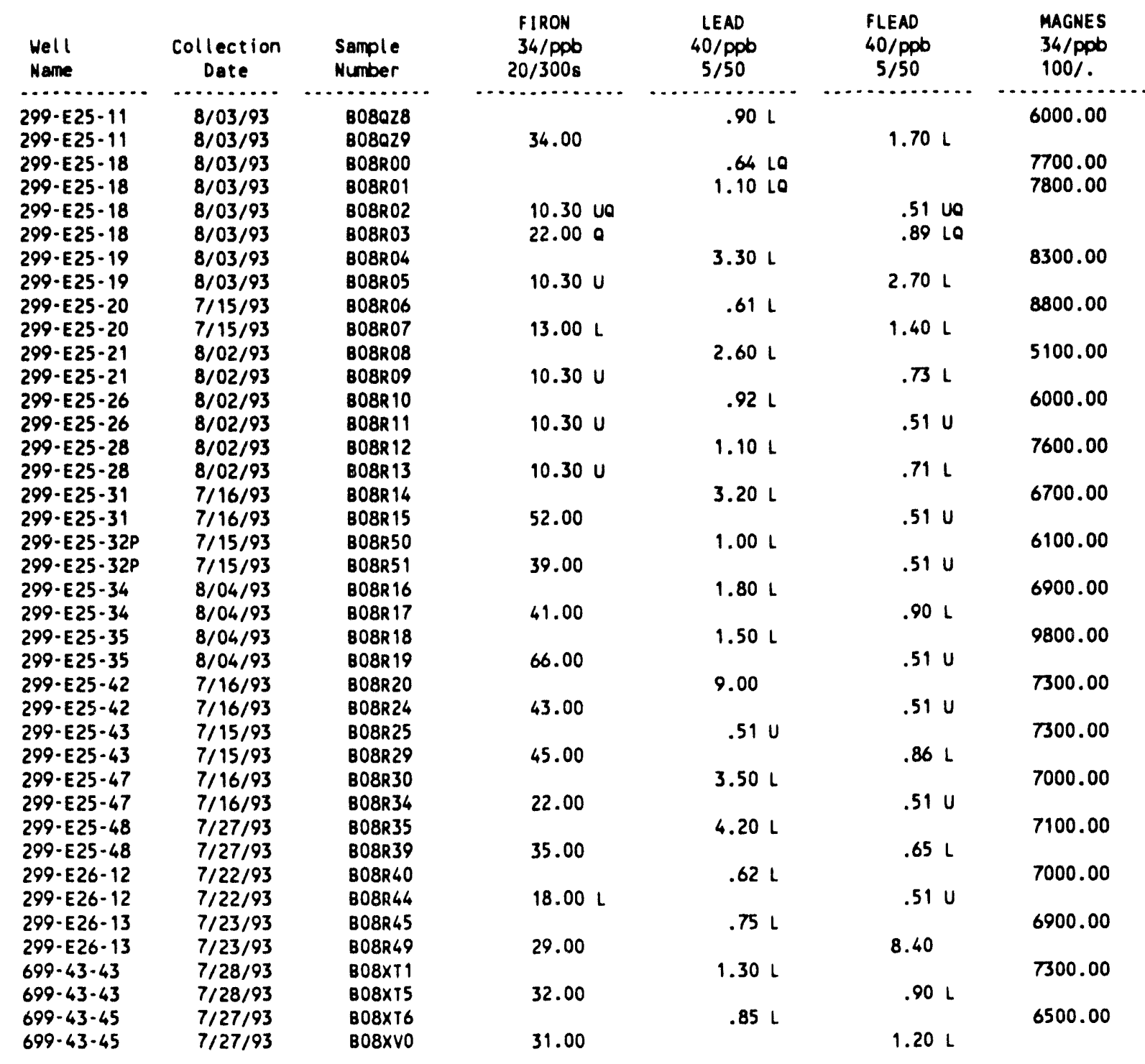

\begin{tabular}{|c|c|c|c|c|c|c|}
\hline $\begin{array}{l}\text { Well } \\
\text { Name }\end{array}$ & $\begin{array}{c}\text { Collection } \\
\text { Date }\end{array}$ & $\begin{array}{l}\text { Sample } \\
\text { Number }\end{array}$ & $\begin{array}{c}\text { FMAGNES } \\
34 / \text { Ppb } \\
100 \%\end{array}$ & $\begin{array}{r}\text { MANGESE } \\
34 / \mathrm{ppb} \\
10 / 50 \mathrm{~s}\end{array}$ & $\begin{array}{l}\text { FMANGAN } \\
34 / \text { ppb } \\
10 / 50 \mathrm{~s}\end{array}$ & $\begin{array}{c}\text { NICKEL } \\
34 / \mathrm{ppb} \\
30 \%\end{array}$ \\
\hline $299-E 25-11$ & $8 / 03 / 93$ & 808028 & & 16.00 & & $17.90 \mathrm{U}$ \\
\hline $\begin{array}{l}299-E 25-11 \\
299-E 25-18\end{array}$ & $\begin{array}{l}8 / 03 / 93 \\
8 / 03 / 93\end{array}$ & $\begin{array}{l}B 08 Q 29 \\
\text { B08R00 }\end{array}$ & 5800.00 & & $1.35 \mathrm{U}$ & \\
\hline $299-E 25-18$ & $8 / 03 / 93$ & B08k01 & & $1.35 \mathrm{U}$ & & $17.90 \mathrm{U}$ \\
\hline $299-E 25-18$ & $8 / 03 / 93$ & BO8R02 & 7600.00 & & $1.35 \mathrm{U}$ & \\
\hline $299-E 25-18$ & $8 / 03 / 93$ & BO8R03 & 7500.00 & & $1.35 \mathrm{U}$ & \\
\hline $299-E 25-19$ & $8 / 03 / 93$ & BO8R04 & & 40.00 & & $17.90 \mathrm{U}$ \\
\hline $299-E 25-19$ & $8 / 03 / 93$ & BO8R05 & 8400.00 & & $1.35 \mathrm{U}$ & \\
\hline $299-E 25-20$ & $7 / 15 / 93$ & B08R06 & & $9.60 \mathrm{~L}$ & & $17.90 \mathrm{U}$ \\
\hline $299-\varepsilon 25-20$ & $7 / 15 / 93$ & 808R07 & 8800.00 & & $1.35 \mathrm{U}$ & \\
\hline
\end{tabular}


Table 7-4. Constituents with at Least One Value Above the CRQL for the 216-A-29 Ditch Data for Reporting Period July 1 through September 30, 1993. (sheet 5 of 8)

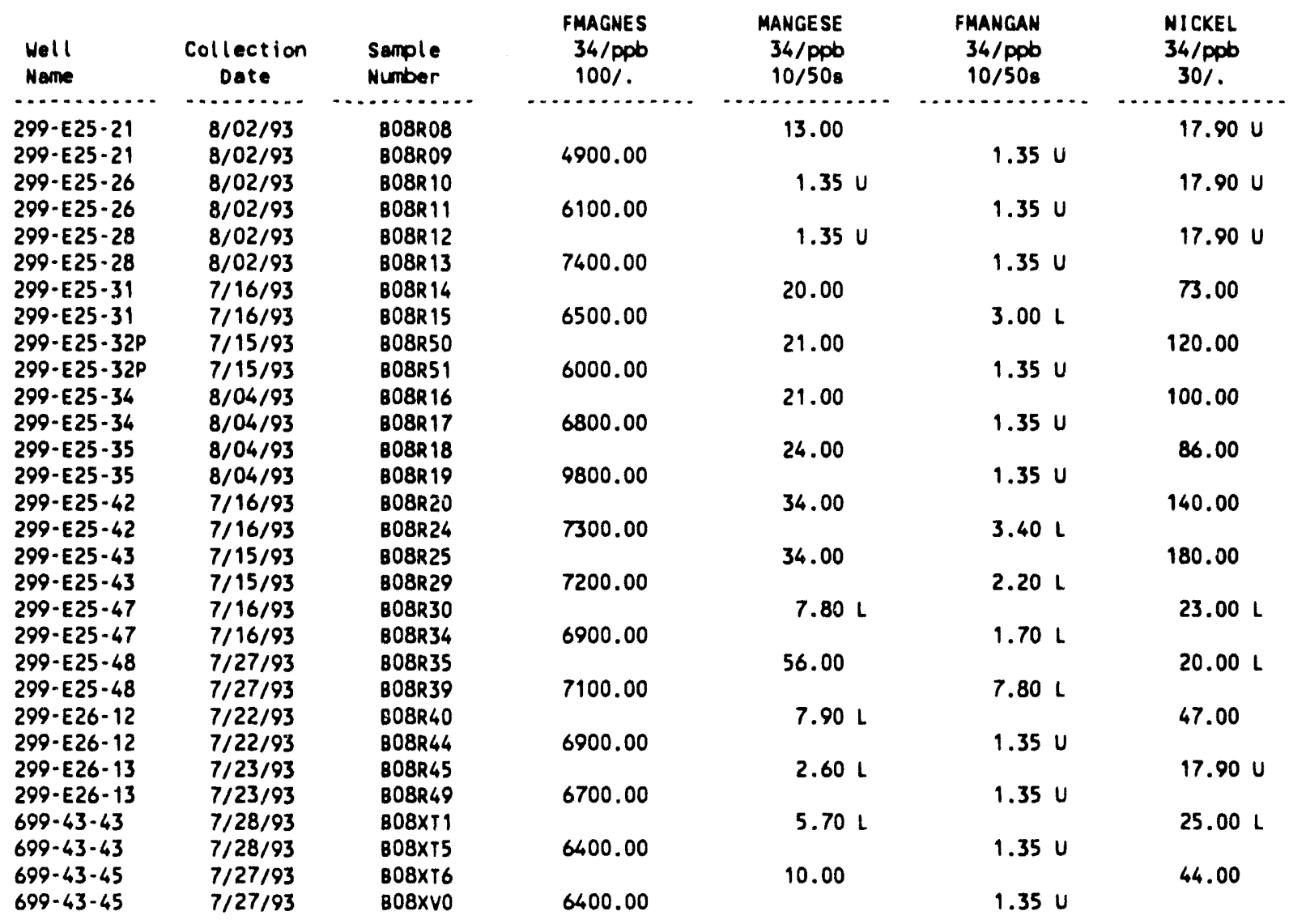

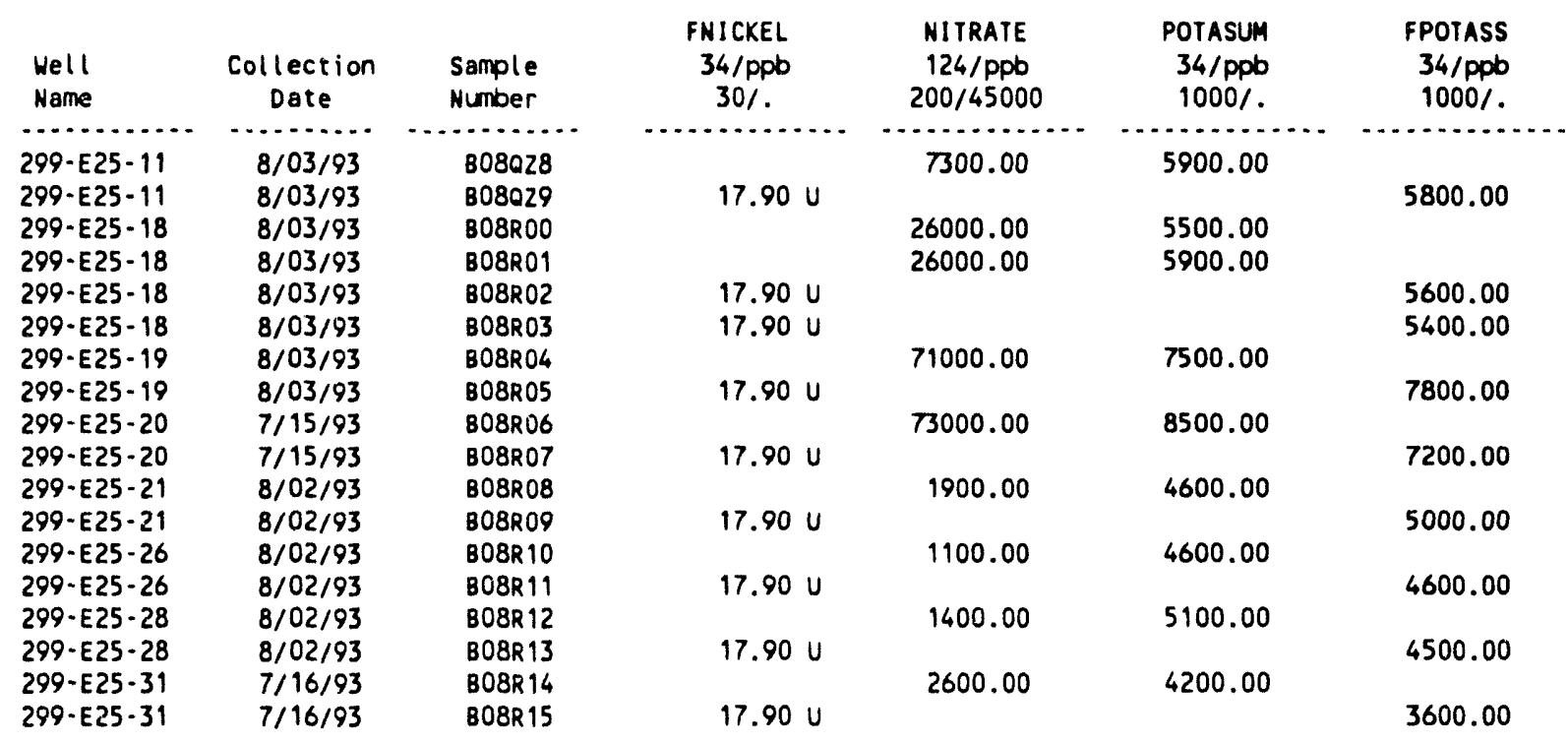


Table 7-4. Constituents with at Least One Value Above the CRQL for the 216-A-29 Ditch Data for Reporting Period July 1 through September 30, 1993. (sheet 6 of 8)

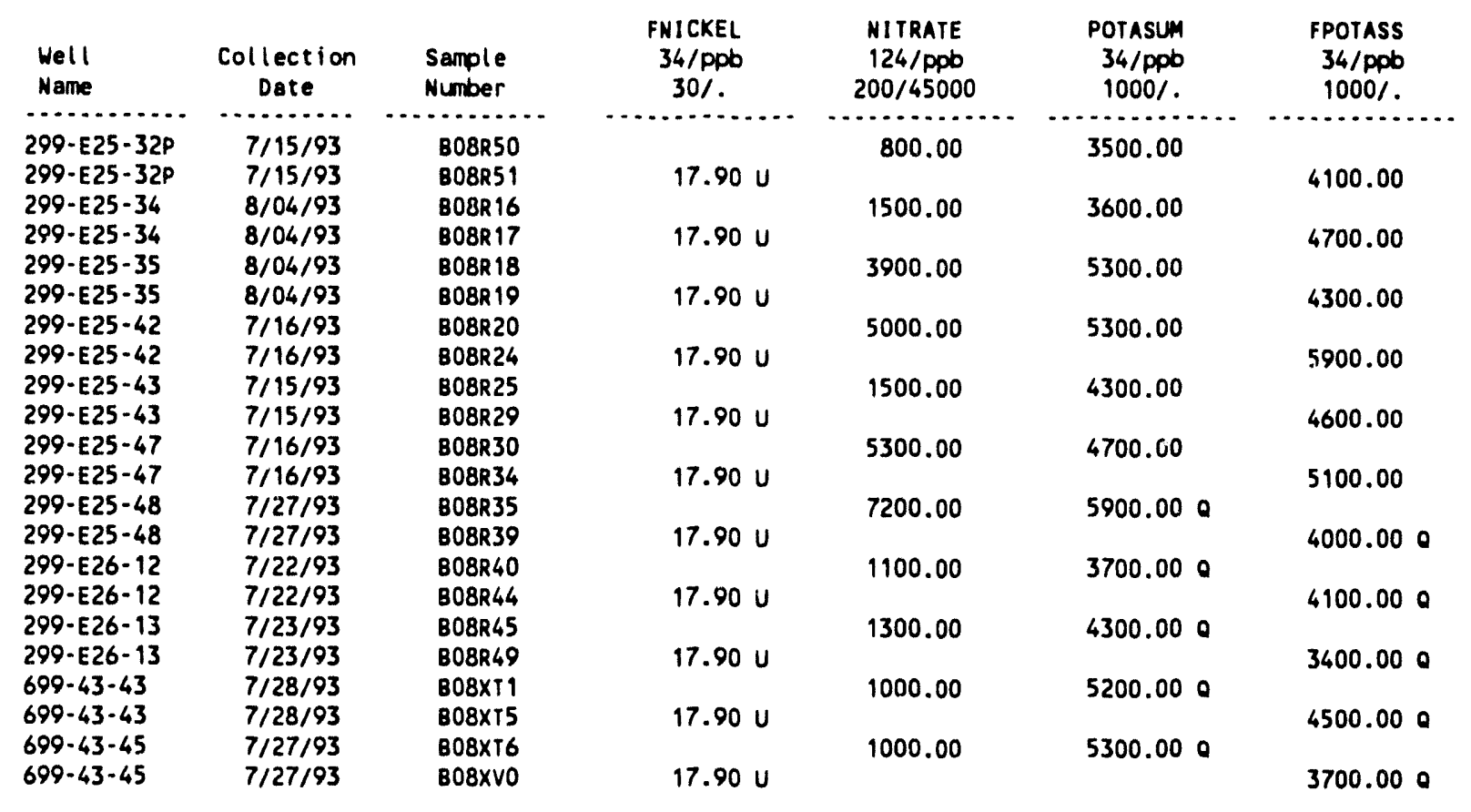

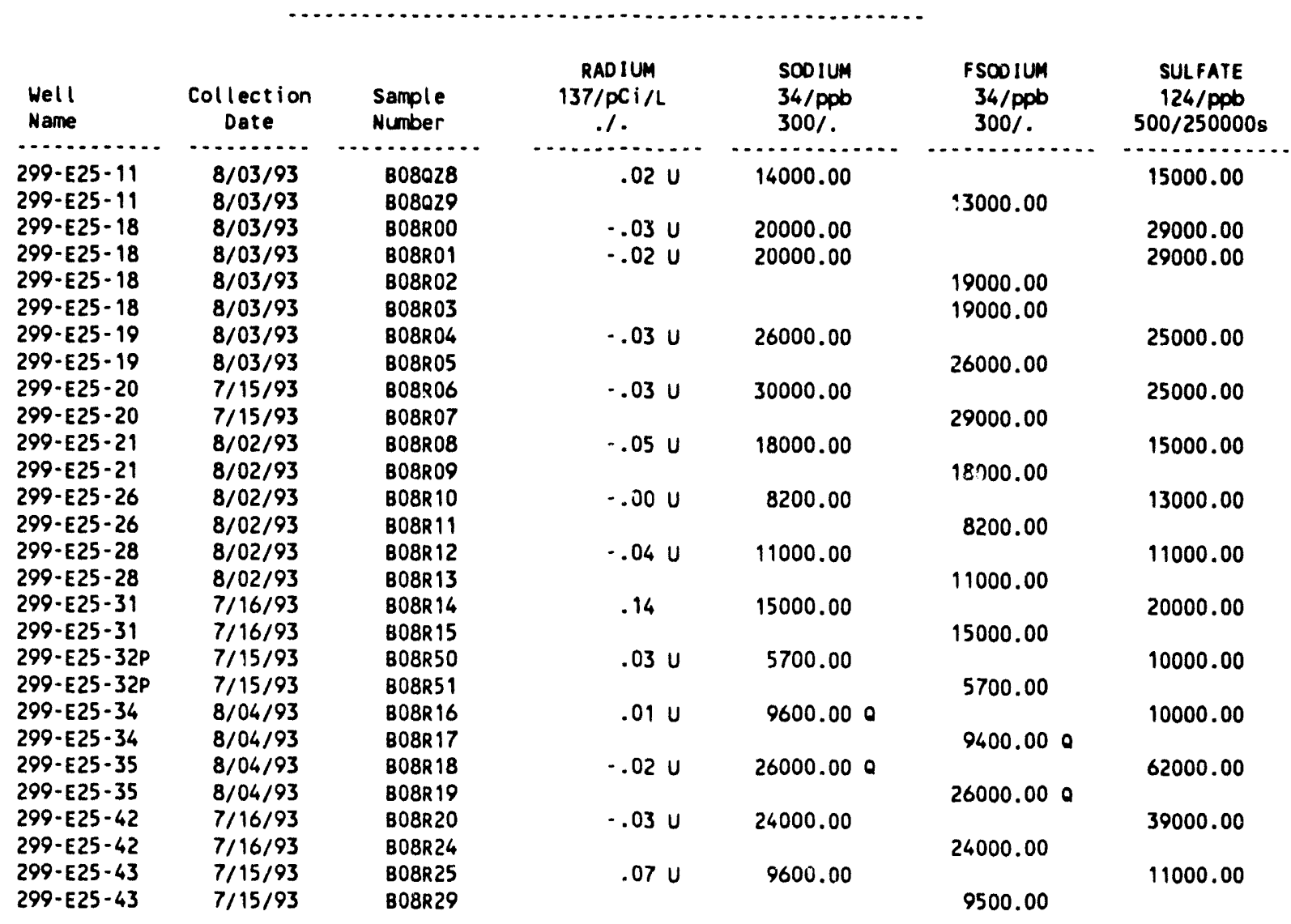


Table 7-4. Constituents with at Least One Value Above the CRQL for the 216-A-29 Ditch Data for Reporting Period July 1 through September 30, 1993. (sheet 7 of 8)

\begin{tabular}{|c|c|c|c|c|c|c|}
\hline $\begin{array}{l}\text { Well } \\
\text { Name }\end{array}$ & $\begin{array}{c}\text { Collection } \\
\text { Date }\end{array}$ & $\begin{array}{l}\text { Sample } \\
\text { Number }\end{array}$ & $\begin{array}{c}\text { RADIUM } \\
137 / \mathrm{PCI} / \mathrm{L} \\
. / .\end{array}$ & $\begin{array}{l}\text { SOD IUM } \\
34 / \mathrm{ppb} \\
300 / .\end{array}$ & $\begin{array}{l}\text { FSOD IUM } \\
34 / \mathrm{ppb} \\
300 / .\end{array}$ & $\begin{array}{c}\text { SULFATE } \\
124 / \mathrm{ppb} \\
500 / 250000 \mathrm{~s}\end{array}$ \\
\hline . . . . . . . . & $\ldots . . .$. & ............ & $\ldots . .6$. & 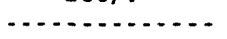 & $\ldots$. & $\ldots+\ldots$ \\
\hline $\begin{array}{l}299-E 25-47 \\
299-E 25-47\end{array}$ & $\begin{array}{l}7 / 16 / 93 \\
7 / 16 / 93\end{array}$ & $\begin{array}{l}\text { BO8R30 } \\
\text { BO8R34 }\end{array}$ & $.03 \mathrm{U}$ & 21000.00 & 22000.00 & 30000.00 \\
\hline $\begin{array}{l}299-E 25-48 \\
299-E 25-48\end{array}$ & $\begin{array}{l}7 / 27 / 93 \\
7 / 27 / 93\end{array}$ & $\begin{array}{l}\text { B08R35 } \\
\text { B08R39 }\end{array}$ & $.09 \mathrm{U}$ & 14000.000 & 14000.000 & 26000.00 \\
\hline $\begin{array}{l}299-E 26-12 \\
299-E 26-12\end{array}$ & $\begin{array}{l}7 / 22 / 93 \\
7 / 22 / 93\end{array}$ & $\begin{array}{l}808 R 40 \\
808 R 44\end{array}$ & $.03 \mathrm{U}$ & 8600.000 & 8500.000 & 7900.00 \\
\hline $\begin{array}{l}299-E 26-13 \\
299-E 26-13\end{array}$ & $\begin{array}{l}7 / 23 / 93 \\
7 / 23 / 93\end{array}$ & $\begin{array}{l}808 R 45 \\
808 R 49\end{array}$ & .15 & 8700.000 & 8500.000 & 8200.00 \\
\hline $\begin{array}{l}699-43-43 \\
699-43-43\end{array}$ & $\begin{array}{l}7 / 28 / 93 \\
7 / 28 / 93\end{array}$ & $\begin{array}{l}\text { B08X11 } \\
808 \times 15\end{array}$ & $.03 \mathrm{U}$ & 9100.000 & 8700.000 & 9200.00 \\
\hline $\begin{array}{l}699-43-45 \\
699-\cdot 3-45\end{array}$ & $\begin{array}{l}7 / 27 / 93 \\
7 / 27 / 93\end{array}$ & $\begin{array}{l}\text { B08X16 } \\
\text { B08xV0 }\end{array}$ & $.13 \mathrm{U}$ & 9100.000 & 9100.000 & 9800.00 \\
\hline
\end{tabular}

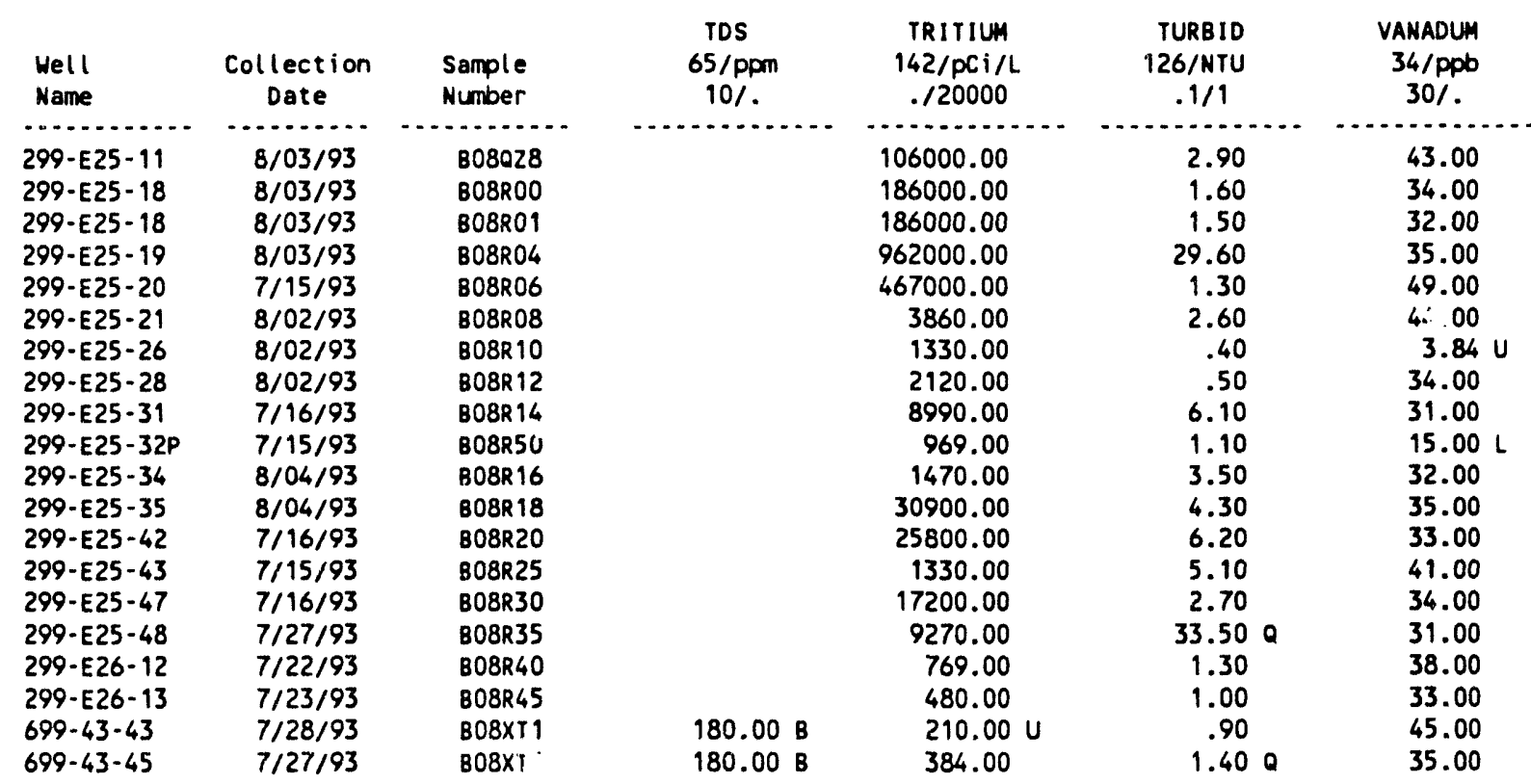

\begin{tabular}{|c|c|c|c|c|c|}
\hline $\begin{array}{l}\text { Well } \\
\text { Mame }\end{array}$ & $\begin{array}{c}\text { rollection } \\
\text { Date }\end{array}$ & $\begin{array}{l}\text { Sample } \\
\text { Number }\end{array}$ & $\begin{array}{c}\text { FVANADI } \\
34 / \mathrm{ppD} \\
30 \%\end{array}$ & $\begin{array}{c}\text { ZINC } \\
34 / \mathrm{ppb} \\
10 \%\end{array}$ & $\begin{array}{c}\text { FZINC } \\
34 / \mathrm{ppb} \\
10 \%\end{array}$ \\
\hline $299-E 25-11$ & $8 / 03 / 93$ & B08Q28 & & $3.44 \mathrm{~L}$ & \\
\hline $299-E 25-11$ & $8 / 03 / 93$ & B08029 & 35.00 & & $3.44 \mathrm{U}$ \\
\hline $299-E 25-18$ & $8 / 03 / 93$ & BOBROO & & 12.00 & \\
\hline $299-E 25-18$ & $8 / 03 / 93$ & B08R01 & & 15.00 & \\
\hline $299-E 25-18$ & $8 / 03 / 93$ & BO8R02 & 3.84 vo & & $3.44 \mathrm{U}$ \\
\hline $299-E 25-18$ & $8 / 03 / 93$ & BO8R03 & 33.000 & & $3.44 \mathrm{U}$ \\
\hline $299-E 25-19$ & $8 / 03 / 93$ & B08R04 & & 130.00 & \\
\hline $299-E 25-19$ & $8 / 03 / 93$ & BC8R05 & 33.00 & & $3.44 \mathrm{U}$ \\
\hline $299-E 25-20$ & $7 / 15 / 93$ & BO8R06 & & 20.00 & \\
\hline $299-E 25-20$ & $7 / 15 / 93$ & BO8R07 & 45.00 & & $3.44 \mathrm{U}$ \\
\hline
\end{tabular}


Table 7-4. Constituents with at Least One Value Above the CRQL for the 216-A-29 Ditch Data for Reporting Period July 1 through September 30,1993 . (sheet 8 of 8 )

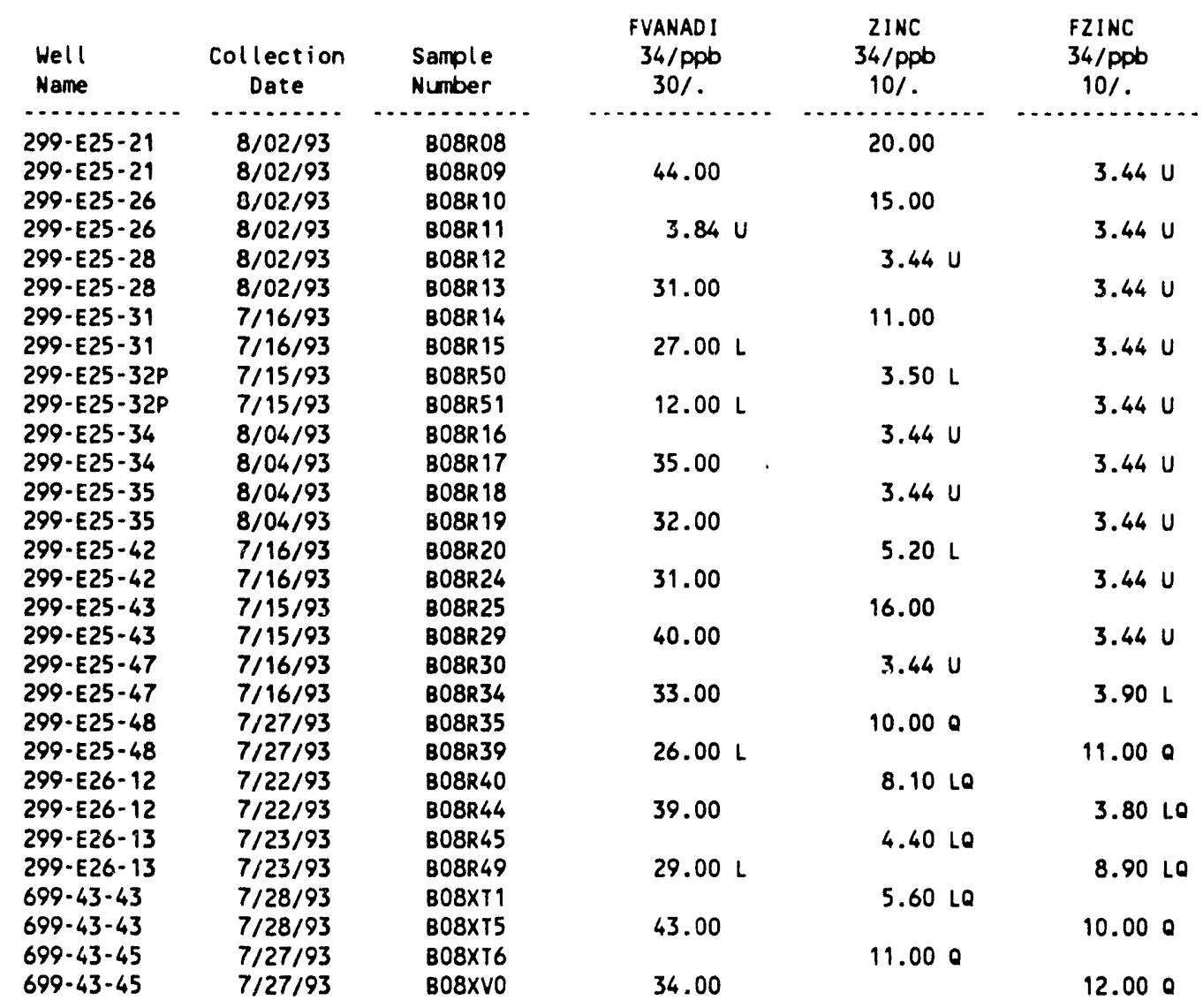

For explanation of this table, see Section 1.4 of report. 
Table 7-5. Contamination Indicator Parameters for the 216-A-29 Ditch Data for Reporting Period July 1 through September 30, 1993.

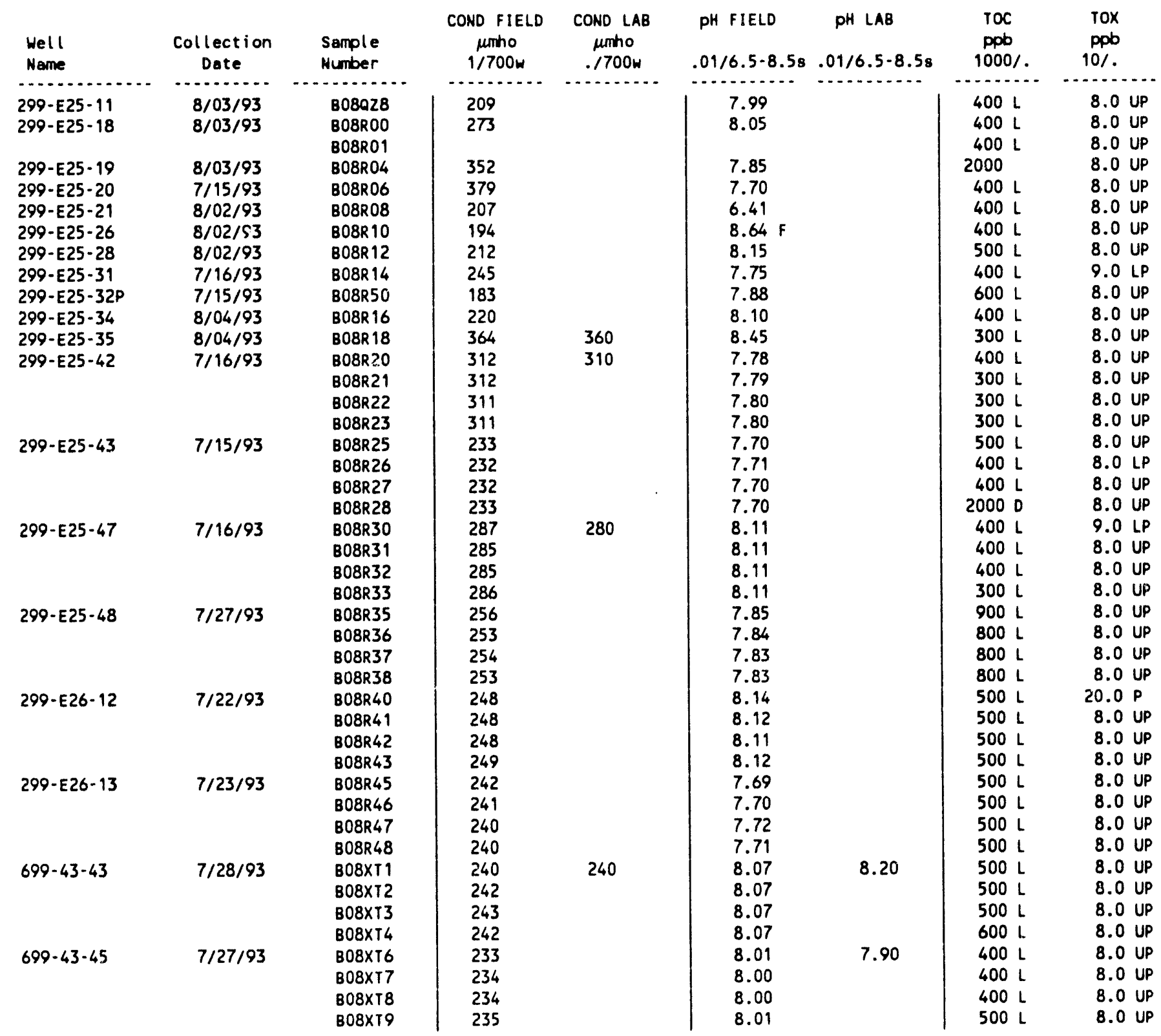

For explanation of this table, see Section 1.4 of report. 
$\mathrm{DOE} / \mathrm{RL}-93-56-3$

This page intentionally left blank. 
DOE/RL-93-56-3

CONTENTS

$8.0216-A-10$ CRIB ..................... . . . . . . . . . .

8.1 INTRODUCTION . . . . . . . . . . . . . . .

8.2 WATER LEVEL MEASUREMENTS . . . . . . . . . . . . . . . 8-1

8.3 WATER CHEMISTRY DATA . . . . . . . . . . . . 8 8-2 


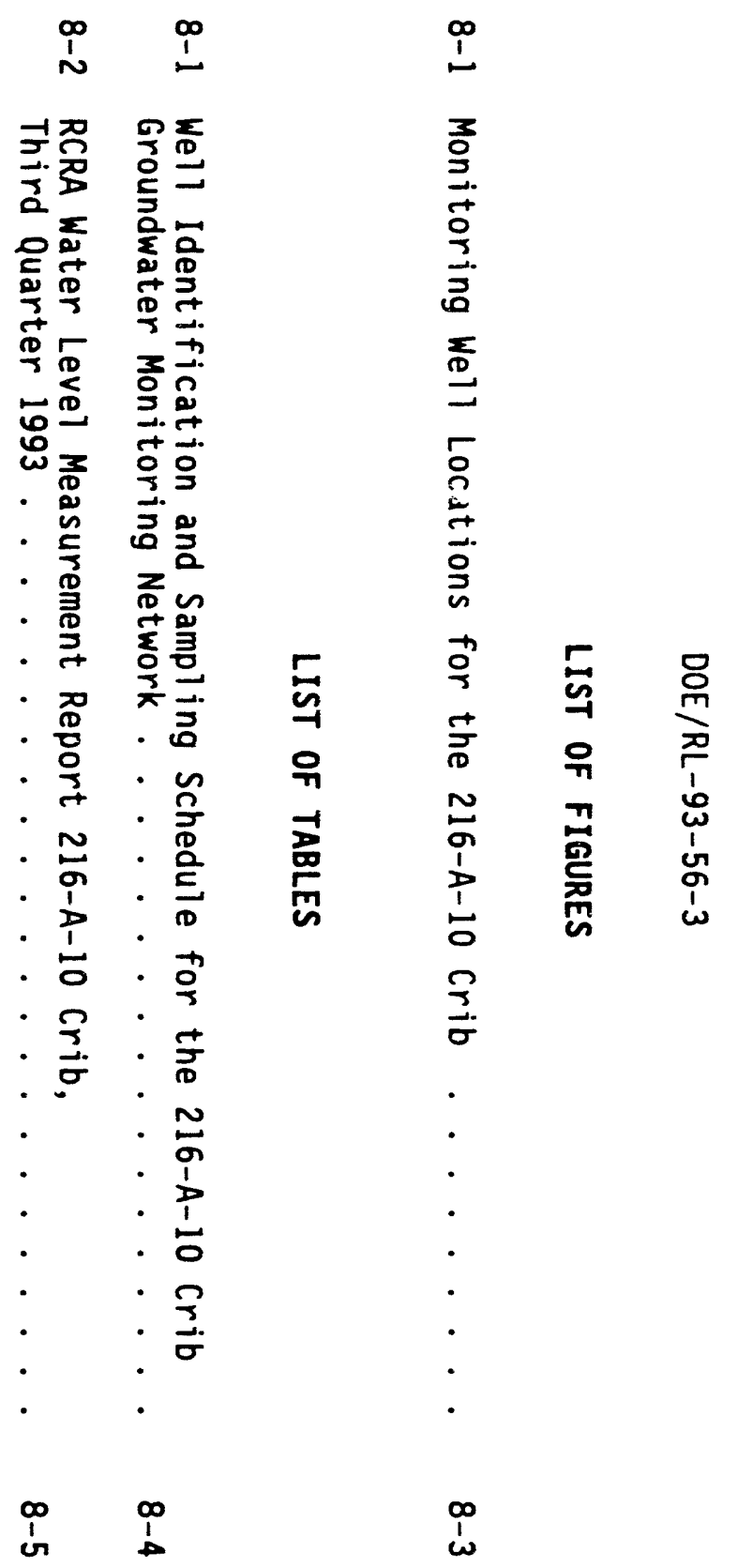




\title{
$8.0216-A-10$ CRIB
}

\author{
G. L. Kasza \\ Westinghouse Hanford Company
}

\subsection{INTRODUCTION}

The 216-A-10 Crib (A-10 Crib) is a retired liquid waste disposal facility that was used during the operation of the PUREX Plant. The crib is located in the 200 East Area approximately $120 \mathrm{~m}(400 \mathrm{ft})$ south of the PUREX Plant (Figures $1-1$ and 8-1). Several waste streams, collectively referred to as the process distillate discharge (PDD), were discharged to the A-10 Crib for soil column disposal during the duration of PUREX Plant operations. PDD waste was first disposed in the crib for a 4-month period during the 1956 PUREX startup. In 1961, the A-10 Crib replaced the 216-A-5 Crib and received PUREX effluent continuously until 1973. Periodic discharges were received in 1977, 1978, and 1981. From 1982 to 1987, effluent discharges to the $A-10 \mathrm{Crib}$ resumed on a continuous basis. Effluent volumes during the period 1981 to 1986 averaged $1 \times 10^{8} \mathrm{~L}\left(2.6 \times 10^{6} \mathrm{gal}\right)$ each year. In 1987 , the $\mathrm{A}-10 \mathrm{Crib}$ was removed from service and replaced by the 216-A-45 Crib.

The PDD waste stream discharged to the A-10 Crib was characteristically acidic and contained concentrated salts. Waste stream constituents included aliphatic hydrocarbon compounds, organic complexants, and radionuclides (plutonium, uranium, strontium-90, cobalt-60, cesium-134 and -137 , ruthenium-103 and -106 , and tritium).

In accordance with the Hanford Federal Facility Agreement and Consent order (Ecology et al. 1992), Milestone M-20-33, RCRA closure/postclosure plans for the A-10 Crib are to be submitted to Ecology and EPA in March 1996. An interim-status, detection-level RCRA groundwater monitoring network has been operational since November 1988.

The RCRA groundwater detection monitoring system consists of six downgradient wells and two upgradient wells, as listed in Table 8-1. Two wells in the 216-A-10 network are shared with the groundwater monitoring network for the 216-A-29 Ditch facility (Tabl? 8-1). Well locations are shown in Figure 8-1. Background monitoring was completed in the fourth quarter of 1989, following the first four quarters of groundwater sampling.

\subsection{WATER LEVEL MEASUREMENTS}

Water level measurements are made on a quarterly basis and during semiannual RCRA sample collection. Water level data collected during the July through September 1993 period are reported in Table 8-2. Measurements from well 299-E24-2 appear consistently anomalous from quarter to quarter, possibly due to survey error. 


\subsection{WATER CHEMISTRY DATA}

Wells in the A-10 Crib groundwater monitoring network are sampled semiannually. No sample collection was scheduled for the July through September 1993 period. In addition, no previously unreported analyses are outstanding. 
$\mathrm{DOE} / \mathrm{RL}-93-56-3$

Figure 8-1. Monitoring We11 Locations for the 216-A-10 Crib.

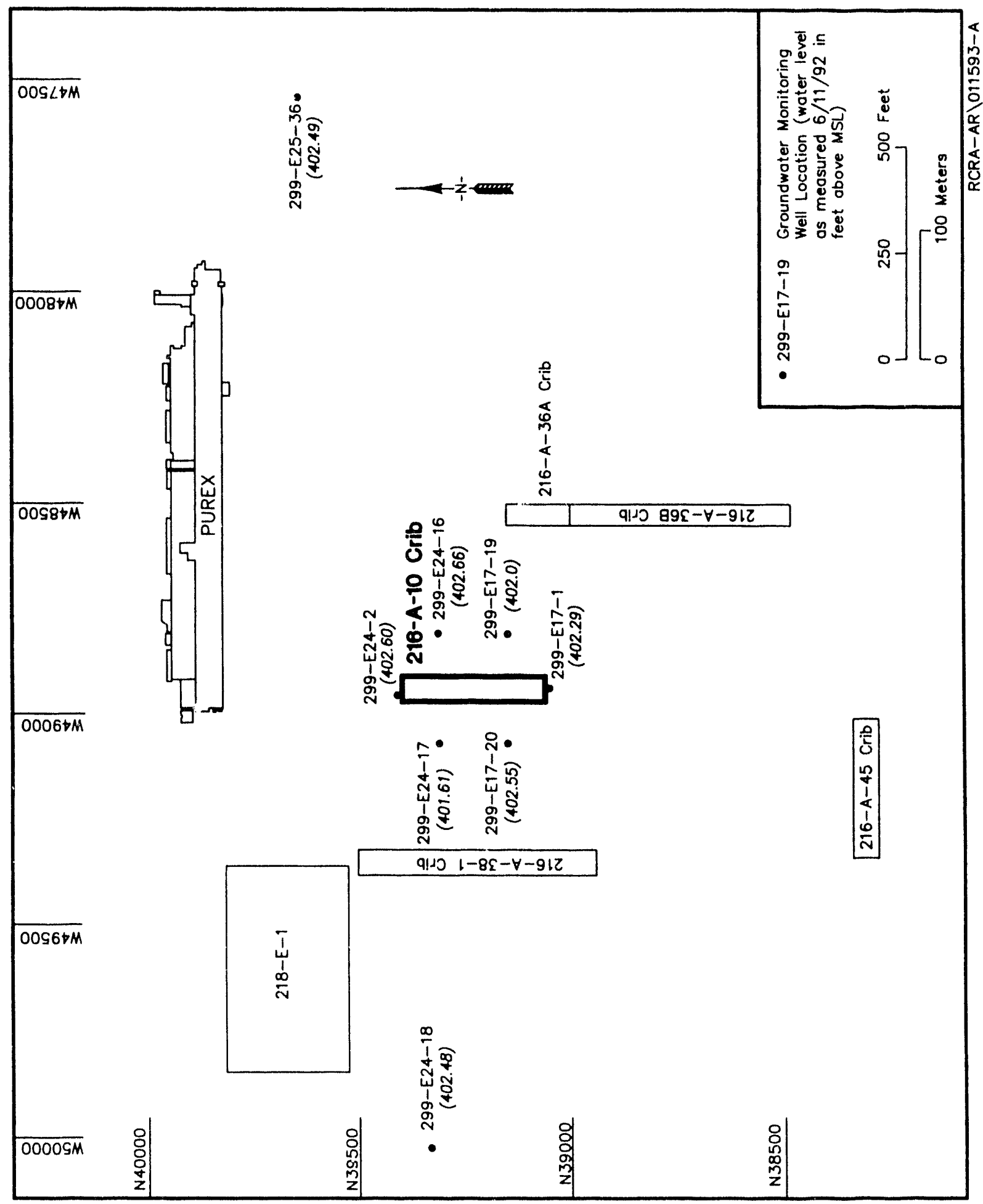


Table 8-1. Well Identification and Sampling Schedule for the 216-A-10 Crib Groundwater Monitoring Network.

\begin{tabular}{|c|c|c|c|c|}
\hline $\begin{array}{c}\text { Well no. } \\
(299-)\end{array}$ & $\begin{array}{l}\text { Relative } \\
\text { position }\end{array}$ & Hydrogeologic unit & $\begin{array}{c}\text { Sample } \\
\text { frequency }\end{array}$ & $\begin{array}{l}\text { Sample date, } \\
\text { 3rd Qtr } 1993\end{array}$ \\
\hline$E 24-18$ & Upgradient & Ringold: Water Table & Semiannually & Not scheduled \\
\hline$E 25-36^{\circ}$ & Upgradient & Ringold: Water Table & Sem:- 'lally & $\bar{b}$ \\
\hline E17-1 & Downgradient & Ringold: Water Table & 'lal1y & Not scheduled \\
\hline E17-19 & Downgradient & Ringold: Water Table & Semi. sally & Not scheduled \\
\hline $\mathrm{E} 17-20^{\circ}$ & Downgradient & Ringold: Water Table & Semiannually & $b$ \\
\hline $\mathrm{E} 24-2$ & Downgradient & Ringold: Water Table & Semiannually & Not scheduled \\
\hline E24-16 & Downgradient & Ringold: Water Table & Semiannually & Not scheduled \\
\hline E24-17 & Downgradient & Ringold: Water Table & Semiannually & Not scheduled \\
\hline
\end{tabular}

Well al so used for 216-A-29 Ditch Groundwater Quality Assessment.

Wells sampled in previous report period. 
Table 8-2. RCRA Water Level Measurement Report 216-A-10 Crib, Third Quarter 1993.

\begin{tabular}{llcl}
\hline We11 & Date & $\begin{array}{c}\text { Depth to } \\
\text { water }(\mathrm{ft})\end{array}$ & $\begin{array}{c}\text { Water level } \\
\text { elevation } \\
\text { above ms }(\mathrm{ft})\end{array}$ \\
\hline $299-\mathrm{E} 17-1$ & $9 / 02 / 93$ & 317.82 & 401.35 \\
$299-\mathrm{E} 17-19$ & $9 / 02 / 93$ & 318.39 & 400.94 \\
$299-E 17-20$ & $9 / 02 / 93$ & 318.05 & 401.18 \\
$299-E 24-2$ & $9 / 02 / 93$ & 313.89 & $403.58+$ \\
$299-E 24-16$ & $9 / 02 / 93$ & 316.69 & 401.58 \\
$299-E 24-17$ & $9 / 02 / 93$ & 318.17 & 400.52 \\
$299-E 24-18$ & $9 / 02 / 93$ & 317.85 & 401.43 \\
$299-E 25-36$ & $9 / 02 / 93$ & 305.76 & 401.63 \\
\hline
\end{tabular}

Notes: 1. Water level elevations are calculated by subtracting the measured depth to water from the surveyed elevation for the well.

2. Depth-to-water values are transcribed from field records.

3. Elevations marked with a ' $t$ ' are outside of the expected range, and are suspected of error.

4. To convert feet to meters multiply by 0.3048 . 
DOE/RL-93-56-3

This page intentionally left blank.

8-6 
$\mathrm{DOE} / \mathrm{RL}-93-56-3$

\section{CONTENTS}

$9.0216-A-36 B$ CRIB ... . . . . . . . . . . . . . . . . . . . . . 9-1

9.1 INTRODUCTION . . . . . . . . . . . . . . . . . . . . . 9-1

9.2 WATER LEVEL MEASUREMENTS . . . . . . . . . . . . . . . . . . . $9-1$

9.3 WATER CHEMISTRY DATA . . . . . . . . . . . . . . . . . . . . 9-2 


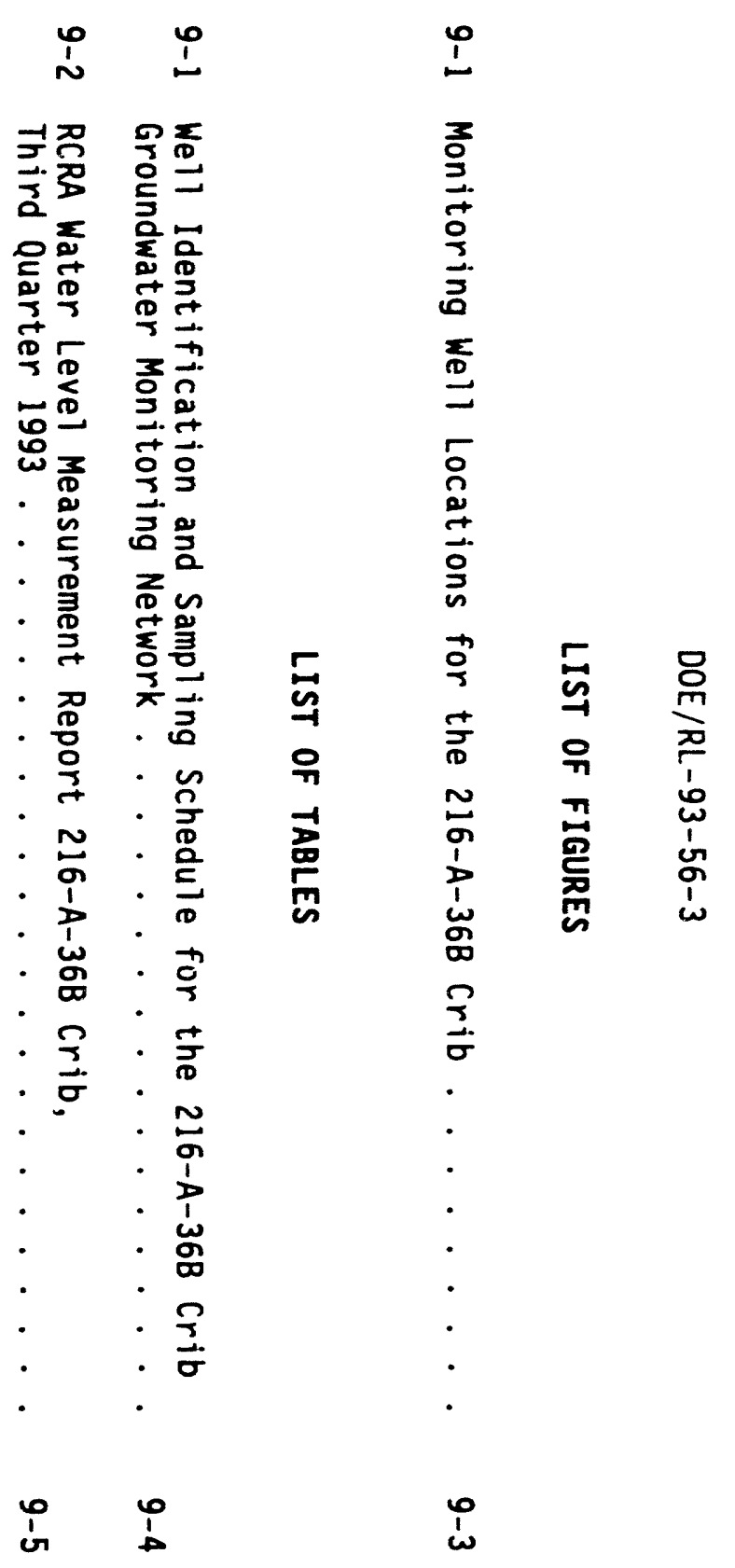




\title{
$9.0216-A-36 B$ CRIB
}

\author{
G. L. Kasza \\ Westinghouse Hanford Company
}

\subsection{INTRODUCTION}

The 216-A-36B Crib (A-36B Crib) is a retired liquid waste disposal facility for the PUREX Plant. The crib is located in the 200 East Area approximately $365 \mathrm{~m}(1,200 \mathrm{ft}$ ) south of the PUREX PIant (Figures $1-1$ and 9-1). The A-36B Crib is the southernmost $152 \mathrm{~m}(500 \mathrm{ft})$ of the original crib (216-A-36). The ammonia scrubber distillate (ASD) waste stream that resulted from PUREX operations was discharged to the crib to percolate through the soil column. The original crib received liquid effluent from September 1965 to March 1966. Because a substantial inventory of radionuclides was disposed to the crib and was assumed to have infiltrated the sediments near the inlet of the crib, a grout curtain was emplaced to partition the crib into two sections, known as $A$ and $B$. Discharge to the $A-36 B$ Crib section resumed in March 1966 and continued until 1972 when the crib was temporarily removed from service. The crib was placed back in service in November 1982 and operated until it was retired from use in October 1987.

ASD effluent disposed in the A-36B Crib was the condensate from the fuel rod decladding operation. In this process, the zircalloy cladding was removed from the irradiated fuel by dissolving the cladding in a boiling solution of ammonium fluoride and ammonium nitrate. Waste stream constituents are known to have included radionuclides (tritium, strontium-90, cesium-137, ruthenium-106, cobait-60, and uranium) and hazardous substances (ammonium, fluoride, and nitrate).

In accordance with the Hanford Federal Facility Agreement and Consent order (Ecology et a1. 1992), Milestone M-20-34, RCRA closure/postclosure plans for the A-36B Crib are to be submitted to Ecology and the EPA in March 1996. An interim-status RCRA groundwater monitoring network has been in place at the crib since May 1988.

The RCRA groundwater detection monitoring system consists of six downgradient wells and one upgradient well, as listed in Table 9-1. Well locations are shown in Figure 9-1. Background monitoring was performed for seven quarters. Indicator parameter critical means were calculated using four quarters of data from September 1988 to June 1989. As shown in Table 9-1, data from well 299-E17-15 are al so used by the 216-A-29 Ditch Groundwater Quality Assessment.

\subsection{WATER LEVEL MEASUREMENTS}

Water level measurements are made on a quarterly basis and during semiannual RCRA sample collection. Water level data collected during the January through March 1993 period are reported in Table 9-2. 


\subsection{WATER CHEMISTRY DATA}

Wells in the A-36B Crib Groundwater Monitoring Network are sampled semiannually; however, the network was not scheduled for sampling in the July through September 1993 period. No unreported analytical data from prior sampling periods are outstanding. 


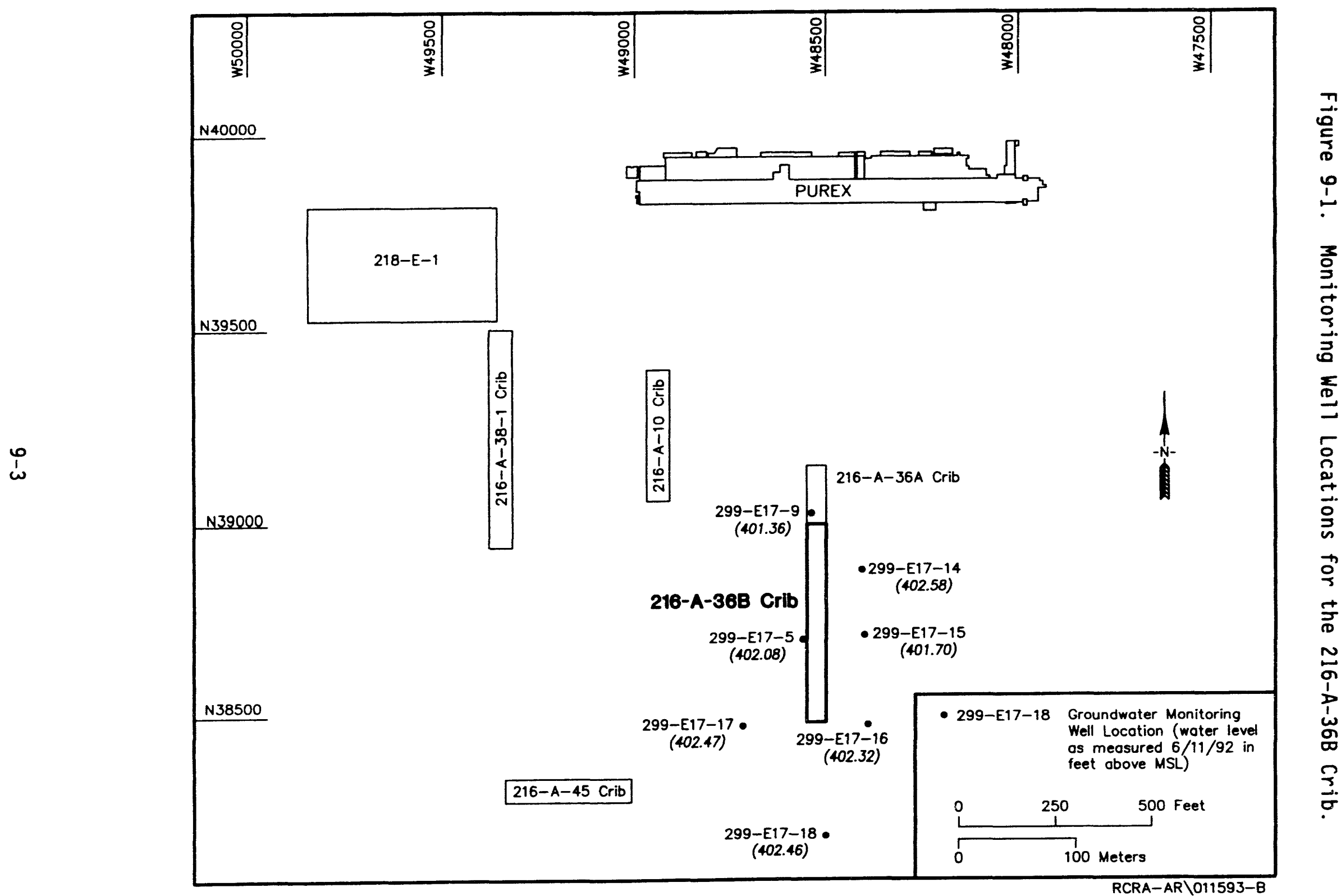


Table 9-1. Well Identification and Sampling Schedule for the 216-A-36B Crib Groundwater Monitoring Network.

\begin{tabular}{|c|c|c|c|c|}
\hline $\begin{array}{c}\text { Well no. } \\
(299-)\end{array}$ & $\begin{array}{c}\text { Relativo } \\
\text { position }\end{array}$ & Hydrogeologic unit & $\begin{array}{c}\text { Sample } \\
\text { frequency }\end{array}$ & $\begin{array}{c}\text { Sample date, } \\
\text { 3rd Qtr 1993 }\end{array}$ \\
\hline E17-17 & Upgradient & Ringold: Water Table & Semiannually & Not scheduled \\
\hline E17-5 & Downgradient & Ringold: Water Table & Semiannually & Not scheduled \\
\hline E17-9 & Downgradient & Ringold: Water Table & Semiannually & Not scheduled \\
\hline E17-14 & Downgradient & Ringold: Water Table & Semiannually & Not scheduled \\
\hline E17-15 & Downgradient & Ringold: Water Table & Semiannually & b \\
\hline E17-16 & Downgradient & Ringold: Water Table & Semiannually & Not scheduled \\
\hline E17-18 & Downgradient & Ringold: Water Table & Semiannually & Not scheduled \\
\hline
\end{tabular}

"Well shared with the 216-A-29 Ditch network.

Well sampled in previous report period. 
Table 9-2. RCRA Water Level Measurement Report 216-A-36B Crib, Third Quarter 1993.

\begin{tabular}{llcc}
\hline We11 & Date & $\begin{array}{c}\text { Wepth to } \\
\text { water }(\mathrm{ft})\end{array}$ & $\begin{array}{c}\text { Water level } \\
\text { elevation } \\
\text { above ms }\end{array}$ \\
\hline $299-\mathrm{ft})$
\end{tabular}

Notes: 1. Water level elevations are calculated by subtracting the measured depth to water from the surveyed elevation for the well.

2. Depth-to-water values are transcribed from field records.

3. To convert feet to meters multiply by 0.3048 . 
$\mathrm{DOE} / \mathrm{RL}-93-56-3$

This page intentionally left blank. 
DOE/RL-93-56-3

\section{CONTENTS}

$10.0216-$ B-63 TRENCH . . . . . . . . . . . . . . 10-1

10.1 INTRODUCTION ...................... 10-1

10.2 WATER LEVEL MEASUREMENTS . . . . . . . . . . . 10-1

10.3 WATER CHEMISTRY DATA .............. 10-1 
$\mathrm{DOE} / \mathrm{RL}-93-56-3$

\section{LIST OF FIGURES}

10-1 Well Location Map for the 216-B-63 Trench. . . . . . . . . . 10-3

\section{LIST OF TABLES}

10-1 Monitoring Well Purpose and Sampling Schedule for the

216-B-63 Trench Network . . . . . . . . . . . . . . . . . . 10-4

10-2 RCRA Water Level Measurement Report 216-B-63 Trench,

Third Quarter 1993 . . . . . . . . . . . . . . . 10-5

10-3 Constituent List and Summary of Results for the 216-B-63 Trench

Data for Reporting Period July 1 through September 30, 1993 . . . 10-6

10-4 Constituents with at Least One Value Above the CRQL for the 216-B-63 Trench Data for Reporting Period July 1 through

September 30,1993 ................ 10-12

10-5 Contamination Indicator Parameters for the 216-B-63 Trench Data

for Reporting Period July 1 through September 30, 1993 . . . . 10-15 


\title{
10.0 216-B-63 TRENCH
}

\author{
M. D. Sweeney \\ Westinghouse Hanford Company
}

\subsection{INTRODUCTION}

A RCRA detection-level groundwater monitoring network is being established for the 216-B-63 Trench. The 216-B-63 Trench received wastewater containing hazardous waste and radioactive materials from the $B$ Plant, located in the 200 East Area (Figures $1-1$ and 10-1). Liquid effluent discharge to the 216-B-63 Trench ceased in February 1992. However, the trench is being retained to receive $B$ Plant discharges in the event of an emergency.

The 216-B-63 is an open, unlined, earthen trench in servjce from March 1970 until February 1992. The trench received $4.0 \times 10^{5}$ to $1.5 \times 10^{6} \mathrm{~L} /$ day $(100,000$ to $400,000 \mathrm{gal} / \mathrm{day})$ of 1 iquid effluent from the $B$ Plant chemical sewer, none of which is now designated as dangerous waste. The effluent was a mixture of steam condensate and raw water. Documented hazardous discharges occurred from 1970 to October 1985 and consisted of aqueous sulfuric acid and sodium hydroxide solutions exceeding the pH limits of 2.0 and 12.5, respectively. Radioactive soils were dredged from the trench in August 1970. In 1985, physical controls, radiation monitors, and operating procedures were modified to avoid inadvertent discharge of chemicals or radioactive substances to the wastewater stream.

Four quarters of groundwater background sampling have been completed for all of the wells in the network. Background sampling is done to establish background water quality, in accordance with the requirements outlined in 40 CFR 265 Interim-Status Indicator-Evaluation Groundwater Monitoring Plan for the 216-B-63 Trench (PNL 1989). The nine wells within the network are now on a semiannual sampling program. Based on the present groundwater flow direction, seven of the wells are downgradient and five are upgradient of the trench (Figure 10-1). The purpose of the wells, their sampling schedule, and the dates sampled are summarized in Table 10-1. As indicated in Table 10-1, some wells in the 216-B-63 network are shared with the Low-Level Burial Grounds (LLBG) and single-shell tanks (SST) groundwater monitoring networks.

\subsection{WATER LEVEL MEASUREMENTS}

Water level measurements obtained during the July through September 1993 period are presented in Table 10-2. Wells 299-E33-36, 299-E34-8, 299-E34-10, and 299-E33-37 were resurveyed to verify these elevations. Water in well 299-E33-36 remains lower than in nearby wells in the network. Reasons for this apparent discrepancy are currently under investigation.

\subsection{WATER CHEMISTRY DATA}

Groundwater samples were collected from the network from July to September 1993. The se data are shown in Tables 10-3 through 10-5. The constituent list and summary of results are provided in Table 10-3. 
Constituents with at least one value above the CRQL for all available data are presented in Table 10-4. Several results for analytes from two wells have not been received at the writing of this report (299-E27-18 and 299-E27-19).

Constituents found in concentrations above DWS in samples from the July through September 1993 sampling period include chromium in unfiltered samples from wells 299-E27-11, 299-E27-17, 299-E27-8, 299-E27-9, and 299-E34-10;

coliform in well 299-E27-19; iron in unfiltered samples from wells 299-E27-8, 299-E27-9, 299-E34-10, 299-E27-11, and 299-E27-17; and turbidity in

wells 299-E27-19 and 299-E27-18. A RADE has been submitted for these results. Results for filtered samples for both iron and chromium continue to be well below DWS.

A critical mean has not been calculated due to laboratory problems (see Section 1.4). No exceedances for any of the parameters were noted in any of the wells.

Non-DWS constituents with laboratory qualifiers and QC flags indicating data problems include sodium and TC. Over $50 \%$ of the sodium results have $Q$ flags, indicating suspect $Q C$ data.

Over $50 \%$ of TC results are flagged with 'B.' This flag indicates that the blanks associated with these samples were found to be contaminated. It is unknown at this time whether the analyte concentrations were corrected for these blank concentrations. This issue is currently under investigation. 


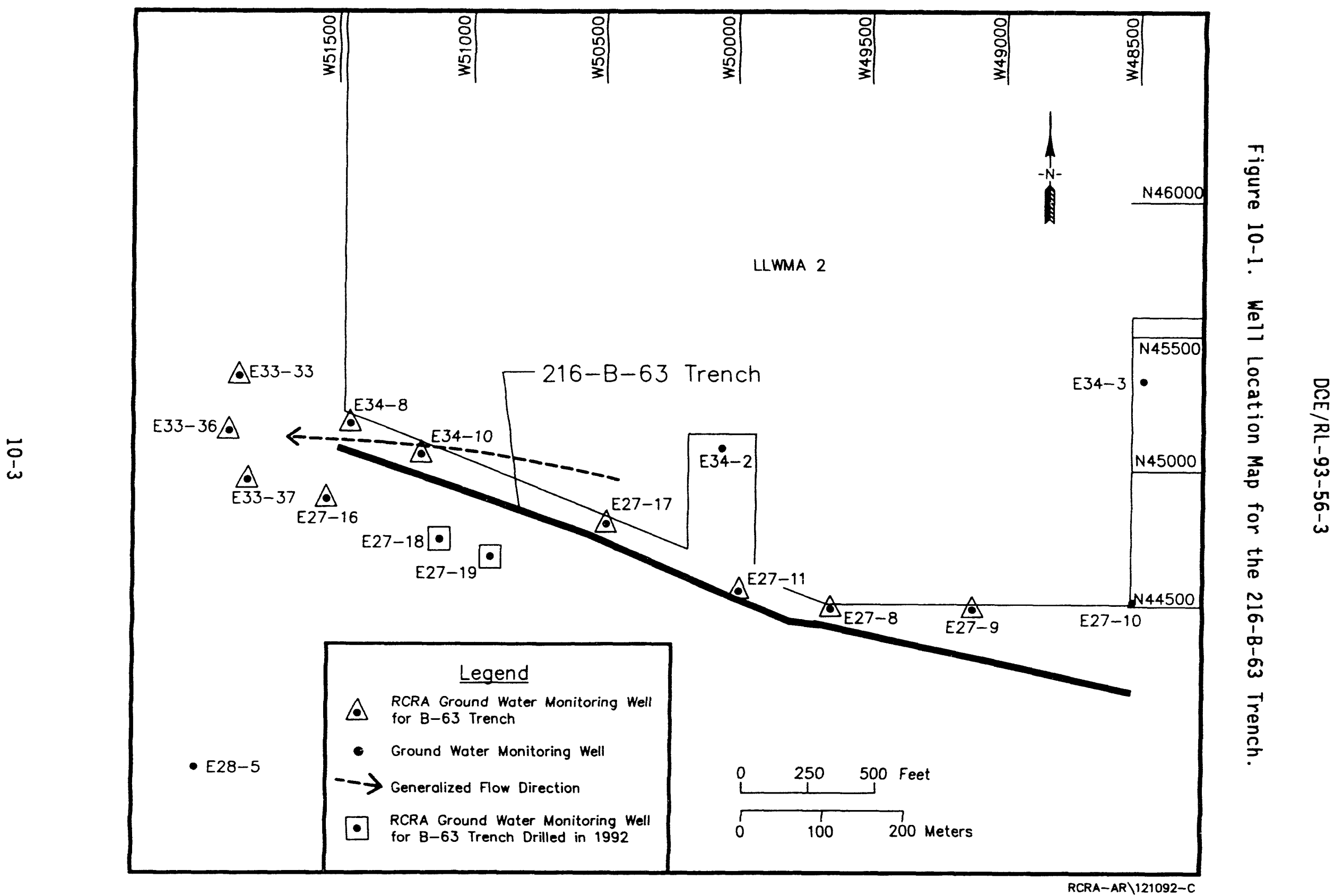


Table 10-1. Monitoring Well Purpose and Sampling Schedule for the 216-B-63 Trench Network.

\begin{tabular}{|c|c|c|c|c|}
\hline $\begin{array}{c}\text { Well no. } \\
(299-)\end{array}$ & $\begin{array}{l}\text { Relative } \\
\text { position }\end{array}$ & Hydrogeologic unit & $\begin{array}{c}\text { Sample } \\
\text { frequency }\end{array}$ & Sample date \\
\hline$E 27-8^{a}$ & Upgradient & Hanford: Water Table & Semiannually & $8 / 04 / 93$ \\
\hline$E 27-9^{\circ}$ & Upgradient & Hanford: Water Table & Semiannually & $8 / 04 / 93$ \\
\hline $\mathrm{E} 27-11^{\circ}$ & Upgradient & Hanford: Water Table & Semiannually & $7 / 14 / 93$ \\
\hline$E 27-16$ & Downgradient & Hanford: Water Table & Semiannually & $6 / 07 / 93$ \\
\hline$E 27-17^{\circ}$ & Upgradient & Hanford: Water Table & Semiannually & $7 / 14 / 93$ \\
\hline E27-18 & Downgradient & Hanford: Water Table & Quarterly & $9 / 15 / 93$ \\
\hline E27-19 & Downgradient & Hanford: Water Table & Quarterly & $9 / 15 / 93$ \\
\hline$E 33-33^{b}$ & Downgradient & Hanford: Water Table & Semiannually & $3 / 01 / 93$ \\
\hline$E 33-36^{b}$ & Downgradient & Hanford: Water Table & Semiannually & $3 / 11 / 93$ \\
\hline E33-37 & Downgradient & Hanford: Water Table & Semiannually & $6 / 14 / 93$ \\
\hline$E 34-8$ & Downgradient & Hanford: Water Table & Semiannually & $6 / 07 / 93$ \\
\hline$E 34-10^{\circ}$ & Upgradient & Hanford: Water Table & Semiannually & $7 / 14 / 93$ \\
\hline
\end{tabular}

Well shared with the Low-Level Burial Grounds facility network.

Well shared with the single-shell tanks facility network. 
Table 10-2. RCRA Water Level Measurement Report 216-B-63 Trench, Third Quarter 1993.

\begin{tabular}{|c|c|c|c|}
\hline Well & Date & $\begin{array}{l}\text { Depth to } \\
\text { water (ft) }\end{array}$ & $\begin{array}{l}\text { Water level } \\
\text { elevation } \\
\text { above msl (ft) }\end{array}$ \\
\hline \multicolumn{4}{|c|}{ Wells Monitoring the Top of the Unconfined Aquifer } \\
\hline $299-E 27-8$ & $\begin{array}{l}8 / 04 / 93 \\
9 / 09 / 93\end{array}$ & $\begin{array}{l}236.00 \\
236.02\end{array}$ & $\begin{array}{l}401.83^{\star} \\
401.81\end{array}$ \\
\hline $299-E 27-9$ & $\begin{array}{l}8 / 04 / 93 \\
9 / 09 / 93\end{array}$ & $\begin{array}{l}227.20 \\
227.34\end{array}$ & $\begin{array}{l}402.01 * \\
401.87\end{array}$ \\
\hline 299-E27-11 & $\begin{array}{l}7 / 14 / 93 \\
9 / 09 / 93\end{array}$ & $\begin{array}{l}241.50 \\
241.70\end{array}$ & $\begin{array}{l}401.79 * \\
401.59\end{array}$ \\
\hline $299-E 27-16$ & $9 / 07 / 93$ & 250.58 & 401.55 \\
\hline $299-E 27-17$ & $\begin{array}{l}7 / 14 / 93 \\
9 / 09 / 93\end{array}$ & $\begin{array}{l}232.59 \\
232.77\end{array}$ & $\begin{array}{l}402.13^{\star} \\
401.95\end{array}$ \\
\hline $299-E 27-18$ & $9 / 15 / 93$ & 248.29 & $401.86 *$ \\
\hline $299-E 27-19$ & $9 / 15 / 93$ & 249.00 & $401.88^{\star}$ \\
\hline $299-E 33-33$ & $\begin{array}{l}7 / 20 / 93 \\
8 / 19 / 93 \\
9 / 21 / 93\end{array}$ & $\begin{array}{l}238.38 \\
238.34 \\
238.53\end{array}$ & $\begin{array}{l}401.79 \\
401.83 \\
401.64\end{array}$ \\
\hline $299-E 33-36$ & $9 / 07 / 93$ & 246.33 & 400.34 \\
\hline $299-E 33-37$ & $9 / 07 / 93$ & 251.30 & 401.71 \\
\hline $299-E 34-8$ & $9 / 07 / 93$ & 239.20 & 401.32 \\
\hline $299-E 34-10$ & $\begin{array}{l}7 / 14 / 93 \\
9 / 09 / 93\end{array}$ & $\begin{array}{l}237.62 \\
237.78\end{array}$ & $\begin{array}{l}402.15^{\star} \\
401.99\end{array}$ \\
\hline
\end{tabular}

Notes: 1. Water level elevations are calcu?ated by subtracting the measured depth to water from the surveyed elevation for the well.

2. Depth-to-water values are transcribed from field records.

3. Elevations marked with an ' $*$ ' were measured at the time of sampling.

4. To convert feet to meters multiply by 0.3048 . 
Table 10-3. Constituent List and Summary of Results for the 216-B-63 Trench Data for Reporting Period July 1 through September 30, 1993. (sheet 1 of 6)

CONTAMINATION INDICATOR PARAMETERS

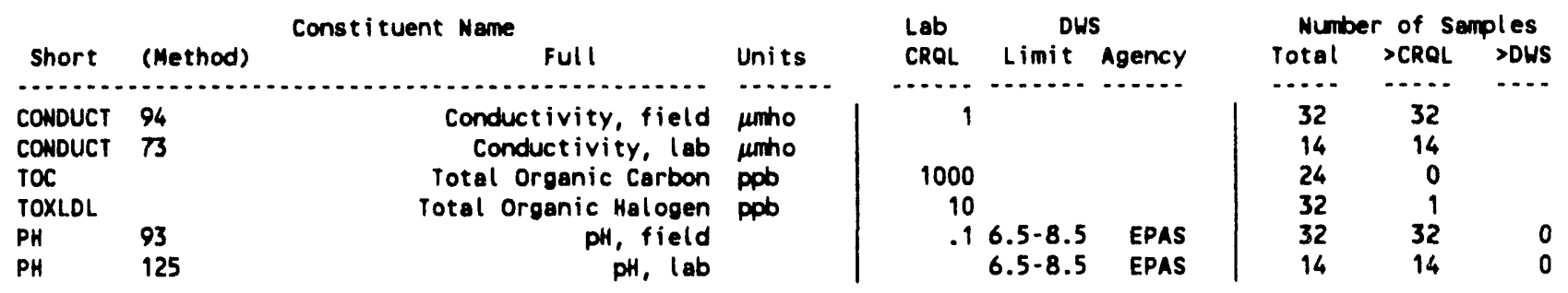

DRINKING WATER PARAMETERS

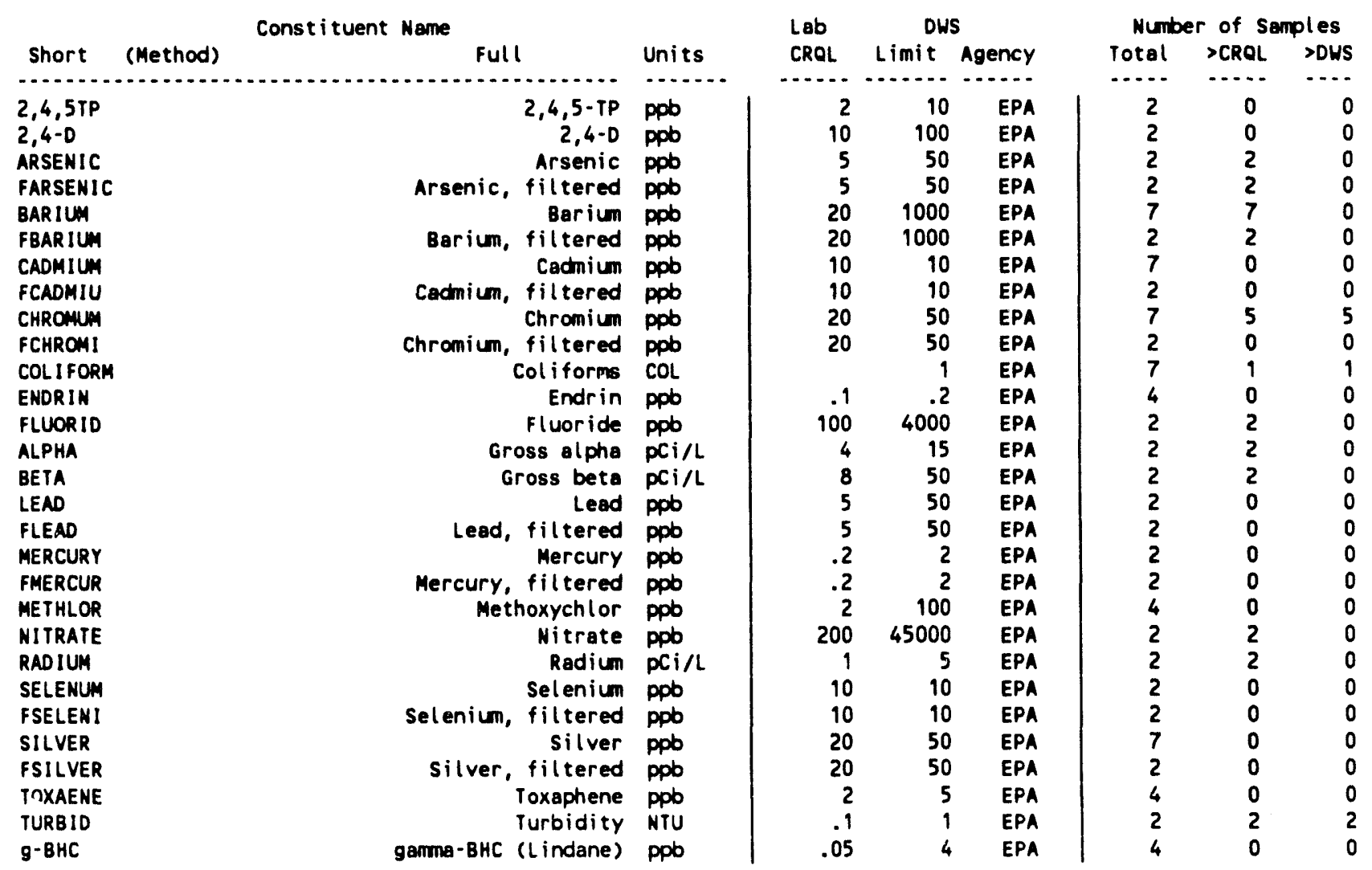

GROUNDWATER QUALITY PARAMETERS

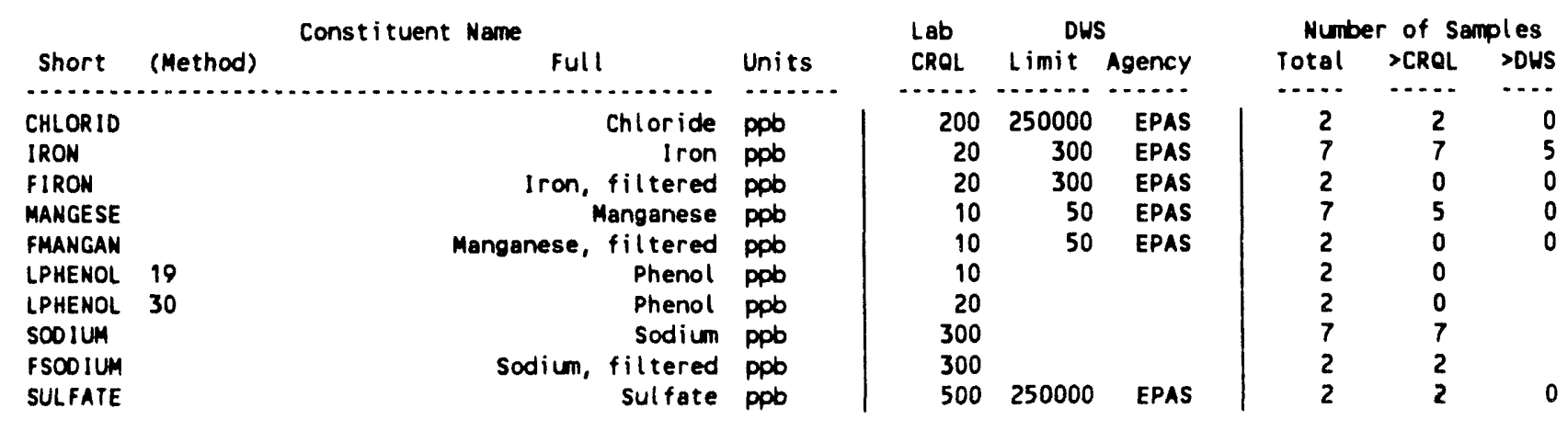


Table 10-3. Constituent List and Summary of Results for the 216-B-63 Trench Data for Reporting Period July 1 through September 30, 1993. (sheet 2 of 6)

SITE SPECIFIC AND OTHER CONSTITUENTS

\begin{tabular}{|c|c|c|c|c|c|c|c|c|}
\hline \multirow[b]{2}{*}{ Short } & \multirow[b]{2}{*}{ (Method) } & \multirow{2}{*}{ Constituent Name } & \multirow[b]{2}{*}{ Units } & \multirow{2}{*}{$\begin{array}{l}\text { Lab } \\
\text { CRQL }\end{array}$} & DHS & \multicolumn{3}{|c|}{ Number of Samples } \\
\hline & & & & & Limit Agency & Total & $>$ CROL & $>$ DWS \\
\hline$\cdots \cdots$ & $\cdots$ & $x^{\prime}$ & $\ldots \ldots$ & $\ldots \ldots$ & $\ldots \ldots \ldots$ & $\cdots \cdots$ & $\ldots$. & $\cdots$ \\
\hline $1112-t c$ & & $1,1,1,2$-Tetrachloroethane & ppob & 5 & & 2 & 0 & \\
\hline $1,1,1-T$ & & 1,1,1-Trichloroethane & ppb & 5 & & 10 & 0 & \\
\hline $1122-t c$ & & $1,1,2,2$-Tetrachloroethane & ppb & 5 & & 2 & 0 & \\
\hline $1,1,2-T$ & & 1,1,2-Trichloroethane & ppb & 5 & & 10 & 0 & \\
\hline $1,1-01 C$ & & 1,1-Dichloroethane & ppob & 5 & & 10 & 0 & \\
\hline DICETHY & & 1,1-Dichloroethene & ppb & 5 & & 2 & 0 & \\
\hline $123-t r p$ & & 1,2,3-Trichloropropane & ppb & 5 & & 2 & 0 & \\
\hline TETRCH8 & & $1,2,4,5-$ Tetrachlorobenzene & ppb & 10 & & 2 & 0 & \\
\hline TRICHLB & & $1,2,4-$ Trichlorobenzene & ppb & 10 & & 2 & 0 & \\
\hline DIBRCHL & & 1,2-0 ibromo-3-chloropropane & ppb & 5 & & 2 & 0 & \\
\hline DIBRETH & & 1,2-Dibromoethane & ppb & 5 & & 2 & 0 & \\
\hline 12-cben & & 1,2-Dichlorobenzene & ppb & 10 & & 2 & 0 & \\
\hline $1,2-01 C$ & & 1,2-Dichloroethane & ppb & 5 & & 10 & 0 & \\
\hline 12-DCE & & 1,2-0ichloroethene & ppb & 5 & & 10 & 0 & \\
\hline DICPANE & & 1,2-o ichloropropane & ppb & 5 & & 2 & 0 & \\
\hline 13-dben & & 1,3-Dichlorobenzene & ppb & 10 & & 2 & 0 & \\
\hline 14-otben & 16 & 1,4-Dichlorobenzene & ppob & 5 & & 8 & 0 & \\
\hline 14-dben & 19 & 1,4-Dichlorobenzene & ppb & 10 & & 2 & 0 & \\
\hline DIOXANE & & 1,4-Dioxane & ppb & 200 & & 2 & 0 & \\
\hline NAPHQUI & & 1,4-Naphtoquinone & ppb & 10 & & 2 & 0 & \\
\hline 1BUTYN & & 1-Butanol & ppb & 1000 & & 10 & 0 & \\
\hline 1-naphe & & 1-Naph thyl amine & ppb & 10 & & 2 & 0 & \\
\hline TETRCHP & & $2,3,4,6$-Tetrachlorophenol & ppb & 10 & & 2 & 0 & \\
\hline $2,4,5-T$ & & $2,4,5-T$ & ppb & 2 & & 2 & 0 & \\
\hline $245-\operatorname{trp}$ & & 2,4,5-Trichlorophenol & ppb & 10 & & 2 & 0 & \\
\hline 246-trp & 19 & 2,4,6-Trichlorophenol & ppb & 10 & & 2 & 0 & \\
\hline $246-\operatorname{trp}$ & 30 & 2,4,6-Trichlorophenol & ppb & 5 & & 2 & 0 & \\
\hline 24-dchp & 19 & 2,4-Dichlorophenol & ppb & 10 & & 2 & 0 & \\
\hline 24-dchp & 30 & 2,4-Dichlorophenol & ppb & 5 & & 2 & 0 & \\
\hline DIMPHEN & 19 & 2,4-0 ime thylphenol & ppb & 10 & & 2 & 0 & \\
\hline DIMPHEN & 30 & 2,4-D imethylphenol & ppb & 5 & & 2 & 0 & \\
\hline DINPHEN & 19 & 2,4-Dinitrophenol & ppb & 50 & & 2 & 0 & \\
\hline DINPHEN & 30 & 2,4-Dinitrophenol & ppb & 150 & & 2 & 0 & \\
\hline 24 -dint & & 2,4-D initrotoluene & ppb & 10 & & 2 & 0 & \\
\hline 26-dchp & 19 & 2,6-0 ichlorophenol & ppb & 10 & & 2 & 0 & \\
\hline 26-dchp & 30 & 2,6-Dichlorophenol & ppb & 5 & & 2 & 0 & \\
\hline 26-dint & & 2,6-Dinitrotoluene & ppb & 10 & & 2 & 0 & \\
\hline ACEFENE & & 2-Acetylaminofluorene & ppb & 10 & & 2 & 0 & \\
\hline CHLNAPH & & 2-Chloronaphthalene & ppb & 10 & & 2 & 0 & \\
\hline CHLPHEN & 19 & 2-Chlorophenol & ppb & 10 & & 2 & 0 & \\
\hline CHLPHEN & 30 & 2-Chlorophenol & ppb & 5 & & 2 & 0 & \\
\hline 2HEXANO & & 2-Hexanone & ppb & 50 & & 2 & 0 & \\
\hline 2MENAPH & & 2-Methylnaph thalene & ppo & 10 & & 2 & 0 & \\
\hline 2METHPH & & 2-Methylphenol & ppb & 10 & & 2 & 0 & \\
\hline 2-napha & & 2-Naph thyl amine & ppb & 10 & & 2 & 0 & \\
\hline ONITANI & & 2-Nitroaniline & ppb & 50 & & 2 & 0 & \\
\hline 2NITPH & 19 & 2-Nitrophenol & ppb & 10 & & 2 & 0 & \\
\hline 2NITPH & 30 & 2-Nitrophenol & ppb & 5 & & 2 & 0 & \\
\hline PICOLIN & & 2-picoline & ppb & 10 & & 2 & 0 & \\
\hline BUTDINP & 30 & 2-sec-Butyl-4,6-dinitrophenol (ON & ppb & 5 & & 2 & 0 & \\
\hline BUTDINP & 49 & 2-sec-Butyl-4,6-dinitrophenol (DN & ppb & 1 & & 2 & 0 & \\
\hline DICHBEN & & $3,3^{\prime}-\mathrm{Dich}$ lorobenzidine & ppb & 20 & & 2 & 0 & \\
\hline DIMEYLB & & 3,3'-D imethyl benzidine & ppb & 10 & & 2 & 0 & \\
\hline METCHAN & & 3-Methylcholanthrene & ppb & 10 & & 2 & 0 & \\
\hline MNITANI & & 3-Nitroanil ine & ppb & 50 & & 2 & 0 & \\
\hline
\end{tabular}


Table 10-3. Constituent List and Summary of Results for the 216-B-63 Trench Data for Reporting Period July 1 through September 30, 1993. (sheet 3 of 6)

\begin{tabular}{|c|c|c|c|c|c|c|c|c|}
\hline \multirow[b]{2}{*}{ Short } & \multirow[b]{2}{*}{ (Method) } & \multirow[t]{2}{*}{ Constituent Name } & \multirow[b]{2}{*}{ Units } & \multirow{2}{*}{$\begin{array}{l}\text { Lab } \\
\text { CRQL }\end{array}$} & DWS & \multicolumn{3}{|c|}{ Number of Semples } \\
\hline & & & & & Limit Agency & Total & $>C R Q L$ & $>$ DUS \\
\hline$\ldots \ldots$ & & (n) & $\ldots \ldots$ & 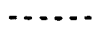 & $\ldots \ldots \ldots$ & $\ldots \ldots$ & $\cdots$ & $\ldots$ \\
\hline DDD & & $4,4^{\prime}-D D D$ & ppb & .1 & & 4 & 0 & \\
\hline DDE & & $4,4^{\prime}-$ DDE & ppb & .05 & & 4 & 0 & \\
\hline DDT & & $4,4^{\prime}-$ DDT & ppb & .1 & & 4 & 0 & \\
\hline 460N2MP & 19 & 4,6-0 initro-2-methylphenol & ppb & 50 & & 2 & 0 & \\
\hline 460N2MP & 30 & 4,6-Dinitro-2-methylphenol & ppb & 200 & & 2 & 0 & \\
\hline AMINUYL & & 4-Aminobiphenyl & ppb & 10 & & 2 & 0 & \\
\hline BROPHEN & & 4-Bromophenylphenyl ether & ppb & 10 & & 2 & 0 & \\
\hline CHLCRES & 19 & 4-Chloro-3-methylphenol & ppb & 20 & & 2 & 0 & \\
\hline CHLCRES & 30 & 4-Chloro-3-methylphenol & ppb & 5 & & 2 & 0 & \\
\hline CHLANIL & & 4-Chloroaniline & ppb & 20 & & 2 & 0 & \\
\hline 4CFPETH & & 4-Chlorophenylphenyl ether & ppb & 10 & & 2 & 0 & \\
\hline MIBK & & 4-Methyl-2-pentanone & ppb & 50 & & 10 & 0 & \\
\hline 4METHPH & & 4-Methylphenol & ppb & 10 & & 2 & 0 & \\
\hline NITRANI & & 4-Nitroaniline & ppb & 50 & & 2 & 0 & \\
\hline NITPHEN & 19 & 4-Nitrophenol & ppob & 50 & & 2 & 0 & \\
\hline NITPHEN & 30 & 4-Nitrophenol & ppb & 30 & & 2 & 0 & \\
\hline 4NITQUI & & 4-Nitroquinol ine-1-oxide & ppb & 10 & & 2 & 0 & \\
\hline NITRTOL & & 5-Nitro-0-toluidine & ppb & 10 & & 2 & 0 & \\
\hline DIMBENZ & & 7, 12-D imethyl benz [a] anthracene & ppb & 10 & & 2 & 0 & \\
\hline ACENAPH & & Aceraph thene & ppb & 10 & & 2 & 0 & \\
\hline ACENATL & & Acenaphthylene & ppb & 10 & & 2 & 0 & \\
\hline ACETONE & & Acetone & ppb & 100 & & 10 & 0 & \\
\hline ACETILE & & Acetonitrile & pob & 200 & & 2 & 0 & \\
\hline ACETOPH & & Acetophenone & ppb & 10 & & 2 & 0 & \\
\hline ACROLIN & & Acrolein & ppb & 5 & & 2 & 0 & \\
\hline ACRYILE & & Acrylonitrile & ppo & 5 & & 2 & 0 & \\
\hline ALDRIN & & Aldrin & ppb & .05 & & 4 & 0 & \\
\hline DIMPHAM & & Alpha, alpha-D imethyl phenethyl ami & ppob & 10 & & 2 & $\mathbf{0}$ & \\
\hline a-BHC & & Alpha-BHC & ppob & .05 & & 4 & $\mathbf{0}$ & \\
\hline ALUMNUM & & Aluminum & ppb & 200 & & 7 & 1 & \\
\hline FALUMIN & & Aluminum & ppb & 200 & & 2 & 0 & \\
\hline AMMONIU & & Ammonium ion & ppb & 100 & & 2 & 0 & \\
\hline ANILINE & & Aniline & ppb & 10 & & 2 & 0 & \\
\hline ANTHRA & & Anthracene & ppb & 10 & & 2 & 0 & \\
\hline ANT IONY & & Ant imony & ppb & 200 & & 7 & 0 & \\
\hline FANTIMO & & Antimony, filtered & ppb & 200 & & 2 & 0 & \\
\hline$S B-125$ & & Ant imony-125 & $\mathrm{pCi} / \mathrm{L}$ & & & 7 & 1 & \\
\hline ARAMITE & & Aramite & ppb & 10 & & 2 & 0 & \\
\hline AR 1016 & & Aroclor-1016 & ppb & 1 & & 1 & 0 & \\
\hline AR1221 & & Aroclor-1221 & ppb & 1 & & 1 & 0 & \\
\hline AR1232 & & Aroclor-1232 & ppb & 1 & & 1 & 0 & \\
\hline AR 1242 & & Aroclor -1242 & ppb & 1 & & 1 & 0 & \\
\hline AR 1248 & & Aroclor -1248 & ppb & 1 & & 1 & 0 & \\
\hline AR1254 & & Aroclor -1254 & ppb & 1 & & 1 & 0 & \\
\hline AR 1260 & & Aroclor -1260 & ppb & 1 & & 1 & 0 & \\
\hline BENZENE & & Benzene & ppb & 5 & & 10 & 0 & \\
\hline BENZAAN & & Benzo (a) anthracene & ppb & 10 & & 2 & 0 & \\
\hline BENZOPY & & Benzo(a)pyrene & ppb & 10 & & 2 & 0 & \\
\hline BENZBFL & & Benzo(b)f luoranthene & ppb & 10 & & 2 & 0 & \\
\hline BGHIPER & & Benzo(ghi)perylene & $p p b$ & 10 & & 2 & 0 & \\
\hline BNZKFLU & & Benzo(k) fluoranthene & ppb & 10 & & 2 & 0 & \\
\hline BENZOTH & & Benzothiazole & ppb & 10 & & 2 & 0 & \\
\hline BENZALC & & Benzyl alcohol & ppb & 20 & & 2 & 0 & \\
\hline BERYLUM & & Beryllium & ppb & 3 & & 7 & 0 & \\
\hline FBERYLL & & Beryllium, filtered & ppb & 3 & & 2 & 0 & \\
\hline b-BHC & & Beta-BHC & ppb & .05 & & 4 & 0 & \\
\hline BIS2CHM & & Bis (2-Choroethoxy)methane & ppb & 10 & & 2 & 0 & \\
\hline BIS2CHE & & Bis(2-chloroethyl) ether & ppb & 10 & & 2 & 0 & \\
\hline
\end{tabular}


Table 10-3. Constituent List and Summary of Results for the 216-B-63 Trench Data for Reporting Period July 1 through September 30, 1993. (sheet 4 of 6)

\begin{tabular}{|c|c|c|c|c|c|c|c|c|}
\hline \multirow[b]{2}{*}{ Short } & Constituent Nene & \multirow[b]{2}{*}{ Units } & \multirow{2}{*}{$\begin{array}{l}\text { Lab } \\
\text { CROL }\end{array}$} & \multicolumn{2}{|l|}{ DWS } & \multicolumn{3}{|c|}{ Number of Semples } \\
\hline & (Method) & & & Limit & Agency & Total & $>$ CRQL & $>$ DWS \\
\hline 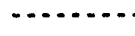 & 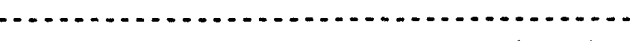 & $\ldots . . .$. & $\ldots .$. & $\ldots \ldots$ & $\cdots \ldots$ & $\ldots .$. & $\ldots$ & $-\cdots$ \\
\hline BISZETH & Bis(2-chloroisopropyl) ether & ppb & 10 & & & 2 & 0 & \\
\hline BISZEPH & Bis(2-ethylhexyl) phthalate & ppb & 10 & & & 2 & 0 & \\
\hline BROMIDE & Bromide & ppb & 500 & & & 2 & 0 & \\
\hline $\mathrm{BOCM}$ & Bromodichloromethane & ppb & 5 & 100 & EPA & 2 & 0 & 0 \\
\hline BROMORM & Bromoform & ppb & 5 & 100 & EPA & 2 & 0 & 0 \\
\hline BUTBENP & Butylbenzylphthalate & ppb & 10 & & & 2 & 0 & \\
\hline CALCIUM & Calcium & ppb & 100 & & & 7 & 7 & \\
\hline FCALCIU & Calcium, filtered & ppb & 100 & & & 2 & 2 & \\
\hline CARBIDE & Carbon disulfide & ppb & 5 & & & 10 & 0 & \\
\hline TETRANE & Carbon tetrachloride & ppb & 5 & & & 10 & 0 & \\
\hline CS-137 & Cesium-137 & $\mathrm{pCi} / \mathrm{L}$ & 20 & & & 7 & 0 & \\
\hline CHLOANE & Chlordane & pob & .1 & & & 4 & 0 & \\
\hline CHLROB & Chlorobenzene & ppb & 5 & & & 2 & 0 & \\
\hline ChLLATE & Chlorobenzilate & ppb & 10 & & & 2 & 0 & \\
\hline CLETHAN & Chloroethane & ppb & 10 & & & 2 & 0 & \\
\hline CHLFORM & Chloroform & ppo & 5 & 100 & EPA & 10 & 0 & 0 \\
\hline CHLPRENE & Chloroprene & ppb & 5 & & & 2 & 0 & \\
\hline CHRYSEN & Chrysene & ppb & 10 & & & 2 & 0 & \\
\hline COBALT & Cobalt & ppb & 20 & & & 7 & 0 & \\
\hline FCOBALT & Cobalt, filtered & ppb & 20 & & & 2 & 0 & \\
\hline$c 0-60$ & Cobalt -60 & $\mathrm{pCi} / \mathrm{L}$ & & & & 7 & 0 & \\
\hline COPPER & Copper & ppb & 20 & 1000 & EPAS & 7 & 1 & 0 \\
\hline FCOPPER & Copper, filtered & ppb & 20 & 1000 & EPAS & 2 & 0 & 0 \\
\hline CRESOLS & Cresols (methylphenols) & ppb & 10 & & & 2 & 0 & \\
\hline$d-B H C$ & Delta-BHC & ppb & .1 & & & 4 & 0 & \\
\hline DIBPHTH & Di-n-butylphthalate & ppb & 10 & & & 2 & 0 & \\
\hline DIOPHTH & Di-n-octylphthalate & ppb & 10 & & & 2 & 0 & \\
\hline DIALLATE & Diallate & ppb & 10 & & & 2 & 0 & \\
\hline DIBAHAN & D ibenz $[a, h]$ anthracene & ppb & 10 & & & 2 & 0 & \\
\hline DIBENFR & Dibenzofuran & ppb & 10 & & & 2 & 0 & \\
\hline BRCHLMET & Dibromochloromethane & ppb & 5 & 100 & EPA & 2 & 0 & 0 \\
\hline DIBRMET & Dibromomethane & ppb & 5 & & & 2 & 0 & \\
\hline DICDIFM & Dishlorodifluorome thane & ppo & 5 & & & 2 & 0 & \\
\hline DIELRIN & Dieldrin & ppb & .05 & & & 4 & 0 & \\
\hline DIEPHTH & Diethyl phthalate & ppb & 10 & & & 2 & 0 & \\
\hline DIMETHO & Dimethoate & ppb & 10 & & & 2 & $\mathbf{0}$ & \\
\hline DIMPHTH & Dimethyl phthalate & ppb & 10 & & & 2 & 0 & \\
\hline DIPHAMI & Diphenyl amine & ppb & 10 & & & 2 & 0 & \\
\hline DISULFO & Disulfoton & ppb & 2 & & & 2 & 0 & \\
\hline ENDO1 & Endosulfan I & ppb & .1 & & & 4 & 0 & \\
\hline ENDO2 & Endosulfan II & ppb & .05 & & & 4 & 0 & \\
\hline ENDSFAN & Endosulfan sulfate & ppb & .5 & & & 4 & 0 & \\
\hline ENDRALD & Endrin Aldehyde & ppb & .2 & & & 4 & 0 & \\
\hline ETHCYAN & Ethyl cyanide & ppb & 10 & & & 10 & 0 & \\
\hline ETHMETH & Ethyl methacrylate & ppb & 5 & & & 2 & 0 & \\
\hline ETHMETS & Ethyl methanesul fonate & ppb & 10 & & & 2 & 0 & \\
\hline ETHBENZ & Ethylbenzene & ppb & 5 & & & 2 & 0 & \\
\hline FAMPHUR & Famphur & ppb & 10 & & & 2 & 0 & \\
\hline FLUORAN & Fluoranthene & ppo & 10 & & & 2 & 0 & \\
\hline FLRENE & Fluorene & ppb & 10 & & & 2 & 0 & \\
\hline HEPTLOR & Heptachlor & ppb & .05 & & & 4 & 0 & \\
\hline HEPT IOE & Heptachlor epoxide & ppb & 1 & & & 4 & 0 & \\
\hline HEXCBEN & Hexachl orobenzene & ppb & 10 & & & 2 & 0 & \\
\hline HEXCBUT & Hexachlorobutadiene & ppb & 10 & & & 2 & 0 & \\
\hline HEXCCYC & Hexachlorocyclopent adi ene & ppb & 10 & & & 2 & 0 & \\
\hline HEXCETH & Hexachloroethane & ppb & 10 & & & 2 & 0 & \\
\hline HEXACHL & Hexachlorophene & ppb & 10 & & & 2 & 0 & \\
\hline HEXAENE & Hexach loropropene & ppb & 10 & & & 2 & 0 & \\
\hline
\end{tabular}


Table 10-3. Constituent List and Summary of Results for the 216-B-63 Trench Data for Reporting Period July 1 through September 30, 1993 . (sheet 5 of 6)

\begin{tabular}{|c|c|c|c|c|c|c|c|c|}
\hline \multicolumn{4}{|c|}{ Const ituent Name } & \multicolumn{2}{|r|}{ DWS } & \multicolumn{3}{|c|}{ Number of Samples } \\
\hline Short & (Method) & Full & Units & CROL & Limit Agency & Total & $>$ CROL & $>$ DWS \\
\hline$\cdots \ldots \ldots$ & $\cdots$ & $n_{1}$ & $\ldots \ldots$ & $\cdots \cdots$ & $\ldots \ldots \ldots$ & $\cdots+-$ & $\ldots \ldots$ & $\cdots$ \\
\hline INDENOP & & Indeno $(1,2,3-\mathrm{cd})$ pyrene & ppb & 10 & & 2 & 0 & \\
\hline ISOBUTY & & I sobutyl alcohol & ppb & 200 & & 2 & 0 & \\
\hline ISOORIN & & Isodrin & ppb & 10 & & 2 & 0 & \\
\hline ISOPHER & & Isophorone & ppb & 10 & & 2 & 0 & \\
\hline ISOSOLE & & Isosafrole & ppb & 10 & & 2 & 0 & \\
\hline KEPONE & & Kepone & ppb & 10 & & 2 & 0 & \\
\hline KEROSEN & & Kerosene & ppb & 10000 & & 2 & 0 & \\
\hline MAGNES & & Magnesium & ppb & 100 & & 7 & 7 & \\
\hline FMAGNES & & Magnesium, filtered & ppo & 100 & & 2 & 2 & \\
\hline METHACR & & Methacrylonitrile & ppb & 5 & & 2 & 0 & \\
\hline METHAPY & & Methapyrilene & ppb & 10 & & 2 & 0 & \\
\hline IOOONET & & Methyl lodide & ppb & 5 & & 2 & 0 & \\
\hline METHBRO & & Methyl bromide & ppb & 10 & & 2 & 0 & \\
\hline METHCHL & & Methyl chloride & ppb & 10 & & 2 & 0 & \\
\hline METHONE & & Methyl ethyl ketone & ppb & 100 & & 10 & 0 & \\
\hline METACRY & & Methyl methacrylate & ppb & 5 & & 2 & 0 & \\
\hline METMSUL & & Methyl methanesulfonate & ppb & 10 & & 2 & 0 & \\
\hline METHPAR & & Methyl parathion & ppb & .5 & & 2 & 0 & \\
\hline METHYCH & & Methylene chloride & ppb & 5 & & 10 & 0 & \\
\hline DIPRNIT & & N-Nitroso-di-n-dipropylamine & ppb & 10 & & 2 & 0 & \\
\hline NNIBUTY & & N-Nitrosodi-n-butylamine & ppb & 10 & & 2 & 0 & \\
\hline NAIDIEY & & N-Nitrosodiethylamine & ppb & 10 & & 2 & 0 & \\
\hline NNIDIME & & $N-N i$ trosodimethylamine & ppb & 10 & & 2 & 0 & \\
\hline NND IPHA & & N-Nitrosodiphenylamine & ppb & 10 & & 2 & 0 & \\
\hline NNIMETH & & $\mathrm{N}$-Nitrosome thylethylamine & ppb & 10 & & 2 & 0 & \\
\hline NNIMORP & & $N-N i t r o s o m o r p h o l$ ine & ppob & 10 & & 2 & 0 & \\
\hline NNIPIPE & & N-Nitrosopiperidine & ppb & 10 & & 2 & 0 & \\
\hline NAPHTHA & & Naphthalene & ppb & 10 & & 2 & 0 & \\
\hline NICKEL & & Nickel & ppb & 30 & & 7 & 5 & \\
\hline FNICKEL & & Nickel, filtered & ppb & 30 & & 2 & 0 & \\
\hline NITRITE & & Nitrite & ppb & 200 & & 2 & 0 & \\
\hline NITBENZ & & Nitrobenzene & ppb & 10 & & 2 & 0 & \\
\hline NITRPYR & & Nitrosopyrrolidine & ppb & 10 & & 2 & 0 & \\
\hline TRIPHOS & & $0,0,0$-Triethyl phosphorothioate & ppb & 10 & & 2 & 0 & \\
\hline DIPHOS & & 0,0-diethyl0-2-pyraz inylphosphor & ppb & 10 & & 2 & 0 & \\
\hline PARATHI & & Parathion & ppb & 10 & & 2 & 0 & \\
\hline PENTCHB & & Pentachlorobenzene & ppb & 10 & & 2 & 0 & \\
\hline PENTACH & & Pentachlor oethane & ppb & 5 & & 2 & 0 & \\
\hline PENTCHN & & Pentachloronitrobenzene (PCNB) & ppb & 10 & & 2 & 0 & \\
\hline PENTCHP & 19 & Pentachlorophenol & ppb & 50 & & 2 & 0 & \\
\hline PENTCHP & 30 & Pentachlorophenol & ppb & 100 & & 2 & 0 & \\
\hline PHENTIN & & Phenacetin & ppb & 10 & & 2 & 0 & \\
\hline PHENANT & & Phenanthrene & ppb & 10 & & 2 & 0 & \\
\hline PHORATE & & Phorate & ppb & 2 & & 2 & 0 & \\
\hline PHOSPHA & & Phosphote & ppb & 400 & & 2 & 0 & \\
\hline POTASUM & & Potassium & ppb & 300 & & 7 & 7 & \\
\hline FPOTASS & & Potassium, filtered & ppb & 300 & & 2 & 2 & \\
\hline PRONIDE & & Pronamide & ppb & 10 & & 2 & 0 & \\
\hline PYRENE & & Pyrene & ppb & 10 & & 2 & 0 & \\
\hline PYRIOIN & & Pyridine & ppb & 10 & & 2 & 0 & \\
\hline$R U-106$ & & Ruthenium-106 & $\mathrm{pCi} / \mathrm{L}$ & & & 7 & 0 & \\
\hline SAFROL & & Safrol & ppb & 10 & & 2 & 0 & \\
\hline STYRENE & & Styrene & ppb & 5 & & 2 & 0 & \\
\hline PERCENE & & Tetrachloroethene & ppb & 5 & & 10 & 0 & \\
\hline TETPHNL & & Tetrachlorophenols & ppb & 10 & & 2 & 0 & \\
\hline PYROPHOS & & Tetraethyldi thiopyrophosphate & ppb & 10 & & 2 & 0 & \\
\hline
\end{tabular}


Table 10-3. Constituent List and Summary of Results for the 216-B-63 Trench Data for Reporting Period July 1 through September 30, 1993. (sheet 6 of 6 )

\begin{tabular}{|c|c|c|c|c|c|c|c|c|c|}
\hline \multirow[b]{2}{*}{ Short } & \multirow[b]{2}{*}{ (Method) } & \multirow{2}{*}{$\begin{array}{l}\text { Constituent Name Full } \\
\text { foll }\end{array}$} & \multirow[b]{2}{*}{ Units } & \multirow{2}{*}{$\begin{array}{l}\text { Lab } \\
\text { CRQL }\end{array}$} & \multicolumn{2}{|c|}{ DWS } & \multicolumn{3}{|c|}{ Number of Samples } \\
\hline & & & & & Limit & Agency & Total & $>\mathrm{CROL}$ & >DWS \\
\hline$\cdots \cdot$ & & (n) & $\cdots$ & (n) & (n) & $\cdots$ & (n) & $\cdots$ & $\cdots$ \\
\hline TAF & & Tetrahydrofuran & ppb & 10 & & & 10 & 0 & \\
\hline TIN & & $\operatorname{Tin}$ & ppb & 100 & & & 7 & 0 & \\
\hline FTIN & & Tin, filtered & ppb & 100 & & & 2 & 0 & \\
\hline TOLUENE & & Toluene & ppb & 5 & & & 10 & 0 & \\
\hline & & Total Carbon & ppb & 2000 & & & 7 & 7 & \\
\hline TDS & & Total Dissolved Solids & ppm & 10 & & & 7 & 7 & \\
\hline TRIBUTPH & & Tributyl Phosphate & ppb & 10 & & & 2 & 0 & \\
\hline TRICENE & & Trichloroethene & ppb & 5 & & & 10 & 0 & \\
\hline TRCMFLM & & Trichl or omonof luor ome thane & ppb & 5 & & & 2 & 0 & \\
\hline TRIPHNL & & Trichlorophenols & ppb & 5 & & & 2 & 0 & \\
\hline TOICHPH & & Tris-2-chloroethyl phosphate & ppb & 10 & & & 2 & 0 & \\
\hline TRITIUM & & Tritium & $\mathrm{pCi} / \mathrm{L}$ & 500 & 20000 & EPA & 2 & 2 & 0 \\
\hline URANIUM & & Uranium & ppo & .5 & & & 2 & 2 & \\
\hline VANADUM & & Vanadium & ppb & 30 & & & 7 & 3 & \\
\hline FVANADI & & Vanadium, filtered & ppb & 30 & & & 2 & $\mathbf{0}$ & \\
\hline VIHYLAC & & Vinyl acetate & ppb & 5 & & & 2 & 0 & \\
\hline VINYIDE & & Vinyl chloride & ppb & 10 & 2 & EPA & 10 & 0 & 0* \\
\hline XYLENE & & Xylenes (total) & ppo & 5 & & & 10 & 0 & \\
\hline ZINC & & zinc & ppb & 10 & & & 7 & 4 & \\
\hline FZINC & & zine, filtered & ppb & 10 & & & 2 & 0 & \\
\hline ALLYLCL & & allylchloride & ppb & 100 & & & 2 & 0 & \\
\hline TOICPENE & & cis-1,3-Dichloropropene & ppb & 5 & & & 2 & 0 & \\
\hline MCRESOL & & $m$-Cresol & ppb & 10 & & & 2 & 0 & \\
\hline DINBENZ & & m-dinitrobenzene & ppb & 10 & & & 2 & 0 & \\
\hline OTOLHYD & & o-Toluidine & $\mathrm{ppb}$ & 10 & & & 2 & 0 & \\
\hline DIMEAMB & & p-Dimethylaminoazobenzene & ppo & 10 & & & 2 & 0 & \\
\hline PHENDIA & & p-Phenylenediamine & ppb & 10 & & & 2 & 0 & \\
\hline SYMTRIN & & sym-Trinitrobenzene & ppb & 10 & & & 2 & 0 & \\
\hline DICPENE & & trans-1,3-Dichloropropene & ppb & 5 & & & 2 & 0 & \\
\hline TOIBUTEN & & trans-1,4-dichloro-2-butene & ppb & 5 & & & 2 & 0 & \\
\hline
\end{tabular}

For explanation of this table, see section 1.4 of report. 
Table 10-4. Constituents with at Least One Value Above the CRQL for the 216-B-63 Trench Data for Reporting Period July 1 through September 30, 1993. (sheet 1 of 3)

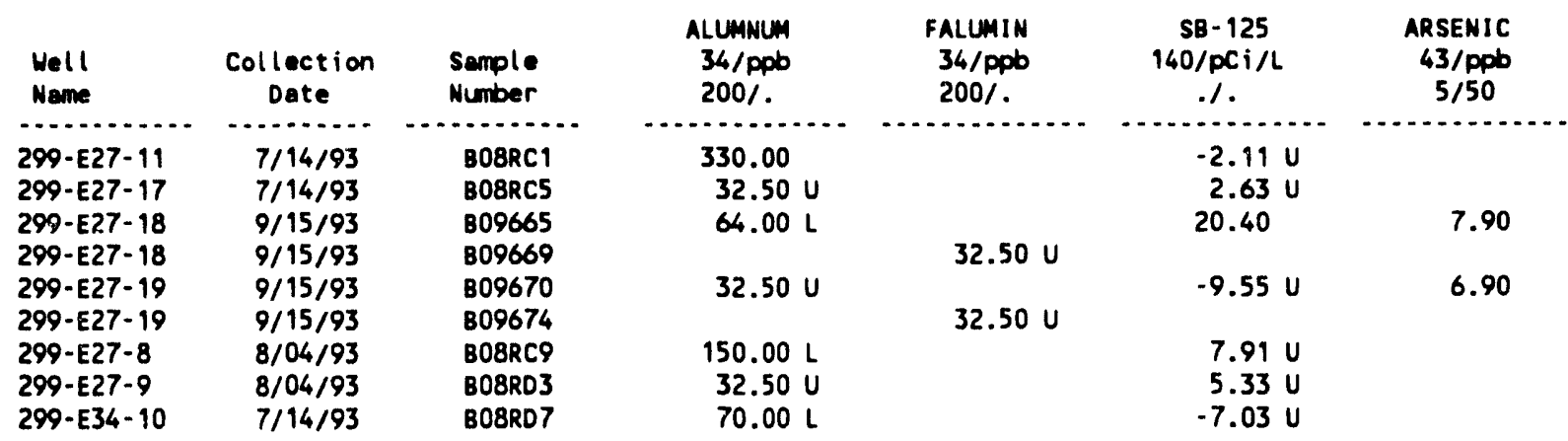

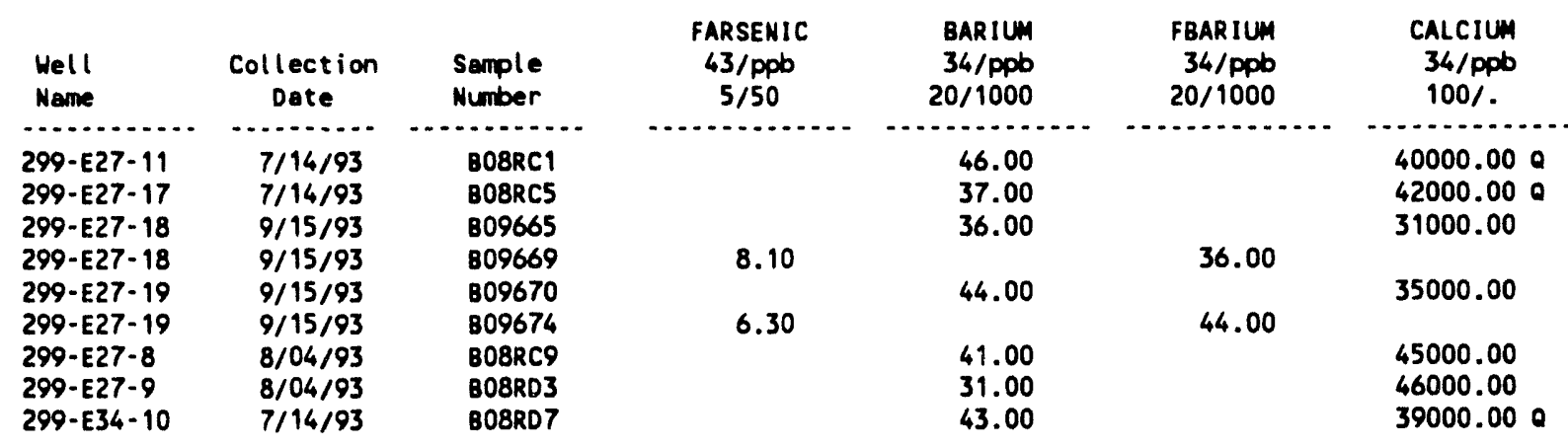

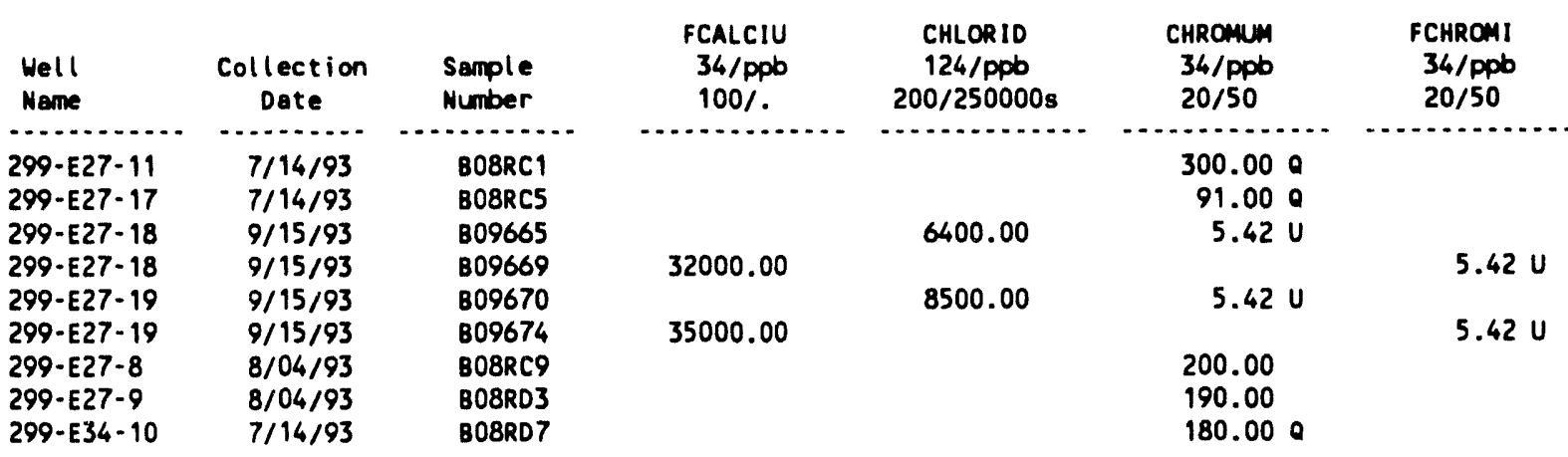

\begin{tabular}{|c|c|c|c|c|c|c|}
\hline $\begin{array}{l}\text { Well } \\
\text { Nane }\end{array}$ & $\begin{array}{c}\text { Collection } \\
\text { Date }\end{array}$ & $\begin{array}{l}\text { Semple } \\
\text { Number }\end{array}$ & $\begin{array}{c}\text { COL I FORM } \\
144 / \text { COL } \\
1 / 1\end{array}$ & $\begin{array}{c}\text { COPPER } \\
34 / \text { Ppb } \\
20 / 1000 \mathrm{~s}\end{array}$ & $\begin{array}{c}\text { FCOPPER } \\
\text { 34/pob } \\
20 / 1000 \mathrm{~s}\end{array}$ & $\begin{array}{l}\text { FLUOR ID } \\
\text { 124/ppb } \\
100 / 4000\end{array}$ \\
\hline$\cdots+\cdots$ & $\ldots \ldots$ & $\ldots \ldots \ldots$ & , & $\ldots$ & 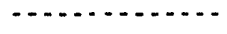 & $\cdots \cdots$ \\
\hline $\begin{array}{l}299-E 27-11 \\
299-E 27-17\end{array}$ & $\begin{array}{l}7 / 14 / 93 \\
7 / 14 / 93\end{array}$ & $\begin{array}{l}\text { BO8RC1 } \\
\text { B08RC5 }\end{array}$ & $\begin{array}{l}1.00 \mathrm{U} \\
1.00 \mathrm{U}\end{array}$ & $\begin{array}{l}26.00 \\
10.00 \mathrm{~L}\end{array}$ & & \\
\hline $\begin{array}{l}299-E 27-18 \\
299-E 27-18\end{array}$ & $\begin{array}{l}9 / 15 / 93 \\
9 / 15 / 93\end{array}$ & $\begin{array}{l}\text { B09665 } \\
809669\end{array}$ & $1.00 \mathrm{U}$ & $2.65 \mathrm{U}$ & $2.65 \mathrm{U}$ & 300.00 \\
\hline $\begin{array}{l}\text { 299-E27- } 19 \\
299-E 27-19\end{array}$ & $\begin{array}{l}9 / 15 / 93 \\
9 / 15 / 93\end{array}$ & $\begin{array}{l}B 09670 \\
B 09674\end{array}$ & 14.00 & $2.65 \mathrm{U}$ & $2.65 \mathrm{U}$ & 400.00 \\
\hline $\begin{array}{l}299-E 27-8 \\
299-E 27-9 \\
299-E 34-10\end{array}$ & $\begin{array}{l}8 / 04 / 93 \\
8 / 04 / 93 \\
7 / 14 / 93\end{array}$ & $\begin{array}{l}\text { B08RC9 } \\
\text { B08RD3 } \\
\text { B08RD7 }\end{array}$ & $\begin{array}{l}1.00 \mathrm{U} \\
1.00 \mathrm{U} \\
1.00 \mathrm{U}\end{array}$ & $\begin{array}{r}2.65 \mathrm{U} \\
2.65 \mathrm{U} \\
17.00 \mathrm{~L}\end{array}$ & & \\
\hline
\end{tabular}


Table 10-4. Constituents with at Least One Value Above the CRQL for the 216-B-63 Trench Data for Reporting Period July 1

through September 30, 1993. (sheet 2 of 3)

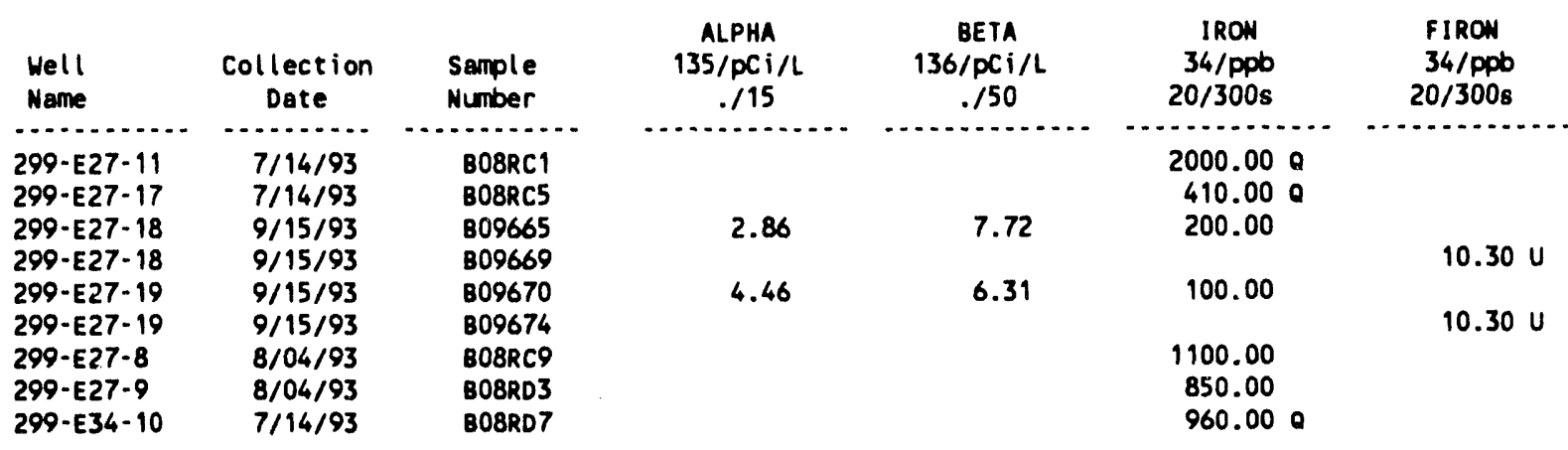

\begin{tabular}{|c|c|c|c|c|c|c|}
\hline $\begin{array}{l}\text { Well } \\
\text { Name }\end{array}$ & $\begin{array}{l}\text { Collection } \\
\text { Date }\end{array}$ & $\begin{array}{l}\text { Sample } \\
\text { Number }\end{array}$ & $\begin{array}{l}\text { MASNES } \\
34 / \text { ppb } \\
100 \%\end{array}$ & $\begin{array}{l}\text { FMAGNES } \\
34 / \mathrm{ppb} \\
100 \%\end{array}$ & $\begin{array}{r}\text { MANGESE } \\
34 / \mathrm{ppb} \\
10 / 50 \mathrm{~s}\end{array}$ & $\begin{array}{l}\text { FMANGAN } \\
34 / \mathrm{ppb} \\
10 / 50 \mathrm{~s}\end{array}$ \\
\hline $\begin{array}{l}299-E 27-11 \\
299-E 27-17 \\
299-E 27-18\end{array}$ & $\begin{array}{l}7 / 14 / 93 \\
7 / 14 / 93 \\
9 / 15 / 93\end{array}$ & $\begin{array}{l}\text { B08RC1 } \\
\text { B08RC5 } \\
\text { B09665 }\end{array}$ & $\begin{array}{r}11000.00 \\
11000.00 \\
8800.00\end{array}$ & & $\begin{array}{r}37.00 \\
10.00 \\
1.35\end{array}$ & \\
\hline 299-E27-18 & $9 / 15 / 93$ & B09669 & & 8900.00 & & $1.35 \mathrm{U}$ \\
\hline $\begin{array}{l}299-E 27-19 \\
299-E 27-19\end{array}$ & $\begin{array}{l}9 / 15 / 93 \\
9 / 15 / 93\end{array}$ & $\begin{array}{l}B 09670 \\
B 09674\end{array}$ & 9700.00 & & 1.35 & \\
\hline $\begin{array}{l}299-E 27-8 \\
299-E 27-9 \\
299-E 34-10\end{array}$ & $\begin{array}{l}8 / 04 / 93 \\
8 / 04 / 93 \\
7 / 14 / 93\end{array}$ & $\begin{array}{l}\text { BO8RC9 } \\
\text { B08RD3 } \\
\text { B08RD7 }\end{array}$ & $\begin{array}{l}12000.00 \\
13000.00 \\
10000.00\end{array}$ & & $\begin{array}{l}21.00 \\
17.00 \\
19.00\end{array}$ & \\
\hline
\end{tabular}

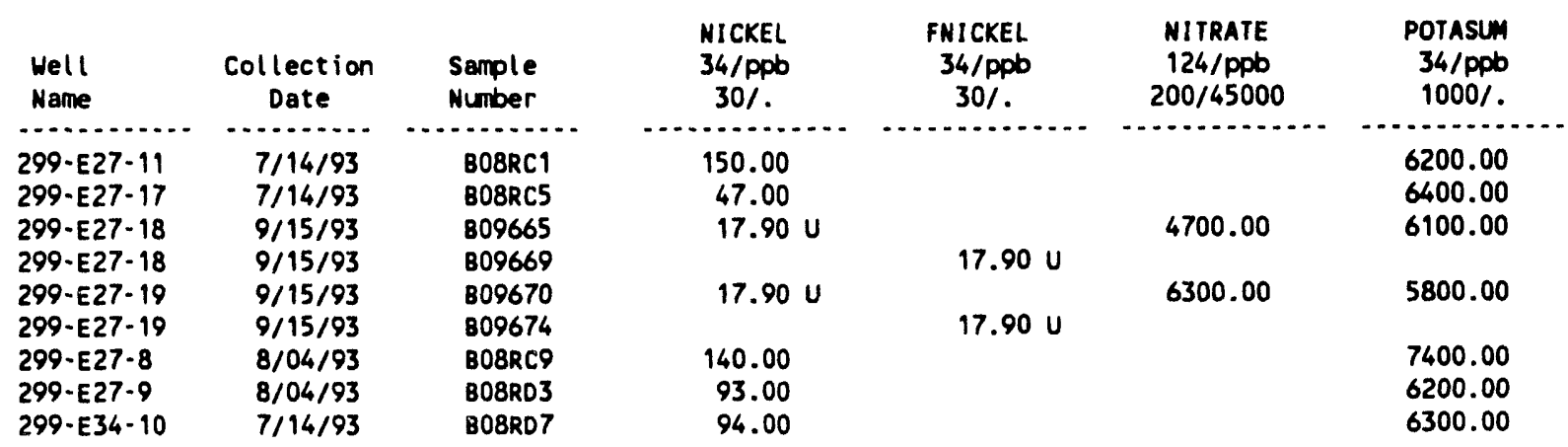

\begin{tabular}{|c|c|c|c|c|c|c|}
\hline $\begin{array}{l}\text { Well } \\
\text { Name }\end{array}$ & $\begin{array}{c}\text { Collection } \\
\text { Date }\end{array}$ & $\begin{array}{l}\text { Sample } \\
\text { Number }\end{array}$ & $\begin{array}{c}\text { FPOTASS } \\
34 / \mathrm{ppb} \\
1000 / .\end{array}$ & $\begin{array}{c}\text { RADIUM } \\
137 / \mathrm{pCi} / \mathrm{L} \\
. /\end{array}$ & $\begin{array}{l}\text { SOD IUM } \\
34 / \mathrm{ppb} \\
300 /\end{array}$ & $\begin{array}{l}\text { FSO0 IUM } \\
34 / \text { ppt } \\
300 /\end{array}$ \\
\hline$\ldots \ldots$ & $\ldots \ldots$ & $\ldots \ldots \ldots$ & $\cdots$ & - & $\ldots+\ldots$ & \\
\hline $\begin{array}{l}299-E 27-11 \\
299-E 27-17\end{array}$ & $\begin{array}{l}7 / 14 / 93 \\
7 / 14 / 93\end{array}$ & $\begin{array}{l}\text { B08RC1 } \\
\text { B08RC5 }\end{array}$ & & & $\begin{array}{l}15000.000 \\
16000.000\end{array}$ & \\
\hline $\begin{array}{l}299-E 27-18 \\
299-E 27-18\end{array}$ & $\begin{array}{l}9 / 15 / 93 \\
9 / 15 / 93\end{array}$ & $\begin{array}{l}B 09665 \\
809669\end{array}$ & 5800.00 & .49 & 13000.00 & 13000.00 \\
\hline 299-E27-19 & $9 / 15 / 93$ & B09670 & & .19 & 14000.00 & \\
\hline 299-E27-19 & $9 / 15 / 93$ & 809674 & 6200.00 & & & 14000.00 \\
\hline $\begin{array}{l}299-E 27-8 \\
299-E 27-9\end{array}$ & $\begin{array}{l}8 / 04 / 93 \\
8 / 04 / 93\end{array}$ & $\begin{array}{l}\text { BOBRC9 } \\
\text { BOBRD3 }\end{array}$ & & & $\begin{array}{l}14000.000 \\
14000.000\end{array}$ & \\
\hline $299-E 34-10$ & $7 / 14 / 93$ & B08RD7 & & & 16000.000 & \\
\hline
\end{tabular}


Table 10-4. Constituents with at Least One Value Above the CRQL for the 216-B-63 Trench Data for Reporting Period July 1 through September 30,1993 . (sheet 3 of 3 )

\begin{tabular}{|c|c|c|c|c|c|c|}
\hline $\begin{array}{l}\text { Well } \\
\text { Name }\end{array}$ & $\begin{array}{c}\text { Collection } \\
\text { Date }\end{array}$ & $\begin{array}{l}\text { Sample } \\
\text { Number }\end{array}$ & $\begin{array}{c}\text { SULFATE } \\
\text { iZ4/Ppb } \\
500 / 2500008\end{array}$ & $\begin{array}{c}\text { TC } \\
127 / \mathrm{ppb} \\
2000 \%\end{array}$ & $\begin{array}{l}\text { TDS } \\
\text { 65/ppm } \\
10 /\end{array}$ & $\begin{array}{l}\text { TRITIUM } \\
\text { 142/pCi/L } \\
.120000\end{array}$ \\
\hline $\begin{array}{l}299-E 27-11 \\
299-E 27-17 \\
299-E 27-18 \\
299-E 27-19 \\
299-E 27-8 \\
299-E 27-9 \\
299-E 34-10\end{array}$ & $\begin{array}{l}7 / 14 / 93 \\
7 / 14 / 93 \\
9 / 15 / 93 \\
9 / 15 / 93 \\
8 / 04 / 93 \\
8 / 04 / 93 \\
7 / 14 / 93\end{array}$ & $\begin{array}{l}\text { B08RC1 } \\
\text { B08RC5 } \\
\text { B09665 } \\
\text { B09670 } \\
\text { B08RC9 } \\
\text { B08RD3 } \\
\text { B08RD7 }\end{array}$ & $\begin{array}{ll}35000.00 & 0 \\
50000.00 & D\end{array}$ & $\begin{array}{l}24000.00 \\
25000.00 \\
24000.00 \mathrm{~B} \\
25000.00 \mathrm{~B} \\
23000.00 \mathrm{~B} \\
22000.00 \mathrm{~B} \\
25000.00\end{array}$ & $\begin{array}{l}260.00 \\
250.00 \\
200.00 \\
240.00 \\
300.00 \\
310.00 \\
230.00\end{array}$ & $\begin{array}{l}5330.00 \\
5820.00\end{array}$ \\
\hline $\begin{array}{l}\text { Well } \\
\text { Name }\end{array}$ & $\begin{array}{c}\text { Collection } \\
\text { Date }\end{array}$ & $\begin{array}{l}\text { Sample } \\
\text { Number }\end{array}$ & $\begin{array}{c}\text { TURB ID } \\
\text { 126/NTU } \\
.1 / 1\end{array}$ & $\begin{array}{l}\text { URANIUM } \\
\text { 145/ppb } \\
.1 .\end{array}$ & $\begin{array}{c}\text { VANADUM } \\
34 / \mathrm{ppb} \\
30 \%\end{array}$ & $\begin{array}{c}\text { FVANADI } \\
34 / \mathrm{ppb} \\
30 \%\end{array}$ \\
\hline $\begin{array}{l}299-E 27-11 \\
299-E 27-17 \\
299-E 27-18 \\
299-E 27-18 \\
299-E 27-19 \\
299-E 27-19 \\
299-E 27-8 \\
299-E 27-9 \\
299-E 34-10\end{array}$ & $\begin{array}{l}7 / 14 / 93 \\
7 / 14 / 93 \\
9 / 15 / 93 \\
9 / 15 / 93 \\
9 / 15 / 93 \\
9 / 15 / 93 \\
8 / 04 / 93 \\
8 / 04 / 93 \\
7 / 14 / 93\end{array}$ & $\begin{array}{l}\text { B08RC1 } \\
\text { B08RC5 } \\
\text { B09665 } \\
\text { B09669 } \\
\text { B09670 } \\
\text { B09674 } \\
\text { B08RC9 } \\
\text { B08RD3 } \\
\text { B08RD 7 }\end{array}$ & $\begin{array}{l}6.700 \\
2.900\end{array}$ & $\begin{array}{l}2.47 \\
2.53\end{array}$ & $\begin{array}{l}40.00 \\
38.00 \\
3.84 U \\
3.84 U \\
3.84 U \\
3.84 U \\
40.00\end{array}$ & $\begin{array}{l}3.84 \mathrm{U} \\
3.84 \mathrm{U}\end{array}$ \\
\hline $\begin{array}{l}\text { Well } \\
\text { Name }\end{array}$ & $\begin{array}{c}\text { Collection } \\
\text { Date }\end{array}$ & $\begin{array}{l}\text { Sample } \\
\text { Number }\end{array}$ & $\begin{array}{c}\text { 2INC } \\
34 / \text { ppb } \\
10 \%\end{array}$ & $\begin{array}{l}\text { F2INC } \\
34 / \mathrm{ppb} \\
10 \%\end{array}$ & & \\
\hline $\begin{array}{l}299-E 27-11 \\
299-E 27-17 \\
299-E 27-18 \\
299-E 27-18 \\
299-E 27-19 \\
299-E 27-19 \\
299-E 27-8 \\
299-E 27-9 \\
299-E 34-10\end{array}$ & $\begin{array}{l}7 / 14 / 93 \\
7 / 14 / 93 \\
9 / 15 / 93 \\
9 / 15 / 93 \\
9 / 15 / 93 \\
9 / 15 / 93 \\
8 / 04 / 93 \\
8 / 04 / 93 \\
7 / 14 / 93\end{array}$ & $\begin{array}{l}\text { B08RC1 } \\
\text { B08RC5 } \\
\text { B09665 } \\
\text { B09669 } \\
\text { B09670 } \\
\text { B09674 } \\
\text { B08RC9 } \\
\text { B08RD3 } \\
\text { B08RD 7 }\end{array}$ & $\begin{array}{l}33.00 \mathrm{Q} \\
8.80 \mathrm{LQ} \\
44.00 \\
15.00 \\
3.44 \mathrm{U} \\
3.44 \mathrm{U} \\
19.00 \mathrm{Q}\end{array}$ & $\begin{array}{l}3.44 \mathrm{U} \\
3.44 \mathrm{U}\end{array}$ & & \\
\hline
\end{tabular}

For explanation of this table, see Section 1.4 of report. 
Table 10-5. Contamination Indicator Parameters for the 216-B-63 Trench Data for Reporting Period July 1 through September 30, 1993.

\begin{tabular}{|c|c|c|c|c|c|c|c|c|}
\hline $\begin{array}{l}\text { Well } \\
\text { Name }\end{array}$ & $\begin{array}{c}\text { Collection } \\
\text { Date }\end{array}$ & $\begin{array}{l}\text { Sample } \\
\text { Number }\end{array}$ & $\begin{array}{c}\text { COND FIELD } \\
\mu \mathrm{mhO} \\
1 / 700 \mathrm{~W}\end{array}$ & $\begin{array}{l}\text { COND LAB } \\
\mu \text { mho } \\
. / 700 \mathrm{~W}\end{array}$ & $\begin{array}{l}\text { PH FIELD } \\
.01 / 6.5-8.5 \mathrm{~s}\end{array}$ & $\begin{array}{l}\text { PH LAB } \\
.01 / 6.5-8.58\end{array}$ & $\begin{array}{l}\text { TOC } \\
\text { ppb } \\
1000 \%\end{array}$ & $\begin{array}{c}\text { rox } \\
\text { ppb } \\
10 \%\end{array}$ \\
\hline $299-E 27-11$ & $7 / 14 / 93$ & $\begin{array}{l}\text { B08RC1 } \\
\text { B08RC2 } \\
\text { B08RC3 } \\
\text { B08RC4 }\end{array}$ & $\begin{array}{l}390 \\
388 \\
389 \\
389\end{array}$ & 390 & $\begin{array}{l}7.93 \\
7.93 \\
7.92 \\
7.91\end{array}$ & 7.80 & $\begin{array}{l}500 \mathrm{~L} \\
500 \mathrm{~L} \\
400 \mathrm{~L} \\
400 \mathrm{~L}\end{array}$ & $\begin{array}{l}8.0 \text { UP } \\
8.0 \text { UP } \\
8.0 \text { UP } \\
8.0 \text { UP }\end{array}$ \\
\hline $299-E 27-17$ & $7 / 14 / 93$ & $\begin{array}{l}\text { B08RC5 } \\
\text { B08RC6 } \\
\text { B08RC7 } \\
\text { B08RC8 }\end{array}$ & $\begin{array}{l}369 \\
370 \\
371 \\
372\end{array}$ & 370 & $\begin{array}{l}7.83 \\
7.84 \\
7.84 \\
7.85\end{array}$ & 7.90 & $\begin{array}{l}300 \mathrm{~L} \\
400 \mathrm{~L} \\
400 \mathrm{~L} \\
400 \mathrm{~L}\end{array}$ & $\begin{array}{l}8.0 \text { UP } \\
8.0 \text { UP } \\
8.0 \text { UP } \\
8.0 \text { UP }\end{array}$ \\
\hline 299-E27-18 & $9 / 15 / 93$ & $\begin{array}{l}B 09665 \\
B 09666 \\
B 09667 \\
B 09668\end{array}$ & $\begin{array}{l}306 \\
306 \\
307 \\
307\end{array}$ & $\begin{array}{l}310 \\
310 \\
310 \\
310\end{array}$ & $\begin{array}{l}8.08 \\
8.08 \\
8.08 \\
8.08\end{array}$ & $\begin{array}{l}8.30 \\
8.30 \\
8.30 \\
8.30\end{array}$ & & $\begin{array}{l}8.0 \text { UP } \\
8.0 \text { UP } \\
8.0 \text { UP } \\
8.0 \text { UP }\end{array}$ \\
\hline 299-E27-19 & $9 / 15 / 93$ & $\begin{array}{l}B 09670 \\
B 09671 \\
B 09672 \\
B 09673\end{array}$ & $\begin{array}{l}349 \\
346 \\
346 \\
345\end{array}$ & $\begin{array}{l}350 \\
350 \\
350 \\
350\end{array}$ & $\begin{array}{l}8.07 \\
8.07 \\
8.07 \\
8.07\end{array}$ & $\begin{array}{l}8.30 \\
8.30 \\
8.30 \\
8.30\end{array}$ & & $\begin{array}{l}20.0 \mathrm{P} \\
8.0 \text { UP } \\
8.0 \text { UP } \\
8.0 \text { UP }\end{array}$ \\
\hline $299-E 27-8$ & $8 / 04 / 93$ & $\begin{array}{l}\text { BO8RC9 } \\
\text { BO8RDO } \\
\text { B08RD } 1 \\
\text { BO8RD2 }\end{array}$ & $\begin{array}{l}418 \\
418 \\
417 \\
417\end{array}$ & 410 & $\begin{array}{l}7.79 \\
7.79 \\
7.79 \\
7.79\end{array}$ & 7.80 & $\begin{array}{l}400 L \\
400 L \\
800 L \\
500 L\end{array}$ & $\begin{array}{l}8.0 \text { UP } \\
9.0 \mathrm{LP} \\
9.0 \mathrm{LP} \\
8.0 \text { UP }\end{array}$ \\
\hline 299-E27-9 & $8 / 04 / 93$ & $\begin{array}{l}\text { B08RD } 3 \\
\text { B08RD4 } \\
\text { B08RD5 } \\
\text { 808RD6 }\end{array}$ & $\begin{array}{l}419 \\
420 \\
420 \\
421\end{array}$ & 410 & $\begin{array}{l}7.88 \\
7.88 \\
7.88 \\
7.88\end{array}$ & 7.80 & $\begin{array}{l}400 L \\
500 L \\
400 L \\
400 L\end{array}$ & $\begin{array}{l}8.0 \text { UP } \\
8.0 \text { UP } \\
8.0 \text { UP } \\
8.0 \text { UP }\end{array}$ \\
\hline 299-E33-33 & $6 / 16 / 93$ & $\begin{array}{l}\text { B08N64 } \\
\text { B08N65 } \\
\text { B08N66 } \\
\text { B08N67 }\end{array}$ & $\begin{array}{l}289 \\
290 \\
289 \\
289\end{array}$ & 280 & $\begin{array}{l}7.90 \\
7.90 \\
7.90 \\
7.89\end{array}$ & 8.20 & $\begin{array}{l}300 \mathrm{~L} \\
300 \mathrm{~L} \\
300 \mathrm{~L} \\
400 \mathrm{~L}\end{array}$ & $\begin{array}{l}10.0 \text { UP } \\
10.0 \text { UP } \\
10.0 \text { UP } \\
10.0 \text { UP }\end{array}$ \\
\hline $299-E 34-10$ & $7 / 14 / 93$ & $\begin{array}{l}\text { B08RD7 } \\
\text { B0BRD8 } \\
\text { BO8RD9 } \\
\text { B08RFO }\end{array}$ & $\begin{array}{l}349 \\
349 \\
348 \\
348\end{array}$ & 340 & $\begin{array}{l}7.90 \\
7.91 \\
7.90 \\
7.90\end{array}$ & 7.90 & $\begin{array}{l}400 \mathrm{~L} \\
300 \mathrm{~L} \\
300 \mathrm{~L} \\
300 \mathrm{~L}\end{array}$ & $\begin{array}{l}8.0 \text { UP } \\
8.0 \text { UP } \\
8.0 \text { UP } \\
8.0 \text { UP }\end{array}$ \\
\hline
\end{tabular}

For explanation of this table, see Section 1.4 of report. 
$\mathrm{DOE} / \mathrm{RL}-93-56-3$

This page intentionally left blank.

10-16 
$\mathrm{DOE} / \mathrm{RL}-93-56-3$

CONTENTS

$11.0216-5-10$ POND AND DITCH . . . . . . . . . . . . . . . 11-1

11.1 INTRODUCTION . . . . . . . . . . . . . . . . . . . . 11-1

11.2 WATER LEVEL MEASUREMENTS . . . . . . . . . . . . . . . . 11-2

11.3 WATER CHEMISTRY DATA . . . . . . . . . . . . . . . 11-2 
DOE / RL-93-56-3

\section{LIST OF FIGURES}

11-1 Monitoring Well Location for the 216-S-10 Facility . . . . . 11-4

\section{LIST OF TABLES}

11-1 Monitoring Well Purpose and Sampling Schedule for the 216-S-10 Facility................. 11-5

11-2 RCRA Water Leve1 Measurement Report 216-S-10 Facility, Third Quarter $1993 \ldots . . \ldots$. . . . . . . . . . . . .

11-3 Constituent List and Summary of Results for the 216-S-10 Facility Data for Reporting Period July 1 through September $30,1993 \ldots \ldots$. . . . . . . . 11-7

11-4 Constituents with at Least One Value Above the CRQL for the 216-S-10 Facility Data for Reporting Period July 1 through September 30,1993 ................ 11-13

11-5 Contamination Indicator Parameters for the 216-S-10 Facility Data for Reporting Period July 1 through September 30, 1993 . . 11-16 
DOE/RL-93-56-3

\title{
11.0 216-S-10 POND AND DITCH
}

\author{
J. W. Lindberg \\ Hestinghouse Hanford Company
}

\subsection{INTRODUCTION}

The 216-S-10 Pond and Ditch (S-10 Facility) are located south-southwest of the 200 West Area, directly outside the perimeter fence. The S-10 Facility received wastewater from the Reduction-0xidation (REDOX) Plant (202-S Building) located in the 200 West Area. In the past, wastewater disposed of at the S-10 Facility has contained hazardous waste and radioactive materials. Since 1985, physical controls and operating procedures have been modified to avoid inadvertent discharge of hazardous chemicals to the wastewater stream. The facility has been monitored under the interim-status, detection-level monitoring program since August 1991. In October 1991, the effluent waste stream, including the water source, to the 216-S-10 Ditch was deactivated. Because the S-10 Facility is not expected to receive additional hazardous substances, the facility will be closed under RCRA interim status.

The S-10 Facility initially comprised an open, unlined ditch $1.8 \mathrm{~m}(6 \mathrm{ft})$ deep, $1.2 \mathrm{~m}(4 \mathrm{ft})$ wide at the bottom, and $686 \mathrm{~m}(2,250 \mathrm{ft})$ long. In addition, an open, unlined percolation pond constructed at the southwest end of the ditch, which is approximately 2 hectares ( 5 acres) in size, was active during part of the time the ditch was receiving wastes.

The 216-S-10 Ditch began receiving wastewater from the REDOX Plant in August 1951, and the 216-S-10 Pond was dug and placed in service in February 1954. In October 1985, the 216-S-10 Pond and portions of the 216-S-10 Ditch were decommissioned, backfilled, and stabilized. The remaining portion of the 216-S-10 Ditch (approximately $594 \mathrm{~m}[1,950 \mathrm{ft}]$ ) received ncihazardous, nonregulated waste from the 202-S Building chemical sewer until deactivation in October 1991. This waste stream was composed of cooling water, steam condensate, water tower overflow, and drain effluent. These source streams had been routed so that they would not come into contact with hazardous materials.

Releases of hazardous materials and constituents to the S-10 Facility are poorly documented. Radioactive waste was reportedly disposed of at the S-10 Facility resulting from contaminated floor and sewer drains at the REDOX Plant, and hazardous chemical releases are documented in 1954 and 1983.

The annual volume of effluent discharged to the 216-S-10 Ditch was between $1.9 \times 10^{8}$ and $5.7 \times 10^{8} \mathrm{~L}\left(5.0 \times 10^{7}\right.$ and $1.5 \times 10^{8}$ gal $)$ during 1991. This volume of discharge had created a localized recharge mound and an associated perched water table directly below the receiving (north) end of the ditch. This recharge mound has since dissipated, and the perched water table has been dry since shortly after the ditch was deactivated. 
Groundwater beneath the 216-S-10 Ditch is sampled semiannually (except for well 299-W27-2, which is sampled quarterly) in accordance with the Interim-Status Groundwater Monitoring Plan for the 216-5-10 Pond and Ditch (WHC 1990b). The site is currently in the indicator parameters evaluation program.

The monitoring network currently consists of two upgradient wells, three downgradient wells, and one downgradient well that monitors the base of the unconfined aquifer (Figure 11-1). Table 11-1 lists the wells and their sampling status. All of the wells now have at least four quarters of analytical data. The results of the background statistical calculations for the upgradient monitoring wells (based on the first four quarters of analytical data) were presented in the RCRA annual report (DOE-RL 1993).

\subsection{WATER LEVEL MEASUREMENTS}

Water level measurements obtained during the third quarter of 1993 are presented in Table 11-2. Water level measurements are obtained at all wells before sampling. Also, quarterly water level measurements are made by WHC at four wells at the S-10 Facility (299-W26-8, 299-W26-9, 299-W26-10, and 299-W26-12).

\subsection{WATER CHEMISTRY DATA}

Groundwater sampling during the third quarter of 1993 included all of the wells in the monitoring network. Wells 299-W26-7, 299-W26-8, 299-W26-9, and 299-W26-10 were sampled on June 28, 1993. We1ls 299-W26-12 and 299-W27-2 were sampled on July 8, 1993. The samples collected from the five wells on semiannual status represents the final sampling of these wells for FY 1993. Available data for the reporting period July 1 (June 28) through September 30 , 1993, are shown in Tables 11-3 through 11-5. Table 11-3 includes a 1ist of reported constituents and a summary of results. Tables 11-4 and 11-5 show results for specific constituents.

Critical mean values for the indicator parameters were not exceeded in any of the wells that monitor the top of the unconfined aquifer. A critical mean value for TOX is not determined; this value will be calculated upon resolution of data quality issues. The critical mean value for field specific conductance was exceeded in well 299-W27-2. However, well 299-W27-2

(downgradient) monitors the base of the unconfined aquifer and is not used for statistical evaluation purposes. As more data become available from well 299-W27-2, time versus concentration plots will be developed to monitor the groundwater quality at the deeper horizon.

Constituents in Tables 11-3 and 11-4 that showed concentrations above the DWS include chromium (unfiltered), manganese (unfiltered), iron (unfiltered), $\mathrm{pH}$, and turbidity (see Table 1-6 for a complete listing of DWSs). Chromium (unfiltered) concentraticus above the 100-ppb primary DWS occurred in downgradient wells 299-W26-12 (160 ppb) and 299-W27-2 (140 and $120 \mathrm{ppb}$ ). Filtered chromium concentrations were all below this DWS. Because the 
turbidity in these same two wells was also above DWS, the high level of unfiltered chromium may be due to constituents remaining in the ground from well construction.

Unfiltered manganese concentrations above the secondary DWS of $50 \mathrm{ppb}$ occurred in well 299-W27-2 (70 ppb). Filtered manganese concentrations were all below this DWS. Like the higher levels of chromium in this well, the higher levels of unfiltered manganese may be due to constituents remaining in the ground from well construction.

Unfiltered iron concentrations above the secondary DWS of $300 \mathrm{ppb}$ occurred in wells 299-W26-12 (1,300 ppb) and 299-W27-2 (1,900 and 1,800 ppb). Filtered iron concentrations were all below this DWS. This constituent, like chromium and manganese, may be due to well construction because the higher levels occurred in the same wells and were associated with higher levels of turbidity.

Results for field $\mathrm{pH}$ in well 299-W26-8 from the June 28th sampling were slightly above the DWS of 8.5. However, these results were below the statistically calculated critical mean value of 9.04 .

Blank contamination was noted in many samples analyzed for TC and TOC in this quarter. More than $50 \%$ of the TC blank data were flagged by ' $B$ ' and ' $Q$.' Blank contamination is being investigated to determine its cause and potential effect on the samples. 
$\mathrm{DOE} / \mathrm{RL}-93-56-3$

Figure 11-1. Monitoring Well Location for the 216-S-10 Facility.

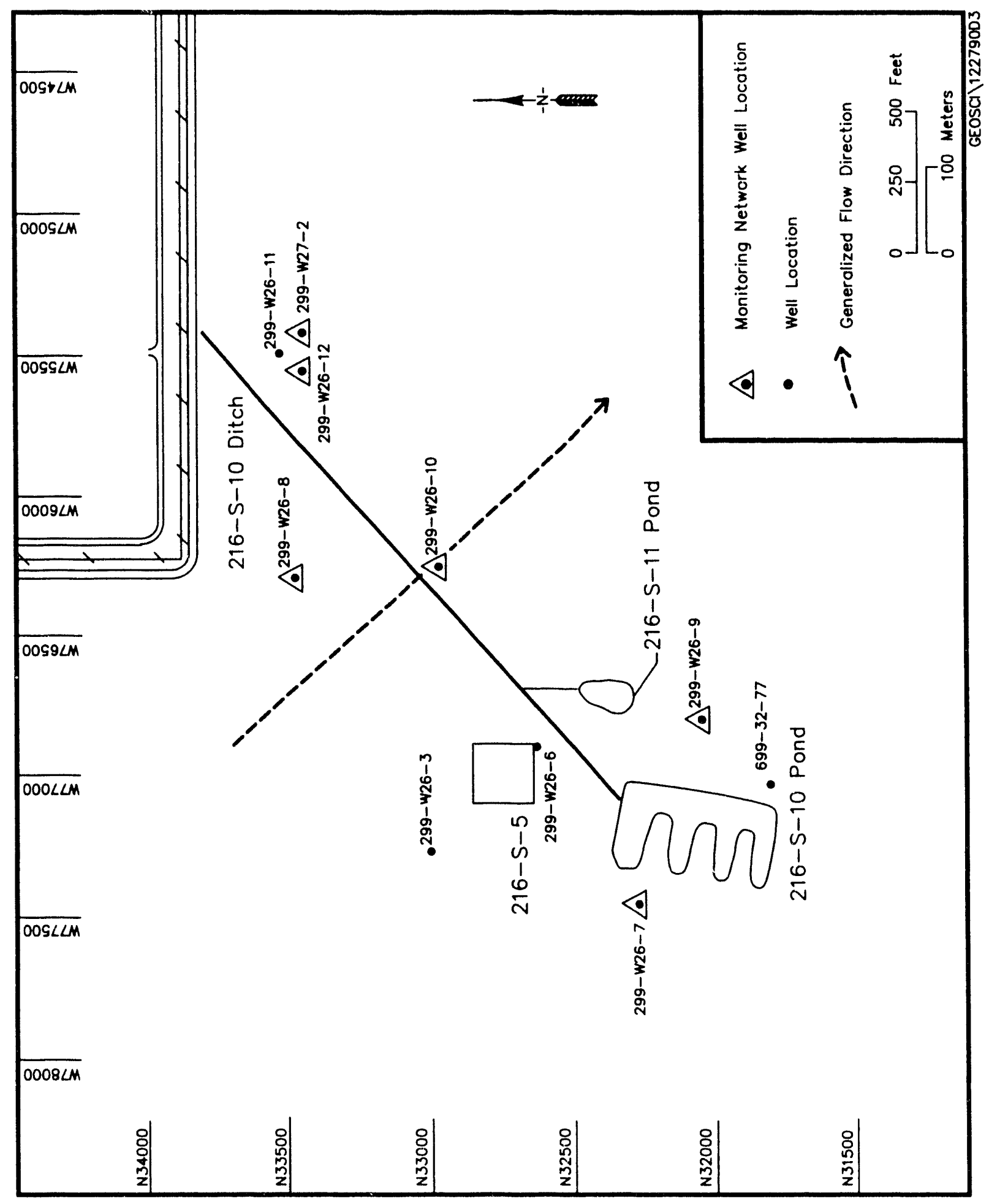


Table 11-1. Monitoring Well Purpose and Sampling Schedule for the 216-S-10 Facility.

\begin{tabular}{|c|c|c|c|c|}
\hline $\begin{array}{c}\text { Well no. } \\
(299-)\end{array}$ & $\begin{array}{c}\text { Reiative } \\
\text { position }\end{array}$ & \multicolumn{1}{|c|}{ Hydrogeologic unit } & $\begin{array}{c}\text { Sample } \\
\text { frequency }\end{array}$ & Sample date \\
\hline W26-7 & Upgradient & Ringold: Water Table & Semiannually & $6 / 28 / 93$ \\
\hline W26-8 & Upgradient & Ringold: Water Table & Semiannually & $6 / 28 / 93$ \\
\hline W26-9 & Downgradient & Ringold: Water Table & Semiannually & $6 / 28 / 93$ \\
\hline W26-10 & Downgradient & Ringold: Water Table & Semiannually & $6 / 28 / 93$ \\
\hline W26-12 & Downgradient & Ringold: Water Table & Semiannually & $7 / 08 / 93$ \\
\hline W27-2 & Downgradient & Ringold: $\begin{array}{l}\text { Base of } \\
\text { Unconfined }\end{array}$ & Quarterly & $\begin{array}{l}7 / 08 / 93 \\
9 / 27 / 93\end{array}$ \\
\hline
\end{tabular}


Table 11-2. RCRA Water Level Measurement Report 216-S-10 Facility, Third Quarter 1993.

\begin{tabular}{llll}
\hline We11 & Date & $\begin{array}{c}\text { Depth to } \\
\text { water }(\mathrm{ft})\end{array}$ & $\begin{array}{c}\text { Water level } \\
\text { elevation } \\
\text { above ms } 1 \text { (ft) }\end{array}$ \\
\hline \multicolumn{1}{c}{ Wells Monitoring the Top of the Unconfined Aquifer } \\
\hline $299-W 26-8$ & $9 / 07 / 93$ & 208.99 & 457.32 \\
$299-W 26-9$ & $9 / 07 / 93$ & 196.29 & 457.87 \\
$299-W 26-10$ & $9 / 07 / 93$ & 214.41 & 456.46 \\
& $9 / 07 / 93$ & 214.38 & 456.49 \\
$299-W 26-12$ & $7 / 08 / 93$ & 218.29 & $457.40 \star$ \\
$9 / 07 / 93$ & 218.74 & 456.95 \\
\hline
\end{tabular}

Notes: 1. Water level elevations are calculated by subtracting the measured depth to water from the surveyed elevation for the well.

2. Depth-to-water values are transcribed from field records.

3. Elevations marked with an ' $*$ ' were measured at the time of sampling.

4. To convert feet to meters multiply by 0.3048 . 
Table 11-3. Constituent List and Summary of Results for the 216-S-10 Facility Data for Reporting Period July 1 through September 30, 1993. (sheet 1 of 6)

CONTAMINATION INDICATOR PARAMETERS

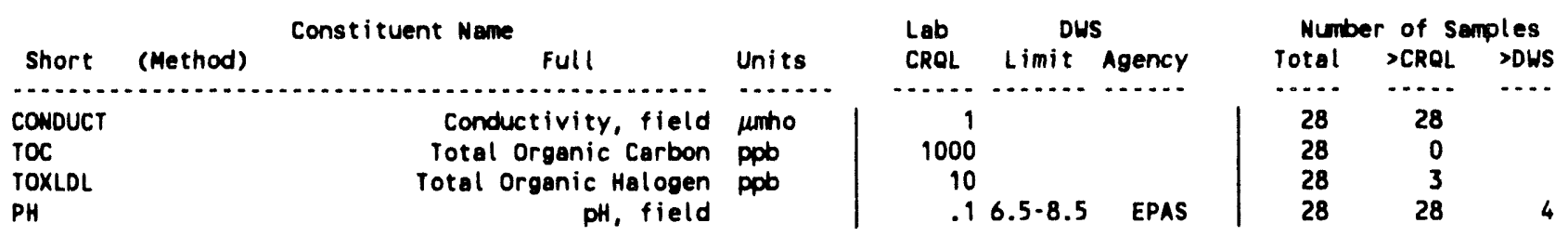

DRINKING WATER PARAMETERS

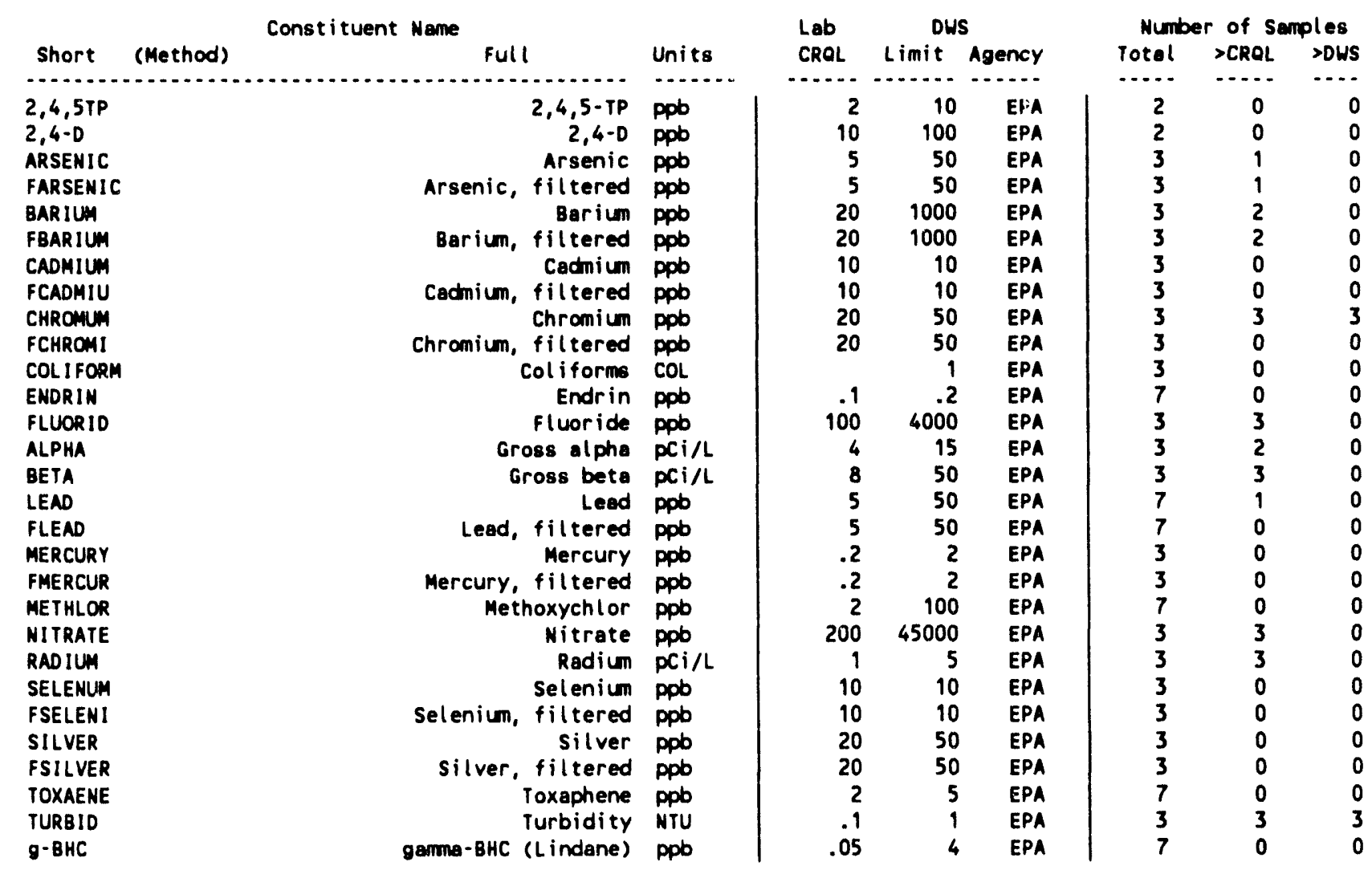

GROUNDWATER QUALITY PARAMETERS

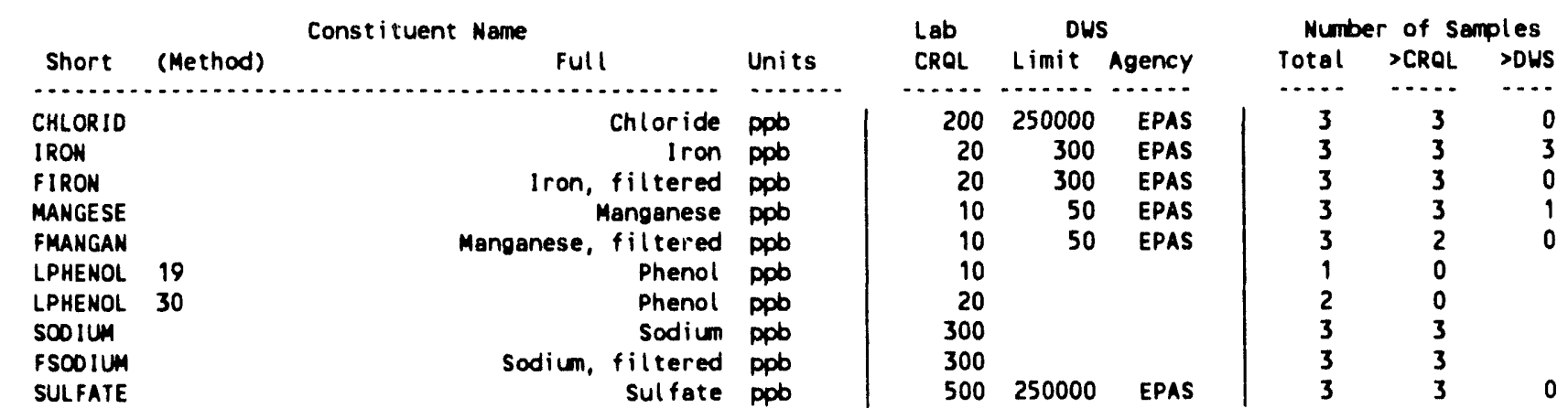


Table 11-3. Constituent List and Summary of Results for the 216-S-10 Facility Data for Reporting Period July 1 through September 30, 1993. (sheet 2 of 6)

SITE SPECIFIC AND OTHER CONSTITUENTS

\begin{tabular}{|c|c|c|c|c|c|c|c|c|}
\hline \multirow[b]{2}{*}{ Short } & \multicolumn{2}{|r|}{ Constituent Name } & \multirow[b]{2}{*}{ Units } & \multirow{2}{*}{$\begin{array}{l}\text { Lab } \\
\text { CROL }\end{array}$} & DUS & \multicolumn{3}{|c|}{ Number of Samples } \\
\hline & (Method) & Full & & & Limit Agency & Total & >CRQL & $>$ DWS \\
\hline$\cdots \ldots$ & $\cdots$ & . & $\ldots \ldots$ & $\ldots \ldots$ & $\ldots \ldots \ldots$ & $\ldots$. & $\ldots$. & $\ldots$ \\
\hline $1112-t c$ & & $1,1,1,2$ - Tetrachloroethane & ppb & 5 & & 1 & 0 & \\
\hline $1,1,1-T$ & & 1,1,1-Trichloroethane & ppb & 5 & & 6 & 0 & \\
\hline $1122-t c$ & & $1,1,2,2$-1etrachloroethane & ppob & 5 & & 1 & 0 & \\
\hline $1,1,2-T$ & & 1,1,2-Trichloroethene & ppb & 5 & & 6 & 0 & \\
\hline $1,1-01 C$ & & 1,1-Dichloroethane & ppb & 5 & & 6 & 0 & \\
\hline DICETHY & & 1,1-0 ichloroethene & ppo & 5 & & 1 & 0 & \\
\hline $123-\operatorname{trp}$ & & 1,2,3-Trichloropropane & ppob & 5 & & 1 & 0 & \\
\hline TETRCHB & & $1,2,4,5-$ Tetrachl orobenzene & ppb & 10 & & 1 & 0 & \\
\hline TRICHLB & & 1,2,4-Trichlorobenzene & ppo & 10 & & 1 & 0 & \\
\hline DIBRCHL & & 1,2-Dibromo-3-chloropropane & ppb & 5 & & 1 & 0 & \\
\hline DIBRETH & & 1,2-0 ibromoethane & ppb & 5 & & 1 & 0 & \\
\hline 12-cben & & 1,2-Dichlorobenzene & ppb & 10 & & 1 & 0 & \\
\hline $1,2-D I C$ & & 1,2-Dichloroethane & ppb & 5 & & 6 & 0 & \\
\hline 12-DCE & & 1,2-Dichloroethene & ppb & 5 & & 6 & 0 & \\
\hline DICPANE & & 1,2-Dichloropropane & ppb & 5 & & 1 & 0 & \\
\hline 13-dben & & 1,3-Dichlorobenzene & ppo & 10 & & 1 & 0 & \\
\hline 14 - dben & 16 & 1,4-Dichlorobenzene & ppo & 5 & & 5 & 0 & \\
\hline 14-ctben & 19 & 1,4-Dichlorobenzene & ppb & 10 & & 1 & 0 & \\
\hline DIOXANE & & 1,4-Dioxane & ppob & 200 & & 1 & 0 & \\
\hline NAPHOUI & & 1,4-Naph toquinone & ppb & 10 & & 1 & 0 & \\
\hline 1BUTYN & & 1-Butanol & ppb & 1000 & & 6 & 0 & \\
\hline 1-napha & & 1-Naphthylamine & ppb & 10 & & 1 & 0 & \\
\hline TETRCHP & & $2,3,4,6$ - Tetrachlorophenol & ppb & 10 & & 1 & 0 & \\
\hline $23781 C D$ & & $2,3,7,8-\mathrm{TCDD}$ & ppo & .005 & & 1 & 0 & \\
\hline $2,4,5-T$ & & $2,4,5-T$ & ppb & 2 & & 2 & 0 & \\
\hline $245-\operatorname{trp}$ & & $2,4,5$-Trichlorophenol & ppb & 10 & & 1 & D & \\
\hline 246-trp & 19 & 2,4,6-Trichlorophenol & ppb & 10 & & 1 & 0 & \\
\hline $246-\operatorname{trp}$ & 30 & 2,4,6-Trichlorophenol & ppb & 5 & & 2 & 0 & \\
\hline 24 -dchp & 19 & 2,4-0ichlorophenol & ppb & 10 & & 1 & 0 & \\
\hline 24-dchp & 30 & 2,4-Dichlorophenol & ppb & 5 & & 2 & 0 & \\
\hline DIMPHEN & 19 & 2,4-D ime thylphenol & ppo & 10 & & 1 & 0 & \\
\hline DIMPHEN & 30 & 2,4-D imethylphenol & ppo & 5 & & 2 & 0 & \\
\hline DINPHEN & 19 & 2,4-0 initrophenol & ppo & 50 & & 1 & 0 & \\
\hline DINPHEN & 30 & 2,4-D initrophenol & ppo & 150 & & 2 & 0 & \\
\hline 24 -dint & & 2,4-Dinitrotoluene & ppo & 10 & & 1 & 0 & \\
\hline 26-dchp & 19 & 2,6-Dich lorophenol & ppb & 10 & & 1 & 0 & \\
\hline 26-dchp & 30 & 2,6-Dichlorophenol & ppb & 5 & & 2 & 0 & \\
\hline 26-dint & & 2,6-D initrotoluene & pob & 10 & & 1 & 0 & \\
\hline ACEFENE & & 2-Acetyl aminof luorene & ppb & 10 & & 1 & 0 & \\
\hline CHLNAPH & & 2-Chloronaphthalene & pob & 10 & & 1 & 0 & \\
\hline CHLPHEN & 19 & 2-Chlorophenol & pob & 10 & & 1 & 0 & \\
\hline CHLPHEN & 30 & 2-Chlorophenol & pob & 5 & & 2 & 0 & \\
\hline 2HEXANO & & 2-Hexanone & ppob & 50 & & 1 & 0 & \\
\hline 2MENAPH & & 2-Methyl naph thal ene & pob & 10 & & 1 & 0 & \\
\hline 2METHPH & & 2-Methylphenol & ppb & 10 & & 1 & 0 & \\
\hline 2-napha & & 2-Naphthylamine & ppb & 10 & & 1 & 0 & \\
\hline ONITANI & & 2-Nitroaniline & ppb & 50 & & 1 & 0 & \\
\hline 2NITPH & 19 & 2-Nitrophenol & ppob & 10 & & 1 & 0 & \\
\hline 2NITPH & 30 & 2-Nitrophenol & ppb & 5 & & 2 & 0 & \\
\hline PICOLIN & & 2-Picoline & ppb & 10 & & 1 & 0 & \\
\hline BUTOINP & 30 & 2-sec-Butyl-4,6-dinitrophenol (DN & ppb & 5 & & 2 & 0 & \\
\hline BUTOINP & 49 & 2-sec-Butyl-4,6-dinitrophenol (DN & ppb & 1 & & 2 & 0 & \\
\hline DICHBEN & & $3,3^{\prime}-0$ ichl or obenzidine & ppo & 20 & & 1 & 0 & \\
\hline DIMEYLB & & 3,3'-D imethylbenzidine & ppb & 10 & & 1 & 0 & \\
\hline METCHAN & & 3-Methylchol anthrene & ppb & 10 & & 1 & 0 & \\
\hline MNITANI & & 3-Nitroaniline & ppb & 50 & & 1 & 0 & \\
\hline
\end{tabular}


Table 11-3. Constituent List and Summary of Results for the 216-S-10 Facility Data for Reporting Period July 1 through September 30, 1993. (sheet 3 of 6)

\begin{tabular}{|c|c|c|c|c|c|c|c|c|}
\hline & & Const ituent Name & & Lab & DWS & Nunt & of $\mathrm{Se}$ & mples \\
\hline Short & (Method) & Full & Units & CROL & Limit Agency & Total & $>\mathrm{CROL}$ & >DWS \\
\hline$\cdots \cdots$ & $\cdots$ & 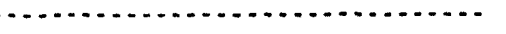 & $\ldots \ldots$ & $\cdots \cdots$ & $\cdots+\cdots$ & $\cdots$ & $\cdots \cdots$ & $\cdots$ \\
\hline DOD & & $4,4^{\prime}-D D D$ & ppob & .1 & & 7 & 0 & \\
\hline DDE & & $4,4^{\prime}-$ DDE & ppo & .05 & & 7 & 0 & \\
\hline DDT & & $4,4^{\prime}-D D T$ & ppob & .1 & & 7 & 0 & \\
\hline 460N2MP & 19 & 4,6-Dinitro-2-methylphenol & ppb & 50 & & 1 & 0 & \\
\hline 46DN2MP & 30 & 4,6-0 initro-2-methylphenol & ppb & 200 & & 2 & 0 & \\
\hline AMINOYL & & 4-Aminobiphenyl & ppb & 10 & & 1 & 0 & \\
\hline BROPHEN & & 4-Bromophenylphenyl ether & ppob & 10 & & 1 & 0 & \\
\hline CHLCRES & 19 & 4-Chloro-3-methyl phenol & ppo & 20 & & 1 & 0 & \\
\hline CHLCRES & 30 & 4-Chloro-3-methylphenol & ppb & 5 & & 2 & 0 & \\
\hline CHLANIL & & 4-Chloroeniline & ppob & 20 & & 1 & 0 & \\
\hline ४CPPETH & & 4-Chlorophenylphenyl ether & ppob & 10 & & 1 & 0 & \\
\hline MIBK & & 4-Methyl-2-pentenone & ppb & 50 & & 6 & 0 & \\
\hline 4METHPH & & 4-Methylphenol & ppb & 10 & & 1 & 0 & \\
\hline NITRANI & & 4-Nitroeniline & ppb & 50 & & 1 & 0 & \\
\hline NITPHEN & 19 & 4-Nitrophenol & ppob & 50 & & 1 & 0 & \\
\hline NITPHEN & 30 & 4-Nitrophenol & ppb & 30 & & 2 & 0 & \\
\hline 4NITQUI & & 4-Nitroquinol ine-1-0xide & ppob & 10 & & 1 & 0 & \\
\hline NITRTOL & & 5-Nitro-o-toluidine & ppob & 10 & & 1 & 0 & \\
\hline DIMBENZ & & 7,12-D imethylbenz [a] anthracene & ppb & 10 & & 1 & 0 & \\
\hline ACENAPH & & Acenaphthene & ppb & 10 & & 1 & 0 & \\
\hline ACENATL & & Acenaph thylene & ppob & 10 & & 1 & 0 & \\
\hline ACETONE & & Acetone & ppb & 100 & & 6 & 0 & \\
\hline ACETILE & & Acetonitrile & ppb & 200 & & 1 & 0 & \\
\hline ACETOPH & & Acetophenone & ppb & 10 & & 1 & 0 & \\
\hline ACROLIN & & Acrolein & ppb & 5 & & 1 & 0 & \\
\hline ACRYILE & & Acrylonitrile & ppb & 5 & & 1 & 0 & \\
\hline ALDRIN & & Aldrin & ppb & .05 & & 7 & 0 & \\
\hline DIMPHAM & & Alpha, alpha-D imethylphene thyl ami & ppb & 10 & & 1 & 0 & \\
\hline a-BHC & & Alpha-BHC & ppo & .05 & & 7 & 0 & \\
\hline ALUMNUM & & Aluminum & ppo & 200 & & 3 & 3 & \\
\hline FALUMIN & & Aluminum & ppb & 200 & & 3 & 0 & \\
\hline ANILINE & & Aniline & ppb & 10 & & 1 & 0 & \\
\hline ANTHRA & & Anthracene & ppo & 10 & & 1 & 0 & \\
\hline ANTIONY & & Ant imony & ppb & 200 & & 3 & 0 & \\
\hline FANTIMO & & Antimony, filtered & ppb & 200 & & 3 & 0 & \\
\hline ARAMITE & & Aramite & ppb & 10 & & 1 & 0 & \\
\hline AR1016 & & Aroclor-1016 & ppb & 1 & & 1 & 0 & \\
\hline AR1221 & & Aroclor- 1221 & ppb & 1 & & 1 & 0 & \\
\hline AR 1232 & & Aroclor- 1232 & ppb & 1 & & 1 & 0 & \\
\hline AR 1242 & & Aroclor -1242 & ppb & 1 & & 1 & 0 & \\
\hline AR 1248 & & Aroclor -1248 & ppb & 1 & & 1 & 0 & \\
\hline AR1254 & & Aroclor -1254 & ppb & 1 & & 1 & 0 & \\
\hline AR1260 & & Aroclor -1260 & ppb & 1 & & 1 & 0 & \\
\hline BENZENE & & Benzene & ppo & 5 & & 6 & 0 & \\
\hline BENZAAN & & Benzo (a) anthracene & ppb & 10 & & 1 & 0 & \\
\hline BENZOPY & & Benzo(a)pyrene & ppb & 10 & & 1 & 0 & \\
\hline BENZBFL & & Benzo(b)fluoranthene & ppb & 10 & & 1 & 0 & \\
\hline BGHIPER & & Benzo(ghi)perylene & ppb & 10 & & 1 & 0 & \\
\hline BNZKFLU & & Benzo $(k)$ fluoranthene & ppb & 10 & & $i$ & 0 & \\
\hline BENZOTH & & Benzothiazole & ppb & 10 & & 1 & 0 & \\
\hline BENZALC & & Benzyl alcohol & ppb & 20 & & 1 & 0 & \\
\hline BERYLUM & & Beryllium & ppb & 3 & & 3 & 0 & \\
\hline FBERYLL & & Beryllium, filtered & ppb & 3 & & 3 & 0 & \\
\hline b-BHC & & Beta-BHC & ppb & .05 & & 7 & 0 & \\
\hline BIS2CHM & & Bis (2-Choroethoxy)methane & ppb & 10 & & 1 & 0 & \\
\hline BIS2CHE & & Bis(2-chloroethyl) ether & ppb & 10 & & 1 & 0 & \\
\hline
\end{tabular}


Table 11-3. Constituent List and Summary of Results for the 216-S-10 Facility Data for Reporting Period July 1 through September 30, 1993. (sheet 4 of 6)

\begin{tabular}{|c|c|c|c|c|c|c|c|c|}
\hline \multirow[b]{2}{*}{ Short } & Constituent Name & \multirow[b]{2}{*}{ Units } & \multirow{2}{*}{$\begin{array}{l}\text { Lab } \\
\text { CROL }\end{array}$} & \multicolumn{2}{|l|}{ DUS } & \multicolumn{3}{|c|}{ Number of Semples } \\
\hline & (Method) & & & Limit & Agency & Total & $>$ CROL & $>$ DUS \\
\hline$\ldots \ldots$ & $n_{1}$ & ....... & $\ldots . .$. & $\ldots \ldots$ & $\ldots \ldots$ & ..... & $\ldots$. & $\ldots$ \\
\hline BISZETH & Bis(2-chloroisopropyl) ether & ppb & 10 & & & 1 & 0 & \\
\hline BIS2EPH & Bis(2-ethylhexyl) phthalate & ppb & 10 & & & 1 & 0 & \\
\hline BROMIDE & Bromide & ppb & 500 & & & 3 & 0 & \\
\hline BDCM & Bromodichloromethane & ppob & 5 & 100 & EPA & 1 & 0 & 0 \\
\hline BROMORM & Bromoform & ppb & 5 & 100 & EPA & 1 & 0 & 0 \\
\hline BUTBENP & Butylbenzylphthalate & ppb & 10 & & & 1 & 0 & \\
\hline CALCIUM & Calcium & ppb & 100 & & & 3 & 3 & \\
\hline FCALCIU & Calcium, filtered & ppb & 100 & & & 3 & 3 & \\
\hline CARBIDE & Carbon disulfide & ppb & 5 & & & 6 & 0 & \\
\hline TETRANE & Carbon tetrachloride & ppb & 5 & & & 6 & 0 & \\
\hline CHLOANE & Chlordane & ppb & .1 & & & 7 & 0 & \\
\hline CHLROB & Chlorobenzene & ppb & 5 & & & 1 & 0 & \\
\hline CHLLATE & Chlorobenz i late & ppb & 10 & & & 1 & 0 & \\
\hline CLETHAN & Chloroethane & ppob & 10 & & & 1 & 0 & \\
\hline CHLFORM & Chloroform & ppb & 5 & 100 & EPA & 6 & 0 & 0 \\
\hline CHLPRENE & Chloroprene & ppo & 5 & & & 1 & 0 & \\
\hline CHRYSEN & Chrysene & ppb & 10 & & & 1 & 0 & \\
\hline COBALT & Cobalt & ppob & 20 & & & 3 & 0 & \\
\hline FCOBALT & Cobalt, filtered & ppb & 20 & & & 3 & 0 & \\
\hline COPPER & Copper & ppo & 20 & 1000 & EPAS & 3 & 0 & 0 \\
\hline FCOPPER & Copper, filtered & ppo & 20 & 1000 & EPAS & 3 & 0 & 0 \\
\hline CRESOLS & Cresols (methylphenols) & ppb & 10 & & & 2 & 0 & \\
\hline CYANIDE & Cyanide & ppob & 20 & & & 1 & 0 & \\
\hline d-BHC & Delta-BHC & ppo & .1 & & & 7 & 0 & \\
\hline DIBPHTH & Di-n-butylphthalate & ppb & 10 & & & 1 & 0 & \\
\hline DIOPHTH & Di-n-octylphthalate & ppb & 10 & & & 1 & 0 & \\
\hline DIALlate & Diallate & ppb & 10 & & & 1 & 0 & \\
\hline DIBAHAN & D ibenz $[a, h]$ anthracene & ppb & 10 & & & 1 & 0 & \\
\hline DIBENFR & Dibenzofuran & ppb & 10 & & & 1 & 0 & \\
\hline BRCHLMET & Dibromochlorome thane & ppob & 5 & 100 & EPA & 1 & 0 & 0 \\
\hline DIBRMET & Dibromome thane & ppb & 5 & & & 1 & 0 & \\
\hline DICDIFM & Dichlorodif lworome thane & ppob & 5 & & & 1 & 0 & \\
\hline DIELRIN & Dieldrin & ppb & .05 & & & 7 & 0 & \\
\hline DIEPHTH & Diethyl phthalate & ppb & 10 & & & 1 & 0 & \\
\hline DIMETHO & Dimethoste & ppo & 10 & & & 1 & 0 & \\
\hline DIMPHTH & Dimethyl phthalate & ppo & 10 & & & 1 & 0 & \\
\hline DIPHAMI & Diphenyl amine & ppb & 10 & & & 1 & 0 & \\
\hline DISULFO & Disulfoton & ppb & 2 & & & 1 & 0 & \\
\hline ENDO1 & Endosulfan I & ppb & .1 & & & 7 & 0 & \\
\hline ENDO2 & Endosultan II & ppb & .05 & & & 7 & 0 & \\
\hline ENDSFAN & Endosulfan sulfate & ppb & .5 & & & 7 & 0 & \\
\hline ENDRALD & Endr in Aldehyde & ppo & .2 & & & 7 & 0 & \\
\hline ETHCYAN & Ethyl cyanide & nob & 10 & & & 6 & 0 & \\
\hline ETHMETH & Ethyl methacrylate & ppo & 5 & & & 1 & 0 & \\
\hline ETHMETS & Ethyl methanesulfonate & ppb & 10 & & & 1 & 0 & \\
\hline ETHBENZ & Ethylbenzene & ppb & 5 & & & 1 & 0 & \\
\hline FAMPHUR & Famphur & ppob & 10 & & & 1 & 0 & \\
\hline FLUORAN & Fluoranthene & ppob & 10 & & & 1 & 0 & \\
\hline FLRENE & Fluorene & ppb & 10 & & & 1 & 0 & \\
\hline HEPTLOR & Heptachlor & ppb & .05 & & & 7 & 0 & \\
\hline HEPTIDE & Heptachlor epoxide & ppb & 1 & & & 7 & 0 & \\
\hline HEXCBEN & Hexach lorobenzene & ppb & 10 & & & 1 & 0 & \\
\hline HEXCBUT & Hexachlorobutadiene & ppb & 10 & & & 1 & 0 & \\
\hline HEXCCYC & Hexach l or ocyclopentadiene & ppb & 10 & & & 1 & 0 & \\
\hline HEXCETH & Hexachloroethane & ppb & 10 & & & 1 & 0 & \\
\hline HEXACHL & Hexachl orophene & ppo & 10 & & & 1 & 0 & \\
\hline REXAENE & Hexachloropropene & ppb & 10 & & & 1 & 0 & \\
\hline
\end{tabular}


Table 11-3. Constituent List and Summary of Results for the 216-S-10 Facility Data for Reporting Period July 1 through September 30, 1993. (sheet 5 of 6)

\begin{tabular}{|c|c|c|c|c|c|c|c|c|}
\hline \multicolumn{4}{|c|}{ Constituent Name } & \multicolumn{2}{|r|}{ DWS } & \multicolumn{3}{|c|}{ Number of Semples } \\
\hline Short & (Method) & Full & Units & CRQL & Limit Agency & Total & $>$ CROL & $>$ DUS \\
\hline$\cdots \ldots$ & $\ldots \ldots \ldots$ & $\ldots+\ldots$ & $\ldots \ldots$ & $\cdots \ldots$ & $\cdots \ldots+\cdots$ & $\cdots \cdots$ & $\cdots$ & $\cdots$ \\
\hline INDENOP & & Indeno $(1,2,3 \cdot \mathrm{cd})$ prrene & ppb & 10 & & 1 & 0 & \\
\hline I SOBUTY & & Isobutyl alcohol & ppb & 200 & & 1 & 0 & \\
\hline ISOORIN & & Isodrin & ppb & 10 & & 1 & 0 & \\
\hline ISOPHER & & Isophorone & ppb & 10 & & 1 & 0 & \\
\hline ISOSOLE & & Isosafrole & ppo & 10 & & 1 & 0 & \\
\hline KEPONE & & Kepone & ppb & 10 & & 1 & 0 & \\
\hline KEROSEN & & Kerosene & ppb & 10000 & & 1 & 0 & \\
\hline MAGNES & & Magnesium & ppb & 100 & & 3 & 3 & \\
\hline FMAGNES & & Megnesium, filtered & ppb & 100 & & 3 & 3 & \\
\hline METHACR & & Methacrylonitrile & ppb & 5 & & 1 & 0 & \\
\hline METHAPY & & Methapyrilene & ppb & 10 & & 1 & 0 & \\
\hline IOOOMET & & Methyl Iodide & ppb & 5 & & 1 & 0 & \\
\hline METHBRO & & Methyl bromide & ppb & 10 & & 1 & 0 & \\
\hline METHCHL & & Methyl chloride & ppb & 10 & & 1 & 0 & \\
\hline METHONE & & Methyl ethyl ketone & ppb & 100 & & 6 & 0 & \\
\hline METACRY & & Methyl methacrylate & ppb & 5 & & 1 & 0 & \\
\hline METMSUL & & Methyl methanesulfonate & ppb & 10 & & 1 & 0 & \\
\hline METHPAR & & Methyl parathion & ppb & .5 & & 1 & 0 & \\
\hline METHYCH & & Methylene chloride & ppb & 5 & & 6 & 0 & \\
\hline DIPRNIT & & N-Nitroso-di-n-dipropylamine & ppb & 10 & & 1 & 0 & \\
\hline NNIBUTY & & N-Nitrosodi-n-butylamine & ppb & 10 & & 1 & 0 & \\
\hline NNIDIEY & & N-Nitrosodiethylamine & ppb & 10 & & 1 & 0 & \\
\hline NHIDIME & & N-Nitrosodimethyl amine & ppb & 10 & & 1 & 0 & \\
\hline NND IPHA & & N-Nitrosodiphenylamine & ppo & 10 & & 1 & 0 & \\
\hline NNIMETH & & $N$-Nitrosomethylethylamine & ppb & 10 & & 1 & $\mathbf{0}$ & \\
\hline NNIMORP & & N-Nitrosomorphol ine & ppb & 10 & & 1 & 0 & \\
\hline NNIPIPE & & N-Nitrosopiperidine & ppb & 10 & & 1 & $\mathbf{0}$ & \\
\hline MAPHTHA & & Naphthalene & ppb & 10 & & 1 & 0 & \\
\hline NICKEL & & Nickel & ppb & 30 & & 3 & 3 & \\
\hline FNICKEL & & Nickel, filtered & ppo & 30 & & 3 & 0 & \\
\hline NITRITE & & Nitrite & ppb & 200 & & 3 & 0 & \\
\hline NITBENZ & & Nitrobenzene & ppb & 10 & & 1 & $\mathbf{0}$ & \\
\hline NITRPYR & & Nitrosopyrrolidine & ppo & 10 & & 1 & 0 & \\
\hline TRIPHOS & & $0,0,0-$ Triethyl phosphorothioate & ppob & 10 & & 1 & 0 & \\
\hline DIPHOS & & 0,0-diethyl0-2-pyraz inylphosphor & ppob & 10 & & 1 & 0 & \\
\hline PCDDS & & PCDOs & ppob & .01 & & 1 & 0 & \\
\hline PCDFS & & PCDFs & ppob & .01 & & 1 & 0 & \\
\hline PARATHi & & Parathion & ppb & 10 & & 1 & 0 & \\
\hline PENTCHB & & Pentachlorobenzene & ppob & 10 & & 1 & 0 & \\
\hline PENTACH & & Pentachloroethane & ppb & 5 & & 1 & 0 & \\
\hline PENTCHN & & Pentachloronitrobenzene (PCNB) & ppb & 10 & & 1 & 0 & \\
\hline PENTCHP & 19 & Pentachlorophenol & ppo & 50 & & 1 & 0 & \\
\hline PENTCKP & 30 & Pentachlorophenol & ppob & 100 & & 2 & 0 & \\
\hline PHENTIN & & Phenacet in & ppo & 10 & & 1 & 0 & \\
\hline PHENANT & & Phenanthrene & ppo & 10 & & 1 & 0 & \\
\hline PHORATE & & Phorate & ppb & 2 & & 1 & 0 & \\
\hline PHOSPHA & & Phosphate & ppb & 400 & & 3 & 0 & \\
\hline POTASUM & & Potassium & ppo & 300 & & 3 & 3 & \\
\hline FPOTASS & & Potassium, filtered & ppb & 300 & & 3 & 3 & \\
\hline PRONIOE & & Pronamide & ppb & 10 & & 1 & 0 & \\
\hline PYRENE & & Pyrene & ppb & 10 & & 1 & 0 & \\
\hline PYRIDIN & & Pyridine & ppb & 10 & & 1 & 0 & \\
\hline SAFROL & & Safrol & ppb & 10 & & 1 & 0 & \\
\hline STYRENE & & Styrene & ppb & 5 & & 1 & 0 & \\
\hline SULFIDE & & Sulfide & ppb & 10000 & & 1 & 0 & \\
\hline TC- 99 & & Technetium-99 & $\mathrm{pCi} / \mathrm{L}$ & 15 & & 7 & 2 & \\
\hline
\end{tabular}


Table 11-3. Constituent List and Summary of Results for the 216-S-10 Facility Data for Reporting Period July 1 through September 30, 1993. (sheet 6 of 6)

\begin{tabular}{|c|c|c|c|c|c|c|c|c|}
\hline \multicolumn{3}{|c|}{ Constituent Name } & \multicolumn{3}{|c|}{ DWS } & \multicolumn{3}{|c|}{ Number of Samples } \\
\hline Short & (Method) & Units & CRQL & Limit & Agency & Total & $>C R Q L$ & $>$ DWS \\
\hline$\cdots \ldots \ldots$ & $\ldots \ldots \ldots \ldots$ & $\ldots \ldots$ & $\ldots \ldots$ & $\ldots \ldots$ & $\ldots \ldots$ & $\ldots \ldots$ & $\cdots \cdots$ & $\cdots$ \\
\hline PERCENE & Tetrachloroethene & ppb & 5 & & & 6 & 0 & \\
\hline TETPHNL & Tetrachlorophenols & ppo & 10 & & & 2 & 0 & \\
\hline PYROPHOS & Tetraethyldi thi opyrophosphate & ppb & 10 & & & 1 & 0 & \\
\hline TAF & Tetrahydrofuran & ppb & 10 & & & 6 & 0 & \\
\hline THALLIUM & Thallium & ppo & 5 & & & 1 & 0 & \\
\hline FTHALIUM & Thallium, filtered & ppb & 5 & & & 1 & 0 & \\
\hline TIN & Tin & ppb & 100 & & & 3 & 0 & \\
\hline FTIN & Tin, filtered & ppb & 100 & & & 3 & 0 & \\
\hline TOLUENE & Tolvene & ppo & 5 & & & 6 & 0 & \\
\hline TC & Total Carbon & ppb & 2000 & & & 7 & 7 & \\
\hline TRIBUTPH & Tributyl Phosphate & ppob & 10 & & & 1 & 0 & \\
\hline TRICENE & Trichloroethene & ppo & 5 & & & 6 & 0 & \\
\hline TRCMFLM & Trich l or ononof l wor cme thane & ppb & 5 & & & 1 & 0 & \\
\hline TRIPHNL & Trichlorophenols & ppb & 5 & & & 2 & 0 & \\
\hline TOICHPH & Tris-2-chloroethyl phosphate & pob & 10 & & & 1 & 1 & \\
\hline TRITIUM & Tritiun & $\mathrm{pCi} / \mathrm{L}$ & 500 & 20000 & EPA & 2 & 0 & 0 \\
\hline URANIUM & Uranium & ppo & .5 & & & 7 & 7 & \\
\hline VANADUM & Vanadium & ppb & 30 & & & 3 & 1 & \\
\hline FVANAD I & Vanadium, filtered & ppob & 30 & & & 3 & 1 & \\
\hline VINYLAC & Vinyl acetate & ppb & 5 & & & 1 & 0 & \\
\hline VINYIOE & Vinyl chloride & ppb & 10 & 2 & EPA & 6 & 0 & 0 * \\
\hline XYLENE & $x y($ enes (total) & ppo & 5 & & & 6 & 0 & \\
\hline ZINC & zinc & ppo & 10 & & & 3 & 3 & \\
\hline FZINC & zinc, filtered & ppo & 10 & & & 3 & 1 & \\
\hline ALLYLCL & allylchloride & ppo & 100 & & & 1 & 0 & \\
\hline TOICPENE & c is-1,3-D ichloropropene & ppb & 5 & & & 1 & 0 & \\
\hline MCRESOL & m-Cresol & ppb & 10 & & & 1 & 0 & \\
\hline DINBENZ & m-dinitrobenzene & ppb & 10 & & & 1 & 0 & \\
\hline OTOLHYD & o-Toluidine & ppo & 10 & & & 1 & 0 & \\
\hline DIMEAMB & p-D imethylaminoazobenzene & ppb & 10 & & & 1 & 0 & \\
\hline PHENDIA & p-Phenylenedienine & ppb & 10 & & & 1 & 0 & \\
\hline SYMTRIN & sym-Trinitrobenzene & ppob & 10 & & & 1 & 0 & \\
\hline DICPENE & trans-1,3-Dichloropropene & ppb & 5 & & & 1 & 0 & \\
\hline TOIBUTEN & trans-1,4-dichloro-2-butene & ppob & 5 & & & 1 & 0 & \\
\hline
\end{tabular}

For explanation of this table, see section 1.4 of report. 
Table 11-4. Constituents with at Least One Value Above the CRQL for the 216-S-10 Facility Data for Reporting Period July 1

through September 30, 1993. (sheet 1 of 3)

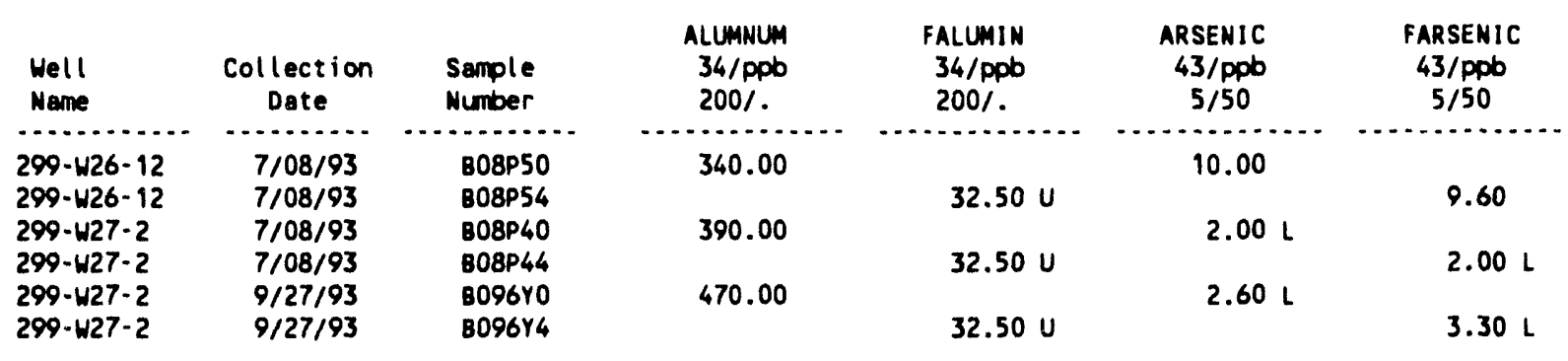

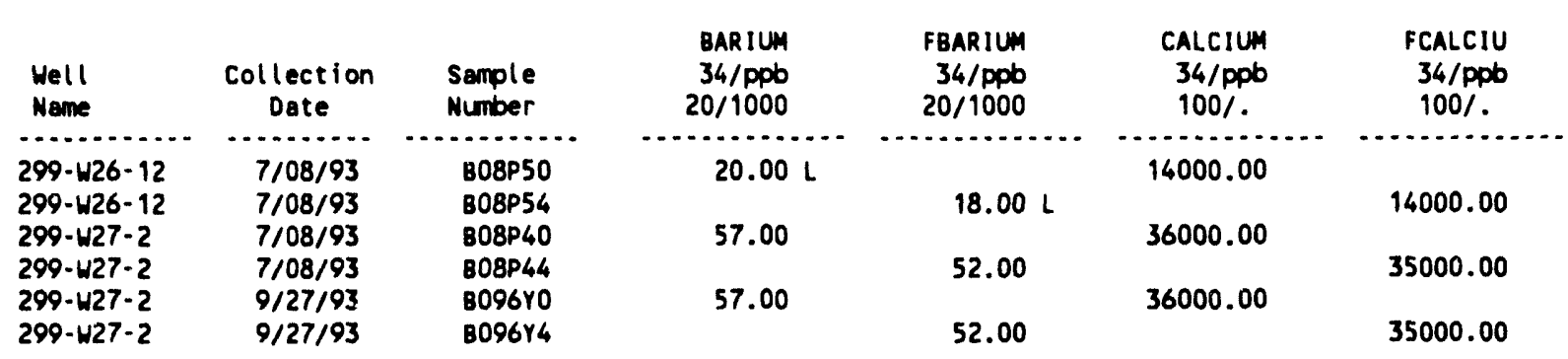

$\begin{array}{lcc}\begin{array}{l}\text { Well } \\ \text { Name }\end{array} & \begin{array}{c}\text { Collection } \\ \text { Date }\end{array} & \begin{array}{c}\text { Sample } \\ \text { Number }\end{array} \\ 299-W 26-12 & 7 / 08 / 93 & \text { BO8P50 } \\ 299-W 26-12 & 7 / 08 / 93 & \text { BO8P54 } \\ 299-W 27-2 & 7 / 08 / 93 & \text { BO8P40 } \\ 299-W 27-2 & 7 / 08 / 93 & \text { BO8P44 } \\ 299-W 27-2 & 9 / 27 / 93 & \text { BOS6YO }\end{array}$

299-W27-2 $9 / 27 / 93 \quad$ B096Y4

\begin{tabular}{|c|c|c|c|}
\hline $\begin{array}{c}\text { CHLORID } \\
124 / \text { ppb } \\
200 / 250000 \text { s }\end{array}$ & $\begin{array}{l}\text { CHROAUN } \\
34 / \text { ppb } \\
20 / 50\end{array}$ & $\begin{array}{l}\text { FCHROMI } \\
34 / \text { ppb } \\
20 / 50\end{array}$ & $\begin{array}{l}\text { FLUOR ID } \\
124 / p p b \\
100 / 4000\end{array}$ \\
\hline 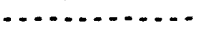 & $\ldots$ & 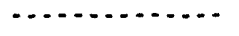 & $\ldots$ \\
\hline 1500.00 & 160.00 & $7.20 \mathrm{~L}$ & 1000.00 \\
\hline 19000.00 & 140.00 & $5.42 \mathrm{U}$ & 700.00 \\
\hline 20000.000 & 120.00 & $5.42 \mathrm{U}$ & 400.00 \\
\hline
\end{tabular}

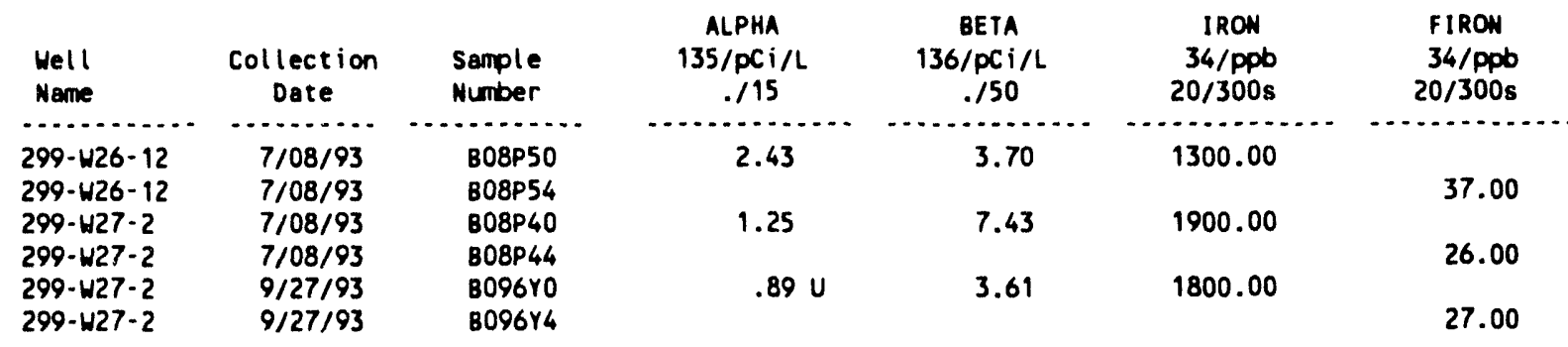

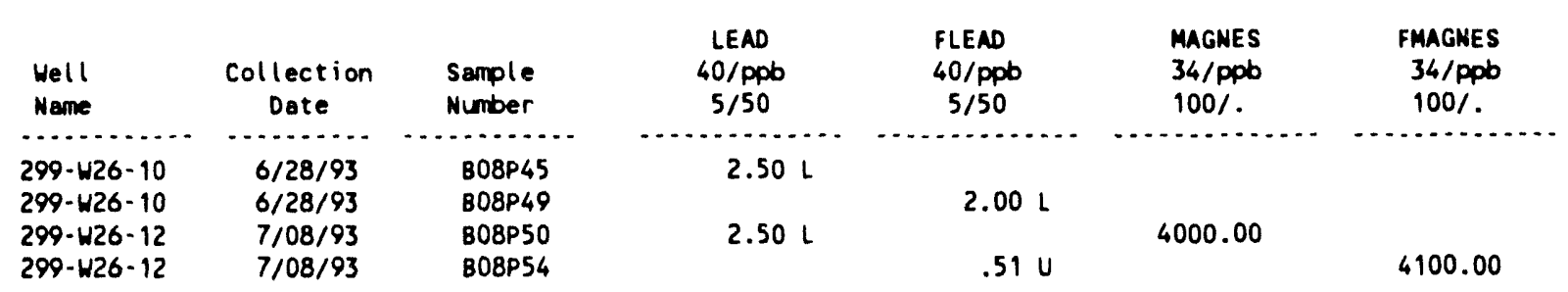


Table 11-4. Constituents with at Least One Value Above the CRQL for the 216-S-10 Facility Data for Reporting Period July 1 through September 30, 1993. (sheet 2 of 3)

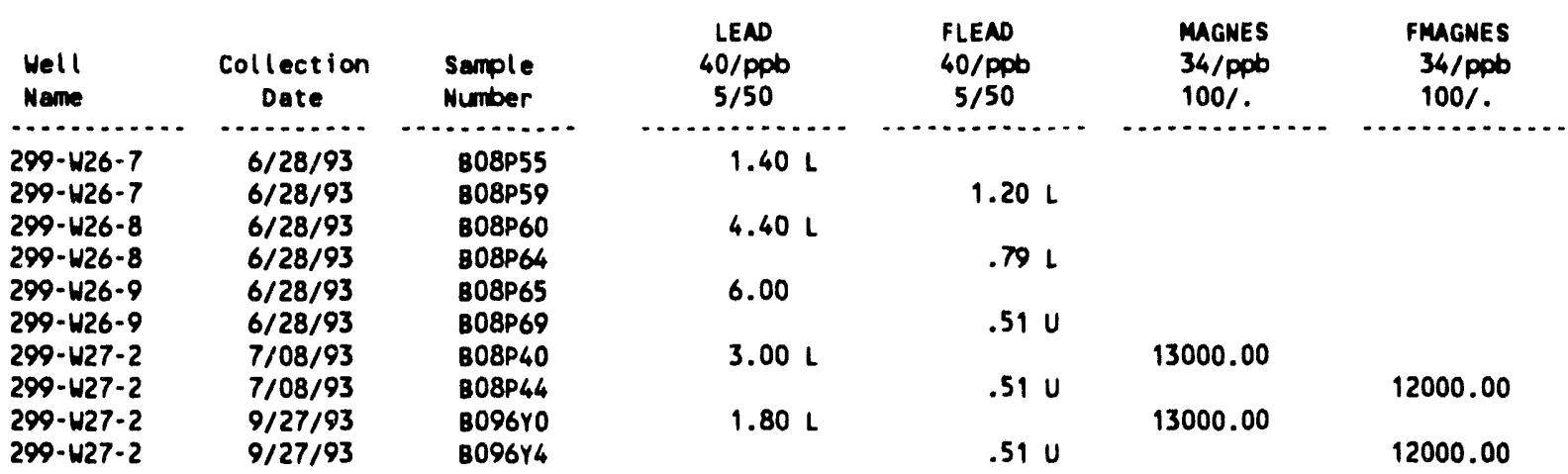

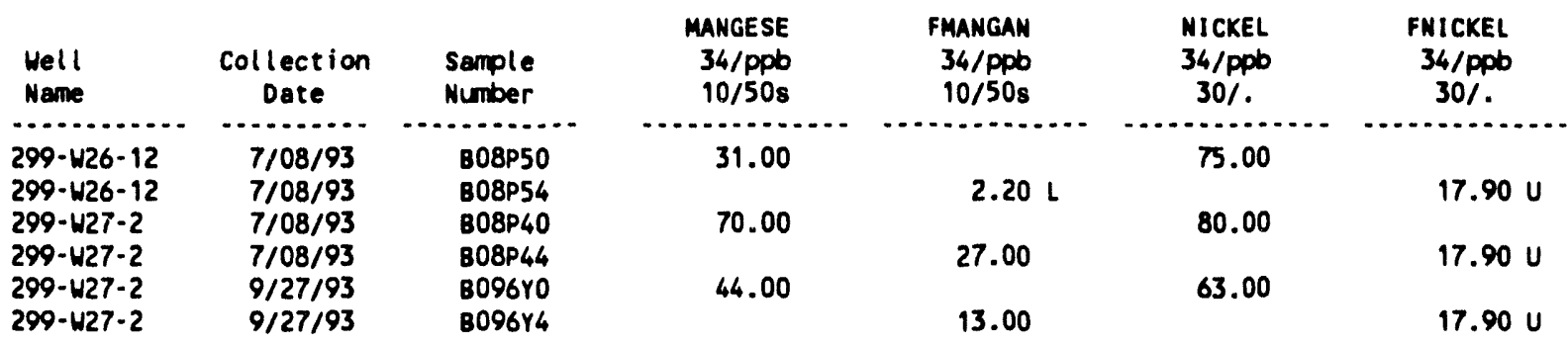

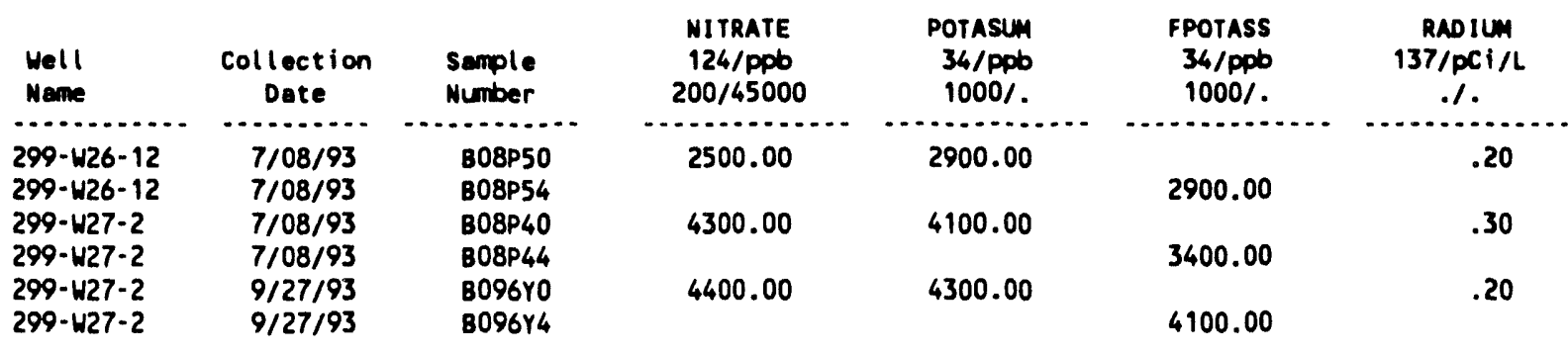

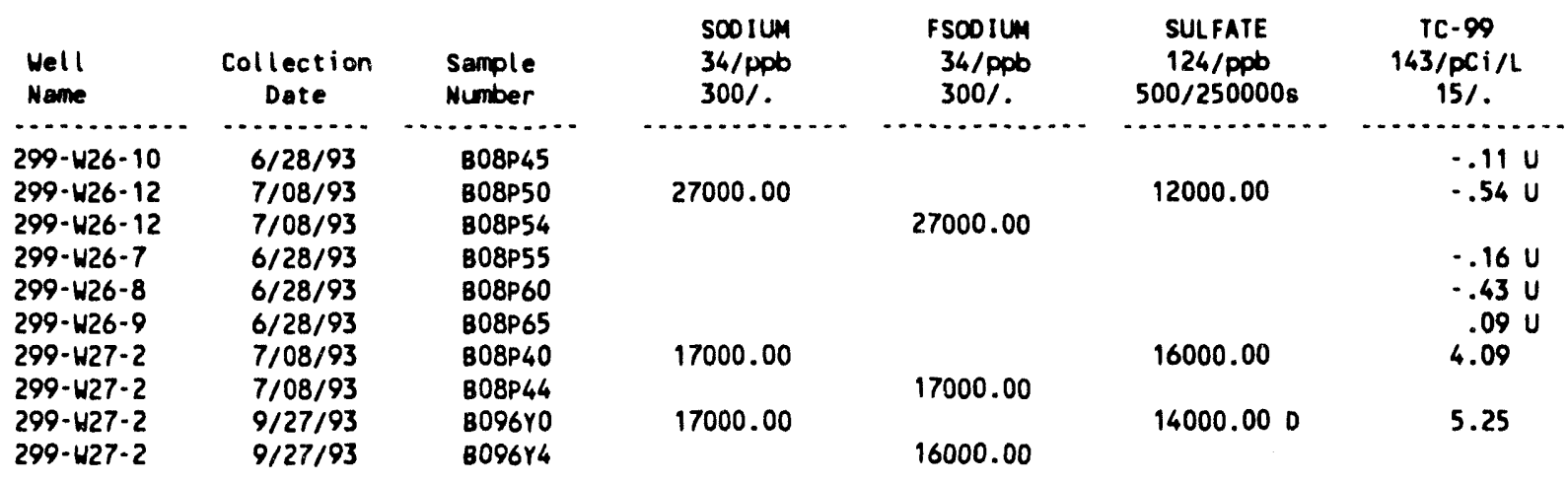


Table 11-4. Constituents with at Least One Value Above the CRQL for the 216-S-10 Facility Data for Reporting Period July 1 through September 30, 1993. (sheet 3 of 3 )

\begin{tabular}{|c|c|c|c|c|c|c|}
\hline $\begin{array}{l}\text { Well } \\
\text { Mame }\end{array}$ & $\begin{array}{c}\text { Collection } \\
\text { Date }\end{array}$ & $\begin{array}{l}\text { Sample } \\
\text { Number }\end{array}$ & $\begin{array}{c}\text { TC } \\
127 / \mathrm{ppb} \\
2000 / .\end{array}$ & $\begin{array}{c}\text { TOICHPH } \\
19 / \mathrm{ppb} \\
.1 .\end{array}$ & $\begin{array}{c}\text { TURBID } \\
\text { 126/NTU } \\
.1 / 1\end{array}$ & $\begin{array}{c}\text { URANIUM } \\
\text { 145/ppb } \\
.1 .\end{array}$ \\
\hline $\begin{array}{l}299-W 26-10 \\
299-W 26-12 \\
299-W 26-7 \\
299-W 26-8 \\
299-W 26-9 \\
299-W 27-2 \\
299-W 27-2\end{array}$ & $\begin{array}{l}6 / 28 / 93 \\
7 / 08 / 93 \\
6 / 28 / 93 \\
6 / 28 / 93 \\
6 / 28 / 93 \\
7 / 08 / 93 \\
9 / 27 / 93\end{array}$ & $\begin{array}{l}\text { 808P45 } \\
808 P 50 \\
808 P 55 \\
808 P 60 \\
\text { B08P65 } \\
\text { 808P40 } \\
\text { B096Y0 }\end{array}$ & $\begin{array}{l}25000.00 \mathrm{BO} \\
22000.00 \\
24000.00 \mathrm{BO} \\
27000.00 \mathrm{BO} \\
25000.00 \mathrm{BO} \\
30000.00 \\
31000.00 \mathrm{~B}\end{array}$ & 47.00 & $\begin{array}{l}13.80 \\
17.00\end{array}$ & $\begin{array}{r}1.71 \\
3.64 \\
2.23 \\
22.80 \\
1.80 \\
.59 \\
.64\end{array}$ \\
\hline $\begin{array}{l}\text { Well } \\
\text { Name }\end{array}$ & $\begin{array}{c}\text { Collection } \\
\text { Date }\end{array}$ & $\begin{array}{l}\text { Sample } \\
\text { Number }\end{array}$ & $\begin{array}{c}\text { VANADUM } \\
34 / \mathrm{ppb} \\
30 \%\end{array}$ & $\begin{array}{c}\text { FVANADI } \\
34 / \mathrm{ppb} \\
30 \%\end{array}$ & $\begin{array}{c}21 N C \\
34 / \mathrm{ppb} \\
10 \%\end{array}$ & $\begin{array}{c}\text { FZINC } \\
34 / \text { ppo } \\
10 \%\end{array}$ \\
\hline $\begin{array}{l}299-W 26-12 \\
299-W 26-12 \\
299-W 27-2 \\
299-W 27-2 \\
299-W 27-2 \\
299-W 27-2\end{array}$ & $\begin{array}{l}7 / 08 / 93 \\
7 / 08 / 93 \\
7 / 08 / 93 \\
7 / 08 / 93 \\
9 / 27 / 93 \\
9 / 27 / 93\end{array}$ & $\begin{array}{l}\text { B08P50 } \\
\text { B08P54 } \\
\text { B08P40 } \\
\text { B08P44 } \\
\text { B096YO } \\
\text { B096Y4 }\end{array}$ & $\begin{array}{l}35.00 \\
19.00 \mathrm{~L} \\
25.00 \mathrm{~L}\end{array}$ & $\begin{array}{l}35.00 \\
20.00 \mathrm{~L} \\
22.00 \mathrm{~L}\end{array}$ & $\begin{array}{r}12.00 \\
110.00 \\
200.00\end{array}$ & $\begin{array}{l}3.44 \mathrm{U} \\
4.00 \mathrm{~L} \\
16.00\end{array}$ \\
\hline
\end{tabular}

For explanation of this table, see Section 1.4 of report. 
Table 11-5. Contamination Indicator Parameters for the 216-S-10 Facility Data for Reporting Period July 1 through September 30, 1993.

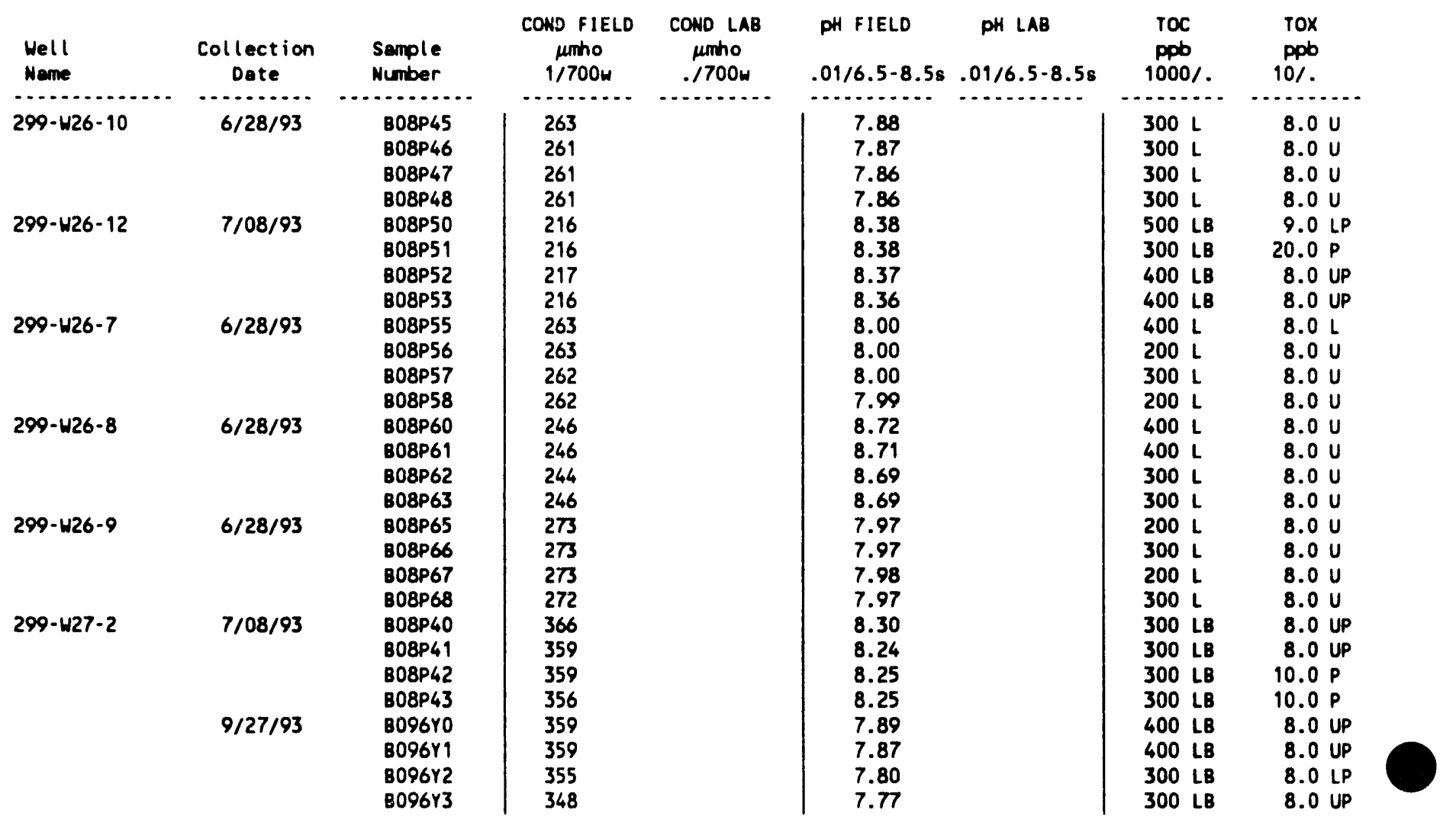

For explanation of this table, see Section 1.4 of report. 


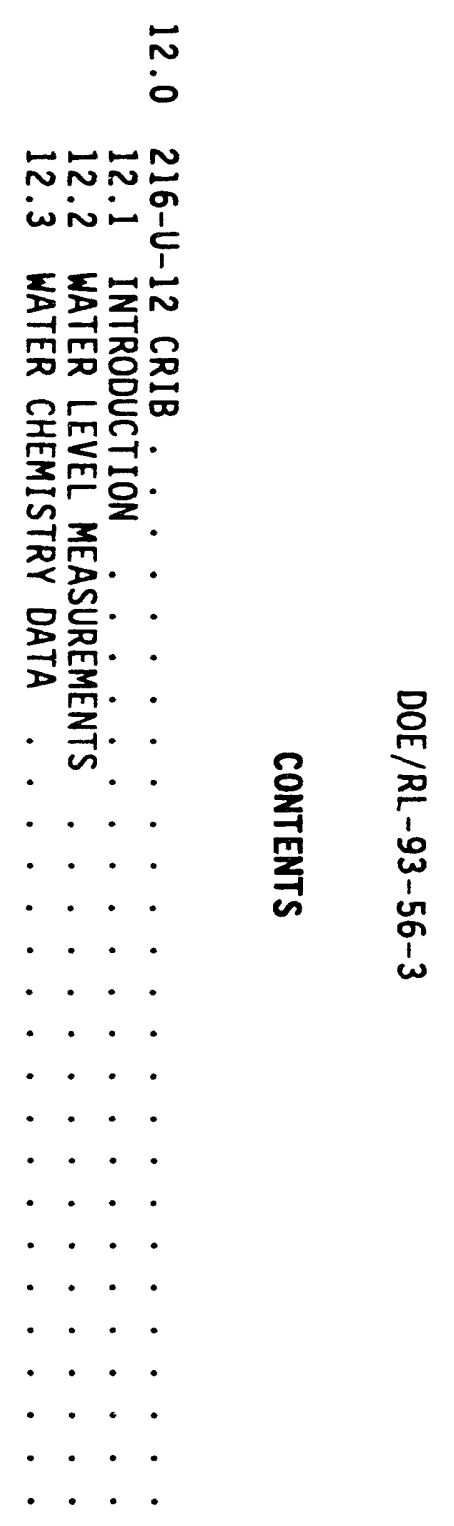

ลักล

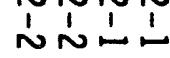


$\mathrm{DOE} / \mathrm{RL}-93-56-3$

\section{LIST OF FIGURES}

12-1 Well Location Map for 216-U-12 Crib............... 12-3

\section{LIST OF TABLES}

12-1 Monitoring Well Purpose and Sampling Schedule for the 216-U-12 Crib..................... 12-4

12-2 RCRA Water Level Measurement Report 216-U-12 Crib, Third Quarter 1993 .................. 12-5

12-3 Constituent List and Summary of Results for the 216-U-12 Crib Data for Reporting Period July 1 through September 30, 1993 . . . 12-6

12-4 Constituents with at Least One Value Above the CRQL for the 216-U-12 Crib Data for Reporting Period July 1 through September 30, 1993 ................. 12-9

12-5 Contamination Indicator Parameters for the 216-U-12 Crib Data for Reporting Period July 1 through September 30, 1993 . . . . 12-14 


\title{
$12.0216-U-12$ CRIB
}

\author{
B. A. Williams \\ Westinghouse Hanford Company
}

\subsection{INTRODUCTION}

A RCRA assessment-level groundwater monitoring network is established for the 216-U-12 Crib (U-12 Crib). The U-12 Crib received wastewater from the $U$ Plant in the 200 West Area from April 1960 until February 1988, when it was permanently retired and replaced by the 216-U-17 $\mathrm{Crib}$. Wastewater disposed of in the U-12 Crib contained hazardous waste and radioactive materials. DOE, Richland Operations Office has proposed that the facility be closed under RCRA interim status ( 40 CFR 265).

The U-12 Crib is located approximately $609 \mathrm{~m}(1,998 \mathrm{ft})$ south of the $U$ Plant in the 200 West Area (Figure 1-1). The crib is an unlined, gravelbottom, percolation crib that is 3 by $30.5 \mathrm{~m}$ (10 by $100 \mathrm{ft}$ ) wide and $4 \mathrm{~m}$ $(13 \mathrm{ft})$ deep. The crib has a plastic barrier cover and is backfilled with the original excavated soil. A vitrified clay distribution pipe, buried in the gravel, dispersed the effluent across the bottom of the crib.

The waste was composed of effluent from the $U$ Plant and included 291-U-1 Stack drainage and process condensate from the 224-U Building. The U-12 Crib received this waste stream from April 1960 until 1972, when it was deactivated. The U-12 Crib was reactivated in November 1981 and received waste until it was permanently retired in February 1988. An average of over $1,000,000 \mathrm{~L}(264,200 \mathrm{gal})$ of effluent each year were disposed of in the crib during its active life. The $U-12$ Crib has received low-level radioactive wastes that are known to have included chemicals such as dilute nitric acid, as well as radioactive wastes of plutonium, strontium, ruthenium, and uranium. Since 1985, physical controls and operating procedures have been modified to avoid inadvertent discharge of hazardous chemicals to the wastewater stream. Hydrogeologic evaluation (as of 1983) below the crib has indicated radioactive contamination to a depth of a least $43 \mathrm{~m}(140 \mathrm{ft})$. The depth to water below the crib is approximately $64 \mathrm{~m}(210 \mathrm{ft})$.

RCRA Interim-Status Groundwater Monitoring Plan for the 216-U-12 Crib (WHC 1990C) presents the program used to establish the background levels for the CIPs. The document summarizes the available data for the disposal facility. In accordance with the plan, four RCRA groundwater monitoring wells were installed in 1990. The monitoring network consisted of one upgradient and three downgradient wells (Figure 12-1).

The sample results for the third quarter of 1993 represent the $n$ inth calendar quarter of data collected at the U-12 Crib. Data collected during the third quarter of 1992 from downgradient wells 299-W22-41 and 299-W22-42 showed elevated levels of specific conductivity. Verification sampling and analysis during that and subsequent quarters corroborated these findings. The U-12 Crib groundwater monitoring well network currently is sampled quarterly for assessment monitoring in accordance with the U-12 Crib groundwater assessment plan (WHC 1993a). 
Two existing we11s, 299-W22-22 and 299-W22-23, have been remediated for inclusion in the monitoring network and were sampled for the first time last quarter (second quarter CY 1993). Table 12-1 lists the network wells and their sampling status during the reporting period. Currently the network consists of six wells.

\subsection{WATER LEVEL MEASUREMENTS}

Water levels in each network well are measured at least quarterly and when water samples are collected. Water level measurements obtained during the quarter are presented in Table 12-2. Water levels were measured up to three times during this quarter.

\subsection{WATER CHEMISTRY DATA}

Groundwater samples were collected in July from wells 299-W22-22, 299-W22-40, and 299-W22-41, and in August for well 299-W22-23 and represent the April through June (second quarter CY 1993) quarterly sampling period. Groundwater samples representative of this quarter (July through September) were collected in September for all the wells except 299-W22-22, which is being evaluated for inadequate water sample volume. All data currently available from this and the previous sampling period are shown in Tables 12-3 through 12-5. Table 12-3 includes the constituent 1 ist and a summary of results. Tables 12-4 and 12-5 show results for specific constituents. Analytical data unavailable at the time this report was prepared will be included in future quarterly reports.

Constituents that showed concentrations at or above DWS include unfiltered chromium in wells 299-W22-22, 299-W22-23, 299-W22-40, 299-W22-41, and upgradient well 299-W22-43. The DWS for unfiltered iron was exceeded in wells 299-W22-22, 299-W22-23, 299-W22-40, and 299-W22-43. Results for filtered chromium and filtered iron in these wells were below DWS. Elevated concentrations of these metals in unfiltered samples are believed to be an artifact of well installation, and are not representative of groundwater quality. Many constituent concentrations for remediated well 299-W22-23 are elevated above DWS. These numbers are believed to result from old well construction techniques. Currently well 299-W22-23 is under investigation.

Specific conductivity in downgradient wells 299-W22-41 and 299-W22-42 exceeded the critical mean of $437 \mu \mathrm{mho} / \mathrm{cm}$. Results for nitrate (DWS $=45,000 \mathrm{ppb}$ ), the principal constituent suspected of causing elevated conductivity, are also elevated in these wells; 299-W22-41 had a value of $230,000 \mathrm{ppb}$ and well 299-W22-42 had a value of 200,000 ppb. Carbon tetrachloride values were above the 5-ppb DWS in all four wells in the network. This is consistent with previous data results.

An explanation of any data flags can be found in Section 1.4.5. 


\section{-}

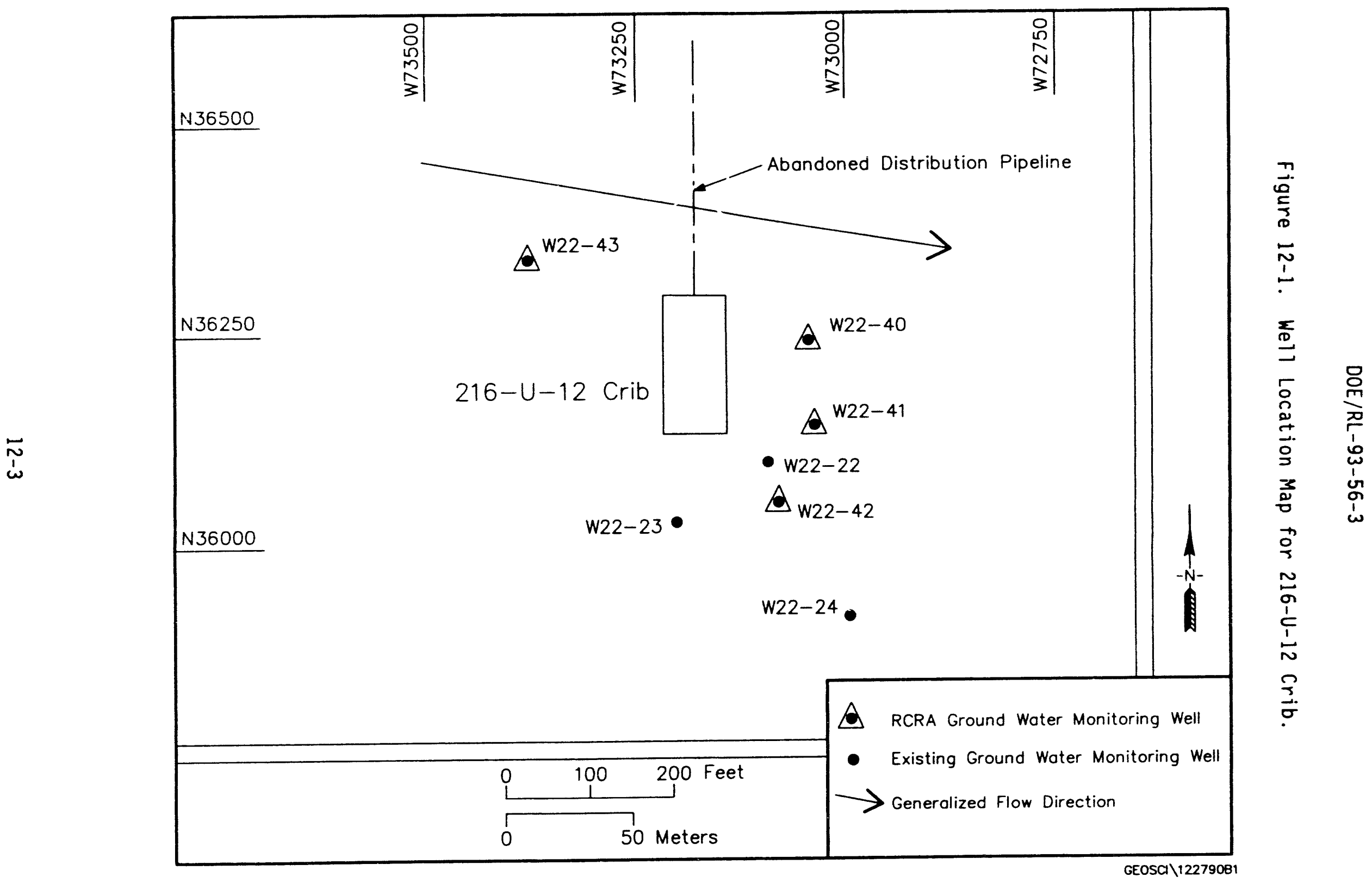


Table 12-1. Monitoring Well Purpose and Sampling Schedule for the 216-U-12 Crib.

\begin{tabular}{|c|c|c|c|c|}
\hline $\begin{array}{c}\text { Well no. } \\
(229-)\end{array}$ & $\begin{array}{c}\text { Relative } \\
\text { position }\end{array}$ & \multicolumn{1}{|c|}{ Hydrogeologic unit } & $\begin{array}{c}\text { Sample } \\
\text { frequency }\end{array}$ & $\begin{array}{c}\text { Sample date, } \\
\text { 3rd Qtr 1993 }\end{array}$ \\
\hline W22-43 & Upgradient & Ringold: Water Table & Quarterly & $9 / 20 / 93$ \\
\hline W22-40 & Downgradient & Ringold: Water Table & Quarterly & $9 / 20 / 93$ \\
\hline W22-41 & Downgradient & Ringold: Water Table & Quarterly & $9 / 20 / 93$ \\
\hline W22-42 & Downgradient & Ringold: Water Table & Quarterly & $9 / 20 / 93$ \\
\hline W22-22 & Downgradient & Ringold: Water Table & Quarterly & Not sampled \\
\hline W22-23 & Upgradient & Ringold: Water Table & Quarterly & $9 / 30 / 93$ \\
\hline
\end{tabular}

Note: Hydrogeologic units include the sandy gravels of the Hanford formation and silty sands of the Ringold Formation. 
Table 12-2. RCRA Water Level Measurement Report 216-U-12 Crib, Third Quarter 1993.

\begin{tabular}{|c|c|c|c|}
\hline Well & Date & $\begin{array}{l}\text { Depth to } \\
\text { water }(\mathrm{ft})\end{array}$ & $\begin{array}{l}\text { Water level } \\
\text { elevation } \\
\text { above msl }(\mathrm{ft})\end{array}$ \\
\hline \multicolumn{4}{|c|}{ Wells Monitoring the Top of the Unconfined Aquifer } \\
\hline $299-W 22-40$ & $\begin{array}{l}7 / 01 / 93 \\
9 / 10 / 93 \\
9 / 20 / 93\end{array}$ & $\begin{array}{l}236.36 \\
236.61 \\
236.64\end{array}$ & $\begin{array}{l}455.87^{\star} \\
455.62 \\
455.59 \star\end{array}$ \\
\hline $299-W 22-41$ & $\begin{array}{l}7 / 01 / 93 \\
9 / 10 / 93 \\
9 / 20 / 93\end{array}$ & $\begin{array}{l}235.97 \\
236.15 \\
236.18\end{array}$ & $\begin{array}{l}455.77^{\star} \\
455.59 \\
455.56^{\star}\end{array}$ \\
\hline $299-W 22-42$ & $\begin{array}{l}9 / 10 / 93 \\
9 / 20 / 93\end{array}$ & $\begin{array}{l}235.62 \\
235.71\end{array}$ & $\begin{array}{l}455.54 \\
455.45^{\star}\end{array}$ \\
\hline $299-W 22-43$ & $\begin{array}{l}9 / 07 / 93 \\
9 / 20 / 93\end{array}$ & $\begin{array}{l}235.13 \\
234.92\end{array}$ & $\begin{array}{l}456.22 \\
456.43^{\star}\end{array}$ \\
\hline
\end{tabular}

Notes: 1. Water level elevations are calculated by subtracting the measured depth to water from the surveyed elevation for the well.

2. Depth-to-water values are transcribed from field records.

3. Elevations marked with an ' $*$ ' were measured at the time of sampling.

4. To convert feet to meters multiply by 0.3048 . 
Table 12-3. Constituent List and Summary of Results for the 216-U-12 Crib Data for Reporting Period July 1 through September 30, 1993. (sheet 1 of 3)

CONTAMINATION INDICATOR PARAMETERS

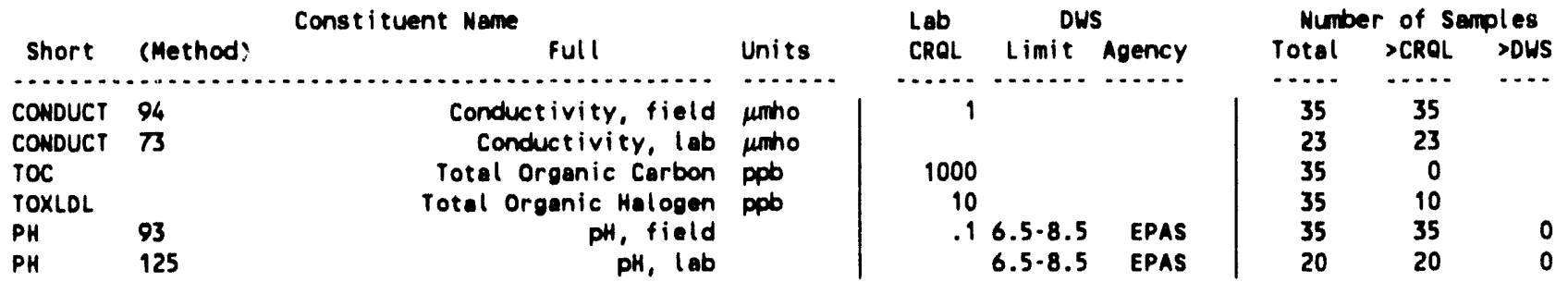

DRINKING WATER PARAMETERS

\begin{tabular}{|c|c|c|c|c|c|c|c|c|}
\hline \multicolumn{3}{|c|}{ Constituent Name } & \multicolumn{3}{|c|}{ DWS } & \multicolumn{3}{|c|}{ Number of Semples } \\
\hline (Method) & Full & Units & CROL & Limit & Agency & Total & $>$ CROL & $>$ DUS \\
\hline$\ldots \ldots . . .$. & n & $\ldots \ldots$ & $\ldots .$. & $\cdots \cdots$ & $\ldots . .$. & $\cdots \cdot$ & $\ldots \ldots$ & $\ldots$ \\
\hline $2,4,5 \mathrm{TP}$ & $2,4,5-T P$ & ppb & 2 & 10 & EPA & 6 & 0 & 0 \\
\hline $2,4-0$ & $2,4-0$ & ppo & 10 & 100 & EPA & 6 & 0 & 0 \\
\hline ARSENIC & Arsenic & ppo & 5 & 50 & EPA & 6 & 0 & 0 \\
\hline FARSENIC & Arsenic, filtered & ppb & 5 & 50 & EPA & 6 & 0 & 0 \\
\hline BARIUM & Bariun & ppo & 20 & 1000 & EPA & 9 & 9 & 0 \\
\hline FBARIUM & Berium, filtered & ppb & 20 & 1000 & EPA & 9 & 9 & 0 \\
\hline CADMIUM & Cadnium & ppb & 10 & 10 & EPA & 9 & 0 & 0 \\
\hline FCADMIU & Cadmium, filtered & ppb & 10 & 10 & EPA & 9 & 0 & 0 \\
\hline CHROMUM & Chromium & ppb & 20 & 50 & EPA & 9 & 6 & 6 \\
\hline FCHROMI & Chromiun, filtered & ppb & 20 & 50 & EPA & 9 & 0 & 0 \\
\hline COLIFORM & Coliforms & COL & & 1 & EPA & 6 & 0 & 0 \\
\hline ENDRIN & Endrin & ppb & .1 & .2 & EPA & 8 & 0 & 0 \\
\hline FLUORID & Fluoride & ppb & 100 & 4000 & EPA & 8 & 8 & 0 \\
\hline ALPHA & Gross alphe & $\mathrm{pCi} / \mathrm{L}$ & 4 & 15 & EPA & 9 & 8 & 0 \\
\hline BETA & Gross bete & $\mathrm{PCi} / \mathrm{L}$ & 8 & 50 & EPA & 9 & 9 & 0 \\
\hline LEAD & Lead & ppo & 5 & 50 & EPA & 6 & 1 & 0 \\
\hline FLEND & Lead, filtered & ppb & 5 & 50 & EPA & 6 & 0 & 0 \\
\hline MERCURY & Mercury & ppo & .2 & 2 & EPA & 6 & 0 & 0 \\
\hline FMERCUR & Mercury, filtered & ppb & .2 & 2 & EPA & 6 & 0 & 0 \\
\hline METHLOR & Methoxychlor & ppo & 2 & 100 & EPA & 8 & 0 & 0 \\
\hline NITRATE & Nitrate & ppb & 200 & 45000 & EPA & 8 & 8 & 3 \\
\hline RADIUM & Radium & $\mathrm{pCi} / \mathrm{L}$ & 1 & 5 & EPA & 6 & 5 & 0 \\
\hline SELENUM & Selenium & ppo & 10 & 10 & EPA & 6 & 0 & 0 \\
\hline FSELENI & Selenium, filtered & ppb & 10 & 10 & EPA & 6 & 0 & 0 \\
\hline SILVER & silver & ppo & 20 & 50 & EPA & 9 & 0 & 0 \\
\hline FSILVER & silver, filtered & ppb & 20 & 50 & EPA & 9 & 0 & 0 \\
\hline TOXAENE & Toxaphene & ppb & 2 & 5 & EPA & 8 & 0 & 0 \\
\hline TURBID & Turbidity & NTU & .1 & 1 & EPA & 6 & 6 & 6 \\
\hline$g \cdot B H C$ & gamma-BHC (L indane) & ppb & .05 & 4 & EPA & 8 & 0 & 0 \\
\hline
\end{tabular}

GROUNDWATER QUALITY PARAMETERS

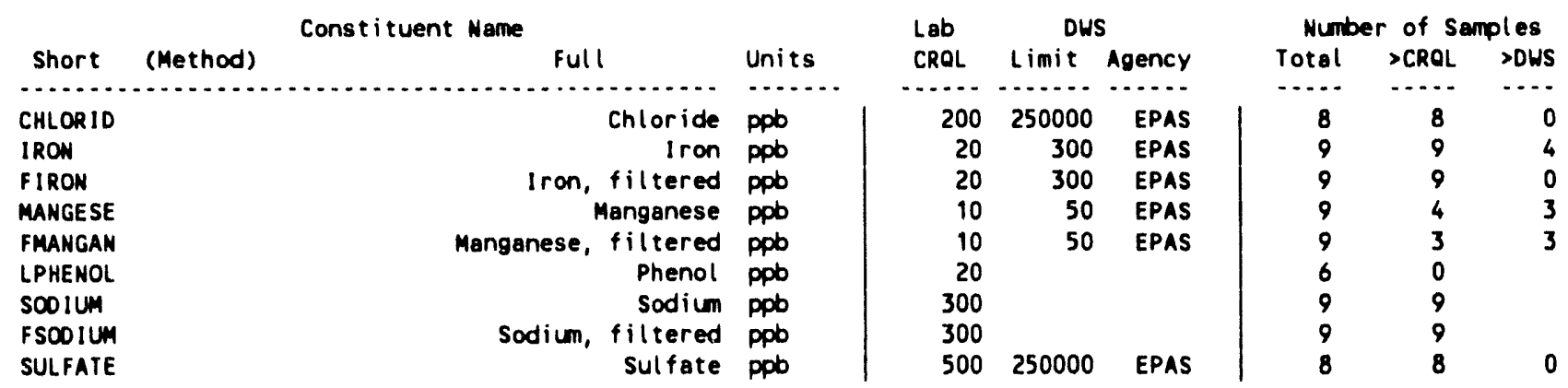


Table 12-3. Constituent List and Summary of Results for the 216-U-12 Crib Data for Reporting Period July 1 through September 30, 1993. (sheet 2 of 3)

SITE SPECIFIC AND OTHER CONSTITUENTS

\begin{tabular}{|c|c|c|c|c|c|c|c|c|c|}
\hline \multirow[b]{2}{*}{ Short } & \multicolumn{2}{|r|}{ Constituent Name } & \multirow[b]{2}{*}{ Units } & \multirow{2}{*}{$\begin{array}{l}\text { Lab } \\
\text { CROL }\end{array}$} & \multicolumn{2}{|c|}{ DWS } & \multicolumn{3}{|c|}{ Number of Semples } \\
\hline & (Method) & Full & & & Limit & Agency & Total & $>$ CRQL & $>0$ WS \\
\hline$\cdots \ldots$ & $\ldots \ldots$ & , & $\ldots \ldots$ & $\cdots \cdots$ & $\ldots \ldots$ & $\ldots \ldots$ & $\cdots$. & $\cdots+$ & $\ldots$ \\
\hline $1,1,1-T$ & & 1,1,1-Trichloroethane & ppo & 5 & & & 10 & 0 & \\
\hline $1,1,2-1$ & & 1,1,2-Trichloroethane & ppb & 5 & & & 10 & 0 & \\
\hline $1,1-01 C$ & & 1,1-Dichloroethane & ppo & 5 & & & 10 & 0 & \\
\hline $1,2-01 C$ & & 1,2-D ichloroethane & ppb & 5 & & & 10 & 0 & \\
\hline 12-DCE & & 1,2-0 ichloroethene & ppo & 5 & & & 10 & 0 & \\
\hline 14-ctben & & 1,4-Dichlorobenzene & ppb & 5 & & & 10 & 0 & \\
\hline 1BUTYN & & 1-Butanol & ppob & 1000 & & & 10 & 0 & \\
\hline $2,4,5-r$ & & $2,4,5 \cdot T$ & ppob & 2 & & & 6 & 0 & \\
\hline $246-t r p$ & & 2,4,6-Trichlorophenol & ppb & 5 & & & 6 & 0 & \\
\hline 24-dchp & & 2,4-0 ichlorophenol & ppob & 5 & & & 6 & 0 & \\
\hline DIMPHEN & & 2,4-0 imethylphenol & ppob & 5 & & & 6 & 0 & \\
\hline DINPHEN & & 2,4-D initrophenol & ppob & 150 & & & 6 & 0 & \\
\hline 26-dchp & & 2,6-Dichlorophenol & ppob & 5 & & & 6 & 0 & \\
\hline CHLPHEN & & 2-Chlorophenol & ppb & 5 & & & 6 & 0 & \\
\hline 2NITPH & & 2-Nitrophenol & ppob & 5 & & & 6 & 0 & \\
\hline BUTOINP & 30 & 2-sec-Butyl-4,6-dinitrophenol CDN & ppob & 5 & & & 6 & 0 & \\
\hline BUTDINP & 49 & 2-sec-8utyl-4,6-dinitrophenol CDN & ppb & 1 & & & 6 & 0 & \\
\hline DDD & & $4,4^{\prime}-D D D$ & ppob & .1 & & & 8 & 0 & \\
\hline DDE & & $4,4 !-D D E$ & ppb & .05 & & & 8 & 0 & \\
\hline DOT & & $4,4 \cdot-D D T$ & ppob & .1 & & & 8 & 0 & \\
\hline 460N2MP & & 4,6-D initro-2-methylphenol & ppb & 200 & & & 6 & 0 & \\
\hline CHLCRES & & 4-Chloro-3-methylphenol & ppo & 5 & & & 6 & 0 & \\
\hline MIBK & & 4-Methyl-2-pentanone & ppb & 50 & & & 10 & 0 & \\
\hline NITPHEN & & 4-Nitrophenol & ppb & 30 & & & 6 & 0 & \\
\hline ACETONE & & Acetone & ppb & 100 & & & 10 & 0 & \\
\hline ALDRIN & & Aldrin & ppb & .05 & & & 8 & 0 & \\
\hline a-BHC & & Alpha-BHC & ppb & .05 & & & 8 & 0 & \\
\hline ALUMNUM & & Aluminum & ppb & 200 & & & 9 & 2 & \\
\hline FALUMIN & & Aluminum & ppb & 200 & & & 9 & 0 & \\
\hline ANTIONY & & Antimony & ppb & 200 & & & 9 & 0 & \\
\hline FANT IMO & & Antimony, filtered & ppb & 200 & & & 9 & 0 & \\
\hline BENZENE & & Benzene & ppb & 5 & & & 10 & 0 & \\
\hline BERYLUM & & Beryllium & ppb & 3 & & & 9 & 0 & \\
\hline FBERYLL & & Beryllium, filtered & ppb & 3 & & & 9 & 0 & \\
\hline b-BHC & & Beta-BHC & ppb & .05 & & & 8 & 0 & \\
\hline BROMIDE & & Bromide & ppo & 500 & & & 8 & 0 & \\
\hline CALCIUM & & Calcium & ppo & 100 & & & 9 & 9 & \\
\hline FCALCIU & & Calcium, filtered & ppb & 100 & & & 9 & 9 & \\
\hline CARBIDE & & Carbon disulfide & ppb & 5 & & & 10 & 0 & \\
\hline TETRANE & & Carbon tetrachloride & ppb & 5 & & & 10 & 8 & \\
\hline CHLOANE & & Chlordane & ppb & .1 & & & 8 & 0 & \\
\hline CHLFORM & & Chloroform & ppb & 5 & 100 & EPA & 10 & 0 & 0 \\
\hline COBALT & & Cobalt & ppb & 20 & & & 9 & 0 & \\
\hline FCOBALT & & Cobalt, filtered & ppb & 20 & & & 9 & 0 & \\
\hline COPPER & & Copper & ppb & 20 & 1000 & EPAS & 9 & 2 & 0 \\
\hline FCOPPER & & Copper, filtered & ppb & 20 & 1000 & EPAS & 9 & 0 & 0 \\
\hline CRESOLS & & Cresols (methylphenols) & ppb & 10 & & & 6 & 0 & \\
\hline d-BHC & & Delt $8-B H C$ & ppb & .1 & & & 8 & 0 & \\
\hline DIELRIN & & Dieldrin & ppb & .05 & & & 8 & 0 & \\
\hline ENDO1 & & Endosulfan I & ppb & .1 & & & 8 & 0 & \\
\hline ENDO2 & & Endosulfan II & ppb & .05 & & & 8 & 0 & \\
\hline ENDSFAN & & Endosulfan sulfate & ppb & .5 & & & 8 & 0 & \\
\hline ENDRALD & & Endrin Aldehyde & ppb & .2 & & & 8 & 0 & \\
\hline ETHCYAN & & Ethyl cyanide & ppb & 10 & & & 10 & 0 & \\
\hline HEPTLOR & & Heptachlor & ppb & .05 & & & 8 & 0 & \\
\hline HEPTIDE & & Heptachlor epoxide & ppb & 1 & & & 8 & 0 & \\
\hline $1-129$ & & lodine-129 & $\mathrm{pCi} / \mathrm{L}$ & 1 & & & 3 & 3 & \\
\hline
\end{tabular}


Table 12-3. Constituent List and Summary of Results for the 216-U-12 Crib Data for Reporting Period July 1 through September 30, 1993. (sheet 3 of 3)

\begin{tabular}{|c|c|c|c|c|c|c|c|c|}
\hline \multicolumn{9}{|c|}{ Number of Semples } \\
\hline short & (Method) & Units & CROL & Limit & Agency & Total & $>\mathrm{CROL}$ & $>$ DUS \\
\hline$\ldots \ldots \ldots$ & $\ldots \ldots \ldots \ldots \ldots$ & $\ldots \ldots$ & $\ldots \ldots$ & $\ldots \ldots$ & $\ldots \ldots$ & $\ldots \ldots$ & $\ldots \ldots$ & $\ldots$ \\
\hline MAGNES & Magnesium & ppo & 100 & & & 9 & 9 & \\
\hline FMAGNES & Magnesium, filtered & Ppo & 100 & & & 9 & 9 & \\
\hline METHONE & Methyl ethyl ketone & $p p b$ & 100 & & & 10 & 0 & \\
\hline METHYCH & Methylene chloride & ppb & 5 & & & 10 & 0 & \\
\hline NICKEL & Nickel & ppb & 30 & & & 9 & 5 & \\
\hline FNICKEL & Nickel, filtered & ppb & 30 & & & 9 & 1 & \\
\hline NITRITE & Nitrite & ppb & 200 & & & 8 & 0 & \\
\hline PENTCHP & Pentachlorophenol & ppob & 100 & & & 6 & 0 & \\
\hline PHOSPHA & Phosphate & ppo & 400 & & & 8 & 0 & \\
\hline POTASUM & Potassium & ppb & 300 & & & 9 & 9 & \\
\hline FPOTASS & Potassium, filtered & ppo & 300 & & & 9 & 9 & \\
\hline TC. $\varnothing 9$ & Technetium- 99 & $\mathrm{pCi} / \mathrm{L}$ & 15 & & & 11 & 10 & \\
\hline PERCENE & Tetrachloroethene & ppob & 5 & & & 10 & 0 & \\
\hline TETPHNL & Tetrachlorophenols & ppb & 10 & & & 6 & 0 & \\
\hline TAF & Tetrahydrofuran & ppob & 10 & & & 10 & 0 & \\
\hline TIN & Tin & ppb & 100 & & & 9 & 0 & \\
\hline FTIN & Tin, filtered & ppb & 100 & & & 9 & 0 & \\
\hline TOLUENE & Toluene & ppb & & & & 10 & 0 & \\
\hline rC & Total Carbon & ppb & 2000 & & & 8 & 8 & \\
\hline TRICENE & Trichloroethene & ppob & 5 & & & 10 & 0 & \\
\hline TRIPHNL & Trichlorophenols & ppb & 5 & & & 6 & 0 & \\
\hline TRITIUM & Tritiun & $\mathrm{pCi} / \mathrm{L}$ & 500 & 20000 & EPA & 9 & 9 & 2 \\
\hline URANIUM & Uranium & ppo & .5 & & & 8 & 8 & \\
\hline$U-234$ & Uranium-234 & $\mathrm{PCi} / \mathrm{L}$ & .1 & & & 3 & 3 & \\
\hline$U-235$ & Uranium-235 & $\mathrm{pci/l}$ & .1 & & & 3 & 1 & \\
\hline$U-238$ & Uranium-238 & $\mathrm{PCi} / \mathrm{L}$ & .1 & & & 3 & 3 & \\
\hline VANADUM & Vanadium & ppo & 30 & & & 9 & 2 & \\
\hline FVANAD I & Vanadium, filtered & ppb & 30 & & & 9 & 2 & \\
\hline VINYIDE & Vinyl chloride & ppo & 10 & 2 & EPA & 10 & 0 & $0^{*}$ \\
\hline XYLENE & Xylenes (total) & ppo & 5 & & & 10 & 0 & \\
\hline ZINC & 2 inc & pob & 10 & & & 9 & 5 & \\
\hline FZINC & zinc, filtered & ppob & 10 & & & 9 & 2 & \\
\hline
\end{tabular}

For explanation of this table, see Section 1.4 of report. 
Table 12-4. Constituents with at Least One Value Above the CRQL for the 216-U-12 Crib Data for Reporting Period July 1

through September 30, 1993. (sheet 1 of 5)

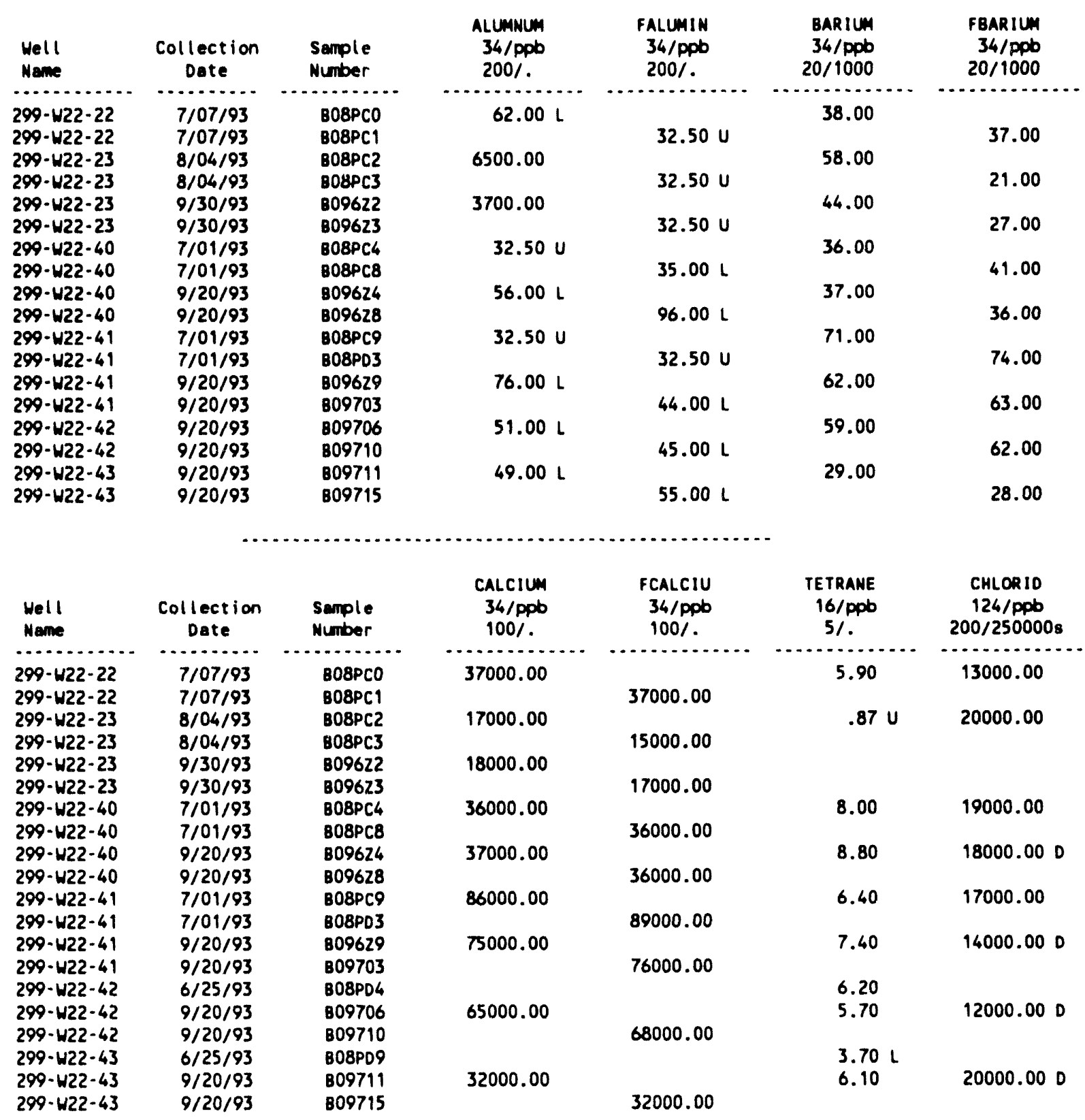


Table 12-4. Constituents with at Least One Value Above the CRQL for the 216-U-12 Crib Data for Reporting Period July 1

through September 30, 1993. (sheet 2 of 5)

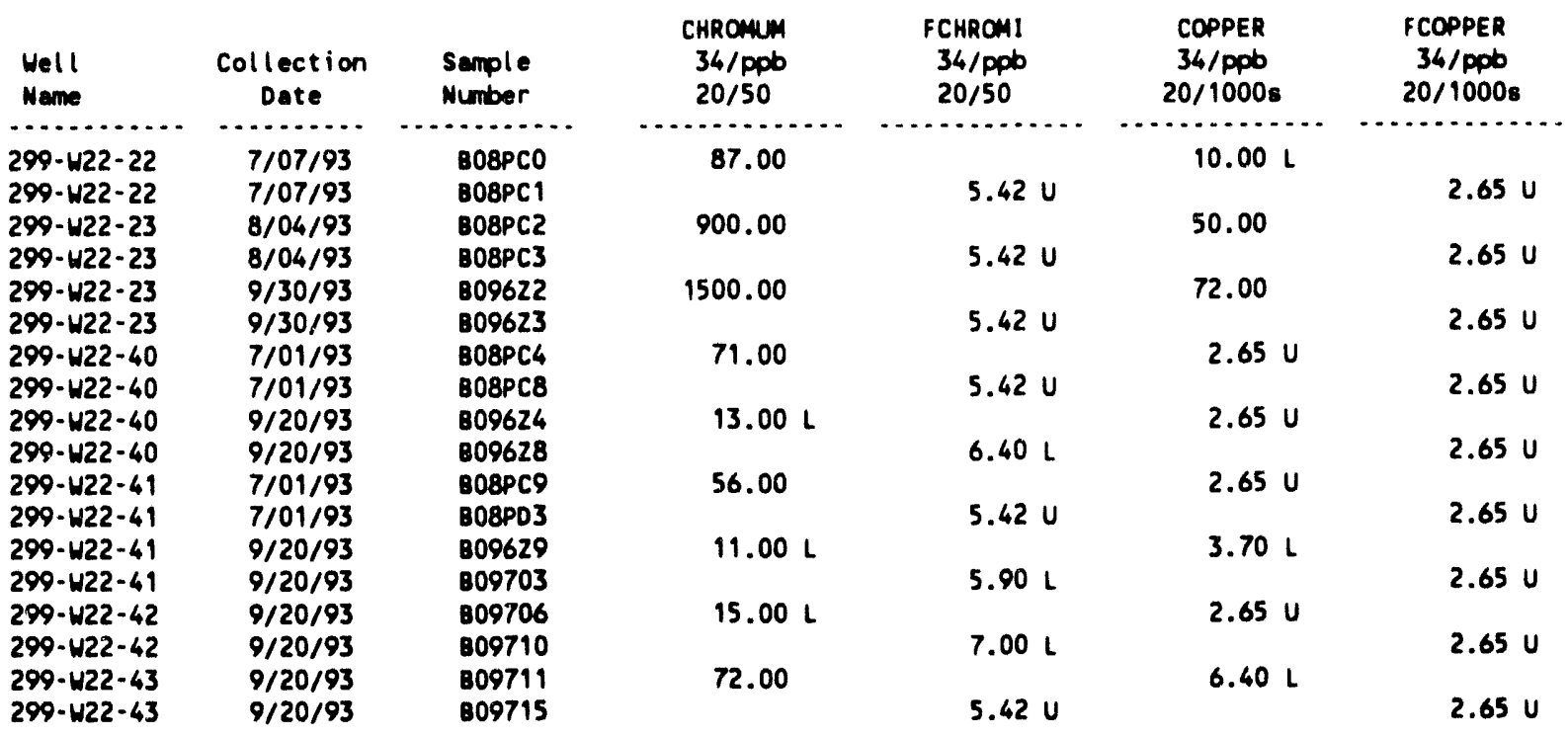

\begin{tabular}{|c|c|c|c|c|c|c|}
\hline $\begin{array}{l}\text { Mell } \\
\text { Name }\end{array}$ & $\begin{array}{c}\text { Collection } \\
\text { Date }\end{array}$ & $\begin{array}{l}\text { Sample } \\
\text { Number }\end{array}$ & $\begin{array}{l}\text { FLUOR ID } \\
124 / \mathrm{ppb} \\
100 / 4000\end{array}$ & $\begin{array}{c}\text { ALPHA } \\
135 / \mathrm{PCi} / \mathrm{L} \\
. / 15\end{array}$ & $\begin{array}{c}\text { BETA } \\
136 / \text { PCi/L } \\
.150\end{array}$ & $\begin{array}{c}1-129 \\
139 / \mathrm{pCi} / \mathrm{L} \\
. /\end{array}$ \\
\hline $\begin{array}{l}299-W 22-22 \\
299-W 22-23 \\
299-W 22-23 \\
299-W 22-40 \\
299-W 22-40 \\
299-W 22-41 \\
299-W 22-41 \\
299-W 22-42 \\
299-W 22-43\end{array}$ & $\begin{array}{l}7 / 07 / 93 \\
8 / 04 / 93 \\
9 / 30 / 93 \\
7 / 01 / 93 \\
9 / 20 / 93 \\
7 / 01 / 93 \\
9 / 20 / 93 \\
9 / 20 / 93 \\
9 / 20 / 93\end{array}$ & $\begin{array}{l}\text { B08PCO } \\
\text { B08PC2 } \\
\text { B09622 } \\
\text { B08PC4 } \\
\text { B09624 } \\
\text { B08PC9 } \\
\text { B09629 } \\
\text { B09706 } \\
\text { B09711 }\end{array}$ & $\begin{array}{l}600.00 \\
1100.008 \\
800.00 \\
500.00 \\
900.00 \\
500.00 \\
500.00 \\
600.00\end{array}$ & $\begin{array}{c}.88 U \\
10.20 \\
3.89 \\
4.41 \\
3.22 \\
3.59 \\
3.43 \\
5.13 \\
2.34\end{array}$ & $\begin{array}{c}8.13 \\
10.00 \\
8.19 \\
7.31 \\
4.97 \\
27.90 \\
26 \\
22.00 \\
6.43\end{array}$ & $\begin{array}{r}1.33 \\
13.30 \\
14.90\end{array}$ \\
\hline
\end{tabular}

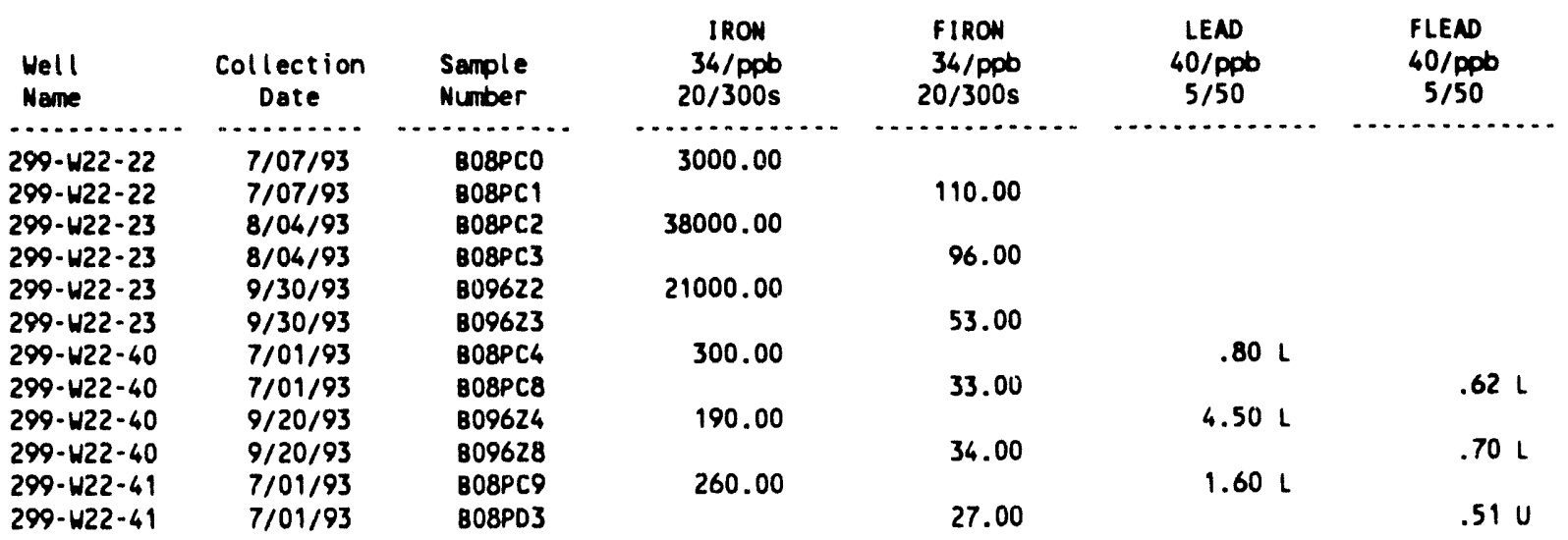


Table 12-4. Constituents with at Least One Value Above the CRQL for the 216-U-12 Crib Data for Reporting Period July 1

through September 30,1993 . (sheet 3 of 5)

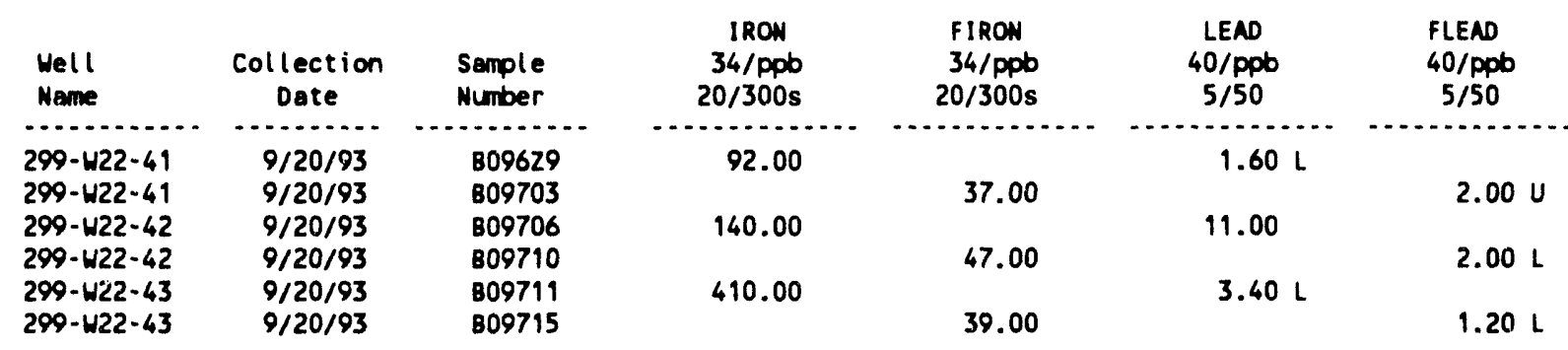

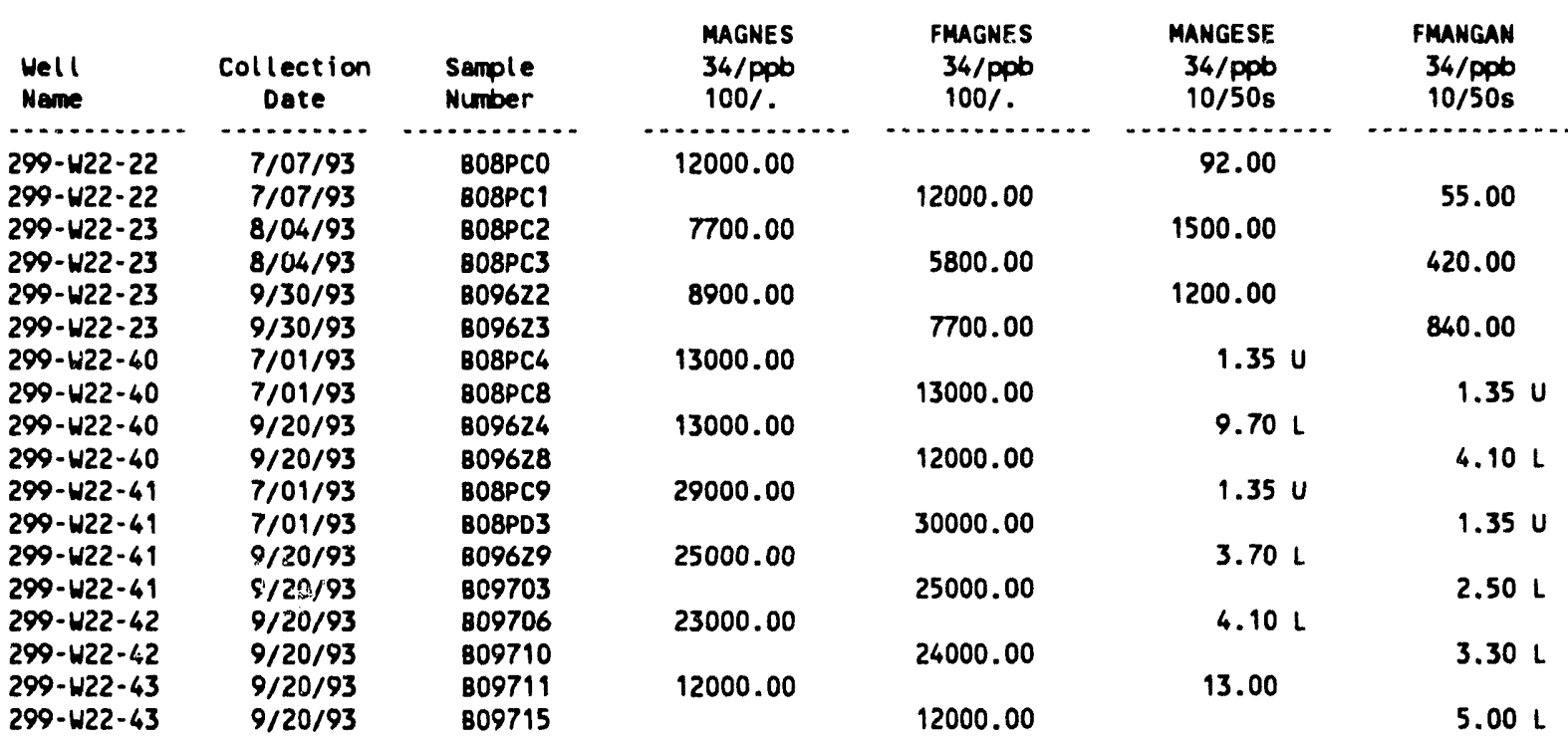

\begin{tabular}{|c|c|c|c|c|c|c|}
\hline $\begin{array}{l}\text { Well } \\
\text { Name }\end{array}$ & $\begin{array}{l}\text { Collection } \\
\text { Date }\end{array}$ & $\begin{array}{l}\text { Sample } \\
\text { Number }\end{array}$ & $\begin{array}{l}\text { NICKEL } \\
34 / \mathrm{ppb} \\
30 \%\end{array}$ & $\begin{array}{c}\text { FNICKEL } \\
34 / \text { ppb } \\
30 \%\end{array}$ & $\begin{array}{l}\text { NITRATE } \\
124 / \text { ppb } \\
200 / 45000\end{array}$ & $\begin{array}{l}\text { POTASUM } \\
34 / \mathrm{ppDb} \\
1000 \%\end{array}$ \\
\hline $299-$ W22- 22 & $7 / 07 / 93$ & BO8PCO & 48.00 & & 23000.00 & 4700.00 \\
\hline 299-W22- 22 & $7 / 07 / 93$ & BOBPC1 & & $17.90 \mathrm{U}$ & & \\
\hline $\begin{array}{l}299-W 22-23 \\
299-W 22-23\end{array}$ & $\begin{array}{l}8 / 04 / 93 \\
8 / 04 / 93\end{array}$ & $\begin{array}{l}\text { B08PC2 } \\
\text { B08PC3 }\end{array}$ & 450.00 & $17.90 \mathrm{U}$ & $1200.00 \mathrm{~F}$ & 3500.00 \\
\hline $\begin{array}{l}299-W 22-23 \\
299-W 22-23\end{array}$ & $\begin{array}{l}9 / 30 / 93 \\
9 / 30 / 93\end{array}$ & $\begin{array}{l}809622 \\
809623\end{array}$ & 700.00 & $17.90 \mathrm{U}$ & & 2700.00 \\
\hline $\begin{array}{l}2.99-W 22-40 \\
299-W 22-40\end{array}$ & $\begin{array}{l}7 / 01 / 93 \\
7 / 01 / 93\end{array}$ & $\begin{array}{l}\text { BOBPC4 } \\
\text { BO8PC8 }\end{array}$ & 47.00 & $17.90 \mathrm{U}$ & 25000.00 & 4200.00 \\
\hline $\begin{array}{l}299-W 22-40 \\
299-W 22-40\end{array}$ & $\begin{array}{l}9 / 20 / 93 \\
9 / 20 / 93\end{array}$ & $\begin{array}{l}B 09624 \\
B 09628\end{array}$ & $29.00 \mathrm{~L}$ & $22.00 \mathrm{~L}$ & 24000.000 & 4100.00 \\
\hline $\begin{array}{l}299-W 22-41 \\
299-W 22-41\end{array}$ & $\begin{array}{l}7 / 01 / 93 \\
7 / 01 / 93\end{array}$ & $\begin{array}{l}\text { BO8PC9 } \\
\text { BOBPD3 }\end{array}$ & $17.90 \mathrm{U}$ & $17.90 \mathrm{U}$ & 270000.00 & 4900.00 \\
\hline $\begin{array}{l}299-W 22-41 \\
299-W 22-41\end{array}$ & $\begin{array}{l}9 / 20 / 93 \\
9 / 20 / 93\end{array}$ & $\begin{array}{l}809629 \\
809703\end{array}$ & $17.90 \mathrm{U}$ & $17.90 \mathrm{U}$ & 230000.000 & 4800.00 \\
\hline $\begin{array}{l}299-W 22-42 \\
299-W 22-42\end{array}$ & $\begin{array}{l}9 / 20 / 93 \\
9 / 20 / 93\end{array}$ & $\begin{array}{l}809706 \\
809710\end{array}$ & $27.00 \mathrm{~L}$ & $20.00 \mathrm{~L}$ & $200000.00 \mathrm{D}$ & 5300.00 \\
\hline $\begin{array}{l}299-W 22-43 \\
299-W 22-43\end{array}$ & $\begin{array}{l}9 / 20 / 93 \\
9 / 20 / 93\end{array}$ & $\begin{array}{l}B 09711 \\
B 09715\end{array}$ & 72.00 & 42.00 & $.00 \mathrm{D}$ & 3900.00 \\
\hline
\end{tabular}


Table 12-4. Constituents with at Least One Value Above the CRQL for the 216-U-12 Crib Data for Reporting Period July 1 through September 30, 1993. (sheet 4 of 5)

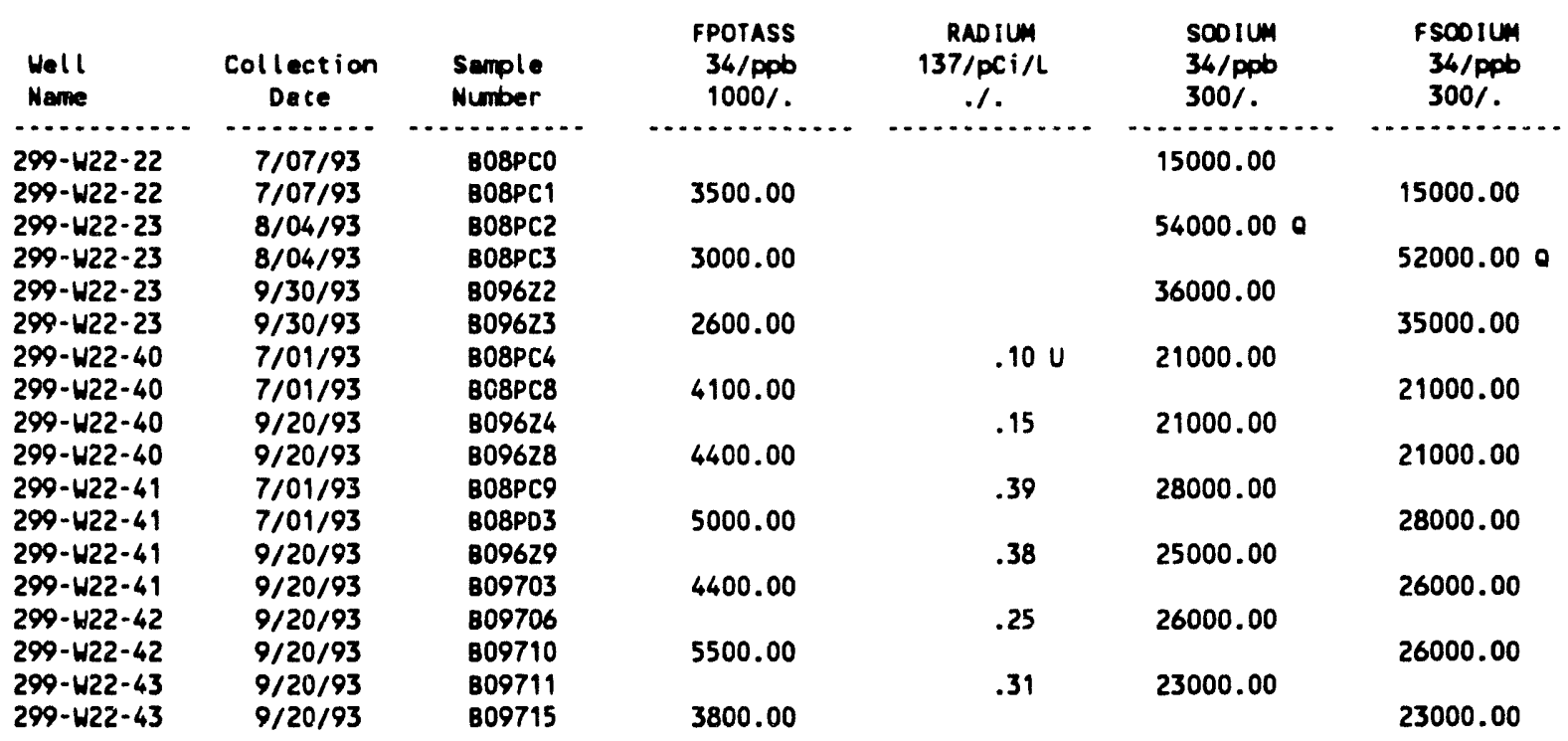

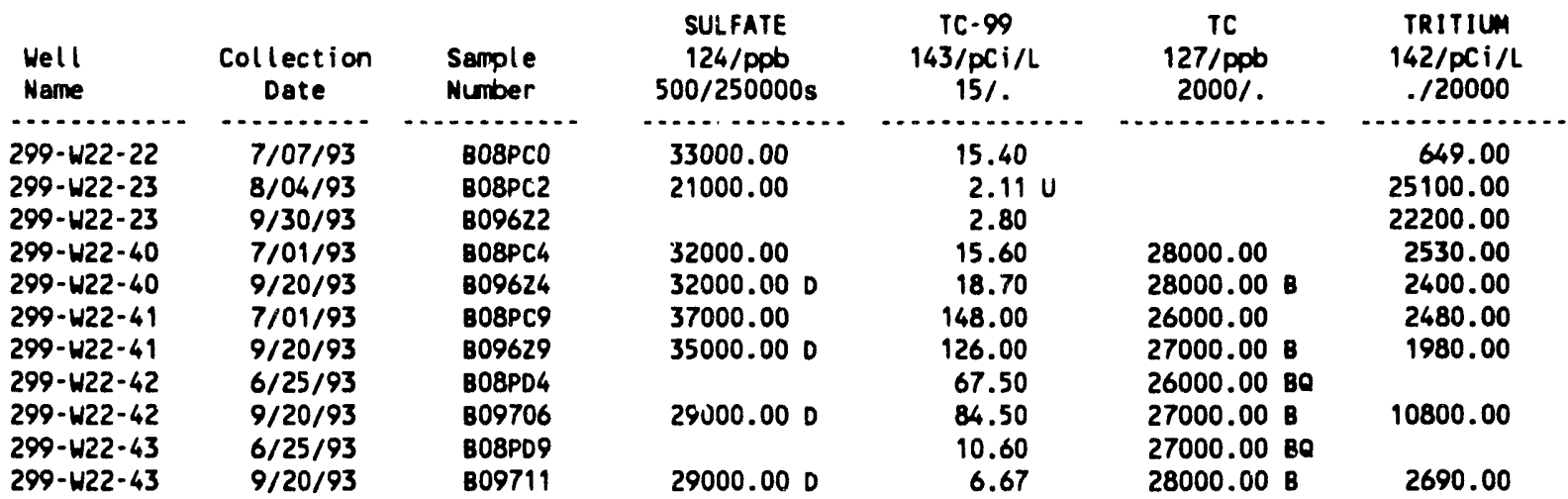

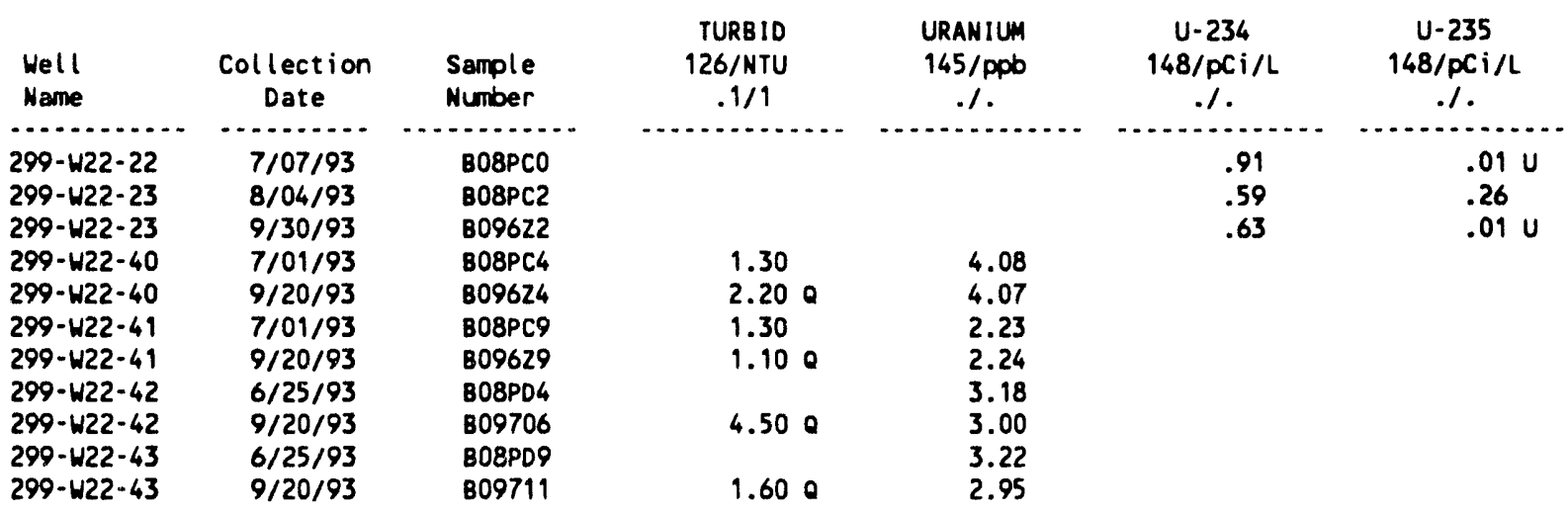


Table 12-4. Constituents with at Least One Value Above the CRQL for the 216-U-12 Crib Data for Reporting Period July 1 through September 30, 1993. (sheet 5 of 5)

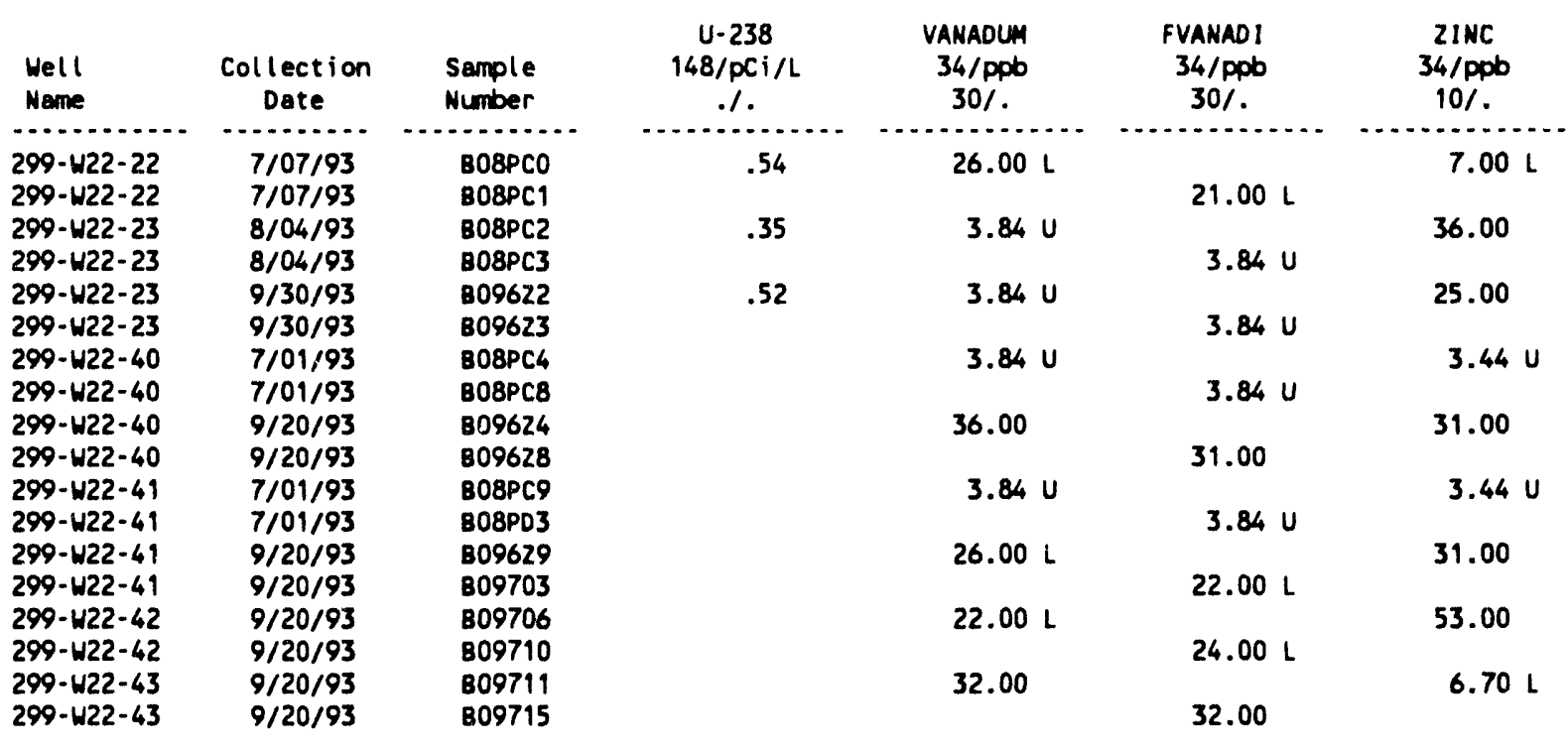

\begin{tabular}{|c|c|c|c|}
\hline $\begin{array}{l}\text { Hell } \\
\text { Name }\end{array}$ & $\begin{array}{c}\text { Collection } \\
\text { Date }\end{array}$ & $\begin{array}{l}\text { Sample } \\
\text { Number }\end{array}$ & $\begin{array}{c}\text { FZINC } \\
34 / \mathrm{ppb} \\
10 \%\end{array}$ \\
\hline $\begin{array}{l}299-W 22-22 \\
299-W 22-23 \\
299-W 22-23 \\
299-W 22-40 \\
299-W 22-40 \\
299-W 22-41 \\
299-W 22-41 \\
299-W 22-42 \\
299-W 22-43\end{array}$ & $\begin{array}{l}7 / 07 / 93 \\
8 / 04 / 93 \\
9 / 30 / 93 \\
7 / 01 / 93 \\
9 / 20 / 93 \\
7 / 01 / 93 \\
9 / 20 / 93 \\
9 / 20 / 93 \\
9 / 20 / 93\end{array}$ & $\begin{array}{l}\text { BO8PC1 } \\
\text { B08PC3 } \\
B 09623 \\
B 08 P C 8 \\
B 09628 \\
B 08 P 03 \\
B 09703 \\
B 09710 \\
B 09715\end{array}$ & $\begin{array}{l}3.44 \mathrm{U} \\
3.44 \mathrm{U} \\
3.44 \mathrm{U} \\
3.44 \mathrm{U} \\
3.44 \mathrm{U} \\
3.44 \mathrm{U} \\
29.00 \\
29.00 \\
5.00 \mathrm{~L}\end{array}$ \\
\hline
\end{tabular}

For explanation of this table, see section 1.4 of report. 
Table 12-5. Contamination Indicator Parameters for the 216-U-12 Crib Data for Reporting Period July 1 through September 30, 1993.

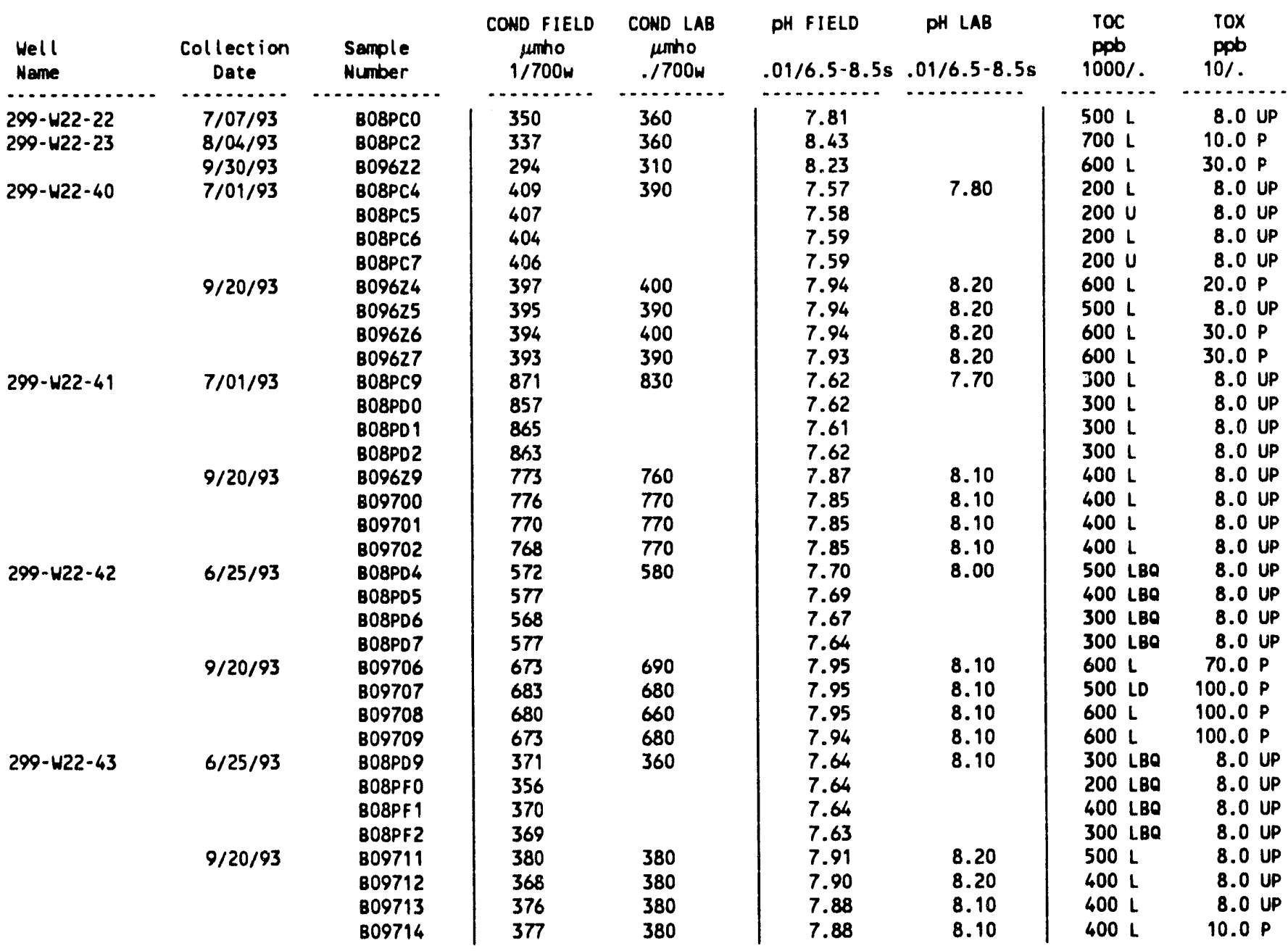

For explanation of this table, see Section 1.4 of report. 


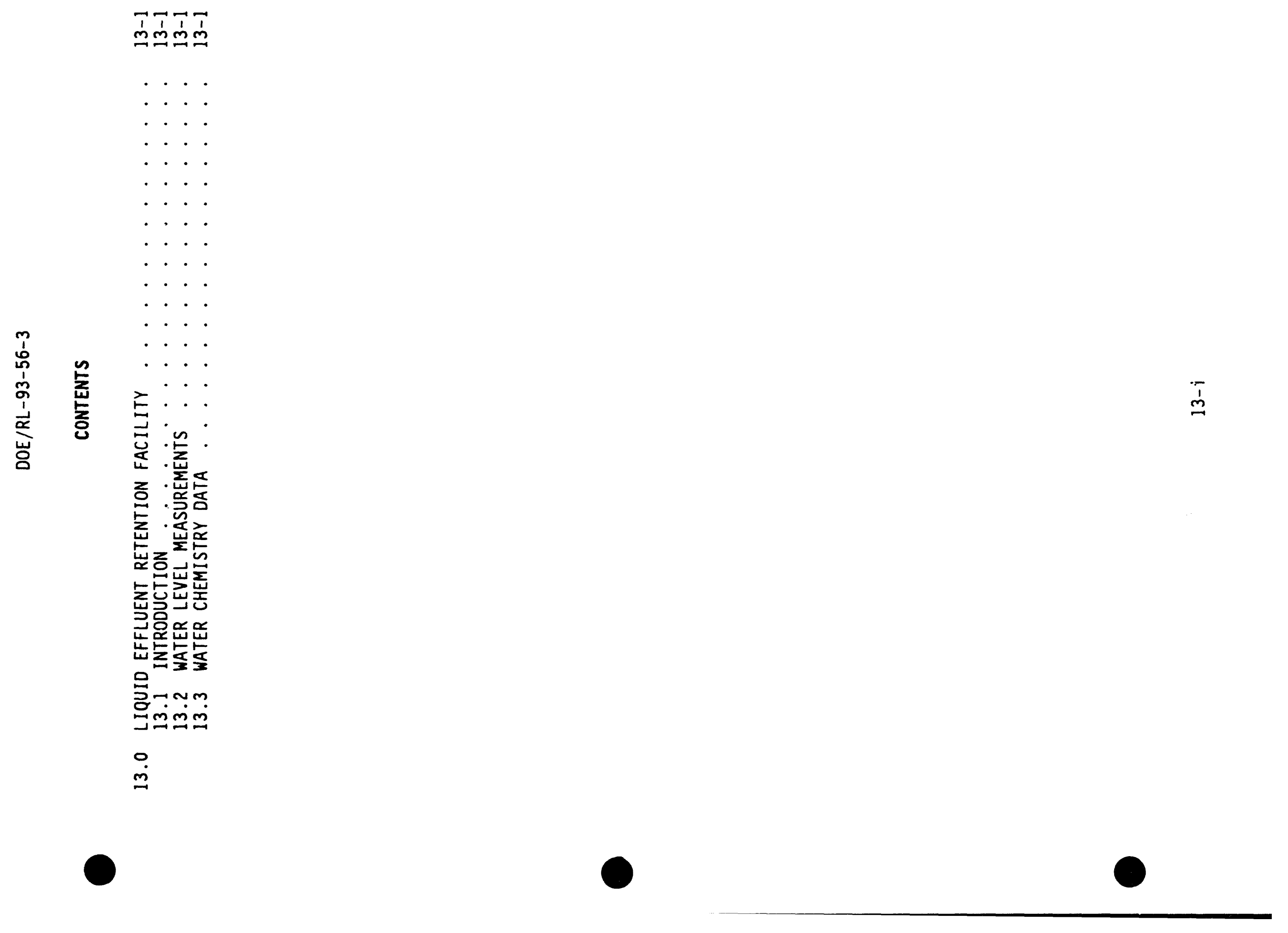




\section{LIST OF FIGURES}

13-1 Monitoring Well Locations for the Liquid Effluent Retention Facility .................. 13-3

\section{LIST OF TABLES}

13-1 Monitoring Well Purpose and Sampling Schedule for the Liquid Effluent Retention Facility Network. . . . . . . . . . . . 13-4

13-2 RCRA Water Level Measurement Report LERF, Third Quarter 1993 • 13-5

13-3 Constituent List and Summary of Results for the Liquid Effluent Retention Facility Data for Reporting Period July l through September 30,1993 . . . . . . . . . . . . . . . 13-6

13-4 Constituents with at Least One Value Above the CRQL for the Liquid Effluent Retention Facility Data for Reporting Period July 1 through September 30,1993 . . . . . . . . . 13-8

13-5 Contamination Indicator Parameters for the Liquid Effluent Retention Facility Data for Reporting Period July l through September 30,1993 ............... . 13-10

13-6 Critical Means Table for the Liquid Effluent Retention Facility.................. 13-11 
$\mathrm{DOE} / \mathrm{RL}-93-56-3$

\title{
13.0 LIQUIO EFFLUENT RETENTION FACILITY
}

\author{
M. D. Sweeney \\ Hestinghouse Hanford Company
}

\subsection{INTRODUCTION}

The Liquid Effluent Retention Facility (LERF) was constructed to provide interim storage of 242-A Evaporator process condensate effluent suspected of containing listed and dangerous (ammonia) waste constituents. Construction of the LERF began in May 1990 as a facility expansion under interim-status regulations. The LERF, classified as a surface impoundment for mixed waste storage, will be permitted in accordance with WAC 173-303. The evaporator was shut down in 1989 and is scheduled for restart. A treatment unit for the evaporator liquid waste is being designed and is scheduled for operation in 1994. The LERF basins will serve as temporary storage for the waste until that time.

The facility is classified as a surface impoundment and was originally planned to be four basins arranged side by side. Four excavations were made; only three of the four basins are lined and currently planned for use. The basins are located at the northeast corner of the 200 East Area. The liquid effluent may contain spent nonhalogenated solvents (F003 and F005, WAC 173-303-9004, "Dangerous Waste Source List"), namely acetone, methyl ethyl ketone, and methyl isobutyl ketone. The ammonia content in the liquid effluent may also be cause for handling the effluent as a dangerous waste.

A groundwater monitoring network was installed, in accordance with the groundwater monitoring plan (WHC 1991), around the LERF in 1990. Four quarters of background groundwater sampling collection and analys is were completed. The facility is currently in the detection-level indicator parameter evaluation program until closure or other action. Three downgradient wells and one upgradient well constitute the monitoring network. Figure 13-1 shows the well locations. Table 13-1 provides the sampling event and last sampling date and interval, and defines the upgradient and downgradient wells.

\subsection{WATER LEVEL MEASUREMENTS}

Water levels are measured quarterly and when groundwater samples are collected at each of the four wells in the network. Water level measurements obtained during the quarter are presented in Table 13-2.

\subsection{WATER CHEMISTRY DATA}

The samples for third quarter of 1993 were collected on July 14 and July 15, 1993. One of the downgradient wells, 299-E26-9, has been removed from the sampling schedule. This well has gone dry; less than $0.3 \mathrm{~m}(1 \mathrm{ft})$ of water is left in the well casing. 
The data received before September 30, 1993, are reported in Tables 13-2 through 13-5. Unfiltered chromium and iron continue to indicate fluctuating concentrations above the DWS for these constituents. Filtered analys is for these parameters are well below the DWS. A statistical evaluation has been completed for indicator parameters for the LERF. This evaluation was

performed for upgradient/downgradient comparisons and the results are given in Table 13-6.

There were no exceedances for the current sampling period. 


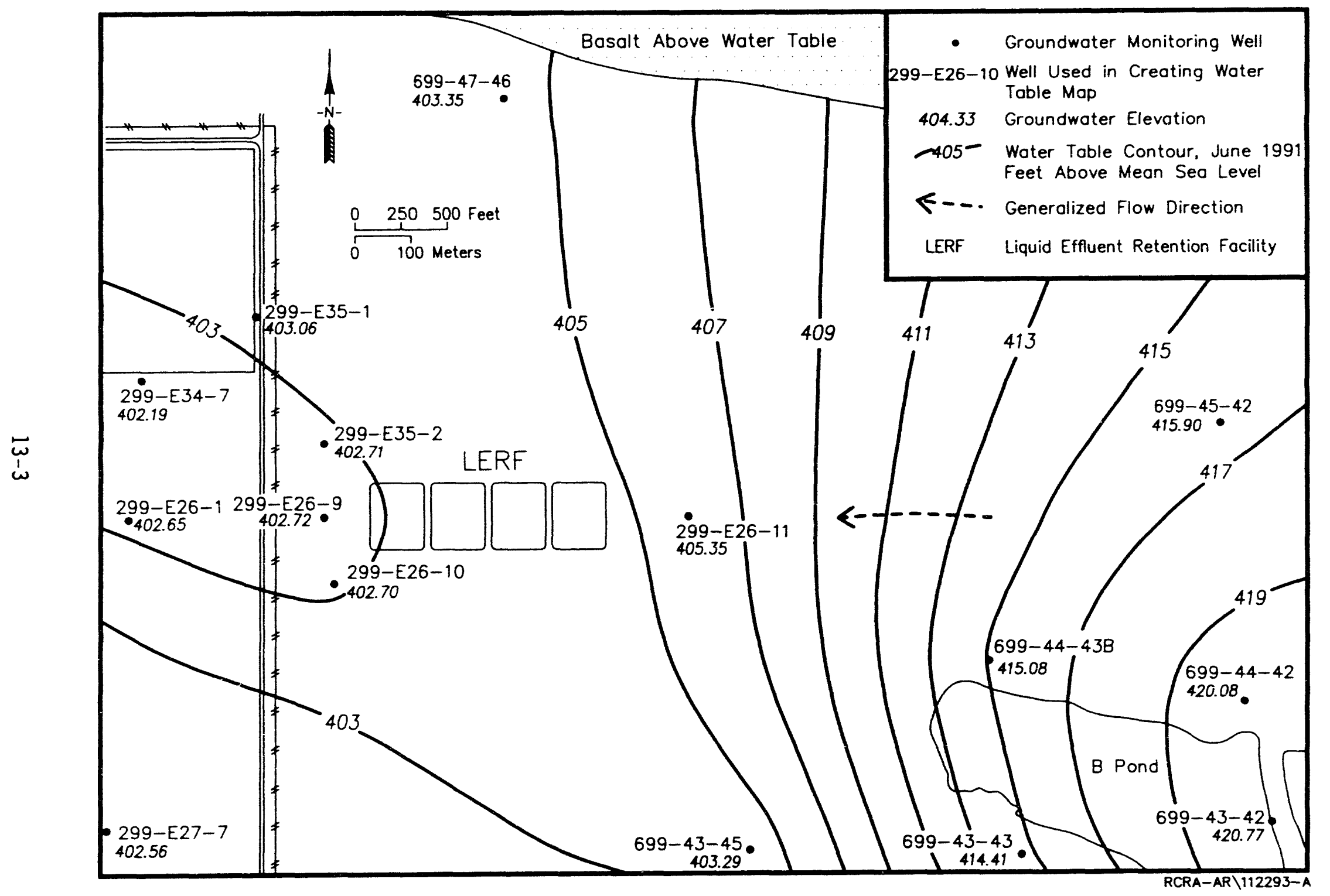

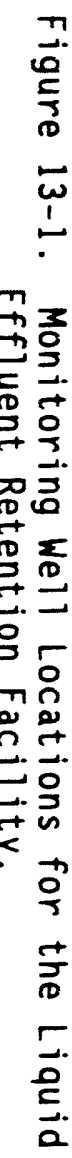


Table 13-1. Monitoring Well Purpose and Sampling Schedule for the Liquid Effluent Retention Facility Network.

\begin{tabular}{|c|c|c|c|c|}
\hline $\begin{array}{c}\text { Well no. } \\
(299-)\end{array}$ & $\begin{array}{c}\text { Relative } \\
\text { position }\end{array}$ & Hydrogeologic unit & $\begin{array}{c}\text { Sample } \\
\text { frequency }\end{array}$ & $\begin{array}{c}\text { Sample date, } \\
\text { 3rd Qtr 1993 }\end{array}$ \\
\hline E26-9 & Downgradient & Hanford: Water Table & Semiannually & Not sampled \\
\hline E26-10 & Downgradient & Hanford: Water Table & Semiannually & $7 / 15 / 93$ \\
\hline E26-11 & Upgradient & Hanford: Water Table & Semiannually & $7 / 14 / 93$ \\
\hline E35-2 & Downgradient & Hanford: Water Table & Semiannually & $7 / 14 / 93$ \\
\hline
\end{tabular}


Table 13-2. RCRA Water Level Measurement Report LERF, Third Quarter 1993.

\begin{tabular}{llll}
\hline We11 & Date & $\begin{array}{c}\text { Depth to } \\
\text { water }(\mathrm{ft})\end{array}$ & $\begin{array}{c}\text { Water level } \\
\text { elevation } \\
\text { above ms }(\mathrm{ft})\end{array}$ \\
\hline $299-E 26-9$ & $9 / 02 / 93$ & 200.54 & 402.35 \\
$299-E 26-10$ & $7 / 15 / 93$ & 198.28 & $403.19 *$ \\
& $9 / 02 / 93$ & 199.14 & 402.33 \\
& $9 / 02 / 93$ & 199.08 & $402.39^{\star}$ \\
& $7 / 14 / 93$ & 194.29 & $405.39^{\star}$ \\
& $9 / 02 / 93$ & 194.38 & 405.30 \\
& $9 / 02 / 93$ & 194.35 & $405.33^{\star}$ \\
& $7 / 14 / 93$ & 199.65 & $402.45^{\star}$ \\
$9 / 02 / 93$ & 199.69 & $402.41^{\star}$ \\
& $9 / 02 / 93$ & 199.57 & 402.53 \\
\hline
\end{tabular}

Notes: 1. Water level elevations are calculated by subtracting the measured depth to water from the surveyed elevation for the well.

2. Depth-to-water values are transcribed from field records.

3. Elevations marked with an ' $*$ ' were measured at the time of sampling.

4. To convert feet to meters multiply by 0.3048 . 
Table 13-3. Constituent List and Summary of Results for the Liquid Effluent Retention Facility Data for Reporting Period July 1 through September 30,1993 . (sheet 1 of 2)

CONTAMINATION INOICATOR PARAMETERS

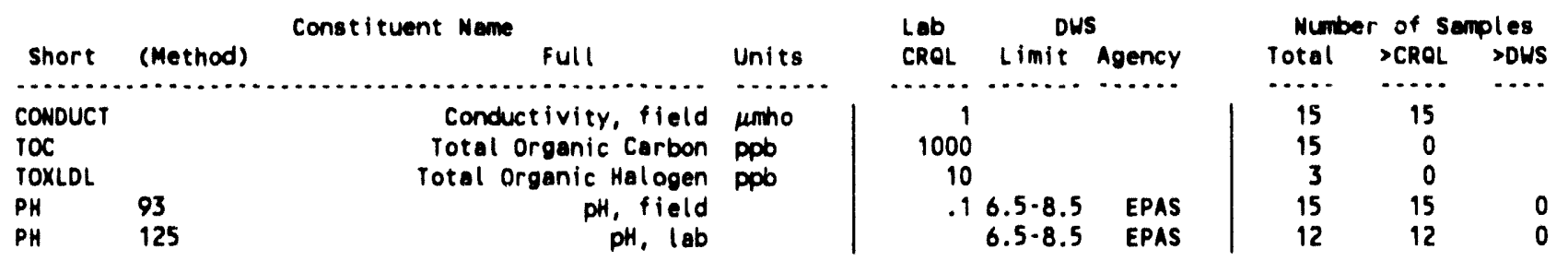

DRINKING WATER PARAMETERS

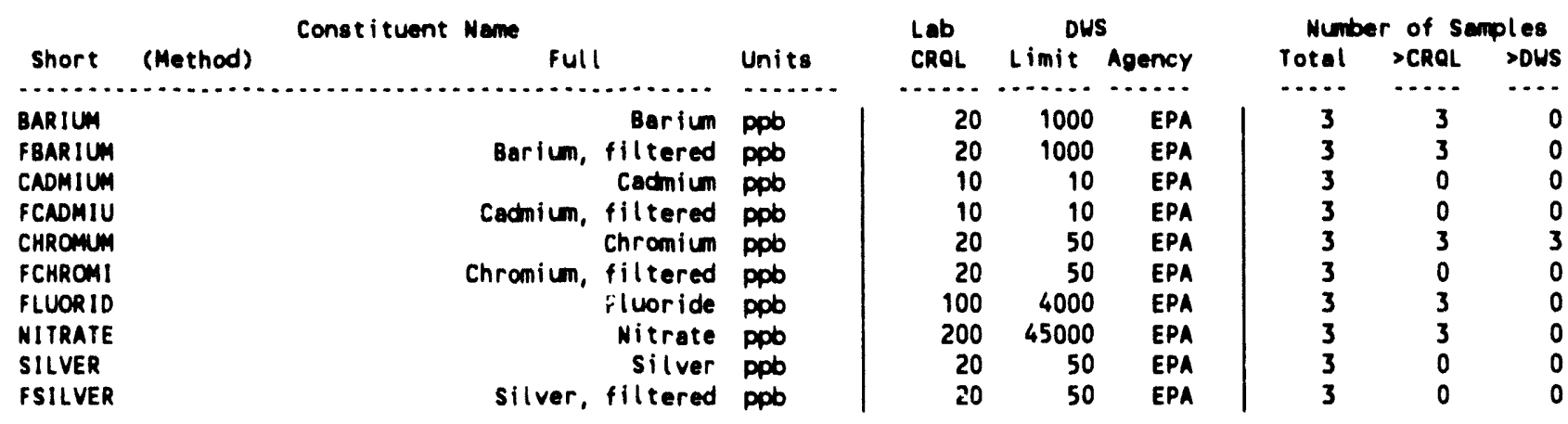

GROUNDWATER OUALITY PARAMETERS

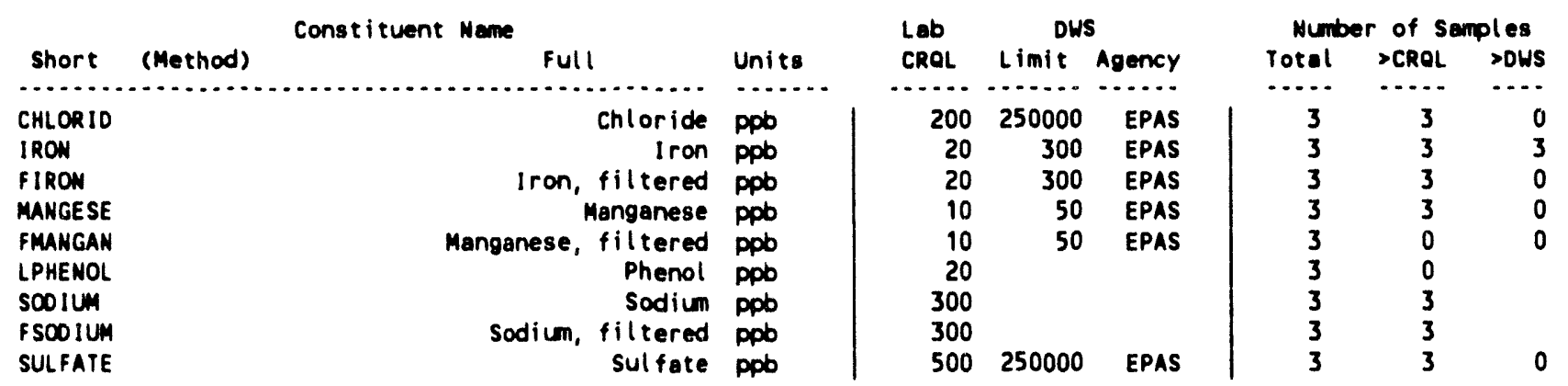

SITE SPECIFIC AND OTHER CONSTITUENTS

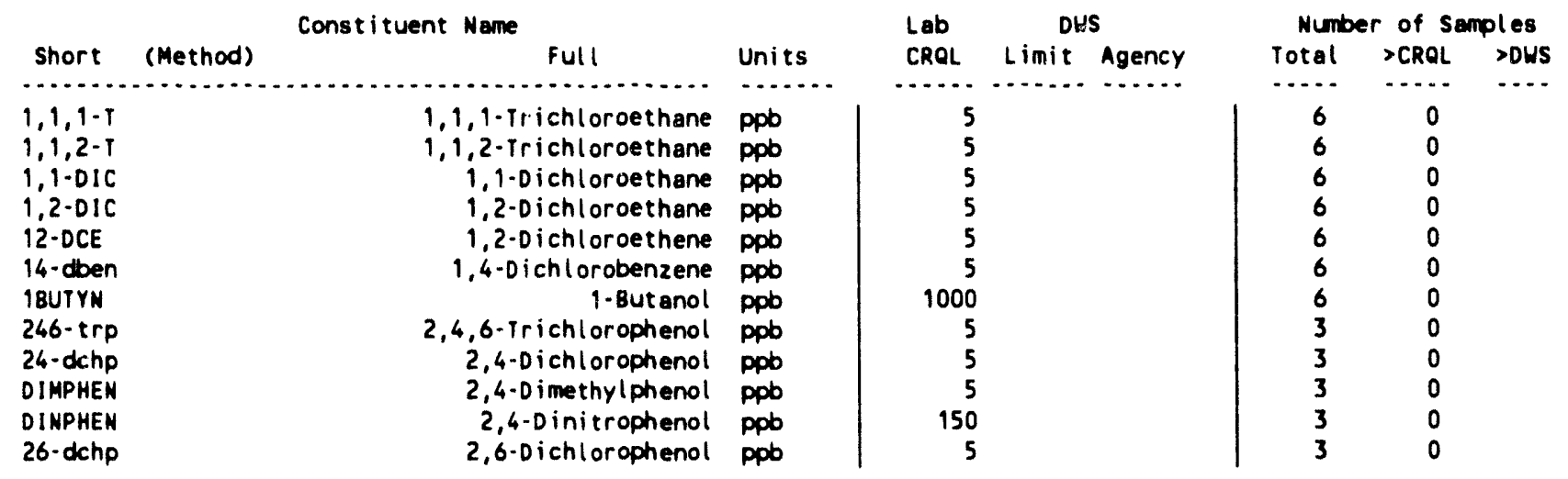


Table 13-3. Constituent List and Summary of Results for the Liquid Effluent Retention Facility Data for Reporting Period July 1 through September 30,1993 . (sheet 2 of 2 )

\begin{tabular}{|c|c|c|c|c|c|c|c|c|}
\hline \multicolumn{3}{|c|}{ Constituent Neme } & \multicolumn{3}{|c|}{ Dus } & \multicolumn{3}{|c|}{ Number of Semples } \\
\hline Short & (Method) & Units & CROL & Limit & Agency & Total & $>$ CROL & $>$ DUS \\
\hline$\ldots \ldots$ & $\ldots \ldots \ldots \ldots \ldots$ & $\ldots \ldots$ & $\ldots . .$. & $\ldots \ldots$ & $\ldots \ldots$ & $\ldots$. & $\ldots$. & $\cdots$ \\
\hline CHLPHEN & 2-Chlorophenol & ppb & 5 & & & 3 & 0 & \\
\hline 2NITPH & 2-Nitrophenol & ppob & 5 & & & 3 & 0 & \\
\hline BUTDINP & 2-sec-Butyl-4,6-dinitrophenol (DN & ppb & 5 & & & 3 & 0 & \\
\hline 460N2MP & 4,6-0 initro-2-methylphenol & ppb & 200 & & & 3 & 0 & \\
\hline CHLCRES & 4-Chloro-3-methylphenol & ppb & 5 & & & 3 & 0 & \\
\hline MIBK & 4-Methyl-2-pentanone & ppb & 50 & & & 6 & 0 & \\
\hline NITPHEN & 4-Nitrophenol & ppb & 30 & & & 3 & 0 & \\
\hline ACE TONE & Acetone & ppb & 100 & & & 6 & 0 & \\
\hline ALLMNUM & Aluminum & ppb & 200 & & & 3 & 0 & \\
\hline FALUMIN & Aluminum & ppb & 200 & & & 3 & 0 & \\
\hline AMMONIU & Ammonium ion & ppo & 100 & & & 3 & 1 & \\
\hline ANTIONY & Ant imony & ppb & 200 & & & 3 & 0 & \\
\hline FANT IMO & Ant imony, filtered & ppb & 200 & & & 3 & 0 & \\
\hline BENZENE & Benzene & ppo & 5 & & & 6 & 0 & \\
\hline BERYLUM & Berylliun & ppb & 3 & & & 3 & 0 & \\
\hline FBERYLL & Beryllicm, filtered & ppb & 3 & & & 3 & 0 & \\
\hline BROMIOE & Bromide & ppb & 500 & & & 3 & 0 & \\
\hline CALCIUM & Calciun & ppb & 100 & & & 3 & 3 & \\
\hline FCALCIU & Calcium, filtered & ppb & 100 & & & 3 & 3 & \\
\hline CARBIDE & Carbon disulfide & ppb & 5 & & & 6 & 0 & \\
\hline TETRANE & Carbon tetrachloride & ppb & 5 & & & 6 & 0 & \\
\hline CHLFORM & Chloroform & ppb & 5 & 100 & EPA & 6 & 0 & 0 \\
\hline COBALT & Cobalt & ppob & 20 & & & 3 & 0 & \\
\hline FCOBALT & Cobelt, filtered & ppb & 20 & & & 3 & 0 & \\
\hline COPPER & Copper & ppo & 20 & 1000 & EPAS & 3 & 0 & 0 \\
\hline FCOPPER & Copper, filtered & ppb & 20 & 1000 & EPAS & 3 & 0 & 0 \\
\hline CRESOLS & Cresols (methylphenols) & ppo & 10 & & & 3 & 0 & \\
\hline ETHCYAN & Ethyl cyanide & ppb & 10 & & & 6 & 0 & \\
\hline MAGNES & Magnes ium & ppob & 100 & & & 3 & 3 & \\
\hline FMAGNES & Magnesium, filtered & ppb & 100 & & & 3 & 3 & \\
\hline METHONE & Methyl ethyl ketone & ppb & 100 & & & 6 & 0 & \\
\hline METHYCH & Methylene chloride & popb & 5 & & & 6 & 0 & \\
\hline NICKEL & Nickel & ppb & 30 & & & 3 & 3 & \\
\hline FNICKEL & Nickel, filtered & ppb & 30 & & & 3 & 0 & \\
\hline NITRITE & Nitrite & ppb & 200 & & & 3 & 0 & \\
\hline PENTCHP & Pent achl orophenol & ppob & 100 & & & 3 & 0 & \\
\hline PHOSPHA & Phosphate & ppob & 400 & & & 3 & 0 & \\
\hline POTASUM & Potessium & pob & 300 & & & 3 & 3 & \\
\hline FPOTASS & Potessium, filtered & ppb & 300 & & & 3 & 3 & \\
\hline PERCENE & Tetrachloroethene & ppb & 5 & & & 6 & 0 & \\
\hline TETPHNL & Tetrachlorophenols & ppo & 10 & & & 3 & 0 & \\
\hline TAF & Tetrahydrofuran & ppb & 10 & & & 6 & 0 & \\
\hline TIN & Tin & ppb & 100 & & & 3 & 0 & \\
\hline FTIN & Tin, filtered & ppb & 100 & & & 3 & 0 & \\
\hline TOLUENE & roluene & ppb & 5 & & & 6 & 0 & \\
\hline TRICENE & Trichloroethene & ppob & 5 & & & 6 & 0 & \\
\hline TRIPHNL & Trichlorophenols & ppb & 5 & & & 3 & 0 & \\
\hline TRITIUM & Tritium & $\mathrm{PCi} / \mathrm{L}$ & 500 & 20000 & EPA & 3 & 2 & 0 \\
\hline VANADUM & Vanadium & ppb & 30 & & & 3 & 2 & \\
\hline FVANADI & Vanadium, filtered & ppb & 30 & & & 3 & 2 & \\
\hline VINYIDE & Vinyl chloride & ppo & 10 & 2 & EPA & 6 & 0 & $0^{*}$ \\
\hline XYLENE & $x y($ enes (total) & ppb & 5 & & & 6 & 0 & \\
\hline ZINC & Zinc & ppb & 10 & & & 3 & 2 & \\
\hline FZINC & zinc, filtered & ppb & 10 & & & 3 & 1 & \\
\hline
\end{tabular}


Table 13-4. Constituents with at Least One Value Above the CRQL for the Liquid Effluent Retention Facility Data for Reporting Period July 1 through September 30, 1993. (sheet 1 of 2)

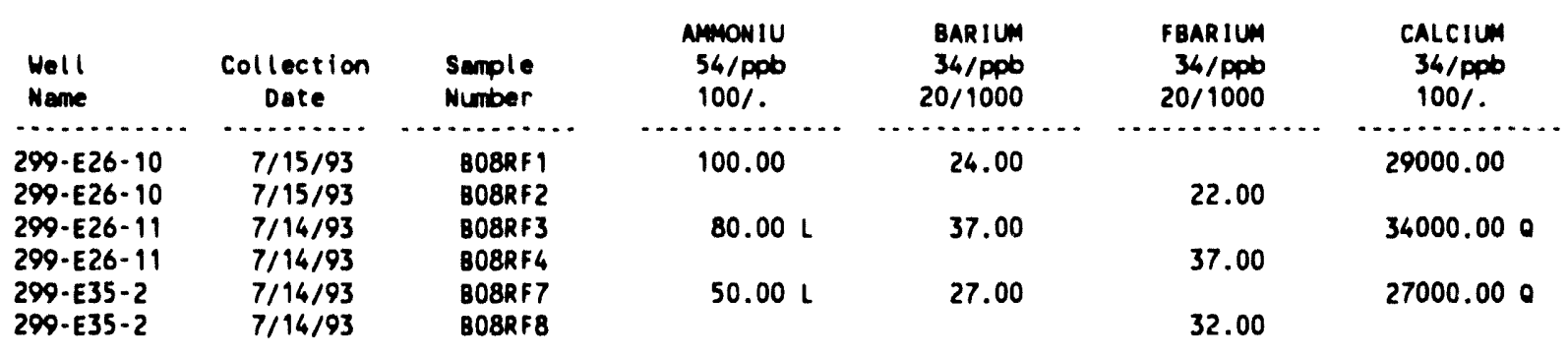

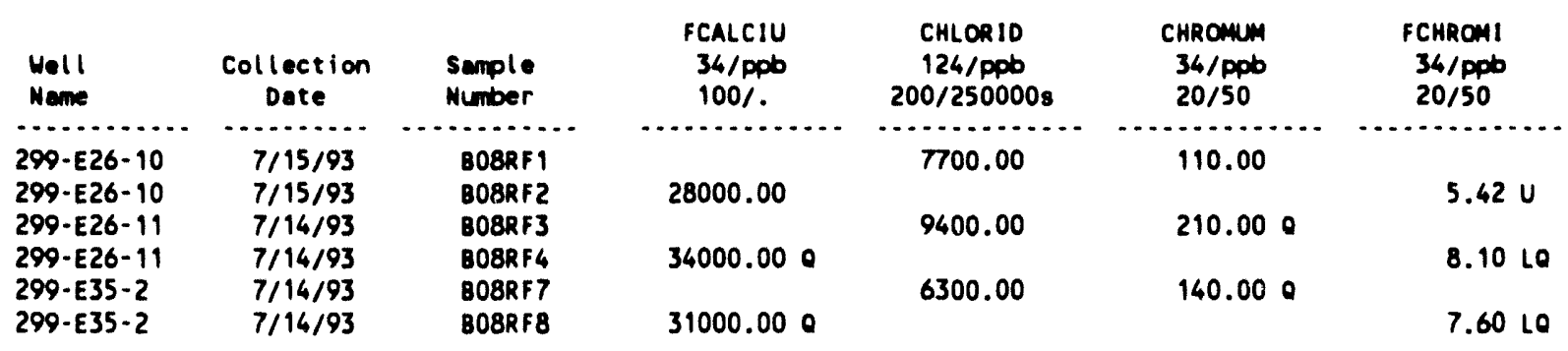

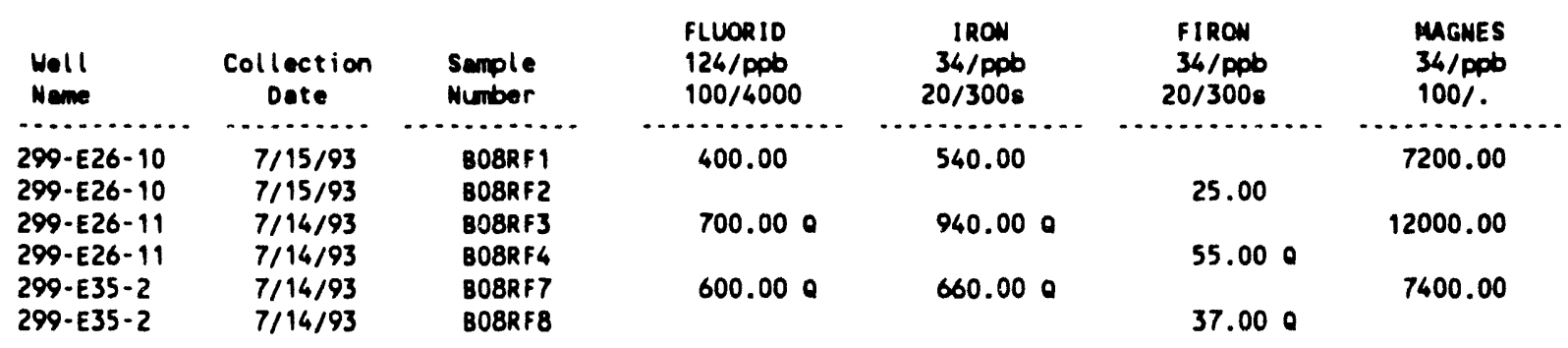

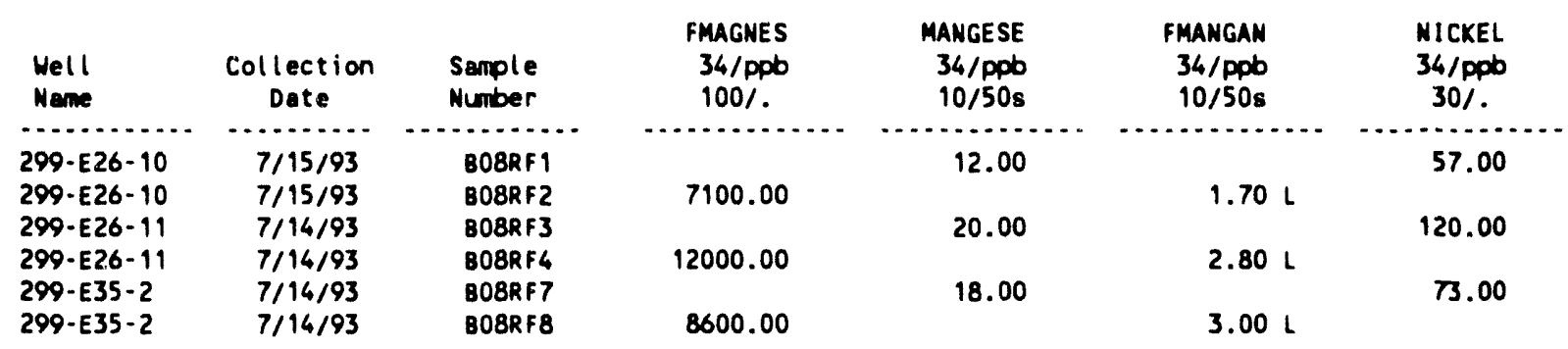

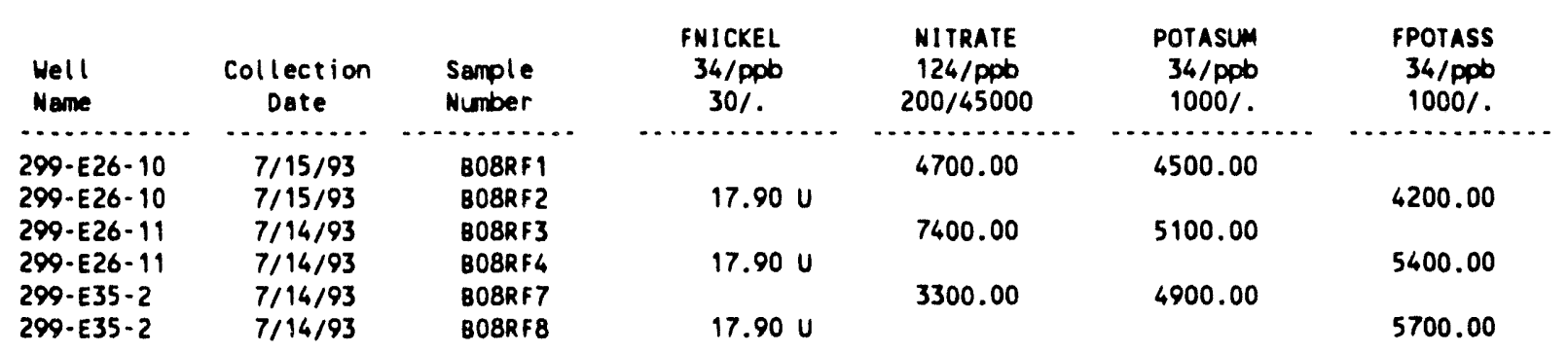


Table 13-4. Constituents with at Least One Value Above the CRQL for the Liquid Effluent Retention Facility Data for Reporting Period July 1 through September 30, 1993. (sheet 2 of 2)

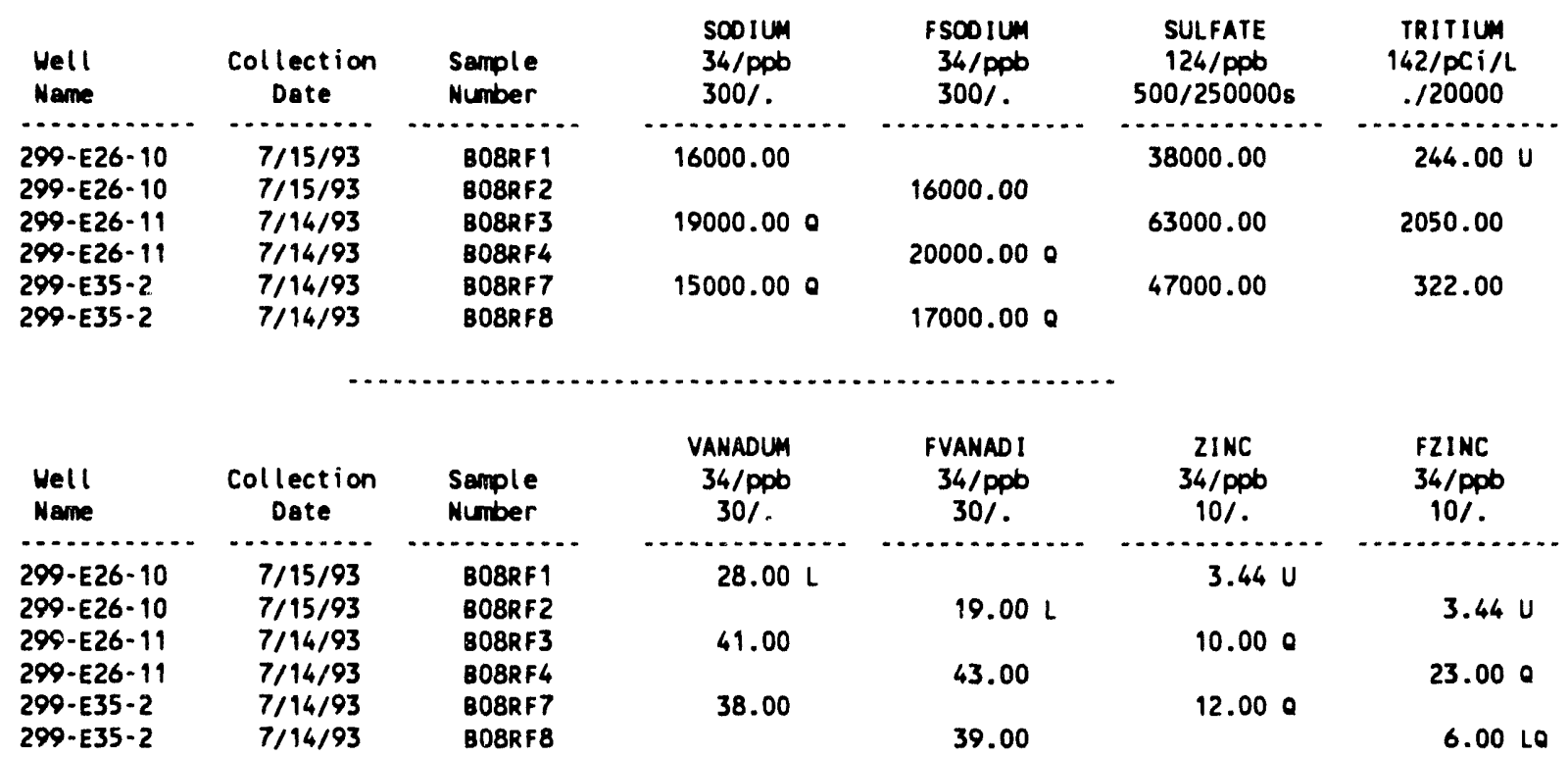

For explanation of this table, see Section 1.4 of report. 
Table 13-5. Contamination Indicator Parameters for the Liquid Effluent Retention Facility Data for Reporting Period July 1 through September 30, 1993.

\begin{tabular}{|c|c|c|c|c|c|c|c|c|}
\hline $\begin{array}{l}\text { Well } \\
\text { Name }\end{array}$ & $\begin{array}{c}\text { Collection } \\
\text { Date }\end{array}$ & $\begin{array}{l}\text { Sample } \\
\text { Number }\end{array}$ & $\begin{array}{c}\text { COND FIELD } \\
\mu \text { mho } \\
1 / 700 \mathrm{~W}\end{array}$ & $\begin{array}{c}\text { COND LAB } \\
\mu m h o \\
. / 700 \mathrm{~W}\end{array}$ & $\begin{array}{l}\text { PH FIELD } \\
.01 / 6.5-8.5 \mathrm{~s}\end{array}$ & $\begin{array}{c}\text { PH LAB } \\
.01 / 6.5-8.5 \mathrm{~s}\end{array}$ & $\begin{array}{c}\text { TOC } \\
\text { ppb } \\
1000 / .\end{array}$ & $\begin{array}{l}\text { Tox } \\
\text { ppb } \\
10 \%\end{array}$ \\
\hline 299-E26-10 & $\begin{array}{l}7 / 15 / 93 \\
9 / 02 / 93\end{array}$ & $\begin{array}{l}\text { B08RF } 1 \\
\text { B095N4 } \\
\text { B095N5 } \\
\text { B095N6 } \\
\text { B095N7 }\end{array}$ & $\begin{array}{l}285 \\
291 \\
291 \\
291 \\
291\end{array}$ & & $\begin{array}{l}7.68 \\
7.59 \\
7.58 \\
7.58 \\
7.58\end{array}$ & $\begin{array}{l}8.20 \\
8.20 \\
8.20 \\
8.20\end{array}$ & $\begin{array}{l}400 \mathrm{~L} \\
400 \mathrm{~L} \\
400 \mathrm{~L} \\
400 \mathrm{~L} \\
400 \mathrm{~L}\end{array}$ & 8.0 UP \\
\hline 299-E26-11 & $\begin{array}{l}7 / 14 / 93 \\
9 / 02 / 93\end{array}$ & $\begin{array}{l}\text { B08RF3 } \\
\text { B095N8 } \\
\text { B095N9 } \\
\text { B095PO } \\
\text { B095P1 }\end{array}$ & $\begin{array}{l}360 \\
363 \\
363 \\
363 \\
360\end{array}$ & & $\begin{array}{l}7.92 \\
7.84 \\
7.81 \\
7.83 \\
7.84\end{array}$ & $\begin{array}{l}8.10 \\
8.10 \\
8.10 \\
8.10\end{array}$ & $\begin{array}{l}300 \mathrm{~L} \\
300 \mathrm{~L} \\
300 \mathrm{~L} \\
300 \mathrm{~L} \\
200 \mathrm{~L}\end{array}$ & 8.0 UP \\
\hline 299-E35-2 & $\begin{array}{l}7 / 14 / 93 \\
9 / 02 / 93\end{array}$ & $\begin{array}{l}\text { B08RF7 } \\
\text { B095P2 } \\
\text { B095P3 } \\
\text { B095P4 } \\
\text { B095P5 }\end{array}$ & $\begin{array}{l}305 \\
316 \\
316 \\
315 \\
316\end{array}$ & & $\begin{array}{l}7.70 \\
7.82 \\
7.80 \\
7.80 \\
7.80\end{array}$ & $\begin{array}{l}8.30 \\
8.40 \\
8.40 \\
8.40\end{array}$ & $\begin{array}{l}500 \mathrm{~L} \\
500 \mathrm{~L} \\
500 \mathrm{~L} \\
500 \mathrm{~L} \\
500 \mathrm{~L}\end{array}$ & 8.0 UP \\
\hline
\end{tabular}

For explanation of this table, see Section 1.4 of report. 
Table 13-6. Critical Means Table for the Liquid Effluent Retention Facility.

\begin{tabular}{|l|c|}
\hline \multicolumn{1}{|c|}{ Constituent (unit) } & Critical mean \\
\hline Field conductivity $(\mu \mathrm{mho} / \mathrm{cm})$ & 474.7 \\
\hline Field $\mathrm{pH}$ & {$[2.96,12.52]$} \\
\hline TOC & $4308.0 \mathrm{ppb}$ \\
\hline
\end{tabular}

TOC = total organic carbon. 
DOE/RL-93-56-3

This page intentionally left blank. 
$\mathrm{DOE} / \mathrm{RL}-93-56-3$

\section{CONTENTS}

14.0 2101-M POND . . . . . . . . . . . . . . . . . . . . . . . . . 14 . . . . 14

14.1 INTRODUCTION .................... 14-1

14.2 WATER LEVEL DATA ..................... 14-1

14.3 WATER CHEMISTRY DATA . . . . . . . . . . . . . . . . . . 14-1 
DOE/RL-93-56-3

\section{LIST OF FIGURES}

14-1 Monitoring Well Locations for the 2101-M Pond . . . . . . . . . 14-2

\section{LIST OF TABLES}

14-1 Monitoring We11 Purpose and Sampling Schedule for the 2101-M Pond . . . . . . . . . . . . . . . . . 14-3

14-2 RCRA Water Level Measurement Report 2101-M Pond, Third Quarter 1993 . . . . . . . . . . . . . . . . 14-4

14-3 Constituent List and Summary of Results for the 2101-M Pond Data for Reporting Period July 1 through September 30, 1993 . . . 14-5 


\subsection{1-M POND}

E. C. Thornton

Westinghouse Hanford Company

\subsection{INTRODUCTION}

The 2101-M Pond is a U-shaped, unlined trench located in the 200 East Area. Since August 1988, the pond has been monitored under interim-status, detection-level groundwater monitoring on a semiannual basis using a four-well monitoring network (Figure 14-1). Table 14-1 provides information on the purpose of each well, the unit monitored, and sampling information.

Upgradient well 299-E18-1 is also used as an upgradient monitoring well for the $B$ Pond project.

\subsection{WATER LEVEL DATA}

Water level measurements were performed in the July through September 1993 period for all four wells. The data are presented in Table 14-2. Water levels have been corrected for vertical deviations for each well.

\subsection{WATER CHEMISTRY DATA}

Semiannual sampling was not scheduled for the third quarter of 1993. Groundwater samples were most recently collected on June 4, 1993, for wells 299-E18-2, 299-E18-3, and 299-E18-4, while well 299-E18-1 was sampled on June 11, 1993. Most of the associated analytical data were reported in the second quarterly report. Some pesticide data were received later, however, and are reported in Table 14-3. All values were at or below the CRQL. 
Figure 14-1. Monitoring Wel1 Locations for the 2101-M Pond.

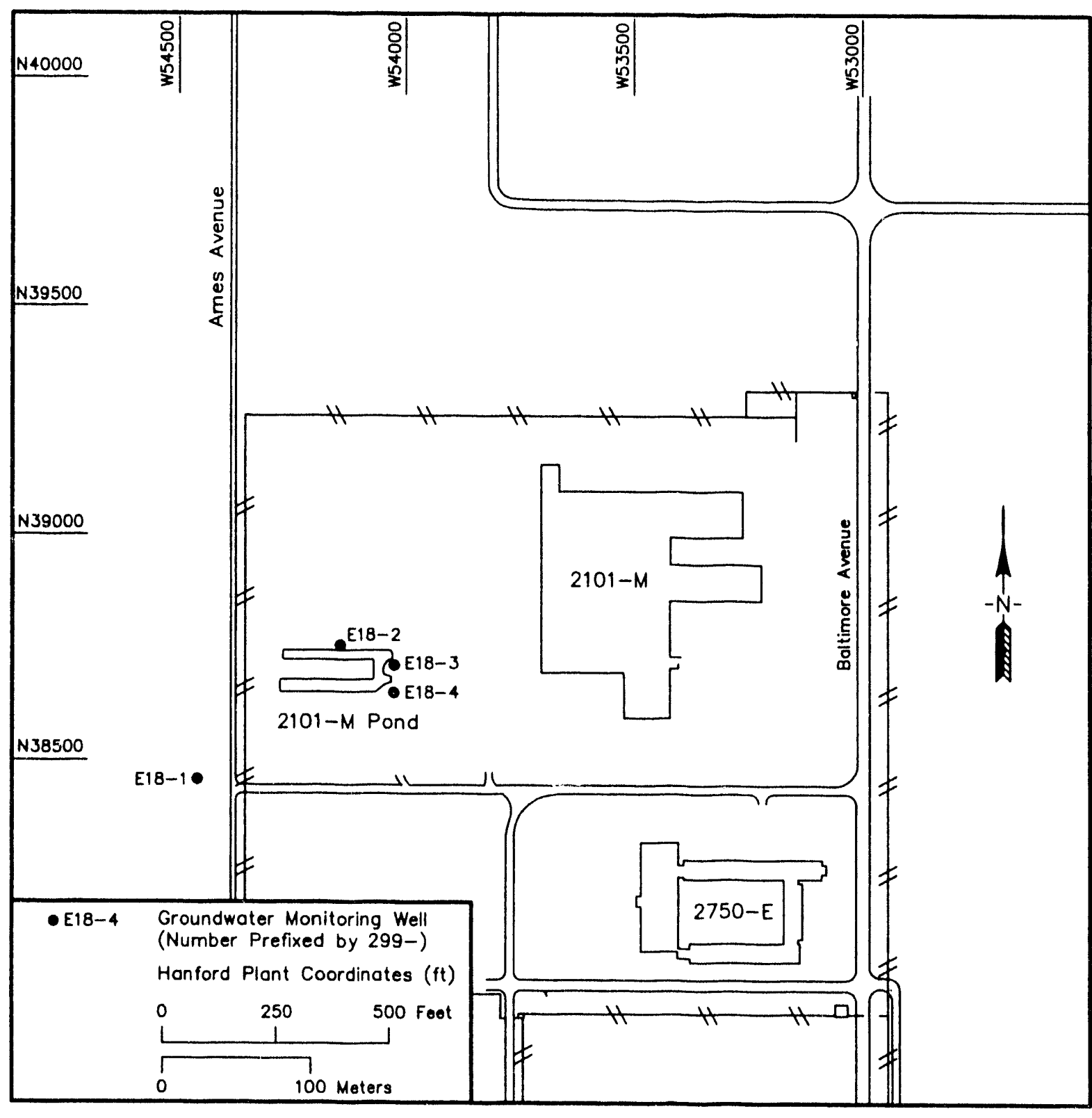

RCRA-AR $\backslash 010794-C$ 
Table 14-1. Monitoring Well Purpose and Sampling Schedule for the 2101-M Pond.

\begin{tabular}{|c|c|c|c|c|}
\hline $\begin{array}{c}\text { Well no. } \\
(299-)\end{array}$ & $\begin{array}{c}\text { Relative } \\
\text { position }\end{array}$ & \multicolumn{1}{|c|}{ Hydrogeologic unit } & $\begin{array}{c}\text { Sample } \\
\text { frequency }\end{array}$ & Sample date \\
\hline E18-1 & Upgradient & Ringold: Water Table & Semiannually & $6 / 11 / 93$ \\
\hline E18-2 & Downgradient & Ringold: Water Table & Semiannually & $6 / 04 / 93$ \\
\hline E18-3 & Downgradient & Ringold: Water Table & Semiannually & $6 / 04 / 93$ \\
\hline E18-4 & Downgradient & Ringold: Water Table & Semiannually & $6 / 04 / 93$ \\
\hline
\end{tabular}

"Ringold Formation at this site is a muddy, sandy gravel to a slightly muddy gravelly sand over the screened interval from approximately 92 to $101 \mathrm{~m}$ (302 to $331 \mathrm{ft}$ ) below top of casing. 
Table 14-2. RCRA Water Level Measurement Report 2101-M Pond, Third Quarter 1993.

\begin{tabular}{llll}
\hline We11 & Date & $\begin{array}{c}\text { Depth to } \\
\text { water }(\mathrm{ft})\end{array}$ & $\begin{array}{c}\text { Water leve } \\
\text { elevation } \\
\text { above ms }\end{array}$ \\
\hline 299-E18-1 & $7 / 19 / 93$ & 318.27 & 402.03 \\
& $8 / 16 / 93$ & 318.34 & 401.96 \\
& $9 / 20 / 93$ & 318.32 & 401.98 \\
& $7 / 19 / 93$ & 319.57 & 401.86 \\
& $8 / 16 / 93$ & 319.64 & 401.79 \\
& $9 / 20 / 93$ & 319.58 & 401.85 \\
& $7 / 19 / 93$ & 320.21 & 401.86 \\
& $8 / 16 / 93$ & 320.27 & 401.80 \\
& $9 / 20 / 93$ & 320.09 & 401.98 \\
& $7 / 19 / 93$ & 319.02 & 402.55 \\
& $8 / 16 / 93$ & 319.69 & 401.88 \\
& $9 / 20 / 93$ & 319.57 & 402.00 \\
\hline
\end{tabular}

Notes: 1. Water level elevations are calculated by subtracting the measured depth to water from the surveyed elevation corrected for vertical deviations for the well.

2. Depth-to-water values are transcribed from field records.

3. To convert feet to meters multiply by 0.3048 . 
Table 14-3. Constituent List and Summary of Results for the 2101-M Pond Data for Reporting Period July 1 through September 30, 1993.

DRINKING WATER PARAMETERS

\begin{tabular}{|c|c|c|c|c|c|c|c|c|c|}
\hline \multirow[b]{2}{*}{ Short } & \multicolumn{2}{|c|}{ Const ituent Name } & \multirow[b]{2}{*}{ Units } & \multirow{2}{*}{$\begin{array}{l}\text { Lab } \\
\text { CROL }\end{array}$} & \multicolumn{2}{|c|}{ DWS } & \multicolumn{3}{|c|}{ Number of Semples } \\
\hline & (Method) & Full & & & Limit & Agency & Total & $>$ CROL & $>$ OWS \\
\hline$\ldots \ldots$ & - & $\ldots \ldots \ldots$ & $\ldots .$. & $\ldots \ldots$ & $\ldots \ldots$ & $\ldots \ldots$ & $\cdots \cdots$ & $\ldots$ & $\ldots$ \\
\hline ENDRIN & & Endrin & pob & .1 & .2 & EPA & 1 & 0 & 0 \\
\hline METHLOR & & Methoxychlor & ppb & 2 & 100 & EPA & 1 & 0 & 0 \\
\hline TOXAENE & & Toxaphene & Ppb & 2 & 5 & EPA & 1 & C & 0 \\
\hline$g-B H C$ & & genma-BHC (L indene) & ppb & .05 & 4 & EPA & 1 & 0 & 0 \\
\hline
\end{tabular}

SITE SPECIFIC ANO OTHER CONSTITUENTS

\begin{tabular}{|c|c|c|c|c|c|c|c|c|}
\hline \multicolumn{4}{|c|}{ Const ituent Name } & \multicolumn{2}{|r|}{ DHS } & \multicolumn{3}{|c|}{ Number of Semples } \\
\hline short & (Method) & Full & Units & CROL & Limit Agency & Total & $>C R Q L$ & $>0$ WS \\
\hline$\ldots \ldots$ & $\ldots \ldots$ & $\ldots \ldots \ldots$ & $\ldots \ldots$ & $\ldots \ldots$ & $\ldots \ldots \ldots$ & $\cdots \ldots$ & $\cdots \cdots$ & $\cdots$ \\
\hline DDD & & $4,4^{\prime}-D D D$ & ppb & .1 & & 1 & 0 & \\
\hline DDE & & $4,4^{\prime}-D D E$ & ppb & .05 & & 1 & 0 & \\
\hline DOT & & $4,4^{\prime}-$ DDT & ppb & .1 & & 1 & 0 & \\
\hline ALORIN & & Aldrin & ppb & .05 & & 1 & 0 & \\
\hline a-BHC & & Alpha-BHC & ppb & .05 & & 1 & 0 & \\
\hline b-BHC & & Beta-BHC & ppob & .05 & & 1 & 0 & \\
\hline CHLOANE & & Chlordane & ppb & .1 & & 1 & 0 & \\
\hline$d-B H C$ & & Delta-BHC & ppo & .1 & & 1 & 0 & \\
\hline DIELRIN & & Dieldrin & ppb & .05 & & 1 & 0 & \\
\hline ENDO1 & & Endosulfan 1 & ppb & .1 & & 1 & 0 & \\
\hline ENDO2 & & Endosulfan II & ppb & .05 & & 1 & 0 & \\
\hline ENDSFAN & & Endosulfan sulfate & ppb & .5 & & 1 & 0 & \\
\hline ENDRALD & & Endr in Aldehyde & ppo & .2 & & 1 & 0 & \\
\hline HEPTLOR & & Heptachior & ppb & .05 & & 1 & 0 & \\
\hline HEPTIDE & & Heptachlor epoxide & ppob & 1 & & 1 & 0 & \\
\hline
\end{tabular}

for explanation of this table, see Section 1.4 of report. 
DOE/RL-93-56-3

This page intentionally left blank. 
DOE/RL-93-56-3

\section{CONTENTS}

15.0 LOW-LEVEL BURIAL GROUNDS . . . . . . . . . . . 15-1

15.1 INTRODUCTION . . . . . . . . . . . . . . . . 15-1

15.2 WATER LEVEL MEASUREMENTS . . . . . . . . . . . . . . . . 15-1

15.3 WATER CHEMISTRY DATA ............... 15-1 


\section{LIST OF FIGURES}

15-1 Monitoring Well Locations for Low-Level Waste Management Area 1 ....... . . . . . . . 15-3

15-2 Monitoring Well Locations for Low-Level Waste Management Area 2. . . . . . . . . . . . . . . . . 15-4

15-3 Monitoring Well Locations for Low-Level Waste Management Area 3 ... . . . . . . . . . . . 15-5

15-4 Monitoring Well Locations for Low-Level Waste Management Area 4 . . . . . . . . . . . . . . 15-6

15-5 Monitoring Well Locations for Low-Level Waste Management Area 5 . . . . . . . . . . . . . . 15-7

\section{LIST OF TABLES}

15-1 Low-Level Burial Grounds . . . . . . . . . . . . . . . . 15-8

15-2 Monitoring Well Purpose and Sampling Schedule for Low-Leve1 Waste Management Area 1.... . . . . . . . . . . 15-9

15-3 Monitoring We11 Purpose and Sampling Schedule for Low-Level Waste Management Area 2............... 15-10

15-4 Monitoring Well Purpose and Sampling Schedule for Low-Level Waste Management Area 3 .............. 15-11

15-5 Monitoring Well Purpose and Sampling Schedule for Low-Level Waste Management Area 4.............. 15-12

15-6 Monitoring Well Purpose and Sampling Schedule for Low-Level Waste Management Area 5. . . . . . . . . . . . . 15-13

15-7 RCRA Water Level Measurement Report LLWMA 1,

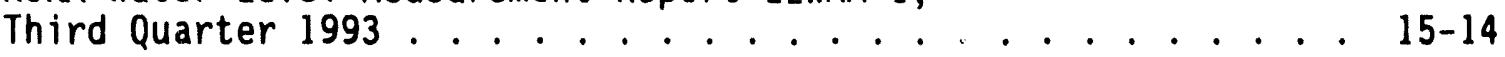

15-8 RCRA Water Leve1 Measurement Report LLWMA 2, Third Quarter 1993.......... . . . . . . . . 15-17

15-9 RCRA Water Level Measurement Report LLWMA 3, Third Quarter 1993................... 15-19

15-10 RCRA Water Level Measurement Report LLWMA 4, Third Quarter 1993 ................... 15-22

15-11 RCRA Water Level Measurement Report LLWMA 5, Third Quarter $1993 \ldots . . .15-24$ 
15-12 Constituent List and Summary of Results for the Low-Level

Waste Burial Grounds WMA 1 Data for Reporting Period

July 1 through September 30, 1993 ............ . . 15-26

15-13 Constituents with at Least One Value Above the CRQL for the

Low-Level Waste Burial Grounds WMA 1 Data for Reporting

Period July 1 through September 30, 1993 . . . . . . . . . . 15-29

15-14 Contamination Indicator Parameters for the Low-Level Waste

Burial Grounds WMA 1 Data for Reporting Period July 1

through September 30,1993............. 15-37

15-15 Constituent List and Summary of Results for the Low-Level

Waste Burial Grounds WMA 2 Data for Reporting Period

July 1 through September 30, 1993

15-16 Constituents with at Least one Value Above the CRQL for the

Low-Level Waste Burial Grounds WMA 2 Data for Reporting

Period July 1 through September 30, 1993 ......... . 15-41

15-17 Contamination Indicator Parameters for the Low-Level Waste

Burial Grounds WMA 2 Data for Reporting Period July 1

through September $30,1993 . . . . . . . . . .15-44$

15-18 Constituent :ist and Swary of Results for the Low-Leve1

Waste Burial Grounds ata for Reporting Period

July 1 through Septer

1993

15-19 Constitlients with at Least One Value Above the CRQL for the

Low-Level Waste Burial Grounds WMA 3 Data for Reporting

Period July 1 through September 30, 1993 . . . . . . . . . 15-51

15-20 Contamination Indicator Parameters for the Low-Level Waste

Burial Grounds WMA 3 Data for Reporting Period July 1

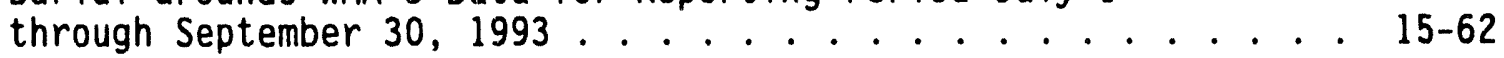

15-21 Constituent List and Summary of Results for the Low-Level

Waste Burial Grounds WMA 4 Data for Reporting Period

July 1 through September 30, 1993

15-22 Constituents with at Least One Value Above the CRQL for the

Low-Level Waste Burial Grounds WMA 4 Data for Reporting

Period July 1 through September 30, 1993 . . . . . . . . . 15-68

15-23 Contamination Indicator Parameters for the Low-Level Waste

Burial Grounds WMA 4 Data for Reporting Period July 1

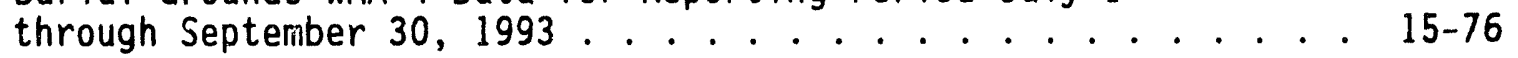

15-24 Constituent List and Summary of Results for the Low-Level

Waste Burial Grounds WMA 5 Data for Reporting Period

July 1 through September 30, 1993 


\section{LIST OF TABLES (cont.)}

15-25 Constituents with at Least One Value Above the CRQL for the Low-Level Waste Burial Grounds WMA 5 Data for Reporting Period July 1 through September 30, 1993 ........ . . 15-84

15-26 Contamination Indicator Parameters for the Low-Level Waste Burial Grounds WMA 5 Data for Reporting Period July 1

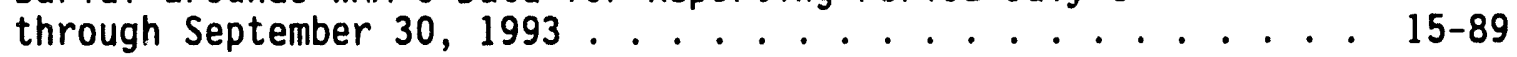

15-27 Water Quality Standards Exceeded at Low-Level Waste Management Area 1. . . . . . . . . . . . . . . 15-90

15-28 Water Quality Standards Exceeded at Low-Level Waste Management Area 2........ . . . . . . . . 15-90

15-29 Water Quality Standards Exceeded at Low-Level Waste Management Area 3. . . . . . . . . . . . . . . 15-91

15-30 Water Quality Standards Exceeded at Low-Level Waste Management Area 4. . . . . . . . . . . . . . 15-92

15-31 Water Quality Standards Exceeded at Low-Level Waste Management Area 5. . . . . . . . . . . . . . . . 15-93 


\title{
15.0 LOW-LEVEL BURIAL GROUNDS
}

\author{
R. B. Mercer \\ Westinghouse Hanford Company
}

\subsection{INTRODUCTION}

The 200 Areas LLBGs consist of five Low-Level Waste Management Areas (LLWMA). LLWMA-1 and LLWMA-2 are located in the 200 East Area and LLWMAs-3, -4 , and -5 are located in the 200 West Area (Figures 15-1 through 15-5). A description of the waste contained in each burial ground is provided in Table 15-1.

The groundwater monitoring program for the LLBG began with the installation of 35 monitoring wells in 1987 . Additional wells have been installed in subsequent years. The present monitoring networks are listed in Tables 15-2 through 15-6.

Currently, groundwater quality assessment programs are under way for LLWMA-1 and LLWMA-3. Groundwater sampling is conducted on a quarterly basis under the assessment programs. Semiannual detection-level sampling is being conducted at LLWMA-2 and LLWMA-4. The first four quarters of data have been collected at some of the LLWMA-5 monitoring wells, and background values have been established of the indicator parameters (except TOX). This site is now in a detection monitoring program with semiannual sampling.

\subsection{MATER LEVEL MEASUREMENTS}

In addition to measurements taken at the time of sampling, water levels are measured monthly at LLWMA-1 and LLWMA-3 (areas in assessment) and quarterly at LLWMAS-2, -4 , and -5 . Water leve 1 measurements are presented in Tables 15-7 through 15-11. Additional water level measurements are reported for some wells if they are monitored for other projects. Suspected errors in the water level measurements occurred at several wells this quarter as indicated in the tables. Water level measurements were not taken at well 299-W6-5 because of an obstructed casing. Water levels continue to declining beneath LLWMA-2. Well 299-E34-4 has been dry since it was constructed. Water levels in wells 299-E34-6, 299-E34-7, and 299-E35-1 have fallen to the point that measurements may not be representative.

\subsection{WATER CHEMISTRY DATA}

Scheduled groundwater samples were collected as scheduled at all five LLWMAs. Five wells scheduled for semiannual sampling at LLWMA-2 were sampled this quarter for the 216-B-63 RCRA monitoring program. Samples were analyzed for CIPs, interim primary drinking water parameters, water quality parameters, and site-specific parameters. Results of analyses are listed in Tables 15-12 through 15-26. These tables also contain any previously unreported data. The replicate averages for the indicator parameters did not exceed the critical means at LLWMAs-2, -4 or -5 . 
An NCR was issued during the construction of well 299-W18-32 because a portion of a broken casing shoe left in the screened interval. A P flag is being assigned to the chemistry data for metals from this well. Results flagged with a ' $Q$ ' are associated with suspect $Q C$ data. All TOX results are flagged with a 'P,' indicating a problem with data. Data quality and data flags are discussed in Sections 1.2 and 1.4 , respectively. Tables 15-27 through 15-31 list the water quality standards that were exceeded for the data reported this quarter. 
Figure 15-1. Monitoring Well Locations for Low-Level Waste Management Area 1.
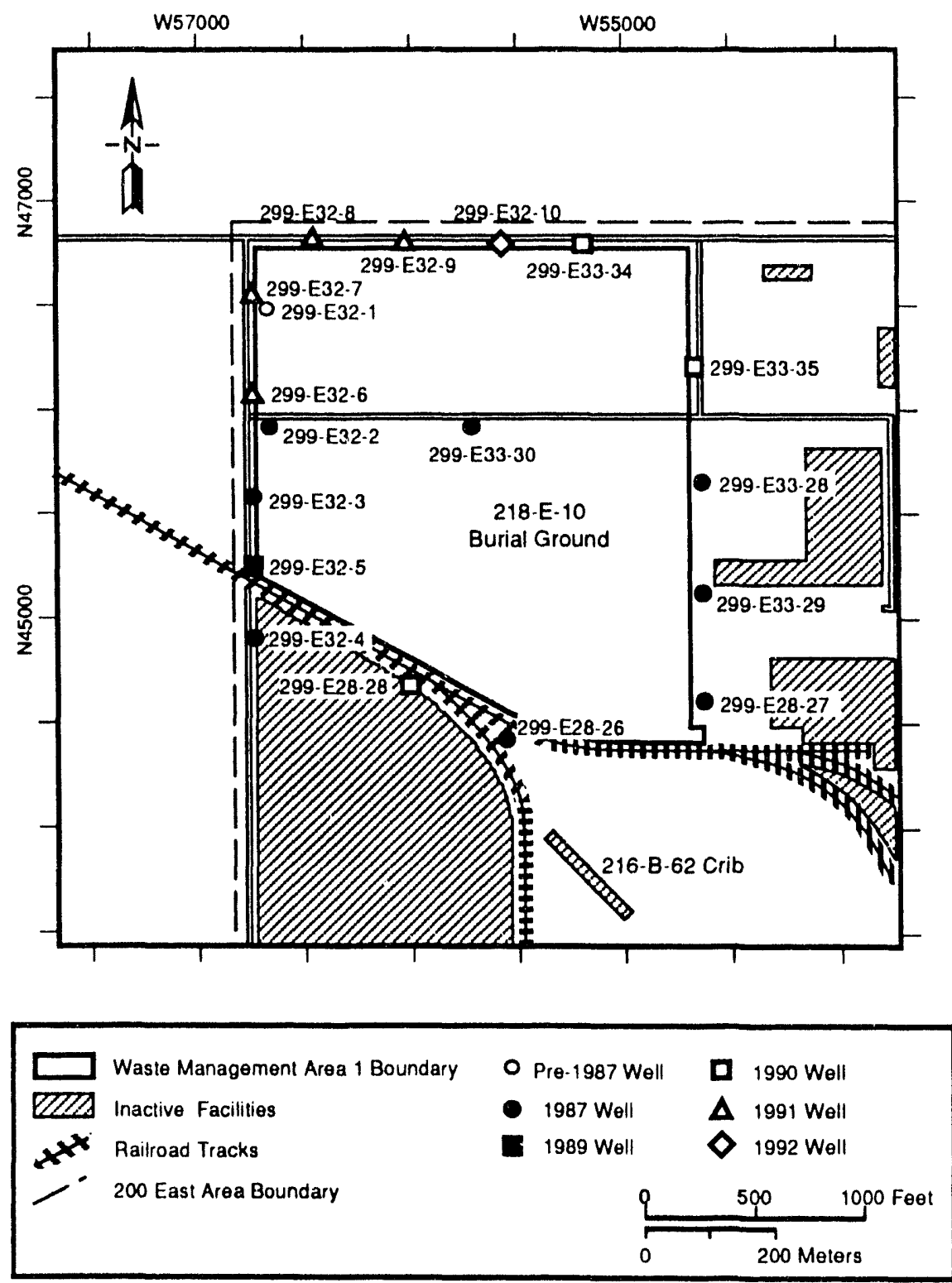

H9012007.102 
Figure 15-2. Monitoring Well Locations for Low-Level Waste Management Area 2.
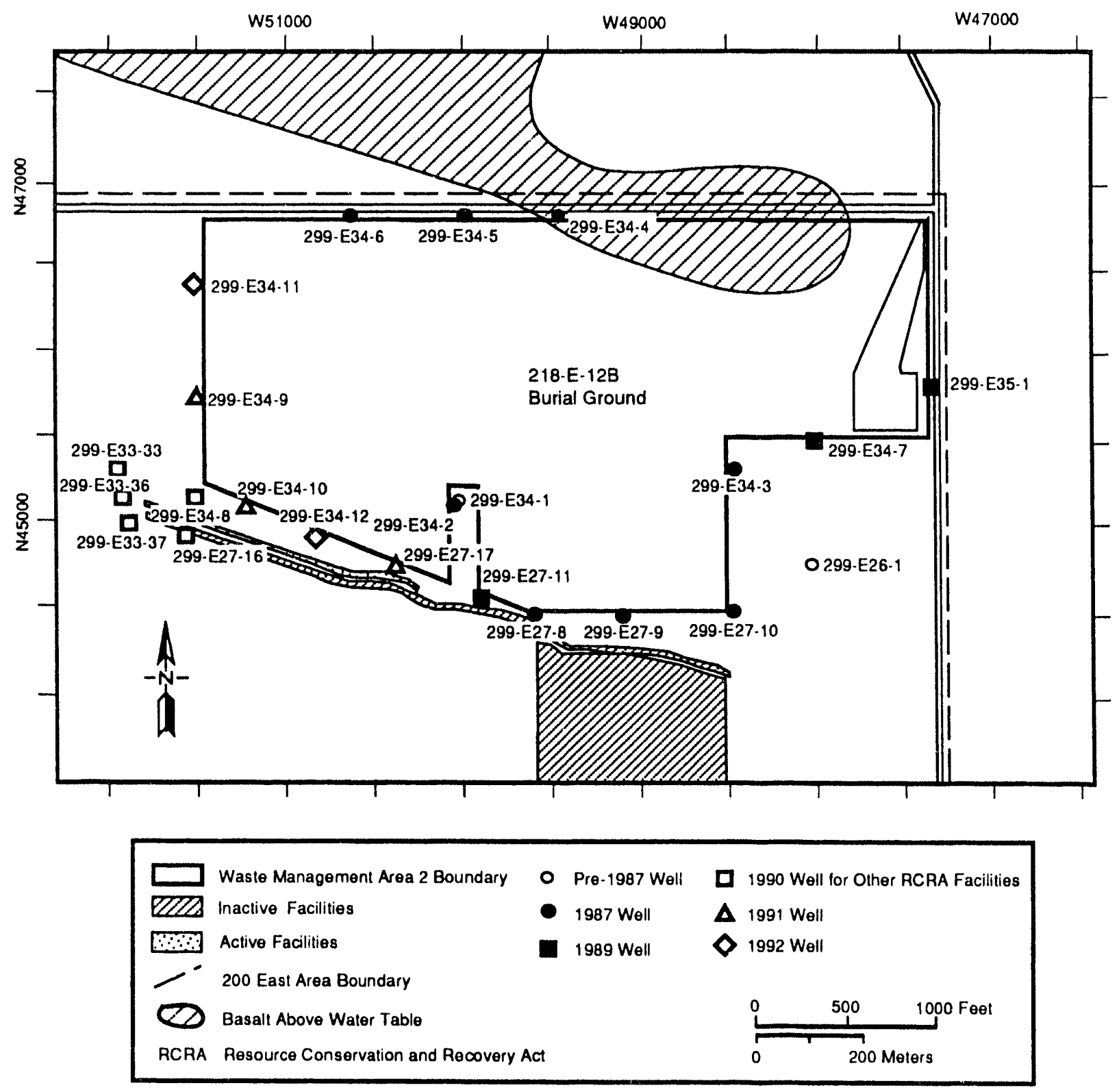

H9012007.103 
Figure 15-3. Monitoring Well Locations for Low-Level Waste Management Area 3.
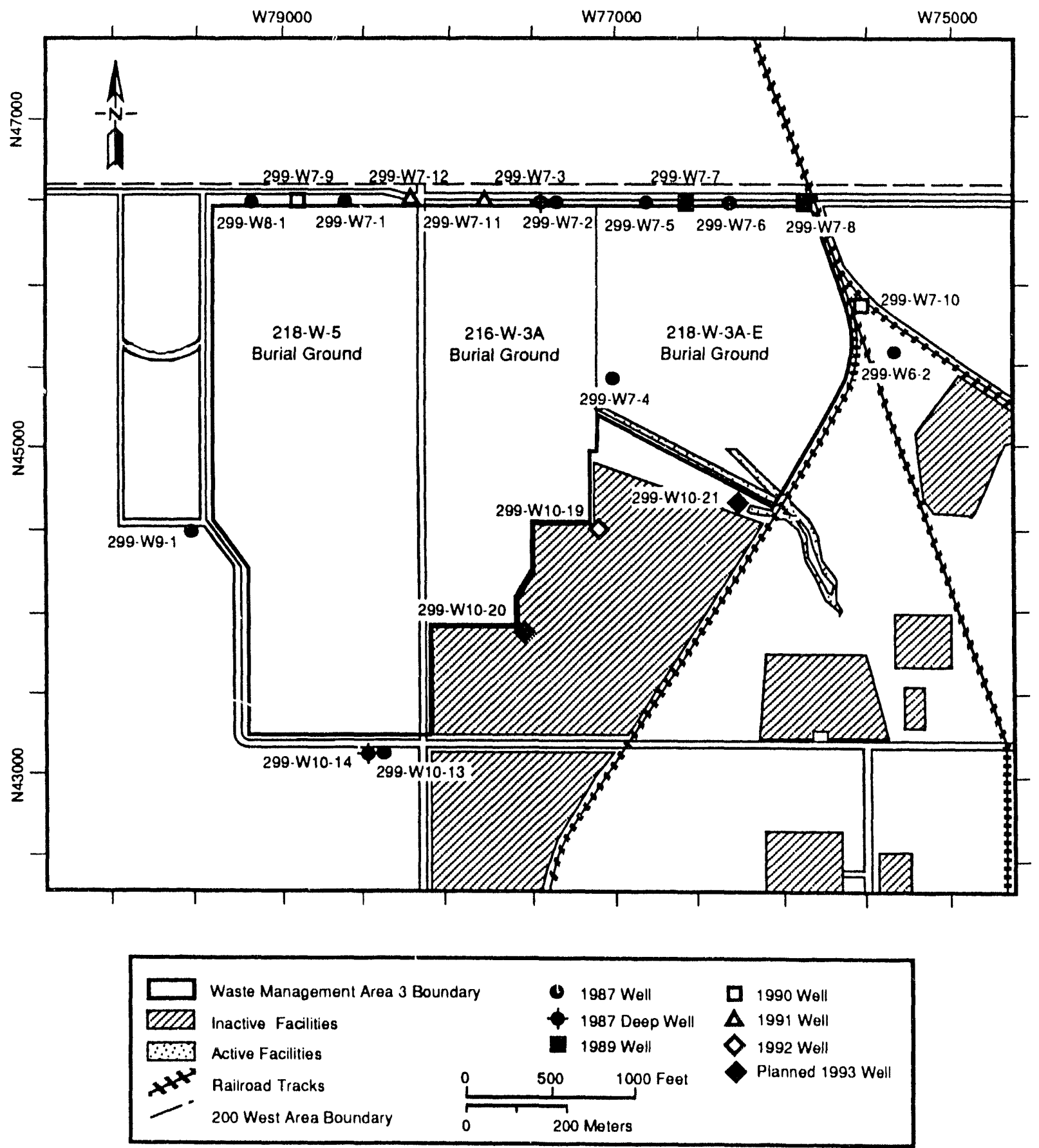

H9012007.104 
Figure 15-4. Monitoring Well Locations for Low-Level Waste Management Area 4.
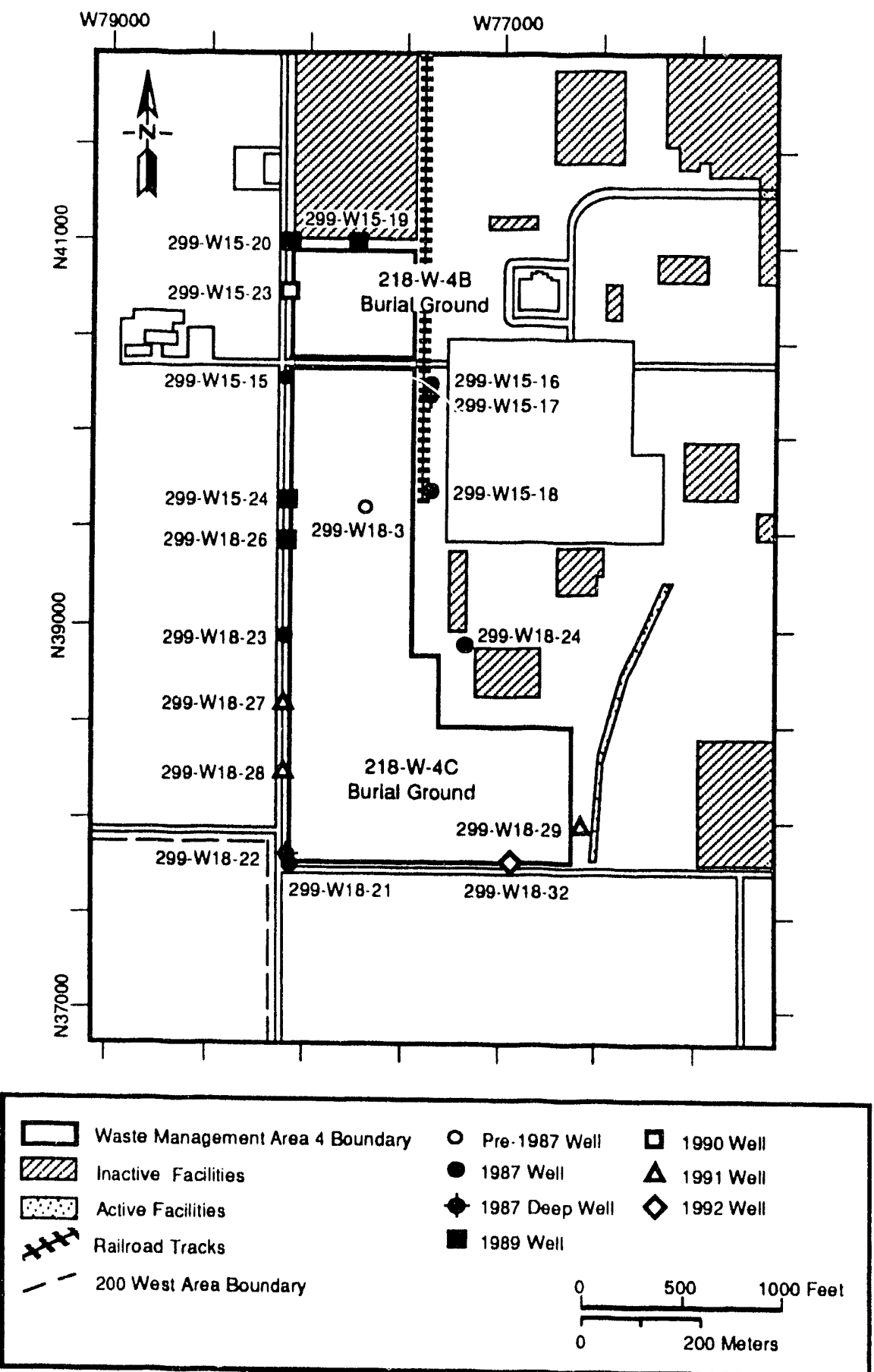

H9012007.105b 
Figure 15-5. Monitoring Well Locations for Low-Level Waste Management Area 5.
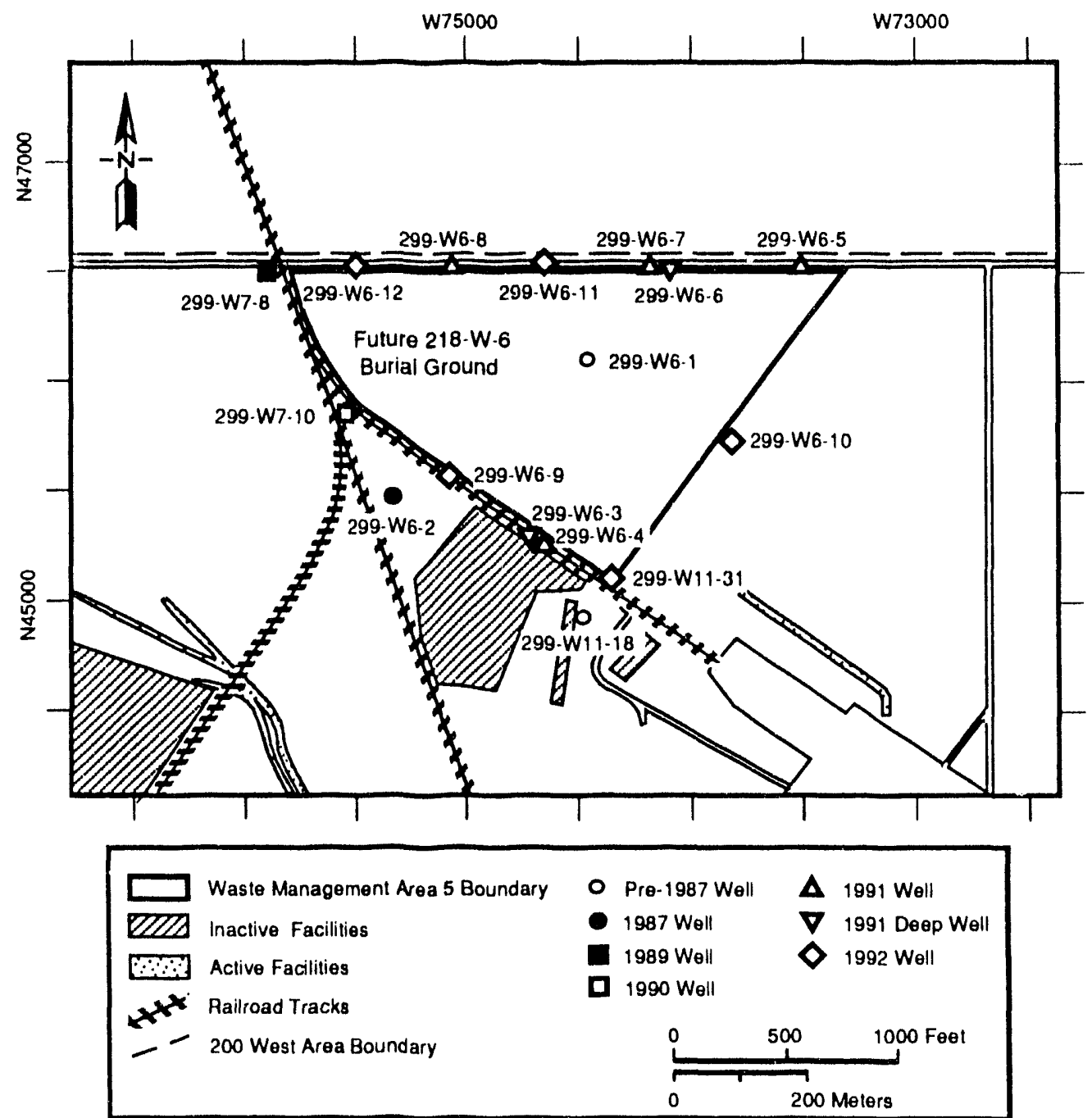

H9012007.106 
Table 15-1. Low-Level Burial Grounds.

\begin{tabular}{|c|c|c|c|}
\hline LLWMA- & Burial Ground & First use & Contents \\
\hline 1 & $218-E-10$ & 1960 & $\begin{array}{l}\text { Dragoff material } \\
\text { Failed equipment } \\
\text { Mixed waste }\end{array}$ \\
\hline 2 & $218-E-12 B$ & 1968 & $\begin{array}{l}\text { Miscellaneous dry waste } \\
\text { Transuranic waste } \\
\text { Submarine reactor compartments }\end{array}$ \\
\hline \multirow[t]{3}{*}{3} & $218-W-3 A$ & 1970 & $\begin{array}{l}\text { Ion exchange resins } \\
\text { Failed equipment }\end{array}$ \\
\hline & $218-W-3 A E$ & 1981 & $\begin{array}{l}\text { Low-level waste } \\
\text { Low-level mixed waste }\end{array}$ \\
\hline & $218-W-5$ & 1986 & $\begin{array}{l}\text { Low-level waste } \\
\text { Low-level mixed waste }\end{array}$ \\
\hline \multirow[t]{2}{*}{4} & $218-W-4 B$ & 1968 & $\begin{array}{l}\text { Mixed waste } \\
\text { Retrievable transuranic waste }\end{array}$ \\
\hline & $218-W-4 C$ & 1978 & $\begin{array}{l}\text { Transuranic waste } \\
\text { Mixed waste } \\
\text { Low-level waste }\end{array}$ \\
\hline 5 & $218-W-6$ & $N / A$ & None to date \\
\hline
\end{tabular}

$N / A=$ not applicable. 
Table 15-2. Monitoring Well Purpose and Sampling Schedule for Low-Leve1 Waste Management Area 1.

\begin{tabular}{|c|c|c|c|c|}
\hline $\begin{array}{l}\text { Well no. } \\
(299-)\end{array}$ & $\begin{array}{l}\text { Relative } \\
\text { position }\end{array}$ & Hydrogeologic unit & $\begin{array}{c}\text { Sample } \\
\text { frequency }\end{array}$ & $\begin{array}{l}\text { Sample date, } \\
\text { 3rd Qtr } 1993\end{array}$ \\
\hline$E 28-26^{87}$ & Upgradient & $\begin{array}{l}\text { Hanford/Ringold: } \\
\text { Water Table }\end{array}$ & Quarterly & $8 / 05 / 93$ \\
\hline$E 28-27^{87}$ & Upgradient & Hanford: Water Table & Quarterly & $8 / 05 / 93$ \\
\hline$E 28-28^{90}$ & Upgradient & $\begin{array}{l}\text { Hanford/Ringold: } \\
\text { Water Table }\end{array}$ & Quarterly & $7 / 28 / 93$ \\
\hline$E 32-2^{87}$ & Downgradient & Hanford: Water Table & Quarterly & $7 / 30 / 93$ \\
\hline$E 32-3^{87}$ & Downgradient & $\begin{array}{l}\text { Hanford/Ringold: } \\
\text { Water Table }\end{array}$ & Quarterly & $7 / 30 / 93$ \\
\hline$E 32-4^{87}$ & Upgradient & $\begin{array}{l}\text { Hanford/Ringold: } \\
\text { Water Table }\end{array}$ & Quarterly & $9 / 02 / 93$ \\
\hline$E 32-5^{89}$ & Downgradient & $\begin{array}{l}\text { Hanford/Ringold: } \\
\text { Water Table }\end{array}$ & Quarterly & $7 / 30 / 93$ \\
\hline$E 32-6^{91}$ & Downgradient & Hanford: Water Table & Quarterly & $7 / 23 / 93$ \\
\hline$E 32-7^{91}$ & Downgradient & Hanford: Water Table & Quarterly & $7 / 26 / 93$ \\
\hline$E 32-8^{91}$ & Downgradient & Hanford: Water Table & Quarterly & $7 / 26 / 93$ \\
\hline$E 32-9^{91}$ & Downgradient & Hanford: Water Table & Quarterly & $7 / 26 / 9 \hat{3}$ \\
\hline$E 32-10^{92}$ & Downgradient & Hanford: Water Table & Quarterly & $7 / 28 / 93$ \\
\hline$E 33-28^{87}$ & Upgradient & Hanford: Water Table & Quarterly & $7 / 30 / 93$ \\
\hline$E 33-29^{87}$ & Upgradient & Hanford: Water Table & Quarterly & $7 / 30 / 93$ \\
\hline$E 33-30^{87}$ & Downgradient & Hanford: Water Table & Quarterly & $8 / 06 / 93$ \\
\hline$E 33-34^{90}$ & Downgradient & Hanford: Water Table & Quarterly & $8 / 06 / 93$ \\
\hline$E 33-35^{90}$ & Upgradient & Hanford: Water Table & Quarterly & $8 / 06 / 93$ \\
\hline
\end{tabular}

Note: Superscript following well number indicates year of installation. 
Table 15-3. Monitoring Well Purpose and Sampling Schedule for Low-Level Waste Management Area 2.

\begin{tabular}{|c|c|c|c|c|c|}
\hline $\begin{array}{c}\text { Wel1 no. } \\
(299-)\end{array}$ & $\begin{array}{l}\text { Relative } \\
\text { position }\end{array}$ & \multicolumn{2}{|c|}{ Hydrogeologic unit } & $\begin{array}{c}\text { Sample } \\
\text { frequency }\end{array}$ & $\begin{array}{l}\text { Sample date, } \\
\text { 3rd Qtr } 1993\end{array}$ \\
\hline$E 27-8^{87}$ & Downgradient & Hanford: & Water Table & Semiannually & $8 / 04 / 93$ \\
\hline$E 27-9^{87}$ & Downgradient & Hanford: & Water Table & Semiannually & $8 / 04 / 93$ \\
\hline$E 27-10^{87}$ & Upgradient & Hanford: 1 & Water Table & Semiannually & NS \\
\hline$E 27-11^{89}$ & Downgradient & Hanford: & Water Table & Semiannually & $7 / 14 / 53$ \\
\hline$E 27-17^{91}$ & Downgradient & Hanford: 1 & Water Table & Semiannually & $7 / 14 / 93$ \\
\hline$E 34-2^{87}$ & Downgradient & Hanford: & Water Table & Semiannually & NS \\
\hline$E 34-3^{87}$ & Upgradient & Hanford: 1 & Water Table & Semiannually & NS \\
\hline$E 34-4^{87}$ & Upgradient & Hanford: 1 & Water Table & $N / A$ & Dry \\
\hline$E 34-5^{87}$ & Upgradient & Hanford: & Water Table & Semiannually & NS \\
\hline$E 34-6^{87}$ & Upgradient & Hanford: 1 & Water Table & $N / A$ & Dry \\
\hline$E 34-7^{89}$ & Upgradient & Hanford: & Water Table & Semiannually & NS \\
\hline$E 34-9^{91}$ & Downgradient & Hanford: 1 & Water Table & Semiannually & NS \\
\hline$E 34-10^{91}$ & Downgradient & Hanford: 1 & Water Table & Semiannually & $7 / 14 / 93$ \\
\hline$E 34-11^{92}$ & Downgradient & Hanford: & Nater Table & Quarterly & $7 / 20 / 93$ \\
\hline$E 34-12^{92}$ & Downgradient & Hanford: & Water Table & Quarterly & $7 / 20 / 93$ \\
\hline$E 35-1^{89}$ & Upgradient & Hanford: & Water Table & $N / A$ & Dry \\
\hline
\end{tabular}

$N / A=$ not available.

NS = not sampled.

Note: Superscript following well number indicates year of installation. 
Table 15-4. Monitoring Well Purpose and Sampling Schedule for Low-Level Waste Management Area 3.

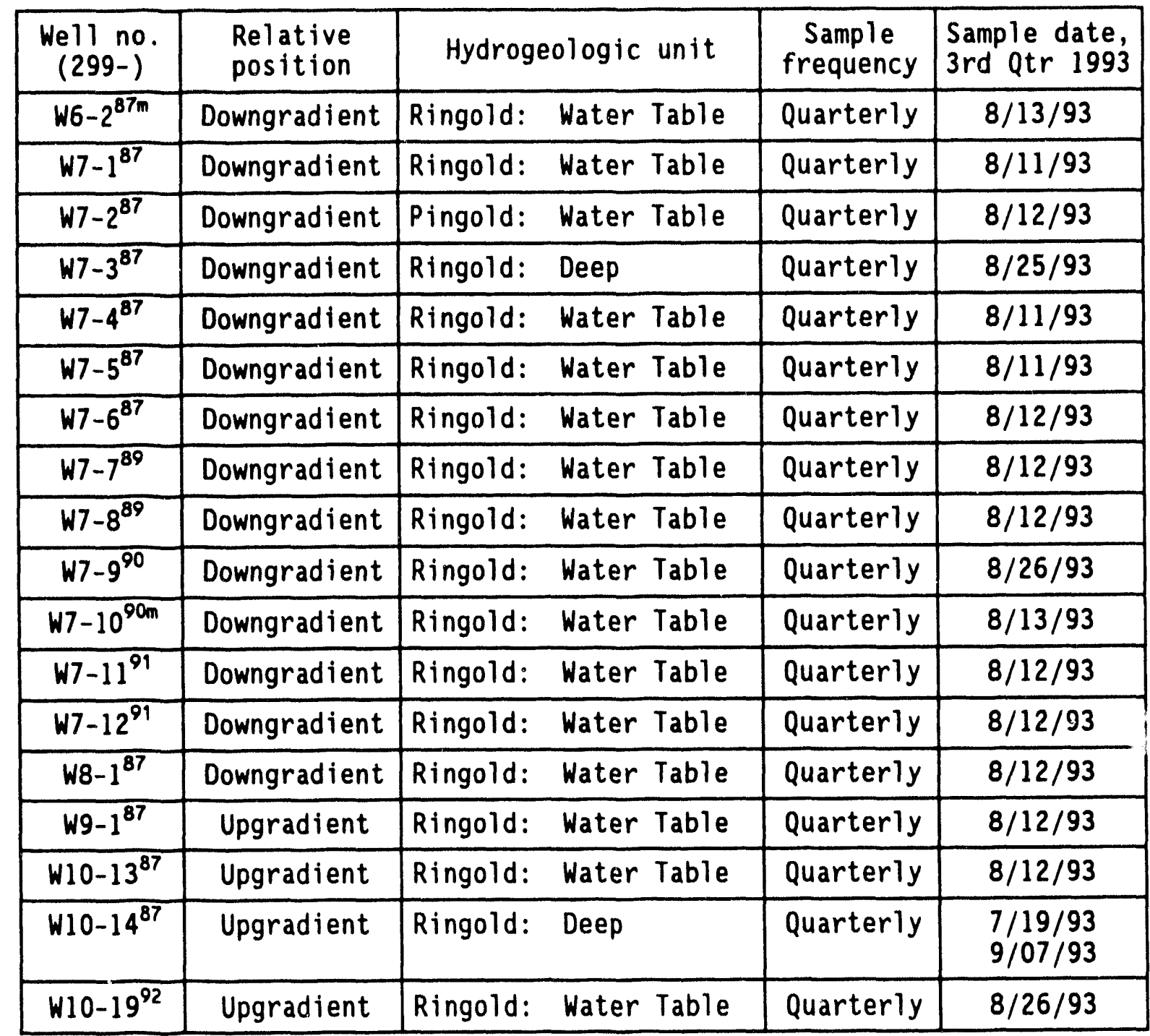

Notes: 1. Superscript following well number indicates year of installation.

2. Well monitors multiple Low-Level Waste Management Areas.

3. Deep indicates well monitors bottom of the unconfined aquifer. 
Table 15-5. Monitoring Well Purpose and Sampling Schedule for Low-Level Waste Management Area 4.

\begin{tabular}{|c|c|c|c|c|}
\hline $\begin{array}{c}\text { Well no. } \\
(299-)\end{array}$ & $\begin{array}{l}\text { Relative } \\
\text { position }\end{array}$ & Hydrogeologic unit & $\begin{array}{c}\text { Sample } \\
\text { frequency }\end{array}$ & $\begin{array}{l}\text { Sample date, } \\
\text { 3rd Qtr } 1993\end{array}$ \\
\hline$W 15-15^{87}$ & Downgradient & Ringold: Water Table & Semiannually & $8 / 16 / 93$ \\
\hline$W 15-16^{87}$ & Upgradient & Ringold: Water Table & Semiannually & $8 / 16 / 93$ \\
\hline$W 15-17^{87}$ & Upgradient & Ringold: Deep & Semiannually & $9 / 08 / 93$ \\
\hline$W 15-18^{87}$ & Upgradient & Ringold: Water Table & Semiannually & $8 / 16 / 93$ \\
\hline$W 15-19^{89}$ & Downgradient & Ringold: Water Table & Semiannually & $9 / 03 / 93$ \\
\hline$W 15-20^{89}$ & Downgradient & Ringold: Water Table & Semiannually & $8 / 13 / 93$ \\
\hline$W 15-23^{90}$ & Downgradient & Ringold: Water Table & Semiannually & $8 / 26 / 93$ \\
\hline$W 15-24^{89}$ & Downgradient & Ringold: Water Table & Semiannually & $8 / 23 / 93$ \\
\hline$W 18-21^{87}$ & Downgradient & Ringold: Water Table & Semiannualiy & $8 / 23 / 93$ \\
\hline$W 18-22^{87}$ & Downgradient & Ringold: Deep & Semiannually & $9 / 01 / 93$ \\
\hline$W 18-23^{87}$ & Downgradient & Ringold: Water Table & Semiannually & $8 / 27 / 93$ \\
\hline$W 18-24^{87}$ & Upgradient & Ringold: Water Table & Semiannually & $8 / 27 / 93$ \\
\hline$W 18-26^{89}$ & Downgradient & Ringold: Water Table & Semiannually & $8 / 13 / 93$ \\
\hline$W 18-27^{91}$ & Downgradient & Ringold: Water Table & Quarterly & $8 / 23 / 93$ \\
\hline$W 18-28^{91}$ & Downgradient & Ringold: Water Table & Quarterly & $8 / 26 / 93$ \\
\hline$W 18-29^{91}$ & Upgradient & Hanford: Perched & erly & $\begin{array}{l}8 / 26 / 93 \\
9 / 20 / 93\end{array}$ \\
\hline$W 18-32^{92}$ & Upgradient & Ringold: Water Table & Quarterly & $8 / 26 / 93$ \\
\hline
\end{tabular}

Notes: 1. Superscript following well number indicates year of installation.

2. Deep indicates well monitors bottom of the unconfined aquifer. 
Table 15-6. Monitoring Well Purpose and Sampling Schedule for Low-Level Waste Management Area 5.

\begin{tabular}{|c|c|c|c|c|}
\hline $\begin{array}{c}\text { Well no. } \\
(299-)\end{array}$ & $\begin{array}{l}\text { Relative } \\
\text { position }\end{array}$ & Hydrogeologic unit & $\begin{array}{c}\text { Sample } \\
\text { frequency }\end{array}$ & $\begin{array}{l}\text { Sample date, } \\
\text { 3rd Qtr } 1993\end{array}$ \\
\hline$W 6-2^{87 m}$ & Upgradient & Ringold: Water Table & Quarterly & $8 / 13 / 93$ \\
\hline W6- $3^{91}$ & Upgradient & Ringold: Deep & Semiannually & NS \\
\hline W6- $4^{91}$ & Upgradient & Ringold: Water Table & Semiannually & NS \\
\hline W6- $5^{91}$ & Downgradient & Ringold: Water Table & Semiannually & NS \\
\hline W6- $6^{91}$ & Downgradient & Ringold: Deep & Semiannually & $9 /: 0 ; y 3$ \\
\hline W6- $7^{91}$ & Downgradient & Ringold: Water Table & Semiannually & $9 / 09 / 93$ \\
\hline W6- $8^{91}$ & Downgradient & Ringold: Water Table & Semiannually & $9 / 09 / 93$ \\
\hline W6- $9^{92}$ & Upgradient & Ringold: Water Table & Quarterly & $9 / 10 / 93$ \\
\hline W6- $10^{92}$ & Upgradient & Ringold: Water Table & Quarterly & $9 / 09 / 93$ \\
\hline W6- $11^{92}$ & Downgradient & Ringold: Water Table & Quarterly & $9 / 09 / 93$ \\
\hline$w 6-12^{92}$ & Downgradient & Ringold: Water Table & Quarterly & $9 / 09 / 93$ \\
\hline$W 7-10^{90 m}$ & Upgradient & Ringold: Water Table & Quarterly & $8 / 13 / 93$ \\
\hline$W 11-31^{92}$ & Upgradient & Ringold: Water Table & Quarterly & $9 / 09 / 93$ \\
\hline
\end{tabular}

NS = not sampled.

Notes: 1. Superscript following well number indicates year of installation.

2. mell monitors multiple Low-Level Waste Management Areas.

3. Deep indicates well monitors bottom of the unconfined aquifer. 
Table 15-7. RCRA Water Level Measurement Report

LLWMA 1, Third Quarter 1993. (sheet 1 of 3)

We11 Date $\quad \begin{gathered}\text { Depth to } \\ \text { water (ft) }\end{gathered} \begin{gathered}\text { Water level } \\ \text { elevation } \\ \text { above ms }\end{gathered}$

LLWMA-1 Wells Monitoring the Top of the Unconfined Aquifer

\begin{tabular}{|c|c|c|c|}
\hline 299-E28-26 & $\begin{array}{l}7 / 27 / 93 \\
8 / 05 / 93 \\
8 / 20 / 93 \\
9 / 22 / 93\end{array}$ & $\begin{array}{l}285.50 \\
285.54 \\
285.48 \\
285.60\end{array}$ & $\begin{array}{l}401.76 \\
401.72 \star \\
401.78 \\
401.66\end{array}$ \\
\hline 299-E28-27 & $\begin{array}{l}7 / 27 / 93 \\
8 / 05 / 93 \\
8 / 20 / 93 \\
9 / 22 / 93\end{array}$ & $\begin{array}{l}278.68 \\
278.78 \\
278.70 \\
278.78\end{array}$ & $\begin{array}{l}401.69 \\
401.59 * \\
401.67 \\
401.59\end{array}$ \\
\hline 299-E28-28 & $\begin{array}{l}7 / 27 / 93 \\
7 / 28 / 93 \\
8 / 20 / 93 \\
9 / 22 / 93\end{array}$ & $\begin{array}{l}284.92 \\
284.76 \\
284.97 \\
285.03\end{array}$ & $\begin{array}{l}401.63 \\
401.79 \star \\
401.58 \\
401.52\end{array}$ \\
\hline 299-E32-2 & $\begin{array}{l}7 / 27 / 93 \\
7 / 30 / 93 \\
8 / 20 / 93 \\
9 / 22 / 93\end{array}$ & $\begin{array}{l}268.58 \\
268.50 \\
268.53 \\
268.60\end{array}$ & $\begin{array}{l}401.48 \\
401.56^{\star} \\
401.53 \\
401.46\end{array}$ \\
\hline 299-E32-3 & $\begin{array}{l}7 / 27 / 93 \\
7 / 30 / 93 \\
8 / 20 / 93 \\
9 / 22 / 93\end{array}$ & $\begin{array}{l}274.85 \\
274.90 \\
274.88 \\
275.96\end{array}$ & $\begin{array}{l}401.66 \\
401.61 * \\
401.63 \\
400.55+\end{array}$ \\
\hline $299-E 32-4$ & $\begin{array}{l}7 / 27 / 93 \\
8 / 20 / 93 \\
9 / 02 / 93 \\
9 / 22 / 93\end{array}$ & $\begin{array}{l}284.35 \\
284.37 \\
284.48 \\
284.45\end{array}$ & $\begin{array}{l}401.53 \\
401.51 \\
401.40 \star \\
401.43\end{array}$ \\
\hline 299-E32-5 & $\begin{array}{l}7 / 27 / 93 \\
7 / 30 / 93 \\
8 / 20 / 93 \\
9 / 22 / 93\end{array}$ & $\begin{array}{l}280.49 \\
280.50 \\
280.54 \\
280.63\end{array}$ & $\begin{array}{l}401.65 \\
401.64 * \\
401.60 \\
401.51\end{array}$ \\
\hline 299-E32-6 & $\begin{array}{l}7 / 23 / 93 \\
9 / 09 / 93\end{array}$ & $\begin{array}{l}265.56 \\
265.65\end{array}$ & $\begin{array}{l}401.89 \star \\
401.80\end{array}$ \\
\hline 299-E32-7 & $\begin{array}{l}7 / 26 / 93 \\
7 / 27 / 93 \\
8 / 20 / 93 \\
9 / 22 / 93\end{array}$ & $\begin{array}{l}256.66 \\
256.57 \\
256.59 \\
256.65\end{array}$ & $\begin{array}{l}401.76^{\star} \\
401.85 \\
401.83 \\
401.77\end{array}$ \\
\hline
\end{tabular}


Table 15-7. RCRA Water Level Measurement Report LLWMA 1, Third Quarter 1993. (sheet 2 of 3)

\begin{tabular}{|c|c|c|c|}
\hline Well & Date & $\begin{array}{l}\text { Depth to } \\
\text { water (ft) }\end{array}$ & $\begin{array}{l}\text { Water level } \\
\text { elevation } \\
\text { above msl (ft) }\end{array}$ \\
\hline LLWMA-1 & itoring $t$ & Top of the & ined Aquifer \\
\hline 299-E32-8 & $\begin{array}{l}7 / 26 / 93 \\
7 / 27 / 93 \\
8 / 20 / 93 \\
9 / 22 / 93\end{array}$ & $\begin{array}{l}243.80 \\
243.83 \\
243.84 \\
243.91\end{array}$ & $\begin{array}{l}401.79 \star \\
401.76 \\
401.75 \\
401.68\end{array}$ \\
\hline 299-E32-9 & $\begin{array}{l}7 / 26 / 93 \\
7 / 27 / 93 \\
8 / 20 / 93 \\
9 / 22 / 93\end{array}$ & $\begin{array}{l}241.40 \\
241.43 \\
241.52 \\
241.58\end{array}$ & $\begin{array}{l}401.93^{\star} \\
401.90 \\
401.81 \\
401.75\end{array}$ \\
\hline $299-E 32-10$ & $\begin{array}{l}7 / 28 / 93 \\
9 / 22 / 93\end{array}$ & $\begin{array}{l}234.40 \\
236.17\end{array}$ & $\begin{array}{l}403.48^{\star}+ \\
401.71\end{array}$ \\
\hline 299-E33-28 & $\begin{array}{l}7 / 27 / 93 \\
7 / 30 / 93 \\
8 / 20 / 93 \\
9 / 22 / 93\end{array}$ & $\begin{array}{l}262.50 \\
262.85 \\
262.51 \\
262.60\end{array}$ & $\begin{array}{l}401.73 \\
401.38 \star \\
401.72 \\
401.63\end{array}$ \\
\hline 299-E33-29 & $\begin{array}{l}7 / 27 / 93 \\
7 / 30 / 93 \\
8 / 20 / 93 \\
9 / 22 / 93\end{array}$ & $\begin{array}{l}272.04 \\
271.40 \\
272.06 \\
272.14\end{array}$ & $\begin{array}{l}401.73 \\
402.37^{\star}+ \\
401.71 \\
401.63\end{array}$ \\
\hline 299-E33-30 & $\begin{array}{l}7 / 27 / 93 \\
8 / 06 / 93 \\
8 / 20 / 93 \\
9 / 22 / 93\end{array}$ & $\begin{array}{l}261.97 \\
261.94 \\
262.00 \\
262.07\end{array}$ & $\begin{array}{l}401.73 \\
401.76 \star \\
401.70 \\
401.63\end{array}$ \\
\hline 299-E33-34 & $\begin{array}{l}7 / 27 / 93 \\
8 / 06 / 93 \\
8 / 20 / 93 \\
9 / 22 / 93\end{array}$ & $\begin{array}{l}231.68 \\
231.65 \\
231.50 \\
231.82\end{array}$ & $\begin{array}{l}401.65 \\
401.68 \star \\
401.83 \\
401.51\end{array}$ \\
\hline
\end{tabular}


Table 15-7. RCRA Water Level Measurement Report LLWMA 1, Third Quarter 1993. (sheet 3 of 3)

Wel1 Date $\begin{gathered}\text { Depth to } \\ \text { water (ft) }\end{gathered} \begin{gathered}\text { Water level } \\ \text { elevation } \\ \text { above ms }(\mathrm{ft})\end{gathered}$

LLWMA-1 Wells Monitoring the Top of the Unconfined Aquifer

\begin{tabular}{llll}
\hline $299-E 33-35$ & $7 / 27 / 93$ & 241.35 & 401.66 \\
& $8 / 06 / 93$ & 241.34 & $401.67 \star$ \\
$8 / 20 / 93$ & 241.40 & 401.61 \\
& $9 / 22 / 93$ & 241.46 & 401.55 \\
\hline
\end{tabular}

Notes: 1. Water level elevations are calculated by subtracting the measured depth to water from the surveyed elevation for the well.

2. Depth-to-water values are transcribed from field records.

3. Elevations marked with an ' $*$ ' were measured at the time of sampling.

4. Elevations marked with a ' + ' are outside of the expected range, and are suspected of error.

5. To convert feet to meters multiply by 0.3048 . 
Table 15-8. RCRA Water Level Measurement Report LLWMA 2, Third Quarter 1993. (sheet 1 of 2)

\begin{tabular}{|c|c|c|c|}
\hline Well & Date & $\begin{array}{l}\text { Depth to } \\
\text { water }(\mathrm{ft})\end{array}$ & $\begin{array}{l}\text { Water level } \\
\text { elevation } \\
\text { above msl (ft) }\end{array}$ \\
\hline \multicolumn{4}{|c|}{ LLWMA-2 Wells } \\
\hline 299-E27-8 & $\begin{array}{l}8 / 04 / 93 \\
9 / 09 / 93\end{array}$ & $\begin{array}{l}236.00 \\
236.02\end{array}$ & $\begin{array}{l}401.83^{\star} \\
401.81\end{array}$ \\
\hline 299-E27-9 & $\begin{array}{l}8 / 04 / 93 \\
9 / 09 / 93\end{array}$ & $\begin{array}{l}227.20 \\
227.34\end{array}$ & $\begin{array}{l}402.01 * \\
401.87\end{array}$ \\
\hline 299-E27-10 & $9 / 09 / 93$ & 222.34 & 402.13 \\
\hline 299-E27-11 & $\begin{array}{l}7 / 14 / 93 \\
9 / 09 / 93\end{array}$ & $\begin{array}{l}241.50 \\
241.70\end{array}$ & $\begin{array}{l}401.79 \star \\
401.59\end{array}$ \\
\hline 299-E27-17 & $\begin{array}{l}7 / 14 / 93 \\
9 / 09 / 93\end{array}$ & $\begin{array}{l}232.59 \\
232.77\end{array}$ & $\begin{array}{l}402.13^{\star} \\
401.95\end{array}$ \\
\hline 299-E34-2 & $9 / 01 / 93$ & 228.99 & 401.81 \\
\hline 299-E34-3 & $9 / 09 / 93$ & 209.40 & 402.12 \\
\hline $299-E 34-5$ & $9 / 01 / 93$ & 187.88 & 402.91 \\
\hline $299-E 34-7$ & $9 / 09 / 93$ & 202.46 & 401.79 \\
\hline 299-E34-9 & $9 / 09 / 93$ & 226.83 & 401.86 \\
\hline 299-E34-10 & $\begin{array}{l}7 / 14 / 93 \\
9 / 09 / 93\end{array}$ & $\begin{array}{l}237.62 \\
237.78\end{array}$ & $\begin{array}{l}402.15^{\star} \\
401.99\end{array}$ \\
\hline
\end{tabular}


Table 15-8. RCRA Water Level Measurement Report

LLWMA 2, Third Quarter 1993. (sheet 2 of 2)

LLWMA-2 Wells Monitoring the Top of the Unconfined Aquifer

\begin{tabular}{llll}
\hline $299-E 34-11$ & $7 / 20 / 93$ & 215.75 & $402.18^{\star}$ \\
& $9 / 09 / 93$ & 216.02 & 401.91 \\
$299-E 34-12$ & $7 / 20 / 93$ & 236.89 & $401.94^{\star}$ \\
& $9 / 09 / 93$ & 237.17 & 401.66 \\
\hline
\end{tabular}

Notes: 1. Water level elevations are calculated by subtracting the measured depth to water from the surveyed elevation for the well.

2. Depth-to-water values are transcribed from field records.

3. Elevations marked with an '*' were measured at the time of sampling.

4. To convert feet to meters multiply by 0.3048 . 
Table 15-9. RCRA Water Level Measurement Report LLWMA 3, Third Quarter 1993. (sheet 1 of 3)

\begin{tabular}{|c|c|c|c|}
\hline Well & Date & $\begin{array}{l}\text { Depth to } \\
\text { water }(f t)\end{array}$ & $\begin{array}{l}\text { Water level } \\
\text { elevation } \\
\text { above msl (ft) }\end{array}$ \\
\hline \multicolumn{4}{|c|}{ LLWMA-3 } \\
\hline 299-W6-2 & $\begin{array}{l}7 / 27 / 93 \\
8 / 13 / 93 \\
8 / 20 / 93 \\
9 / 22 / 93\end{array}$ & $\begin{array}{l}234.77 \\
234.89 \\
234.86 \\
235.13\end{array}$ & $\begin{array}{l}457.68 \\
457.56 \star \\
457.59 \\
457.32\end{array}$ \\
\hline 299-W7-1 & $\begin{array}{l}7 / 27 / 93 \\
8 / 11 / 93 \\
8 / 20 / 93 \\
9 / 22 / 93\end{array}$ & $\begin{array}{l}232.36 \\
232.45 \\
232.43 \\
232.68\end{array}$ & $\begin{array}{l}458.35 \\
458.26^{\star} \\
458.28 \\
458.03\end{array}$ \\
\hline $299-W 7-2$ & $\begin{array}{l}7 / 27 / 93 \\
8 / 12 / 93 \\
8 / 20 / 93 \\
9 / 22 / 93\end{array}$ & $\begin{array}{l}218.35 \\
218.38 \\
218.44 \\
218.61\end{array}$ & $\begin{array}{l}457.24 \\
457.21^{\star} \\
457.15 \\
456.98\end{array}$ \\
\hline 299-W7-4 & $\begin{array}{l}7 / 27 / 93 \\
8 / 11 / 93 \\
8 / 20 / 93 \\
9 / 22 / 93\end{array}$ & $\begin{array}{l}212.62 \\
212.55 \\
212.71 \\
212.90\end{array}$ & $\begin{array}{l}459.07 \\
459.14^{\star} \\
458.98 \\
458.79\end{array}$ \\
\hline 299-W7-5 & $\begin{array}{l}7 / 27 / 93 \\
8 / 11 / 93 \\
8 / 20 / 93 \\
9 / 22 / 93\end{array}$ & $\begin{array}{l}216.26 \\
216.40 \\
216.36 \\
216.56\end{array}$ & $\begin{array}{l}456.79 \\
456.65^{\star} \\
456.69 \\
456.49\end{array}$ \\
\hline 299-W7-6 & $\begin{array}{l}7 / 27 / 93 \\
8 / 12 / 93 \\
8 / 20 / 93 \\
9 / 22 / 93\end{array}$ & $\begin{array}{l}221.99 \\
222.04 \\
222.05 \\
222.29\end{array}$ & $\begin{array}{l}456.65 \\
456.60^{\star} \\
456.59 \\
456.35\end{array}$ \\
\hline $299-W 7-7$ & $\begin{array}{l}7 / 27 / 93 \\
8 / 12 / 93 \\
8 / 20 / 93 \\
9 / 22 / 93\end{array}$ & $\begin{array}{l}218.11 \\
218.12 \\
218.19 \\
218.41\end{array}$ & $\begin{array}{l}456.83 \\
456.82^{\star} \\
456.75 \\
456.53\end{array}$ \\
\hline 299-W7-8 & $\begin{array}{l}7 / 27 / 93 \\
8 / 12 / 93 \\
8 / 20 / 93 \\
9 / 22 / 93\end{array}$ & $\begin{array}{l}231.93 \\
231.91 \\
232.28 \\
232.18\end{array}$ & $\begin{array}{l}455.42 \\
455.44^{\star} \\
455.07 \\
455.17\end{array}$ \\
\hline
\end{tabular}


Table 15-9. RCRA Water Level Measurement Report LLWMA 3, Third Quarter 1993. (sheet 2 of 3)

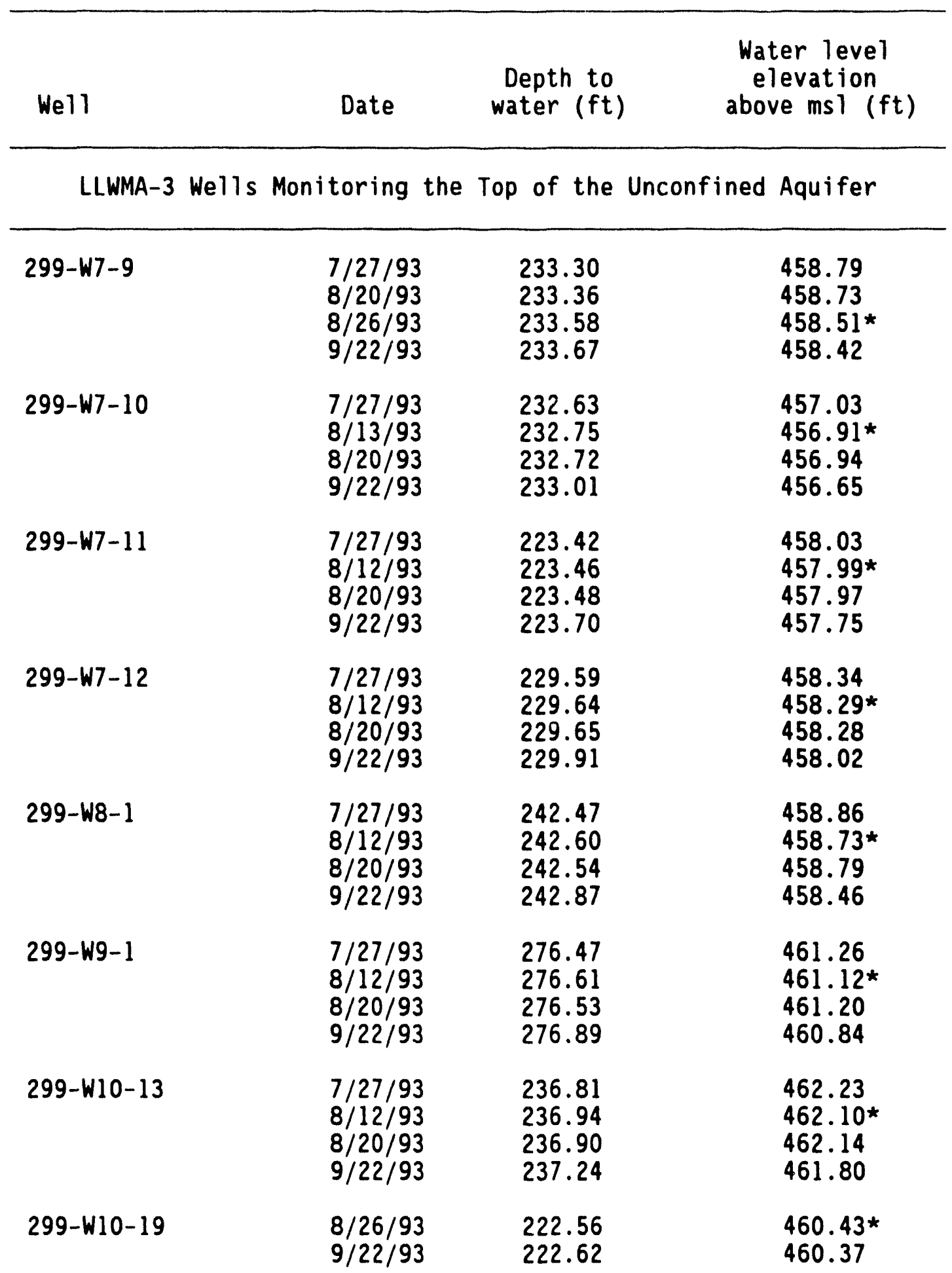


$\mathrm{DOE} / \mathrm{RL}-93-56-3$

Table 15-9. RCRA Water Level Measurement Report

LLWMA 3, Third Quarter 1993. (sheet 3 of 3)

\begin{tabular}{|c|c|c|c|}
\hline Well & Date & $\begin{array}{l}\text { Depth to } \\
\text { water ( } f t)\end{array}$ & $\begin{array}{l}\text { Water level } \\
\text { elevation } \\
\text { above msl (ft) }\end{array}$ \\
\hline \multicolumn{4}{|l|}{ LLWMA-3 W } \\
\hline 299-W7-3 & $\begin{array}{l}7 / 27 / 93 \\
8 / 20 / 93 \\
8 / 25 / 93 \\
9 / 22 / 93\end{array}$ & $\begin{array}{l}219.96 \\
219.99 \\
220.17 \\
220.27\end{array}$ & $\begin{array}{l}456.18 \\
456.15 \\
455.97 \star \\
455.87\end{array}$ \\
\hline 299-W10-14 & $\begin{array}{l}7 / 19 / 93 \\
7 / 27 / 93 \\
7 / 27 / 93 \\
8 / 20 / 93 \\
9 / 07 / 93 \\
9 / 22 / 93\end{array}$ & $\begin{array}{l}238.01 \\
237.63 \\
236.81 \\
237.71 \\
237.97 \\
238.02\end{array}$ & $\begin{array}{l}461.42 \star \\
461.80 \\
462.62 \\
461.72 \\
461.46 \star \\
461.41\end{array}$ \\
\hline
\end{tabular}

Notes: 1. Water level elevations are calculated by subtracting the measured depth to water from the surveyed elevation for the well.

2. Depth-to-water values are transcribed from field records.

3. Elevations marked with an '*' were measured at the time of sampling.

4. To convert feet to meters multiply by 0.3048 . 
Table 15-10. RCRA Water Level Measurement Report LLWMA 4, Third Quarter 1993. (sheet 1 of 2)

\begin{tabular}{|c|c|c|c|}
\hline Well & Date & $\begin{array}{l}\text { Depth to } \\
\text { water (ft) }\end{array}$ & $\begin{array}{c}\text { Water level } \\
\text { elevation } \\
\text { above msl (ft) }\end{array}$ \\
\hline \multirow{2}{*}{$\frac{\text { LLWMA-4 }}{\text { W15-15 }}$} & itoring $t$ & Top of the $U$ & ined Aquifer \\
\hline & $\begin{array}{l}8 / 16 / 93 \\
9 / 09 / 93\end{array}$ & $\begin{array}{l}234.46 \\
234.57\end{array}$ & $\begin{array}{l}463.50 * \\
463.39\end{array}$ \\
\hline 299-W15-16 & $\begin{array}{l}8 / 16 / 93 \\
9 / 09 / 93\end{array}$ & $\begin{array}{l}220.78 \\
220.91\end{array}$ & $\begin{array}{l}464.11 * \\
463.98\end{array}$ \\
\hline $299-W 15-18$ & $\begin{array}{l}8 / 16 / 93 \\
9 / 09 / 93\end{array}$ & $\begin{array}{l}222.17 \\
221.62\end{array}$ & $\begin{array}{l}463.54^{\star} \\
464.09\end{array}$ \\
\hline $299-W 15-19$ & $\begin{array}{l}9 / 03 / 93 \\
9 / 09 / 93\end{array}$ & $\begin{array}{l}228.28 \\
228.35\end{array}$ & $\begin{array}{l}463.32^{\star} \\
463.25\end{array}$ \\
\hline $299-W 15-20$ & $\begin{array}{l}8 / 13 / 93 \\
9 / 09 / 93\end{array}$ & $\begin{array}{l}235.66 \\
235.22\end{array}$ & $\begin{array}{l}462.70^{*}+ \\
463.14\end{array}$ \\
\hline $299-W 15-23$ & $\begin{array}{l}8 / 26 / 93 \\
9 / 09 / 93\end{array}$ & $\begin{array}{l}236.29 \\
236.21\end{array}$ & $\begin{array}{l}463.20 * \\
463.28\end{array}$ \\
\hline $299-W 15-24$ & $\begin{array}{l}8 / 23 / 93 \\
9 / 09 / 93\end{array}$ & $\begin{array}{l}235.86 \\
235.92\end{array}$ & $\begin{array}{l}463.51 * \\
463.45\end{array}$ \\
\hline $299-W 18-21$ & $\begin{array}{l}8 / 23 / 93 \\
9 / 09 / 93\end{array}$ & $\begin{array}{l}205.36 \\
205.18\end{array}$ & $\begin{array}{l}463.26^{\star} \\
463.44\end{array}$ \\
\hline $299-W 18-23$ & $\begin{array}{l}8 / 27 / 93 \\
9 / 09 / 93\end{array}$ & $\begin{array}{l}233.26 \\
233.33\end{array}$ & $\begin{array}{l}463.55^{\star} \\
463.48\end{array}$ \\
\hline 299-W18-24 & $\begin{array}{l}8 / 27 / 93 \\
9 / 09 / 93\end{array}$ & $\begin{array}{l}220.09 \\
220.11\end{array}$ & $\begin{array}{l}464.26^{\star} \\
464.24\end{array}$ \\
\hline $299-W 18-26$ & $\begin{array}{l}8 / 13 / 93 \\
9 / 09 / 93\end{array}$ & $\begin{array}{l}235.39 \\
235.54\end{array}$ & $\begin{array}{l}463.66^{\star} \\
463.51\end{array}$ \\
\hline $299-W 18-27$ & $\begin{array}{l}8 / 23 / 93 \\
9 / 09 / 93\end{array}$ & $\begin{array}{l}226.61 \\
226.74\end{array}$ & $\begin{array}{l}463.64^{\star} \\
463.51\end{array}$ \\
\hline
\end{tabular}


Table 15-10. RCRA Water Level Measurement Report LLWMA 4, Third Quarter 1993. (sheet 2 of 2)

\begin{tabular}{|c|c|c|c|}
\hline Well & Date & $\begin{array}{l}\text { Depth to } \\
\text { water (ft) }\end{array}$ & $\begin{array}{l}\text { Water level } \\
\text { elevation } \\
\text { above msl (ft) }\end{array}$ \\
\hline \multicolumn{4}{|l|}{ LLWMA-4 } \\
\hline $299-W 18-28$ & $\begin{array}{l}8 / 26 / 93 \\
9 / 09 / 93\end{array}$ & $\begin{array}{l}216.29 \\
216.24\end{array}$ & $\begin{array}{l}463.70 \star \\
463.75\end{array}$ \\
\hline $299-W 18-32$ & $\begin{array}{l}8 / 26 / 93 \\
9 / 09 / 93\end{array}$ & $\begin{array}{l}212.04 \\
211.83\end{array}$ & $\begin{array}{l}464.61^{\star} \\
464.82\end{array}$ \\
\hline
\end{tabular}

LLWMA-4 Wells Monitoring the Bottom of the Unconfined Aquifer

\begin{tabular}{llll}
\hline $299-W 15-17$ & $9 / 08 / 93$ & 220.05 & $464.59 \star^{\prime}+$ \\
& $9 / 09 / 93$ & 220.99 & 463.65 \\
$299-W 18-22$ & $9 / 01 / 93$ & 205.25 & $463.24 \star$ \\
& $9 / 09 / 93$ & 205.55 & 462.94 \\
\hline
\end{tabular}

LLWMA-4 Wells Monitoring the Perched Zone

\begin{tabular}{llll}
\hline $299-W 18-29$ & $8 / 26 / 93$ & 125.70 & $548.44^{\star}$ \\
& $9 / 09 / 93$ & 125.64 & 548.50 \\
& $9 / 20 / 93$ & 126.02 & $548.12^{\star}$ \\
\hline
\end{tabular}

Notes: 1. Water level elevations are calculated by subtracting the measured depth to water from the surveyed elevation for the well.

2. Depth-to-water values are transcribed from field records.

3. Elevations marked with an ' $*$ ' were measured at the time of sampling.

4. Elevations marked with a ' + ' are outside of the expected range, and are suspected of error.

5. To convert feet to meters multiply by 0.3048 . 
Table 15-11. RCRA Water Level Measurement Report LLWMA 5, Third Quarter 1993. (sheet 1 of 2)

\begin{tabular}{cccc}
\hline We11 & Date & $\begin{array}{c}\text { Depth to } \\
\text { water }(\mathrm{ft})\end{array}$ & $\begin{array}{c}\text { Water level } \\
\text { elevation } \\
\text { above ms }\end{array}$ \\
\hline LLWMA-5 Wells
\end{tabular}


Table 15-11. RCRA Water Level Measurement Report LLWMA 5, Third Quarter 1993. (sheet 2 of 2)

\begin{tabular}{llll}
\hline We11 & Date & $\begin{array}{c}\text { Depth to } \\
\text { water }(\mathrm{ft})\end{array}$ & $\begin{array}{c}\text { Water level } \\
\text { elevation } \\
\text { above ms } 1 \mathrm{ft})\end{array}$ \\
\hline LLWMA-5 Wells Monitoring the Bottom of the Unconfined Aquifer \\
\hline $299-$ W6-3 & $9 / 09 / 93$ & 243.11 & 456.72 \\
$299-W 6-6$ & $9 / 09 / 93$ & 256.23 & $\begin{array}{l}453.77 \\
453.95 *\end{array}$ \\
\hline
\end{tabular}

Notes: 1. Water level elevations aro calculated by subtracting the measured depth to water from the surveyed elevation for the well.

2. Depth-to-water values are transcribed from field records.

3. Elevations marked with an ' $*$ ' were measured at the time of sampling.

4. To convert feet to meters multiply by 0.3048 . 
Table 15-12. Constituent List and Summary of Results for the Low-Level Waste Burial Grounds WMA 1 Data for Reporting Period July 1 through September 30, 1993. (sheet 1 of 3)

CONTAMINATION INDICATOR PARAMETERS

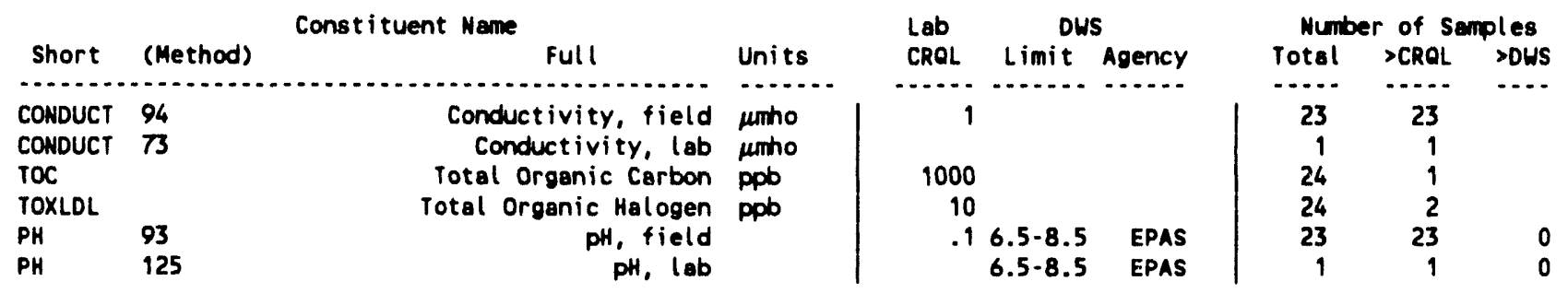

DRINKING WATER PARAMETERS

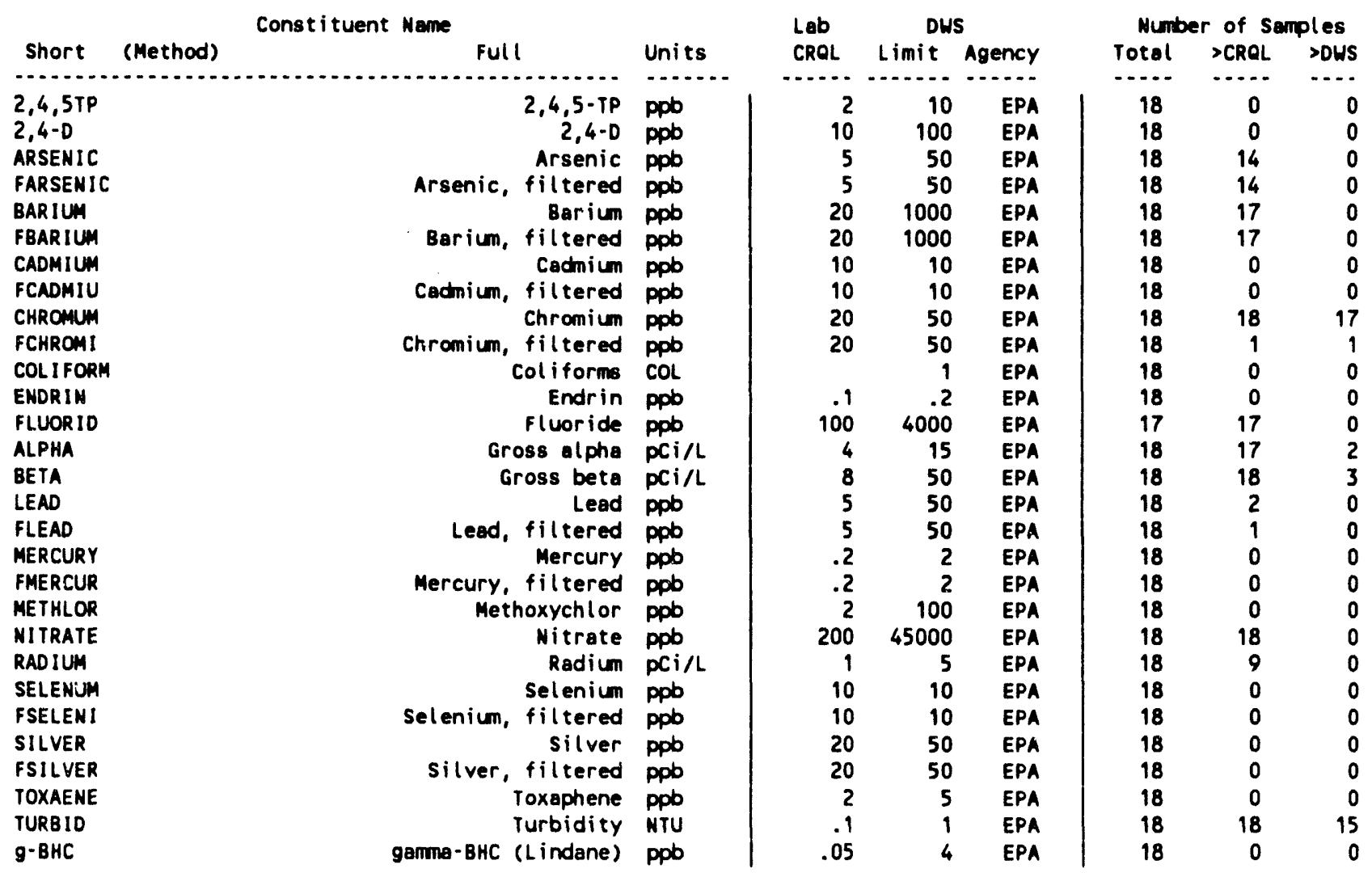

GROUNDWATER QUALITY PARAMETERS

\begin{tabular}{|c|c|c|c|c|c|c|c|c|c|}
\hline \multicolumn{4}{|c|}{ Const ituent Name } & Lab & \multicolumn{2}{|c|}{ DUS } & \multicolumn{3}{|c|}{ Number of Semples } \\
\hline Short & (Method) & Full & Units & CROL & Limit & Agency & Total & $>$ CROL & $>$ DUS \\
\hline$\cdots \ldots$ & 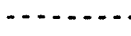 & $\ldots \ldots \ldots$ & $\cdots \cdots$ & $\ldots \ldots$ & $\ldots \ldots$ & $\ldots \ldots$ & $\cdots$ & - . & $\ldots$ \\
\hline $\begin{array}{l}\text { CHLOR ID } \\
\text { IRON }\end{array}$ & & $\begin{array}{r}\text { Chloride } \\
\text { Iron }\end{array}$ & $\begin{array}{l}\text { ppb } \\
\text { ppb }\end{array}$ & $\begin{array}{r}200 \\
20\end{array}$ & $\begin{array}{r}250000 \\
300\end{array}$ & $\begin{array}{l}\text { EPAS } \\
\text { EPAS }\end{array}$ & $\begin{array}{l}18 \\
18\end{array}$ & $\begin{array}{l}18 \\
18\end{array}$ & $\begin{array}{r}0 \\
17\end{array}$ \\
\hline FIRON & & Iron, filtered & ppb & 20 & 300 & EPAS & 18 & 13 & 0 \\
\hline MANGESE & & Manganese & ppb & 10 & 50 & EPAS & 18 & 10 & 0 \\
\hline FMANGAN & & Manganese, filtered & ppb & 10 & 50 & EPAS & 18 & 0 & 0 \\
\hline
\end{tabular}


Table 15-12. Constituent List and Summary of Results for the Low-Level Waste Burial Grounds WMA 1 Data for Reporting Period July 1 through September 30, 1993. (sheet 2 of 3)

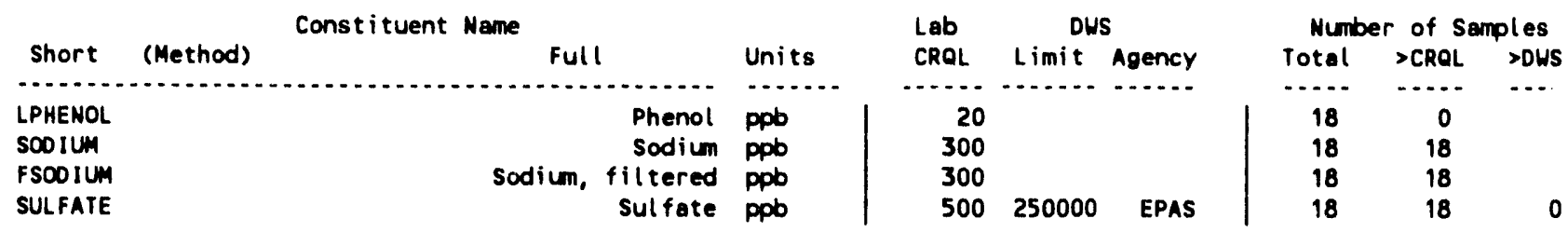

SITE SPECIFIC AND OTHER CONSTITUENTS

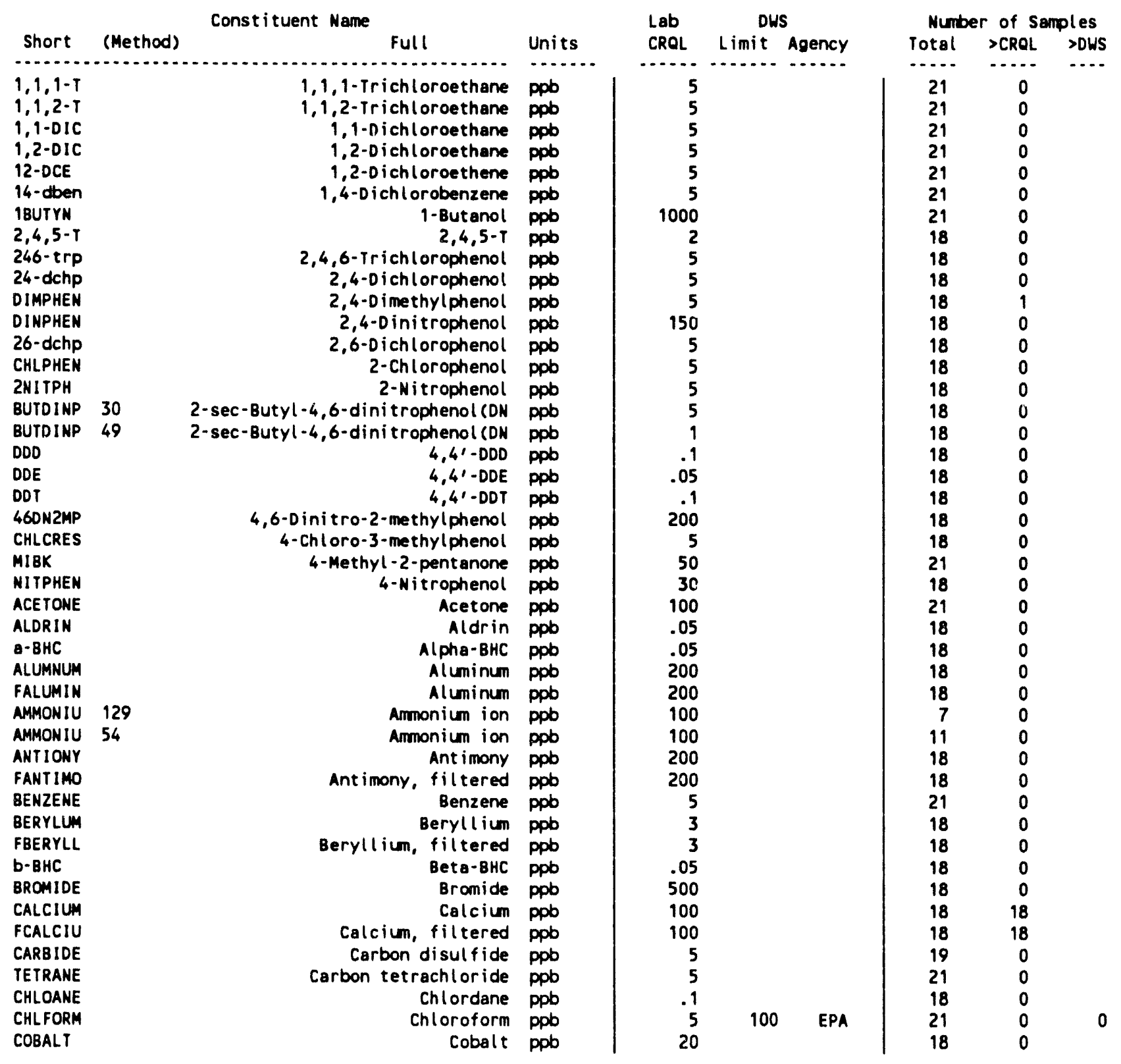


Table 15-12. Constituent List and Summary of Results for the Low-Level Waste Burial Grounds WMA 1 Data for Reporting

Period July 1 through September 30, 1993. (sheet 3 of 3)

\begin{tabular}{|c|c|c|c|c|c|c|c|c|c|}
\hline \multirow[b]{2}{*}{ Short } & \multirow[b]{2}{*}{ (Method) } & \multirow[t]{2}{*}{ Constituent Mame } & \multirow[b]{2}{*}{ Units } & \multirow{2}{*}{$\begin{array}{l}\text { Lab } \\
\text { CROL }\end{array}$} & \multicolumn{2}{|c|}{ DUS } & \multicolumn{3}{|c|}{ Number of Samples } \\
\hline & & & & & Limit & Agency & Total & $>$ CROL & $>$ DWS \\
\hline$\cdots \cdots \cdots$ & $\cdots+\ldots . .$. & . & $\ldots \ldots$ & $\ldots \ldots$ & $\ldots \ldots$ & $\ldots . .$. & $\ldots$. & $\ldots$ & $\ldots$ \\
\hline FCOBALT & & Cobalt, filtered & ppo & 20 & & & 18 & 0 & \\
\hline COPPER & & Copper & ppo & 20 & 1000 & EPAS & 18 & 1 & 0 \\
\hline FCOPPER & & Copper, filtered & ppb & 20 & 1000 & EPAS & 18 & 0 & 0 \\
\hline CRESOLS & & Cresols (methylphenols) & ppo & 10 & & & 18 & 0 & \\
\hline CYANIDE & 358 & Cyanide & ppb & 20 & & & 14 & 0 & \\
\hline CYANIDE & 56 & Cyanide & ppb & 20 & & & 4 & 0 & \\
\hline d-BHC & & Delta-BHC & ppb & .1 & & & 18 & 0 & \\
\hline DIELRIN & & Dieldrin & ppb & .05 & & & 18 & 0 & \\
\hline ENDO1 & & Endosulfan 1 & ppb & .1 & & & 18 & 0 & \\
\hline ENDOZ & & Endosulfan II & ppo & .05 & & & 18 & 0 & \\
\hline ENDSFAN & & Endosulfan sulfate & ppb & .5 & & & 18 & 0 & \\
\hline ENDRALD & & Endrin Aldehyde & ppb & .2 & & & 18 & 0 & \\
\hline ETHCYAN & & Ethyl cyanide & ppb & 10 & & & 19 & 0 & \\
\hline HEPTLOR & & Heptechlor & ppb & .05 & & & 18 & 0 & \\
\hline HEPTIDE & & Heptachlor epoxide & ppb & 1 & & & 18 & 0 & \\
\hline LHYDRAZ & & Hydrezine & ppo & 30 & & & 1 & 0 & \\
\hline MAGNES & & Magnesium & ppo & 100 & & & 18 & 18 & \\
\hline FMAGNES & & Megnesium, filtered & ppb & 100 & & & 18 & 18 & \\
\hline METHONE & & Methyl ethyl ketone & ppb & 100 & & & 21 & 0 & \\
\hline METHYCH & & Methylene chloride & ppo & 5 & & & 21 & 0 & \\
\hline NICKEL & & Nickel & ppb & 30 & & & 18 & 16 & \\
\hline FNICKEL & & Nickel, filtered & ppb & 30 & & & 18 & 1 & \\
\hline NITRITE & & Nitrite & ppb & 200 & & & 18 & 0 & \\
\hline PENTCHP & & Pent achlorophenol & ppto & 100 & & & 18 & 0 & \\
\hline PHOSPHA & & Phosphate & ppb & 400 & & & 18 & 0 & \\
\hline POTASUM & & Potassium & ppb & 300 & & & 18 & 18 & \\
\hline FPOTASS & & Potassium, filtered & ppb & 300 & & & 18 & 18 & \\
\hline PERCENE & & Tetrachloroethene & ppb & 5 & & & 21 & 0 & \\
\hline TETPHNL & & Tetrachlorophenols & ppo & 10 & & & 18 & 0 & \\
\hline TAF & & Tetrahydrofuran & ppb & 10 & & & 21 & 2 & \\
\hline TIN & & Tin & ppb & 100 & & & 18 & 0 & \\
\hline FTIN & & Tin, filtered & ppb & 100 & & & 18 & 0 & \\
\hline TOLUENE & & Toluene & ppb & 5 & & & 21 & i & \\
\hline TDS & & Total Dissolved Solids & ppm & 10 & & & 1 & 1 & \\
\hline TRICENE & & Trichloroethene & ppob & 5 & & & 21 & 0 & \\
\hline TRIPHNL & & Trichlorophenols & ppb & 5 & & & 18 & 0 & \\
\hline TRITIUM & & Tritium & $\mathrm{pCi} / \mathrm{L}$ & 500 & 20000 & EPA & 18 & 18 & 6 \\
\hline URANIUM & & Uranium & ppo & .5 & & & 18 & 18 & \\
\hline VAKADUM & & Vanadium & ppo & 30 & & & 18 & 0 & \\
\hline FVANAD I & & Vanedium, filtered & ppb & 30 & & & 18 & 0 & \\
\hline VINYIDE & & vinyl chloride & ppb & 10 & 2 & EPA & 21 & 0 & 0 * \\
\hline XYLENE & & $x y l e n e s(\operatorname{tota}()$ & ppb & 5 & & & 21 & 0 & \\
\hline ZINC & & 2 inc & ppo & 10 & & & 18 & 3 & \\
\hline FZINC & & Zinc, filtered & ppb & 10 & & & 18 & 2 & \\
\hline
\end{tabular}

For explanation of this table, see Section 1.4 of report. 
Table 15-13. Constituents with at Least One Value Above the CRQL for the Low-Level Waste Burial Grounds WMA 1 Data for Reporting Period July 1 through September 30, 1993. (sheet 1 of 8)

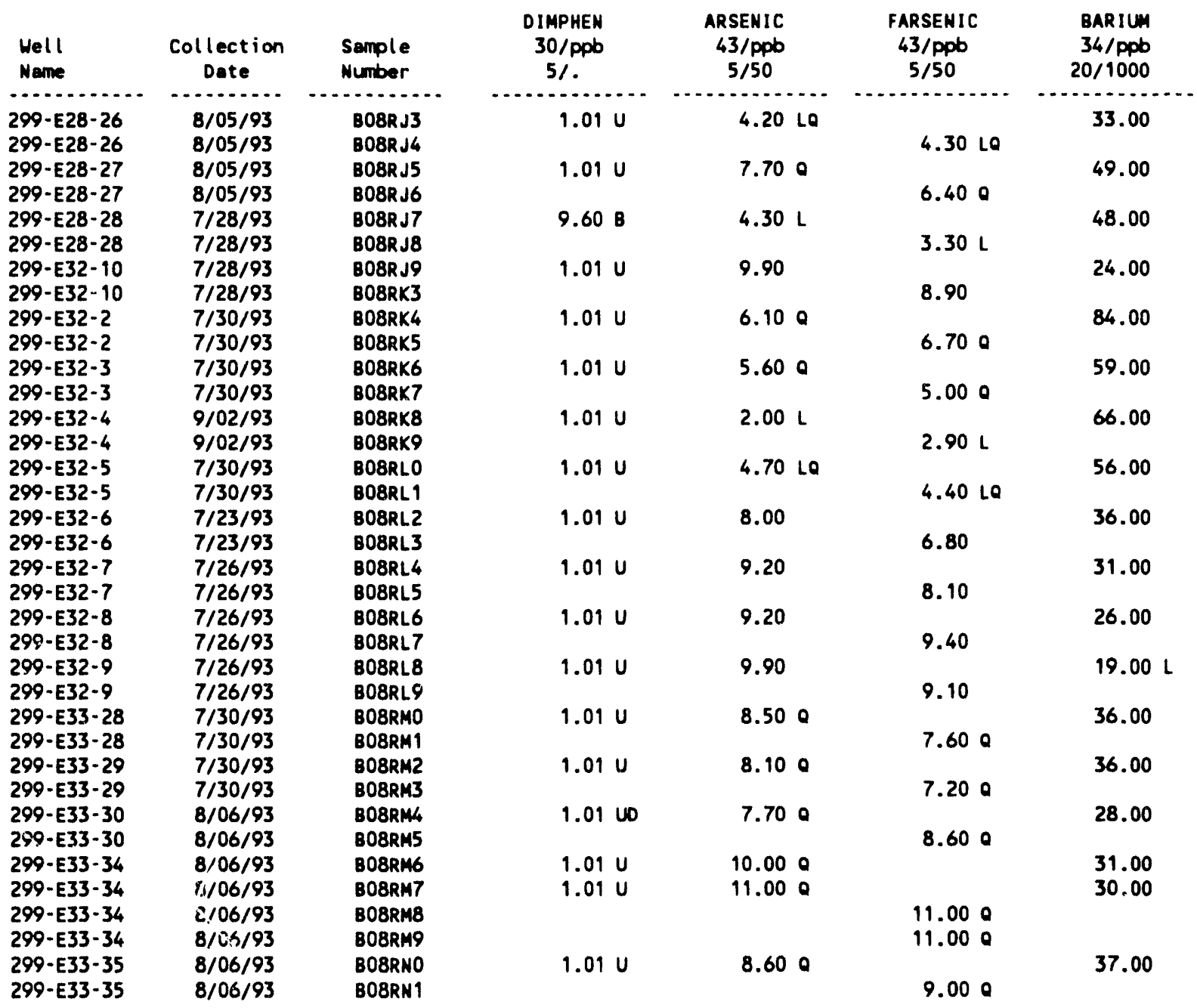

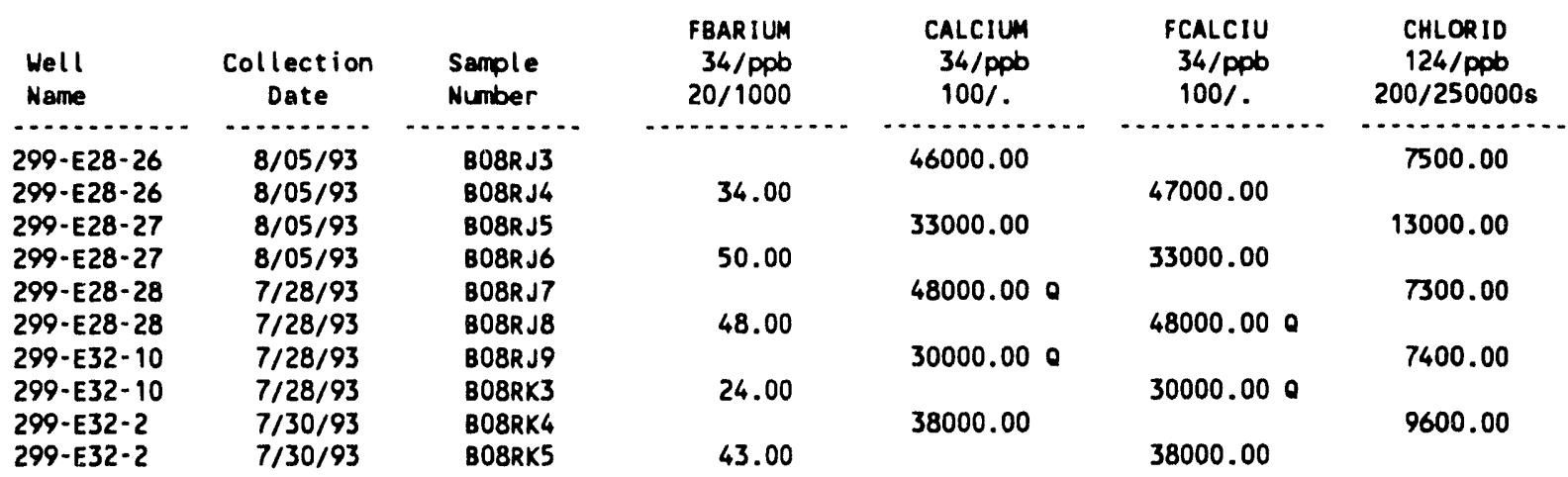


Table 15-13. Constituents with at Least One Value Above the CRQL for the Low-Level Waste Burial Grounds WMA 1 Data for Reporting Period July 1 through September 30, 1993. (sheet 2 of 8)

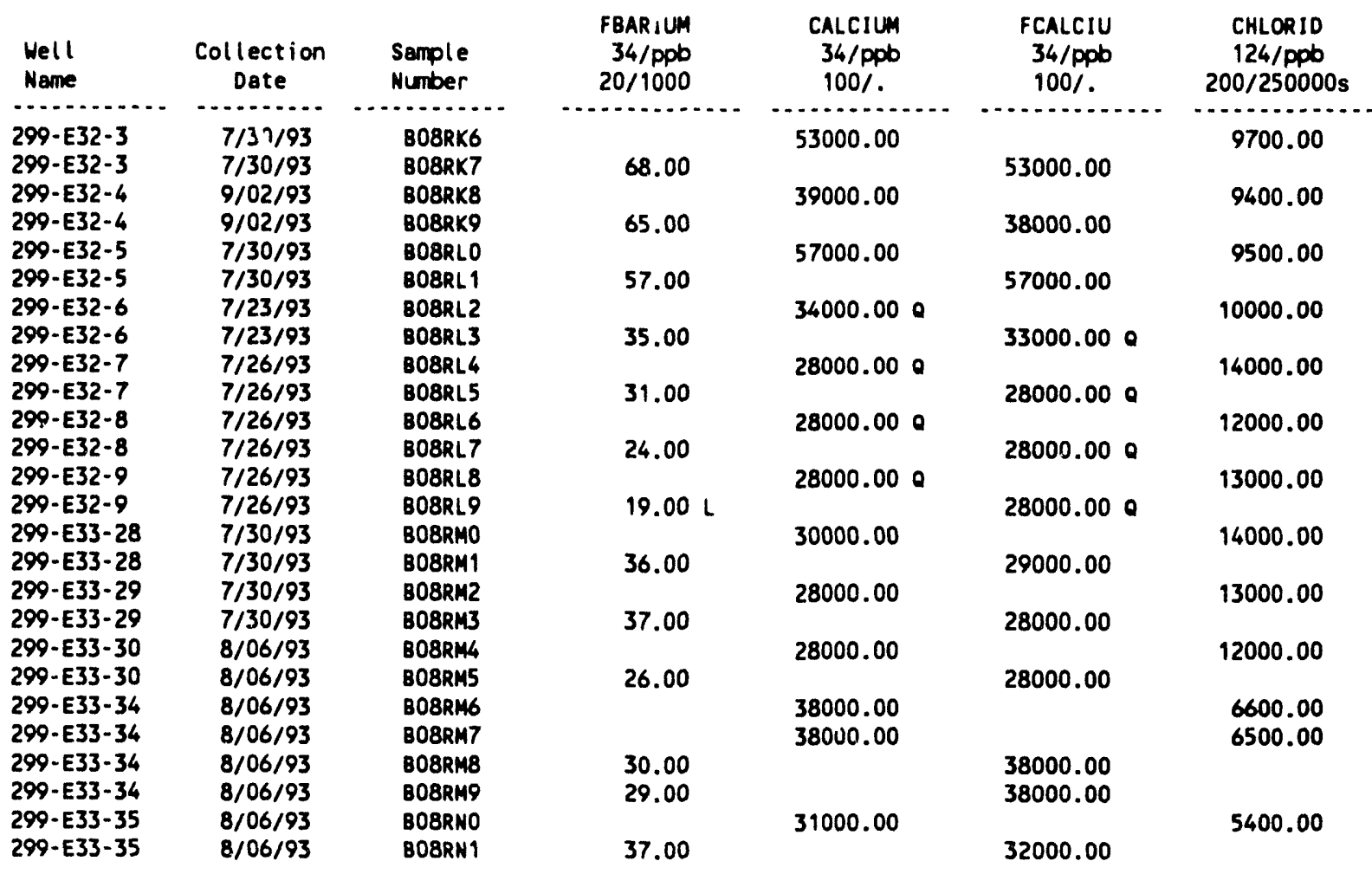

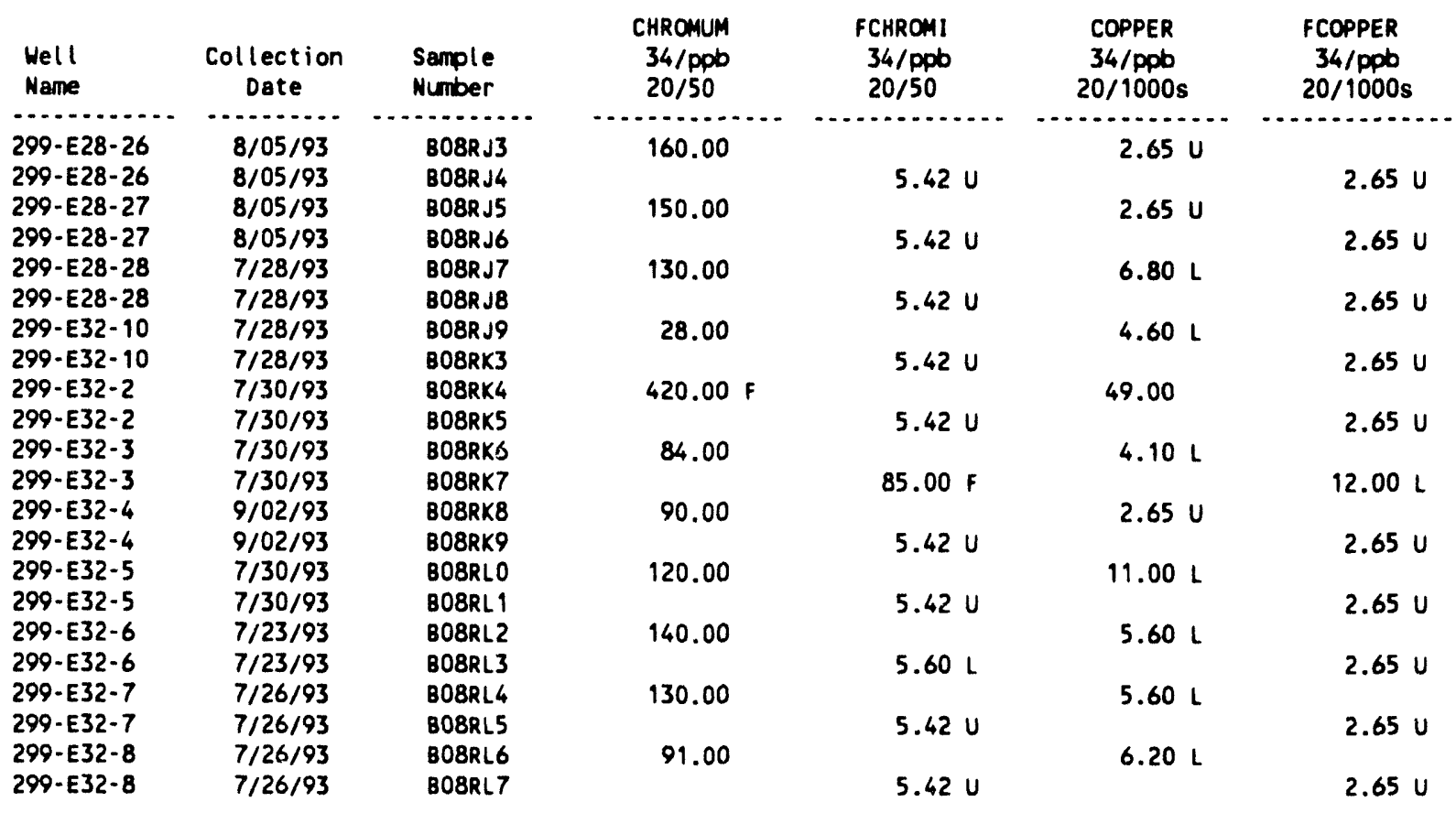


Table 15-13. Constituents with at Least One Value Above the CRQL for the Low-Level Waste Burial Grounds WMA 1 Data for Reporting Period July 1 through September 30, 1993. (sheet 3 of 8)

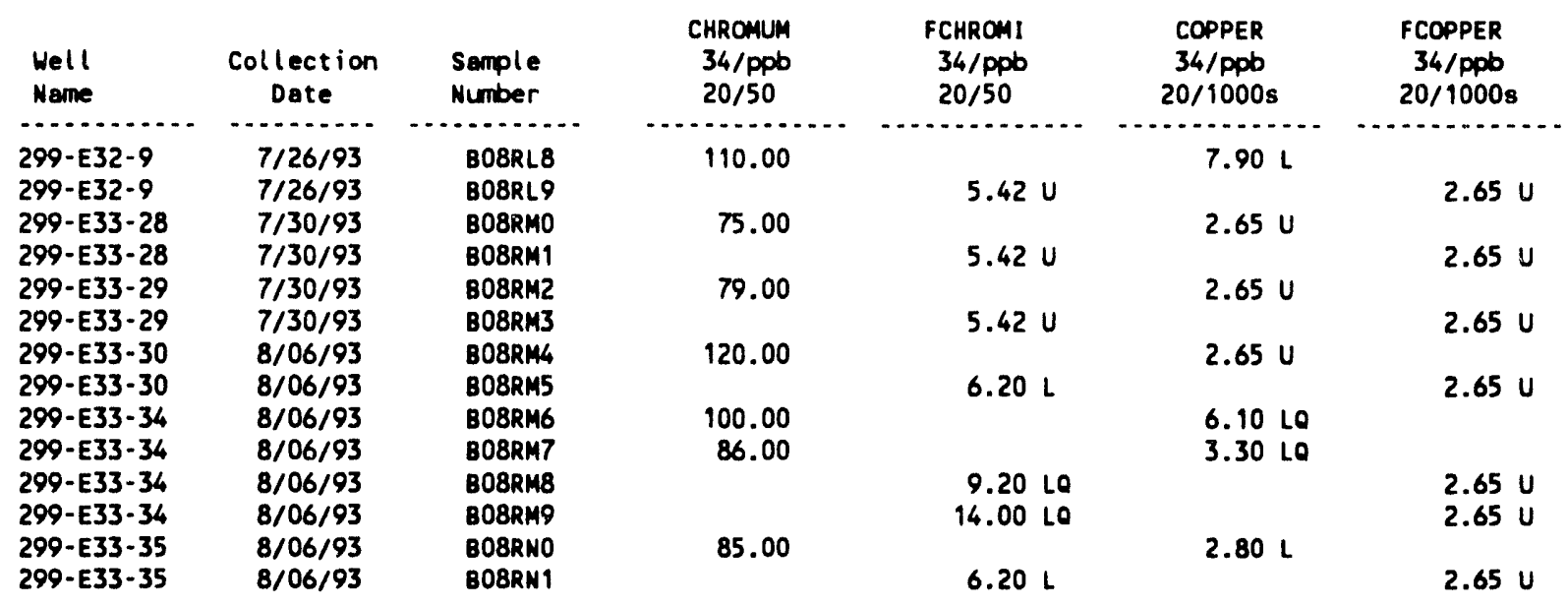

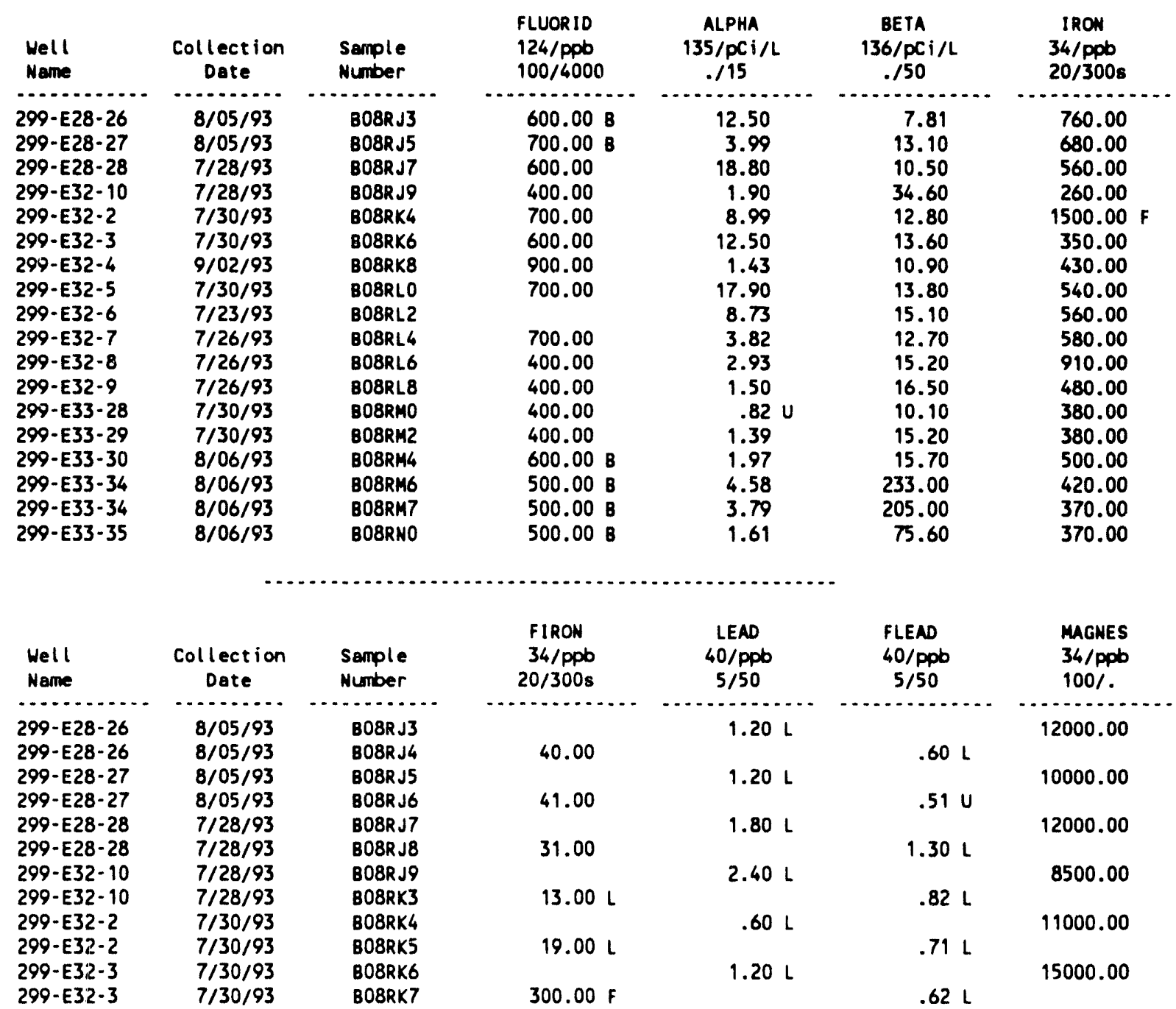


Table 15-13. Constituents with at Least One Value Above the CRQL for the Low-Level Waste Burial Grounds WMA 1 Data for Reporting Period July 1 through September 30, 1993. (sheet 4 of 3 )

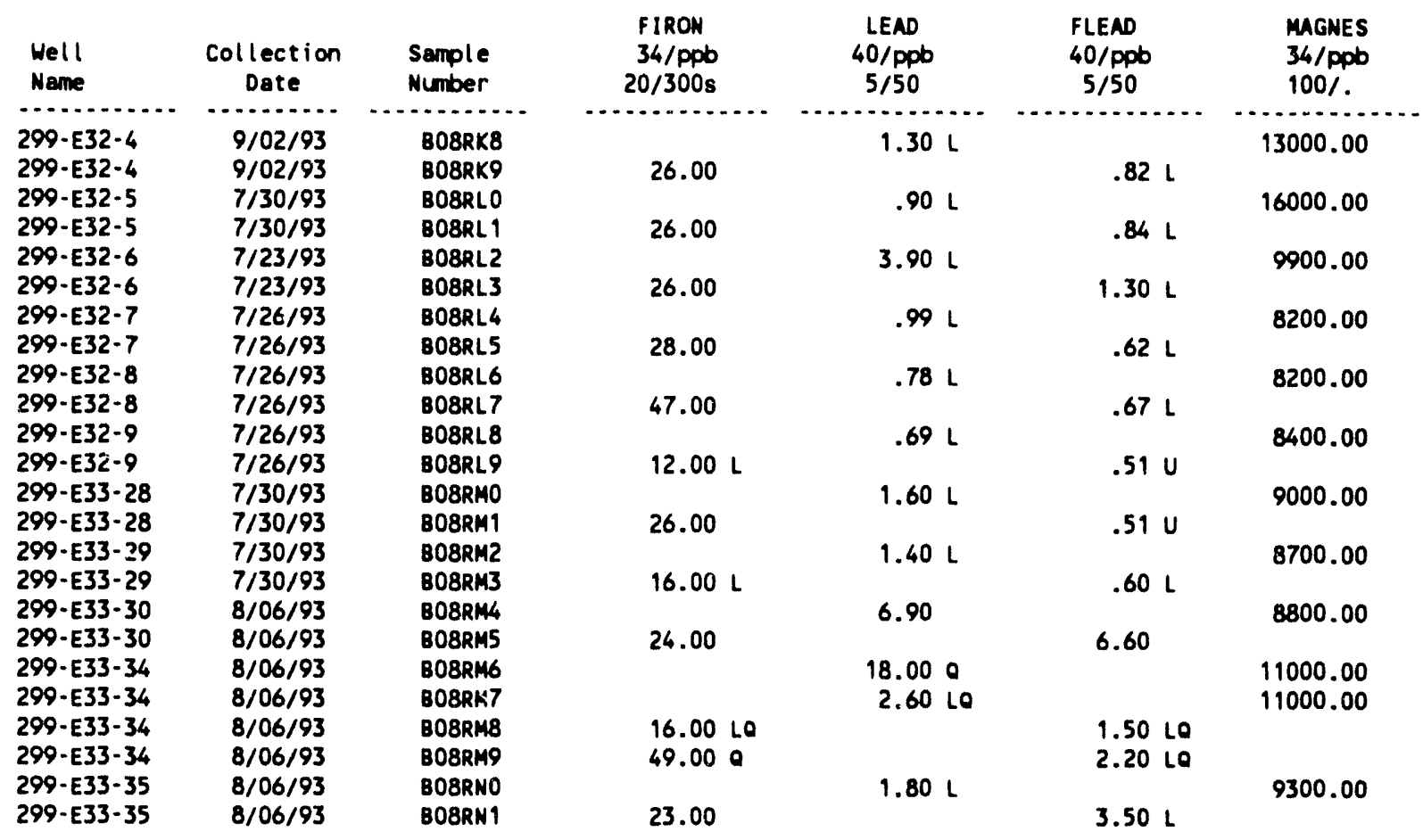

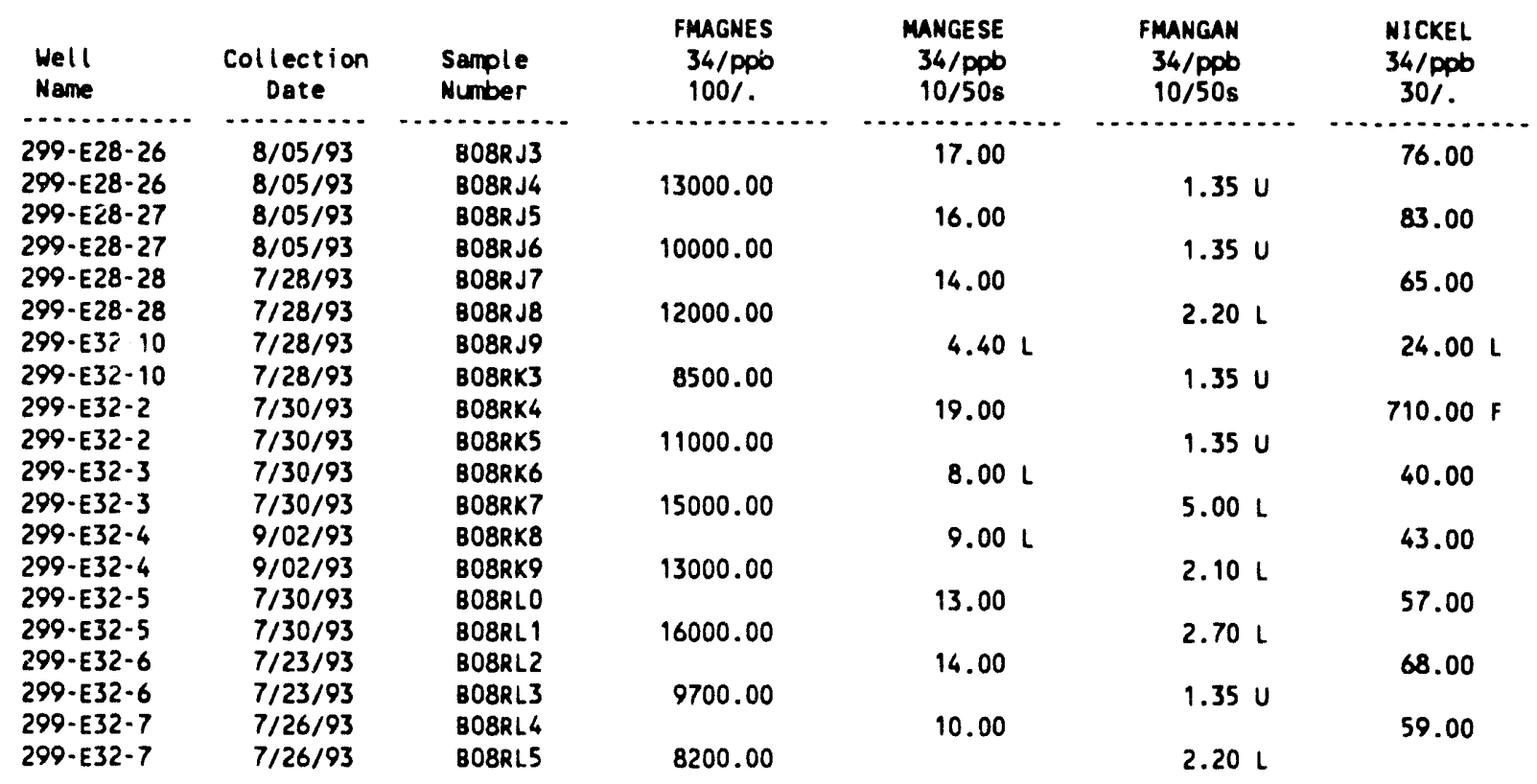


Table 15-13. Constituents with at Least One Value Above the CRQL for the Low-Level Waste Burial Grounds WMA 1 Data for Reporting Period July 1 through September 30, 1993. (sheet 5 of 8)

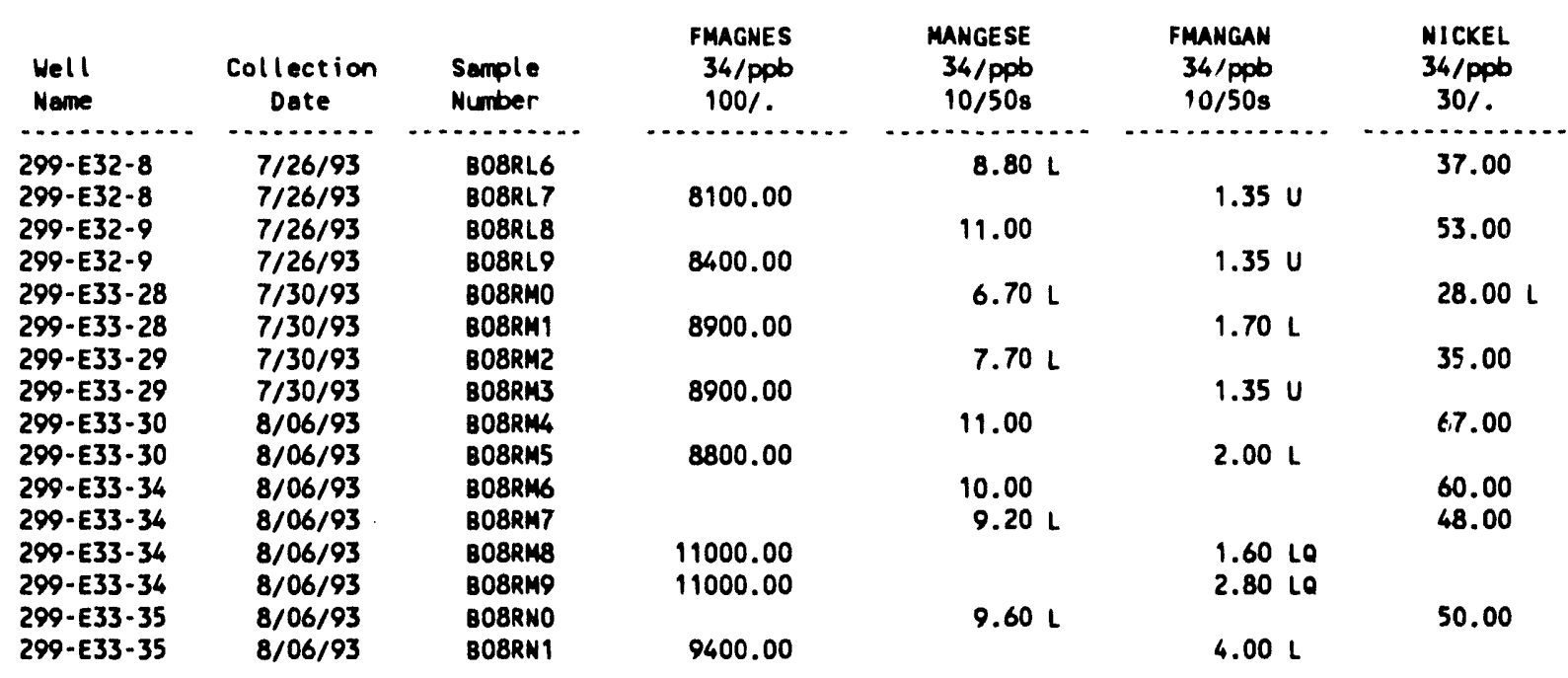

\begin{tabular}{|c|c|c|c|c|c|c|}
\hline $\begin{array}{l}\text { Well } \\
\text { Name }\end{array}$ & $\begin{array}{c}\text { Collection } \\
\text { Date }\end{array}$ & $\begin{array}{l}\text { Sample } \\
\text { Number }\end{array}$ & $\begin{array}{l}\text { FNICKEL } \\
34 / \text { PpD } \\
30 \%\end{array}$ & $\begin{array}{c}\text { NITRATE } \\
124 / \mathrm{ppb} \\
200 / 45000\end{array}$ & $\begin{array}{l}\text { POTASUM } \\
34 / \mathrm{ppb} \\
1000 \%\end{array}$ & $\begin{array}{c}\text { FPOTASS } \\
34 / \mathrm{ppb} \\
1000 \%\end{array}$ \\
\hline 299-E28-26 & $8 / 05 / 93$ & BO8RJ3 & & 33000.00 & 4600.00 & \\
\hline 299-E28-26 & $8 / 05 / 93$ & B08R J4 & $17.90 \mathrm{U}$ & & & 5100.00 \\
\hline 299-E28-27 & $8 / 05 / 93$ & B08R.J5 & & 18000.00 & 5400.00 & \\
\hline $\begin{array}{l}299-E 28-27 \\
299-E 28-28\end{array}$ & $\begin{array}{l}8 / 05 / 93 \\
7 / 28 / 93\end{array}$ & $\begin{array}{l}\text { BO8R.J6 } \\
\text { B08RJ7 }\end{array}$ & $17.90 \mathrm{U}$ & 36000.00 & 4800.000 & 5800.00 \\
\hline 299-E28-28 & $7 / 28 / 93$ & BO8R J8 & $17.90 \mathrm{U}$ & & & $6200.00 a$ \\
\hline 299-E32-10 & $7 / 28 / 93$ & BO8RJ9 & & 9500.00 & 5100.000 & \\
\hline $299-E 32-10$ & $7 / 28 / 93$ & B08RK3 & $17.90 \mathrm{U}$ & & & 4500.000 \\
\hline $299-E 32-2$ & $7 / 30 / 93$ & B08RK4 & & 24000.00 & 7100.00 & \\
\hline 299-E32-2 & $7 / 30 / 93$ & BO8RK5 & $17.90 \mathrm{U}$ & & & 6100.00 \\
\hline 299-E32-3 & $7 / 30 / 93$ & B08RK6 & & 44000.00 & 6000.00 & \\
\hline $299-E 32-3$ & $7 / 30 / 93$ & B08RK7 & $160.00 \mathrm{~F}$ & & & 5400.00 \\
\hline $299-E 32-4$ & $9 / 02 / 93$ & B08RK8 & & $25000.00 \mathrm{DH}$ & 6000.00 & \\
\hline 299-E32-4 & 9/02/93 & BO8RK9 & $17.90 \mathrm{U}$ & & & 6100.00 \\
\hline $299-E 32-5$ & $7 / 30 / 93$ & BOBRLO & & 42000.00 & 5000.00 & \\
\hline 299-E32-5 & $7 / 30 / 93$ & B08RLI & $17.90 \mathrm{U}$ & & & 5500.00 \\
\hline 299-E32-6 & $7 / 23 / 93$ & B08RL2 & & 18000.00 & 6500.000 & \\
\hline 299-E32-6 & $7 / 23 / 93$ & B08RL3 & $17.90 \mathrm{U}$ & & & 5700.000 \\
\hline $299-E 32-7$ & $7 / 26 / 93$ & B08RL4 & & 8900.00 & 5300.000 & \\
\hline $299-E 32-7$ & $7 / 26 / 93$ & B08RL5 & $17.90 \mathrm{U}$ & & & 5200.000 \\
\hline 299-E32-8 & $7 / 26 / 93$ & B08RL6 & & 5100.00 & $4000.00 \Omega$ & \\
\hline $299-E 32-8$ & $7 / 26 / 93$ & B08RL7 7 & $17.90 \mathrm{U}$ & & & $5600.00 Q$ \\
\hline 299-E32-9 & $7 / 26 / 93$ & B08RL8 & & 4500.00 & 4100.000 & \\
\hline 299-E32-9 & $7 / 26 / 93$ & B08RL9 & $17.90 \mathrm{U}$ & & & 5000.000 \\
\hline 299-E33- 28 & $7 / 30 / 93$ & BO8RMO & & 4600.00 & 5800.00 & \\
\hline 299-E33-28 & $7 / 30 / 93$ & B08RM1 & $17.90 \mathrm{U}$ & & & 6100.00 \\
\hline $299-E 33-29$ & $7 / 30 / 93$ & BO8RM2 & & 8200.00 & 6000.00 & \\
\hline $299-E 33-29$ & $7 / 30 / 93$ & B08RM3 & $u$ & & & 6200.00 \\
\hline
\end{tabular}


Table 15-13. Constituents with at Least One Value Above the CRQL for the Low-Level Waste Burial Grounds WMA 1 Data for Reporting Period July 1 through September 30, 1993. (sheet 6 of 8)

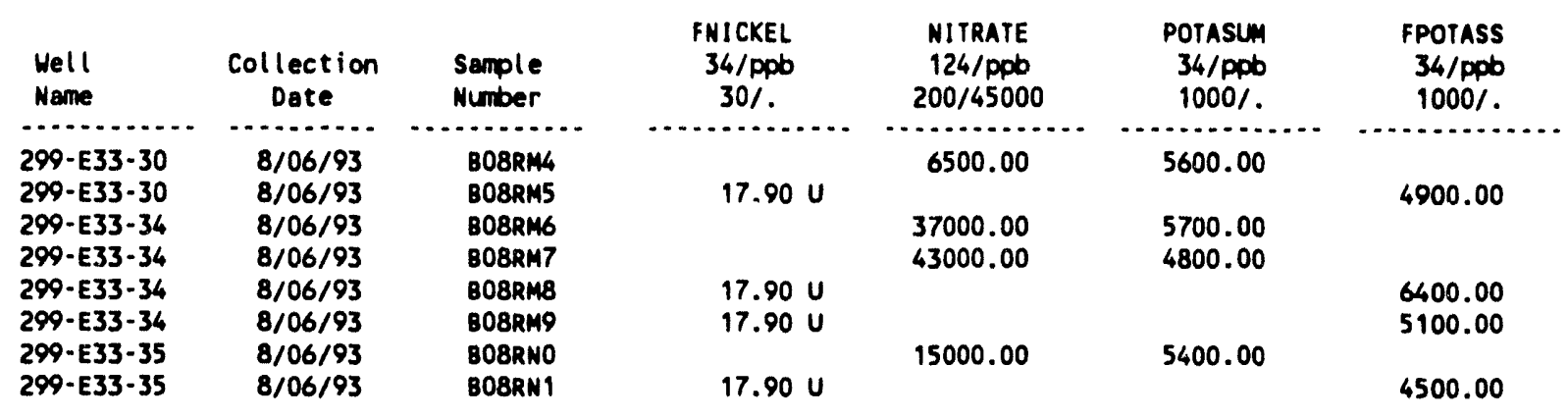

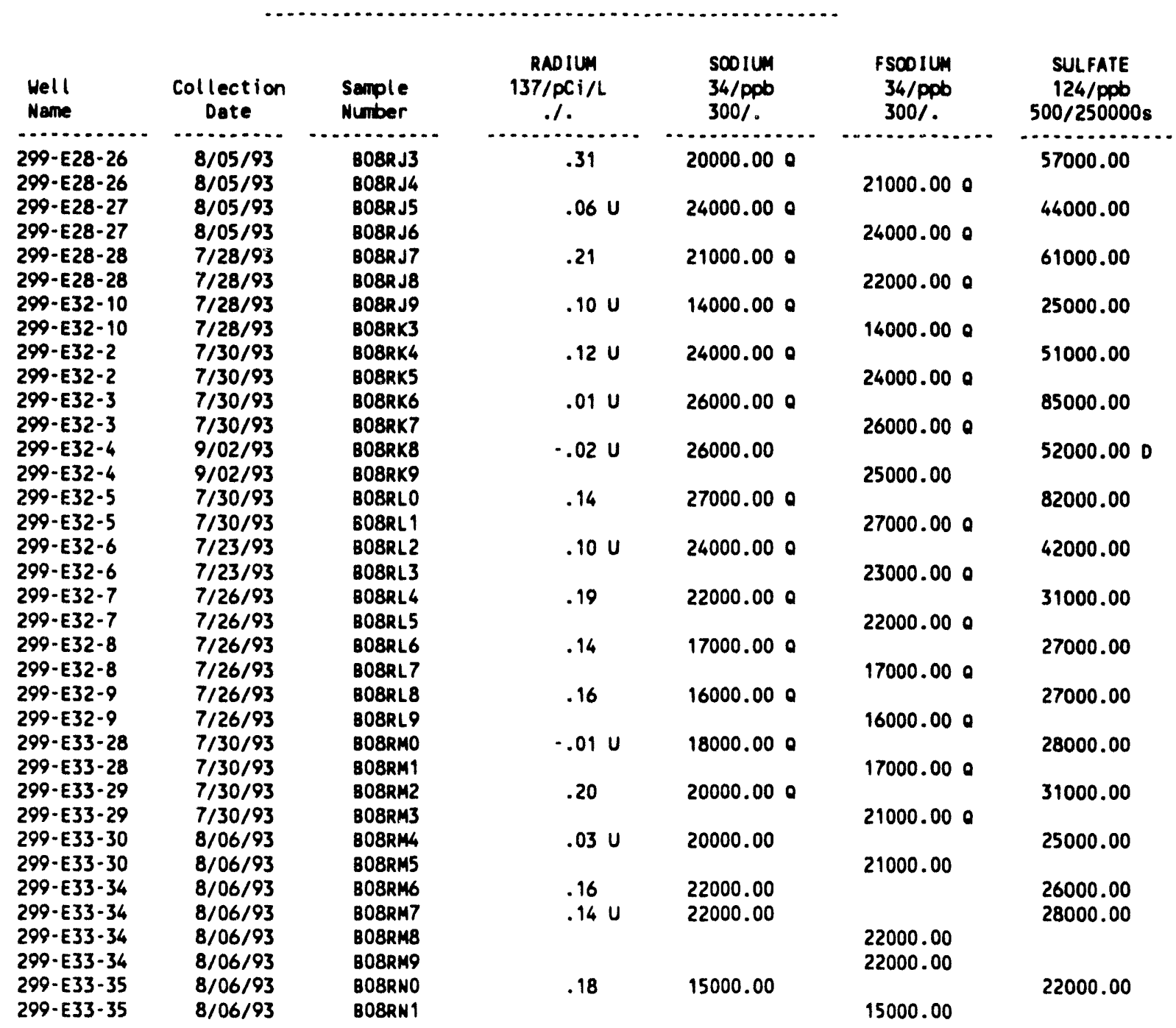


Table 15-13. Constituents with at Least One Value Above the CRQL for the Low-Level Waste Burial Grounds WMA 1 Data for Reporting Period July 1 through September 30, 1993. (sheet 7 of 8)

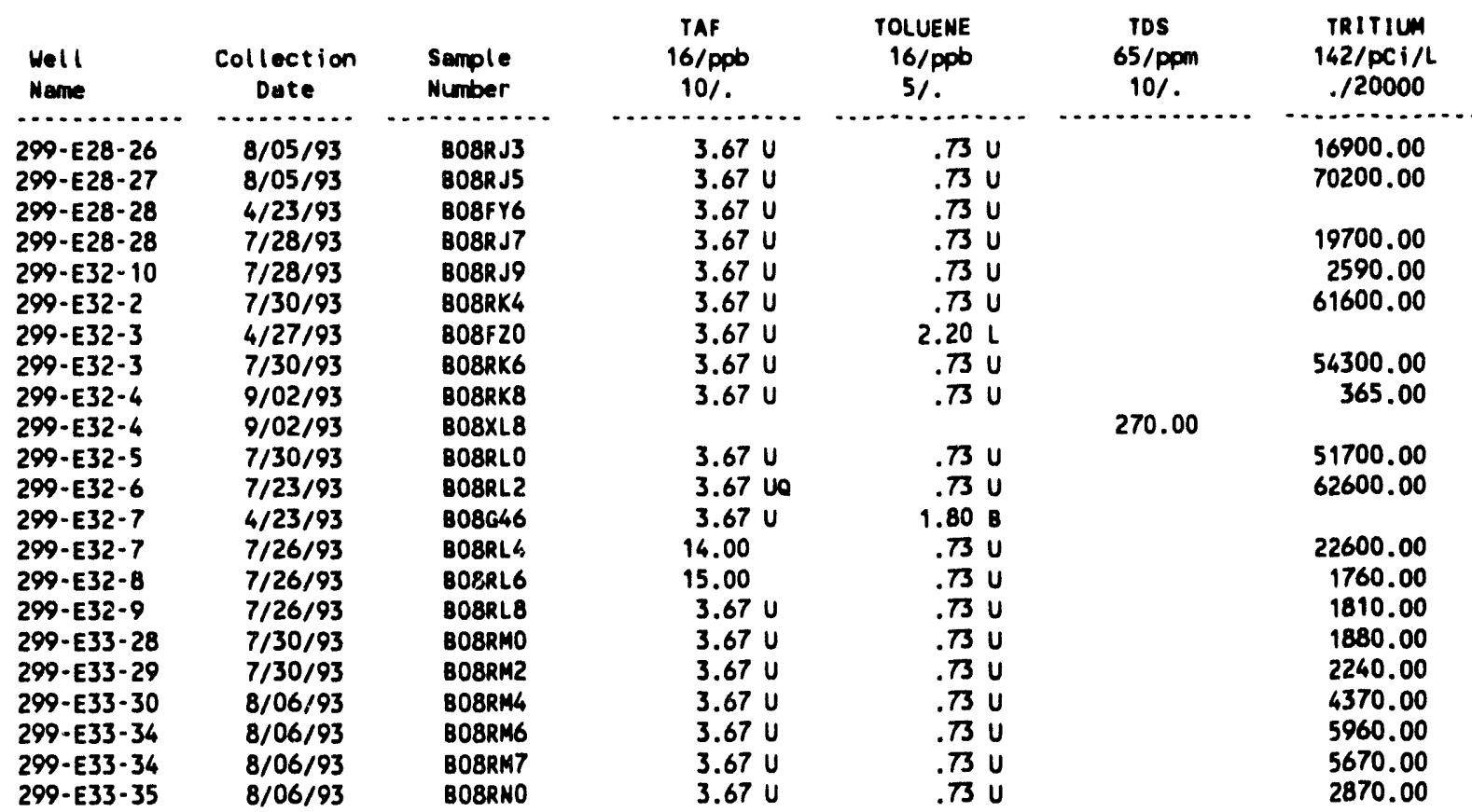

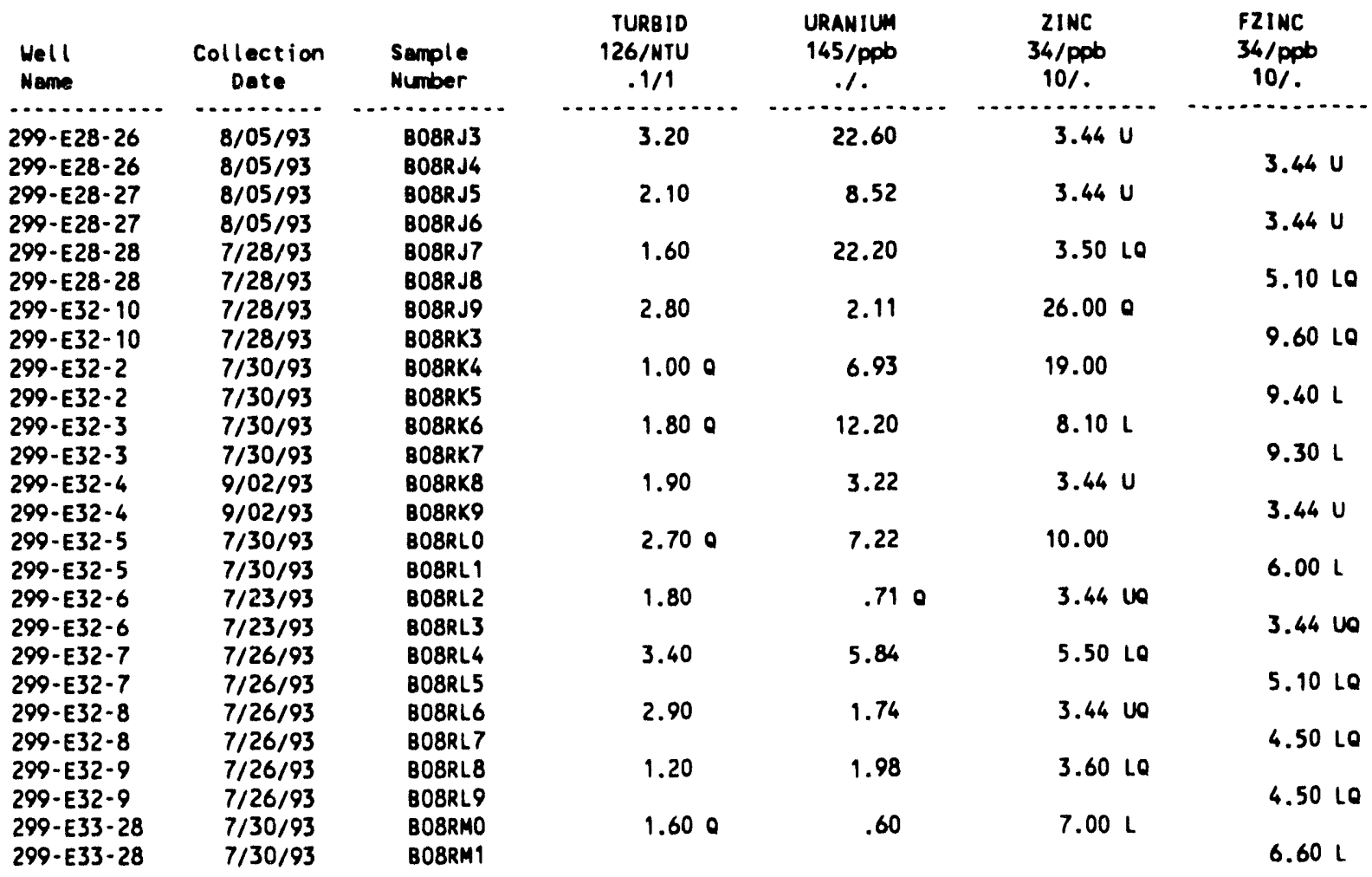


Table 15-13. Constituents with at Least One Value Above the CRQL for the Low-Level Waste Burial Grounds WMA 1 Data for Reporting Period July 1 through September 30, 1993. (sheet 8 of 8)

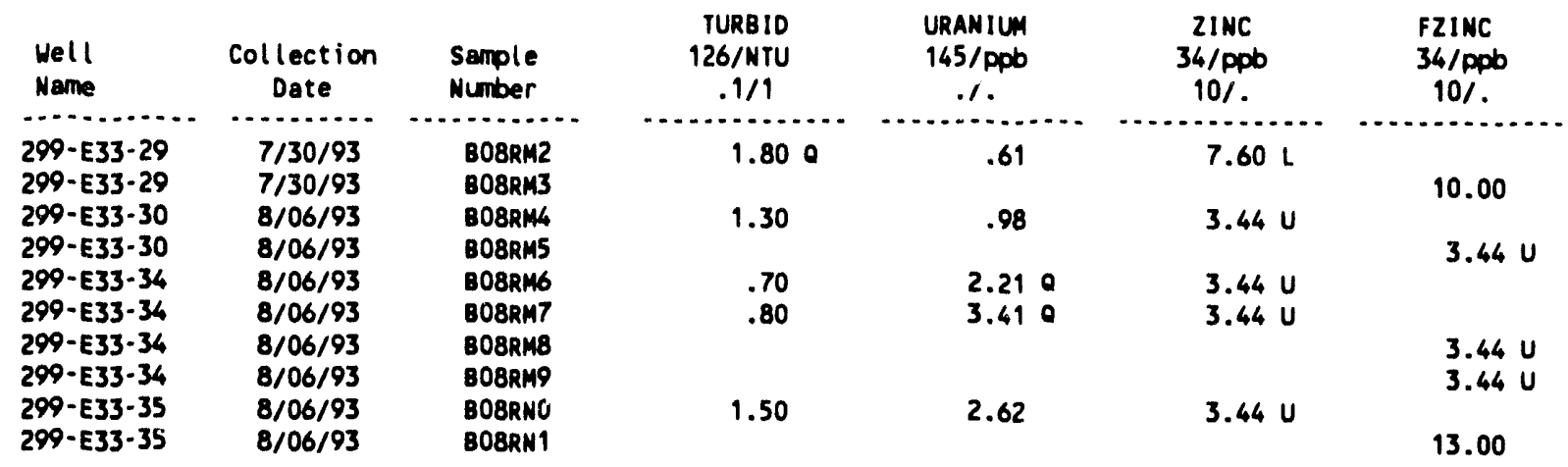

For explanation of this table, see Section 1.4 of report. 
Table 15-14. Contamination Indicator Parameters for the Low-Level Waste Burial Grounds WMA 1 Data for Reporting Period July 1

through September 30, 1993.

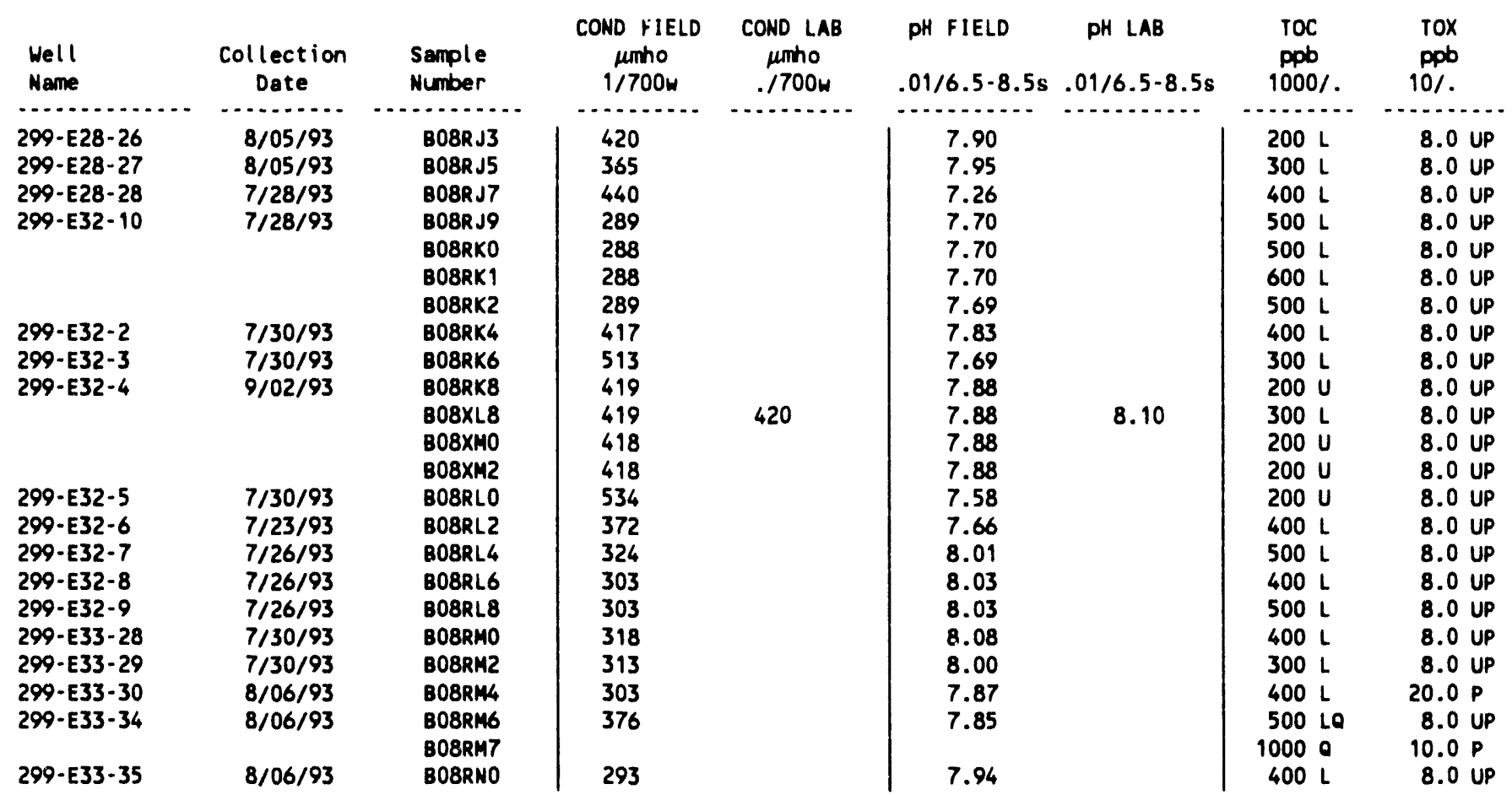

For explanation of this table, see section 1.4 of report. 
Table 15-15. Constituent List and Summary of Results for the Low-Level Waste Burial Grounds WMA 2 Data for Reporting Period July 1 through September 30,1993 . (sheet 1 of 3 )

CONTAMINATION INDICATOR PARAMETERS

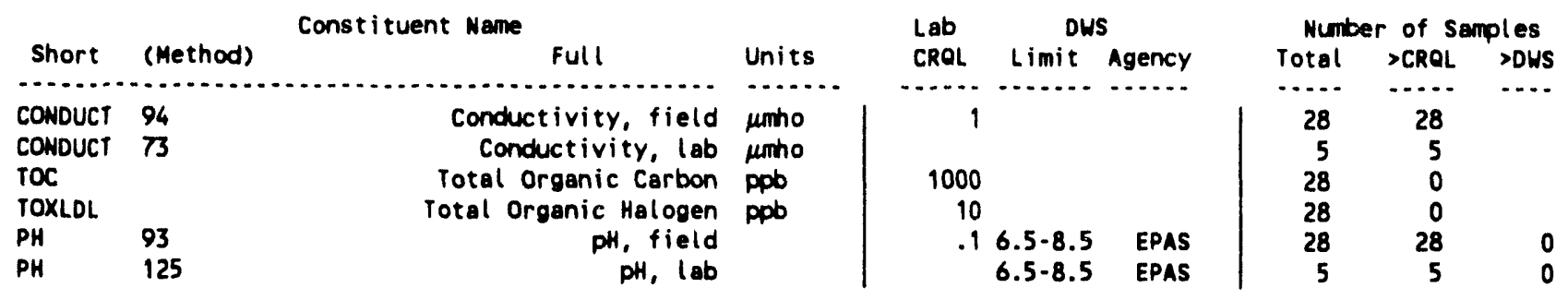

DRINKING WATER PARAMETERS

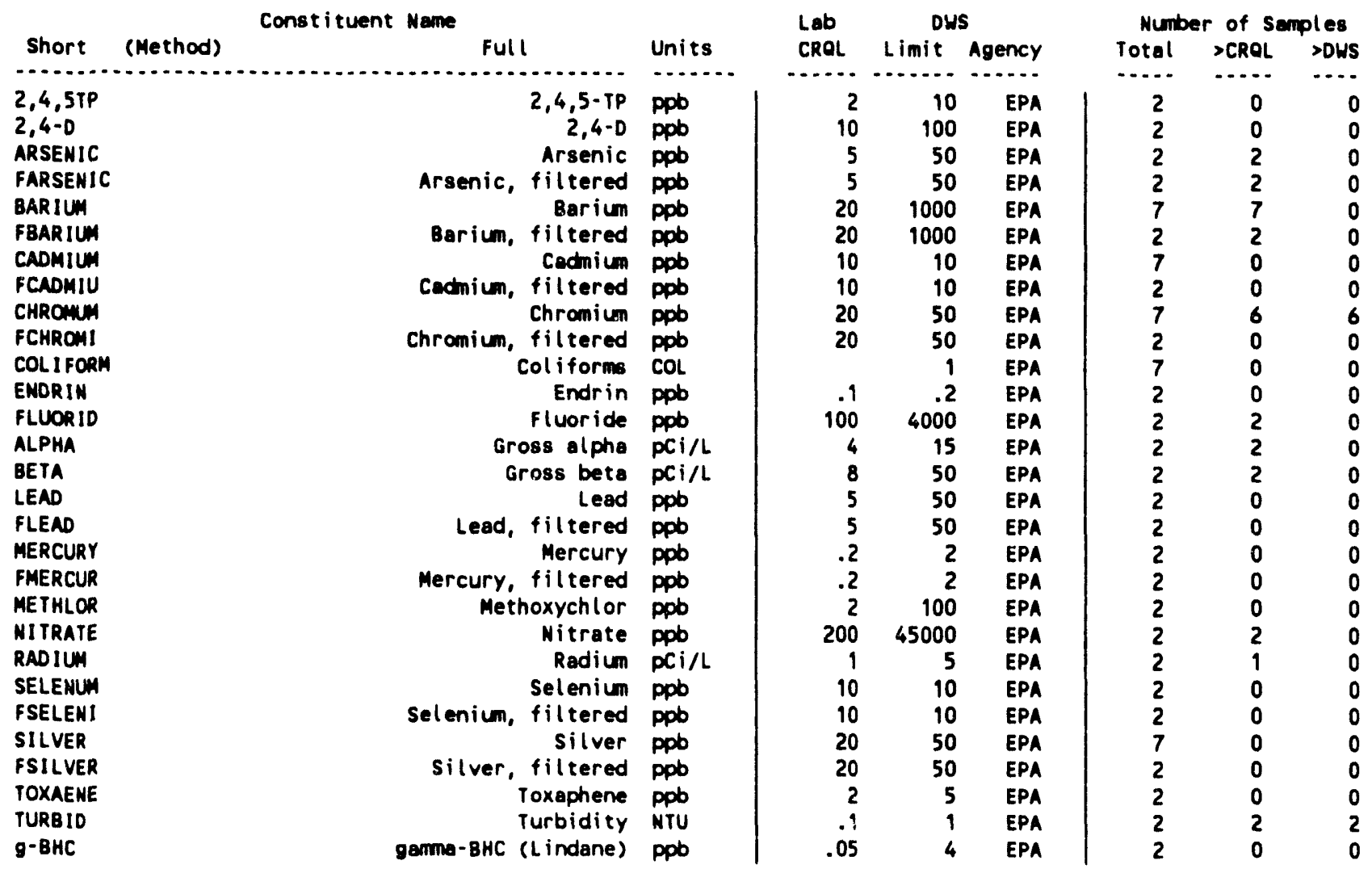

GROUNDWATER QUALITY PARAMETERS

\begin{tabular}{|c|c|c|c|c|c|c|c|c|c|}
\hline \multicolumn{4}{|c|}{ Constituent Name } & \multicolumn{3}{|c|}{ DWS } & \multicolumn{3}{|c|}{ Number of Samples } \\
\hline short & (Method) & Full & Units & CROL & Limit & Agency & Total & $>C R Q L$ & $>$ DUS \\
\hline$\ldots \ldots$ & $\ldots \ldots . . . .$. & $\ldots \ldots$ & $\ldots \ldots$ & $\ldots . .$. & $\ldots . .$. & $\ldots .$. & $\cdots$ & $\ldots$. & $\ldots$ \\
\hline $\begin{array}{l}\text { CHLOR ID } \\
\text { IRON }\end{array}$ & & $\begin{array}{r}\text { Chloride } \\
\text { Iron }\end{array}$ & $\begin{array}{l}\text { ppb } \\
p p b\end{array}$ & $\begin{array}{r}200 \\
20\end{array}$ & $\begin{array}{r}250000 \\
300\end{array}$ & $\begin{array}{l}\text { EPAS } \\
\text { EPAS }\end{array}$ & $\begin{array}{l}2 \\
7\end{array}$ & $\begin{array}{l}2 \\
7\end{array}$ & $\begin{array}{l}0 \\
6\end{array}$ \\
\hline $\begin{array}{l}\text { FIRON } \\
\text { MANGESE }\end{array}$ & & $\begin{array}{l}\text { Iron, filtered } \\
\text { Manganese }\end{array}$ & $\begin{array}{l}\text { ppb } \\
\text { ppb }\end{array}$ & $\begin{array}{l}20 \\
10\end{array}$ & $\begin{array}{r}300 \\
50\end{array}$ & $\begin{array}{l}\text { EPAS } \\
\text { EPAS }\end{array}$ & $\begin{array}{l}2 \\
7\end{array}$ & $\begin{array}{l}2 \\
5\end{array}$ & $\begin{array}{l}0 \\
0\end{array}$ \\
\hline FMANGAN & & Manganese, filtered & ppb & 10 & 50 & EPAS & 2 & 0 & 0 \\
\hline
\end{tabular}


Table 15-15. Constituent List and Summary of Results for the Low-Level Waste Burial Grounds WMA 2 Data for Reporting Period July 1 through September 30, 1993. (sheet 2 of 3)

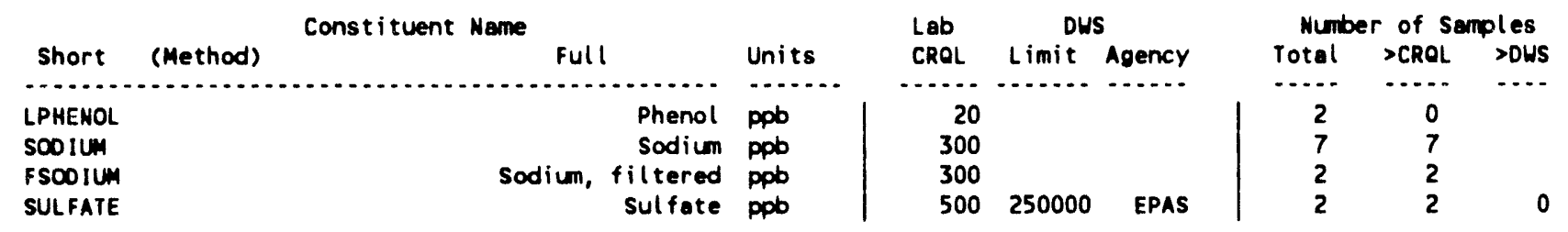

SITE SPECIFIC AND OTHER CONSTITUENTS

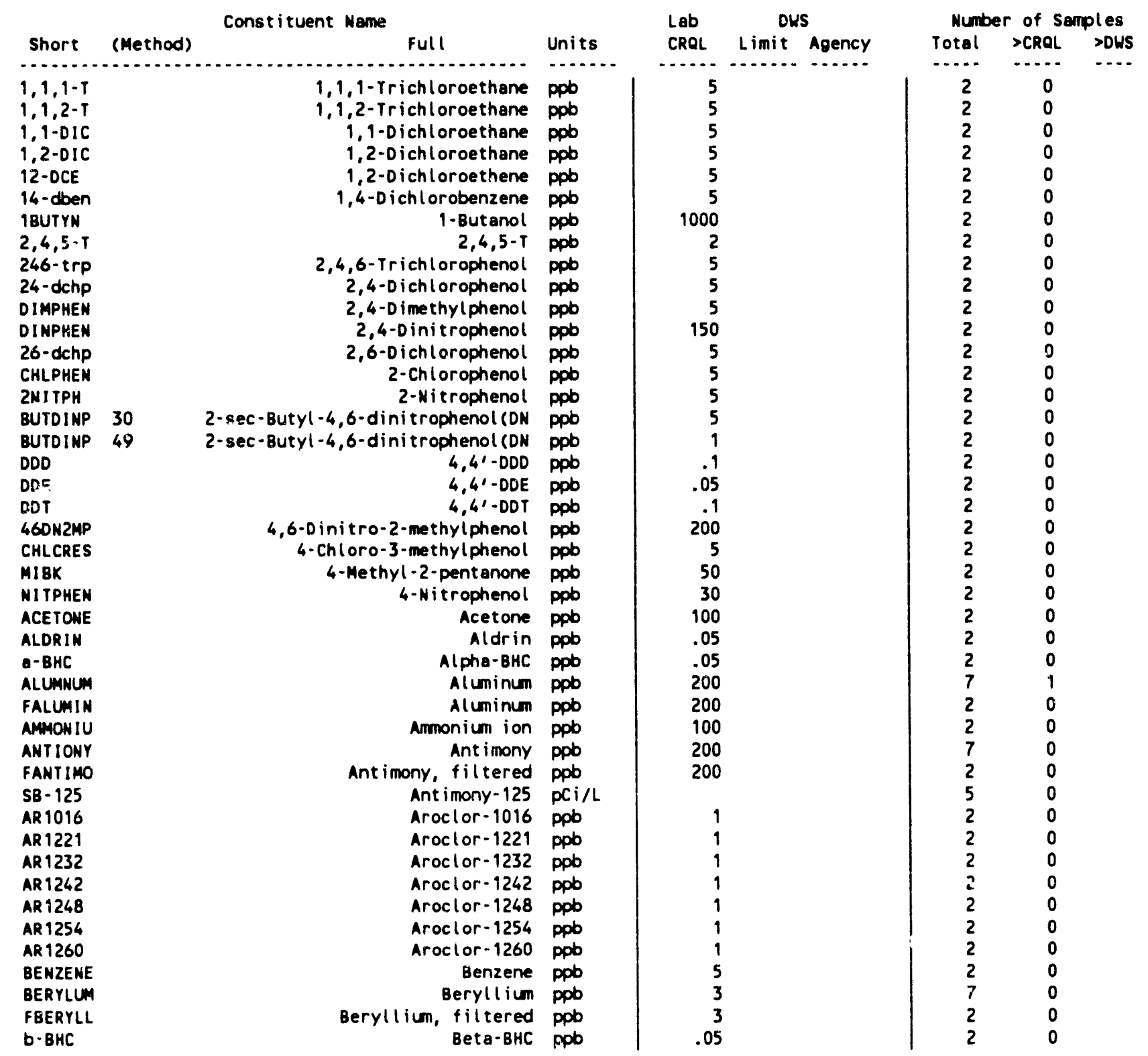


Table 15-15. Constituent List and Summary of Results for the Low-Level Waste Burial Grounds WMA 2 Data for Reporting Period July 1 through September 30, 1993. (sheet 3 of 3)

\begin{tabular}{|c|c|c|c|c|c|c|c|c|}
\hline \multirow[b]{2}{*}{ Short } & \multicolumn{2}{|l|}{ Constituent Nane } & \multirow{2}{*}{$\begin{array}{l}\text { Lab } \\
\text { CROL }\end{array}$} & \multicolumn{2}{|c|}{ DUS } & \multicolumn{3}{|c|}{ Number of Semples } \\
\hline & (Method) & Units & & Limit & Agency & Total & $>C R Q L$ & $>$ DWS \\
\hline$\ldots \ldots \ldots$ & - & $\cdots \cdots$ & $\ldots$. & $\ldots \ldots$ & $\ldots \ldots$ & $\cdots \cdots$ & $\ldots$. & $\ldots$ \\
\hline BROMIDE & Bromide & ppb & 500 & & & 2 & 0 & \\
\hline CALCIUM & Calcium & ppb & 100 & & & 7 & 7 & \\
\hline FCALCIU & Calcium, filtered & ppo & 100 & & & 2 & 2 & \\
\hline CARBIDE & Carbon disulfide & ppb & 5 & & & 2 & 0 & \\
\hline TETRANE & Carbon tetrachloride & ppb & 5 & & & 2 & 0 & \\
\hline CS- 137 & Cesium- 137 & $\mathrm{PCi} / \mathrm{L}$ & 20 & & & 5 & 0 & \\
\hline CHLOANE & Chlordane & ppb & .1 & & & 2 & 0 & \\
\hline CHLFORM & Chloroform & ppb & 5 & 100 & EPA & 2 & 0 & 0 \\
\hline COBALT & Cobalt & ppb & 20 & & & 7 & 0 & \\
\hline FCOBALT & Cobalt, filtered & ppob & 20 & & & 2 & 0 & \\
\hline$c 0-60$ & Cobelt $t-60$ & $\mathrm{pCi} / \mathrm{L}$ & & & & 5 & 0 & \\
\hline COPPER & Copper & ppb & 20 & 1000 & EPAS & 7 & 1 & 0 \\
\hline FCOPPER & Copper, filtered & ppb & 20 & 1000 & EPAS & 2 & 0 & 0 \\
\hline CRESOLS & Cresols (methylphenols) & ppb & 10 & & & 2 & 0 & \\
\hline CYANIDE & Cyanide & ppob & 20 & & & 2 & 0 & \\
\hline d-BHC & Delta-BHC & ppb & .1 & & & 2 & 0 & \\
\hline DIELRIN & Dieldrin & ppb & .05 & & & 2 & 0 & \\
\hline ENDO1 & Endosulfan I & ppb & .1 & & & 2 & 0 & \\
\hline ENDO2 & Endosulfan II & ppb & .05 & & & 2 & 0 & \\
\hline ENDSFAN & Endosulfan sulfate & ppo & .5 & & & 2 & 0 & \\
\hline ENDRALD & Endrin Aldehyde & ppb & .2 & & & 2 & 0 & \\
\hline ETHCYAN & Ethyl cyanide & ppb & 10 & & & 2 & 0 & \\
\hline HEPTLOR & Heptachlor & ppb & .05 & & & 2 & 0 & \\
\hline HEPTIDE & Heptachlor epoxide & ppb & 1 & & & 2 & 0 & \\
\hline MAGNES & Magnesiun & ppb & 100 & & & 7 & 7 & \\
\hline FMAGNES & Magnesium, filtered & ppb & 100 & & & 2 & 2 & \\
\hline METHONE & Methyl ethyl ketone & ppob & 100 & & & 2 & 0 & \\
\hline METHYCH & Methylene chloride & ppb & 5 & & & 2 & 0 & \\
\hline NICKEL & Nickel & ppb & 30 & & & 7 & 6 & \\
\hline FNICKEL & Nickel, filtered & ppb & 30 & & & 2 & 0 & \\
\hline NITRITE & Nitrite & ppb & 200 & & & 2 & 0 & \\
\hline PENTCHP & Pentachl orophenol & ppb & 100 & & & 2 & 0 & \\
\hline PHCSPHA & Phosphate & ppo & 400 & & & 2 & 0 & \\
\hline POTASUM & Potassiun & ppb & 300 & & & 7 & 7 & \\
\hline FPOTASS & Potassium, filtered & ppb & 300 & & & 2 & 2 & \\
\hline RU- 106 & Ruthenium-106 & $\mathrm{pCi} / \mathrm{L}$ & & & & 5 & 0 & \\
\hline PERCENE & Tetrachloroethene & ppo & 5 & & & 2 & 0 & \\
\hline TETPHNL & Tetrachl orophenols & ppob & 10 & & & 2 & 0 & \\
\hline TAF & Tetrahydrofuran & ppb & 10 & & & 2 & 0 & \\
\hline TIN & $\operatorname{Tin}$ & ppb & 100 & & & 7 & 0 & \\
\hline FTIN & Tin, filtered & ppb & 100 & & & 2 & 0 & \\
\hline TOLUENE & Tolvene & ppb & 5 & & & 2 & 0 & \\
\hline TC & Total Carbon & ppb & 2000 & & & 5 & 5 & \\
\hline TOS & Total Dissolved Solids & ppm & 10 & & & 5 & 5 & \\
\hline TRICENE & Trichloroethene & ppb & 5 & & & 2 & 0 & \\
\hline TRIPHNL & Trichlorophenols & ppo & 5 & & & 2 & 0 & \\
\hline TRITIUM & Tritium & $\mathrm{pCi} / \mathrm{L}$ & 500 & 20000 & EPA & 2 & 2 & 0 \\
\hline URANIUM & Uranium & ppb & .5 & & & 2 & 2 & \\
\hline VANADUM & Vanadium & ppb & 30 & & & 7 & 5 & \\
\hline FVANADI & Vanadium, filtered & ppb & 30 & & & 2 & 2 & \\
\hline VINYIDE & Vinyl chloride & ppo & 10 & 2 & EPA & 2 & 0 & 0 \\
\hline XYLENE & xylenes (total) & ppob & 5 & & & 2 & 0 & \\
\hline ZINC & zinc & ppb & 10 & & & 7 & 2 & \\
\hline FZINC & zinc, filtered & ppob & 10 & & & 2 & 0 & \\
\hline
\end{tabular}

For explanation of this table, see Section 1.4 of report. 
Table 15-16. Constituents with at Least One Value Above the CRQL for the Low-Level Waste Burial Grounds WMA 2 Data for Reporting Period July 1 through September 30, 1993. (sheet 1 of 3)

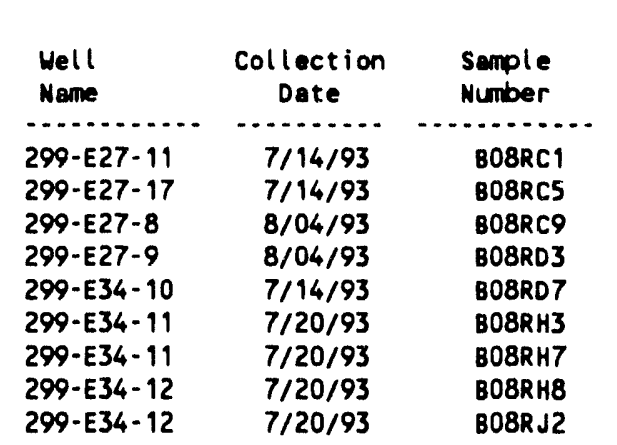

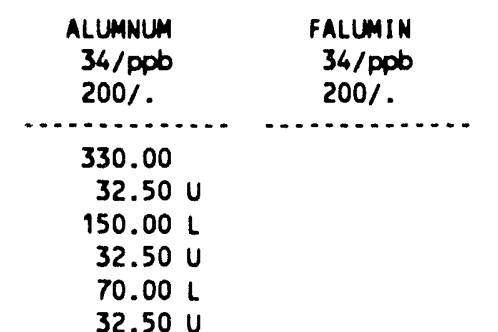

$32.50 \mathrm{U}$

$44.00 \mathrm{~L}$

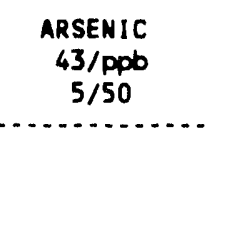

5.90

12.00

$32.50 \mathrm{U}$

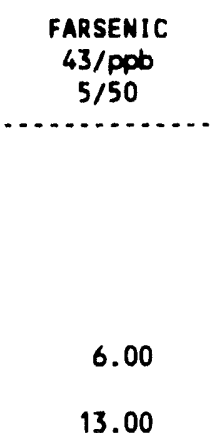

13.00

\begin{tabular}{|c|c|c|c|c|c|c|}
\hline $\begin{array}{l}\text { Well } \\
\text { Name }\end{array}$ & $\begin{array}{c}\text { Collection } \\
\text { Date }\end{array}$ & $\begin{array}{l}\text { Sample } \\
\text { Number }\end{array}$ & $\begin{array}{l}\text { BARIUM } \\
34 / \mathrm{ppb} \\
20 / 1000\end{array}$ & $\begin{array}{l}\text { FBARIUM } \\
34 / \mathrm{ppb} \\
20 / 1000\end{array}$ & $\begin{array}{l}\text { CALCIUM } \\
34 / \text { ppb } \\
100 \%\end{array}$ & $\begin{array}{c}\text { FCALCIU } \\
34 / \text { ppb } \\
100 \%\end{array}$ \\
\hline $\begin{array}{l}299-E 27-11 \\
299-E 27-17 \\
299-E 27-8 \\
299-E 27-9 \\
299-E 34-10 \\
299-E 34-11\end{array}$ & $\begin{array}{l}7 / 14 / 93 \\
7 / 14 / 93 \\
8 / 04 / 93 \\
8 / 04 / 93 \\
7 / 14 / 93 \\
7 / 20 / 93\end{array}$ & $\begin{array}{l}\text { BOBRC1 } \\
\text { BOBRC5 } \\
\text { BOBRC9 } \\
\text { BOBRD } 3 \\
\text { B08RD } 7 \\
\text { BOBRH3 }\end{array}$ & $\begin{array}{l}46.00 \\
37.00 \\
41.00 \\
31.00 \\
43.00 \\
35.00\end{array}$ & & $\begin{array}{l}40000.000 \\
42000.000 \\
45000.00 \\
46000.00 \\
39000.00 \\
42000.00\end{array}$ & \\
\hline $\begin{array}{l}299-E 34-11 \\
299-E 34-12 \\
299-E 34-12\end{array}$ & $\begin{array}{l}7 / 20 / 93 \\
7 / 20 / 93 \\
7 / 20 / 93\end{array}$ & $\begin{array}{l}\text { BO8RH7 } \\
\text { B08RH8 } \\
\text { B08R J2 }\end{array}$ & 47.00 & $\begin{array}{l}34.00 \\
40.00\end{array}$ & 35000.000 & $\begin{array}{l}41000.000 \\
32000.000\end{array}$ \\
\hline
\end{tabular}

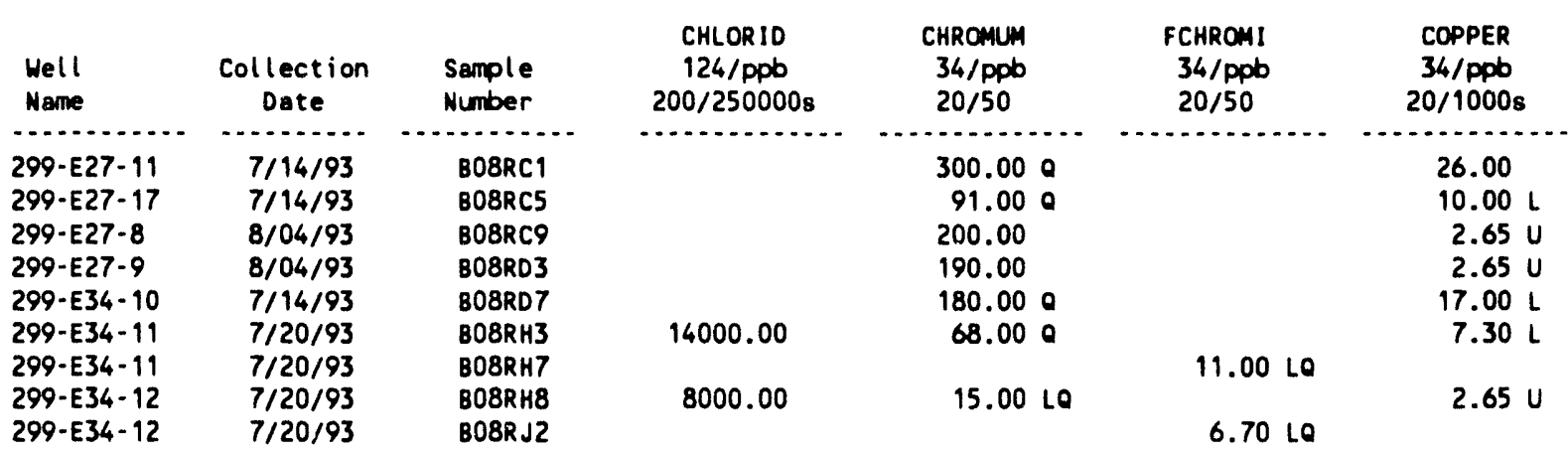

\begin{tabular}{|c|c|c|c|c|c|c|}
\hline $\begin{array}{l}\text { Well } \\
\text { Name }\end{array}$ & $\begin{array}{c}\text { Collection } \\
\text { Date }\end{array}$ & $\begin{array}{l}\text { Sample } \\
\text { Number }\end{array}$ & $\begin{array}{c}\text { FCOPPER } \\
34 / \mathrm{ppb} \\
20 / 1000 \mathrm{~s}\end{array}$ & $\begin{array}{l}\text { FLUORID } \\
124 / \mathrm{ppb} \\
100 / 4000\end{array}$ & $\begin{array}{c}\text { ALPHA } \\
135 / \text { PCi } / L \\
. / 15\end{array}$ & $\begin{array}{c}\text { BETA } \\
136 / \mathrm{PCi} / \mathrm{L} \\
. / 50\end{array}$ \\
\hline $\begin{array}{l}299-E 34-11 \\
299-E 34-11\end{array}$ & $\begin{array}{l}7 / 20 / 93 \\
7 / 20 / 93\end{array}$ & $\begin{array}{l}\text { B08RH3 } \\
\text { B08RH7 }\end{array}$ & $2.65 \mathrm{U}$ & 700.00 & 5.19 & 4.62 \\
\hline $\begin{array}{l}299-E 34-12 \\
299-E 34-12\end{array}$ & $\begin{array}{l}7 / 20 / 93 \\
7 / 20 / 93\end{array}$ & $\begin{array}{l}\text { B08RH8 } \\
\text { B08RJ2 }\end{array}$ & $2.65 \mathrm{U}$ & 400.00 & 1.74 & 5.60 \\
\hline
\end{tabular}


Table 15-16. Constituents with at Least One Value Above the CRQL for the Low-Level Waste Burial Grounds WMA 2 Data for Reporting Period July 1 through September 30, 1993. (sheet 2 of 3)

\begin{tabular}{|c|c|c|c|c|c|c|}
\hline $\begin{array}{l}\text { Mell } \\
\text { Name }\end{array}$ & $\begin{array}{l}\text { Collection } \\
\text { Date }\end{array}$ & $\begin{array}{l}\text { Sample } \\
\text { Number }\end{array}$ & $\begin{array}{c}\text { IRON } \\
34 / \text { ppb } \\
20 / 300 \text { s }\end{array}$ & $\begin{array}{l}\text { FIRON } \\
34 / \mathrm{ppb} \\
20 / 300 \mathrm{~s}\end{array}$ & $\begin{array}{l}\text { MAGNES } \\
34 / \text { ppb } \\
100 \%\end{array}$ & $\begin{array}{l}\text { FMAGNES } \\
34 / \text { ppD } \\
100 \%\end{array}$ \\
\hline $\begin{array}{l}299-E 27-11 \\
299-E 27-17 \\
299-E 27-8 \\
299-E 27-9 \\
299-E 34-10 \\
299-E 34-11\end{array}$ & $\begin{array}{l}7 / 14 / 93 \\
7 / 14 / 93 \\
8 / 04 / 93 \\
8 / 04 / 93 \\
7 / 14 / 93 \\
7 / 20 / 93\end{array}$ & $\begin{array}{l}\text { B08RC1 } \\
\text { B08RC5 } \\
\text { B08RC9 } \\
\text { B08RD 3 } \\
\text { B08RD7 } \\
\text { B08RH3 }\end{array}$ & $\begin{array}{r}2000.000 \\
410.000 \\
1100.00 \\
850.00 \\
960.000 \\
310.000\end{array}$ & $\cdots$ & $\begin{array}{l}11000.00 \\
11000.00 \\
12000.00 \\
13000.00 \\
10000.00 \\
12000.00\end{array}$ & \\
\hline $\begin{array}{l}299-E 34-11 \\
299-E 34-12 \\
200-E 34-12\end{array}$ & $\begin{array}{l}7 / 20 / 93 \\
7 / 20 / 93\end{array}$ & $\begin{array}{l}\text { BO8RH7 } \\
\text { BO8RH8 }\end{array}$ & 120.00 o & 35.000 & 8700.00 & 12000.00 \\
\hline
\end{tabular}

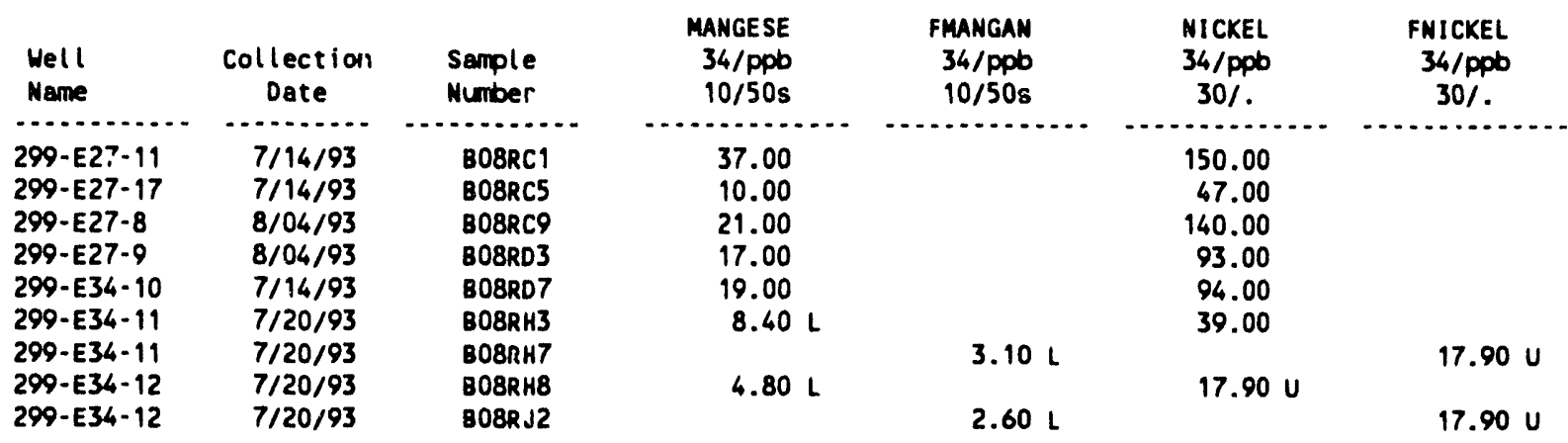

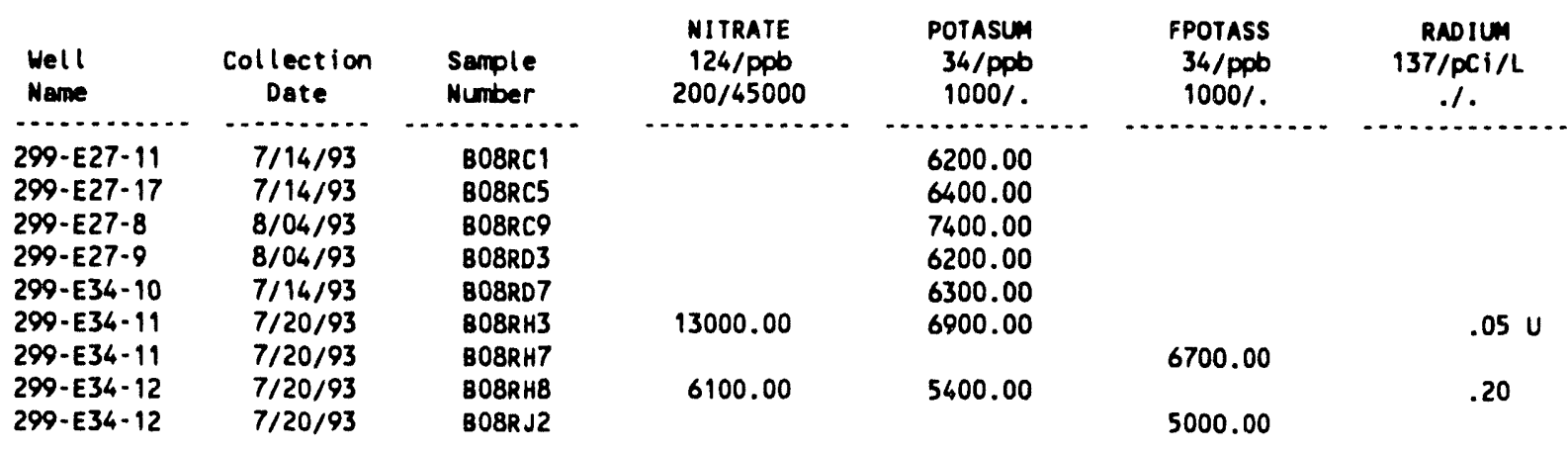

\begin{tabular}{|c|c|c|c|c|c|c|}
\hline $\begin{array}{l}\text { Well } \\
\text { Name }\end{array}$ & $\begin{array}{c}\text { Collection } \\
\text { Date }\end{array}$ & $\begin{array}{l}\text { Sample } \\
\text { Number }\end{array}$ & $\begin{array}{l}\text { s00IUM } \\
34 / \mathrm{ppb} \\
300 \%\end{array}$ & $\begin{array}{l}\text { FSODIUM } \\
34 / \mathrm{ppb} \\
300 \%\end{array}$ & $\begin{array}{c}\text { SULFATE } \\
124 / \mathrm{ppb} \\
500 / 250000 \mathrm{~s}\end{array}$ & $\begin{array}{c}\text { TC } \\
127 / p p b \\
2000 \% .\end{array}$ \\
\hline $\begin{array}{l}299-E 27-11 \\
299-E 27-17 \\
299-E 27-8 \\
299-E 27-9\end{array}$ & $\begin{array}{l}7 / 14 / 93 \\
7 / 14 / 93 \\
8 / 04 / 93 \\
8 / 04 / 93\end{array}$ & $\begin{array}{l}\text { B08RC1 } \\
\text { BO8RC5 } \\
\text { B08RC9 } \\
\text { B08RD } 3\end{array}$ & $\begin{array}{l}15000.000 \\
16000.000 \\
14000.000 \\
14000.000\end{array}$ & & & $\begin{array}{l}24000.00 \\
25000.00 \\
23000.00 \\
22000.00\end{array}$ \\
\hline
\end{tabular}


Table 15-16. Constituents with at Least One Value Above the CRQL for the Low-Level Waste Burial Grounds WMA 2 Data for Reporting Period July 1 through September 30, 1993. (sheet 3 of 3)

\begin{tabular}{|c|c|c|c|c|c|c|}
\hline $\begin{array}{l}\text { Well } \\
\text { Neme }\end{array}$ & $\begin{array}{c}\text { Collection } \\
\text { Dete }\end{array}$ & $\begin{array}{l}\text { Sample } \\
\text { Number }\end{array}$ & $\begin{array}{l}\text { SO0IUM } \\
34 / \mathrm{ppb} \\
300 / .\end{array}$ & $\begin{array}{l}\text { FSO0 IIM } \\
34 / \mathrm{ppb} \\
300 / .\end{array}$ & $\begin{array}{c}\text { SULFATE } \\
124 / \mathrm{ppb} \\
500 / 250000 \mathrm{~s}\end{array}$ & $\begin{array}{c}\text { TC } \\
127 / \mathrm{ppb} \\
2000 / .\end{array}$ \\
\hline$\ldots \ldots \ldots . . . .$. & $\ldots \ldots \ldots$ & $\ldots \ldots \ldots$ & 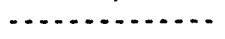 & $\ldots \ldots \ldots \ldots$ & $\ldots \ldots \ldots$ & $\ldots \ldots$ \\
\hline $\begin{array}{l}299-E 34-10 \\
299-E 34-11\end{array}$ & $\begin{array}{l}7 / 14 / 93 \\
7 / 20 / 93\end{array}$ & $\begin{array}{l}\text { B08RD7 } \\
\text { B08RH3 }\end{array}$ & $\begin{array}{l}16000.00 \\
23000.00\end{array}$ & & 69000.00 & 25000.00 \\
\hline $\begin{array}{l}299-\text { E34- } 11 \\
299-E 34-12\end{array}$ & $\begin{array}{l}7 / 20 / 93 \\
7 / 20 / 93\end{array}$ & $\begin{array}{l}\text { B08RH7 } \\
\text { B08RH8 }\end{array}$ & 13000.000 & 23000.000 & 46000.00 & \\
\hline
\end{tabular}

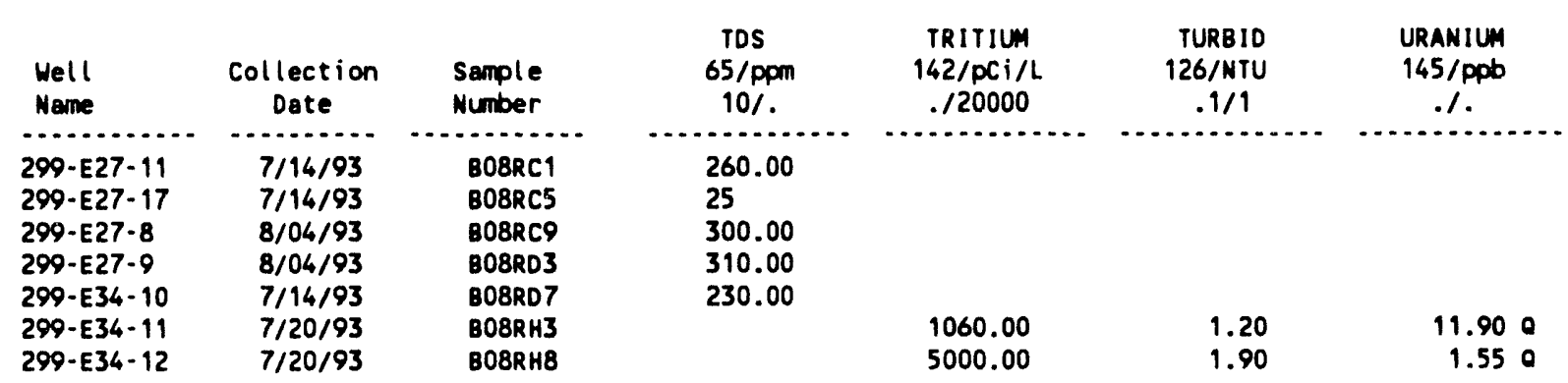

\begin{tabular}{|c|c|c|c|c|c|c|}
\hline $\begin{array}{l}\text { Well } \\
\text { Name }\end{array}$ & $\begin{array}{l}\text { Collection } \\
\text { Date }\end{array}$ & $\begin{array}{l}\text { Sample } \\
\text { Number }\end{array}$ & $\begin{array}{c}\text { VANADUM } \\
34 / \text { ppb } \\
30 \%\end{array}$ & $\begin{array}{c}\text { FVANAD1 } \\
34 / p \text { p } \\
30 \%\end{array}$ & $\begin{array}{c}\text { ZINC } \\
34 / p p b \\
10 \%\end{array}$ & $\begin{array}{c}\text { FZINC } \\
34 / \text { ppb } \\
10 \%\end{array}$ \\
\hline $\begin{array}{l}299-E 27-11 \\
299-E 27-17 \\
299-E 27-8 \\
299-E 27-9 \\
299-E 34-10 \\
299-E 34-11\end{array}$ & $\begin{array}{l}7 / 14 / 93 \\
7 / 14 / 93 \\
8 / 04 / 93 \\
8 / 04 / 93 \\
7 / 14 / 93 \\
7 / 20 / 93\end{array}$ & $\begin{array}{l}\text { B08RC1 } \\
\text { B08RC5 } \\
\text { BO8RC9 } \\
\text { BO8RD3 } \\
\text { B08RD7 } \\
\text { BOBRH3 }\end{array}$ & $\begin{array}{l}40.00 \\
38.00 \\
3.84 U \\
3.84 U \\
40.00 \\
34.00\end{array}$ & & $\begin{array}{r}33.00 \mathrm{O} \\
8.80 \mathrm{LO} \\
3.44 \mathrm{U} \\
3.44 \mathrm{U} \\
19.00 \mathrm{O} \\
7.30 \mathrm{LO}\end{array}$ & \\
\hline 299-E34-11 & $7 / 20 / 93$ & BOBRH7 & & 34.00 & & $5.00 \mathrm{LO}$ \\
\hline $299-E 34-12$ & $\begin{array}{l}7 / 20 / 93 \\
7 / 20 / 93\end{array}$ & $\begin{array}{l}\text { B08RH8 } \\
\text { BOBRJ2 }\end{array}$ & 39.00 & & $9.00 \mathrm{LO}$ & \\
\hline
\end{tabular}

For explanation of this table, see Section 1.4 of report. 
Table 15-17. Contamination Indicator Parameters for the Low-Level Waste Burial Grounds WMA 2 Data for Reporting Period July 1 through September 30, 1993.

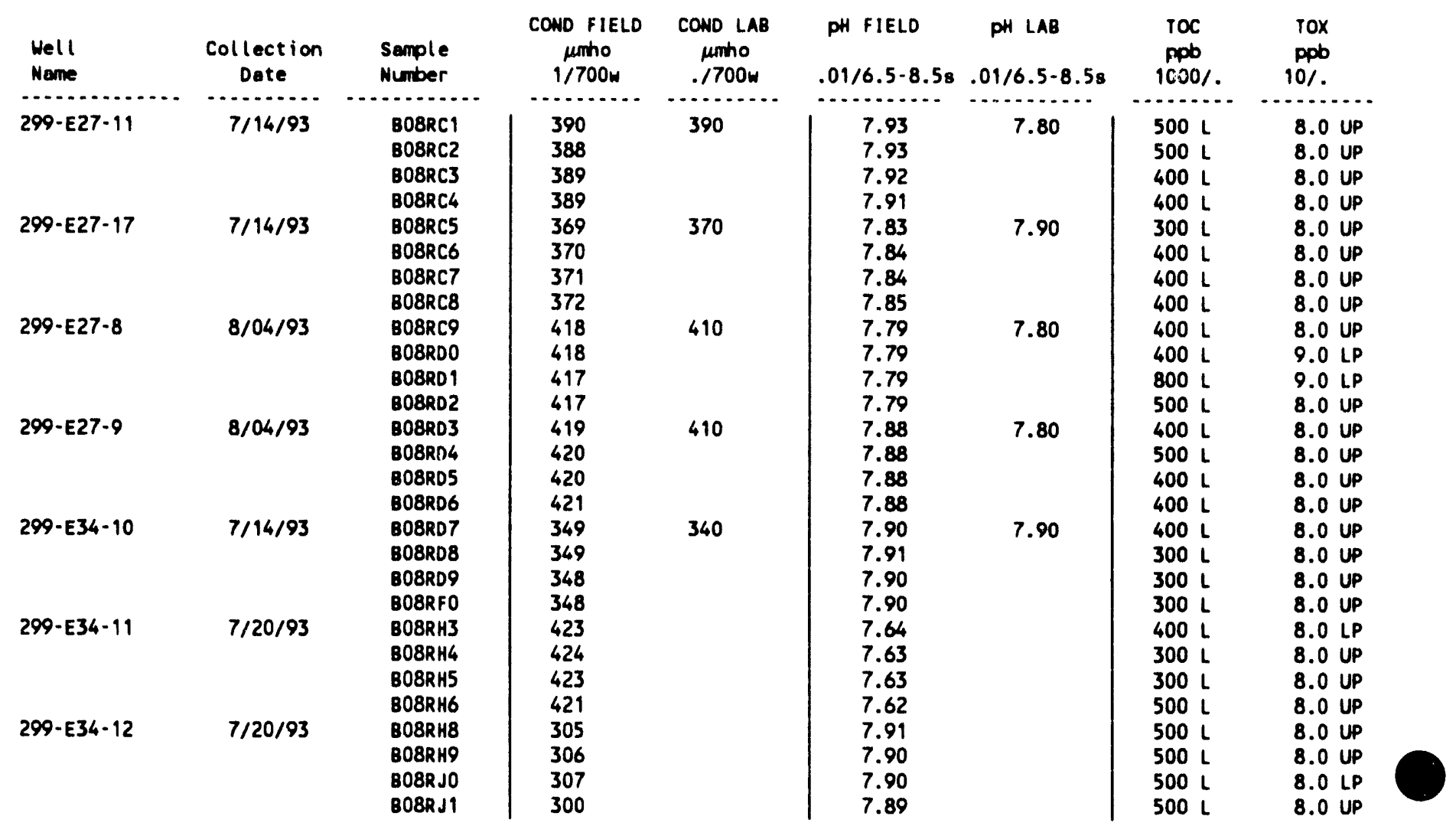

For explanation of this table, see Section 1.4 of report. 
Table 15-18. Constituent List and Summary of Results for the Low-Level Waste Burial Grounds WMA 3 Data for Reporting Period July 1 through September 30, 1993. (sheet 1 of 6)

CONTAMINATION INDICATOR PARAMETERS

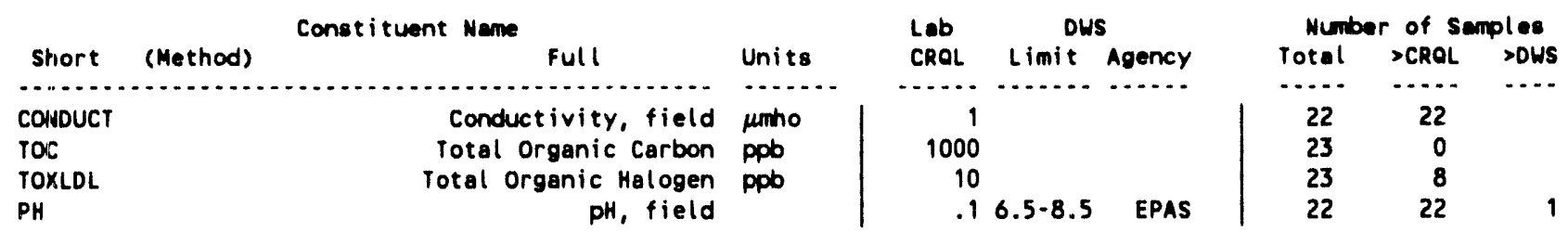

\section{DRINKING WATER PARAMETERS}

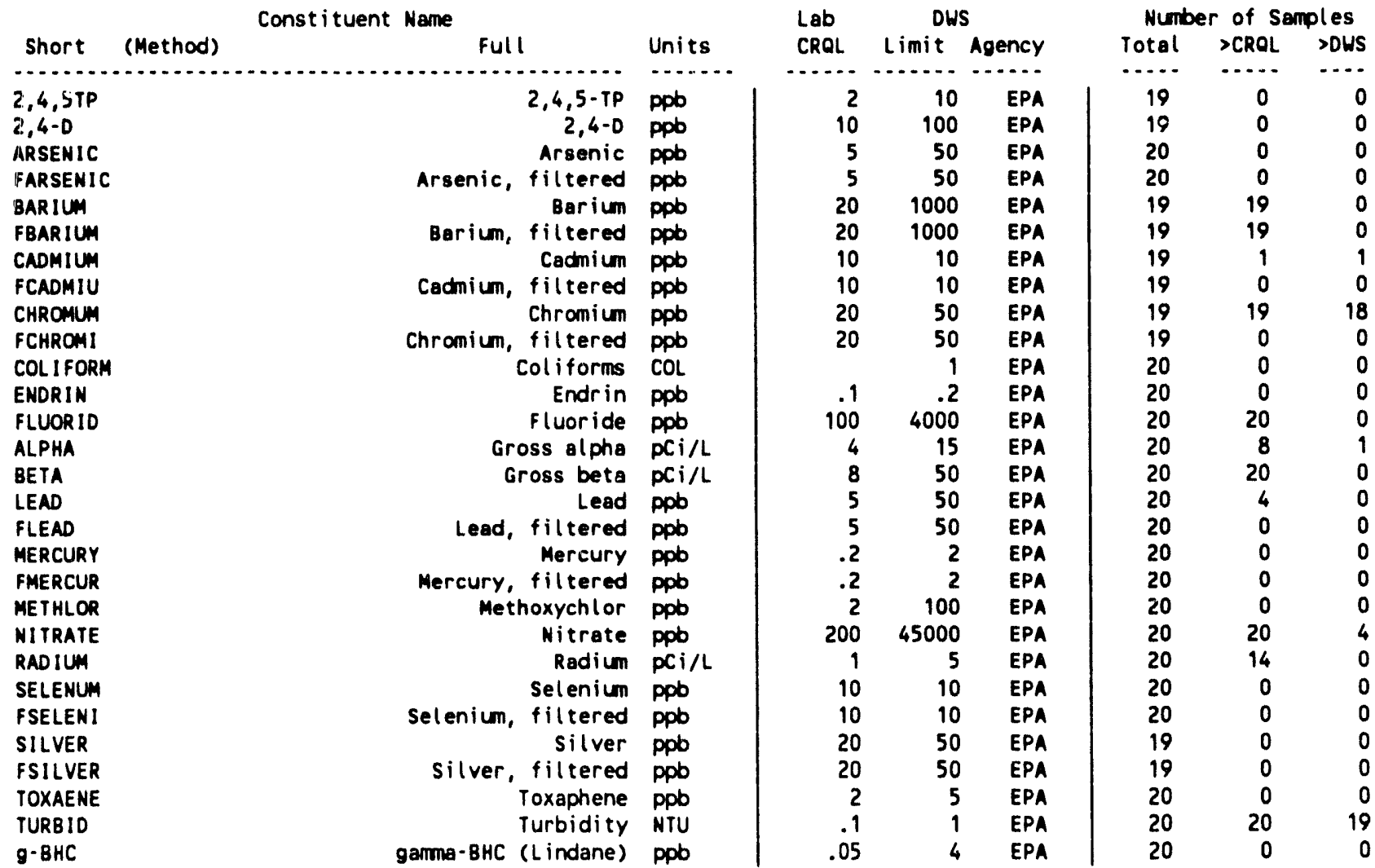

GROUNDWATER OUALITY PARAMETERS

\begin{tabular}{|c|c|c|c|c|c|c|c|c|c|}
\hline \multirow[b]{2}{*}{ Short } & \multirow[b]{2}{*}{ (Method) } & \multirow[t]{2}{*}{ Constituent Name } & \multirow[b]{2}{*}{ Units } & \multirow{2}{*}{$\begin{array}{l}\text { Lab } \\
\text { CROL }\end{array}$} & \multicolumn{2}{|c|}{ DWS } & \multicolumn{3}{|c|}{ Number of Semples } \\
\hline & & & & & Limit & Agency & Total & $>$ CRQL & $>$ DWS \\
\hline$\ldots \ldots$ & $\cdots$ & $\ldots \ldots$ & $\ldots . .$. & $\ldots \ldots$ & $\cdots \ldots$ & $\ldots \ldots$ & $\ldots \ldots$ & $\ldots$ & $\cdots$ \\
\hline CHLORID & & Chloride & ppb & 200 & 250000 & EPAS & 20 & 20 & 0 \\
\hline IRON & & Iron & ppo & 20 & 300 & EPAS & 19 & 19 & 19 \\
\hline FIRON & & Iron, filtered & ppb & 20 & 300 & EPAS & 19 & 14 & 0 \\
\hline MANGESE & & Manganese & ppb & 10 & 50 & EPAS & 19 & 17 & 5 \\
\hline FMANGAN & & Manganese, filtered & ppb & 10 & 50 & EPAS & 19 & 2 & 0 \\
\hline LPHENOL & 19 & Phenol & $\mathrm{ppb}$ & 10 & & & 18 & 0 & \\
\hline LPHENOL & 30 & Phenol & ppb & 20 & & & 20 & 1 & \\
\hline SOOIUM & & Sodium & ppb & 300 & & & 19 & 19 & \\
\hline FSOOIUM & & Sodium, filtered & ppb & 300 & & & 19 & 19 & \\
\hline SULFATE & & Sulfate & ppb & 500 & 250000 & EPAS & 20 & 20 & 0 \\
\hline
\end{tabular}


Table 15-18. Constituent List and Summary of Results for the Low-Level Waste Burial Grounds WMA 3 Data for Reporting Period July 1

through September 30, 1993. (sheet 2 of 6 )

SITE SPECIFIC AND OTHER CONSTITUENTS

\begin{tabular}{|c|c|c|c|c|c|c|c|c|}
\hline \multirow{2}{*}{ Short } & & \multirow{2}{*}{ Constituent Name Full } & \multirow[b]{2}{*}{ Units } & \multicolumn{2}{|r|}{ DWS } & \multicolumn{3}{|c|}{ Number of Samples } \\
\hline & (Method) & & & CROL & Limit Agency & Total & $>C R Q L$ & $>$ DWS \\
\hline $1112-t c$ & & $1,1,2$-Totrcohlarothan & $\cdots$ & 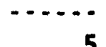 & $\cdots \ldots . . . .$. & $\cdots$ & $\cdots$ & $\cdots$ \\
\hline $\begin{array}{l}1112-t c \\
1,1,1-T\end{array}$ & & $\begin{array}{r}\text { 1,1,1,2-1etrachloroethane } \\
1,1,1-\text { Trichloroethane }\end{array}$ & $\begin{array}{l}\text { ppo } \\
\text { pob }\end{array}$ & $\begin{array}{l}5 \\
5\end{array}$ & & $\begin{array}{r}2 \\
26\end{array}$ & $\begin{array}{l}0 \\
0\end{array}$ & \\
\hline $1122-t c$ & & 1,1,2,2-Tetrachloroethane & ppob & 5 & & 2 & 0 & \\
\hline 1,1,2-T & & 1,1,2-Trichloroethene & ppob & 5 & & 26 & 0 & \\
\hline $1,1-01 C$ & & 1,1-Dichloroethane & ppob & 5 & & 26 & 0 & \\
\hline DICETHY & & 1,1-D ichloroethene & ppb & 5 & & 2 & 0 & \\
\hline $123-t r p$ & & 1,2,3-Trichloropropene & ppb & 5 & & 2 & 0 & \\
\hline TETRCHB & & $1,2,4,5-$ Tetrachl orobenzene & ppb & 10 & & 2 & 0 & \\
\hline TRICHLB & & 1,2,4-Trichl orobenzene & ppb & 10 & & 2 & 0 & \\
\hline DIBRCHL. & & 1,2-D ibromo-3-chloropropane & ppb & 5 & & 2 & 0 & \\
\hline DIBRETH & & 1,2-0 ibromoethane & ppb & 5 & & 2 & 0 & \\
\hline 12-cben & & 1,2-0 ichlorobenzene & ppo & 10 & & 2 & 0 & \\
\hline 1,2-DIC & & 1,2-0ichloroethane & ppob & 5 & & 26 & 0 & \\
\hline 12-DCE & & 1,2-Dichloroethene & ppob & 5 & & 26 & 0 & \\
\hline DICPANE & & 1,2-Dichl oropropane & ppb & 5 & & 2 & 0 & \\
\hline 13-cben & & 1,3-0 ichlorobenzene & ppb & 10 & & 2 & 0 & \\
\hline 14-dben & 16 & 1,4-0 ichlor obenzene & ppb & 5 & & 24 & 0 & \\
\hline 14-cben & 19 & 1,4-0 ichlorobenzene & ppb & 10 & & 2 & 0 & \\
\hline DIOXANE & & 1,4-Dioxane & ppb & 200 & & 2 & 0 & \\
\hline NAPHQUI & & 1,4-Naphtoquinone & ppb & 10 & & 2 & 0 & \\
\hline 1BUTYN & & 1-Butanol & ppb & 1000 & & 26 & 0 & \\
\hline 1-napha & & 1-Naphthylamine & ppb & 10 & & 2 & 0 & \\
\hline TETRCHP & & $2,3,4,6$ - Tetrachlorophenol & ppb & 10 & & 2 & 0 & \\
\hline $2,4,5-T$ & & $2,4,5-T$ & ppb & 2 & & 19 & 0 & \\
\hline $245-\mathrm{trp}$ & & $2,4,5-$ Trichlorophenol & ppb & 10 & & 2 & 0 & \\
\hline $246-t r p$ & 19 & 2,4,6-Trichlorophenol & ppb & 10 & & 2 & 0 & \\
\hline $246-t r p$ & 30 & 2,4,6-Trichlorophenol & ppo & 5 & & 20 & 0 & \\
\hline 24-dchp & 19 & 2,4-Dichlorophenol & ppob & 10 & & 18 & 0 & \\
\hline $24-d c h p$ & 30 & 2,4-0 ichlorophenol & ppb & 5 & & 20 & 0 & \\
\hline DIMPHEN & 19 & 2,4-0 imethylphenol & ppob & 10 & & 2 & 0 & \\
\hline DIMPHEN & 30 & 2,4-D imethylphenol & ppb & 5 & & 20 & 0 & \\
\hline DINPHEN & 19 & 2,4-D initrophenol & ppb & 50 & & 2 & 0 & \\
\hline DINPHEN & 30 & $2,4-0$ initrophenol & ppo & 150 & & 20 & 0 & \\
\hline 24 -dint & & 2,4-D initrotoluene & ppob & 10 & & 2 & 0 & \\
\hline 26-dchp & 19 & 2,6-D ichlorophenol & ppob & 10 & & 2 & 0 & \\
\hline 26-dchp & 30 & 2,6-0 ichlorophenol & ppb & 5 & & 20 & 0 & \\
\hline 26-dint & & 2,6-D initrotoluene & ppb & 10 & & 2 & 0 & \\
\hline ACEFENE & & 2-Acetylaminof luorene & ppo & 10 & & 2 & 0 & \\
\hline CHLNAPH & & 2-Chloronaphthalene & ppo & 10 & & 2 & 0 & \\
\hline CHLPHEN & 19 & 2-Chlorophenol & ppb & 10 & & 2 & 0 & \\
\hline CHLPHEN & 30 & 2-Chlorophenol & ppo & 5 & & 20 & 0 & \\
\hline 2HEXAMO & & 2-Hexanone & $p p b$ & 50 & & 2 & 0 & \\
\hline ZMENAPH & & 2-Methyl naphthal ene & ppb & 10 & & 2 & 0 & \\
\hline 2METHPH & & 2-Methylphenol & ppo & 10 & & 18 & 0 & \\
\hline 2-napha & & 2-Naphthylamine & ppb & 10 & & 2 & 0 & \\
\hline ONITANI & & 2-Nitroaniline & ppb & 50 & & 2 & 0 & \\
\hline 2NITPH & 19 & 2-Nitrophenol & ppo & 10 & & 18 & 0 & \\
\hline 2NITPH & 30 & 2-Nitrophenol & ppb & 5 & & 20 & 0 & \\
\hline PICOLIN & & 2-Picoline & ppb & 10 & & 2 & 0 & \\
\hline BUTDINP & 30 & nitrophenol (ON & ppb & 5 & & 20 & 0 & \\
\hline BUTOINP & 49 & 2-sec-Butyl-4,6-dinitrophenol (DN & ppb & 1 & & 19 & 0 & \\
\hline
\end{tabular}


Table 15-18. Constituent List and Summary of Results for the Low-Level Waste Burial Grounds WMA 3 Data for Reporting Period July 1

through September 30, 1993. (sheet 3 of 6)

\begin{tabular}{|c|c|c|c|c|c|c|c|c|}
\hline & & Constituent Name & & Lab & DWS & Numb & $\mathrm{r}$ of $\mathrm{Sa}$ & \\
\hline Short & (Method) & $\begin{array}{l}\text { Full } \\
\ldots . . . . . .\end{array}$ & Units & CROL & Limit Agency & Total & $>$ CROL & >OWS \\
\hline DICHBEN & & 3,31-Dichlorobenz idine & ppb & 20 & . & 2 & 0 & $\cdots$ \\
\hline DIMEYLB & & 3,3'-D imethylbenz idine & ppb & 10 & & 2 & 0 & \\
\hline METCHAN & & 3-Methylcholanthrene & ppo & 10 & & $\overline{2}$ & 0 & \\
\hline MNITANI & & 3-Nitroaniline & ppb & 50 & & 2 & 0 & \\
\hline DOD & & $4,4^{\prime}-000$ & ppb & .1 & & 20 & 0 & \\
\hline DDE & & $4,4^{\prime}-$ DDE & ppob & .05 & & 20 & 0 & \\
\hline DOT & & $4,41-0 D T$ & ppb & .1 & & 20 & 0 & \\
\hline 460N2MP & 19 & 4,6-0 initro-2-methylphenol & ppb & 50 & & 2 & 0 & \\
\hline $460 N 2 M P$ & 30 & 4,6-0 initro-2-methylphenol & ppb & 200 & & 20 & 0 & \\
\hline $\begin{array}{l}\text { AMINOYL } \\
\text { BROPHEN }\end{array}$ & & 4-Aminobiphenyl & ppb & 10 & & 2 & 0 & \\
\hline $\begin{array}{l}\text { BROPHEN } \\
\text { CHLCRES }\end{array}$ & & 4-8romophenylphenyl ether & ppb & 10 & & 2 & 0 & \\
\hline $\begin{array}{l}\text { CHLCRES } \\
\text { CHLCRES }\end{array}$ & $\begin{array}{l}19 \\
30\end{array}$ & 4-Chloro-3-methylphenol & ppb & 20 & & 2 & 0 & \\
\hline $\begin{array}{l}\text { CHLCRES } \\
\text { CHLANIL }\end{array}$ & 30 & 4-Chloro-3-methylphenol & ppb & 5 & & 20 & 0 & \\
\hline $\begin{array}{l}\text { CHLANIL } \\
\text { 4CPPETH }\end{array}$ & & 4-Chloroaniline & ppb & 20 & & 2 & 0 & \\
\hline & & 4-Chlorophenylphenyl ether & ppb & 10 & & 2 & 0 & \\
\hline $\begin{array}{l}\text { MIBK } \\
\text { 4METHPH }\end{array}$ & & 4-Methyl-2-pentanone & ppb & 50 & & 26 & 0 & \\
\hline & & 4-Methylphenol & ppb & 10 & & 18 & 0 & \\
\hline $\begin{array}{l}\text { NITRANI } \\
\text { NITPHEN }\end{array}$ & & 4-Nitroaniline & ppb & 50 & & 2 & 0 & \\
\hline $\begin{array}{l}\text { NITPHEN } \\
\text { NITPHEN }\end{array}$ & $\begin{array}{l}19 \\
30\end{array}$ & 4-Nitrophenol & ppb & 50 & & 2 & 0 & \\
\hline $\begin{array}{l}\text { NITPHEN } \\
\text { GNITOUI }\end{array}$ & 30 & 4-Nitrophenol & ppb & 30 & & 20 & 0 & \\
\hline $\begin{array}{l}\text { 4NITOUI } \\
\text { NITRTOL }\end{array}$ & & 4-Nitroquinol ine-1-oxide & ppb & 10 & & 2 & 0 & \\
\hline NITRTOL & & 5-Nitro-0-toluidine & ppb & 10 & & 2 & 0 & \\
\hline DIMBENZ & & 7, 12-D ime thylbenz (a) anthracene & ppb & 10 & & 2 & 0 & \\
\hline ACENAPH & & Acenaph thene & ppb & 10 & & 2 & 0 & \\
\hline ACENATL & & Acenaphthylene & ppo & 10 & & 2 & 0 & \\
\hline ACETONE & & Acetone & ppb & 100 & & 26 & 0 & \\
\hline ACETILE & & Acetonitrile & ppb & 200 & & 2 & 0 & \\
\hline ACETOPH & & Acetophenone & ppo & 10 & & 2 & 0 & \\
\hline ACROL IN & & Acrolein & ppb & 5 & & 2 & 0 & \\
\hline ACRYILE & & Acrylonitrile & ppb & 5 & & 2 & 0 & \\
\hline ALDRIN & & Aldrin & ppob & .05 & & 20 & 0 & \\
\hline DIMPHAM & & Alpha, ol pha-D ime thylphenethyl ami & ppb & 10 & & 2 & 0 & \\
\hline O-BHC & & Alpha-BHC & ppb & .05 & & 20 & 0 & \\
\hline ALLUMNUM & & Aluminum & ppo & 200 & & 19 & 4 & \\
\hline FALUMIN & & Aluminum & ppb & 200 & & 19 & 0 & \\
\hline AMMONIU & 129 & Ammonium ion & ppb & 100 & & 19 & 0 & \\
\hline AMMONIU & 54 & Ammonium ion & ppb & 100 & & 1 & 0 & \\
\hline ANILINE & & Aniline & ppo & 10 & & 2 & 0 & \\
\hline ANTHRA & & Anthracene & ppb & 10 & & 2 & 0 & \\
\hline ANTIONY & & Ant imony & ppb & 200 & & 19 & 0 & \\
\hline FANTIMO & & Ant imony, filtered & ppob & 200 & & 19 & 0 & \\
\hline ARAMITE & & Aramite & ppb & 10 & & 2 & 0 & \\
\hline BENZENE & & Benzene & ppb & 5 & & 26 & 0 & \\
\hline BENZAAN & & Benzo( 8 ) anthracene & ppb & 10 & & 2 & 0 & \\
\hline BENZOPY & & Benzo(a)pyrene & ppb & 10 & & 2 & 0 & \\
\hline BENZBFL & & Benzo(b)fluoranthene & ppb & 10 & & 2 & 0 & \\
\hline BGHIPER & & Benzo(ghi)perylene & ppb & 10 & & 2 & 0 & \\
\hline BNZKFLU & & Benzo $(k)$ fluoranthene & ppb & 10 & & 2 & 0 & \\
\hline BENZOTH & & Benzothiazole & ppb & 10 & & 18 & 0 & \\
\hline BENZALC & & Benzyl alcohol & ppos & 20 & & 2 & 0 & \\
\hline BERYLUM & & Beryllium & ppb & 3 & & 19 & 0 & \\
\hline FBERYLL & & Beryllium, filtered & ppo & 3 & & 19 & 0 & \\
\hline b-BHC & & Beta-BHC & $\mathrm{ppb}$ & .05 & & 20 & 0 & \\
\hline BIS2CHM & & Bis (2-Chor oe thoxy)methane & ppb & 10 & & 2 & 0 & \\
\hline BISZCHE & & Bis(2-chloroethyl) ether & ppb & 10 & & 2 & 0 & \\
\hline BISZETH & & Bis(2-chloroisopropyl) ether & ppb & 10 & & 2 & 0 & \\
\hline BISZEPH & & Bis (2-ethylhexyl) ph thalate & ppb & 10 & & 18 & 0 & \\
\hline
\end{tabular}


Table 15-18. Constituent List and Summary of Results for the Low-Level Waste Burial Grounds WMA 3 Data for Reporting Period July 1

through September 30, 1993. (sheet 4 of 6)

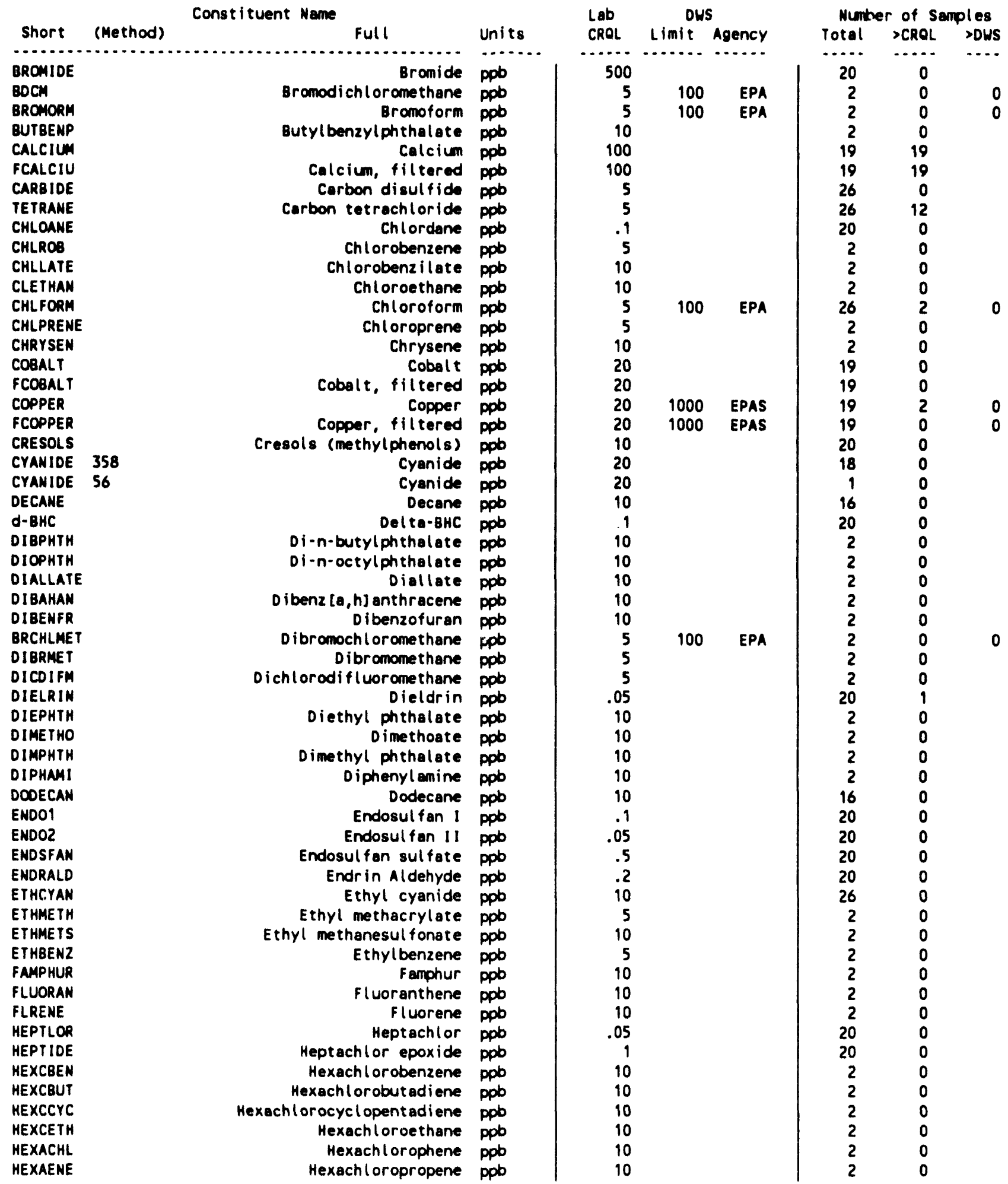




\section{Table 15-18. Constituent List and Summary of Results for the Low-Level Waste Burial Grounds WMA 3 Data for Reporting Period July 1 through September 30, 1993. (sheet 5 of 6)}

\begin{tabular}{|c|c|c|c|c|c|c|c|c|}
\hline & & Constituent Name & & Lab & Dus & Num & $r$ of $s$ & mples \\
\hline Short & (Method) & Full & Units & CROL & Limit Agency & Total & $>$ CROL & $>$ WWS \\
\hline INDENOP & & Indeno(1, 23 -cd)pyrene & $\infty$ & 10 & & ? & 0 & $\cdots$ \\
\hline : SOBUTY & & I sobutyl alcohol & ppo & 200 & & 2 & $\begin{array}{l}0 \\
0\end{array}$ & \\
\hline ISODR IN & & Isodrin & ppb & 10 & & 2 & 0 & \\
\hline I SOPHER & & Isophorone & ppo & 10 & & 2 & 0 & \\
\hline I SOSOLE & & Isosafrole & ppo & 10 & & 2 & 0 & \\
\hline KEPONE & & Kepone & ppb & 10 & & 2 & 0 & \\
\hline KEROSEN & & Kerosene & ppb & 10000 & & 2 & 0 & \\
\hline MAGNES & & Magnesium & ppb & 100 & & 19 & 19 & \\
\hline FMAGNES & & Magnesium, filtered & ppb & 100 & & 19 & 19 & \\
\hline METHACR & & Methacrylonitrile & ppo & 5 & & 2 & 0 & \\
\hline METHAPY & & Methapyrilene & ppb & 10 & & 2 & 0 & \\
\hline IOOOMET & & Methyl lodide & ppb & 5 & & 2 & 0 & \\
\hline METHBRO & & Methyl bromide & ppb & 10 & & 2 & 0 & \\
\hline METHCHL & & Methyl chloride & ppb & 10 & & 2 & 0 & \\
\hline METHONE & & Methyl ethyl ketone & ppob & 100 & & 26 & 0 & \\
\hline METACRY & & Methyl methacrylate & ppob & 5 & & 2 & 0 & \\
\hline METMSUL & & Methyl methanesul fonate & ppo & 10 & & 2 & 0 & \\
\hline METHYCH & & Methylene chloride & ppo & 5 & & 26 & 1 & \\
\hline DIPRNIT & & N-Nitroso-di-n-dipropylamine & ppo & 10 & & 2 & 0 & \\
\hline MNIBUTY & & $N$-Nitrosodi-n-butylamine & ppo & 10 & & 2 & 0 & \\
\hline MNIDIEY & & N-Nitrosodiethylamine & ppb & 10 & & 2 & 0 & \\
\hline NNIDIME & & $\mathrm{N}$-Nitrosodimethylamine & ppb & 10 & & 2 & 0 & \\
\hline NNDIPHA & & N-Nitrosodiphenylamine & ppb & 10 & & 2 & 0 & \\
\hline NAIMETH & & $N-N i$ trosome thylethylamine & ppb & 10 & & 2 & 0 & \\
\hline NNIMORP & & N-Nitrosomorphol ine & ppo & 10 & & 2 & 0 & \\
\hline NMIPIPE & & N-Nitrosopiperidine & ppb & 10 & & 2 & 0 & \\
\hline MAPHTHA & & Naph thal ene & ppb & 10 & & 18 & 0 & \\
\hline NICKEL & & Nickel & ppb & 30 & & 19 & 18 & \\
\hline FNICKEL & & Nickel, filtered & ppo & 30 & & 19 & 2 & \\
\hline NITRITE & & Nitrite & ppb & 200 & & 20 & 2 & \\
\hline NITBENZ & & Nitrobenzene & ppb & 10 & & 2 & 0 & \\
\hline NITRPYR & & Nitrosopyrrolidine & ppb & 10 & & 2 & 0 & \\
\hline TRIPHOS & & 0,0,0-Triethyl phosphorothioste & ppb & 10 & & 2 & 0 & \\
\hline DIPHOS & & 0,0-diethyl0-2-pyrazinylphosphor & ppb & 10 & & 2 & 0 & \\
\hline PARATHI & & Parathion & ppb & 10 & & 2 & 0 & \\
\hline PENTCHB & & Pentachlorobenzene & ppb & 10 & & 2 & 0 & \\
\hline PENTACH & & Pentachloroethane & ppb & 5 & & 2 & 0 & \\
\hline PENTCHN & & Pentachloronitrobenzene (PCNB) & ppb & 10 & & 2 & 0 & \\
\hline PENTCHP & 19 & Pentachlorophenol & ppb & 50 & & 18 & 0 & \\
\hline PENTCHP & 30 & Pentachlorophenol & ppb & 100 & & 20 & 0 & \\
\hline PHENTIN & & Phenacet in & ppb & 10 & & 2 & 0 & \\
\hline PHENANT & & Phenanthrene & ppb & 10 & & 2 & 0 & \\
\hline PHOSPHA & & Phosphate & ppb & 400 & & 20 & 0 & \\
\hline POTASUM & & Potassium & ppb & 300 & & 19 & 19 & \\
\hline FPOTASS & & Potassium, filtered & ppo & 300 & & 19 & 19 & \\
\hline PRONIDE & & Pronamide & ppb & 10 & & 2 & 0 & \\
\hline PYRENE & & Pyrene & ppb & 10 & & 2 & 0 & \\
\hline PYRIDIN & & Pyridine & ppob & 10 & & 2 & 0 & \\
\hline SAFROL & & Safrol & ppb & 10 & & 2 & 0 & \\
\hline STYRENE & & Styrene & ppb & 5 & & 2 & 0 & \\
\hline PERCENE & & Tetrachloroethene & ppob & 5 & & 26 & 0 & \\
\hline TETPHNL & & Tetrachlorophenols & ppo & 10 & & 20 & 0 & \\
\hline TETRADE & & Tetradecane & ppb & 10 & & 16 & 0 & \\
\hline PYROPHOS & & Tetraethyldithiopyrophosphate & ppb & 10 & & 2 & 0 & \\
\hline
\end{tabular}


Table 15-18. Constituent List and Summary of Results for the Low-Level Waste Burial Grounds WMA 3 Data for Reporting Period July 1

through September 30, 1993. (sheet 6 of 6)

\begin{tabular}{|c|c|c|c|c|c|c|c|c|c|}
\hline \multirow{3}{*}{\multicolumn{2}{|c|}{$\begin{array}{c}\text { Short (Method) } \\
\ldots \ldots\end{array}$}} & \multirow{2}{*}{$\begin{array}{l}\text { Constituent Name full } \\
\text { fore }\end{array}$} & \multirow[b]{2}{*}{ Units } & \multicolumn{3}{|c|}{ DWS } & \multicolumn{3}{|c|}{ Number of Semples } \\
\hline & & & & CROL & Limit & Agency & Total & $>C R Q L$ & $>$ DWS \\
\hline & & (1) & $\cdots$ & $\cdots \cdot$ & 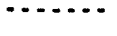 & $\cdots$ & $\cdots$ & $\cdots \cdot$ & $\cdots$ \\
\hline TAF & & Tetrahydrofuran & ppb & 10 & & & 26 & 0 & \\
\hline TIN & & Tin & ppb & 100 & & & 19 & 0 & \\
\hline FTIN & & Tin, filtered & ppb & 100 & & & 19 & 0 & \\
\hline TOLUENE & & Toluene & ppb & 5 & & & 26 & 1 & \\
\hline TRIBUTPH & & Tributyl Phosphate & ppob & 10 & & & 18 & 0 & \\
\hline TRICENE & & Trichloroethene & pppb & 5 & & & 26 & 1 & \\
\hline TRCMFLM & & Trichloromonof luor ome thane & ppb & 5 & & & 2 & 0 & \\
\hline TRIPHNL & & Irichlorophenols & ppb & 5 & & & 20 & 0 & \\
\hline TOICHPH & & Tris-2-chloroethyl phosphate & ppb & 10 & & & 18 & 0 & \\
\hline TRITIUM & & Iritiun & $\mathrm{PCi} / \mathrm{L}$ & 500 & 20000 & EPA & 20 & 8 & 0 \\
\hline URANIUM & & Uranium & ppob & .5 & & & 20 & 20 & \\
\hline VANADUM & & Vanadium & ppb & 30 & & & 19 & 1 & \\
\hline FVANADI & & Vanadium, filtered & ppb & 30 & & & 19 & 1 & \\
\hline VINYLAC & & Vinyl acetate & ppb & 5 & & & 2 & 0 & \\
\hline VINYIDE & & Vinyl chloride & ppb & 10 & 2 & EPA & 26 & 0 & 0 * \\
\hline XYLENE & & xylenes (total) & ppo & 5 & & & 26 & 0 & \\
\hline ZINC & & Zinc & ppb & 10 & & & 19 & 11 & \\
\hline FZINC & & Zinc, filtered & ppb & 10 & & & 19 & 4 & \\
\hline ALLYLCL & & - llylchloride & ppb & 100 & & & 2 & 0 & \\
\hline TOICPENE & & cis-1,3-Dichloropropene & ppb & 5 & & & 2 & 0 & \\
\hline MCRESOL & & $m$-Cresol & ppb & 10 & & & 18 & 0 & \\
\hline DINBENZ & & m-dini trabenzene & ppob & 10 & & & 2 & 0 & \\
\hline OTOLHYD & & 0-Toluidine & ppb & 10 & & & 2 & 0 & \\
\hline DIMEAMB & & p-Dimethylaminoazobenzene & ppb & 10 & & & 2 & 0 & \\
\hline PHENDIA & & p-Phenylenediemine & ppb & 10 & & & 2 & 0 & \\
\hline SYMTRIN & & sym-irinitrobenzene & ppo & 10 & & & 2 & $\mathbf{0}$ & \\
\hline DICPENE & & trans-1,3-0ichloropropene & ppt & $\mathbf{5}$ & & & 2 & 0 & \\
\hline TOIBUTEN & & trans-1,4-dichloro-2-butene & ppb & 5 & & & 2 & 0 & \\
\hline
\end{tabular}

For explanation of this table, see section 1.4 of report. 
Table 15-19. Constituents with at Least One Value Above the CRQL for the Low-Level Waste Burial Grounds WMA 3 Data for Reporting Period July 1 through September 30, 1993. (sheet 1 of 11)

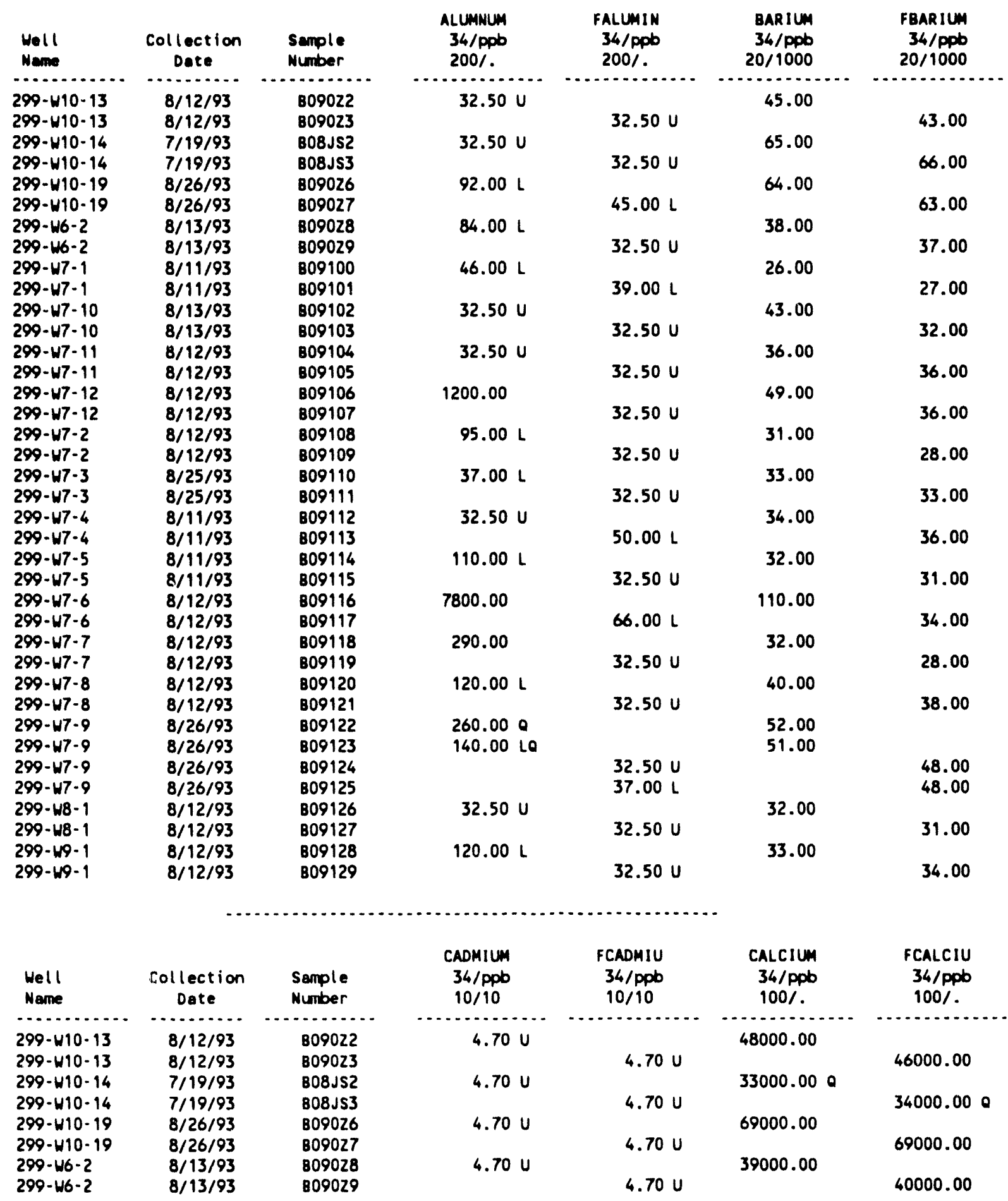


Table 15-19. Constituents with at Least One Value Above the CRQL for the Low-Level Waste Burial Grounds WMA 3 Data for Reporting Period July 1 through September 30, 1993. (sheet 2 of 11)

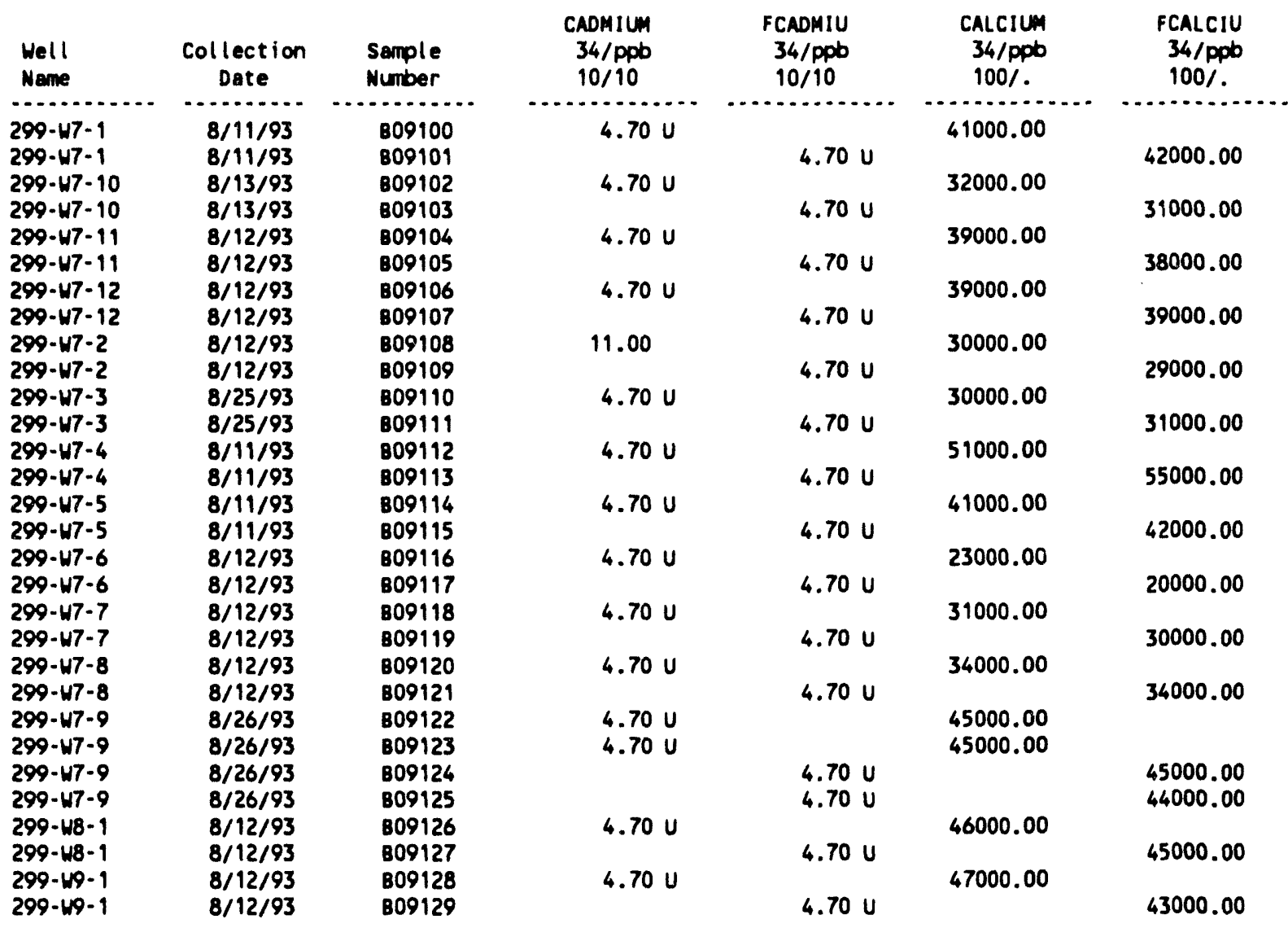

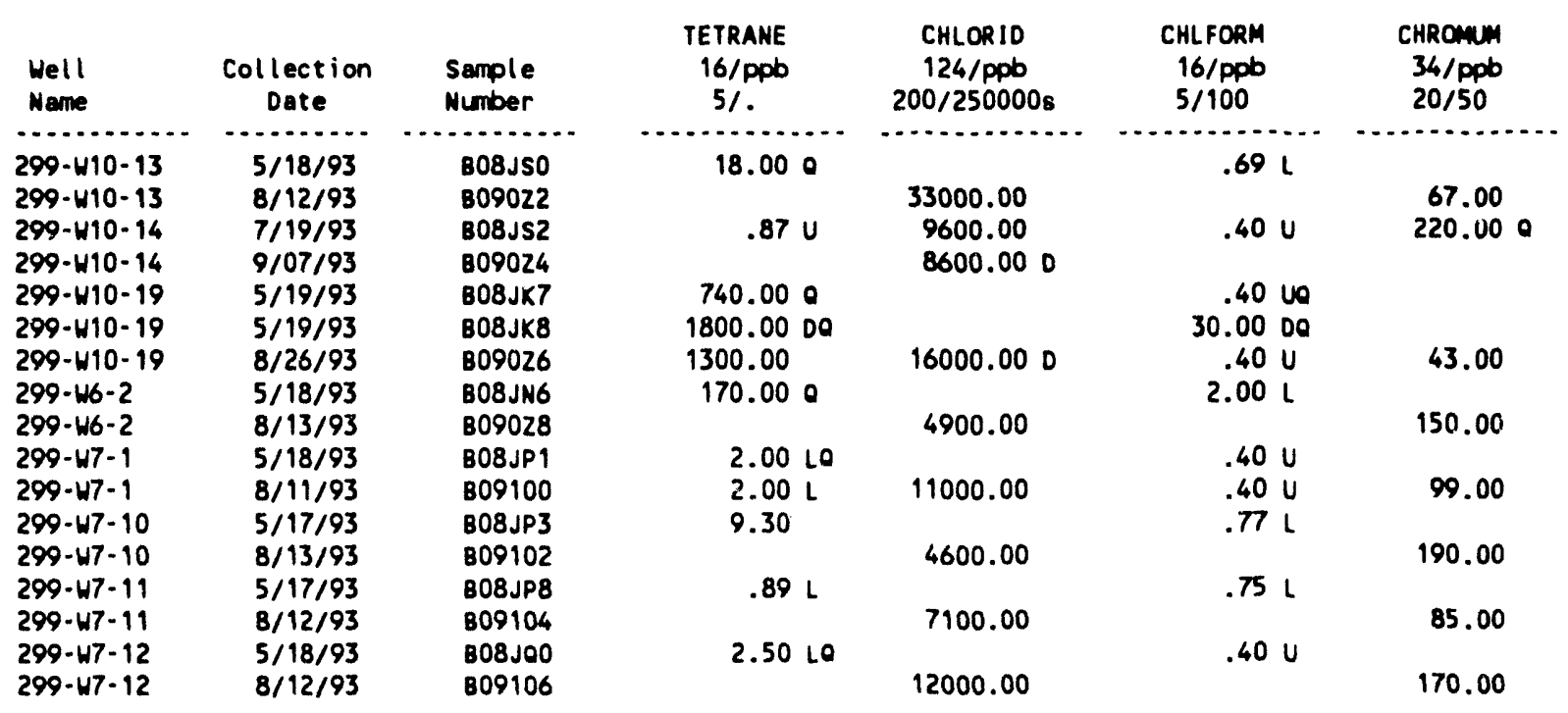


Table 15-19. Constituents with at Least One Value Above the CRQL for the Low-Level Waste Burial Grounds WMA 3 Data for Reporting Period July 1 through September 30, 1993. (sheet 3 of 11)

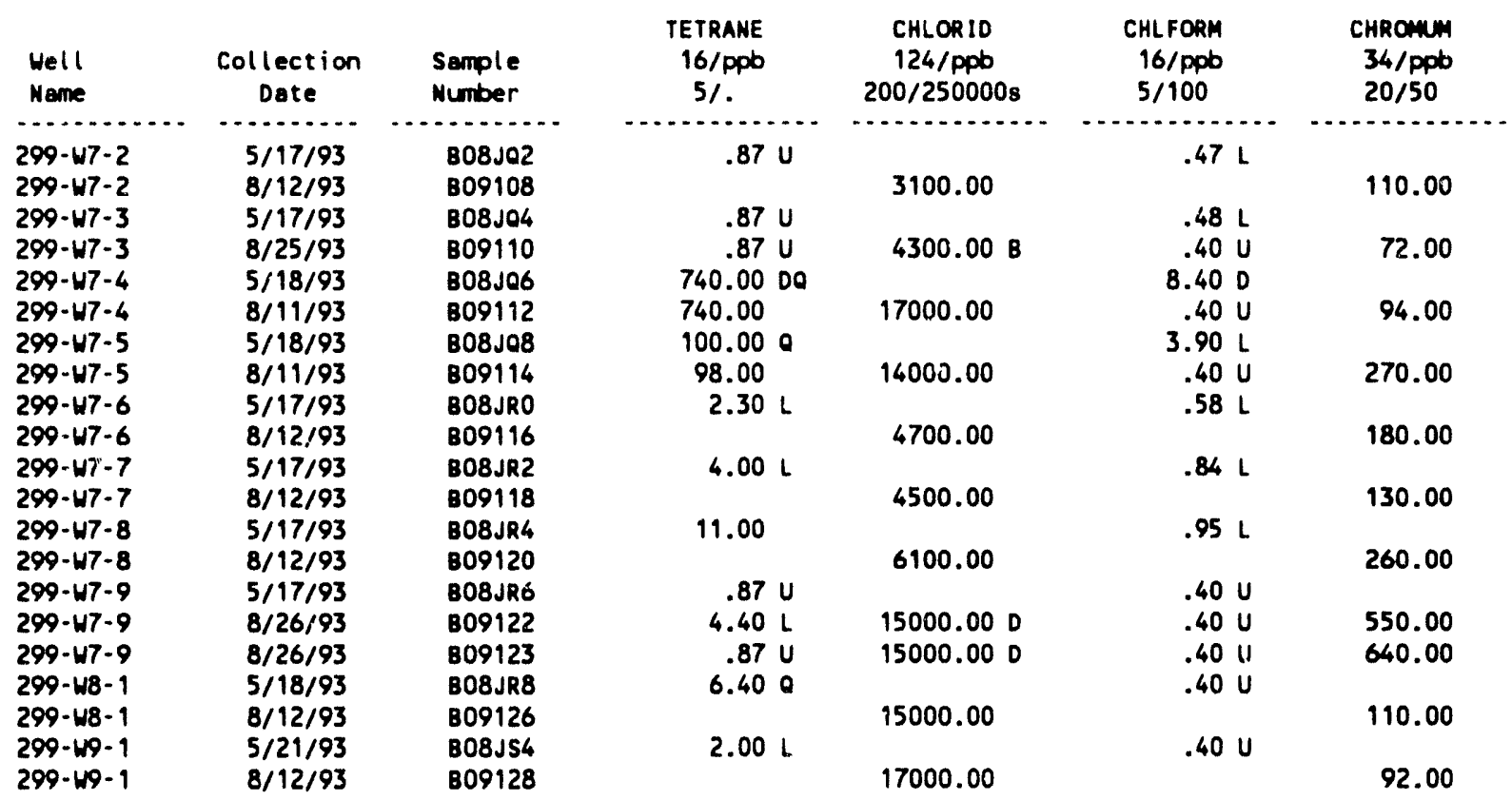

\begin{tabular}{|c|c|c|c|c|c|c|}
\hline $\begin{array}{l}\text { Well } \\
\text { Neme }\end{array}$ & $\begin{array}{c}\text { Collection } \\
\text { Date }\end{array}$ & $\begin{array}{l}\text { Sample } \\
\text { Number }\end{array}$ & $\begin{array}{l}\text { FCHROMI } \\
34 / \text { PpD } \\
20 / 50\end{array}$ & $\begin{array}{c}\text { COPPER } \\
34 / \text { ppb } \\
20 / 10008\end{array}$ & $\begin{array}{c}\text { FCOPPER } \\
34 / \text { Ppb } \\
20 / 10008\end{array}$ & $\begin{array}{l}\text { DIELRIN } \\
17 / \text { PPD } \\
.05 \% .\end{array}$ \\
\hline $\begin{array}{l}299-w 10-13 \\
299-w 10-13\end{array}$ & $\begin{array}{l}8 / 12 / 93 \\
8 / 12 / 93\end{array}$ & $\begin{array}{l}809022 \\
809023\end{array}$ & $5.42 \mathrm{U}$ & $2.65 U$ & $2.65 \mathrm{U}$ & $.02 U$ \\
\hline $299-\$ 10-14$ & $7 / 19 / 93$ & B08Js2 & & $4.10 \mathrm{~L}$ & & $.02 u$ \\
\hline $299-\$ 10-14$ & $7 / 19 / 93$ & B08JS3 & $8.40 \mathrm{LQ}$ & & $2.65 \mathrm{U}$ & \\
\hline $299 \cdot W 10-14$ & $9 / 07 / 93$ & B09024 & & & & $.02 \mathrm{U}$ \\
\hline $299-W 10-19$ & $8 / 26 / 93$ & B09026 & & $2.65 \mathrm{U}$ & & $.02 \mathrm{U}$ \\
\hline $299-w 10-19$ & $8 / 26 / 93$ & B09027 & $16.00 \mathrm{~L}$ & & $2.65 \mathrm{U}$ & \\
\hline $299-146-2$ & $8 / 13 / 93$ & 809028 & & $2.65 U$ & & $.02 \mathrm{U}$ \\
\hline $299-166-2$ & $8 / 13 / 93$ & 809029 & $5.42 \mathrm{U}$ & & $2.65 U$ & \\
\hline $299-w 7-1$ & $8 / 11 / 93$ & B09100 & & $2.65 U$ & & $.02 \mathrm{U}$ \\
\hline $299-47-1$ & $8 / 11 / 93$ & B09101 & $5.42 \mathrm{U}$ & & $2.65 \mathrm{U}$ & \\
\hline $299-47-10$ & $8 / 13 / 93$ & 809102 & & $2.65 \mathrm{U}$ & & $.02 \mathrm{U}$ \\
\hline $299-W 7-10$ & $8 / 13 / 93$ & B09103 & $5.42 \mathrm{U}$ & & $2.65 \mathrm{U}$ & \\
\hline $299-47-91$ & $8 / 12 / 93$ & 809104 & & $2.65 \mathrm{U}$ & & $.02 \mathrm{U}$ \\
\hline $299-w 7-11$ & $8 / 12 / 93$ & 809105 & $5.42 \mathrm{U}$ & & $2.65 \mathrm{U}$ & \\
\hline $299-w 7-12$ & $8 / 12 / 93$ & B09106 & & $2.65 \mathrm{U}$ & & $.02 \mathrm{U}$ \\
\hline $299-47-12$ & $8 / 12 / 93$ & 809107 & $5.42 \mathrm{U}$ & & $2.65 \mathrm{U}$ & \\
\hline $299-47-2$ & $8 / 12 / 93$ & B09108 & & $2.65 \mathrm{U}$ & & .0200 \\
\hline $299-47-2$ & $8 / 12 / 93$ & 809109 & $5.42 \mathrm{U}$ & & $2.65 \mathrm{U}$ & \\
\hline $299-47-3$ & $8 / 25 / 93$ & 809110 & & $2.65 \mathrm{U}$ & & $.02 \mathrm{U}$ \\
\hline $299-47-3$ & $8 / 25 / 93$ & B09111 & $5.42 \mathrm{U}$ & & $2.65 \mathrm{U}$ & \\
\hline $299-\$ 7-4$ & $8 / 11 / 93$ & B09112 & & $2.65 \mathrm{U}$ & & $.02 \mathrm{U}$ \\
\hline $299-67-4$ & $8 / 11 / 93$ & 809113 & $11.00 \mathrm{~L}$ & & $2.65 \mathrm{U}$ & \\
\hline $299-47-5$ & $8 / 11 / 93$ & B09114 & & $7.40 \mathrm{~L}$ & & $.02 \mathrm{U}$ \\
\hline $99-W 7-5$ & $8 / 11 / 93$ & B09115 & $9.50 \mathrm{~L}$ & & $2.65 \mathrm{U}$ & \\
\hline
\end{tabular}


Table 15-19. Constituents with at Least One Value Above the CRQL for the Low-Level Waste Burial Grounds WMA 3 Data for Reporting Period July 1 through September 30, 1993. (sheet 4 of 11)

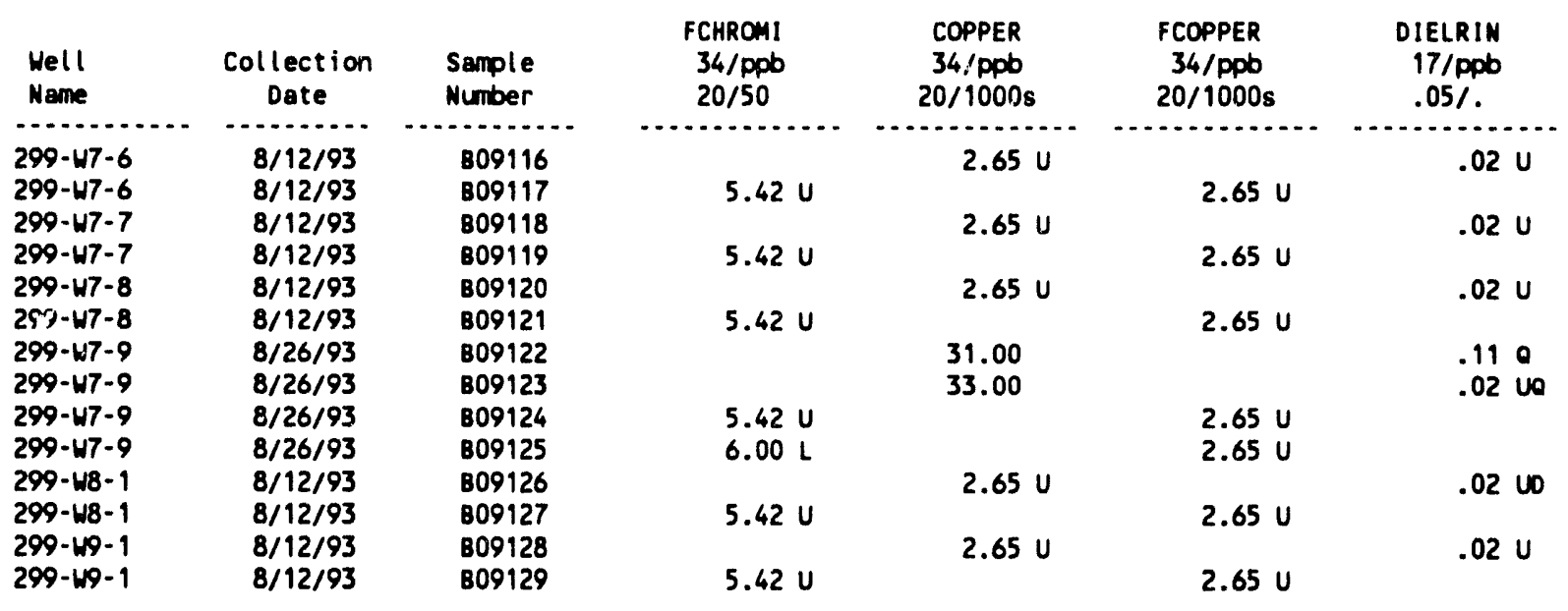

\begin{tabular}{|c|c|c|c|c|c|c|c|}
\hline $\begin{array}{l}\text { Well } \\
\text { Name }\end{array}$ & $\begin{array}{c}\text { Collection } \\
\text { Date }\end{array}$ & $\begin{array}{l}\text { Semple } \\
\text { Number }\end{array}$ & $\begin{array}{l}\text { FLUOR ID } \\
124 / \mathrm{ppb} \\
100 / 4000\end{array}$ & $\begin{array}{c}\text { ALPHA } \\
135 / \mathrm{pC} \mathrm{i} / \mathrm{L} \\
. / 15\end{array}$ & $\begin{array}{c}\text { BETA } \\
136 / \mathrm{pCi} / \mathrm{L} \\
. / 50\end{array}$ & $\begin{array}{c}\text { IRON } \\
34 / \mathrm{ppb} \\
20 / 300 \mathrm{~s}\end{array}$ & \\
\hline 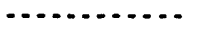 & $\ldots \ldots \ldots$ & $\ldots \ldots \ldots$ & , & 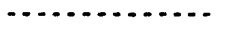 & $\ldots \ldots$ & 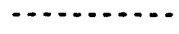 & \\
\hline $299-W 10-13$ & $8 / 12 / 93$ & 809022 & 500.00 & $1.03 \mathrm{U}$ & 6.94 & 340.00 & \\
\hline $\begin{array}{l}299-410-14 \\
299-410-14\end{array}$ & $\begin{array}{l}7 / 19 / 93 \\
9 / 07 / 03\end{array}$ & $\begin{array}{l}\text { B08JS2 } \\
809074\end{array}$ & 600.00 & $.36 U$ & 4.67 & 930.00 & 0 \\
\hline $299-W 10-14$ & $9 / 07 / 93$ & 809024 & 400.00 & $1.27 \mathrm{U}$ & 6.63 & & \\
\hline $299-410-19$ & $8 / 26 / 93$ & B09026 & 400.00 & 6.02 & 18.90 & 310.00 & \\
\hline $299-146-2$ & $8 / 13 / 93$ & B09028 & 400.00 & $.79 \mathrm{U}$ & 9.24 & 770.00 & \\
\hline $299-47-1$ & $8 / 11 / 93$ & 809100 & 400.00 & $-.31 \mathrm{U}$ & 4.05 & 480.00 & a \\
\hline $299-47-10$ & $8 / 13 / 93$ & B09102 & 500.00 & 2.76 & 6.21 & 910.00 & \\
\hline $299-47-11$ & $8 / 12 / 93$ & B09104 & 400.00 & 1.32 & 6.50 & 390.00 & \\
\hline $299-47-12$ & $8 / 12 / 93$ & B09106 & 400.00 & 1.63 & 8.17 & 3800.00 & \\
\hline $299-w 7-2$ & $8 / 12 / 93$ & B09108 & 400.00 & $.78 \mathrm{U}$ & 5.23 & 910.00 & \\
\hline $299-47-3$ & $8 / 25 / 93$ & 809110 & $400.00 \mathrm{~B}$ & $-.36 \mathrm{U}$ & 5.55 & 390.00 & \\
\hline $299-47-4$ & $8 / 11 / 93$ & B09112 & 500.00 & 1.96 & 13.10 & 380.00 & 0 \\
\hline $299-W 7-5$ & $8 / 11 / 93$ & $B 09114$ & 400.00 & $.63 \mathrm{U}$ & 6.90 & 1200.00 & 0 \\
\hline $299-47-6$ & $8 / 12 / 93$ & $B 09116$ & 500.00 & 15.50 & 15.60 & 7600.00 & \\
\hline $299-W 7-7$ & $8 / 12 / 93$ & $B 09118$ & 400.00 & 1.24 & 4.21 & 1200.00 & \\
\hline $299-47-8$ & $8 / 12 / 93$ & 809120 & 400.00 & $1.25 \mathrm{U}$ & 4.55 & 1400.00 & \\
\hline $299-47-9$ & $8 / 26 / 93$ & 809122 & 300.000 & $.68 \mathrm{va}$ & 8.210 & 3100.00 & \\
\hline $299-47-9$ & $8 / 26 / 93$ & 809123 & $400.00 a$ & 3.690 & 5.010 & 3100.00 & \\
\hline $299-48-1$ & $8 / 12 / 93$ & 809126 & 400.00 & $.85 \mathrm{U}$ & 5.43 & 470.00 & \\
\hline $299-149-1$ & $8 / 12 / 93$ & $B 09128$ & 400.00 & $.37 \mathrm{U}$ & 6.88 & 1100.00 & \\
\hline $\begin{array}{l}\text { Well } \\
\text { Name }\end{array}$ & $\begin{array}{c}\text { Collection } \\
\text { Date }\end{array}$ & $\begin{array}{l}\text { Semple } \\
\text { Number }\end{array}$ & $\begin{array}{c}\text { FIRON } \\
34 / \mathrm{ppb} \\
20 / 300 \mathrm{~s}\end{array}$ & $\begin{array}{l}\text { LEAD } \\
\text { 40/ppb } \\
5 / 50\end{array}$ & $\begin{array}{l}\text { FLEAD } \\
40 / \mathrm{ppb} \\
5 / 50\end{array}$ & $\begin{array}{l}\text { MAGNES } \\
34 / \text { ppb } \\
100 / .\end{array}$ & \\
\hline$\ldots \ldots \ldots$ & $\ldots \ldots \ldots$ & 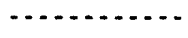 & $\ldots \ldots \ldots$ & - & $\ldots \ldots$ & $\ldots \ldots$ & \\
\hline $299-W 10-13$ & $8 / 12 / 93$ & B090Z2 & & $2.80 \mathrm{~L}$ & & 16000.00 & \\
\hline $299-w 10-13$ & $8 / 12 / 93$ & 809023 & 44.00 & & $.71 \mathrm{~L}$ & & \\
\hline $299-W 10-14$ & $7 / 19 / 93$ & B08JS2 & & $1.20 \mathrm{~L}$ & & 12000.00 & \\
\hline $299-W 10-14$ & $7 / 19 / 93$ & B08JS3 & 70.000 & & $.92 \mathrm{~L}$ & & \\
\hline $299-W 10-14$ & $9 / 07 / 93$ & 809024 & & $.85 \mathrm{LB}$ & & & \\
\hline $299-410-14$ & $9 / 07 / 93$ & 809025 & & & $.73 \mathrm{LB}$ & & \\
\hline $299-410-19$ & $8 / 26 / 93$ & B09026 & & $1.40 \mathrm{~L}$ & & 21000.00 & \\
\hline $299 \cdot H 10-19$ & $8 / 26 / 93$ & B09027 & $17.00 \mathrm{~L}$ & & $.51 \mathrm{U}$ & & \\
\hline
\end{tabular}


Table 15-19. Constituents with at Least One Value Above the CRQL for the Low-Level Waste Burial Grounds WMA 3 Data for Reporting Period July 1 through September 30,1993. (sheet 5 of 11)

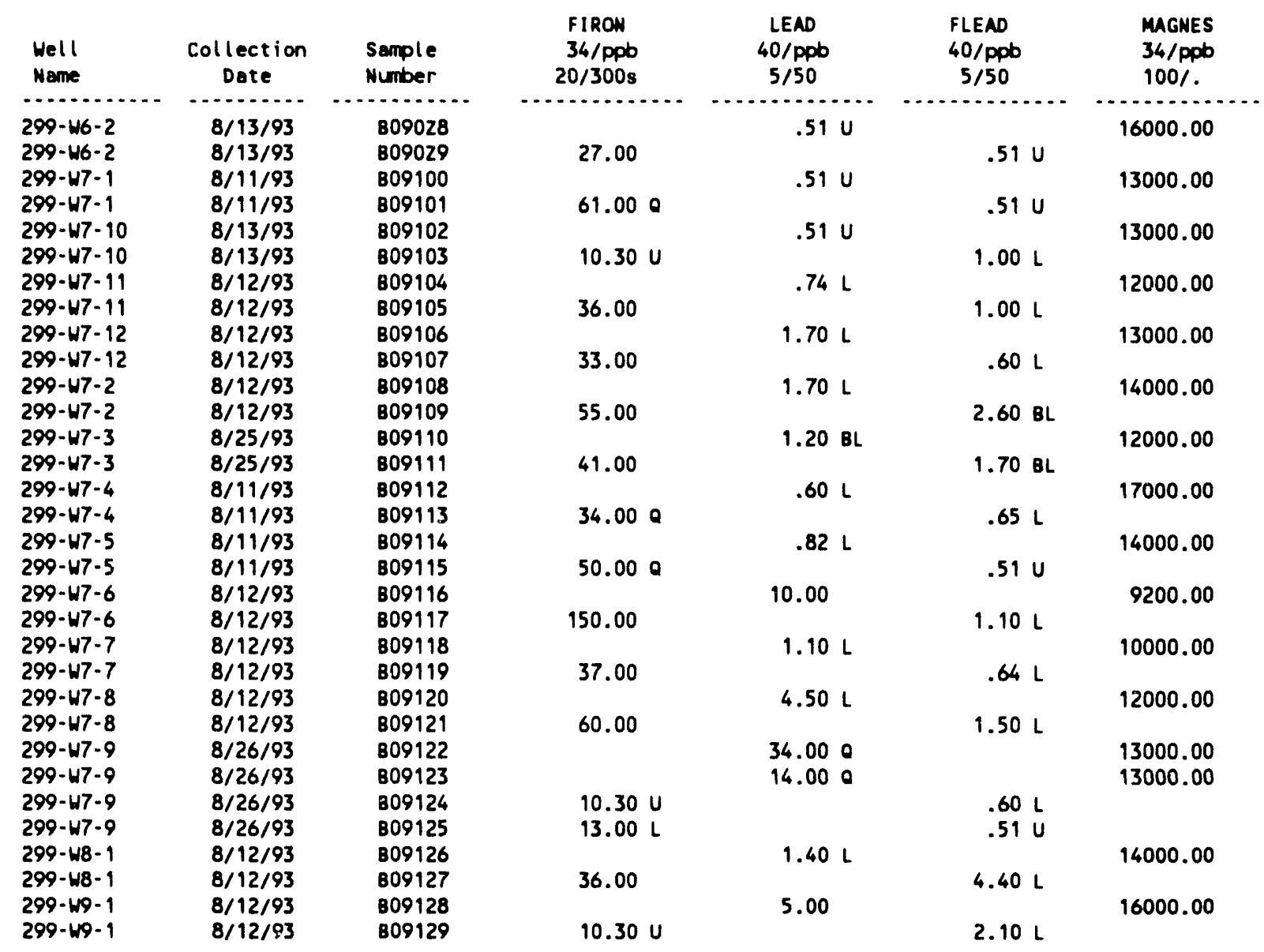

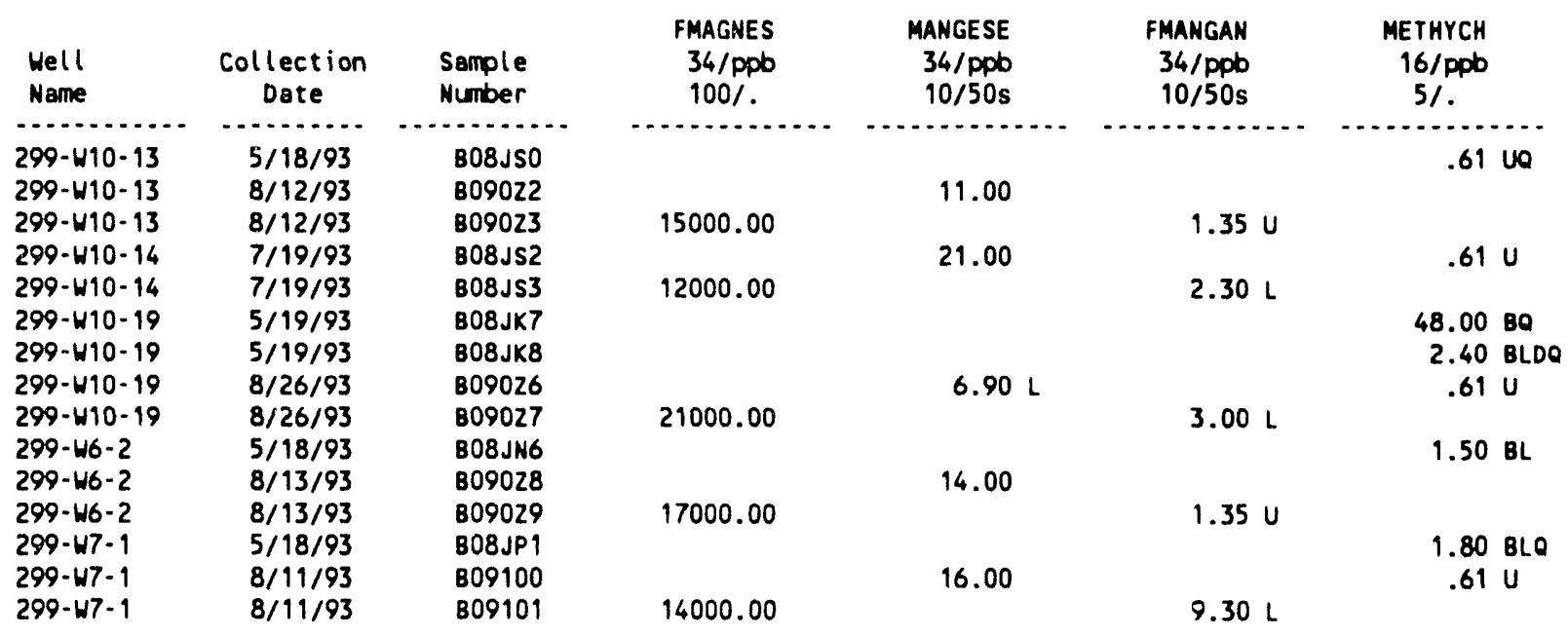


Table 15-19. Constituents with at Least One Value Above the CRQL for the Low-Level Waste Burial Grounds WMA 3 Data for Reporting Period July 1 through September 30, 1993. (sheet 6 of 11)

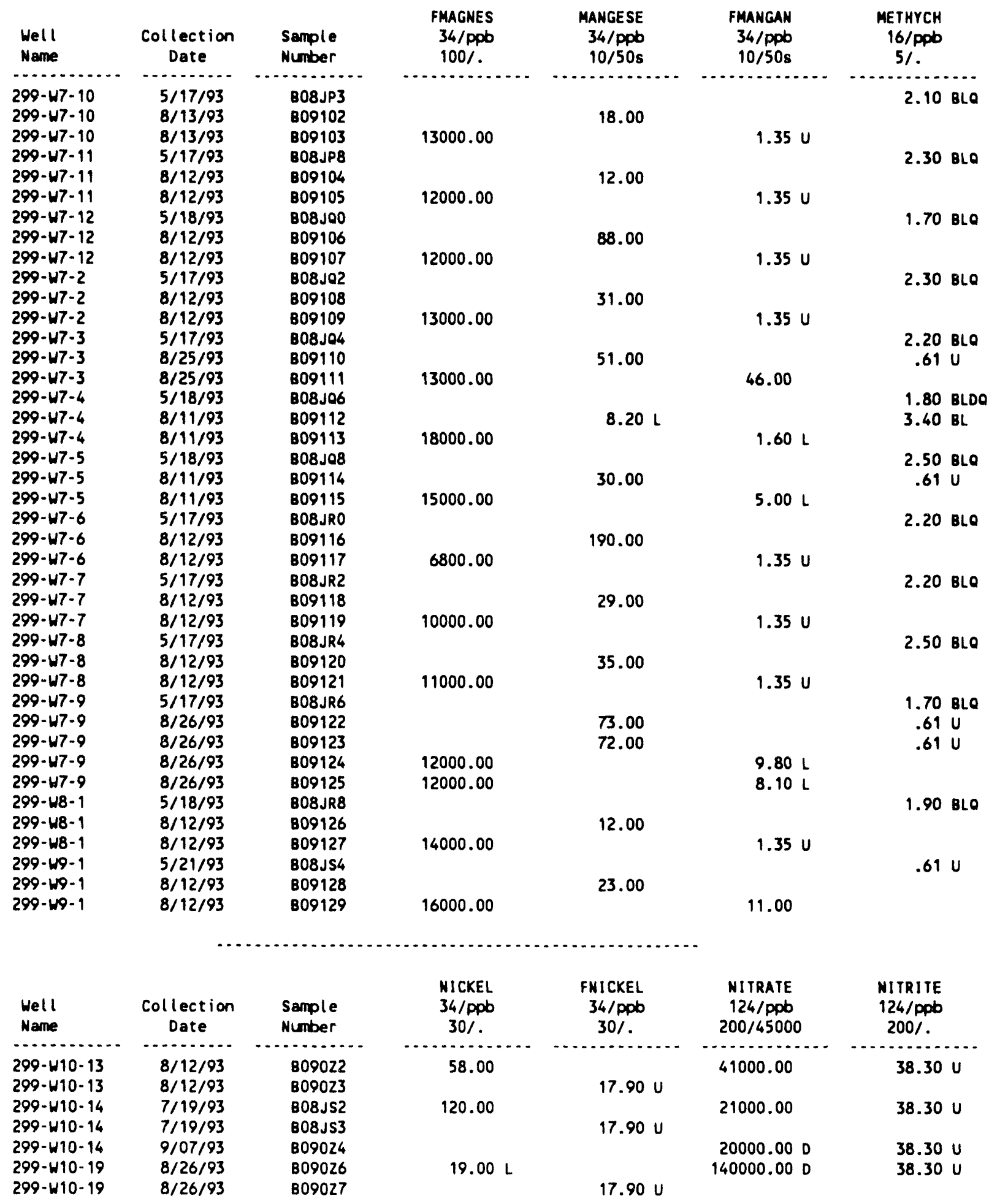


Table 15-19. Constituents with at Least One Value Above the CRQL for the Low-Level Waste Burial Grounds WMA 3 Data for Reporting Period July 1 through September 30, 1993. (sheet 7 of 11)

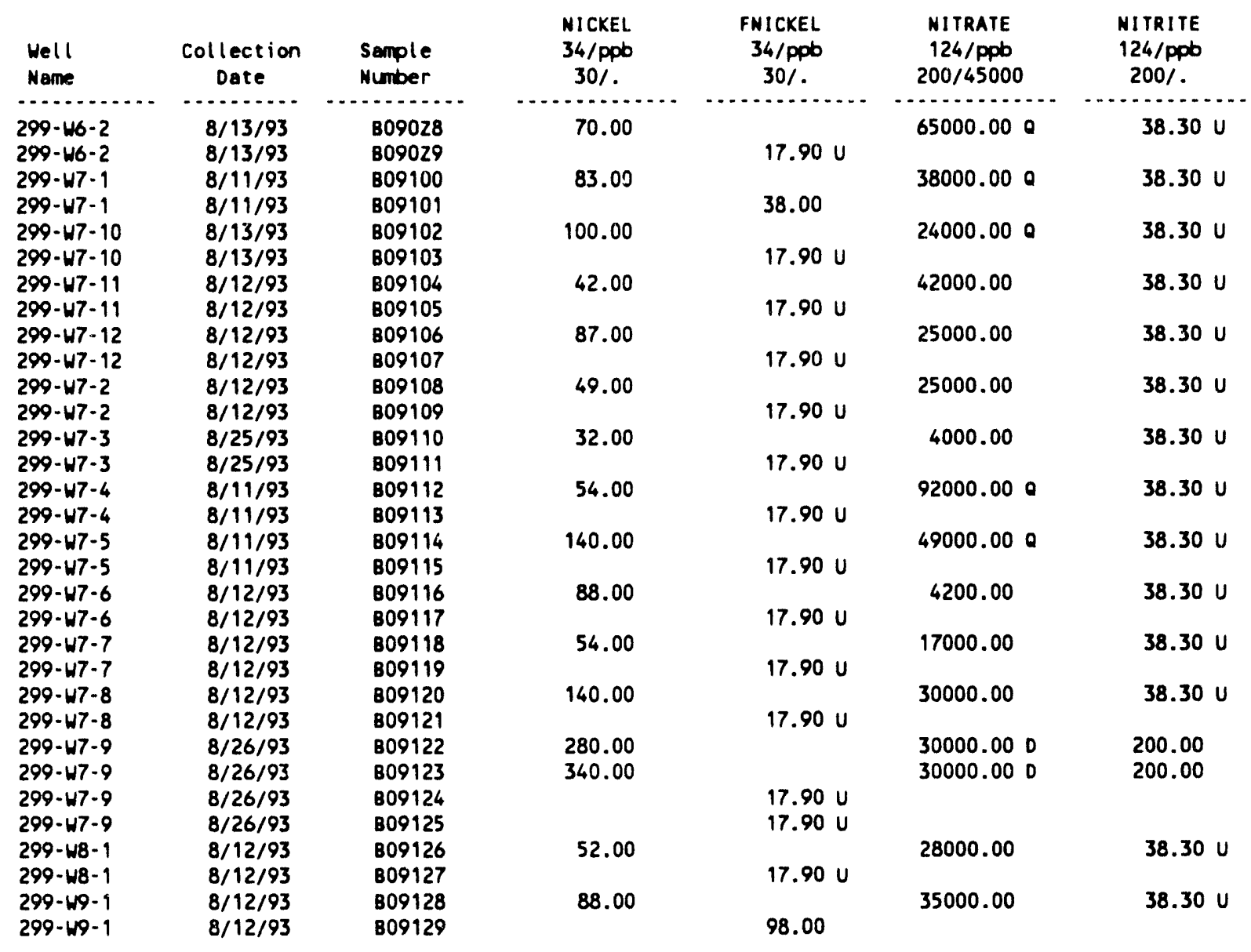

\begin{tabular}{|c|c|c|c|c|c|c|}
\hline $\begin{array}{l}\text { Well } \\
\text { Name }\end{array}$ & $\begin{array}{c}\text { Collection } \\
\text { Date }\end{array}$ & $\begin{array}{l}\text { Sample } \\
\text { Number }\end{array}$ & $\begin{array}{c}\text { LPHENOL } \\
30 / p p b \\
1 / .\end{array}$ & $\begin{array}{l}\text { POTASUM } \\
34 / \mathrm{ppD} \\
1000 / .\end{array}$ & $\begin{array}{l}\text { FPOTASS } \\
34 / \mathrm{ppb} \\
1000 / .\end{array}$ & $\begin{array}{c}\text { RADIUM } \\
137 / \mathrm{pCi} / \mathrm{L} \\
. / .\end{array}$ \\
\hline $299-w 10-13$ & $8 / 12 / 93$ & 809022 & 310 & 4700.00 & & 22 \\
\hline $299-w 10-13$ & $8 / 12 / 93$ & 809023 & & $4 r 00.00$ & 4600.00 & \\
\hline $299-W 10-14$ & $7 / 19 / 93$ & B08JS2 & $.31 \mathrm{U}$ & 4200.00 & & .18 \\
\hline $299-W 10-14$ & $7 / 19 / 93$ & B08.JS3 & & & 4300.00 & \\
\hline $299-W 10-14$ & $9 / 07 / 93$ & 809024 & $.31 \mathrm{U}$ & & & $.05 \mathrm{U}$ \\
\hline $\begin{array}{l}299-W 10-19 \\
299-W 10-19\end{array}$ & $\begin{array}{l}8 / 26 / 93 \\
8 / 26 / 93\end{array}$ & $\begin{array}{l}\text { B09026 } \\
\text { B09027 }\end{array}$ & 1.20 & 4600.00 & 4600.00 & .30 \\
\hline $\begin{array}{l}299-116-2 \\
299-166-2\end{array}$ & $\begin{array}{l}8 / 13 / 93 \\
8 / 13 / 93\end{array}$ & $\begin{array}{l}809028 \\
809029\end{array}$ & $.31 \mathrm{U}$ & 2700.00 & 2900.00 & $.09 \mathrm{U}$ \\
\hline $\begin{array}{l}299-67-1 \\
299-67-1\end{array}$ & $\begin{array}{l}8 / 11 / 93 \\
8 / 11 / 93\end{array}$ & $\begin{array}{l}\text { B09100 } \\
\text { B09101 }\end{array}$ & $.31 \mathrm{U}$ & 2900.00 & 3300.00 & $.04 \mathrm{U}$ \\
\hline $\begin{array}{l}299-W 7-10 \\
299-W 7-10\end{array}$ & $\begin{array}{l}8 / 13 / 93 \\
8 / 13 / 93\end{array}$ & $\begin{array}{l}\text { B09102 } \\
\text { B09103 }\end{array}$ & $.31 \mathrm{U}$ & 2600.00 & 2200.00 & .29 \\
\hline $\begin{array}{l}299-W 7-11 \\
299-W 7-11\end{array}$ & $\begin{array}{l}8 / 12 / 93 \\
8 / 12 / 93\end{array}$ & $\begin{array}{l}\text { B09104 } \\
\text { B09105 }\end{array}$ & $.31 \mathrm{U}$ & 4000.00 & 3700.00 & .14 \\
\hline $\begin{array}{l}299-W 7-12 \\
299-47-12\end{array}$ & $\begin{array}{l}8 / 12 / 93 \\
8 / 12 / 93\end{array}$ & $\begin{array}{l}809106 \\
809107\end{array}$ & $.31 \mathrm{U}$ & 3800.00 & 4100.00 & .16 \\
\hline
\end{tabular}


Table 15-19. Constituents with at Least One Value Above the CRQL for the Low-Level Waste Burial Grounds WMA 3 Data for Reporting Period July 1 through September 30, 1993. (sheet 8 of 11)

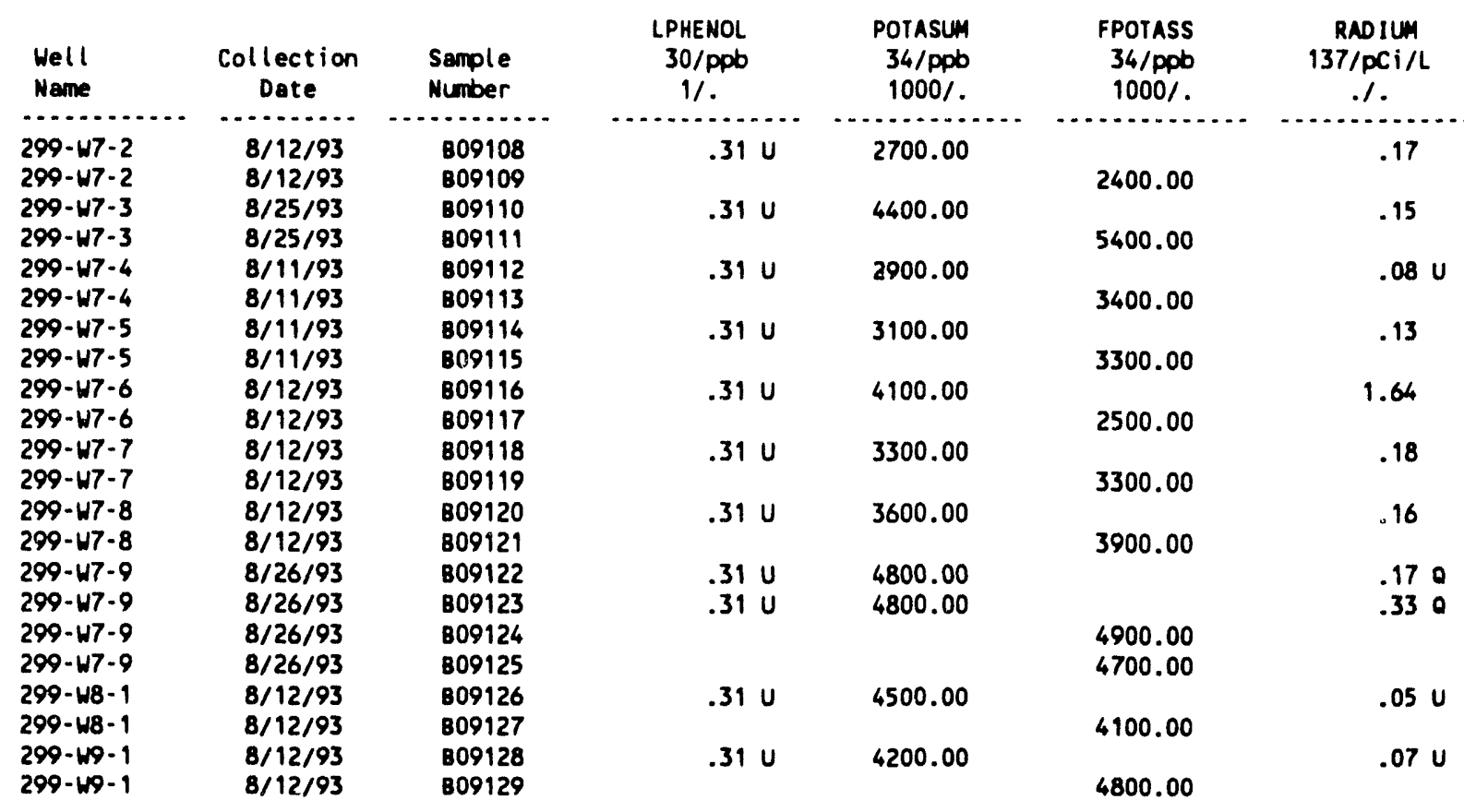

\begin{tabular}{|c|c|c|c|c|c|c|}
\hline $\begin{array}{l}\text { Well } \\
\text { Name }\end{array}$ & $\begin{array}{c}\text { Collection } \\
\text { Date }\end{array}$ & $\begin{array}{l}\text { Sample } \\
\text { Number }\end{array}$ & $\begin{array}{l}\text { S00IUM } \\
34 / \text { ppb } \\
300 \%\end{array}$ & $\begin{array}{l}\text { FSODIUM } \\
34 / \mathrm{ppb} \\
300 \%\end{array}$ & $\begin{array}{c}\text { SULFATE } \\
124 / \mathrm{ppb} \\
500 / 250000 \mathrm{~s}\end{array}$ & $\begin{array}{l}\text { TOLUENE } \\
16 / \text { ppb } \\
5 / .\end{array}$ \\
\hline $299-w 10-13$ & $5 / 18 / 93$ & B08JSO & . & . & . & $.73 \mathrm{U}$ \\
\hline $\begin{array}{l}299-W 10-13 \\
299-W 10-13\end{array}$ & $\begin{array}{l}8 / 12 / 93 \\
8 / 12 / 93\end{array}$ & $\begin{array}{l}\text { B09022 } \\
\text { B09023 }\end{array}$ & 12000.00 & 12000.00 & 39000.00 & \\
\hline $299-W 10-14$ & $7 / 19 / 93$ & B08JS2 & $15000.00 a$ & & 24000.00 & $.73 \mathrm{U}$ \\
\hline $299-W 10-14$ & $7 / 19 / 93$ & B08JS3 & & 15000.000 & & \\
\hline $299-W 10-14$ & $9 / 07 / 93$ & 809024 & & & $24000.00 \mathrm{D}$ & \\
\hline $299-410-19$ & $5 / 19 / 93$ & B08JK7 & & & & 23.0080 \\
\hline $299-W 10-19$ & $5 / 19 / 93$ & B08JK8 & & & & .73 voe \\
\hline $299-W 10-19$ & $8 / 26 / 93$ & B09026 & 13000.00 & & $42000.00 \mathrm{D}$ & $.73 \mathrm{U}$ \\
\hline $\begin{array}{l}299-W 10-19 \\
299-W 6-2\end{array}$ & $\begin{array}{l}8 / 26 / 93 \\
5 / 18 / 93\end{array}$ & $\begin{array}{l}\text { B09027 } \\
\text { B08JN6 }\end{array}$ & & 13000.00 & & $.73 \mathrm{u}$ \\
\hline 299-W6-2 & $8 / 13 / 93$ & B09028 & 9800.00 & & 26000.00 & ע ת \\
\hline $299-116-2$ & $8 / 13 / 93$ & B09029 & & 10000.00 & & \\
\hline $299-47-1$ & $5 / 18 / 93$ & B08JP1 & & & & $.73 \mathrm{U}$ \\
\hline $299-W 7-1$ & $8 / 11 / 93$ & B09100 & 8400.000 & & 49000.00 & $.73 \mathrm{U}$ \\
\hline $299-W 7-1$ & $8 / 11 / 93$ & B09101 & & 8700.000 & & \\
\hline $299-W 7-10$ & $5 / 17 / 93$ & B08JP3 & & & & $.73 \mathrm{U}$ \\
\hline $299-W 7-10$ & $8 / 13 / 93$ & 809102 & 8200.00 & & 22000.00 & \\
\hline $299-W 7-10$ & $8 / 13 / 93$ & 809103 & & 8000.00 & & \\
\hline $299-W 7-11$ & $5 / 17 / 93$ & B08JP8 & & & & $.73 \mathrm{U}$ \\
\hline $299-w 7-11$ & $8 / 12 / 93$ & 809104 & 10000.00 & & 31000.00 & \\
\hline $299-w 7-11$ & $8 / 12 / 93$ & 809105 & & 9900.00 & & \\
\hline $299-W 7-12$ & $5 / 18 / 93$ & $808 \mathrm{~J} 00$ & & & & $.73 \mathrm{U}$ \\
\hline $299-67-12$ & $8 / 12 / 93$ & 809106 & 9000.00 & & 31000.00 & \\
\hline $299-W 7-12$ & $8 / 12 / 93$ & B09107 & & 8900.00 & & \\
\hline
\end{tabular}


Table 15-19. Constituents with at Least One Value Above the CRQL for the Low-Level Waste Burial Grounds WMA 3 Data for Reporting Period July 1 through September 30, 1993. (sheet 9 of 11)

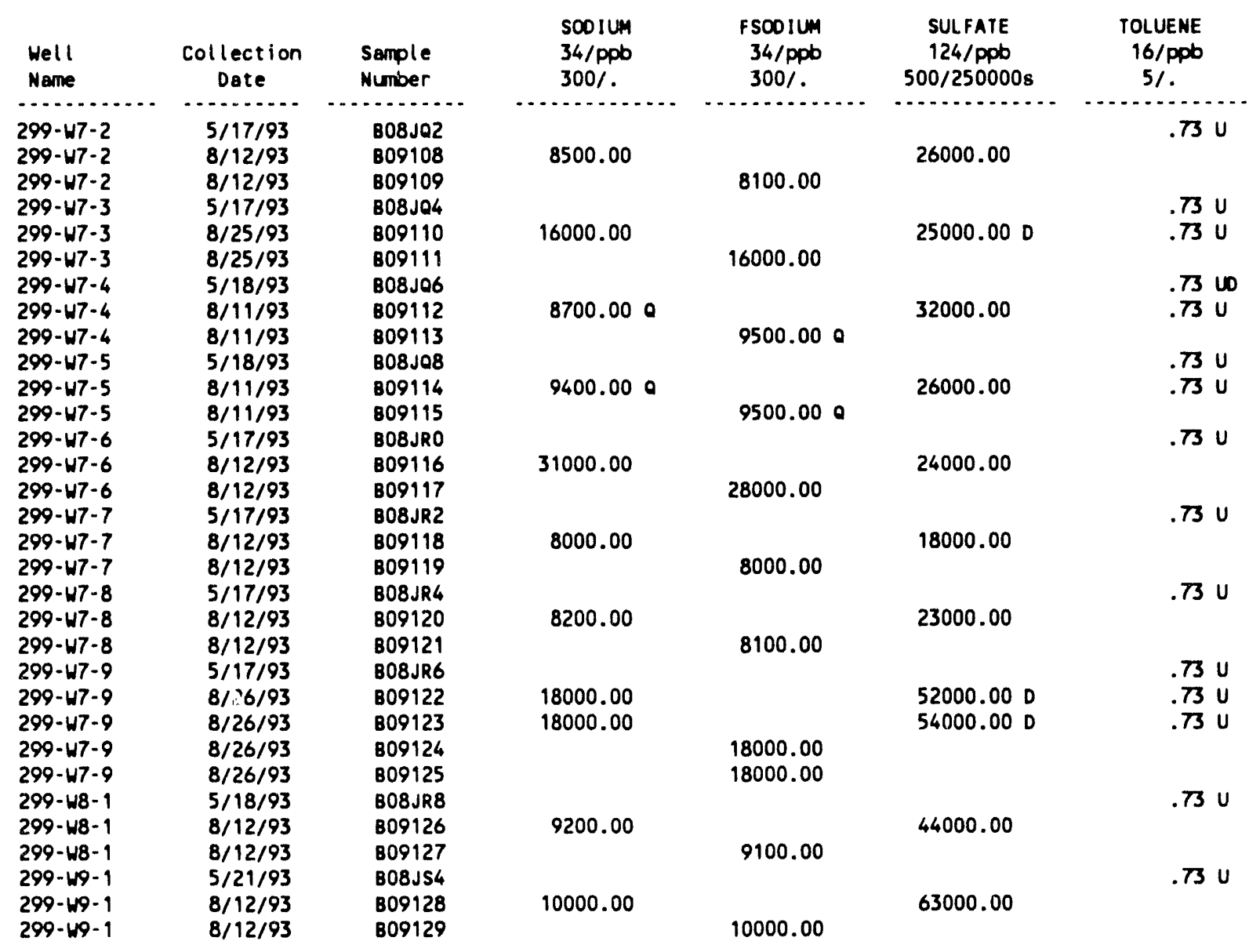

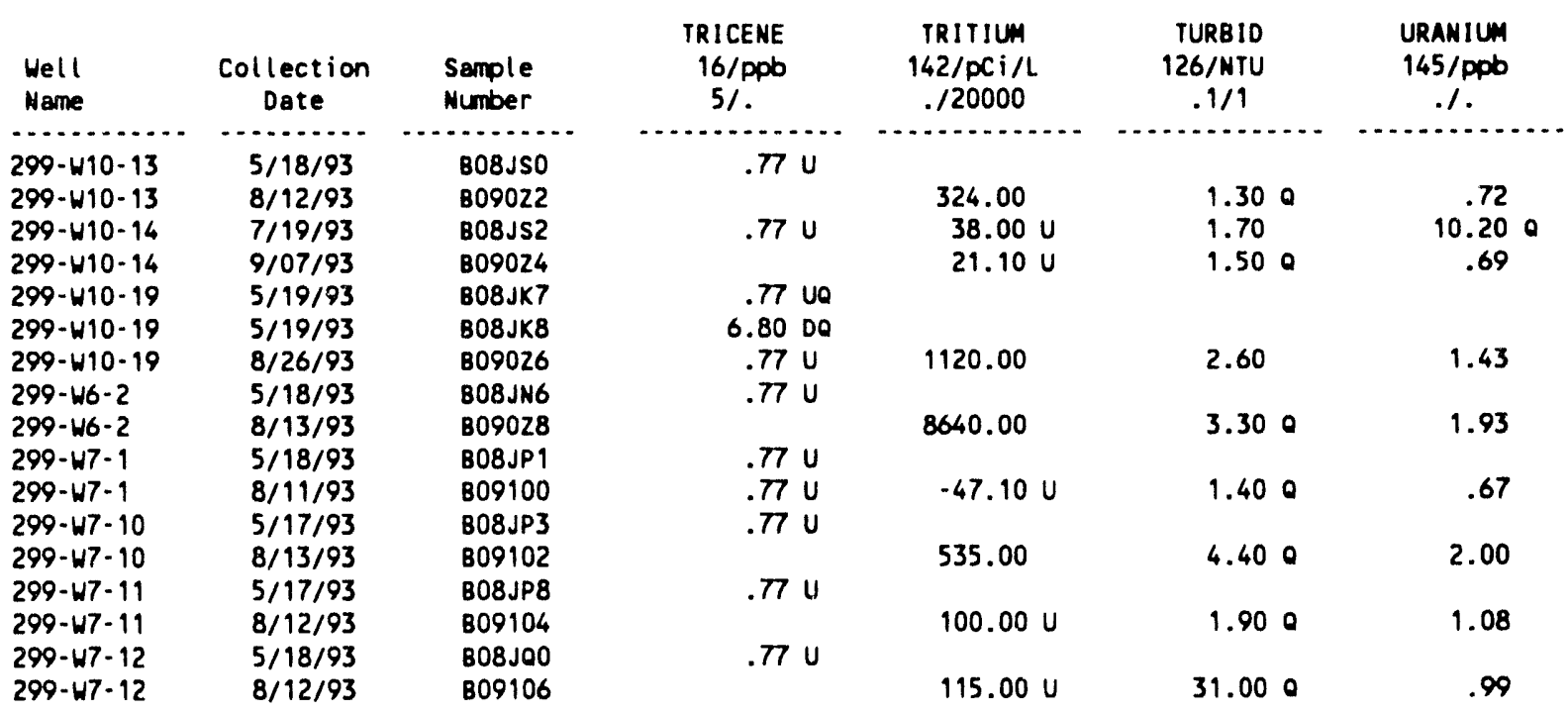


Table 15-19. Constituents with at Least One Value Above the CRQL for the Low-Level Waste Burial Grounds WMA 3 Data for Reporting Period July 1 through September 30, 1993. (sheet 10 of 11)

\begin{tabular}{|c|c|c|c|c|c|c|}
\hline $\begin{array}{l}\text { Well } \\
\text { Nane }\end{array}$ & $\begin{array}{c}\text { Collection } \\
\text { Date }\end{array}$ & $\begin{array}{l}\text { Semple } \\
\text { Number }\end{array}$ & $\begin{array}{c}\text { TRICENE } \\
16 / \text { PPb } \\
5 / .\end{array}$ & $\begin{array}{l}\text { TRLTIUM } \\
142 / \mathrm{pCi} / \mathrm{L} \\
.120000\end{array}$ & $\begin{array}{c}\text { TURBID } \\
\text { 126/NTU } \\
.1 / 1\end{array}$ & $\begin{array}{l}\text { URANIUN } \\
145 / \text { pDb } \\
.1 .\end{array}$ \\
\hline 299-W7-2 & $5 / 17 / 93$ & 808J02 & $.77 \mathrm{u}$ & & & \\
\hline $299-47-2$ & $8 / 12 / 93$ & B09108 & & $77.90 \mathrm{U}$ & 4.600 & .79 \\
\hline $299-W 7-3$ & $5 / 17 / 93$ & B08J04 & $.77 \mathrm{U}$ & & & \\
\hline $299-47-3$ & $8 / 25 / 93$ & 809110 & $.77 \mathrm{U}$ & $145.00 \mathrm{U}$ & 1.40 & 1.40 \\
\hline $299-47-4$ & $5 / 18 / 93$ & 808J06 & $2.70 \mathrm{LD}$ & & & \\
\hline $299-W 7-4$ & $8 / 11 / 93$ & 809112 & $.77 \mathrm{U}$ & 611.00 & .800 & 1.48 \\
\hline $299-47-5$ & $5 / 18 / 93$ & 808J08 & $.77 \mathrm{U}$ & & & \\
\hline $299-47-5$ & $8 / 11 / 93$ & B09114 & $.77 \mathrm{U}$ & $195.00 \mathrm{U}$ & 2.800 & 1.38 \\
\hline $299-47-6$ & $5 / 17 / 93$ & 808JRO & $.77 \mathrm{U}$ & & & \\
\hline $299-47-6$ & $8 / 12 / 93$ & B09116 & & 613.00 & 180.000 & 3.80 \\
\hline $299-W 7-7$ & $5 / 17 / 93$ & 808JR2 & $.77 \mathrm{U}$ & & & \\
\hline $299-47-7$ & $8 / 12 / 93$ & 809118 & & 325.00 & 13.000 &.$\pi$ \\
\hline $299-47-8$ & $5 / 17 / 93$ & B08JR4 & $.77 \mathrm{U}$ & & & \\
\hline $299-47-8$ & $8 / 12 / 93$ & 809120 & & 456.00 & 6.500 & $.96 \mathrm{H}$ \\
\hline $299-47-9$ & $5 / 17 / 93$ & 808JR6 & $.77 \mathrm{U}$ & & & \\
\hline 299-W7-9 & $8 / 26 / 93$ & 809122 & $.77 \mathrm{U}$ & $4.62 \mathrm{U}$ & 5.60 & 1.49 \\
\hline $\begin{array}{l}299-47-9 \\
200-48-9\end{array}$ & $8 / 26 / 93$ & 809123 & $.77 \mathrm{U}$ & $.93 .40 \mathrm{U}$ & 5.90 & 1.48 \\
\hline $299-48-1$ & $8 / 12 / 93$ & 809126 & & $165.00 \mathrm{U}$ & 2.000 & .84 \\
\hline $299-49-1$ & $5 / 21 / 93$ & B08JS4 & $.77 \mathrm{U}$ & & & \\
\hline $299-w 9-1$ & $8 / 12 / 93$ & 809128 & & $110.00 \mathrm{U}$ & 10.000 & .77 \\
\hline
\end{tabular}

\begin{tabular}{|c|c|c|c|c|c|c|}
\hline $\begin{array}{l}\text { Hell } \\
\text { Name }\end{array}$ & $\begin{array}{c}\text { Collection } \\
\text { Date }\end{array}$ & $\begin{array}{l}\text { Sample } \\
\text { Number }\end{array}$ & $\begin{array}{c}\text { VANADUM } \\
34 / \text { ppD } \\
30 \%\end{array}$ & $\begin{array}{c}\text { FVANADI } \\
34 / \mathrm{ppb} \\
30 \%\end{array}$ & $\begin{array}{c}\text { ZINC } \\
34 / \text { ppo } \\
10 \%\end{array}$ & $\begin{array}{c}\text { FZINC } \\
34 / \text { ppb } \\
10 \%\end{array}$ \\
\hline $299-410-13$ & $8 / 12 / 93$ & 809022 & $3.84 U$ & & $3.44 \mathrm{U}$ & \\
\hline $299-W 10-13$ & $8 / 12 / 93$ & 809023 & & $3.84 \mathrm{U}$ & & $3.44 \mathrm{U}$ \\
\hline $299-w 10-14$ & $7 / 19 / 93$ & B08JS2 & 43.00 & & 10.000 & \\
\hline $299--110-14$ & $7 / 19 / 93$ & B08J53 & & 43.00 & & 11.000 \\
\hline $299-410-19$ & $8 / 26 / 93$ & 809026 & $13.00 \mathrm{~L}$ & & 21.00 & \\
\hline $299-W 10-19$ & $8 / 26 / 93$ & $B 09027$ & & $15.00 \mathrm{~L}$ & & $3.90 \mathrm{~L}$ \\
\hline $299-146-2$ & $8 / 13 / 93$ & B09028 & $3.84 \mathrm{U}$ & & 21.00 & \\
\hline $299-46-2$ & $8 / 13 / 93$ & B09029 & & $3.84 \mathrm{U}$ & & 16.00 \\
\hline $299-w 7-1$ & $8 / 11 / 93$ & B09100 & $11.00 \mathrm{~L}$ & & $4.90 \mathrm{LO}$ & \\
\hline $299-w 7-1$ & $8 / 11 / 93$ & 809101 & & $11.00 \mathrm{~L}$ & & $7.60 \mathrm{LO}$ \\
\hline $299-W 7-10$ & $8 / 13 / 93$ & B09102 & $3.84 \mathrm{U}$ & & 17.00 & \\
\hline $299-W 7-10$ & $8 / 13 / 93$ & B09103 & & $3.84 \mathrm{U}$ & & 13.00 \\
\hline $299-w 7-11$ & $8 / 12 / 93$ & B09104 & $3.84 \mathrm{U}$ & & $3.44 \mathrm{U}$ & \\
\hline $299-47-11$ & $8 / 12 / 93$ & 809105 & & $3.84 \mathrm{U}$ & & $3.44 \mathrm{U}$ \\
\hline $299-w 7-12$ & $8 / 12 / 93$ & 809106 & $3.84 \mathrm{U}$ & & $3.44 \mathrm{U}$ & \\
\hline $299-W 7-12$ & $8 / 12 / 93$ & B09107 & & $3.84 \mathrm{U}$ & & $3.44 \mathrm{U}$ \\
\hline $299-47-2$ & $8 / 12 / 93$ & B09108 & $3.84 \mathrm{U}$ & & $3.44 \mathrm{U}$ & \\
\hline $299-47-2$ & $8 / 12 / 93$ & 809109 & & $3.84 \mathrm{U}$ & & $3.44 \mathrm{U}$ \\
\hline $299 \cdot 47-3$ & $8 / 25 / 93$ & B09110 & $22.00 \mathrm{~L}$ & & 20.00 & \\
\hline $299-47-3$ & $8 / 25 / 93$ & B09111 & & $26.00 \mathrm{~L}$ & & $3.44 \mathrm{U}$ \\
\hline $299-67-4$ & $8 / 11 / 93$ & B09112 & $11.00 \mathrm{~L}$ & 20.006 & $9.40 \mathrm{La}$ & 1.7. \\
\hline $299-117-4$ & $8 / 11 / 93$ & B09113 & & $12.00 \mathrm{~L}$ & & $12.00 \mathrm{Q}$ \\
\hline $299-W 7-5$ & $8 / 11 / 93$ & 809114 & $11.00 \mathrm{~L}$ & & 19.000 & \\
\hline $299-47-5$ & $8 / 11 / 93$ & 809115 & & $11.00 \mathrm{~L}$ & & $7.60 \mathrm{LO}$ \\
\hline $299-w 7-6$ & $8 / 12 / 93$ & B09116 & $3.84 \mathrm{U}$ & & 66.00 & \\
\hline $299-47-6$ & $8 / 12 / 93$ & 809117 & & $3.84 \mathrm{U}$ & & $3.44 \mathrm{U}$ \\
\hline $299-167-7$ & $8 / 12 / 93$ & 809118 & $3.84 \mathrm{U}$ & & $3.44 \mathrm{U}$ & \\
\hline $299-177 \cdot 7$ & $8 / 12 / 93$ & 809119 & & $3.84 U$ & & $3.44 \mathrm{U}$ \\
\hline
\end{tabular}


Table 15-19. Constituents with at Least One Value Above the CRQL for the Low-Level Waste Burial Grounds WMA 3 Data for Reporting Period July 1 through September 30, 1993. (sheet 11 of 11)

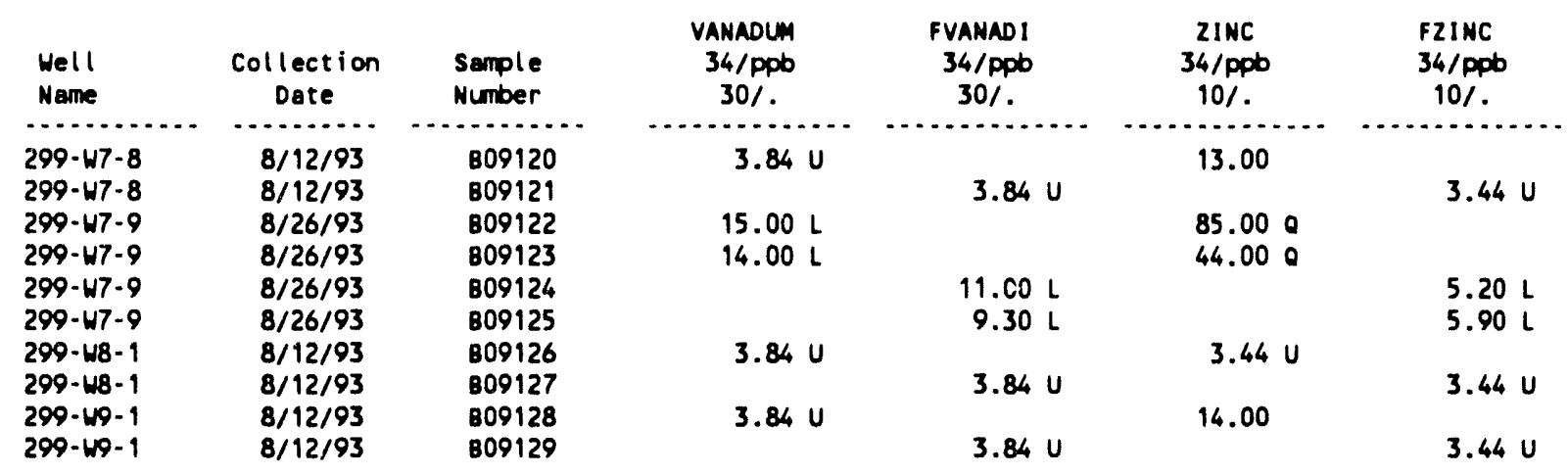

For explanation of this table, see Section 1.4 of report. 
Table 15-20. Contamination Indicator Parameters for the Low-Level Waste Burial Grounds WMA 3 Data for Reporting Period July 1 through September 30, 1993.

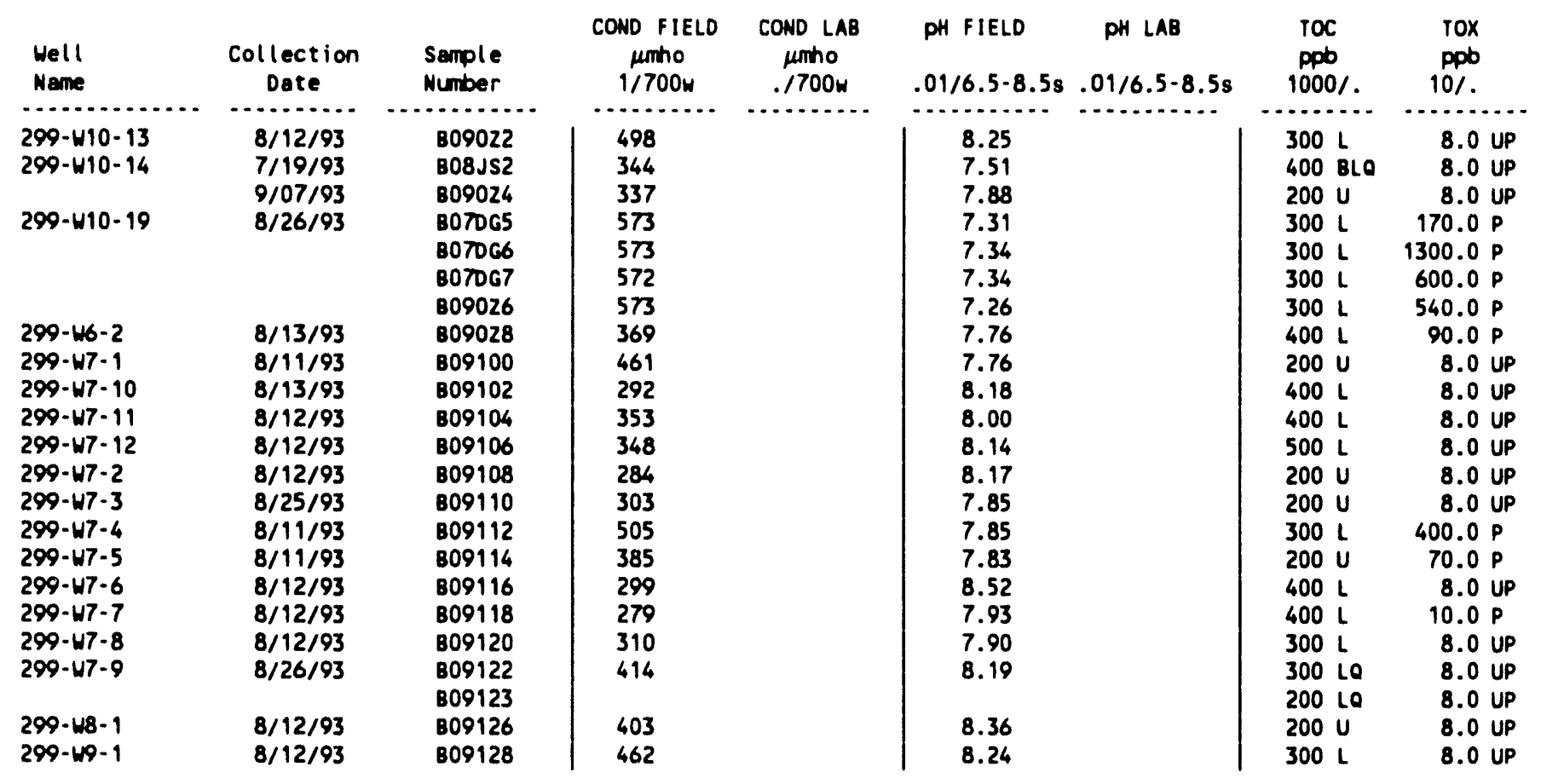

For explanation of this table, see Section 1.4 of report. 
Table 15-21. Constituent List and Summary of Results for the Low-Level Waste Burial Grounds WMA 4 Data for Reporting Period July 1 through September 30, 1993. (sheet 1 of 5)

CONTAMINATION INDICATOR PARAMETERS

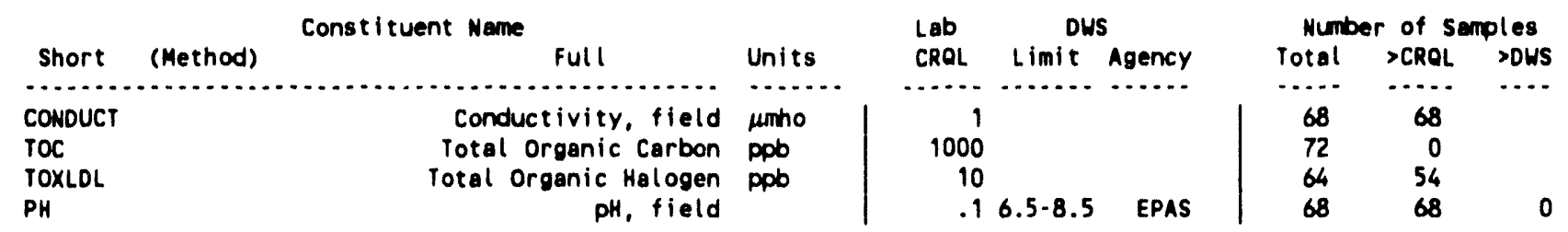

DRINKING WATER PARAMETERS

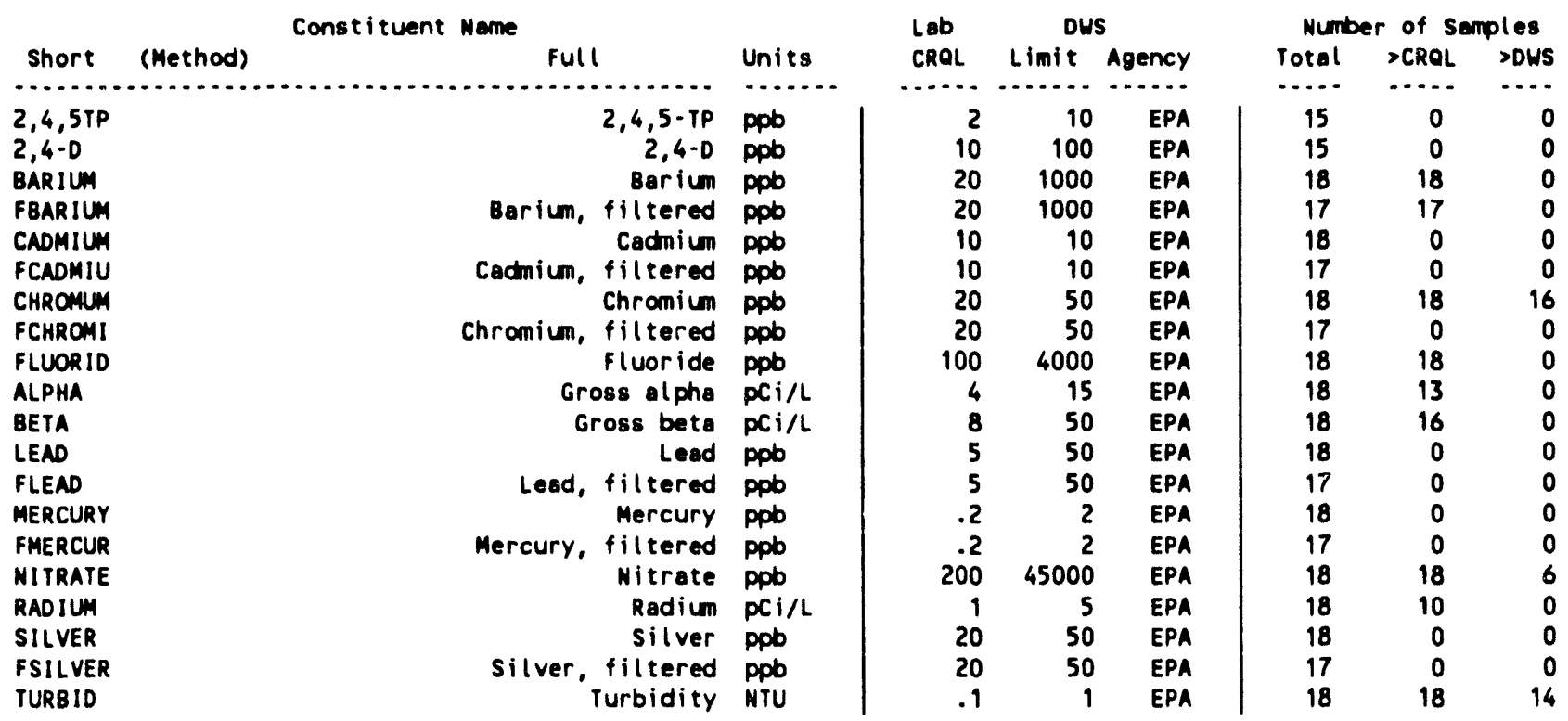

GROUNDWATER QUALITY PARAMETERS

\begin{tabular}{|c|c|c|c|c|c|c|c|c|c|}
\hline \multirow[b]{2}{*}{ Short } & \multirow{3}{*}{ 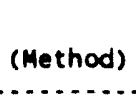 } & \multirow[t]{2}{*}{ Const ituent Name } & \multirow[b]{2}{*}{ Units } & \multirow{2}{*}{$\begin{array}{l}\text { Lab } \\
\text { CROL }\end{array}$} & \multicolumn{2}{|c|}{ DWS } & \multicolumn{3}{|c|}{ Number of Semples } \\
\hline & & & & & Limit & Agency & Total & $>C R Q L$ & $>$ DUS \\
\hline$\ldots \ldots$. & & $\ldots$ & $\ldots \ldots$ & $\ldots \ldots$ & $\ldots \ldots$ & $\ldots \ldots$ & $\ldots$. & $\ldots$. & $-\cdots$ \\
\hline CHLORID & & Chloride & ppb & 200 & 250000 & EPAS & 18 & 18 & 0 \\
\hline IRON & & Iron & ppob & 20 & 300 & EPAS & 18 & 18 & 16 \\
\hline FIRON & & Iron, filtered & ppo & 20 & 300 & EPAS & 17 & 14 & 0 \\
\hline MANGESE & & Manganese & pob & 10 & 50 & EPAS & 18 & 14 & 5 \\
\hline FMANGAN & & Manganese, filtered & pob & 10 & 50 & EPAS & 17 & 1 & 0 \\
\hline LPHENOL & & Phenol & ppo & 10 & & & 12 & 0 & \\
\hline soolum & & Sodium & ppb & 300 & & & 18 & 18 & \\
\hline FSODIUM & & Sodium, filtered & ppb & 300 & & & 17 & 17 & \\
\hline SULFATE & & Sulfate & ppb & 500 & 250000 & EPAS & 18 & 18 & 0 \\
\hline
\end{tabular}


Table 15-21. Constituent List and Summary of Results for the Low-Level Waste Burial Grounds WMA 4 Data for Reporting Period July 1

through September 30, 1993. (sheet 2 of 5)

SITE SPECIFIC AND OTHER CONSTITUENTS

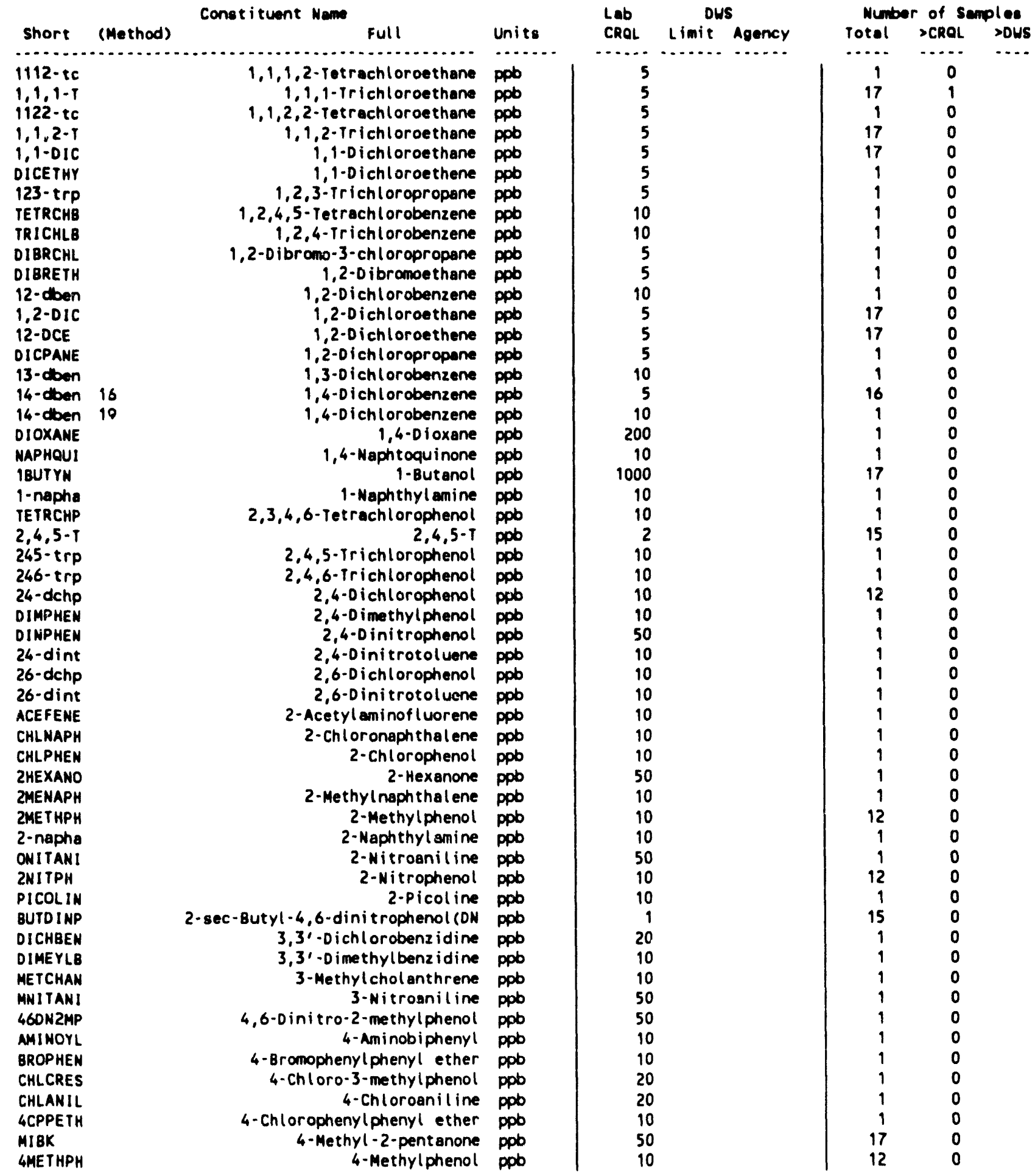


Table 15-21. Constituent List and Summary of Results for the Low-Level Waste Burial Grounds WMA 4 Data for Reporting Period July 1

through September 30, 1993. (sheet 3 of 5)

\begin{tabular}{|c|c|c|c|c|c|c|c|c|}
\hline \multirow[b]{2}{*}{ Short } & \multirow[t]{2}{*}{ Constituent Name } & \multirow[b]{2}{*}{ Units } & \multirow{2}{*}{$\begin{array}{l}\text { Lab } \\
\text { CRQL }\end{array}$} & \multicolumn{2}{|c|}{ DWS } & \multicolumn{3}{|c|}{ Number of Samples } \\
\hline & & & & Limit & Agency & Total & $>$ CROL & $>$ DWS \\
\hline MITRANI & 4-Nitroeniline & $\mathrm{mb}$ & $\cdots$ & $\cdots$ & & $\cdots$ & $\cdots$ & $\cdots$ \\
\hline NITPHEN & $\begin{array}{l}\text { 4-Mitroantiline } \\
\text { 4-Nitrophenol }\end{array}$ & ppb & $\begin{array}{l}50 \\
50\end{array}$ & & & 1 & $\begin{array}{l}0 \\
0\end{array}$ & \\
\hline 4N!TOUI & 4 -Nitroquinol ine-1-oxide & $\begin{array}{l}p p 0 \\
p p o b\end{array}$ & $\begin{array}{l}50 \\
10\end{array}$ & & & 1 & $\begin{array}{l}0 \\
0\end{array}$ & \\
\hline NITRTOL & 5-Nitro-0-toluidine & ppb & 10 & & & 1 & 0 & \\
\hline DIMBENZ & 7,12-D ime thy lbenz $[a]$ anthracene & ppob & 10 & & & 1 & 0 & \\
\hline ACENAPH & Acenaph thene & ppb & 10 & & & 1 & 0 & \\
\hline ACENATL & Acenaphthylene & ppb & 10 & & & 1 & 0 & \\
\hline ACETONE & Acetone & ppb & 100 & & & 17 & 0 & \\
\hline ACEIILE & Acetonitrile & ppob & 200 & & & 1 & 0 & \\
\hline ACETOPH & Acetophenone & ppob & 10 & & & 1 & 0 & \\
\hline ACROL IN & Acrolein & ppb & 5 & & & 1 & 0 & \\
\hline ACRYILE & Acrylonitrile & ppb & 5 & & & 1 & 0 & \\
\hline DIMPHAM & Alpha, al pha-D imethylphene thylami & ppb & 10 & & & 1 & 0 & \\
\hline ALUMNUM & Aluminum & ppb & 200 & & & 18 & 3 & \\
\hline FALUMIN & Aluminum & ppb & 200 & & & 17 & 0 & \\
\hline ANILINE & Aniline & ppb & 10 & & & 1 & 0 & \\
\hline ANTHRA & Anthracene & ppb & 10 & & & 1 & 0 & \\
\hline ANTIONY & Ant imony & ppb & 200 & & & 18 & 0 & \\
\hline FANTIMO & Antimony, filtered & ppb & 200 & & & 17 & 0 & \\
\hline ARAMITE & Aramite & ppb & 10 & & & 1 & 0 & \\
\hline BENZENE & Benzene & ppb & 5 & & & 17 & 0 & \\
\hline BENZAAN & Benzo( 8 ) anthracene & ppb & 10 & & & 1 & 0 & \\
\hline BENZOPY & Benzo(a)pyrene & ppb & 10 & & & 1 & 0 & \\
\hline BENZBFL & Benzo(b)fluoranthene & ppb & 10 & & & 1 & 0 & \\
\hline BGHIPER & Benzo(ghi)perylene & ppb & 10 & & & 1 & 0 & \\
\hline BNZKFLU & Benzo $(k)$ fluoranthene & ppb & 10 & & & 1 & 0 & \\
\hline BENZOTH & Benzothiazole & ppob & 10 & & & 12 & 0 & \\
\hline BENZALC & Benzyl alcohol & ppb & 20 & & & 1 & 0 & \\
\hline BERYLUM & Beryllium & ppb & 3 & & & 18 & 0 & \\
\hline FBERYLL & Beryllium, filtered & ppob & 3 & & & 17 & 0 & \\
\hline BIS2CHM & Bis(2-Choroethoxy)methane & ppb & 10 & & & 1 & 0 & \\
\hline BIS2CHE & Bis(2-chloroethyl) ether & ppb & 10 & & & 1 & 0 & \\
\hline BISLETH & Bis(2-chloroisopropyl) ether & ppb & 10 & & & 1 & 0 & \\
\hline BIS2EPH & Bis(2-ethylhexyl) phthalate & ppb & 10 & & & 12 & 0 & \\
\hline BROMIDE & Bromide & ppb & 500 & & & 18 & 0 & \\
\hline $80 \mathrm{~cm}$ & Bromodichloromethane & ppb & 5 & 100 & EPA & 1 & 0 & 0 \\
\hline BROMORM & Bromoform & ppob & 5 & 100 & EPA & 1 & 0 & 0 \\
\hline BUTBENP & Butylbenzylphthalate & ppb & 10 & & & 1 & 0 & \\
\hline CALCIUM & Calcium & ppob & 100 & & & 18 & 18 & \\
\hline FCALCIU & Calcium, filtered & ppb & 100 & & & 17 & 17 & \\
\hline CARB IDE & Carbon disulfide & ppob & 5 & & & 17 & 0 & \\
\hline TETRANE & Carbon tetrachloride & ppob & 5 & & & 17 & 17 & \\
\hline CHLROB & Chlorobenzene & ppb & 5 & & & 1 & 0 & \\
\hline Chllate & Chlorobenzilate & ppb & 10 & & & 1 & 0 & \\
\hline CLETHAN & Chloroethane & ppb & 10 & & & 1 & 0 & \\
\hline CHLFORM & Chloroform & ppo & 5 & 100 & EPA & 17 & 10 & 0 \\
\hline CHLPRENE & Chloroprene & ppb & 5 & & & 1 & 0 & \\
\hline CHRYSEN & Chrysene & ppb & 10 & & & 1 & 0 & \\
\hline COBALT & Cobalt & ppb & 20 & & & 18 & 0 & \\
\hline FCOBALT & Cobalt, filtered & ppb & 20 & & & 17 & 0 & \\
\hline COPPER & Copper & ppb & 20 & 1000 & EPAS & 18 & 5 & 0 \\
\hline FCOPPER & Copper, filtered & ppb & 20 & 1000 & EPAS & 17 & 0 & 0 \\
\hline CYANIDE & Cyanide & ppb & 20 & & & 17 & 0 & \\
\hline DECANE & Decane & ppb & 10 & & & 11 & 0 & \\
\hline DIBPHTH & Di-n-butylphthalate & ppob & 10 & & & 1 & 0 & \\
\hline DIOPHTH & Di-n-octylphthalate & ppob & 10 & & & 1 & 0 & \\
\hline DIALLATE & Diallate & ppb & 10 & & & 1 & 0 & \\
\hline
\end{tabular}


Table 15-21. Constituent List and Summary of Results for the Low-Level Waste Burial Grounds WMA 4 Data for Reporting Period July 1 through September 30, 1993. (sheet 4 of 5)

\begin{tabular}{|c|c|c|c|c|c|c|c|c|c|}
\hline \multicolumn{4}{|c|}{ Constituent Name } & \multicolumn{3}{|c|}{ Dus } & \multicolumn{3}{|c|}{ Number of Samples } \\
\hline Short & (Method) & Full & Units & CROL & Limit & Agency & Total & $>C R O L$ & $>$ DUS \\
\hline$\cdots \ldots$ & $\ldots \ldots$ & $\ldots \ldots$ & $\ldots . .$. & $\ldots . .$. & $\ldots . .$. & $\ldots \ldots$ & $\ldots \ldots$ & $\ldots$. & $\ldots$ \\
\hline DIBAHAN & & D ibenz $[a, h]$ anthracene & ppb & 10 & & & 1 & 0 & \\
\hline DIBENFR & & Dibenzofuran & ppb & 10 & & & 1 & 0 & \\
\hline BRCHLMET & & Dibronochlor ome thane & ppo & 5 & 100 & EPA & 1 & 0 & 0 \\
\hline DIBRMET & & D ibromone thane & ppb & 5 & & & 1 & 0 & \\
\hline DICOIFA & & Dichlorodif luor ome thane & ppb & 5 & & & 1 & 0 & \\
\hline DIEPHTH & & Diethyl phthalate & ppb & 10 & & & 1 & 0 & \\
\hline DIMETHO & & Dimethoate & ppb & 10 & & & 1 & 0 & \\
\hline DIMPHTH & & Dimethyl phthalate & ppb & 10 & & & 1 & 0 & \\
\hline DIPHAMI & & Diphenyl amine & ppb & 10 & & & 1 & 0 & \\
\hline DOOECAN & & Dodecane & ppb & 10 & & & 11 & 0 & \\
\hline ETHCYAN & & Ethyl cyanide & ppb & 10 & & & 17 & 0 & \\
\hline ETHMETH & & Ethyl methacrylate & ppb & 5 & & & 1 & 0 & \\
\hline ETHMETS & & Ethyl methanesulfonate & ppob & 10 & & & 1 & 0 & \\
\hline ETHBENZ & & Ethylbenzene & ppb & 5 & & & 1 & 0 & \\
\hline FAMPHUR & & Famphur & ppb & 10 & & & 1 & 0 & \\
\hline FLUORAK & & Fluorenthene & ppb & 10 & & & 1 & 0 & \\
\hline FLRENE & & Fluorene & ppb & 10 & & & 1 & 0 & \\
\hline HEXCBEN & & Hexachlorobenzene & ppb & 10 & & & 1 & 0 & \\
\hline HEXCBUT & & Hexachl orobut adiene & ppb & 10 & & & 1 & 0 & \\
\hline HEXCCYC & & Hexach lorocyclopent adiene & ppb & 10 & & & 1 & 0 & \\
\hline HEXCETH & & Hexachloroethane & ppb & 10 & & & 1 & 0 & \\
\hline HEXACHL & & Hexachlorophene & ppo & 10 & & & 1 & 0 & \\
\hline HEXAENE & & Hexachloropropene & ppo & 10 & & & 1 & 0 & \\
\hline INDENOP & & Indeno $(1,2,3$-cd)pyrene & ppb & 10 & & & 1 & 0 & \\
\hline ISOBUTY & & Isobutyl alcohol & ppb & 200 & & & 1 & 0 & \\
\hline ISOORIN & & Isodrin & ppb & 10 & & & 1 & 0 & \\
\hline ISOPHER & & Isophorone & ppo & 10 & & & 1 & 0 & \\
\hline ISOSOLE & & Isosafrole & ppb & 10 & & & 1 & 0 & \\
\hline KEPONE & & Kepone & ppb & 10 & & & 1 & 0 & \\
\hline KEROSEN & & Kerosene & ppb & 10000 & & & 1 & 0 & \\
\hline MAGNES & & Megnesium & ppb & 100 & & & 18 & 18 & \\
\hline FMAGNES & & Magnesium, filtered & ppb & 100 & & & 17 & 17 & \\
\hline METHACR & & Methacrylonitrile & ppob & 5 & & & 1 & 0 & \\
\hline METHAPY & & Methapyrilene & ppo & 10 & & & 1 & 0 & \\
\hline IOOOMET & & Methyl Iodide & ppo & 5 & & & 1 & 0 & \\
\hline METHBRO & & Methyl bromide & ppb & 10 & & & 1 & 0 & \\
\hline METHCHL & & Methyi chloride & ppb & 10 & & & 1 & 0 & \\
\hline METHONE & & Methyl ethyl ketone & ppb & 100 & & & 17 & 0 & \\
\hline METACRY & & Methyl methacrylate & ppb & 5 & & & 1 & 0 & \\
\hline METMSUL & & Methyl methanesul fonate & ppb & 10 & & & 1 & 0 & \\
\hline METHYCH & & Methylene chloride & ppb & 5 & & & 17 & 0 & \\
\hline DIPRNIT & & $N$-Nitroso-di-n-dipropylamine & ppb & 10 & & & 1 & 0 & \\
\hline NNIBUTY & & $N$-Nitrosodi-n-butylamine & ppb & 10 & & & 1 & 0 & \\
\hline NHIDIEY & & N-Nitrosodiethylanine & ppb & 10 & & & 1 & 0 & \\
\hline NNIDIME & & N-Nitrosodimethylamine & ppb & 10 & & & 1 & 0 & \\
\hline NND IPHA & & N-Nitrosodiphenyl amine & ppb & 10 & & & 1 & 0 & \\
\hline NNIMETH & & N-Nitrosomethylethyl anine & ppb & 10 & & & 1 & 0 & \\
\hline NNIMORP & & $N$-Nitrosomorphol ine & ppb & 10 & & & 1 & 0 & \\
\hline NNIPIPE & & N-Mitrosopiperidine & ppb & 10 & & & 1 & 0 & \\
\hline NAPHTHA & & Naphthalene & ppob & 10 & & & 12 & 0 & \\
\hline NICKEL. & & Nickel & ppb & 30 & & & 18 & 16 & \\
\hline FHICKEL & & Nickel, filtered & ppob & 30 & & & 17 & 0 & \\
\hline NITRITE & & Nitrite & ppb & 200 & & & 18 & 1 & \\
\hline NITBENZ & & Nitrobenzene & ppb & 10 & & & 1 & 0 & \\
\hline NITRPYR & & Nitrosopyrrolidine & ppb & 10 & & & 1 & 0 & \\
\hline
\end{tabular}


Table 15-21. Constituent List and Summary of Results for the Low-Level Waste Burial Grounds WMA 4 Data for Reporting Period July 1

through September 30,1993 . (sheet 5 of 5 )

\begin{tabular}{|c|c|c|c|c|c|c|c|c|}
\hline \multirow[b]{2}{*}{ Short } & \multirow[t]{2}{*}{ Const ituent Nane } & \multirow[b]{2}{*}{ Units } & \multirow{2}{*}{$\begin{array}{l}\text { Lab } \\
\text { CROL }\end{array}$} & \multicolumn{2}{|l|}{ DWS } & \multicolumn{3}{|c|}{ Number of Semples } \\
\hline & & & & Limit & Agency & Total & $>$ CRQL & $>$ OWS \\
\hline$\cdots \ldots$ & 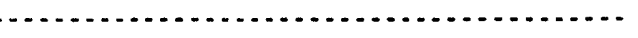 & $\ldots \ldots$ & $\ldots \ldots$ & $\ldots . .$. & $\ldots \ldots$ & $\ldots \ldots$ & $\cdots$ & $\cdots$ \\
\hline TRIPHOS & $0,0,0-$ Triethyl phosphorothioate & ppb & 10 & & & 1 & 0 & \\
\hline DIPHOS & 0,0 -diethyl0-2-pyraz inylphosphor & ppo & 10 & & & 1 & 0 & \\
\hline PARATHI & Parathion & ppo & 10 & & & 1 & 0 & \\
\hline PENTCHB & Pentachl orobenzene & ppb & 10 & & & 1 & 0 & \\
\hline PENTACH & Pentach l oroethane & ppob & 5 & & & 1 & 0 & \\
\hline PENTCHN & Pentachloronitrobenzene (PCNB) & ppb & 10 & & & 1 & 0 & \\
\hline PENTCHP & Pentachlorophenol & ppb & 50 & & & 12 & 0 & \\
\hline PHENTIN & Phenacet in & ppb & 10 & & & 1 & 0 & \\
\hline PHENANT & Phenanthrene & ppb & 10 & & & 1 & 0 & \\
\hline PHOSPHA & Phosphate & ppob & 400 & & & 18 & 0 & \\
\hline POTASUM & Potassium & ppb & 300 & & & 18 & 18 & \\
\hline FPOTASS & Potassium, filtered & ppb & 300 & & & 17 & 17 & \\
\hline PRONIDE & Pronamide & ppb & 10 & & & 1 & 0 & \\
\hline PYRENE & Pyrene & ppob & 10 & & & 1 & 0 & \\
\hline PYRIDIN & Pyridine & ppo & 10 & & & 1 & 0 & \\
\hline SAFROL & Safrol & ppob & 10 & & & 1 & 0 & \\
\hline STYRENE & Styrene & ppb & 5 & & & 1 & 0 & \\
\hline PERCENE & Tetrachloroethene & ppo & 5 & & & 17 & 0 & \\
\hline TETRADE & Tetradecane & ppb & 10 & & & 11 & 0 & \\
\hline PYROPHOS & Tetraethyldi thiopyrophosphate & ppb & 10 & & & 1 & 0 & \\
\hline TAF & Tetrahydrofuran & ppb & 10 & & & 17 & 0 & \\
\hline TIN & $\operatorname{Tin}$ & ppb & 100 & & & 18 & 0 & \\
\hline FTIN & Tin, filtered & ppb & 100 & & & 17 & 0 & \\
\hline TOLUENE & Tolvene & ppb & 5 & & & 17 & 0 & \\
\hline TRIBUTPH & Tributyl Phosphate & ppb & 10 & & & 12 & 0 & \\
\hline TRICENE & Trichloroethene & ppb & 5 & & & 17 & 0 & \\
\hline TRCMFLM & Trichloromonof luor ome thane & ppb & 5 & & & 1 & 0 & \\
\hline TOICHPH & Tris-2-chloroethyl phosphate & ppb & 10 & & & 12 & 0 & \\
\hline TRITIUM & Tritium & $\mathrm{pCi} / \mathrm{L}$ & 500 & 20000 & EPA & 18 & 6 & 3 \\
\hline URANIUA & Uranium & ppb & .5 & & & 18 & 18 & \\
\hline VANADUM & Vanadium & ppob & 30 & & & 18 & 5 & \\
\hline FVANADI & Vanadiun, filtered & ppb & 30 & & & 17 & 5 & \\
\hline VINYLAC & Vinyl acetate & ppb & 5 & & & 1 & 0 & \\
\hline VINYIDE & Vinyl chloride & ppb & 10 & 2 & EPA & 17 & 0 & 0 * \\
\hline XYLENE & Xylenes (total) & ppb & 5 & & & 17 & 0 & \\
\hline ZINC & Zinc & ppb & 10 & & & 18 & 4 & \\
\hline FZINC & zinc, filtered & ppb & 10 & & & 17 & 1 & \\
\hline ALLYLCL & allylchloride & ppb & 100 & & & 1 & 0 & \\
\hline TDICPENE & cis-1,3-0 ichloropropene & ppb & 5 & & & 1 & 0 & \\
\hline MCRESOL. & m-Cresol & ppb & 10 & & & 12 & 0 & \\
\hline DINBENZ & m-dinitrobenzene & ppb & 10 & & & 1 & 0 & \\
\hline OTOLHYO & o-Tolvidine & ppb & 10 & & & 1 & 0 & \\
\hline DIMEAMB & p-o imethylami noazobenzene & ppo & 10 & & & 1 & 0 & \\
\hline PHENDIA & P-Phenylenediamine & ppb & 10 & & & 1 & 0 & \\
\hline SYMTRIN & sym-Trinitrobenzene & ppo & 10 & & & 1 & 0 & \\
\hline DICPENE & trans-1,3-Dichloropropene & ppb & 5 & & & 1 & 0 & \\
\hline TDIBUTEN & trans-1,4-dichloro-2-butene & ppb & 5 & & & 1 & 0 & \\
\hline
\end{tabular}

For explanation of this table, see Section 1.4 of report. 
Table 15-22. Constituents with at Least One Value Above the CRQL for the Low-Level Waste Burial Grounds WMA 4 Data for Reporting Period July 1 through September 30, 1993. (sheet 1 of 8 )

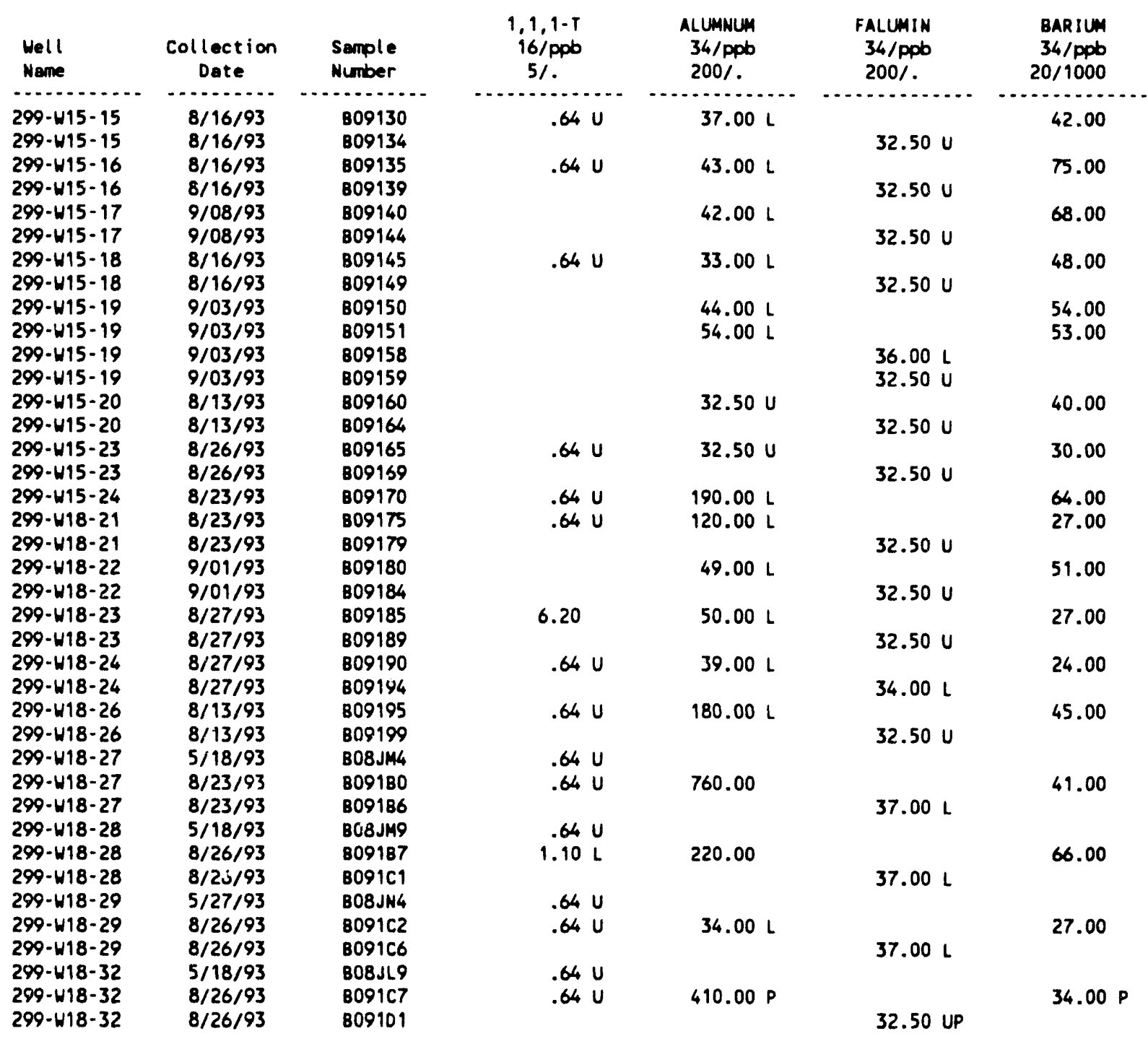

\begin{tabular}{|c|c|c|c|c|c|c|}
\hline $\begin{array}{l}\text { Well } \\
\text { Name }\end{array}$ & $\begin{array}{c}\text { Collection } \\
\text { Date }\end{array}$ & $\begin{array}{l}\text { Sample } \\
\text { Number }\end{array}$ & $\begin{array}{r}\text { FBARIUM } \\
34 / \text { ppb } \\
20 / 1000\end{array}$ & $\begin{array}{c}\text { CALCIUM } \\
34 / \mathrm{ppb} \\
100 / .\end{array}$ & $\begin{array}{l}\text { FCALCIU } \\
34 / \mathrm{ppb} \\
100 /\end{array}$ & $\begin{array}{c}\text { TETRANE } \\
16 / \mathrm{ppb} \\
5 / .\end{array}$ \\
\hline$\ldots \ldots \ldots$ & $\ldots \ldots \ldots$ & $\ldots \ldots$ & 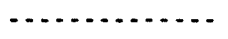 & $\ldots \ldots \ldots$ & $\ldots \ldots \ldots$ & \\
\hline $\begin{array}{l}299-W 15-15 \\
299-415-15\end{array}$ & $\begin{array}{l}8 / 16 / 93 \\
8 / 16 / 93\end{array}$ & $\begin{array}{l}809130 \\
809134\end{array}$ & 42.00 & 39000.000 & 39000.000 & 1400.00 \\
\hline $\begin{array}{l}299-W 15-16 \\
299-W 15-16\end{array}$ & $\begin{array}{l}8 / 16 / 93 \\
8 / 16 / 93\end{array}$ & $\begin{array}{l}809135 \\
809139\end{array}$ & 75.00 & 69000.000 & 69000.000 & 7000.00 \\
\hline $\begin{array}{l}299-W 15-17 \\
299-W 15-17\end{array}$ & $\begin{array}{l}9 / 08 / 93 \\
9 / 08 / 93\end{array}$ & $\begin{array}{l}\text { B09140 } \\
\text { B09144 }\end{array}$ & 68.00 & 38000.00 & 37000.00 & \\
\hline $\begin{array}{l}299-W 15-18 \\
299-W 15-18\end{array}$ & $\begin{array}{l}8 / 16 / 93 \\
8 / 16 / 93\end{array}$ & $\begin{array}{l}809145 \\
809149\end{array}$ & 47.00 & 42000.000 & 42000.000 & 1900.00 \\
\hline
\end{tabular}


Table 15-22. Constituents with at Least One Value Above the CRQL for the Low-Level Waste Burial Grounds WMA 4 Data for Reporting Period July 1 through September 30, 1993. (sheet 2 of 8 )

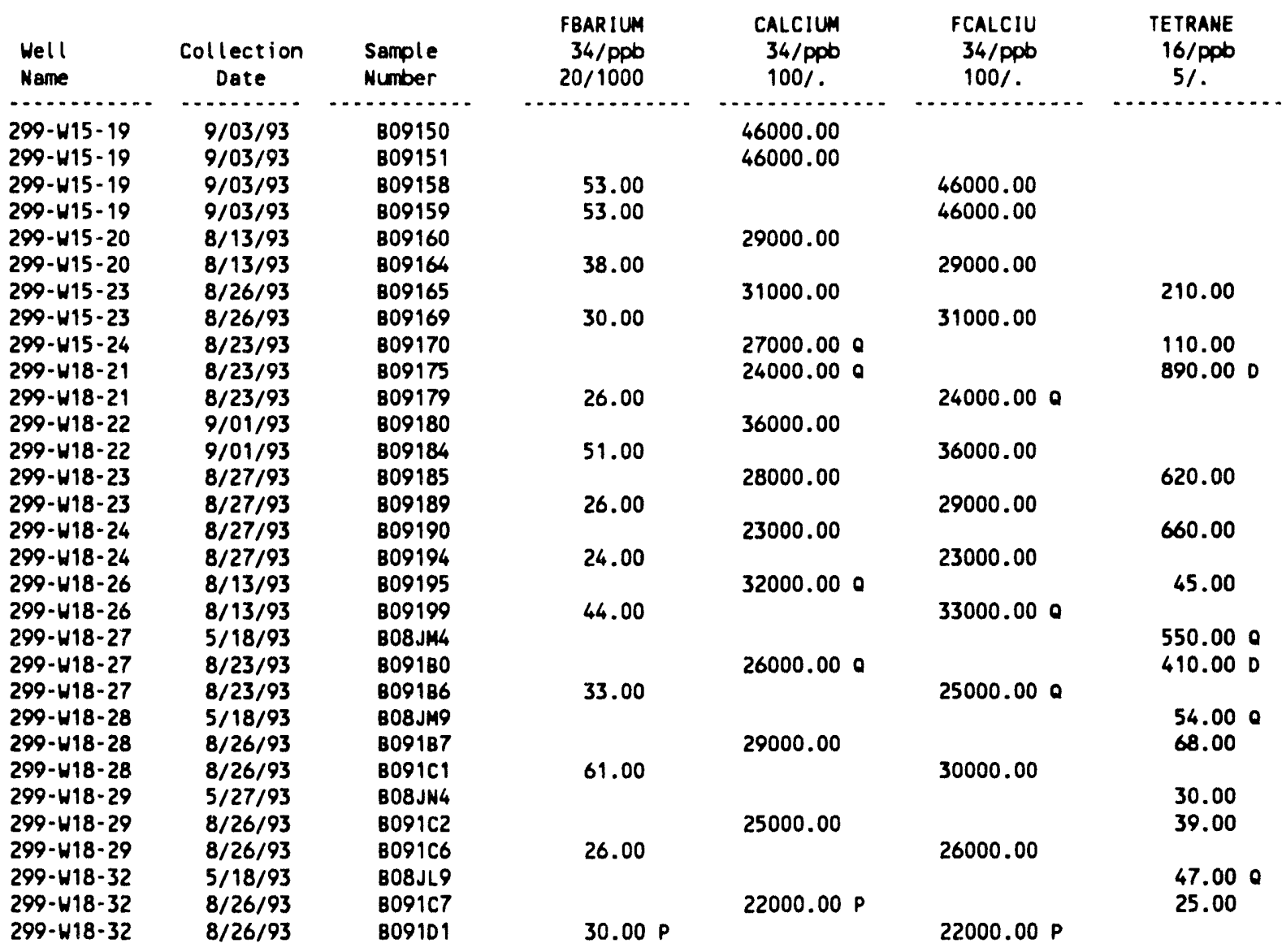

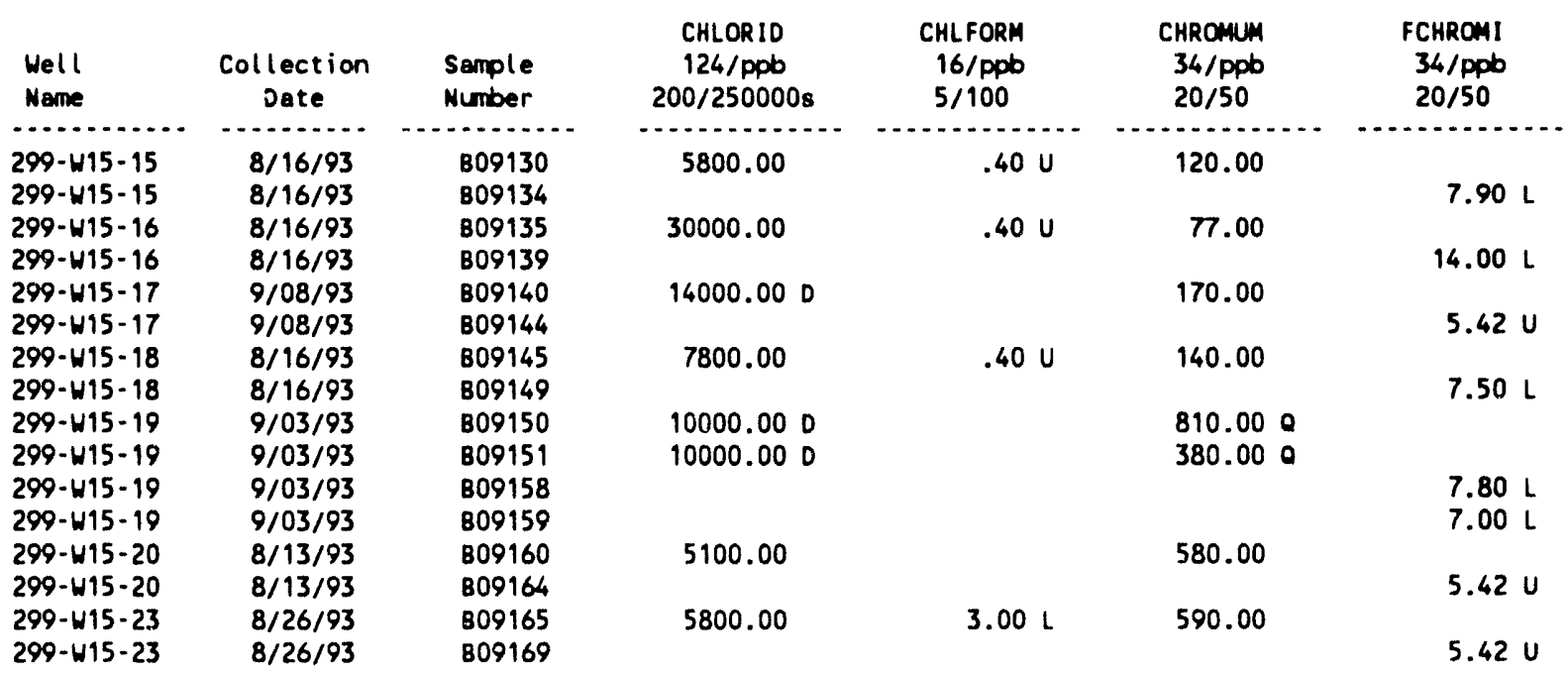


Table 15-22. Constituents with at Least One Value Above the CRQL for the Low-Level Waste Burial Grounds WMA 4 Data for Reporting Period July 1 through September 30, 1993. (sheet 3 of 8 )

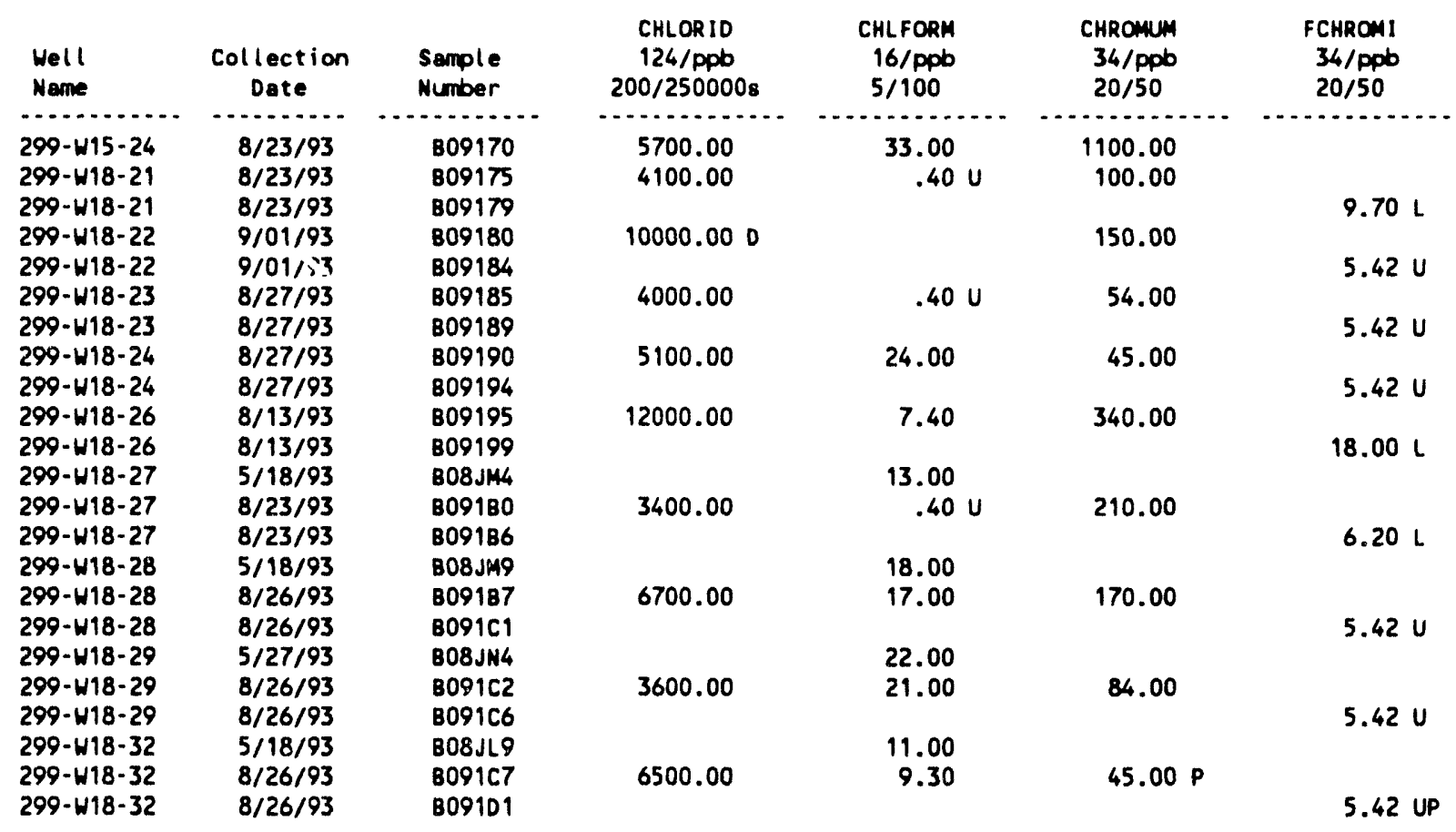

\begin{tabular}{|c|c|c|c|c|c|c|}
\hline $\begin{array}{l}\text { Well } \\
\text { Nane }\end{array}$ & $\begin{array}{c}\text { Collection } \\
\text { Date }\end{array}$ & $\begin{array}{l}\text { Sample } \\
\text { Number }\end{array}$ & $\begin{array}{l}\text { COPPER } \\
34 / \mathrm{ppb} \\
20 / 1000 \mathrm{~s}\end{array}$ & $\begin{array}{c}\text { FCOPPER } \\
34 / \text { ppb } \\
20 / 1000 \mathrm{~s}\end{array}$ & $\begin{array}{l}\text { FLUORID } \\
124 / \mathrm{ppb} \\
100 / 4000\end{array}$ & $\begin{array}{c}\text { ALPHA } \\
135 / \mathrm{pCi} / \mathrm{L} \\
. / 15\end{array}$ \\
\hline$\ldots \ldots \ldots$ & $\cdots$ & $\ldots \ldots \ldots$ & $\cdots$ & 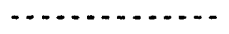 & $\ldots$ & (25) \\
\hline $\begin{array}{l}299-W 15-15 \\
299-W 15-15\end{array}$ & $\begin{array}{l}8 / 16 / 93 \\
8 / 16 / 93\end{array}$ & $\begin{array}{l}B 09130 \\
B 09134\end{array}$ & $8.00 \mathrm{~L}$ & $2.70 \mathrm{~L}$ & 500.00 & 2.85 \\
\hline $\begin{array}{l}299-W 15-16 \\
299-W 15-16\end{array}$ & $\begin{array}{l}8 / 16 / 93 \\
8 / 16 / 93\end{array}$ & $\begin{array}{l}B 09135 \\
809139\end{array}$ & $2.65 \mathrm{U}$ & $2.65 \mathrm{U}$ & 700.00 & 1.67 \\
\hline $\begin{array}{l}299-W 15-17 \\
299-W 15-17\end{array}$ & $\begin{array}{l}9 / 08 / 93 \\
9 / 08 / 93\end{array}$ & $\begin{array}{l}809140 \\
809144\end{array}$ & $2.65 \mathrm{U}$ & $2.65 \mathrm{U}$ & 300.00 & $.45 \mathrm{U}$ \\
\hline $\begin{array}{l}299-W 15-18 \\
299-H 15-18\end{array}$ & $\begin{array}{l}8 / 16 / 93 \\
8 / 16 / 93\end{array}$ & $\begin{array}{l}809145 \\
809149\end{array}$ & $3.50 \mathrm{~L}$ & $2.65 \mathrm{U}$ & 600.00 & $.28 \mathrm{U}$ \\
\hline $\begin{array}{l}299-W 15-19 \\
299-W 15-19\end{array}$ & $\begin{array}{l}9 / 03 / 93 \\
9 / 03 / 93\end{array}$ & $\begin{array}{l}\mathrm{B} 09150 \\
\mathrm{~B} 09151\end{array}$ & $\begin{array}{l}28.000 \\
20.000\end{array}$ & & $\begin{array}{l}500.00 \\
500.00\end{array}$ & $\begin{array}{l}1.780 \\
1.12 \text { va }\end{array}$ \\
\hline $\begin{array}{l}299-W 15-19 \\
299-W 15-19\end{array}$ & $\begin{array}{l}9 / 03 / 93 \\
9 / 03 / 93\end{array}$ & $\begin{array}{l}B 09158 \\
B 09159\end{array}$ & & $\begin{array}{l}2.65 \mathrm{U} \\
2.65 \mathrm{U}\end{array}$ & & \\
\hline $\begin{array}{l}299-W 15-20 \\
299-W 15-20\end{array}$ & $\begin{array}{l}8 / 13 / 93 \\
8 / 13 / 93\end{array}$ & $\begin{array}{l}809160 \\
809164\end{array}$ & $2.65 \mathrm{U}$ & $2.65 \mathrm{U}$ & 400.00 & 1.45 \\
\hline $\begin{array}{l}299-W 15-23 \\
299-W 15-23\end{array}$ & $\begin{array}{l}8 / 26 / 93 \\
8 / 26 / 93\end{array}$ & $\begin{array}{l}809165 \\
809169\end{array}$ & 23.00 & $3.40 \mathrm{~L}$ & 500.00 & 2.09 \\
\hline $\begin{array}{l}299-W 15-24 \\
299-W 18-21\end{array}$ & $\begin{array}{l}8 / 23 / 93 \\
8 / 23 / 93\end{array}$ & $\begin{array}{l}809170 \\
809175\end{array}$ & $\begin{array}{l}42.00 \\
2.65 \mathrm{U}\end{array}$ & & $\begin{array}{l}500.00 \\
500.00\end{array}$ & $\begin{array}{r}1.90 \\
11.20\end{array}$ \\
\hline $299-W 18-21$ & $8 / 23 / 93$ & 809179 & & $2.65 \mathrm{U}$ & & \\
\hline $299-W 18-22$ & $9 / 01 / 93$ & 809180 & $2.65 \mathrm{U}$ & & 400.00 & 1.32 \\
\hline $299-418-22$ & $9 / 01 / 93$ & B09184 & & $2.65 \mathrm{U}$ & & \\
\hline $\begin{array}{l}299-W 18-23 \\
299-W 18-23\end{array}$ & $\begin{array}{l}8 / 27 / 93 \\
8 / 27 / 93\end{array}$ & $\begin{array}{l}B 09185 \\
B 09189\end{array}$ & $2.65 \mathrm{U}$ & $2.65 \mathrm{U}$ & $500.00 \mathrm{~B}$ & 1.50 \\
\hline
\end{tabular}


Table 15-22. Constituents with at Least One Value Above the CRQL for the Low-Level Waste Burial Grounds WMA 4 Data for Reporting Period July 1 through September 30, 1993. (sheet 4 of 8 )

\begin{tabular}{|c|c|c|c|c|c|c|}
\hline $\begin{array}{l}\text { Well } \\
\text { Name }\end{array}$ & $\begin{array}{c}\text { Collection } \\
\text { Date }\end{array}$ & $\begin{array}{l}\text { Sample } \\
\text { Number }\end{array}$ & $\begin{array}{c}\text { COPPER } \\
\text { 34/Ppb } \\
20 / 1000 \mathrm{~s}\end{array}$ & $\begin{array}{c}\text { FCOPPER } \\
34 / \text { ppb } \\
20 / 1000 \mathrm{~s}\end{array}$ & $\begin{array}{l}\text { FLUOR ID } \\
124 / p p b \\
100 / 4000\end{array}$ & $\begin{array}{c}\text { ALPHA } \\
135 / \text { PC i } / L \\
. / 15\end{array}$ \\
\hline$\cdots$ & $\cdots \ldots \ldots$ & $\ldots \ldots \ldots+\cdots$ & $-\ldots$ & , & $\ldots \ldots$ & \\
\hline $\begin{array}{l}299-W 18-24 \\
299-W 18-24\end{array}$ & $\begin{array}{l}8 / 27 / 93 \\
8 / 27 / 93\end{array}$ & $\begin{array}{l}809190 \\
809194\end{array}$ & $2.65 \mathrm{U}$ & $2.65 \mathrm{U}$ & $700.00 \mathrm{~B}$ & 2.23 \\
\hline $\begin{array}{l}299-W 18-26 \\
299-W 18-26\end{array}$ & $\begin{array}{l}8 / 13 / 93 \\
8 / 13 / 93\end{array}$ & $\begin{array}{l}809195 \\
809199\end{array}$ & 21.00 & $5.40 \mathrm{~L}$ & 500.00 & 2.07 \\
\hline $\begin{array}{l}299-W 18-27 \\
299-W 18-27\end{array}$ & $\begin{array}{l}8 / 23 / 93 \\
8 / 23 / 93\end{array}$ & $\begin{array}{l}B 09180 \\
809186\end{array}$ & $12.00 \mathrm{~L}$ & $2.65 \mathrm{U}$ & 400.00 & 2.28 \\
\hline $\begin{array}{l}299-W 18-28 \\
299-W 18-28\end{array}$ & $\begin{array}{l}8 / 26 / 93 \\
8 / 26 / 93\end{array}$ & $\begin{array}{l}8091 \mathrm{~B} 7 \\
8091 \mathrm{Cl}\end{array}$ & $10.00 \mathrm{~L}$ & $5.50 \mathrm{~L}$ & 500.00 & $.57 \mathrm{U}$ \\
\hline $\begin{array}{l}299-W 18-29 \\
299-W 18-29\end{array}$ & $\begin{array}{l}8 / 26 / 93 \\
8 / 26 / 93\end{array}$ & $\begin{array}{l}8091 \mathrm{C2} \\
8091 \mathrm{C6}\end{array}$ & $5.00 \mathrm{~L}$ & $2.65 \mathrm{U}$ & 300.00 & $.83 \mathrm{U}$ \\
\hline $\begin{array}{l}299-H 18-32 \\
299-418-32\end{array}$ & $\begin{array}{l}8 / 26 / 93 \\
8 / 26 / 93\end{array}$ & $\begin{array}{l}8091 \mathrm{C} 7 \\
809101\end{array}$ & $4.70 \mathrm{LP}$ & 2.65 UP & 500.00 & 4.19 \\
\hline
\end{tabular}

\begin{tabular}{|c|c|c|c|c|c|c|}
\hline $\begin{array}{l}\text { Well } \\
\text { Name }\end{array}$ & $\begin{array}{l}\text { Collection } \\
\text { Date }\end{array}$ & $\begin{array}{l}\text { Sample } \\
\text { Number }\end{array}$ & $\begin{array}{c}\text { BETA } \\
136 / \mathrm{DCI} / \mathrm{L} \\
.150\end{array}$ & $\begin{array}{c}\text { IRON } \\
34 / \mathrm{ppb} \\
20 / 300 \mathrm{~s}\end{array}$ & $\begin{array}{c}\text { FIRON } \\
\text { 34/Ppb } \\
20 / 300 \mathrm{~s}\end{array}$ & $\begin{array}{l}\text { MAGNES } \\
34 / \text { ppb } \\
100 \%\end{array}$ \\
\hline $299-415-15$ & $8 / 16103$ & & & & & \\
\hline $\begin{array}{l}299-W 15-15 \\
299-W 15-15\end{array}$ & $\begin{array}{l}8 / 16 / 93 \\
8 / 16 / 93\end{array}$ & $\begin{array}{l}809130 \\
809134\end{array}$ & 7.51 & 460.00 & 63.00 & 11000.00 \\
\hline $299-W 15-16$ & $8 / 16 / 93$ & B09135 & 7.90 & 360.00 & & 19000.00 \\
\hline $299-W 15-16$ & $8 / 16 / 93$ & B09139 & & & 54.00 & \\
\hline $299-W 15-17$ & $9 / 08 / 93$ & B0\$140 & 6.75 & 770.00 & & 13000.00 \\
\hline $299-W 15-17$ & $9 / 08 / 93$ & B09144 & & & 35.00 & \\
\hline $299-W 15-18$ & $8 / 16 / 93$ & 809145 & 7.83 & 610.00 & & 12000.00 \\
\hline $299-W 15-18$ & $8 / 16 / 93$ & B09149 & & & 30.00 & \\
\hline $299-+15-19$ & $9 / 03 / 93$ & 809150 & 11.20 & 3400.000 & & 12000.00 \\
\hline $299-W 15-19$ & $9 / 03 / 93$ & 809151 & 9.56 & 1700.000 & & 11000.00 \\
\hline $\begin{array}{l}299-W 15-19 \\
299-W 15-19\end{array}$ & $\begin{array}{l}9 / 03 / 93 \\
9 / 03 / 93\end{array}$ & $\begin{array}{l}809158 \\
809159\end{array}$ & & & $\begin{array}{l}25.000 \\
95.000\end{array}$ & \\
\hline $299-W 15-20$ & $8 / 13 / 93$ & 809160 & 2.90 & 2300.00 & & 8200.00 \\
\hline $299-W 15-20$ & $8 / 13 / 93$ & B09164 & & & $10.30 \mathrm{U}$ & \\
\hline $299-W 15-23$ & $8 / 26 / 93$ & 809165 & 3.75 & 2500.00 & & 8300.00 \\
\hline 299-W15-23 & $8 / 26 / 93$ & B09169 & & & 31.00 & \\
\hline $299-W 15-24$ & $8 / 23 / 93$ & 809170 & 5.93 & 4700.00 & & 8000.00 \\
\hline $299-418-21$ & $8 / 23 / 93$ & 809175 & $1.90 \mathrm{U}$ & 620.00 & & 7600.00 \\
\hline $299-W 18-21$ & $8 / 23 / 93$ & 809179 & & & 50.00 & \\
\hline $\begin{array}{l}299-W 18-22 \\
299-W 18-22\end{array}$ & $\begin{array}{l}9 / 01 / 93 \\
9 / 01 / 93\end{array}$ & $\begin{array}{l}B 09180 \\
809184\end{array}$ & 5.81 & 660.00 & 37.00 & 12000.00 \\
\hline $\begin{array}{l}299-W 18-23 \\
299-W 18-23\end{array}$ & $\begin{array}{l}8 / 27 / 93 \\
8 / 27 / 93\end{array}$ & $\begin{array}{l}809185 \\
\text { B09189 }\end{array}$ & 3.86 & 270.00 & $15.00 \mathrm{~L}$ & 7500.00 \\
\hline $\begin{array}{l}299-W 18-24 \\
299-W 18-24\end{array}$ & $\begin{array}{l}8 / 27 / 93 \\
8 / 27 / 93\end{array}$ & $\begin{array}{l}\text { B09190 } \\
\text { B09194 }\end{array}$ & $1.34 \mathrm{U}$ & 210.00 & 21.00 & 6700.00 \\
\hline $299-W 18-26$ & $8 / 13 / 93$ & B09195 & 4.22 & 1700.00 & & 8300.00 \\
\hline $299-418-26$ & $8 / 13 / 93$ & 809199 & & & 77.00 & \\
\hline $\begin{array}{l}299-W 18-27 \\
299-W 18-27\end{array}$ & $\begin{array}{l}8 / 23 / 93 \\
8 / 23 / 93\end{array}$ & $\begin{array}{l}B 091 B 0 \\
B 09186\end{array}$ & 4.75 & 2400.00 & 22.00 & 7900.00 \\
\hline $\begin{array}{l}299-W 18-28 \\
299-W 18-28\end{array}$ & $\begin{array}{l}8 / 26 / 93 \\
8 / 26 / 93\end{array}$ & $\begin{array}{l}\text { B091B? } \\
\text { B091C1 }\end{array}$ & 4.99 & 1100.00 & 29.00 & 8500.00 \\
\hline $299-418-29$ & $8 / 26 / 93$ & B091C2 & 4.56 & 390.00 & & 5000.00 \\
\hline $299-418-29$ & $8 / 26 / 93$ & B091C6 & & & $10.30 \mathrm{U}$ & \\
\hline $\begin{array}{l}299-W 18-32 \\
299-W 18-32\end{array}$ & $\begin{array}{l}8 / 26 / 93 \\
8 / 26 / 93\end{array}$ & $\begin{array}{l}B 091 C 7 \\
809101\end{array}$ & 2.86 & $1400.00 \mathrm{P}$ & $31.00 \mathrm{P}$ & 6800.00 \\
\hline
\end{tabular}


Table 15-22. Constituents with at Least One Value Above the CRQL for the Low-Level Waste Burial Grounds WMA 4 Data for Reporting Period July 1 through September 30, 1993. (sheet 5 of 8 )

\begin{tabular}{|c|c|c|c|c|c|c|}
\hline $\begin{array}{l}\text { Well } \\
\text { Name }\end{array}$ & $\begin{array}{c}\text { Collection } \\
\text { Dote }\end{array}$ & $\begin{array}{l}\text { Sample } \\
\text { Number }\end{array}$ & $\begin{array}{c}\text { FMAGNES } \\
34 / \mathrm{ppb} \\
100 \%\end{array}$ & $\begin{array}{l}\text { MANGESE } \\
34 / \mathrm{ppb} \\
10 / 50 \mathrm{~s}\end{array}$ & $\begin{array}{r}\text { FMANGAN } \\
34 / \mathrm{ppb} \\
10 / 50 \mathrm{~s}\end{array}$ & $\begin{array}{c}\text { NICKEL } \\
34 / \text { ppb } \\
30 \%\end{array}$ \\
\hline $299-W 15-15$ & $8 / 16 / 93$ & B09130 & & 11.00 & & 60.00 \\
\hline $299-W 15-15$ & $8 / 16 / 93$ & 809134 & 11000.00 & & $2.80 \mathrm{~L}$ & \\
\hline $299-W 15-16$ & $8 / 16 / 93$ & B09135 & & $7.90 \mathrm{~L}$ & & 37.00 \\
\hline $299-W 15-16$ & $8 / 16 / 93$ & 809139 & 19000.00 & & $2.80 \mathrm{~L}$ & \\
\hline $299-615-17$ & $9 / 08 / 93$ & $B 09140$ & & 19.00 & & 110.00 \\
\hline 299-W15-17 & $9 / 08 / 93$ & B09144 & 13000.00 & & $5.60 \mathrm{~L}$ & \\
\hline $299-W 15-18$ & $8 / 16 / 93$ & 809145 & & 14.00 & & 69.00 \\
\hline $299-W 15-18$ & $8 / 16 / 93$ & B09149 & 12000.00 & & $2.80 \mathrm{~L}$ & \\
\hline $\begin{array}{l}299-W 15-19 \\
299-W 15-19\end{array}$ & $\begin{array}{l}9 / 03 / 93 \\
9 / 03 / 93\end{array}$ & $\begin{array}{l}\text { B09150 } \\
\text { B09151 }\end{array}$ & & $\begin{array}{l}73.000 \\
39.000\end{array}$ & & $\begin{array}{l}410.000 \\
200.000\end{array}$ \\
\hline $\begin{array}{l}299-W 15-19 \\
299-W 15-19\end{array}$ & $\begin{array}{l}9 / 03 / 93 \\
9 / 03 / 93\end{array}$ & $\begin{array}{l}309158 \\
B 09159\end{array}$ & $\begin{array}{l}11000.00 \\
11000.00\end{array}$ & & $\begin{array}{l}3.90 \mathrm{~L} \\
3.40 \mathrm{~L}\end{array}$ & \\
\hline $\begin{array}{l}299-W 15-20 \\
299-W 15-20\end{array}$ & $\begin{array}{l}8 / 13 / 93 \\
8 / 13 / 93\end{array}$ & $\begin{array}{l}\text { B09160 } \\
809164\end{array}$ & 8100.00 & 51.00 & $1.35 \mathrm{U}$ & 280.00 \\
\hline $\begin{array}{l}299-W 15-23 \\
299-w 15-23\end{array}$ & $\begin{array}{l}8 / 26 / 93 \\
8 / 26 / 93\end{array}$ & $\begin{array}{l}809165 \\
809169\end{array}$ & 8100.00 & 58.00 & $7.70 \mathrm{~L}$ & 320.00 \\
\hline $\begin{array}{l}299-w 15-24 \\
299-w 18-21\end{array}$ & $\begin{array}{l}8 / 23 / 93 \\
8 / 23 / 93\end{array}$ & $\begin{array}{l}B 09170 \\
B 09175\end{array}$ & & $\begin{array}{r}110.00 \\
14.00\end{array}$ & & $\begin{array}{r}520.00 \\
48.00\end{array}$ \\
\hline $299-418-21$ & $8 / 23 / 93$ & 809179 & 7600.00 & & $5.30 \mathrm{~L}$ & \\
\hline $299-W 18-22$ & $9 / 01 / 93$ & B09180 & & 12.00 & & 76.00 \\
\hline $\begin{array}{l}299-W 18-22 \\
299-W 18-23\end{array}$ & $\begin{array}{l}9 / 01 / 93 \\
8 / 27 / 93\end{array}$ & $\begin{array}{l}809184 \\
809185\end{array}$ & 12000.00 & $7.30 \mathrm{~L}$ & $1.35 \mathrm{U}$ & $27.00 \mathrm{~L}$ \\
\hline $299-W 18-23$ & $8 / 27 / 93$ & B09189 & 7500.00 & & $3.00 \mathrm{~L}$ & \\
\hline $299 \cdot-W 18-24$ & $8 / 27 / 93$ & 809190 & & $8.20 \mathrm{~L}$ & & $27.00 \mathrm{~L}$ \\
\hline $299-W 18-24$ & $8 / 27 / 93$ & B09194 & 6600.00 & & $3.40 \mathrm{~L}$ & \\
\hline $299-W 18-26$ & $8 / 13 / 93$ & 809195 & & 36.00 & & 170.00 \\
\hline $299-W 18-26$ & $8 / 13 / 93$ & B09199 & 8300.00 & & $7.60 \mathrm{~L}$ & \\
\hline $299-W 18-27$ & $8 / 23 / 93$ & B091BO & & 84.00 & & 110.00 \\
\hline $299-W 18-27$ & $8 / 23 / 93$ & B091B6 & 7600.00 & & $5.10 \mathrm{~L}$ & \\
\hline $299-W 18-28$ & $8 / 26 / 93$ & B091B 7 & & 47.00 & & 80.00 \\
\hline $299-W 18-28$ & $8 / 26 / 93$ & B091C1 & 8400.00 & & 28.00 & \\
\hline $299-W 18-29$ & $8 / 26 / 93$ & B091C2 & & $9.00 \mathrm{~L}$ & & 50.00 \\
\hline $299-W 18-29$ & $8 / 26 / 93$ & $8091 \mathrm{C} 6$ & 5100.00 & & $3.00 \mathrm{~L}$ & \\
\hline $299-W 18-32$ & $8 / 26 / 93$ & $8091 C 7$ & & $18.00 \mathrm{P}$ & & 35.00 \\
\hline $299-418-32$ & $8 / 26 / 93$ & 809101 & $6700.00 \mathrm{P}$ & & $3.90 \mathrm{LP}$ & \\
\hline
\end{tabular}

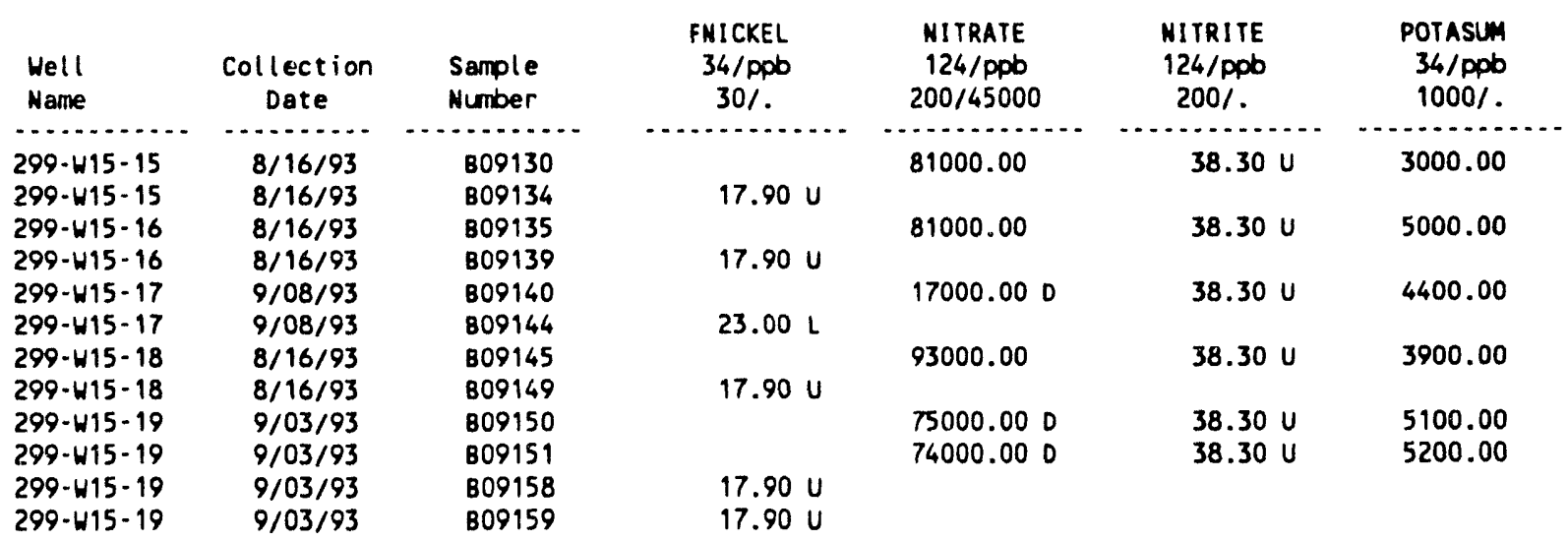


Table 15-22. Constituents with at Least One Value Above the CRQL for the Low-Level Waste Burial Grounds WMA 4 Data for Reporting Period July 1 through September 30, 1993. (sheet 6 of 8)

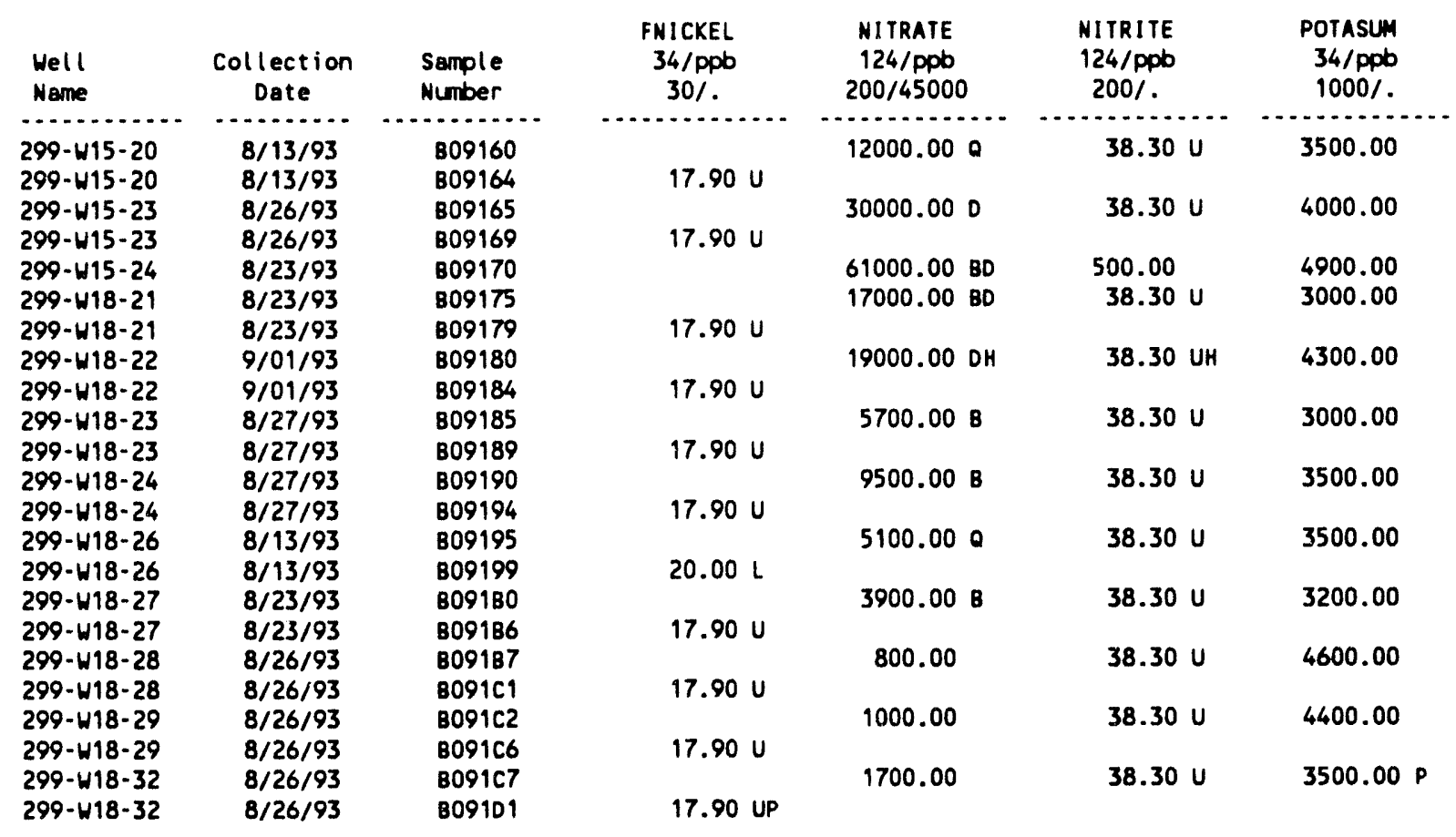

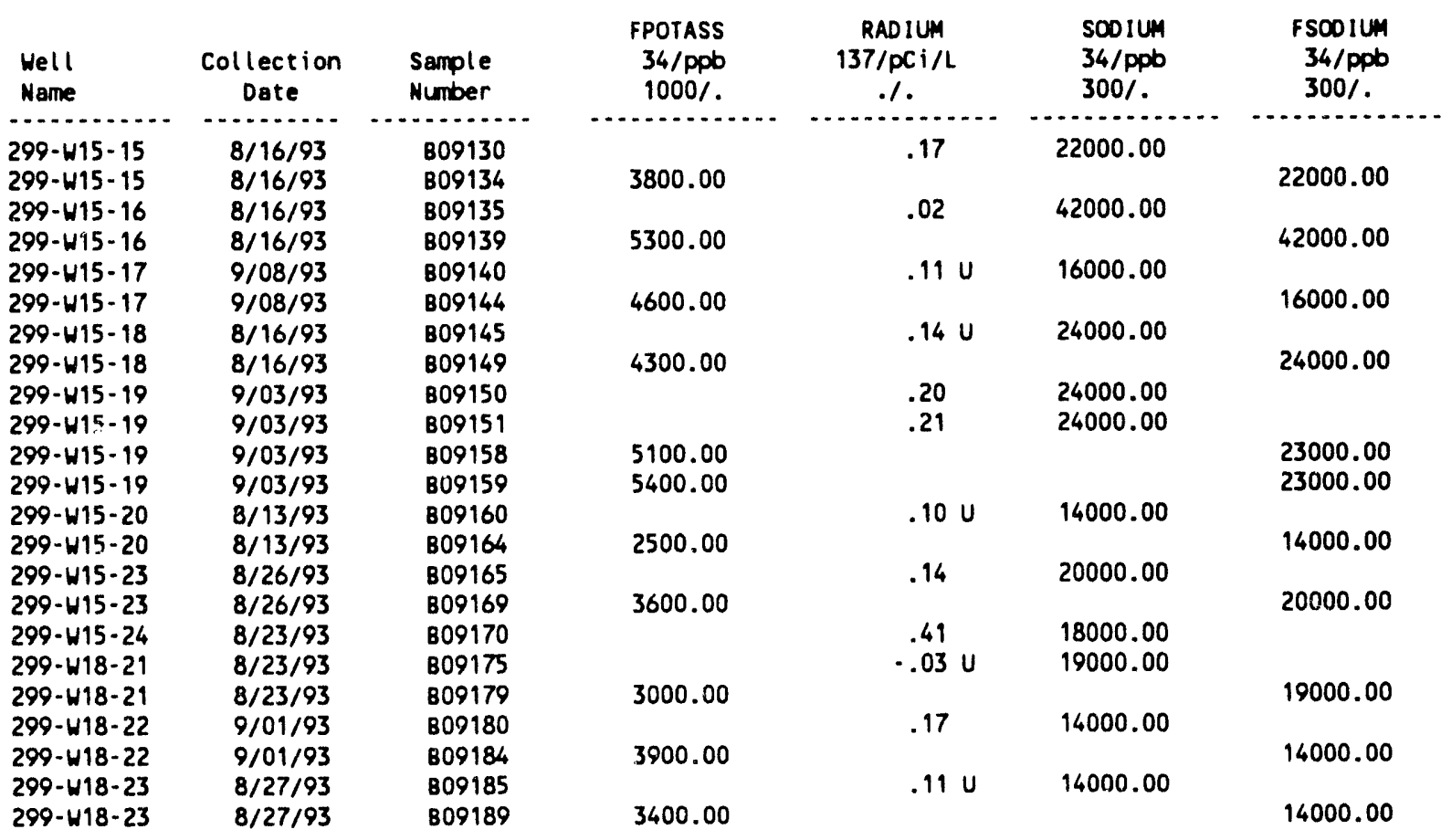


Table 15-22. Constituents with at Least One Value Above the CRQL for the Low-Level Waste Burial Grounds WMA 4 Data for Reporting Period July 1 through September 30, 1993. (sheet 7 of 8 )

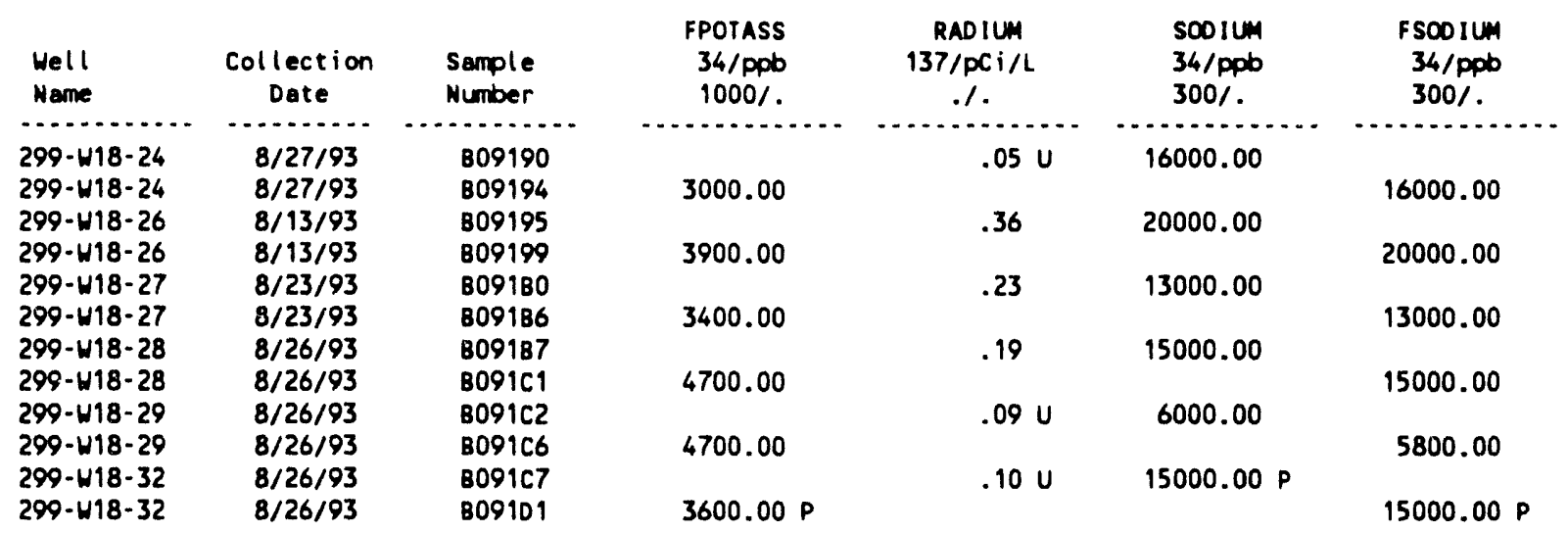

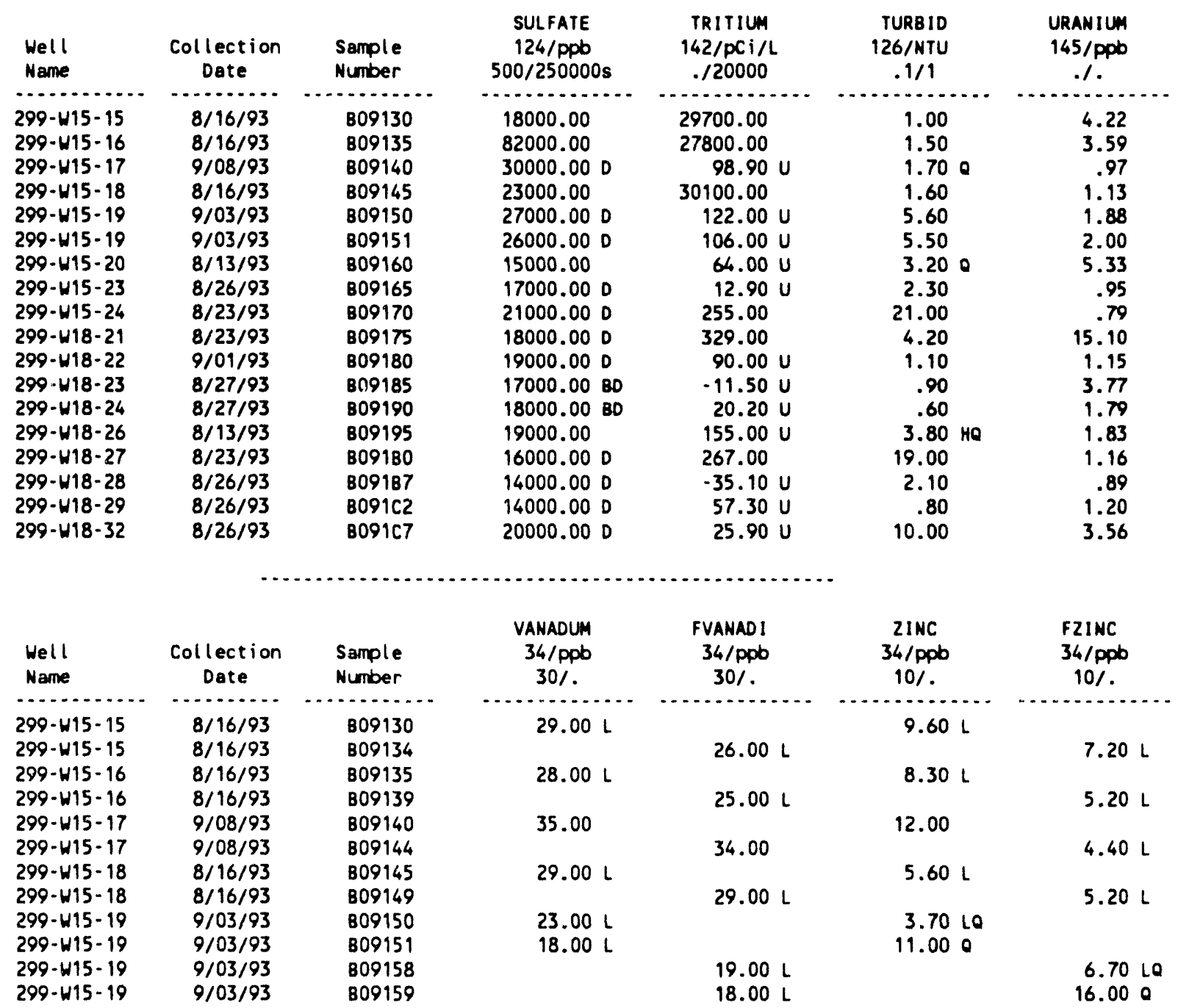


Table 15-22. Constituents with at Least One Value Above the CRQL for the Low-Level Waste Burial Grounds WMA 4 Data for Reporting Period July 1 through September 30, 1993. (sheet 8 of 8 )

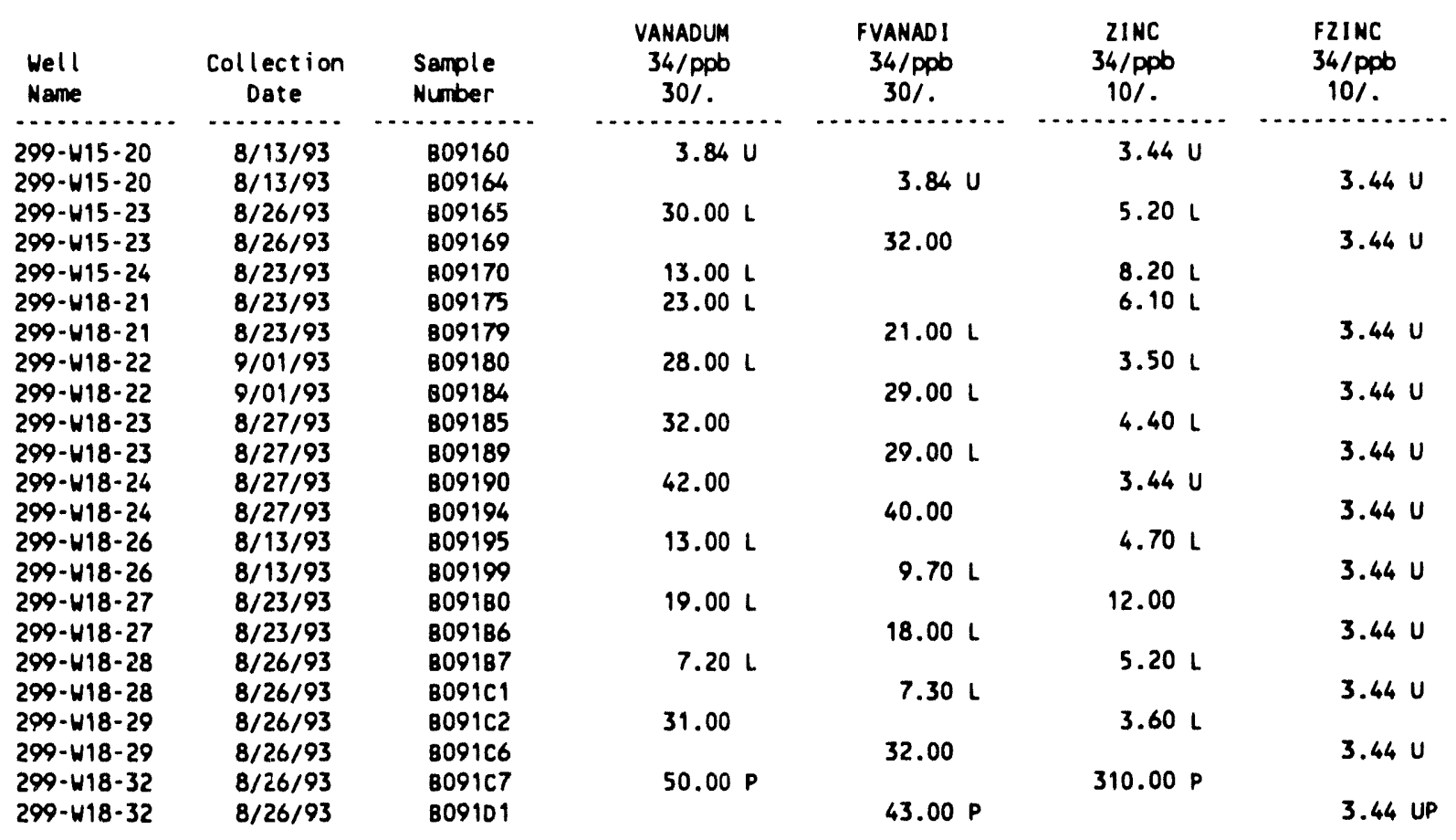

For explanation of this table, see Section 1.4 of report. 
Table 15-23. Contamination Indicator Parameters for the Low-Level Waste Burial Grounds WMA 4 Data for Reporting Period July 1 through September 30, 1993. (sheet 1 of 2)

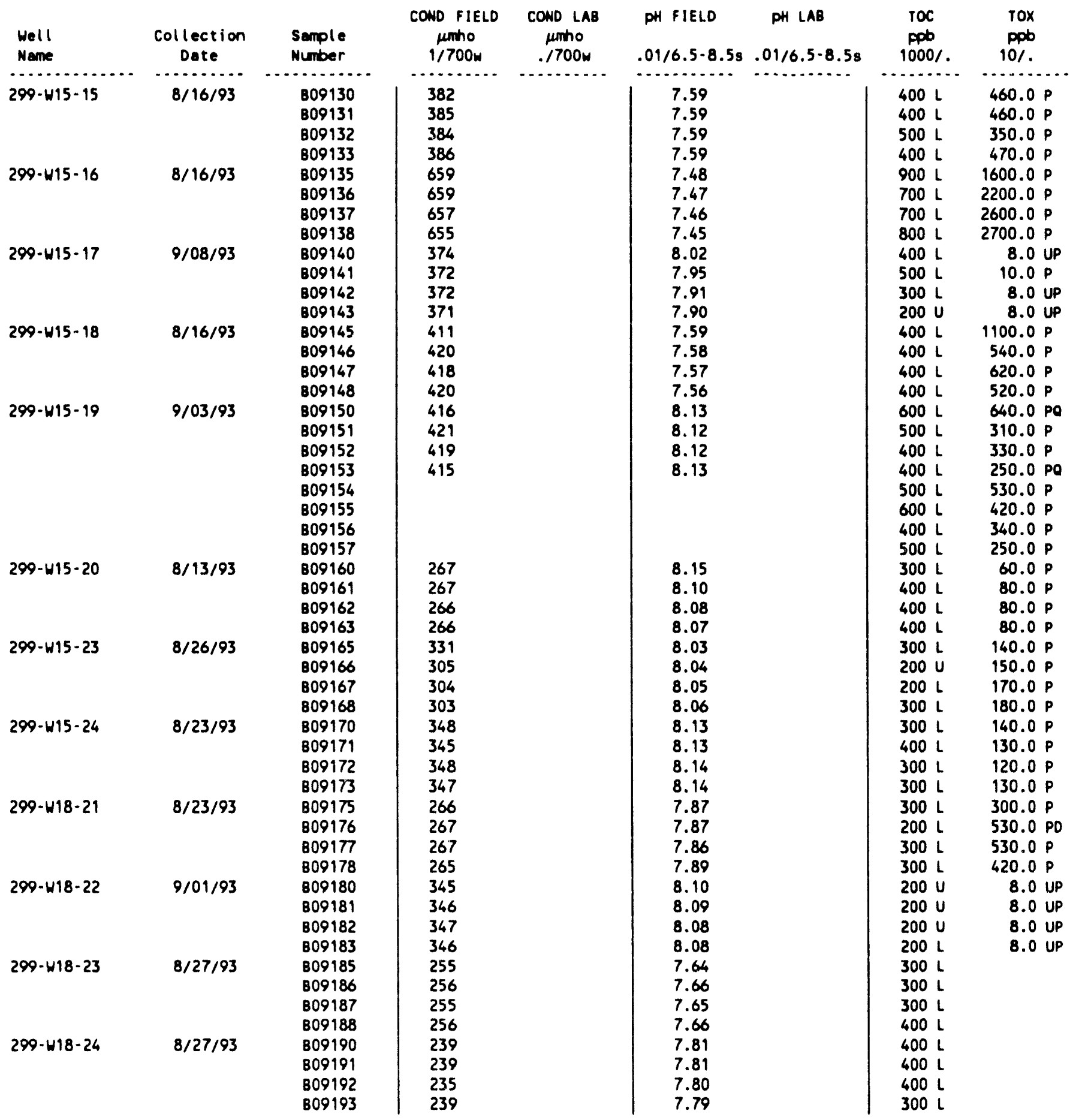


Table 15-23. Contamination Indicator Parameters for the Low-Level Waste Burial Grounds WMA 4 Data for Reporting Period July 1 through September 30,1993 . (sheet 2 of 2)

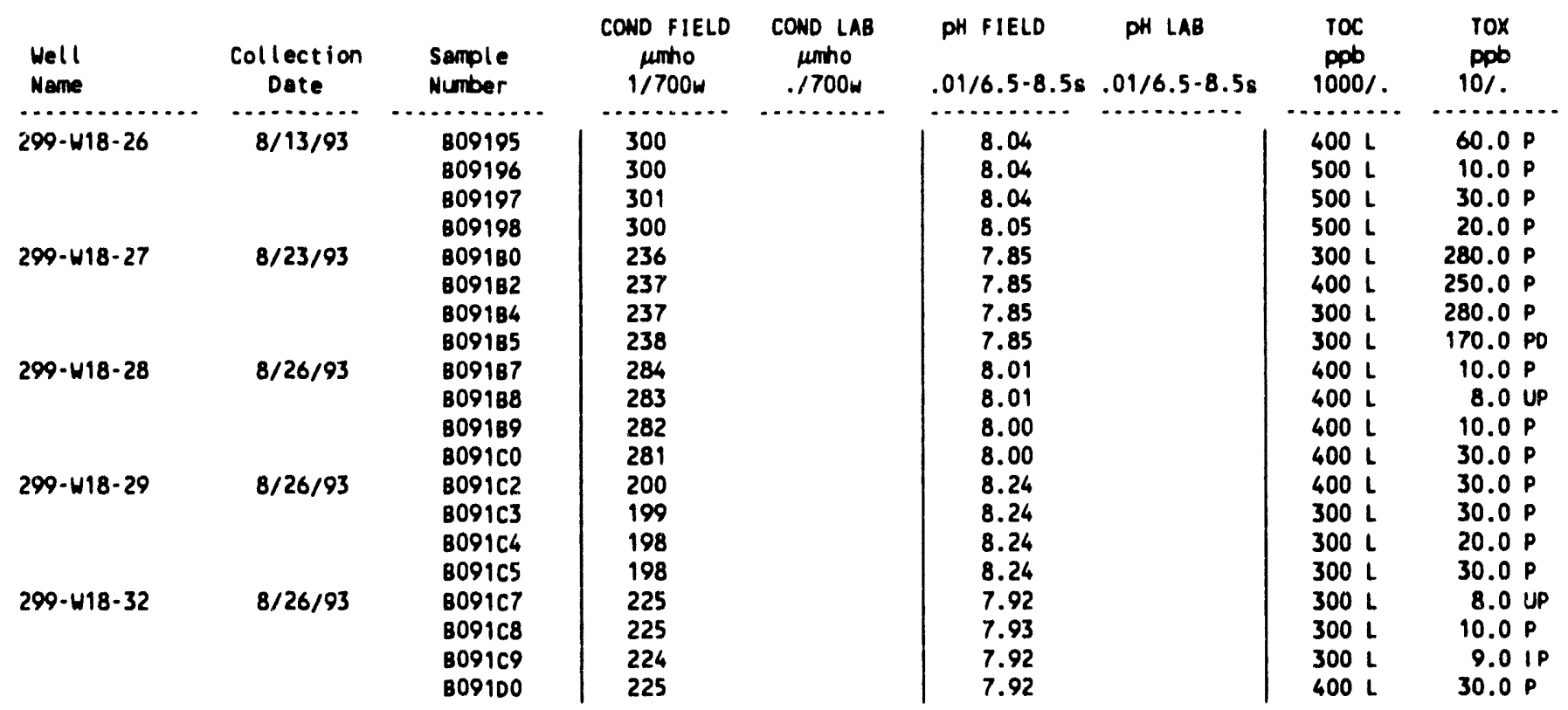

For explanation of this table, see Section 1.4 of report. 
Table 15-24. Constituent List and Summary of Results for the Low-Level Waste Burial Grounds WMA 5 Data for Reporting Period July 1 through September 30,1993. (sheet 1 of 6)

CONTAMINATION INDICATOR PARAMETERS

\begin{tabular}{|c|c|c|c|c|c|c|c|c|c|}
\hline \multicolumn{4}{|c|}{ Constituent Name } & \multicolumn{3}{|c|}{ DWS } & \multicolumn{3}{|c|}{ Number of Semples } \\
\hline Short & (Method) & Full & Units & CRQL & Limit & Agency & Total & $>$ CROL & $>$ DUS \\
\hline$\ldots \ldots$ & 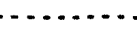 & $\ldots \ldots \ldots$ & $\ldots \ldots$ & $\ldots \ldots$ & $\ldots \ldots$ & $\ldots \ldots$ & $\cdots$ & $\ldots$. & $\cdots$ \\
\hline $\begin{array}{l}\text { CONDUCT } \\
\text { TOC } \\
\text { TOXLDL }\end{array}$ & & $\begin{array}{l}\text { Conductivity, field } \\
\text { Total Organic Carbon } \\
\text { Total Organic Halogen }\end{array}$ & $\begin{array}{l}\text { umho } \\
\text { ppb } \\
\text { ppb }\end{array}$ & $\begin{array}{r}1 \\
1000 \\
10\end{array}$ & & & $\begin{array}{l}46 \\
22 \\
46\end{array}$ & $\begin{array}{r}46 \\
0 \\
33\end{array}$ & \\
\hline PH & & pH, field & & .1 & $6.5 \cdot 8.5$ & EPAS & 46 & 46 & 0 \\
\hline
\end{tabular}

DRINKING WATER PARAMETERS

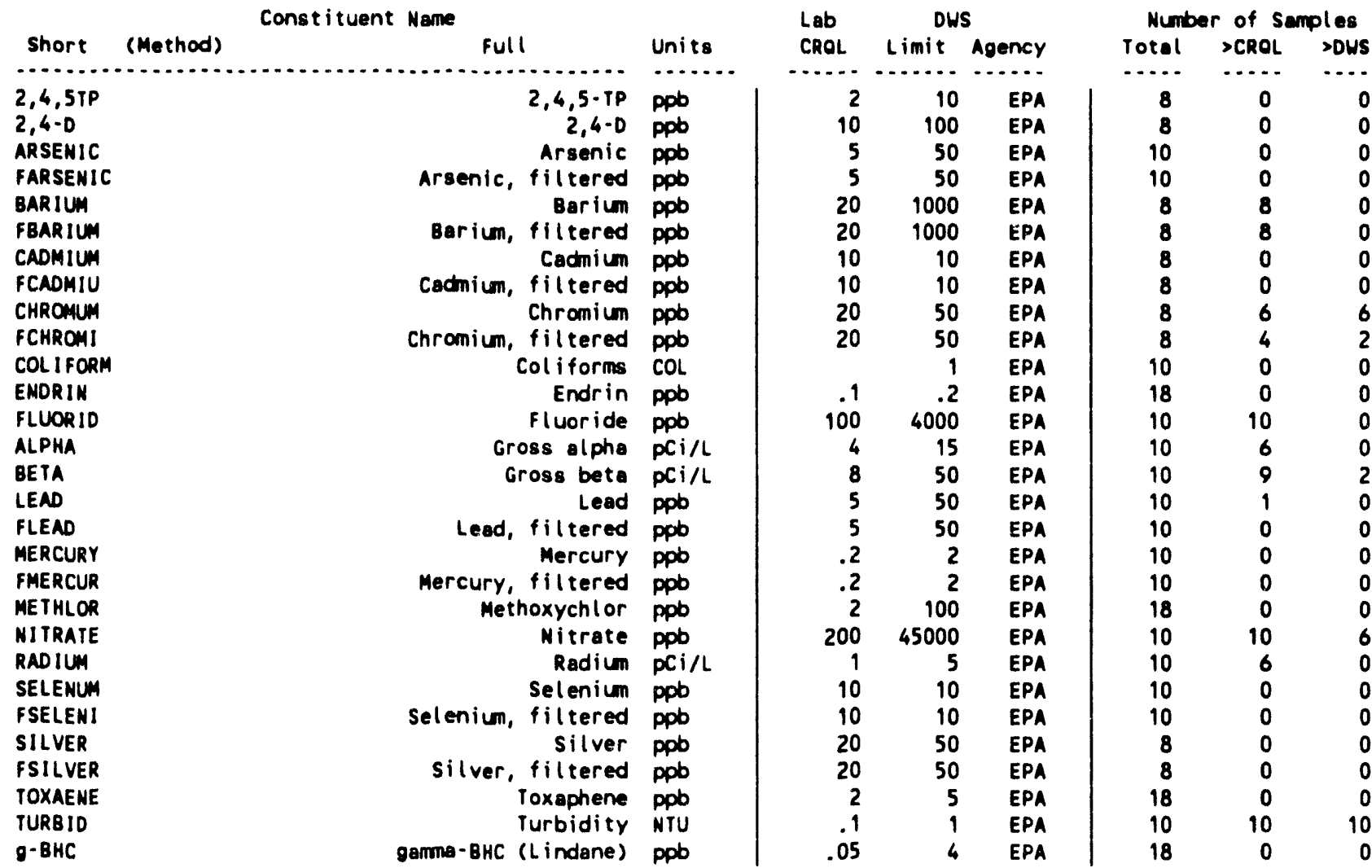

GROUNDWATER QUALITY PARAMETERS

\begin{tabular}{|c|c|c|c|c|c|c|c|c|c|}
\hline \multicolumn{4}{|c|}{ Const ituent Name } & \multicolumn{3}{|c|}{ DWS } & \multicolumn{3}{|c|}{ Nunber of Semples } \\
\hline Short & (Method) & Full & Units & CRQL & Limit & Agency & Total & $>$ CROL & $>0$ WS \\
\hline$\ldots \ldots$ & $\ldots \ldots \ldots$ & $\ldots \ldots \ldots$ & $\ldots \ldots$ & $\ldots .$. & $\ldots \ldots$ & $\ldots \ldots$ & $\ldots \ldots$ & $\ldots$. & $\ldots$ \\
\hline CHLORID & & Chloride & ppo & 200 & 250000 & EPAS & 10 & 10 & 0 \\
\hline IRON & & Iron & ppo & 20 & 300 & EPAS & 8 & 8 & 7 \\
\hline FIRON & & Iron, filtered & ppb & 20 & 300 & EPAS & 8 & 3 & 0 \\
\hline MANGESE & & Manganese & ppb & 10 & 50 & EPAS & 8 & 7 & 0 \\
\hline FMANGAN & & Manganese, filtered & ppb & 10 & 50 & EPAS & 8 & 2 & 0 \\
\hline LPHENOL & 19 & Phenol & ppb & 10 & & & 7 & 0 & \\
\hline LPHENOL & 30 & Phenol & ppb & 20 & & & 8 & 0 & \\
\hline SODIUM & & Sodium & ppb & 300 & & & 8 & 8 & \\
\hline FSODIUM & & Sodium, filtered & ppb & 300 & & & 8 & 8 & \\
\hline SULFATE & & Sulfate & ppb & 500 & 250000 & EPAS & 10 & 10 & 0 \\
\hline
\end{tabular}




\section{Table 15-24. Constituent List and Summary of Results for the Low-Level Waste Burial Grounds WMA 5 Data for Reporting Period July 1 through September 30, 1993. (sheet 2 of 6 )}

SITE SPECIFIC AND OTHER CONSTITUENTS

\begin{tabular}{|c|c|c|c|c|c|c|c|c|}
\hline Short & (Method) & $\begin{array}{l}\text { Constituent Neme Full } \\
\text { Ful }\end{array}$ & Units & $\begin{array}{l}\text { Lab } \\
\text { CROL }\end{array}$ & $\begin{array}{c}\text { DWS } \\
\text { Limit Agency }\end{array}$ & $\begin{array}{l}\text { Numt } \\
\text { Total }\end{array}$ & $\begin{array}{l}\text { of } \mathrm{Se} \\
>\mathrm{CROL}\end{array}$ & $\begin{array}{l}\text { mples } \\
>\text { DWS }\end{array}$ \\
\hline (112...... & & . & $\cdots$ & $\cdots .$. & $\cdots$ & $\cdots$ & & \\
\hline $1112-t c$ & & 1,1,1,2-Tetrachlor oethane & ppb & 5 & & 5 & 0 & \\
\hline $1,1,1-T$ & & 1,1,1-Trichloroethane & ppob & 5 & & 18 & 0 & \\
\hline $1122 \cdot t c$ & & $1,1,2,2-$ Tetrachlor oe thane & ppb & 5 & & 5 & 0 & \\
\hline $1,1,2-1$ & & 1,1,2-Irichloroethane & ppb & 5 & & 18 & 0 & \\
\hline $1,1-D I C$ & & 1,1-Dichloroethane & ppb & 5 & & 18 & 0 & \\
\hline DICETHY & & 1,1-Dichloroethene & ppo & 5 & & 5 & 0 & \\
\hline 123-trp & & 1,2,3-Trichloropropane & ppb & 5 & & 5 & 0 & \\
\hline TETRCHB & & $1,2,4,5$ - Tetrachlorobenzene & ppb & 10 & & 5 & 0 & \\
\hline TRICHLB & & 1,2,4-irichlor obenzene & Ppb & 10 & & 5 & 0 & \\
\hline $\begin{array}{l}\text { DIBRCHL } \\
\text { DIBRETH }\end{array}$ & & $\begin{array}{r}\text { 1,2-D ibromo-3-chloropropane } \\
\text { 1,2-D ibromoethane }\end{array}$ & $\begin{array}{l}\text { ppb } \\
\text { ppb }\end{array}$ & $\begin{array}{l}5 \\
5\end{array}$ & & $\begin{array}{l}5 \\
5\end{array}$ & $\begin{array}{l}0 \\
0\end{array}$ & \\
\hline 12-ctben & & 1,2-0ichlorobenzene & ppb & 10 & & 5 & 0 & \\
\hline $1,2-01 C$ & & 1,2-Dichloroethane & ppo & 5 & & 18 & 0 & \\
\hline 12-DCE & & 1,2-Dichloroethene & ppob & 5 & & 18 & 0 & \\
\hline DICPANE & & 1,2-Dichloropropane & ppo & 5 & & $\begin{array}{l}5 \\
5\end{array}$ & 0 & \\
\hline 13-cben & & 1,3-Dichlorobenzene & ppb & 10 & & 5 & 0 & \\
\hline 14-dben & 16 & 1,4-Dichlorobenzene & ppb & 5 & & 13 & 0 & \\
\hline 14-cben & 19 & 1,4-Dichlorobenzene & ppb & 10 & & 5 & 0 & \\
\hline DIOXANE & & 1,4-Dioxane & ppb & 200 & & 5 & 0 & \\
\hline NAPHQUI & & 1,4-Nophtoquinone & ppob & 10 & & 5 & 0 & \\
\hline 1BUTYN & & 1-Butanol & ppb & 1000 & & 18 & 0 & \\
\hline 1-napha & & 1-Naphthylanine & ppb & 10 & & 5 & 0 & \\
\hline TETRCHP & & $2,3,4,6$ - Tetrachlorophenol & ppb & 10 & & 5 & 0 & \\
\hline $2378 \mathrm{rCD}$ & & $2,3,7,8-T C D D$ & ppb & .005 & & 1 & 0 & \\
\hline $2,4,5-1$ & & $2,4,5-T$ & ppb & 2 & & 8 & 0 & \\
\hline $245 \cdot \operatorname{trp}$ & & 2,4,5-Trichlorophenol & ppob & 10 & & 5 & 0 & \\
\hline $246-\operatorname{trp}$ & 19 & 2,4,6-Trichlorophenol & ppob & 10 & & 5 & 0 & \\
\hline 246-trp & 30 & 2,4,6-Trichlorophenol & ppb & 5 & & 8 & 0 & \\
\hline 24-dchp & 19 & 2,4-Dichlorophenol & ppb & 10 & & 7 & 0 & \\
\hline 24 -dchp & 30 & 2,4-D ichlorophenol & ppb & 5 & & 8 & 0 & \\
\hline DIMPHEN & 19 & 2,4-D imethylphenol & ppb & 10 & & 5 & 0 & \\
\hline DIMPHEN & 30 & 2,4-D ime thylphenol & ppb & 5 & & 8 & 0 & \\
\hline DINPHEN & 19 & 2,4-Dinitrophenol & ppb & 50 & & 5 & 0 & \\
\hline DINPHEN & 30 & 2,4-D initrophenol & ppb & 150 & & 8 & 0 & \\
\hline 24 -dint & & $2,4-0$ initrotoluene & ppb & 10 & & 5 & 0 & \\
\hline 26-dchp & 19 & 2,6-Dichlorophenol & ppb & 10 & & 5 & 0 & \\
\hline 26-dchp & 30 & 2,6-Dichlorophenol & ppb & 5 & & 8 & 0 & \\
\hline 26-dint & & 2,6-0 initrotoluene & ppb & 10 & & 5 & 0 & \\
\hline ACEFENE & & 2-Acetylaminof luorene & ppb & 10 & & 5 & 0 & \\
\hline CHLNAPH & & 2-Chloronaph thalene & ppb & 10 & & 5 & 0 & \\
\hline CHLPHEN & 19 & 2-Chlorophenol & ppb & 10 & & 5 & 0 & \\
\hline CHLPHEN & 30 & 2-Chlorophenol & ppo & 5 & & 8 & 0 & \\
\hline 2HEXANO & & 2-Hexanone & ppb & 50 & & 5 & 0 & \\
\hline 2MENAPH & & 2-Methylnaph thal ene & ppo & 10 & & 5 & 0 & \\
\hline 2METHPH & & 2-Methylphenol & ppb & 10 & & 7 & 0 & \\
\hline 2-naphe & & 2-Naphthylamine & ppob & 10 & & 5 & 0 & \\
\hline ONITANI & & 2-Nitroaniline & ppb & 50 & & 5 & 0 & \\
\hline 2NITPH & 19 & 2-Nitrophenol & ppb & 10 & & 7 & 0 & \\
\hline 2NITPH & 30 & 2-Nitrophenol & ppo & 5 & & 8 & 0 & \\
\hline PICOLIN & & 2-Picoline & ppob & 10 & & 5 & 0 & \\
\hline BUTDINP & 30 & 2-sec-Butyl-4,6-dinitrophenol (DN & ppb & 5 & & 8 & 0 & \\
\hline BUTDINP & 49 & 2-sec-Butyl-4,6-dinitrophenol (DN & ppb & 1 & & 8 & 0 & \\
\hline DICHBEN & & 3,3i-Dichlorobenz idine & ppob & 20 & & 5 & 0 & \\
\hline DIMEYLB & & 3,3'-D ime thylbenzidine & ppb & 10 & & 5 & 0 & \\
\hline
\end{tabular}


Table 15-24. Constituent List and Summary of Results for the Low-Level Waste Burial Grounds WMA 5 Data for Reporting Period July 1 through September 30, 1993. (sheet 3 of 6)

\begin{tabular}{|c|c|c|c|c|c|c|c|c|}
\hline \multicolumn{4}{|c|}{ Constituent Name } & \multicolumn{2}{|r|}{ DWS } & \multicolumn{3}{|c|}{ Number of Semples } \\
\hline Short & (Method) & Full & Units & CROL & Limit Agency & Totel & $>\mathrm{CROL}$ & $>$ DUS \\
\hline$\ldots \ldots$ & $\ldots \ldots$ & 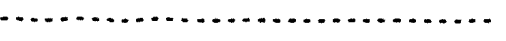 & $\ldots \ldots$ & $\ldots \ldots$ & $\ldots \ldots \ldots$ & $\ldots \ldots$ & $\cdots \cdots$ & $\cdots$ \\
\hline METCHAN & & 3-Methylcholanthrene & ppb & 10 & & 5 & 0 & \\
\hline MNITAN! & & 3-Nitroaniline & ppb & 50 & & 5 & 0 & \\
\hline DDD & & $4,4^{\prime}-D D D$ & ppo & .1 & & 18 & 0 & \\
\hline DOE & & $4,4^{\prime}-$ DDE & ppo & .05 & & 18 & 0 & \\
\hline DOT & & $4.41 .00 T$ & ppb & .1 & & 18 & 0 & \\
\hline 460N2MP & 19 & 4,6-0 initro-2-methylphenol & ppb & 50 & & 5 & 0 & \\
\hline 460N2NP & 30 & 4,6-Dinitro-2-methylphenol & ppb & 200 & & 8 & 0 & \\
\hline MINOYL & & 4-Aminobiphenyi & ppb & 10 & & 5 & 0 & \\
\hline BROPKEN & & 4-Bromophenylphenyl ether & ppb & 10 & & 5 & 0 & \\
\hline CHLCRES & 19 & 4-Chloro-3-methylphenol & ppb & 20 & & 5 & 0 & \\
\hline CHLCRES & 30 & 4-Chloro-3-methylphenol & ppb & 5 & & 8 & 0 & \\
\hline CHLANIL & & 4-Chloroaniline & ppob & 20 & & 5 & 0 & \\
\hline 4CPPETH & & 4-Chlorophenylphenyl ether & ppo & 10 & & 5 & 0 & \\
\hline MIOK & & 4-Methyl-2-pentanone & ppb & 50 & & 18 & 0 & \\
\hline СMETHPH & & 4-Methylphenol & ppb & 10 & & 7 & 0 & \\
\hline NITRANI & & 4-Nitroaniline & ppb & 50 & & 5 & 0 & \\
\hline NITPHEN & 19 & 4-Nitrophenol & ppb & 50 & & 5 & 0 & \\
\hline NITPHEN & 30 & 4-Nitrophenol & ppb & 30 & & 8 & 0 & \\
\hline 4NITOUI & & 4-Nitroquinoline-1-oxide & ppo & 10 & & 5 & 0 & \\
\hline NITRTOL & & 5-Nitro-0-toluidine & ppb & 10 & & 5 & 0 & \\
\hline DIMBENZ & & 7, 12-D imethylbenz (B) anthracene & ppb & 10 & & 5 & 0 & \\
\hline ACENAPH & & Acenaph thene & ppb & 10 & & 5 & 0 & \\
\hline ACENATL & & Acenaphthylene & ppb & 10 & & 5 & 0 & \\
\hline ACETONE & & Acetone & ppo & 100 & & 18 & 0 & \\
\hline ACETILE & & Acetonitrile & ppb & 200 & & 5 & 0 & \\
\hline ACETOPH & & Acetophenone & ppb & 10 & & 5 & 0 & \\
\hline ACROLIN & & Acrolein & ppb & 5 & & 5 & 0 & \\
\hline ACRYILE & & Acrylonitrile & ppb & 5 & & 5 & 0 & \\
\hline ALDRIN & & Aldrin & ppb & .05 & & 18 & 0 & \\
\hline DIMPHAM & & Alpha, alpha-D imethylphenethyl ami & ppb & 10 & & 5 & 0 & \\
\hline a-BHC & & Alpha-BHC & ppob & .05 & & 18 & 0 & \\
\hline ALUMNUM & & Aluminum & ppo & 200 & & 8 & 2 & \\
\hline FALUMIN & & Aluminum & ppb & 200 & & 8 & 0 & \\
\hline AMMONIU & & Ammonium ion & ppo & 100 & & 10 & 2 & \\
\hline ANILINE & & Aniline & ppo & 10 & & 5 & 0 & \\
\hline ANTHRA & & Anthracene & ppb & 10 & & 5 & 0 & \\
\hline ANT IONY & & Ant imony & pob & 200 & & 8 & 0 & \\
\hline FANTIMO & & Ant imony, filtered & ppb & 200 & & 8 & 0 & \\
\hline ARAMITE & & Aranite & ppb & 10 & & 5 & 0 & \\
\hline AR 1016 & & Aroclor -1016 & ppb & 1 & & 5 & 0 & \\
\hline AR1221 & & Aroclor-1221 & ppb & 1 & & 5 & 0 & \\
\hline AR 1232 & & Aroclor -1232 & ppb & 1 & & 5 & 0 & \\
\hline AR 1242 & & Aroclor -1242 & ppb & 1 & & 5 & 0 & \\
\hline AR 1248 & & Aroclor -1248 & ppb & 1 & & 5 & 0 & \\
\hline AR1254 & & Aroclor -1254 & ppb & 1 & & 5 & 0 & \\
\hline$A R 1260$ & & Aroclor -1260 & ppb & 1 & & 5 & 0 & \\
\hline BENZENE & & Benzene & ppo & 5 & & 18 & 0 & \\
\hline BENZAAN & & Benzo( 8 ) anthracene & ppb & 10 & & 5 & 0 & \\
\hline BENZOPY & & Benzo(a)pyrene & ppb & 10 & & 5 & 0 & \\
\hline BENZBFL & & Benzo(b) fluor anthene & ppb & 10 & & 5 & 0 & \\
\hline BGHIPER & & Benzo(ghi)perylene & ppb & 10 & & 5 & 0 & \\
\hline BNZKFLU & & Benzo(k) fluoranthene & ppb & 10 & & 5 & 0 & \\
\hline BENZOTH & & Benzothiazole & ppb & 10 & & 7 & 0 & \\
\hline BENZALC & & Benzyl alcohol & ppb & 20 & & 5 & 0 & \\
\hline BERYLUM & & Beryllium & ppb & 3 & & 8 & 0 & \\
\hline FBERYLL & & Beryllium, filtered & ppb & 3 & & 8 & 0 & \\
\hline b-BHC & & Beta-BHC & ppb & .05 & & 18 & 0 & \\
\hline
\end{tabular}




\section{Table 15-24. Constituent List and Summary of Results for the Low-Level Waste Burial Grounds WMA 5 Data for Reporting Period July 1 \\ through September 30,1993 . (sheet 4 of 6 )}

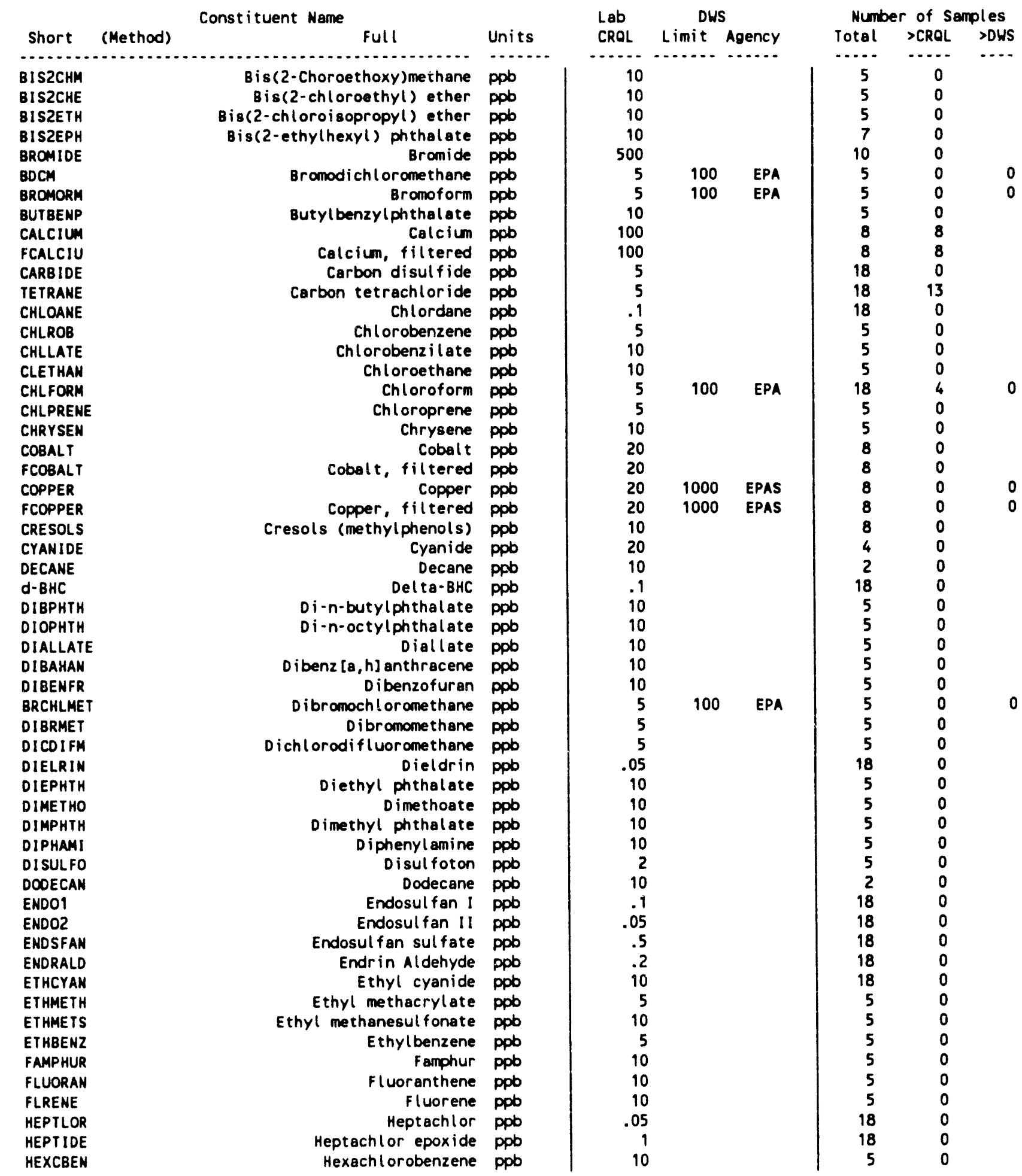


Table 15-24. Constituent List and Summary of Results for the Low-Level Waste Burial Grounds WMA 5 Data for Reporting Period July 1

through September 30, 1993. (sheet 5 of 6)

\begin{tabular}{|c|c|c|c|c|c|c|c|c|}
\hline & & Constituent Name & & Lab & DWS & Num & r of $\mathrm{Se}$ & ples \\
\hline Short & (Method) & Full & Units & CROL & Limit Agency & Total & $>$ CRQL & $>$ DWS \\
\hline$\cdots \cdots$ & 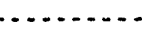 & 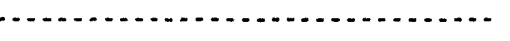 & $\ldots$ & 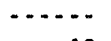 & $\cdots \ldots$ & $\cdots \cdot$ & $\cdots$ & $\cdots$ \\
\hline HEXCBUT & & Hexachlorobutadiene & ppb & 10 & & 5 & 0 & \\
\hline HEXCCYC & & Hexach lorocyclopent adiene & ppo & 10 & & 5 & 0 & \\
\hline HEXCETH & & Hexachloroe thane & ppb & 10 & & 5 & 0 & \\
\hline HEXACHL & & Hexach lorophene & ppb & 10 & & 5 & 0 & \\
\hline HEXAENE & & Hexach l oropropene & ppob & 10 & & 5 & 0 & \\
\hline INDENOP & & Indeno(1,2,3-cd)pyrene & ppb & 10 & & 5 & 0 & \\
\hline I SOBUTY & & Isobutyl alcohol & ppob & 200 & & 5 & 0 & \\
\hline ISOOR IN & & Isodrin & ppob & 10 & & 5 & 0 & \\
\hline ISOPHER & & I sophorone & ppb & 10 & & 5 & 0 & \\
\hline ISOSOLE & & I sosafrole & ppob & 10 & & 5 & 0 & \\
\hline KEPONE & & Kepone & ppb & 10 & & 5 & 0 & \\
\hline KEROSEN & & Kerosene & ppb & 10000 & & 5 & 0 & \\
\hline MAGNES & & Magnesium & ppb & 100 & & 8 & 8 & \\
\hline FMAGNES & & Magnesium, filtered & ppb & 100 & & 8 & 8 & \\
\hline METHACR & & Methacrylonitrile & ppb & 5 & & 5 & 0 & \\
\hline METHAPY & & Methapyrilene & ppo & 10 & & 5 & 0 & \\
\hline IODOMET & & Methyl lodide & ppb & 5 & & 5 & 0 & \\
\hline METHBRO & & Methyl bromide & ppb & 10 & & 5 & 0 & \\
\hline METHCHL & & Methyl chloride & ppb & 10 & & 5 & 0 & \\
\hline METHONE & & Methyl ethyl ketone & ppb & 100 & & 18 & 0 & \\
\hline METACRY & & Methyl methacrylate & ppb & 5 & & 5 & 0 & \\
\hline METMSUL & & Methyl methanesul fonate & ppb & 10 & & 5 & 0 & \\
\hline METHPAR & & Methyl parathion & ppb & .5 & & 5 & 0 & \\
\hline METHYCH & & Methylene chloride & ppb & 5 & & 18 & 2 & \\
\hline DIPRNIT & & N-Nitroso-di-n-dipropylanine & ppb & 10 & & 5 & 0 & \\
\hline NNIBUTY & & $N$-Nitrosodi-n-butylamine & ppb & 10 & & 5 & 0 & \\
\hline NNIDIEY & & N-Nitrosodiethylamine & ppo & 10 & & 5 & 0 & \\
\hline NNIDIME & & N-Nitrosodimethylamine & ppob & 10 & & 5 & 0 & \\
\hline NND IPHA & & $\mathrm{N}-\mathrm{Nit}$ rosodiphenylamine & ppb & 10 & & 5 & 0 & \\
\hline NNIMETH & & N-Nitrosomethylethylamine & ppb & 10 & & 5 & 0 & \\
\hline NNIMORP & & $\mathrm{N}$-Nitrosomorpholine & ppb & 10 & & 5 & 0 & \\
\hline NHIPIPE & & N-Nitrosopiperidine & ppb & 10 & & 5 & 0 & \\
\hline NAPHTHA & & Haphthalene & ppb & 10 & & 7 & 0 & \\
\hline NICKEL & & Nickel & ppb & 30 & & 8 & 4 & \\
\hline FNICKEL & & Nickel, filtered & ppb & 30 & & 8 & 0 & \\
\hline NITRITE & & Nitrite & ppb & 200 & & 10 & 0 & \\
\hline NITBENZ & & Nitrobenzene & ppb & 10 & & 5 & 0 & \\
\hline NITRPYR & & Nitrosopyrrolidine & ppb & 10 & & 5 & 0 & \\
\hline TRIPHOS & & $0,0,0$-Triethyl phosphorothioate & ppb & 10 & & 5 & 0 & \\
\hline DIPHOS & & 0,0-diethyl0-2-pyrazinylphosphor & ppb & 10 & & 5 & 0 & \\
\hline PCDDS & & PCDDs & ppb & .01 & & 1 & 0 & \\
\hline PCOFS & & PCDF8 & ppb & .01 & & 1 & 0 & \\
\hline PARATHI & & Parathion & ppb & 10 & & 5 & 0 & \\
\hline PENTCHB & & Pentschlorobenzene & ppo & 10 & & 5 & 0 & \\
\hline PENTACH & & Pentachloroethane & ppb & 5 & & 5 & 0 & \\
\hline PENTCHN & & Pentachloronitrobenzene (PCNB) & ppb & 10 & & 5 & 0 & \\
\hline PENTCHP & 19 & Pentachlorophenol & ppb & 50 & & 7 & 0 & \\
\hline PENTCHP & 30 & Pentachlorophenol & ppb & 100 & & 8 & 0 & \\
\hline PHENTIN & & Phenacetin & ppb & 10 & & 5 & 0 & \\
\hline PHENANT & & Phenanthrene & ppb & 10 & & 5 & 0 & \\
\hline PHORATE & & Phorate & ppb & 2 & & 5 & 0 & \\
\hline PKOSPHA & & Phosphate & ppo & 400 & & 10 & 0 & \\
\hline POTASUM & & Potassium & ppb & 300 & & 8 & 8 & \\
\hline FPOTASS & & Potassium, filtered & ppo & 300 & & 8 & 8 & \\
\hline PRONIDE & & Pronamide & ppb & 10 & & 5 & 0 & \\
\hline PYRENE & & Pyrene & ppb & 10 & & 5 & 0 & \\
\hline PYRIDIN & & Pyridine & ppb & 10 & & 5 & 0 & \\
\hline
\end{tabular}


Table 15-24. Constituent List and Summary of Results for the Low-Level Waste Burial Grounds WMA 5 Data for Reporting Period July 1 through September 30, 1993. (sheet 6 of 6)

\begin{tabular}{|c|c|c|c|c|c|c|c|c|c|}
\hline \multirow[b]{2}{*}{ Short } & \multirow[b]{2}{*}{ (Method) } & \multirow{2}{*}{ Const ituent Name } & \multirow[b]{2}{*}{ Units } & \multirow{2}{*}{$\begin{array}{l}\text { Lab } \\
\text { CRQL }\end{array}$} & \multicolumn{2}{|c|}{ DWS } & \multicolumn{3}{|c|}{ Number of Samples } \\
\hline & & & & & Limit & Agency & Total & $>$ CROL & $>$ DWS \\
\hline SAFROL & & & & & $\cdots$ & 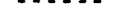 & 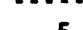 & 0 & $\cdots$ \\
\hline $\begin{array}{l}\text { SAFRUL } \\
\text { STYRENE }\end{array}$ & & Safrol & ppo & 10 & & & 5 & 0 & \\
\hline STYRENE & & Styrene & ppb & $\mathbf{5}$ & & & 5 & 0 & \\
\hline PERCENE & & Tetrachloroe thene & ppb & 5 & & & 18 & 0 & \\
\hline TETPHNL & & Tetrachlorophenols & ppb & 10 & & & 8 & 0 & \\
\hline TETRADE & & Tetradecane & ppob & 10 & & & 2 & 0 & \\
\hline PYROPHOS & & Tetraethyldi thiopyrophosphate & ppb & 10 & & & 5 & 0 & \\
\hline TAF & & Tetrahydrofuran & ppb & 10 & & & 18 & 0 & \\
\hline TIN & & Tin & ppb & 100 & & & 8 & 0 & \\
\hline FTIN & & Tin, filtered & ppb & 100 & & & 8 & 0 & \\
\hline TOLUENE & & Toluene & ppb & 5 & & & 18 & 1 & \\
\hline TRIBUTPH & & Tributyl Phosphate & ppb & 10 & & & 7 & 0 & \\
\hline TRICENE & & Trichloroe thene & ppb & 5 & & & 18 & 4 & \\
\hline TRCMFLM & & Trichloromonoflunrome thane & ppb & 5 & & & 5 & 0 & \\
\hline TRIPHNL & & Trichlorophenols & ppb & 5 & & & 8 & 0 & \\
\hline TOICHPH & & Tris-2-chloroethyl phosphate & ppob & 10 & & & 7 & 0 & \\
\hline TRITIUA & & Tritium & $\mathrm{pCi} / \mathrm{L}$ & 500 & 20000 & EPA & 10 & 9 & 3 \\
\hline URANIUM & & Uranium & ppb & .5 & & & 10 & 10 & \\
\hline VANADUH & & Vanadium & ppb & 30 & & & 8 & 0 & \\
\hline FVANADI & & Vanedium, filtered & ppob & 30 & & & 8 & 0 & \\
\hline VINYLAC & & Vinyl acetate & ppb & 5 & & & 5 & 0 & \\
\hline VINYIDE & & Vinyl chloride & ppb & 10 & 2 & EPA & 18 & 0 & 0* \\
\hline XYLENE & & Xylenes (total) & ppb & 5 & & & 18 & 0 & \\
\hline ZINC & & Zine & ppb & 10 & & & 8 & 4 & \\
\hline FZINC & & Zine, filtered & ppob & 10 & & & 8 & 3 & \\
\hline ALLYLCL & & allylchloride & ppb & 100 & & & 5 & 0 & \\
\hline TDICPENE & & cis-1,3-Dichl oropropene & ppb & 5 & & & 5 & 0 & \\
\hline MCRESOL & & m-Cresol & ppb & 10 & & & 7 & 0 & \\
\hline DINBENZ & & m-dinitrobenzene & ppb & 10 & & & 5 & 0 & \\
\hline OTOLHYD & & 0-Toluidine & ppb & 10 & & & 5 & 0 & \\
\hline DIMEAMB & & p-Dimethylaminoazobenzene & ppb & 10 & & & 5 & 0 & \\
\hline PHENDIA & & p-Phenylenediamine & ppb & 10 & & & 5 & 0 & \\
\hline SYMTRIN & & sym-Trinitrobenzerve & ppb & 10 & & & 5 & 0 & \\
\hline DICPENE & & trans-1,3-Dichloropropene & ppb & 5 & & & 5 & 0 & \\
\hline TDIBUTEN & & rans-1,4-dichloro-2-butene & ppb & 5 & & & 5 & 0 & \\
\hline
\end{tabular}

For explanation of this table, see Section 1.4 of report. 
Table 15-25. Constituents with at Least One Value Above the CRQL for the Low-Level Waste Burial Grounds WMA 5 Data for Reporting Period July 1 through September 30, 1993. (sheet 1 of 5)

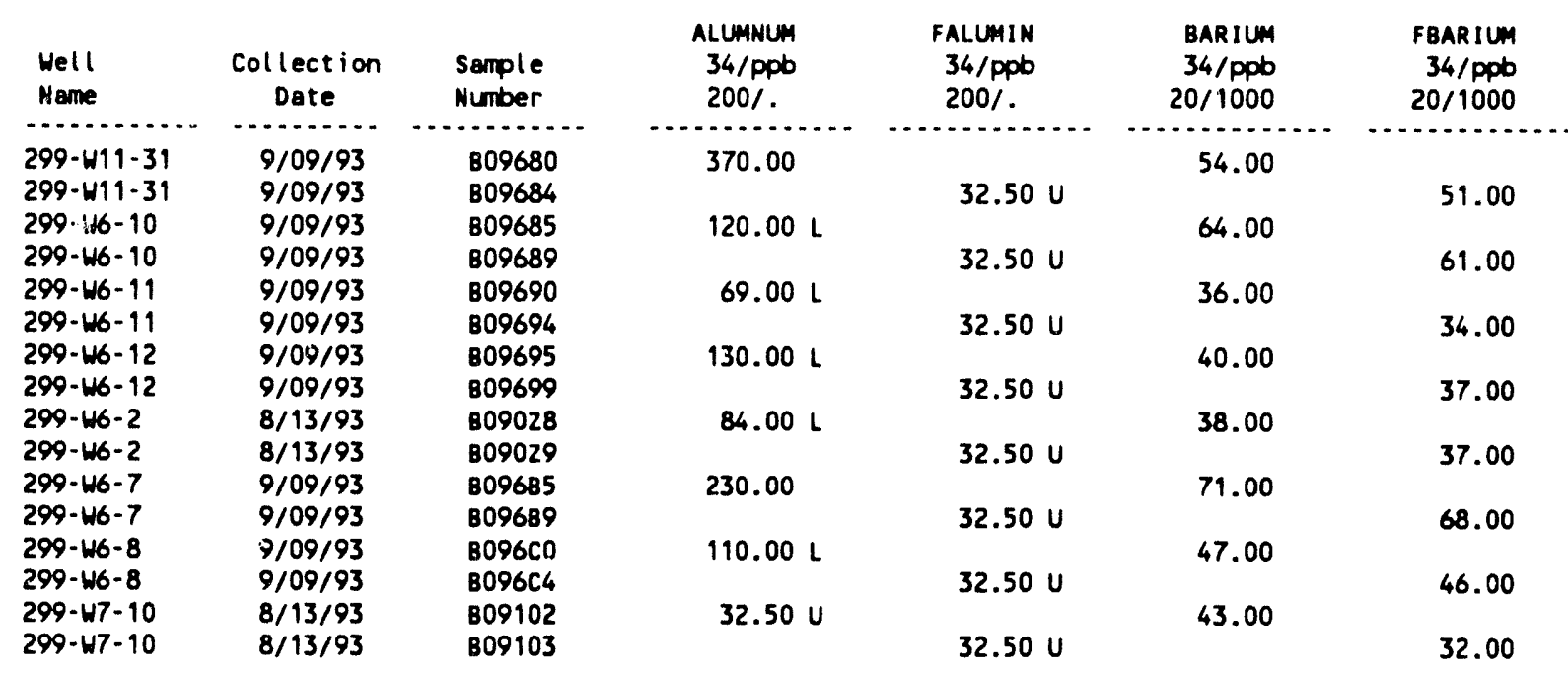

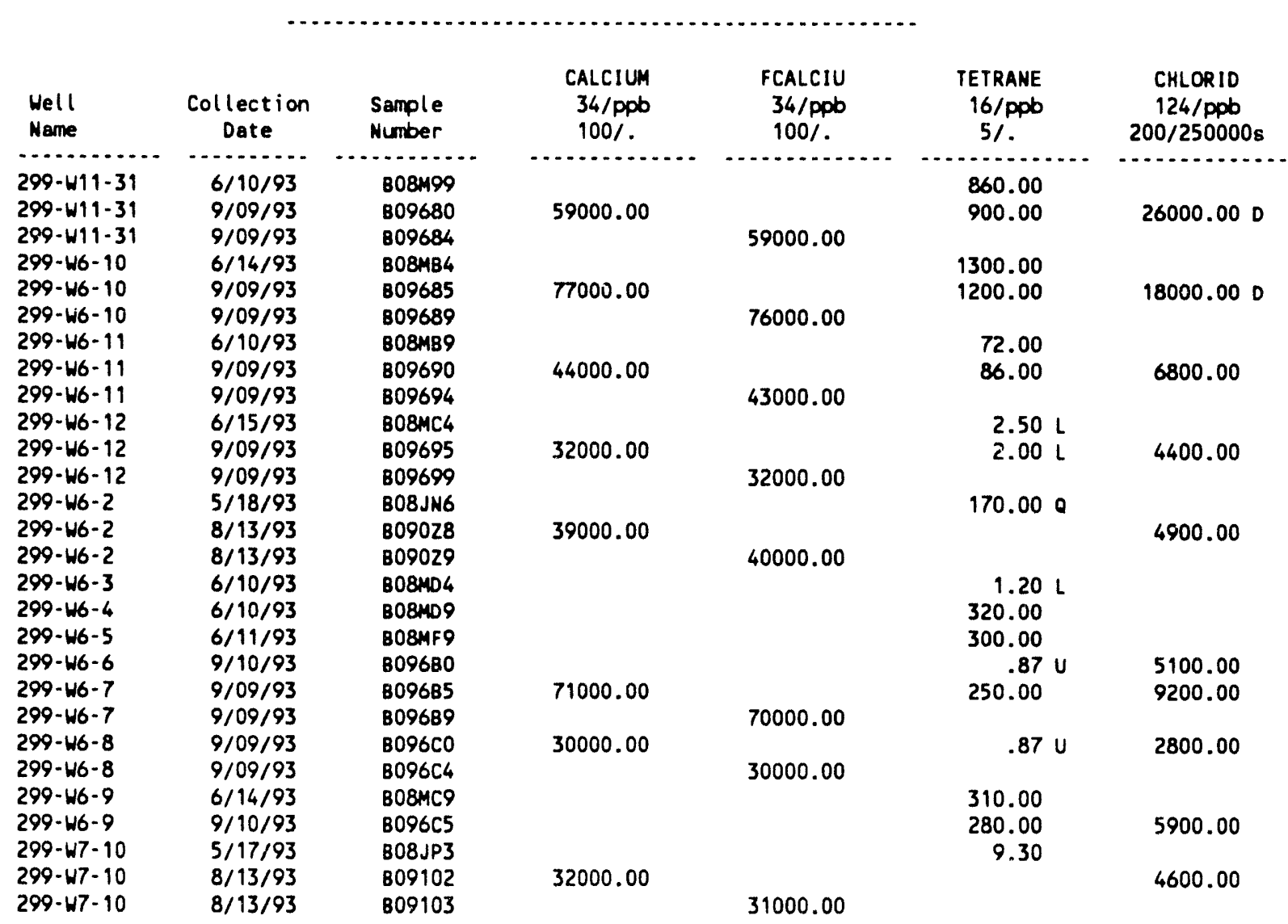


Table 15-25. Constituents with at Least One Value Above the CRQL for the Low-Level Waste Burial Grounds WMA 5 Data for Reporting Period July 1 through September 30, 1993. (sheet 2 of 5)

\begin{tabular}{|c|c|c|c|c|c|c|}
\hline $\begin{array}{l}\text { Well } \\
\text { Name }\end{array}$ & $\begin{array}{c}\text { Collection } \\
\text { Date }\end{array}$ & $\begin{array}{l}\text { Sample } \\
\text { Number }\end{array}$ & $\begin{array}{c}\text { CHLFORM } \\
16 / \text { ppb } \\
5 / 100\end{array}$ & $\begin{array}{c}\text { CHROMUM } \\
34 / \text { ppb } \\
20 / 50\end{array}$ & $\begin{array}{c}\text { FCHROMI } \\
34 / \mathrm{ppb} \\
20 / 50\end{array}$ & $\begin{array}{l}\text { FLUOR ID } \\
124 / \text { ppb } \\
100 / 4000\end{array}$ \\
\hline $299-$-11-31 & $6 / 10 / 93$ & B08M99 & $.40 \mathrm{U}$ & & & \\
\hline $299-W 11-31$ & $9 / 09 / 93$ & B09680 & $.40 \mathrm{U}$ & 92.00 & & 1000.00 \\
\hline $299-W 11-31$ & $9 / 09 / 93$ & 809684 & & & 39.00 & \\
\hline $299-166-10$ & $6 / 14 / 93$ & B08MB4 & 13.00 & & & \\
\hline $299-w 6-10$ & $9 / 09 / 93$ & 809685 & $.40 \mathrm{U}$ & 68.00 & & 400.00 \\
\hline $299-w 6-10$ & $9 / 09 / 93$ & B09689 & & & 60.00 & \\
\hline 299-W6-11 & $6 / 10 / 93$ & В08MB9 & $1.90 \mathrm{~L}$ & & & \\
\hline $299-w 6-11$ & $9 / 09 / 93$ & 809690 & $.40 \mathrm{U}$ & 69.00 & & 400.00 \\
\hline $299-W 6-11$ & $9 / 09 / 93$ & B09694 & & & 27.00 & \\
\hline $299-w 6-12$ & $6 / 15 / 93$ & $808 M C 4$ & $.58 \mathrm{~L}$ & & & \\
\hline 299-W6-12 & $9 / 09 / 93$ & B09695 & $.40 \mathrm{U}$ & $5.42 \mathrm{U}$ & & 400.00 \\
\hline $299-46-12$ & $9 / 09 / 93$ & 809699 & & & $5.42 \mathrm{U}$ & \\
\hline $299-46-2$ & $5 / 18 / 93$ & B08JN6 & $2.00 \mathrm{~L}$ & & & \\
\hline $299-16-2$ & $8 / 13 / 93$ & B090Z8 & & 150.00 & & 400.00 \\
\hline $299-166-2$ & $8 / 13 / 93$ & 809029 & & & $5.42 \mathrm{U}$ & \\
\hline $299-w 6-3$ & $6 / 10 / 93$ & B08MD4 & $1.40 \mathrm{~L}$ & & & \\
\hline $299-166-4$ & $6 / 10 / 93$ & B08MD9 & 20.00 & & & \\
\hline $299-W 6-5$ & $6 / 11 / 93$ & B08MF9 & 12.00 & & & \\
\hline $\begin{array}{l}299-w 6-6 \\
200-46-7\end{array}$ & $9 / 10 / 93$ & $\begin{array}{l}809680 \\
809685\end{array}$ & $.40 \mathrm{U}$ & & & $\begin{array}{l}300.00 \\
400.00\end{array}$ \\
\hline 299-W6-7 & $9 / 09 / 93$ & 809685 & 6.50 & 220.00 & & 400.00 \\
\hline $299-116-7$ & $9 / 09 / 93$ & 809689 & & & 67.00 & \\
\hline $299-46-8$ & $9 / 09 / 93$ & $8096 C 0$ & $.40 \mathrm{U}$ & $5.42 U$ & & 400.00 \\
\hline $299-46-8$ & $9 / 09 / 93$ & $\mathrm{~B} 096 \mathrm{C4}$ & & & $5.42 \mathrm{U}$ & \\
\hline 299-W6-9 & $6 / 14 / 93$ & В08MC9 & $3.10 \mathrm{~L}$ & & & \\
\hline $299-116-9$ & $9 / 10 / 93$ & $8096 C 5$ & $.40 \mathrm{U}$ & & & 400.00 \\
\hline $299-W 7-10$ & $5 / 17 / 93$ & B08JP3 & $.77 \mathrm{~L}$ & & & \\
\hline $299-47-10$ & $8 / 13 / 93$ & B09102 & & 190.00 & & 500.00 \\
\hline $299-\omega 7-10$ & $8 / 13 / 93$ & B09103 & & & $5.42 \mathrm{U}$ & \\
\hline
\end{tabular}

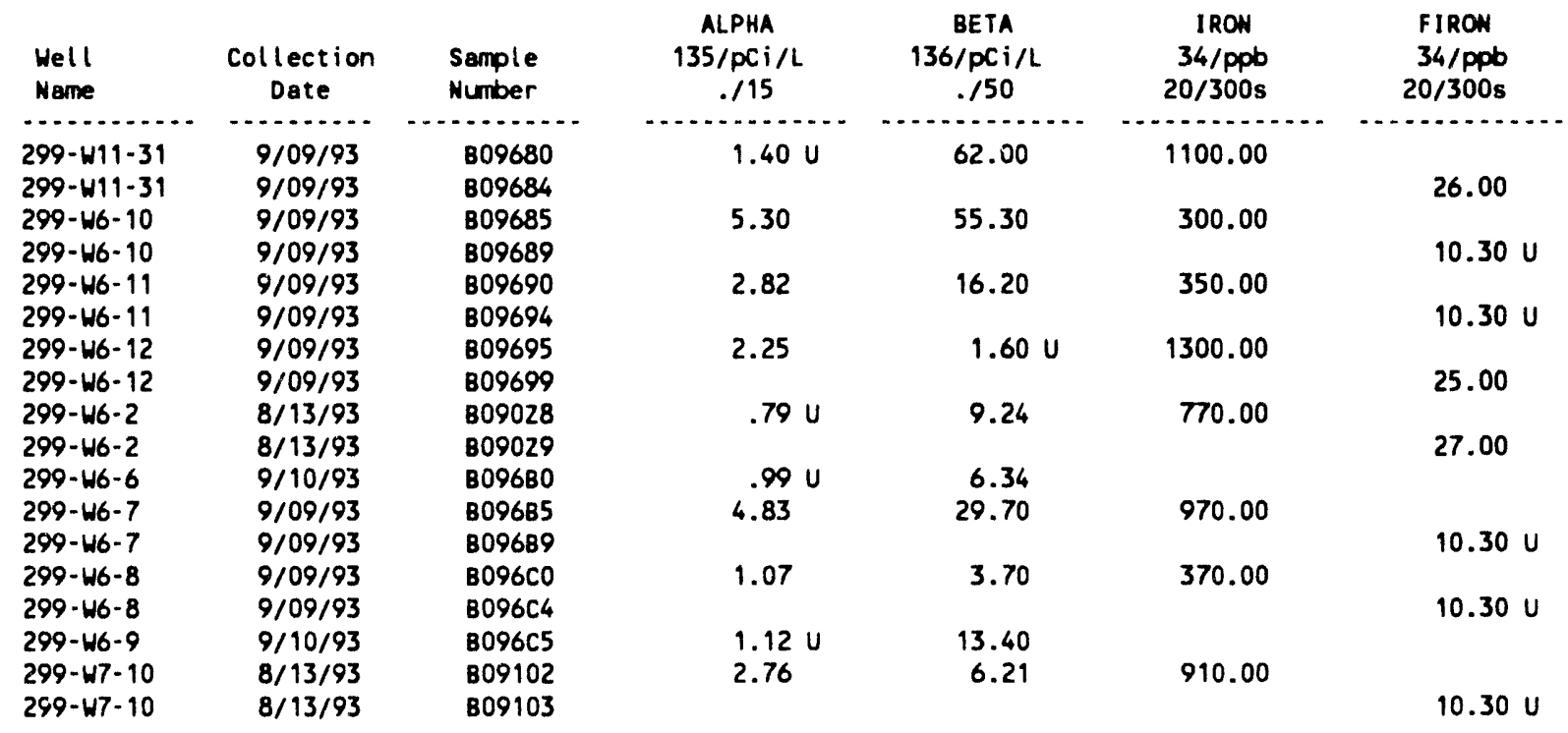


Table 15-25. Constituents with at Least One Value Above the CRQL for the Low-Level Waste Burial Grounds WMA 5 Data for Reporting Period July 1 through September 30,1993 . (sheet 3 of 5)

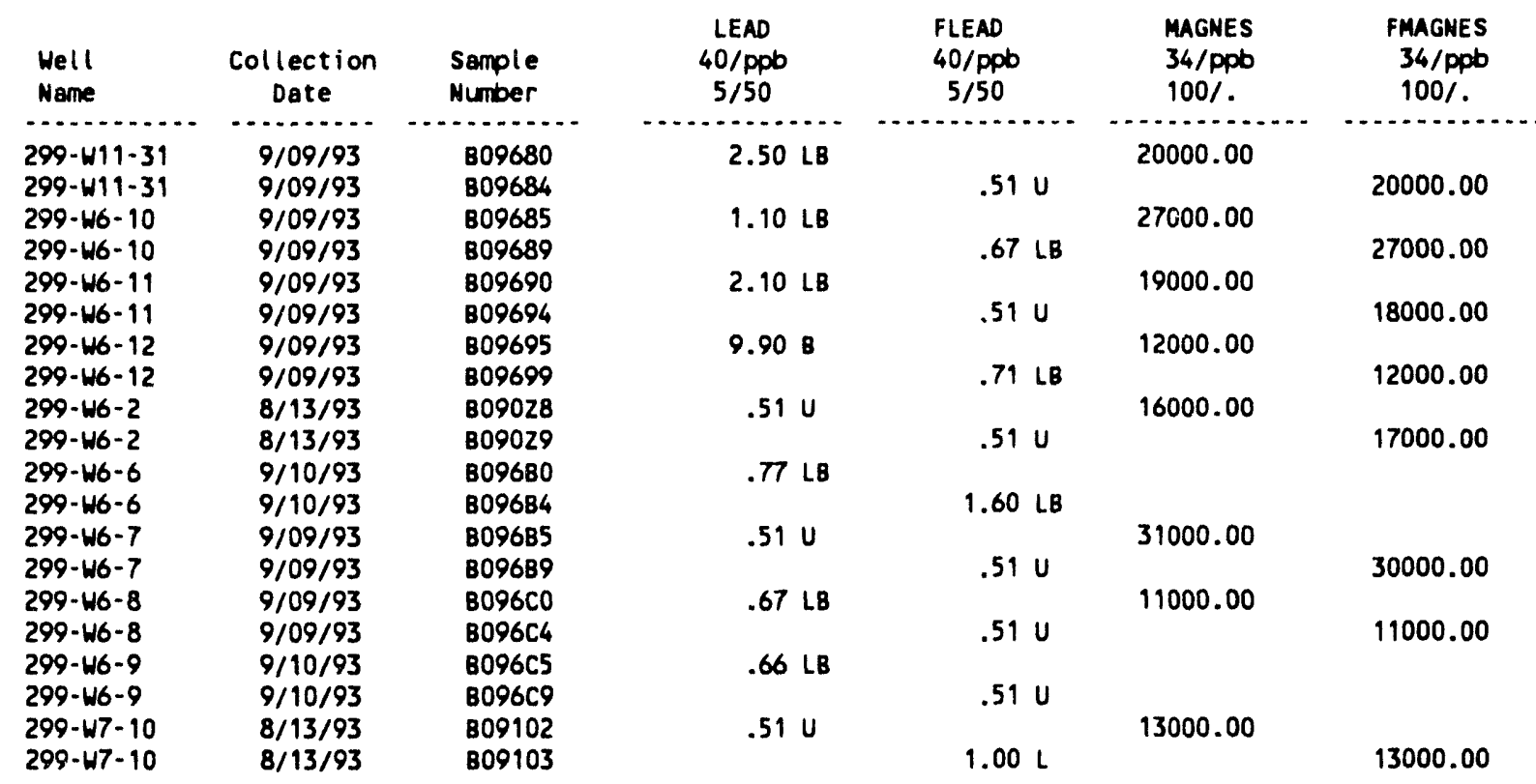

\begin{tabular}{|c|c|c|c|c|c|c|}
\hline $\begin{array}{l}\text { Well } \\
\text { Name }\end{array}$ & $\begin{array}{c}\text { Collection } \\
\text { Date }\end{array}$ & $\begin{array}{l}\text { Sample } \\
\text { Number }\end{array}$ & $\begin{array}{r}\text { MANGESE } \\
34 / \mathrm{ppb} \\
10 / 50 \mathrm{~s}\end{array}$ & $\begin{array}{c}\text { FMANGAN } \\
34 / \text { ppb } \\
10 / 50 \text { s }\end{array}$ & $\begin{array}{c}\text { METHYCH } \\
16 / \mathrm{ppb} \\
5 / .\end{array}$ & $\begin{array}{c}\text { NICKEL } \\
34 / \mathrm{ppb} \\
30 /\end{array}$ \\
\hline$\cdots$ & $\cdots$ & $\ldots \ldots$ & n & $\ldots \ldots \ldots$ & $\cdots$ & - \\
\hline $299-H 11-31$ & $6 / 10 / 93$ & 808м99 & & & $.61 \mathrm{U}$ & \\
\hline $299-W 11-31$ & $9 / 09 / 93$ & 809680 & 18.00 & & $.61 \mathrm{U}$ & 32.00 \\
\hline $299-W 11-31$ & $9 / 09 / 93$ & B09684 & & $1.35 \mathrm{U}$ & & \\
\hline $299-w 6-10$ & $6 / 14 / 93$ & BOBMB 4 & & & 19.00 & \\
\hline $299-W 6-10$ & $9 / 09 / 93$ & 809685 & 11.00 & & 20.00 & $17.90 \mathrm{U}$ \\
\hline $299-16-10$ & $9 / 09 / 93$ & B09689 & & $1.35 \mathrm{U}$ & & \\
\hline $299-w 6-11$ & $6 / 10 / 93$ & B08NB9 & & & $.61 \mathrm{U}$ & \\
\hline $299-1+6-11$ & $9 / 09 / 93$ & B09690 & $1.35 \mathrm{U}$ & & $.61 \mathrm{U}$ & $17.90 \mathrm{U}$ \\
\hline $299-W 6-11$ & $9 / 09 / 93$ & 809694 & & $1.35 \mathrm{U}$ & & \\
\hline $299-166-12$ & $6 / 15 / 93$ & BO8MC4 & & & $.61 \mathrm{U}$ & \\
\hline $299-W 6-12$ & $9 / 09 / 93$ & 809695 & 30.00 & & $.61 \mathrm{U}$ & $17.90 \mathrm{U}$ \\
\hline $299-46-12$ & $9 / 09 / 93$ & B09699 & & 24.00 & & \\
\hline $299-16-2$ & $5 / 18 / 93$ & B08JN6 & & & $1.50 \mathrm{BLO}$ & \\
\hline $299-16-2$ & $8 / 13 / 93$ & B09028 & 14.00 & & & 70.00 \\
\hline 299-W6-2 & $8 / 13 / 93$ & 809029 & & $1.35 \mathrm{U}$ & & \\
\hline $\begin{array}{l}299-W 6-3 \\
299-166-4\end{array}$ & $\begin{array}{l}6 / 10 / 93 \\
6 / 10 / 93\end{array}$ & $\begin{array}{l}\text { B08MD4 } \\
\text { B08MD9 }\end{array}$ & & & $\begin{array}{l}.61 \mathrm{U} \\
2.80 \mathrm{BL}\end{array}$ & \\
\hline $299-W 6-5$ & $6 / 11 / 93$ & BO8MF9 & & & .61 vo & \\
\hline $299-46-6$ & $9 / 10 / 93$ & B09680 & & & $.61 \mathrm{U}$ & \\
\hline $299-W 6-7$ & $9 / 09 / 93$ & B09685 & 23.00 & & $.61 \mathrm{U}$ & 67.00 \\
\hline $299-166-7$ & $9 / 09 / 93$ & B09689 & & $1.35 \mathrm{U}$ & & \\
\hline $299-146-8$ & $9 / 09 / 93$ & $\mathrm{~B} 096 \mathrm{CO}$ & 26.00 & & $.61 \mathrm{U}$ & $17.90 \mathrm{U}$ \\
\hline $299-46-8$ & $9 / 09 / 93$ & $8096 \mathrm{C} 4$ & & 17.00 & & \\
\hline 299-W6-9 & $6 / 14 / 93$ & BO8MC9 & & & $.61 \mathrm{U}$ & \\
\hline $299-116-9$ & $9 / 10 / 93$ & $8096 \mathrm{C} 5$ & & & $1.50 \mathrm{BL}$ & \\
\hline $299-W 7-10$ & $5 / 17 / 93$ & B08JP3 & & & $2.10 \mathrm{BLO}$ & \\
\hline $299-47-10$ & $8 / 13 / 93$ & B09102 & 18.00 & & & 100.00 \\
\hline $299-47-10$ & $8 / 13 / 93$ & 809103 & & $1.35 \mathrm{U}$ & & \\
\hline
\end{tabular}


Table 15-25. Constituents with at Least One Value Above the CRQL for the Low-Level Waste Burial Grounds WMA 5 Data for Reporting Period July 1 through September 30, 1993. (sheet 4 of 5)

\begin{tabular}{|c|c|c|c|c|c|c|}
\hline $\begin{array}{l}\text { Well } \\
\text { Name }\end{array}$ & $\begin{array}{c}\text { Collection } \\
\text { Date }\end{array}$ & $\begin{array}{l}\text { Sample } \\
\text { Number }\end{array}$ & $\begin{array}{c}\text { FNICKEL } \\
34 / \mathrm{ppb} \\
30 \%\end{array}$ & $\begin{array}{l}\text { NITRATE } \\
\text { 124/ppb } \\
200 / 45000\end{array}$ & $\begin{array}{c}\text { POTASUM } \\
34 / \text { ppb } \\
1000 / .\end{array}$ & $\begin{array}{c}\text { FPOTASS } \\
34 / \mathrm{ppb} \\
1000 /\end{array}$ \\
\hline$\ldots \ldots \ldots$ & $\ldots \ldots \ldots$ & $\ldots \ldots \ldots$ & $\ldots$ & $\cdots$ & $\cdots+\cdots$ & $\cdots \ldots$ \\
\hline $299-411-31$ & $9 / 09 / 93$ & B09680 & & $150000.00 \mathrm{D}$ & 4400.00 & \\
\hline $299-W 11-31$ & $9 / 09 / 93$ & B09684 & $17.90 \mathrm{U}$ & & & 4500.00 \\
\hline $299-146-10$ & $9 / 09 / 93$ & B09685 & & $170000.00 \mathrm{D}$ & 4000.00 & \\
\hline $299-46-10$ & $9 / 09 / 93$ & B09689 & $17.90 \mathrm{U}$ & & & 4200.00 \\
\hline $299-46-11$ & $9 / 09 / 93$ & B09690 & & $86000.00 \mathrm{D}$ & 3400.00 & \\
\hline $299-W 6-11$ & $9 / 09 / 93$ & 809694 & $17.90 \mathrm{U}$ & & & 3100.00 \\
\hline $299-w 6-12$ & $9 / 09 / 93$ & 809695 & & $15000.00 \mathrm{D}$ & 2600.00 & \\
\hline $299-46-12$ & $9 / 09 / 93$ & 809699 & $17.90 \mathrm{U}$ & & & 2700.00 \\
\hline $299-116-2$ & $8 / 13 / 93$ & 809028 & & 65000.000 & 2700.00 & \\
\hline $299-46-2$ & $8 / 13 / 93$ & B09029 & $17.90 \mathrm{U}$ & & & 2900.00 \\
\hline $\begin{array}{l}299-146-6 \\
299-146-7\end{array}$ & $\begin{array}{l}9 / 10 / 93 \\
9 / 09 / 93\end{array}$ & $\begin{array}{l}\mathrm{B} 096 \mathrm{BO} \\
8096 \mathrm{B5}\end{array}$ & & $\begin{array}{c}2600.00 \\
220000.000\end{array}$ & 3900.00 & \\
\hline $299-146-7$ & $9 / 09 / 93$ & B09689 & $17.90 \mathrm{U}$ & 260000.000 & JYUU.00 & 4300.00 \\
\hline $299-W 6-8$ & $9 / 09 / 93$ & B096CO & & 5600.00 & 4000.00 & \\
\hline $299-116-8$ & $9 / 09 / 93$ & B096C4 & $17.90 \mathrm{U}$ & & & 3800.00 \\
\hline $299-116-9$ & $9 / 10 / 93$ & B096C5 & & $67000.00 \mathrm{D}$ & & \\
\hline $299-47-10$ & $8 / 13 / 93$ & 809102 & & 24000.000 & 2600.00 & \\
\hline $299-W 7-10$ & $8 / 13 / 93$ & 809103 & $17.90 \mathrm{U}$ & & & 2200.00 \\
\hline & & & RADIUM & SOOIUA & FSODIUM & SULFATE \\
\hline $\begin{array}{l}\text { Well } \\
\text { Nane }\end{array}$ & $\begin{array}{c}\text { Collection } \\
\text { Date }\end{array}$ & $\begin{array}{l}\text { Sample } \\
\text { Number }\end{array}$ & $\begin{array}{c}137 / p C i / L \\
. /\end{array}$ & $\begin{array}{l}34 / p p b \\
300 / .\end{array}$ & $\begin{array}{l}\text { 34/ppt } \\
300 / .\end{array}$ & $\begin{array}{c}124 / p p b \\
500 / 250000 \text { s }\end{array}$ \\
\hline$\ldots \ldots$ & $\ldots \ldots \ldots$ & $\cdots+\infty \cdots$ & $\cdots$ & 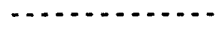 & $+\cdots$ & $\cdots$ \\
\hline $299-W 11-31$ & $9 / 09 / 93$ & $\begin{array}{l}809680 \\
809684\end{array}$ & .20 & 57000.00 & 5700000 & 69000.000 \\
\hline $\begin{array}{l}299-W 17-31 \\
299-w 6-10\end{array}$ & $9 / 09 / 95$ & $\begin{array}{l}809684 \\
809685\end{array}$ & .45 & 24000.00 & 37000.00 & $55000.00 \mathrm{D}$ \\
\hline $299-W 6-10$ & $9 / 09 / 93$ & B09689 & & & 24000.00 & \\
\hline $299-46-11$ & $9 / 09 / 93$ & B09690 & .27 & 12000.00 & & $28000.00 \mathrm{D}$ \\
\hline $299-46-11$ & $9 / 09 / 93$ & 809694 & & & 11000.00 & \\
\hline $299-46-12$ & $9 / 09 / 93$ & 809695 & .25 & 8500.00 & & $22000.00 \mathrm{D}$ \\
\hline $299-w 6-12$ & $9 / 09 / 93$ & B09699 & & & 8500.00 & \\
\hline $299-166-2$ & $8 / 13 / 93$ & B09028 & $.09 \mathrm{U}$ & 9800.00 & & 26000.00 \\
\hline $299-w 6-2$ & $8 / 13 / 93$ & 809029 & & & 10000.00 & \\
\hline $299-116-6$ & $9 / 10 / 93$ & B09680 & $.05 \mathrm{U}$ & & & $28000.00 \mathrm{D}$ \\
\hline $299-W 6-7$ & $9 / 09 / 93$ & B096B5 & .46 & 12000.00 & & $39000.00 \mathrm{D}$ \\
\hline $299-146-7$ & $9 / 09 / 93$ & B09689 & & & 12000.00 & \\
\hline $\begin{array}{l}299-46-8 \\
299-46-8\end{array}$ & $\begin{array}{l}9 / 09 / 93 \\
9 / 09 / 93\end{array}$ & $\begin{array}{l}8096 C 0 \\
8096 C 4\end{array}$ & $.06 \mathrm{U}$ & 9300.00 & 9400.00 & $17000.00 \mathrm{D}$ \\
\hline 299-W6-9 & $9 / 10 / 93$ & $8096 C 5$ & $.10 \mathrm{U}$ & & & 26000.000 \\
\hline $299-W 7-10$ & $8 / 13 / 93$ & 809102 & .29 & 8200.00 & & 22000.00 \\
\hline $299-47-10$ & $8 / 13 / 93$ & B09103 & & & 8000.00 & \\
\hline
\end{tabular}


Table 15-25. Constituents with at Least One Value Above the CRQL for the Low-Level Waste Burial Grounds WMA 5 Data for Reporting Period July 1 through September 30, 1993. (sheet 5 of 5)

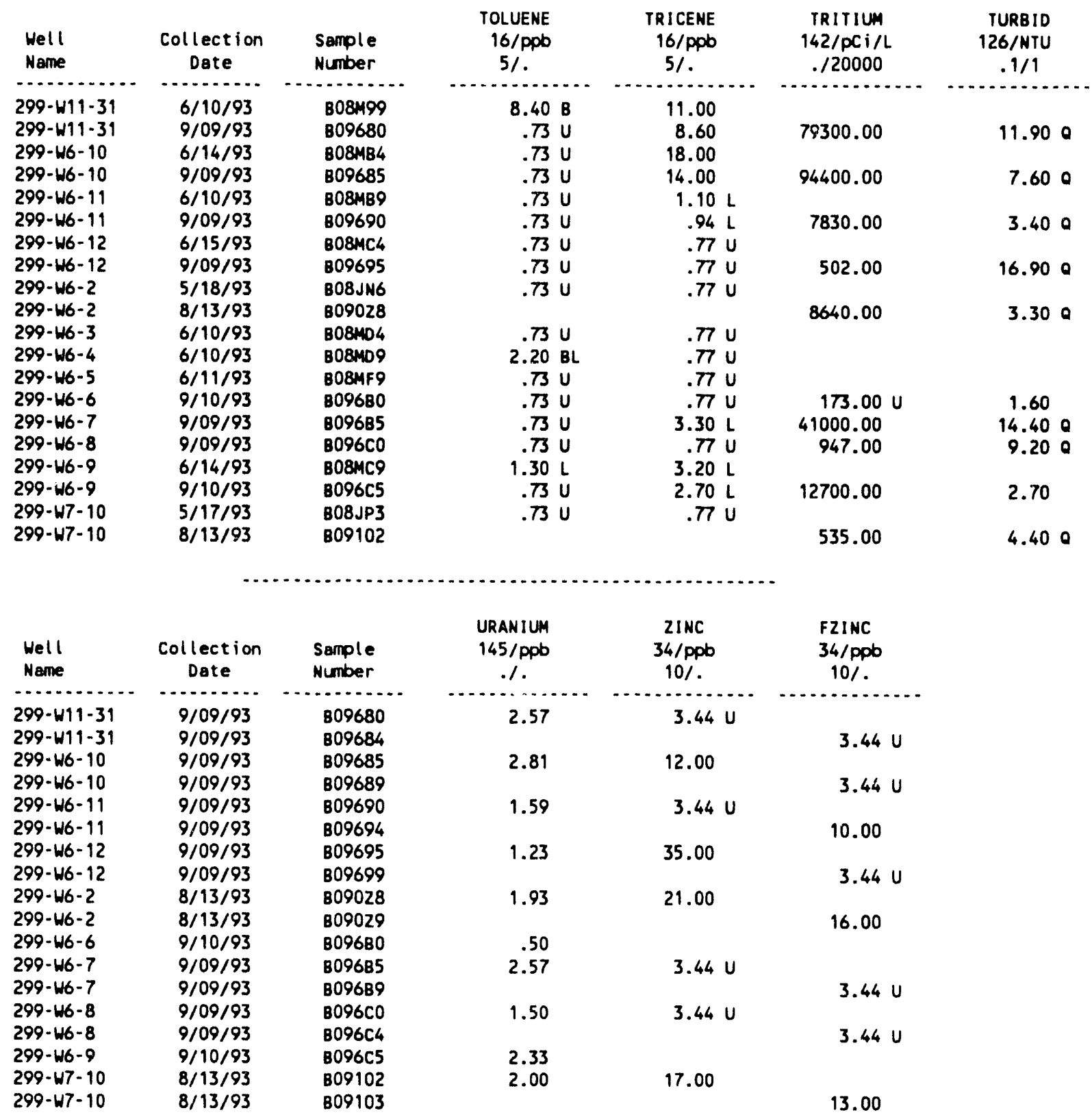

For explanation of this table, see Section 1.4 of report. 
Table 15-26. Contamination Indicator Parameters for the Low-Level Waste Burial Grounds WMA 5 Data for Reporting Period July 1 through September 30, 1993.

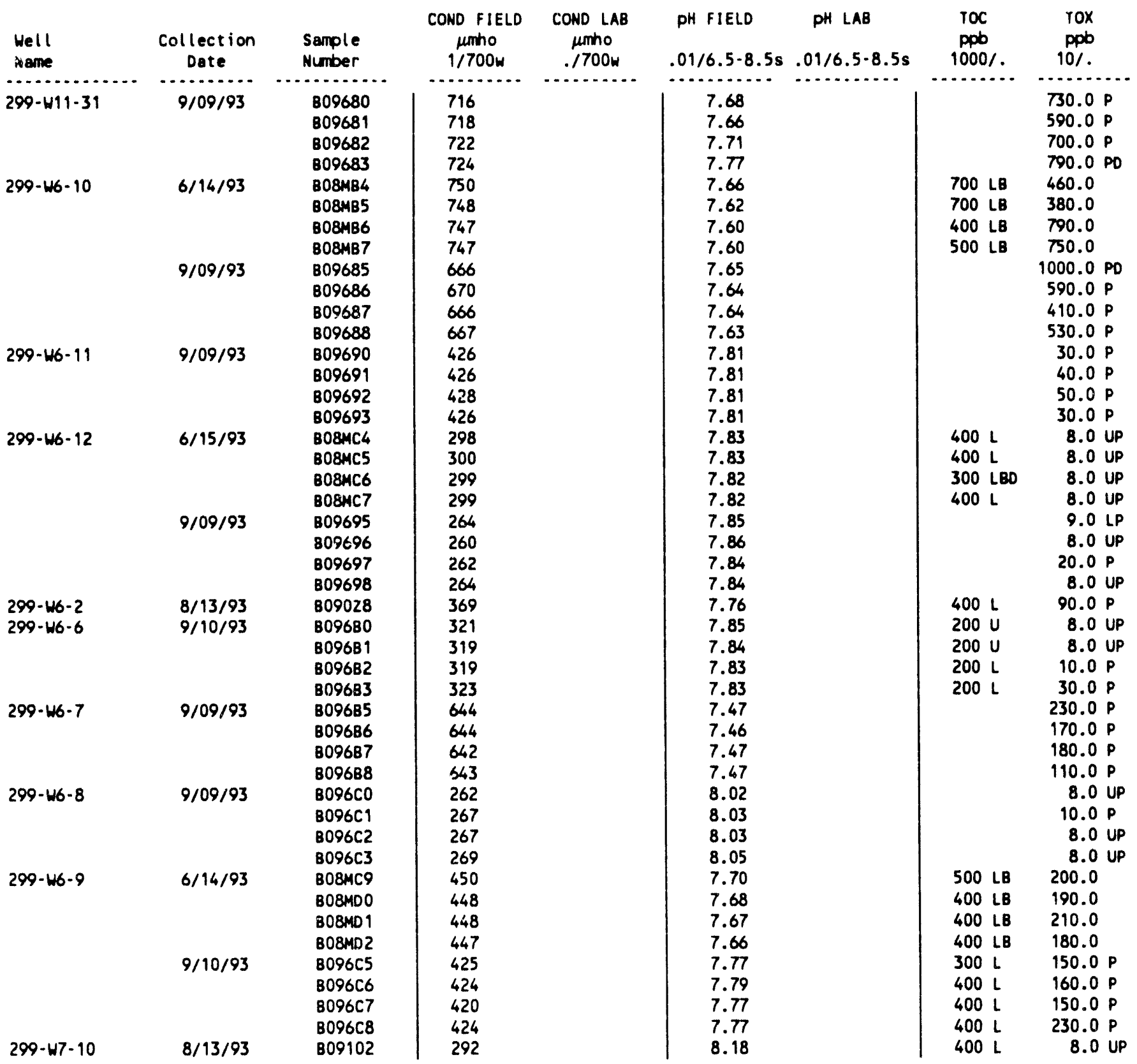

For explanation of this table, see Section 1.4 of report. 
Table 15-27. Water Quality Standards Exceeded at Low-Level Waste Management Area 1.

\begin{tabular}{|c|c|c|c|}
\hline $\begin{array}{l}\text { Chromium } \\
(50 \mathrm{ppb})\end{array}$ & $\begin{array}{l}299-E 28-26 \\
299-E 32-2 \\
299-E 32 \cdot-5 \\
299-E 32-8 \\
299-E 33-29 \\
299-E 33-35\end{array}$ & $\begin{array}{l}299-E 28-27 \\
299-E 32-3 \\
299-E 32-6 \\
299-E 32-9 \\
299-E 33-30\end{array}$ & $\begin{array}{l}299-E 28-28 \\
299-E 32-4 \\
299-E 32-7 \\
299-E 33-28 \\
299-E 33-34\end{array}$ \\
\hline $\begin{array}{l}\text { Gross alpha } \\
(15 \mathrm{pC} i / L)\end{array}$ & $299-E 28-28$ & $299-E 32-5$ & - \\
\hline $\begin{array}{l}\text { Gross beta } \\
(50 \mathrm{pC} i / L)\end{array}$ & 299-E33-34 & 299-E33-35 & -- \\
\hline $\begin{array}{l}\text { Turbidity } \\
\text { (1 NTU) }\end{array}$ & $\begin{array}{l}299-E 28-26 \\
299-E 32-3^{\circ} \\
299-E 32-6 \\
299-E 32-9 \\
299-E 33-29^{\circ}\end{array}$ & $\begin{array}{l}299-E 28-27 \\
299-E 32-4 \\
299-E 32-7 \\
299-E 32-10 \\
299-E 33-30\end{array}$ & $\begin{array}{l}299-E 28-28 \\
299-E 32-5^{\circ} \\
299-E 32-8 \\
299-E 33-28^{8} \\
299-E 33-35\end{array}$ \\
\hline $\begin{array}{l}\text { Iron } \\
(300 \mathrm{ppb})\end{array}$ & $\begin{array}{l}299-E 28-26 \\
299-E 32-2 \\
299-E 32-5 \\
299-E 32-8 \\
299-E 33-29 \\
299-E 33-35\end{array}$ & $\begin{array}{l}299-E 28-27 \\
299-E 32-3 \\
299-E 32-6 \\
299-E 32-9 \\
299-E 33-30\end{array}$ & $\begin{array}{l}299-E 28-28 \\
299-E 32-4 \\
299-E 32-7 \\
299-E 33-28 \\
299-E 33-34\end{array}$ \\
\hline $\begin{array}{l}\text { Tritium } \\
(20,000 \mathrm{pC} i / \mathrm{L})\end{array}$ & $\begin{array}{l}299-E 28-27 \\
299-E 32-5\end{array}$ & $\begin{array}{l}299-E 32-2 \\
299-E 32-6\end{array}$ & $\begin{array}{l}299-E 32-3 \\
299-E 32-7\end{array}$ \\
\hline
\end{tabular}

NTU = nephelometric turbidity unit.

'Field QC samples associated with this data exceeded the reliable detection limits.

Table 15-28. Water Quality Standards Exceeded at Low-Level Waste Management Area 2.

\begin{tabular}{|llll|}
\hline $\begin{array}{l}\text { Chromium } \\
(50 \mathrm{ppb})\end{array}$ & $\begin{array}{l}299-\mathrm{E} 27-8 \\
299-E 27-17^{\circ}\end{array}$ & $\begin{array}{l}299-\mathrm{E} 27-9 \\
299-E 34-10^{\circ}\end{array}$ & $\begin{array}{l}299-\mathrm{E} 27-11^{\circ} \\
299-E 34-11^{\circ}\end{array}$ \\
\hline $\begin{array}{l}\text { Turbidity } \\
(1 \mathrm{NTU})\end{array}$ & $299-\mathrm{E} 34-11$ & $299-\mathrm{E} 34-12$ & -- \\
\hline Iron & & & \\
$(300 \mathrm{ppb})$ & $299-\mathrm{E} 27-8$ & $299-E 27-9$ & $299-\mathrm{E} 27-11^{\circ}$ \\
\hline
\end{tabular}

NTU = nephelometric turbidity unit.

${ }^{\circ}$ Field QC samples associated with this data exceeded the reliable detection limits. 
Table 15-29. Water Quality Standards Exceeded at Low-Level Waste Management Area 3.

\begin{tabular}{|c|c|c|c|}
\hline $\begin{array}{l}\text { Field } \mathrm{pH} \\
(>8.5)\end{array}$ & $299-W 7-6$ & -- & -- \\
\hline $\begin{array}{l}\text { Cadmium } \\
(10 \mathrm{ppb})\end{array}$ & $299-W 7-2$ & -- & -- \\
\hline $\begin{array}{l}\text { Chromium } \\
(50 \mathrm{ppb})\end{array}$ & $\begin{array}{l}299-W 6-2 \\
299-W 7-3 \\
299-W 7-6 \\
299-W 7-9 \\
299-W 7-12 \\
299-W 10-13\end{array}$ & $\begin{array}{l}299-W 7-1 \\
299-W 7-4 \\
299-W 7-7 \\
299-W 7-10 \\
299-W 8-1 \\
299-W 10-14^{\circ}\end{array}$ & $\begin{array}{l}299-W 7-2 \\
299-W 7-5 \\
299-W 7-8 \\
299-W 7-11 \\
299-W 9-1\end{array}$ \\
\hline $\begin{array}{l}\text { Gross alpha } \\
(15 \mathrm{pCi} / \mathrm{L})\end{array}$ & $299-W 7-6$ & -- & -- \\
\hline $\begin{array}{l}\text { Nitrate } \\
(45,000 \mathrm{ppb})\end{array}$ & $\begin{array}{l}299-W 6-2^{\circ} \\
299-W 10-19\end{array}$ & $299-W 7-4^{\circ}$ & $299-W 7-5^{\circ}$ \\
\hline $\begin{array}{l}\text { Turbidity } \\
\text { (1 NTU) }\end{array}$ & $\begin{array}{l}299-W 6-2^{a} \\
299-W 7-3 \\
299-W 7-7^{a} \\
299-W 7-10^{a} \\
299-W 8-1^{a} \\
299-W 10-14^{a}\end{array}$ & $\begin{array}{l}299-W 7-1^{a} \\
299-W 7-5^{a} \\
299-W 7-8^{a} \\
299-W 7-11^{a} \\
299-W 9-1^{a} \\
299-W 10-19\end{array}$ & $\begin{array}{l}299-W 7-2^{a} \\
299-W 7-6^{\circ} \\
299-W 7-9^{\circ} \\
299-W 7-12^{\circ} \\
299-W 10-13^{\circ}\end{array}$ \\
\hline $\begin{array}{l}\text { Iron } \\
(300 \mathrm{ppb})\end{array}$ & $\begin{array}{l}299-W 6-2 \\
299-W 7-3 \\
299-W 7-6 \\
299-W 7-9 \\
299-W 7-12 \\
299-W 10-13\end{array}$ & $\begin{array}{l}299-W 7-1^{\circ} \\
299-W 7-4^{\circ} \\
299-W 7-7 \\
299-W 7-10 \\
299-W 8-1 \\
299-W 10-14^{\circ}\end{array}$ & $\begin{array}{l}299-W 7-2 \\
299-W 7-5^{\circ} \\
299-W 7-8 \\
299-W 7-11 \\
299-W 9-1 \\
299-W 10-19\end{array}$ \\
\hline $\begin{array}{l}\text { Manganese } \\
(50 \mathrm{ppb})\end{array}$ & $\begin{array}{l}299-W 7-3 \\
299-W 7-12\end{array}$ & $299-W 7-6$ & $299-W 7-9$ \\
\hline $\begin{array}{l}\text { Carbon tetrachloride } \\
(5 \mathrm{ppb})\end{array}$ & $\begin{array}{l}299-W 6-2^{a} \\
299-W 7-5^{a} \\
299-W 10-13^{\circ}\end{array}$ & $\begin{array}{l}299-W 7-4^{\circ} \\
299-W 7-8 \\
299-W 10-19^{a}\end{array}$ & $\begin{array}{l}299-W 7-4^{\circ} \\
299-W 8-1\end{array}$ \\
\hline
\end{tabular}

NTU = nephelometric turbidity unit.

- Field QC samples associated with this data exceeded the reliable detection limits.

${ }^{b}$ Constituent standard omitted from chemistry tables. 
Table 15-30. Water Quality Standards Exceeded at Low-Level Waste Management Area 4.

\begin{tabular}{|llll|}
\hline $\begin{array}{l}\text { Chromium } \\
(50 \mathrm{ppb})\end{array}$ & $299-W 15-15$ & $299-W 15-16$ & $299-W 15-17$ \\
& $299-W 15-18$ & $299-W 15-19^{\circ}$ & $299-W 15-20$ \\
& $299-W 15-23$ & $299-W 15-24$ & $299-W 18-21$ \\
& $299-W 18-22$ & $299-W 18-23$ & $299-W 18-26$ \\
& $299-W 18-27$ & $299-W 18-28$ & $299-W 18-29$ \\
\hline $\begin{array}{l}\text { Nitrate } \\
(45,000 \mathrm{ppb})\end{array}$ & $299-W 15-15$ & $299-W 15-16$ & $299-W 15-18$ \\
\hline Turbidity & $299-W 15-19$ & $299-W 15-24$ & \\
(1 NTU) & $299-W 15-16$ & $299-W 15-17^{\circ}$ & $299-W 15-18$ \\
& $299-W 15-19$ & $299-W 15-20^{\circ}$ & $299-W 15-23$ \\
& $299-W 15-24^{\circ}$ & $299-W 18-21$ & $299-W 18-22$ \\
& $299-W 18-26$ & $299-W 18-27$ & $299-W 18-28$ \\
\hline Iron & $299-W 18-32$ & & \\
(300 ppb) & $299-W 15-15$ & $299-W 15-16$ & $299-W 15-17$ \\
& $299-W 15-18$ & $299-W 15-19^{\circ}$ & $299-W 15-20$ \\
& $299-W 15-23$ & $299-W 15-24$ & $299-W 18-21$ \\
& $299-W 18-22$ & $299-W 18-26$ & $299-W 18-27$ \\
& $299-W 18-28$ & $299-W 18-29$ & $299-W 18-32$ \\
\hline Manganese & $299-W 15-19^{\circ}$ & $299-W 15-20$ & $299-W 15-23$ \\
(50 ppb) & $299-W 15-24$ & $299-W 18-27$ & \\
\hline Carbon tetrachlorideb & $299-W 15-15$ & $299-W 15-16$ & $299-W 15-18$ \\
(5 ppb) & $299-W 15-23$ & $299-W 15-24$ & $299-W 18-21$ \\
& $299-W 18-23$ & $299-W 18-24$ & $299-W 18-26$ \\
& $299-W 18-27^{\circ}$ & $299-W 18-28^{\circ}$ & $299-W 18-29$ \\
\hline Tritium & $299-W 18-32$ & & \\
(20,000 pCi/L) & $299-W 15-15$ & $299-W 15-16$ & $299-W 15-18$ \\
\hline
\end{tabular}

NTU = nephelometric turbidity unit.

${ }^{\circ}$ Field QC samples associated with this data exceeded the reliable detection limits.

${ }^{b}$ Constituent standard omitted from chemistry tables. 
Table 15-31. Water Quality Standards Exceeded at Low-Level Waste Management Area 5.

\begin{tabular}{|c|c|c|c|}
\hline $\begin{array}{l}\text { Chromium } \\
(50 \mathrm{ppb})\end{array}$ & $\begin{array}{l}299-W 6-2 \\
299-W 6-11\end{array}$ & $\begin{array}{l}299-W 6-7 \\
299-W 7-10\end{array}$ & $\begin{array}{l}299-W 6-10 \\
299-W 11-31\end{array}$ \\
\hline $\begin{array}{l}\text { Filtered chromium } \\
(50 \mathrm{ppb})\end{array}$ & $299-W 6-7$ & 299-W6-7 & -- \\
\hline $\begin{array}{l}\text { Gross beta } \\
(50 \mathrm{pCi} / \mathrm{L})\end{array}$ & $299-W 6-10$ & $299-W 11-31$ & -- \\
\hline $\begin{array}{l}\text { Nitrate } \\
(45,000 \mathrm{ppb})\end{array}$ & $\begin{array}{l}299-W 6-2^{0} \\
299-W 6-10\end{array}$ & $\begin{array}{l}299-W 6-7 \\
299-W 6-11\end{array}$ & $\begin{array}{l}299-W 6-9 \\
299-W 11-31\end{array}$ \\
\hline $\begin{array}{l}\text { Turbidity } \\
\text { (1 NTU) }\end{array}$ & $\begin{array}{l}299-W 6-2^{a} \\
299-W 6-8^{a} \\
299-W 6-11^{a} \\
299-W 11-31^{a}\end{array}$ & $\begin{array}{l}299-W 6-6 \\
299-W 6-9 \\
299-W 6-12^{a}\end{array}$ & $\begin{array}{l}299-W 6-7^{a} \\
299-W 6-10^{a} \\
299-W 7-10^{a}\end{array}$ \\
\hline $\begin{array}{l}\text { Iron } \\
(300 \mathrm{ppb})\end{array}$ & $\begin{array}{l}299-W 6-2 \\
299-W 6-11 \\
299-W 11-31\end{array}$ & $\begin{array}{l}299-W 6-7 \\
299-W 6-12\end{array}$ & $\begin{array}{l}299-W 6-8 \\
299-W 7-10\end{array}$ \\
\hline $\begin{array}{l}\text { Carbon tetrachloride } \\
(5 \mathrm{ppb})\end{array}$ & $\begin{array}{l}299-W 6-2^{\circ} \\
299-W 6-7 \\
299-W 6-11\end{array}$ & $\begin{array}{l}299-W 6-4 \\
299-W 6-9 \\
299-W 7-10\end{array}$ & $\begin{array}{l}299-W 6-5 \\
299-W 6-10 \\
299-W 11-31\end{array}$ \\
\hline $\begin{array}{l}\text { Trichloroethylene } \\
(5 \mathrm{ppb})\end{array}$ & $299-W 6-10$ & $299-W 11-31$ & -- \\
\hline $\begin{array}{l}\text { Tritium } \\
(20,000 \mathrm{pC} i / L)\end{array}$ & 299-W6-7 & $299-W 6-10$ & $299-W 11-31$ \\
\hline
\end{tabular}

NTU = nephelometric turbidity unit.

"Field QC samples associated with this data exceeded the reliable detection limits.

${ }^{b}$ Constituent standard omitted from chemistry tables. 
DOE /RL-93-56-3

This page intentionally left blank. 
$\bar{\sim} \mathrm{J}$. RL $-93-56-3$

\section{CONTENTS}

16.0 SINGLE-SHELL TANKS ...................... . . . 16-1

16.1 INTRODUCTION .. . . . . . . . . . . . . . . . . . . . . . 16-1

16.2 WATER LEVEL MEASUREMENTS . . . . . . . . . . . . . . . 16-2

16.3 WATER CHEMISTRY DATA . . . . . . . . . . . . . . . . . . . 16-3

16.3.1 Waste Management Area A-AX . . . . . . . . . . . . 16-4

16.3.2 Waste Management Area B-BX-BY . . . . . . . . . . . 16-4

16.3.3 Waste Management Area $C$. . . . . . . . . . . . . . 16-4

16.3.4 Waste Management Area S-SX . . . . . . . . . . . . 16-4

16.3.5 Waste Management Area $T$. . . . . . . . . . . . . 16-5

16.3.6 Waste Management Area TX-TY . . . . . . . . . . . . 16-6

16.3.7 Waste Management Area U. . . . . . . . . . . . . 16-7 
$\begin{array}{llll}\sigma & \sigma & \sigma & \sigma \\ 1 & \omega & 1 & 1\end{array}$

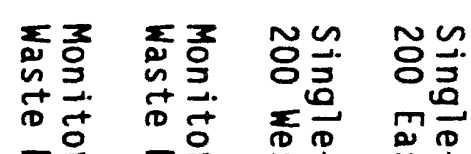

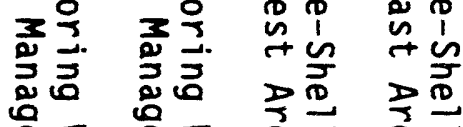

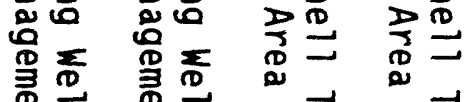

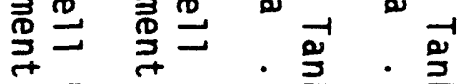

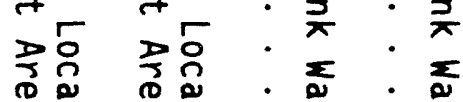

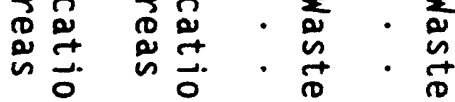

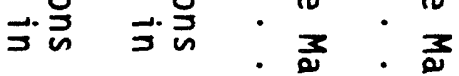

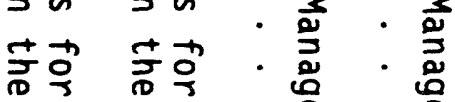

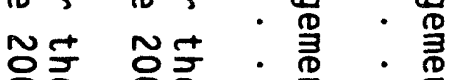

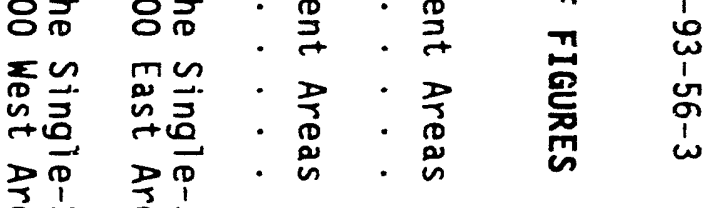

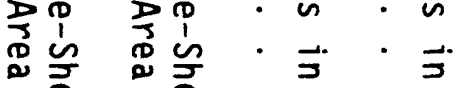

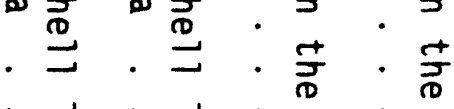

- $\frac{1}{2} \cdot \overrightarrow{\frac{1}{x}}$

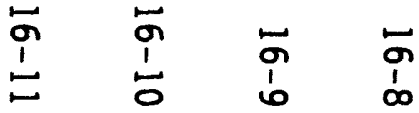

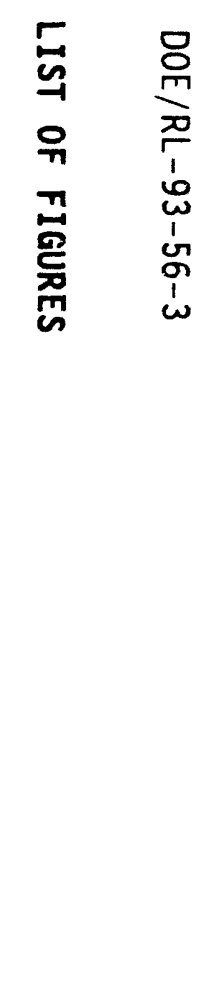




\section{LIST OF TABLES}

16-1 Groundwater Monitoring Wells for the Single-Shell Tanks . . . 16-12

16-2 RCRA Water Level Measurement Report Single-She11 Tanks, Third Quarter 1993............... . 16-14

16-3 Constituent List and Summary of Results for the Single-Shell

Tank Waste Management Area A-AX Data for Reporting Period Juiy 1 through September $30,1993 \ldots$. . . . . . . . . 16-22

16-4 Constituents with at Least One Value Above the CRQL for the Single-Shell Tank Waste Management Area A-AX Data for Reporting Period July 1 through September 30, 1993 . . . . . . 16-28

16-5 Contamination Indicator Parameters for the Single-Shell Tank Waste Management Area A-AX Data for Reporting Period July 1 through September $30,1993 \ldots$. . . . . . . . 16-30

16-6 Constituent List and Summary of Results for the Single-Shell Tank Waste Management Area B-BY-BX Data for Reporting Period July 1 through September 30,1993 .......... 16-31

16-7 Constituents with at Least One Value Above the CRQL for the Single-Shell Tank Waste Management Area B-BY-BX Data for Reporting Period July 1 through September 30, 1993 ..... . 16-34

16-8 Contamination Indicator Parameters for the Single-Shell Tank Waste Management Area B-BY-BX Data for Reporting Period July 1 through September $30,1993 \ldots$ 16-36

16-9 Constituent List and Summary of Results for the Single-Shell Tank Waste Management Area C Data for Reporting Period July 1 through September 30, $1993 \ldots$. . . . . . . . . 16-37

16-10 Constituents with at Least One Value Above the CRQL for the Single-Shell Tank Waste Management Area C Data for Reporting Period July 1 through September 30, $1993 \ldots$. . . . 16-40

16-11 Contamination Indicator Parameters for the Single-Shell Tank Waste Management Area C Data for Reporting Period July 1 through September 30, $1993 \ldots$............. 16-41

16-12 Constituent List and Summary of Results for the Single-Shell Tank Waste Management Area S-SX Data for Reporting Period July 1 through September 30,1993 ........... 16-42

16-13 Constituents with at Least One Value Above the CRQL for the Single-Shell Tank Waste Management Area S-SX Data for Reporting Period July 1 through September 30, 1993 . . . . . . 16-48 


\section{LIST OF TABLES (cont.)}

16-14 Contamination Indicator Parameters for the Single-Shell Tank

Waste Management Area S-SX Data for Reporting Period

July 1 through September 30,1993 . . . . . . . . . . . 16-51

16-15 Constituent List and Summary of Results for the Single-Shell

Tank Waste Management Area T Data for Reporting Period

July 1 through September 30, 1993 . . . . . . . . . . . 16-52

16-16 Constituents with at Least One Value Above the CRQL for

the Single-Shell Tank Waste Management Area T Data for

Reporting Period July 1 through September 30, 1993...... . 16-55

16-17 Contamination Indicator Parameters for the Single-Shell Tank

Waste Management Area T Data for Reporting Period July 1

through September 30, 1993 ............... 16-57

16-18 Constituent List and Summary of Results for the Single-Shell

Tank Waste Management Area TX-TY Data for Reporting Period

July 1 through September 30,1993 ............. 16-58

16-19 Constituents with at Least One Value Above the CRQL for the

Single-Shell Tank Waste Management Area TX-TY Data for

Reporting Period July 1 through September 30, 1993....... . 16-61

16-20 Contamination Indicator Parameters for the Single-Shell Tank Waste Management Area TX-TY Data for Reporting Period July 1 through September 30,1993 . . . . . . . . . . . 16-64

16-21 Constituent List and Summary of Results for the Single-Shell Tank Waste Management Area U Data for Reporting Period July 1 through September 30, 1993 . . . . . . . . . . 16-65

16-22 Constituents with at Least One Value Above the CRQL for the Single-Shell Tank Waste Management Area U Data for Reporting Period July 1 through September 30, 1993 .. . . . . 16-68

16-23 Contamination Indicator Parameters for the Single-Shell Tank Waste Management Area U Data for Reporting Period July 1 through September 30, 1993. 
$\mathrm{DOE} / \mathrm{RL}-93-56-3$

\title{
16.0 SINGLE-SHELL TANKS
}

\author{
J. A. Caggiano \\ Westinghouse Hanford Company
}

\subsection{INTRODUCTION}

Although decommissioned in 1980, the SSTs are considered to be actively storing hazardous and radioactive waste and have been designated as RCRA facilities. The SSTs received metal and first-cycle waste from the chemical processing of uranium-bearing spent fuel rods. Spent fuel rods from reactors in the 100 Areas were processed using the bismuth phosphate, REDOX, or PUREX process. Metal waste is that which was produced in the first step of the process of dissolving spent fuels and contains about $90 \%$ of fission product radionuclides as well as uranium and some plutonium. Other waste streams (e.g., coating, noncomplexed, and deionizing wastes) also were sent to SSTs at some time in their history (see Anderson 1990 for a discussion of types of waste sent to various SSTs from startup until the tank was no longer used). Chemical and radiological contents of the various SSTs were summarized in July 1993 (WHC 1993C).

The 149 SSTs are located in 7 waste management areas (WMA) containing 1 or more tank farms (Figures 16-1 and 16-2). Three WMAs are in the 200 East Area (A-AX, B-BX-BY, and C) and four are in the 200 West Area (S-SX, T, TX-TY, and $U$ ). Each tank farm contains from 4 (AX Tank Farm) to 18 (TX Tank Farm) tanks, each of which is an underground, reinforced-concrete tank with a single liner of carbon steel. The larger tanks have a diameter of $23 \mathrm{~m}(75 \mathrm{ft})$, varying height, and are buried at least $2 \mathrm{~m}(6 \mathrm{ft})$ below the ground surface. The capacity of these tanks varies from $2,006,000$ to $3,785,000 \mathrm{~L}(530,000$ to $1,000,000 \mathrm{gal})$. There are sixteen 200-series tanks, each of which hold $208,175 \mathrm{~L}(55,000 \mathrm{gal})$. Four 200-series tanks are located in each of the original four tank farms: 241-B, 241-C, 241-T, and 241-U. The original four tank farms were built in 1944; SSTs were built from 1944 to 1964.

Interim-status, detection-level groundwater monitoring was initiated at the SSTs in 1989 with the construction of 12 RCRA standard wells. Sampling was initiated in February 1990 at WMAs A-AX, B-BX-BY, C, and T, but was suspended until July 1991 because of the absence of analytical laboratory support. Sampling resumed in July 1991 at WMAs A-AX, B-BX-BY, and T, and continued quarterly through early 1993. By October 1991, all existing RCRA standard wells in all WMAs were being sampled quarterly. Most wells have been sampled quarterly more than four times. All WMAs except for $T$ and TX-TY are now in detection-level, semiannual indicator parameter evaluation status. WMAs $T$ and TX-TY continue to be sampled quarterly, as these sites are now in assessment monitoring status because of elevated specific conductance. A groundwater quality assessment monitoring plan (Caggiano and Chou 1993) for WMAs T and TX-TY was submitted to Ecology in July 1993. The SST WMA well locations are shown in Figures 16-3 and 16-4. Wells in the SST monitoring networks are listed in Table 16-1.

Numerous older wells in tank farms and in the vicinity are constructed of carbon steel casing that is perforated and open to different levels in the unconfined aquifer. These wells were part of an operational groundwater 
monitoring system at some point in their history and currently are used only for water level measurements or to collect samples for operational monitoring. Some are used for environmental surveillance monitoring to check for conformance to DOE orders (e.g., Schmidt et al. 1992). These older wells do not comply with the construction specifications of WAC 173-160 and are not used for collection of RCRA samples. One exception is well 2-E27-7, a well constructed in 1982 that has a carbon steel casing, an annular seal, and a stainless steel screen below the carbon steel casing. There is no carbon steel in contact with groundwater in well 2-E27-7. Samples are collected from this well, which is upgradient of WMA $C$.

\subsection{WATER LEVEL MEASUREMENTS}

Water levels measured in 67 wells for this quarter are given in Table 16-2. These data include routine water level measurements taken when sampling, as well as nonroutine monthly measurements made by Environmental Field Services personnel. Geodetic survey elevations used to calculate water levels may have changed this quarter because of new surveys or because the survey datum was changed to National Geodetic Vertical Datum 1929 Standard (NGVD29) or to a corrected NGVD29 (previous surveys used locally established benchmarks as the reference). Some water level elevations depart from historical trends and are flagged in Table 16-2 (e.g., 2-E27-14 water level measurement on September 21, 1993). Several wells exhibited a drop of about $0.15 \mathrm{~m}(0.5 \mathrm{ft})$ between June and July 1993; these drops are suspicious because monthly water level declines in these wells have historically been less than $0.06 \mathrm{~m}(0.2 \mathrm{ft})$. Wells exhibiting this pattern include 2-E25-2, 2-E33-18, 2-E27-15, and 2-E27-7.

Water levels in upgradient wells 2-W18-25 and 2-W18-31 appear to be declining faster than in downgradient we11 2-W19-32 at WMA U. A possible change in flow direction is suggested and this trend will be watched. Additional wells have been added to the water level monitoring network in the vicinity of the 241-U Tank Farm to determine the extent and possible source for this change. Background values will be modified if this is a permanent change in groundwater flow direction.

Water levels in a number of older carbon steel wells located inside the perimeter fences of tank farms have not been measured since July 1993 because of limited access to tank farms. Concern for safety of personnel following several incidents within tank farms has limited access into tank farms to projects/activities deemed to be highest priority. These wells have been removed from the water level network for each WMA until they are evaluated to determine whether the data produced by these measurements add any value to the data provided by the RCRA standard wells. Access restrictions occurred in August 1993; water levels for these older wells inside tank farms are reported for July 1993.

We11 2-E33-24, located adjacent to the 241-BY Tank Farm (WMA B-BX-BY), was decommissioned in September 1993 so that a cover could be constructed over the adjacent 216-B-57 Crib as part of remedial measures for operable unit 200-BP-1. Water levels had been measured monthly in this well until its decommissioning. 


\subsection{WATER CHEMISTRY DATA}

Data received to date from analyses of samples collected during the third quarter of 1993 are provided in Tables 16-3 through 16-23. All wells in all WMAs were sampled this quarter except for 2-W10-9 (WMA T). Wel1 2-W10-9 is not a RCRA standard well; remediation may not be performed to bring the well back into the sampling network because it is very close to RCRA standard well 2-W10-15. Remediation of well 2-W11-28 is being completed and it is scheduled to be sampled in December 1993. It has not been sampled since May 1992 because of problems with the well and the sampling pump. Some samples from SST wells were collected in late September 1993, and the data were not received in time to be included in this quarterly report. Missing data should appear in the next quarterly report.

All TOX values for the third quarter of 1993 are still flagged (with a ' $P$ ') because of an audit that discovered $Q C$ problems in the laboratory (see Section 1.2.5 and Tables 16-5, 16-8, 16-11, 16-14, 16-17, 16-20, and 16-23). Exceedance of holding times for constituents are flagged in the analytical data tables. Values for analyses of unfiltered samples of iron, chromium, and, in some cases, manganese, exceed DWS or the secondary maximum containment level (SMCL) in a number of wells in all WMAs. In most cases, the values for analyses of filtered samples for these constituents are below standards. These constituents were not an integral part of any waste stream discharged to the SSTs. These constituents are also common in results of unfiltered sample analyses in many RCRA wells at the Hanford Site, and are believed to result from common practices during construction of the wells. In many of these wells with elevated metals in unfiltered analyses, turbidity of the sampled groundwater exceeded 1 NTU, suggesting that the metals are present in particulate form. In wells where turbidity is excessively high (e.g., wells 2-Wio-18 and 2-W15-22 at WMA TX-TY), analyses of unfiltered samples are high in aluminum, suggesting that the turbidity may be due to an alumino-silicate (e.g., clays). Blank contamination was noted in many samples analyzed for TOC and TC in the third quarter of 1993 (see Section 1.2 for details).

Four quarters of background data have been obtained from wells in all WMAs and the data have been statistically analyzed to calculate a critical mean for TOC, specific conductance, and critical range (for pH) for each site. Data are compared to the critical me on for these values in each of these WMAs. Values for TOX were not used to calculate a critical mean for each WMA because of QC problems in the laboratory. Replicate average values from downgradient wells are compared to the critical means for indicator parameters to determine whether the facilities in any SST WMAs may be affecting groundwater quality.

Additional wells to supplement the groundwater quality assessment program are being identified and checked before having any remedial work performed and adding them to the SST network for WMAs $T$ and TX-TY. These two WMAs are in groundwater quality assessment status because of elevated specific conductance. Additional wells are being added to the network for each of these sites to provide data for determining the possible extent of the high conductivity plume in groundwater beneath these two WMAs, and whether the SSTS are the source responsible for this elevated specific conductance. 


\subsubsection{Waste Management Area A-AX}

At WMA A-AX, iron is above the SMCL in we11 2-E25-46 for unfiltered analyses, but is below standard for filtered analyses. Constituents found in various QC samples are discussed in Section 1.2 of this report. We11 2-E25-46 was the only well sampled during the third quarter of 1993, as four quarters of background data are still being obtained on this well. All other wells are scheduled to be sampled in December 1993.

There were no exceedances of the critical mean or critical range during the third quarter of 1993 for specific conductance, TOC, or pH at WMA A-AX. Values for these parameters for June 1993 samples also did not exceed the critical mean. Values for conductivity and ph were reported from both field and laboratory measurements.

\subsubsection{Waste Management Area B-BX-BY}

Unfiltered analyses of chromium and iron exceed the DWS and SMCL, respectively, in well 2-E33-43, but are below these limits in analyses of filtered samples. Gross beta again exceeds the $50-\mathrm{pCi} / \mathrm{L}$ limit in wel1 2-E33-42. Technetium-99 is the likely source of elevated gross beta.

Values for $\mathrm{pH}$, specific conductance, and TOC did not exceed the critical mean or critical range for wells for this quarter or for data reported from the June 1993 sampling. Both field and laboratory measurements of pH and specific conductance were made. TOC was below the CRQL for all wells for the previous quarter, but TOC was only reported for wells 2-E33-42 and 2-E33-43 in WMA B-BX-BY this quarter.

\subsubsection{Waste Management Area C}

There were no exceedances of standards in samples analyzed from well 2-E27-7, the only well sampled this quarter.

Values for $\mathrm{pH}$, specific conductance, and TOC did not exceed the critical mean or critical range $(\mathrm{pH})$ for samples collected in June and September 1993. Both field and laboratory measurements of these two parameters were reported. TOC was below the CRQL for all wells for the second quarter of 1993, but TOC was reported only for well 2-E27-7 in WMA $C$ this quarter. TOX data for the last quarter were all below the CRQL except for one sample from well 2-E27-12.

\subsubsection{Waste Management Area S-SX}

Iron is elevated above standards in unfiltered analyses for wells 2-W22-44 and 2-W22-45, but is below standards in analyses of filtered samples for all wells at WMA S-SX. Unfiltered sample results for manganese and chromium were also above the SMCL in we11 2-W22-44, but below the 1 imit in all wells for filtered analyses. 
Values for $\mathrm{pH}$ and TOC did not exceed the critical mean or critical range $(\mathrm{pH})$ for this quarter. There was an exceedance of the critical mean for specific conductance in well 2-Wč2-44, but the data are erroneous. A request for review of these four values has been submitted. Without these anomalous data for well 2-W22-44, there is no exceedance of the critical mean for specific conductance. The initial reading for specific conductance at well 2-W22-44 was $204 \mu \mathrm{mho} / \mathrm{cm}$ recorded at 0948 on September 29, 1993. This reading is well within the range of previous measurements of specific conductance for this we 11 , which range between 190 and $220 \mu \mathrm{mho} / \mathrm{cm}$. Seven additional readings beginning at 0954 on September 29 are 1332, 1382, 1327, $1358,1346,1352$, and $1370 \mu \mathrm{mho} / \mathrm{cm}$. A handwritten note beneath the entry on the field record sheet reads, "after adjusting $\mathrm{pH}$ meter, 197/197." A specific conductance of $197 \mu \mathrm{mho} / \mathrm{cm}$ would be consistent with previous values obtained on this well. Laboratory measurement of specific conductance for this well is $190 \mu \mathrm{mho} / \mathrm{cm}$, lending further suspicion to the field values for conductivity. Additional field measurements of specific conductance were made in this well for verification and the eight values (obtained after purging) averaged $200 \mu \mathrm{mho} / \mathrm{cm}$, thus confirming that the values recorded on September 29, 1993 are erroneous. Correspondence subsequently received from PNL in December 1993 indicated that the September 29, 1993 values were suspect and that the value of $197 \mu \mathrm{mho} / \mathrm{cm}$ was likely correct because of instrument malfunction caused by a faulty battery. Therefore, no further action is required regarding any change in status of monitoring. TOC data were all below the CRQL for the second quarter of 1993, but were reported only for wells 2-W22-44 and 2-W22-45 for this quarter. Neither well exceeds the critical mean.

\subsubsection{Waste Management Area $T$}

Iron and chromium are elevated in analyses of unfiltered samples for most wells at WMA T. Filtered chromium exceeds the DWS of $50 \mathrm{ppb}$ in well 2-W10-15. Filtered analyses values for iron are below the SMCL in all wells. Gross beta is elevated above the 50-pCi/L limit in downgradient well 2-W10-15. Field conductivity is elevated in wells 2-W10-15 and 2-W10-16 in WMA T, continuing a previously noted trend. Tritium is elevated above the 20,000-pCi/L standard in wells 2-W10-15 and 2-W10-16. Data for wells 2-W10-15 and 2-W11-27 have been flagged because of problems during well construction. Carbon steel was left in well 2-W10-15 during completion when backpulling resulted in parting of the temporary casing some $15 \mathrm{~m}$ (50 ft) above the water table. A comparison of the principal constituents of the carbon steel and several quarters of data for ICP metals reveals no significant difference in water quality that might be attributed to the carbon steel remaining in well 2-W10-15. The P flags have been removed from these data.

A stainless steel weight and $0.76 \mathrm{~m}(2.5 \mathrm{ft})$ of carbon steel measuring tape broke off during completion of wells 2-W11-27 and 2-W11-28. These weights and tapes remain in the filter pack below the water table. A similar check on the metals composing the steel tapes revealed no significant differences attributable to the presence of these steel tapes in the filter pack. Consequently, the $P$ flag has been removed from the data for well 2-W11-27. 
Remediation of well 2-W11-28 to repair the casing damaged during completion of the well in December 1991 was completed. However, the inside diameter of the well in the damaged interval (between 52 and $53 \mathrm{~m}$ [171.8 and $175.2 \mathrm{ft}]$ deep) has been narrowed to less than $6 \mathrm{~cm}(2.5 \mathrm{in}$.); consequently, a steel tape will not pass between the riser pipes and the narrowed casing. Therefore, water levels cannot be measured using a steel tape. Consideration is being given to use a dedicated $E$ tape installed in a pump riser pipe that would be used to measure water levels. Carbon steel casing rollers used in the remediation of this well broke from the drill string and now rest at the bottom of the screen. Data will be flagged to evaluate whether any effects of this included steel on groundwater quality.

Values for all TOC samples for the second and third quarters of 1993 are below the CRQL. All reported values for $\mathrm{pH}$ are within the critical range for all wells.

The average of quadruplicate measurements of field-specific conductance continues to exceed the critical mean for specific conductance in downgradient well 2-W10-15, following a pattern that triggered this site into assessment monitoring. Measurements of field- and laboratory-specific conductance in all other wells are below the critical mean for conductivity.

Total dissolved solid values for wells 2-W10-15 and 2-W10-16 are 790 and $520 \mathrm{ppm}$, respectively.

\subsubsection{Waste Management Area TX-TY}

Tritium is elevated in all downgradient wells (2-W10-17, 2-W14-12, and 2-W10-18) at WMA TX-TY, but is below the 20,000-pCi/L 1 imit in upgradient well 2-W15-22. Turbidity is elevated in wells 2-W10-18 and 2-W15-22 at WMA TX-TY. Gross beta of $1,220 \mathrm{pCi} / \mathrm{L}$ in well $2-W 14-12$ is apparently due to elevated technetium-99 at $12,200 \mathrm{pCi} / \mathrm{L}$. Gross beta al so exceeds the standard in upgradient wel1 2-W15-22 at $53.3 \mathrm{pCi} / \mathrm{L}$ and is al so because of technetium-99 at $219 \mathrm{pCi} / \mathrm{L}$. Iodine-129 is elevated in downgradient well 2-W14-12 at $64.20 \mathrm{pCi} / \mathrm{L}$. Analyses of filtered samples for iron are generally below standards, but the analyses of unfiltered samples are above the SMCL of $300 \mathrm{ppb}$ in all wells. Unfiltered analyses of manganese are also elevated above the 50-ppb standard in upgradient we 11 2-W15-22 and downgradient wells 2-W10-18 and 2-W14-12. Analyses of unfiltered samples for chromium are above the 50-ppb standard in all wells but are below the standard for filtered analyses in all wells except well 2-W14-12. TDS is elevated in downgradient wells 2-W10-17 and 2-W14-12 at 520 and $1,200 / 1,100 \mathrm{ppm}$, respectively.

Field conductivities measured at downgradient wells 2-W10-17 and 2-W14-12 continue to exceed the SMCL and the critical mean. Elevated specific conductance has triggered this site into assessment monitoring. Measurements of $\mathrm{pH}$ are within the critical range. Data from TOC for the June and September 1993 samples are below the CRQL. 


\subsubsection{Waste Management Area U}

Results of analyses for TOC were reported for the second and third quarters of 1993 and all are below the CRQL. TOC values for this quarter are above the MDL of $200 \mathrm{ppb}$. Values for TOX for wells 2-W18-25, 2-W18-31, and 2-W19-31 appear elevated for the June and September 1993 sample events. Most TOX data are flagged because of unsatisfactory audit findings related to $Q C$ in the laboratory.

Values for specific conductance are consistent with previous quarters and do not exceed the SMCL for conductivity. Values of field pH agree with those uone in the laboratory. Values for $\mathrm{pH}$ do not exceed the critical range; values for specific conductance and TOC do not exceed the critical mean. quarter.

No indicator parameter data were reported for well 2-W19-32 for this 
Figure 16-1. Single-Shell Tank Waste Management Areas in the 200 East Area.

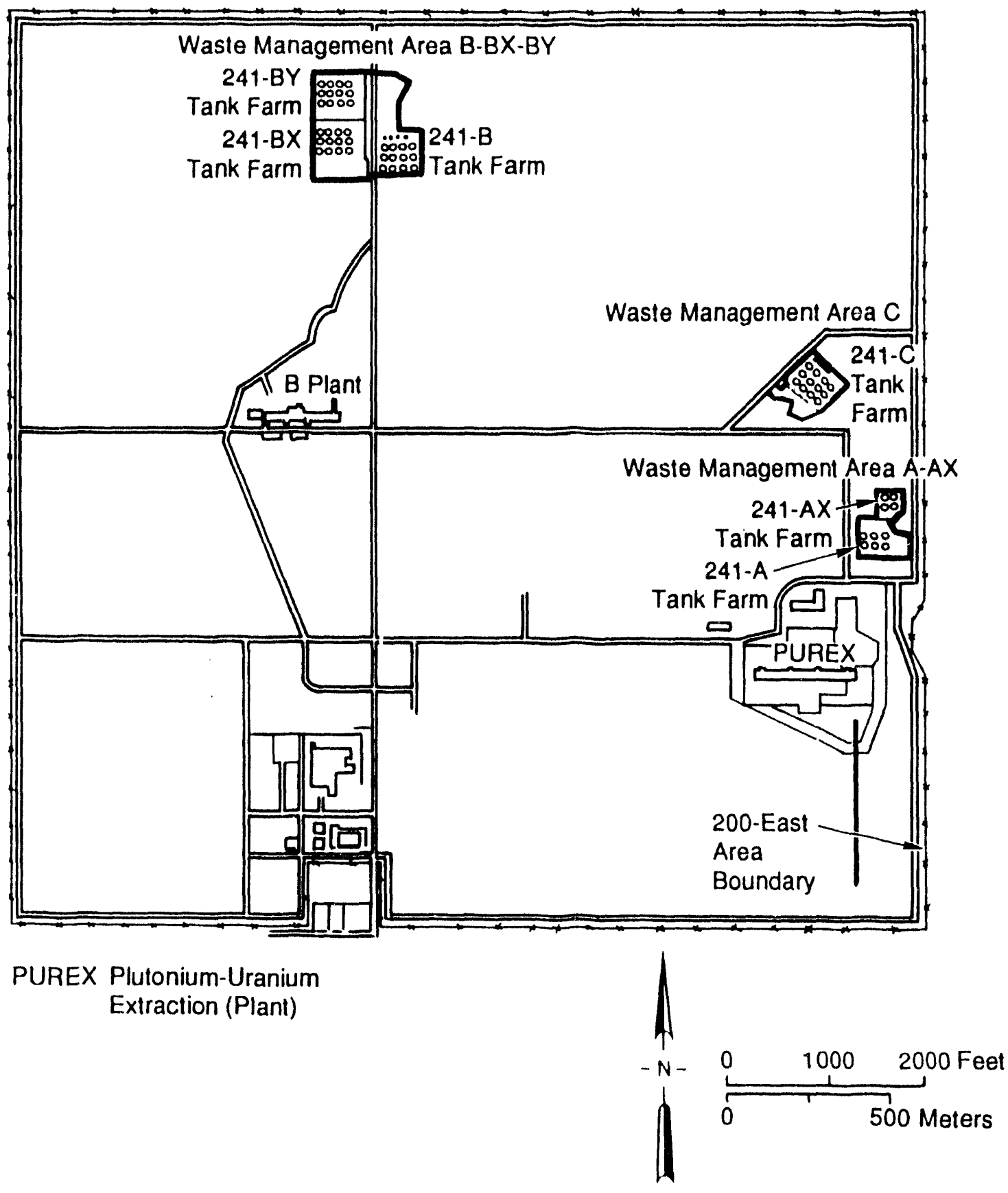

PS.90-71 
DOE/RL-93-56-3

Figure 16-2. Single-Shell Tank Waste Management Areas in the 200 West Area.

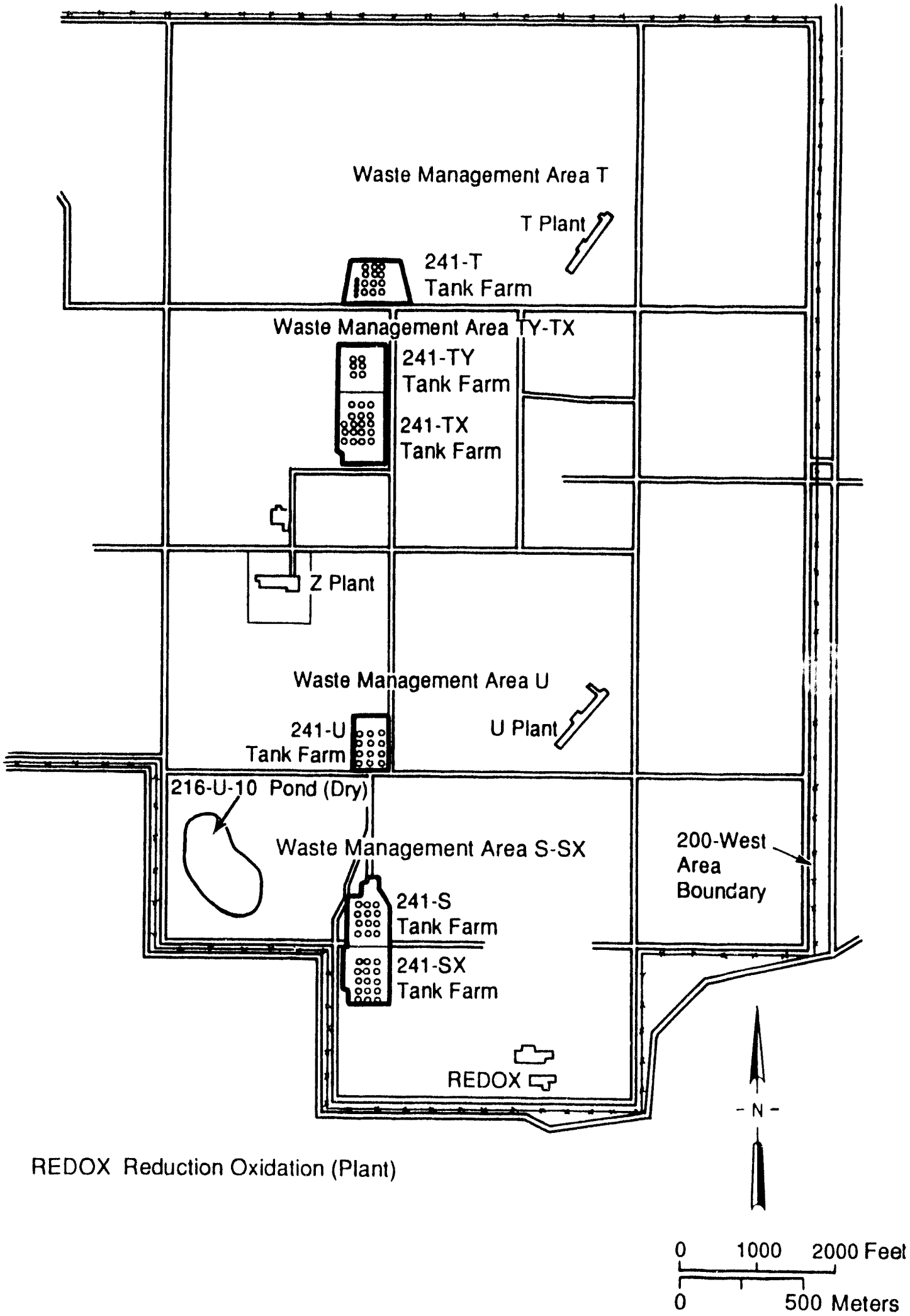

PS- $90-69$ 


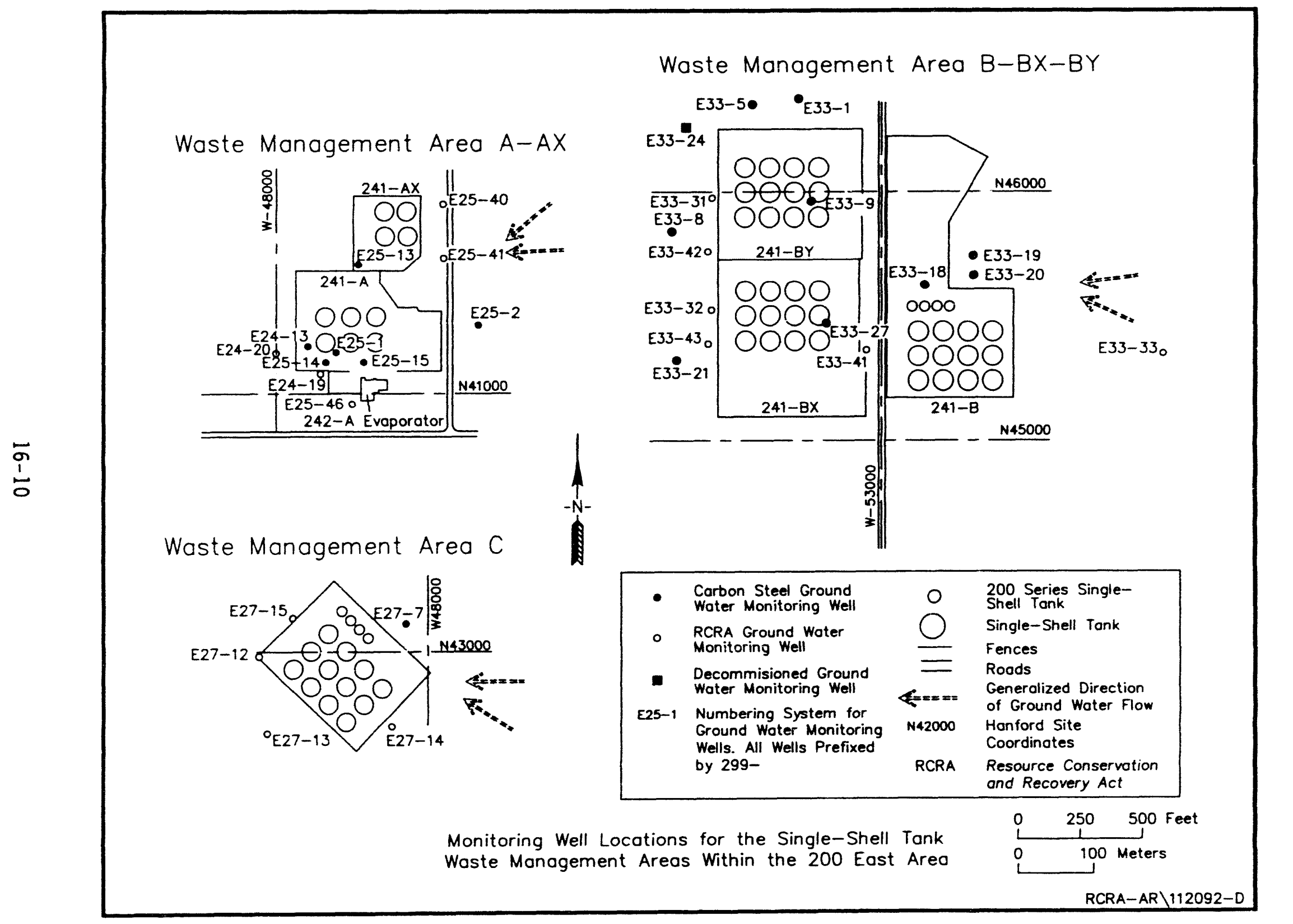

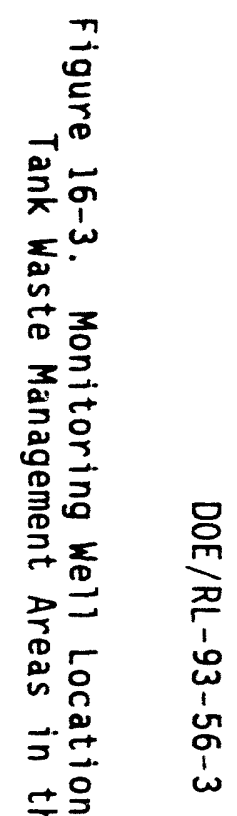

家票

ㅇㅇㅇㅇํㄱ

需宁

品

字范

赵

i

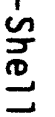

Monitoring Well Locations for the Single-Shell Tank $\stackrel{2}{0 \quad 250 \quad 500 \mathrm{Feet}}$

RCRA-AR\112092-D 


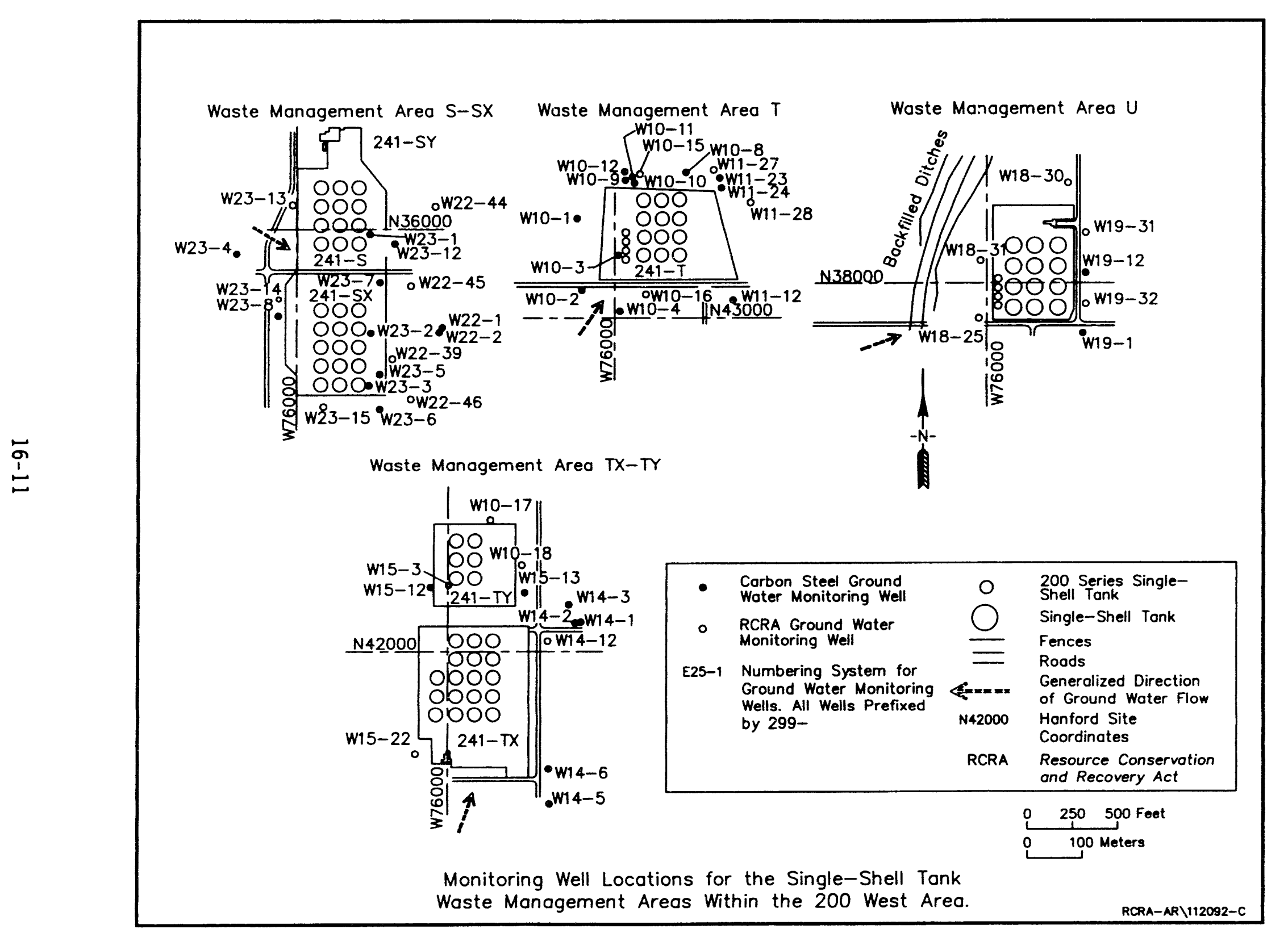


Table 16-1. Groundwater Monitoring Wells for the Single-Shell Tanks. (sheet 1 of 2)

\begin{tabular}{|c|c|c|c|}
\hline $\begin{array}{c}\text { Well no. } \\
(299-)\end{array}$ & Aquifer & $\begin{array}{c}\text { Sample } \\
\text { frequency }\end{array}$ & $\begin{array}{l}\text { Sample date, } \\
\text { 3rd Qtr } 1993\end{array}$ \\
\hline \multicolumn{4}{|c|}{ Single-Shell Tanks Waste Management Area A-AX } \\
\hline$E 24-19^{89}$ & Ringold: Water Table & Semiannually & NS \\
\hline$E 24-20^{90}$ & Ringold: Water Tatle & Semiannually & NS \\
\hline$E 25-40^{89}$ & Ringold: Water Table & Semiannually & NS \\
\hline$E 25-41^{89}$ & Ringold: Water Table & Semiannually & NS \\
\hline$E 25-46^{92}$ & Ringold: Water Table & Semiannually & $9 / 29 / 93$ \\
\hline \multicolumn{4}{|c|}{ Single-Shell Tanks Waste Management Area B-BX-BY } \\
\hline$E 33-31^{89}$ & Hanford: Water Table & Semiannually & NS \\
\hline$E 33-32^{89}$ & Hanford: Water Table & Semiannually & NS \\
\hline$E 33-33^{89}$ & Hanford: Water Table & Semiannually & NS \\
\hline$E 33-36^{90}$ & Hanford: Water Table & Semiannually & NS \\
\hline$E 33-41^{906}$ & Hanford: Water Table & Semiannually & NS \\
\hline$E 33-38^{90 c}$ & Hanford: Water Table & Semiannually & NS \\
\hline$E 33-39^{90 c}$ & Hanford: Water Table & Semiannually & NS \\
\hline$E 33-42^{91}$ & Hanford: Water Table & Semiannual 1y & $9 / 21 / 93$ \\
\hline$E 33-43^{91}$ & Hanford: Water Table & Semiannually & $9 / 21 / 93$ \\
\hline \multicolumn{4}{|c|}{ Single-Shell Tank Waste Management Area C } \\
\hline$E 27-12^{89}$ & Ringold: Water Table & Semiannually & NS \\
\hline$E 27-13^{89}$ & Ringold: Water Table & Semiannually & NS \\
\hline$E 27-14^{89}$ & Ringold: Water Table & Semiannually & NS \\
\hline$E 27-15^{89}$ & Ringold: Water Table & Semiannually & NS \\
\hline$E 27-7^{82}$ & Ringold: Water Table & Semiannually & $9 / 21 / 93$ \\
\hline \multicolumn{4}{|c|}{ Single-Shel1 Tanks Waste Management Area S-SX } \\
\hline$W 22-39^{90}$ & Ringold: Water Table & Semiannually & NS \\
\hline W22-44 & Ringold: Water Table & Semiannually & $9 / 29 / 93$ \\
\hline$W 22-45^{92}$ & Ringold: Water Table & Semiannually & $9 / 29 / 93$ \\
\hline W22-46 & Ringold: Water Table & Semiannually & $\overline{N S}$ \\
\hline W23-139t & Ringold: Water Table & Semiannually & NS \\
\hline$W 23-14^{90}$ & Ringold: Water Table & Semiannually & NS \\
\hline W23-15 & Ringold: Water Table & Semiannually & NS \\
\hline
\end{tabular}


Table 16-1. Groundwater Monitoring Wells for the Single-Shell Tanks. (sheet 2 of 2)

\begin{tabular}{|c|c|c|c|}
\hline $\begin{array}{c}\text { We } 11 \text { no. } \\
(299-)\end{array}$ & Aquifer ${ }^{\circ}$ & $\begin{array}{c}\text { Sample } \\
\text { frequency }\end{array}$ & $\begin{array}{l}\text { Sample date, } \\
\text { 3rd Qtr } 1993\end{array}$ \\
\hline \multicolumn{4}{|c|}{ Single-Shell Tanks Waste Management Area T } \\
\hline$W 10-15^{89}$ & Ringold: Water Table & Quarterly & $9 / 28 / 93$ \\
\hline $410-16^{89}$ & Ringold: Water Table & Quarterly & $9 / 28 / 93$ \\
\hline$W 11-27^{91}$ & Ringold: Water Table & Quarterly & $9 / 28 / 93$ \\
\hline$W 11-28^{91}$ & Ringold: Water Table & Quarterly & $\mathrm{NS}^{\mathbf{d}}$ \\
\hline \multicolumn{4}{|c|}{ Single-Shell Tanks Waste Management Area TX-TY } \\
\hline$W 10-17^{90}$ & Ringold: Water Table & Quarterly & $9 / 28 / 93$ \\
\hline$W 10-18^{90}$ & Ringold: Water Table & Quarterly & $9 / 28 / 93$ \\
\hline$W 14-12^{91}$ & Ringold: Water Table & Quarterly & $9 / 28 / 93$ \\
\hline$W 15-22^{90}$ & Ringold: Water Table & Quarterly & $9 / 28 / 93$ \\
\hline \multicolumn{4}{|c|}{ Single-She 11 Tanks Waste Management Area U } \\
\hline$W 18-25^{90}$ & Ringold: Water Table & Semiannually & NS \\
\hline$W 18-30^{91}$ & Ringold: Water Table & Semiannually & $9 / 20 / 93$ \\
\hline$W 18-31^{91}$ & Ringold: Water Table & Semiannually & $9 / 20 / 93$ \\
\hline$W 19-31^{90}$ & Ringold: Water Table & Semiannually & NS \\
\hline$W 19-32^{90}$ & Ringold: Water Table & Semiannually & NS \\
\hline$W 19-12^{83}$ & Ringold: Water Table & Semiannually & NS \\
\hline
\end{tabular}

NS = not sampled.

Notes: Shading denotes upgradient wells. Superscript following well number denotes the year of installation.

'Wells are screened either in sand and gravel of the Hanford formation or in matrix-supported silty sandy gravel of the Ringold Formation.

Well in middle of WMA B-BX-BY and not clearly upgradient or downgradient. Therefore, data from sampling/analyses in this well are not used in statistical evaluations.

'Part of 200-BP-1 network. Not sampled regularly as RCRA wells.

Well being repaired. No sample collected. 
Table 16-2. RCRA Water Level Measurement Report Single-Shell Tanks, Third Quarter 1993.

(sheet 1 of 8 )

\begin{tabular}{|c|c|c|c|}
\hline Well & Date & $\begin{array}{l}\text { Depth to } \\
\text { water }(f t)\end{array}$ & $\begin{array}{l}\text { Water level } \\
\text { elevation } \\
\text { above msl (ft) }\end{array}$ \\
\hline \multicolumn{4}{|c|}{ 241-A/AX Tank Farm Wells } \\
\hline 299-E24-13 & $7 / 20 / 93$ & 289.10 & 402.03 \\
\hline 299-E24-19 & $\begin{array}{l}7 / 20 / 93 \\
8 / 19 / 93 \\
9 / 21 / 93\end{array}$ & $\begin{array}{l}291.74 \\
291.74 \\
291.97\end{array}$ & $\begin{array}{l}401 \cdot 91 \\
401.91 \\
401.68\end{array}$ \\
\hline 299-E24-20 & $\begin{array}{l}7 / 20 / 93 \\
8 / 19 / 93 \\
9 / 21 / 93\end{array}$ & $\begin{array}{l}287.09 \\
287.08 \\
287.33\end{array}$ & $\begin{array}{l}402.19 \\
402.20 \\
401.95\end{array}$ \\
\hline 299-E25-1 & $7 / 20 / 93$ & 288.52 & 402.05 \\
\hline 299-E25-2 & $\begin{array}{l}7 / 20 / 93 \\
8 / 19 / 93 \\
9 / 21 / 93\end{array}$ & $\begin{array}{l}273.36 \\
273.32 \\
273.56\end{array}$ & $\begin{array}{l}402.09 \\
402.13 \\
401.89\end{array}$ \\
\hline $299-E 25-13$ & $7 / 20 / 93$ & 280.26 & 402.17 \\
\hline 299-E25-40 & $\begin{array}{l}7 / 20 / 93 \\
8 / 19 / 93 \\
9 / 21 / 93\end{array}$ & $\begin{array}{l}263.70 \\
263.66 \\
263.88\end{array}$ & $\begin{array}{l}402.01 \\
402.05 \\
401.83\end{array}$ \\
\hline $299-E 25-41$ & $\begin{array}{l}7 / 20 / 93 \\
8 / 19 / 93 \\
9 / 21 / 93\end{array}$ & $\begin{array}{l}269.19 \\
269.15 \\
269.37\end{array}$ & $\begin{array}{l}402.07 \\
402.11 \\
401.89\end{array}$ \\
\hline $299-E 25-46$ & $\begin{array}{l}7 / 20 / 93 \\
8 / 20 / 93 \\
9 / 21 / 93 \\
9 / 29 / 93\end{array}$ & $\begin{array}{l}292.56 \\
292.56 \\
292.57 \\
292.70\end{array}$ & $\begin{array}{l}402.25 \\
402.25 \\
402.24 \\
402.11^{\star}\end{array}$ \\
\hline \multicolumn{4}{|c|}{ 241-B/BX/BY Tank Farm Wells } \\
\hline 299-E33-1 & $\begin{array}{l}7 / 20 / 93 \\
8 / 19 / 93 \\
9 / 21 / 93\end{array}$ & $\begin{array}{l}230.06 \\
230.08 \\
230.21\end{array}$ & $\begin{array}{l}402.05 \\
402.03 \\
401.90\end{array}$ \\
\hline
\end{tabular}


Table 16-2. RCRA Water Level Measurement Report

Single-Shell Tanks, Third Quarter 1993.

(sheet 2 of 8 )

\begin{tabular}{|c|c|c|c|}
\hline Well & Date & $\begin{array}{l}\text { Depth to } \\
\text { water (ft) }\end{array}$ & $\begin{array}{l}\text { Water level } \\
\text { elevation } \\
\text { above msl (ft) }\end{array}$ \\
\hline \multicolumn{4}{|c|}{ 241-B/BX/BY Tank Farm Wells } \\
\hline 299-E33-5 & $\begin{array}{l}7 / 20 / 93 \\
8 / 19 / 93 \\
9 / 21 / 93\end{array}$ & $\begin{array}{l}232.61 \\
232.66 \\
232.80\end{array}$ & $\begin{array}{l}402.11 \\
402.06 \\
401.92\end{array}$ \\
\hline 299-E33-8 & $\begin{array}{l}7 / 20 / 93 \\
8 / 19 / 93 \\
9 / 21 / 93\end{array}$ & $\begin{array}{l}248.92 \\
248.92 \\
249.38\end{array}$ & $\begin{array}{l}401.81 \\
401.81 \\
401.35\end{array}$ \\
\hline 299-E33-18 & $\begin{array}{l}7 / 20 / 93 \\
8 / 19 / 93 \\
9 / 21 / 93\end{array}$ & $\begin{array}{l}249.74 \\
249.78 \\
249.89\end{array}$ & $\begin{array}{l}402.12 \\
402.08 \\
401.97\end{array}$ \\
\hline $299-E 33-21$ & $\begin{array}{l}7 / 20 / 93 \\
8 / 19 / 93 \\
9 / 21 / 93\end{array}$ & $\begin{array}{l}266.28 \\
266.31 \\
266.48\end{array}$ & $\begin{array}{l}401.85 \\
401.82 \\
401.65\end{array}$ \\
\hline $299-E 33-24$ & $\begin{array}{l}7 / 20 / 93 \\
8 / 19 / 93\end{array}$ & $\begin{array}{l}236.33 \\
236.34\end{array}$ & $\begin{array}{l}401.64 \\
401.63\end{array}$ \\
\hline 299-E33-31 & $\begin{array}{l}7 / 20 / 93 \\
8 / 19 / 93 \\
9 / 21 / 93\end{array}$ & $\begin{array}{l}245.46 \\
245.50 \\
245.64\end{array}$ & $\begin{array}{l}401.82 \\
401.78 \\
401.64\end{array}$ \\
\hline 299-E33-32 & $\begin{array}{l}7 / 20 / 93 \\
8 / 19 / 93 \\
9 / 21 / 93\end{array}$ & $\begin{array}{l}258.10 \\
258.17 \\
258.24\end{array}$ & $\begin{array}{l}401.73 \\
401.66 \\
401.59\end{array}$ \\
\hline 299-E33-33 & $\begin{array}{l}7 / 20 / 93 \\
8 / 19 / 93 \\
9 / 21 / 93\end{array}$ & $\begin{array}{l}238.38 \\
238.34 \\
238.53\end{array}$ & $\begin{array}{l}401.79 \\
401.83 \\
401.64\end{array}$ \\
\hline $299-E 33-36$ & $9 / 07 / 93$ & 246.33 & 400.34 \\
\hline $299-E 33-38$ & $9 / 01 / 93$ & 230.16 & 401.79 \\
\hline 299-E33-39 & $9 / 01 / 93$ & 221.67 & 401.65 \\
\hline $299-E 33-41$ & $\begin{array}{l}7 / 20 / 93 \\
8 / 20 / 93 \\
9 / 21 / 93\end{array}$ & $\begin{array}{l}252.96 \\
252.95 \\
253.15\end{array}$ & $\begin{array}{l}401.99 \\
402.00 \\
401.80\end{array}$ \\
\hline
\end{tabular}


Table 16-2. RCRA Water Level Measurement Report Single-Shell Tanks, Third Quarter 1993. (sheet 3 of 8 )

\begin{tabular}{|c|c|c|c|}
\hline Well & Date & $\begin{array}{l}\text { Depth to } \\
\text { water (ft) }\end{array}$ & $\begin{array}{l}\text { Water level } \\
\text { elevation } \\
\text { above msl (ft) }\end{array}$ \\
\hline \multicolumn{4}{|c|}{ 241-B/BX/BY Tank Farm Wells } \\
\hline 299-E33-42 & $\begin{array}{l}7 / 20 / 93 \\
8 / 19 / 93 \\
9 / 21 / 93 \\
9 / 21 / 93\end{array}$ & $\begin{array}{l}252.26 \\
252.32 \\
252.39 \\
252.24\end{array}$ & $\begin{array}{l}402.04 \\
401.98 \\
401.91^{\star} \\
402.06\end{array}$ \\
\hline $299-E 33-43$ & $\begin{array}{l}7 / 20 / 93 \\
8 / 19 / 93 \\
9 / 21 / 93\end{array}$ & $\begin{array}{l}260.76 \\
260.93 \\
260.93\end{array}$ & $\begin{array}{l}401.92 \\
401.75 \\
401.75\end{array}$ \\
\hline \multicolumn{4}{|c|}{ 241-C Tank Farm Wells } \\
\hline 299-E27-7 & $\begin{array}{l}7 / 20 / 93 \\
8 / 19 / 93 \\
9 / 21 / 93 \\
9 / 21 / 93\end{array}$ & $\begin{array}{l}232.45 \\
232.52 \\
232.80 \\
232.64\end{array}$ & $\begin{array}{l}402.22 \\
402.15 \\
401.87 \\
402.03^{*}+\end{array}$ \\
\hline 299-E27-12 & $\begin{array}{l}7 / 20 / 93 \\
8 / 19 / 93 \\
9 / 21 / 93\end{array}$ & $\begin{array}{l}259.03 \\
259.02 \\
257.25\end{array}$ & $\begin{array}{l}401.92 \\
401.93 \\
403.70+\end{array}$ \\
\hline $299-E 27-13$ & $\begin{array}{l}7 / 20 / 93 \\
8 / 19 / 93 \\
9 / 21 / 93\end{array}$ & $\begin{array}{l}267.86 \\
266.92 \\
267.08\end{array}$ & $\begin{array}{l}401.13+ \\
402.07 \\
401.91+\end{array}$ \\
\hline $299-E 27-14$ & $\begin{array}{l}7 / 20 / 93 \\
8 / 19 / 93 \\
9 / 21 / 93\end{array}$ & $\begin{array}{l}256.40 \\
256.40 \\
257.48\end{array}$ & $\begin{array}{l}401.94 \\
401.94 \\
400.86\end{array}$ \\
\hline $299-E 27-15$ & $\begin{array}{l}7 / 20 / 93 \\
8 / 19 / 93 \\
9 / 21 / 93\end{array}$ & $\begin{array}{l}251.56 \\
251.67 \\
251.33\end{array}$ & $\begin{array}{l}401.11 \\
401.00 \\
401.34\end{array}$ \\
\hline
\end{tabular}


Table 16-2. RCRA Water Level Measurement Report Single-Shell Tanks, Third Quarter 1993.

(sheet 4 of 8 )

\begin{tabular}{|c|c|c|c|}
\hline Well & Date & $\begin{array}{l}\text { Depth to } \\
\text { water (ft) }\end{array}$ & $\begin{array}{l}\text { Water level } \\
\text { elevation } \\
\text { above mst ( } f t)\end{array}$ \\
\hline \multicolumn{4}{|c|}{ 241-S/SX Tank Farm Wells } \\
\hline $299-W 22-39$ & $\begin{array}{l}7 / 22 / 93 \\
8 / 19 / 93 \\
9 / 21 / 93\end{array}$ & $\begin{array}{l}209.58 \\
209.54 \\
209.91\end{array}$ & $\begin{array}{l}458.68 \\
458.72 \\
458.35\end{array}$ \\
\hline 299-W22-44 & $\begin{array}{l}7 / 22 / 93 \\
8 / 20 / 93 \\
9 / 21 / 93 \\
9 / 29 / 93\end{array}$ & $\begin{array}{l}217.10 \\
217.17 \\
217.52 \\
217.42\end{array}$ & $\begin{array}{l}461.03 \\
460.96 \\
460.61 \\
460.71^{\star}\end{array}$ \\
\hline 299-W22-45 & $\begin{array}{l}7 / 21 / 93 \\
8 / 20 / 93 \\
9 / 21 / 93 \\
9 / 29 / 93\end{array}$ & $\begin{array}{l}206.70 \\
206.81 \\
207.12 \\
206.95\end{array}$ & $\begin{array}{l}459.51 \\
459.40 \\
459.09 \\
459.26^{\star}\end{array}$ \\
\hline $299-W 22-46$ & $\begin{array}{l}7 / 22 / 93 \\
8 / 19 / 93 \\
9 / 21 / 93\end{array}$ & $\begin{array}{l}212.67 \\
212.67 \\
213.09\end{array}$ & $\begin{array}{l}458.51 \\
458.51 \\
458.09\end{array}$ \\
\hline 299-W23-1 & $7 / 21 / 93$ & 204.89 & 461.25 \\
\hline 299-W23-2 & $7 / 21 / 93$ & 203.64 & 459.84 \\
\hline $299-W 23-3$ & $7 / 21 / 93$ & 204.62 & 458.94 \\
\hline $299-W 23-6$ & $\begin{array}{l}7 / 21 / 93 \\
8 / 20 / 93 \\
9 / 21 / 93\end{array}$ & $\begin{array}{l}208.21 \\
208.28 \\
208.58\end{array}$ & $\begin{array}{l}458.55 \\
458.48 \\
458.18\end{array}$ \\
\hline 299-W23-7 & $7 / 21 / 93$ & 203.35 & 460.32 \\
\hline 299-W23-8 & $\begin{array}{l}7 / 21 / 93 \\
8 / 19 / 93 \\
9 / 21 / 93\end{array}$ & $\begin{array}{l}203.09 \\
203.05 \\
203.48\end{array}$ & $\begin{array}{l}460.86 \\
460.90 \\
460.47\end{array}$ \\
\hline $299-W 23-12$ & $7 / 21 / 93$ & 205.26 & 460.89 \\
\hline $299-W 23-13$ & $\begin{array}{l}7 / 21 / 93 \\
8 / 19 / 93 \\
9 / 21 / 93\end{array}$ & $\begin{array}{l}204.79 \\
204.83 \\
205.25\end{array}$ & $\begin{array}{l}461.54 \\
461.50 \\
461.08\end{array}$ \\
\hline
\end{tabular}


Table 16-2. RCRA Water Level Measurement Report Single-Shell Tanks, Third Quarter 1993.

(sheet 5 of 8 )

\begin{tabular}{|c|c|c|c|}
\hline We11 & Date & $\begin{array}{l}\text { Depth to } \\
\text { water }(f t)\end{array}$ & $\begin{array}{l}\text { Water level } \\
\text { elevation } \\
\text { above mst (ft) }\end{array}$ \\
\hline \multicolumn{4}{|c|}{ 241-S/SX Tank Farm Wells } \\
\hline 299-W23-14 & $\begin{array}{l}7 / 21 / 93 \\
8 / 19 / 93 \\
9 / 21 / 93\end{array}$ & $\begin{array}{l}203.29 \\
203.26 \\
203.70\end{array}$ & $\begin{array}{l}460.71 \\
460.74 \\
460.30\end{array}$ \\
\hline $299-W 23-15$ & $\begin{array}{l}7 / 21 / 93 \\
8 / 19 / 93 \\
9 / 21 / 93\end{array}$ & $\begin{array}{l}196.06 \\
196.04 \\
196.38\end{array}$ & $\begin{array}{l}459.38 \\
459.40 \\
459.06\end{array}$ \\
\hline \multicolumn{4}{|c|}{ 241-T Tank Farm Wells } \\
\hline $299-W 10-3$ & $7 / 21 / 93$ & 209.59 & 461.87 \\
\hline 299-W10-8 & $\begin{array}{l}7 / 21 / 93 \\
8 / 19 / 93 \\
9 / 21 / 93\end{array}$ & $\begin{array}{l}218.73 \\
218.91 \\
219.25\end{array}$ & $\begin{array}{l}461.32 \\
461.14 \\
460.80\end{array}$ \\
\hline 299-W10-9 & $\begin{array}{l}7 / 21 / 93 \\
8 / 19 / 93 \\
9 / 21 / 93\end{array}$ & $\begin{array}{l}212.94 \\
213.09 \\
213.45\end{array}$ & $\begin{array}{l}461.73 \\
461.58 \\
461.22\end{array}$ \\
\hline $299-W 10-10$ & $\begin{array}{l}7 / 21 / 93 \\
8 / 19 / 93 \\
9 / 21 / 93\end{array}$ & $\begin{array}{l}213.54 \\
213.70 \\
214.05\end{array}$ & $\begin{array}{l}461.55 \\
461.39 \\
461.04\end{array}$ \\
\hline $299-W 10-11$ & $\begin{array}{l}7 / 22 / 93 \\
8 / 19 / 93 \\
9 / 21 / 93\end{array}$ & $\begin{array}{l}213.40 \\
213.54 \\
213.93\end{array}$ & $\begin{array}{l}461.86 \\
461.72 \\
461.33\end{array}$ \\
\hline $299-W 10-12$ & $\begin{array}{l}7 / 21 / 93 \\
8 / 19 / 93 \\
9 / 21 / 93\end{array}$ & $\begin{array}{l}213.13 \\
213.30 \\
213.65\end{array}$ & $\begin{array}{l}461.62 \\
461.45 \\
461.10\end{array}$ \\
\hline $299-W 10-15$ & $\begin{array}{l}7 / 21 / 93 \\
8 / 19 / 93 \\
9 / 21 / 93 \\
9 / 28 / 93\end{array}$ & $\begin{array}{l}214.14 \\
214.24 \\
214.62 \\
214.91\end{array}$ & $\begin{array}{l}461.50 \\
461.40 \\
461.02 \\
460.73^{\star}\end{array}$ \\
\hline
\end{tabular}


$\mathrm{DOE} / \mathrm{RL}-93-56-3$

Table 16-2. RCRA Water Level Measurement Report Single-Shell Tanks, Third Quarter 1993.

(sheet 6 of 8 )

\begin{tabular}{|c|c|c|c|}
\hline Well & Date & $\begin{array}{l}\text { Depth to } \\
\text { water (ft) }\end{array}$ & $\begin{array}{l}\text { Water level } \\
\text { elevation } \\
\text { above msl (ft) }\end{array}$ \\
\hline \multicolumn{4}{|c|}{ 241-T Tank Farm Wells } \\
\hline $299-W 10-16$ & $\begin{array}{l}7 / 21 / 93 \\
8 / 19 / 93 \\
9 / 21 / 93 \\
9 / 28 / 93\end{array}$ & $\begin{array}{l}210.92 \\
210.98 \\
211.33 \\
211.26\end{array}$ & $\begin{array}{l}461.84 \\
461.78 \\
461.43 \\
461.50 \star\end{array}$ \\
\hline $299-W 11-23$ & $\begin{array}{l}7 / 21 / 93 \\
8 / 19 / 93 \\
9 / 21 / 93\end{array}$ & $\begin{array}{l}227.01 \\
227.10 \\
227.46\end{array}$ & $\begin{array}{l}460.87 \\
460.78 \\
460.42\end{array}$ \\
\hline $299-W 11-24$ & $\begin{array}{l}7 / 21 / 93 \\
8 / 19 / 93 \\
9 / 21 / 93\end{array}$ & $\begin{array}{l}225.86 \\
225.95 \\
226.30\end{array}$ & $\begin{array}{l}461.31 \\
461.22 \\
460.87\end{array}$ \\
\hline $299-W 11-27$ & $\begin{array}{l}7 / 21 / 93 \\
8 / 19 / 93 \\
9 / 21 / 93 \\
9 / 28 / 93\end{array}$ & $\begin{array}{l}223.88 \\
223.99 \\
224.36 \\
224.22\end{array}$ & $\begin{array}{l}461.39 \\
461.28 \\
460.91 \\
461.05^{\star}\end{array}$ \\
\hline
\end{tabular}

241-TX/TY Tank Farm Wells

\begin{tabular}{llll}
\hline $299-W 10-17$ & $7 / 21 / 93$ & 208.57 & 462.27 \\
& $8 / 19 / 93$ & 208.70 & 462.14 \\
$9 / 21 / 93$ & 209.12 & 461.72 \\
$9 / 28 / 93$ & 208.87 & $461.97 \star$ \\
$299-W 1 C-18$ & & & \\
& $7 / 21 / 93$ & 207.94 & 462.99 \\
& $8 / 19 / 93$ & 208.08 & 462.85 \\
$299-W 14-12$ & $9 / 21 / 93$ & 208.43 & 462.50 \\
& $9 / 28 / 93$ & 208.32 & $462.61^{\star}$ \\
& $7 / 21 / 93$ & 206.86 & 463.66 \\
& $8 / 19 / 93$ & 206.95 & 463.57 \\
& $9 / 21 / 93$ & 207.31 & 463.21 \\
& $9 / 28 / 93$ & 207.13 & $463.39 \star$
\end{tabular}


Table 16-2. RCRA Water Level Measurement Report Single-Shell Tanks, Third Quarter 1993. (sheet 7 of 8 )

\begin{tabular}{|c|c|c|c|}
\hline Well & Date & $\begin{array}{l}\text { Depth to } \\
\text { water }(f t)\end{array}$ & $\begin{array}{l}\text { Water level } \\
\text { elevat } 7 \\
\text { above msl }(\mathrm{ft})\end{array}$ \\
\hline \multicolumn{4}{|c|}{ 241-TX/TY Tank Farm Wells } \\
\hline $299-W 15-3$ & $7 / 21 / 93$ & 207.40 & 463.10 \\
\hline 299-W15-12 & $\begin{array}{l}7 / 21 / 93 \\
8 / 19 / 93 \\
9 / 21 / 93\end{array}$ & $\begin{array}{l}206.89 \\
206.99 \\
207.37\end{array}$ & $\begin{array}{l}463.18 \\
463.08 \\
462.70\end{array}$ \\
\hline $299-W 15-13$ & $\begin{array}{l}7 / 21 / 93 \\
8 / 19 / 93 \\
9 / 21 / 93\end{array}$ & $\begin{array}{l}206.99 \\
207.08 \\
207.48\end{array}$ & $\begin{array}{l}463.13 \\
463.04 \\
462.64\end{array}$ \\
\hline $299-W 15-22$ & $\begin{array}{l}7 / 21 / 93 \\
8 / 19 / 93 \\
9 / 21 / 93 \\
9 / 28 / 93\end{array}$ & $\begin{array}{l}205.89 \\
205.94 \\
206.28 \\
206.07\end{array}$ & $\begin{array}{l}464.88 \\
464.83 \\
464.49 \\
464.70 \star\end{array}$ \\
\hline \multicolumn{4}{|c|}{ 241-U Tank Farm Wells } \\
\hline $299-W 18-25$ & $\begin{array}{l}7 / 21 / 93 \\
8 / 19 / 93 \\
9 / 21 / 93\end{array}$ & $\begin{array}{l}200.76 \\
200.85 \\
201.32\end{array}$ & $\begin{array}{l}465.28 \\
465.19 \\
464.72\end{array}$ \\
\hline $299-W 18-30$ & $\begin{array}{l}7 / 21 / 93 \\
8 / 19 / 93 \\
9 / 20 / 93 \\
9 / 21 / 93\end{array}$ & $\begin{array}{l}207.57 \\
207.67 \\
207.00 \\
208.11\end{array}$ & $\begin{array}{l}465.27 \\
465.17 \\
465.84^{\star} \\
464.73\end{array}$ \\
\hline $299-W 18-31$ & $\begin{array}{l}7 / 21 / 93 \\
8 / 19 / 93 \\
9 / 20 / 93 \\
9 / 21 / 93\end{array}$ & $\begin{array}{l}198.89 \\
198.98 \\
199.61 \\
199.45\end{array}$ & $\begin{array}{l}465.27 \\
465.18 \\
464.55^{\star} \\
464.71\end{array}$ \\
\hline 299-W19-12 & $\begin{array}{l}7 / 21 / 93 \\
8 / 19 / 93 \\
9 / 21 / 93\end{array}$ & $\begin{array}{l}208.00 \\
207.96 \\
208.44\end{array}$ & $\begin{array}{l}465.25 \\
465.29 \\
464.81\end{array}$ \\
\hline
\end{tabular}


Table 16-2. RCRA Water Level Measurement Report Single-Shell Tanks, Third Quarter 1993.

(sheet 8 of 8 )

\begin{tabular}{llll}
\hline & \multicolumn{2}{c}{ 241-U Tank Farm Wells } & \\
\hline $299-W 19-31$ & $7 / 21 / 93$ & 208.95 & 465.24 \\
& $8 / 19 / 93$ & 209.03 & 465.16 \\
& $9 / 21 / 93$ & 209.45 & 464.74 \\
$299-W 19-32$ & $7 / 20 / 93$ & 209.26 & $465.64^{\star}$ \\
& $7 / 21 / 93$ & 209.29 & 465.61 \\
& $8 / 19 / 93$ & 209.36 & 465.54 \\
& $9 / 21 / 93$ & 209.88 & 465.02 \\
\hline
\end{tabular}

Notes: 1. Water level elevations are calculated by subtracting the measured depth to water from the surveyed elevation for the well.

2. Depth-to-water values are transcribed from field records.

3. Elevations marked with an $1 * 1$ were measured at the time of sampling.

4. Elevations marked with a ' + ' are outside of the expected range, and are suspected of error.

5. To convert feet to meters multiply by 0.3048 . 
Table 16-3. Constituent List and Summary of Results for the Single-Shell Tank Waste Management Area A-AX Data for Reporting Period July 1 through September 30, 1993. (sheet 1 of 6)

CONTAMINATION INDICATOR PARAMETERS

\begin{tabular}{|c|c|c|c|c|c|c|c|c|c|}
\hline \multicolumn{4}{|c|}{ Const ituent Name } & \multicolumn{3}{|c|}{ DUS } & \multicolumn{3}{|c|}{ Number of Samples } \\
\hline Short & (Method) & Full & Units & CROL & Limit & Agency & Total & $>$ CROL & $>$ DUS \\
\hline$\cdots \ldots$. & $\ldots \ldots \ldots$ & $\ldots \ldots \ldots$ & $\ldots \ldots$ & $\ldots \ldots$ & $\ldots \ldots$ & $\ldots \ldots$ & $\ldots$. & $-\ldots$ & .... \\
\hline CONDUCT & 94 & Conductivity, field & $\mu \operatorname{mho}$ & 1 & & & 24 & 24 & \\
\hline CONDUCT & 73 & Conductivity, lab & unho & & & & 9 & 9 & \\
\hline TOC & & Total Organic Carbon & ppb & 1000 & & & 24 & 0 & \\
\hline TOXLDL & & Total Organic Halogen & ppb & 10 & & & 24 & 4 & \\
\hline PH & 93 & pH, field & & .1 & $6.5-8.5$ & EPAS & 24 & 24 & 0 \\
\hline PH & 125 & pH, lab & & & $6.5-8.5$ & EPAS & 9 & 9 & 0 \\
\hline
\end{tabular}

DRINKING WATER PARAMETERS

\begin{tabular}{|c|c|c|c|c|c|c|c|c|}
\hline \multicolumn{3}{|c|}{ Constituent Nane } & \multicolumn{3}{|c|}{ DUS } & \multicolumn{3}{|c|}{ Number of Semples } \\
\hline (Method) & Full & Units & CRQL & Limit & Agency & Total & $>C R Q L$ & $>$ DUS \\
\hline 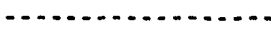 & - & $\ldots \ldots$ & $\ldots \ldots$ & $\ldots . .$. & $\ldots \ldots$ & $\ldots$. & $\ldots$ & $\ldots$ \\
\hline $2,4,5 T P$ & $2,4,5-T P$ & ppb & 2 & 10 & EPA & 1 & 0 & 0 \\
\hline $2,4-0$ & $2,4-0$ & ppb & 10 & 100 & EPA & 1 & 0 & 0 \\
\hline BARIUM & Berium & ppb & 20 & 1000 & EPA & 1 & 1 & 0 \\
\hline FBARIUM & Berium, filtered & ppb & 20 & 1000 & EPA & 1 & 1 & 0 \\
\hline CADMIUM & Cacmium & ppo & 10 & 10 & EPA & 1 & 0 & 0 \\
\hline FCADMIU & Cadnium, filtered & ppb & 10 & 10 & EPA & 1 & 0 & 0 \\
\hline CHROMUM & Chromium & ppo & 20 & 50 & EPA & 1 & 1 & 0 \\
\hline FCHROMI & Chromium, filtered & ppb & 20 & 50 & EPA & 1 & 0 & 0 \\
\hline COL IFORM & Coliforms & COL & & 1 & EPA & 1 & 0 & 0 \\
\hline ENDRIN & Endrin & ppb & .1 & .2 & EPA & 6 & 0 & 0 \\
\hline ALPHA & Gross alpha & $\mathrm{pCi} / \mathrm{L}$ & 4 & 15 & EPA & 1 & 1 & 0 \\
\hline BETA & Gross beta & $\mathrm{pCi} / \mathrm{L}$ & 8 & 50 & EPA & 1 & 1 & 0 \\
\hline MERCURY & Mercury & ppob & .2 & 2 & EPA & 1 & 0 & 0 \\
\hline FMERCUR & Mercury, filtered & ppo & .2 & 2 & EPA & 1 & 0 & 0 \\
\hline METHLOR & Methoxychlor & ppb & 2 & 100 & EPA & 6 & 0 & 0 \\
\hline RADIUM & Radium & $\mathrm{pCi} / \mathrm{L}$ & 1 & 5 & EPA & 1 & 0 & 0 \\
\hline SILVER & silver & ppo & 20 & 50 & EPA & 1 & 0 & 0 \\
\hline FSILVER & silver, filtered & ppb & 20 & 50 & EPA & 1 & 0 & 0 \\
\hline TOXAENE & Toxaphene & ppo & 2 & 5 & EPA & 6 & 0 & 0 \\
\hline TURBID & Turbidity & NTU & .1 & 1 & EPA & 1 & 1 & 1 \\
\hline 9-BHC & gamma-BHC (L indene) & ppb & .05 & 4 & EPA & 6 & 0 & 0 \\
\hline
\end{tabular}

GROUHDWATER QUALITY PARAMETERS

\begin{tabular}{|c|c|c|c|c|c|c|c|c|c|}
\hline \multicolumn{4}{|c|}{ Constituent Name } & \multicolumn{3}{|c|}{ DWS } & \multicolumn{3}{|c|}{ Number of Samples } \\
\hline Short & (Method) & full & Units & CRQL & Limit & Agency & Total & $>$ CRQL & $>$ OUS \\
\hline$\ldots \ldots$ & $\ldots \ldots+\cdots$ & - & $\ldots \ldots$ & $\ldots \ldots$ & $\cdots \cdots$ & $\ldots \ldots$ & $\cdots$ & $\cdots \cdots$ & $\cdots$ \\
\hline IRON & & Iron & ppb & 20 & 300 & EPAS & 1 & 1 & 0 \\
\hline FIRON & & Iron, filtered & ppb & 20 & 300 & EPAS & 1 & 1 & 0 \\
\hline MANGESE & & Manganese & ppb & 10 & 50 & EPAS & 1 & 1 & 0 \\
\hline FMANGAN & & Manganese, filtered & ppb & 10 & 50 & EPAS & 1 & 0 & 0 \\
\hline LPHENOL & 19 & Phenol & ppb & 10 & & & 1 & 0 & \\
\hline LPHENOL & 30 & Phenol & ppb & 20 & & & 1 & 0 & \\
\hline SODIUM & & Sodium & ppb & 300 & & & 1 & 1 & \\
\hline FSOOIUM & & Sodium, filtered & ppb & 300 & & & 1 & 1 & \\
\hline
\end{tabular}


Table 16-3. Constituent List and Summary of Results for the Single-Shell Tank Waste Management Area A-AX Data for Reporting Period July 1 through September 30, 1993. (sheet 2 of 6)

SITE SPECIFIC AND OTHER CONSTITUENTS

\begin{tabular}{|c|c|c|c|c|c|c|c|c|}
\hline \multirow{2}{*}{ Short } & & \multirow{2}{*}{$\begin{array}{l}\text { Constituent Nane Full } \\
\text { Ful }\end{array}$} & \multirow[b]{2}{*}{ Units } & \multicolumn{2}{|r|}{ Dus } & \multicolumn{3}{|c|}{ Number of Semples } \\
\hline & (Method) & & & CRQL & Limit Agency & Total & & \\
\hline $1112-t c$ & & 1,1,1,2-Tetrachloroe thane & ppb & 5 & & 1 & 0 & \\
\hline $1,1,1-T$ & & $1,1,1$-Trichloroethane & ppb & 5 & & 1 & 0 & \\
\hline $1122-t c$ & & $1,1,2,2$-Tetrachloroe thane & ppob & 5 & & 1 & 0 & \\
\hline $1,1,2-1$ & & 1,1,2-Trichloroethane & pot & 5 & & 1 & 0 & \\
\hline $1,1-01 C$ & & 1,1-Dichloroethane & pob & 5 & & 1 & 0 & \\
\hline DICETHY & & 1,1-Dichloroethene & ppob & 5 & & 1 & 0 & \\
\hline $123-\operatorname{trp}$ & & 1,2,3-Trichloropropane & ppob & 5 & & 1 & 0 & \\
\hline TETRCHO & & $1,2,4,5$ - Tetrachlorobenzene & ppob & 10 & & 1 & 0 & \\
\hline TRICHLB & & 1,2,4-Trichlorobenzene & ppob & 10 & & 1 & 0 & \\
\hline DIBRCHL & & 1,2-Dibrjmo-3-chloropropane & ppb & 5 & & 1 & 0 & \\
\hline DIBRETH & & 1,2-0 ibromoethane & ppob & 5 & & 1 & 0 & \\
\hline 12-cben & & 1,2-Dichlorobenzene & ppob & 10 & & 1 & 0 & \\
\hline $1,2-01 C$ & & 1,2-Dichloroethane & ppo & 5 & & 1 & 0 & \\
\hline 12-DCE & & 1,2-Dichloroethene & ppo & 5 & & 1 & 0 & \\
\hline DICPANE & & 1,2-Dichloropropane & ppb & 5 & & 1 & 0 & \\
\hline 13-aben & & 1,3-Dichlorobenzene & ppb & 10 & & 1 & 0 & \\
\hline 16-dben & & 1,4-Dichlorabenzene & ppob & 10 & & 1 & 0 & \\
\hline DIOXANE & & 1,4-Dioxane & ppob & 200 & & 1 & 0 & \\
\hline MAPHQUI & & 1,4-Maphtoquinone & ppb & 10 & & 1 & 0 & \\
\hline 1BUTYN & & 1-Butanol & ppob & 1000 & & 1 & 0 & \\
\hline 1-naphe & & 1-Naphthyl anine & ppob & 10 & & 1 & 0 & \\
\hline TETRCHP & & 2,3,4,6-Tetrachlorophenol & ppo & 10 & & 1 & 0 & \\
\hline 2378TCD & & $2,3,7,8-T C D D$ & ppob & .005 & & 1 & 0 & \\
\hline $2,4,5-T$ & & $2,4,5-T$ & ppb & 2 & & 1 & 0 & \\
\hline $245-\operatorname{trp}$ & & 2,4,5-Trichlorophenol & ppo & 10 & & 1 & 0 & \\
\hline 246-trp & 19 & 2,4,6-Trichlorophenol & ppo & 10 & & 1 & 0 & \\
\hline $246-\operatorname{trp}$ & 30 & 2,4,6-Trichlor ophenol & ppob & 5 & & 1 & 0 & \\
\hline $24-$ dchp & 19 & 2,4-0 ichlorophenol & ppo & 10 & & 1 & 0 & \\
\hline $24-d c h p$ & 30 & 2,4-D ichlorophenol & ppob & 5 & & 1 & 0 & \\
\hline DIMPHEN & 19 & 2,4-D ime thylphenol & ppb & 10 & & 1 & 0 & \\
\hline DIMPHEN & 30 & 2,4-D imethylphenol & ppb & 5 & & 1 & 0 & \\
\hline DINPHEN & 19 & 2,4-D initrophenol & ppo & 50 & & 1 & 0 & \\
\hline DINPHEN & 30 & 2,4-Dinitrophenol & ppb & 150 & & $i$ & 0 & \\
\hline 24 -dint & & 2,4-D initrotolvene & ppb & 10 & & 1 & 0 & \\
\hline 26-dchp & 19 & 2,6-Dichlorophenol & ppb & 10 & & 1 & 0 & \\
\hline 26-dchp & 30 & 2,6-D ichlorophenol & ppb & 5 & & 1 & 0 & \\
\hline 26-dint & & 2,6-0 initrotoluene & ppb & 10 & & 1 & 0 & \\
\hline ACEFENE & & 2-Acetyleminof luorene & ppo & 10 & & 1 & 0 & \\
\hline CHLNAPH & & 2-Chloronaphthal ene & ppb & 10 & & 1 & 0 & \\
\hline CHLPHEN & 19 & 2-Chlorophenol & ppo & 10 & & 1 & 0 & \\
\hline CHLPHEN & 30 & 2-Chlorophenol & ppb & 5 & & $i$ & 0 & \\
\hline 2HEXANO & & 2-Hexanone & ppb & 50 & & 1 & 0 & \\
\hline 2MENAPH & & 2-Methylnaphthalene & ppo & 10 & & 1 & 0 & \\
\hline 2ME THPH & & 2-Methylphenol & ppo & 10 & & 1 & 0 & \\
\hline 2-napha & & 2-Naphthylamine & ppo & 10 & & 1 & 0 & \\
\hline ONITANI & & 2-Nitroaniline & ppb & 50 & & 1 & 0 & \\
\hline 2NITPH & 19 & 2-Nitrophenol & ppb & 10 & & 1 & 0 & \\
\hline 2NITPH & 30 & 2-Nitrophenol & ppb & 5 & & 1 & 0 & \\
\hline PICOLIN & & 2-Picoline & ppb & 10 & & 1 & 0 & \\
\hline BUTDINP & 30 & nitrophenol CON & ppob & 5 & & 1 & 0 & \\
\hline BUTDINP & 49 & -sec-Butyl-4,6-dinitrophenol (ON & ppb & 1 & & 1 & 0 & \\
\hline
\end{tabular}


Table 16-3. Constituent List and Summary of Results for the Single-Shell Tank Waste Management Area A-AX Data for Reporting Period July 1 through September 30, 1993. (sheet 3 of 6 )

\begin{tabular}{|c|c|c|c|c|c|c|c|c|}
\hline \multirow{2}{*}{ Short } & \multirow[b]{2}{*}{ (Method) } & \multirow{2}{*}{$\begin{array}{l}\text { Constituent Nane Full } \\
\text { Ful }\end{array}$} & \multirow[b]{2}{*}{ Units } & \multirow{2}{*}{$\begin{array}{l}\text { Lab } \\
\text { CRQL }\end{array}$} & DWS & \multicolumn{3}{|c|}{ Number of Semples } \\
\hline & & & & & Limit Agency & Total & $>C R Q L$ & $L \quad>$ DWS \\
\hline & & (a) & $\cdots$ & $\cdots$ & (n) & $\cdots \cdot$ & $\cdots$ & $\cdots$ \\
\hline DICHBEN & & 3,3'-Dichlorobenzidine & ppb & 20 & & 1 & 0 & \\
\hline DIMEYLB & & 3,3'-D imethylbenz idine & ppb & 10 & & 1 & 0 & \\
\hline METCHAN & & 3-Methylchol anthrene & ppb & 10 & & 1 & 0 & \\
\hline MNITANI & & 3-Nitroeniline & ppb & 50 & & 1 & 0 & \\
\hline DDD & & $4,4^{\prime}-D D D$ & ppb & .1 & & 6 & 0 & \\
\hline DDE & & $4,4^{\prime}-D D E$ & ppb & .05 & & 6 & 0 & \\
\hline DDT & & $4,4^{\prime}-00 T$ & ppb & .1 & & 6 & 0 & \\
\hline 460N2MP & 19 & 4,6-0 ini tro-2-methylphenol & ppb & 50 & & 1 & 0 & \\
\hline 460N2MP & 30 & 4,6-D initro-2-methylphenol & ppb & 200 & & 1 & 0 & \\
\hline AMINOYL & & 4-Aminobiphenyl & ppb & 10 & & 1 & 0 & \\
\hline BROPHEN & & 4-Bromophenylphenyl ether & ppb & 10 & & 1 & 0 & \\
\hline CHLCRES & 19 & 4-Chloro-3-methylphenol & ppb & 20 & & 1 & 0 & \\
\hline CHLCRES & 30 & 4-chloro-3-methylphenol & ppb & 5 & & 1 & 0 & \\
\hline CHLANIL & & 4-Chloroaniline & ppb & 20 & & 1 & 0 & \\
\hline 4CPPETH & & 4-Chlorophenylphenyl ether & ppb & 10 & & 1 & 0 & \\
\hline MIBK & & 4-Methyl-2-pentanone & ppb & 50 & & 1 & 0 & \\
\hline 4METHPH & & 4-Methylphenol & ppb & 10 & & 1 & 0 & \\
\hline NITRANI & & 4-Witroenil ine & $p p b$ & 50 & & 1 & 0 & \\
\hline NITPHEN & 19 & 4-Nitrophenol & ppb & 50 & & 1 & 0 & \\
\hline NITPHEN & 30 & 4-Nitrophenol & ppb & 30 & & 1 & 0 & \\
\hline 4NITQUI & & 4-Nitroquinol ine-1-oxide & ppb & 10 & & 1 & 0 & \\
\hline NITRTOL & & 5-Nitro-0-toluidine & ppb & 10 & & 1 & 0 & \\
\hline DIMBENZ & & 7, 12-D imethylbenz [a] anthracene & ppb & 10 & & 1 & 0 & \\
\hline ACENAPH & & Acenaph thene & ppb & 10 & & 1 & 0 & \\
\hline ACENATL & & Acenaph thylene & ppb & 10 & & 1 & 0 & \\
\hline ACETONE & & Acetone & $\mathrm{ppb}$ & 100 & & 1 & 0 & \\
\hline ACETILE & & Acetonitrile & ppo & 200 & & 1 & 0 & \\
\hline ACETOPH & & Ace tophenone & ppb & 10 & & 1 & 0 & \\
\hline ACROLIN & & Acrolein & ppb & 5 & & 1 & 0 & \\
\hline ACRYILE & & Acrylonitrile & ppb & 5 & & 1 & 0 & \\
\hline ALDRIN & & Aldrin & ppob & .05 & & 6 & 0 & \\
\hline DIMPHAM & & Alphe, alphe-Dimethylphenethylami & ppob & 10 & & 1 & 0 & \\
\hline Q-BHC & & Alphe-BHC & ppb & .05 & & 6 & 0 & \\
\hline ALUMNUM & & Aluminum & ppb & 200 & & 1 & 0 & \\
\hline FALUMIN & & Aluminum & ppb & 200 & & 1 & 0 & \\
\hline AMMONIU & & Ammonium ion & ppb & 100 & & 1 & 0 & \\
\hline ANILINE & & Aniline & $p p b$ & 10 & & 1 & 0 & \\
\hline ANTHRA & & Anthracene & ppob & 10 & & 1 & 0 & \\
\hline ANT IONY & & Ant imony & ppb & 200 & & 1 & 0 & \\
\hline FANTIMO & & Antimony, filtered & ppo & 200 & & 1 & 0 & \\
\hline SB-125 & & Ant imony- 125 & $\mathrm{pCi} / \mathrm{L}$ & & & 1 & 0 & \\
\hline ARAMITE & & Aramite & ppob & 10 & & 1 & 0 & \\
\hline AR1016 & & Aroclor-1016 & ppb & 1 & & 1 & 0 & \\
\hline AR1221 & & Aroclor-1221 & ppb & 1 & & 1 & 0 & \\
\hline AR1232 & & Aroctor-1232 & ppb & 1 & & 1 & 0 & \\
\hline AR1242 & & Aroclor-1242 & ppb & 1 & & 1 & 0 & \\
\hline AR1248 & & Aroctor -1248 & ppb & 1 & & 1 & 0 & \\
\hline AR1254 & & Aroclor-1254 & ppob & 1 & & 1 & 0 & \\
\hline AR1260 & & Aroclor -1260 & ppb & 1 & & 1 & 0 & \\
\hline BENZENE & & Benzene & ppb & 5 & & 1 & 0 & \\
\hline BENZAAN & & Benzo (a) anthracene & ppb & 10 & & 1 & 0 & \\
\hline BENZOPY & & Benzo(a)pyrene & ppb & 10 & & 1 & 0 & \\
\hline BENZBFL & & Benzo(b)fluoranthene & ppb & 10 & & 1 & 0 & \\
\hline BGHIPER & & Benzo(ghi)perylene & ppb & 10 & & 1 & 0 & \\
\hline BNZKFLU & & Benzo(k)fluoranthene & ppb & 10 & & 1 & 0 & \\
\hline BENZOTH & & Benzothiazole & ppb & 10 & & 1 & 0 & \\
\hline
\end{tabular}


Table 16-3. Constituent List and Summary of Results for the Single-Shell Tank Waste Management Area A-AX Data for Reporting Period July 1 through September 30,1993 . (sheet 4 of 6 )

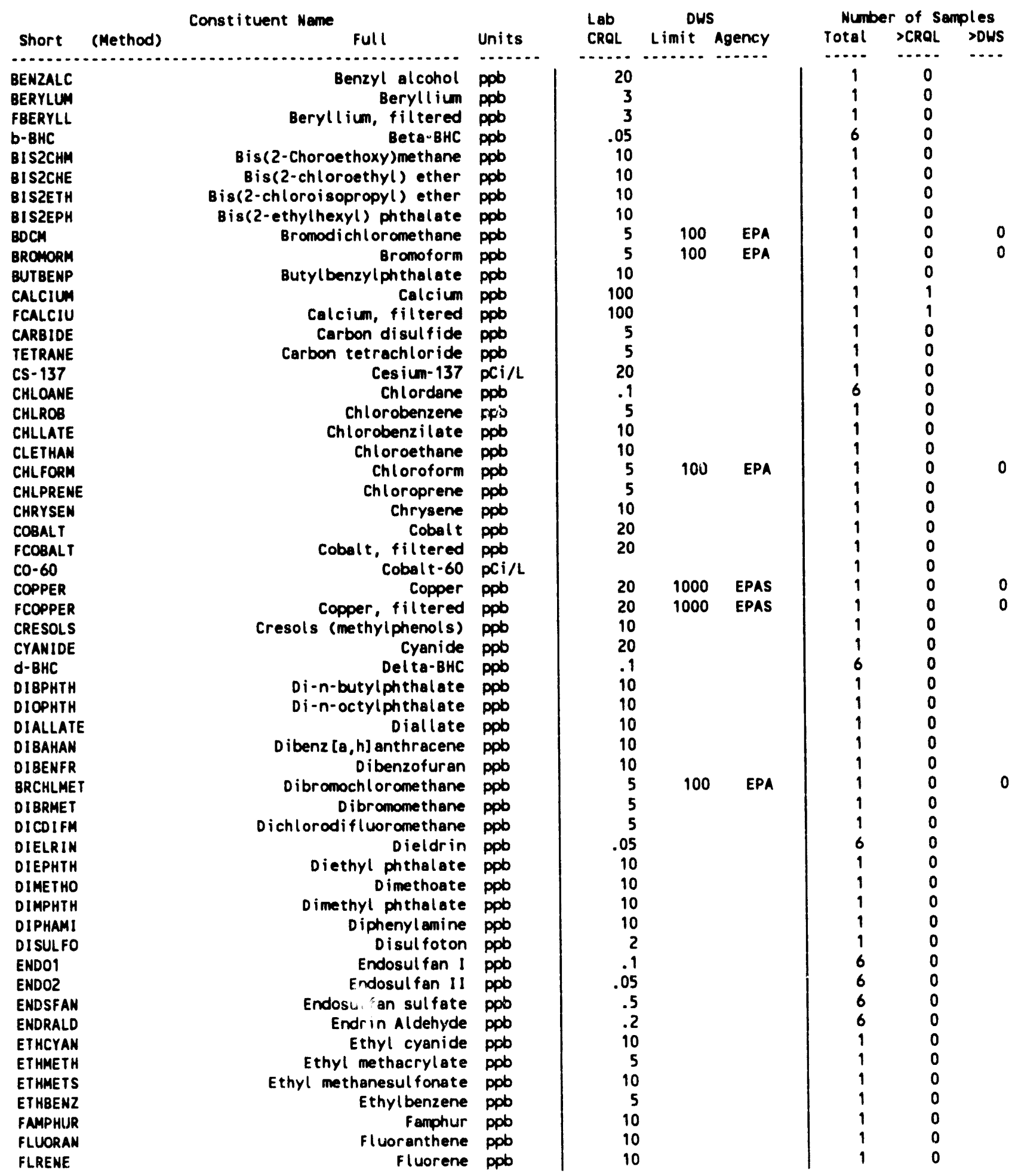


Table 16-3. Constituent List and Summary of Results for the Single-Shell

Tank Waste Management Area A-AX Data for Reporting Period July 1 through September 30, 1993. (sheet 5 of 6)

\begin{tabular}{|c|c|c|c|c|c|c|c|c|c|}
\hline \multicolumn{4}{|c|}{ Constituent Name } & \multicolumn{3}{|c|}{ DWS } & \multicolumn{3}{|c|}{ Number of Semples } \\
\hline Short & (Method) & Full & Units & CRQL & Limit & Agency & Total & $>\mathrm{CROL}$ & $>$ DUS \\
\hline$\ldots \ldots$ & - & - & $\ldots \ldots$ & $\ldots \ldots$ & $\ldots \ldots$ & $\ldots .$. & $\ldots$. & $\ldots$. & -... \\
\hline HEPTLOR & & Heptachlor & ppb & .05 & & & 6 & 0 & \\
\hline MEPTIDE & & Heptachlor epoxide & ppb & 1 & & & 6 & 0 & \\
\hline HEXCBEN & & Hexach lorobenzene & ppb & 10 & & & 1 & 0 & \\
\hline HEXCBUT & & Hexachl orobutadiene & ppo & 10 & & & 1 & 0 & \\
\hline HEXCCYC & & Hexach lor ocycl opentadiene & ppb & 10 & & & 1 & 0 & \\
\hline HEXCETH & & Mexachloroethane & ppb & 10 & & & 1 & 0 & \\
\hline HEXACHL & & Hexach lorophene & ppb & 10 & & & 1 & 0 & \\
\hline HEXAENE & & Hexachl oropropene & ppb & 10 & & & 1 & 0 & \\
\hline LHYDRAZ & & Hydrazine & ppb & 30 & & & 1 & 0 & \\
\hline INDENOP & & Indeno (1,2,3-cd)pyrene & ppo & 10 & & & 1 & 0 & \\
\hline $1-129$ & & lodine-129 & $\mathrm{pCi} / \mathrm{L}$ & 1 & & & 1 & 1 & \\
\hline I SOBUTY & & Isobutyl alcohol & ppb & 200 & & & 1 & 0 & \\
\hline ISOORIN & & Isodrin & ppob & 10 & & & 1 & 0 & \\
\hline ISOPHER & & Isophorone & ppb & 10 & & & 1 & 0 & \\
\hline ISOSOLE & & Isosafrole & ppb & 10 & & & 1 & 0 & \\
\hline KEPONE & & Kepone & ppo & 10 & & & 1 & 0 & \\
\hline KEROSEN & & Kerosene & ppb & 10000 & & & 1 & 0 & \\
\hline MAGNES & & Magnesium & ppb & 100 & & & 1 & 1 & \\
\hline FMAGNES & & Magnesium, filtered & ppb & 100 & & & 1 & 1 & \\
\hline METHACR & & Methacrylonitrile & ppb & 5 & & & 1 & 0 & \\
\hline METHAPY & & Methapyrilene & ppb & 10 & & & 1 & 0 & \\
\hline IODOMET & & Methyl lodide & ppb & 5 & & & 1 & 0 & \\
\hline METHBRO & & Methyl bromide & ppb & 10 & & & 1 & 0 & \\
\hline METHCHL & & Methyi chloride & ppb & 10 & & & 1 & 0 & \\
\hline METHONE & & Methyl ethyl ketone & ppb & 100 & & & 1 & 0 & \\
\hline METACRY & & Methyl methacrylate & ppb & 5 & & & 1 & 0 & \\
\hline METMSUL & & Methyl methanesulfonate & ppb & 10 & & & 1 & 0 & \\
\hline METHPAR & & Methyl parathion & ppb & .5 & & & 1 & 0 & \\
\hline METHYCH & & Methylene chloride & ppb & 5 & & & 1 & 0 & \\
\hline DIPRNIT & & N-Nitroso-di-n-dipropylemine & ppb & 10 & & & 1 & 0 & \\
\hline NNIBUTY & & N-Nitrosodi-n-butylemine & ppb & 10 & & & 1 & 0 & \\
\hline NNIDIEY & & N-Nitrosodiethylamine & ppob & 10 & & & 1 & 0 & \\
\hline NNIDIME & & N-Nitrosodimethylamine & ppb & 10 & & & 1 & 0 & \\
\hline NNDIPHA & & $N$-Nitrosodiphenylamine & ppb & 10 & & & 1 & 0 & \\
\hline NNIMETH & & N-Nitrosomethylethylamine & ppb & 10 & & & 1 & 0 & \\
\hline WNIMORP & & N-Nitrosomorphol ine & ppb & 10 & & & 1 & 0 & \\
\hline NNIPIPE & & N-Nitrosopiperidine & ppb & 10 & & & 1 & 0 & \\
\hline NAPHTHA & & Naphthalene & ppb & 10 & & & 1 & 0 & \\
\hline NICKEL & & Nickel & ppb & 30 & & & 1 & 0 & \\
\hline FNICKEL & & Nickel, filtered & ppb & 30 & & & 1 & 0 & \\
\hline NITBENZ & & Nitrobenzene & ppb & 10 & & & 1 & 0 & \\
\hline NITRPYR & & Nitrosopyrrolidine & ppb & 10 & & & 1 & 0 & \\
\hline TRIPHOS & & $0,0,0$-Triethyl phosphorothioate & ppb & 10 & & & 1 & 0 & \\
\hline DIPHOS & & 0,0-diethyl0-2-pyraz inylphosphor & ppb & 10 & & & 1 & 0 & \\
\hline PCDDS & & PCDDs & ppb & .01 & & & 1 & 1 & \\
\hline PCDFS & & PCDFs & ppb & .01 & & & 1 & 1 & \\
\hline PARATHI & & Parathion & ppb & 10 & & & 1 & 0 & \\
\hline PENTCHB & & Pentachl orobenzene & ppb & 10 & & & 1 & 0 & \\
\hline PENTACH & & Pentachloroethane & ppb & 5 & & & 1 & 0 & \\
\hline PENTCHN & & Pentachloronitrobenzene (PCNB) & ppb & 10 & & & 1 & 0 & \\
\hline PENTCHP & 19 & Pentachlorophenol & ppb & 50 & & & 1 & 0 & \\
\hline PENTCHP & 30 & Pentachlorophenol & ppb & 100 & & & 1 & 0 & \\
\hline PERCHLO & & Perchlorate & ppb & 500 & & & 1 & 0 & \\
\hline PHENTIN & & Phenacetin & ppb & 10 & & & 1 & 0 & \\
\hline PHENANT & & Phenanthrene & ppo & 10 & & & 1 & 0 & \\
\hline PHORATE & & Phorate & ppb & 2 & & & 1 & 0 & \\
\hline
\end{tabular}


Table 16-3. Constituent List and Summary of Results for the Single-Shell Tank Waste Management Area A-AX Data for Reporting Period July 1 through September 30, 1993. (sheet 6 of 6)

\begin{tabular}{|c|c|c|c|c|c|c|c|c|}
\hline \multirow[b]{2}{*}{ Short } & Constituent Name & \multirow[b]{2}{*}{ Units } & \multirow{2}{*}{$\begin{array}{l}\text { Lab } \\
\text { CROL }\end{array}$} & \multicolumn{2}{|l|}{ DWS } & \multicolumn{3}{|c|}{ Number of Samples } \\
\hline & (Method) & & & Limit & Agency & Total & $>C R Q L$ & $>$ DUS \\
\hline 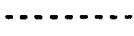 & $\ldots \ldots \ldots \ldots$ & $\ldots \ldots$ & $\ldots .$. & $\ldots \ldots$ & $\ldots \ldots$ & $\cdots$ & $\ldots \ldots$ & $\cdots$ \\
\hline PU-238 & Plutonium-238 & $\mathrm{pCi} / \mathrm{L}$ & .1 & & & 1 & 0 & \\
\hline PU39-40 & Plutonium-239/40 & $\mathrm{pCi} / \mathrm{L}$ & .1 & & & 1 & 0 & \\
\hline POTASUM & Potassium & ppb & 300 & & & 1 & 1 & \\
\hline FPOTASS & Potassiun, filtered & ppo & 300 & & & 1 & 1 & \\
\hline PRONIDE & Pronamide & ppob & 10 & & & 1 & 0 & \\
\hline PYRENE & Pyrene & ppob & 10 & & & 1 & 0 & \\
\hline PYRIDIN & Pyridine & ppob & 10 & & & 1 & 0 & \\
\hline$R U-106$ & Ruthenium-106 & $\mathrm{pCi} / \mathrm{L}$ & & & & 1 & 0 & \\
\hline SAFROL & Safrol & ppo & 10 & & & 1 & 0 & \\
\hline $5 R-90$ & stront ium-90 & $\mathrm{PCi} / \mathrm{L}$ & 5 & 8 & EPA & 1 & 0 & 0 \\
\hline STYRENE & Styrene & ppb & 5 & & & 1 & 0 & \\
\hline TC- 99 & Technet ium-99 & $\mathrm{PCi} / \mathrm{L}$ & 15 & & & 1 & 1 & \\
\hline PERCENE & Tetrachloroethene & ppb & 5 & & & 1 & 0 & \\
\hline TETPHNL & Tetrachlorophenols & ppb & 10 & & & 1 & 0 & \\
\hline PYROPHOS & Tetraethyldithiopyrophosphate & ppob & 10 & & & 1 & 0 & \\
\hline TAF & Tetrahydrofuran & ppb & 10 & & & 1 & 0 & \\
\hline TIN & Tin & ppb & 100 & & & 1 & 0 & \\
\hline FTIN & Tin, filtered & ppb & 100 & & & 1 & 0 & \\
\hline TOLUENE & Tolvene & ppb & 5 & & & 1 & 0 & \\
\hline TC & Total Carbon & ppo & 2000 & & & 6 & 6 & \\
\hline TOS & Totel Dissolved Solids & ppm & 10 & & & 1 & 1 & \\
\hline TRIBUTPH & Tributyl Phosphate & ppb & 10 & & & 1 & 0 & \\
\hline TRICENE & Trichloroethene & ppb & 5 & & & 1 & 0 & \\
\hline TRCAFLM & Trichl oromonof luorome thane & ppb & 5 & & & 1 & 0 & \\
\hline TRIPHNL & Trichlorophenols & ppb & 5 & & & 1 & 0 & \\
\hline TDICHPH & Tris-2-chloroethyl phosphate & ppb & 10 & & & 1 & 0 & \\
\hline TRITIUM & Tritium & $\mathrm{PCi} / \mathrm{L}$ & 500 & 20000 & EPA & 1 & 1 & 0 \\
\hline URANIUM & Uraniun & ppo & .5 & & & 1 & 1 & \\
\hline VANADUM & Vanadium & ppb & 30 & & & 1 & 0 & \\
\hline FVANADI & Vanadium, filtered & ppb & 30 & & & 1 & 0 & \\
\hline VINYLAC & Vinyl acetate & ppb & 5 & & & 1 & 0 & \\
\hline VINYIDE & Vinyl chloride & ppo & 10 & 2 & EPA & 1 & 0 & $0^{*}$ \\
\hline XYLENE & Xylsnes (total) & ppb & 5 & & & 1 & 0 & \\
\hline ZINC & Zinc & ppb & 10 & & & 1 & 0 & \\
\hline FZINC & zinc, filtered & ppb & 10 & & & 1 & 0 & \\
\hline ALLYLCL & allylchloride & ppob & 100 & & & 1 & 0 & \\
\hline TOICPENE & cis-1,3-D ichloropropene & ppob & 5 & & & 1 & 0 & \\
\hline MCRESOL & $m$-Cresol & ppb & 10 & & & 1 & 0 & \\
\hline DINBENZ & m-dinitrobenzene & ppb & 10 & & & 1 & 0 & \\
\hline OTOLHYO & o-Toluidine & ppob & 10 & & & 1 & 0 & \\
\hline DIMEAMB & p-D imethyl aminoazobenzene & ppb & 10 & & & 1 & 0 & \\
\hline PHENDIA & p-Phenylenediamine & ppb & 10 & & & 1 & 0 & \\
\hline SYMTRIN & sym-Trinitrobenzene & ppb & 10 & & & 1 & 0 & \\
\hline DICPENE & trons-1,3-Dichloropropene & ppb & 5 & & & 1 & 0 & \\
\hline TDIBUTEN & trans-1,4-dichloro-2-butene & ppb & 5 & & & 1 & 0 & \\
\hline
\end{tabular}

For explanation of this table, see Section 1.4 of report. 
Table 16-4. Constituents with at Least One Value Above the CRQL for the Single-She 11 Tank Waste Management Area A-AX Data for Reporting Period July 1 through September 30, 1993. (sheet 1 of 2)

\begin{tabular}{|c|c|c|c|c|c|c|}
\hline $\begin{array}{l}\text { Hell } \\
\text { Name }\end{array}$ & $\begin{array}{c}\text { Collection } \\
\text { Date }\end{array}$ & $\begin{array}{l}\text { Semple } \\
\text { Number }\end{array}$ & $\begin{array}{l}\text { BARIUM } \\
34 / \mathrm{PpD} \\
20 / 1000\end{array}$ & $\begin{array}{l}\text { FBARIUM } \\
34 / \text { ppb } \\
20 / 1000\end{array}$ & $\begin{array}{l}\text { CALCIU: } \\
34 / \text { ppb } \\
100 \%\end{array}$ & $\begin{array}{l}\text { FCALCIU } \\
34 / \text { ppb } \\
100 / .\end{array}$ \\
\hline $\begin{array}{l}299-E 25-46 \\
299-E 25-46\end{array}$ & $\begin{array}{l}9 / 29 / 93 \\
9 / 29 / 93\end{array}$ & $\begin{array}{l}8096 \times 5 \\
8096 \times 9\end{array}$ & 25.00 & 25.00 & 28000.00 & 28000.00 \\
\hline
\end{tabular}

\begin{tabular}{|c|c|c|c|c|c|c|}
\hline $\begin{array}{l}\text { Well } \\
\text { Nane }\end{array}$ & $\begin{array}{c}\text { Collection } \\
\text { Date }\end{array}$ & $\begin{array}{l}\text { Sample } \\
\text { Number }\end{array}$ & $\begin{array}{c}\text { CHROMUM } \\
34 / \mathrm{ppb} \\
20 / 50\end{array}$ & $\begin{array}{l}\text { FCHRONI } \\
34 / \text { ppb } \\
20 / 50\end{array}$ & $\begin{array}{c}\text { ALPHA } \\
135 / \mathrm{pCi} / \mathrm{L} \\
. / 15\end{array}$ & $\begin{array}{c}\text { BETA } \\
136 / \mathrm{PCi} / \mathrm{L} \\
. / 50\end{array}$ \\
\hline $\begin{array}{l}299-E 25-46 \\
299-E 25-46\end{array}$ & $\begin{array}{l}9 / 29 / 93 \\
9 / 29 / 93\end{array}$ & $\begin{array}{l}8096 K 5 \\
8096 K 9\end{array}$ & 29.00 & $5.42 \mathrm{U}$ & 2.05 & 26.20 \\
\hline
\end{tabular}

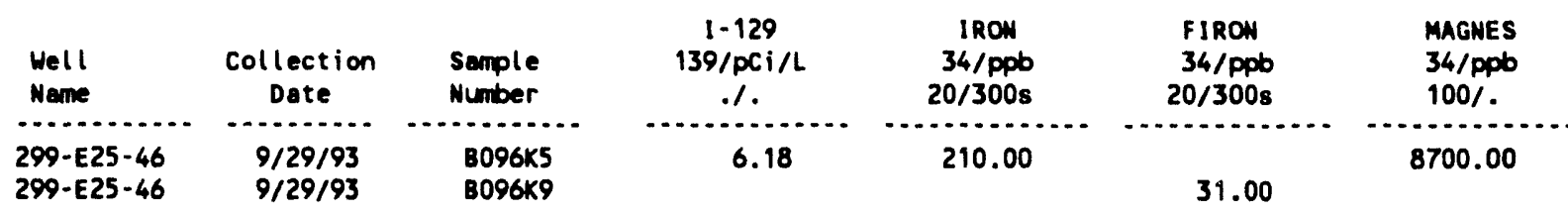

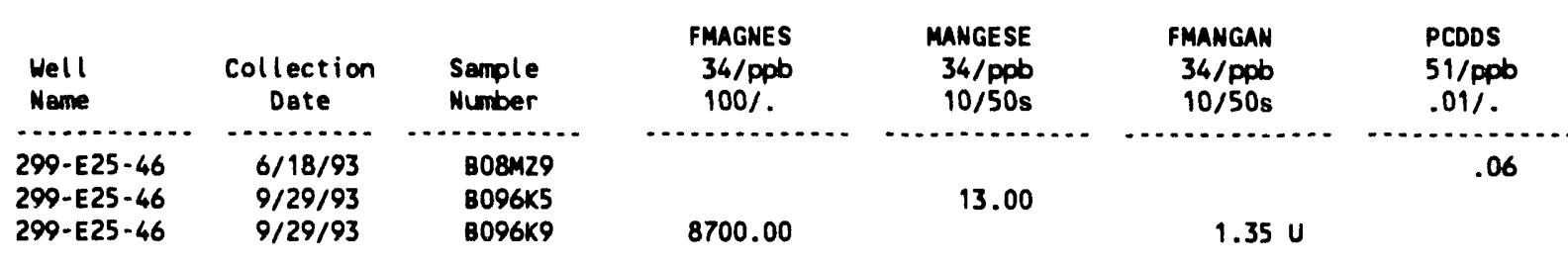

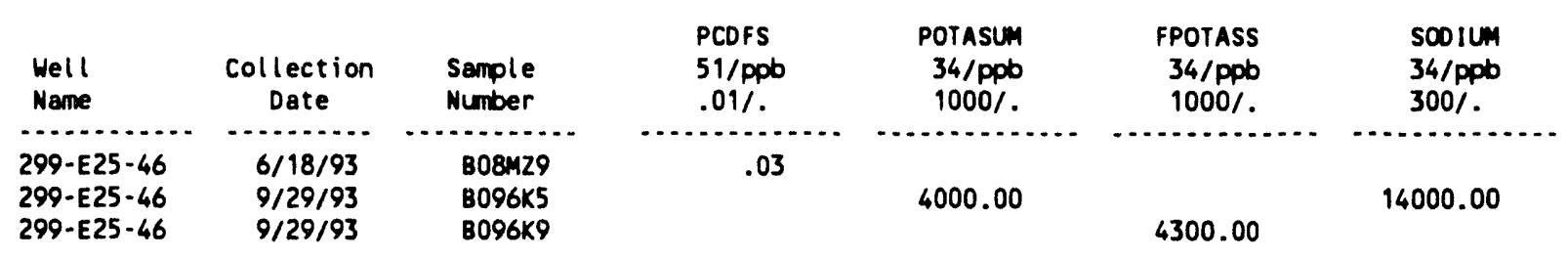

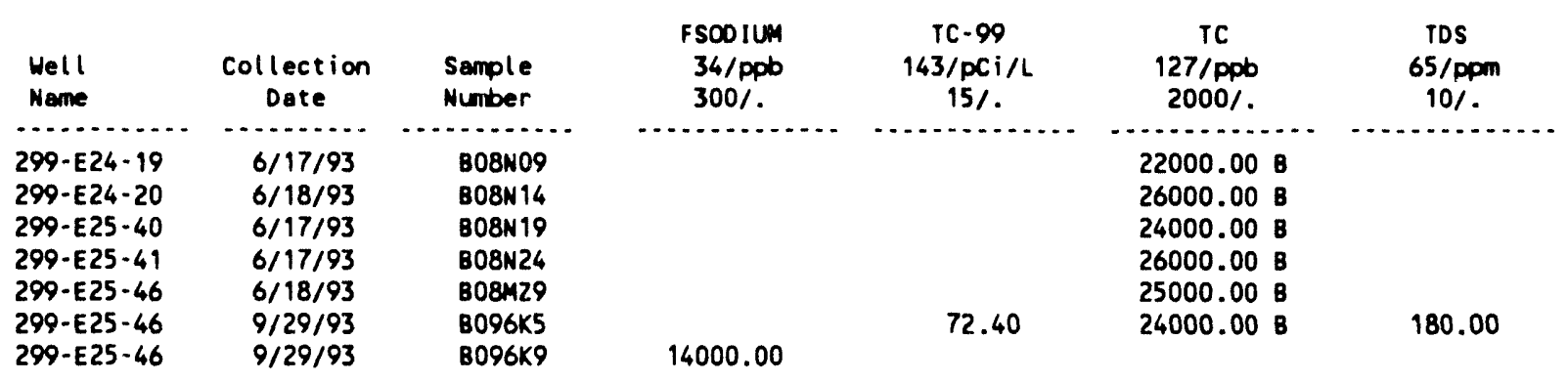


Table 16-4. Constituents with at Least One Value Above the CRQL for the Single-Shell Tank Waste Management Area A-AX Data for Reporting

Period July 1 through September 30, 1993. (sheet 2 of 2)

\begin{tabular}{|c|c|c|c|c|c|}
\hline $\begin{array}{l}\text { Well } \\
\text { Name }\end{array}$ & $\begin{array}{c}\text { Collection } \\
\text { Date }\end{array}$ & $\begin{array}{l}\text { Semple } \\
\text { Number }\end{array}$ & $\begin{array}{l}\text { TRITIUM } \\
\text { 142/pCi/L } \\
. / 20000\end{array}$ & $\begin{array}{c}\text { TURBID } \\
\text { 126/NTU } \\
.1 / 1\end{array}$ & $\begin{array}{l}\text { URANIUM } \\
\text { 145/ppob } \\
.1 .\end{array}$ \\
\hline $299-E 25-46$ & $9 / 29 / 93$ & $8096 \times 5$ & 3350.00 & 2.20 & 2.68 \\
\hline
\end{tabular}

For explanation of this table, see Section 1.4 of report. 
Table 16-5. Contamination Indicator Parameters for the Single-Shell Tank Waste Management Area A-AX Data for Reporting Period July 1 through September 30, 1993.

\begin{tabular}{|c|c|c|c|c|c|c|c|c|}
\hline $\begin{array}{l}\text { Well } \\
\text { Nane }\end{array}$ & $\begin{array}{c}\text { Collection } \\
\text { Date }\end{array}$ & $\begin{array}{l}\text { Sample } \\
\text { Number }\end{array}$ & $\begin{array}{c}\text { COND FIELD } \\
\substack{\mu \mathrm{mho} \\
1 / 700 \mathrm{w}}\end{array}$ & $\begin{array}{c}\text { COND LAB } \\
\mu \text { mho } \\
. / 700 \mathrm{~W}\end{array}$ & $\begin{array}{l}\text { PH FIELD } \\
.01 / 6.5-8.58\end{array}$ & $\begin{array}{c}\text { PH LAB } \\
.01 / 6.5-8.5 \mathrm{~s}\end{array}$ & $\begin{array}{l}\text { TOC } \\
\text { ppb } \\
1000 /\end{array}$ & $\begin{array}{l}\text { rox } \\
\text { ppb } \\
10 \%\end{array}$ \\
\hline 299-E24-19 & $6 / 17 / 93$ & $\begin{array}{l}\text { B08N09 } \\
\text { B08N10 } \\
\text { B08N11 } \\
\text { B08N12 }\end{array}$ & $\begin{array}{l}257 \\
259 \\
259 \\
258\end{array}$ & 260 & $\begin{array}{l}7.17 \\
7.15 \\
7.15 \\
7.15\end{array}$ & 8.00 & $\begin{array}{l}700 \text { LB } \\
600 \text { LB } \\
600 \text { LB } \\
600 \text { LB }\end{array}$ & $\begin{array}{c}8.0 \mathrm{U} \\
10.0 \\
8.0 \mathrm{U} \\
8.0 \mathrm{U}\end{array}$ \\
\hline $299-E 24-20$ & $6 / 18 / 93$ & $\begin{array}{l}\text { B08N14 } \\
\text { B08N15 } \\
\text { B08N16 } \\
\text { B08N17 }\end{array}$ & $\begin{array}{l}381 \\
374 \\
369 \\
371\end{array}$ & 350 & $\begin{array}{l}7.89 \\
7.88 \\
7.89 \\
7.88\end{array}$ & 8.10 & $\begin{array}{l}400 \text { LB } \\
400 \text { LB } \\
400 \text { LB } \\
500 \text { LB }\end{array}$ & $\begin{array}{l}8.0 \text { UP } \\
8.0 \text { UP } \\
8.0 \text { UP } \\
8.0 \text { UP }\end{array}$ \\
\hline $299-E 25-40$ & $6 / 17 / 93$ & $\begin{array}{l}\text { B08N19 } \\
\text { B08N20 } \\
\text { B08N21 } \\
.08 N 22\end{array}$ & $\begin{array}{l}324 \\
321 \\
321 \\
321\end{array}$ & 310 & $\begin{array}{l}7.98 \\
7.98 \\
7.98 \\
7.97\end{array}$ & 8.00 & $\begin{array}{ll}500 & \text { LB } \\
500 & L 8 \\
500 & L B \\
600 & L B\end{array}$ & $\begin{array}{r}8.0 U \\
8.0 U \\
8.0 U \\
10.0\end{array}$ \\
\hline 299-E25-41 & $6 / 17 / 93$ & $\begin{array}{l}\text { B08N24 } \\
\text { B08N25 } \\
\text { BO8N26 } \\
\text { B08N27 }\end{array}$ & $\begin{array}{l}480 \\
472 \\
468 \\
470\end{array}$ & 370 & $\begin{array}{l}7.96 \\
7.96 \\
7.95 \\
7.95\end{array}$ & 8.00 & $\begin{array}{ll}500 & \text { LB } \\
500 & \text { LB } \\
500 & \text { LB } \\
500 & \text { LB }\end{array}$ & $\begin{array}{l}8.0 U \\
8.0 U \\
8.0 U \\
8.0 U\end{array}$ \\
\hline $299-E 25-46$ & $6 / 18 / 93$ & $\begin{array}{l}\text { B08N29 } \\
\text { B08N00 } \\
\text { B08N01 } \\
\text { B08N02 }\end{array}$ & $\begin{array}{l}267 \\
266 \\
269 \\
268\end{array}$ & 260 & $\begin{array}{l}6.92 \\
6.91 \\
6.92 \\
6.92\end{array}$ & 7.70 & $\begin{array}{l}400 \mathrm{LB} \\
500 \mathrm{BL} \\
400 \mathrm{LB} \\
400 \mathrm{LB}\end{array}$ & $\begin{array}{rl}20.0 & \mathrm{P} \\
8.0 & \text { UP } \\
20.0 \mathrm{P} \\
8.0 \text { UP }\end{array}$ \\
\hline & $9 / 29 / 93$ & $\begin{array}{l}\text { B096K5 } \\
8096 K 6 \\
8096 K 7 \\
B 096 K 8\end{array}$ & $\begin{array}{l}277 \\
277 \\
277 \\
278\end{array}$ & $\begin{array}{l}270 \\
270 \\
280 \\
280\end{array}$ & $\begin{array}{l}7.96 \\
7.96 \\
7.96 \\
7.96\end{array}$ & $\begin{array}{l}8.30 \\
8.30 \\
8.30 \\
8.30\end{array}$ & $\begin{array}{l}500 \text { LB } \\
500 \text { LB } \\
500 \text { LB } \\
600 \text { LB }\end{array}$ & $\begin{array}{l}8.0 \text { UP } \\
8.0 \text { UP } \\
8.0 \text { UP } \\
8.0 \text { UP }\end{array}$ \\
\hline
\end{tabular}

For explanation of this table, see Section 1.4 of report. 
Table 16-6. Constituent List and Summary of Results for the Single-Shell Tank Waste Management Area B-BY-BX Data for Reporting Period July 1 through September 30, 1993. (sheet 1 of 3 )

CONTAMINATION INDICATOR PARAMETERS

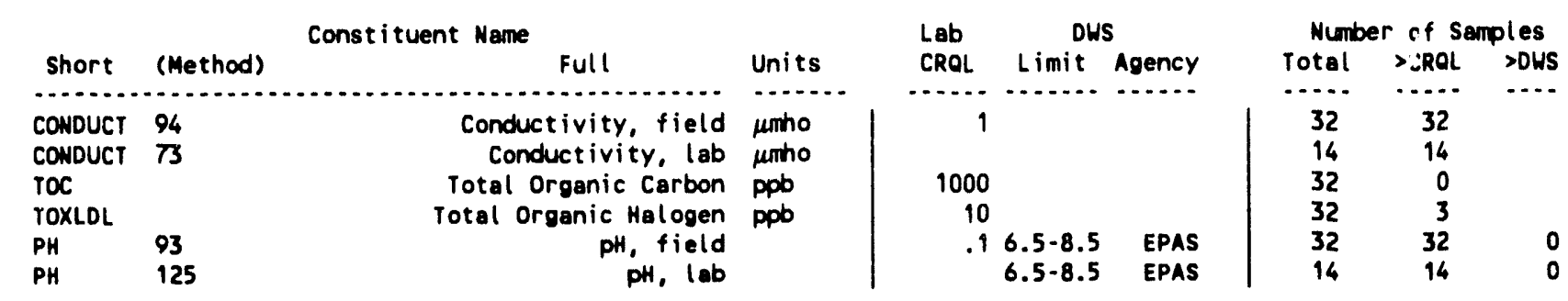

DRINKING WATER PARAMETERS

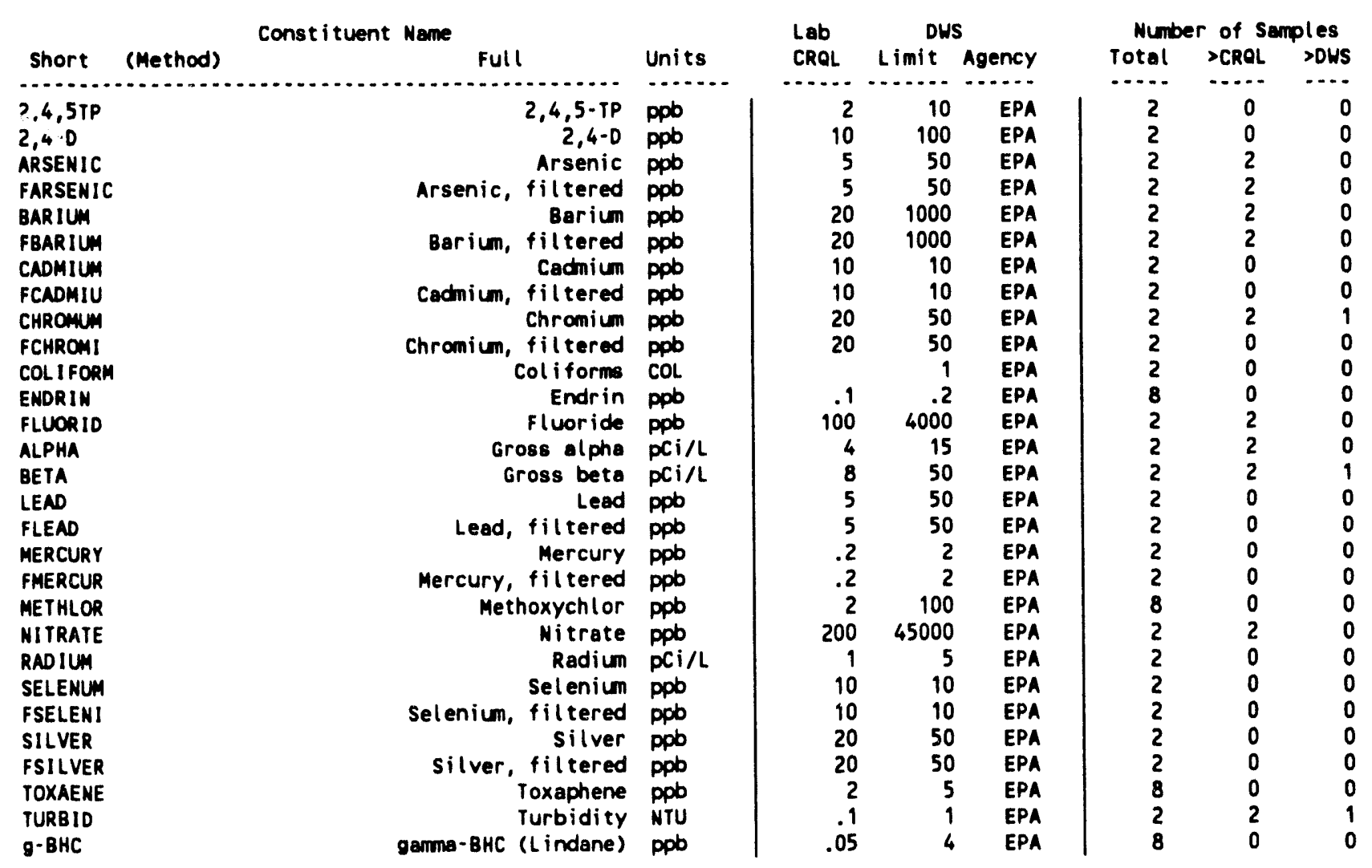

GROUNDWATER QUALITY PARAMETERS

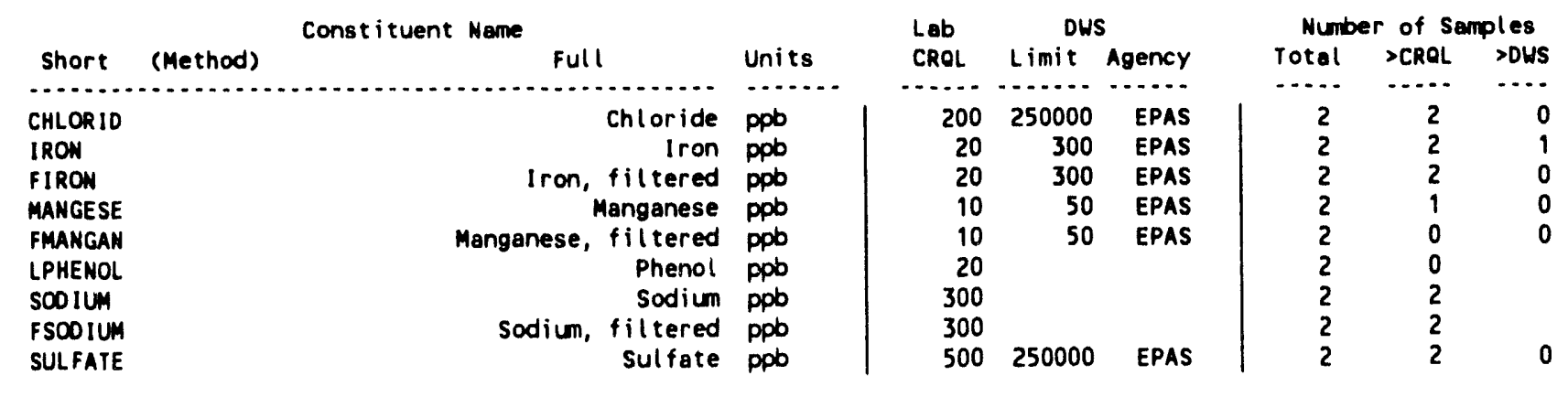


Table 16-6. Constituent List and Summary of Results for the Single-Shell Tank Waste Management Area B-BY-BX Data for Reporting Period July 1 through September 30,1993 . (sheet 2 of 3 )

SITE SPECIFIC AMD OTHER CONSTITUENTS

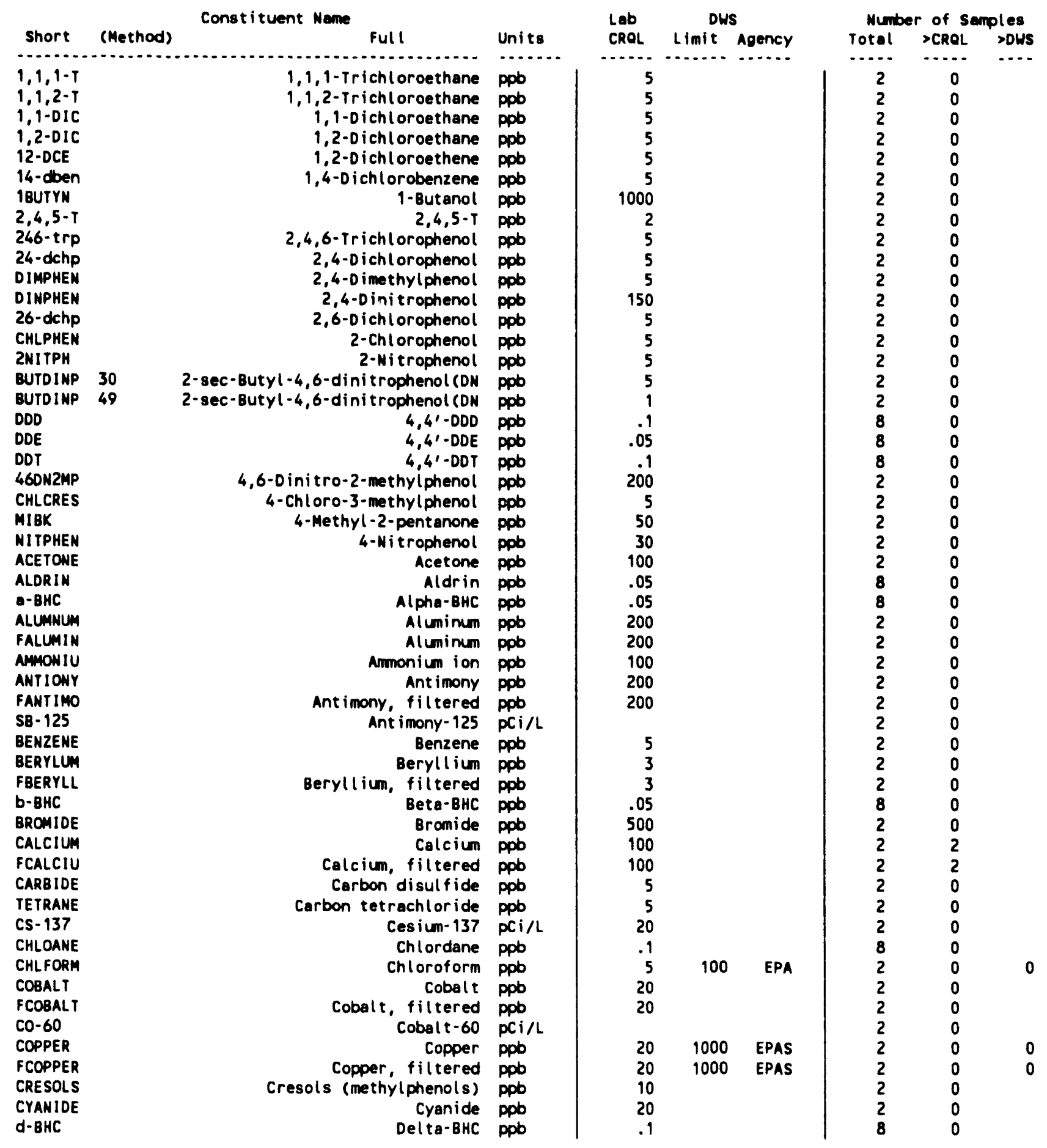


Table 16-6. Constituent List and Summary of Results for the Single-Shell Tank Waste Management Area B-BY-BX Data for Reporting Period July 1 through September 30, 1993. (sheet 3 of 3 )

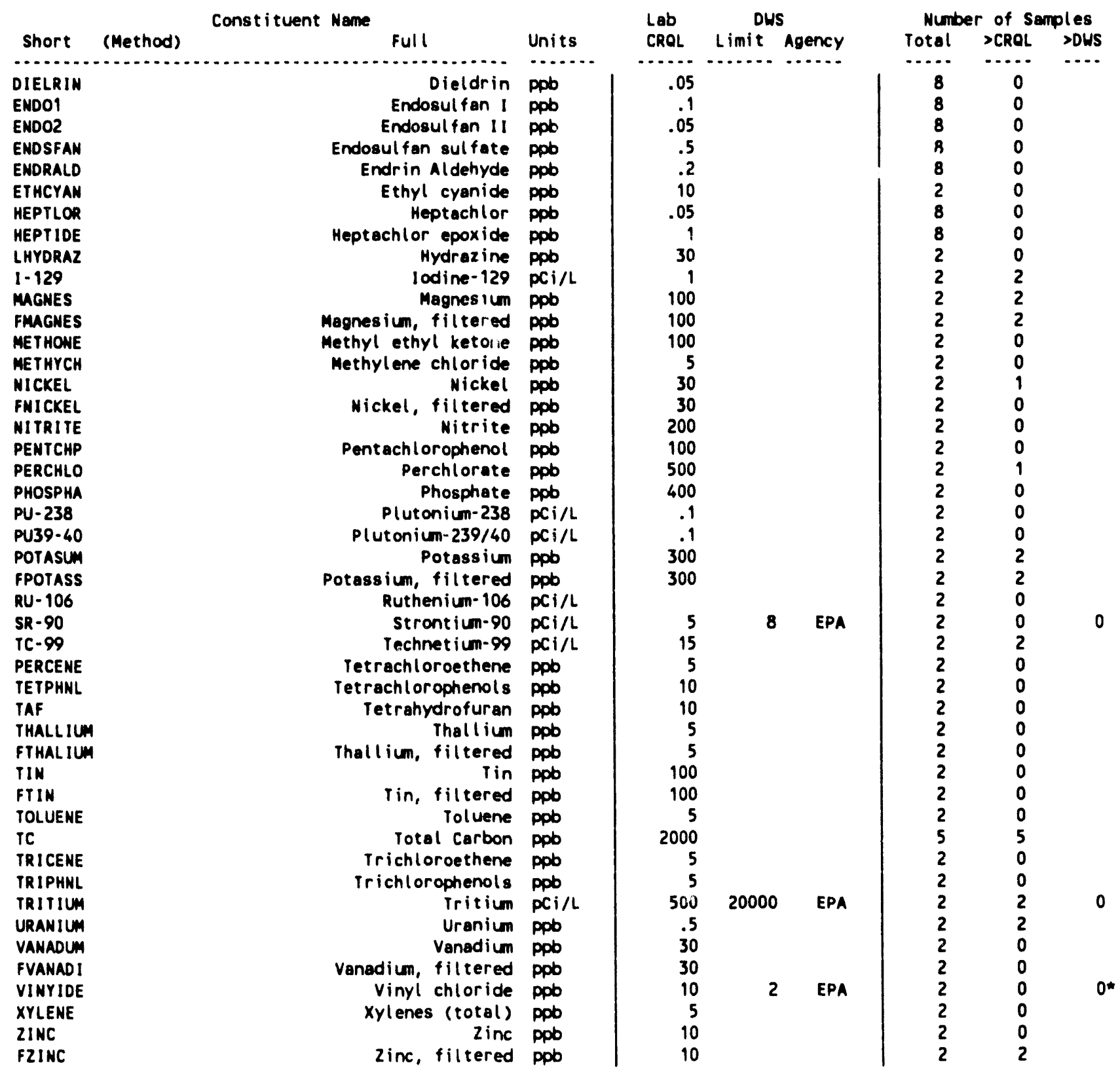

For explanation of this table, see Section 1.4 of report. 
Table 16-7. Constituents with at Least One Value Above the CRQL for the Single-Shell Tank Waste Management Area B-BY-BX Data for Reporting Period July 1 through September 30, 1993. (sheet 1 of 2)

\begin{tabular}{|c|c|c|c|c|c|c|}
\hline $\begin{array}{l}\text { Well } \\
\text { Name }\end{array}$ & $\begin{array}{c}\text { Collection } \\
\text { Dete }\end{array}$ & $\begin{array}{l}\text { Semple } \\
\text { Number }\end{array}$ & $\begin{array}{c}\text { ARSENIC } \\
43 / \text { ppb } \\
5 / 50\end{array}$ & $\begin{array}{c}\text { FARSENIC } \\
43 / \mathrm{ppb} \\
5 / 50\end{array}$ & $\begin{array}{l}\text { BARIUM } \\
34 / \text { PpD } \\
2.0 / 1000\end{array}$ & $\begin{array}{r}\text { FBARIUM } \\
34 / \text { Ppb } \\
20 / 1000\end{array}$ \\
\hline $\begin{array}{l}299-E 33-42 \\
299-E 33-42 \\
299-E 33-43 \\
299-E 33-43\end{array}$ & $\begin{array}{l}9 / 21 / 93 \\
9 / 21 / 93 \\
9 / 21 / 93 \\
9 / 21 / 93\end{array}$ & $\begin{array}{l}\text { B096L5 } \\
\text { B096L9 } \\
8096 M 0 \\
8096 \times 44\end{array}$ & $\begin{array}{r}11.00 \\
9.20\end{array}$ & $\begin{array}{l}11.00 \\
10.00\end{array}$ & $\begin{array}{l}29.00 \\
33.00\end{array}$ & $\begin{array}{l}29.00 \\
33.00\end{array}$ \\
\hline
\end{tabular}

\begin{tabular}{|c|c|c|c|c|c|c|}
\hline $\begin{array}{l}\text { Hell } \\
\text { Name }\end{array}$ & $\begin{array}{c}\text { Collection } \\
\text { Date }\end{array}$ & $\begin{array}{l}\text { Sample } \\
\text { Number }\end{array}$ & $\begin{array}{l}\text { CALCIUM } \\
34 / \text { ppb } \\
100 \%\end{array}$ & $\begin{array}{l}\text { FCALCIU } \\
34 / \text { ppb } \\
100 / .\end{array}$ & $\begin{array}{c}\text { CHLORID } \\
124 / \text { ppb } \\
200 / 2500008\end{array}$ & $\begin{array}{l}\text { CHROMum } \\
34 / \text { ppb } \\
20 / 50\end{array}$ \\
\hline $\begin{array}{l}299-E 33-42 \\
299-E 33-42 \\
299-E 33-43 \\
299-E 33-43\end{array}$ & $\begin{array}{l}9 / 21 / 93 \\
9 / 21 / 93 \\
9 / 21 / 93 \\
9 / 21 / 93\end{array}$ & $\begin{array}{l}B 096 L 5 \\
8096 L 9 \\
8096 M 0 \\
B 096 M 4\end{array}$ & $\begin{array}{l}32000.00 \\
31000.00\end{array}$ & $\begin{array}{l}32000.00 \\
31000.00\end{array}$ & $\begin{array}{l}5500.00 \\
9100.000\end{array}$ & $\begin{array}{l}25.00 \\
73.00\end{array}$ \\
\hline
\end{tabular}

\begin{tabular}{|c|c|c|c|c|c|c|}
\hline $\begin{array}{l}\text { Well } \\
\text { Nane }\end{array}$ & $\begin{array}{c}\text { Collection } \\
\text { Date }\end{array}$ & $\begin{array}{l}\text { Sample } \\
\text { Number }\end{array}$ & $\begin{array}{l}\text { FCHROMI } \\
34 / \text { ppb } \\
20 / 50\end{array}$ & $\begin{array}{l}\text { FLUORID } \\
124 / \mathrm{pp6} \\
100 / 4000\end{array}$ & $\begin{array}{c}\text { ALPHA } \\
135 / \mathrm{PCi} / \mathrm{L} \\
. / 15\end{array}$ & $\begin{array}{c}\text { BETA } \\
136 / \mathrm{PCi} / \mathrm{L} \\
. / 50\end{array}$ \\
\hline $\begin{array}{l}299-E 33-42 \\
299-E 33-42 \\
299-E 33-43 \\
299-E 33-43\end{array}$ & $\begin{array}{l}9 / 21 / 93 \\
9 / 21 / 93 \\
9 / 21 / 93 \\
9 / 21 / 93\end{array}$ & $\begin{array}{l}809615 \\
809619 \\
8096 M 0 \\
8096114\end{array}$ & $\begin{array}{l}5.42 \mathrm{U} \\
5.42 \mathrm{U}\end{array}$ & $\begin{array}{l}400.00 \\
400.00\end{array}$ & $\begin{array}{l}2.13 \\
3.63\end{array}$ & $\begin{array}{l}108.00 \\
11.50\end{array}$ \\
\hline
\end{tabular}

\begin{tabular}{|c|c|c|c|c|c|c|}
\hline $\begin{array}{l}\text { Well } \\
\text { Name }\end{array}$ & $\begin{array}{c}\text { Collection } \\
\text { Date }\end{array}$ & $\begin{array}{l}\text { Sample } \\
\text { Mumber }\end{array}$ & $\begin{array}{c}1-129 \\
139 / \mathrm{pCi} / \mathrm{L} \\
. /\end{array}$ & $\begin{array}{c}\text { IRON } \\
34 / \mathrm{ppb} \\
20 / 300 \mathrm{~s}\end{array}$ & $\begin{array}{c}\text { FIRON } \\
\text { 34/ppb } \\
20 / 300 \text { s }\end{array}$ & $\begin{array}{l}\text { MAGMES } \\
34 / \text { ppb } \\
100 \%\end{array}$ \\
\hline a.............. & $\ldots \ldots$ & …........ & …........... & $\ldots \ldots \ldots$ & $\cdots \cdot$ & \\
\hline $\begin{array}{l}299-E 33-42 \\
299-E 33-42 \\
299-E 33-43 \\
299-E 33-43\end{array}$ & $\begin{array}{l}9 / 21 / 93 \\
9 / 21 / 93 \\
9 / 21 / 93 \\
9 / 21 / 93\end{array}$ & $\begin{array}{l}\text { B096L5 } \\
\text { B096L9 } \\
\text { B096MO } \\
\text { B096M4 }\end{array}$ & 6.28 & $\begin{array}{l}110.00 \\
360.00\end{array}$ & $\begin{array}{l}24.00 \\
34.00\end{array}$ & $\begin{array}{l}8600.00 \\
8500.00\end{array}$ \\
\hline
\end{tabular}

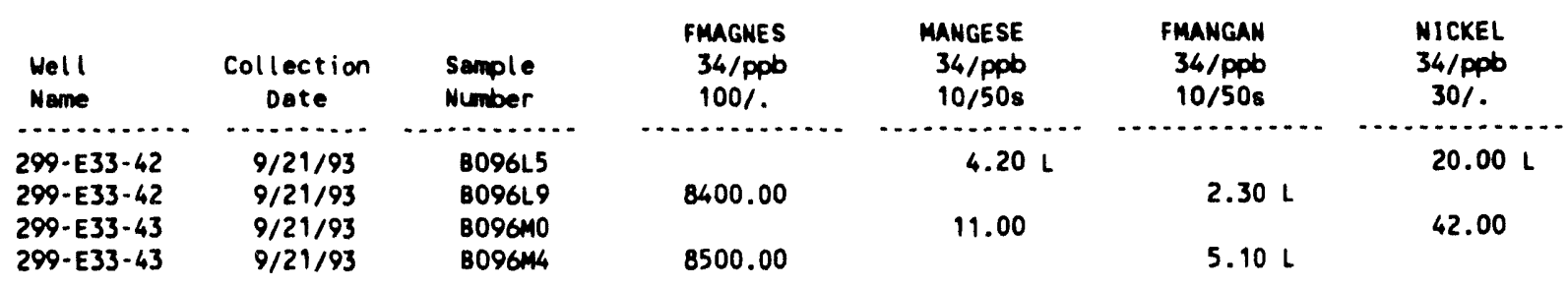


Table 16-7. Constituents with at Least One Value Above the CRQL for the Single-Shell Tank Waste Management Area B-BY-BX Data for Reporting Period July 1 through September 30, 1993. (sheet 2 of 2)

\begin{tabular}{|c|c|c|c|c|c|c|}
\hline $\begin{array}{l}\text { Well } \\
\text { Nane }\end{array}$ & $\begin{array}{c}\text { Collection } \\
\text { Date }\end{array}$ & $\begin{array}{l}\text { Semple } \\
\text { Number }\end{array}$ & $\begin{array}{c}\text { FNICKEL } \\
34 / \text { ppb } \\
30 \%\end{array}$ & $\begin{array}{l}\text { NITRATE } \\
124 / \mathrm{ppb} \\
200 / 45000\end{array}$ & $\begin{array}{l}\text { PERCHLO } \\
62 / \mathrm{ppb} \\
500 / .\end{array}$ & $\begin{array}{c}\text { POTASUN } \\
34 / \text { ppb } \\
1000 \% .\end{array}$ \\
\hline $\begin{array}{l}299-E 33-42 \\
299-E 33-42\end{array}$ & $\begin{array}{l}9 / 21 / 93 \\
9 / 21 / 93\end{array}$ & $\begin{array}{l}\text { B09615 } \\
809619\end{array}$ & 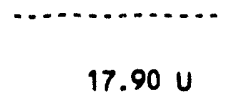 & $13000.00 \mathrm{D}$ & $400.00 \mathrm{~L}$ & 5700.00 \\
\hline $\begin{array}{l}299-E 33-43 \\
299-E 33-43\end{array}$ & $\begin{array}{l}9 / 21 / 93 \\
9 / 21 / 93\end{array}$ & $\begin{array}{l}\text { B096M0 } \\
\text { B096M4 }\end{array}$ & $17.90 \mathrm{U}$ & 5200.00 & 500.00 & 5200.00 \\
\hline
\end{tabular}

\begin{tabular}{|c|c|c|c|c|c|c|}
\hline $\begin{array}{l}\text { Well } \\
\text { Name }\end{array}$ & $\begin{array}{c}\text { Collection } \\
\text { Date }\end{array}$ & $\begin{array}{l}\text { Sample } \\
\text { Number }\end{array}$ & $\begin{array}{l}\text { FPOTASS } \\
34 / \mathrm{ppb} \\
1000 / .\end{array}$ & $\begin{array}{l}\text { s001um } \\
34 / \mathrm{ppb} \\
300 \%\end{array}$ & $\begin{array}{l}\text { FSO0 IUm } \\
34 / \text { ppb } \\
300 \%\end{array}$ & $\begin{array}{c}\text { SULFATE } \\
124 / \text { ppb } \\
500 / 250000 \mathrm{~s}\end{array}$ \\
\hline $\begin{array}{l}299-E 33-42 \\
299-E 33-42 \\
299-E 33-43 \\
299-E 33-43\end{array}$ & $\begin{array}{l}9 / 21 / 93 \\
9 / 21 / 93 \\
9 / 21 / 93 \\
9 / 21 / 93\end{array}$ & $\begin{array}{l}\text { B096L.5 } \\
\text { B096L.9 } \\
\text { B096N0 } \\
\text { B096N4 }\end{array}$ & $\begin{array}{l}5500.00 \\
5300.00\end{array}$ & 15000.00 & $\begin{array}{l}14000.00 \\
15000.00\end{array}$ & 28000.000 \\
\hline
\end{tabular}

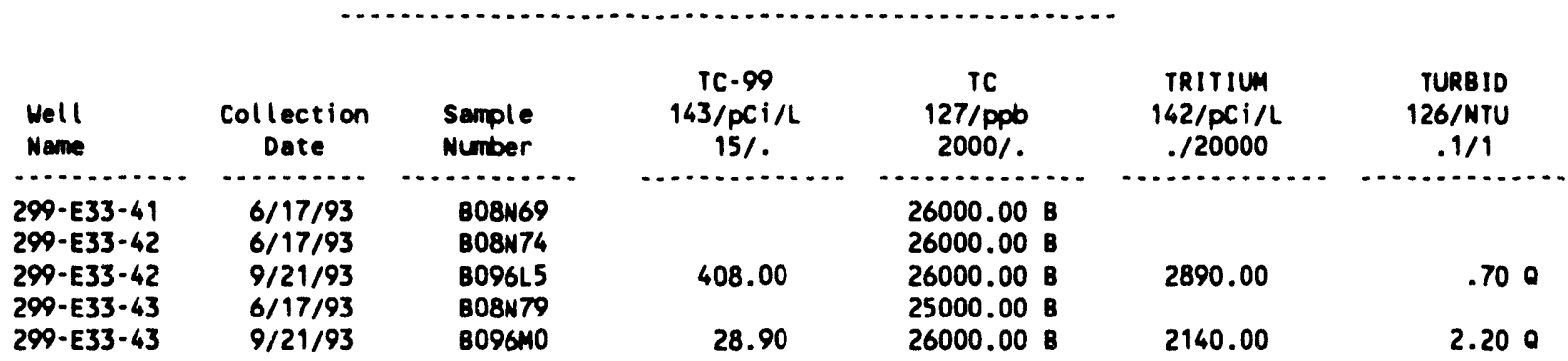

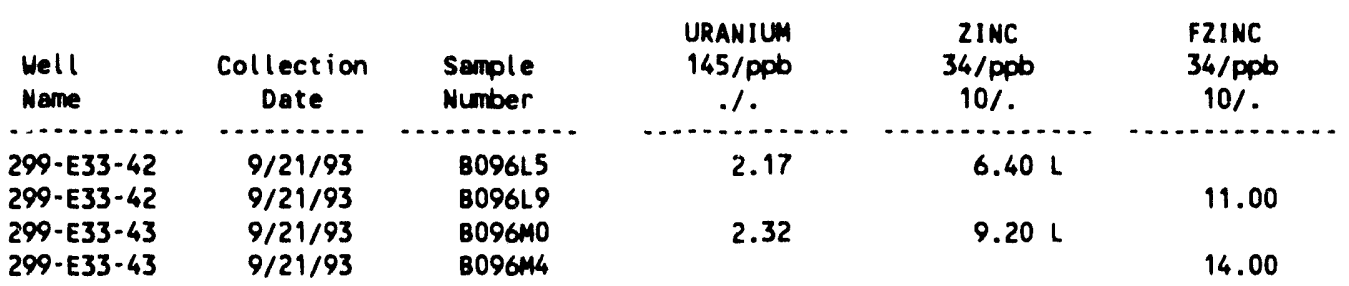

For explanation of this table, see Section 1.4 of report. 
Table 16-8. Contamination Indicator Parameters for the Single-Shell Tank Waste Management Area B-BY-BX Data for Reporting Period July 1 through September 30, 1993.

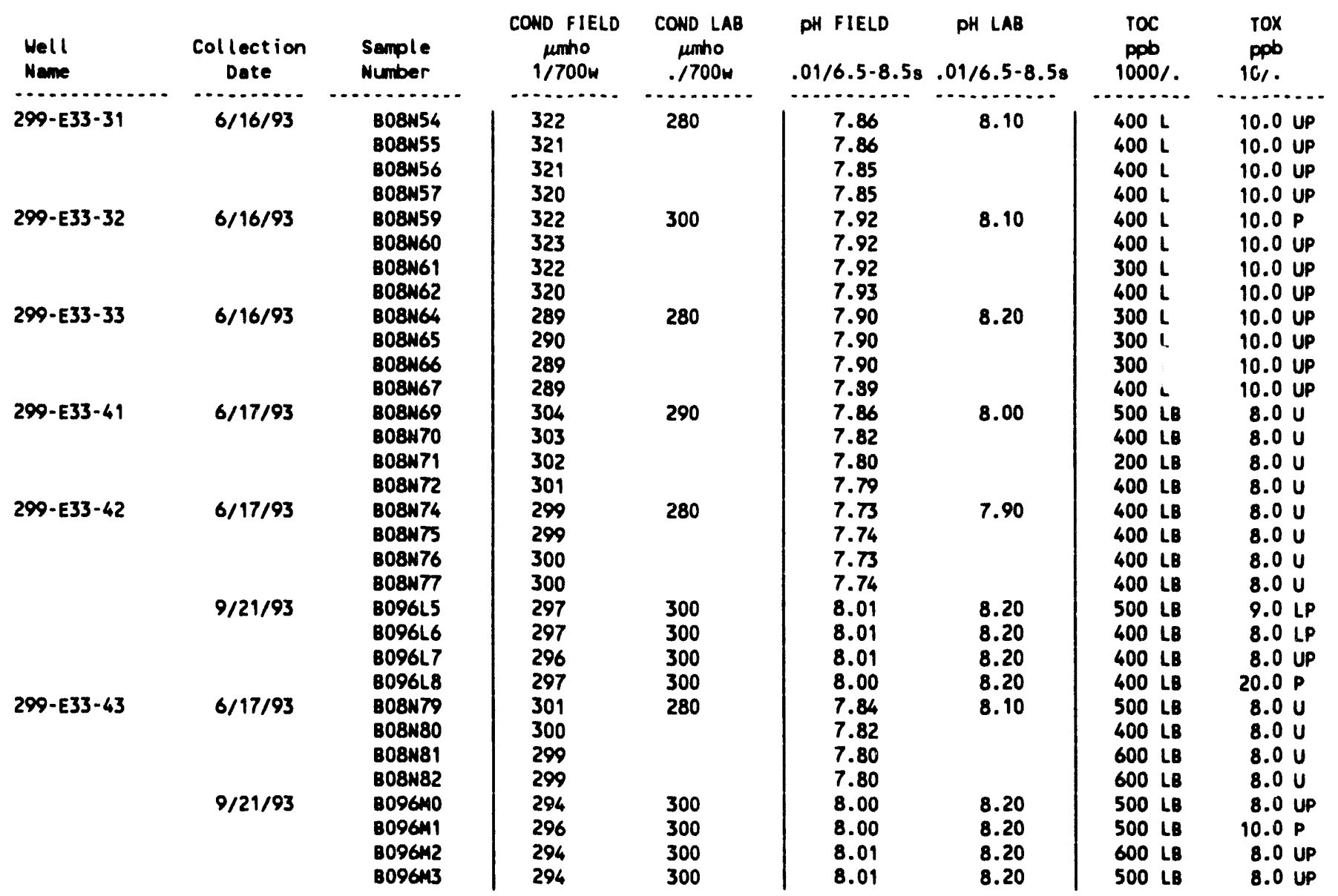

For explanation of this table, see Section 1.4 of report. 
Table 16-9. Constituent List and Summary of Results for the Single-Shell Tank Waste Management Area C Data for Reporting Period

July 1 through September 30, 1993. (sheet 1 of 3 )

CONTAMIMATION INDICATOR PARAMETERS

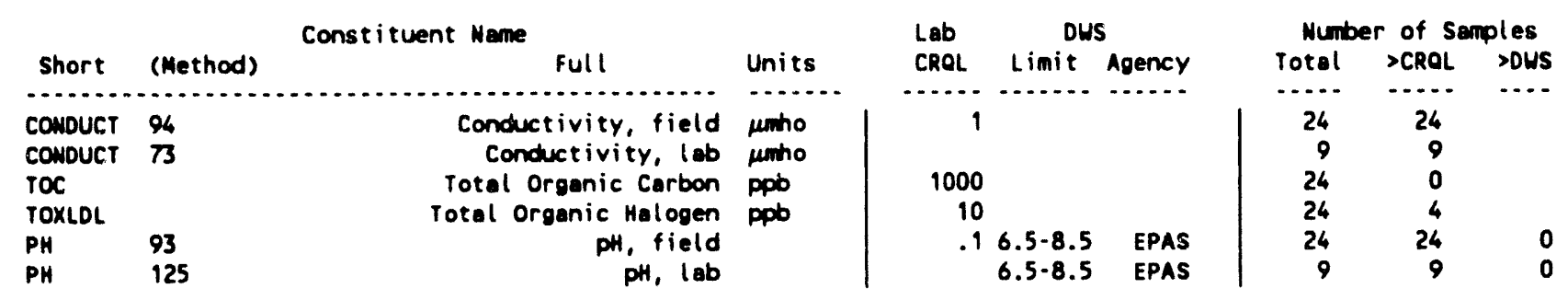

DRINKING HATER PARAMETERS

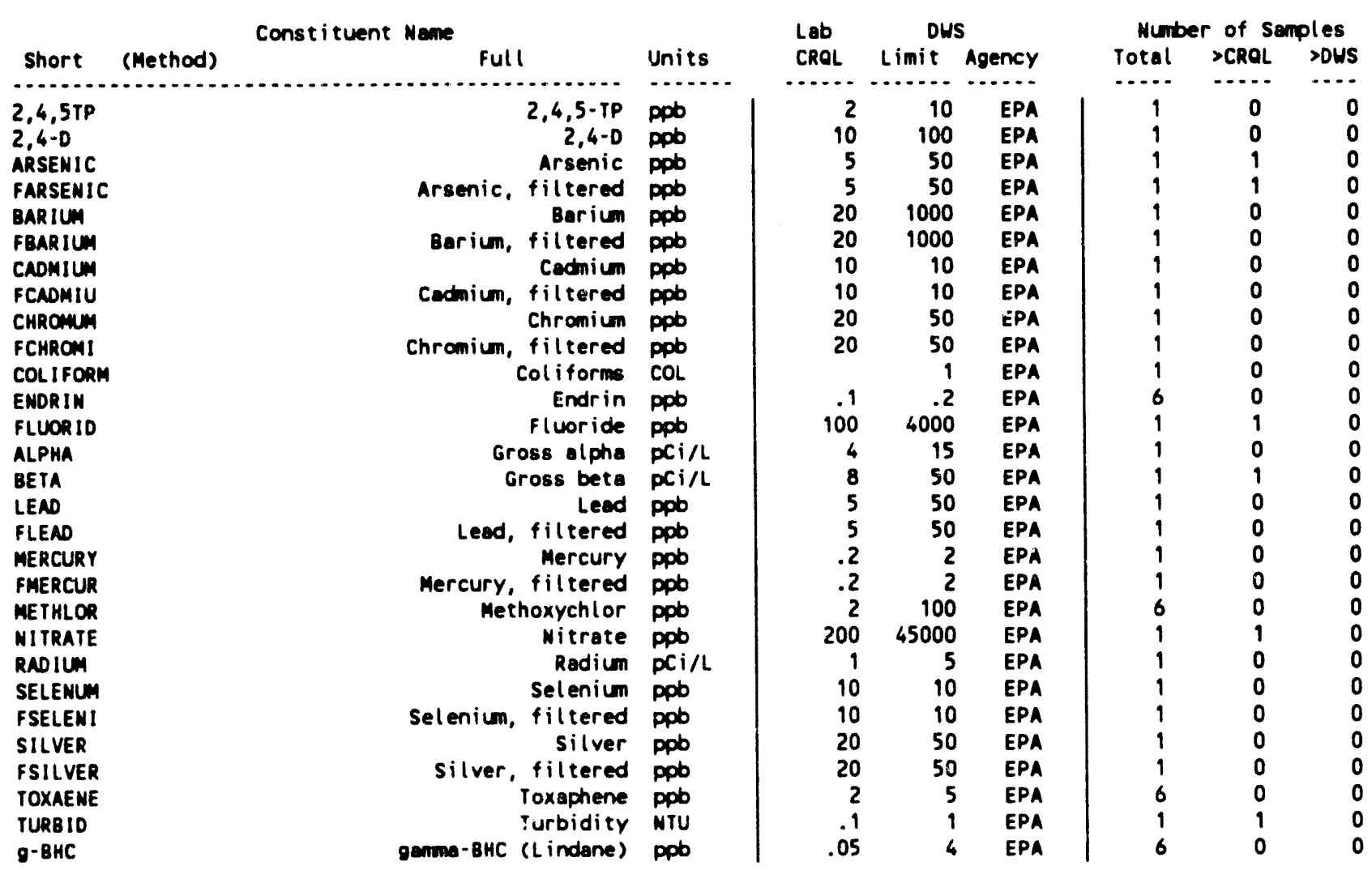

GROUNDWATER QUALITY PARAMETERS

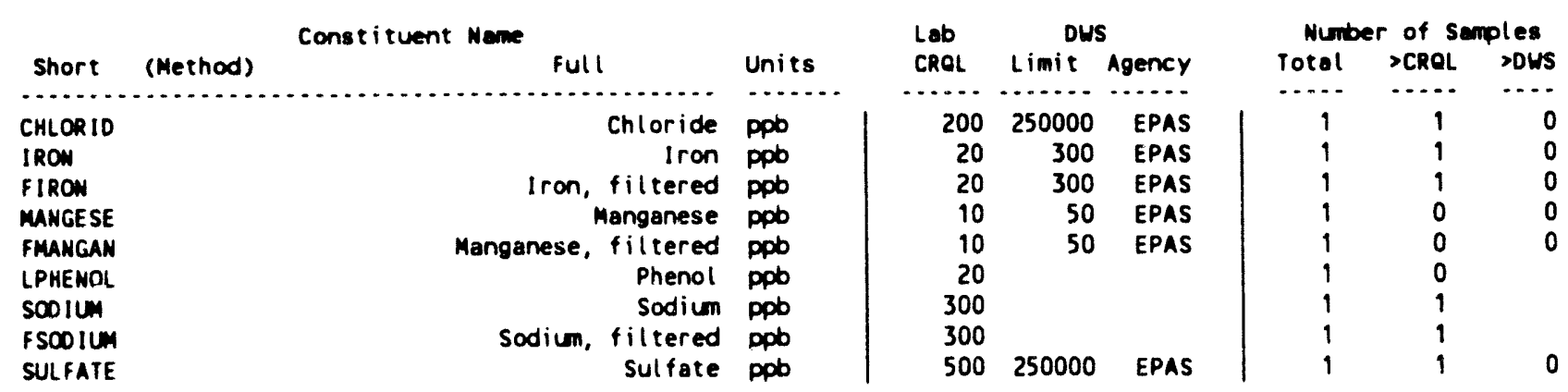




\section{Table 16-9. Constituent List and Summary of Results for the Single-Shell Tank Waste Management Area C Data for Reporting Period July 1 through September 30, 1993. (sheet 2 of 3)}

SITE SPECIFIC AND OTHER CONSTITUENTS

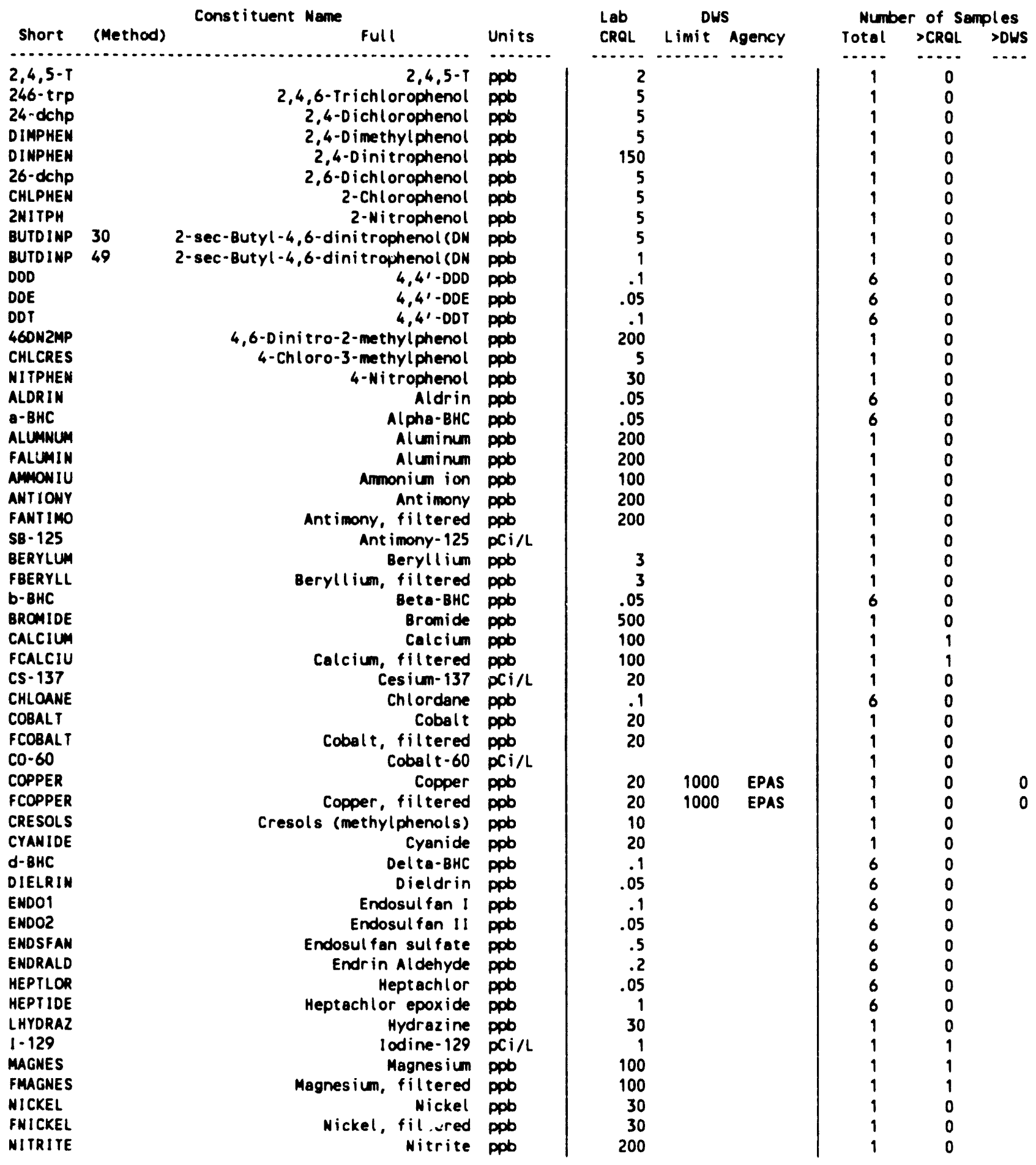


Table 16-9. Constituent List and Summary of Results for the Single-Shell Tank Waste Management Area C Data for Reporting Period July 1 through September 30,1993. (sheet 3 of 3 )

\begin{tabular}{|c|c|c|c|c|c|c|c|c|c|}
\hline \multirow[b]{2}{*}{ Short } & \multirow[b]{2}{*}{ (Method) } & \multirow{2}{*}{$\begin{array}{l}\text { Constituent Nane Full } \\
\text { fune }\end{array}$} & \multirow[b]{2}{*}{ Units } & \multirow{2}{*}{$\begin{array}{l}\text { Lab } \\
\text { CRQL }\end{array}$} & \multicolumn{2}{|c|}{ Dus } & \multicolumn{3}{|c|}{ Number of Samples } \\
\hline & & & & & Limit & Agency & Total & $>$ CROL & $>$ DWS \\
\hline - & & , & (n) & (n.... & (n........ & $\ldots$ & $\cdots$ & $\cdots$ & $\cdots$ \\
\hline PENTCHP & & Pentachlorophenol & ppb & 100 & & & 1 & 0 & \\
\hline PERCHLO & & Perchlorate & ppb & 500 & & & 1 & 1 & \\
\hline PHOSPHA & & Phosphate & ppob & 400 & & & 1 & 0 & \\
\hline PU-238 & & Plutonium-238 & $\mathrm{PCi} / \mathrm{L}$ & .1 & & & 1 & 0 & \\
\hline PU39-40 & & Plutonium-239/40 & $\mathrm{PCi} / \mathrm{L}$ & .1 & & & 1 & 0 & \\
\hline POTASUM & & Potassium & ppb & 300 & & & 1 & 1 & \\
\hline FPOTASS & & Potassium, filtered & ppo & 300 & & & 1 & 1 & \\
\hline$R U-106$ & & Ruthenium- 106 & $\mathrm{PCi} / \mathrm{L}$ & & & & 1 & 0 & \\
\hline SR-90 & & strontium-90 & $\mathrm{PC} i / L$ & 5 & 8 & EPA & 1 & 0 & 0 \\
\hline$T C-99$ & & Technet ium- 99 & $\mathrm{PCi} / \mathrm{L}$ & 15 & & & 1 & 0 & \\
\hline TETPHNL & & Tetrachlorophenols & ppb & 10 & & & 1 & 0 & \\
\hline THALLIUN & & Thallium & ppb & 5 & & & 1 & 0 & \\
\hline FTHALIUM & & Thallium, filtered & ppo & 5 & & & 1 & 0 & \\
\hline TIN & & $\operatorname{Tin}$ & ppb & 100 & & & 1 & 0 & \\
\hline FTIN & & Tin, filtered & ppb & 100 & & & 1 & 0 & \\
\hline TC & & Total Carbon & ppob & 2000 & & & 1 & 1 & \\
\hline TRIPHNL & & Trichlorophenols & ppo & 5 & & & 1 & 0 & \\
\hline TRITIUM & & Tritiun & $\mathrm{pCi} / \mathrm{L}$ & 500 & 20000 & EPA & 1 & 1 & 0 \\
\hline URANIUM & & Uranium & ppb & .5 & & & 1 & 1 & \\
\hline VANADUM & & Vanadium & ppob & 30 & & & 1 & 0 & \\
\hline FVANADI & & Vanadium, filtered & ppb & 30 & & & 1 & 0 & \\
\hline ZINC & & 2 ine & ppob & 10 & & & 1 & 1 & \\
\hline FZINC & & Zinc, filtered & ppb & 10 & & & 1 & 0 & \\
\hline
\end{tabular}

For explanation of this table, see Section 1.4 of report. 
Table 16-10. Constituents with at Least One Value Above the CRQL for the Single-Shell Tank Waste Management Area C Data for Reporting Period July 1 through September 30, 1993.

\begin{tabular}{|c|c|c|c|c|c|c|}
\hline $\begin{array}{l}\text { Well } \\
\text { Name }\end{array}$ & $\begin{array}{c}\text { Collection } \\
\text { Date }\end{array}$ & $\begin{array}{l}\text { Semple } \\
\text { Number }\end{array}$ & $\begin{array}{c}\text { ARSENIC } \\
43 / \mathrm{ppb} \\
5 / 50\end{array}$ & $\begin{array}{c}\text { FARSENIC } \\
43 / \mathrm{ppb} \\
5 / 50\end{array}$ & $\begin{array}{c}\text { CALCIUM } \\
34 / \text { ppb } \\
100 \%\end{array}$ & $\begin{array}{l}\text { FCALCIU } \\
34 / \mathrm{ppb} \\
100 \%\end{array}$ \\
\hline $\begin{array}{l}299-E 27-7 \\
299-E 27-7\end{array}$ & $\begin{array}{l}9 / 21 / 93 \\
9 / 21 / 93\end{array}$ & $\begin{array}{l}B 096 L 0 \\
B 096 L 4\end{array}$ & 8.80 & 8.20 & 28000.00 & 29000.00 \\
\hline
\end{tabular}

\begin{tabular}{|c|c|c|c|c|c|c|}
\hline $\begin{array}{l}\text { Mell } \\
\text { Name }\end{array}$ & $\begin{array}{c}\text { Collection } \\
\text { Date }\end{array}$ & $\begin{array}{l}\text { Sample } \\
\text { Number }\end{array}$ & $\begin{array}{c}\text { CHLORID } \\
124 / \mathrm{ppb} \\
200 / 250000 \mathrm{~s}\end{array}$ & $\begin{array}{l}\text { FLUOR1D } \\
124 / \mathrm{ppD} \\
100 / 4000\end{array}$ & $\begin{array}{c}\text { BETA } \\
136 / \mathrm{pCi} / \mathrm{L} \\
.150\end{array}$ & $\begin{array}{c}1.129 \\
139 / \mathrm{pCi} / \mathrm{L} \\
. /\end{array}$ \\
\hline 299-E27-7 & $9 / 21 / 93$ & $8096 \angle 0$ & 3900.00 & 300.00 & 5.92 & 2.50 \\
\hline
\end{tabular}

\begin{tabular}{|c|c|c|c|c|c|c|}
\hline $\begin{array}{l}\text { Well } \\
\text { Name }\end{array}$ & $\begin{array}{c}\text { Collection } \\
\text { Date }\end{array}$ & $\begin{array}{l}\text { Semple } \\
\text { Number }\end{array}$ & $\begin{array}{c}\text { IRON } \\
34 / \mathrm{Ppb} \\
20 / 300 \mathrm{~s}\end{array}$ & $\begin{array}{l}\text { FIRON } \\
\text { 34/Ppb } \\
20 / 300 \mathrm{~s}\end{array}$ & $\begin{array}{l}\text { MAGNES } \\
34 / \text { ppb } \\
100 \% .\end{array}$ & $\begin{array}{l}\text { FMAGNES } \\
34 / \mathrm{ppb} \\
100 \%\end{array}$ \\
\hline $\begin{array}{l}299-E 27-7 \\
299-E 27-7\end{array}$ & $\begin{array}{l}9 / 21 / 93 \\
9 / 21 / 93\end{array}$ & $\begin{array}{l}8096 L 10 \\
8096 L 4\end{array}$ & 20.00 & 23.00 & 7400.00 & 7500.00 \\
\hline
\end{tabular}

\begin{tabular}{|c|c|c|c|c|c|c|}
\hline $\begin{array}{l}\text { Hell } \\
\text { Name }\end{array}$ & $\begin{array}{c}\text { Collection } \\
\text { Date }\end{array}$ & $\begin{array}{l}\text { Sample } \\
\text { Number }\end{array}$ & $\begin{array}{c}\text { NITRATE } \\
124 / \mathrm{ppb} \\
200 / 45000\end{array}$ & $\begin{array}{l}\text { PERCHLO } \\
62 / \mathrm{ppb} \\
500 \%\end{array}$ & $\begin{array}{c}\text { POTASUM } \\
34 / \mathrm{ppb} \\
1000 \%\end{array}$ & $\begin{array}{l}\text { FPOTASS } \\
34 / \mathrm{ppb} \\
1000 / .\end{array}$ \\
\hline $\begin{array}{l}\text { 299-E27-7 } \\
299-E 27-7\end{array}$ & $\begin{array}{l}9 / 21 / 93 \\
9 / 21 / 93\end{array}$ & $\begin{array}{l}\text { B096LL } \\
\text { B096LL }\end{array}$ & 3000.00 & 600.00 & 4600.00 & 5000.00 \\
\hline
\end{tabular}

\begin{tabular}{|c|c|c|c|c|c|c|}
\hline $\begin{array}{l}\text { Well } \\
\text { Name }\end{array}$ & $\begin{array}{c}\text { Collection } \\
\text { Date }\end{array}$ & $\begin{array}{l}\text { Semple } \\
\text { Number }\end{array}$ & $\begin{array}{l}\text { s001um } \\
34 / p p b \\
300 \%\end{array}$ & $\begin{array}{l}\text { FSODIUN } \\
34 / \text { ppb } \\
300 \%\end{array}$ & $\begin{array}{c}\text { SULFATE } \\
124 / \mathrm{ppb} \\
500 / 250000 \mathrm{~s}\end{array}$ & $\begin{array}{c}\text { TC } \\
127 / p p b \\
2000 \%\end{array}$ \\
\hline $\begin{array}{l}299-E 27-7 \\
299-E 27-7\end{array}$ & $\begin{array}{l}9 / 21 / 93 \\
9 / 21 / 93\end{array}$ & $\begin{array}{l}B 09610 \\
B 09614\end{array}$ & 8800.00 & 8900.00 & 25000.000 & $23000.00 \mathrm{~B}$ \\
\hline
\end{tabular}

\begin{tabular}{|c|c|c|c|c|c|c|}
\hline $\begin{array}{l}\text { Hell } \\
\text { Name }\end{array}$ & $\begin{array}{l}\text { Collection } \\
\text { Date }\end{array}$ & $\begin{array}{l}\text { Sample } \\
\text { Number }\end{array}$ & $\begin{array}{l}\text { TRITIUM } \\
\text { 142/PCi/L } \\
. / 20000\end{array}$ & $\begin{array}{c}\text { TURBID } \\
\text { 126/NTU } \\
.1 / 1\end{array}$ & $\begin{array}{c}\text { URANIUM } \\
\text { 145/ppb } \\
.1 .\end{array}$ & $\begin{array}{c}\text { ZINC } \\
34 / \mathrm{ppD} \\
10 \%\end{array}$ \\
\hline 299-E27-7 & $9 / 21 / 93$ & BO96LO & 656.00 & $.20 c$ & 1.75 & 16.00 \\
\hline
\end{tabular}

\begin{tabular}{|c|c|c|c|}
\hline $\begin{array}{l}\text { Well } \\
\text { Name }\end{array}$ & $\begin{array}{c}\text { Collection } \\
\text { Dete }\end{array}$ & $\begin{array}{l}\text { Sample } \\
\text { Number }\end{array}$ & $\begin{array}{c}\text { F21NC } \\
34 / \mathrm{ppb} \\
10 \%\end{array}$ \\
\hline 299-E27-7 & $9 / 21 / 93$ & 809614 & 4.30 \\
\hline
\end{tabular}

For explanation of this table, see Section 1.4 of report. 
Table 16-11. Contamination Indicator Parameters for the Single-Shel1 Tank Waste Management Area C Data for Reporting Period July 1 through September 30, 1993.

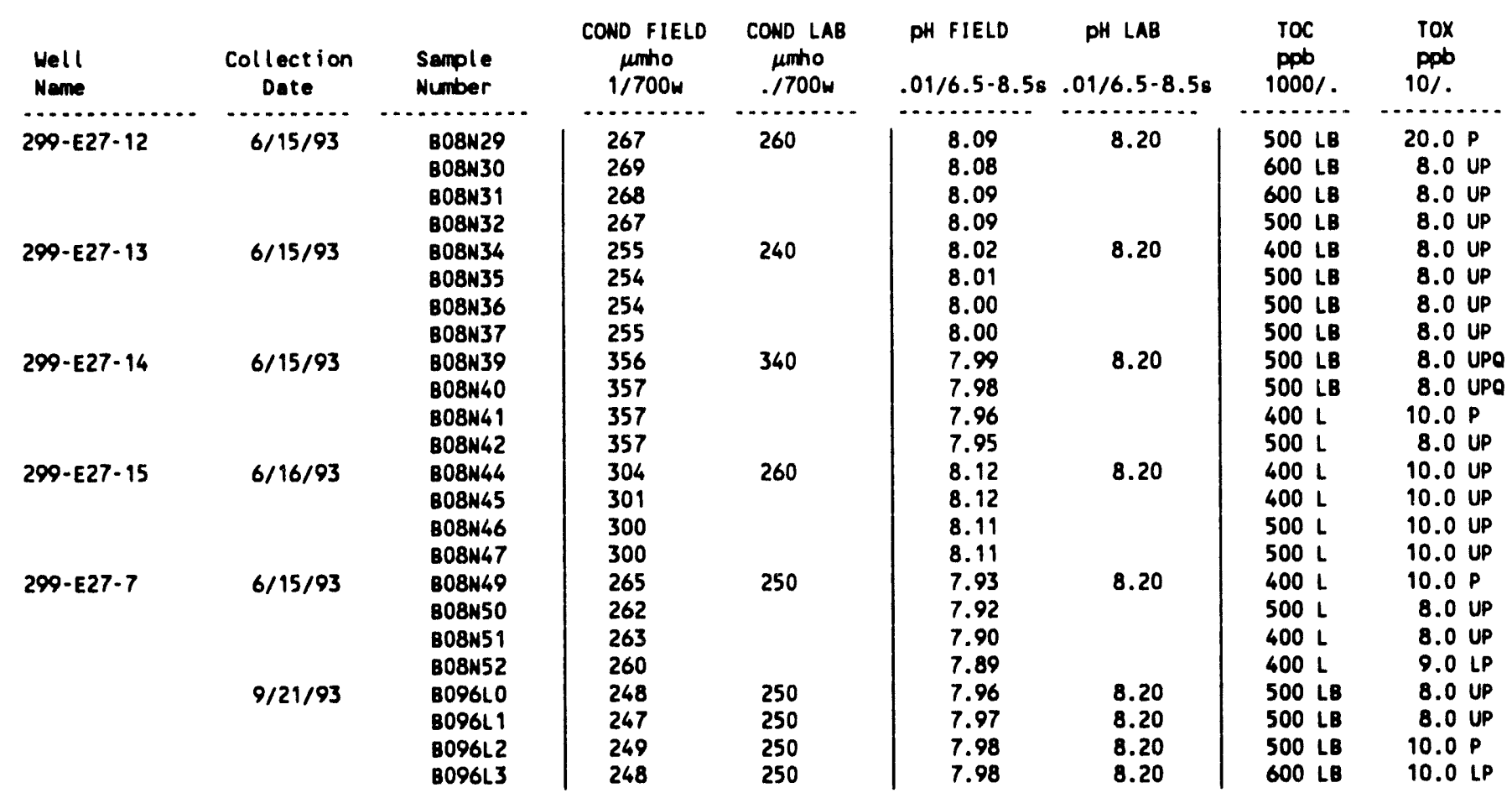

For explanation of this table, see Section 1.4 of report. 
Table 16-12. Constituent List and Summary of Results for the Single-Shell

Tank Waste Management Area S-SX Data for Reporting Period July 1 through September 30, 1993. (sheet 1 of 6)

CONTAMINATION INDICATOR PARAMETERS

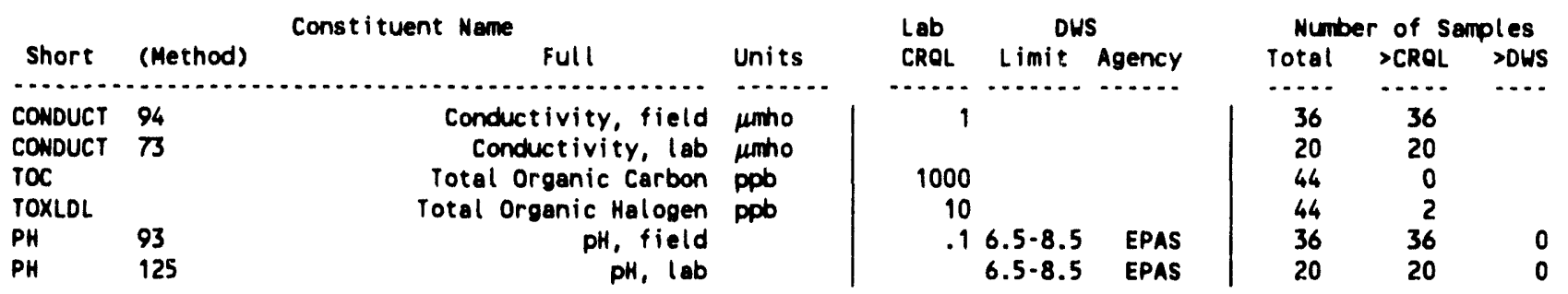

DRINKING WATER PARAMETERS

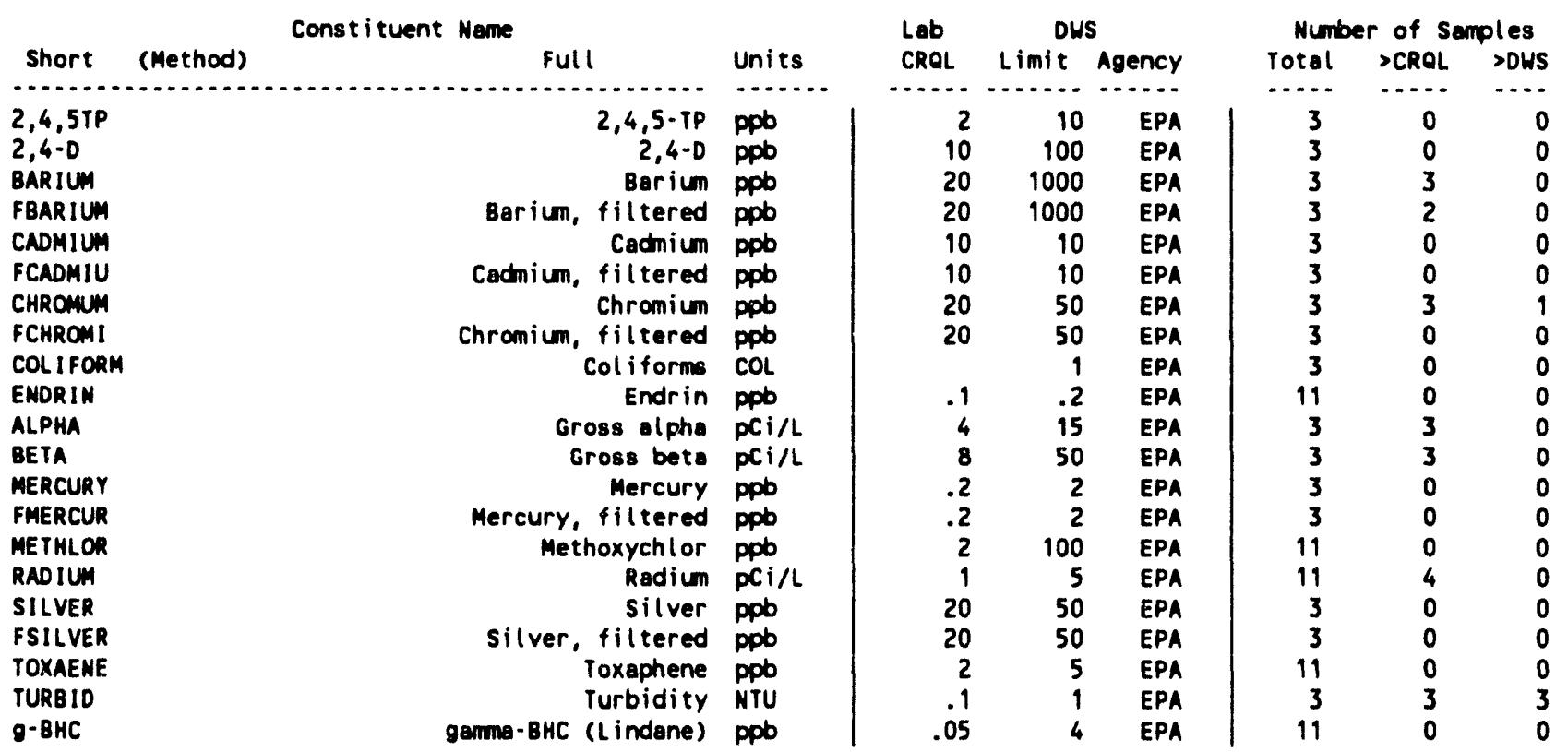

GROUNOWATER QUALITY PARAMETERS

\begin{tabular}{|c|c|c|c|c|c|c|c|c|c|}
\hline \multicolumn{4}{|c|}{ Const ituent Name } & \multicolumn{3}{|c|}{ DUS } & \multicolumn{3}{|c|}{ Number of Samples } \\
\hline Short & (Method) & Full & Units & CROL & Limit & Agency & Total & $>C R O L$ & $>$ DWS \\
\hline$\ldots \ldots \ldots$ & $\cdots$ & $\ldots \ldots$ & $\ldots \ldots$ & $\cdots-\cdots$ & $\cdots \ldots$ & $\cdots \cdots$ & $\ldots$. & $\ldots$ & $\ldots$ \\
\hline IRON & & Iron & ppob & 20 & 300 & EPAS & 3 & 3 & 3 \\
\hline FIRON & & Iron, filtered & ppb & 20 & 300 & EPAS & 3 & 0 & 0 \\
\hline MANGESE & & Manganese & ppb & 10 & 50 & EPAS & 3 & 3 & 1 \\
\hline FMANGAN & & Manganese, filtered & ppb & 10 & 50 & EPAS & 3 & 0 & 0 \\
\hline LPHENOL & 19 & Phenol & ppb & 10 & & & 1 & 0 & \\
\hline LPHENOL & 30 & Phenol & ppb & 20 & & & 3 & 0 & \\
\hline SODIUN & & Sodium & ppb & 300 & & & 3 & 3 & \\
\hline FSODIUM & & Sodiun, filtered & ppb & 300 & & & 3 & 3 & \\
\hline
\end{tabular}



Table 16-12. Constituent List and Summary of Results for the Single-Shell Tank Waste Management Area S-SX Data for Reporting Period July 1 through September 30,1993 . (sheet 2 of 6 )

SITE SPECIFIC AND OTHER CONSTITUENTS

\begin{tabular}{|c|c|c|c|c|c|c|c|c|}
\hline & & Constituent Name & & Lab & DWS & Numb & $r$ of $S t$ & mples \\
\hline Short & (Method) & Full & Units & CROL & Limit Agency & Total & $>$ CROL & $>$ DWS \\
\hline $1112-t c$ & & $1,1,1,2$-Tetrachloroethane & ppo & 5 & $\cdots$ & 1 & 0 & $\cdots \cdot$ \\
\hline $1,1,1-T$ & & $1,1,1$-Trichloroethane & ppb & 5 & & 1 & 0 & \\
\hline $1122-\mathrm{tc}$ & & 1,1,2,2-Tetrachloroethane & ppb & 5 & & 1 & 0 & \\
\hline 1,1,2-T & & 1,1,2-Trichloroethane & ppb & 5 & & 1 & 0 & \\
\hline $1,1-01 C$ & & 1,1-Dichloroethane & ppb & 5 & & 1 & 0 & \\
\hline DICETHY & & 1,1-Dichloroethene & ppb & 5 & & 1 & 0 & \\
\hline 123-trp & & 1,2,3-Trichloropropane & ppb & 5 & & 1 & 0 & \\
\hline TETRCHB & & $1,2,4,5-$ Tetrachlorobenzene & ppob & 10 & & 1 & 0 & \\
\hline TRICHLB & & 1,2,4-Trichlorobenzene & ppb & 10 & & 1 & 0 & \\
\hline $\begin{array}{l}\text { DIBRCHL } \\
\text { DIBRETH }\end{array}$ & & 1,2-Dibromo-3-chloropropane & ppob & $\begin{array}{l}5 \\
5\end{array}$ & & 1 & $\begin{array}{l}0 \\
0\end{array}$ & \\
\hline $\begin{array}{l}\text { DIBRETH } \\
\text { 12-cben }\end{array}$ & & $\begin{array}{l}\text { 1,2-0ibromoe thane } \\
\text { 1,2-Dichlorobenzene }\end{array}$ & ppob & 10 & & 1 & 0 & \\
\hline $1,2-D I C$ & & 1,2-Dichloroethane & ppob & 5 & & 1 & 0 & \\
\hline 12-DCE & & 1,2-Dichloroethene & ppb & 5 & & 1 & 0 & \\
\hline DICPANE & & 1,2-Dichloropropane & ppob & 5 & & 1 & 0 & \\
\hline 13-dben & & 1,3-0 ich lorobenzene & ppb & 10 & & 1 & 0 & \\
\hline 14-dben & & 1,4-Dichlor obenzene & ppb & 10 & & 1 & 0 & \\
\hline DIOXANE & & 1,4-Dioxane & ppb & 200 & & 1 & 0 & \\
\hline NAPHOUI & & 1,4-Naph toquinone & ppb & 10 & & 1 & 0 & \\
\hline 1BUTYN & & 1-Butanol & ppb & 1000 & & 1 & 0 & \\
\hline 1-napho & & 1-Naphthylamine & ppb & 10 & & 1 & 0 & \\
\hline TETRCHP & & $2,3,4,6$ - Tetrachlorophenol & ppo & 10 & & 1 & 0 & \\
\hline 2378TCD & & $2,3,7,8-T C D D$ & ppb & .005 & & 1 & 0 & \\
\hline $2,4,5-T$ & & $2,4,5-T$ & ppo & 2 & & 3 & 0 & \\
\hline $245-\operatorname{trp}$ & & $2,4,5$ - Trichlorophenol & ppob & 10 & & 1 & 0 & \\
\hline $246-\operatorname{trp}$ & 19 & 2,4,6-Trichlorophenol & ppb & 10 & & 1 & 0 & \\
\hline $246-\operatorname{trp}$ & 30 & 2,4,6-Trichlorophenol & ppob & 5 & & 3 & 0 & \\
\hline 24 -dchp & 19 & 2,4-Dichlorophenol & ppo & 10 & & 1 & 0 & \\
\hline $24-d c h p$ & 30 & 2,4-0 ichlorophenol & ppob & 5 & & 3 & 0 & \\
\hline DIMPHEN & 19 & 2,4-D ime thy (phenol & ppb & 10 & & 1 & 0 & \\
\hline DIMPHEN & 30 & 2,4-0 imethylphenol & ppb & 5 & & 3 & 0 & \\
\hline DINPHEN & 19 & 2,4-Dinitrophenol & ppb & 50 & & 1 & 0 & \\
\hline DINPHEN & 30 & 2,4-D initrophenol & ppb & 150 & & 3 & 0 & \\
\hline 24 -dint & & $2,4-0$ initrotoluene & ppo & 10 & & 1 & 0 & \\
\hline 26-dchp & 19 & 2,6-Dichlorophenol & ppb & 10 & & 1 & 0 & \\
\hline 26-dchp & 30 & 2,6-0 ichlorophenol & ppob & 5 & & 3 & 0 & \\
\hline 26-dint & & 2,6-Dinitrotoluene & ppb & 10 & & 1 & 0 & \\
\hline ACEFENE & & 2-Acetyl aminof luorene & ppb & 10 & & 1 & 0 & \\
\hline CHLNAPH & & 2-Chloronaph thalene & ppob & 10 & & 1 & 0 & \\
\hline CHLPHEN & 19 & 2-Chlorophenol & ppb & 10 & & 1 & 0 & \\
\hline CHLPHEN & 30 & 2-Chlorophenol & ppb & 5 & & 3 & 0 & \\
\hline 2HEXANO & & 2-Hexanone & ppob & 50 & & 1 & 0 & \\
\hline 2MENAPH & & 2-Methyl naph thal ene & ppb & 10 & & 1 & 0 & \\
\hline 2METHPH & & 2-Methylphenol & ppb & 10 & & 1 & 0 & \\
\hline 2-naphe & & 2-Naphthylamine & ppb & 10 & & 1 & 0 & \\
\hline ONITANI & & 2-Nitroaniline & ppb & 50 & & 1 & 0 & \\
\hline 2NITPH & 19 & 2-Nitrophenol & ppo & 10 & & 1 & 0 & \\
\hline 2NITPH & 30 & 2-Nitrophenol & ppb & 5 & & 3 & 0 & \\
\hline PICOLIN & & 2-Picoline & ppb & 10 & & 1 & 0 & \\
\hline BUTDINP & 30 & 2-sec-Butyl-4,6-dinitrophenol (DN & ppb & 5 & & 3 & 0 & \\
\hline BUTDINP & 49 & 2-sec-Butyl-4,6-dinitrophenol (DN & ppb & 1 & & 3 & 0 & \\
\hline DICHBEN & & 3,3'-Dichlorobenzidine & ppb & 20 & & 1 & 0 & \\
\hline DIMEYLB & & 3,3'-D ime thylbenzidine & ppo & 10 & & 1 & 0 & \\
\hline
\end{tabular}


Table 16-12. Constituent List and Summary of Results for the Single-Shell

Tank Waste Management Area S-SX Data for Reporting Period

July 1 through September 30, 1993. (sheet 3 of 6)

\begin{tabular}{|c|c|c|c|c|c|c|c|c|}
\hline \multicolumn{4}{|c|}{ Constituent Name } & \multicolumn{2}{|r|}{ Dus } & \multicolumn{3}{|c|}{ Number of Semples } \\
\hline Short & (Method) & Full & Units & CROL & Limit Agency & Total & $>\mathrm{CROL}$ & $>$ DWS \\
\hline$\ldots \ldots$ & $\ldots \ldots$ & $\ldots \ldots \ldots$ & $\ldots \ldots$ & $\cdots \cdots$ & $\ldots \ldots \ldots$ & $\ldots \ldots$ & $\cdots \cdots$ & $\cdots$ \\
\hline METCHAM & & 3-Methylcholanthrene & ppo & 10 & & 1 & 0 & \\
\hline MNITANI & & 3-Nitroaniline & ppb & 50 & & 1 & 0 & \\
\hline DDO & & $4,4^{\prime}-000$ & ppo & .1 & & 11 & 0 & \\
\hline DDE & & $4,4^{\prime}-$ DDE & ppb & .05 & & 11 & 0 & \\
\hline DDT & & $4,4^{\prime}-0 D T$ & ppo & .1 & & 11 & 0 & \\
\hline 460N2MP & 19 & 4,6-Dinitro-2-methylphenol & ppb & 50 & & 1 & 0 & \\
\hline 460N2MP & 30 & 4,6-D initro-2-methylphenol & ppb & 200 & & 3 & 0 & \\
\hline AMINOYL & & 4-Aminobiphenyl & ppb & 10 & & 1 & 0 & \\
\hline BROPHEN & & 4-Bromophenylphenyl ether & ppob & 10 & & 1 & 0 & \\
\hline CHLCRES & 19 & 4-Chloro-3-methylphenol & ppb & 20 & & 1 & 0 & \\
\hline CHLCRES & 30 & 4-Chloro-3-methylphenol & ppo & 5 & & 3 & 0 & \\
\hline CHLANIL & & 4-Chloroaniline & ppo & 20 & & 1 & 0 & \\
\hline 4CPPETH & & 4-Chlorophenylphenyl ether & ppb & 10 & & 1 & 0 & \\
\hline MIBK & & 4-Methyl-2-pent anone & ppo & 50 & & 1 & 0 & \\
\hline ४METHPH & & 4-Methylphenol & ppob & 10 & & 1 & 0 & \\
\hline NITRANI & & 4-Nitroaniline & ppb & 50 & & 1 & 0 & \\
\hline NITPHEN & 19 & 4-Nitrophenol & ppb & 50 & & 1 & 0 & \\
\hline NITPHEN & 30 & 4-Nitrophenol & ppb & 30 & & 3 & 0 & \\
\hline 4NITOUI & & 4-Nitroquinol ine-1-oxide & ppo & 10 & & 1 & 0 & \\
\hline NITRTOL & & 5-Nitro-o-toluidine & ppo & 10 & & 1 & 0 & \\
\hline DIMBENZ & & 7, 12-D imethylbenz [a] anthracene & ppo & 10 & & 1 & 0 & \\
\hline ACENAPH & & Acenaph thene & ppb & 10 & & 1 & 0 & \\
\hline ACENATL & & Acenaph thylene & ppb & 10 & & 1 & 0 & \\
\hline ACETONE & & Acetone & ppb & 100 & & 1 & 0 & \\
\hline ACETILE & & Acetonitrile & ppob & 200 & & 1 & 0 & \\
\hline ACETOPH & & Acetophenone & ppb & 10 & & 1 & 0 & \\
\hline ACROLIN & & Acrolein & ppo & 5 & & 1 & 0 & \\
\hline ACRYILE & & Acrylonitrile & ppo & 5 & & 1 & 0 & \\
\hline ALDRIN & & Aldrin & ppo & .05 & & 11 & 0 & \\
\hline DIMPHAM & & Alpha, alpha-D imethyl phenethyl ami & ppb & 10 & & 1 & 0 & \\
\hline a-BHC & & Alpha-BHC & ppb & .05 & & 11 & 0 & \\
\hline ALUMNUM & & Aluminum & ppb & 200 & & 3 & 3 & \\
\hline FALUMIN & & Aluminum & ppb & 200 & & 3 & 0 & \\
\hline AMMONIU & & Ammonium ion & ppb & 100 & & 3 & 0 & \\
\hline ANILINE & & Aniline & ppb & 10 & & 1 & 0 & \\
\hline ANTHRA & & Antínracene & ppb & 10 & & 1 & 0 & \\
\hline ANTIONY & & Ant imony & ppb & 200 & & 3 & 0 & \\
\hline FANTIMO & & Antimony, filtered & ppob & 200 & & 3 & 0 & \\
\hline$S B-125$ & & Ant imony-125 & $\mathrm{pCi} / \mathrm{L}$ & & & 5 & 1 & \\
\hline ARAMITE & & Aramite & ppb & 10 & & 1 & 0 & \\
\hline AR 1016 & & Aroclor-1016 & ppb & 1 & & 1 & 0 & \\
\hline AR1221 & & Aroclor-1221 & ppb & 1 & & 1 & 0 & \\
\hline AR 1232 & & Aroclor-1232 & ppb & 1 & & 1 & 0 & \\
\hline AR 1242 & & Aroclor-1242 & ppb & 1 & & 1 & 0 & \\
\hline AR1248 & & Aroclor- 1248 & ppo & 1 & & 1 & 0 & \\
\hline AR1254 & & Aroclor -1254 & ppb & 1 & & 1 & 0 & \\
\hline AR1260 & & Aroclor -1260 & ppo & 1 & & 1 & 0 & \\
\hline BENZENE & & Benzene & ppb & 5 & & 1 & 0 & \\
\hline BENZAAN & & Benzo( $(a)$ anthracene & ppb & 10 & & 1 & 0 & \\
\hline BENZOPY & & Benzo(a)pyrene & ppb & 10 & & 1 & 0 & \\
\hline BENZBFL & & Benzo(b) fluoranthene & ppb & 10 & & 1 & 0 & \\
\hline BGHIPER & & Benzo(ghi)perylene & ppb & 10 & & 1 & 0 & \\
\hline BNZKFLU & & Benzo $(k)$ f luor anthene & ppb & 10 & & 1 & 0 & \\
\hline BENZOTH & & Benzothiazole & pob & 10 & & 1 & 0 & \\
\hline BENZALC & & Benzyl alcohol & ppb & 20 & & 1 & 0 & \\
\hline BERYLUM & & Beryllium & ppb & 3 & & 3 & 0 & \\
\hline FBERYLL & & Beryllium, filtered & pp'o & 3 & & 3 & 0 & \\
\hline b-BHC & & Beta-BHC & ppb & .05 & & 11 & 0 & \\
\hline
\end{tabular}


Table 16-12. Constituent List and Summary of Results for the Single-Shell Tank Waste Management Area S-SX Data for Reporting Period July 1 through September 30, 1993. (sheet 4 of 6)

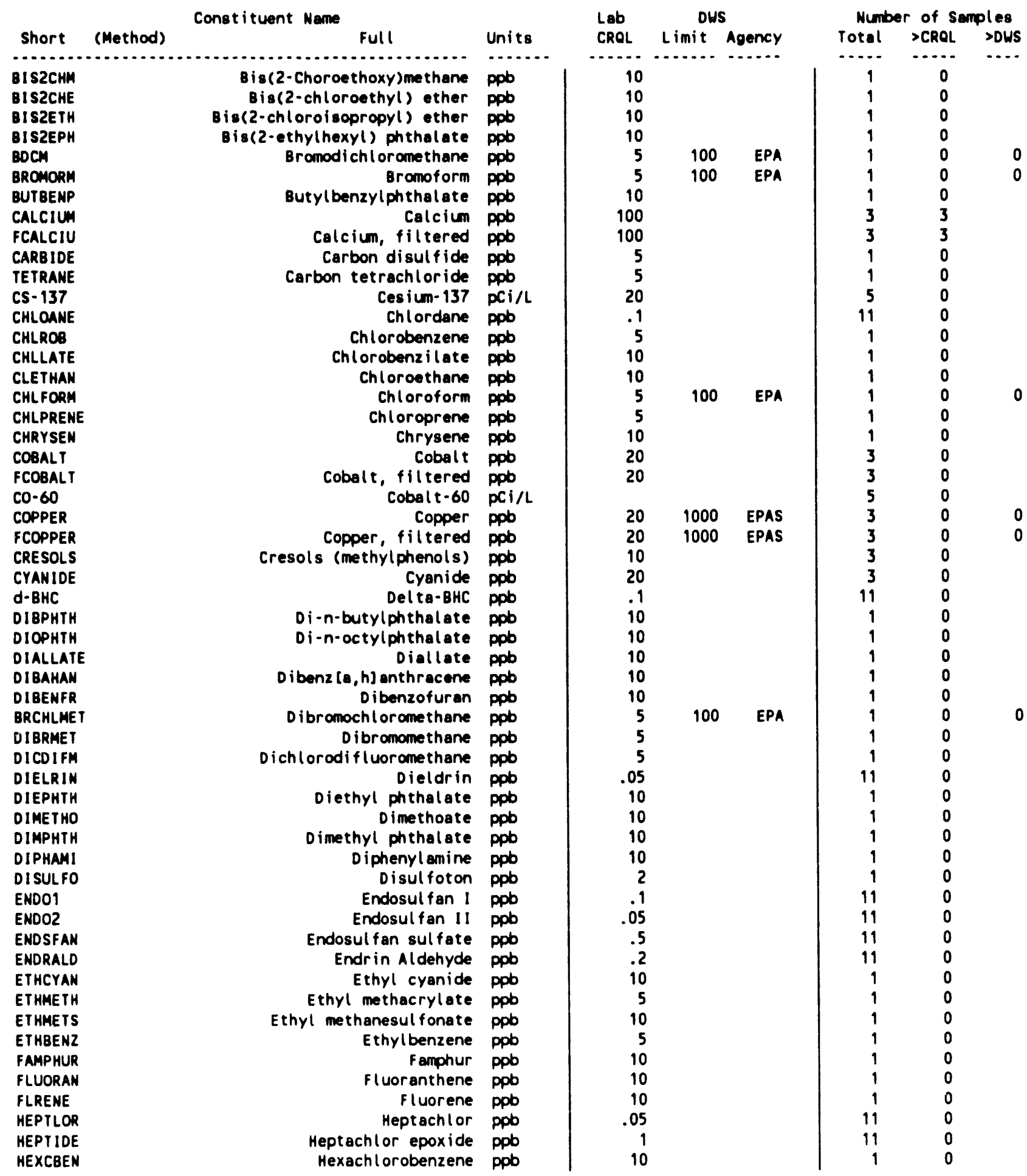


Table 16-12. Constituent List and Summary of Results for the Single-Shell Tank Waste Management Area S-SX Data for Reporting Period July 1 through September 30, 1993. (sheet 5 of 6)

\begin{tabular}{|c|c|c|c|c|c|c|c|c|}
\hline \multicolumn{4}{|c|}{ Constituent Name } & \multicolumn{2}{|r|}{ DWS } & \multicolumn{3}{|c|}{ Number of Semples } \\
\hline Short & (Method) & Full & Units & CROL & Limit Agency & Total & $>$ CRQL & $>$ DWS \\
\hline$\ldots \ldots \ldots$ & $\cdots$ & (n, & $\ldots \ldots$ & $\ldots . .$. & $\cdots \ldots \ldots$ & $\cdots .$. & $\ldots .$. & $\ldots$ \\
\hline HEXCBUT & & Hexach l orobut adiene & ppb & 10 & & 1 & 0 & \\
\hline HEXCCYC & & Hexachlorocyc lopent adiene & ppo & 10 & & 1 & 0 & \\
\hline HEXCETH & & Hexachi oroethane & ppo & 10 & & 1 & 0 & \\
\hline HEXACHL & & Hexachlorophene & ppb & 10 & & 1 & 0 & \\
\hline HEXAENE & & Hexach lor opropene & ppb & 10 & & 1 & 0 & \\
\hline LHYDRAZ & & Hydrazine & ppob & 30 & & 3 & 0 & \\
\hline INDENOP & & Indeno $(1,2,3-\mathrm{cd})$ pyrene & ppb & 10 & & 1 & 0 & \\
\hline $1-129$ & & Iodine-129 & $\mathrm{PCi} / \mathrm{L}$ & 1 & & 11 & 1 & \\
\hline I SOBUTY & & Isobutyl alcohol & ppb & 200 & & 1 & 0 & \\
\hline ISOORIN & & Isodrin & ppo & 10 & & 1 & 0 & \\
\hline ISOPHER & & I sophorone & ppob & 10 & & 1 & 0 & \\
\hline ISOSOLE & & Isosafrole & ppb & 10 & & 1 & 0 & \\
\hline KEPONE & & Kepone & ppb & 10 & & 1 & 0 & \\
\hline KEROSEN & & Kerosene & ppb & 10000 & & 1 & 0 & \\
\hline MAGNES & & Magnesium & ppo & 100 & & 3 & 3 & \\
\hline FMAGNES & & Magnesium, filtered & ppo & 100 & & 3 & 3 & \\
\hline METHACR & & Methacrylonitrile & ppb & 5 & & 1 & 0 & \\
\hline METHAPY & & Methapyrilene & ppb & 10 & & 1 & 0 & \\
\hline IODONET & & Methyl lodide & ppb & 5 & & 1 & 0 & \\
\hline METHBRO & & Methyl bromide & ppb & 10 & & 1 & 0 & \\
\hline METHCHL & & Methyl chloride & ppb & 10 & & 1 & 0 & \\
\hline METHONE & & Methyl ethyl ketone & ppb & 100 & & 1 & 0 & \\
\hline METACRY & & Methyl methacrylate & ppb & $\mathbf{5}$ & & 1 & 0 & \\
\hline METMSUL & & Methyl methenesulfonate & ppb & 10 & & 1 & 0 & \\
\hline METHPAR & & Methyl parathion & ppb & .5 & & 1 & 0 & \\
\hline METHYCH & & Methylene chloride & ppb & 5 & & 1 & 0 & \\
\hline DIPRNIT & & N-Nitroso-di-n-dipropylamine & ppb & 10 & & 1 & 0 & \\
\hline NNIBUTY & & N-Nitrosodi-n-butylamine & ppb & 10 & & 1 & 0 & \\
\hline NHIDIEY & & $N$-Nitrosodiethylanine & ppb & 10 & & 1 & 0 & \\
\hline NNIDIME & & N-Mitrosodimethylanine & ppb & 10 & & 1 & 0 & \\
\hline NNO IPHA & & N-Nitrosodiphenylamine & ppb & 10 & & 1 & 0 & \\
\hline NNIMETH & & N-Nitrosomethylethylamine & ppb & 10 & & 1 & 0 & \\
\hline NNIMORP & & $N$-Nitrosomorphol ine & ppb & 10 & & 1 & 0 & \\
\hline NNIPIPE & & N-Nitrosopiperidine & ppb & 10 & & 1 & 0 & \\
\hline NAPHTHA & & Naphthalene & ppo & 10 & & 1 & 0 & \\
\hline NICKEL & & Nickel & ppb & 30 & & 3 & 1 & \\
\hline FNICKEL & & Nickel, filtered & ppb & 30 & & 3 & 0 & \\
\hline NI TBENZ & & Nitrobenzene & ppb & 10 & & 1 & 0 & \\
\hline NITRPYR & & Nitrosopyrrolidine & ppb & 10 & & 1 & 0 & \\
\hline TRIPHOS & & $0,0,0$-Triethyl phosphorothioate & ppo & 10 & & 1 & 0 & \\
\hline DIPHOS & & 0,0-diethyl0-2-pyraz inylphosphor & ppb & 10 & & 1 & 0 & \\
\hline PCDDS & & PCDD8 & ppb & .01 & & 1 & 0 & \\
\hline PCDFS & & PCOF 8 & ppo & .01 & & 1 & 0 & \\
\hline PARATHI & & Parathion & ppb & 10 & & 1 & 0 & \\
\hline PENTCHB & & Pentachlorobenzene & ppb & 10 & & 1 & 0 & \\
\hline PENTACH & & Pentachloroethane & ppo & 5 & & 1 & 0 & \\
\hline PENTCHN & & Pentachloronitrobenzene (PCNB) & ppb & 10 & & 1 & 0 & \\
\hline PENTCHP & 19 & Pentachl orophenol & ppb & 50 & & 1 & 0 & \\
\hline PENTCHP & 30 & Pent achl orophenol & ppb & 100 & & 3 & 0 & \\
\hline PERCHLO & & Perchlorate & ppb & 500 & & 3 & 0 & \\
\hline PHENTIN & & Phenacet in & ppb & 10 & & 1 & 0 & \\
\hline PHENANT & & Phenanthrene & ppb & 10 & & 1 & 0 & \\
\hline PHORATE & & Phorate & ppb & 2 & & 1 & 0 & \\
\hline PU-238 & & Plutonium-238 & pcill & .1 & & 3 & 0 & \\
\hline PU39-40 & & Plutonium-239/40 & $\mathrm{pCi} / \mathrm{L}$ & .1 & & 3 & 0 & \\
\hline POTASUM & & Potassium & ppb & 300 & & 3 & 3 & \\
\hline FPOTASS & & Potassium, filtered & ppb & 300 & & 3 & 3 & \\
\hline
\end{tabular}




\section{Table 16-12. Constituent List and Summary of Results for the Single-Shell Tank Waste Management Area S-SX Data for Reporting Period July 1 through September 30, 1993. (sheet 6 of 6)}

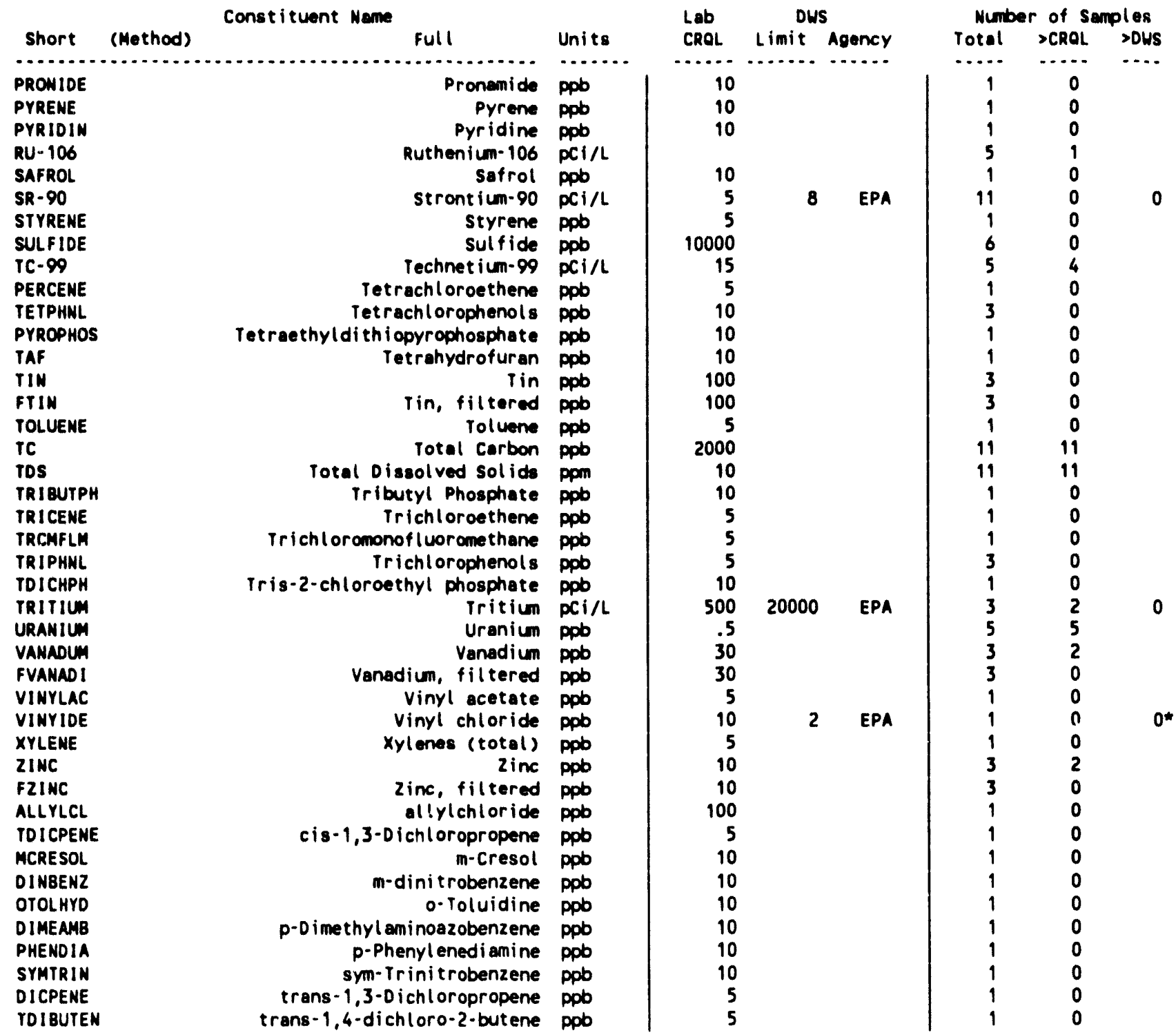

For explanation of this table, see Section 1.4 of report. 
Table 16-13. Constituents with at Least One Value Above the CRQL for the Single-Shel1 Tank Waste Management Area S-SX Data for Reporting Period July 1 through September 30, 1993. (sheet 1 of 3)

\begin{tabular}{|c|c|c|c|c|c|c|}
\hline $\begin{array}{l}\text { Well } \\
\text { Name }\end{array}$ & $\begin{array}{c}\text { Collection } \\
\text { Date }\end{array}$ & $\begin{array}{l}\text { Semple } \\
\text { Number }\end{array}$ & $\begin{array}{l}\text { ALUMNUM } \\
\text { 34/ppb } \\
200 / .\end{array}$ & $\begin{array}{l}\text { FALUMIN } \\
34 / \text { ppb } \\
200 / .\end{array}$ & $\begin{array}{c}\text { SB-125 } \\
140 / \mathrm{pCi} / \mathrm{L} \\
. /\end{array}$ & $\begin{array}{l}\text { BARIUN } \\
34 / \text { ppb } \\
20 / 1000\end{array}$ \\
\hline$\ldots$ & $\ldots \ldots$ & $\cdots \cdots$ & - & $\cdots$ & 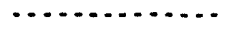 & $\cdots$ \\
\hline $\begin{array}{l}299-W 22-44 \\
299-W 22-44\end{array}$ & $\begin{array}{l}9 / 29 / 93 \\
9 / 29 / 93\end{array}$ & $\begin{array}{l}809650 \\
809654\end{array}$ & 840.00 & $32.50 \mathrm{U}$ & $-26.70 \mathrm{U}$ & 31.00 \\
\hline $\begin{array}{l}299-422-45 \\
299-422-45\end{array}$ & $\begin{array}{l}9 / 29 / 93 \\
9 / 29 / 93\end{array}$ & $\begin{array}{l}809655 \\
809656\end{array}$ & $\begin{array}{l}220.00 \\
330.00\end{array}$ & & $\begin{array}{l}5.17 \mathrm{U} \\
9.24 \mathrm{U}\end{array}$ & $\begin{array}{l}28.00 \\
29.00\end{array}$ \\
\hline $\begin{array}{l}299-W 22-45 \\
299-W 22-45\end{array}$ & $\begin{array}{l}9 / 29 / 93 \\
9 / 29 / 93\end{array}$ & $\begin{array}{l}\text { B09613 } \\
\text { B09614 }\end{array}$ & & $\begin{array}{l}32.50 \mathrm{U} \\
32.50 \mathrm{U}\end{array}$ & & \\
\hline $\begin{array}{l}299-423-15 \\
299-423-15\end{array}$ & $\begin{array}{l}6 / 25 / 93 \\
6 / 25 / 93\end{array}$ & $\begin{array}{l}\text { B08NC9 } \\
\text { BO8NDO }\end{array}$ & & & $\begin{array}{c}18.300 \\
-11.50 \mathrm{va}\end{array}$ & \\
\hline
\end{tabular}

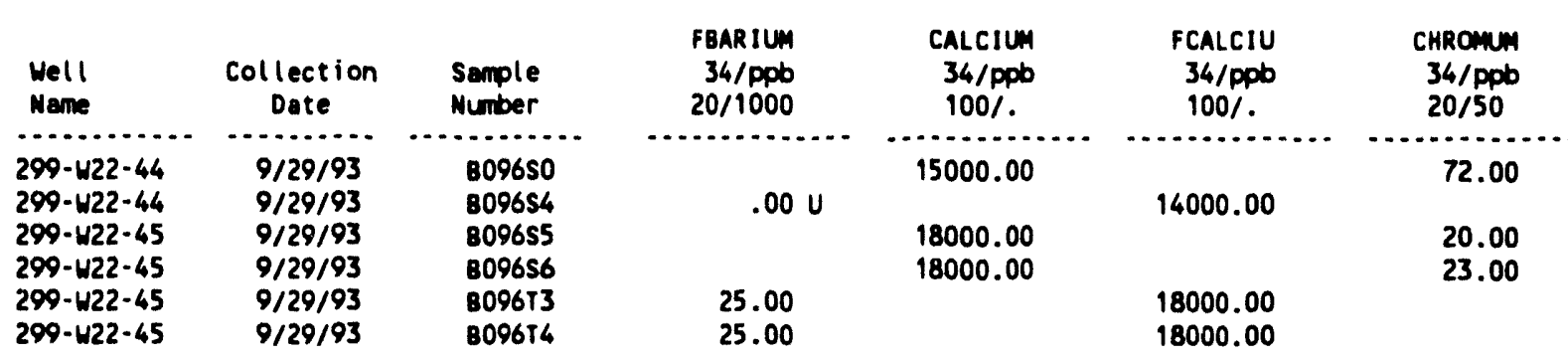

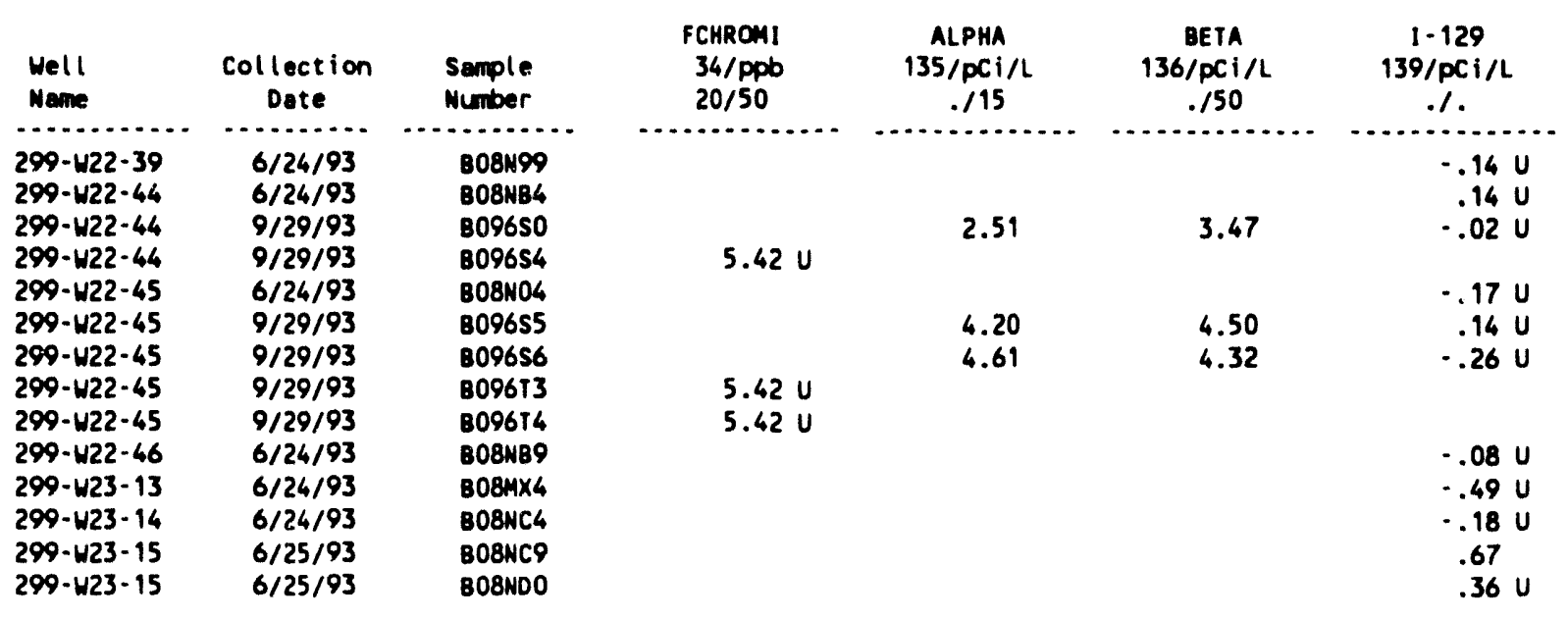

\begin{tabular}{|c|c|c|c|c|c|c|}
\hline $\begin{array}{l}\text { Well } \\
\text { Nane }\end{array}$ & $\begin{array}{c}\text { Collection } \\
\text { Date }\end{array}$ & $\begin{array}{l}\text { Sample } \\
\text { Number }\end{array}$ & $\begin{array}{c}\text { IRON } \\
\text { 34/ppb } \\
20 / 300 \mathrm{~s}\end{array}$ & $\begin{array}{c}\text { FIRON } \\
\text { 34/ppb } \\
20 / 300 \mathrm{~s}\end{array}$ & $\begin{array}{l}\text { MAGNES } \\
34 / \text { ppb } \\
100 / .\end{array}$ & $\begin{array}{l}\text { FMAGNES } \\
34 / \mathrm{ppb} \\
100 /\end{array}$ \\
\hline $299-122-44$ & $9 / 29 / 93$ & $\begin{array}{c}\text { 8096s0 } \\
\text { cons. }\end{array}$ & 1600.00 & 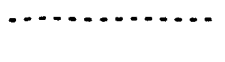 & 4900.00 & $\cdots \ldots$ \\
\hline $\begin{array}{l}299-W 22-44 \\
299-W 22-45 \\
299-W 22-45\end{array}$ & $\begin{array}{l}9 / 29 / 93 \\
9 / 29 / 93 \\
9 / 29 / 93\end{array}$ & $\begin{array}{l}809654 \\
809655 \\
809656\end{array}$ & $\begin{array}{l}690.00 \\
880.00\end{array}$ & $10.30 \mathrm{U}$ & $\begin{array}{l}5600.00 \\
5700.00\end{array}$ & 4600.00 \\
\hline $\begin{array}{l}299-422-45 \\
299-1422-45\end{array}$ & $9 / 29 / 93$ & 809656 & 000.00 & $10.30 \mathrm{U}$ & 3700.00 & 5700.00 \\
\hline $299-122-45$ & $9 / 29 / 93$ & B09614 & & $10.30 \mathrm{U}$ & & 5700.00 \\
\hline
\end{tabular}


Table 16-13. Constituents with at Least One Value Above the CRQL for the Single-She 11 Tank Waste Management Area S-SX Data for Reporting Period July 1 through September 30, 1993. (sheet 2 of 3)

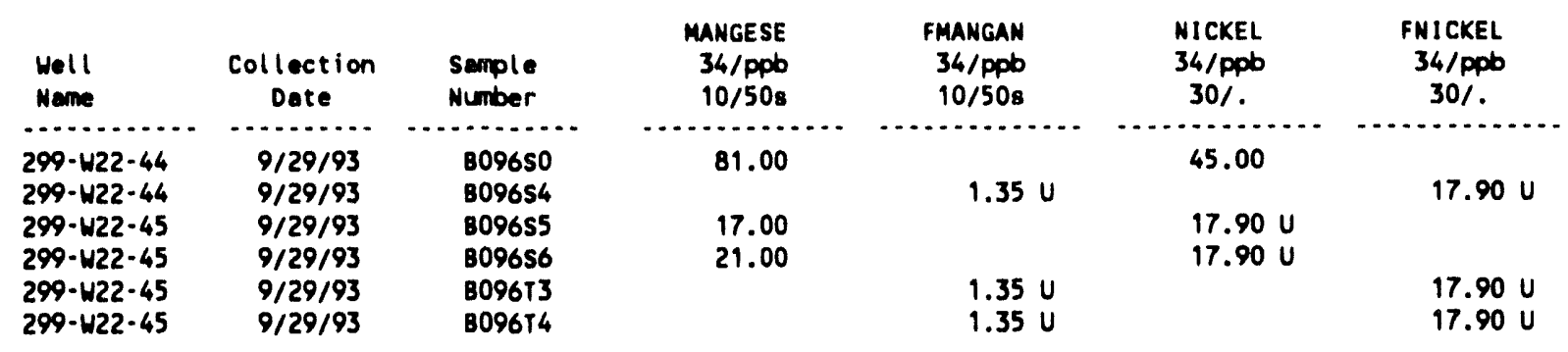

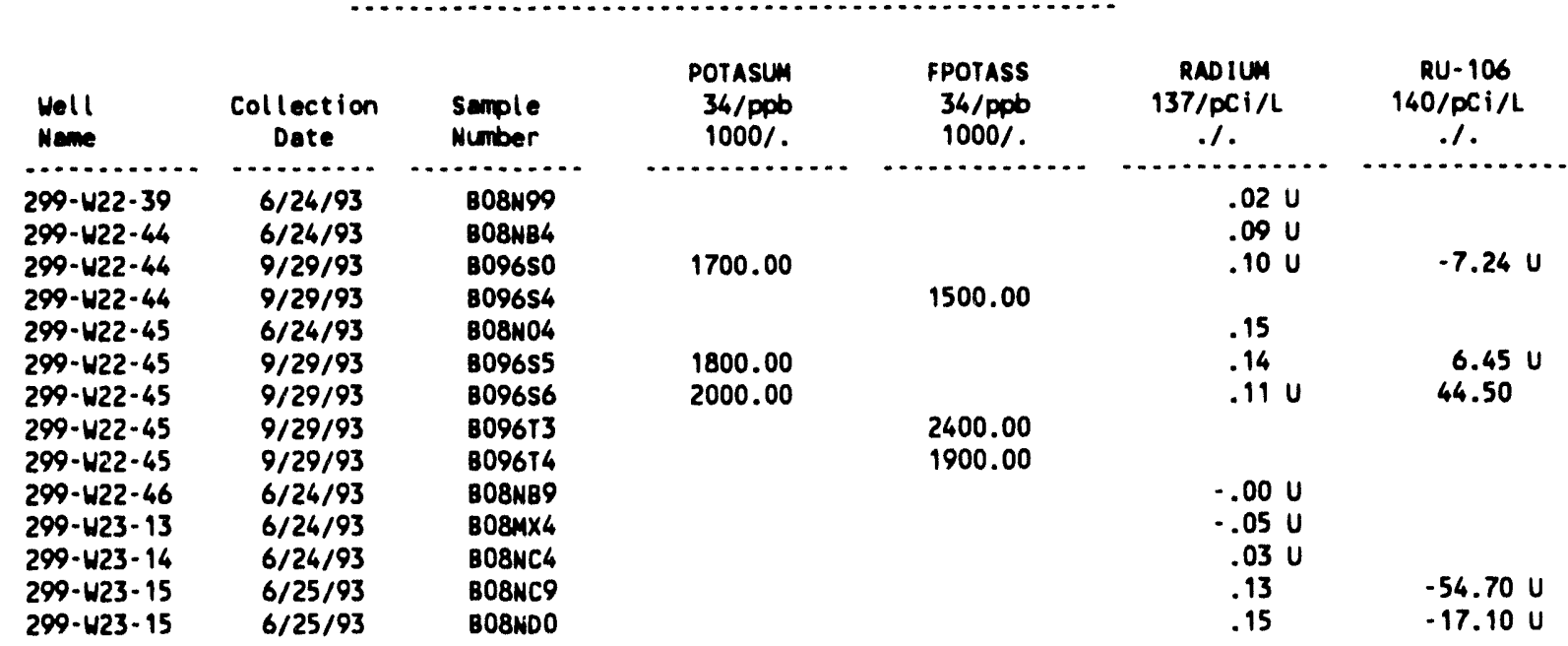

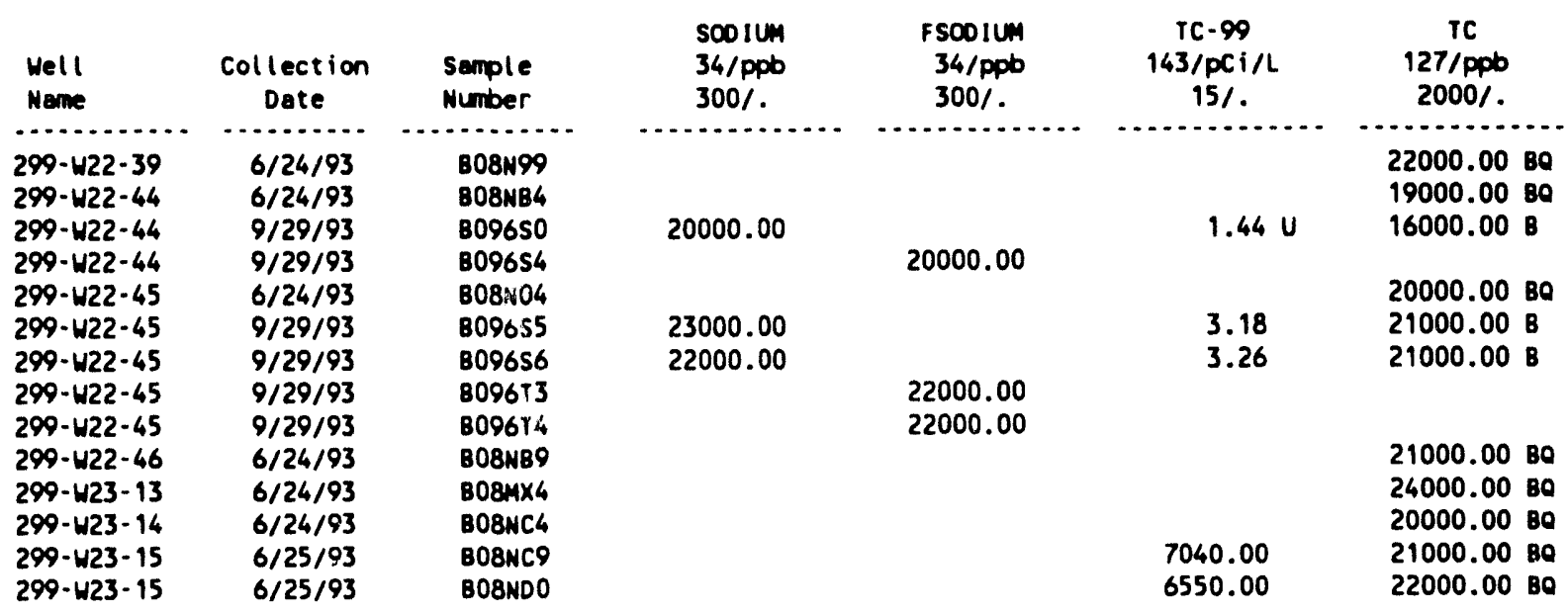


Table 16-13. Constituents with at Least One Value Above the CRQL for the Single-Shel1 Tank Waste Management Area S-SX Data for Reporting Period July 1 through September 30, 1993. (sheet 3 of 3)

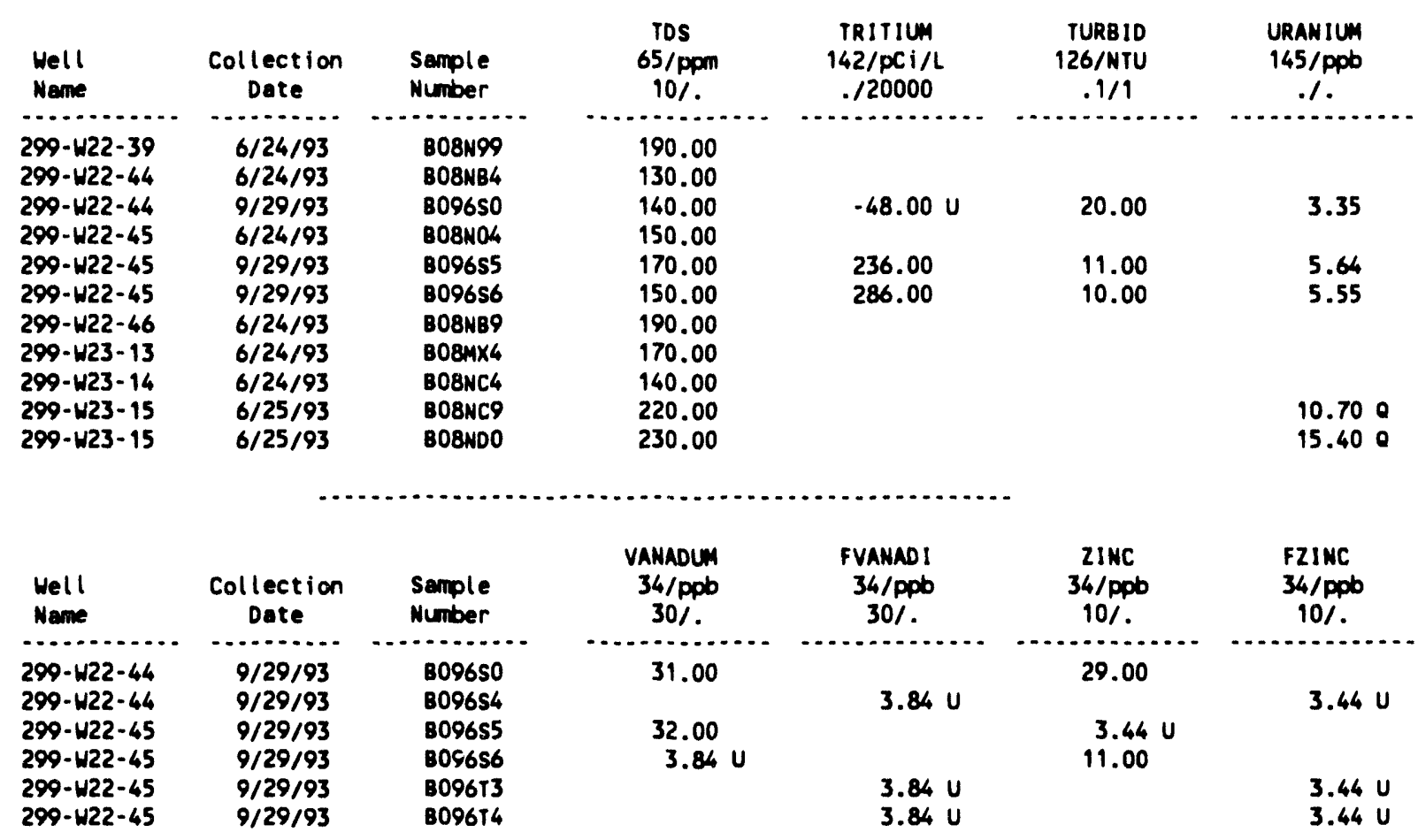

For explanation of this table, see Section 1.4 of report. 
Table 16-14. Contamination Indicator Parameters for the Single-Shell Tank Waste Management Area S-SX Data for Reporting Period July 1 through September 30, 1993.

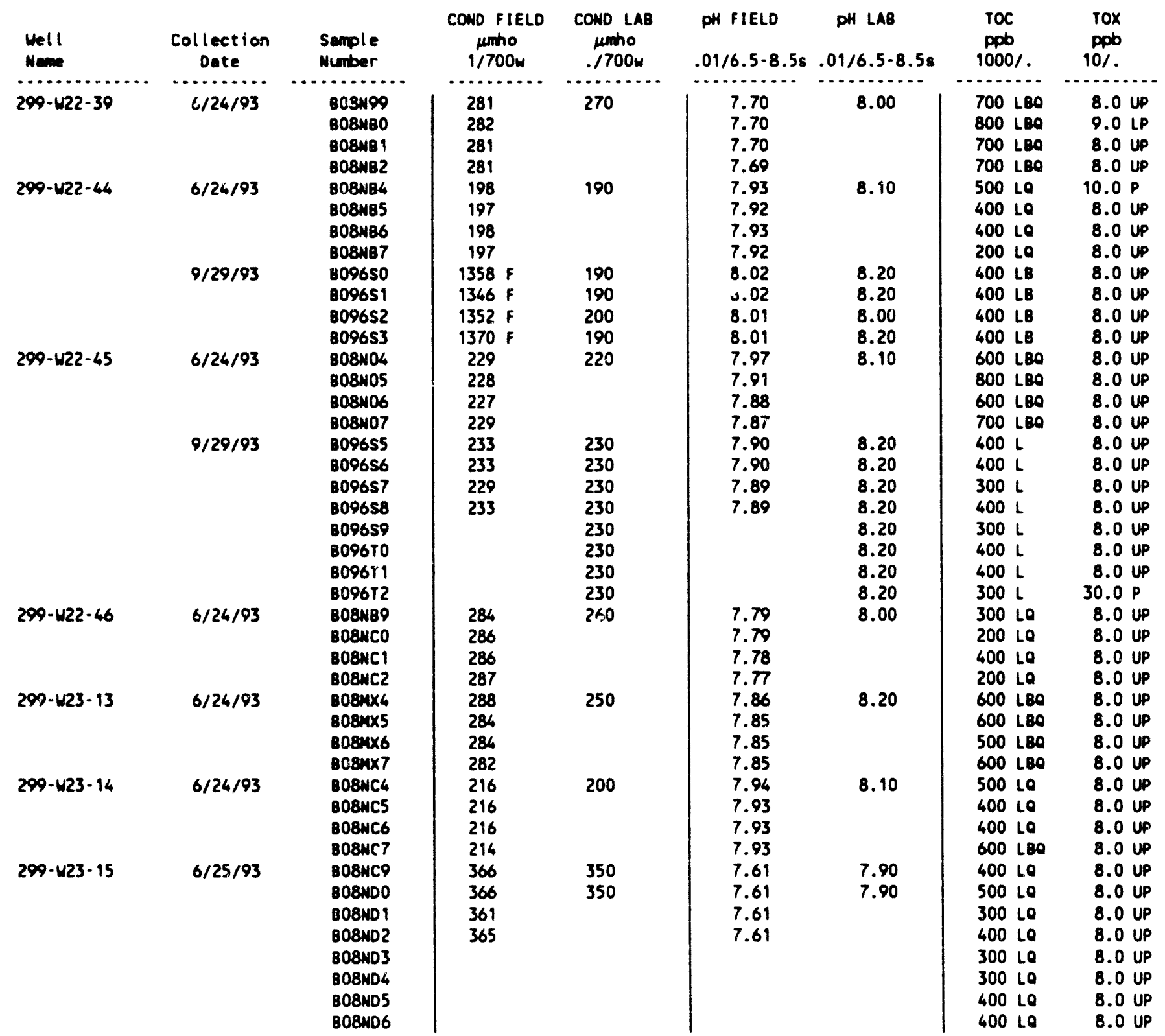

For explenation of this table, see Section 1.4 of report. 
Table 16-15. Constituent List and Summary of Results for the Single-Shell Tank Waste Management Area T Data for Reporting Period July 1 through September 30, 1993. (sheet 1 of 3)

CONTAMINATION INDICATOR PARAMETERS

\begin{tabular}{|c|c|c|c|c|c|c|c|c|c|}
\hline \multirow[b]{2}{*}{ Short } & \multirow[b]{2}{*}{ (Method) } & \multirow[t]{2}{*}{ Constituent Nane } & \multirow[b]{2}{*}{ Units } & \multirow{2}{*}{$\begin{array}{l}\text { Lab } \\
\text { CROL }\end{array}$} & \multicolumn{2}{|c|}{ DWS } & \multicolumn{3}{|c|}{ Number of Semples } \\
\hline & & & & & Limit & Agency & Total & $>$ CROL & $>$ DWS \\
\hline$\ldots \ldots$ & 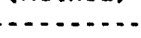 & $\ldots \ldots$ & ...... & $\ldots .$. & $\ldots . .$. & $\ldots . .$. & $\ldots$. & ..... & $\ldots$ \\
\hline CONDUCT & 94 & Conductivity, field & umho & 1 & & & 24 & 24 & \\
\hline CONDUCT & 73 & Conductivity, lab & umho & & & & 15 & 15 & \\
\hline Toc & & Total Organic Carbon & ppob & 1000 & & & 24 & 0 & \\
\hline TOXLOL & & Total Organic Halogen & ppob & 10 & & & 24 & 17 & \\
\hline PH & 93 & pH, tield & & .1 & $6.5-8.5$ & EPAS & 24 & 24 & 0 \\
\hline PH & 125 & pH, lab & & & $6.5 \cdot 8.5$ & EPAS & 15 & 15 & 0 \\
\hline
\end{tabular}

DRINKING WATER PARAMETERS

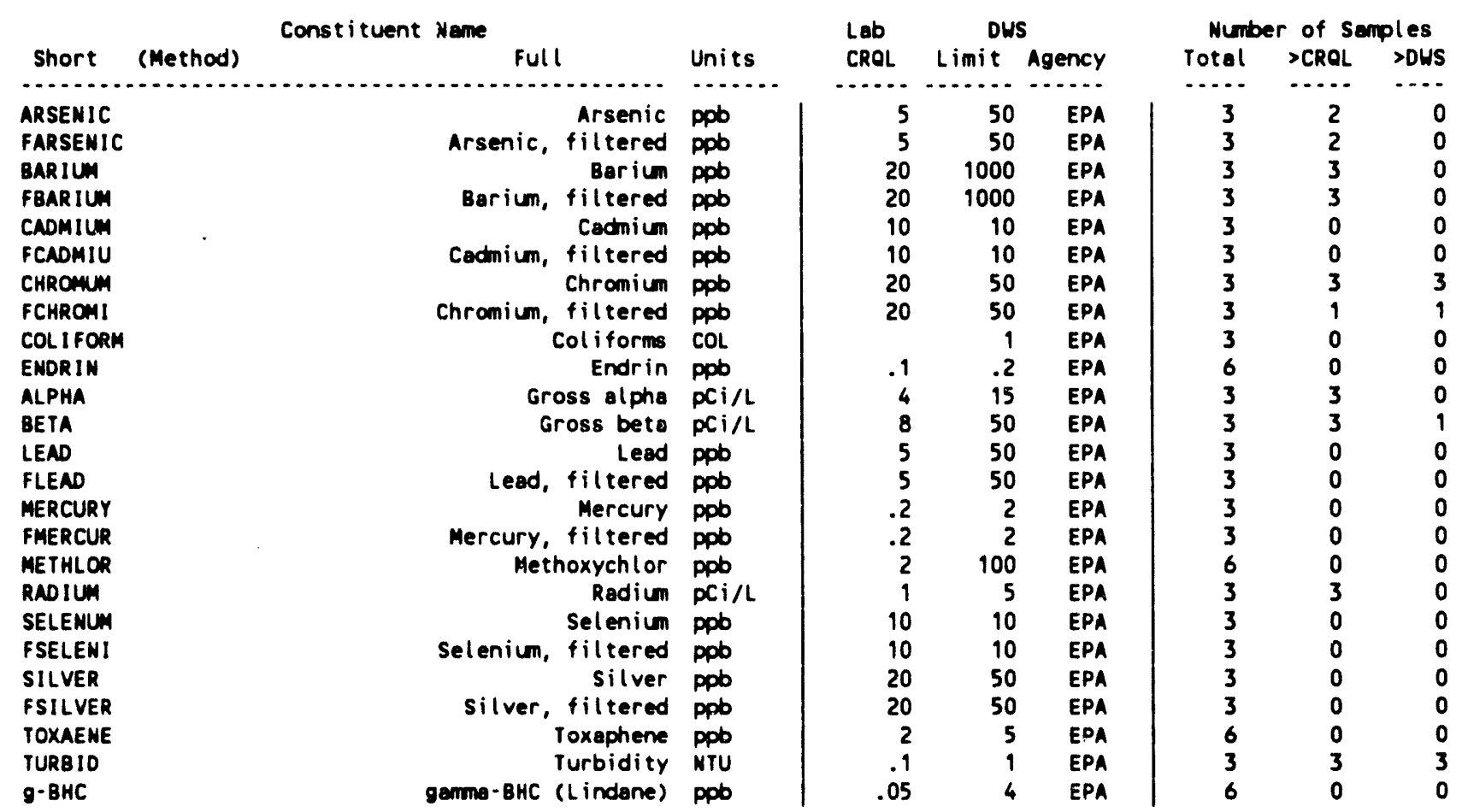

GROUNDWATER QUALITY PARAMETERS

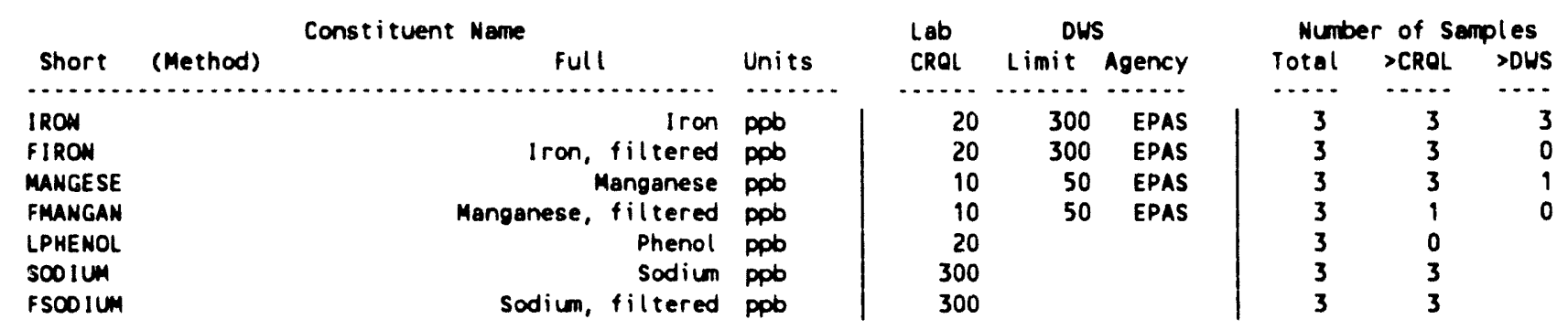


Table 16-15. Constituent List and Summary of Results for the Single-She 11 Tank Waste Management Area T Data for Reporting Period July 1 through September 30, 1993. (sheet 2 of 3)

SITE SPECIFIC AND OTHER CONSTITUENTS

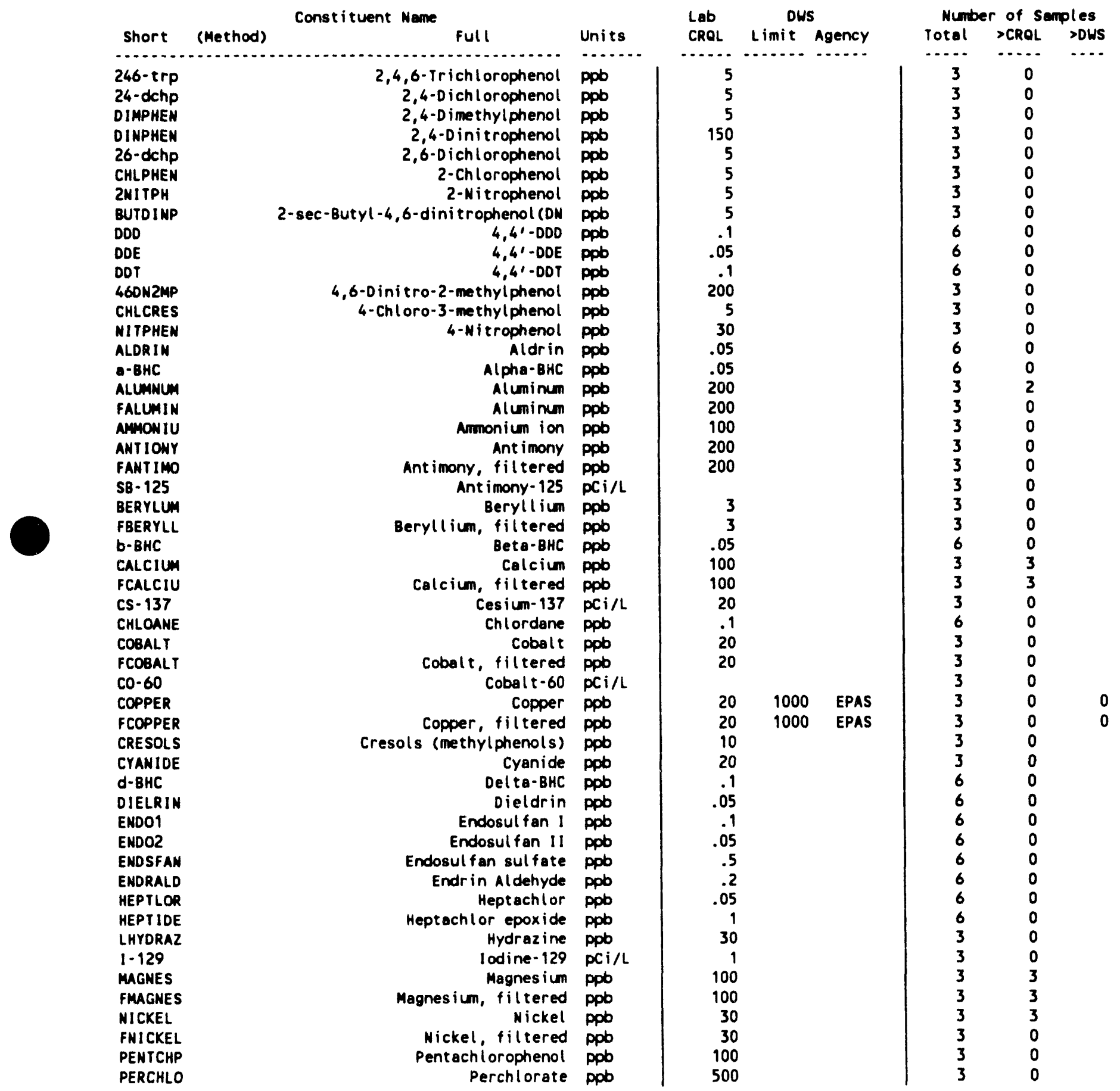


Table 16-15. Constituent List and Summary of Results for the Single-Shell Tank Waste Management Area T Data for Reporting Period July 1 through September 30,1993 . (sheet 3 of 3 )

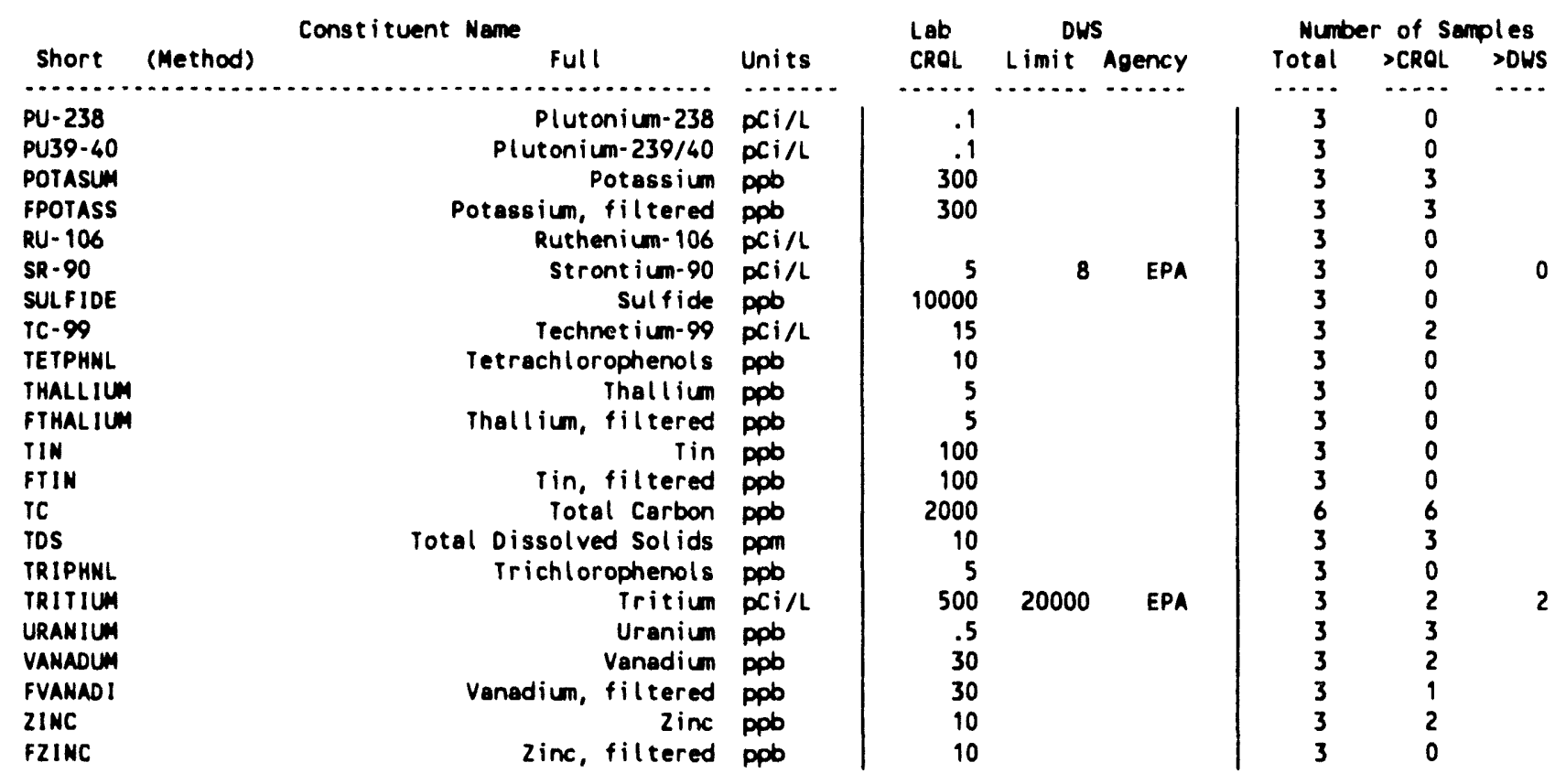

For explanation of this table, see Section 1.4 of report. 
Table 16-16. Constituents with at Least One Value Above the CRQL for the Single-Shell Tank Waste Management Area T Data for Reporting Period July 1 through September 30, 1993. (sheet 1 of 2)

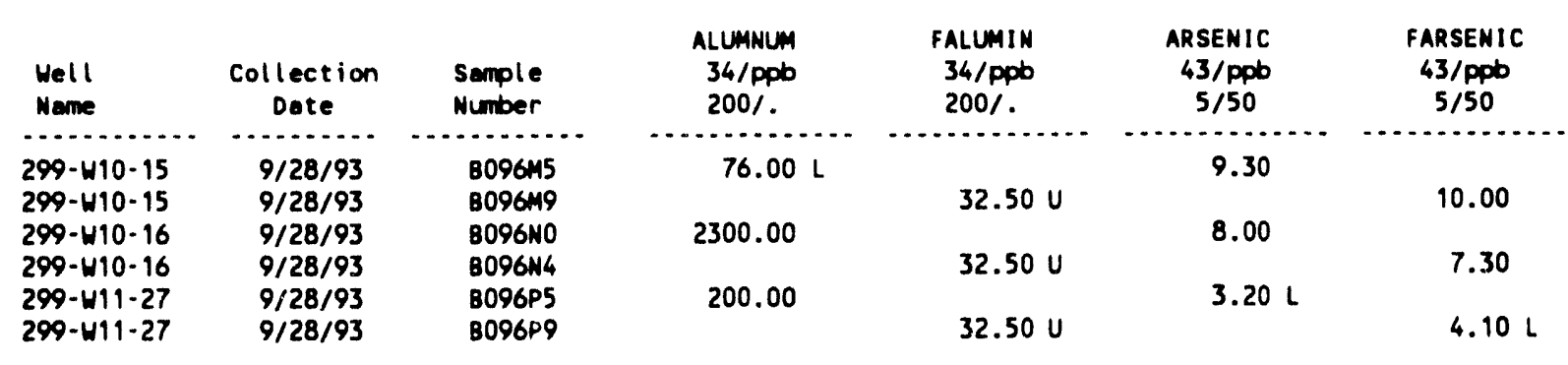

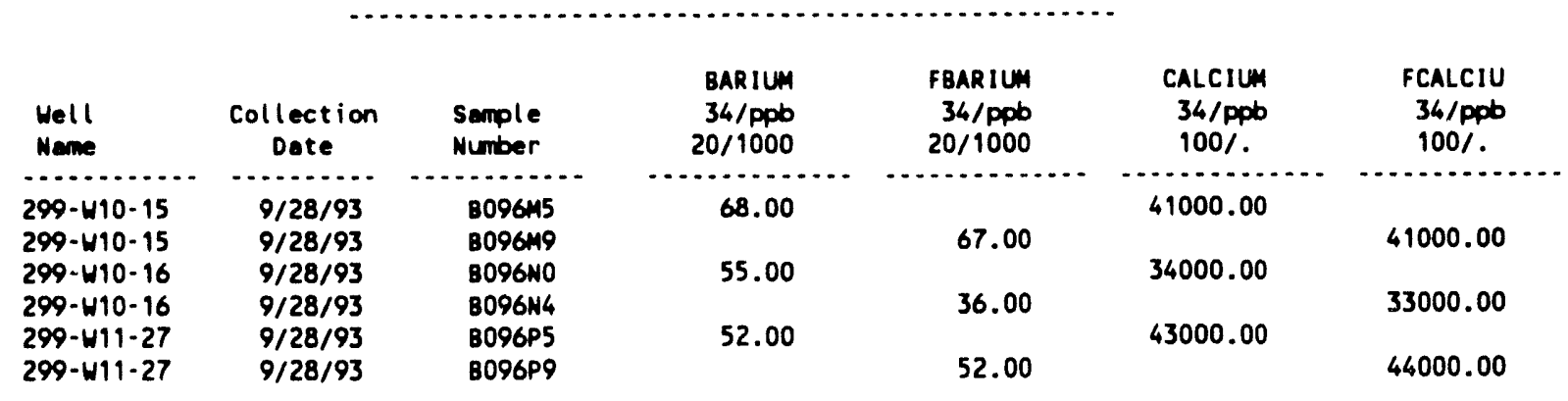

\begin{tabular}{|c|c|c|c|c|c|c|}
\hline $\begin{array}{l}\text { Well } \\
\text { Nene }\end{array}$ & $\begin{array}{c}\text { Collection } \\
\text { Dete }\end{array}$ & $\begin{array}{l}\text { Sample } \\
\text { Number }\end{array}$ & $\begin{array}{l}\text { CHROMUM } \\
34 / \text { ppb } \\
20 / 50\end{array}$ & $\begin{array}{l}\text { FCHROMI } \\
34 / \text { ppb } \\
20 / 50\end{array}$ & $\begin{array}{c}\text { ALPHA } \\
135 / \mathrm{PCi} / \mathrm{L} \\
. / 15\end{array}$ & $\begin{array}{c}\text { BETA } \\
136 / \mathrm{PCi} / \mathrm{L} \\
. / 50\end{array}$ \\
\hline $\begin{array}{l}299-410-15 \\
299-410-15\end{array}$ & $9 / 28 / 93$ & 8096415 & 230.00 & 130.00 & 3.42 & 108.00 \\
\hline $\begin{array}{l}299-\$ 10-16 \\
299-W 10-16\end{array}$ & $\begin{array}{l}9 / 28 / 93 \\
9 / 28 / 93\end{array}$ & $\begin{array}{l}\text { B096NO } \\
\text { B096N4 }\end{array}$ & 230.00 & $5.42 \mathrm{U}$ & 1.95 & 34.10 \\
\hline $\begin{array}{l}299-W 11-27 \\
299-W 11-27\end{array}$ & $\begin{array}{l}9 / 28 / 93 \\
9 / 28 / 93\end{array}$ & $\begin{array}{l}\text { B096P5 } \\
\text { 8096P9 }\end{array}$ & 200.00 & $5.42 \mathrm{U}$ & 2.07 & 3.87 \\
\hline
\end{tabular}

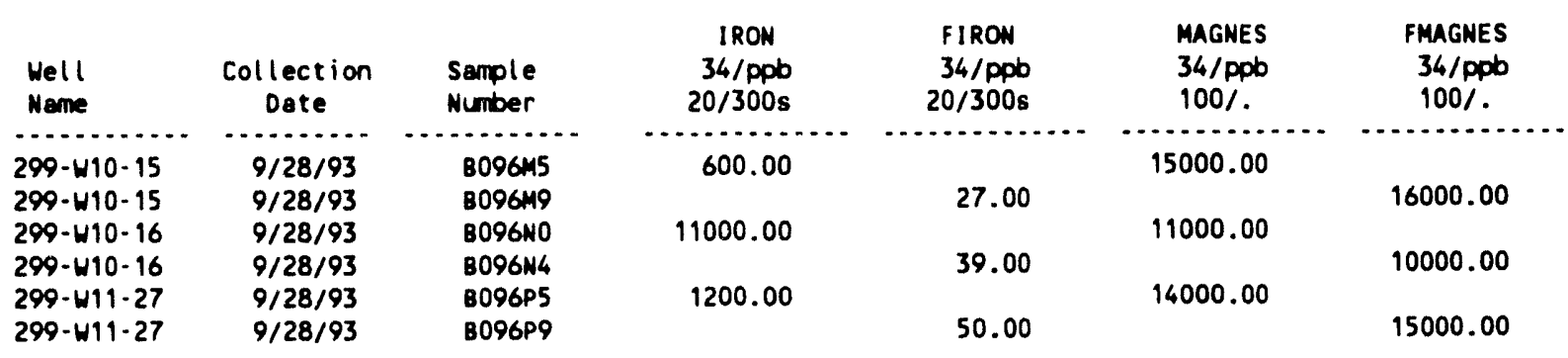

\begin{tabular}{|c|c|c|c|c|c|c|}
\hline $\begin{array}{l}\text { Well } \\
\text { Name }\end{array}$ & $\begin{array}{l}\text { Collection } \\
\text { Date }\end{array}$ & $\begin{array}{l}\text { Sample } \\
\text { Number }\end{array}$ & $\begin{array}{r}\text { MANGESE } \\
34 / \mathrm{ppD} \\
10 / 50 \mathrm{~s}\end{array}$ & $\begin{array}{l}\text { FMANGAN } \\
34 / \text { ppb } \\
10 / 50 \mathrm{~s}\end{array}$ & $\begin{array}{c}\text { NICKEL } \\
34 / \text { PpD } \\
30 \%\end{array}$ & $\begin{array}{c}\text { FNICKEL } \\
34 / \text { ppD } \\
30 \%\end{array}$ \\
\hline $\begin{array}{l}299-w 10-15 \\
299-w 10-15\end{array}$ & $\begin{array}{l}9 / 28 / 93 \\
9 / 28 / 93\end{array}$ & $\begin{array}{l}\text { B096M5 } \\
\text { B096M9 }\end{array}$ & 14.00 & $1.35 \mathrm{U}$ & 48.00 & $17.90 \mathrm{U}$ \\
\hline $\begin{array}{l}299-W 10-16 \\
299-+10-16\end{array}$ & $\begin{array}{l}9 / 28 / 93 \\
9 / 28 / 93\end{array}$ & $\begin{array}{l}\text { B096NO } \\
\text { B096N4 }\end{array}$ & 100.00 & 18.00 & 130.00 & $17.90 \mathrm{U}$ \\
\hline $\begin{array}{l}299-W 11-27 \\
299-W 11-27\end{array}$ & $\begin{array}{l}9 / 28 / 93 \\
9 / 28 / 93\end{array}$ & $\begin{array}{l}\text { B096P5 } \\
\text { B096P9 }\end{array}$ & 31.00 & $1.35 \mathrm{U}$ & 92.00 & $17.90 U$ \\
\hline
\end{tabular}


Table 16-16. Constituents with at Least One Value Above the CRQL for the Single-Shell Tank Waste Management Area T Data for Reporting Period July 1 through September 30, 1993. (sheet 2 of 2)

\begin{tabular}{|c|c|c|c|c|c|c|}
\hline $\begin{array}{l}\text { Well } \\
\text { Nane }\end{array}$ & $\begin{array}{c}\text { Collection } \\
\text { Date }\end{array}$ & $\begin{array}{l}\text { Sample } \\
\text { Number }\end{array}$ & $\begin{array}{c}\text { POTASUM } \\
34 / \mathrm{ppb} \\
1000 / .\end{array}$ & $\begin{array}{l}\text { FPOTASS } \\
34 / \mathrm{ppb} \\
1000 \%\end{array}$ & $\begin{array}{c}\text { RADIUM } \\
\text { 137/PCi/L } \\
. \%\end{array}$ & $\begin{array}{l}\text { SO0IUM } \\
34 / \mathrm{ppb} \\
300 \%\end{array}$ \\
\hline $\begin{array}{l}299-W 10-15 \\
299-W 10-15\end{array}$ & $\begin{array}{l}9 / 28 / 93 \\
9 / 28 / 93\end{array}$ & $\begin{array}{l}\text { B096M5 } \\
\text { B096M9 }\end{array}$ & 4900.00 & 5200.00 & .74 & 180000.00 \\
\hline $\begin{array}{l}299-W 10-16 \\
299-W 10-16\end{array}$ & $\begin{array}{l}9 / 28 / 93 \\
9 / 28 / 93\end{array}$ & $\begin{array}{l}\text { B096NO } \\
\text { B096N4 }\end{array}$ & 4400.00 & 4100.00 & .56 & 110000.00 \\
\hline $\begin{array}{l}299-W 11-27 \\
299-W 11-27\end{array}$ & $\begin{array}{l}9 / 28 / 93 \\
9 / 28 / 93\end{array}$ & $\begin{array}{l}\text { B096P5 } \\
\text { B096P9 }\end{array}$ & 4100.00 & 4100.00 & .72 & 19000.00 \\
\hline
\end{tabular}

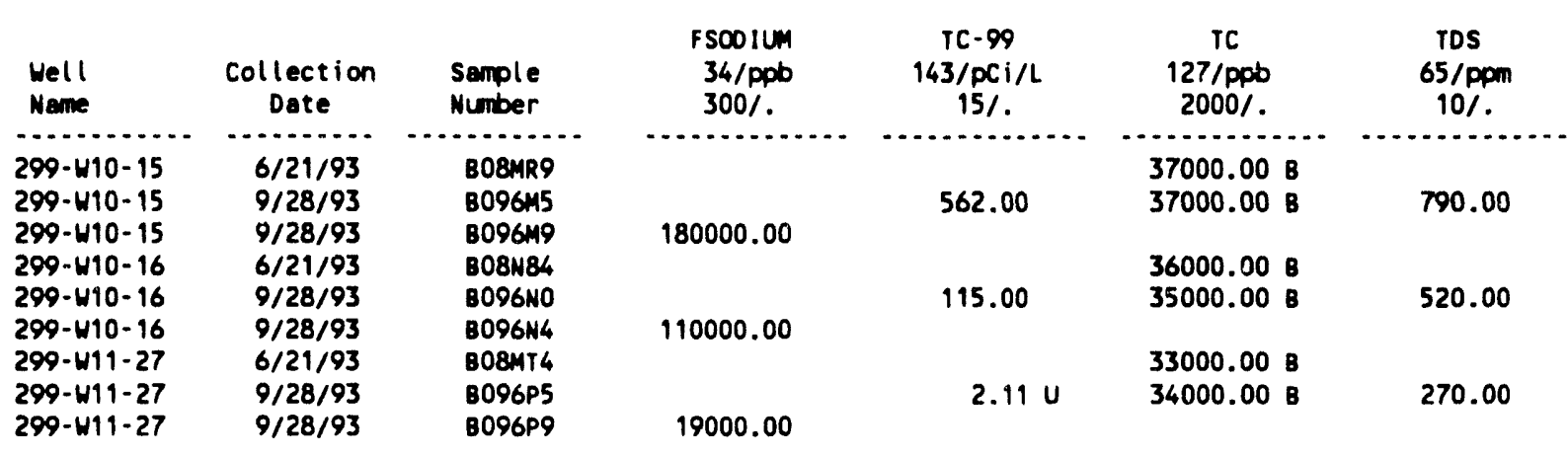

\begin{tabular}{|c|c|c|c|c|c|c|}
\hline $\begin{array}{l}\text { Well } \\
\text { Name }\end{array}$ & $\begin{array}{c}\text { Collection } \\
\text { Date }\end{array}$ & $\begin{array}{l}\text { Sample } \\
\text { Number }\end{array}$ & $\begin{array}{l}\text { TRITIUM } \\
142 / \mathrm{pCi} / \mathrm{L} \\
. / 20000\end{array}$ & $\begin{array}{c}\text { TURBID } \\
\text { 126/NTU } \\
.1 / 1\end{array}$ & $\begin{array}{l}\text { URANIUM } \\
\text { 145/ppob } \\
.1 .\end{array}$ & $\begin{array}{c}\text { VANADUM } \\
34 / \text { ppb } \\
30 \%\end{array}$ \\
\hline $\begin{array}{l}299-W 10-15 \\
299-W 10-16 \\
299-W 11-27\end{array}$ & $\begin{array}{l}9 / 28 / 93 \\
9 / 28 / 93 \\
9 / 28 / 93\end{array}$ & $\begin{array}{l}\text { B096M5 } \\
\text { B096NO } \\
\text { B096P5 }\end{array}$ & $\begin{array}{l}35300.00 \\
46100.00 \\
171.00 \mathrm{U}\end{array}$ & $\begin{array}{r}3.50 \\
19.00 \\
7.30\end{array}$ & $\begin{array}{l}2.47 \\
1.49 \\
1.95\end{array}$ & $\begin{array}{l}38.00 \\
48.00 \\
3.84\end{array}$ \\
\hline
\end{tabular}

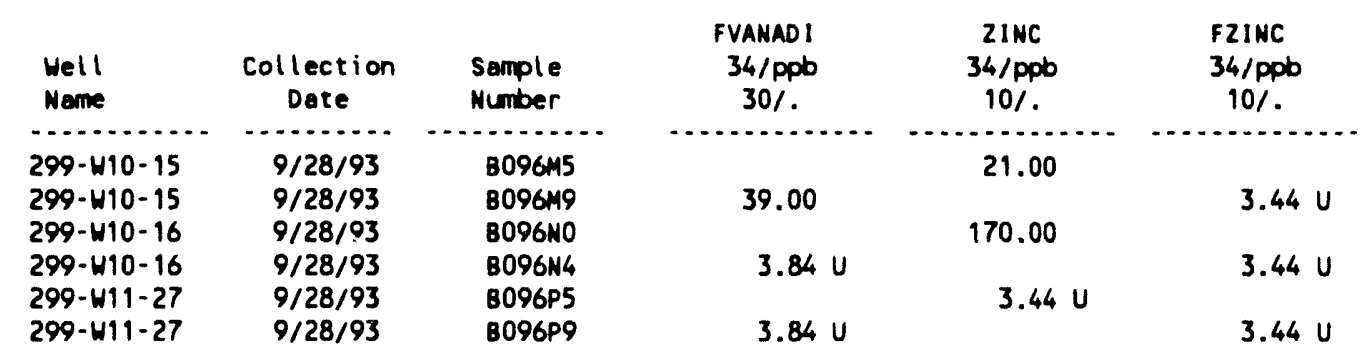

\footnotetext{
For explanation of this table, see Section 1.4 of report.
} 
Table 16-17. Contamination Indicator Parameters for the Single-Shell Tank Waste Management Area T Data for Reporting Period July 1 through September 30, 1993.

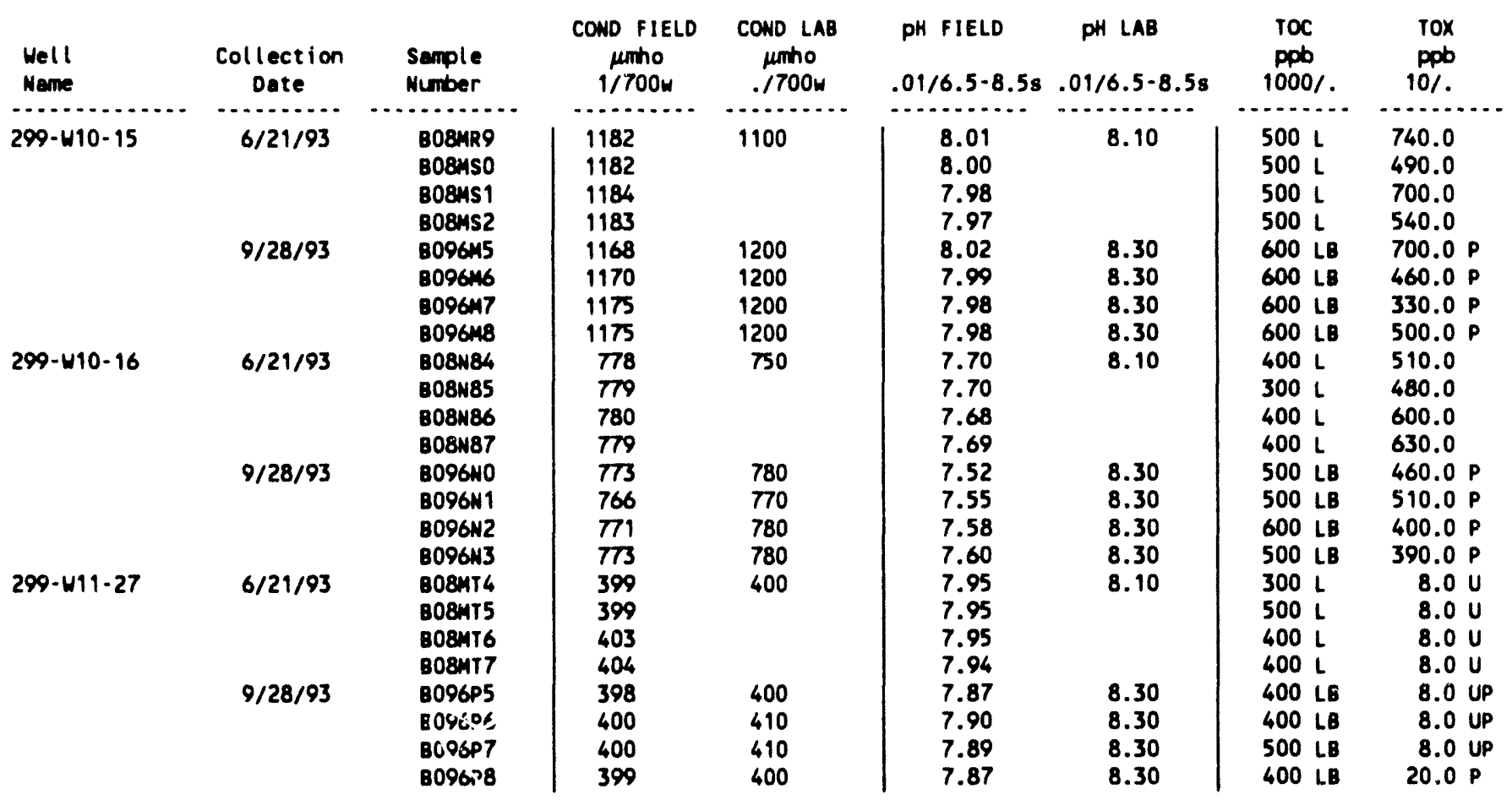

For explanation of this table, see Section 1.4 of report. 
Table 16-18. Constituent List and Summary of Results for the Single-She1l Tank Waste Management Area TX-TY Data for Reporting Period July 1 through September 30,1993. (sheet 1 of 3)

CONTAMINATION INDICATOR PARAMETERS

\begin{tabular}{|c|c|c|c|c|c|c|c|c|c|}
\hline \multirow[b]{2}{*}{ Short } & \multicolumn{2}{|r|}{ Constituent Name } & \multirow[b]{2}{*}{ Units } & \multirow{2}{*}{$\begin{array}{l}\text { Lab } \\
\text { CROL }\end{array}$} & \multicolumn{2}{|c|}{ DWS } & \multicolumn{3}{|c|}{ Number of Samples } \\
\hline & (Method) & full & & & Limit & Agency & Total & $>\mathrm{CROL}$ & $>$ DWS \\
\hline$\ldots \ldots$ & 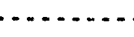 & $\ldots \ldots$ & $\ldots \ldots$ & $\ldots \ldots$ & $\ldots \ldots$ & $\ldots \ldots$ & $\ldots$ & $\ldots$. & $\cdots$ \\
\hline CONDUCT & 94 & Conductivity, field & $\mu$ mho & 1 & & & 29 & 29 & \\
\hline CONOUCT & 73 & Conductivity, lab & umho & & & & 20 & 20 & \\
\hline TOC & & Total Organic Carbon & ppo & 1000 & & & 32 & 0 & \\
\hline TOXLDL & & Total Organic Halogen & ppo & 10 & & & 32 & 32 & \\
\hline PH & 93 & pH, field & & .1 & $6.5-8.5$ & EPAS & 29 & 29 & 0 \\
\hline PH & 125 & pH, lab & & & $6.5-8.5$ & EPAS & 20 & 20 & 0 \\
\hline
\end{tabular}

DRINKING WATER PARAMETERS

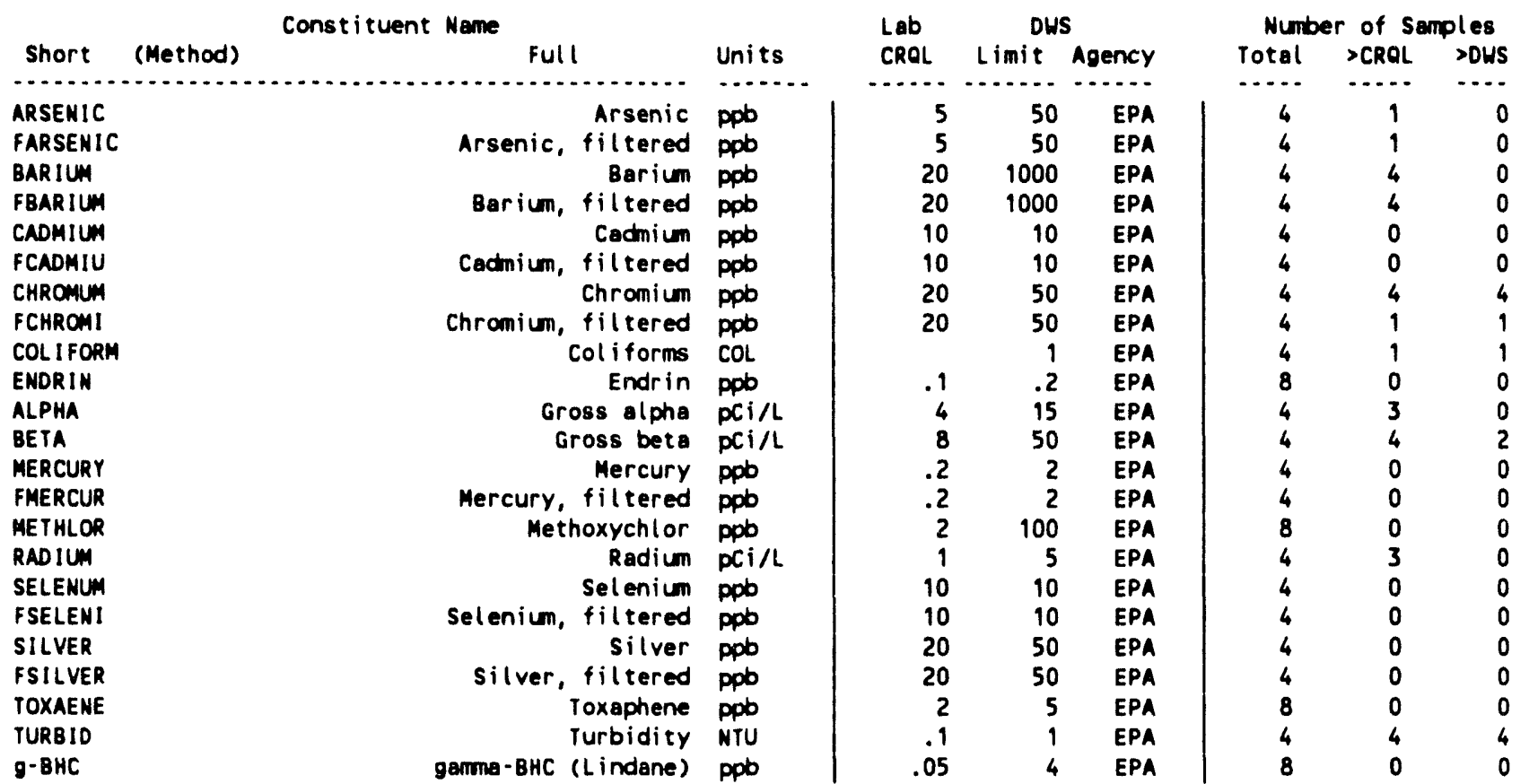

GROUNDWATER QUALITY PARAMETERS

\begin{tabular}{|c|c|c|c|c|c|c|c|c|c|}
\hline \multicolumn{4}{|c|}{ Constituent Name } & \multicolumn{3}{|c|}{ DWS } & \multicolumn{3}{|c|}{ Number of Samples } \\
\hline Short & (Method) & Full & Units & CROL & Limit & Agency & Total & $>$ CROL & $>$ DUS \\
\hline$\ldots \ldots$ & $\ldots$ & 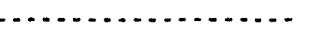 & $\ldots \ldots$ & $\ldots .$. & $\ldots \ldots$ & $\ldots \ldots$ & $\cdots$ & $\ldots$. & $\cdots$ \\
\hline IRON & & Iron & ppb & 20 & 300 & EPAS & 4 & 4 & 4 \\
\hline FIRON & & Iron, filtered & ppo & 20 & 300 & EPAS & 4 & 2 & 0 \\
\hline MANGESE & & Manganese & ppo & 10 & 50 & EPAS & 4 & 3 & 3 \\
\hline FMANGAN & & Manganese, filtered & ppb & 10 & 50 & EPAS & 4 & 2 & 0 \\
\hline LPHENOL & & Phenol & ppb & 20 & & & 4 & 0 & \\
\hline SOOIUM & & Sodium & ppb & 300 & & & 4 & 4 & \\
\hline FSOO IUM & & Sodium, filtered & ppb & 300 & & & 4 & 4 & \\
\hline
\end{tabular}


Table 16-18. Constituent List and Summary of Results for the Single-Shell Tank Waste Management Area TX-TY Data for Reporting Period July 1 through September 30, 1993. (sheet 2 of 3 )

SITE SPECIFIC AND OTHER CONSTITUENTS

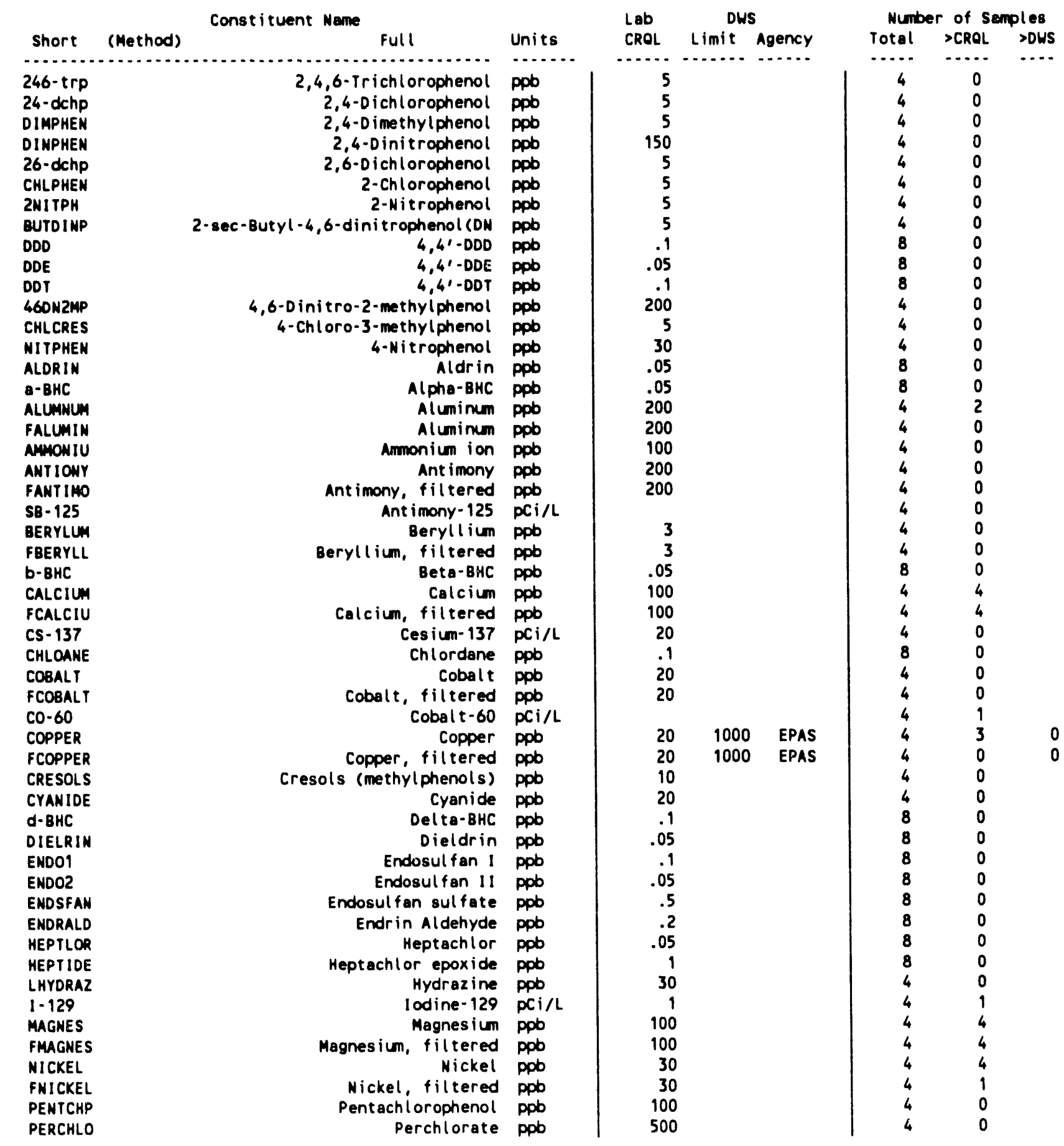


Table 16-18. Constituent List and Summary of Results for the Single-Shell

Tank Waste Management Area TX-TY Data for Reporting Period July 1 through September 30, 1993. (sheet 3 of 3)

\begin{tabular}{|c|c|c|c|c|c|c|c|c|c|}
\hline & & Constituent Name & & Lab & DWS & & Numt & of $s$ & oles \\
\hline Short & (Method) & Full & Units & CROL & Limit & Agency & Total & $>$ CROL & >DWS \\
\hline$\ldots \ldots . .$. & $\ldots . . . .$. & $\ldots \ldots \ldots \ldots$ & $\ldots . .$. & $\ldots \ldots$ & $\ldots \ldots$ & $\ldots \ldots$ & $\ldots$. & $\ldots .$. & $\ldots$ \\
\hline PU-238 & & Plutonium-238 & $\mathrm{pCi} / \mathrm{L}$ & .1 & & & 4 & 0 & \\
\hline PU39-40 & & Plutonium-239/40 & $\mathrm{pi} i \mathrm{~L}$ & .1 & & & 4 & 0 & \\
\hline POTASUM & & Potassium & ppo & 300 & & & 4 & 4 & \\
\hline FPOTASS & & Potassium, filtered & ppo & 300 & & & 4 & 4 & \\
\hline RU- 106 & & Ruthenium-106 & $\mathrm{pCi} / \mathrm{L}$ & & & & 4 & 0 & \\
\hline SR-90 & & strontium-90 & $\mathrm{pci} / \mathrm{l}$ & 5 & 8 & EPA & 4 & 0 & 0 \\
\hline SULFIDE & & sulfide & ppo & 10000 & & & 4 & 0 & \\
\hline $\mathrm{TC} .99$ & & Technetium-99 & $\mathrm{pci} / \mathrm{L}$ & 15 & & & 4 & 4 & \\
\hline TETPHNL & & Tetrachlorophenols & ppo & 10 & & & 4 & 0 & \\
\hline TIN & & Tin & ppob & 100 & & & 4 & 0 & \\
\hline FTIN & & Tin, filtered & ppo & 100 & & & 4 & 0 & \\
\hline TC & & Total Carbon & ppo & 2000 & & & 8 & 8 & \\
\hline TOS & & Total Dissolved Solids & ppm & 10 & & & 8 & 8 & \\
\hline TRIPHNL & & Trichlorophenols & ppo & 5 & & & 4 & 0 & \\
\hline TRITIUM & & Tritiun & $\mathrm{pCi} / \mathrm{L}$ & 500 & 20000 & EPA & 4 & 4 & 3 \\
\hline URANIUA & & Uranium & ppb & .5 & & & 4 & 4 & \\
\hline VANADUM & & Vanadium & ppob & 30 & & & 4 & 3 & \\
\hline FVANADI & & Vanadium, filtered & ppb & 30 & & & 4 & 1 & \\
\hline ZINC & & Zinc & ppb & 10 & & & 4 & 2 & \\
\hline FZINC & & zinc, filtered & ppb & 10 & & & 4 & 0 & \\
\hline
\end{tabular}

For explanation of this table, see Section 1.4 of report. 
Table 16-19. Constituents with at Least One Value Above the CRQL for the Single-Shell Tank Waste Management Area TX-TY Data for Reporting Period July 1 through September 30, 1993. (sheet 1 of 3)

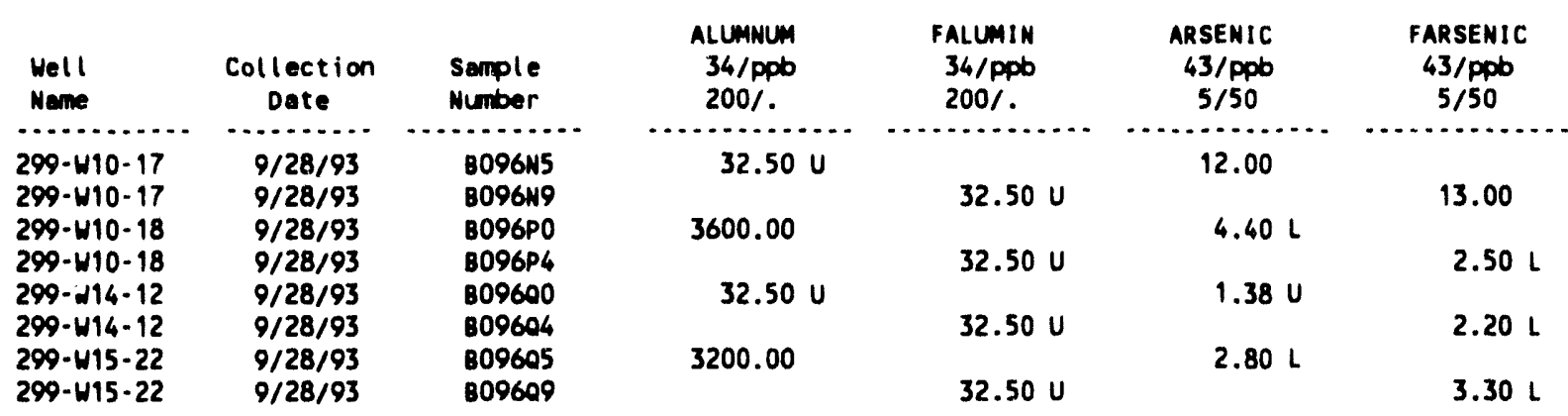

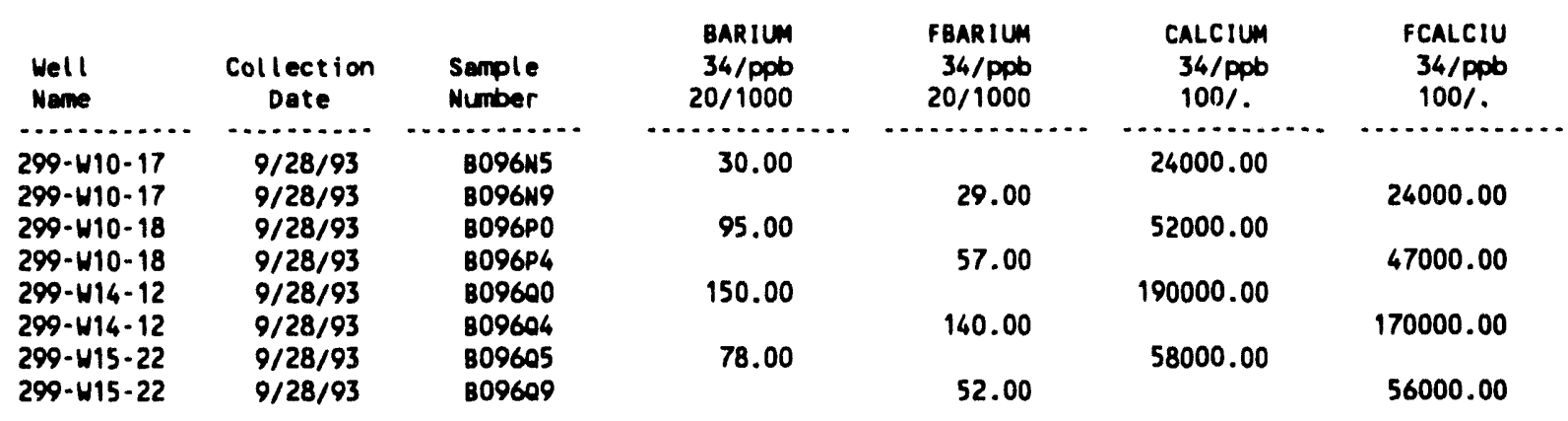

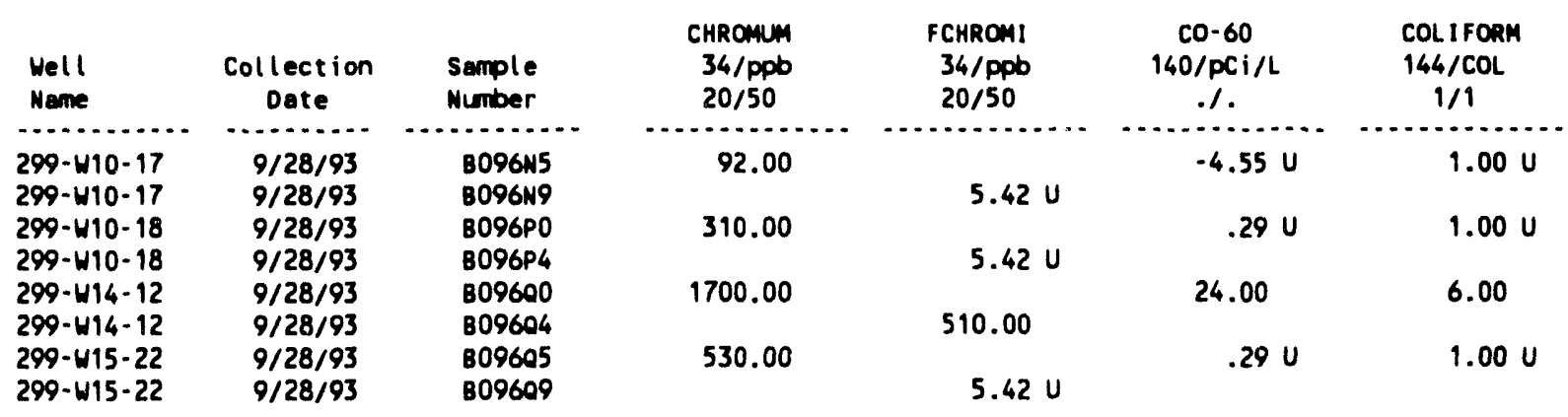

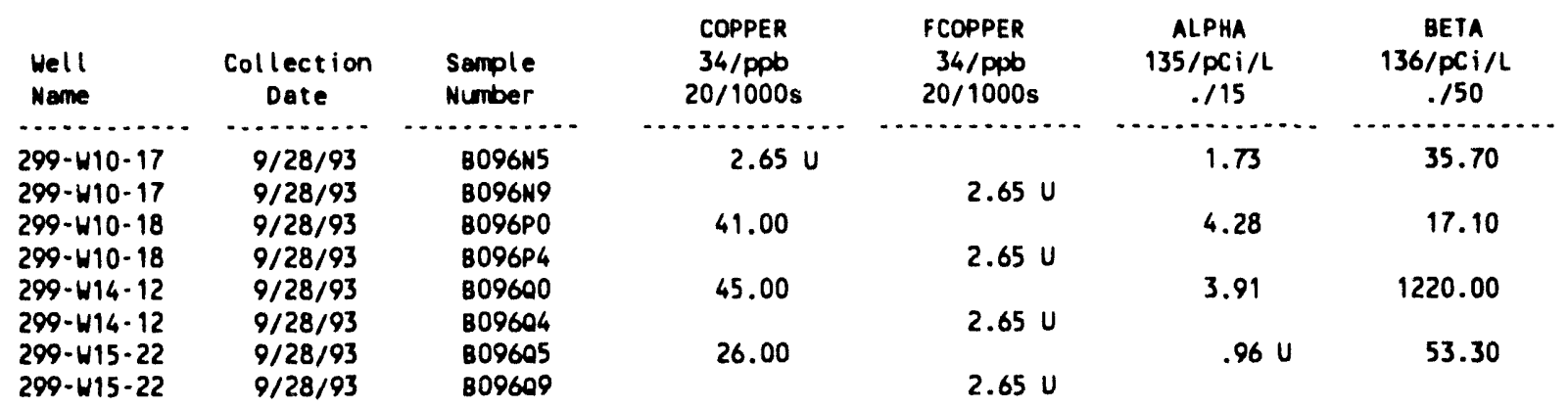


Table 16-19. Constituents with at Least One Value Above the $G$ RQ for the Single-Shell Tank Waste Management Area TX-TY Data for Reporting

Period July 1 through September 30, 1993. (sheet 2 of 3)

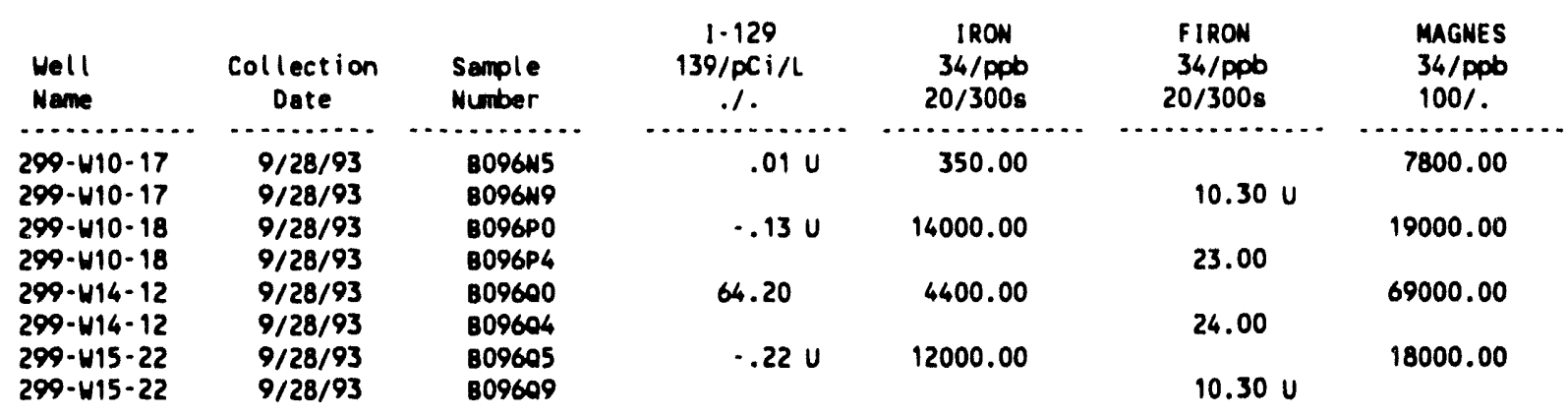

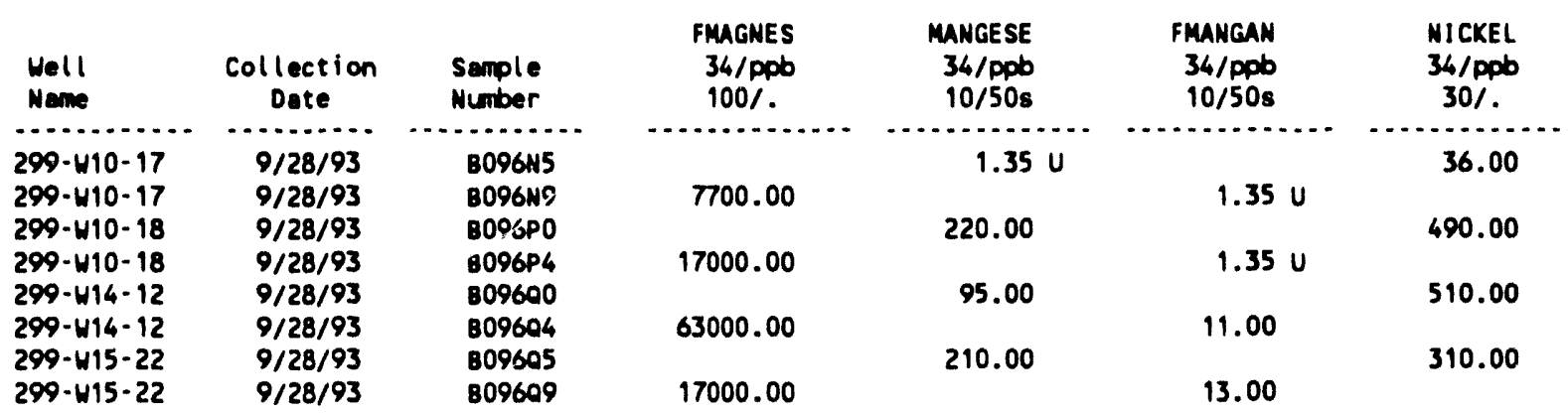

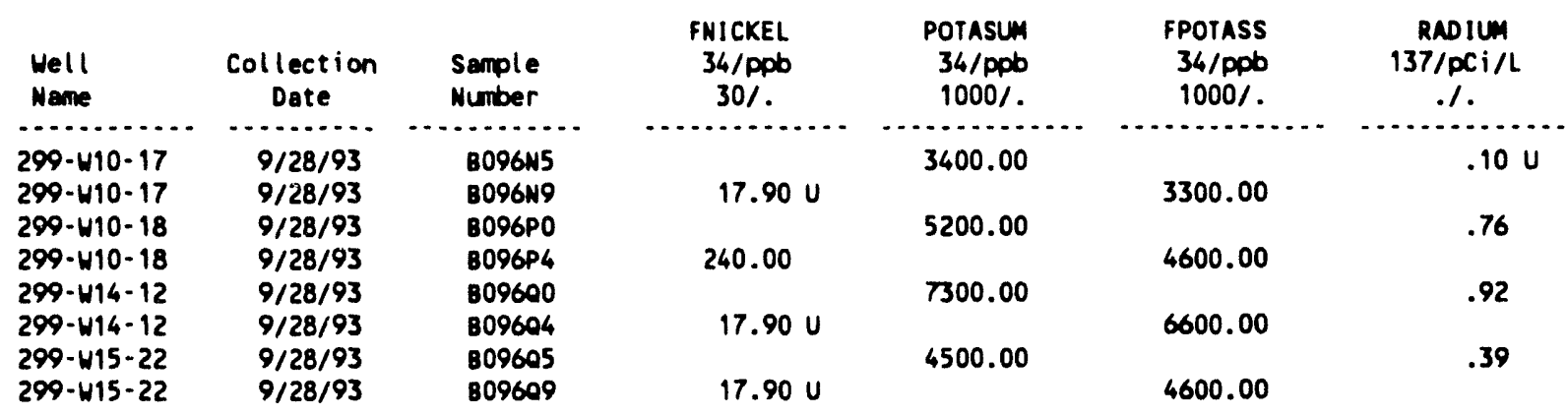

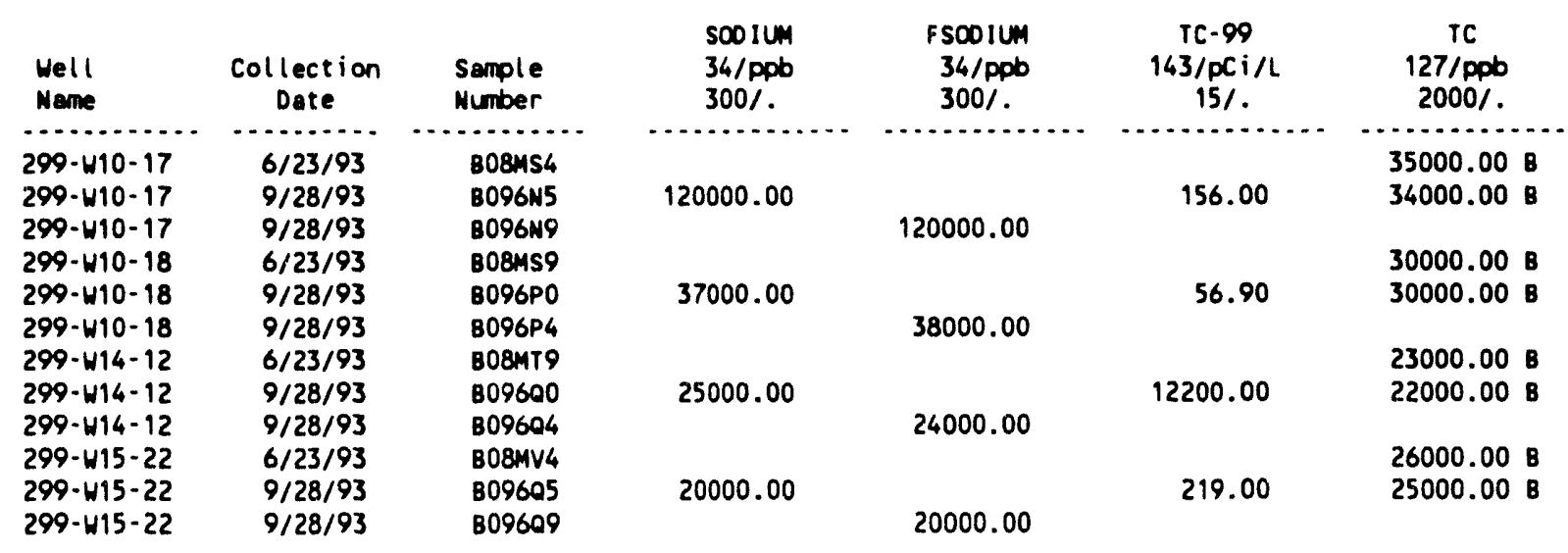


Table 16-19. Constituents with at Least One Value Above the CRQL for the Single-She 11 Tank Waste Management Area TX-TY Data for Reporting Period July 1 through September 30, 1993. (sheet 3 of 3)

\begin{tabular}{|c|c|c|c|c|c|c|}
\hline $\begin{array}{l}\text { Well } \\
\text { Neme }\end{array}$ & $\begin{array}{c}\text { Collection } \\
\text { Date }\end{array}$ & $\begin{array}{l}\text { Sample } \\
\text { Number }\end{array}$ & $\begin{array}{l}\text { TDS } \\
65 / \mathrm{ppm} \\
10 \%\end{array}$ & $\begin{array}{l}\text { TRITIUM } \\
\text { 142/pCI/L } \\
. / 20000\end{array}$ & $\begin{array}{c}\text { TURBID } \\
\text { 126/NTU } \\
.1 / 1\end{array}$ & $\begin{array}{c}\text { URANIUM } \\
\text { 145/ppb } \\
.1 .\end{array}$ \\
\hline $\begin{array}{l}299-W 10-17 \\
299-W 10-17 \\
299-W 10-18 \\
299-W 10-18 \\
299-W 14-12 \\
299-W 14-12 \\
299-W 15-22 \\
299-W 15-22\end{array}$ & $\begin{array}{l}6 / 23 / 93 \\
9 / 28 / 93 \\
6 / 23 / 93 \\
9 / 28 / 93 \\
6 / 23 / 93 \\
9 / 28 / 93 \\
6 / 23 / 93 \\
9 / 28 / 93\end{array}$ & $\begin{array}{l}\text { B08MS4 } \\
\text { B096N5 } \\
\text { B08MSO } \\
\text { B096PO } \\
\text { B08MT9 } \\
\text { B09600 } \\
\text { B08MV4 } \\
\text { B09605 }\end{array}$ & $\begin{array}{c}520.00 \mathrm{~B} \\
490.00 \\
390.00 \mathrm{~B} \\
360.00 \\
1200.00 \mathrm{~B} \\
1100.00 \\
340.00 \mathrm{~B} \\
330.00\end{array}$ & $\begin{array}{r}38600.00 \\
22800.00 \\
564000.00 \\
9600.00\end{array}$ & $\begin{array}{r}1.10 \\
150.00 \\
9.60 \\
75.00\end{array}$ & $\begin{array}{l}1.39 \\
1.56 \\
1.75 \\
1.17\end{array}$ \\
\hline $\begin{array}{l}\text { Well } \\
\text { Name }\end{array}$ & $\begin{array}{c}\text { Collection } \\
\text { Date }\end{array}$ & $\begin{array}{l}\text { Sample } \\
\text { Number }\end{array}$ & $\begin{array}{c}\text { VANADUM } \\
34 / \text { PpD } \\
30 \%\end{array}$ & $\begin{array}{c}\text { FVANADI } \\
34 / \mathrm{ppD} \\
30 \%\end{array}$ & $\begin{array}{c}\text { 2INC } \\
34 / \mathrm{ppb} \\
10 \%\end{array}$ & $\begin{array}{c}\text { FZINC } \\
34 / \text { ppb } \\
10 \%\end{array}$ \\
\hline $\begin{array}{l}299-W 10-17 \\
299-W 10-17 \\
299-W 10-18 \\
299-W 10-18 \\
299-W 14-12 \\
299-W 14-12 \\
299-W 15-22 \\
299-W 15-22\end{array}$ & $\begin{array}{l}9 / 28 / 93 \\
9 / 28 / 93 \\
9 / 28 / 93 \\
9 / 28 / 93 \\
9 / 28 / 93 \\
9 / 28 / 93 \\
9 / 28 / 93 \\
9 / 28 / 93\end{array}$ & $\begin{array}{l}8096 N 5 \\
8096 N 9 \\
8096 P 0 \\
8096 P 4 \\
809600 \\
809604 \\
809605 \\
809609\end{array}$ & $\begin{array}{l}49.00 \\
49.00 \\
3.84 \mathrm{U} \\
33.00\end{array}$ & $\begin{array}{l}50.00 \\
3.84 \mathrm{U} \\
3.84 \mathrm{U} \\
3.84 \mathrm{U}\end{array}$ & $\begin{array}{l}3.44 \mathrm{U} \\
49.00 \\
3.44 \mathrm{U} \\
76.00\end{array}$ & $\begin{array}{l}3.44 \mathrm{U} \\
3.44 \mathrm{U} \\
3.44 \mathrm{U} \\
3.44 \mathrm{U}\end{array}$ \\
\hline
\end{tabular}

For explanation of this table, see Section 1.4 of report. 
Table 16-20. Contamination Indicator Parameters for the Single-Shell Tank Waste Management Area TX-TY Data for Reporting Period July 1 through September 30, 1993.

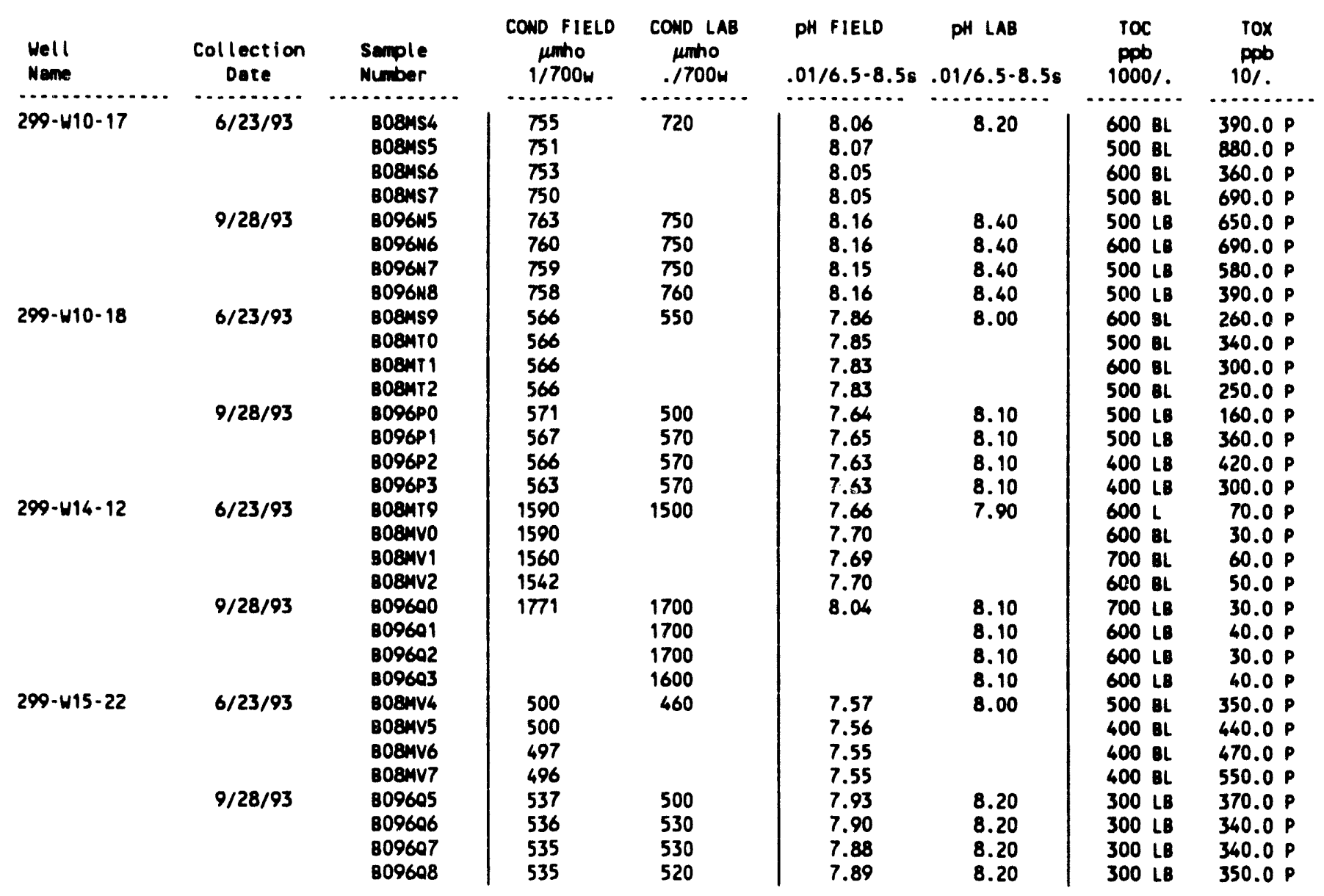


Table 16-21. Constituent List and Summary of Results for the Single-Shell Tank Waste Management Area U Data for Reporting Period July 1 through September 30, 1993. (sheet 1 of 3)

CONTAMINATION INDICATOR PARAMETERS

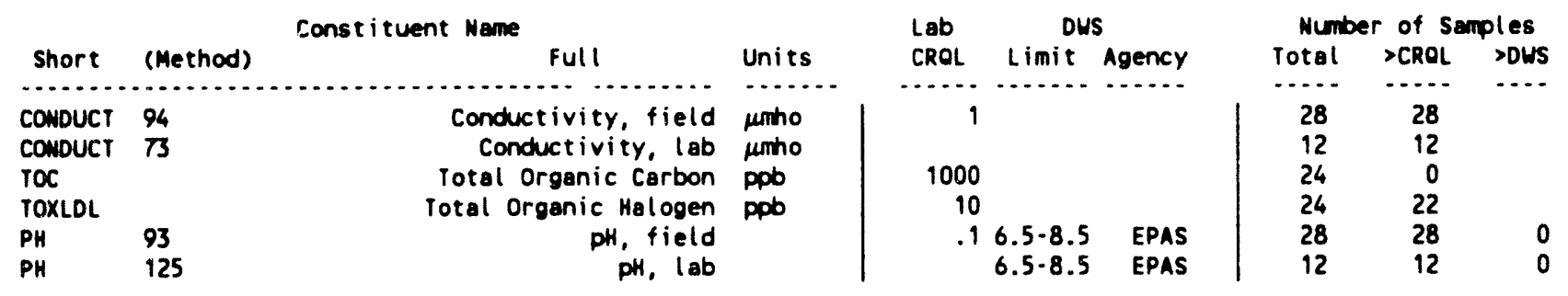

DRINKING WATER PARAMETERS

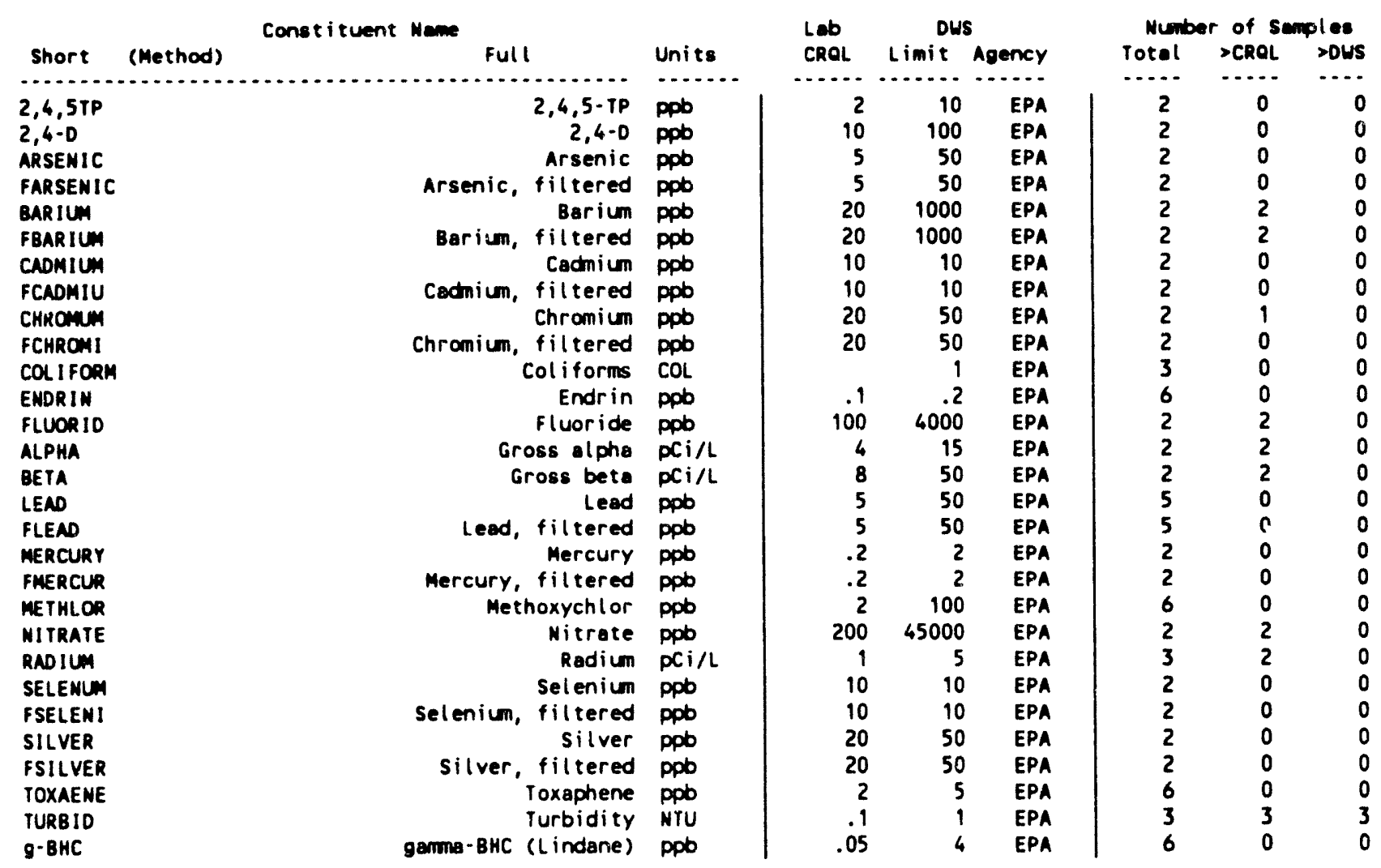

GROUNDWATER QUALITY PARAMETERS

\begin{tabular}{|c|c|c|c|c|c|c|c|c|c|}
\hline \multirow[b]{2}{*}{ Short } & \multirow[b]{2}{*}{ (Method) } & \multirow{2}{*}{ Constituent Name } & \multirow[b]{2}{*}{ Units } & \multirow{2}{*}{$\begin{array}{l}\text { Lab } \\
\text { CROL }\end{array}$} & \multicolumn{2}{|c|}{ DWS } & \multicolumn{3}{|c|}{ Number of Samples } \\
\hline & & & & & Limit & Agency & Total & $>$ CRQL & $>$ DWS \\
\hline$\ldots \ldots \ldots$ & $\cdots$ & $\ldots \ldots \ldots \ldots$ & $\ldots \ldots$ & $\ldots \ldots$ & $\cdots \ldots$ & $\ldots \ldots$ & $\ldots \ldots$ & 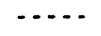 & $\ldots$ \\
\hline CHLORID & & Chloride & ppb & 200 & 250000 & EPAS & 2 & 2 & 0 \\
\hline IRON & & Iron & ppo & 20 & 300 & EPAS & 2 & 2 & 0 \\
\hline FIRON & & Iron, filtered & ppo & 20 & 300 & EPAS & 2 & 2 & 0 \\
\hline MANGESE & & Manganese & ppob & 10 & 50 & EPAS & 2 & 1 & 0 \\
\hline FMANGAN & & Manganese, filtered & ppb & 10 & 50 & EPAS & 2 & 1 & 0 \\
\hline LPHENOL & & Phenol & ppo & 20 & & & 3 & 0 & \\
\hline SOOIUM & & Sodium & ppo & 300 & & & 2 & 2 & \\
\hline FSOOIUM & & Sodium, filtered & ppb & 300 & & & 2 & 2 & \\
\hline SULFATE & & Sulfate & ppb & 500 & 250000 & EPAS & 2 & 2 & 0 \\
\hline
\end{tabular}




\section{Table 16-21. Constituent List and Summary of Results for the Single-Shell Tank Waste Management Area U Data for Reporting Period July 1 through September 30, 1993. (sheet 2 of 3)}

SITE SPECIFIC AND OTHER CONSTITUENTS

\begin{tabular}{|c|c|c|c|c|c|c|c|c|c|}
\hline \multirow[b]{2}{*}{ Short } & \multirow{3}{*}{ (Method) } & \multirow{2}{*}{$\begin{array}{l}\text { Constituent Name Full } \\
\text { Fene }\end{array}$} & \multirow[b]{2}{*}{ Units } & \multirow{2}{*}{$\begin{array}{l}\text { Lab } \\
\text { CRaL }\end{array}$} & \multicolumn{2}{|c|}{ Dus } & \multicolumn{3}{|c|}{ Number of Semples } \\
\hline & & & & & Limit & Agency & Total & $>\mathrm{CRQL}$ & $>$ DUS \\
\hline$\ldots \ldots \ldots$ & & 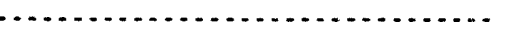 & $\ldots \ldots$ & $\ldots \ldots$ & $\ldots \ldots$ & $\ldots .$. & $\ldots$. & $\ldots$. & $\ldots$ \\
\hline $2,4,5-T$ & & $2,4,5-T$ & ppo & 2 & & & 2 & 0 & \\
\hline $266-\operatorname{trp}$ & & $2,4,6-T r i c h l o r o p h e n o l$ & ppb & 5 & & & 3 & 0 & \\
\hline 24-dchp & & 2,4-0 ichlorophenol & ppo & 5 & & & 3 & 0 & \\
\hline DIMPHEN & & 2,4-D ime thylphenol & ppo & 5 & & & 3 & 0 & \\
\hline DINPHEN & & 2,4-D initrophenol & ppb & 150 & & & 3 & 0 & \\
\hline 26-dchp & & 2,6-0 ichlorophenol & ppo & 5 & & & 3 & 0 & \\
\hline CHLPHEN & & 2-Chlorophenol & ppo & 5 & & & 3 & 0 & \\
\hline 2NITPH & & 2-Nitrophenol & ppb & 5 & & & 3 & 0 & \\
\hline BUTOINP & 30 & 2-sec-Butyl-4,6-dinitrophenol (DN & ppob & 5 & & & 3 & 0 & \\
\hline BUTDINP & 49 & 2-sec-Butyl-4,6-dinitrophenol (DN & ppob & 1 & & & 2 & 0 & \\
\hline DDD & & $4.41-000$ & ppo & .1 & & & 6 & 0 & \\
\hline DOE & & $4,41-$ DDE & ppb & .05 & & & 6 & 0 & \\
\hline DDT & & $4.4^{\prime}-$ DDT & ppb & .1 & & & 6 & 0 & \\
\hline 460N2MP & & 4,6-0 initro-2-methylphenol & ppo & 200 & & & 3 & 0 & \\
\hline CHLCRES & & 4-Chloro-3-methylphenol & ppo & 5 & & & 3 & 0 & \\
\hline NITPHEN & & 4-Nitrophenol & ppob & 30 & & & 3 & 0 & \\
\hline ALDR IN & & Aldrin & ppo & .05 & & & 6 & 0 & \\
\hline a-BHC & & Alpha-BHC & ppo & .05 & & & 6 & 0 & \\
\hline ALUMNUM & & Aiminum & ppo & 200 & & & 2 & 0 & \\
\hline FALUMIN & & Aluminum & ppo & 200 & & & 2 & 0 & \\
\hline AMMONIU & & Ammonium ion & ppo & 100 & & & 2 & 0 & \\
\hline ANT IONY & & Ant imony & ppb & 200 & & & 2 & 0 & \\
\hline FANTIIMO & & Ant imony, filtered & ppo & 200 & & & 2 & 0 & \\
\hline BERYLUM & & Beryllium & ppo & 3 & & & 2 & 0 & \\
\hline FBERYLL & & Beryllium, filtered & ppo & 3 & & & 2 & 0 & \\
\hline b-BHC & & Beta-BHC & ppb & .05 & & & 6 & 0 & \\
\hline BROMIOE & & Bromide & ppb & 500 & & & 2 & 0 & \\
\hline CALCIUN & & Calcium & $k, b$ & 100 & & & 2 & 2 & \\
\hline FCALCIU & & Calcium, filtered & ppo & 100 & & & 2 & 2 & \\
\hline CS- 137 & & Cesium-137 & $\mathrm{pCi} / \mathrm{L}$ & 20 & & & 2 & 1 & \\
\hline CHLOANE & & Chlordane & ppo & .1 & & & 6 & 0 & \\
\hline COBALT & & Cobalt & ppo & 20 & & & 2 & 0 & \\
\hline FCOBALT & & Cobelt, filtered & ppo & 20 & & & 2 & 0 & \\
\hline $\mathrm{CO} .60$ & & Cobal $t-60$ & $\mathrm{pCi} / \mathrm{L}$ & & & & 2 & 0 & \\
\hline COPPER & & Copper & ppb & 20 & 1000 & EPAS & 2 & 0 & 0 \\
\hline FCOPPER & & Copper, filtered & ppb & 20 & 1000 & EPAS & 2 & 0 & 0 \\
\hline CRESOLS & & Cresols (methylphenols) & ppb & 10 & & & 3 & 0 & \\
\hline CYANIDE & & Cyanide & ppob & 20 & & & 2 & 0 & \\
\hline d-BHC & & Delta-BHC & ppb & .1 & & & 6 & 0 & \\
\hline DIELRIN & & Dieldrin & ppb & .05 & & & 6 & 0 & \\
\hline ENDO1 & & Endosulfan I & ppb & .1 & & & 6 & 0 & \\
\hline ENDO2 & & Endosulfan II & ppob & .05 & & & 6 & 0 & \\
\hline ENDSFAN & & Endosulfan sulfate & ppo & .5 & & & 6 & 0 & \\
\hline ENDRALD & & Endr in Aldehyde & ppb & .2 & & & 6 & 0 & \\
\hline HEPTLOR & & Heptachlor & ppb & .05 & & & 6 & 0 & \\
\hline HEPTIDE & & Heptachlor epoxide & ppb & 1 & & & 6 & 0 & \\
\hline LHYORAZ & & Hydrazine & ppo & 30 & & & 3 & 0 & \\
\hline $1-129$ & & lodine-129 & pCi/L & 1 & & & 3 & 0 & \\
\hline MAGNES & & Magnesium & ppo & 100 & & & 2 & 2 & \\
\hline FMAGNES & & Magnesium, filtered & ppb & 100 & & & 2 & 2 & \\
\hline NICKEL & & Nickel & ppo & 30 & & & 2 & 0 & \\
\hline FNICKEL & & Nickel, filtered & ppo & 30 & & & 2 & 0 & \\
\hline NITRITE & & Nitrite & ppo & 200 & & & 2 & 1 & \\
\hline
\end{tabular}


Table 16-21. Constituent List and Summary of Results for the Single-Shell Tank Waste Management Area U Data for Reporting Period

July 1 through September 30, 1993. (sheet 3 of 3)

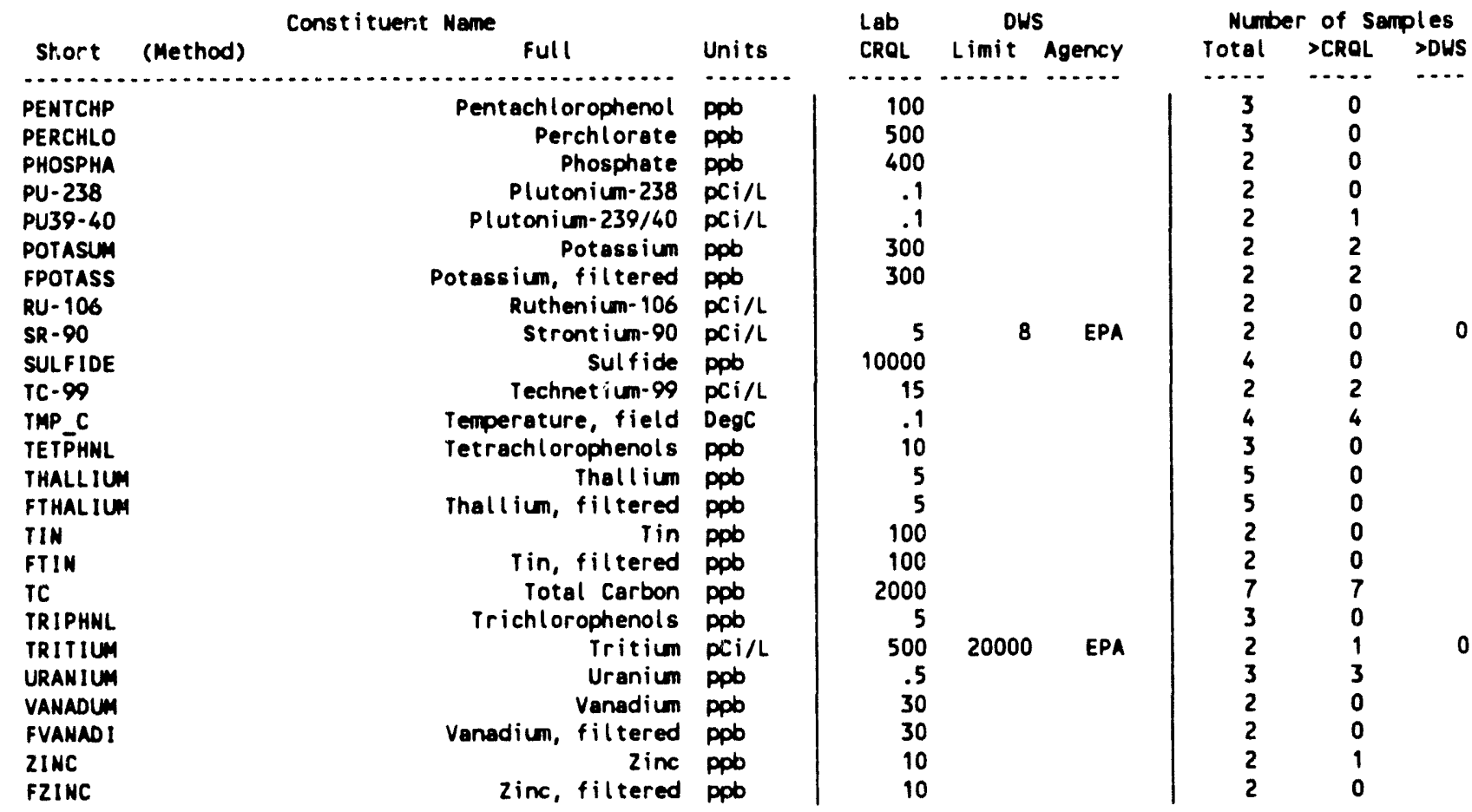

For explanation of this table, see Section 1.4 of report. 
Table 16-22. Constituents with at Least One Value Above the CRQL for the Single-She1l Tank Waste Management Area U Data for Reporting Period July 1 through September 30, 1993. (sheet 1 of 2)

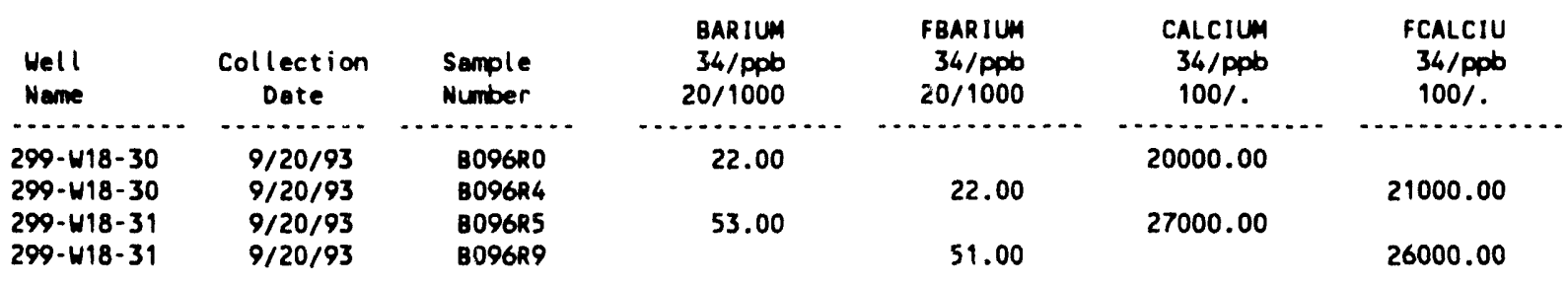

\begin{tabular}{|c|c|c|c|c|c|c|}
\hline $\begin{array}{l}\text { Well } \\
\text { Nane }\end{array}$ & $\begin{array}{c}\text { Collection } \\
\text { Date }\end{array}$ & $\begin{array}{l}\text { Sample } \\
\text { Number }\end{array}$ & $\begin{array}{l}\text { FLUORID } \\
124 / \mathrm{ppb} \\
100 / 4000\end{array}$ & $\begin{array}{c}\text { ALPHA } \\
135 / P C i / L \\
. / 15\end{array}$ & $\begin{array}{c}\text { BETA } \\
136 / \mathrm{PCi} / \mathrm{L} \\
. / 50\end{array}$ & $\begin{array}{c}\text { IRON } \\
34 / \mathrm{ppb} \\
20 / 300 \mathrm{~s}\end{array}$ \\
\hline $\begin{array}{l}299-W 18-30 \\
299-W 18-31\end{array}$ & $\begin{array}{l}9 / 20 / 93 \\
9 / 20 / 93\end{array}$ & $\begin{array}{l}\text { B096RO } \\
\text { B096R5 }\end{array}$ & $\begin{array}{l}400.00 \\
400.00\end{array}$ & $\begin{array}{l}2.21 \\
3.95\end{array}$ & $\begin{array}{l}6.79 \\
3.70\end{array}$ & $\begin{array}{l}120.00 \\
200.00\end{array}$ \\
\hline
\end{tabular}

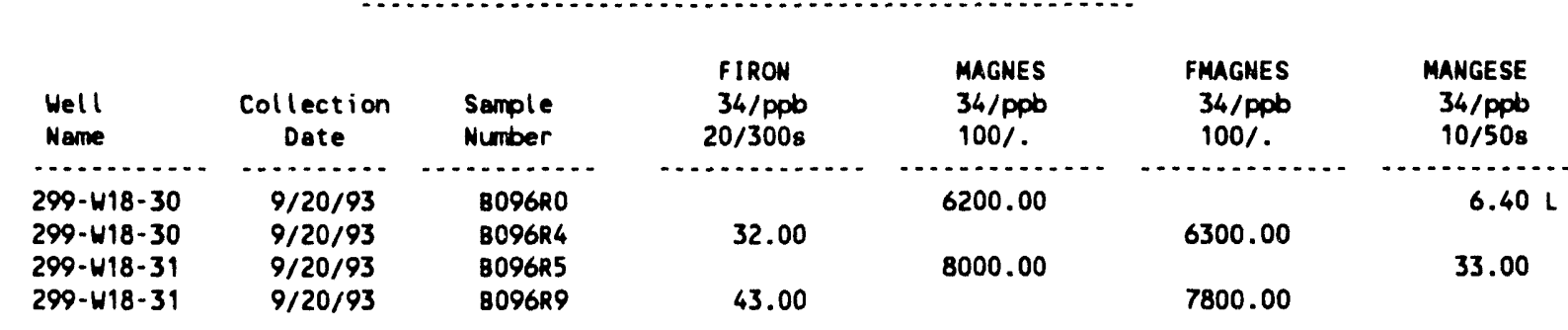

\begin{tabular}{|c|c|c|c|c|c|c|}
\hline $\begin{array}{l}\text { Well } \\
\text { Name }\end{array}$ & $\begin{array}{c}\text { Collection } \\
\text { Date }\end{array}$ & $\begin{array}{l}\text { Sample } \\
\text { Number }\end{array}$ & $\begin{array}{l}\text { FMANGAN } \\
34 / \text { ppb } \\
10 / 50 \mathrm{~s}\end{array}$ & $\begin{array}{l}\text { NITRATE } \\
124 / \mathrm{ppb} \\
200 / 45000\end{array}$ & $\begin{array}{l}\text { NITRITE } \\
124 / \mathrm{ppb} \\
200 / .\end{array}$ & $\begin{array}{c}\text { PU39-40 } \\
146 / \text { PCi/L } \\
. / .\end{array}$ \\
\hline $\begin{array}{l}299-W 18-30 \\
299-W 18-30\end{array}$ & $9 / 20 / 93$ & $\begin{array}{l}\text { B096RO } \\
\text { B096R4 }\end{array}$ & $1.70 \mathrm{~L}$ & 4900.00 & $38.30 \mathrm{U}$ & .05 \\
\hline $\begin{array}{l}299-w 18-31 \\
299-w 18-31\end{array}$ & $\begin{array}{l}9 / 20 / 93 \\
9 / 20 / 93\end{array}$ & $\begin{array}{l}\text { B096R5 } \\
\text { 8096R9 }\end{array}$ & 30.00 & 6900.00 & 200.00 & $-.00 \mathrm{U}$ \\
\hline
\end{tabular}


Table 16-22. Constituents with at Least One Value Above the CRQL for the Single-Shell Tank Waste Management Area U Data for Reporting Period July 1 through September 30, 1993. (sheet 2 of 2)

\begin{tabular}{|c|c|c|c|c|c|c|}
\hline $\begin{array}{l}\text { Well } \\
\text { Name }\end{array}$ & $\begin{array}{c}\text { Collection } \\
\text { Dote }\end{array}$ & $\begin{array}{l}\text { Semple } \\
\text { Number }\end{array}$ & $\begin{array}{l}\text { POTASUM } \\
\text { 34/ppb } \\
1000 \%\end{array}$ & $\begin{array}{l}\text { FPOTASS } \\
34 / \mathrm{ppb} \\
1000 \%\end{array}$ & $\begin{array}{c}\text { RADIUM } \\
137 / \mathrm{PCi} / \mathrm{L} \\
. / .\end{array}$ & $\begin{array}{l}\text { so0 Ium } \\
34 / \text { ppb } \\
300 \%\end{array}$ \\
\hline $\begin{array}{l}299-W 18-30 \\
299-W 18-30 \\
299-W 18-31 \\
299-W 18-31 \\
299-W 19-32\end{array}$ & $\begin{array}{l}9 / 20 / 93 \\
9 / 20 / 93 \\
9 / 20 / 93 \\
9 / 20 / 93 \\
7 / 20 / 93\end{array}$ & $\begin{array}{l}\text { B096RO } \\
\text { B096R4 } \\
\text { BO96R5 } \\
\text { BO96R9 } \\
\text { BO8MR8 }\end{array}$ & $\begin{array}{l}3000.00 \\
4200.00\end{array}$ & $\begin{array}{l}3000.00 \\
4000.00\end{array}$ & $\begin{array}{l}.11 \mathrm{U} \\
.18 \\
.41\end{array}$ & $\begin{array}{l}17000.00 \\
14000.00\end{array}$ \\
\hline $\begin{array}{l}\text { Well } \\
\text { Name }\end{array}$ & $\begin{array}{c}\text { Collection } \\
\text { Date }\end{array}$ & $\begin{array}{l}\text { Sample } \\
\text { Number }\end{array}$ & $\begin{array}{l}\text { Fs001um } \\
34 / \mathrm{ppb} \\
300 \%\end{array}$ & $\begin{array}{c}\text { SULFATE } \\
124 / \mathrm{ppb} \\
500 / 250000 \mathrm{~s}\end{array}$ & $\begin{array}{c}\text { TC- } 99 \\
143 / \mathrm{pCi} / \mathrm{L} \\
15 \%\end{array}$ & $\begin{array}{c}\text { TC } \\
127 / \mathrm{ppb} \\
2000 / .\end{array}$ \\
\hline $\begin{array}{l}299-W 18-25 \\
299-W 18-30 \\
299-W 18-30 \\
299-W 18-30 \\
299-W 18-31 \\
299-W 18-31 \\
299-W 18-31 \\
299-W 19-31 \\
299-W 19-32\end{array}$ & $\begin{array}{l}6 / 21 / 93 \\
6 / 22 / 93 \\
9 / 20 / 93 \\
9 / 20 / 93 \\
6 / 21 / 93 \\
9 / 20 / 93 \\
9 / 20 / 93 \\
6 / 21 / 93 \\
7 / 20 / 93\end{array}$ & $\begin{array}{l}\text { BO8MV9 } \\
\text { BO8MW4 } \\
\text { BO96RO } \\
\text { BO96R4 } \\
\text { B08N89 } \\
\text { BO96R5 } \\
\text { BO96R9 } \\
\text { BOBN94 } \\
\text { BOBMR8 }\end{array}$ & $\begin{array}{l}17000.00 \\
14000.00\end{array}$ & $15000.00 \mathrm{D}$ & 8.57 & $\begin{array}{l}24000.00 \mathrm{~B} \\
23000.00 \mathrm{~B} \\
22000.00 \mathrm{~B} \\
25000.00 \mathrm{~B} \\
25000.00 \mathrm{~B} \\
32000.00 \mathrm{~B} \\
25000.00 \mathrm{~B}\end{array}$ \\
\hline
\end{tabular}

\begin{tabular}{|c|c|c|c|c|c|c|}
\hline $\begin{array}{l}\text { Hell } \\
\text { Neme }\end{array}$ & $\begin{array}{c}\text { Collection } \\
\text { Dete }\end{array}$ & $\begin{array}{l}\text { Semple } \\
\text { Number }\end{array}$ & $\begin{array}{c}\text { TRITIUM } \\
\text { 142/pCi/L } \\
. / 20000\end{array}$ & $\begin{array}{c}\text { TURBID } \\
126 / N T U \\
.1 / 1\end{array}$ & $\begin{array}{c}\text { URANIUM } \\
\text { 145/ppb } \\
.1 .\end{array}$ & $\begin{array}{c}\text { ZINC } \\
34 / p p b \\
10 /\end{array}$ \\
\hline - & $\ldots \ldots$ & - & 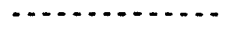 & $\cdots$ & $\cdots$ & 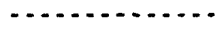 \\
\hline $\begin{array}{l}299-W 18-30 \\
299-W 18-31 \\
299-W 19-32\end{array}$ & $\begin{array}{l}9 / 20 / 93 \\
9 / 20 / 93 \\
7 / 20 / 93\end{array}$ & $\begin{array}{l}\text { BO96RO } \\
\text { BO96R5 } \\
\text { BOBMR8 }\end{array}$ & $\begin{array}{l}281.00 \\
-79.60 U\end{array}$ & $\begin{array}{l}3.200 \\
5.200 \\
42.50\end{array}$ & $\begin{array}{l}1.87 \\
5.61 \\
6.450\end{array}$ & $\begin{array}{l}10.00 \\
3.44 \mathrm{U}\end{array}$ \\
\hline
\end{tabular}

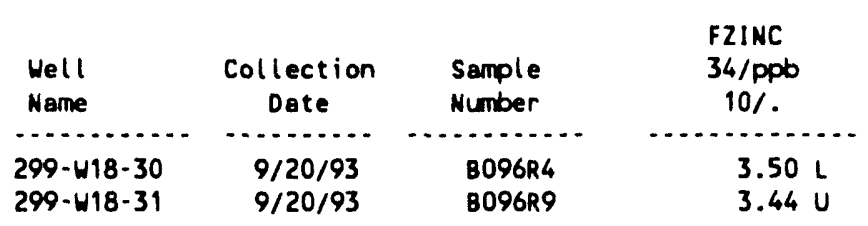

For explanation of this table, see Section 1.4 of report. 
Table 16-23. Contamination Indicator Parameters for the Single-Shell Tank Waste Management Area U Data for Reporting Period July 1 through September 30, 1993.

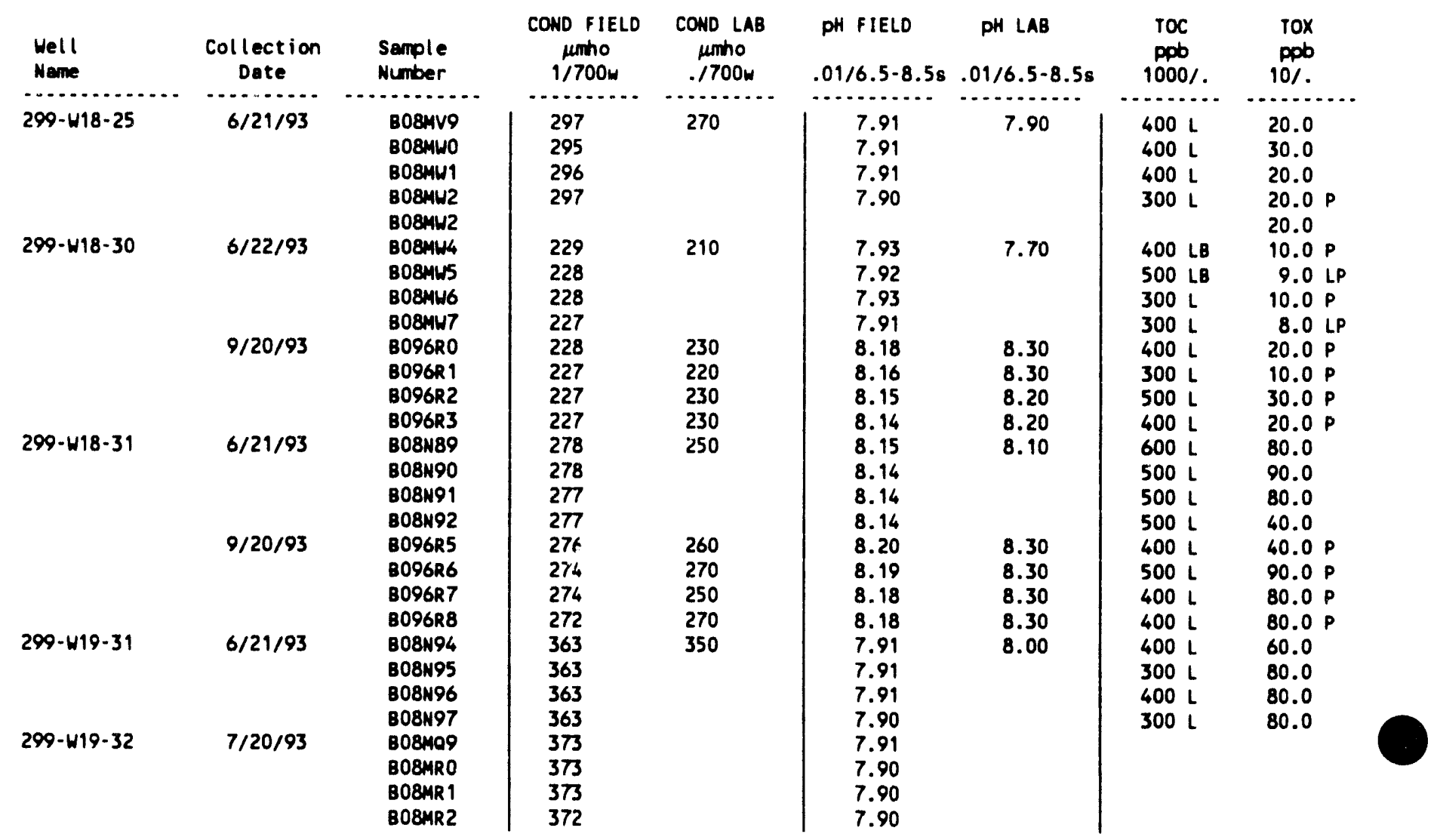

For explanation of this table, see Section 1.4 of report. 
$\mathrm{DOE} / \mathrm{RL}-93-56-3$

\section{CONTENTS}

17.0300 AREA PROCESS TRENCHES . . . . . . . . . . . . . . . 17-1

17.1 INTRODUCTION . . . . . . . . . . . . . . . . . 17-1

17.2 WATER LEVEL MEASUREMENTS .................. . . 17-1

17.3 WATER CHEMISTRY DATA . . . . . . . . . . . . . . . . . . . 17-1 
DOE/RL-93-56-3

LIST OF FIGURES

17-1 Monitoring We11 Locations for the 300 Area Process Trenches . . 17-3

\section{LIST OF TABLES}

17-1 Monitoring Well Purpose and Sampling Schedule for the 300 Area

Process Trenches Network . . . . . . . . . . . . 17-4

17-2 RCRA Water Level Measurement Report 300 Area Process Trenches, Third Quarter 1993 .............. 17-5

17-3 Constituent List and Summary of Results for the 300 Area Process Trenches Data for Reporting Period July 1 through September 30,1993 ............. 17-10

17-4 Constituents with at Least One Value Above the CRQL for the 300 Area Process Trenches Data for Reporting Period

July 1 through September 30, $1993 \ldots$. . . . . . . . . 17-13

17-5 Contamination Indicator Parameters for the 300 Area Process

Trenches Data for Reporting Period July 1 through

September 30, 1993 


\title{
17.0300 AREA PROCESS TRENCHES
}

\author{
J. V. Borghese
}

Pacific Northwest Laboratory

\subsection{INTRODUCTION}

The groundwater near the 300 Area Process Trenches (316-5) has been monitored by a RCRA well network since June 1985. The groundwater monitoring is being conducted under interim-status regulations (40 CFR 265) and is an assessment-level program (Schalla 1988). The process trenches are located in the northern portion of the 300 Area and are used for the disposal of wastewater from various 300 Area operations. The wastewater consists primarily of cooling water, with small quantities of nonhazardous maintenance and process waste.

Monitoring wells were constructed in response to a Consent and Compliance order (Ecology and EPA 1986). Groundwater wells currently used to monitor the facility are shown in Figure 17-1 and are listed in Table 17-1; they consist of both pre-RCRA and RCRA standard wells.

\subsection{WATER LEVEL MEASUREMENTS}

Water levels are measured monthly and at the time of sampling in selected 300 Area wells. Water level measurements obtained during the third quarter of 1993 are presented in Table 17-2.

\subsection{WATER CHEMISTRY DATA}

Water chemistry samples are collected semiannually from the 300 Area process trenches monitoring wells. In addition to semiannual samples, samples are collected quarterly from well 399-1-17A to provide near-trench monitoring of contaminants. Sampling dates are listed in Table 17-1. Analytical results received for the sampling events are presented in Tables 17-3 through 17-5. An explanation of the data flags is given in Section 1.4. A discussion of the data quality can be found in Section 1.2 . 
Constituents that exceeded the DWS and the associated wells are listed below. The DWS for 1,2-dichloroethene and trichloroethene is $5 \mathrm{ppb}$ for both constituents. These DWSs were inadvertently left off the constituent 1 ist and summary table. The DWS for chromium is $100 \mathrm{ppb}$. The standard is listed incorrectly in Tables 17-3 and 17-4.

\begin{tabular}{|l|l|}
\hline \multicolumn{1}{|c|}{ Constituent } & \multicolumn{1}{c|}{ Well number } \\
\hline Iron & $399-1-16 \mathrm{~B}$ \\
\hline Manganese & $399-1-16 \mathrm{~B}$ \\
\hline Manganese, filtered & $399-1-16 \mathrm{~B}$ \\
\hline 1,2-Dichloroethene & $399-1-16 \mathrm{~A}, 399-1-16 \mathrm{~B}$ \\
\hline Trichloroethene & $399-1-16 \mathrm{~B}$ \\
\hline Gross alpha & $399-1-10 \mathrm{~A}, 399-1-11,399-1-12$, \\
& $399-1-16 \mathrm{~A}, 399-2-1$ \\
\hline
\end{tabular}


Figure 17-1. Monitoring We11 Locations for the 300 Area Process Trenches.

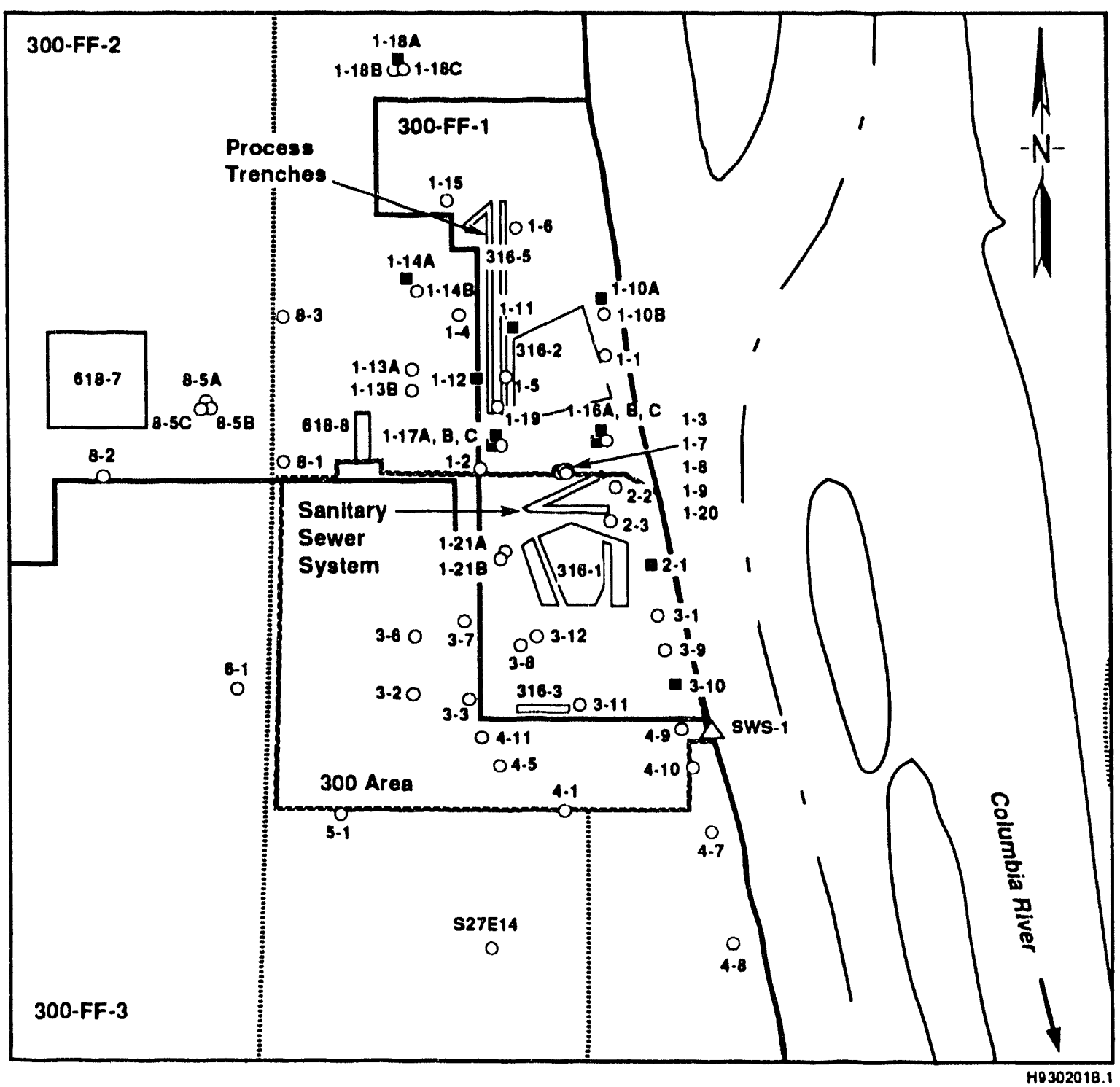

1.12 Well Location and Number (Wells Prelixed by 399-, Except Those Beginning with $S$ are Preflxed with 699-)

- 4.7 Monltoring Network Well

$\triangle$ sws-1 Surface-Water Monltoring Station

...n............ Roads 
Table 17-1. Monitoring Well Purpose and Sampling Schedule for the 300 Area Process Trenches Network.

\begin{tabular}{|c|c|c|c|c|}
\hline \begin{tabular}{|c|} 
Weil no. \\
$\left(399_{-}\right)$
\end{tabular} & $\begin{array}{l}\text { Relative } \\
\text { position }\end{array}$ & Hydrogeologic unit & $\begin{array}{c}\text { Sample } \\
\text { frequency }\end{array}$ & $\begin{array}{l}\text { Sample date, } \\
\text { 3rd Qtr } 1993\end{array}$ \\
\hline $1-10 A$ & Downgradient & Hanford/Ringold: Water Table & Semiannually & $9 / 14 / 93$ \\
\hline $1-11$ & cent & Hanford/Ringold: Water Table & Semia & $9 / 14$ \\
\hline $1-12$ & owngradient & Hanford/Ringold: Water Table & Semiannually & $9 / 14 / 93$ \\
\hline $1-14 A$ & Adjacent & Hanford: Water Table & Semiannually & $9 / 14 / 93$ \\
\hline $1-16 A$ & Owngradient & Ringold: Water Table & Semiannually & $9 / 29 / 93$ \\
\hline $1-16 B$ & Downgradient & $\begin{array}{l}\text { Ringold: Bottom of } \\
\text { Unconfined Aquifer }\end{array}$ & Semian & $9 / 29 / 93$ \\
\hline $1-17 A$ & Downgradient & Ringold: Water Table & terly & 93 \\
\hline $1-178$ & Downs & $\begin{array}{l}\text { Ringold: Bottom of } \\
\text { Unconfined Aquifer }\end{array}$ & Sem & 3 \\
\hline $1-18 A$ & Upgradient & Ringold: Water Table & Semiannually & $9 / 07 / 93$ \\
\hline $2-1$ & Downgradient & Hanford/Ringold: Water Table & Semiannually & $9 / 16 / 93$ \\
\hline $3-9$ & Downgradient & --: Water Table & -- & $9 / 27 / 93^{\circ}$ \\
\hline $3-10$ & Downgradient & Hanford: Water Table & Semiannually & $9 / 03 / 93$ \\
\hline $1-21 A$ & Downgradient & --: Water Table & -- & $9 / 14 / 93^{\circ}$ \\
\hline
\end{tabular}

Note: Hydrogeologic units include the sandy gravels of the Hanford formation and silty sands of the Ringold Formation. Water levels are measured in all wells monthly.

"Special ethylene glycol sampling event. 
Table 17-2. RCRA Water Level Measurement Report

300 Area Process Trenches, Third Quarter 1993. (sheet 1 of 5 )

\begin{tabular}{|c|c|c|c|}
\hline Well & Date & $\begin{array}{l}\text { Depth to } \\
\text { water (ft) }\end{array}$ & $\begin{array}{l}\text { Water level } \\
\text { elevation } \\
\text { above } \mathrm{ms} \text { l }(\mathrm{ft})\end{array}$ \\
\hline
\end{tabular}

Wells Monitoring the Top of the Unconfined Aquifer

\begin{tabular}{|c|c|c|c|}
\hline $399-1-1$ & $\begin{array}{l}7 / 28 / 93 \\
8 / 18 / 93 \\
9 / 27 / 93\end{array}$ & $\begin{array}{l}34.38 \\
34.96 \\
37.05\end{array}$ & $\begin{array}{l}342.28 \\
341.70 \\
339.61\end{array}$ \\
\hline $399-1-3$ & $7 / 28 / 93$ & 42.74 & 341.97 \\
\hline $399-1-4$ & $\begin{array}{l}7 / 28 / 93 \\
8 / 18 / 93 \\
9 / 27 / 93\end{array}$ & $\begin{array}{l}37.78 \\
38.33 \\
39.61\end{array}$ & $\begin{array}{l}342.80 \\
342.25 \\
340.97\end{array}$ \\
\hline $399-1-5$ & $\begin{array}{l}7 / 28 / 93 \\
8 / 18 / 93 \\
9 / 27 / 93\end{array}$ & $\begin{array}{l}37.39 \\
37.88 \\
39.39\end{array}$ & $\begin{array}{l}342.38 \\
341.89 \\
340.38\end{array}$ \\
\hline $399-1-7$ & $\begin{array}{l}7 / 28 / 93 \\
8 / 18 / 93 \\
9 / 27 / 93\end{array}$ & $\begin{array}{l}43.36 \\
43.87 \\
45.61\end{array}$ & $\begin{array}{l}342.24 \\
341.73 \\
339.99\end{array}$ \\
\hline $399-1-8$ & $\begin{array}{l}7 / 28 / 93 \\
8 / 18 / 93 \\
9 / 27 / 93\end{array}$ & $\begin{array}{l}42.61 \\
43.14 \\
44.85\end{array}$ & $\begin{array}{l}342.27 \\
341.74 \\
340.03\end{array}$ \\
\hline $399-1-10 A$ & $\begin{array}{l}7 / 28 / 93 \\
8 / 18 / 93 \\
9 / 14 / 93 \\
9 / 27 / 93\end{array}$ & $\begin{array}{l}30.92 \\
31.51 \\
32.97 \\
33.62\end{array}$ & $\begin{array}{l}342.65 \\
342.06 \\
340.60^{\star} \\
339.95\end{array}$ \\
\hline $399-1-11$ & $\begin{array}{l}7 / 28 / 93 \\
8 / 18 / 93 \\
9 / 14 / 93 \\
9 / 27 / 93\end{array}$ & $\begin{array}{l}35.04 \\
35.57 \\
36.66 \\
36.99\end{array}$ & $\begin{array}{l}342.68 \\
342.15 \\
341.06 \star \\
340.73\end{array}$ \\
\hline $399-1-12$ & $\begin{array}{l}7 / 28 / 93 \\
8 / 18 / 93 \\
9 / 14 / 93 \\
9 / 27 / 93\end{array}$ & $\begin{array}{l}41.97 \\
42.48 \\
43.68 \\
43.99\end{array}$ & $\begin{array}{l}342.44 \\
341.93 \\
340.73 \\
340.42\end{array}$ \\
\hline $399-1-14 A$ & $\begin{array}{l}7 / 28 / 93 \\
8 / 18 / 93 \\
9 / 14 / 93 \\
9 / 27 / 93\end{array}$ & $\begin{array}{l}40.28 \\
40.90 \\
41.77 \\
42.05\end{array}$ & $\begin{array}{l}342.90 \\
342.28 \\
341.41^{\star} \\
341.13\end{array}$ \\
\hline
\end{tabular}


Table 17-2. RCRA Water Level Measurement Report 300 Area Process Trenches, Third Quarter 1993. (sheet 2 of 5 )

Well Date $\begin{gathered}\text { Depth to } \\ \text { water }(\mathrm{ft})\end{gathered} \begin{gathered}\text { Water level } \\ \text { elevation } \\ \text { above ms }\end{gathered}$

Wells Monitoring the Top of the Unconfined Aquifer

\begin{tabular}{|c|c|c|c|}
\hline $399-1-15$ & $\begin{array}{l}7 / 28 / 93 \\
8 / 18 / 93 \\
9 / 27 / 93\end{array}$ & $\begin{array}{l}36.57 \\
37.14 \\
38.38\end{array}$ & $\begin{array}{l}342.96 \\
342.39 \\
341.15\end{array}$ \\
\hline $399-1-16 A$ & $\begin{array}{l}7 / 28 / 93 \\
9 / 27 / 93 \\
9 / 29 / 93\end{array}$ & $\begin{array}{l}39.20 \\
41.61 \\
41.18\end{array}$ & $\begin{array}{l}342.27 \\
339.86 \\
340.29\end{array}$ \\
\hline $399-1-17 A$ & $\begin{array}{l}7 / 28 / 93 \\
8 / 18 / 93 \\
9 / 03 / 93 \\
9 / 27 / 93\end{array}$ & $\begin{array}{l}35.19 \\
35.69 \\
35.95 \\
37.15\end{array}$ & $\begin{array}{l}342.25 \\
341.75 \\
341.49 * \\
340.29\end{array}$ \\
\hline $399-1-18 A$ & $\begin{array}{l}7 / 28 / 93 \\
8 / 18 / 93 \\
9 / 07 / 93 \\
9 / 27 / 93\end{array}$ & $\begin{array}{l}47.30 \\
47.89 \\
48.76 \\
49.10\end{array}$ & $\begin{array}{l}343.48 \\
342.89 \\
342.02 \star \\
341.68\end{array}$ \\
\hline $399-1-19$ & $\begin{array}{l}7 / 28 / 93 \\
8 / 18 / 93 \\
9 / 27 / 93\end{array}$ & $\begin{array}{l}32.20 \\
32.67 \\
34.17\end{array}$ & $\begin{array}{l}342.41 \\
341.97 \\
340.44\end{array}$ \\
\hline $399-2-1$ & $\begin{array}{l}7 / 28 / 93 \\
8 / 18 / 93 \\
9 / 16 / 93 \\
9 / 27 / 93\end{array}$ & $\begin{array}{l}33.38 \\
33.86 \\
35.35 \\
35.97\end{array}$ & $\begin{array}{l}341.85 \\
341.37 \\
339.88 \star \\
339.29\end{array}$ \\
\hline $399-2-2$ & $\begin{array}{l}7 / 28 / 93 \\
8 / 18 / 93 \\
9 / 27 / 93\end{array}$ & $\begin{array}{l}35.29 \\
35.88 \\
37.75\end{array}$ & $\begin{array}{l}342.23 \\
341.64 \\
339.77\end{array}$ \\
\hline $399-2-3$ & $\begin{array}{l}7 / 28 / 93 \\
8 / 18 / 93 \\
9 / 27 / 93\end{array}$ & $\begin{array}{l}33.20 \\
33.70 \\
36.27\end{array}$ & $\begin{array}{l}342.22 \\
341.72 \\
339.15\end{array}$ \\
\hline
\end{tabular}


Table 17-2. RCRA Water Level Measurement Report 300 Area Process Trenches, Third Quarter 1993. (sheet 3 of 5 )

\begin{tabular}{|c|c|c|c|}
\hline Well & Date & $\begin{array}{l}\text { Depth to } \\
\text { water ( } f t)\end{array}$ & $\begin{array}{l}\text { Water level } \\
\text { elevation } \\
\text { above ms l (ft) }\end{array}$ \\
\hline \multicolumn{4}{|c|}{ Wells Monitoring the Top of the Unconfined Aquifer } \\
\hline $399-3-1$ & $\begin{array}{l}7 / 28 / 93 \\
8 / 18 / 93\end{array}$ & $\begin{array}{l}42.64 \\
41.11\end{array}$ & $\begin{array}{l}341.75 \\
343.28\end{array}$ \\
\hline $399-3-6$ & $\begin{array}{l}7 / 28 / 93 \\
8 / 18 / 93 \\
9 / 27 / 93\end{array}$ & $\begin{array}{l}50.62 \\
51.13 \\
52.58\end{array}$ & $\begin{array}{l}342.18 \\
341.67 \\
340.22\end{array}$ \\
\hline $399-3-7$ & $\begin{array}{l}7 / 28 / 93 \\
8 / 18 / 93 \\
9 / 27 / 93\end{array}$ & $\begin{array}{l}52.02 \\
52.49 \\
54.05\end{array}$ & $\begin{array}{l}342.16 \\
341.69 \\
340.13\end{array}$ \\
\hline $399-3-9$ & $\begin{array}{l}7 / 28 / 93 \\
8 / 18 / 93 \\
9 / 27 / 93 \\
9 / 27 / 93\end{array}$ & $\begin{array}{l}46.39 \\
46.73 \\
48.87 \\
48.80\end{array}$ & $\begin{array}{l}341.69 \\
341.35 \\
339.21 \\
339.28^{\star}\end{array}$ \\
\hline $399-3-10$ & $\begin{array}{l}7 / 28 / 93 \\
8 / 18 / 93 \\
9 / 28 / 93\end{array}$ & $\begin{array}{l}43.42 \\
43.83 \\
45.62\end{array}$ & $\begin{array}{l}341.93 \\
341.52 \\
339.73\end{array}$ \\
\hline $399-3-12$ & $\begin{array}{l}7 / 29 / 93 \\
9 / 27 / 93\end{array}$ & $\begin{array}{l}45.79 \\
48.29\end{array}$ & $\begin{array}{l}342.25 \\
339.75\end{array}$ \\
\hline $399-4-1$ & $\begin{array}{l}7 / 28 / 93 \\
8 / 18 / 93 \\
9 / 27 / 93\end{array}$ & $\begin{array}{l}54.05 \\
54.47 \\
56.47\end{array}$ & $\begin{array}{l}341.55 \\
341.13 \\
339.13\end{array}$ \\
\hline $399-4-7$ & $\begin{array}{l}7 / 28 / 93 \\
8 / 18 / 93 \\
9 / 27 / 93\end{array}$ & $\begin{array}{l}37.07 \\
37.42 \\
39.95\end{array}$ & $\begin{array}{l}341.49 \\
341.14 \\
338.61\end{array}$ \\
\hline $399-4-9$ & $\begin{array}{l}7 / 28 / 93 \\
8 / 18 / 93\end{array}$ & $\begin{array}{l}40.53 \\
40.92\end{array}$ & $\begin{array}{l}341.63 \\
341.24\end{array}$ \\
\hline $399-4-10$ & $\begin{array}{l}7 / 28 / 93 \\
8 / 18 / 93 \\
9 / 27 / 93\end{array}$ & $\begin{array}{l}36.69 \\
37.02 \\
39.56\end{array}$ & $\begin{array}{l}341.85 \\
341.52 \\
338.98\end{array}$ \\
\hline
\end{tabular}


Table 17-2. RCRA Water Level Measurement Report 300 Area Process Trenches, Third Quarter 1993. (sheet 4 of 5 )

\begin{tabular}{|c|c|c|c|}
\hline Well & Date & $\begin{array}{l}\text { Depth to } \\
\text { water (ft) }\end{array}$ & $\begin{array}{l}\text { Water level } \\
\text { elevation } \\
\text { above msl (ft) }\end{array}$ \\
\hline \multicolumn{4}{|c|}{ Wells } \\
\hline $399-4-11$ & $\begin{array}{l}7 / 28 / 93 \\
8 / 18 / 93 \\
9 / 27 / 93\end{array}$ & $\begin{array}{l}62.45 \\
62.91 \\
64.65\end{array}$ & $\begin{array}{l}342.00 \\
341.54 \\
339.80\end{array}$ \\
\hline $399-5-1$ & $\begin{array}{l}7 / 28 / 93 \\
8 / 18 / 93 \\
9 / 27 / 93\end{array}$ & $\begin{array}{l}53.54 \\
54.14 \\
55.25\end{array}$ & $\begin{array}{l}341.93 \\
341.39 \\
340.28\end{array}$ \\
\hline $399-6-1$ & $\begin{array}{l}7 / 28 / 93 \\
8 / 18 / 93 \\
9 / 27 / 93\end{array}$ & $\begin{array}{l}46.50 \\
47.21 \\
48.20\end{array}$ & $\begin{array}{l}342.29 \\
341.58 \\
340.59\end{array}$ \\
\hline $399-8-1$ & $\begin{array}{l}7 / 28 / 93 \\
8 / 18 / 93 \\
9 / 27 / 93\end{array}$ & $\begin{array}{l}53.58 \\
54.25 \\
55.31\end{array}$ & $\begin{array}{l}342.55 \\
341.88 \\
340.82\end{array}$ \\
\hline $399-8-2$ & $\begin{array}{l}7 / 28 / 93 \\
8 / 18 / 93 \\
9 / 27 / 93\end{array}$ & $\begin{array}{l}54.69 \\
55.48 \\
56.22\end{array}$ & $\begin{array}{l}343.33 \\
342.54 \\
341.80\end{array}$ \\
\hline $399-8-3$ & $\begin{array}{l}7 / 28 / 93 \\
8 / 18 / 93 \\
9 / 27 / 93\end{array}$ & $\begin{array}{l}51.90 \\
52.59 \\
53.56\end{array}$ & $\begin{array}{l}342.85 \\
342.16 \\
341.19\end{array}$ \\
\hline $699-S 27-E 14$ & $\begin{array}{l}7 / 28 / 93 \\
8 / 18 / 93 \\
9 / 27 / 93\end{array}$ & $\begin{array}{l}58.20 \\
58.57 \\
60.54\end{array}$ & $\begin{array}{l}341.48 \\
341.11 \\
339.14\end{array}$ \\
\hline
\end{tabular}

Wells Monitoring the Bottom of the Unconfined Aquifer

\begin{tabular}{llll}
\hline $399-1-16 B$ & $7 / 28 / 93$ & 38.70 & 342.38 \\
& $8 / 18 / 93$ & 39.32 & 341.76 \\
$9 / 27 / 93$ & 41.18 & 339.90 \\
$399-1-17 B$ & $7 / 28 / 93$ & 35.44 & 342.36 \\
& $8 / 18 / 93$ & 35.89 & 341.91 \\
& $9 / 07 / 93$ & 37.18 & $340.62^{\star}$ \\
$9 / 27 / 93$ & 37.46 & 340.34
\end{tabular}


Table 17-2. RCRA Water Level Measurement Report 300 Area Process Trenches, Third Quarter 1993. (sheet 5 of 5 )

Well Date $\quad \begin{gathered}\text { Depth to } \\ \text { water ( } \mathrm{ft})\end{gathered} \begin{gathered}\text { Water level } \\ \text { elevation } \\ \text { above msi ( } \mathrm{ft} \text { ) }\end{gathered}$

Wells Monitoring the Bottom of the Unconfined Aquifer

\begin{tabular}{llll}
\hline $399-1-18 B$ & $7 / 28 / 93$ & 46.12 & 343.77 \\
& $8 / 18 / 93$ & 46.71 & 343.18 \\
& $9 / 27 / 93$ & 48.03 & 341.86 \\
\hline
\end{tabular}

Wells Monitoring the Confined Aquifer

\begin{tabular}{lccc}
\hline $399-1-9$ & $7 / 28 / 93$ & 26.10 & 358.66 \\
& $8 / 18 / 93$ & 23.19 & 361.57 \\
$9 / 27 / 93$ & 28.78 & 355.98 \\
$399-1-16 C$ & $9 / 27 / 93$ & 8.33 & 373.90 \\
$399-1-17 C$ & $7 / 28 / 93$ & 1.40 & 376.66 \\
& $8 / 18 / 93$ & 2.00 & 376.06 \\
$399-1-18 C$ & $9 / 27 / 93$ & 3.17 & 374.89 \\
& $7 / 28 / 93$ & 43.72 & 344.29 \\
& $8 / 18 / 93$ & 44.25 & 343.76 \\
& $9 / 27 / 93$ & 45.41 & 342.60 \\
\hline
\end{tabular}

Notes: 1. Water level elevations are calculated by subtracting the measured depth to water from the surveyed elevation for the well.

2. Depth-to-water values are transcribed from field records.

3. Elevations marked with an ' $*$ ' were measured at the time of sampling.

4. To convert feet to meters multiply by 0.3048 .

5. Elevations of reference points were surveyed in meters. The datum was converted to feet to calculate water level measurements. 
Table 17-3. Constituent List and Summary of Results for the 300 Area Process Trenches Data for Reporting Period July 1

through September 30,1993 . (sheet 1 of 3 )

CONTAMINATION INDICATOR PARAMETERS

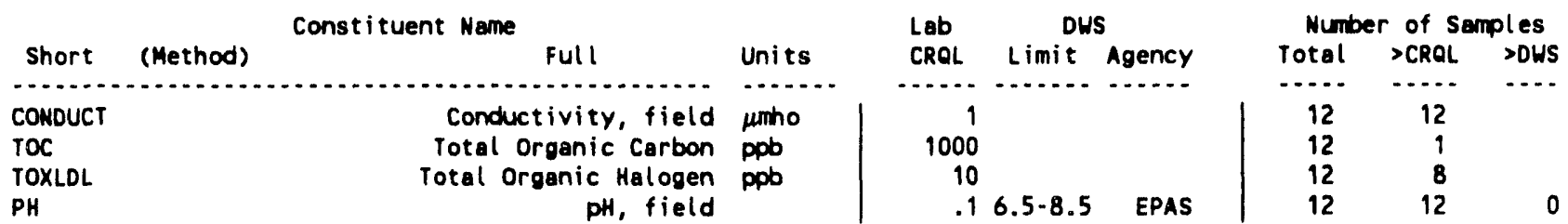

DRINKING WATER PARAMETERS

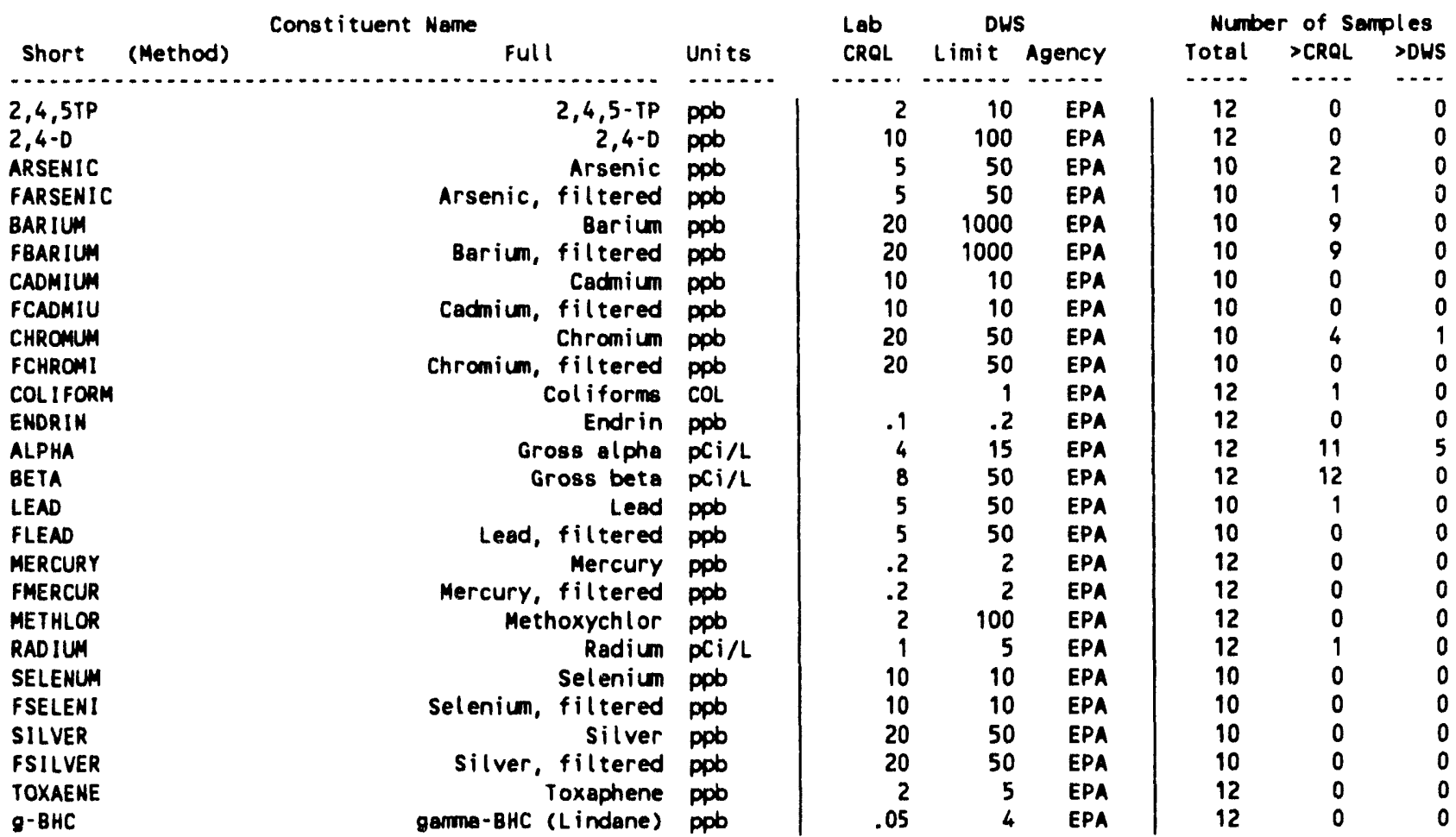

GROUNDWATER QUALITY PARAMETERS

\begin{tabular}{|c|c|c|c|c|c|c|c|c|c|}
\hline \multirow[b]{2}{*}{ Short } & \multicolumn{2}{|c|}{ Constituent Name } & \multirow[b]{2}{*}{ Units } & \multirow{2}{*}{$\begin{array}{l}\text { Lab } \\
\text { CROL }\end{array}$} & \multicolumn{2}{|c|}{ DWS } & \multicolumn{3}{|c|}{ Number of Samples } \\
\hline & (Method) & Full & & & Limit & Agency & Total & $>C R Q L$ & $>$ DWS \\
\hline$\ldots$. & $\ldots \ldots$ & $\ldots \ldots$ & $\ldots \ldots$ & $\ldots . .$. & $\ldots$ & $\ldots \ldots$ & $\cdots$ & $\cdots-\cdots$ & $\ldots$ \\
\hline IRON & & Iron & ppb & 20 & 300 & EPAS & 10 & 10 & 1 \\
\hline FIRON & & Iron, filtered & ppb & 20 & 300 & EPAS & 10 & 7 & 0 \\
\hline MANGESE & & Manganese & ppo & 10 & 50 & EPAS & 10 & 2 & 1 \\
\hline FMANGAN & & Manganese, filtered & ppb & 10 & 50 & EPAS & 10 & 1 & 1 \\
\hline LPHENOL & & Phenol & ppb & 20 & & & 12 & 0 & \\
\hline SOOIUN & & Sodium & ppb & 300 & & & 10 & 10 & \\
\hline FSOOIUM & & Sodium, filtered & ppo & 300 & & & 10 & 10 & \\
\hline
\end{tabular}


Table 17-3. Constituent List and Summary of Results for the 300 Area Process Trenches Data for Reporting Period July 1

through September 30, 1993. (sheet 2 of 3)

SITE SPECIFIC AND OTHER CONSTITUENTS

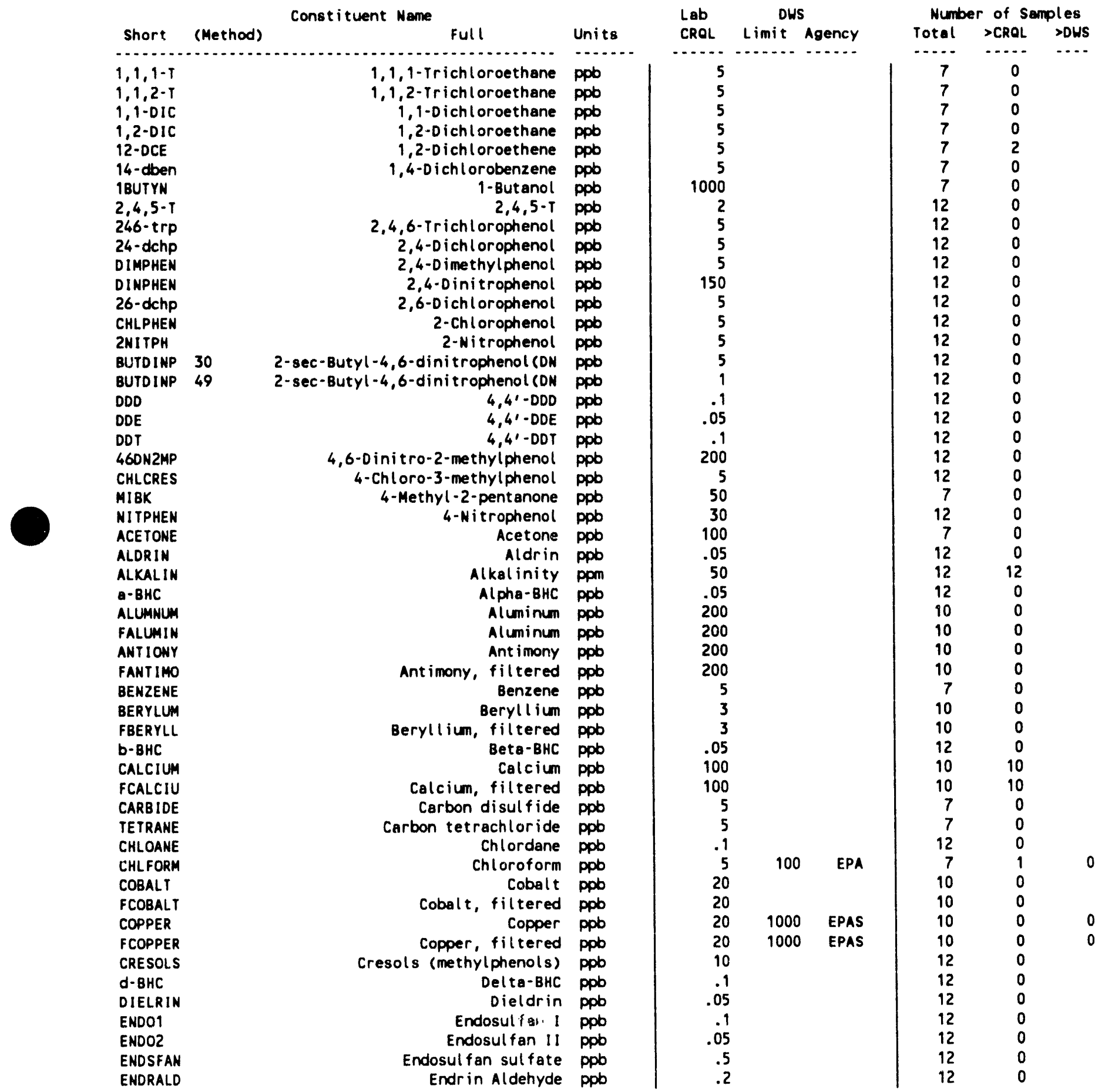


Table 17-3. Constituent List and Summary of Results for the 300 Area Process Trenches Data for Reporting Period July 1 through September 30, 1993. (sheet 3 of 3)

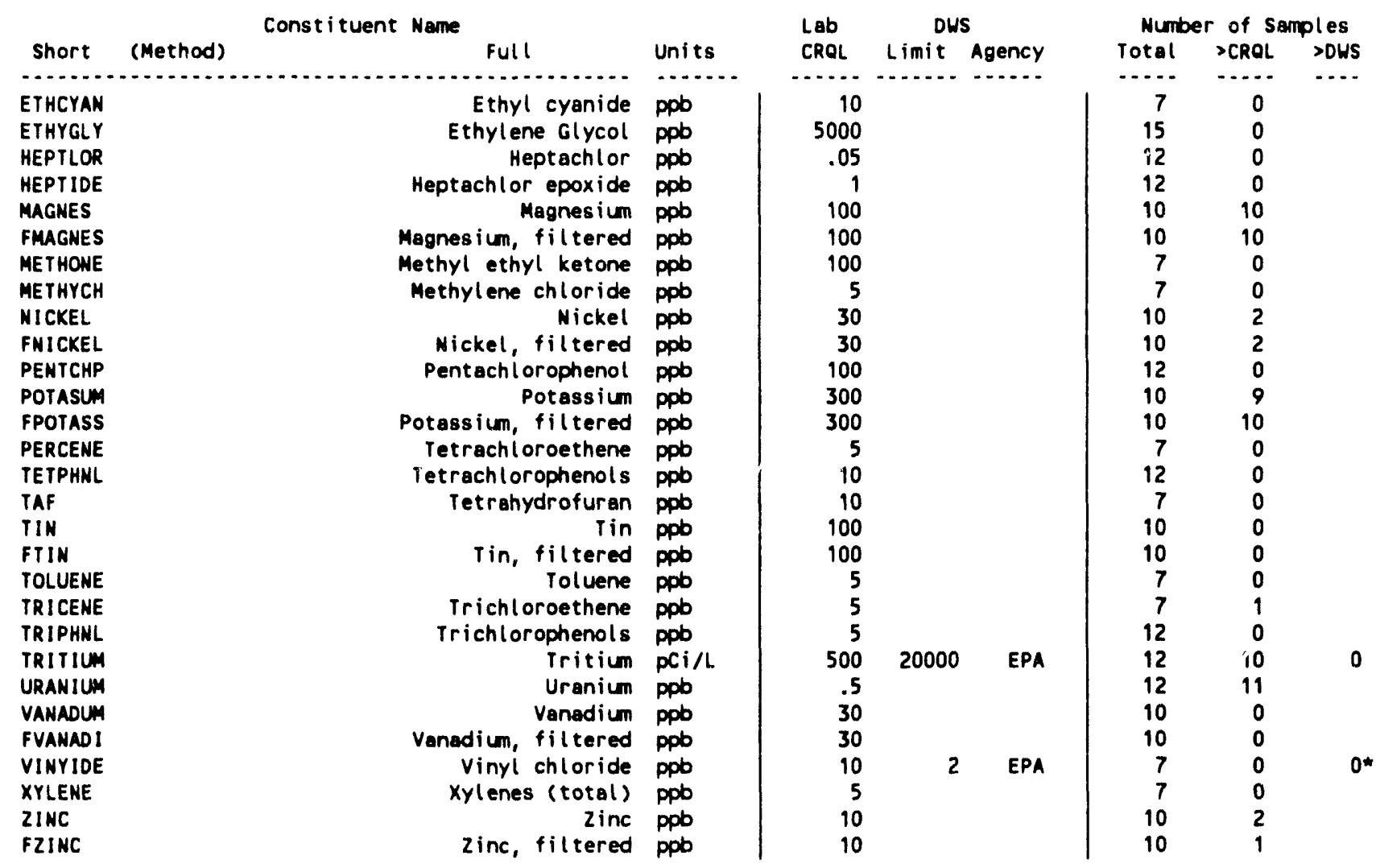

For explanation of this table, see section 1.4 of report. 
Table 17-4. Constituents with at Least One Value Above the CRQL for the 300 Area Process Trenches Data for Reporting Period July 1 through September 30, 1993. (sheet 1 of 5)

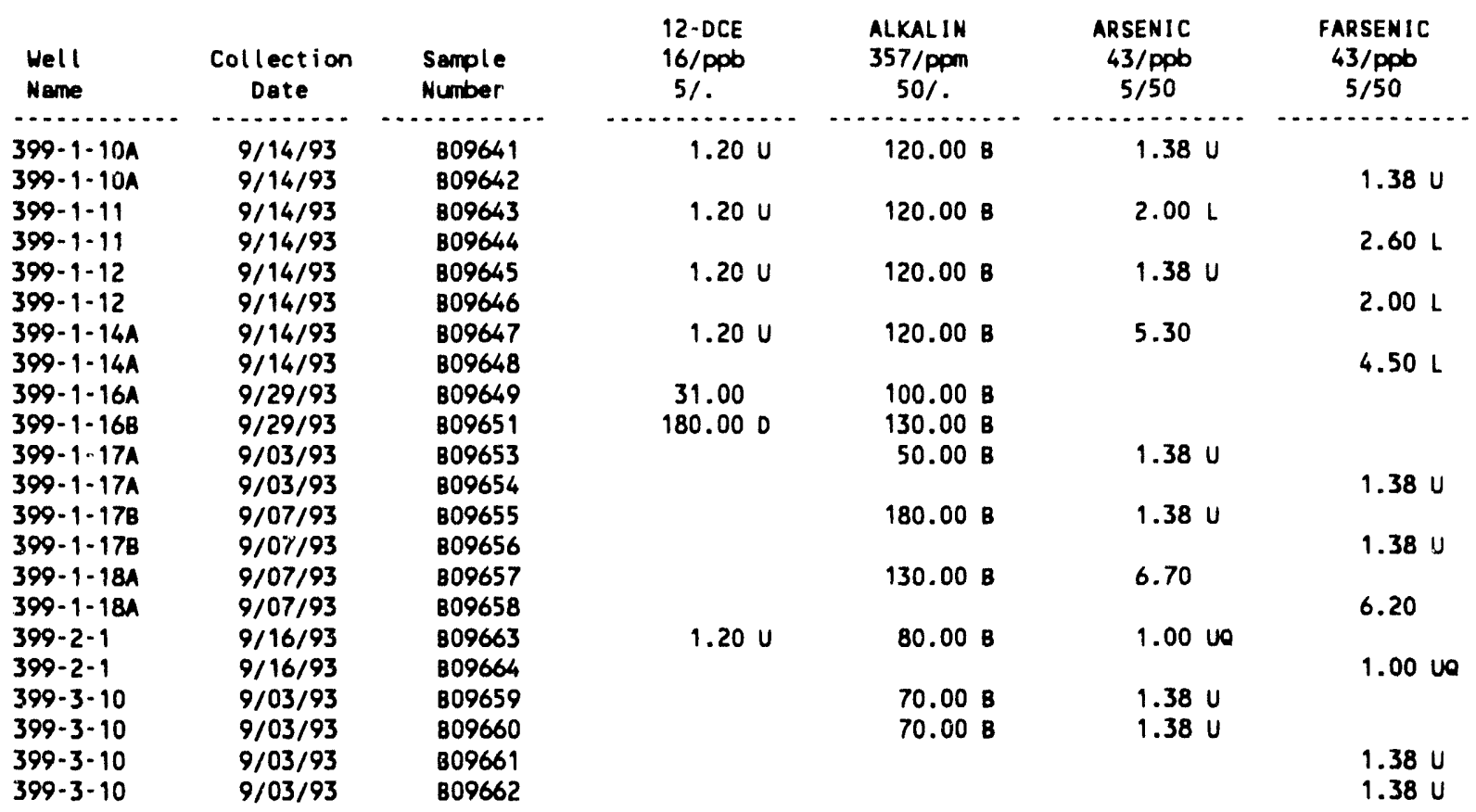

\begin{tabular}{|c|c|c|c|c|c|c|}
\hline $\begin{array}{l}\text { Well } \\
\text { Name }\end{array}$ & $\begin{array}{c}\text { Collection } \\
\text { Date }\end{array}$ & $\begin{array}{l}\text { Sample } \\
\text { Number }\end{array}$ & $\begin{array}{l}\text { BARIUM } \\
34 / \text { ppb } \\
20 / 1000\end{array}$ & $\begin{array}{l}\text { FBARIUM } \\
34 / \mathrm{ppb} \\
20 / 1000\end{array}$ & $\begin{array}{c}\text { CALCIUM } \\
34 / \mathrm{ppb} \\
100 \%\end{array}$ & $\begin{array}{l}\text { FCALCIU } \\
34 / \text { ppb } \\
100 \%\end{array}$ \\
\hline $399-1-10 A$ & $9 / 14 / 93$ & 809641 & $5 i .00$ & & 45000.00 & \\
\hline $399-1-10 A$ & $9 / 14 / 93$ & B09642 & & 50.00 & & 46000.00 \\
\hline $399-1-11$ & $9 / 14 / 93$ & 809643 & 38.00 & & 46000.00 & \\
\hline $399-1-11$ & $9 / 14 / 93$ & 809644 & & 38.00 & & 46000.00 \\
\hline $399-1-12$ & $9 / 14 / 93$ & 809645 & 38.00 & & 46000.00 & \\
\hline $399-1-12$ & $9 / 14 / 93$ & 809646 & & 38.00 & & 45000.00 \\
\hline $399-1-14 A$ & $9 / 14 / 93$ & 809647 & 40.00 & & 45000.00 & \\
\hline $399-1-14 A$ & $9 / 14 / 93$ & B09648 & & 40.00 & & 45000.00 \\
\hline $399-1-16 A$ & $9 / 29 / 93$ & 809649 & 56.00 & & 45000.00 & \\
\hline $399-1-16 A$ & $9 / 29 / 93$ & B09650 & & 55.00 & & 44000.00 \\
\hline $399 \cdot 1 \cdot 168$ & $9 / 29 / 93$ & B09651 & 48.00 & & 17000.00 & \\
\hline $399-1-168$ & $9 / 29 / 93$ & B09652 & & 49.00 & & 17000.00 \\
\hline $399-1-17 \mathrm{~A}$ & $9 / 03 / 93$ & B09653 & $15.00 \mathrm{~L}$ & & 18000.00 & \\
\hline $399-1-17 A$ & $9 / 03 / 93$ & B09654 & & $15.00 \mathrm{~L}$ & & 18000.00 \\
\hline $399-2-1$ & $9 / 16 / 93$ & B09663 & 65.00 & & 37000.00 & \\
\hline $399-2-1$ & $9 / 16 / 93$ & B09664 & & 64.00 & & 36000.00 \\
\hline $399-3-10$ & $9 / 03 / 93$ & B09659 & 52.00 & & 39000.00 & \\
\hline $399 \cdot 3 \cdot 10$ & $9 / 03 / 93$ & B09660 & 53.00 & & 40000.00 & \\
\hline $399-3-10$ & $9 / 03 / 93$ & 809661 & & 53.00 & & 40000.00 \\
\hline $399-3-10$ & $9 / 03 / 93$ & B09662 & & 51.00 & & 38000.00 \\
\hline
\end{tabular}


Table 17-4. Constituents with at Least One Value Above the CRQL for the 300 Area Process Trenches Data for Reporting Period July 1 through September 30, 1993. (sheet 2 of 5)

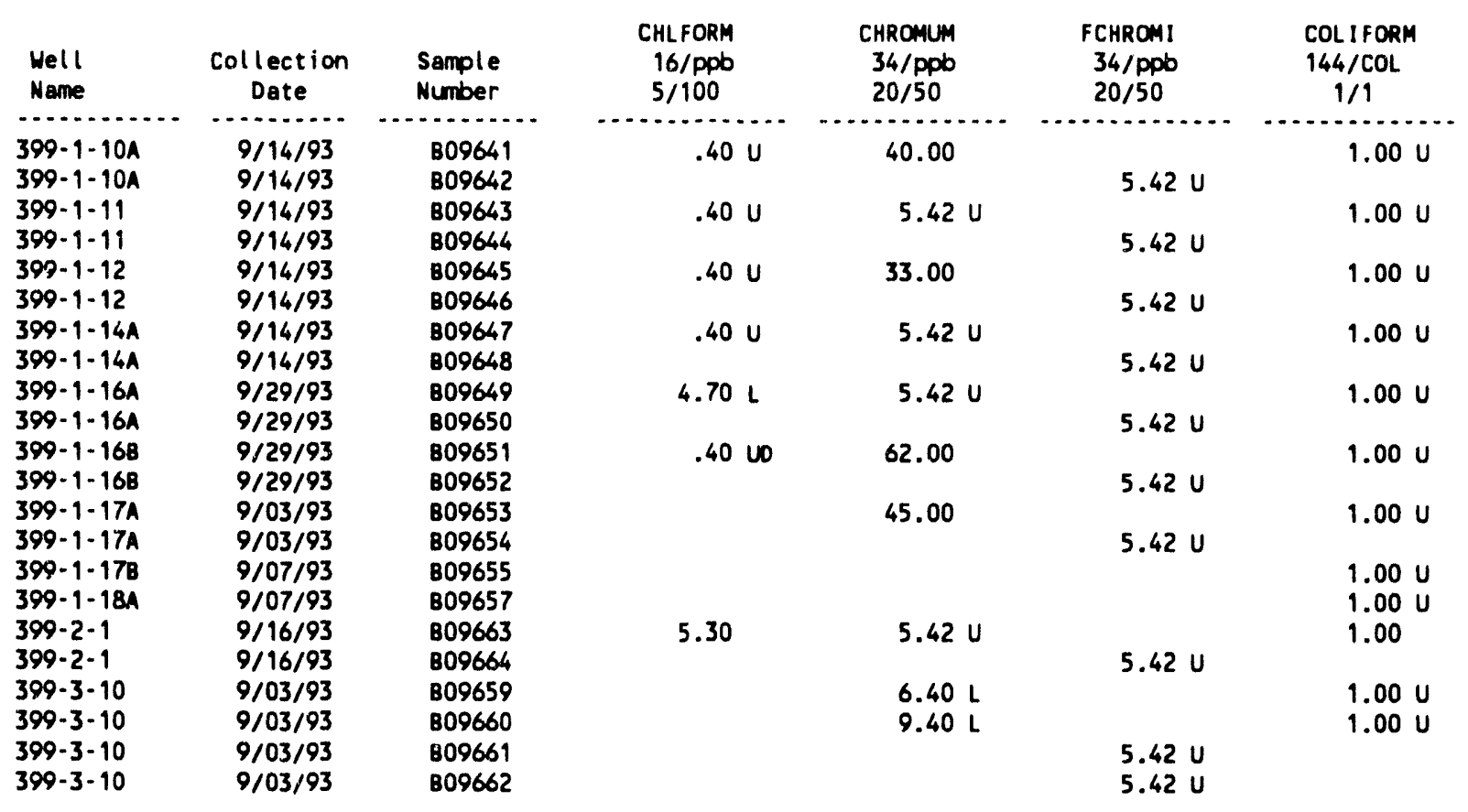

\begin{tabular}{|c|c|c|c|c|c|c|}
\hline $\begin{array}{l}\text { Well } \\
\text { Name }\end{array}$ & $\begin{array}{c}\text { Collection } \\
\text { Date }\end{array}$ & $\begin{array}{l}\text { Sample } \\
\text { Number }\end{array}$ & $\begin{array}{c}\text { ALPHA } \\
135 / \mathrm{pCi} / \mathrm{L} \\
. / 15\end{array}$ & $\begin{array}{c}\text { BETR } \\
\text { 136/PCi I/L } \\
. / 50\end{array}$ & $\begin{array}{c}\text { IRON } \\
34 / \mathrm{ppb} \\
20 / 300 \mathrm{~s}\end{array}$ & $\begin{array}{c}\text { FIROW } \\
\text { 34/ppb } \\
20 / 300 \text { s }\end{array}$ \\
\hline $399-1-10 A$ & oig & 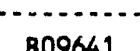 & (1....... & and & (a) & $\cdots$ \\
\hline $399-1-10 A$ & $9 / 14 / 93$ & $\begin{array}{l}809041 \\
809642\end{array}$ & 65.80 & 15.80 & 220.00 & $10.30 \mathrm{U}$ \\
\hline $399-1-11$ & $9 / 14 / 93$ & 809643 & 18.90 & 10.00 & 150.00 & \\
\hline $399-1-11$ & $9 / 14 / 93$ & 809644 & & & & 32.00 \\
\hline $399-1-12$ & $9 / 14 / 93$ & 809645 & 27.70 & 11.50 & 130.00 & \\
\hline $399-1-12$ & $9 / 14 / 93$ & 809646 & & & & 25.00 \\
\hline $399-1-14 A$ & $9 / 14 / 93$ & 809647 & 2.84 & 7.48 & 150.00 & \\
\hline $399-1-14 A$ & $9 / 14 / 93$ & $B 09648$ & & & & $10.30 \mathrm{U}$ \\
\hline $399-1.16 A$ & $9 / 29 / 93$ & 809649 & 92.30 & 25.30 & 71.00 & \\
\hline $399-1-16 A$ & $9 / 29 / 93$ & $B 09650$ & & & & 29.00 \\
\hline $399-1-168$ & $9 / 29 / 93$ & B09651 & 2.14 & 4.71 & 340.00 & \\
\hline $399-1-168$ & $9 / 29 / 93$ & B09652 & & & & 86.00 \\
\hline $399-1-17 A$ & $9 / 03 / 93$ & B09653 & 8.73 & 4.62 & 210.00 & \\
\hline $399-1-17 A$ & $9 / 03 / 93$ & B09654 & & & & $12.00 \mathrm{~L}$ \\
\hline $399-1-178$ & $9 / 07 / 93$ & B09655 & $-.12 \mathrm{U}$ & 8.66 & & \\
\hline $399-1-18 \mathrm{~A}$ & $9 / 07 / 93$ & B09657 & 3.03 & 10.90 & & \\
\hline $399-2-1$ & $9 / 16 / 93$ & B09663 & 67.30 & 20.50 & 210.00 & \\
\hline $399-2-1$ & $9 / 16 / 93$ & B09664 & & & & 74.00 \\
\hline $399-3-10$ & $9 / 03 / 93$ & 809659 & 11.80 & 9.49 & 300.00 & \\
\hline $399-3-10$ & $9 / 03 / 93$ & 809660 & 13.60 & 7.90 & 290.00 & \\
\hline $399 \cdot 3 \cdot 10$ & $9 / 03,93$ & $B 09661$ & & & & 83.00 \\
\hline $399-3-10$ & $9 / 03 / 93$ & 809662 & & & & 70.00 \\
\hline
\end{tabular}


Table 17-4. Constituents with at Least One Value Above the CRQL for the 300 Area Process Trenches Data for Reporting Period July 1 through September 30, 1993. (sheet 3 of 5)

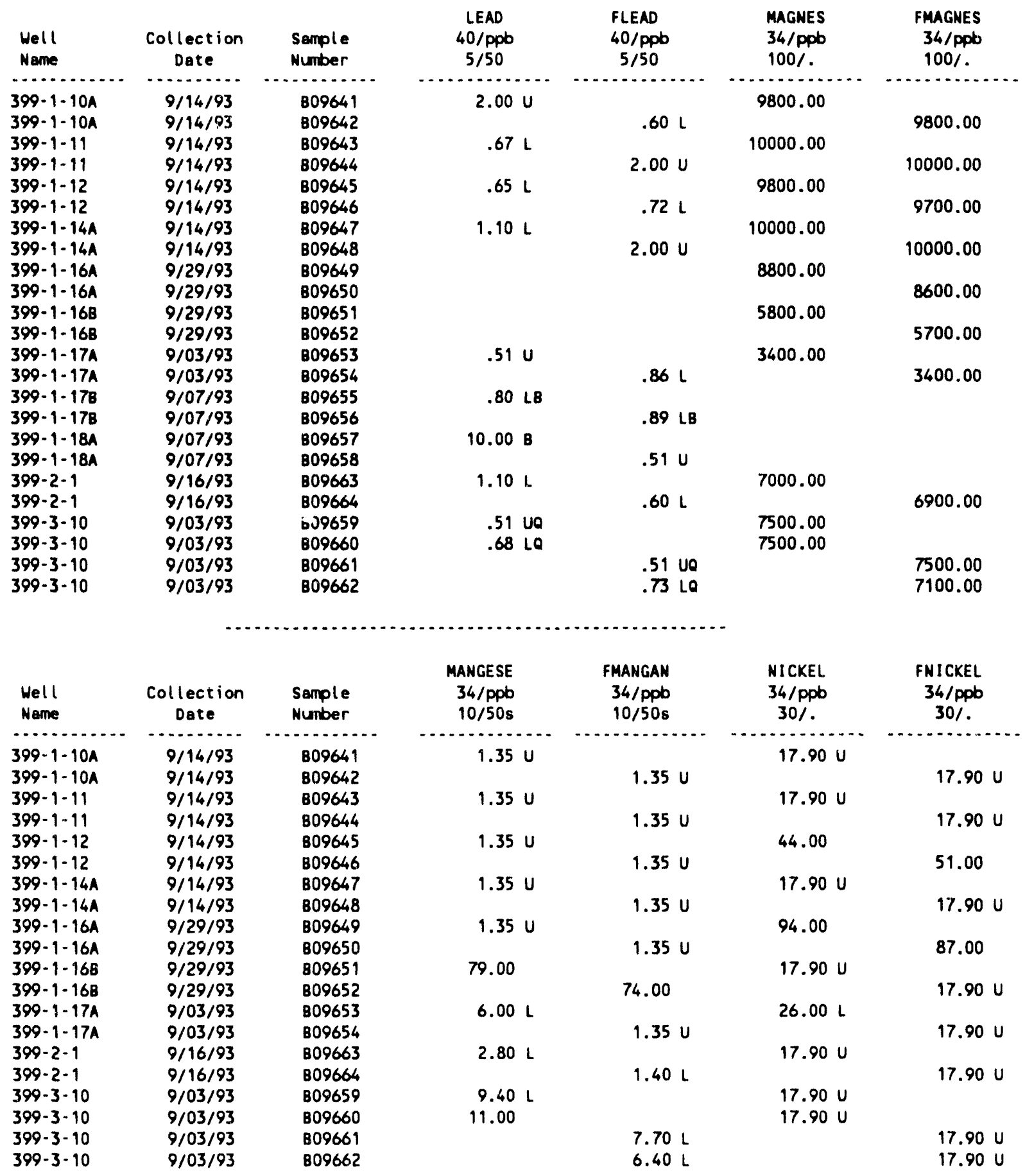


Tabie 17-4. Constituents with at Least One Value Above the CRQL for the 300 Area Process Trenches Data for Reporting Period July 1 through September 30, 1993. (sheet 4 of 5)

\begin{tabular}{|c|c|c|c|c|c|c|}
\hline $\begin{array}{l}\text { Well } \\
\text { Name }\end{array}$ & $\begin{array}{c}\text { Collection } \\
\text { Date }\end{array}$ & $\begin{array}{l}\text { Semple } \\
\text { Number }\end{array}$ & $\begin{array}{c}\text { POTASUM } \\
34 / \text { ppb } \\
1000 / .\end{array}$ & $\begin{array}{l}\text { FPCTASS } \\
34 / \mathrm{ppb} \\
1000 \%\end{array}$ & $\begin{array}{c}\text { RADIUM } \\
137 / \mathrm{pCI} / \mathrm{L} \\
. !\end{array}$ & $\begin{array}{l}\text { so0 Ium } \\
34 / \text { pob } \\
300 / .\end{array}$ \\
\hline$\cdots \ldots$ & $\cdots$ & $\cdots$ & $\ldots \ldots \ldots$ & $\cdots$ & $\ldots$ & $\ldots$ \\
\hline $\begin{array}{l}399-1-10 A \\
399-1-10 A\end{array}$ & $\begin{array}{l}9 / 14 / 93 \\
9 / 14 / 93\end{array}$ & $\begin{array}{l}809641 \\
809642\end{array}$ & 3300.00 & 3700.10 & $.09 \mathrm{U}$ & 20000.00 \\
\hline $\begin{array}{l}399-1-11 \\
399-1-11\end{array}$ & $\begin{array}{l}9 / 14 / 93 \\
9 / 14 / 93\end{array}$ & $\begin{array}{l}809643 \\
809644\end{array}$ & 3600.00 & 3400.00 & .14 & 21000.00 \\
\hline $\begin{array}{l}399-1-12 \\
399-1-12\end{array}$ & $\begin{array}{l}9 / 14 / 93 \\
9 / 14 / 93\end{array}$ & $\begin{array}{l}809645 \\
809646\end{array}$ & 3700.00 & 3000.00 & $-.04 \mathrm{U}$ & 20000.00 \\
\hline $\begin{array}{l}399-1-14 A \\
399-1-14 A\end{array}$ & $\begin{array}{l}9 / 14 / 93 \\
9 / 14 / 93\end{array}$ & $\begin{array}{l}B 09647 \\
809648\end{array}$ & 5600.00 & 5200.00 & $-.00 \mathrm{U}$ & 22000.00 \\
\hline $\begin{array}{l}399 \cdot 1-16 A \\
399 \cdot 1-16 A\end{array}$ & $\begin{array}{l}9 / 29 / 93 \\
9 / 29 / 93\end{array}$ & $\begin{array}{l}B 09649 \\
B 09650\end{array}$ & 2300.00 & 2500.00 & $.08 \mathrm{U}$ & 19000.00 \\
\hline $\begin{array}{l}399-1-168 \\
399-1-168\end{array}$ & $\begin{array}{l}9 / 29 / 93 \\
9 / 29 / 93\end{array}$ & $\begin{array}{l}B 09651 \\
B 09652\end{array}$ & 3600.00 & 3500.00 & $.04 \mathrm{U}$ & 45000.00 \\
\hline $\begin{array}{l}399-1-17 A \\
399-1-17 A\end{array}$ & $\begin{array}{l}9 / 03 / 93 \\
9 / 03 / 93\end{array}$ & $\begin{array}{l}809653 \\
809654\end{array}$ & $860.00 \mathrm{~L}$ & 1900.00 & $-.02 \mathrm{U}$ & 11000.00 \\
\hline $\begin{array}{l}399-1-178 \\
399-1-18 A\end{array}$ & $\begin{array}{l}9 / 07 / 93 \\
9 / 07 / 93\end{array}$ & $\begin{array}{l}809655 \\
809657\end{array}$ & & ( & $\begin{array}{l}.06 \mathrm{U} \\
.05 \mathrm{U}\end{array}$ & \\
\hline $\begin{array}{l}399-2-1 \\
399-2-1\end{array}$ & $\begin{array}{l}9 / 16 / 93 \\
9 / 16 / 93\end{array}$ & $\begin{array}{l}\mathrm{B} 09663 \\
\mathrm{~B} 09664\end{array}$ & 3100.00 & 3300.00 & $.07 \mathrm{U}$ & 19000.00 \\
\hline $\begin{array}{l}399 \cdot 3 \cdot 10 \\
399 \cdot 3 \cdot 10\end{array}$ & $\begin{array}{l}9 / 03 / 93 \\
9 / 03 / 93\end{array}$ & $\begin{array}{l}809659 \\
809660\end{array}$ & $\begin{array}{l}4000.00 \\
3900.00\end{array}$ & & $\begin{array}{r}.00 \mathrm{U} \\
-.04 \mathrm{U}\end{array}$ & $\begin{array}{l}14000.00 \\
14000.00\end{array}$ \\
\hline $\begin{array}{l}399-3-10 \\
399-3-10\end{array}$ & $\begin{array}{l}9 / 03 / 93 \\
9 / 03 / 93\end{array}$ & $\begin{array}{l}B 09661 \\
809662\end{array}$ & & $\begin{array}{l}3900.00 \\
3600.00\end{array}$ & & \\
\hline
\end{tabular}

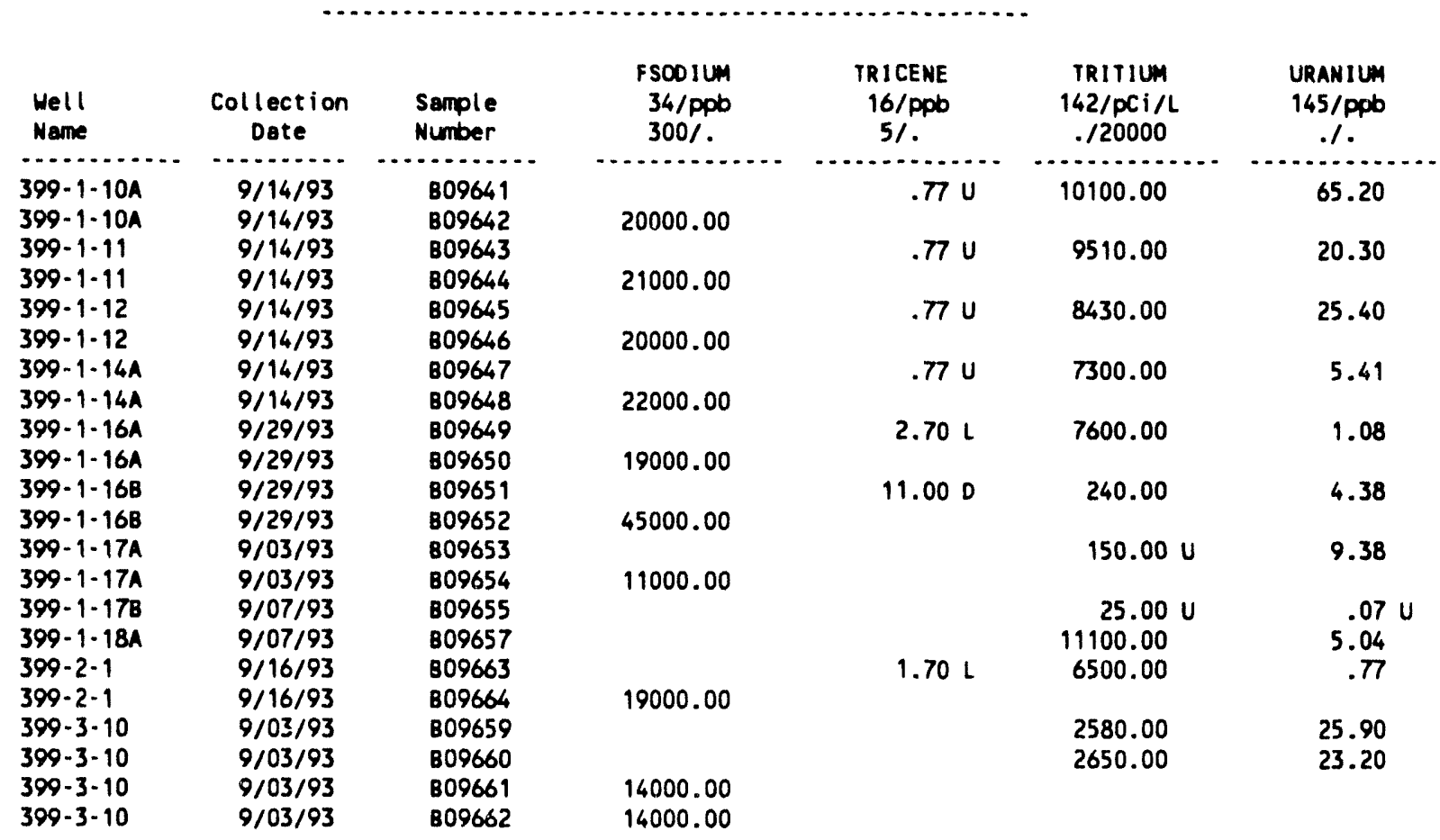


Table 17-4. Constituents with at Least One Value Above the CRQL for the 300 Area Process Trenches Data for Reporting Period July 1 through September 30, 1993. (sheet 5 of 5)

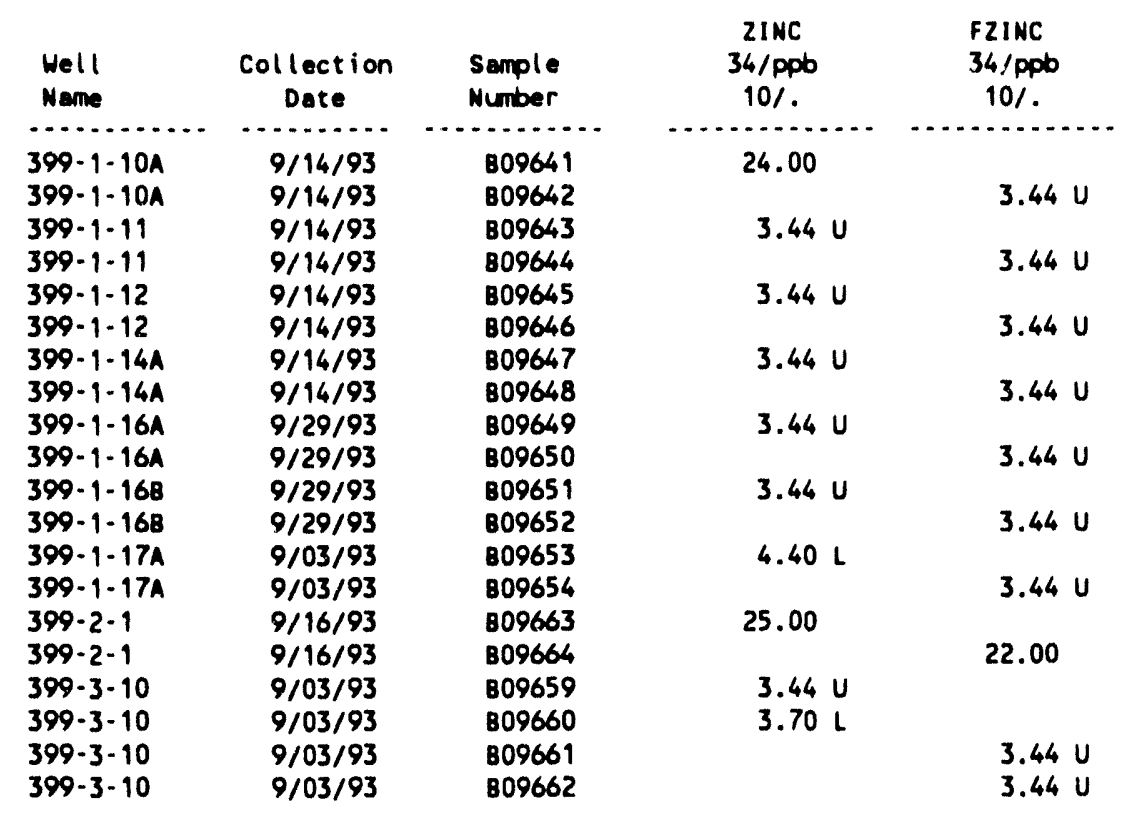

For explanation of this table, see Secticn 1.4 of report. 
Table 17-5. Contamination Indicator Parameters for the 300 Area Process Trenches Data for Reporting Period July 1 through September 30, 1993.

\begin{tabular}{|c|c|c|c|c|c|c|c|c|}
\hline $\begin{array}{l}\text { Well } \\
\text { Name }\end{array}$ & $\begin{array}{c}\text { Collection } \\
\text { Date }\end{array}$ & $\begin{array}{l}\text { Semple } \\
\text { Number }\end{array}$ & $\begin{array}{c}\text { COND FIELD } \\
\text { unho } \\
1 / 700_{W}\end{array}$ & $\begin{array}{c}\text { COND LAB } \\
\mu m \operatorname{mon}^{2} \\
. / 700 \mathrm{~W}\end{array}$ & $\begin{array}{l}\text { PH FIELD } \\
.01 / 6.5-8.5 \mathrm{~s}\end{array}$ & $\begin{array}{c}\text { PH LAB } \\
.01 / 6.5-8.5 \mathrm{~s}\end{array}$ & $\begin{array}{c}\text { roc } \\
p p b \\
1000 / .\end{array}$ & $\begin{array}{l}\text { rox } \\
\text { Ppb } \\
10 \%\end{array}$ \\
\hline $\begin{array}{l}399-1-10 A \\
399-1-11 \\
399-1-12 \\
399-1-14 A \\
399-1-16 A \\
399-1-168 \\
399-1-17 A \\
399-1-17 B \\
399-1-18 A \\
399-2-1 \\
399-3-10 \\
300-3-0\end{array}$ & $\begin{array}{l}9 / 14 / 93 \\
9 / 14 / 93 \\
9 / 14 / 93 \\
9 / 14 / 93 \\
9 / 29 / 93 \\
9 / 29 / 93 \\
9 / 03 / 93 \\
9 / 07 / 93 \\
9 / 07 / 93 \\
9 / 16 / 93 \\
9 / 03 / 93\end{array}$ & $\begin{array}{l}809641 \\
809643 \\
809645 \\
809647 \\
809649 \\
809651 \\
809653 \\
809655 \\
809657 \\
809663 \\
B 09659 \\
809660\end{array}$ & $\begin{array}{l}437 \\
441 \\
436 \\
453 \\
389 \\
332 \\
159 \\
366 \\
452 \\
338 \\
323 \\
\end{array}$ & & $\begin{array}{l}7.48 \\
7.66 \\
7.62 \\
7.79 \\
7.52 \\
8.07 \\
7.01 \\
7.84 \\
7.76 \\
7.19 \\
7.17\end{array}$ & & $\begin{array}{c}400 \mathrm{~L} \\
400 \mathrm{~L} \\
400 \mathrm{~L} \\
400 \mathrm{~L} \\
500 \mathrm{LB} \\
500 \mathrm{LB} \\
1000 \\
400 \mathrm{~L} \\
400 \mathrm{~L} \\
500 \mathrm{~L} \\
600 \mathrm{~L} \\
500 \mathrm{~L}\end{array}$ & $\begin{array}{c}30.0 \mathrm{P} \\
10.0 \mathrm{LP} \\
10.0 \mathrm{P} \\
10.0 \mathrm{P} \\
20.0 \mathrm{P} \\
70.0 \mathrm{P} \\
100.0 \mathrm{P} \\
8.0 \mathrm{UP} \\
8.0 \mathrm{UP} \\
10.0 \mathrm{P} \\
10.0 \mathrm{P} \\
9.0 \mathrm{LP}\end{array}$ \\
\hline
\end{tabular}

For explanation of this table, see Section 1.4 of report. 
DOE/RL-93-56-3

\section{CONTENTS}

18.0 NONRADIOACTIVE DANGEROUS WASTE LANDFILL ........... 18-1

18.1 INTRODUCTION ................... . . . . . . . . . .

18.2 WATER LEVEL DATA . . . . . . . . . . . . . . . . . 18-1

18.3 WATER CHEMISTRY DATA . . . . . . . . . . . . 18-1 


\section{LIST OF FIGURES}

18-1 Monitoring We11 Locations for the Nonradioactive Dangerous Waste Landfill and Solid Waste Landfill . . . . . . . . . . . 18-3

\section{LIST OF TABLES}

18-1 Monitoring Well Purpose and Sampling Schedule for the Nonradioactive Dangerous Waste Landfill Network . . . . . . . . . 18-4

18-2 RCRA Water Level Measurement Report NRDWL, Third Quarter 1993 . 18-5

18-3 Constituent List and Summary of Results for the NRDW Landfill Data for Reporting Period July 1 through September 30, 1993 . . . 18-8

18-4 Constituents with at Least One Value Above the CRQL for the NRDW Landfill Data for Reporting Period July 1 through September 30,1993 .................. 18-10

18-5 Contamination Indicator Parameters for the NRDW Landfill Data for Reporting Period July I through September 30, 1993 . . . . . 18-13 


\title{
18.0 NONRADIOACTIVE DANGEROUS WASTE LANDFILL
}

\author{
F. N. Hodges \\ Westinghouse Hanford Company
}

\subsection{INTRODUCTION}

The Nonradioactive Dangerous Waste Landfill (NRDWL) is part of the Hanford Central Landfill located approximately $5.6 \mathrm{~km}$ (3.5 mi) southeast of the 200 East Area. The NRDWL, covered under the Consent Agreement and Compliance Order (Ecology and EPA 1986), is currently under an interim-status, detection-level groundwater monitoring program (WHC 1993b). The groundwater monitoring network consists of three upgradient and six downgradient wells (Table 18-1). Two upgradient and five downgradient wells are screened at the top of the water table. One upgradient and one downgradient well are screened at the top of a low-permeability unit approximately $21.3 \mathrm{~m}(70 \mathrm{ft}$ ) below the top of the water table. Figure 18-1 shows well locations. It should be noted that well 699-26-34A was previously named 699-26-34.

\subsection{WATER LEVEL DATA}

Nonroutine water level measurements were carried out on a monthly schedule for seven of the NRDWL monitoring wells, the six monitoring wells of the adjacent Solid Waste Landfill, and in several nearby non-RCRA wells. The two new monitoring wells (699-25-34D and 699-26-34B) were placed on a montilly measurement schedule during this quarter. Results of the water level measurements are reported in Table 18-2.

\subsection{WATER CHEMISTRY DATA}

The initial seven-well monitoring network for the NRDWL is on a semiannual sampling schedule, and the two new downgradient monitoring wells (699-25-34D and 699-26-34B) are on a quarterly sampling schedule. A1l wells in the monitoring network were sampled in September 1993. The next scheduled sampling for the two new well is in December 1993. The next scheduled sampling for the remainder of the network is in Marcil 1994. Available data for the September 1993 samplings are included in this report (Tables 18-3 through 18-5).

Tritium exceeded the DWS of $20,000 \mathrm{pCi} / \mathrm{L}$ in all NRDWL wells sampled in September 1993, with the exception of deep downgradient well 699-25-33A. The tritium contamination resulted from upgradient sources in the 200 Areas. A value for gross beta from downgradient well 699-25-35A, sampled on September 16, 1993, exceeded the DWS. This value of $77.6 \mathrm{pCi} / \mathrm{L}$ far exceeds the historical trend for this well and stands in strong contrast to a duplicate sample, from this sampling event, with a reported value of i2.3 $\mathrm{pCi} / \mathrm{L}$. A RADE has been submitted to check this result.

Values of specific conductance, field $\mathrm{pH}$, and TOC reported in this quarter's report do not exceed their critical means for this site. ToX values were not evaluated because the data are suspect. 


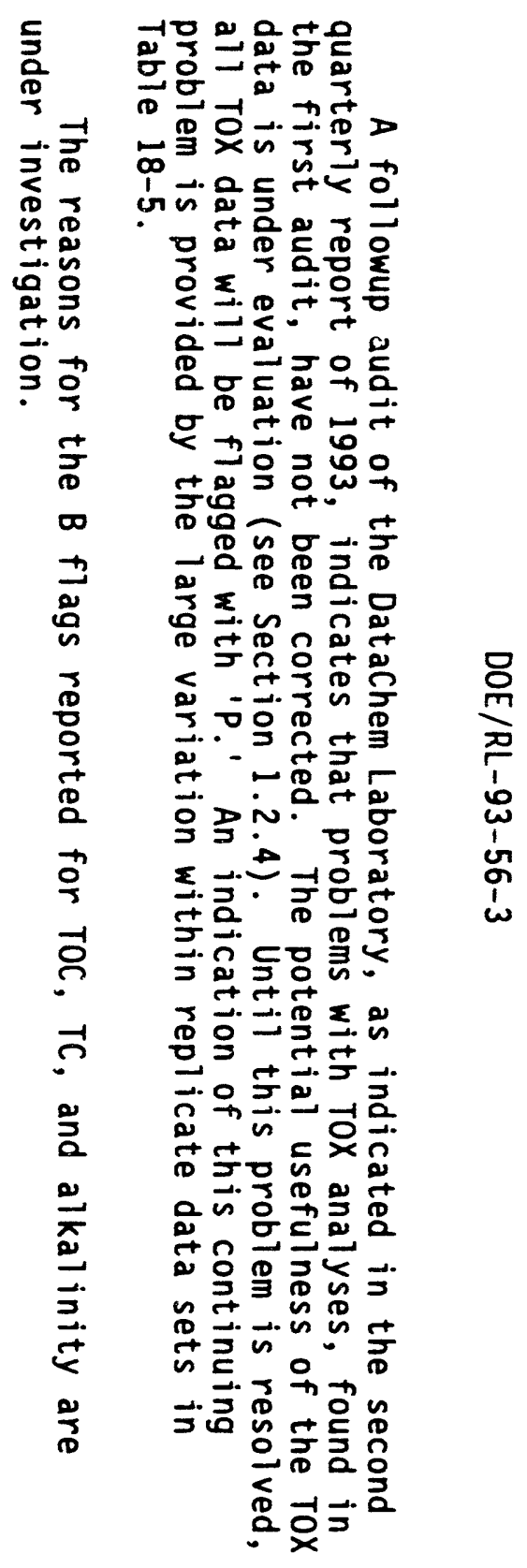


Figure 18-1. Monitoring Well Locations for the Nonradioactive Dangerous Waste Landfill and Solid Waste Landfill.

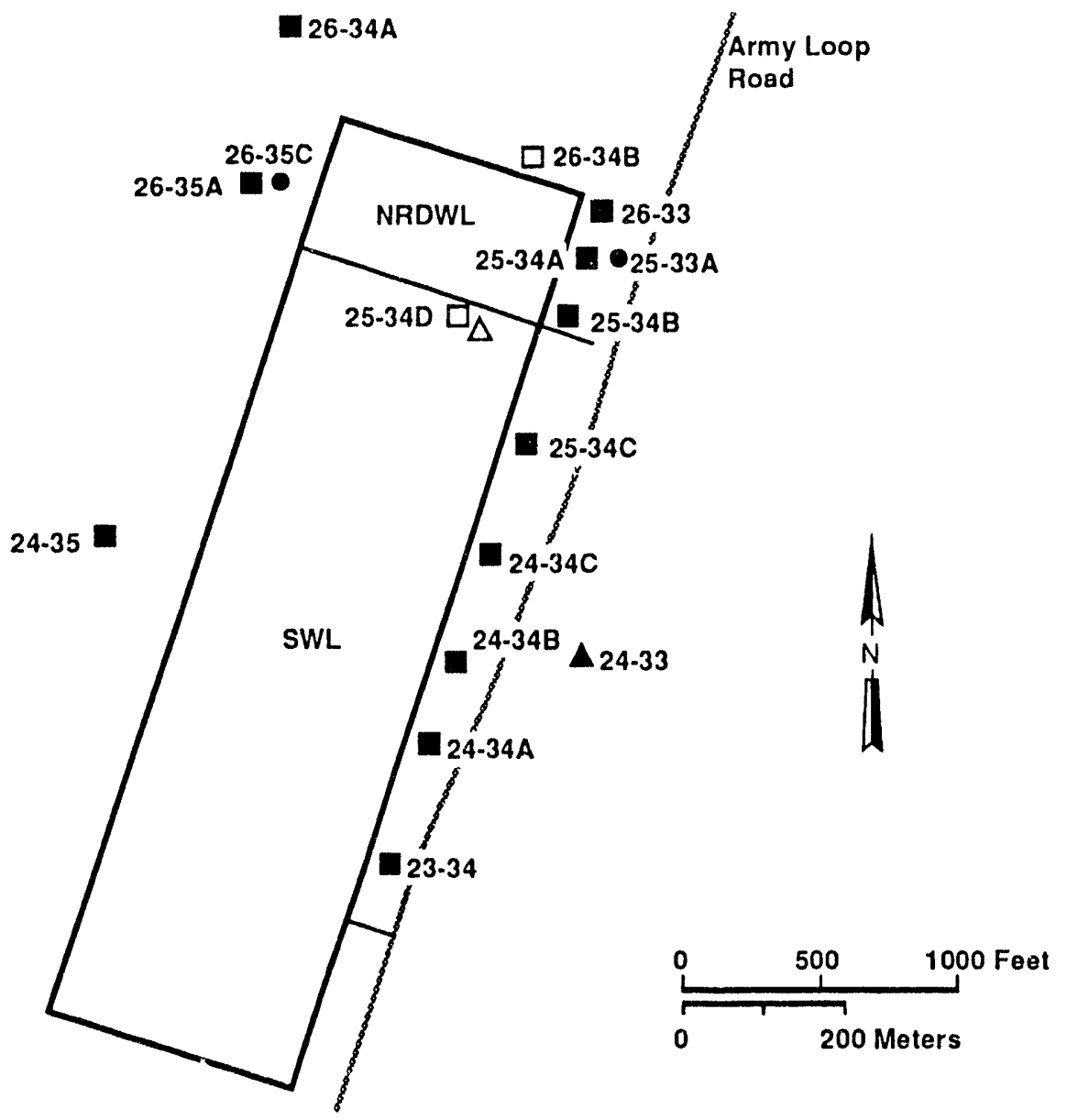

$\triangle \quad$ Proposed '94 Deep Well

- Well completed at the top of the unconfined aquifer

- Well completed in the upper Ringold Formation

- Well not constructed to RCRA specifications

New RCRA wells completed in 1992.

NRDWL Nonradioactive Dangerous Waste Landfill

SWL Solid Waste Landfill

All wells prefixed by 699 . 
Table 18-1. Monitoring Well Purpose and Sampling Scheuule for the Nonradioactive Dangerous Waste Landfill Network.

\begin{tabular}{|c|c|l|c|c|}
\hline $\begin{array}{c}\text { Well no. } \\
(699-)\end{array}$ & $\begin{array}{c}\text { Relative } \\
\text { position }\end{array}$ & \multicolumn{1}{|c|}{ Hydrogeologic unit } & $\begin{array}{c}\text { Sample } \\
\text { frequency }\end{array}$ & $\begin{array}{c}\text { Sample date, } \\
\text { 3rd Qtr 1993 }\end{array}$ \\
\hline $26-34 A$ & Upgradient & Hanford: Water Table & Semiannually & $9 / 08 / 93$ \\
\hline $26-35 A^{\circ}$ & Upgradient & Hanford: Water Table & Semiannually & $9 / 20 / 93$ \\
\hline $26-35 C$ & Upgradient & Top of Ringold & Semiannually & $9 / 08 / 93$ \\
\hline $25-33 A$ & Downgradient & Top of Ringold & Semiannually & $9 / 08 / 93$ \\
\hline $25-34 A$ & Downgradient & Hanford: Water Table & Semiannually & $9 / 16 / 93$ \\
\hline $25-34 B$ & Downgradient & Hanford: Water Table & Semiannually & $9 / 08 / 93$ \\
\hline $25-34 D$ & Downgradient & Hanford: Water Table & Quarterly & $9 / 20 / 93$ \\
\hline $26-33$ & Downgradient & Hanford: Water Table & Semiannually & $9 / 08 / 93$ \\
\hline $26-34 B$ & Downgradient & Hanford: Water Table & Quarterly & $9 / 20 / 93$ \\
\hline
\end{tabular}

Note: Hydrogeologic units include the sandy gravels of the Hanford formation and silty sands of the Ringold Formation. Water levels are measured monthly in all wells.

Well also sampled as part of Solid Waste Landfill network. 
Table 18-2. RCRA Water Level Measurement Report NRDWL, Third Quarter 1993. (sheet 1 of 3)

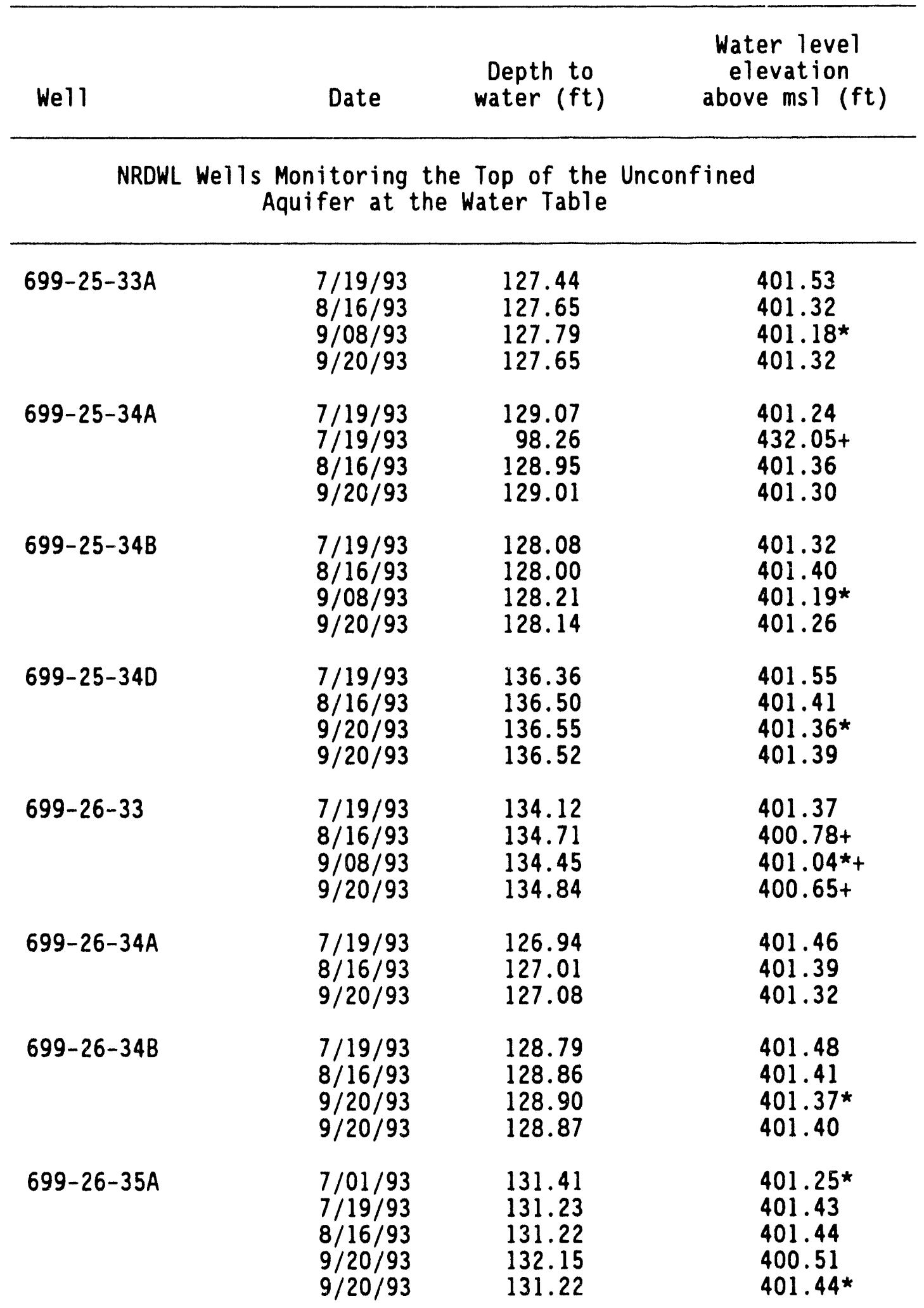


Table 18-2. RCRA Water Level Measurement Report NRDWL, Third Quarter 1993. (sheet 2 of 3)

Well Date $\begin{gathered}\text { Depth to } \\ \text { water }(\mathrm{ft})\end{gathered} \begin{gathered}\text { Water level } \\ \text { elevation } \\ \text { above msl (ft) }\end{gathered}$

NRDWL Wells Monitoring the Upper Ringold

\begin{tabular}{llll}
\hline $699-26-35 C$ & $7 / 19 / 93$ & 131.20 & 401.48 \\
& $8 / 16 / 93$ & 131.27 & 401.41 \\
$9 / 08 / 93$ & 131.24 & $401.44{ }^{\star}$ \\
& $9 / 20 / 93$ & 131.25 & 401.43 \\
\hline
\end{tabular}

Other Wells Associated with NRDWL

\begin{tabular}{|c|c|c|c|}
\hline $699-20-20$ & $\begin{array}{l}7 / 19 / 93 \\
8 / 16 / 93 \\
9 / 20 / 93\end{array}$ & $\begin{array}{l}105.59 \\
105.63 \\
105.64\end{array}$ & $\begin{array}{l}399.99 \\
399.95 \\
399.94\end{array}$ \\
\hline $699-20-39$ & $\begin{array}{l}7 / 19 / 93 \\
8 / 16 / 93 \\
9 / 20 / 93\end{array}$ & $\begin{array}{l}138.22 \\
138.26 \\
138.28\end{array}$ & $\begin{array}{l}401.76 \\
401.72 \\
401.70\end{array}$ \\
\hline $699-23-34$ & $\begin{array}{l}7 / 02 / 93 \\
7 / 19 / 93 \\
8 / 16 / 93 \\
9 / 20 / 93\end{array}$ & $\begin{array}{l}131.39 \\
131.40 \\
131.49 \\
131.46\end{array}$ & $\begin{array}{l}401.47 * \\
401.46 \\
401.37 \\
401.40\end{array}$ \\
\hline $699-24-33$ & $\begin{array}{l}7 / 02 / 93 \\
7 / 19 / 93 \\
8 / 16 / 93 \\
9 / 20 / 93 \\
9 / 29 / 93\end{array}$ & $\begin{array}{l}122.77 \\
122.85 \\
122.88 \\
122.91 \\
122.91\end{array}$ & $\begin{array}{l}401.44^{\star} \\
401.36 \\
401.33 \\
401.30 \\
401.30 \star\end{array}$ \\
\hline $699-24-34 A$ & $\begin{array}{l}7 / 02 / 93 \\
7 / 19 / 93 \\
8 / 16 / 93 \\
9 / 20 / 93 \\
9 / 30 / 93\end{array}$ & $\begin{array}{l}133.05 \\
132.44 \\
132.50 \\
132.48 \\
132.64\end{array}$ & $\begin{array}{l}400.84^{\star} \\
401.45 \\
401.39 \\
401.41 \\
401.25^{\star}\end{array}$ \\
\hline $699-24-34 B$ & $\begin{array}{l}7 / 07 / 93 \\
7 / 19 / 93 \\
8 / 16 / 93 \\
9 / 20 / 93 \\
9 / 20 / 93\end{array}$ & $\begin{array}{l}131.92 \\
132.12 \\
132.07 \\
132.14 \\
132.08\end{array}$ & $\begin{array}{l}401.58^{\star} \\
401.38 \\
401.43 \\
401.36^{\star} \\
401.42\end{array}$ \\
\hline
\end{tabular}


Table 18-2. RCRA Water Level Measurement Report NRDWL, Third Quarter 1993. (sheet 3 of 3)

\begin{tabular}{|c|c|c|c|}
\hline We11 & Date & $\begin{array}{l}\text { Depth to } \\
\text { water (ft) }\end{array}$ & $\begin{array}{l}\text { Water level } \\
\text { elevation } \\
\text { above msl (ft) }\end{array}$ \\
\hline \multicolumn{4}{|c|}{ Other Wells Associated with NRDWL } \\
\hline $699-24-34 C$ & $\begin{array}{l}7 / 07 / 93 \\
7 / 19 / 93 \\
8 / 16 / 93 \\
9 / 20 / 93 \\
9 / 20 / 93\end{array}$ & $\begin{array}{l}131.12 \\
131.13 \\
131.22 \\
131.25 \\
131.18\end{array}$ & $\begin{array}{l}401.46^{\star} \\
401.45 \\
401.36 \\
401.33^{\star} \\
401.40\end{array}$ \\
\hline $699-24-35$ & $\begin{array}{l}7 / 07 / 93 \\
7 / 19 / 93 \\
8 / 16 / 93 \\
9 / 20 / 93 \\
9 / 20 / 93\end{array}$ & $\begin{array}{l}137.29 \\
137.37 \\
137.40 \\
137.49 \\
137.46\end{array}$ & $\begin{array}{l}401.52^{\star} \\
401.44 \\
401.41 \\
401.32 \\
401.35^{\star}\end{array}$ \\
\hline $699-25-34 C$ & $\begin{array}{l}7 / 01 / 93 \\
7 / 19 / 93 \\
8 / 16 / 93 \\
9 / 16 / 93 \\
3 / 20 / 93\end{array}$ & $\begin{array}{l}134.00 \\
134.02 \\
134.49 \\
134.15 \\
134.16\end{array}$ & $\begin{array}{l}401.46^{\star} \\
401.44 \\
400.97+ \\
401.31^{\star} \\
401.30\end{array}$ \\
\hline $699-28-40$ & $\begin{array}{l}7 / 19 / 93 \\
8 / 16 / 93 \\
9 / 20 / 93\end{array}$ & $\begin{array}{l}157.69 \\
157.77 \\
157.89\end{array}$ & $\begin{array}{l}401.75 \\
401.67 \\
401.55\end{array}$ \\
\hline $699-31-31$ & $\begin{array}{l}7 / 19 / 93 \\
8 / 16 / 93 \\
9 / 20 / 93\end{array}$ & $\begin{array}{l}127.90 \\
128.05 \\
128.12\end{array}$ & $\begin{array}{l}401.42 \\
401.27 \\
401.20\end{array}$ \\
\hline $699-34-39 A$ & $\begin{array}{l}7 / 19 / 93 \\
8 / 16 / 93 \\
9 / 20 / 93\end{array}$ & $\begin{array}{l}135.25 \\
135.31 \\
135.36\end{array}$ & $\begin{array}{l}401.82 \\
401.76 \\
401.71\end{array}$ \\
\hline
\end{tabular}

Notes: 1. Water level elevations are calculated by subtracting the measured depth to water from the surveyed elevation for the well.

2. Depth-to-water values are transcribed from field records.

3. Elevations marked with an $1 * 1$ were measured at the time of sampling.

4. Elevations marked with a ' + ' are outside of the expected range and are suspected of error.

5. To convert feet to meters multiply by 0.3048 . 
Table 18-3. Constituent List and Summary of Results for the NRDW Landfill Data for Reporting Period July 1 through September 30, 1993. (sheet 1 of 2)

CONTAMINATION INDICATOR PARAMETERS

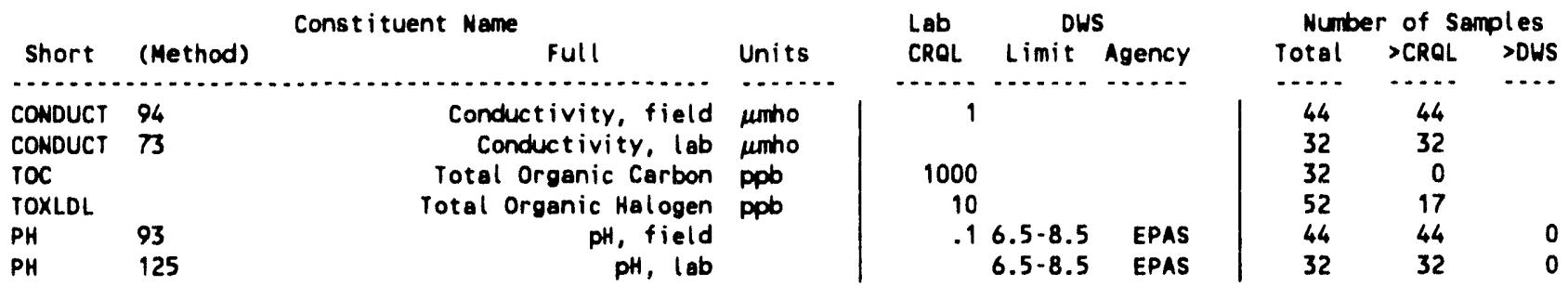

DRINKING WATER PARAMETERS

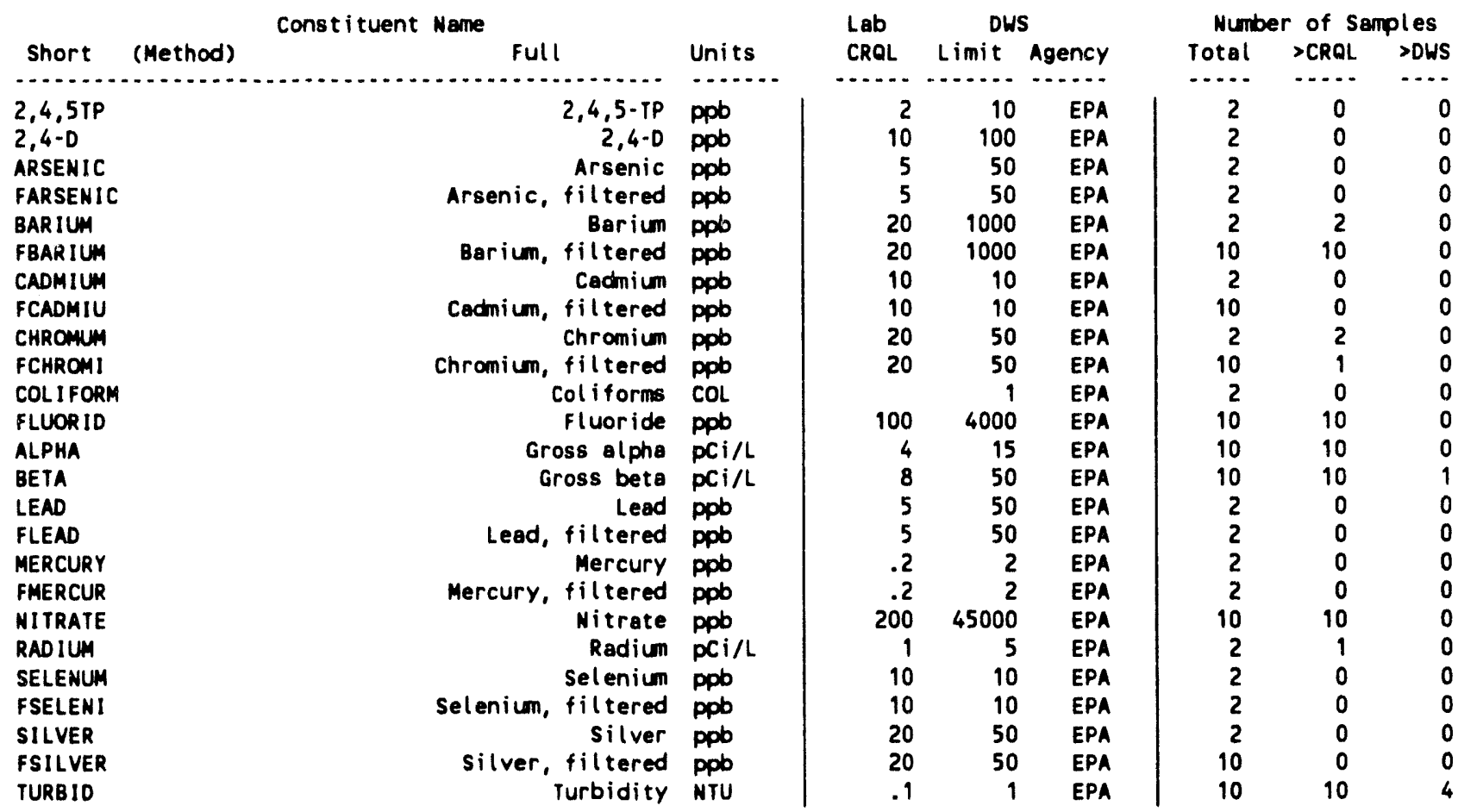

GROUNDWATER QUALITY PARAMETERS

\begin{tabular}{|c|c|c|c|c|c|c|c|c|c|}
\hline \multicolumn{4}{|c|}{ Constituent Name } & \multicolumn{3}{|c|}{ DWS } & \multicolumn{3}{|c|}{ Number of Samples } \\
\hline Short & (Method) & Full & Units & CROL & Limit & Agency & Total & $>\mathrm{CRQL}$ & >DWS \\
\hline$\cdots-\cdots$ & 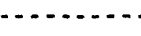 & 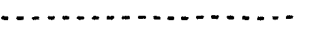 & $\ldots \ldots$ & $\cdots \cdots$ & $\ldots \ldots$ & $\ldots \ldots$ & $\ldots$ & $\cdots \cdots$ & $\cdots$ \\
\hline CHLORID & & Chloride & ppb & 200 & 250000 & EPAS & 10 & 10 & 0 \\
\hline IRON & & Iron & ppb & 20 & 300 & EPAS & 2 & 2 & 0 \\
\hline FIRON & & Iron, filtered & ppb & 20 & 300 & EPAS & 10 & 4 & 0 \\
\hline MANGESE & & Manganese & ppb & 10 & 50 & EPAS & 2 & 0 & 0 \\
\hline FMANGAN & & Manganese, filtered & ppb & 10 & 50 & EPAS & 10 & 1 & 0 \\
\hline LPHENOL & & Phenol & ppb & 20 & & & 2 & 0 & \\
\hline SOOIUM & & Sodium & ppb & 300 & & & 2 & 2 & \\
\hline FSOO IUM & & Sodium, filtered & ppb & 300 & & & 10 & 10 & \\
\hline SULFATE & & Sulfate & ppb & 500 & 250000 & EPAS & 10 & 10 & 0 \\
\hline
\end{tabular}


Table 18-3. Constituent List and Summary of Results for the NRDW Landfill Data for Reporting Period July 1 through September 30, 1993. (sheet 2 of 2)

SITE SPECIFIC ANO OTHER CONSTITUENTS

\begin{tabular}{|c|c|c|c|c|c|c|c|c|c|}
\hline \multicolumn{4}{|c|}{ Constituent Name } & \multicolumn{3}{|c|}{ DWS } & \multicolumn{3}{|c|}{ Number of Samples } \\
\hline Short & (Method) & Full & Units & CRQL & Limit & Agency & Total & $>\mathrm{CROL}$ & $>$ DWS \\
\hline$\ldots \ldots \ldots$ & 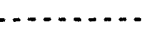 & $\ldots \ldots$ & $\ldots \ldots$ & $\ldots \ldots$ & $\ldots \ldots \ldots$ & $\ldots \ldots$ & $\ldots$. & $\ldots \ldots$ & $\cdots$ \\
\hline $2,4,5-T$ & & $2,4,5-T$ & ppb & 2 & & & 2 & 0 & \\
\hline $246-\operatorname{trp}$ & & $2,4,6$-Trichlorophenol & ppb & 5 & & & 2 & 0 & \\
\hline 24 -dchp & & 2,4-Dichlorophenol & ppob & 5 & & & 2 & 0 & \\
\hline DIMPHEN & & 2,4-D ime thylphenol & ppb & 5 & & & 2 & 0 & \\
\hline DINPHEN & & 2,4-0 initrophenol & ppo & 150 & & & 2 & 0 & \\
\hline 26-dchp & & 2,6-Dichlorophenol & ppb & 5 & & & 2 & 0 & \\
\hline CHLPHEN & & 2-Chlorophenol & ppb & 5 & & & 2 & 0 & \\
\hline 2NITPH & & ?-Nitrophenol & ppb & 5 & & & 2 & 0 & \\
\hline BUTDINP & 30 & 2-sec-8utyl-4,6-dinitrophenol (DN & ppo & 5 & & & 2 & 0 & \\
\hline BUTDINP & 49 & 2-sec-Butyl-4,6-dinitrophenol CDN & ppb & 1 & & & 2 & 0 & \\
\hline 460N2MP & & 4,6-Dinitro-2-methylphenol & ppb & 200 & & & 2 & 0 & \\
\hline CHLCRES & & 4-Chloro-3-me thylphenol & ppo & 5 & & & 2 & 0 & \\
\hline NITPHEN & & 4-Nitrophenol & Ppb & 30 & & & 2 & 0 & \\
\hline ALKALIN & & Alkalinity & ppm & 50 & & & 7 & 7 & \\
\hline ALUMNUM & & Aluminum & ppo & 200 & & & 2 & 0 & \\
\hline FALUMIN & & Aluminum & ppob & 200 & & & 10 & 0 & \\
\hline ANTIONY & & Ant imony & ppb & 200 & & & 2 & 0 & \\
\hline FANTIMO & & Ant imony, filtered & ppb & 200 & & & 10 & 0 & \\
\hline BERYLUM & & Beryllium & ppb & 3 & & & 2 & 0 & \\
\hline FBERYLL & & Beryllium, filtered & ppo & 3 & & & 10 & 0 & \\
\hline BROMIDE & & Bromide & ppb & 500 & & & 10 & 0 & \\
\hline CALCIUM & & Calcium & ppb & 100 & & & 2 & 2 & \\
\hline FCALCIU & & Calcium, filtered & ppob & 100 & & & 10 & 10 & \\
\hline COBALT & & Cobalt & ppb & 20 & & & 2 & 0 & \\
\hline FCOBALT & & Cobalt, filtered & ppb & 20 & & & 10 & 0 & \\
\hline COPPER & & Copper & ppb & 20 & 1000 & EPAS & 2 & 0 & 0 \\
\hline FCOPPER & & Copper, filtered & ppb & 20 & 1000 & EPAS & 10 & 0 & 0 \\
\hline CRESOLS & & Cresols (methylphenols) & ppb & 10 & & & 2 & 0 & \\
\hline MAGNES & & Magnesium & ppb & 100 & & & 2 & 2 & \\
\hline FMAGNES & & Megnesium, filtered & ppo & 100 & & & 10 & 10 & \\
\hline NICKEL & & Nickel & ppb & 30 & & & 2 & 0 & \\
\hline FNICKEL & & Nickel, filtered & ppob & 30 & & & 10 & 0 & \\
\hline NITRITE & & Nitrite & ppb & 200 & & & 10 & 0 & \\
\hline PENTCHP & & Pentachlorophenol & ppb & 100 & & & 2 & 0 & \\
\hline PHOSPHA & & Phosphate & ppb & 400 & & & 10 & 0 & \\
\hline POTASUM & & Potassium & ppb & 300 & & & 2 & 2 & \\
\hline FPOTASS & & Potassium, filtered & ppb & 300 & & & 10 & 10 & \\
\hline TETPHNL & & Tetrachlorophenols & ppb & 10 & & & 2 & 0 & \\
\hline TIN & & Tin & ppb & 100 & & & 2 & 0 & \\
\hline FTIN & & Tin, filtered & ppb & 100 & & & 10 & 0 & \\
\hline TC & & Total Carbon & ppb & 2000 & & & 10 & 10 & \\
\hline TRIPHNL & & Irichlorophenols & ppb & 5 & & & 2 & 0 & \\
\hline TRITIUM & & Tritium & $\mathrm{pCi} / \mathrm{L}$ & 500 & 20000 & EPA & 10 & 10 & 9 \\
\hline VANADUM & & Vanadium & ppb & 30 & & & 2 & 0 & \\
\hline FVANADI & & Vanadium, filtered & ppb & 30 & & & 10 & 0 & \\
\hline ZINC & & zinc & ppb & 10 & & & 2 & 0 & \\
\hline FZINC & & zinc, filtered & ppb & 10 & & & 10 & 2 & \\
\hline
\end{tabular}

For explanation of this table, see Section 1.4 of report. 
Table 18-4. Constituents with at Least One Value Above the CRQL for the NRDW Landfill Data for Reporting Period July 1 through September 30, 1993. (sheet 1 of 3)

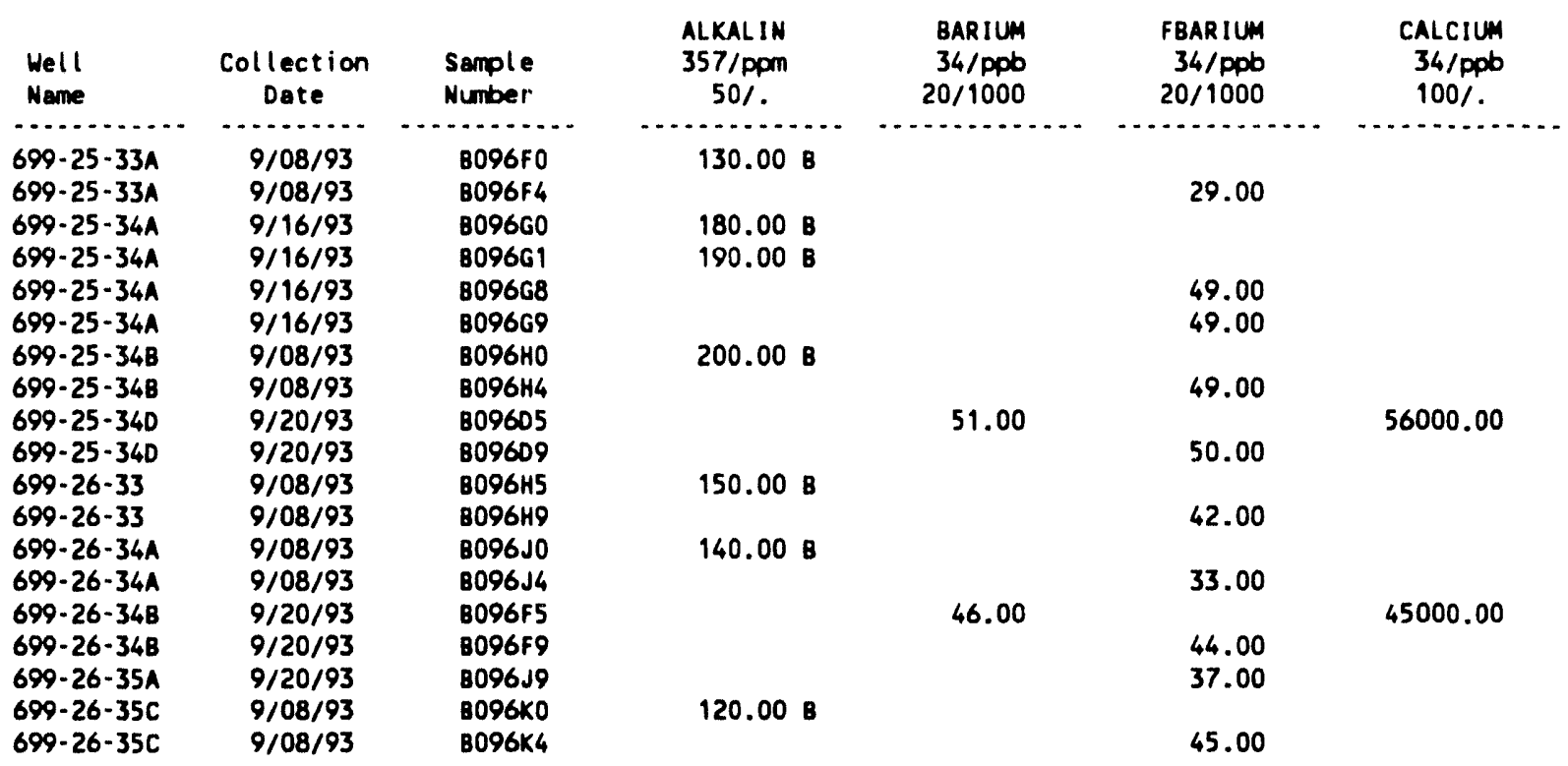

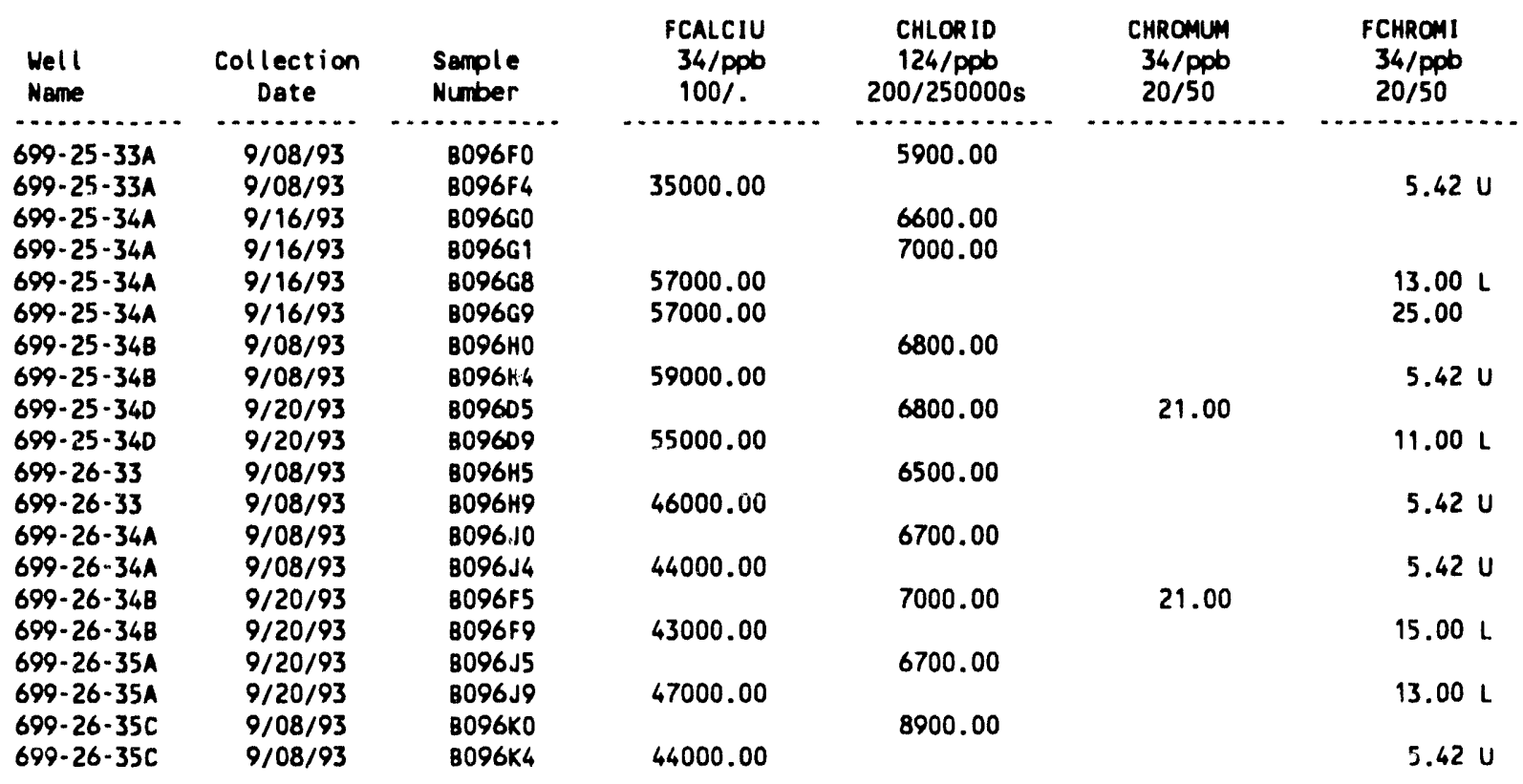

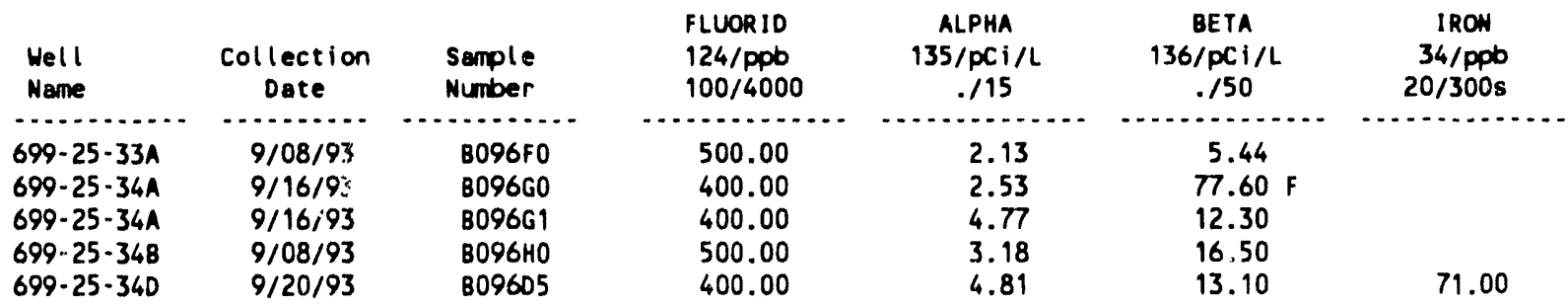


Table 18-4. Constituents with at Least One Value Above the CRQL for the NRDW Landfill Data for Reporting Period July 1

through September 30, 1993. (sheet 2 of 3)

\begin{tabular}{|c|c|c|c|c|c|c|}
\hline $\begin{array}{l}\text { Hell } \\
\text { Name }\end{array}$ & $\begin{array}{c}\text { Collection } \\
\text { Dete }\end{array}$ & $\begin{array}{l}\text { Sample } \\
\text { Number }\end{array}$ & $\begin{array}{l}\text { FLUOR ID } \\
124 / p p b \\
100 / 4000\end{array}$ & $\begin{array}{c}\text { ALPHA } \\
135 / P C i / L \\
. / 15\end{array}$ & $\begin{array}{c}\text { BETA } \\
136 / P C i / L \\
. / 50\end{array}$ & $\begin{array}{c}\text { IRON } \\
34 / \text { ppb } \\
20 / 300 \text { s }\end{array}$ \\
\hline $\begin{array}{l}699-26-33 \\
699-26-34 A \\
699-26-34 B \\
699-26-35 A \\
699-26-35 C\end{array}$ & $\begin{array}{l}9 / 08 / 93 \\
9 / 08 / 93 \\
9 / 20 / 93 \\
9 / 2, / 93 \\
9 / 08 / 93\end{array}$ & $\begin{array}{l}\text { B096H5 } \\
\text { B096J0 } \\
\text { B096F5 } \\
\text { B096J5 } \\
\text { B096KO }\end{array}$ & $\begin{array}{l}500.00 \\
500.00 \\
400.00 \\
400.00 \\
400.00\end{array}$ & $\begin{array}{l}4.03 \\
3.04 \\
4.40 \\
3.84 \\
1.56\end{array}$ & $\begin{array}{l}10.20 \\
12.20 \\
11.10 \\
16.00 \\
20.60\end{array}$ & 62.00 \\
\hline
\end{tabular}

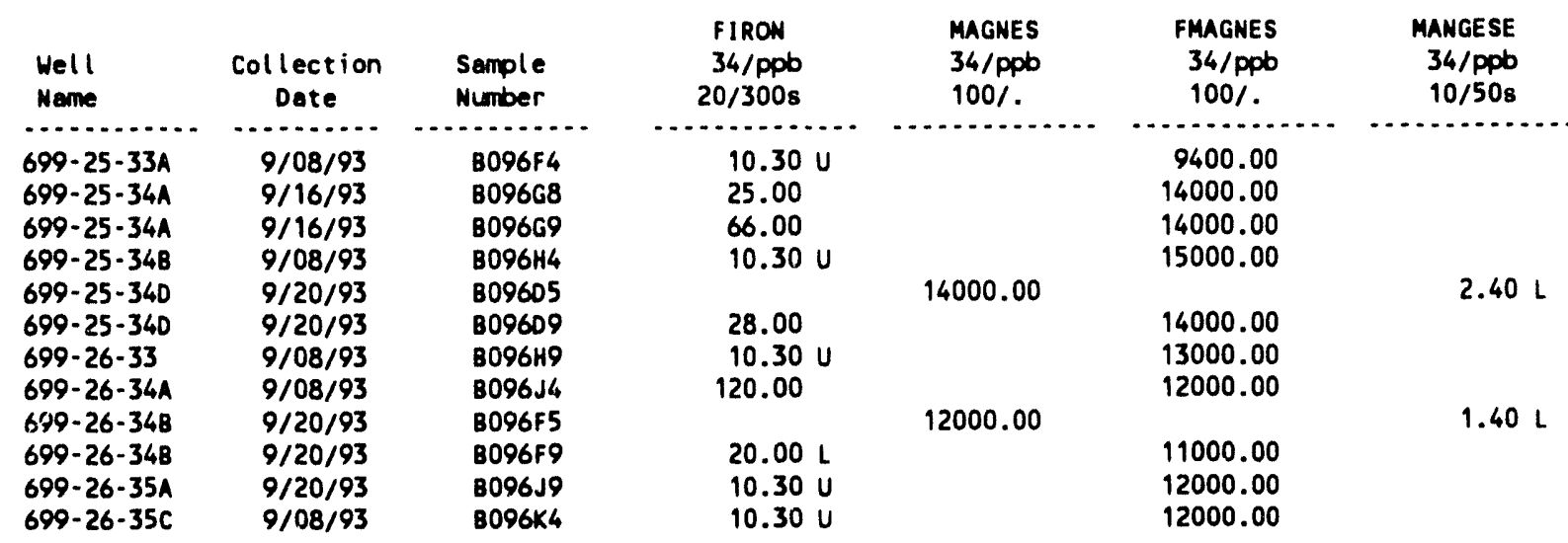

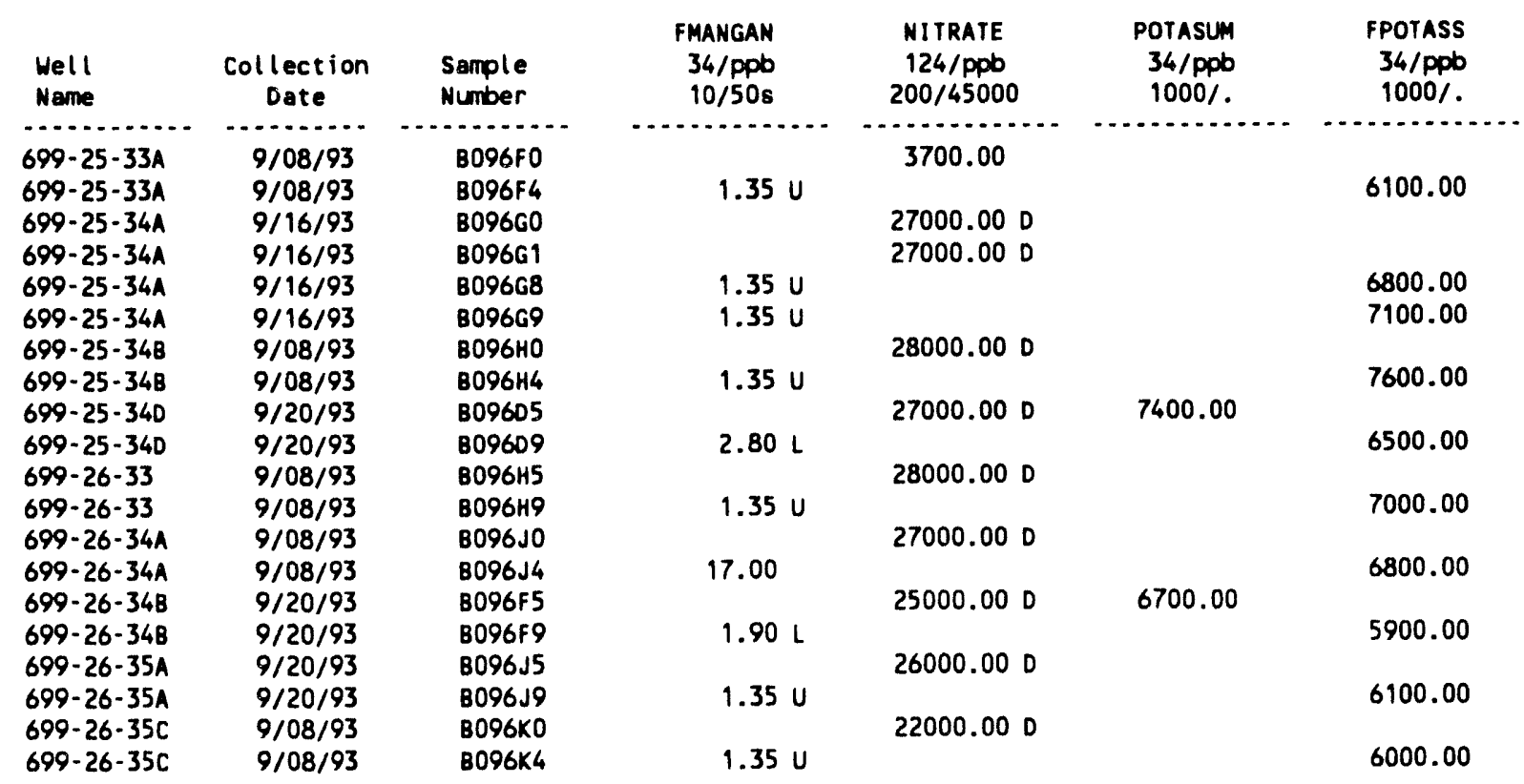


Table 18-4. Constituents with at Least One Value Above the CRQL for the NRDW Landfill Data for Reporting Period July 1 through September 30, 1993. (sheet 3 of 3 )

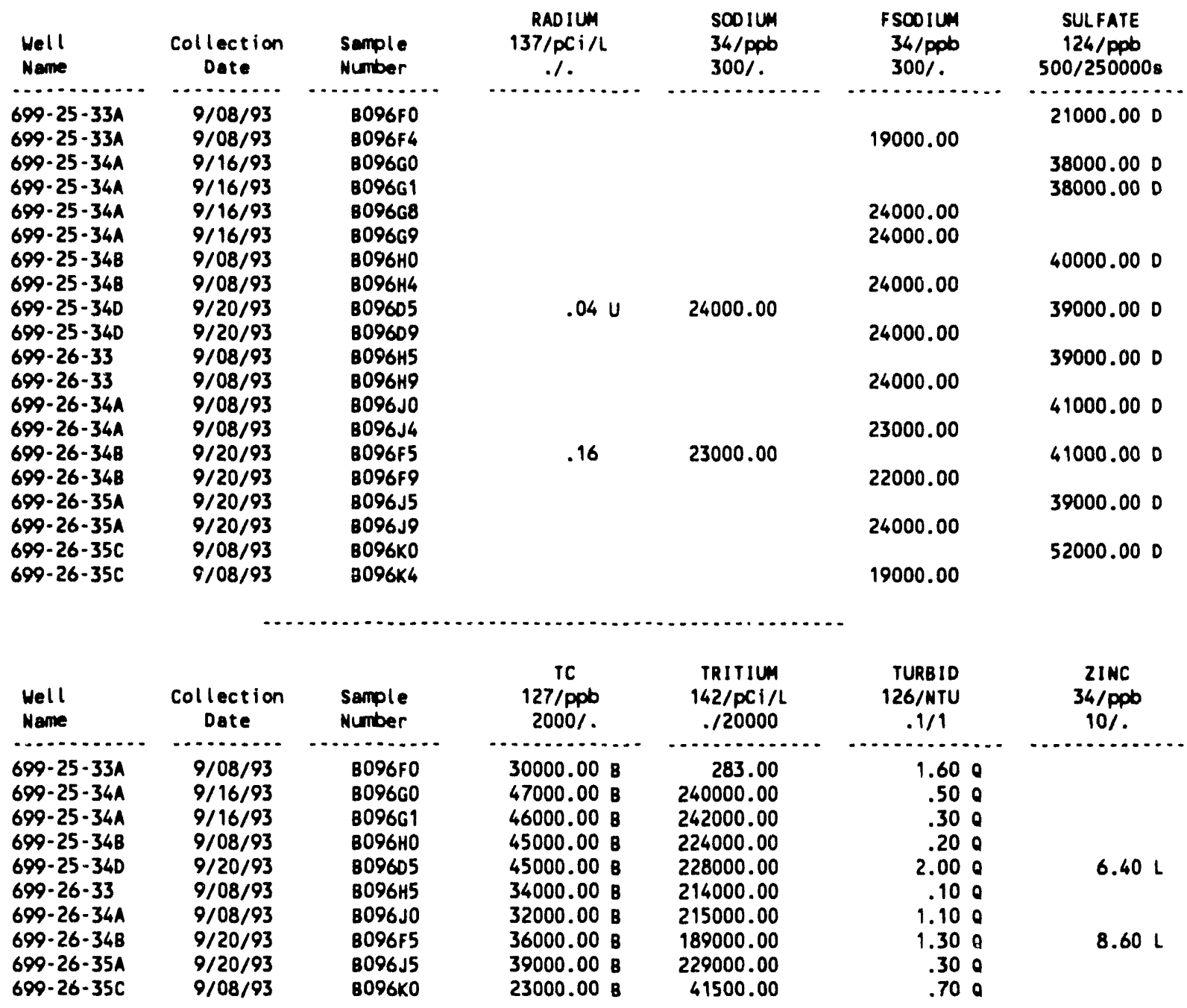

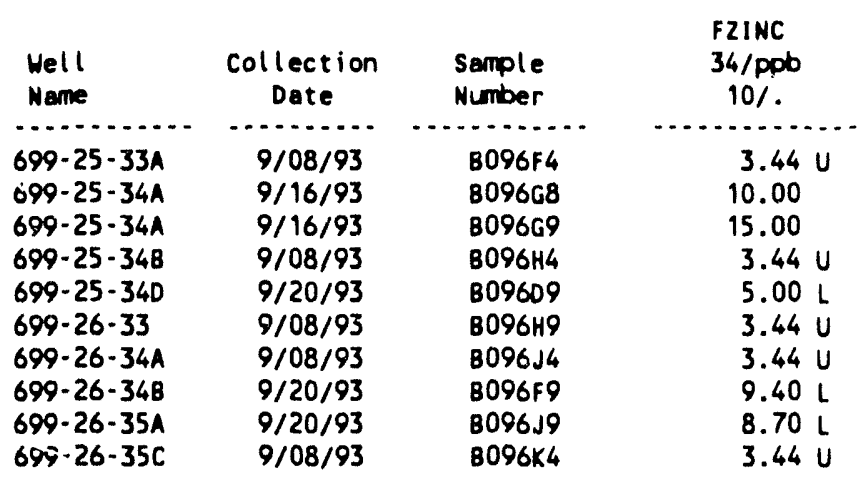

For explanation of this table, see Section 1.4 of report. 
Table 18-5. Contamination Indicator Parameters for the NRDW Landfill Data for Reporting Period July 1 through September 30, 1993.

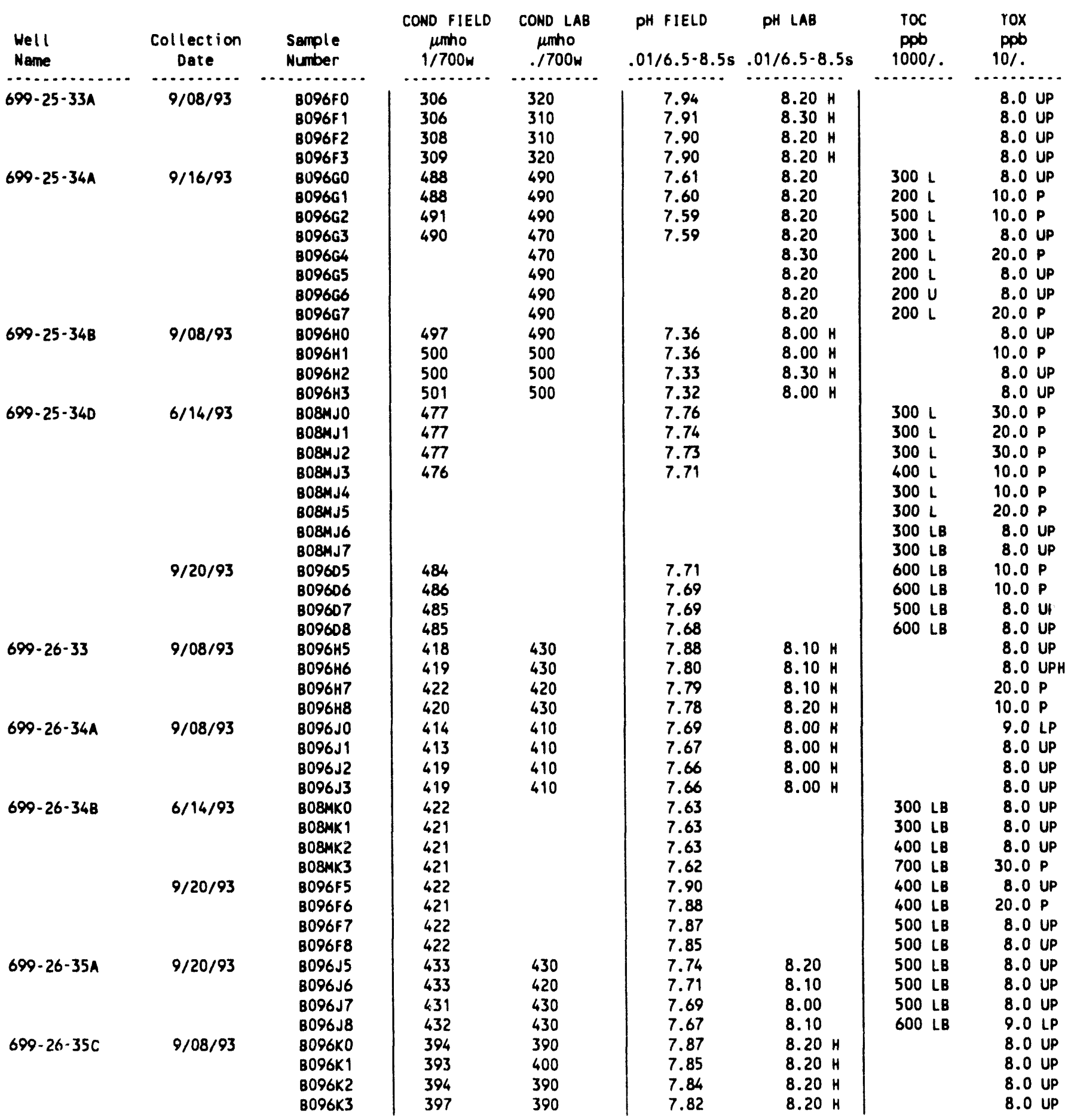

For explanation of this table, see Section 1.4 of report. 
$\stackrel{\varpi}{\stackrel{1}{1}}$

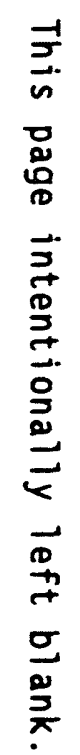

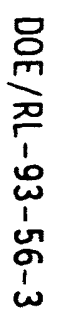




\subsection{REFERENCES}

40 CFR 141, 1992, "National Primary Drinking Water Regulations," Code of Federal Regulations, as amended.

40 CFR 143, 1991, "National Secondary Drinking Water Regulations," Code of Federal Regulations, as amended.

40 CFR 265, 1992, "Interim Status Standards for Owners and Operators of Hazardous Waste Treatment, Storage, and Disposal Facilities, "Code of Federal Regulations, as amended.

Anderson, J. D., 1990, A History of the 200 Area Tank Farms, WHC-MR-0132, Westinghouse Hanford Company, Richland, Washington.

APHA, 1989, Standard Methods for Evaluation of Water and Wastewater, 17 th ed., American Public Health Association, Washington, D.C.

ASTM, 1991, Annual Book of ASTM Standards, Vo1. 11.01, Water and Enviroumental Technology, Philadelphia, Pennsylvania.

Caggiano, J. A., and C. J. Chou, 1993, Interim-Status Groundwater Quality Assessment Plan for the Single Shell Tank Waste Management Areas $T$ and TX-TY: Richland, Washington, Westinghouse Hanford Company, WHC-SD-EN-AP-132, Rev. 0, Westinghouse Hanford Company, Richland, Washington.

Chou, C. J., G. L. Kasza, and R. B. Mercer, 1990, Interim-Status Groundwater Quality Assessment Plan for the 216-A-29 Ditch, WHC-SD-EN-AP-031, Rev. 0 , Westinghouse Hanford Company, Richland, Washington.

DOE, 1987, 216-A-29 Ditch Preliminary Closure/Post Closure Plan, TRAC-0095, U.S. Department of Energy, Richland Operations Office, Richland, Washington.

DOE-RL, 1993, Annual Report for RCRA Groundwater Monitoring Projects at Hanford Site Facilities for 1992, DOE/RL-93-09, U.S. Department of Energy, Richland Operations Office, Richland, Washington.

Ecology and EPA, 1986, Consent Agreement and Compliance Order, Ecology No. DE 86-133, Washington State Department of Ecology and the U.S. Environmental Protection Agency, 01ympia, Washington.

Ecology, EPA, and DOE, 1992, Hanford Federal Facility Agreement and Consent Order, 2 vols, Washington State Department of Ecology, U.S. Environmental Protection Agency, and U.S. Department of Energy, 0lympia, Washington.

EPA, 1979, Methods for Chemical Analysis of Water and Waste, EPA-600/4-79-020, U.S. Environmental Protection Agency, Environmental Monitoring and Support Laboratory, Cincinnati, Ohio. 
EPA, 1986a, RCRA Groundwater Monitoring Technical Enforcement Guidance Document (TEGD), OSWER-9950.1, U.S. Environmental Protection Agency, Washington, D.C.

EPA, 1986b, Test Methods for Evaluating Solid Waste, SW-846, Third Edition, U.S. Environmental Protection Agency, Washington, D.C.

Harris, S. F., 1990, Groundwater Quality Assessment Plan for the 216-8-3 Pond System, WHC-SD-EN-AP-030, Rev. 0, Westinghouse Hanford Company, Richland, Washington.

Hartman, M. J., 1991, Groundwater Monitoring P7an for the 100-D Ponds, WHC-SD-EN-AP-0 18, Rev. 0, Westinghouse Hanford Company, Richland, Washington.

Hartman, M. J., 1993, Groundwater Quality Assessment Plan for the 1324-N/NA Site, WHC-SD-EN-AP-005, Rev. 1, Westinghouse Hanford Company, Richland, Washington.

Kasza, G. L., and S. M. Goodwin, 1991, Groundwater Monitoring Plan for the 216-A-29 Ditch, WHC-SD-EN-AP-045, Rev. 0, Westinghouse Hanford Company, Richl and, Washington.

Peterson, R. E., and M. P. Connelly, 1992, Characterization of Chromium Plume in Groundwater Along the Columbia River Shoreline, Hanford Site, Washington, WHC-SA-1674-VA, Westinghouse Hanford Company, Richland, Washington.

PNL, 1989, 40 CFR 265 Interim-Status Indicator-Evaluation Groundwater Monitoring Plan for the 216-B-63 Trench, PNL-6862, Pacific Northwest Laboratory, Richland, Washington.

Resource Conservation and Recovery Act of 1976, 42 USC 6901, et seq.

Schalla, R., 1988, Revised Ground-Water Monitoring Compliance Plan for the 300 Area Process Trenches, PNL-6671, Pacific Northwest Laboratory, Richland, Washington.

Schmidt, J. W., A. R. Johnson, S. M. Mckinney, C. J. Perkins, and C. R. Webb, 1992, Westinghouse Hanford Company Environmental Surveillance Annual

Report, WHC-EP-0573, West inghouse Hanford Company, Richl and, Washington.

Tee1, S. S., B. N. Bjornstad, and D. R. Newcomer, 1989, Interim-Status Ground Water Monitoring Plan for the Grout Treatment Facility, WHC-SD-EN-AP-006, Rev. 0, Westinghouse Hanford Company, Richland, Washington.

WAC 173-160, 1990, "Minimum Standards for Construction and Maintenance of Wells," Washington Administrative Code, as amended.

WAC 173-303, 1991, "Dangerous Waste Regulations," Washington Administrative Code, as amended. 
WHC, 1990a, 183-D Filter Backwash Wastewater Stream-specific Report, WHC-EP-0342, Addendum 33, Westinghouse Hanford Company, Richland, Washington.

WHC, 1990b, Interim-Status Groundwater Monitoring Plan for the 216-S-10 Pond and Ditch, WHC-SD-EN-AP-018, Rev. 0, Westinghouse Hanford Company, Richland, Washington.

WHC, 1990c, Interim-Status Groundwater Monitoring Plan for the 216-U-12 Crib, WHC-SD-EN-AP-019, Rev. 0, Westinghouse Hanford Company, Richland, Washington.

WHC, 1991, Interim Status Groundwater Monitoring Plan for the 200 East Area Liquid Effluent Retention Facility, WHC-SD-EN-AP-024, Rev. 1, Westinghouse Hanford Company, Richland, Washington.

WHC, 1992, Quality Assurance Project Plan for RCRA Groundwater Monitoring Activities, WHC-SD-EN-QAPP-001, Rev. 1, Westinghouse Hanford Company, Richl and, Washington.

WHC, 1993a, Interim-Status Groundwater Quality Assessment Plan for the 216-U-12 Crib, WHC-SD-EN-AP-108, Rev. 0, Westinghouse Hanford Company, Richland, Washington.

WHC, 1993b, Interim Status Groundwater Monitoring Plan for the Nonradioactive Dangerous Waste Landfill, Hanford, Washington, WHC-SD-EN-AP-026, Rev. 0, Westinghouse Hanford Company, Richland, Washington.

WHC, 1993c, Radionuclide and Chemical Inventories for the Single-Shell Tanks: Report Prepared for Westinghouse Hanford Company by WASTREN, Inc., WHC-SD-WM-TI-565, Rev. 1, Westinghouse Hanford Company, Richland, Washington. 
DOE/RL-93-56-3

This page intentionally left blank.

$19-4$ 
DOE/RL-93-56-3

\section{APPENDIX A}

PREVIOUS DATA FROM 100-NR-2 OPERABLE UNIT

$$
A-i
$$


DOE/RL-93-56-3

This page intentionally left blank. 


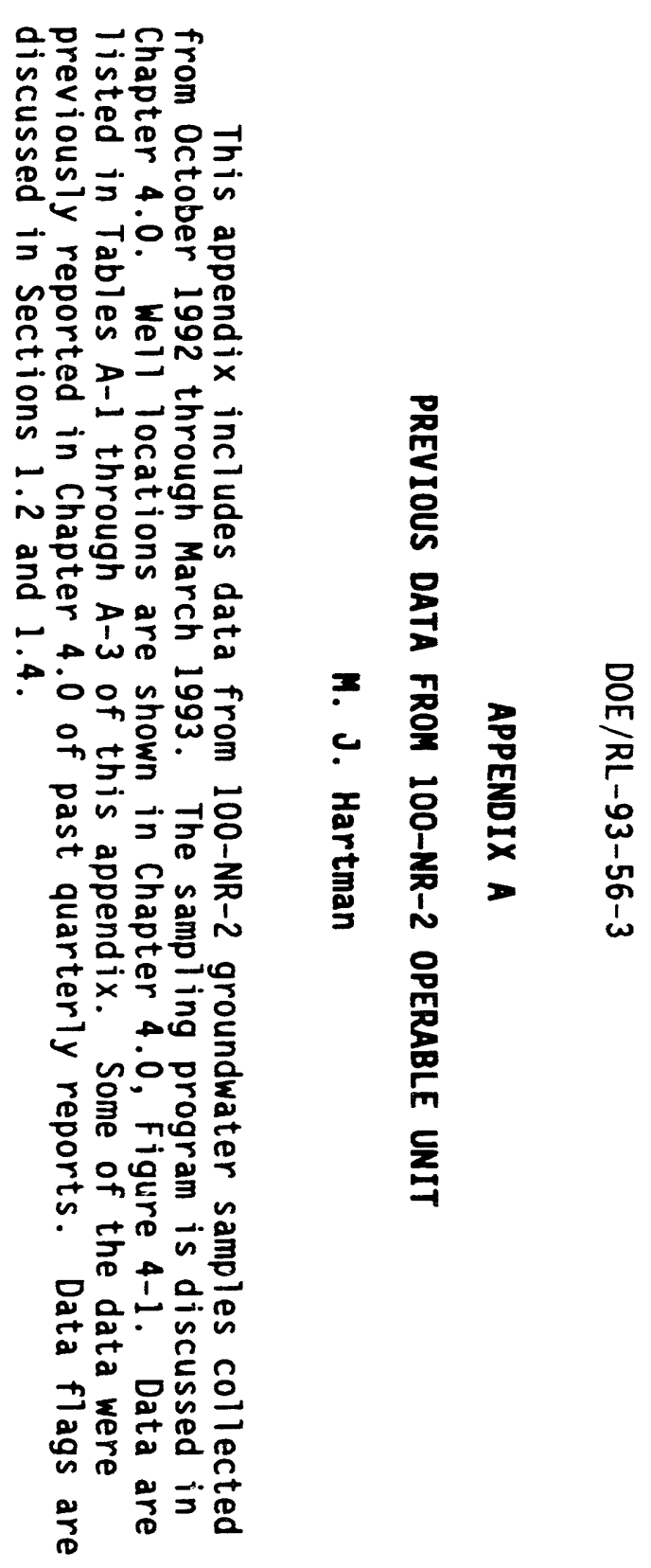


Table A-1. Constituent List and Summary of Results for 100-NR-2 Site Data for Reporting Period October 1, 1992 through March 31, 1993. (sheet 1 of 3)

CONTAMINATION INDICATOR PARAMETERS

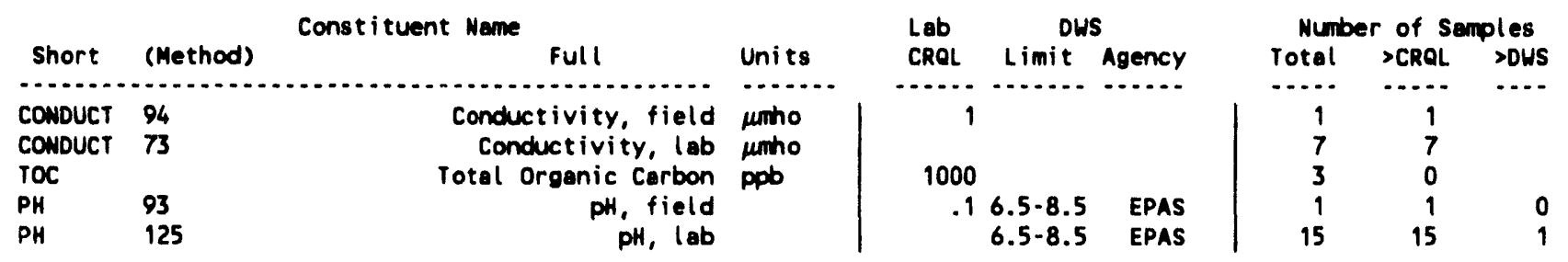

DRINKING WATER PARAMETERS

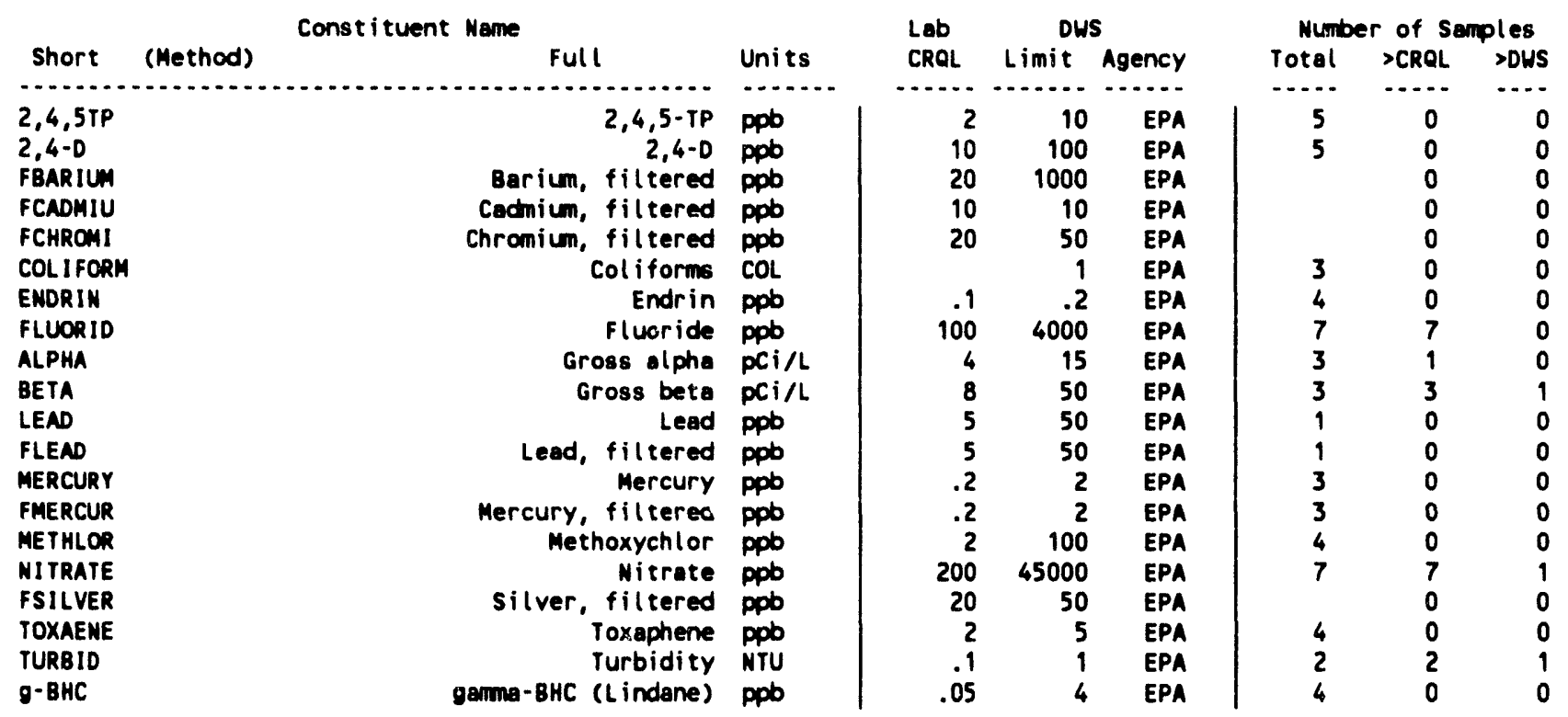

GROUNDWATER QUALITY PARAMETERS

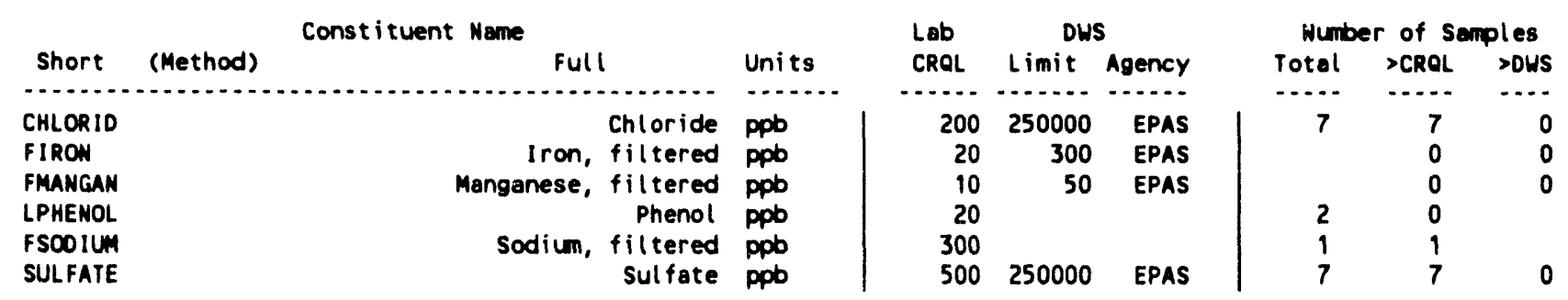


Table A-1. Constituent List and Summary of Results for 100-NR-2 Site Data for Reporting Period October 1, 1992 through March 31, 1993. (sheet 2 of 3)

SITE SPECIFIC AND OTHER CONSTITUENTS

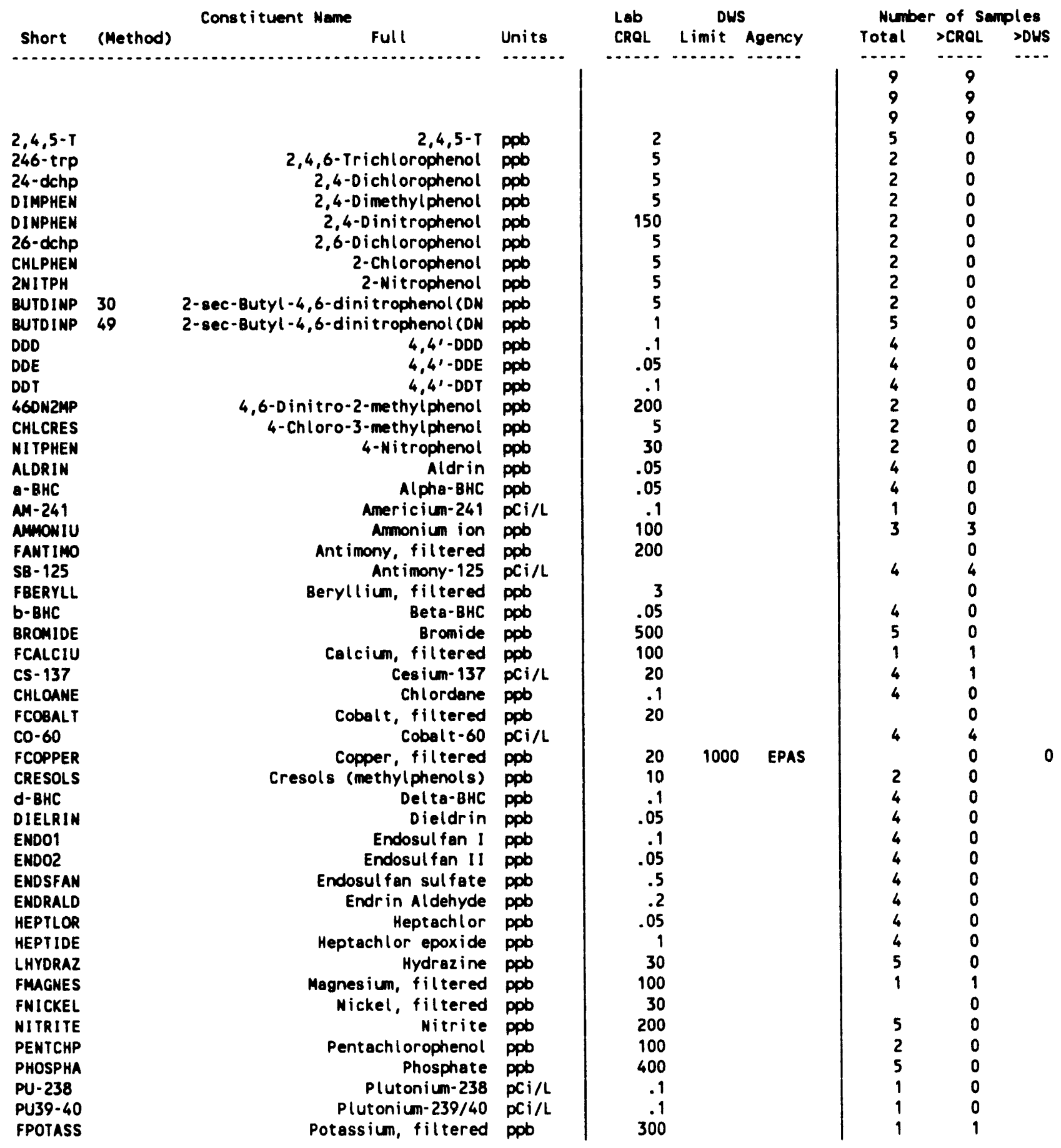


Table A-1. Constituent List and Summary of Results for 100-NR-2 Site Data for Reporting Period October 1, 1992 through March 31, 1993. (sheet 3 of 3)

\begin{tabular}{|c|c|c|c|c|c|c|c|c|c|c|}
\hline \multirow[b]{2}{*}{ Short } & \multirow[b]{2}{*}{ (Method) } & \multirow[t]{2}{*}{ Constituent Name } & \multirow[b]{2}{*}{ Units } & \multirow{2}{*}{$\begin{array}{l}\text { Lab } \\
\text { CRQL }\end{array}$} & \multicolumn{2}{|c|}{ DWS } & & \multicolumn{3}{|c|}{ Number of Samples } \\
\hline & & & & & Limit & Agency & & Total & $>C R Q L$ & $>$ OWS \\
\hline (n) & $\ldots$ & putheniumas & nim & ....... & $\cdots$ & …... & & $\cdots$ & $\cdots$ & $\cdots$ \\
\hline RU-106 & & Ruthenium- 106 & pCi/L & & & & 1 & 4 & 4 & \\
\hline SR-90 & & Strontium-90 & $\mathrm{pCi} / \mathrm{L}$ & 5 & 8 & EPA & & 1 & 1 & 1 \\
\hline TMP_C & & Temperature, field & DegC & .1 & & & & 1 & 1 & \\
\hline TETPENNL & & Tetrachlorophenols & ppob & 10 & & & & 2 & 0 & \\
\hline FIIN & & Tin, filtered & ppb & 100 & & & & & 0 & \\
\hline TRIPHNL & & Trichlorophenols & ppob & 5 & & & & 2 & 0 & \\
\hline TRITIUM & & Tritium & $\mathrm{pCi/L}$ & 500 & 20000 & EPA & & 3 & 3 & 3 \\
\hline FVANADI & & Vanadium, filtered & ppb & 30 & & & & & 0 & \\
\hline FZINC & & zinc, filtered & ppb & 10 & & & & & 0 & \\
\hline
\end{tabular}

For explanation of this table, see Section 1.4 of report. 
Table A-2. Constituents with at Least One Value Above the CRQL for the 100-NR-2 Sites Data for Reporting Period October 1, 1992

through March 31, 1993. (sheet 1 of 2)

\begin{tabular}{|c|c|c|c|c|c|c|}
\hline $\begin{array}{l}\text { Well } \\
\text { Neme }\end{array}$ & $\begin{array}{c}\text { Collection } \\
\text { Date }\end{array}$ & $\begin{array}{l}\text { Semple } \\
\text { Number }\end{array}$ & $\begin{array}{l}\text { AMMONIU } \\
54 / \text { ppo } \\
100 / .\end{array}$ & $\begin{array}{c}\text { SB-125 } \\
140 / \text { PCi/L } \\
. /\end{array}$ & $\begin{array}{l}\text { FCALCIU } \\
34 / \mathrm{ppb} \\
100 \%\end{array}$ & $\begin{array}{c}\text { CS-137 } \\
140 / \mathrm{pCi} / \mathrm{L} \\
. / .\end{array}$ \\
\hline $\begin{array}{l}199-N-32 \\
199-N-62 \\
199-N-70 \\
199-N-76 \\
199-N-76\end{array}$ & $\begin{array}{c}11 / 18 / 92 \\
11 / 17 / 92 \\
11 / 24 / 92 \\
3 / 11 / 93 \\
3 / 11 / 93\end{array}$ & $\begin{array}{l}\text { B07PW5 } \\
\text { B07NS9 } \\
\text { B07PX1 } \\
\text { B086P7 } \\
\text { B086P8 }\end{array}$ & $\begin{array}{l}100.00 \\
200.00 \\
100.00\end{array}$ & $\begin{array}{r}-14.30 \\
1.57 \\
-4.23 \\
-8.10\end{array}$ & 26000.00 & $\begin{array}{c}-.62 U \\
5.29 U \\
1.86 U \\
.46\end{array}$ \\
\hline
\end{tabular}

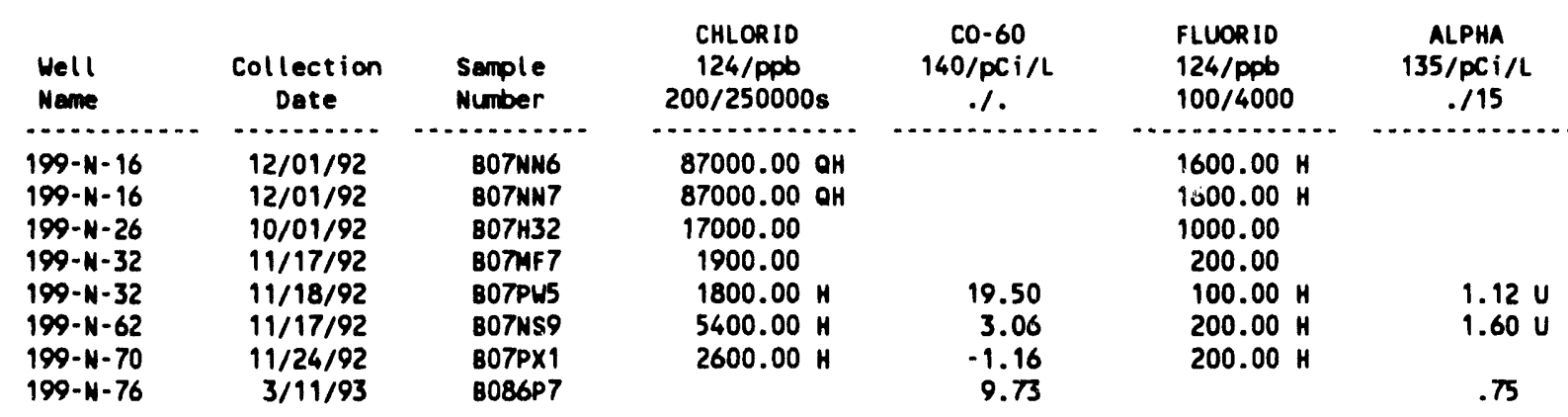

\begin{tabular}{|c|c|c|c|c|c|c|}
\hline $\begin{array}{l}\text { Well } \\
\text { Name }\end{array}$ & $\begin{array}{c}\text { Collection } \\
\text { Date }\end{array}$ & $\begin{array}{l}\text { Sample } \\
\text { Number }\end{array}$ & $\begin{array}{c}\text { BETA } \\
\text { 136/PCi/L } \\
. / 50\end{array}$ & $\begin{array}{c}\text { FMAGNES } \\
34 / \text { pPD } \\
100 \%\end{array}$ & $\begin{array}{c}\text { NITRATE } \\
\text { 124/ppb } \\
200 / 45000\end{array}$ & $\begin{array}{c}\text { FPOTASS } \\
34 / \text { ppb } \\
1000 \% .\end{array}$ \\
\hline $\begin{array}{l}199-N-16 \\
199-N-16 \\
199-N-26 \\
199-N-32 \\
199-N-32 \\
199-N-62 \\
199-N-70 \\
199-N-76\end{array}$ & $\begin{array}{c}12 / 01 / 92 \\
12 / 0192 \\
10 / 01 / 92 \\
11 / 17 / 92 \\
11 / 18 / 92 \\
11 / 17 / 92 \\
11 / 2.4 / 92 \\
3 / 11 / 93\end{array}$ & $\begin{array}{l}\text { B07NN6 } \\
\text { B07NA7 } \\
\text { B07H32 } \\
\text { B07NF7 } \\
\text { B07PW5 } \\
\text { B07NS9 } \\
\text { B07PX1 } \\
\text { B086P7 }\end{array}$ & $\begin{array}{r}23.00 \\
9.52 \\
196.00\end{array}$ & & $\begin{array}{l}22000.00 \mathrm{H} \\
22000.00 \mathrm{H} \\
64000.00 \\
30000.00 \\
30000.00 \mathrm{H} \\
18000.00 \mathrm{H} \\
13000.00 \mathrm{H}\end{array}$ & \\
\hline
\end{tabular}

$\begin{array}{llllll}199-N-76 & 3 / 11 / 93 & B 086 P 7 & 196.00 & 4100.00 & 1600.00 \\ 199-N-76 & 3 / 11 / 93 & B 086 P 8 & & 4100\end{array}$

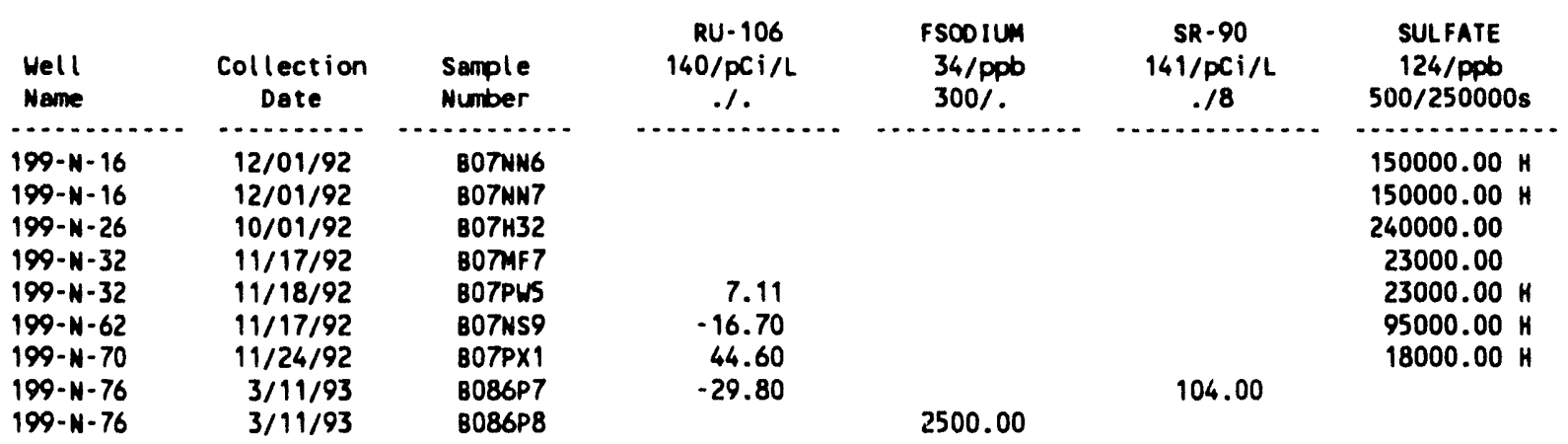




\section{DOE/RL-93-56-3}

Table A-2. Constituents with at Least One Value Above the CRQL for the 100-NR-2 Sites Data for Reporting Period October 1, 1992

through March 31, 1993. (sheet 2 of 2)

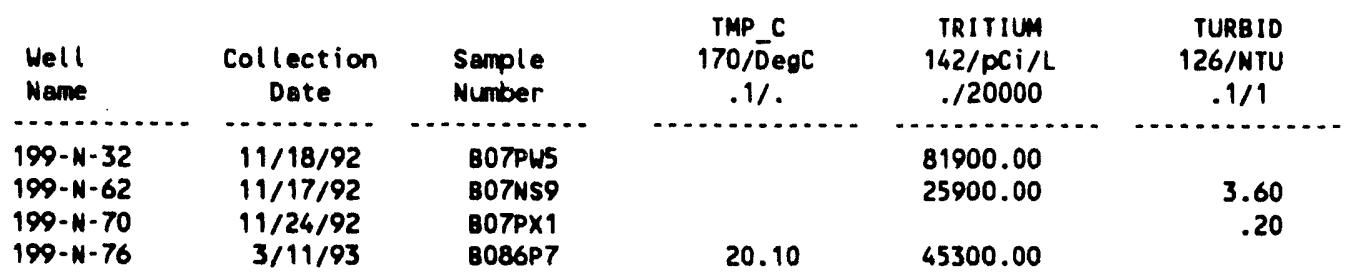

For explanation of this table, see Section 1.4 of report. 
Table A-3. Contamination Indicator Parameters for the 100-NR-2 Site Data for Reporting Period October 1, 1992 through March 31, 1993.

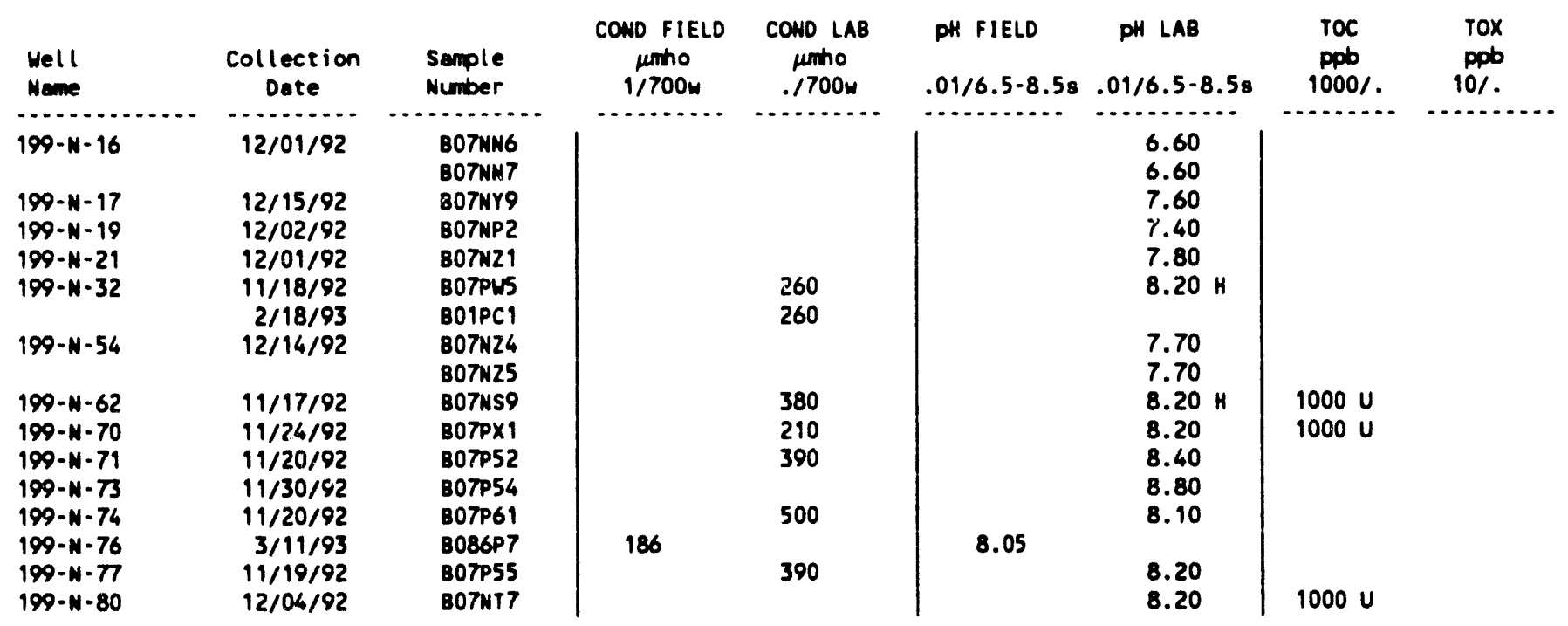

For explanation of this table, see section 1.4 of report. 
DOE/RL-93-56-3

This page intentionally left blank.

A-8 
DOE/RL-93-56-3

\section{DISTRIBUTION}

Number of copies

OFFSITE

2

Washington State Department of

Ecology - Kennewick Branch

7601 W. Clearwater, Suite 102

Kennewick, Washington 99336

J. Wallace

1

Westinghouse Savannah River Company

P. 0. Box A

Aiken, South Carolina 29801

R. C. Tuckfield

ONSITE

10

U.S. Department of Energy. Richland Operations office

R. D. Freeberg

M. J. Furman (4)

A5-19

J. M. Hennig

R3-80

R. G. McLeod

R3-80

R. P. Saget

A5-19

RL Public Reading Room (2)

A5-54

H2-53

4

Pacific Northwest Laboratory

R. W. Bryce

K6-96

B. E. Opitz

D. L. Stewart

K6-79

Technical Files

K6-96

$\mathrm{K} 1-11$

77

Westinghouse Hanford Company

$\begin{array}{ll}\text { M. R. Adams } & H 6-01 \\ \text { L. S. Angerman } & L 6-61 \\ \text { D. B. Barnett } & H 6-06 \\ \text { D. J. Brown } & B 4-63 \\ \text { R. W. Brown } & \mathrm{L} 6-79 \\ \text { W. R. Brown } & H 6-30 \\ \text { J. A. Caggiano } & H 6-06 \\ \text { C. J. Chou } & H 6-06 \\ \text { J. G. Coenenberg } & \mathrm{H6}-24\end{array}$ 
DOE/RL-93-56-3

DISTRIBUTION (continued)

Number of copies

ONSITE

Westinghouse Hanford Company (cont.)

J. J. Dorian

$\mathrm{H} 6-30$

G. C. Evans

H6-23

D. G. Farwick

$\mathrm{H} 4-16$

K. R. Fecht

H6-06

B. H. Ford

H6-06

M. J. Galgoul

H6-03

M. G. Gardner

N3-06

R. D. Gustavson

R1-51

W. M. Hayward

T7-05

F. N. Hodges

H6-06

D. J. Hoff

H6-23

D. G. Horton

H6-06

M. C. Hughes

$\times 5-55$

L. C. Hulstrom

H6-03

R. L. Jackson

H6-06

V. G. Johnson

H6-06

J. C. Johnston

H6-06

D. K. Jungers (10)

H6-06

J. R. Kasper

R2-54

G. L. Kasza

H6-06

A. J. Knepp

H6-06

R. J. Landon

H6-21

A. G. Law

H6-06

L. D. Lockard

S3-90

M. M. McCarthy

N3-13

A. K. McDowell

R1-51

R. B. Mercer

H6- 06

M. A. Mihalic

$\times 5-55$

D. L. Parker

H6-03

R. D. Pierce

H5-11

D. A. Pratt

T4-03

S. M. Price

$\mathrm{H} 6-23$

W. H. Price

N3-05

W. A. Retterer

S2-37

F. A. Ruck III

H6-23

J. T. Sams

J. S. Schmid

H5-14

J. A. Serkowski

H6-06

R. R. Thompson

H6-06

E. C. Thornton

$\mathrm{H} 6-32$

M. J. Van Vleet

H6-06

D. J. Watson

H6-31

R. K. Welty

$X 0-41$

B. A. Williams

$\mathrm{R} 1-80$

H6-06 
DOE/RL-93-56-3

\section{DISTRIBUTION (continued)}

Number of copies

ONSITE

Westinghouse Hanford Company (cont.)

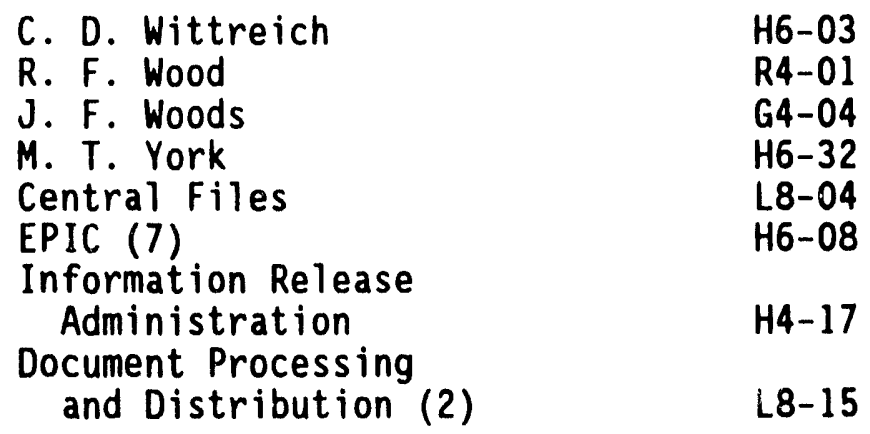

Distr-3 
$\Gamma$
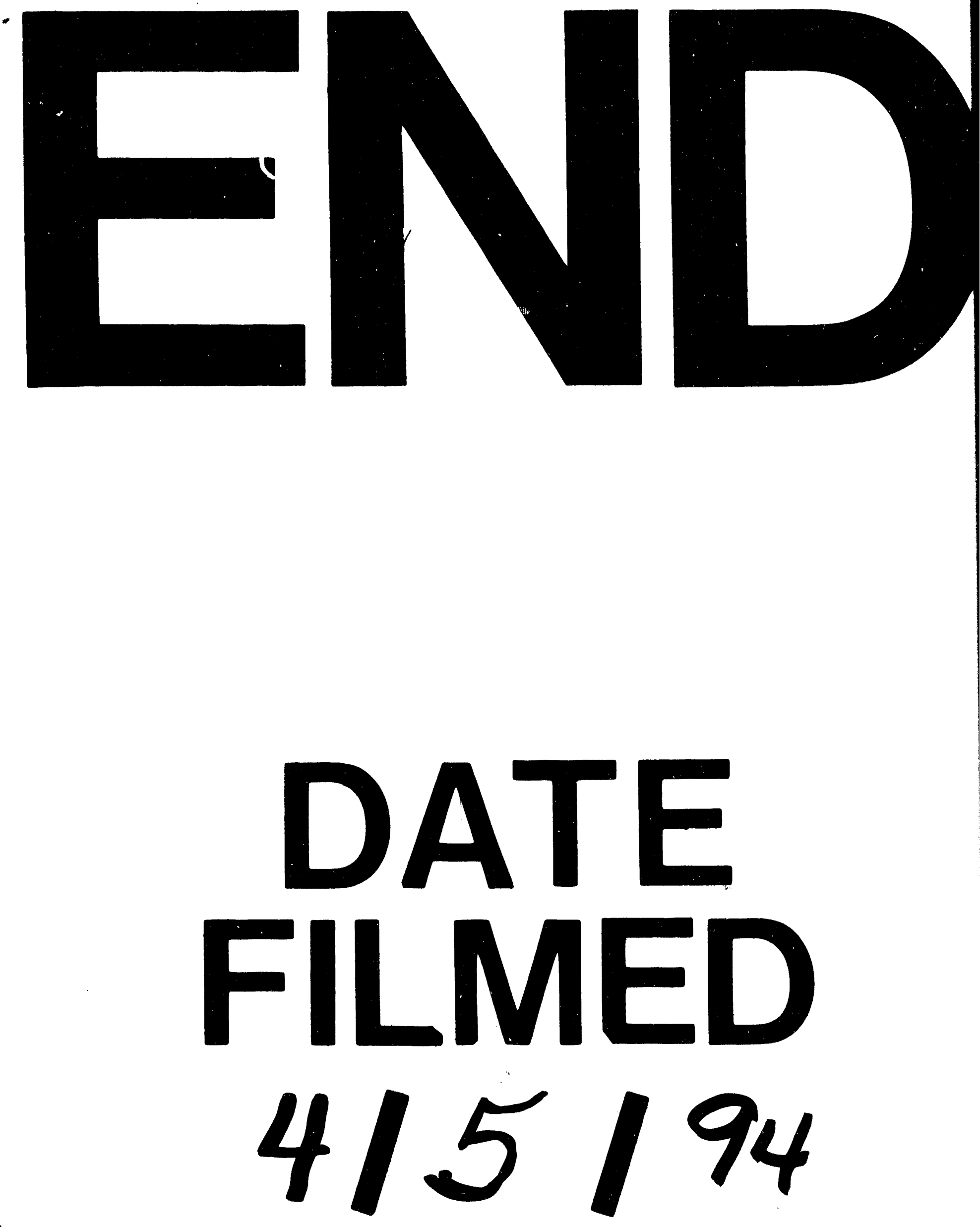
